ORNL/TM-2001/238

\title{
DEVELOPMENT OF IMPROVED BURNABLE POISONS FOR COMMERCIAL NUCLEAR POWER REACTORS
}

\section{October 2001}

J.-P. A. Renier and M. L. Grossbeck 


\section{DOCUMENT AVAILABILITY}

Reports produced after January 1,1996, are generally available free via the U.S. Department of Energy (DOE) Information Bridge:

Web site: http://www.osti.gov/bridge

Reports produced before January 1, 1996, may be purchased by members of the public from the following source:

National Technical Information Service

5285 Port Royal Road

Springfield, VA 22161

Telephone: 703-605-6000 (1-800-553-6847)

TDD: $703-487-4639$

Fax: 703-605-6900

E-mail: info@ntis.fedworld.gov

Web site: http://www.ntis.gov/support/ordernowabout.htm

Reports are available to DOE employees, DOE contractors, Energy Technology Data Exchange (ETDE) representatives, and International Nuclear Information System (INIS) representatives from the following source:

Office of Scientific and Technical Information

P.O. Box 62

Oak Ridge, TN 37831

Telephone: $865-576-8401$

Fax: 865-576-5728

E-mail: reports@adonis.osti.gov

Web site: http://www.osti.gov/contact.html

This report was prepared as an account of work sponsored by an agency of the United States government. Neither the United States government nor any agency thereof, nor any of their employees, makes any warranty, express or implied, or assumes any legal liability or responsibility for the accuracy, completeness, or usefulness of any information, apparatus, product, or process disclosed, or represents that its use would not infringe privately owned rights. Reference herein to any specific commercial product, process, or service by trade name, trademark, manufacturer, or otherwise, does not necessarily constitute or imply its endorsement, recommendation, or favoring by the United States government or any agency thereof. The views and opinions of authors expressed herein do not necessarily state or reflect those of the United States government or any agency thereof. 
ORNL/TM-2001/238

\title{
DEVELOPMENT OF IMPROVED BURNABLE POISONS FOR COMMERCIAL NUCLEAR POWER REACTORS
}

\author{
Report on Phase 1 of NERI Project Number 99-0074 \\ Identification of Candidate Burnable Poison Isotopes and Configurations
}

J.-P. A. Renier, Nuclear Science and Technology Division

M. L. Grossbeck, Metals and Ceramics Division

October 2001

Prepared by the

OAK RIDGE NATIONAL LABORATORY

Oak Ridge, Tennessee 37831-6285

managed by

UT-BATTELLE, LLC

for the

U.S. DEPARTMENT OF ENERGY

under contract DE-AC05-00OR22725 



\section{CONTENTS}

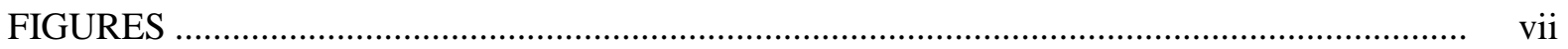

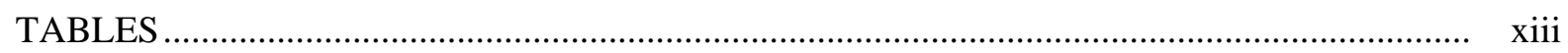

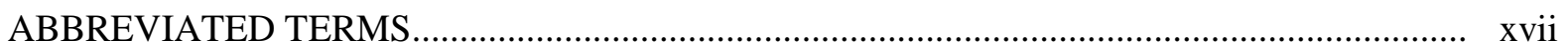

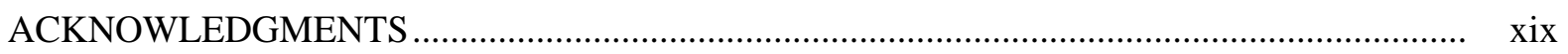

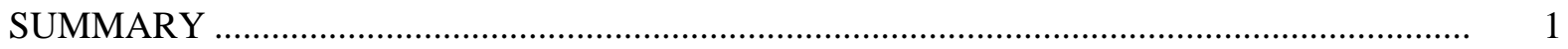

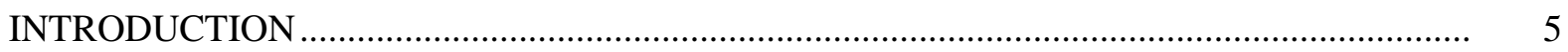

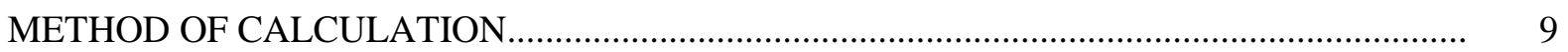

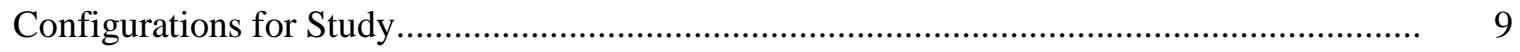

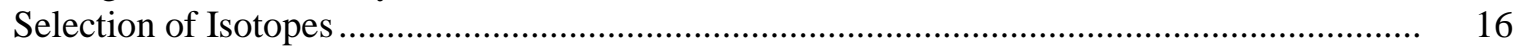

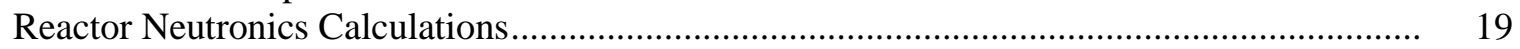

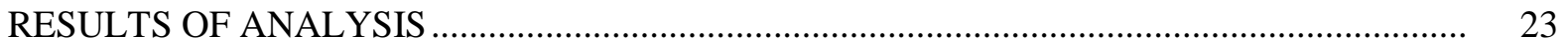

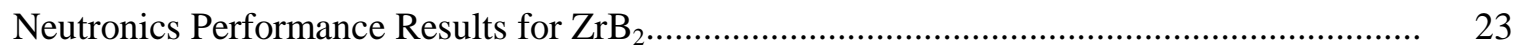

Neutronics Performance Results for Gadolinium ............................................................ 40

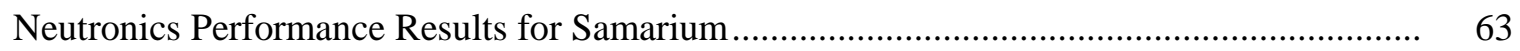

Neutronics Performance Results for Erbium .................................................................. 87

Neutronics Performance Results for Dysprosium......................................................... 107

Neutronics Performance Results for Europium …........................................................... 125

Neutronics Performance Results for Hafnium ............................................................. 140

Neutronics Performance Results for Other Elements ..................................................... 152

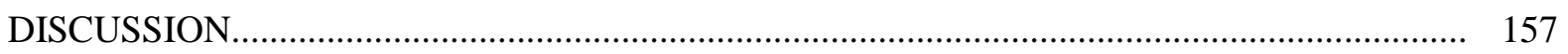

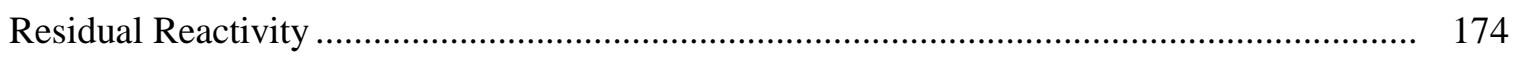

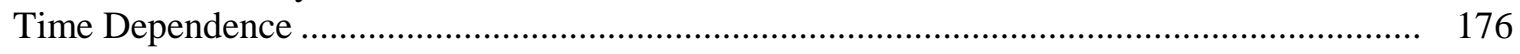

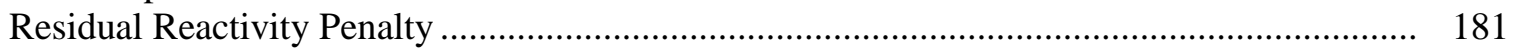

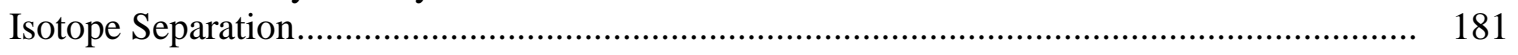

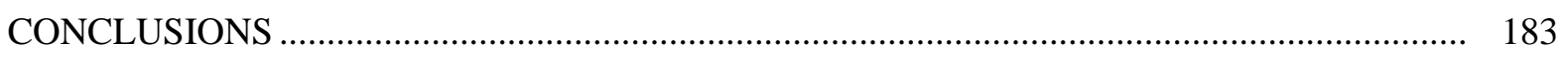

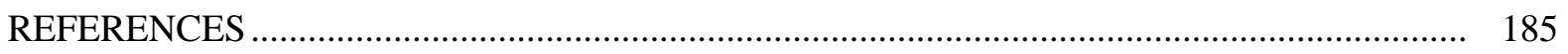

\section{APPENDIXES}

Appendix A1. Reactivity Worths and Masses of $\mathrm{Gd}_{2} \mathrm{O}_{3}$ Burnable Poison

Homogeneously Mixed in the Fuel Pellets ......................................................................... A-1

- Gadolinium with Natural Isotopic Abundances ........................................................ A-3

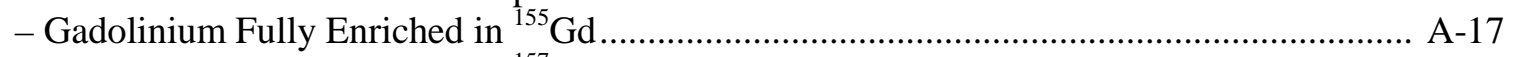

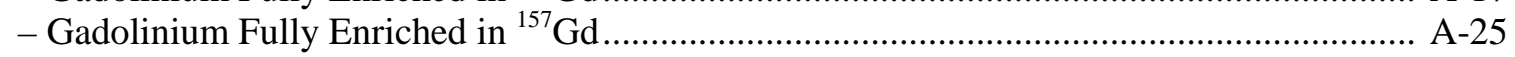


Appendix A2. Reactivity Worths and Masses of $\mathrm{Gd}_{2} \mathrm{O}_{3}$ Burnable Poison

Homogeneously Mixed in the Outer One-Third Volume of the Fuel Pellets .......................... A-39

- Gadolinium with Natural Isotopic Abundances ............................................................ A-41

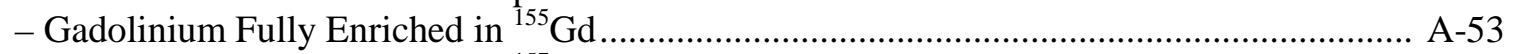

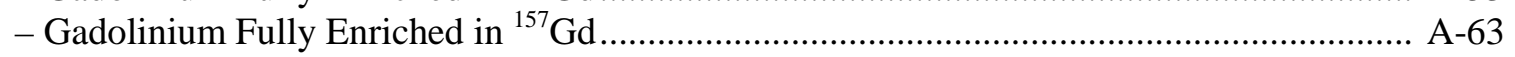

Appendix A3. Reactivity Worths and Masses of $\mathrm{Gd}_{2} \mathrm{O}_{3}$ Coating

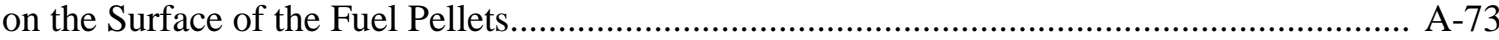

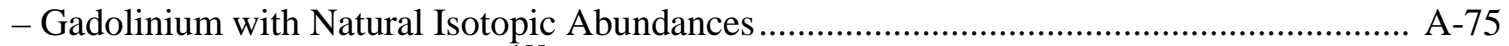

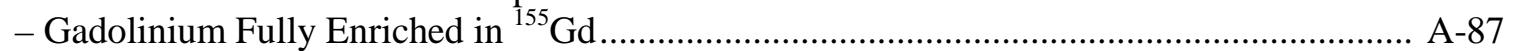

- Gadolinium Fully Enriched in ${ }^{157} \mathrm{Gd}$............................................................... A-9 A

Appendix A4. Reactivity Worths and Masses of Gadolinium Alloyed with the Zircaloy Cladding of Fuel Rods............................................................................... A-113

- Gadolinium with Natural Isotopic Abundances .................................................................. A-115

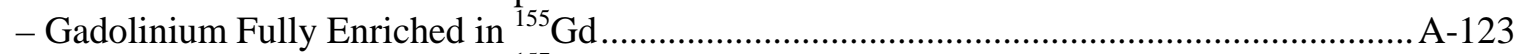

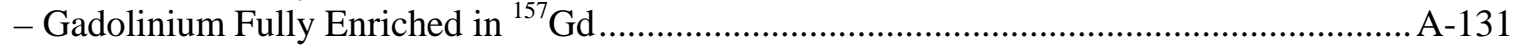

Appendix B1. Reactivity Worths and Masses of $\mathrm{Sm}_{2} \mathrm{O}_{3}$ Burnable Poison

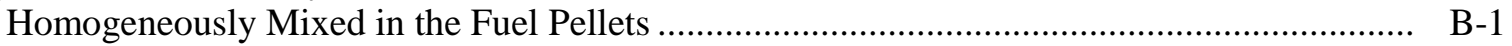

- Samarium with Natural Isotopic Abundances................................................................ B-3

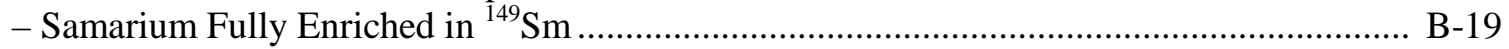

Appendix B2. Reactivity Worths and Masses of $\mathrm{Sm}_{2} \mathrm{O}_{3}$ Burnable Poison

Homogeneously Mixed in the Outer One-Third Volume of the Fuel Pellets ........................... B-37

- Samarium with Natural Isotopic Abundances...................................................................... B-39

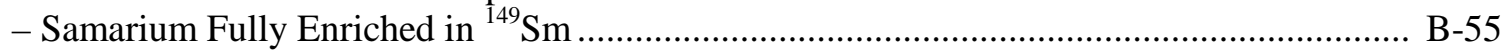

Appendix B3. Reactivity Worths and Masses of $\mathrm{Sm}_{2} \mathrm{O}_{3}$ Coating

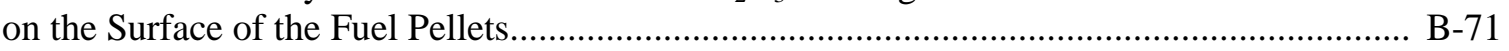

- Samarium with Natural Isotopic Abundances................................................................... B-73

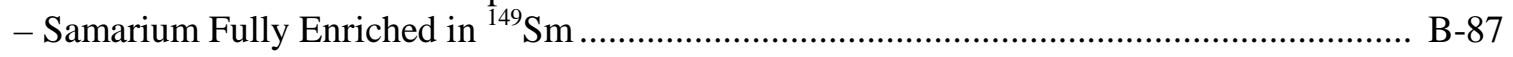

Appendix B4. Reactivity Worths and Masses of Samarium Alloyed

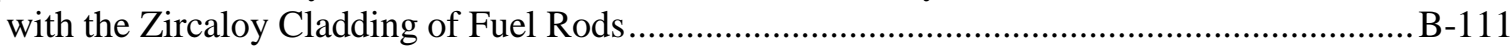

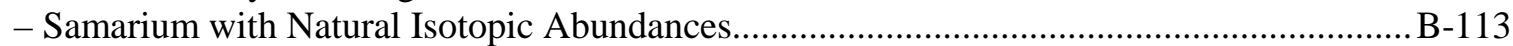

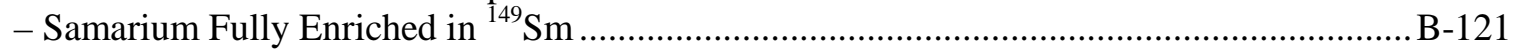

Appendix C1. Reactivity Worths and Masses of $\mathrm{Er}_{2} \mathrm{O}_{3}$ Burnable Poison

Homogeneously Mixed in the Fuel Pellets ..................................................................... C-1

- Erbium with Natural Isotopic Abundances ................................................................... C-3

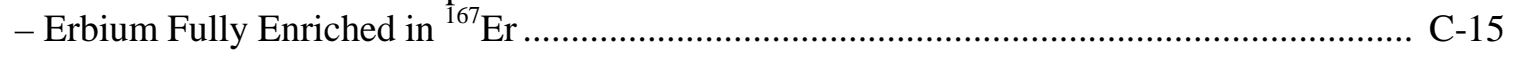

Appendix C2. Reactivity Worths and Masses of $\operatorname{Er}_{2} \mathrm{O}_{3}$ Burnable Poison

Homogeneously Mixed in the Outer One-Third Volume of the Fuel Pellets .......................... C-27

- Erbium with Natural Isotopic Abundances ......................................................................... C-29

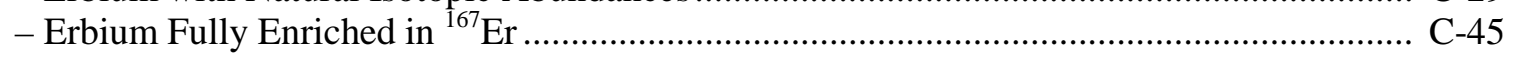


Appendix C3. Reactivity Worths and Masses of $\mathrm{Er}_{2} \mathrm{O}_{3}$ Coating

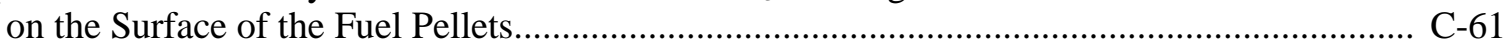

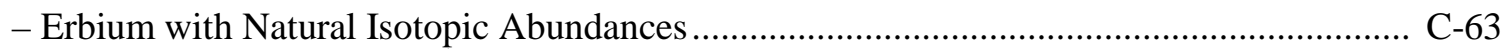

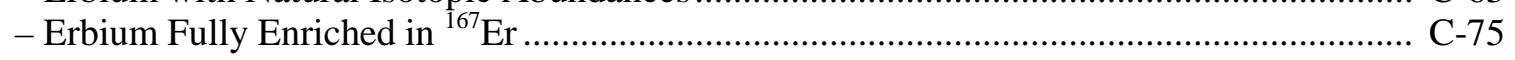

Appendix C4. Reactivity Worths and Masses of Erbium Alloyed

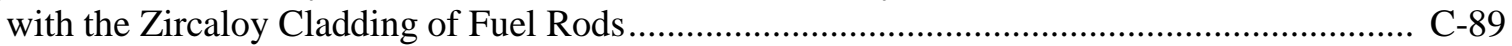

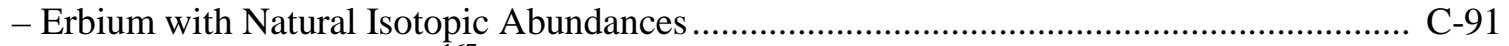

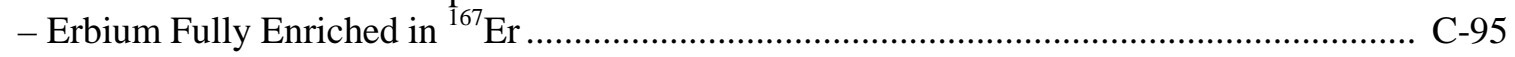

Appendix D1. Reactivity Worths and Masses of $\mathrm{Dy}_{2} \mathrm{O}_{3}$ Burnable Poison

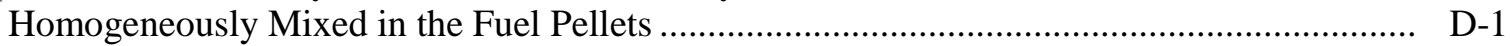

- Dysprosium with Natural Isotopic Abundances ........................................................... D-3

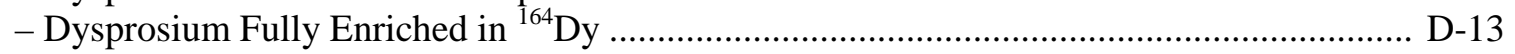

Appendix D2. Reactivity Worths and Masses of $\mathrm{Dy}_{2} \mathrm{O}_{3}$ Burnable Poison

Homogeneously Mixed in the Outer One-Third Volume of the Fuel Pellets .......................... D-23

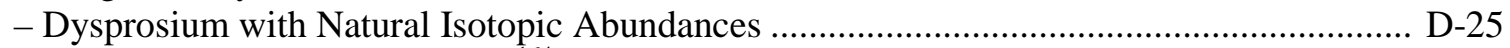

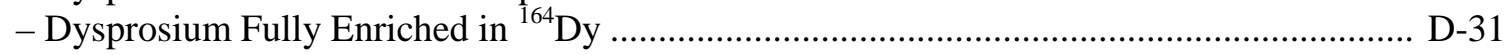

Appendix D3. Reactivity Worths and Masses of $\mathrm{Dy}_{2} \mathrm{O}_{3}$ Coating

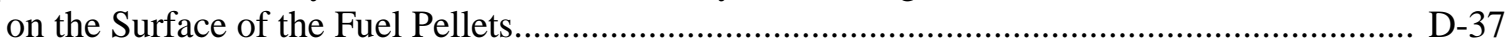

- Dysprosium with Natural Isotopic Abundances .............................................................. D-39

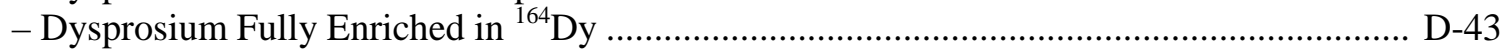

Appendix D4. Reactivity Worths and Masses of Dysprosium Alloyed

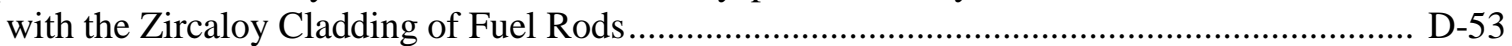

- Dysprosium with Natural Isotopic Abundances .............................................................. D-55

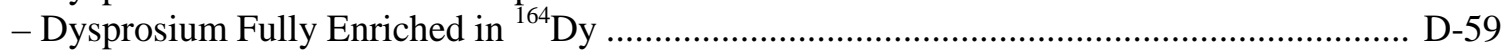

Appendix E1. Reactivity Worths and Masses of $\mathrm{Eu}_{2} \mathrm{O}_{3}$ Burnable Poison

Homogeneously Mixed in the Fuel Pellets ................................................................... E-1

- Europium with Natural Isotopic Abundances ............................................................. E-3

- Europium Fully Enriched in ${ }^{151} \mathrm{Eu}$................................................................. E-7

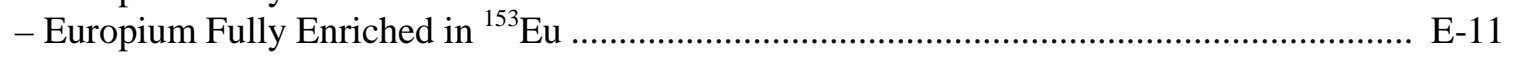

Appendix E2. Reactivity Worths and Masses of $\mathrm{Eu}_{2} \mathrm{O}_{3}$ Burnable Poison

Homogeneously Mixed in the Outer One-Third Volume of the Fuel Pellets ......................... E-15

- Europium with Natural Isotopic Abundances ................................................................... E-17

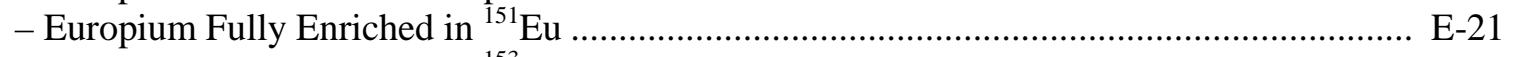

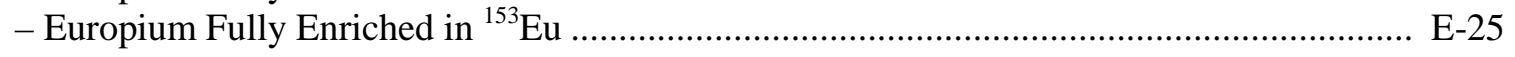

Appendix E3. Reactivity Worths and Masses of $\mathrm{Eu}_{2} \mathrm{O}_{3}$ Coating

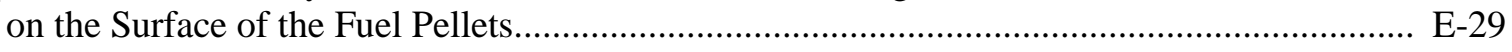

- Europium with Natural Isotopic Abundances .................................................................. E-31

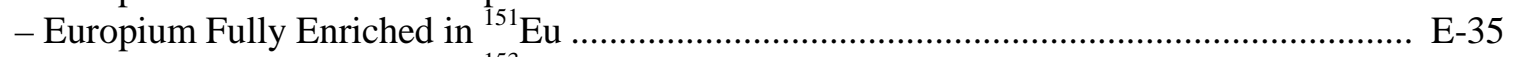

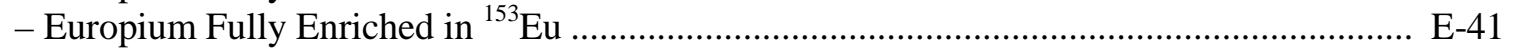

Appendix E4. Reactivity Worths and Masses of Europium Alloyed

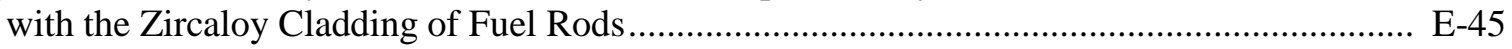

- Europium with Natural Isotopic Abundances ................................................................. E-47

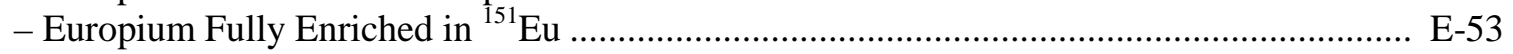


Appendix F1. Reactivity Worths and Masses of $\mathrm{HfO}_{2}$ Burnable Poison

Homogeneously Mixed in the Fuel Pellets ......................................................................... F-1

- Hafnium with Natural Isotopic Abundances................................................................... F-3

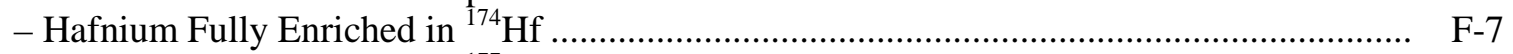

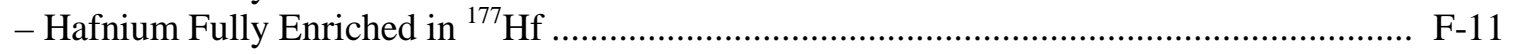

Appendix F2. Reactivity Worths and Masses of $\mathrm{HfO}_{2}$ Burnable Poison

Homogeneously Mixed in the Outer One-third Volume of the Fuel Pellets............................ F-15

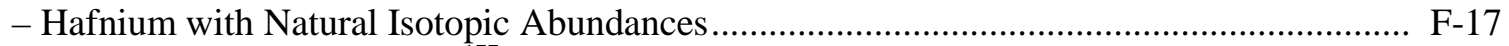

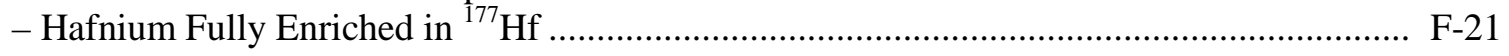

Appendix F3. Reactivity Worths and Masses of $\mathrm{HfO}_{2}$ Coating

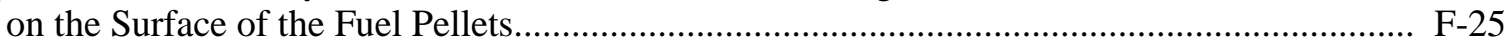

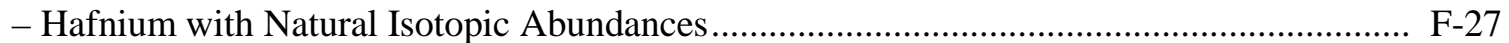

- Hafnium Fully Enriched in ${ }^{177} \mathrm{Hf}$.............................................................................. F-35

Appendix F4. Reactivity Worths and Masses of Hafnium Alloyed

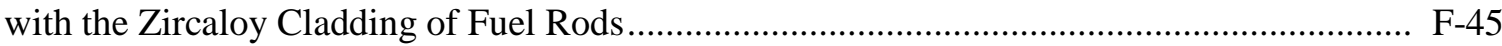

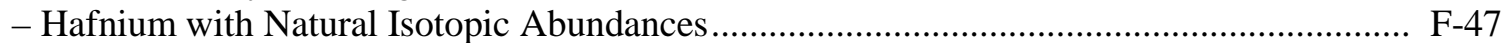

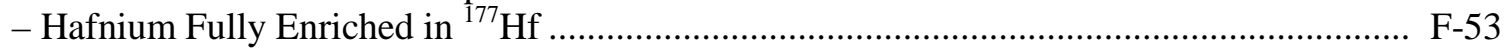

Appendix G1. Reactivity Worths and Masses of $\mathrm{Lu}_{2} \mathrm{O}_{3}$ Coating

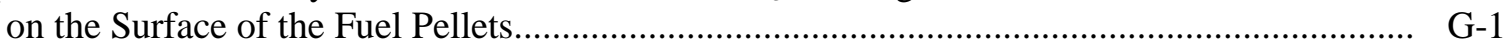

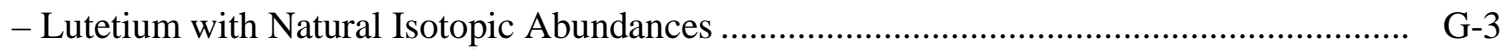

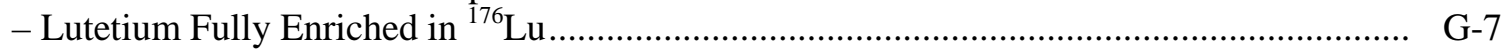




\section{FIGURES}

1 Savings in full power days at the end of a 4 year fuel cycle achieved by using

the single isotopes specified rather than naturally occurring elements

2 Percentage of burnable poison burned at the end of 120 days, 1 year, and

4 years for naturally occurring elements mixed in the outer third of the fuel

rod volume

3 Percentage of burnable poison burned at the end of 120 days, 1 year, and

4 years for single isotopes mixed in the outer third of the fuel rod volume

4 Diagram showing the model of a standard $17 \times 17$ fuel assembly layout

5 Diagram showing the model of a standard $17 \times 17$ fuel assembly layout with 8 non-fueled burnable poison rods

6 Diagram showing the model of a standard $17 \times 17$ fuel assembly layout with 16 non-fueled burnable poison rods

7 Diagram showing the model of a standard $17 \times 17$ fuel assembly layout with 8 integral fuel burnable absorbers

8 Diagram showing the model of a standard $17 \times 17$ fuel assembly layout with 16 integral fuel burnable absorbers

8a Diagram showing the model of a standard $17 \times 17$ fuel assembly layout with 18 integral fuel burnable absorbers

8b Diagram showing the model of a standard $17 \times 17$ fuel assembly layout with 20 integral fuel burnable absorbers

9 Diagram showing the model of a standard $17 \times 17$ fuel assembly layout with 64 integral fuel burnable absorbers

10 Diagram showing the model of a standard $17 \times 17$ fuel assembly layout with 104 integral fuel burnable absorbers

11 Macroscopic cross section of Gd isotopes produced by irradiating naturally occurring Gd

12 Macroscopic cross section of Gd isotopes formed by irradiating Gd with

${ }^{154} \mathrm{Gd}$ and ${ }^{156} \mathrm{Gd}$ initially removed

13 Macroscopic cross section as a function of time for isotopes of samarium produced by irradiation of natural $\mathrm{Sm}$.

14 Macroscopic cross section for samarium isotopes as a function of time produced by irradiation of pure ${ }^{149} \mathrm{Sm}$ 
15 Negative reactivity and masses of BP for B-nat and transmutation daughters as a function of fuel life for $17 \times 17$ fuel assemblies with 104 poison rods, 1.5-mil-thick $\mathrm{ZrB}_{2}$ poison coating on the outer radial surface of the $\mathrm{UO}_{2}$ pellets

16 Negative reactivity and masses of BP for $30 \mathrm{wt} \%$ boron and transmutation daughters as a function of fuel life for $17 \times 17$ fuel assemblies with 104 poison rods, 1.5-mil-thick $\mathrm{ZrB}_{2}$ poison coating on the outer radial surface of the $\mathrm{UO}_{2}$ pellets

17 Negative reactivity and masses of BP for $30 \mathrm{wt} \%$ boron and transmutation daughters as a function of fuel life for $17 \times 17$ fuel assemblies with 64 poison rods, 1.5-mil-thick $\mathrm{ZrB}_{2}$ poison coating on the outer radial surface of the $\mathrm{UO}_{2}$ pellets

18 Negative reactivity and masses of $\mathrm{BP}$ for $30 \mathrm{wt} \%$ boron and transmutation daughters as a function of fuel life for $17 \times 17$ fuel assemblies with 64 poison rods, 2.0-mil-thick $\mathrm{ZrB}_{2}$ poison coating on the outer radial surface of the $\mathrm{UO}_{2}$ pellets

19 Negative reactivity and masses of BP for $30 \mathrm{wt} \%$ boron and transmutation daughters as a function of fuel life for $17 \times 17$ fuel assemblies with 18 poison rods, 1.5-mil-thick $\mathrm{ZrB}_{2}$ poison coating on the outer radial surface of the $\mathrm{UO}_{2}$ pellets

20 Masses of actinides for $30 \mathrm{wt} \%$ boron and transmutation daughters as a function of fuel life for $17 \times 17$ fuel assemblies with 104 poison rods, 1.5 -mil-thick $\mathrm{ZrB}_{2}$ poison coating on the outer radial surface of the $\mathrm{UO}_{2}$ pellets

21 Neutron cross sections as a function of energy, Gd

22 Neutron cross sections as a function of energy, $\mathrm{Tb}$

23 Negative reactivity and masses of BP for Gd-nat and transmutation daughters as a function of fuel life for $17 \times 17$ fuel assemblies with 16 poison rods, $4.0 \mathrm{wt} \%$ $\mathrm{Gd}_{2} \mathrm{O}_{3}$ poison homogeneously mixed in the $\mathrm{UO}_{2}$ pellets

24 Negative reactivity and masses of BP for Gd-nat and transmutation daughters as a function of fuel life for $17 \times 17$ fuel assemblies with 16 poison rods, $8.0 \mathrm{wt} \%$ $\mathrm{Gd}_{2} \mathrm{O}_{3}$ poison homogeneously mixed in the $\mathrm{UO}_{2}$ pellets

25 Negative reactivity and masses of BP for ${ }^{155} \mathrm{Gd}$ and transmutation daughters as a function of fuel life for $17 \times 17$ fuel assemblies with 16 poison rods, $1.0 \mathrm{wt} \%$ $\mathrm{Gd}_{2} \mathrm{O}_{3}$ poison homogeneously mixed in the $\mathrm{UO}_{2}$ pellets

26 Negative reactivity and masses of BP for ${ }^{155} \mathrm{Gd}$ and transmutation daughters as a function of fuel life for $17 \times 17$ fuel assemblies with 16 poison rods, $2.0 \mathrm{wt} \%$ $\mathrm{Gd}_{2} \mathrm{O}_{3}$ poison homogeneously mixed in the $\mathrm{UO}_{2}$ pellets

27 Negative reactivity and masses of BP for ${ }^{157} \mathrm{Gd}$ and transmutation daughters as a function of fuel life for $17 \times 17$ fuel assemblies with 16 poison rods, $0.5 \mathrm{wt} \%$ $\mathrm{Gd}_{2} \mathrm{O}_{3}$ poison homogeneously mixed in the $\mathrm{UO}_{2}$ pellets

28 Negative reactivity and masses of BP for ${ }^{157} \mathrm{Gd}$ and transmutation daughters as a function of fuel life for $17 \times 17$ fuel assemblies with 16 poison rods, $1.0 \mathrm{wt} \%$ $\mathrm{Gd}_{2} \mathrm{O}_{3}$ poison homogeneously mixed in the $\mathrm{UO}_{2}$ pellets 
29 Negative reactivity and masses of BP for ${ }^{157} \mathrm{Gd}$ and transmutation daughters as a function of fuel life for $17 \times 17$ fuel assemblies with 16 poison rods, $2.0 \mathrm{wt} \%$ $\mathrm{Gd}_{2} \mathrm{O}_{3}$ poison homogeneously mixed in the $\mathrm{UO}_{2}$ pellets

30 Negative reactivity and masses of BP for ${ }^{157} \mathrm{Gd}$ and transmutation daughters as a function of fuel life for $17 \times 17$ fuel assemblies with 16 poison rods, $4.0 \mathrm{wt} \%$ $\mathrm{Gd}_{2} \mathrm{O}_{3}$ poison homogeneously mixed in the $\mathrm{UO}_{2}$ pellets

31 Burnable poison initial reactivity worth as a function of FPD penalty-

Gd in 16 IFBA rods

32 Burnable poison initial reactivity worth as a function of FPD penalty-

Gd in 64 IFBA rods

33 Neutron cross sections as a function of energy, $\mathrm{Sm}$

34 Neutron cross sections as a function of energy, Eu

35 Negative reactivity and masses of BP for Sm-nat and transmutation daughters as a function of fuel life for $17 \times 17$ fuel assemblies with 16 poison rods, $4.0 \mathrm{wt} \%$ $\mathrm{Sm}_{2} \mathrm{O}_{3}$ poison homogeneously mixed in the $\mathrm{UO}_{2}$ pellets .

36 Negative reactivity and masses of BP for Sm-nat and transmutation daughters as a function of fuel life for $17 \times 17$ fuel assemblies with 16 poison rods, $8.0 \mathrm{wt} \%$ $\mathrm{Sm}_{2} \mathrm{O}_{3}$ poison homogeneously mixed in the $\mathrm{UO}_{2}$ pellets

37 Negative reactivity and masses of BP for Sm-149 and transmutation daughters as a function of fuel life for $17 \times 17$ fuel assemblies with 16 poison rods, $1.0 \mathrm{wt} \%$ $\mathrm{Sm}_{2} \mathrm{O}_{3}$ poison homogeneously mixed in the $\mathrm{UO}_{2}$ pellets

38 Negative reactivity and masses of BP for Sm-149 and transmutation daughters as a function of fuel life for $17 \times 17$ fuel assemblies with 16 poison rods, $2.0 \mathrm{wt} \%$ $\mathrm{Sm}_{2} \mathrm{O}_{3}$ poison homogeneously mixed in the $\mathrm{UO}_{2}$ pellets

39 Negative reactivity and masses of BP for Sm-149 and transmutation daughters as a function of fuel life for $17 \times 17$ fuel assemblies with 16 poison rods, $4.0 \mathrm{wt} \%$ $\mathrm{Sm}_{2} \mathrm{O}_{3}$ poison homogeneously mixed in the $\mathrm{UO}_{2}$ pellets

40 Burnable poison initial reactivity worth as a function of FPD penaltySm in 16 IFBA rods.

41 Burnable poison initial reactivity worth as a function of FPD penaltySm in 64 IFBA rods.

42 Neutron cross sections as a function of energy, Er

43 Neutron cross sections as a function of energy, Ho

44 Neutron cross sections as a function of energy, Tm 
45 Negative reactivity and masses of BP for Er-nat and transmutation daughters as a function of fuel life for $17 \times 17$ fuel assemblies with 64 poison rods, $4.0 \mathrm{wt} \%$ $\mathrm{Er}_{2} \mathrm{O}_{3}$ poison homogeneously mixed in the $\mathrm{UO}_{2}$ pellets.

46 Negative reactivity and masses of BP for ${ }^{167} \mathrm{Er}$ and transmutation daughters as a function of fuel life for $17 \times 17$ fuel assemblies with 64 poison rods, $1.0 \mathrm{wt} \%$ $\mathrm{Er}_{2} \mathrm{O}_{3}$ poison homogeneously mixed in the $\mathrm{UO}_{2}$ pellets.

47 Burnable poison initial reactivity worth as a function of FPD penalty-

Er in 16 IFBA rods

48 Burnable poison initial reactivity worth as a function of FPD penalty-

Er in 64 IFBA rods

49 Neutron cross sections as a function of energy, Dy

50 Neutron cross sections as a function of energy, Ho

51 Negative reactivity and masses of BP for Dy-nat and transmutation daughters as a function of fuel life for $17 \times 17$ fuel assemblies with 16 poison rods, $4.0 \mathrm{wt} \%$ $\mathrm{Dy}_{2} \mathrm{O}_{3}$ poison homogeneously mixed in the $\mathrm{UO}_{2}$ pellets ......

52 Negative reactivity and masses of BP for ${ }^{164} \mathrm{Dy}$ and transmutation daughters as a function of fuel life for $17 \times 17$ fuel assemblies with 16 poison rods, $4.0 \mathrm{wt} \%$ $\mathrm{Dy}_{2} \mathrm{O}_{3}$ poison homogeneously mixed in the $\mathrm{UO}_{2}$ pellets

53 Negative reactivity and masses of BP for ${ }^{164}$ Dy and transmutation daughters as a function of fuel life for $17 \times 17$ fuel assemblies with 64 poison rods, $1.0 \mathrm{wt} \%$ $\mathrm{Dy}_{2} \mathrm{O}_{3}$ poison homogeneously mixed in the $\mathrm{UO}_{2}$ pellets

54 Negative reactivity and masses of BP for ${ }^{164}$ Dy and transmutation daughters as a function of fuel life for $17 \times 17$ fuel assemblies with 104 poison rods, 0.5 -mil-thick poison coating on the outer radial surface of the $\mathrm{UO}_{2}$ pellets

55 Burnable poison initial reactivity worth as a function of FPD penaltyDy in 64 IFBA rods

56 Neutron cross sections as a function of energy, Eu

57 Negative reactivity and masses of $\mathrm{BP}$ for Eu-nat and transmutation daughters as a function of fuel life for $17 \times 17$ fuel assemblies with 64 poison rods, $2.0 \mathrm{wt} \%$ Eu poison homogeneously mixed in the fuel cladding....

58 Negative reactivity and masses of BP for ${ }^{151} \mathrm{Eu}$ and transmutation daughters as a function of fuel life for $17 \times 17$ fuel assemblies with 64 poison rods, $1.0 \mathrm{wt} \%$ $\mathrm{Eu}$ poison homogeneously mixed in the fuel cladding....

59 Negative reactivity and masses of BP for ${ }^{151} \mathrm{Eu}$ and transmutation daughters as a function of fuel life for $17 \times 17$ fuel assemblies with 104 poison rods, $0.5 \mathrm{wt} \%$ Eu poison homogeneously mixed in the fuel cladding.... 
60 Negative reactivity and masses of BP for ${ }^{151} \mathrm{Eu}$ and transmutation daughters as a function of fuel life for $17 \times 17$ fuel assemblies with 64 poison rods, 0.2-milthick $\mathrm{Eu}_{2} \mathrm{O}_{3}$ poison coating on the outer radial surface of the $\mathrm{UO}_{2}$ pellets

61 Neutron cross sections as a function of energy, $\mathrm{Hf}$

62 Negative reactivity and masses of BP for ${ }^{177} \mathrm{Hf}$ and transmutation daughters as a function of fuel life for $17 \times 17$ fuel assemblies with 64 poison rods, 1.0-milthick $\mathrm{HfO}_{2}$ poison coating on the outer radial surface of the $\mathrm{UO}_{2}$ pellets

63 Negative reactivity and masses of BP for ${ }^{176} \mathrm{Lu}$ and transmutation daughters as a function of fuel life for $17 \times 17$ fuel assemblies with 64 poison rods, 0.5 -milthick $\mathrm{Lu}_{2} \mathrm{O}_{3}$ poison coating on the outer radial surface of the $\mathrm{UO}_{2}$ pellets

64 Negative reactivity and masses of BP for ${ }^{176} \mathrm{Lu}$ and transmutation daughters as a function of fuel life for $17 \times 17$ fuel assemblies with 64 poison rods, 1.0-milthick $\mathrm{Lu}_{2} \mathrm{O}_{3}$ poison coating on the outer radial surface of the $\mathrm{UO}_{2}$ pellets.

65 Ratio of negative reactivity due to burnable poison (BP) at the end of fuel life to that at the beginning of fuel life for various configurations of the BP....

66 Isotope separation benefit shown by the compound ratio of negative reactivity due to the burnable poison (BP) at end of life to that at beginning of life for a single isotope to a similar ratio for the corresponding natural element

67 Burn rate for naturally occurring element burnable poisons homogeneously mixed in the fuel as oxides shown as a percentage of the initial negative reactivity remaining at a given time.....

68 Burn rate for single burnable poisons homogeneously mixed in the fuel as oxides shown as a percentage of the initial negative reactivity remaining at a given time

69 Burn rate for naturally occurring element burnable poisons homogeneously mixed into the outer one-third of the fuel pellets shown as a percentage of the initial negative reactivity remaining at a given time

70 Burn rate for single burnable poisons homogeneously mixed into the outer one-third of the fuel pellets shown as a percentage of the initial negative reactivity remaining at a given time

71 Burn rate for naturally occurring element burnable poisons coated on the outer surface of the fuel pellets as oxides, shown as a percentage of the initial negative reactivity remaining at a given time

72 Burn rate for single burnable poisons coated on the outer surface of the fuel pellets as oxides, shown as a percentage of the initial negative reactivity remaining at a given time 
73 Burn rate for naturally occurring element burnable poisons in the form of a metal alloyed in the Zircaloy cladding, shown as a percentage of the initial negative reactivity remaining at a given time.....

74 Burn rate for single burnable poisons in the form of a metal alloyed in the Zircaloy cladding, shown as a percentage of the initial negative reactivity remaining at a given time 


\section{TABLES}

1 Boron burnable poison in a $\mathrm{ZrB}_{2}$ coating between the $\mathrm{UO}_{2}$ fuel pellet and the $\mathrm{Zr}-4$ cladding of selected fuel rods-Summary results

2 Reactivity worths of boron burnable poison isotopes in the $\mathrm{ZrB}_{2}$ coating of $\mathrm{UO}_{2}$ fuel pellets in 104 Fuel rods-Enrichment: $30 \mathrm{wt} \%{ }^{10} \mathrm{~B}$, initial loading: 30.7 grams ${ }^{10} \mathrm{~B}$.

3 Masses of boron burnable poison isotopes in the $\mathrm{ZrB}_{2}$ coating of $\mathrm{UO}_{2}$ fuel pellets in 104 fuel rods-Enrichment: $30 \mathrm{wt} \%{ }^{10} \mathrm{~B}$, initial loading: 30.7 grams ${ }^{10} \mathrm{~B}$

4a Relative fission power density map at $1 \mathrm{FPD}-\mathrm{ZrB}_{2}$ coating of the $\mathrm{UO}_{2}$ fuel pellets in 104 fuel rods, enrichment: $30 \mathrm{wt} \%{ }^{10} \mathrm{~B}$, initial loading: 30.7 grams ${ }^{10} \mathrm{~B}$

$4 \mathrm{~b} \quad$ Relative fission power density map at $1 \mathrm{FPY}-\mathrm{ZrB}_{2}$ coating of the $\mathrm{UO}_{2}$ fuel pellets in 104 fuel rods, enrichment: $30 \mathrm{wt} \%{ }^{10} \mathrm{~B}$, initial loading: 30.7 grams ${ }^{10} \mathrm{~B}$

$4 \mathrm{c} \quad$ Relative fission power density map at $4 \mathrm{FPY}-\mathrm{ZrB}_{2}$ coating of the $\mathrm{UO}_{2}$ fuel pellets in 104 fuel rods, enrichment: $30 \mathrm{wt} \%{ }^{10} \mathrm{~B}$, initial loading : 30.7 grams ${ }^{10} \mathrm{~B}$.

$5 \quad \mathrm{Gd}_{2} \mathrm{O}_{3}$ burnable poison integrally mixed with the $\mathrm{UO}_{2}$ fuel pellets of selected fuel rods-Summary results

$6 \mathrm{Gd}_{2} \mathrm{O}_{3}$ burnable poison integrally mixed with the outer one-third part of the $\mathrm{UO}_{2}$ fuel pellets of selected fuel rods-Summary results

$7 \quad \mathrm{Gd}_{2} \mathrm{O}_{3}$ burnable poison coating between the $\mathrm{UO}_{2}$ fuel pellets and the $\mathrm{Zr}-4$ cladding of selected fuel rods-Summary results

8 Gd burnable poison integrally mixed with the $\mathrm{Zr}-4$ cladding of selected fuel rods-Summary results

9 Sm burnable poison integrally mixed with the $\mathrm{UO}_{2}$ fuel pellets of selected fuel rods-Summary results

10 Sm burnable poison integrally mixed with the outer one-third part of the $\mathrm{UO}_{2}$ fuel pellets of selected fuel rods-Summary results

11 Sm burnable poison in a $\mathrm{Sm}_{2} \mathrm{O}_{3}$ coating between the $\mathrm{UO}_{2}$ fuel pellet and the $\mathrm{Zr}-4$ cladding of selected fuel rods-Summary results

12 Sm burnable poison integrally mixed with the $\mathrm{Zr}-4$ cladding of selected fuel rods-Summary results

13 Er burnable poison integrally mixed with the $\mathrm{UO}_{2}$ fuel pellets of selected fuel rods-Summary results

14 Er burnable poison integrally mixed with the outer one-third part of the $\mathrm{UO}_{2}$ fuel pellets of selected fuel rods-Summary results 
15 Er burnable poison in an $\mathrm{Er}_{2} \mathrm{O}_{3}$ coating between the $\mathrm{UO}_{2}$ fuel pellet and the $\mathrm{Zr}-4$ cladding of selected fuel rods-Summary results

16 Er burnable poison integrally mixed with the $\mathrm{Zr}-4$ cladding of selected fuel rods-Summary results

17 Dy burnable poison integrally mixed with the $\mathrm{UO}_{2}$ fuel pellets of selected fuel rods-Summary results

18 Dy burnable poison integrally mixed with the outer one-third part of the $\mathrm{UO}_{2}$ fuel pellets of selected fuel rods-Summary results

19 Dy burnable poison in $\mathrm{D}_{2} \mathrm{O}_{3}$ coating between the $\mathrm{UO}_{2}$ fuel pellet and the $\mathrm{Zr}-4$ cladding of selected fuel rods-Summary results

20 Dy burnable poison Integrally mixed with the $\mathrm{Zr}$-4 cladding of selected fuel rods-Summary results

21 Eu burnable poison integrally mixed with the $\mathrm{UO}_{2}$ fuel pellets of selected fuel rods-Summary results

22 Eu burnable poison integrally mixed with the outer one-third part of the $\mathrm{UO}_{2}$ fuel pellets of selected fuel rods-Summary results

23 Eu burnable poison in an $\mathrm{Eu}_{2} \mathrm{O}_{3}$ coating between the $\mathrm{UO}_{2}$ fuel pellet and the $\mathrm{Zr}-4$ cladding of selected fuel rods-Summary results

24 Eu burnable poison integrally mixed with the $\mathrm{Zr}-4$ cladding of selected fuel rods-Summary results

25 Hf burnable poison integrally mixed with the $\mathrm{UO}_{2}$ fuel pellets of selected fuel rods-Summary results

26 Hf burnable poison integrally mixed with the outer one-third part of the $\mathrm{UO}_{2}$ fuel pellets of selected fuel rods-Summary results

27 Hf burnable poison in a $\mathrm{HfO}_{2}$ coating between the $\mathrm{UO}_{2}$ fuel pellet and the $\mathrm{Zr}-4$ cladding of selected fuel rods-Summary results

28 Hf burnable poison integrally mixed with the $\mathrm{Zr}-4$ cladding of selected fuel rods-Summary results

29 Lu burnable poison in a $\mathrm{Lu}_{2} \mathrm{O}_{3}$ coating between the $\mathrm{UO}_{2}$ fuel pellet and the $\mathrm{Zr}-4$ cladding of selected fuel rods-Summary results

30 The effect of isotope separation for the case of burnable poison in the form of gadolinium oxide homogeneously mixed with the fuel.

31 The effect of isotope separation for the case of burnable poison in the form of samarium oxide homogeneously mixed with the fuel. 
32 The effect of isotope separation for the case of burnable poison in the form of erbium oxide homogeneously mixed with the fuel

33 The effect of isotope separation for the case of burnable poison in the form of dysprosium oxide homogeneously mixed with the fuel

34 The effect of isotope separation for the case of burnable poison in the form of gadolinium oxide homogeneously mixed into the outer one-third volume of fuel pellets

35 The effect of isotope separation for the case of burnable poison in the form of samarium oxide homogeneously mixed into the outer one-third volume of fuel pellets

36 The effect of isotope separation for the case of burnable poison in the form of erbium oxide homogeneously mixed into the outer one-third volume of fuel pellets

37 The effect of isotope separation for the case of burnable poison in the form of dysprosium oxide homogeneously mixed into the outer one-third volume of fuel pellets

38 The effect of isotope separation for the case of burnable poison in the form of gadolinium oxide deposited in a thin coating on the lateral surface of the fuel pellets

39 The effect of isotope separation for the case of burnable poison in the form of samarium oxide deposited in a thin coating on the lateral surface of the fuel pellets

40 The effect of isotope separation for the case of burnable poison in the form of erbium oxide deposited in a thin coating on the lateral surface of the fuel pellets.

41 The effect of isotope separation for the case of burnable poison in the form of dysprosium oxide deposited in a thin coating on the lateral surface of the fuel pellets

42 The effect of isotope separation for the case of burnable poison in the form of hafnium oxide deposited in a thin coating on the lateral surface of the fuel pellets

43 The effect of isotope separation for the case of burnable poison in the form of gadolinium alloyed in the Zircaloy cladding.

44 The effect of isotope separation for the case of burnable poison in the form of samarium alloyed in the Zircaloy cladding

45 The effect of isotope separation for the case of burnable poison in the form of erbium alloyed in the Zircaloy cladding

46 The effect of isotope separation for the case of burnable poison in the form of europium alloyed in the Zircaloy cladding.... 
47 The effect of isotope separation for the case of burnable poison in the form of hafnium alloyed in the Zircaloy cladding.

48 The effect of isotope separation for the case of burnable poison in the form of osmium alloyed in the Zircaloy cladding

49 The effect of isotope separation for the case of burnable poison in the form of oxide homogeneously mixed with the fuel.

50 The effect of isotope separation for the case of burnable poison in the form of oxide homogeneously mixed into the outer one-third volume of fuel pellets

51 The effect of isotope separation for the case of burnable poison in the form of oxide deposited in a thin coating on the lateral surface of the fuel pellets

52 The effect of isotope separation for the case of burnable poison in the form of a metal alloyed in the Zircaloy cladding

53 Savings in full-power days achieved by the use of single isotopes over the same naturally occurring elements for $10 \%$ initial negative reactivity

54 Enrichment percentage and annual production for candidate isotopes by plasma separation 


\section{ABBREVIATED TERMS}

BOC
BOL
BP
BWR
CANDU
CCCP
CPU
EOL
FPD
FPY
GWD/MTU
HFIR
IFBA
MHTR
MOX
MVC
PSP
PWR
RAP
RI
STD
VVER

beginning of cycle

beginning of life

burnable poison

boiling-water reactor

Canadian Deuterium Uranium Reactor

current-coupling collision probability

central processing unit

end of life

full-power-day

full-power-year

gigawatt-days per metric ton of uranium

High Flux Isotope Reactor

integral fuel burnable absorber

modular high-temperature reactor

mixed oxide

moderator void coefficient of reactivity

plasma-separating process

pressurized-water reactor

residual absorber penalty

resonance integral

standard deviation

water-cooled, water-moderated atomic reactor (Russian) 



\section{ACKNOWLEDGMENTS}

This work was supported by the U.S. Department of Energy, NERI Project Number IWO MSF 99-0074. 



\title{
DEVELOPMENT OF IMPROVED BURNABLE POISONS FOR COMMERCIAL NUCLEAR POWER REACTORS
}

\begin{abstract}
SUMMARY
Burnable poisons are used in all modern nuclear reactors to permit higher loading of fuel without the necessity of an overly large control rod system. This not only permits a longer core life but can also be used to level the power distribution. Commercial nuclear reactors commonly use $\mathrm{B}_{4} \mathrm{C}$ in separate non-fueled rods and more recently, zirconium boride coatings on the fuel pellets or gadolinium oxide mixed with the fuel. Although the advantages are great, there are problems with using these materials. Boron, which is an effective neutron absorber, transmutes to lithium and helium upon absorption of a neutron. Helium is insoluble and is eventually released to the interior of the fuel rod, where it produces an internal pressure. When sufficiently high, this pressure stress could cause separation of the cladding from the fuel, causing overly high centerline temperatures. Gadolinium has several very strongly absorbing isotopes, but not all have large cross sections and result in residual burnable poison reactivity worth at the end of the fuel life. Even if the amount of this residual absorber is small and the penalty in operation small, the cost of this penalty, even if only several days, can be very high.
\end{abstract}

The objective of this investigation was to study the performance of single isotopes in order to reduce the residual negative reactivity left over at the end of the fuel cycle. Since the behavior of burnable poisons can be strongly influenced by their configuration, four forms for the absorbers were studied: homogeneously mixed with the fuel, mixed with only the outer one-third of the fuel pellet, coated on the perimeter of the fuel pellets, and alloyed with the cladding. In addition, the numbers of fuel rods containing burnable poison were chosen as $8,16,64$, and 104. Other configurations were chosen for a few special cases. An enrichment of $4.5 \mathrm{wt} \%{ }^{235} \mathrm{U}$ was chosen for most cases for study in order to achieve a 4-year fuel cycle. A standard pressurized water reactor fuel core was chosen for the study, and state-of-the-art neutronic reactor core computer codes were used for analysis. Power distribution, fuel burnup, reactivity due to burnable poisons and other fission products, spectrum shift, core reactivity, moderator void coefficients, as well as other parameters were calculated as a function of time and fuel burnup.

The results not only showed advantages of separation of burnable poison isotopes but revealed benefits to be achieved by careful selection of the configuration of even naturally occurring elements used as burnable poisons. The savings in terms of additional days of operation is shown in Figure 1, where the savings is plotted for each of six favorable isotopes in the four configurations. The benefit of isotope separation is most dramatic for dysprosium, but even the time savings in the case of gadolinium is several days. For a modern nuclear plant, one day's worth of electricity is worth about one million dollars, so the resulting savings of only a few days is considerable. It is also apparent that the amount of savings depends upon the configuration of the burnable poison.

The savings in cycle length is not the only factor that must be considered. The following primary factors must all be considered in the choice of a burnable poison:

1. Rate of burnout

2. Initial negative reactivity worth

3. Available volume for burnable poison loading

4. Effect of burnable poison on the moderator void coefficient of reactivity

5. Cost of the burnable poison material

6. Compatibility of the burnable poison with the fuel or cladding 
The rate of burnout of the burnable poison is perhaps the most important factor. A burnable poison that burns away in the first month of operation is not useful even if it burns so completely that its presence is not detectable at the end of the fuel cycle. Gadolinium can be distributed such that over 99\% is burned in the first 120 days, but this is not ideal if a long fuel cycle is desired. Figures 2 and 3 illustrate the time dependence of six elements for the case of the burnable poison distributed in the outer one-third of the fuel for naturally occurring elements and single isotopes, respectively. In this configuration Gd might be used for a 1-year fuel cycle but not for a 4-year cycle. There is a big advantage to using ${ }^{149} \mathrm{Sm}$ over natural samarium, but the time dependence is not favorable in this configuration. However, ${ }^{167} \mathrm{Er}$ burns at an approximately linear rate over 4 years, and there is a significant advantage of separating out ${ }^{167} \mathrm{Er}$, of which only $1 \%$ remains at the end of 4 years. Although the benefit is not as great, ${ }^{164} \mathrm{Dy}$ also demonstrates favorable performance.

Six isotopes were identified as having sufficiently favorable performance to merit study of the efficiency and cost of isotope separation and compatibility with the fuel and cladding. They are ${ }^{157} \mathrm{Gd}$, ${ }^{149} \mathrm{Sm},{ }^{167} \mathrm{Er},{ }^{164} \mathrm{Dy},{ }^{177} \mathrm{Hf}$, and ${ }^{151} \mathrm{Eu}$. Even the lowest savings of isotope separation is on the order of several million dollars over the term of one fuel cycle, and isotope separation costs are expected to be far lower than this value for all six of the isotopes considered.

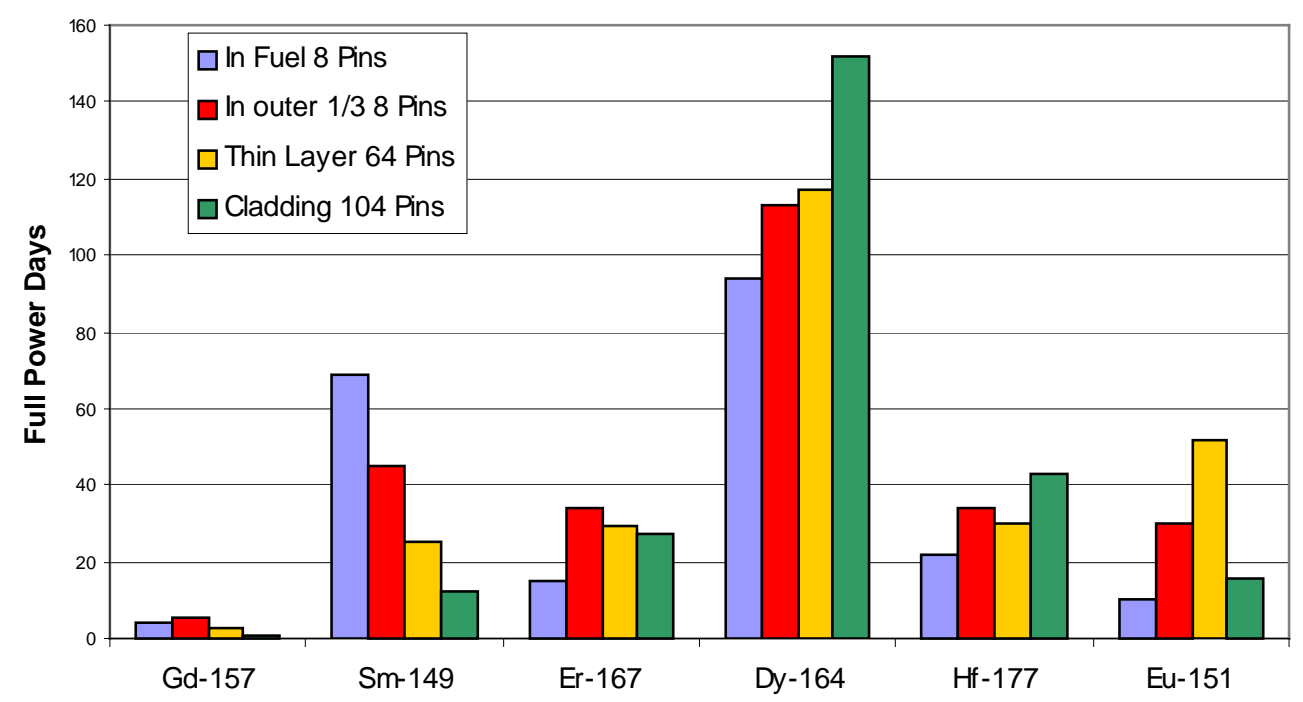

Figure 1. Savings in full power days at the end of a four-year fuel cycle achieved by using the single isotopes specified rather than naturally occurring elements. 


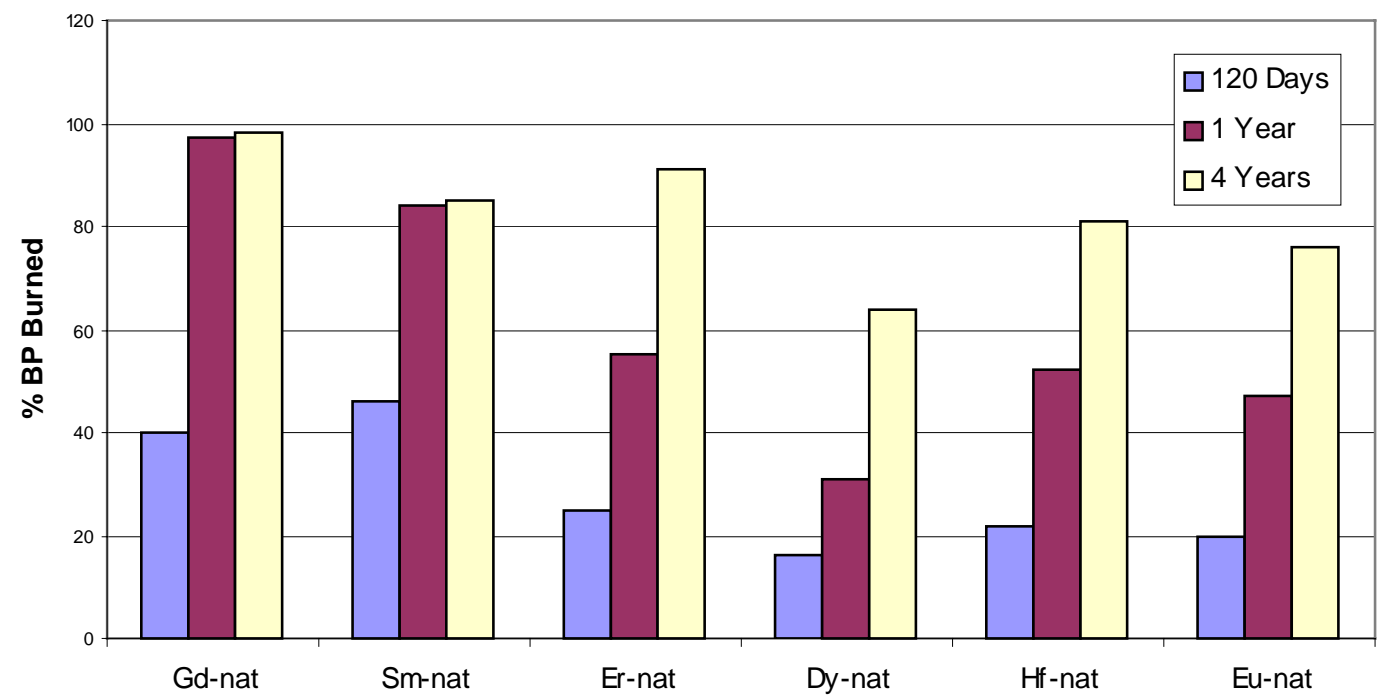

Figure 2. Percentage of burnable poison burned at the end of 120 days, 1 year, and 4 years for naturally occurring elements mixed in the outer third of the fuel rod volume. Eight fuel rods contain burnable poison.

Burn Rate for Separated Isotopes in Outer Third of Fuel, 8 Rods

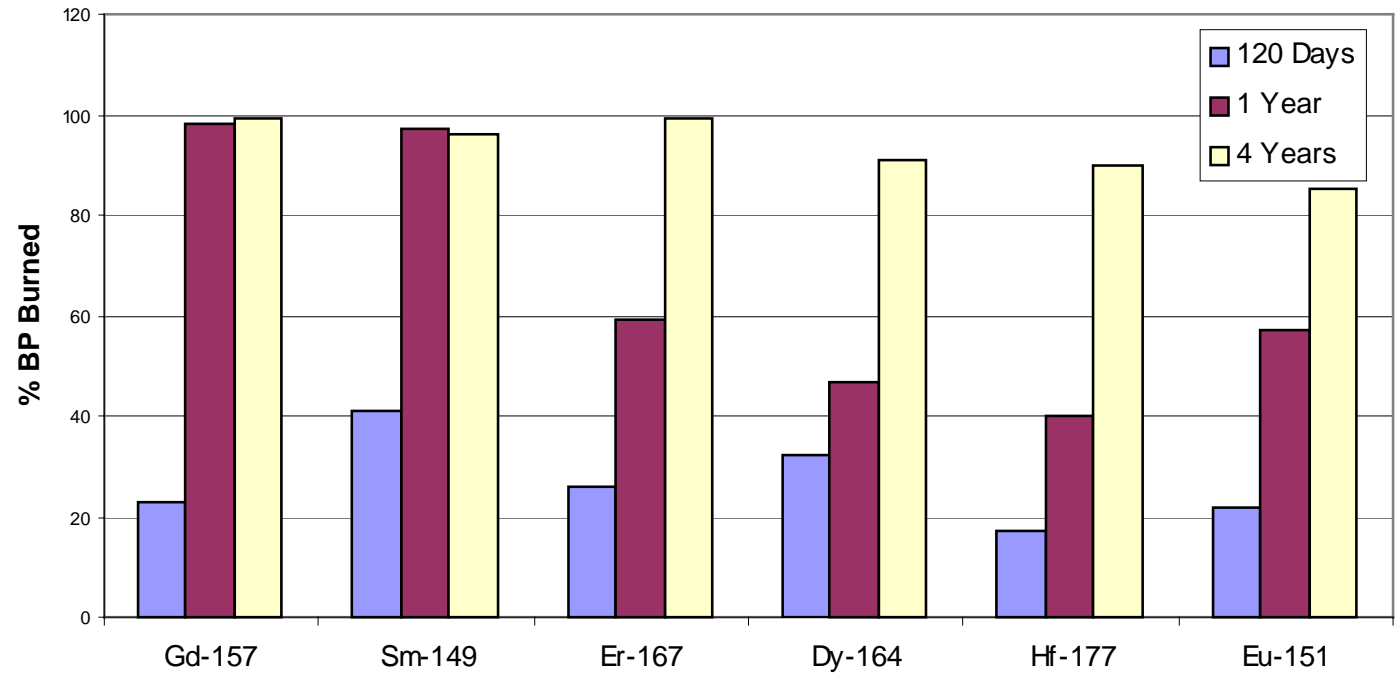

Figure 3. Percentage of burnable poison burned at the end of 120 days, 1 year, and 4 years for single isotopes mixed in the outer third of the fuel rod volume. Eight fuel rods contain burnable poison. 



\section{INTRODUCTION}

A reactor core containing fresh fuel must have excess reactivity to compensate for fuel depletion, production of fission product poisons, and temperature effects that introduce negative reactivity. Control rods can be designed to compensate for this high beginning-of-life (BOL) excess reactivity, but this leads to the requirement of inserting the regulating control rods deeper into the core and/or inserting rods with higher control rod reactivity worths. This leads to the existence of large nonuniform power distributions throughout much of the core life. In pressurized water reactors (PWRs), a soluble boron compound is added to the coolant to compensate for excess reactivity; its concentration is reduced as the fuel is depleted. However, large concentrations of the soluble boron compound lead to an unacceptable reduction in the negative moderator void coefficient of reactivity (MVC). The amount of soluble boron that can be dissolved in the reactor coolant is limited due to the requirement that the MVC must remain negative over the fuel cycle. Too much boron, typically greater than 1,300 ppm at full power, will make the MVC positive.

In order to avoid power peaking in the fuel at full power, the regulating control rods can be inserted only a small distance into the core. In order to compensate for the excess reactivity introduced by freshly loaded fuel and to increase fuel loading and enrichment to extend fuel-cycle length, burnable poisons (BPs) such as $\mathrm{B}_{4} \mathrm{C}, \mathrm{ZrB}_{2}, \mathrm{Gd}_{2} \mathrm{O}_{3}, \mathrm{Er}_{2} \mathrm{O}_{3}$ and other absorbing materials can be incorporated in the fuel rods or can be located in separate non-fueled pins. The methods of choice in most PWRs are $\mathrm{B}_{4} \mathrm{C}$ in separate non-fueled rods and, more recently, a thin coating of $\mathrm{ZrB}_{2}$ surrounding the fuel pellets of a number of fuel rods, or $\mathrm{Gd}_{2} \mathrm{O}_{3}$ and $\mathrm{Er}_{2} \mathrm{O}_{3}$ dispersed inside the fuel pellets of selected fuel rods. In boiling water reactors (BWRs) the method of choice is the incorporation of $\mathrm{Gd}_{2} \mathrm{O}_{3}$ homogeneously mixed in the fuel pellets of a number of fuel rods.

In the proposed use of the Canadian Deuterium Uranium (CANDU) reactor for burning weapons grade plutonium as a mixed oxide (MOX), depleted uranium is used as the matrix material throughout the fuel bundle (ref 1). In the central fuel rod and in the next ring of fuel rods, Dy burnable poison is mixed with the depleted uranium of those fuel rods not containing plutonium. This increases the amount of plutonium required to achieve a given burnup (and thus increases the $\mathrm{Pu}$ disposition rate), reduces the local peak power after refueling, and reduces the local positive moderator void coefficient in the middle of the fuel bundle. As a result, the Dy makes the overall bundle MVC more negative.

As the fuel burns, so does the absorber. Ideally, when the fuel is depleted toward the end-of-life (EOL) of the fuel, so would the combined negative reactivity worth of the burnable poison and its daughters. A BP with a high absorption cross section would be very effective in small concentrations at BOL but would deplete rapidly unless its daughters have a high absorption cross section. A poison with too low an absorption cross section would be present in too high a concentration at EOL of the fuel, thus shortening the fuel life. In a lumped configuration, a shelf-shielding effect can be achieved so that a slower depletion rate of the BP takes place. This can be achieved by incorporating the total required BP inside fewer fuel rods or using a higher concentration of the poison. One way that the depletion rate can be accelerated is by incorporating the poison over a smaller radial volume of the pellets (pellet zoning). The configuration of the BP is limited only by the creativity of the designer. In some cases, self-shielding is achieved on a microscopic scale by precipitation of an absorber-rich phase from a fuel-BP mixture (ref 2). In the present study, alloying the BP with the cladding is suggested, a new configuration, which offers several advantages. The details of self-shielding have been worked out by Radkowsky as part of the U.S. Naval Reactors Program, and the techniques have been in use for many years (ref 3 ). 
The two most commonly used BPs throughout the world are boron and gadolinium (ref 4). Both homogeneous and lumped configurations have been used. Gadolinium has been used in BWRs for many years (ref 5) and has more recently been used in PWRs (ref 6,7) and VVERs (VVER is the Russian acronym for a water-cooled, water-moderated atomic reactor). The gadolinium is dispersed homogeneously throughout the fuel pellets of a number of fuel rods. Another configuration is application of a thin coating of $\mathrm{ZrB}_{2}$ to the perimeter of the fuel pellets (ref 6).

The use of natural gadolinium as a burnable poison, as well as less commonly used absorber materials, suffers from a disadvantage in that it has seven naturally occurring isotopes, several of which have low effective absorption cross sections. These would be present in too high concentrations at EOL of the fuel. The situation is complicated by the fact that some of the burnable poison isotopes transmute or decay into other highly neutron-absorbing isotopes. The result is that the burnable poison isotopes and their daughters do not completely burn up. Some residual reactivity remains at EOL of the fuel, thus shortening the useful life of the fuel. To correct this problem either more fuel must be initially loaded or the initial fissile enrichment must be increased to compensate for this negative reactivity burden.

In the case of boron used as a burnable poison, only ${ }^{10} \mathrm{~B}$ has a significant absorption (n, $\left.\alpha\right)$ cross section, and ${ }^{10} \mathrm{~B}$ does not transmute into highly absorbing isotopes, although residual reactivity is a disadvantage (ref 8). Of more concern, however, is the production of helium by the reaction

$$
{ }^{10} \mathrm{~B}+\mathrm{n} \rightarrow{ }^{4} \mathrm{He}+{ }^{7} \mathrm{Li}
$$

The helium increases the internal pressure of the fuel rods. At a sufficiently high burnup, the combination of fission product gases and helium from boron transmutation can attain a pressure higher than the reactor coolant pressure. When this occurs, the cladding can creep away from the fuel, producing a gap that impedes heat transfer. This results in higher fuel temperatures, and in some cases, fuel melting can occur. The increase in internal pressure can be reduced by spreading the boron burnable absorber over more fuel rods, thus reducing the boron loading in each of the fuel rods containing the poison. This, however, increases cost and increases the burnup rate of the boron.

It can be concluded that all burnable poisons in use suffer from disadvantages that limit the level of initial fissile loading and the level of fuel burnup. One solution to the problem might be to use other absorbers, but the number of useful neutron-absorbing elements is small. However, if isotopes were separated so that only the isotopes with desirable neutron absorption cross sections were used, and the isotopes that result in residual negative reactivity burdens were removed, the disadvantages would vanish.

An example of a candidate for isotope separation is samarium, which has six stable isotopes. Separation of ${ }^{149} \mathrm{Sm}$, which constitutes $13.8 \%$ of natural samarium, would provide a high-crosssection absorber that burns to a much more weakly absorbing isotope. Gadolinium has several stable isotopes, many of which are strong absorbers, but ${ }^{157} \mathrm{Gd}$ (15.8\% natural isotopic abundance) has a very high thermal-absorption cross section, and it transmutes to ${ }^{158} \mathrm{Gd}$, which has a thermal absorption cross section of only a few barns. Separation of this particular isotope has also been suggested by Hove and Spetz, whose calculations have shown significantly reduced residual negative reactivity (ref 9). Erbium presents a similar situation; ${ }^{167} \operatorname{Er}$ (22.9\% natural isotopic abundance) has a 660-barn thermal-capture cross section, producing ${ }^{168} \mathrm{Er}$ with a two-barn thermal cross section. Dysprosium has eight stable isotopes, several of which are strong absorbers. Neutron capture in several of those would lead to another strong absorber, thus resulting in a substantial residual negative reactivity burden at EOL of the fuel. ${ }^{164}$ Dy (28.1\% natural isotopic abundance) with a 2,650-barn thermal absorption cross 
section transmutes to ${ }^{165} \mathrm{Dy}$, which then decays to ${ }^{165} \mathrm{Ho}$ with a half-life of 2.3 hours. ${ }^{165} \mathrm{Ho}$ has a 65 barn capture cross section. This can capture to ${ }^{166} \mathrm{Ho}$, which has a large thermal cross section.

A major disadvantage to using separated isotopes in the past has been the high cost of separation. As an example, using magnetic separation by a calutron, ${ }^{157} \mathrm{Gd}$ is estimated to cost about $\$ 1000$ per gram (ref 10). At this rate, the burnable poison loading for a typical BWR would cost more than the fuel. However newer isotope separation processes are now available that are less expensive. For example, the plasma separation process (PSP) is estimated to cost about a factor of ten less than the calutron process.

The calculations for the first phase of the project have assumed that the isotopes have been separated completely. In reality, the achievable enrichment depends upon the particular isotope, its abundance, and the number of passes in the enrichment process. It is estimated that a single pass in the PSP process to obtain ${ }^{157} \mathrm{Gd}$ would result in $46 \%$ to $73 \%$ enrichment, depending upon the magnetic field strength used. Higher enrichments of ${ }^{157} \mathrm{Gd}$ would, as would other isotopes, require additional passes through the PSP process, which would increase cost. The use of single pass burnable poison enrichments, including the performance of the burnable poisons, will be done in the second phase of the project.

The introduction of burnable poisons in the core hardens the spectrum. Partial voiding of the moderator might reduce the negative reactivity worth of the burnable poison, depending on the presence or absence of low-lying resonances. Voiding of the moderator without the presence of burnable absorbers usually decreases reactivity. This depends on the soluble boron content in the water. The effect of single-pass burnable poison enrichments on the MVC will be further investigated during the second phase. 



\section{METHOD OF CALCULATION}

\section{CONFIGURATIONS FOR STUDY}

In the first phase of this study, emphasis will be placed on the neutronics performance studies of the use of different burnable poisons in PWRs. To reduce the scope and extent of the studies in this project, we will concentrate on one standard reactor design. In particular, we will use the standard Westinghouse $17 \times 17$ fuel assembly design, containing 264 fuel rods, 24 guide tubes (waterholes) and one instrument tube. The Zircaloy-4 clad fuel rods contain $\mathrm{UO}_{2}$-fuel pellets with a density of approximately $95 \%$ of theoretical density. When a burnable poison oxide was homogeneously mixed with the pellet, a density of $95 \%$ of the theoretical density of the combined $\mathrm{UO}_{2}$ - burnable poison compound was used. The active fuel height was assumed to be $3.66 \mathrm{~m}$ (144 in.). A uniform axial fuel enrichment was used. The fuel rod pitch was $12.6 \mathrm{~mm}$ (0.495 in.), the clad outside diameter $9.53 \mathrm{~mm}$ (0.375 in.), the clad inside diameter $8.36 \mathrm{~mm}$ (0.329 in.), the diametrical gap (cold) $0.17 \mathrm{~mm}$ (0.0065 in.), and the pellet outside diameter (cold) $8.192 \mathrm{~mm}$ (0.3225 in.). Each fuel rod contained a plenum in the top region of several inches in height to collect fission-product gases and to reduce the buildup of the internal fuel rod pressure during fuel burnup. The plenum is pre-pressurized with argon gas to avoid densification of the fuel pellets.

The initial fuel enrichment was set at $4.5 \mathrm{wt} \%{ }^{235} \mathrm{U}$ in order to use a fuel lifetime of 4 years at full power. Several additional studies were made for initial fuel enrichments of $6.0 \mathrm{wt} \%$, together with a 5 -year-long fuel lifetime at full power. This enabled us to investigate whether the residual negative reactivity worth of the burnable poisons could be further reduced with longer fuel lifetimes and thus higher burnups. The reactor core contained 193 fuel assemblies, and the rated reactor core power that was used in this study was 3,400 MWth. The average power per fuel assembly was thus $17.6 \mathrm{MWth}$.

Four different types of burnable poison rods were investigated.

1. A homogeneous mixture of the burnable poison as an oxide inside the fuel pellets of a limited number of fuel rods.

2. A homogeneous mixture of the burnable poison inside the outer one-third volume of the fuel pellets. During this study it was found that because of self-shielding effects inside the pellets that contain burnable poisons, the required amount of burnable poisons to achieve a given initial negative reactivity was smaller than for the case where the poisons were mixed over the entire volume of the pellet. As a consequence, the residual negative reactivity worth at EOL of the fuel was smaller for the case of BP in a one-third outer volume than for the cases where the BP was homogeneously mixed over the entire pellet. However, the initial rate of depletion of the burnable absorbers was higher. Although it would make the fuel fabrication process more complex, it is felt that making layered fuel pellets is not unreasonable. Fuel pellets are normally cold-pressed and sintered. A two-compartment die, might be designed for the pressing operation. Alternatively, a smaller core pellet of fuel could be pressed then centered in another die, where the burnable poison would be introduced and pressed around it. As with the entire effort to increase efficiency, the net cost savings will determine feasibility. Making layered fuel pellets might be an easier alternative to coating the fuel pellets with burnable poisons such as $\mathrm{Gd}_{2} \mathrm{O}_{3}, \mathrm{Er}_{2} \mathrm{O}_{3}$, and others.

3. An oxide burnable poison coating on the surface of the pellets in a limited number of fuel rods. This is similar to the technique, recently in use in several PWRs, of using a thin $\mathrm{ZrB}_{2}$ coating on the outer radial surface of the pellets. The use of burnable poisons such as $\mathrm{Gd}_{2} \mathrm{O}_{3}, \mathrm{Sm}_{2} \mathrm{O}_{3}$, and others with natural isotopic abundances, might require too thick a pellet coating, which might impede heat transfer. The use of enriched burnable poisons requires a much thinner coating to achieve a given initial negative reactivity worth. 
4. A homogeneous mixture of the burnable poison in the form of a metal alloyed with the Zircaloy cladding of a number of fuel rods. The use of burnable poisons with natural isotopic abundances would require a significantly higher concentration in the clad than would single isotopes. In some cases, this level of a rare earth would exceed the solubility limit and would precipitate as a second phase, which could harden the metal and reduce its ductility. Even for the cases where the burnable poison remains in solid solution, it could harden and embrittle the clad. The use of enriched burnable poisons would require a much smaller concentration to achieve a given initial negative reactivity. This increases the probability of finding a burnable poison that does not degrade the integrity of the cladding. This issue is to be addressed in the final phase of the project. However, preliminary studies have demonstrated that concentrations of $0.5 \mathrm{wt} \% \mathrm{Gd}$ and 2 wt $\%$ Dy do not embrittle zirconium. The use of burnable absorbers inside the cladding also changes the chemistry and compatibility issue to one of cladding rather than fuel. The results of the neutronics performance studies show that the introduction of enriched burnable poisons into the clad of selected fuel rods is indeed very promising.

The layout of a standard Westinghouse $17 \times 17$ fuel assembly design is shown in Figure 4 .

$\begin{array}{rllllllllllllllllllll} & 1 & 2 & 3 & 4 & 5 & 6 & 7 & 8 & 9 & 10 & 11 & 12 & 13 & 14 & 15 & 16 & 17 \\ 1 & F & F & F & F & F & F & F & F & F & F & F & F & F & F & F & F & F \\ 2 & F & F & F & F & F & F & F & F & F & F & F & F & F & F & F & F & F \\ 3 & F & F & F & F & F & O & F & F & O & F & F & O & F & F & F & F & F \\ 4 & F & F & F & O & F & F & F & F & F & F & F & F & F & O & F & F & F \\ 5 & F & F & F & F & F & F & F & F & F & F & F & F & F & F & F & F & F \\ 6 & F & F & O & F & F & O & F & F & O & F & F & O & F & F & O & F & F \\ 7 & F & F & F & F & F & F & F & F & F & F & F & F & F & F & F & F & F \\ 8 & F & F & F & F & F & F & F & F & F & F & F & F & F & F & F & F & F \\ 9 & F & F & O & F & F & O & F & F & I & F & F & O & F & F & O & F & F \\ 10 & F & F & F & F & F & F & F & F & F & F & F & F & F & F & F & F & F \\ 11 & F & F & F & F & F & F & F & F & F & F & F & F & F & F & F & F & F \\ 12 & F & F & O & F & F & O & F & F & O & F & F & O & F & F & O & F & F \\ 13 & F & F & F & F & F & F & F & F & F & F & F & F & F & F & F & F & F \\ 14 & F & F & F & O & F & F & F & F & F & F & F & F & F & O & F & F & F \\ 15 & F & F & F & F & F & O & F & F & O & F & F & O & F & F & F & F & F \\ 16 & F & F & F & F & F & F & F & F & F & F & F & F & F & F & F & F & F \\ 17 & F & F & F & F & F & F & F & F & F & F & F & F & F & F & F & F & F \\ \text { F: Fuel rod } & & & & & & & & & & & & & & & & \\ \text { O: Guide tube } & & & & & & & & & & & & & & & \\ \text { I: Instrument tube } & & & & & & & & & & & & & & \end{array}$

Figure 4. Diagram showing the model of a standard $17 \times 17$ fuel assembly layout. 
A fuel assembly contains 264 fuel rods, 24 guide tubes (waterholes), and one instrument tube. In normal PWR operation, non-fueled rods containing $\mathrm{B}_{4} \mathrm{C}$ pellets are inserted inside a number of guide tubes of fresh fuel assemblies to compensate for the excess initial reactivity at BOL of the fresh fuel. Depending upon the location of the fuel assemblies in the reactor core, the standard burnable absorber pin configurations can contain 4, 8, 12,16, 20, or 24 non-fueled burnable poison rods per fuel assembly. Layouts of standard fuel assemblies with 8 and 16 non-fueled burnable poison rods are shown in Figures 5 and 6, respectively.

$\begin{array}{cccccccccccccccccc} & 1 & 2 & 3 & 4 & 5 & 6 & 7 & 8 & 9 & 10 & 11 & 12 & 13 & 14 & 15 & 16 & 17 \\ 1 & F & F & F & F & F & F & F & F & F & F & F & F & F & F & F & F & F \\ 2 & F & F & F & F & F & F & F & F & F & F & F & F & F & F & F & F & F \\ 3 & F & F & F & F & F & O & F & F & O & F & F & O & F & F & F & F & F \\ 4 & F & F & F & (O) & F & F & F & F & F & F & F & F & F & (O) & F & F & F \\ 5 & F & F & F & F & F & F & F & F & F & F & F & F & F & F & F & F & F \\ 6 & F & F & O & F & F & O & F & F & (O) & F & F & O & F & F & O & F & F \\ 7 & F & F & F & F & F & F & F & F & F & F & F & F & F & F & F & F & F \\ 8 & F & F & F & F & F & F & F & F & F & F & F & F & F & F & F & F & F \\ 9 & F & F & O & F & F & (O) & F & F & I & F & F & (O) & F & F & O & F & F \\ 10 & F & F & F & F & F & F & F & F & F & F & F & F & F & F & F & F & F \\ 11 & F & F & F & F & F & F & F & F & F & F & F & F & F & F & F & F & F \\ 12 & F & F & O & F & F & O & F & F & (O) & F & F & O & F & F & O & F & F \\ 13 & F & F & F & F & F & F & F & F & F & F & F & F & F & F & F & F & F \\ 14 & F & F & F & (O) & F & F & F & F & F & F & F & F & F & (O) & F & F & F \\ 15 & F & F & F & F & F & O & F & F & O & F & F & O & F & F & F & F & F \\ 16 & F & F & F & F & F & F & F & F & F & F & F & F & F & F & F & F & F \\ 17 & F & F & F & F & F & F & F & F & F & F & F & F & F & F & F & F & F \\ \text { F: Fuel rod } & & & & & & & & & & & & & & & & \\ \text { O: Guide tube } & & & & & & & & & & & & & & & \\ \text { I: Instrument tube } & & & & & & & & & & & & & & \\ \text { (O) Non-fueled burnable poison rod } & & & & & & & & & & \end{array}$

Figure 5. Diagram showing the model of a standard $17 \times 17$ fuel assembly layout with eight non-fueled burnable poison rods. 


$\begin{array}{ccccccccccccccccccc} & 1 & 2 & 3 & 4 & 5 & 6 & 7 & 8 & 9 & 10 & 11 & 12 & 13 & 14 & 15 & 16 & 17 \\ 1 & F & F & F & F & F & F & F & F & F & F & F & F & F & F & F & F & F \\ 2 & F & F & F & F & F & F & F & F & F & F & F & F & F & F & F & F & F \\ 3 & F & F & F & F & F & (O) & F & F & O & F & F & (O) & F & F & F & F & F \\ 4 & F & F & F & (O) & F & F & F & F & F & F & F & F & F & (O) & F & F & F \\ 5 & F & F & F & F & F & F & F & F & F & F & F & F & F & F & F & F & F \\ 6 & F & F & (O) & F & F & O & F & F & (O) & F & F & O & F & F & (O) & F & F \\ 7 & F & F & F & F & F & F & F & F & F & F & F & F & F & F & F & F & F \\ 8 & F & F & F & F & F & F & F & F & F & F & F & F & F & F & F & F & F \\ 9 & F & F & O & F & F & (O) & F & F & I & F & F & (O) & F & F & O & F & F \\ 10 & F & F & F & F & F & F & F & F & F & F & F & F & F & F & F & F & F \\ 11 & F & F & F & F & F & F & F & F & F & F & F & F & F & F & F & F & F \\ 12 & F & F & (O) & F & F & O & F & F & (O) & F & F & O & F & F & (O) & F & F \\ 13 & F & F & F & F & F & F & F & F & F & F & F & F & F & F & F & F & F \\ 14 & F & F & F & (O) & F & F & F & F & F & F & F & F & F & (O) & F & F & F \\ 15 & F & F & F & F & F & (O) & F & F & O & F & F & (O) & F & F & F & F & F \\ 16 & F & F & F & F & F & F & F & F & F & F & F & F & F & F & F & F & F \\ 17 & F & F & F & F & F & F & F & F & F & F & F & F & F & F & F & F & F \\ \text { F: Fuel rod } & & & & & & & & & & & & & & & & \\ \text { O: Guide tube } & & & & & & & & & & & & & & & \\ \text { I: Instrument tube } & & & & & & & & & & & & & & \\ \text { (O): Non-fueled burnable poison rod } & & & & & & & & & & \end{array}$

\section{Figure 6. Diagram showing the model of a standard $17 \times 17$ fuel assembly layout with 16 non-fueled burnable poison rods.}

In the present analyses of the advanced poisons, no burnable absorber rods were inserted inside the guide tubes of the fuel assemblies. The guide tubes were left open for control-rod motion, or they were left open filled with the water coolant. The burnable poisons were placed only inside fuel rods. These are usually referred to as integral fuel burnable absorbers (IFBAs). Four different checkerboard fuel assembly models containing IFBA rods were used in this study. They contained 8, 16, 64, or 104 fuel rods with burnable absorbers. The layouts and the locations of the IFBA rods are shown in Figures 7 through 10. In the configuration with 104 IFBA rods, the fuel rods surrounding the waterholes are replaced with IFBA rods to reduce power peaking. In this configuration each waterhole is surrounded by seven or eight IFBA rods. During the early phases of the research, several cases containing 16 IFBA rods were explored that contained two or four extra IFBA rods. These are reported as cases containing 18 or 20 IFBA rods. The layouts are displayed in Figures $8 \mathrm{a}$ and $8 \mathrm{~b}$, respectively. Note that the presence of water holes in a fuel assembly promote localized innerassembly power density peaks caused by the increase in moderation. To reduce the localized power peaks, the IFBA rods are preferentially placed around the water holes. Note: The layouts displayed in Figures 9 and 10 are nondesign, nonproprietary layouts, not actual ones. 


$\begin{array}{rcccccccccccccccccccc} & 1 & 2 & 3 & 4 & 5 & 6 & 7 & 8 & 9 & 10 & 11 & 12 & 13 & 14 & 15 & 16 & 17 \\ 1 & F & F & F & F & F & F & F & F & F & F & F & F & F & F & F & F & F \\ 2 & F & F & F & F & F & F & F & F & F & F & F & F & F & F & F & F & F \\ 3 & F & F & F & F & F & O & F & F & O & F & F & O & F & F & F & F & F \\ 4 & F & F & F & O & F & F & F & F & (*) & F & F & F & F & O & F & F & F \\ 5 & F & F & F & F & (*) & F & F & F & F & F & F & F & (*) & F & F & F & F \\ 6 & F & F & O & F & F & O & F & F & O & F & F & O & F & F & O & F & F \\ 7 & F & F & F & F & F & F & F & F & F & F & F & F & F & F & F & F & F \\ 8 & F & F & F & F & F & F & F & F & F & F & F & F & F & F & F & F & F \\ 9 & F & F & O & (*) & F & O & F & F & I & F & F & O & F & (*) & O & F & F \\ 10 & F & F & F & F & F & F & F & F & F & F & F & F & F & F & F & F & F \\ 11 & F & F & F & F & F & F & F & F & F & F & F & F & F & F & F & F & F \\ 12 & F & F & O & F & F & O & F & F & O & F & F & O & F & F & O & F & F \\ 13 & F & F & F & F & (*) & F & F & F & F & F & F & F & (*) & F & F & F & F \\ 14 & F & F & F & O & F & F & F & F & (*) & F & F & F & F & O & F & F & F \\ 15 & F & F & F & F & F & O & F & F & O & F & F & O & F & F & F & F & F \\ 16 & F & F & F & F & F & F & F & F & F & F & F & F & F & F & F & F & F \\ 17 & F & F & F & F & F & F & F & F & F & F & F & F & F & F & F & F & F\end{array}$

F: Fuel rod

O: Guide tube

I: Instrument tube

(*): Fuel rod with burnable absorber (IFBA)

Figure 7. Diagram showing the model of a standard $17 \times 17$ fuel assembly layout with eight integral fuel burnable absorbers (IFBAs).

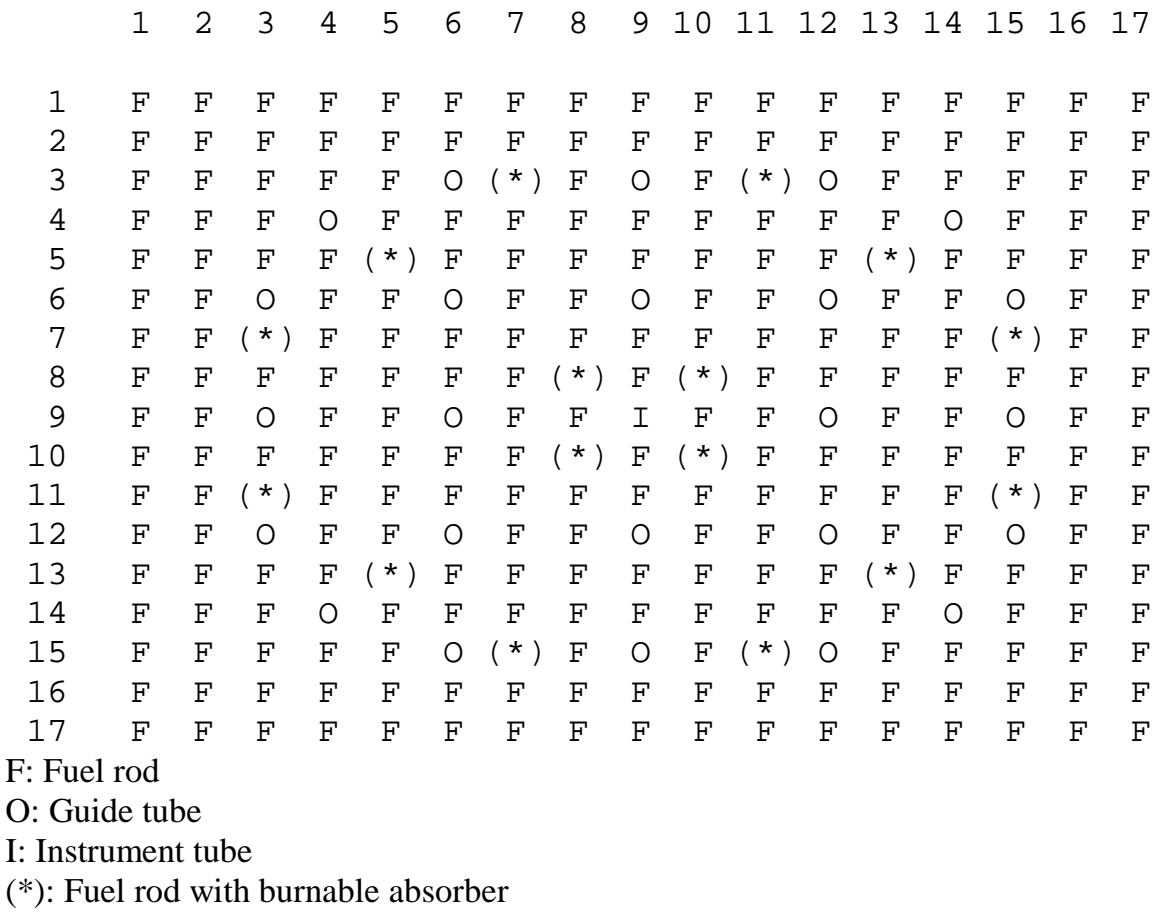

Figure 8. Diagram showing the model of a standard $17 \times 17$ fuel assembly layout with 16 integral fuel burnable absorbers (IFBAs). 


$\begin{array}{cccccccccccccccccc} & 1 & 2 & 3 & 4 & 5 & 6 & 7 & 8 & 9 & 10 & 11 & 12 & 13 & 14 & 15 & 16 & 17 \\ 1 & F & F & F & F & F & F & F & F & F & F & F & F & F & F & F & F & F \\ 2 & F & F & F & F & F & F & F & F & F & F & F & F & F & F & F & F & F \\ 3 & F & F & F & F & F & O & (*) & F & O & F & (*) & O & F & F & F & F & F \\ 4 & F & F & F & O & F & F & F & F & F & F & F & F & F & O & F & F & F \\ 5 & F & F & F & F & (*) & F & F & F & (*) & F & F & F & (*) & F & F & F & F \\ 6 & F & F & O & F & F & O & F & F & O & F & F & O & F & F & O & F & F \\ 7 & F & F & (*) & F & F & F & F & F & F & F & F & F & F & F & (*) & F & F \\ 8 & F & F & F & F & F & F & F & (*) & F & (*) & F & F & F & F & F & F & F \\ 9 & F & F & O & F & F & O & F & F & I & F & F & O & F & F & O & F & F \\ 10 & F & F & F & F & F & F & F & (*) & F & (*) & F & F & F & F & F & F & F \\ 11 & F & F & (*) & F & F & F & F & F & F & F & F & F & F & F & (*) & F & F \\ 12 & F & F & O & F & F & O & F & F & O & F & F & O & F & F & O & F & F \\ 13 & F & F & F & F & (*) & F & F & F & (*) & F & F & F & (*) & F & F & F & F \\ 14 & F & F & F & O & F & F & F & F & F & F & F & F & F & O & F & F & F \\ 15 & F & F & F & F & F & O & (*) & F & O & F & (*) & O & F & F & F & F & F \\ 16 & F & F & F & F & F & F & F & F & F & F & F & F & F & F & F & F & F \\ 17 & F & F & F & F & F & F & F & F & F & F & F & F & F & F & F & F & F \\ \text { F: Fuel rod } & & & & & & & & & & & & & & & & \\ \text { O: Guide tube } & & & & & & & & & & & & & & & \\ \text { I: Instrument tube } & & & & & & & & & & & & & & \\ \text { *): Fuel rod with burnable absorber } & & & & & & & & & & \end{array}$

Figure 8a. Diagram showing the model of a standard $17 \times 17$ fuel assembly layout with 18 integral fuel burnable absorbers (IFBAs).

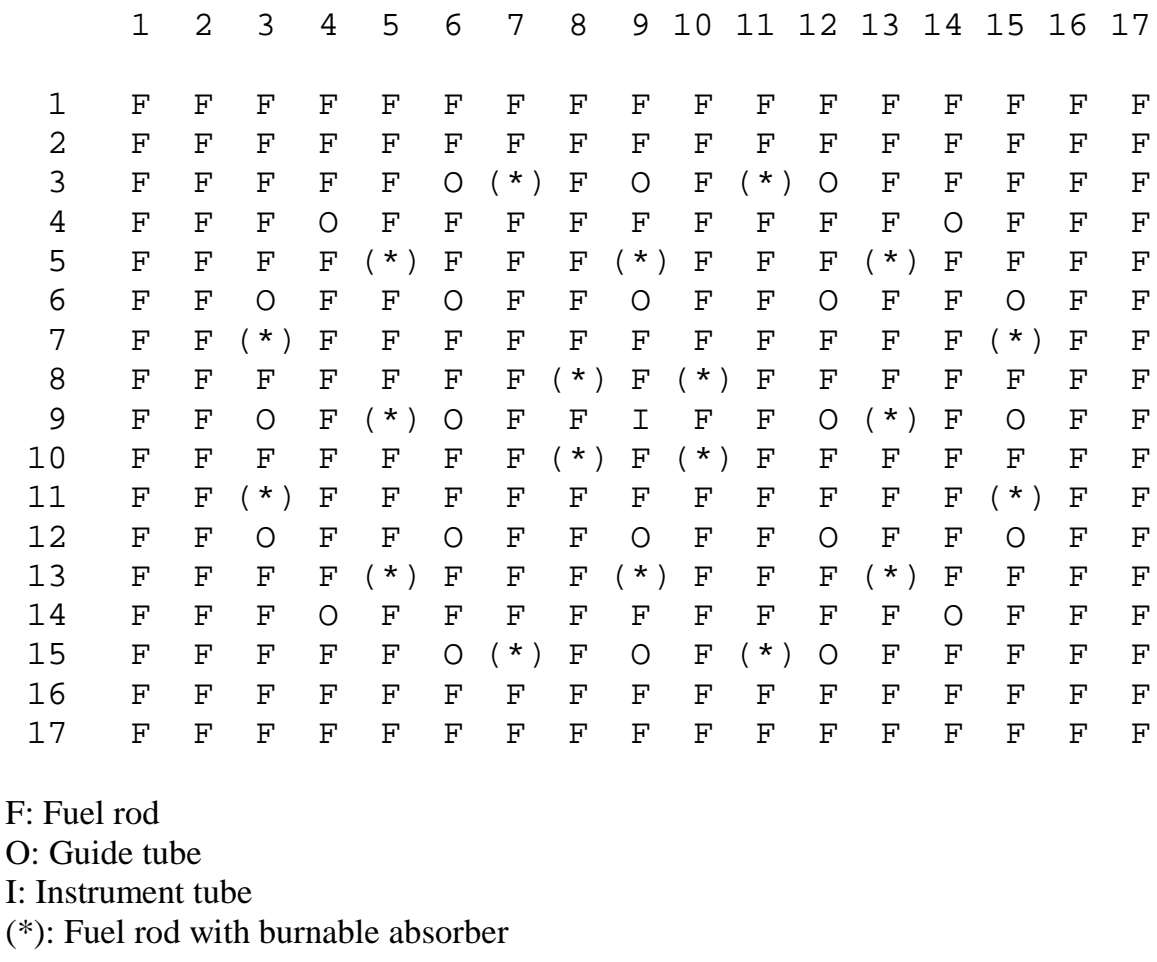

Figure 8b. Diagram showing the model of a standard $17 \times 17$ fuel assembly layout with 20 integral fuel burnable absorbers (IFBAs). 
Figure Not Available

Figure 9. Diagram showing the model of a standard $17 \times 17$ fuel assembly layout with 64 integral fuel burnable absorbers (IFBAs).

Figure Not Available

Figure 10. Diagram showing the model of a standard $17 \times 17$ fuel assembly layout with 104 integral fuel burnable absorbers (IFBAs). 


\section{SELECTION OF ISOTOPES}

Scoping calculations were performed to evaluate candidate isotopes prior to using complex computer codes to select burnable poisons. As an example, gadolinium has two naturally occurring isotopes with very high cross sections. Neglecting isotopes with very small cross sections, the following chain can be used as an approximation for the neutron absorption behavior:

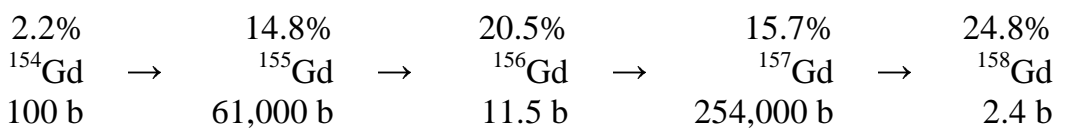

where the natural isotopic abundances are shown above the element symbols, and the 2,200 m/s neutron capture cross sections are shown below the element symbols. The chain is assumed to end with ${ }^{158} \mathrm{Gd}$ because of its low cross section. The time dependence of the concentrations of each isotope can be described by the following series of coupled differential equations:

$$
\begin{aligned}
& \frac{d N^{154}}{d t}=-N^{154} \sigma^{154} \phi \\
& \frac{d N^{155}}{d t}=N^{154} \sigma^{154} \phi-N^{155} \sigma^{155} \phi \\
& \frac{d N^{156}}{d t}=N^{155} \sigma^{155} \phi-N^{156} \sigma^{156} \phi \\
& \frac{d N^{157}}{d t}=N^{156} \sigma^{156} \phi-N^{157} \sigma^{157} \phi
\end{aligned}
$$

where $N$ represents the concentrations of respective isotopes and $\sigma$ represents the respective neutron absorption cross sections.

This series of equations can be solved analytically and the concentrations of each isotope plotted as a function of time. Average one-group spectrum-weighted cross sections were generated for rods of burnable poison at beginning of life using the GP-TALLY code. Figure 11 shows the macroscopic absorption cross section of the four isotopes of Gd beginning with naturally occurring Gd exposed to a neutron flux of $5 \times 10^{+13} \mathrm{n} / \mathrm{cm}^{2} / \mathrm{sec}$. Figure 12 shows a similar plot with ${ }^{154} \mathrm{Gd}$ and ${ }^{156} \mathrm{Gd}$ initially absent. The residual negative reactivity, for which the macroscopic cross section is used as a measure, is reduced by about a factor of five for the case of the separated isotopes.

Such an analysis can be used for all candidate elements in order to obtain a qualitative picture of the behavior and to provide guidance for the complete analyses. However, for many elements, the number of isotopes initially present and the number of daughters created by radioactive decay become so large as to make an analytical solution impractical. The ORIGEN2 program (ref 11), using onegroup spectrum-weighted cross sections for the burnable poison rods at beginning of life, was used for the subsequent preliminary analyses. As an example, the behavior of samarium as a function of time is shown in Figure 13 for the naturally occurring element and in Figure 14 for pure ${ }^{149} \mathrm{Sm}$. In this case, the advantage is not nearly as great, about a factor of two less residual reactivity for the case of pure ${ }^{149} \mathrm{Sm}$. However, all calculations of this type suffer from several oversimplifications, for example: 


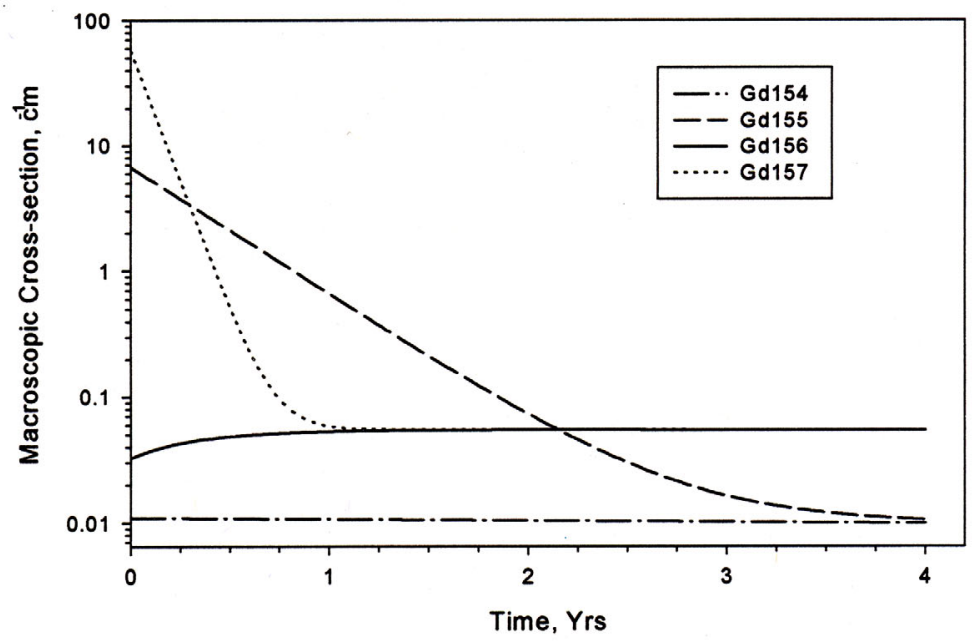

Figure 11. Time-dependent macroscopic cross section of Gd isotopes produced by irradiating naturally occurring Gd.

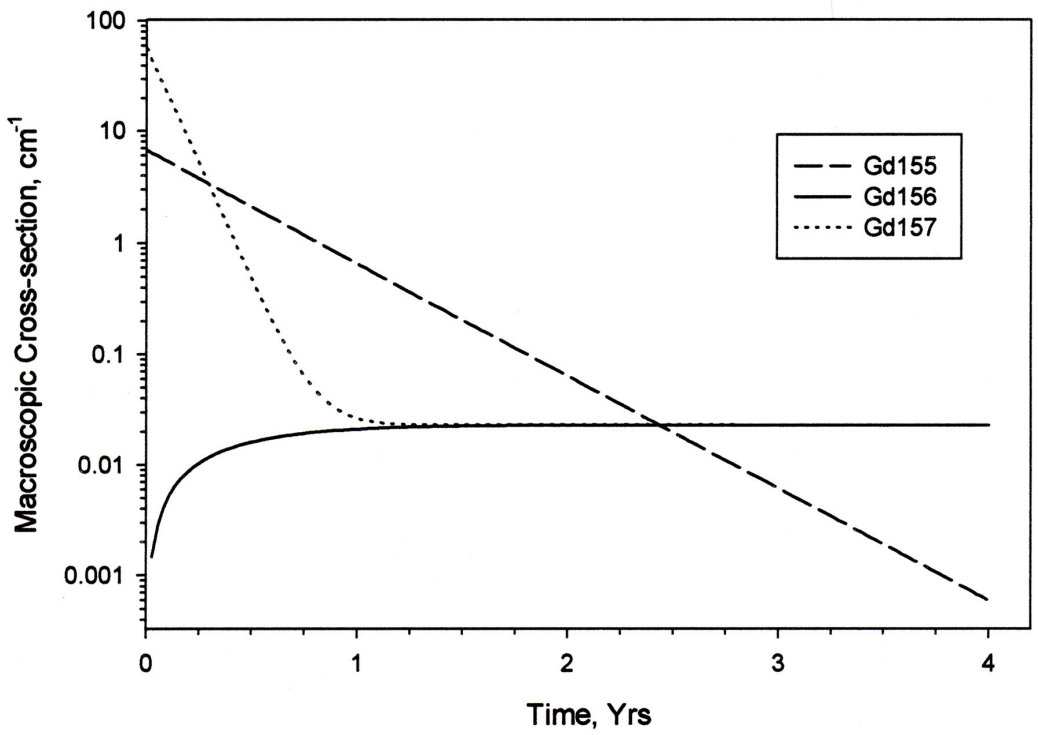

Figure 12. Time-dependent macroscopic cross section of Gd isotopes produced by irradiating $\mathrm{Gd}$ with ${ }^{154} \mathrm{Gd}$ and ${ }^{156} \mathrm{Gd}$ initially removed. 


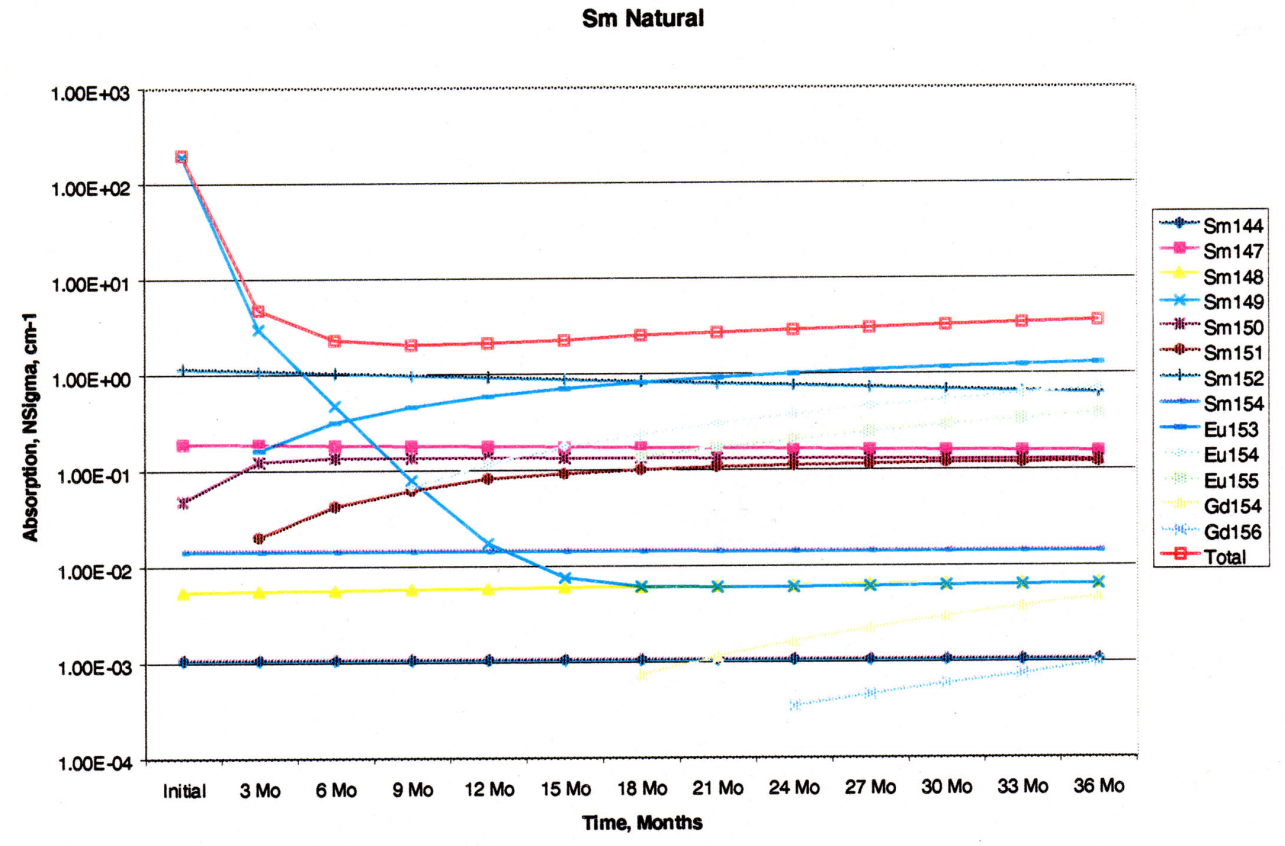

Figure 13. Time-dependent macroscopic cross section of Sm isotopes produced by irradiating naturally occurring Sm.

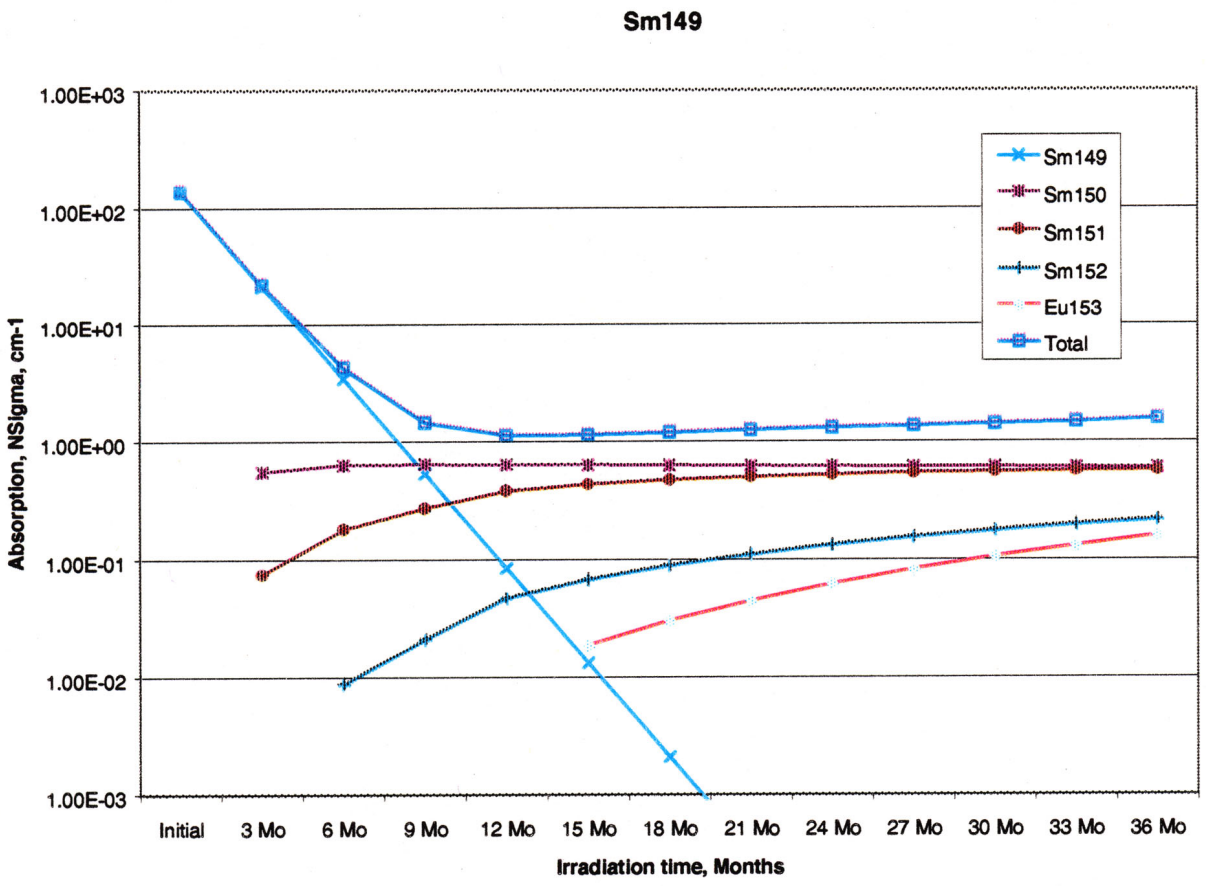

Figure 14. Time-dependent macroscopic cross section of Sm isotopes produced by irradiating pure ${ }^{149} \mathrm{Sm}$. 
1. They assume constant cross sections over the lifetime of the fuel when in fact they vary as the spectrum shifts as the poison burns.

2. The calculation is for one rod only and does not consider the assembly as a whole.

3. Burnout of the fuel is not considered.

Such calculations are useful for guidance, but complex calculations modeling entire detailed assemblies are necessary.

Through the use of both the simple ORIGEN2 calculations and early core-modeling calculations, several stable isotopes were selected for further investigation: ${ }^{149} \mathrm{Sm},{ }^{157} \mathrm{Gd},{ }^{167} \mathrm{Er},{ }^{164} \mathrm{Dy},{ }^{177} \mathrm{Hf},{ }^{151} \mathrm{Eu}$,

${ }^{176} \mathrm{Lu}$. All separated isotopes were modeled as $100 \%$ enriched.

In the cases where the burnable poisons were mixed with fuel or coated on the surface, the burnable poisons were used in the form of sesquioxides, except for $\mathrm{Hf}$, which was used in the form of $\mathrm{HfO}_{2}$. In the case of alloying with the cladding, the burnable poisons were used in the metallic form alloyed with Zircaloy-4. In addition, several calculations of the neutronics performance were done for $\mathrm{ZrB}_{2}$ burnable absorbers for the purpose of comparison. Nondesign nonproprietary dimensions and concentrations were used, although they were made reasonable.

\section{REACTOR NEUTRONICS CALCULATIONS}

Detailed neutronics calculations were performed using state-of-the-art computer codes such as HELIOS version 1.4 and the TALLY-MCNP4C-ORIGEN2.5 Monte Carlo sequence. Initially the two-dimensional HELIOS code version 1.4 (ref 12,13) was used. HELIOS is a neutron and $\gamma$-ray transport code for lattice burnup in generalized two-dimensional geometry. The methods used to obtain fluxes, currents, and number densities were calculation of resonance-shielded microscopic cross sections, calculation of fluxes and currents by the current-coupling collision probability (CCCP) method for particle transport, evaluation of first-flight probabilities, evaluation of the criticality spectrum using the B1 method for the rebalancing of the spectrum of the CCCP solution, and depletion calculations using built-in burnup chains to obtain new number densities after each burnup step. The HELIOS code can be used to calculate detailed neutron spectra in each of the fuel rods. Detailed models of the fuel assemblies can be set up. Each individual fuel rod and burnable poison rod can be specifically incorporated into the computer models. However, fine group cross sections for certain burnable poison isotopes of interest for this study were not available in the HELIOS neutron cross-section libraries. Generation of those cross sections for HELIOS requires proprietary computer codes that are not available to ORNL.

Because of the absence of cross sections for certain burnable poison isotopes of interest in the HELIOS neutron libraries, we switched to the three-dimensional TALLY-MCNP4C-ORIGEN2.5 sequence of computer codes (hereafter called the GP-TALLY code). The generation of pointwise cross sections of the burnable poison isotopes of interest can be performed with the NJOY code (ref 14). This provided more flexibility in investigating the neutronic performance of different burnable poison isotopes. In addition, full three-dimensional models of the fuel assemblies and reactor core could be set up.

The MCNP4C code (ref 15) is a general-purpose Monte Carlo N-Particle code that can be used for neutron, photon, electron, or coupled neutron/photon/electron transport, including the capability to calculate eigenvalues for critical systems. The MCNP4C code can treat any arbitrary three-

dimensional geometry configuration bounded by first- and second-degree surfaces. Pointwise cross sections for different reaction types, such as (n,fission), $(n, \gamma),(n, 2 n),(n, 3 n),(n, p),(n, \alpha)$ and others, 
derived from particular cross-section evaluations such as ENDF/B-VI, are available or can be generated with the NJOY code (ref 14). The thermal scattering $S(\alpha, \beta)$ data as a function of temperature are available for water and other moderators of interest.

The ORIGEN2.5 code is an advanced and updated version of ORIGEN2.1 (ref 11,16). The ORIGEN code is widely used in calculations of the depletion, buildup, and decay of materials during neutron irradiation. It uses the matrix exponential method for burnup and decay. It uses spectrum-weighted one-group cross sections for a variety of neutron reaction types such as (n,fission), $(n, \gamma),(n, 2 n)$, $(n, 3 n),(n, p),(n, \alpha)$ and others. Using different neutron spectra for each of the different regions in the fuel, the burnable poison, and fuel assemblies, region-dependent spectrum-weighted cross sections were used during depletion. The different isotopes in ORIGEN are subdivided into three distinct groups: activation, actinides, and fission products. In order to differentiate the depletion, transmutation, and reactivity worths of the burnable absorbers such as samarium, gadolinium, erbium, and others from the fission products, all the burnable poison isotopes and their decay daughters were placed into the activation products group.

Some of the differences between ORIGEN2.5 and the standard version of ORIGEN2.1 can be summarized as follows. The ORIGEN2.1 code uses ENDF/B-V fission product yields for a limited number of actinides, such as ${ }^{235} \mathrm{U},{ }^{238} \mathrm{U}$, and ${ }^{239} \mathrm{Pu}$. The ORIGEN2.5 code uses ENDF-B/VI fission product yields for 34 actinides, including the fission product yields from the spontaneous fission of several actinides. Since the fission product yields depend on the energy of the neutrons that induce fission, the TALLY module can be used to generate spectrum-weighted fission product yields for each of different depletion regions in the fuel. Several hundred nuclides were also added to the basic ORIGEN libraries. They relate mostly to short-lived isotopes. Note that these capabilities in ORIGEN2.5 were previously implemented in other projects related to the burnup or generation of minor actinides in special-purpose reactor cores or targets.

In the burnable absorber performance studies, each of the fuel and burnable-absorber regions was individually modeled. The depletion of the fuel and burnable poison isotopes was individually followed for each of the different regions of each fuel rod as a function of burnup time. The reactivity worths of the poisons and their daughters were also determined as a function of time. The fuel pellets of the rods containing burnable poison were radially subdivided into three or six equal volume regions. This enabled us to follow the self-shielding effects and depletion in the fuel pellets of each fuel rod due to the presence of the burnable poison isotopes. Typically 27 depletion time points for each full-power-year (FPY) were used. However, in the first year, additional depletion steps were used to better track the larger depletion rates of the burnable poisons at BOL. In most cases a fuel lifetime of 4 years at full power was used. Several additional studies were made for longer fuel lifetimes ( 5 years at full power). At BOL of the fuel, four small depletion time steps of 6 hours each were taken in order to account for equilibrium xenon. The time steps were chosen such that, by doubling the total number of time steps (each time was further subdivided into two equal parts), the change in the residual reactivity worth fraction was less than $2 \%$. Because of the massive amounts of data that were generated at each depletion time step, and in order to reduce the size of the burnupdependent data files, only the data for every nine time points were kept to make tables and figures for this report. While typically 27 depletion time points were used for each FPY, only the data for 3 points were kept per FPY. The GP-TALLY code keeps track of approximately 1,100 individual nuclides for each depletion zone at each depletion time step.

The TALLY module can be considered as a driver, a pre-processor, and a post-processor for the MCNP4C and ORIGEN2.5 modules. It sets up all the necessary inputs to the MCNP4C code that are used for the calculations of the eigenvalue, neutron fluxes, and neutron reaction rates. TALLY also updates all the number densities in the inputs to the MCNP4C and ORIGEN2.5 modules at each 
burnup time step and for each depletion zone. Typically, the TALLY module sets up the necessary MCNP4C "tally" input for each depletion zone and for approximately 200 individual isotopes. This includes all the actinides and all the important fission products. In addition, TALLY sets up the tally input for each of the burnable poison isotopes and their daughters in each of the burnable absorber regions.

The MCNP4C calculated fluxes and spectrum-weighted one-group reaction rates for each of the different fuel and burnable absorber zones are placed into a "tally" data file. This file is then read by the TALLY module. Using the fission rates and their associated power densities and volumes, the TALLY module then normalizes the fluxes to achieve a "user requested" reactor or fuel assembly power, which can be changed at each depletion time step. The neutron fluxes and reaction rates for each isotope and for each depletion zone are then used by TALLY to calculate the region-dependent (n,fission), $(\mathrm{n}, \gamma),(\mathrm{n}, \mathrm{p}),(\mathrm{n}, 2 \mathrm{n}),(\mathrm{n}, 3 \mathrm{n}),(\mathrm{n}, \alpha)$ neutron spectrum-weighted cross sections. If available, the $(\mathrm{n}, \gamma)$ neutron spectrum-weighted cross section into the metastable state of the daughter nuclide was also calculated in TALLY for use in ORIGEN2.5. If needed, TALLY recalculates the spectrumweighted fission product yields for each of the actinides and for each of the fuel regions. The different one-group weighted cross sections for six reaction types are then used by the TALLY module to set up the ORIGEN cross-section libraries and the inputs for a multitude of different ORIGEN2.5 runs, including isotope concentrations (approximately 1,600 isotopes for each depletion zone). After depletion and/or decay, the ORIGEN2.5 code generates updated isotope concentrations for the fuel and burnable absorbers for each of the depletion zones. These are then used by TALLY to update the MCNP4C input of the isotope concentrations. For a fuel assembly with 264 fuel rods, 104 of them containing burnable poisons and a radial subdivision of the fuel into three radial depletion zones, and if fuel assembly material symmetry can be taken into account, a total of approximately 150 depletion zones (each containing more than 200 isotopes) are followed per fuel assembly for each axial zone. Thus at each depletion time step, typically a total of 150 ORIGEN2.5 runs were performed for each axial zone. Assuming six different reaction types for each isotope, TALLY typically sets up the input for approximately $150 \times 200 \times 6=180,000$ different tallies. Because of the limitation on the number of individual tallies that can be set up in one input deck to the MCNP4C code, the input for all the requested tallies is placed within one major neutron tally, into which the sub-tallies for each depletion zone, each isotope, and each reaction type are staged. This TALLY-MCNP4C-ORIGEN2.5 procedure is then repeated for each of the different depletion time steps. Although TALLY has the capability to set up the necessary inputs for photon tallies, this option was not used in the neutronics performance studies of the burnable absorbers. The photon option was previously implemented in ${ }^{238} \mathrm{Pu}$ production studies, in which the effect of the $(\gamma, \mathrm{n})$ reactions in generating parasitic ${ }^{236} \mathrm{Pu}$ was investigated.

It was found that the calculation of 180,000 sub-tallies increased the MCNP4C computer times substantially. In some cases the eigenvalue calculations required central processing unit (CPU) times that were an order of magnitude greater than the CPU times for the same MCNP case but with no tallies. To circumvent this major disadvantage, a new feature was added to the TALLY code. At each depletion time step, TALLY accessed the point-wise cross sections for each of the different isotopes and depletion zones in the model. A unified energy structure was then set up that included all the point-wise energy points, including all the energies that describe the cross sections and each of the resonances for the different reaction types of interest. Typically, a unified neutron energy grid contained approximately 15,000 energy points. The TALLY code then added to the MCNP input a neutron flux tally input containing these 15,000 energy groups. The MCNP4C CPU requirements with this very fine neutron group tally was only slightly higher than the CPU requirements for the MCNP4C cases with no tallies. After completion of an MCNP4C eigenvalue calculation, the TALLY code accessed the MCNP4C tally file, and each of the MCNP4C cross-section files that were used in the MCNP4C problem. TALLY then generated spectrum-weighted one-group cross sections for each 
reaction type, each isotope, and each depletion zone. This feature also enabled us to determine the reactivity worths of each of the burnable absorber isotopes and their daughters.

In this study, we have not analyzed the change in the control rod worth due to the presence of burnable poisons. The loading of burnable poisons result in a shift of the maxwellian spectrum to higher energies, which might change the effective absorption cross section of control rods. In addition, if the control rod worth depends to a considerable extent upon resonance absorption, then the worths might also change with the loading of burnable absorbers.

In phase one of the project, we have strictly performed assembly lattice calculations. The reactor core was filled with the same fuel assemblies, and fuel assembly reshuffling in the reactor core was not performed. An average soluble boron content of $550 \mathrm{ppm}$ was used. The density of the coolant at power was $0.7795 \mathrm{~g} / \mathrm{cm}^{3}$. Typically, the number of source particles per generation was 3,000 to 25,000 , and the number of generations was 250 to 500. The statistical standard deviation (STD) for $\mathrm{k}_{\text {eff }}$ was approximately $0.1 \%$ (at one sigma confidence level). The STD for the weighted cross sections was approximately 1 to $3 \%$.

The MVCs were calculated by decreasing the water coolant density by $10 \%$. The one-sigma STDs of the eigenvalues were better than $0.03 \%$. The MVCs were determined using two different approaches. In the first approach, the "generation" eigenvalues were followed for the MCNP4C run containing the nominal water density at full-power conditions and 2,200 psia, and in the second MCNP4C run the "generation" eigenvalues were followed for the same MCNP4C case, but with a water density reduction of $10 \%$. In the second approach, the "perturbation" option in MCNP4C was used, in which the water density was reduced by $10 \%$. The MCNP4C option with first- and second-order perturbation was used. The differences between the two approaches were usually small and within one STD. 


\section{RESULTS OF ANALYSIS}

\section{NEUTRONICS PERFORMANCE RESULTS FOR ZrB}

In the cases where boron is used as a burnable poison in fuel rods, only the isotope ${ }^{10} \mathrm{~B}$ has a significant absorption $(n, \alpha)$ cross section, and ${ }^{10} \mathrm{~B}$ does not transmute into high absorbing isotopes. Of more concern, however, is the production of helium via the ${ }^{10} \mathrm{~B}(\mathrm{n}, \alpha){ }^{7} \mathrm{Li}$ reaction. The production of helium gas increases the internal pressure of the fuel rods. It is this disadvantage that provides part of the incentive for this project. The methods of choice in most PWRs are the use of $\mathrm{B}_{4} \mathrm{C}$ in separate non-fueled rods, and more recently, the use of a thin coating of $\mathrm{ZrB}_{2}$ surrounding the radial surface of the fuel pellets in a number of fuel rods.

Neutronic performance calculations for $\mathrm{ZrB}_{2}$ burnable absorber were done as part of this project so they could be used for comparison with the proposed burnable absorbers. A summary of the results for $\mathrm{ZrB}_{2}$ is shown in Table 1. Note that the dimensions and concentrations for $\mathrm{ZrB}_{2}$ are non-design values, since the actual values used in commercial reactors are proprietary. The table displays the initial reactivity worth of the burnable absorber for the entire reactor core. The table also displays the residual reactivity worth fraction of the burnable poison at EOL of the fuel. The fraction is defined as the ratio of the negative reactivity worth of the burnable absorber isotopes and their daughters after 4 years at full power, to the initial negative reactivity worth of the burnable poison at EOL of the fuel.

For a fuel assembly with 104 IFBA rods and $30 \mathrm{wt} \%{ }^{10} \mathrm{~B}$-enriched boron, the residual reactivity ratio is $2.404 \times 10^{-4}$. The initial reactivity worth $\left(\delta \mathrm{k}_{\mathrm{eff}} / \mathrm{k}_{\mathrm{eff}}\right)$ of the $\mathrm{ZrB}_{2}$ was $-1.279 \times 10^{-1}$. The residual reactivity worth is mostly due to the zirconium in the $\mathrm{ZrB}_{2}$ compound. This residual reactivity worth ratio is very small, and thus negligible.

Table 1 also shows the effect of using the same $\mathrm{ZrB}_{2}$ coating over a smaller number of IFBA rods, but the residual reactivity ratios are similar even though the initial reactivities are smaller. However, if there is a requirement of having the same total initial reactivity worth spread over a smaller number of rods, then the ratio becomes larger, since the poison rods would have been more self-shielded, and the burnout rate would thus have been smaller. The use of natural boron increases the residual reactivity ratio only very slightly $\left(3.29 \times 10^{-4}\right.$ vs $\left.2.40 \times 10^{-4}\right)$. Note that the residual reactivity ratios are very small for all the cases that use $\mathrm{ZrB}_{2}$ as BP.

If the thickness of the $\mathrm{ZrB}_{2}$ coating is a limiting factor due to heat transfer considerations, then the use of enriched boron would require a smaller number of IFBA rods in order to achieve a given initial reactivity worth of the burnable absorber. This could reduce fabrication cost of the fuel. However, the cost of boron increases. Also, the amount of ${ }^{4} \mathrm{He}$ generation per fuel rod will be higher if the boron is distributed over fewer rods.

Tables 2 and 3 show detailed results for the $\mathrm{ZrB}_{2}$ case with 104 IFBA rods and a ${ }^{10} \mathrm{~B}$ enrichment of $30 \mathrm{wt} \%$. Note that the reactivity worths of the isotopes into which $\mathrm{ZrB}_{2}$ transmutes are very small. For this case, with an initial total loading of 30.7 grams ${ }^{10} \mathrm{~B}$, the ${ }^{4} \mathrm{He}$ production after four full-poweryears (FPYs) is approximately 13.7 grams per fuel assembly. Tables 4a, 4b, and 4c display the relative fission power density maps at one full-power-day (FPD), one FPY, and four FPYs, respectively. For each IFBA location, the tables display four values. The first three values represent the relative fission power densities for the inner one-third, the middle one-third, and the outer onethird of the fuel pellets that are radially surrounded by the $\mathrm{ZrB}_{2}$ coatings. The fourth value represents the relative fission power densities for the burnable absorber $\mathrm{ZrB}_{2}$ coating (which in this case should be zero). This loading provides a fairly flat power distribution over the fuel life, so that the absolute 
rod power density (fuel assembly relative power density multiplied by the relative fuel rod power density) does not violate thermodynamics limits. At BOL of the fuel, the fuel in the IFBA rods is partially self-shielded, and less fission power is thus generated than in the neighboring fuel rods. Toward EOL of the fuel, the relative power is higher since more fissile materials are still present in the fuel of these IFBA rods than in the regular fuel rods.

Figures 15-19 display the values of the reactivity worth of the burnable poison isotopes and their daughters as a function of fuel depletion time. The figures also show the values of the eigenvalues with and without BP. Since ${ }^{10} \mathrm{~B}$ does not transmute into higher absorption isotopes, and because of the use of a large number of IFBA rods, the rate of change of $\delta \mathrm{k}_{\mathrm{eff}} / \mathrm{k}_{\mathrm{eff}}$ of the burnable absorbers is almost linear as a function of depletion time and levels off because of the presence of the zirconium in the $\mathrm{ZrB}_{2}$ coating. The value of $\delta \mathrm{k}_{\text {eff }} / \mathrm{k}_{\text {eff }}$ at which the burnable poison worth levels off is very small and negligible.

Figures 15 and 16 display the results for 104 IFBA rods per fuel assembly for $\mathrm{ZrB}_{2}$ coatings containing natural boron and enriched boron $\left(30 \mathrm{wt} \%{ }^{10} \mathrm{~B}\right)$ respectively. Figures 17 and 18 display the results for 64 IFBA rods per fuel assembly for two different thicknesses of the $\mathrm{ZrB}_{2}$ coatings. Figure 19 displays the results for 16 IFBA rods per assembly.

Figures 15-19 also display the masses of the isotopes of the burnable poison compound and its daughters as a function of depletion time. Note how the ${ }^{4} \mathrm{He}$ builds up rapidly during the first 200 FPDs (approximately 7,350 MWD/MTU burnup). Figure 20 displays the masses of the actinides as a function of fuel burnup. The ratio of fissile to total $\mathrm{Pu}$ is also shown on the right side of the graph.

Because of a relatively fast burnout, the use of $\mathrm{ZrB}_{2}$ coatings limits the time over which the burnable absorbers will have a strong negative reactivity worth, and thus it could not be used for very long cycle times. Mixing of a boron compound inside the fuel pellets suffers from the disadvantage of creating additional gas within the pellet. Comparison of the results for the different cases for $\mathrm{ZrB}_{2}$ displayed in Table 1 show that the initial reactivity worths of the burnable poison are almost proportional to the amount of ${ }^{10} \mathrm{~B}$ in the fuel assembly. The effect of self-shielding by the BP is thus relatively small. Self-shielding could be increased by spreading the $\mathrm{ZrB}_{2}$ in thicker coatings inside fewer IFBA rods. If the thickness of the coating is a limiting factor, then highly enriched boron could be used. However, this increases cost, and also increases the internal pressure in the IFBA rods due to the generation of an increased amount of ${ }^{4} \mathrm{He}$. The use of a sufficient amount of enriched burnable absorber isotopes of gadolinium, samarium, erbium, and others, mixed inside the pellets in a sufficient quantity to create a larger self-shielding effect to delay burnout, together with a $\mathrm{ZrB}_{2}$ coating, might extend appreciably the lifetime over which combined burnable absorbers will have a large negative reactivity worth. However, for cycle times of the order of 1 to 2 years, a combined IFBA is not needed or attractive, but it might be advantageous to use it when very long cycle times with no fuel reloads are required. In this study we have restricted ourselves to analyses of single burnable poisons. No analyses were performed for combined burnable absorbers.

The MVCs were calculated by decreasing the water coolant density by $10 \%$. The one-sigma STDs of the eigenvalues were better than $0.03 \%$. Note that for all the cases displayed in Table 1, the MVCs remain negative. For comparison purposes, we also have displayed the MVC for a case with no BP (see case B-none). 


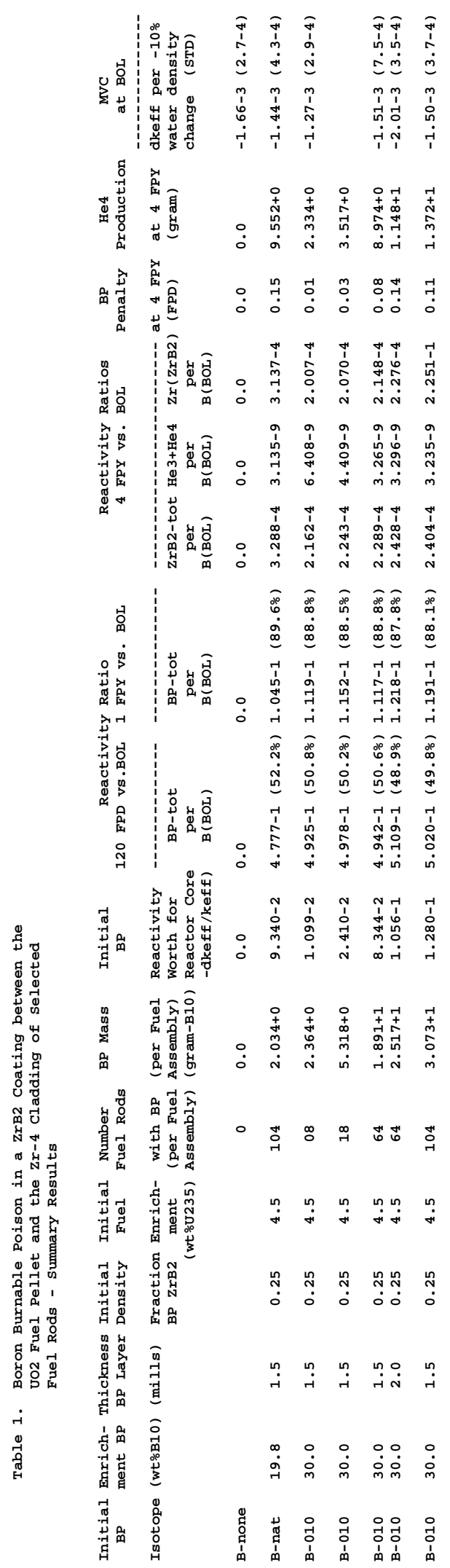




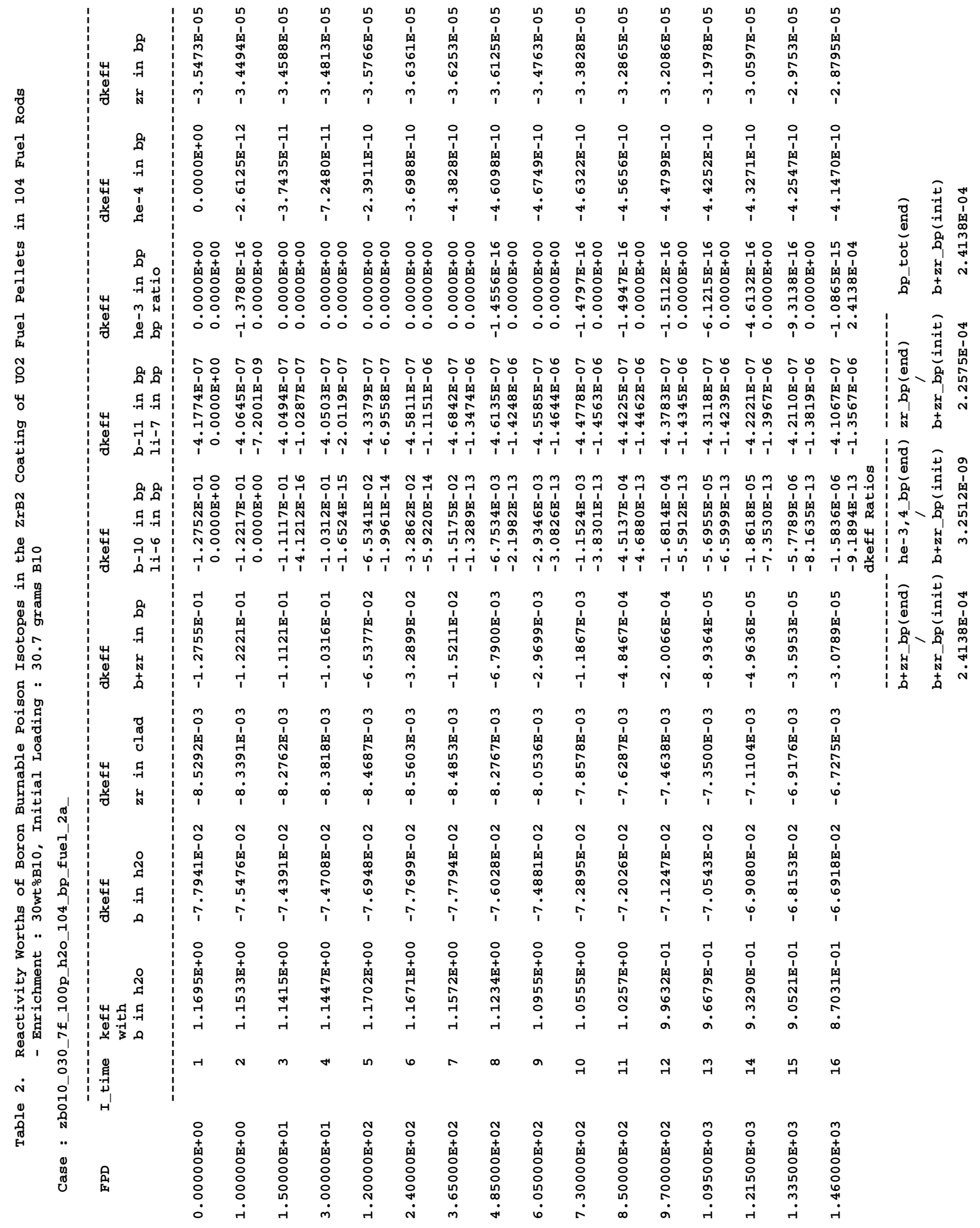




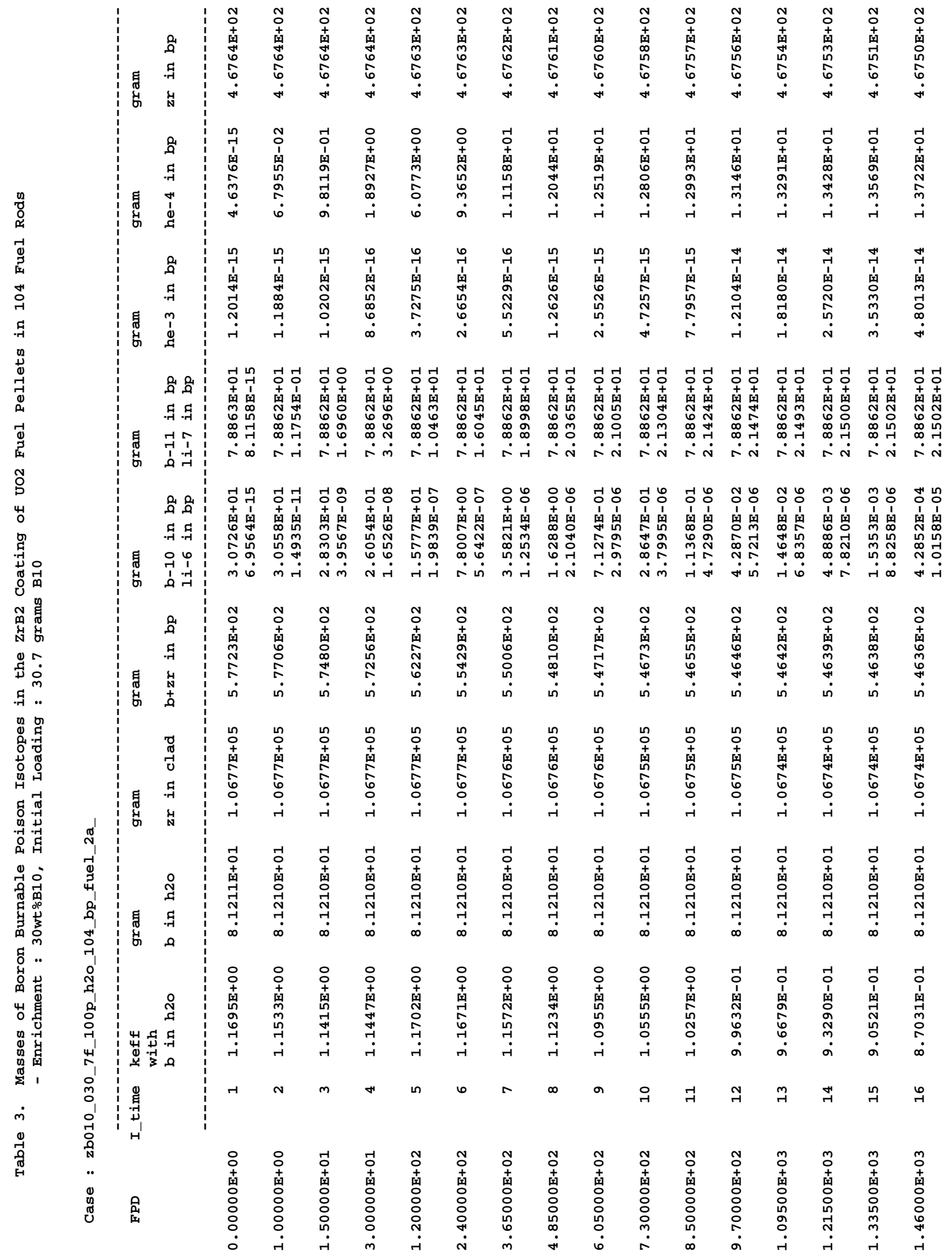




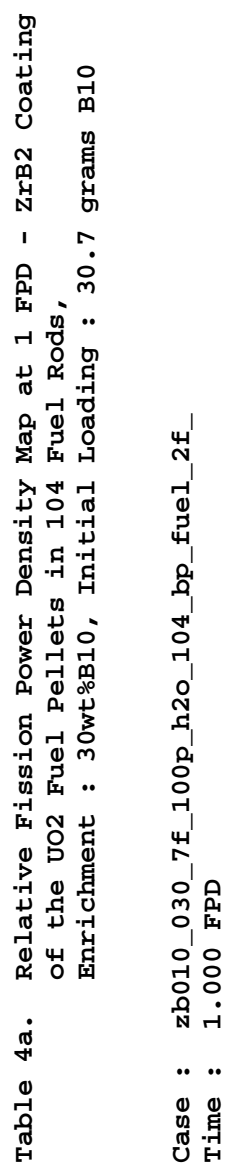

\begin{tabular}{|c|c|c|c|c|c|c|c|}
\hline 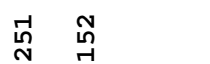 & ูู & $\underset{\sim}{\stackrel{H}{H}}$ & $\underset{H}{ت}$ & ने & ڤn & م & 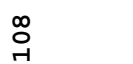 \\
\hline 4. & ri & ri & r & ri & - & i & ri \\
\hline స్ & $\hat{N}$ & พั & $\stackrel{\infty}{N}$ & 총ํㅇㅇํㅇ융 & N & $\hat{N}$ & 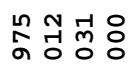 \\
\hline O & - & i & - & ن ن & i. & i & orio \\
\hline స̃ & :े & ํํㅇํํㅇㅇㅇ & ㅇำ & & ํํํํํํํㅇㅇㅇ & 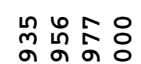 & \\
\hline i & $\stackrel{0}{i}$ & 00 & :0 : & & 00 & 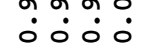 & \\
\hline ปี & ํํㅇํㅇㅇㅇㅇ & & 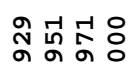 & 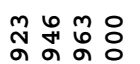 & กั & กู & 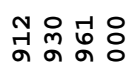 \\
\hline -i & 000 & & $\therefore 00$ & 000 & $\div$ & ! & $\because 000$ \\
\hline$\stackrel{\substack{N \\
\text { N }}}{ }$ & ํำล์ & 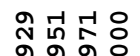 & : & 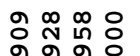 & $\stackrel{\infty}{\circ}$ & ผู & 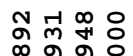 \\
\hline ن. & 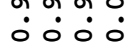 & 1000 & ?. & 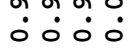 & 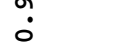 & $\stackrel{?}{0}$ & : \\
\hline 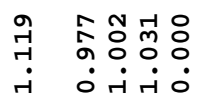 & & 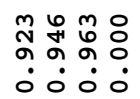 & 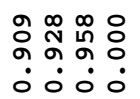 & & 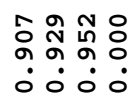 & 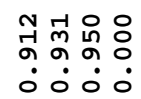 & \\
\hline$\stackrel{N}{N}$ & 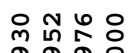 & กั & $\stackrel{\infty}{n}$ & ำํำ ํํㅇㅇํㅇ & กิ & $\hat{n}$ & 잉요 \\
\hline - & 000 & 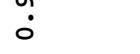 & $\dot{0}$ & $\ddot{0} 000$ & $\stackrel{0}{0}$ & 0 & 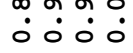 \\
\hline Ñ & 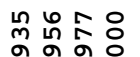 & ถู & ถู & 긍ำ & กิ & : & 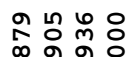 \\
\hline ت. & 0000 & $\because$ & $\ddot{0}$ & 0000 & $\because$ & $\because$ & 0000 \\
\hline 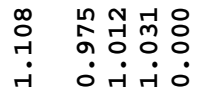 & & 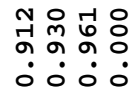 & 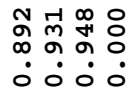 & & 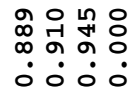 & 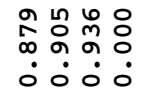 & \\
\hline స̃ & ભํํํํํํㅇㅇㅇ & ถู & ถู & 긍ำ & กิ & : & 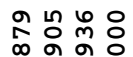 \\
\hline ت. & 000 & $\dot{0}$ & $\circ$ & 000 & $\circ$ & $\dot{0}$ & 0000 \\
\hline ల్ల & 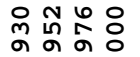 & กั & $\stackrel{\infty}{\stackrel{\infty}{\alpha}}$ & 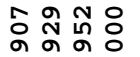 & กू๊ & กू & 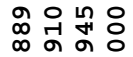 \\
\hline -i & 0000 & $\dot{0}$ & $\dot{0}$ & 0000 & $\dot{0}$ & $\dot{0}$ & $00^{\circ} 0^{\circ}$ \\
\hline 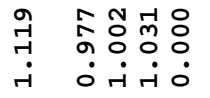 & & 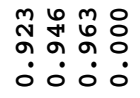 & 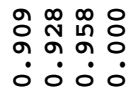 & & 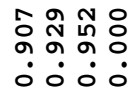 & 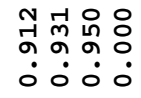 & \\
\hline 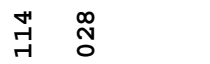 & ด้ำลั & ลู่ & ๕̊ & 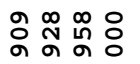 & $\stackrel{\infty}{\stackrel{\infty}{\alpha}}$ & ถู & 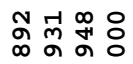 \\
\hline -i & 0000 & 0000 & $\because$ & 0000 & $\div$ & $\dot{0}$ & 0000 \\
\hline స్ & 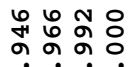 & & 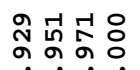 & 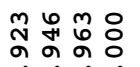 & ్ㅗ & กू & 긍ํํㅇㅇㅇ \\
\hline -i & 0000 & & 0000 & 0000 & $\circ$ & $0^{\circ}$ & 0000 \\
\hline సิ & \&े & సี้ & 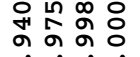 & & 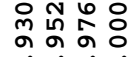 & ભू̆ & \\
\hline - & ri & 0000 & $0000^{\circ}$ & & 0000 & $00^{\circ} 0^{\circ}$ & \\
\hline స̆ & ヘิ & กิ & 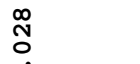 & 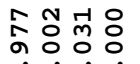 & 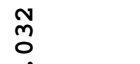 & $\stackrel{\text { ก }}{0}$ & 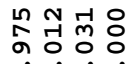 \\
\hline ०० & - & - & -i & $\dot{0}$ - & - & - & 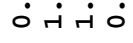 \\
\hline กี & $\stackrel{7}{7}$ & $\underset{\sim}{ت}$ & $\underset{7}{\pi}$ & ने & 号 & ณू & 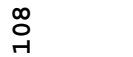 \\
\hline i & -i & ri & $\vec{r}$ & ن & मं & $\dot{r}$ & ri \\
\hline
\end{tabular}




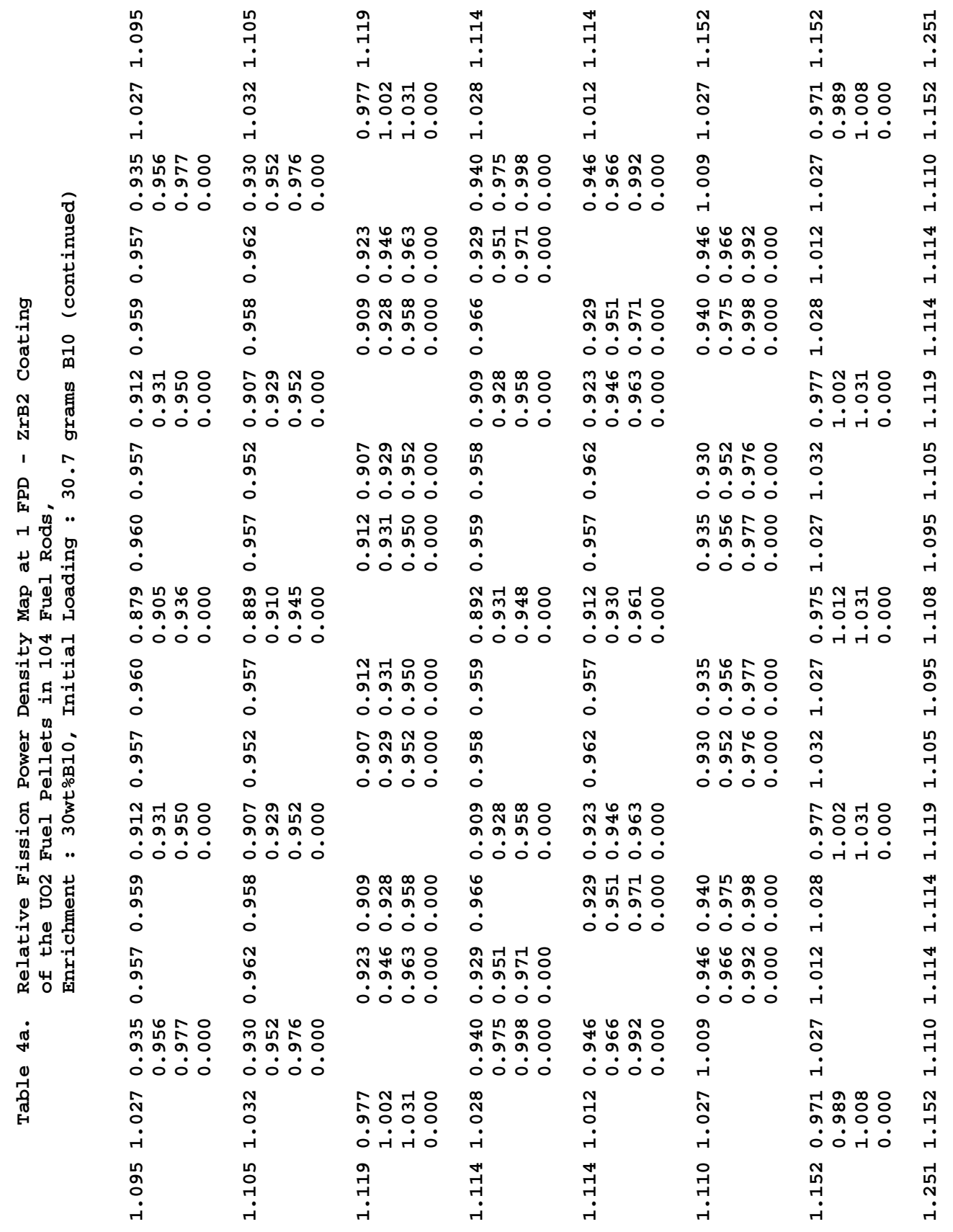




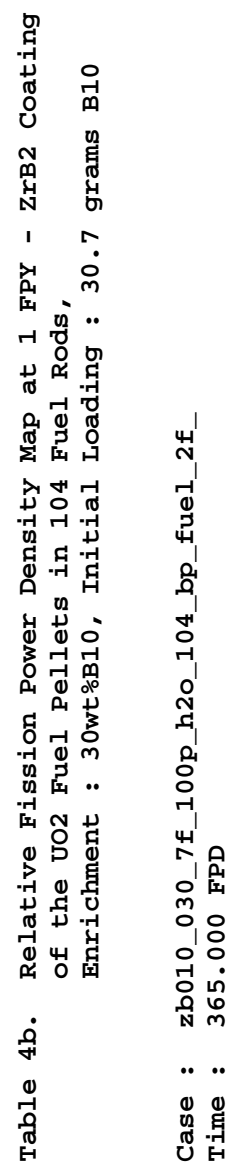

\begin{tabular}{|c|c|c|c|c|c|c|c|}
\hline $\begin{array}{l}\infty \\
\stackrel{-}{\sim} \\
\text { - }\end{array}$ & ฝี & $\stackrel{n}{m}$ & $\stackrel{\circ}{\sim}$ & $\stackrel{\infty}{m}$ & $\stackrel{n}{m}$ & $\stackrel{n}{m}$ & $\begin{array}{l}\text { N゙ } \\
\text { ON }\end{array}$ \\
\hline -i & -i & ri & ri & ri & -i & -i & -i \\
\hline 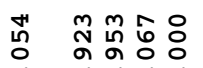 & oิ & $\begin{array}{l}\circ \\
\text { o } \\
\sigma\end{array}$ & ตั & 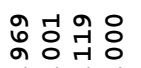 & ลั & ตั & 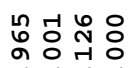 \\
\hline$\dot{0} 0 \dot{0}$ & $\dot{0}$ & 0 & $\dot{0}$ & 0 ન ન & $\dot{0}$ & $\dot{0}$ & ن \\
\hline mo & ล̃ & 꿍ㅇㅇㅇㅇㅇㅇ & 초옹 & & 공ํㅇㅇㅇ & 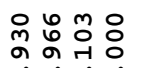 & \\
\hline $0^{\circ}$ & $\circ$ & 0 ન & 0 ت & & $\dot{0} 0$ - & $\dot{0} 0$ - & \\
\hline$\stackrel{n}{m}$ & 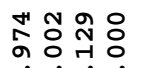 & & 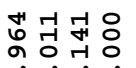 & 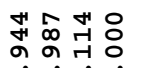 & $\frac{0}{5}$ & 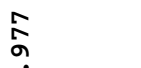 & ฝึ ڤ̆ \\
\hline 0 & 0 ન ન & & 0 & 0 0 न & 0 & 0 & ن \\
\hline ถู & 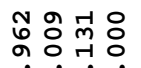 & 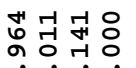 & न & 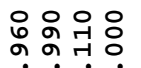 & $\begin{array}{l}\infty \\
\vdots \\
\sigma\end{array}$ & $\begin{array}{l}6 \\
\text { ă }\end{array}$ & ㅊํํㅇํㅇㅇํㅇ \\
\hline 0 & 0 & 0 & - & 0 0 & 0 & 0 & ن \\
\hline 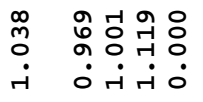 & & 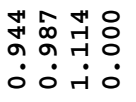 & 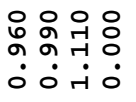 & & 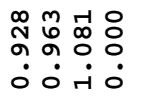 & 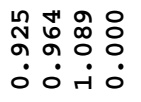 & \\
\hline ล̆ & 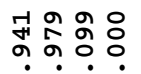 & $\begin{array}{l}0 \\
\stackrel{0}{a} \\
\sigma\end{array}$ & $\begin{array}{l}\infty \\
\stackrel{\infty}{0} \\
\text { ด̆. }\end{array}$ & $\begin{array}{l}\infty \\
\text { N } \\
\text { ŏ } \\
\circ\end{array}$ & חू & กั & 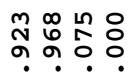 \\
\hline 0 & 00 - & $0^{\circ}$ & 0 & $\dot{0} 0$ - & $0^{\circ}$ & 0 & $\dot{0} 0 \dot{0}^{\circ}$ \\
\hline ตั. & 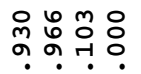 & ấ & $\begin{array}{l}\circ \\
\stackrel{0}{\circ} \\
\text { ?. }\end{array}$ & 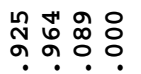 & กั & 웅 & 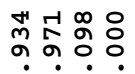 \\
\hline $0^{\circ}$ & ० 0 - & 0 & $\dot{0}$ & 0 0 & $\dot{0}$ & $\dot{0}$ & 00 - \\
\hline 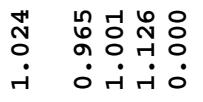 & & 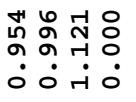 & 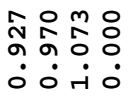 & & 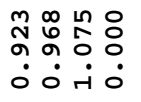 & 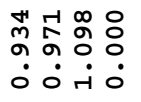 & \\
\hline ตั & 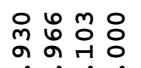 & ล̃ & $\begin{array}{l}\circ \\
\text { ด̆ }\end{array}$ & 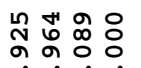 & กั & 号 & ભ゙ 동ㅇㅇ \\
\hline $0^{\circ}$ & 0 0 न & $0^{\circ}$ & $\dot{0}$ & 0 0 & 0 & 0 & $\dot{0} 0 \dot{0}$ \\
\hline ลิ & 걱옹ㅇㅇ & $\frac{0}{5}$ & $\begin{array}{l}\infty \\
0 \\
\sigma\end{array}$ & $\begin{array}{l}\infty \\
\text { N } \\
\text { ŏ }\end{array}$ & ตั & กั & 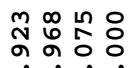 \\
\hline$\dot{0}$ & ० ० न & $0^{\circ}$ & $\dot{0}$ & 0 0 & $0^{\circ}$ & $0^{\circ}$ & 00 ० \\
\hline 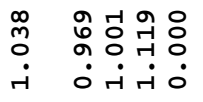 & & 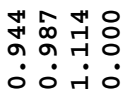 & 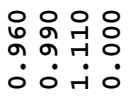 & & 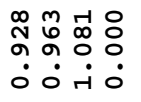 & 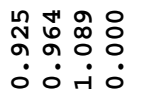 & \\
\hline ถั & 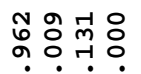 & 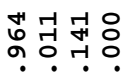 & r. & 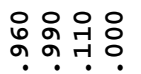 & $\begin{array}{l}\infty \\
\stackrel{0}{\sigma} \\
\sigma\end{array}$ & $\begin{array}{l}\circ \\
\stackrel{\circ}{ }\end{array}$ & 총옹ㅇㅇㅇㅇㅁㅇ \\
\hline 0 & ه ન ન & $\dot{0}$ - & -i & 0 0 & 0 & 0 & $\dot{0} 00^{\circ}$ \\
\hline $\begin{array}{l}\circ \\
\infty \\
o\end{array}$ & 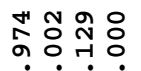 & & 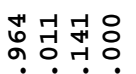 & 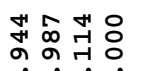 & $\frac{0}{5}$ & ấ & 光 ڤ̆ స్ \\
\hline $0^{\circ}$ & 0 & & 0 & 0 0 & $\dot{0}$ & $\dot{0}$ & 00 - \\
\hline o̊ & 令 & 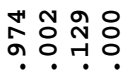 & 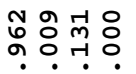 & & 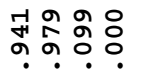 & 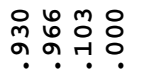 & \\
\hline$\dot{0}$ & $\dot{0}$ & ० & ० & & 0 0 & 0 0 & \\
\hline 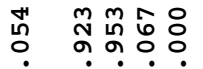 & ô. & \begin{tabular}{l}
$\circ$ \\
$\infty$ \\
$o$ \\
\hdashline
\end{tabular} & ผั & 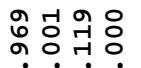 & ลे & ตั & 뉴 겅 ஸ્ \\
\hline 0 0 & 0 & $0^{\circ}$ & $0^{\circ}$ & 0 - & 0 & 0 & ن ن \\
\hline :ึّ & ôn & $\stackrel{\stackrel{n}{m}}{\stackrel{m}{o}}$ & ๙ิ & $\begin{array}{c}\infty \\
m \\
0\end{array}$ & 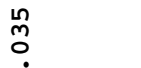 & $\stackrel{\substack{n \\
m}}{o}$ & $\underset{\text { N̦ }}{\text { O̦ }}$ \\
\hline -i & -i & -i & -i & -i & ri & ri & -i \\
\hline
\end{tabular}




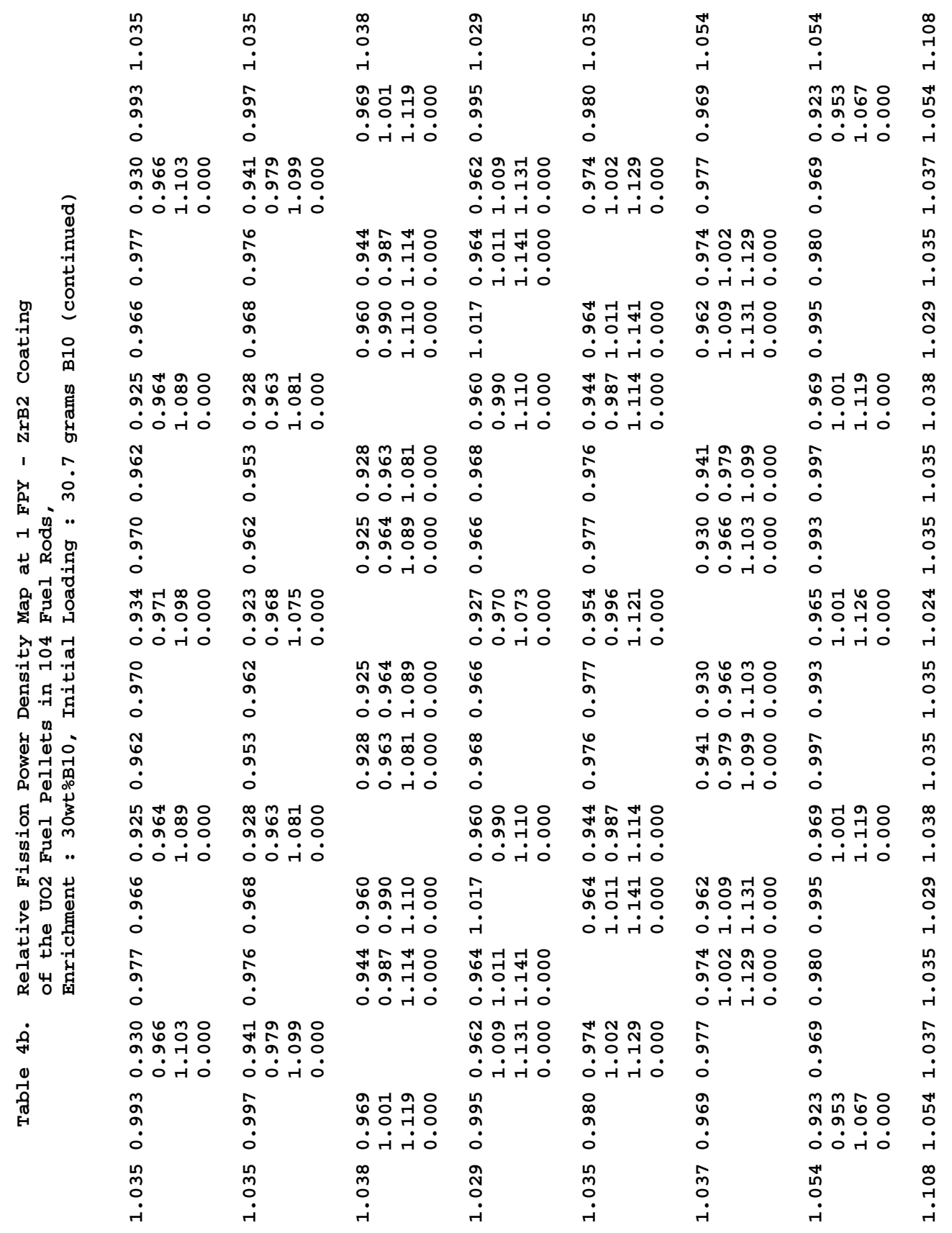




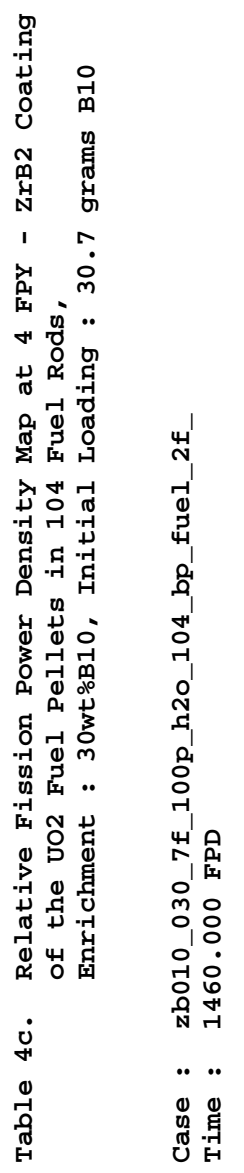

\begin{tabular}{|c|c|c|c|c|c|c|c|}
\hline 옹 & 웅 & $\stackrel{\circ}{\circ}$ & $\begin{array}{l}0 \\
\stackrel{1}{0}\end{array}$ & $\stackrel{n}{\circ}$ & $\begin{array}{l}\infty \\
\stackrel{1}{0}\end{array}$ & $\begin{array}{l}0 \\
\text { - }\end{array}$ & م \\
\hline i & $\dot{r}$ & ri & $\dot{r}$ & -i & $\dot{r}$ & i & 0 \\
\hline 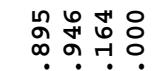 & б̆ & $\stackrel{m}{\circ}$ & 모 & 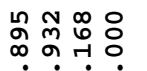 & $\begin{array}{l}0 \\
\text { o } \\
\text { o. }\end{array}$ & oี & 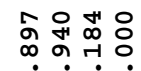 \\
\hline 0000 & 0 & ri & ri & ०० & 0 & $:$ & О \\
\hline बू & ลั & 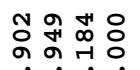 & ન્ન & & 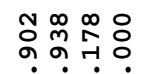 & 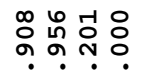 & \\
\hline 0 & $\circ$ & ०० & ० ० & & O० & ०० & \\
\hline$\stackrel{m}{0}$ & 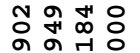 & & 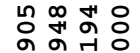 & ㅎํํํํㅇํㅇ응 & $\stackrel{m}{0}$ & స్ & 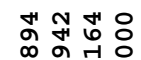 \\
\hline ri & 00 O & & O० & O & i & i & ०० \\
\hline : & 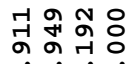 & ڤ̊ํㅇำ & $\stackrel{\infty}{\sim}$ & సี้ & $\stackrel{\text { ก }}{\circ}$ & $\begin{array}{l}\infty \\
\infty \\
\infty\end{array}$ & 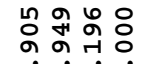 \\
\hline -i & 0 & ० 0 ن & - & 0 0 & - & $\dot{0}$ & ن \\
\hline 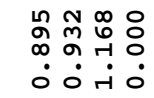 & & 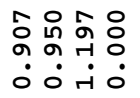 & 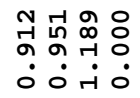 & & 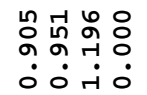 & 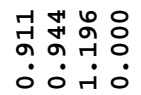 & \\
\hline ఎ & № $\stackrel{\infty}{m} \stackrel{\infty}{\sim}_{\circ}^{\circ}$ & $\stackrel{m}{o}$ & $\stackrel{n}{\circ}$ & ํํㅇำกัㅇㅇㅇ & $\stackrel{\infty}{\infty}$ & s & 끙역ㅇㅇㅇ응 \\
\hline 0 & 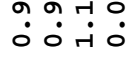 & $\stackrel{\circ}{r}$ & $\stackrel{\circ}{\circ}$ & $\begin{array}{l}0 \\
0 \\
0\end{array}$ & $\stackrel{?}{0}$ & ְ. & 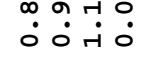 \\
\hline ล̆ & 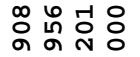 & กิ & $\begin{array}{l}\infty \\
\infty \\
\infty\end{array}$ & નુ & ò & $\stackrel{0}{0}$ & 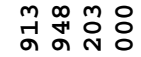 \\
\hline$\dot{0}$ & 00 O & - & $\dot{0}$ & 0 O & $\dot{r}$ & i & 0 \\
\hline 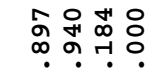 & & 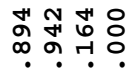 & 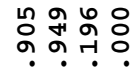 & & 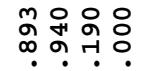 & $\begin{array}{l}m \\
\\
\sigma\end{array}$ & \\
\hline ०० & & 0 ० & 00 - & & 00 - & 00 - & \\
\hline ล̆ & 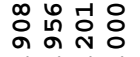 & สิ & $\begin{array}{l}\infty \\
\infty \\
0\end{array}$ & નુ & ò & $\stackrel{0}{\circ}$ & 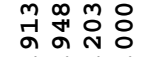 \\
\hline 0 & ० 0 - & -i & 0 & 0 0 & - & $\dot{H}$ & ० ० \\
\hline 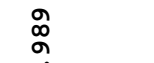 & ㅇํำ & $\stackrel{m}{\circ}$ & $\stackrel{\text { ㅇ }}{\circ}$ & 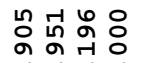 & م્ & : & 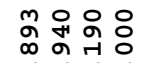 \\
\hline$\circ$ & O० & -i & $\dot{r}$ & ०० Оं० & $\dot{0}$ & i & 0 \\
\hline 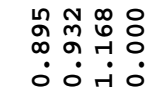 & & 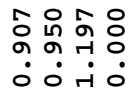 & 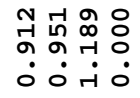 & & 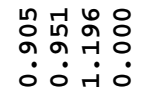 & 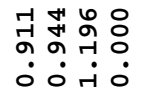 & \\
\hline -1 & 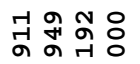 & ด̊̊̆ & $\stackrel{\infty}{\stackrel{\infty}{0}}$ & 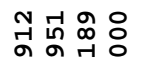 & $\stackrel{n}{\circ}$ & $\begin{array}{l}\infty \\
\infty \\
\infty\end{array}$ & 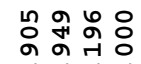 \\
\hline - & 0 0 - & ० 0 ن & - & 0 0 & - & $\dot{0}$ & ن \\
\hline$\stackrel{m}{\circ}$ & 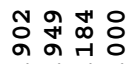 & & 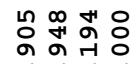 & ㅎํㅇํํㅇํํ융 & $\stackrel{m}{o}$ & సิ & 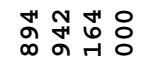 \\
\hline -i & O० & & 00 ० & ०० & i & i & $\dot{0} 0$ \\
\hline ลू & ลू & ํํㅇํำ & નું & & 옹 & 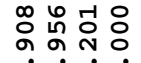 & \\
\hline$\circ$ & $0^{\circ}$ & ० ० न & O O & & O O & ०० & \\
\hline مू & م̆ & $\stackrel{m}{\circ}$ & 요 & 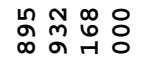 & ळa & ล̆ & 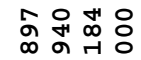 \\
\hline ०० & $\dot{0}$ & - & - & o ० & $\dot{0}$ & $\dot{0}$ & $\dot{0} 0$ - \\
\hline o & $\stackrel{\infty}{\circ}$ & $\stackrel{\circ}{\circ}$ & 옹 & $\stackrel{n}{0}$ & $\stackrel{\infty}{\stackrel{\infty}{0}}$ & 옹 & $\stackrel{5}{\text { a }}$ \\
\hline H & i & -i & $r$ & i & $\dot{r}$ & i & $\dot{0}$ \\
\hline
\end{tabular}




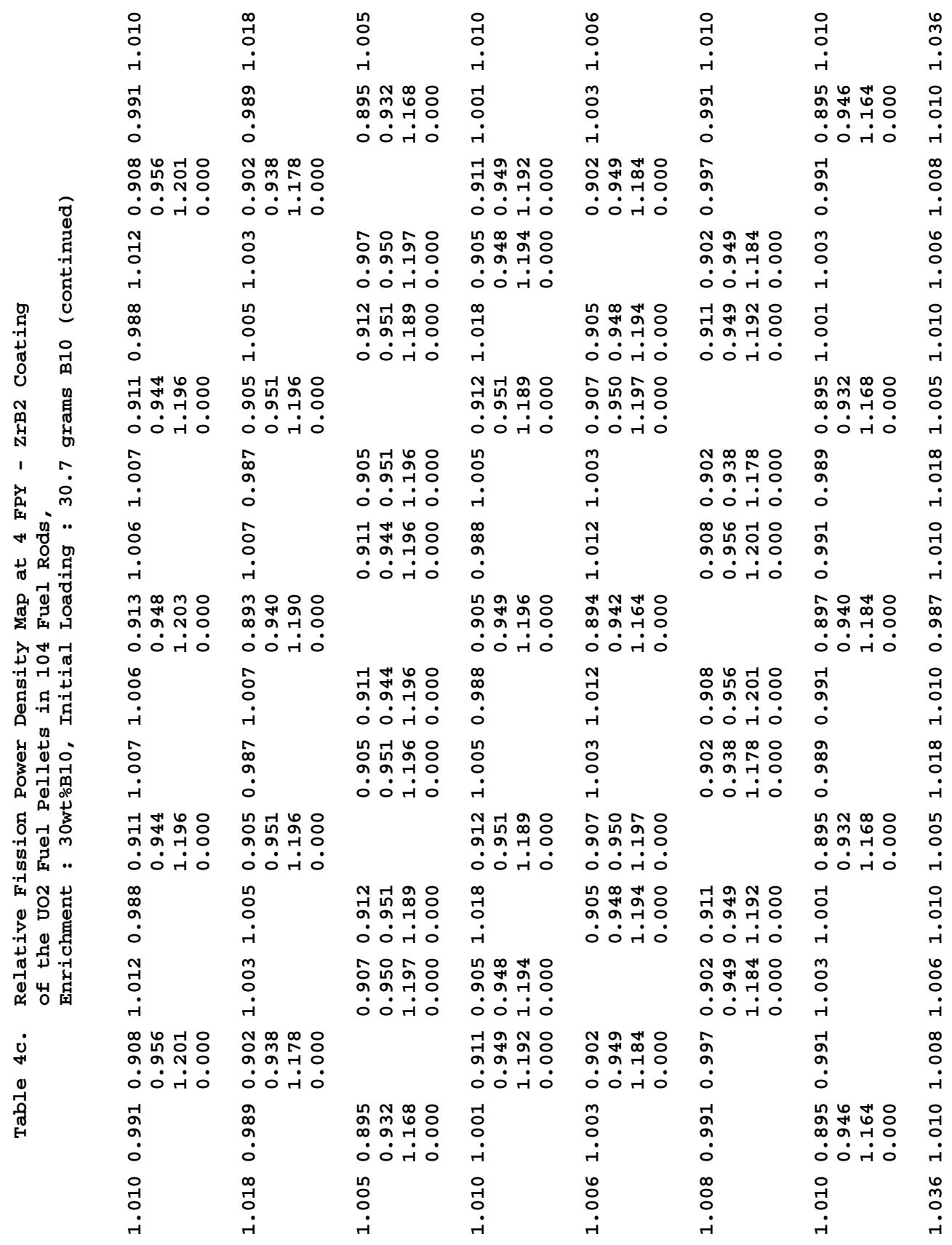


Figure 15. Negative Reactivity and Masses BP for B-nat and transmutation daughters as a function of fuel life for $17 \times 17$ fuel assemblies with 104 poison rods,

1.5 mill thick ZrB2 poison coating on the outer radial surface of the UO2 pellets

Reactor power $3400 \mathrm{MWth}, 193$ fuel assemblies, initial enrichment 4.5wt\%U-235

(Case : zb000_7f_100p_h2o_104_bp_fuel o)
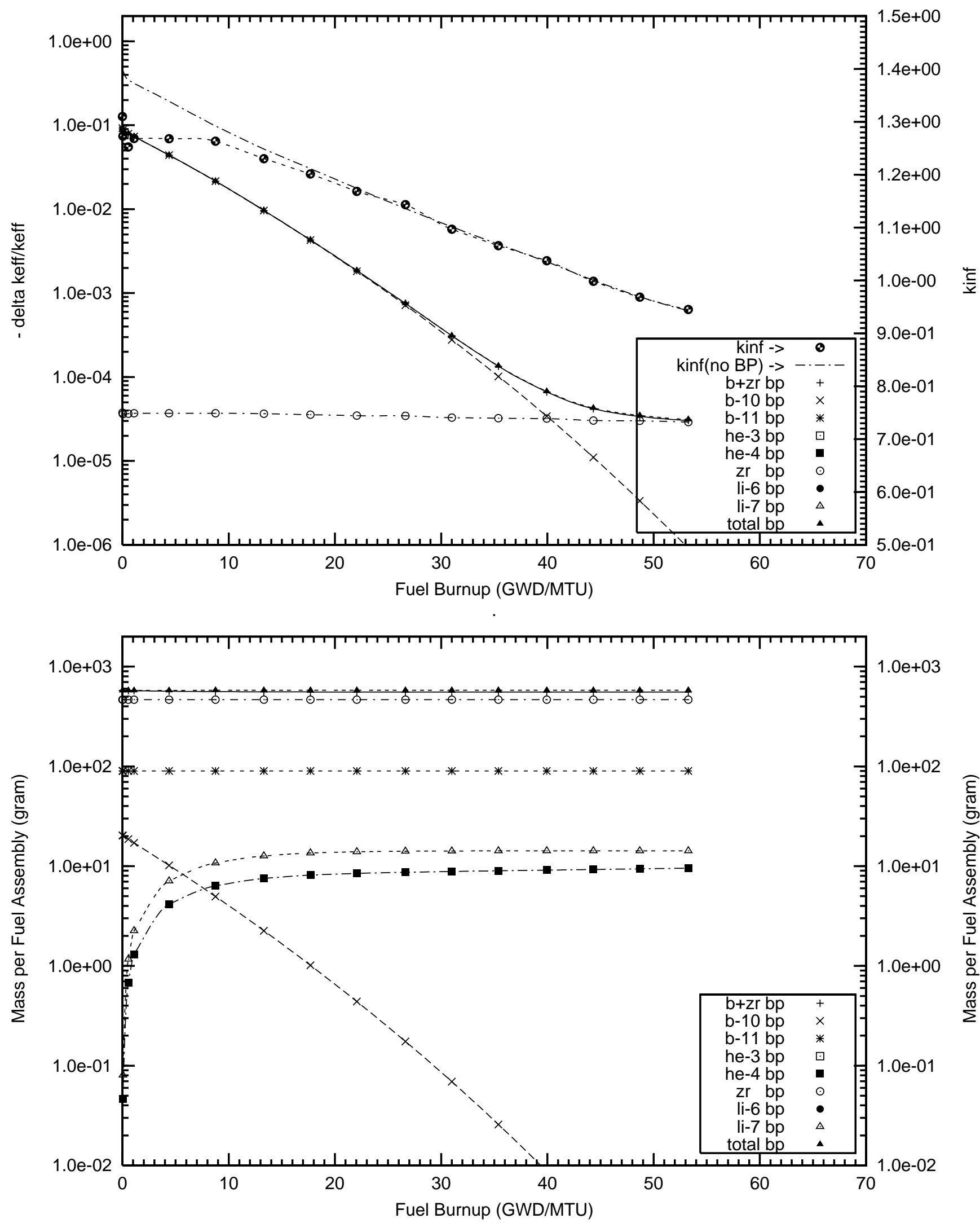
Figure 16. Negative Reactivity and Masses BP for 30wt\% Boron and transmutation daughters as a function of fuel life for $17 \times 17$ fuel assemblies with 104 poison rods,

1.5 mill ZrB2 poison coating on the outer radial surface of the UO2 pellets

Reactor power 3400 MWth, 193 fuel assemblies, initial enrichment 4.5wt\%U-235

(Case : zb010_030_7f_100p_h2o_104_bp_fuel_2a_o)
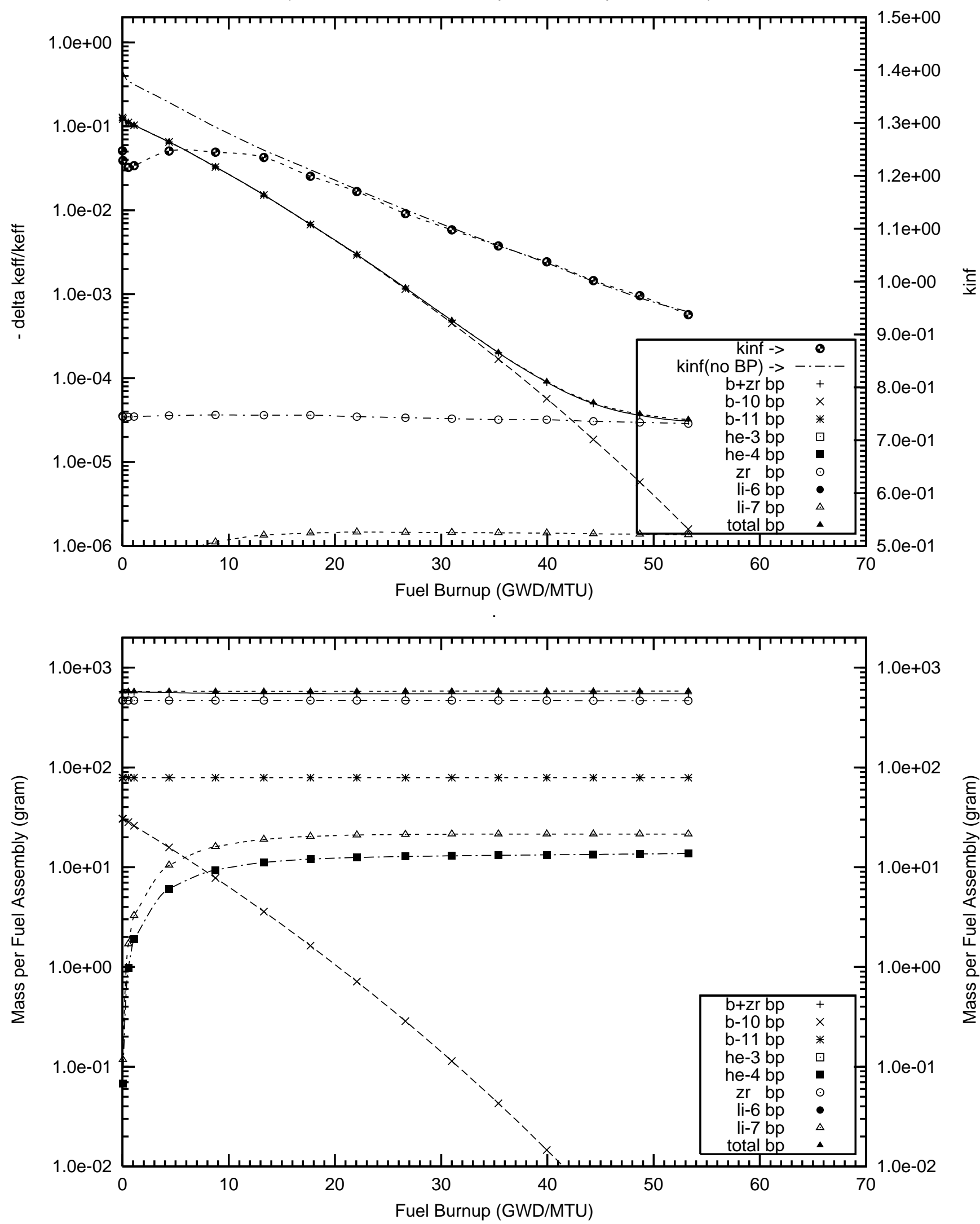
Figure 17. Negative Reactivity and Masses BP for 30wt\% Boron and transmutation daughters as a function of fuel life for $17 \times 17$ fuel assemblies with 64 poison rods,

1.5 mill ZrB2 poison coating on the outer radial surface of the UO2 pellets

Reactor power $3400 \mathrm{MWth}, 193$ fuel assemblies, initial enrichment 4.5wt\%U-235

(Case : zb010_030_7f_64_bp_fuel o)
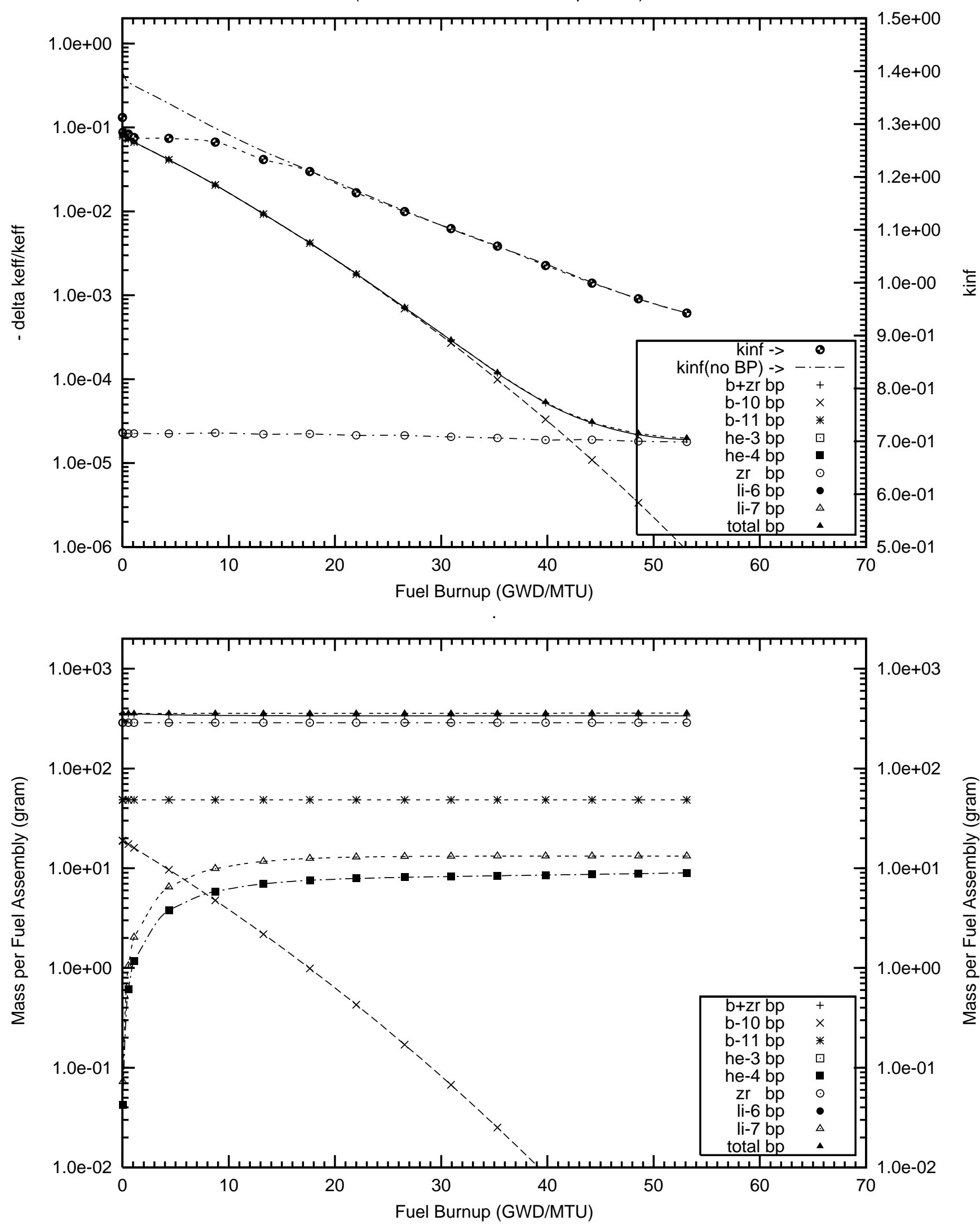
Figure 18. Negative Reactivity and Masses BP for $30 w t \%$ Boron and transmutation daughters as a function of fuel life for $17 \times 17$ fuel assemblies with 64 poison rods,

2.0 mill ZrB2 poison coating on the outer radial surface of the UO2 pellets

Reactor power $3400 \mathrm{MWth}, 193$ fuel assemblies, initial enrichment 4.5wt\%U-235

(Case : zb010_030_2f_64_bp_fuel o)
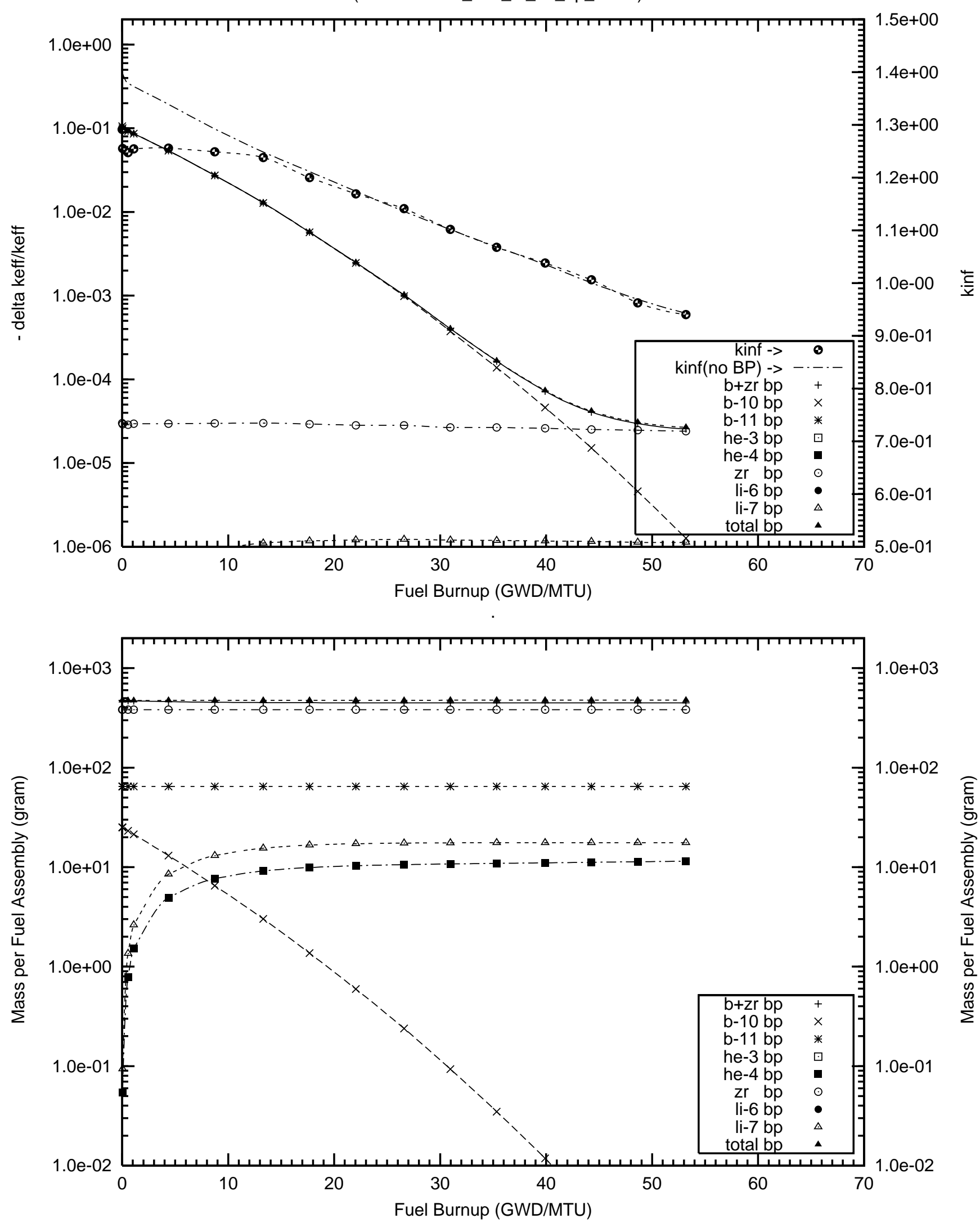
Figure 19. Negative Reactivity and Masses BP for $30 w t \%$ Boron and transmutation daughters as a function of fuel life for $17 \times 17$ fuel assemblies with 18 poison rods,

1.5 mill thick ZrB2 poison coating on the outer radial surface of the UO2 pellets

Reactor power $3400 \mathrm{MWth}, 193$ fuel assemblies, initial enrichment 4.5wt\%U-235

(Case : zb010_030_7f_16_bp_fuel o)
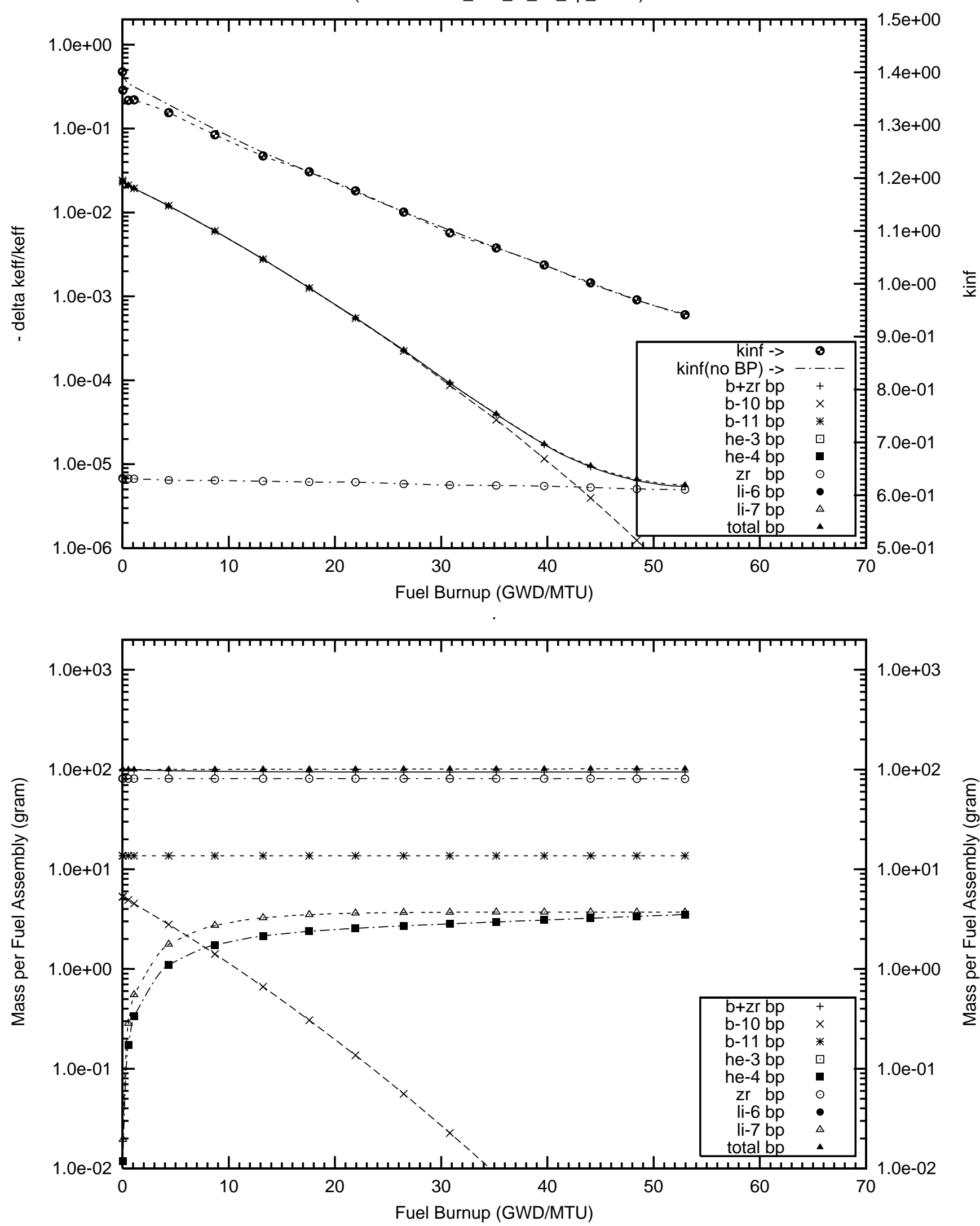
Figure 20. Masses Actinides for $30 w t \%$ Boron and transmutation daughters as a function of fuel life for $17 \times 17$ fuel assemblies with 104 poison rods, 1.5 mill ZrB2 poison coating on the outer radial surface of the UO2 pellets Reactor power $3400 \mathrm{MWth}, 193$ fuel assemblies, initial enrichment 4.5wt\%U-235 (case : zb010_030_7f_100p_h2o_104_bp_fuel_2a_o)

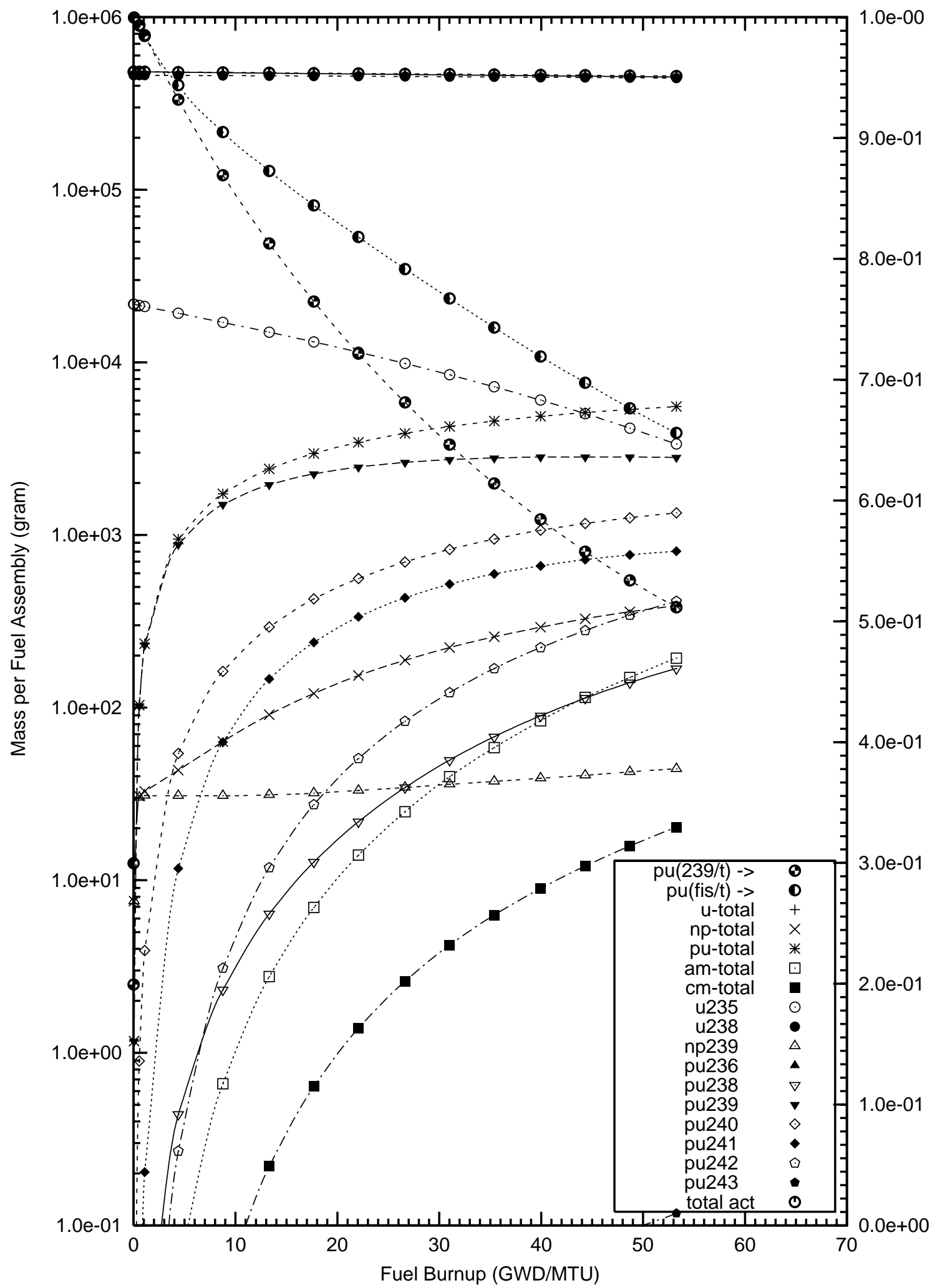

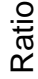




\section{NEUTRONICS PERFORMANCE RESULTS FOR GADOLINIUM}

The method of choice for adding burnable poisons in most PWRs is the use of $\mathrm{B}_{4} \mathrm{C}$ in separate nonfueled rods, and more recently, the application of a thin coating of $\mathrm{ZrB}_{2}$ surrounding the fuel pellets, or the homogeneous dispersal of $\mathrm{Gd}_{2} \mathrm{O}_{3}$ or $\mathrm{Er}_{2} \mathrm{O}_{3}$ inside the fuel pellets of selected fuel rods (ref 6,7). In BWRs the method of choice is the incorporation of $\mathrm{Gd}_{2} \mathrm{O}_{3}$ homogeneously mixed in the $\mathrm{UO}_{2}$ pellets of a number of fuel rods.

In this section, we will investigate the potential benefits of using enriched gadolinium as a burnable absorber in PWRs. Four different configurations of the gadolinium burnable poison were investigated.

1. A homogeneous mixture of the burnable poison as an oxide [gadolinia $\left(\mathrm{Gd}_{2} \mathrm{O}_{3}\right)$ ] inside the fuel pellets of a limited number of fuel rods. A summary of the results is given in Table 5. The plots of reactivity worths and masses of the $\mathrm{BP}$ isotopes and their daughters are displayed in Appendix A1.

2. A homogeneous mixture of the burnable poison gadolinia inside the outer one-third volume of the fuel pellets. A summary of the results is given in Table 6. This configuration of the BP was used to show the effect of zoning the burnable poisons on the burnout times. Plots of the reactivity worths and the masses of the BP isotopes and their daughters are displayed in Appendix A2.

3. A coating of the burnable poison gadolinia on the surface of the fuel pellets, similar to the $\mathrm{ZrB}_{2}$ coatings. A summary of the results is given in Table 7. Plots of the reactivity worths and the masses of the BP isotopes and their daughters are displayed in Appendix A3.

4. A homogeneous mixture of the burnable poison gadolinium in the form of a metal alloyed with the Zircaloy cladding of a number of fuel rods. A summary of the results is given in Table 8 . Plots of the reactivity worths and the masses of the BP isotopes and their daughters are displayed in Appendix A4.

In all the neutronics calculations presented in this section, a theoretical density of $7.41 \mathrm{~g} / \mathrm{cm}^{3}$ was used for gadolinia $\left(\mathrm{Gd}_{2} \mathrm{O}_{3}\right)$, and $7.95 \mathrm{~g} / \mathrm{cm}^{3}$ for metallic gadolinium. A theoretical density of $10.97 \mathrm{~g} / \mathrm{cm}^{3}$ was used for $\mathrm{UO}_{2}$. The actual density fraction of the fuel pellets and of the burnable poison pellets was $95 \%$ of theoretical density.

The half-lives, natural isotopic abundances, and burnup chains related to the depletion and transmutation of gadolinium are given below. Note that the adjacent bands of isotopes such as the Dy isotopes (decay from $\mathrm{Tb}$ isotopes) and $\mathrm{Sm}$ isotopes (decay from Eu isotopes) are not shown. Also, the isomeric states of certain isotopes are not shown. However, the generation of these daughters was taken into account in the BP depletion and decay calculations. 


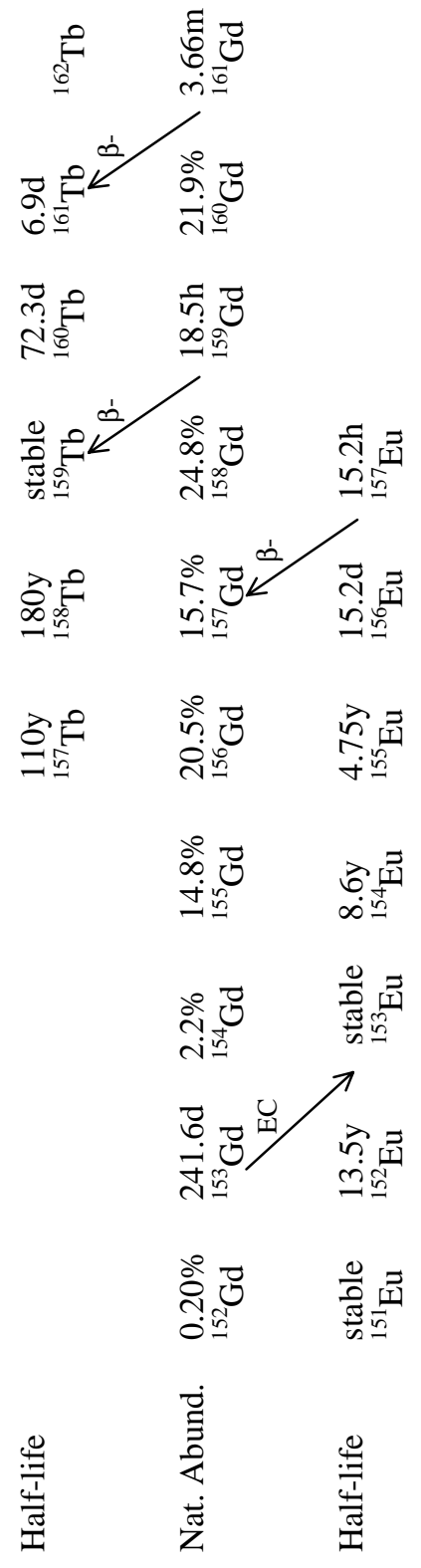




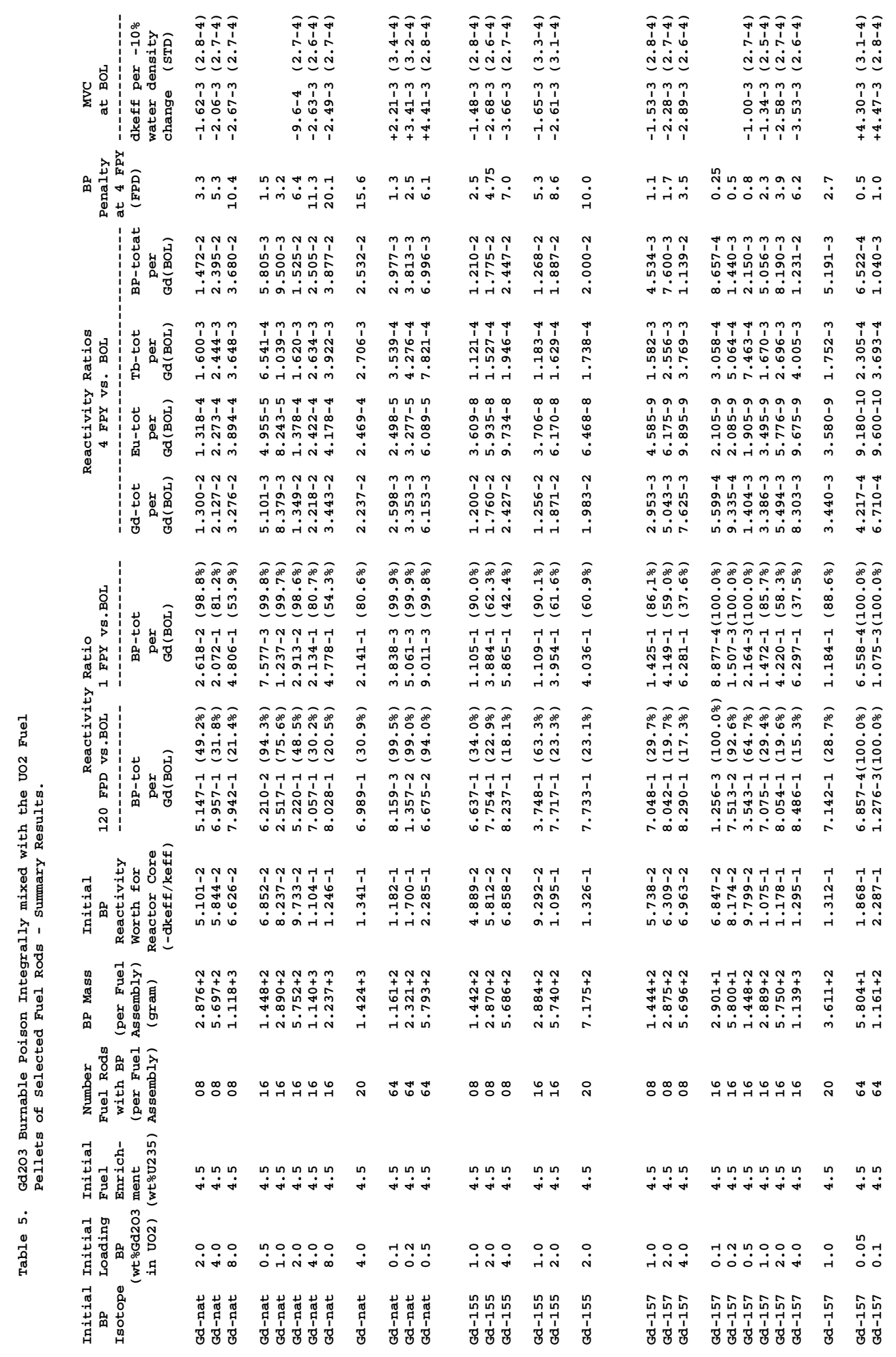




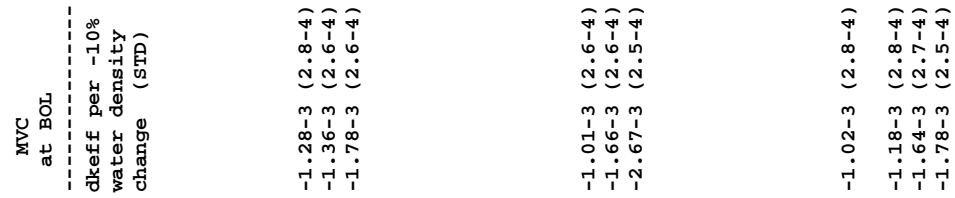

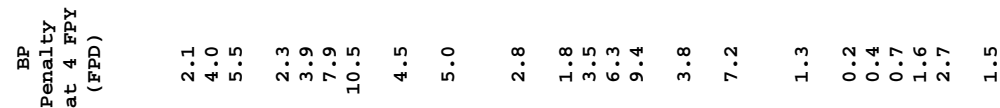

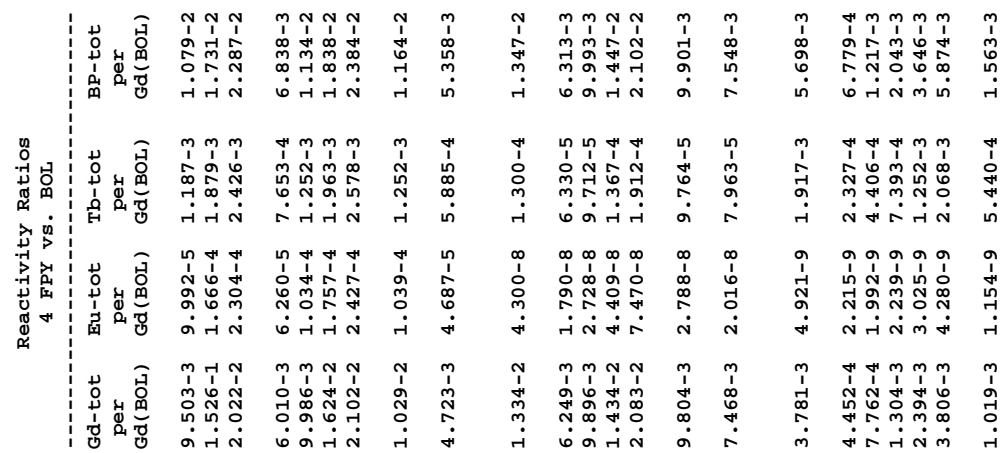

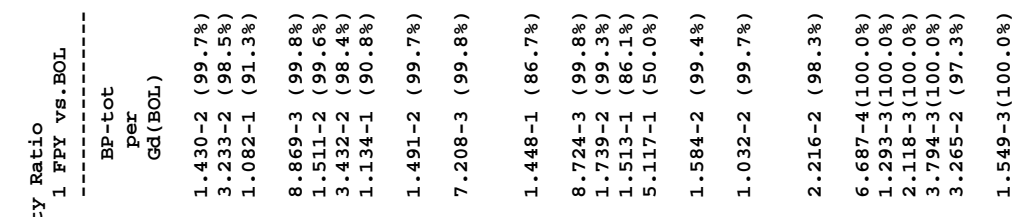

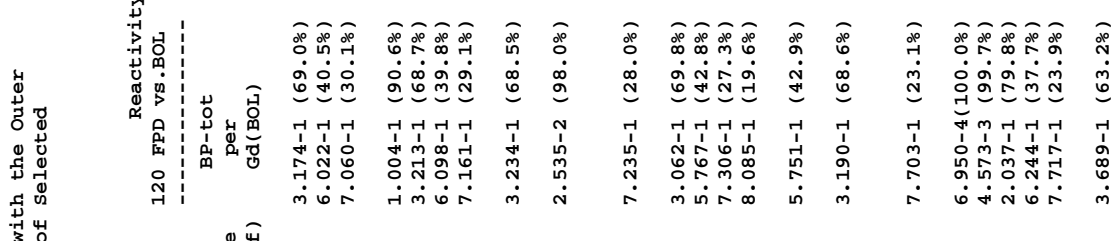

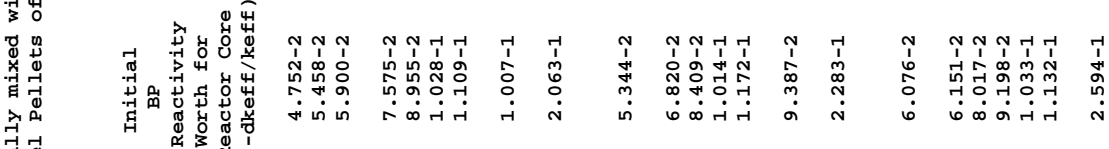

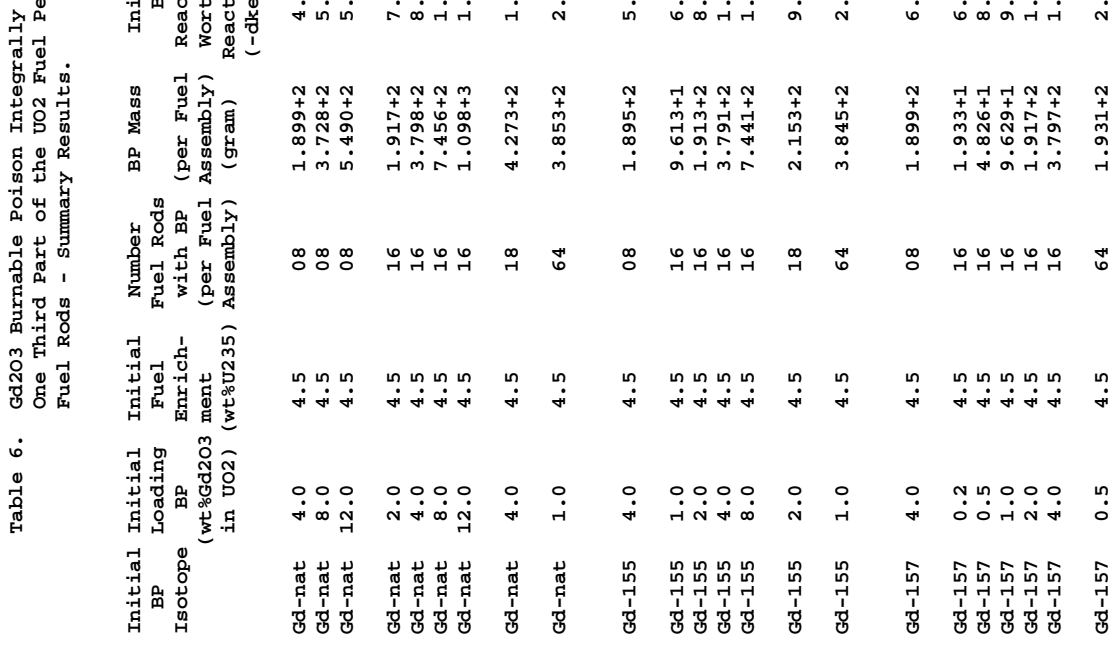




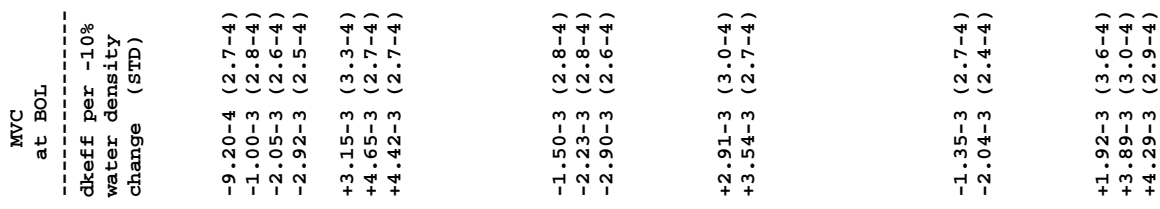

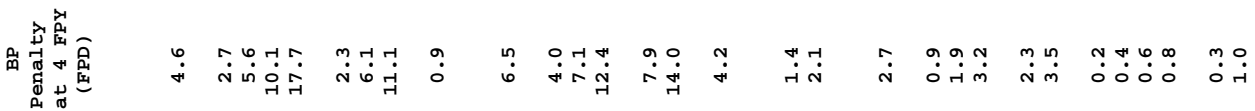

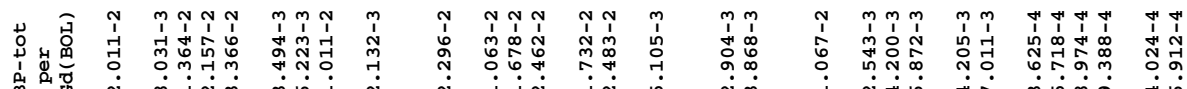

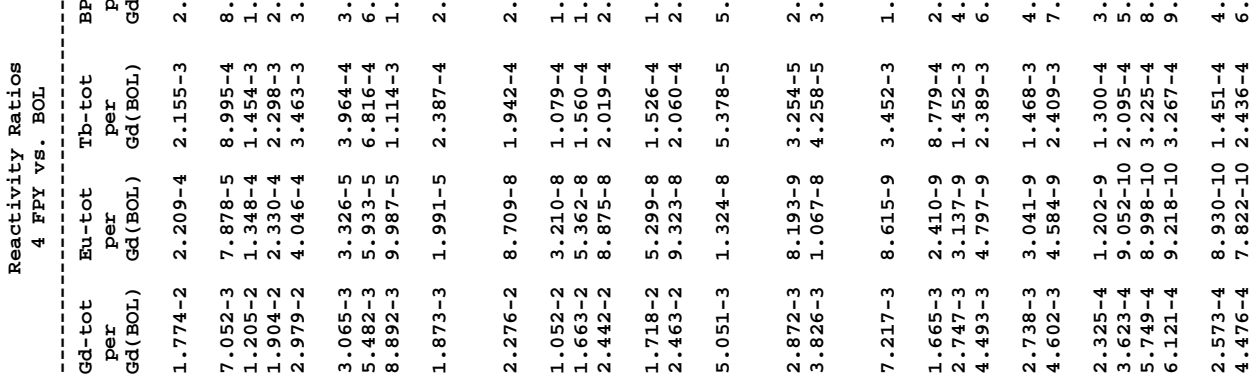

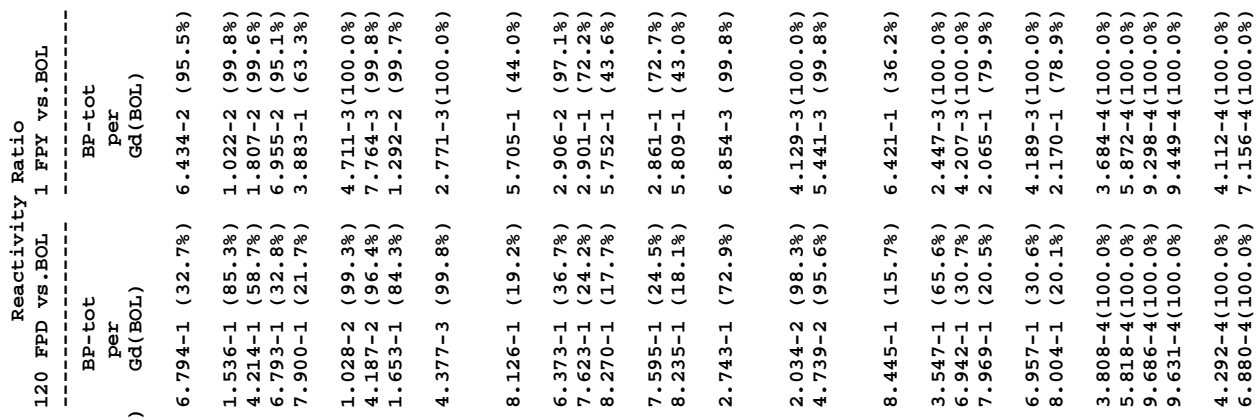

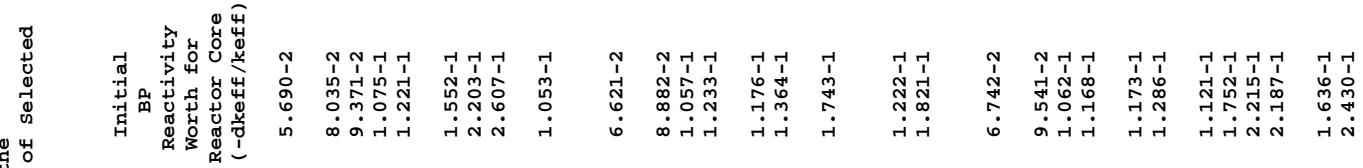

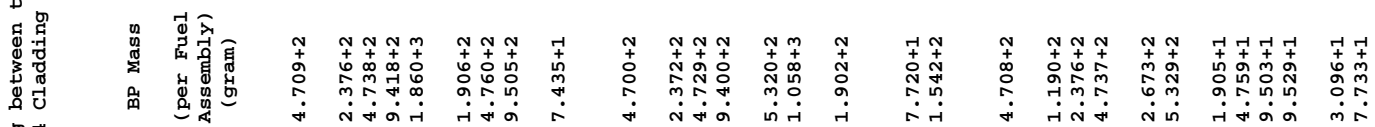

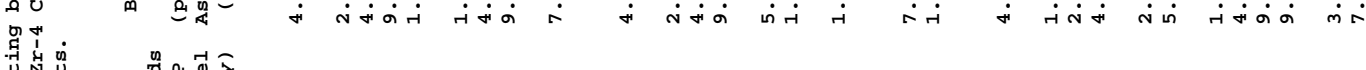

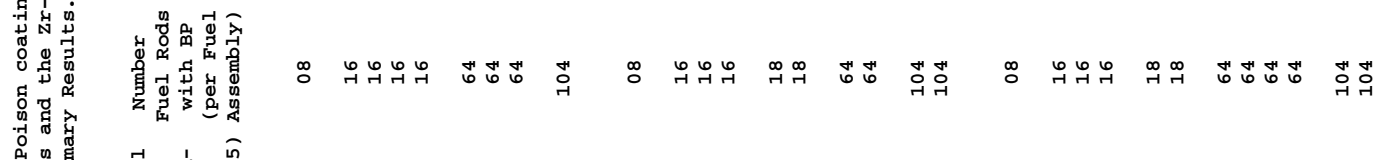

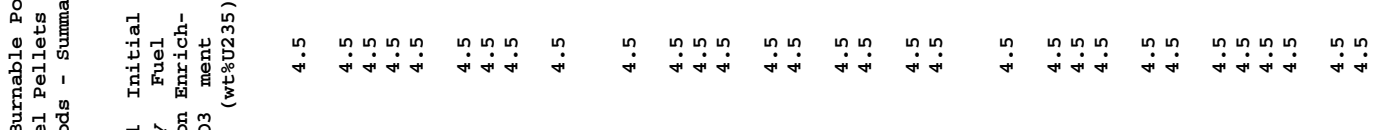

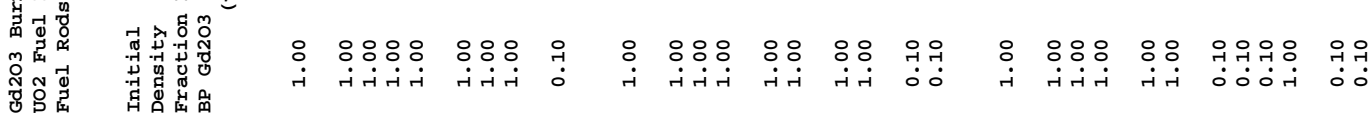

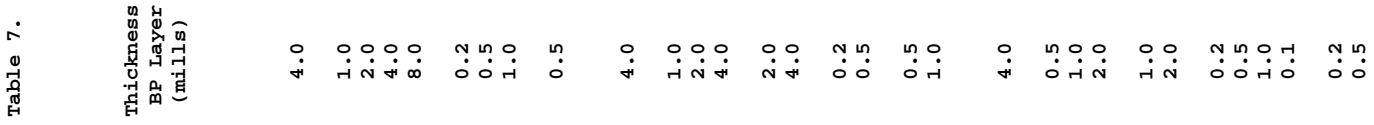

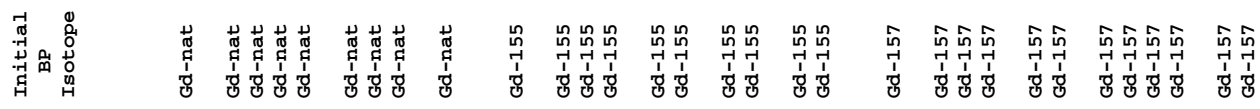




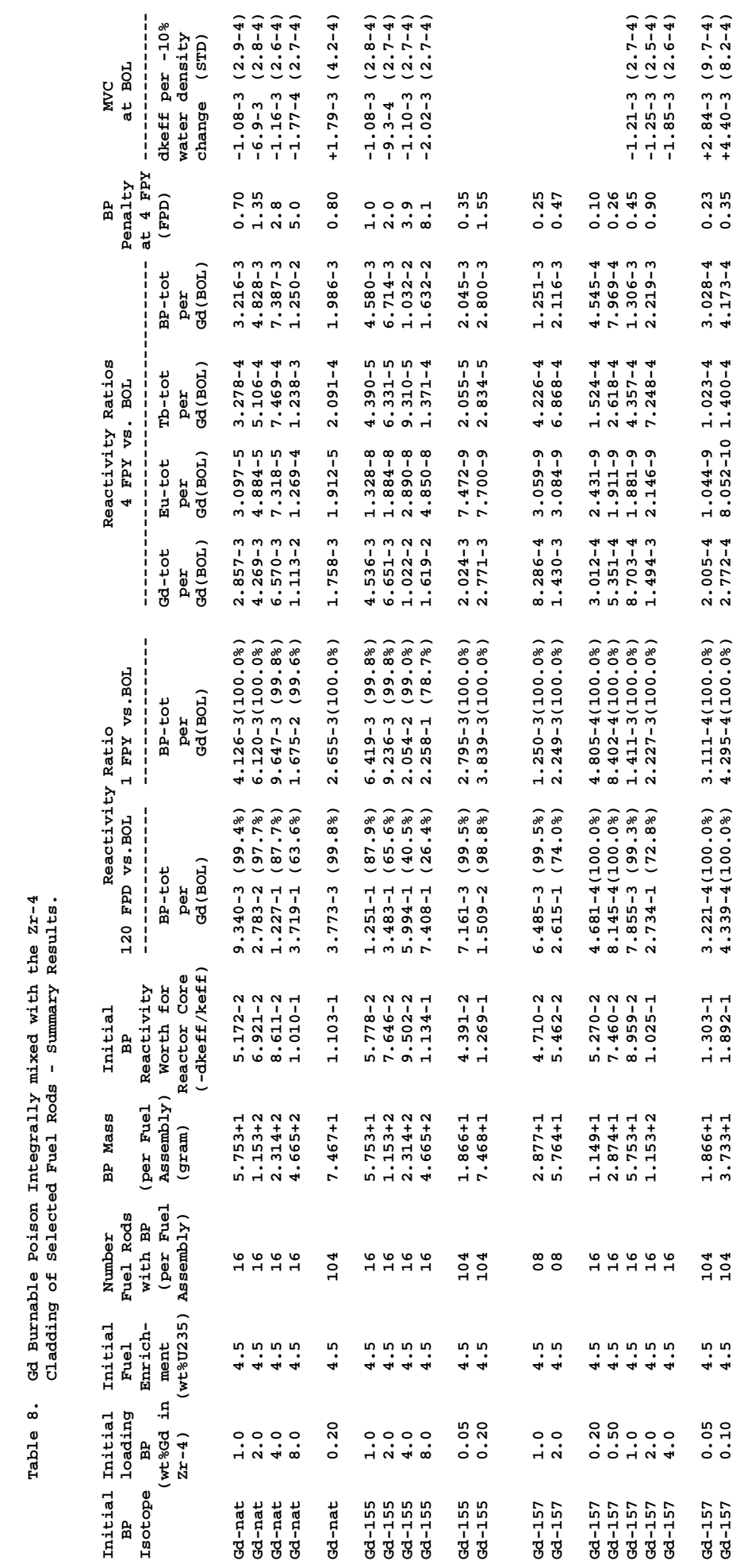


Figure 21 displays the capture cross sections for the gadolinium isotopes. The ${ }^{155} \mathrm{Gd}$ and ${ }^{157} \mathrm{Gd}$ stable isotopes of gadolinium exhibit very large neutron capture cross sections. The natural isotopic abundances are $14.8 \%$ for ${ }^{155} \mathrm{Gd}$ and $15.7 \%$ for ${ }^{157} \mathrm{Gd}$. Other stable isotopes of gadolinium exhibit small capture cross sections. In the use of gadolinium with natural isotopic abundances as a burnable absorber, their presence will cause a residual negative reactivity worth at EOL of the fuel. The removal of these stable isotopes by enriching gadolinium in ${ }^{155} \mathrm{Gd}$ or ${ }^{157} \mathrm{Gd}$, will thus reduce the residual negative reactivity worths. In addition, in order to achieve a given initial negative reactivity worth and for a given number of IFBA rods, the percentage of gadolinium enriched in ${ }^{155} \mathrm{Gd}$ or ${ }^{157} \mathrm{Gd}$ that has to be mixed in the $\mathrm{UO}_{2}$ fuel, coating or cladding will be much smaller. Because of the very large thermal neutron capture cross section of ${ }^{155} \mathrm{Gd}$ and especially of ${ }^{157} \mathrm{Gd}$, a larger self-shielding effect of the poison can be achieved with the same weight percent of gadolinia in the fuel. If the weight percent of gadolinia in the $\mathrm{UO}_{2}$ pellets is limited due to material considerations, then the use of enriched gadolinium could lead to longer burnout times of the burnable poisons.

Note that the ${ }^{158} \mathrm{Gd}(\mathrm{n}, \gamma){ }^{159} \mathrm{Gd}$ reaction produces ${ }^{159} \mathrm{Gd}$ with a half-life of 18.5 days, which then decays into ${ }^{159} \mathrm{~Tb}$. Figure 22 displays the capture cross section for ${ }^{159} \mathrm{~Tb}$. Note that the neutron capture cross section of ${ }^{159} \mathrm{~Tb}$ is approximately 23 barns at $0.0253 \mathrm{eV}$ and that ${ }^{159} \mathrm{~Tb}$ exhibits large resonances in the epithermal energy region [resonance integral (RI) 420 barns]. For the same initial reactivity worth of the BP, the relative residual reactivity worth at EOL due to ${ }^{159} \mathrm{~Tb}$ will thus be larger for ${ }^{157} \mathrm{Gd}$ than for natural $\mathrm{Gd}$ or ${ }^{155} \mathrm{Gd}$.

Table 5 displays the results of using $\mathrm{Gd}_{2} \mathrm{O}_{3}$ mixed homogeneously with the fuel pellets of a number of fuel rods. For three different cases containing 16 IFBA rods per fuel assembly and having a similar initial reactivity worth of the burnable poison $\left(1.10 \times 10^{-1}\right.$ for natural $\mathrm{Gd}$ and 4 wt $\% \mathrm{Gd}_{2} \mathrm{O}_{3}, 1.095 \times$ $10^{-1}$ for ${ }^{155} \mathrm{Gd}$ and $2 \mathrm{wt} \% \mathrm{Gd}_{2} \mathrm{O}_{3}, 1.075 \times 10^{-1}$ for ${ }^{157} \mathrm{Gd}$ and $1.0 \mathrm{wt} \% \mathrm{Gd}_{2} \mathrm{O}_{3}$ ), the residual reactivity worth fraction was $2.51 \times 10^{-2}$ for natural $\mathrm{Gd}, 1.89 \times 10^{-2}$ for ${ }^{155} \mathrm{Gd}$ and $5.06 \times 10^{-3}$ for ${ }^{157} \mathrm{Gd}$. The use of ${ }^{155} \mathrm{Gd}$ gave a slight improvement over natural $\mathrm{Gd}$, and the use of ${ }^{157} \mathrm{Gd}$ gave an improvement by a factor of approximately five. The residual reactivity worth of the BP at EOL of the fuel can be given as an equivalent penalty in FPDs of operation of the reactor: 11.3 FPD for the natural Gd case, 8.6 FPDs for the ${ }^{155} \mathrm{Gd}$ case, and 2.3 FPDs for the ${ }^{157} \mathrm{Gd}$ case. Note also that for these cases (which have a similar initial reactivity worth of the burnable poison), the use of ${ }^{157} \mathrm{Gd}$ decreased the required mass of gadolinia by a factor of four. The residual negative reactivity worth due to the buildup of $\mathrm{Tb}$ in the BP is $10 \%$ of the total for natural Gd, $1 \%$ for ${ }^{155} \mathrm{Gd}$, and $30 \%$ for ${ }^{157} \mathrm{Gd}$. The residual worth due to the buildup of Eu isotopes is zero for the ${ }^{155} \mathrm{Gd}$ and ${ }^{157} \mathrm{Gd}$ burnable poison cases and less than $1 \%$ for the natural $\mathrm{Gd}$ case.

Note that for each case, all the BP isotopes and their daughters (which are not present in the initial loading of the BP) have been added as traces to the BP with a number density of $1.0 \times 10^{-15}$ instead of zero. This is to avoid problems with the calculations at the beginning of cycle (BOC) with the MCNP4C code and to avoid computer exception errors (division by zero) when calculating spectrumweighted cross sections in the GP-TALLY code.

Figures 23-30 display the reactivity worths for several cases that have 16 IFBA rods per fuel assembly. The worths displayed are for a reactor core containing 193 fuel assemblies at a total power level of 3,400 MWth. The IFBA rods contained $4.0 \mathrm{wt} \% \mathrm{Gd}_{2} \mathrm{O}_{3}$ with natural $\mathrm{Gd}$ (natural isotopic abundances of gadolinium) (Figure 23), $8.0 \mathrm{wt} \% \mathrm{Gd}_{2} \mathrm{O}_{3}$ with natural $\mathrm{Gd}$ (Figure 24), $1.0 \mathrm{wt} \% \mathrm{Gd}_{2} \mathrm{O}_{3}$ with ${ }^{155} \mathrm{Gd}$ (gadolinium $100 \mathrm{wt} \%$ enriched in ${ }^{155} \mathrm{Gd}$ ) (Figure 25), $2.0 \mathrm{wt} \% \mathrm{Gd}_{2} \mathrm{O}_{3}$ with ${ }^{155} \mathrm{Gd}$ (Figure 26), 0.5 wt $\% \mathrm{Gd}_{2} \mathrm{O}_{3}$ with ${ }^{157} \mathrm{Gd}$ (gadolinium $100 \mathrm{wt} \%$ enriched in ${ }^{157} \mathrm{Gd}$ ) (Figure 27), $1.0 \mathrm{wt} \%$ $\mathrm{Gd}_{2} \mathrm{O}_{3}$ with ${ }^{157} \mathrm{Gd}$ (Figure 28), 2.0 wt $\% \mathrm{Gd}_{2} \mathrm{O}_{3}$ with ${ }^{157} \mathrm{Gd}$ (Figure 29), and 4.0 wt $\% \mathrm{Gd}_{2} \mathrm{O}_{3}$ with ${ }^{157} \mathrm{Gd}$ (Figure 30), respectively. Figures 23-30 also show the values of the fuel assembly lattice $\mathrm{k}_{\text {inf }}$ with and without BP. Note that during the calculations with the GP-TALLY code, nine depletion time 
steps were taken between each of the symbols displayed on the figures. Figures 23-30 also display the masses of the burnable poison isotopes and their daughters as a function of depletion time. The figures exhibit several interesting features. For example, the case with 16 IFBA rods whose fuel pellets contain 4.0 wt $\% \mathrm{Gd}_{2} \mathrm{O}_{3}$ with natural $\mathrm{Gd}$ (displayed in Figure 23) exhibit several interesting effects. The reactivity worth of the burnable poison isotope ${ }^{157} \mathrm{Gd}$ decreases rapidly with depletion time and reaches a nearly constant value at approximately 600 FPDs [approximately 22 gigawatt days per metric ton of uranium (GWD/MTU) burnup]. The reactivity worth of ${ }^{156} \mathrm{Gd}$ increases slightly with depletion time, due the production of ${ }^{156} \mathrm{Gd}$ through the ${ }^{155} \mathrm{Gd}(\mathrm{n}, \gamma){ }^{156} \mathrm{Gd}$ reaction, and it reaches a nearly equilibrium value. Since the neutron capture cross section of ${ }^{157} \mathrm{Gd}$ is much greater than that of ${ }^{156} \mathrm{Gd}$, the capture reaction rate (and thus the reactivity worth) of ${ }^{157} \mathrm{Gd}$ reaches a secular equilibrium with the capture reaction rate of ${ }^{156} \mathrm{Gd}$. After most of the original ${ }^{157} \mathrm{Gd}$ nuclei have burned out, ${ }^{157} \mathrm{Gd}$ nuclei are produced through the ${ }^{156} \mathrm{Gd}(\mathrm{n}, \gamma){ }^{157} \mathrm{Gd}$ reaction. Since the neutron capture cross section of ${ }^{157} \mathrm{Gd}$ is much larger than that of ${ }^{156} \mathrm{Gd}$, these ${ }^{157} \mathrm{Gd}$ nuclei will burn away at a much higher rate than the ${ }^{156} \mathrm{Gd}$ nuclei, essentially immediately after they were created. This effect is similar to the secular equilibrium effect in the decay of a parent isotope that has a very long half-life, together with its daughter, which has a very short half-life. In the case of ${ }^{156} \mathrm{Gd} /{ }^{157} \mathrm{Gd}$ we reach a secular equilibrium between a parent isotope that has a small neutron capture cross section $\left({ }^{156} \mathrm{Gd}\right)$, together with its daughter, which has a very large cross section $\left({ }^{157} \mathrm{Gd}\right)$. Another secular equilibrium of the reactivity worths is reached between the burnable poison isotopes ${ }^{154} \mathrm{Gd}$ (small absorption cross section) and ${ }^{155} \mathrm{Gd}$ (large absorption cross section).

Another interesting effect is the increase of the reactivity worth of ${ }^{155} \mathrm{Gd}$ as a function of depletion time at the beginning of the fuel cycle (see figure 23), even though the mass of ${ }^{155} \mathrm{Gd}$ decreases (see $\mathrm{BP}$ masses in figure 23). This can be explained as follows: The natural isotopic abundances for ${ }^{155} \mathrm{Gd}$ and ${ }^{157} \mathrm{Gd}$ are similar (14.8\% vs $15.7 \%$ ). However, the neutron capture cross section of ${ }^{157} \mathrm{Gd}$ is much larger than the capture cross section of ${ }^{155} \mathrm{Gd}$. Due to the higher cross section of ${ }^{157} \mathrm{Gd}$, the total mass of ${ }^{157} \mathrm{Gd}$ decreases faster with depletion time than the mass of ${ }^{155} \mathrm{Gd}$. The faster reduction of the amount of ${ }^{157} \mathrm{Gd}$ will make the neutron spectrum softer and thus the spectrum-weighted capture cross section of ${ }^{155} \mathrm{Gd}$ will increase even though the mass of ${ }^{155} \mathrm{Gd}$ decreases. However, after approximately 100 days (approximately 4 GWD/MTU burnup), a sufficient amount of ${ }^{155} \mathrm{Gd}$ has been burned out that the reactivity worth of the ${ }^{155} \mathrm{Gd}$ starts to decrease.

The use of 0.5 wt $\% \mathrm{Gd}_{2} \mathrm{O}_{3}$ fully enriched with ${ }^{157} \mathrm{Gd}$ gives an initial reactivity worth of the BP of $9.800 \times 10^{-2}$ (see Table 5). Doubling the content to $1.0 \mathrm{wt} \% \mathrm{Gd}_{2} \mathrm{O}_{3}$ with ${ }^{157} \mathrm{Gd}$ increases only slightly the initial reactivity worth to $1.075 \times 10^{-1}$, an increase of $9.5 \%$. Increasing the content to $2 \mathrm{wt} \%$ $\mathrm{Gd}_{2} \mathrm{O}_{3}$ increases the initial worth to 0.118 , an increase of only $12 \%$. The large neutron capture cross section of ${ }^{157} \mathrm{Gd}$ makes the IFBA pellets virtually "black," and adding more BP only slightly changes the initial reactivity worth of the BP. In Table 5 we also have displayed the relative BP reactivity ratios at $120 \mathrm{FPD}$ and $1 \mathrm{FPY}$. The burnout times of the BP for the $1.0 \mathrm{wt} \%$ loading are substantially longer than for the $0.5 \mathrm{wt} \%$ loading. The burnout rates of the BP can be controlled with the loading and thus with the "blackness" of the BP. Note however that the residual negative reactivity worth at 4 FPY increases from $2.150 \times 10^{-3}$ to $5.055 \times 10^{-3}$. Comparison of the total worth of the burnable poison for the $0.5 \mathrm{wt} \%, 1.0 \mathrm{wt} \%$, and $2.0 \mathrm{wt} \% \mathrm{Gd}_{2} \mathrm{O}_{3}$ loadings clearly show that the burnout times are much longer for the $1.0 \mathrm{wt} \%$ loading than for the $0.5 \mathrm{wt} \%$ loading, and even longer for the $2.0 \mathrm{wt} \%$ loading. Note also that the trend of the fuel assembly lattice $\mathrm{k}_{\mathrm{inf}}$ as a function of depletion time remains relatively flat for longer depletion times (compare figures 27, 28, and 29).

Comparison of the cases containing 64 IFBA rods with the cases containing 16 or 8 IFBA rods clearly show that, for a given initial reactivity worth, the burnout rates are much greater for 64 IFBA rods than for 16 or 8 IFBA rods. This is clearly due to the self-shielding effect of the neutrons inside the IFBA pellets. Note that the residual BP reactivity worths are smaller for the 64 IFBA cases. In 
Appendix A1, we have displayed the plots of the reactivity worths and the masses for each of the cases displayed in Table 5. The reactivity worths for each of the BP isotopes and their daughters are shown. Note that for a given initial reactivity worth of the BP, the burnout rate is high when the BP is spread over 64 IFBA rods.

The MVCs were calculated by decreasing the water coolant density by $10 \%$. The one-sigma STDs of the fuel assembly lattice $\mathrm{k}_{\text {inf }}$ were better than $0.03 \%$. Note that for all the cases containing 8 and 16 IFBA rods, the MVCs remain negative. For the cases with 64 IFBA rods, the MVC becomes positive for the lattices containing identical standard $17 \times 17$ fuel assemblies with the same BP loadings.

Calculations of the MVC were not performed for checkerboard loadings of fuel assemblies containing 64 IFBA rods in selected reactor core locations, or for fuel assemblies with different fuel-to-water ratios.

Figure 31 displays the initial reactivity worths of the $\mathrm{BP}$ as a function the equivalent penalty (given in FPDs of reactor operation) for several configurations containing 16 IFBA rods. The FPD penalty is directly related to the residual negative reactivity worth of the BPs and their daughters at EOL of the fuel. The trend for the four different forms of the BPs are very similar. Note also the clear advantage of using gadolinium enriched in ${ }^{157} \mathrm{Gd}$ over gadolinium with natural isotopic abundances. Figure 32 displays the initial reactivity worths vs FPD penalty for fuel assembly configurations containing 64 IFBA rods. For the cases where the BP is mixed uniformly with the cladding of a number of fuel rods (see Table 8 and Appendix A4), the required loading using natural gadolinium might be too high (e.g., $8 \mathrm{wt} \%$ ). In order to achieve a given initial reactivity worth of the BP, the use of gadolinium enriched in ${ }^{157} \mathrm{Gd}$ substantially reduces the required loading in the cladding. Further studies of the metallurgical behavior of the Zircaloy cladding in the presence of gadolinium will be done during phase 3 of the project. 
Figure 21. Neutron Cross-Sections as a Function of Energy

Gd-154 (64154.40c)

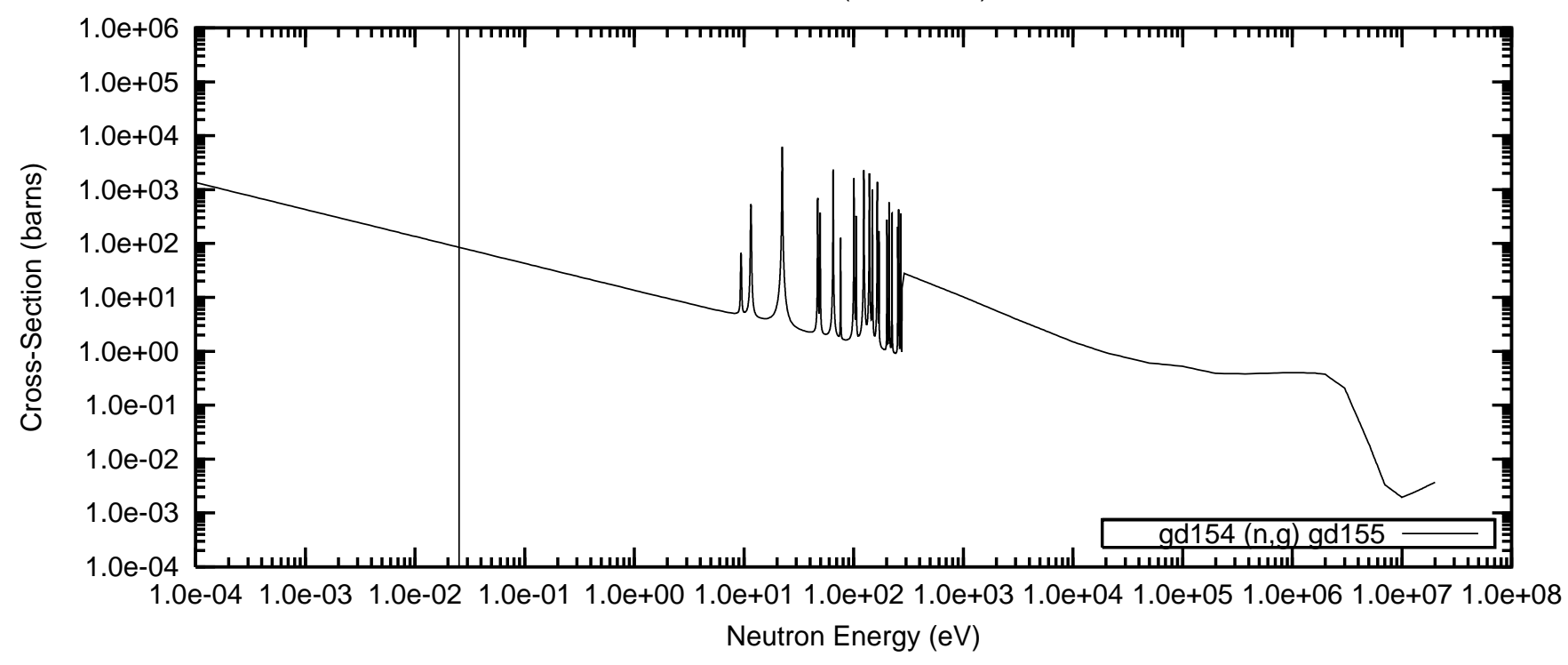

Gd-155 (64155.40c)

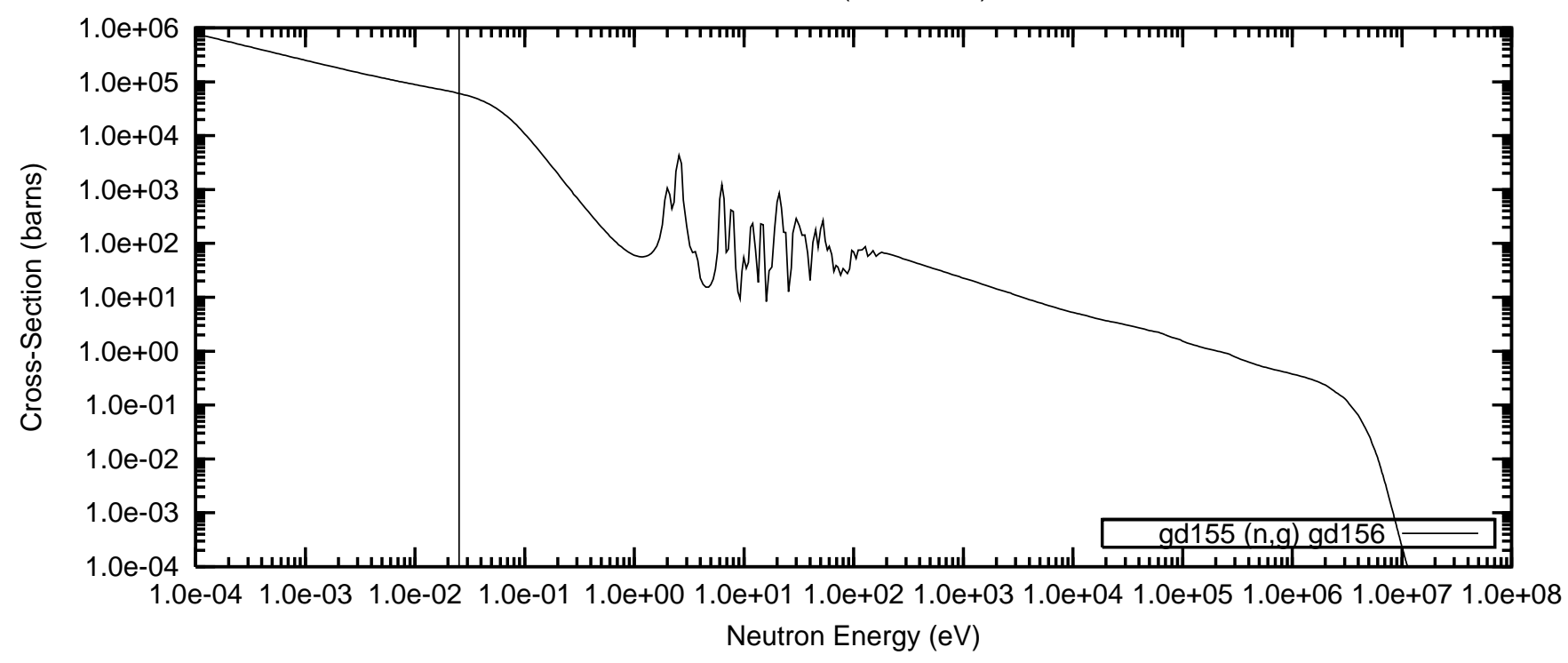

Gd-156 (64156.40c)

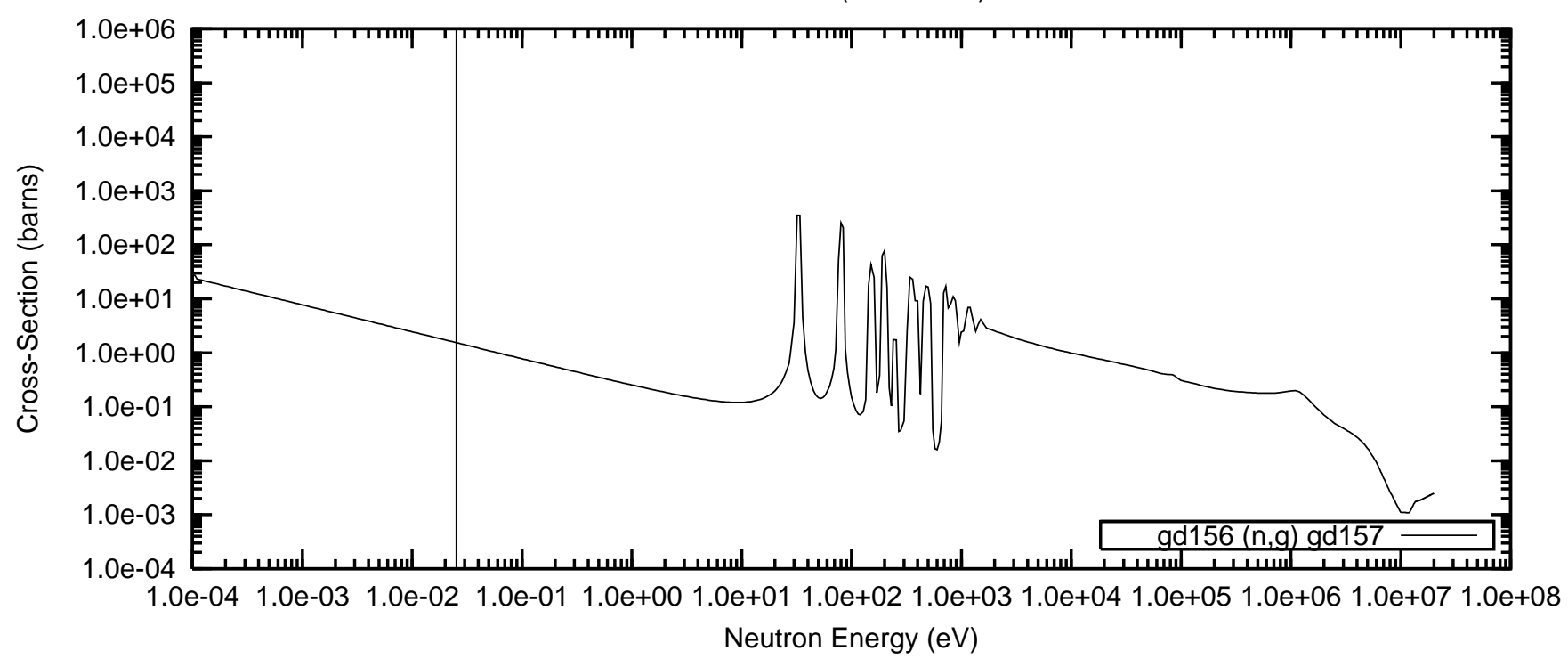


Figure 21 (cont). Neutron Cross-Sections as a Function of Energy Gd-157 (64157.40c)

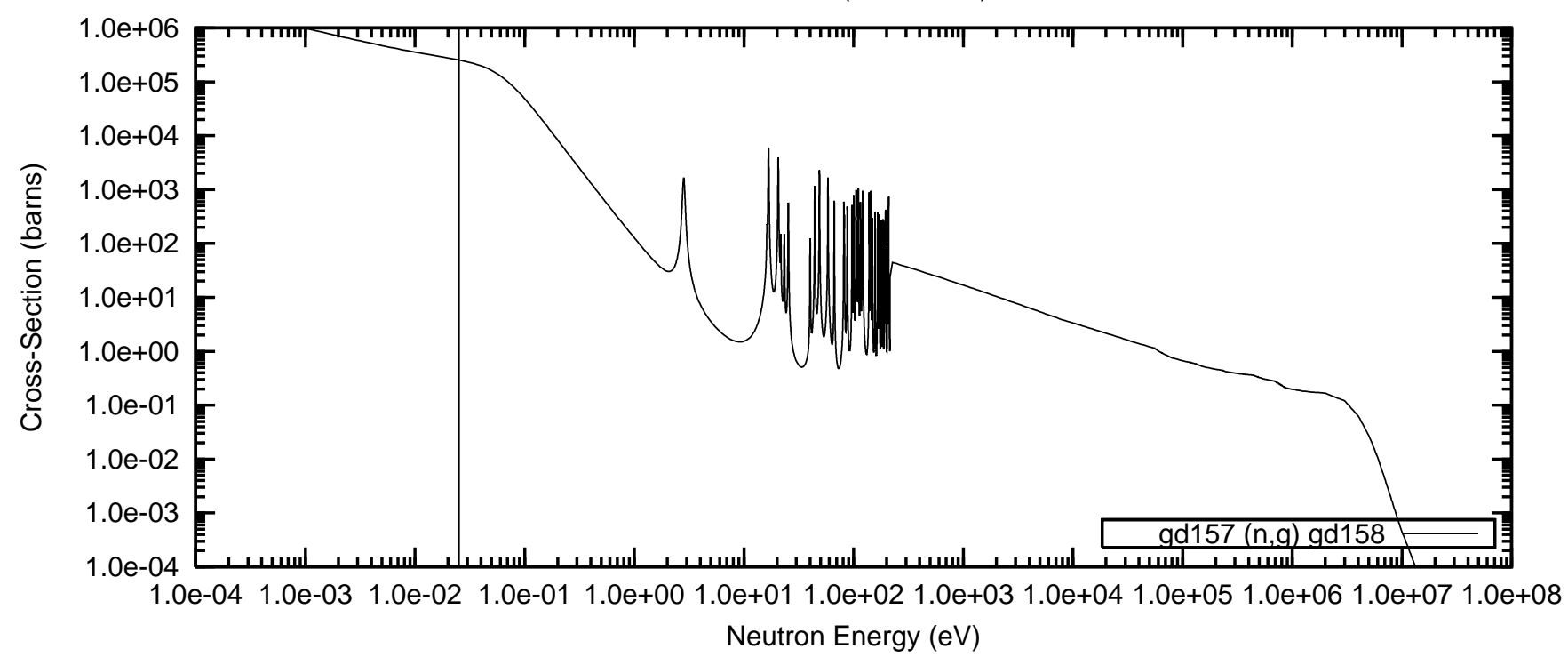

Gd-158 (64158.40c)

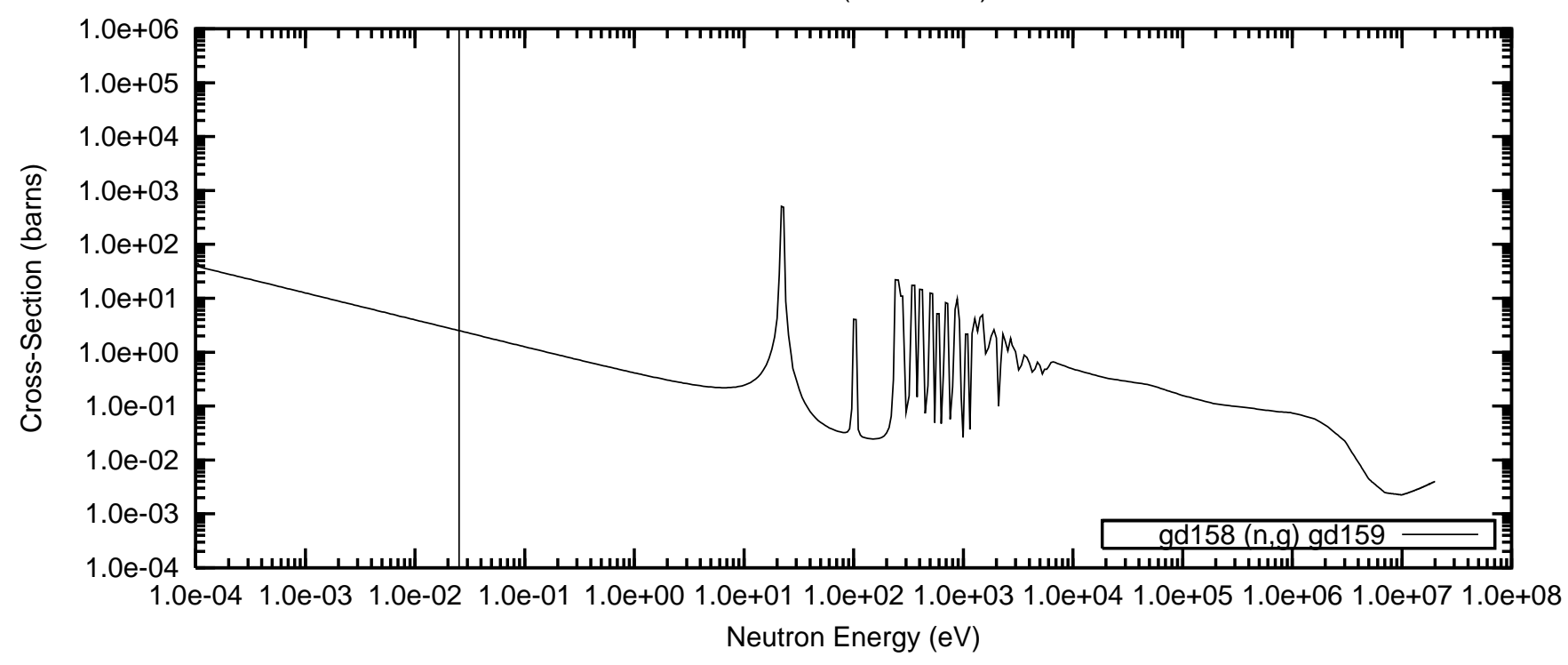

Gd-159 (64159.40c)

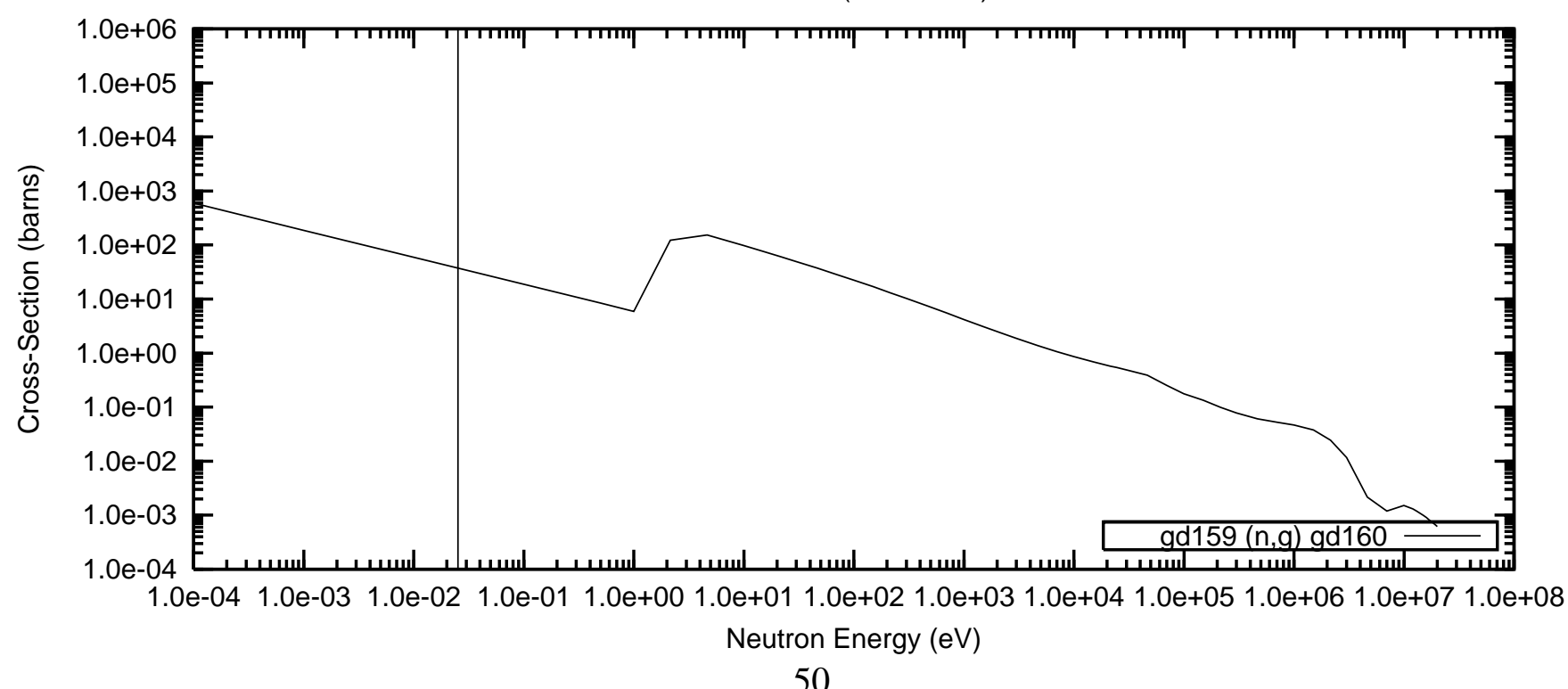


Figure 21 (cont). Neutron Cross-Sections as a Function of Energy Gd-160 (64160.40c)

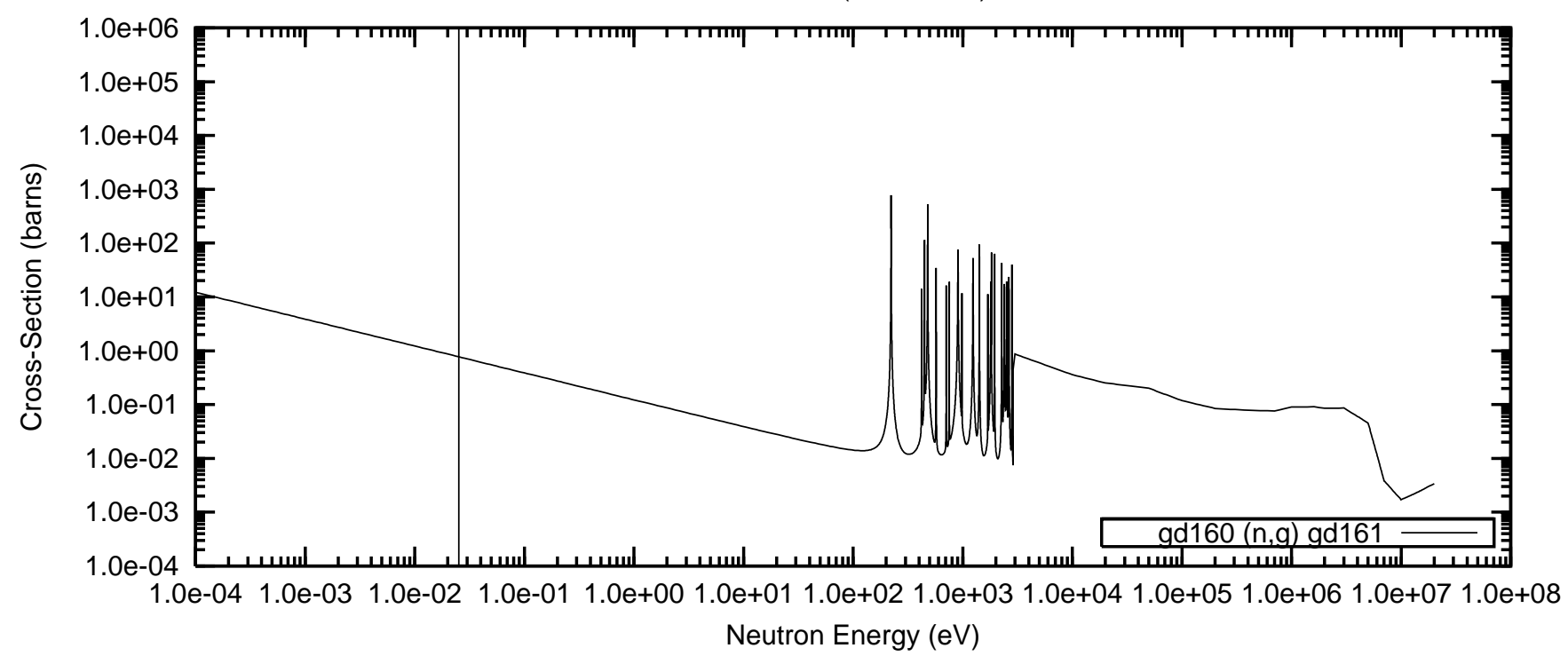


Figure 22. Neutron Cross-Sections as a Function of Energy Tb-159 (65159.40c)

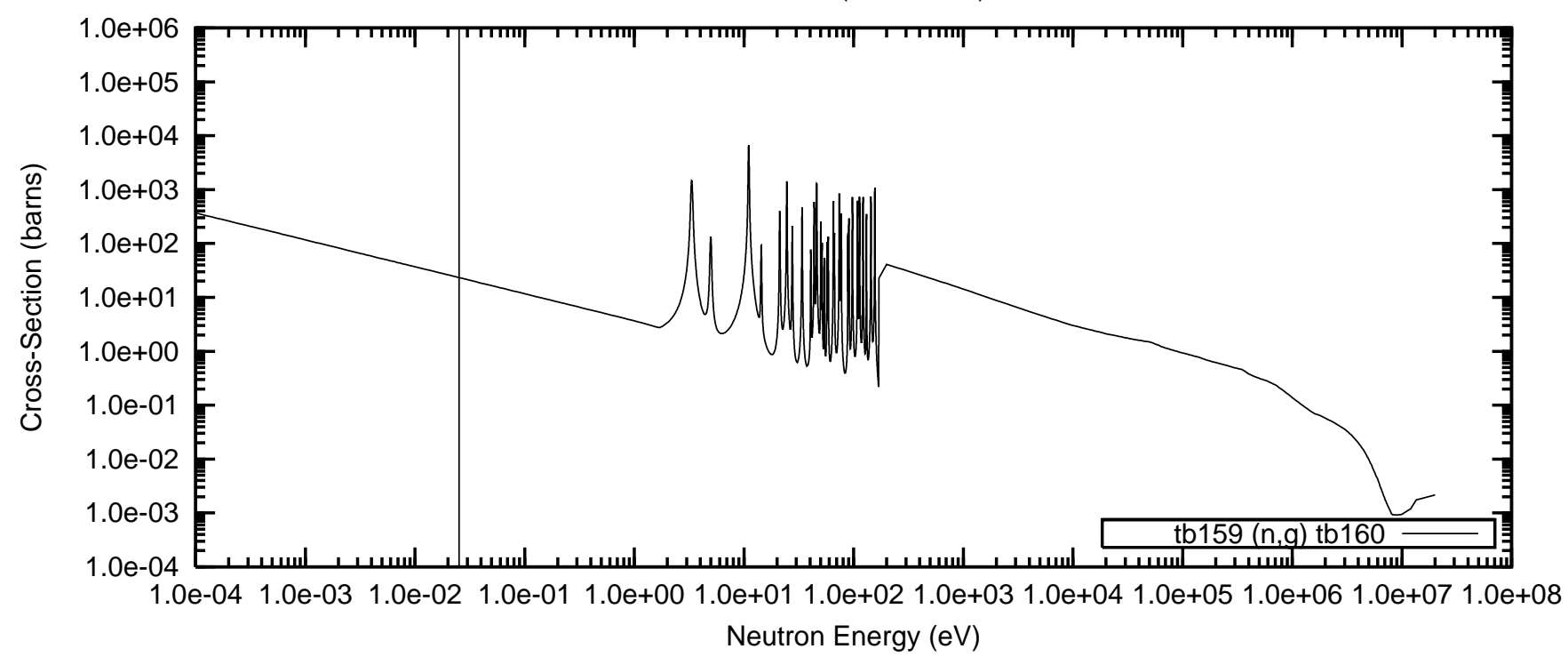

Tb-160 (65160.40c)

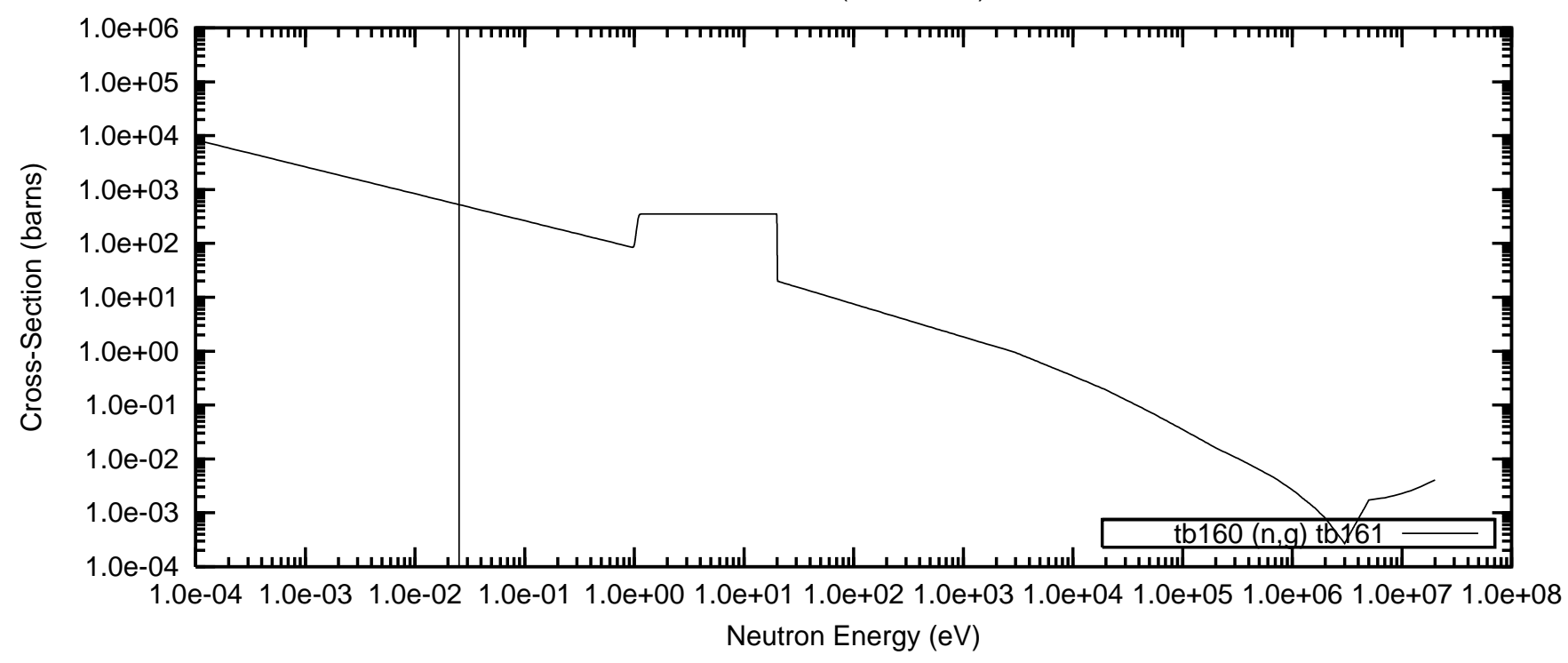

Tb-161 (65161.40c)

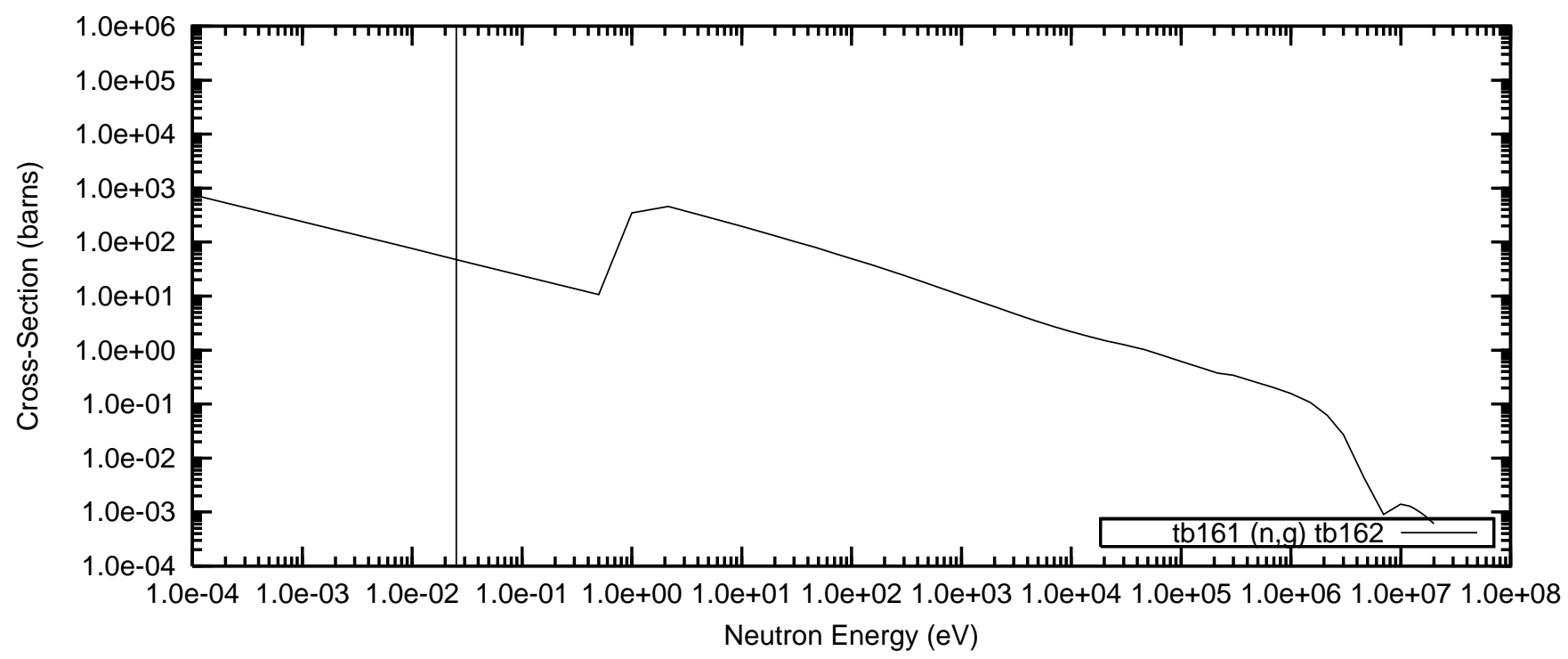


Figure 23. Negative Reactivity and Masses BP for Gd-nat and transmutation daughters as a function of fuel life for $17 \times 17$ fuel assemblies with 16 poison rods, $4.0 \mathrm{wt} \% \mathrm{Gd} 2 \mathrm{O} 3$ poison homogeneously mixed in the UO2 pellets

Reactor power $3400 \mathrm{MWth}, 193$ fuel assemblies, initial enrichment 4.5wt\%U-235

(Case : gd000_3_100p_h2o_16_bp_fuel_2_o)
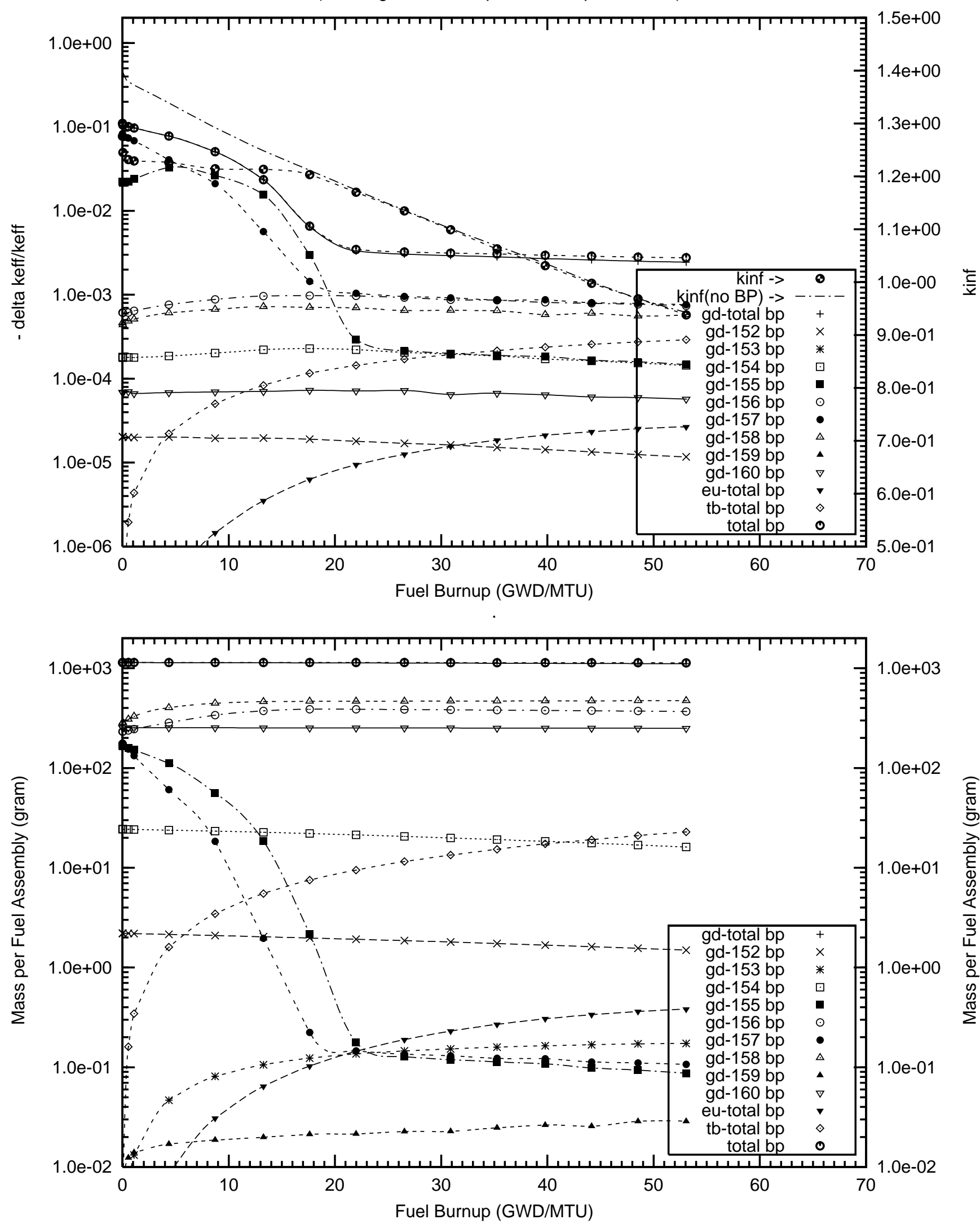
Figure 24. Negative Reactivity and Masses BP for Gd-nat and transmutation daughters as a function of fuel life for $17 \times 17$ fuel assemblies with 16 poison rods, $8.0 \mathrm{wt} \% \mathrm{Gd} 2 \mathrm{O} 3$ poison homogeneously mixed in the UO2 pellets

Reactor power $3400 \mathrm{MWth}, 193$ fuel assemblies, initial enrichment 4.5wt\%U-235

(Case :gd000_4_100p_h2o_16_bp_fuel_2_o)
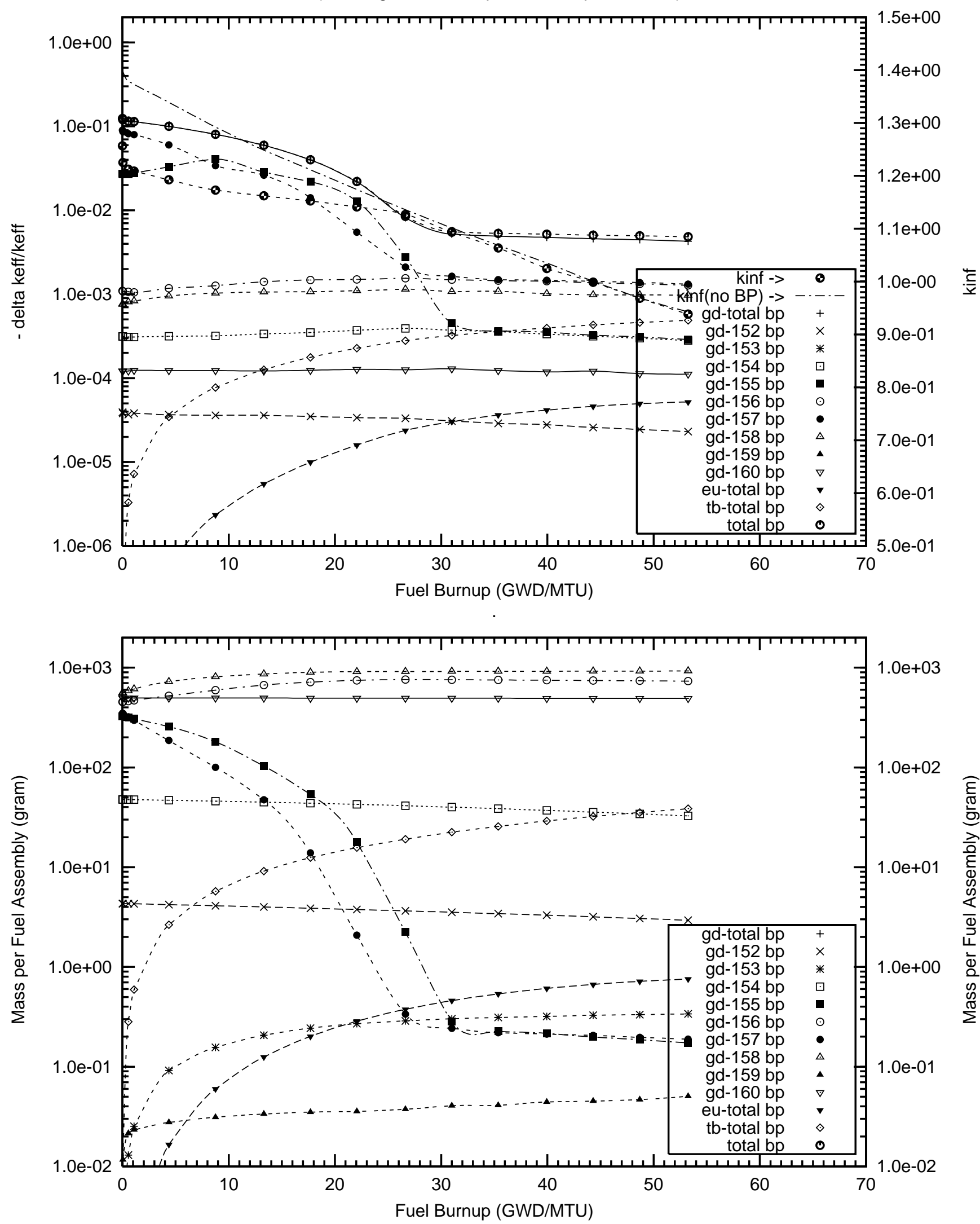
Figure 25. Negative Reactivity and Masses BP for Gd-155 and transmutation daughters as a function of fuel life for $17 \times 17$ fuel assemblies with 16 poison rods, $1.0 \mathrm{wt} \% \mathrm{Gd} 2 \mathrm{O} 3$ poison homogeneously mixed in the UO2 pellets

Reactor power 3400 MWth, 193 fuel assemblies, initial enrichment 4.5wt\%U-235

(Case : gd155_1_100p_h2o_16_bp_fuel_2_o)
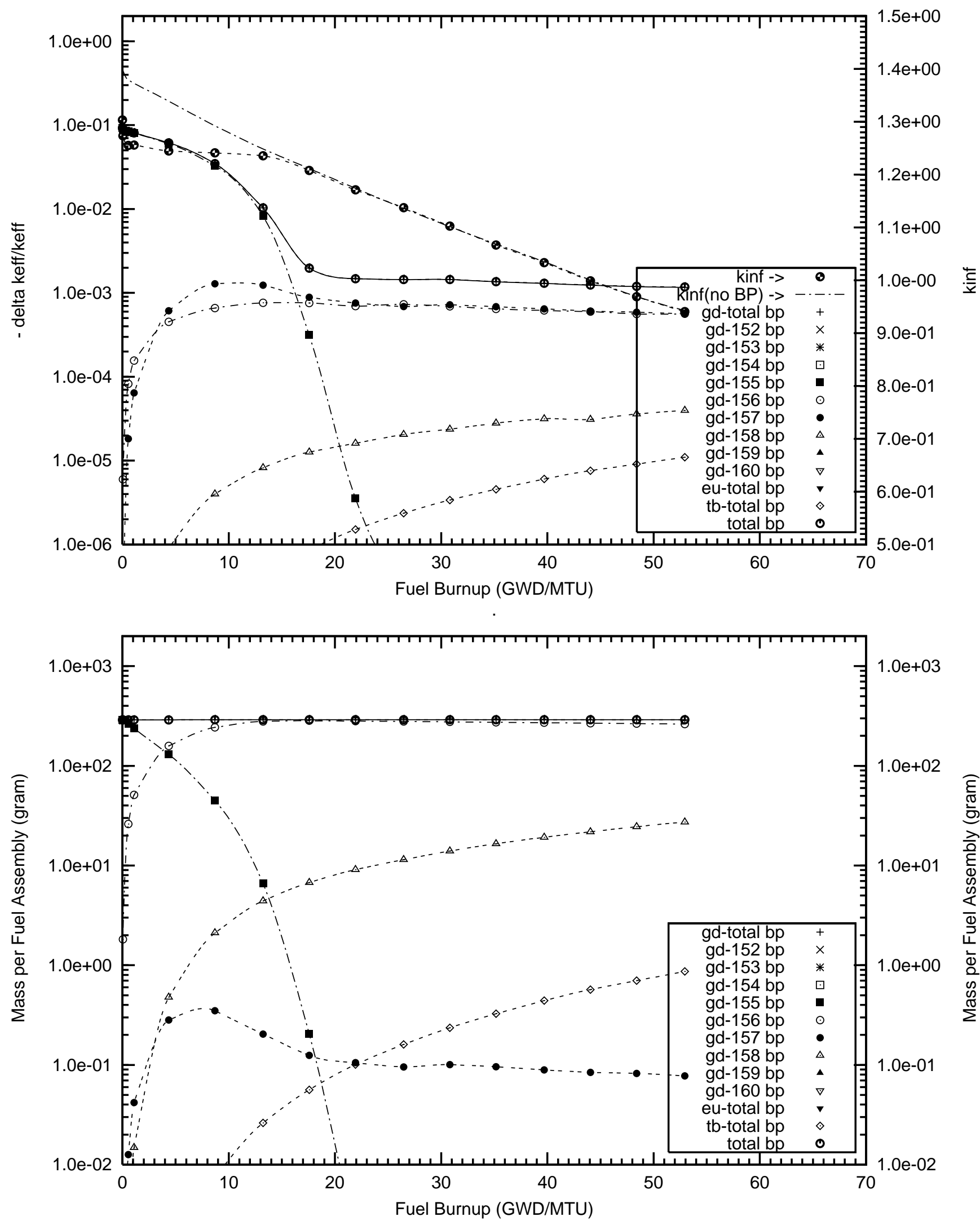
Figure 26. Negative Reactivity and Masses BP for Gd-155 and transmutation daughters as a function of fuel life for $17 \times 17$ fuel assemblies with 16 poison rods, $2.0 \mathrm{wt} \% \mathrm{Gd} 2 \mathrm{O} 3$ poison homogeneously mixed in the UO2 pellets

Reactor power 3400 MWth, 193 fuel assemblies, initial enrichment 4.5wt\%U-235

(Case :gd155_2_100p_h2o_16_bp_fuel_2_o)
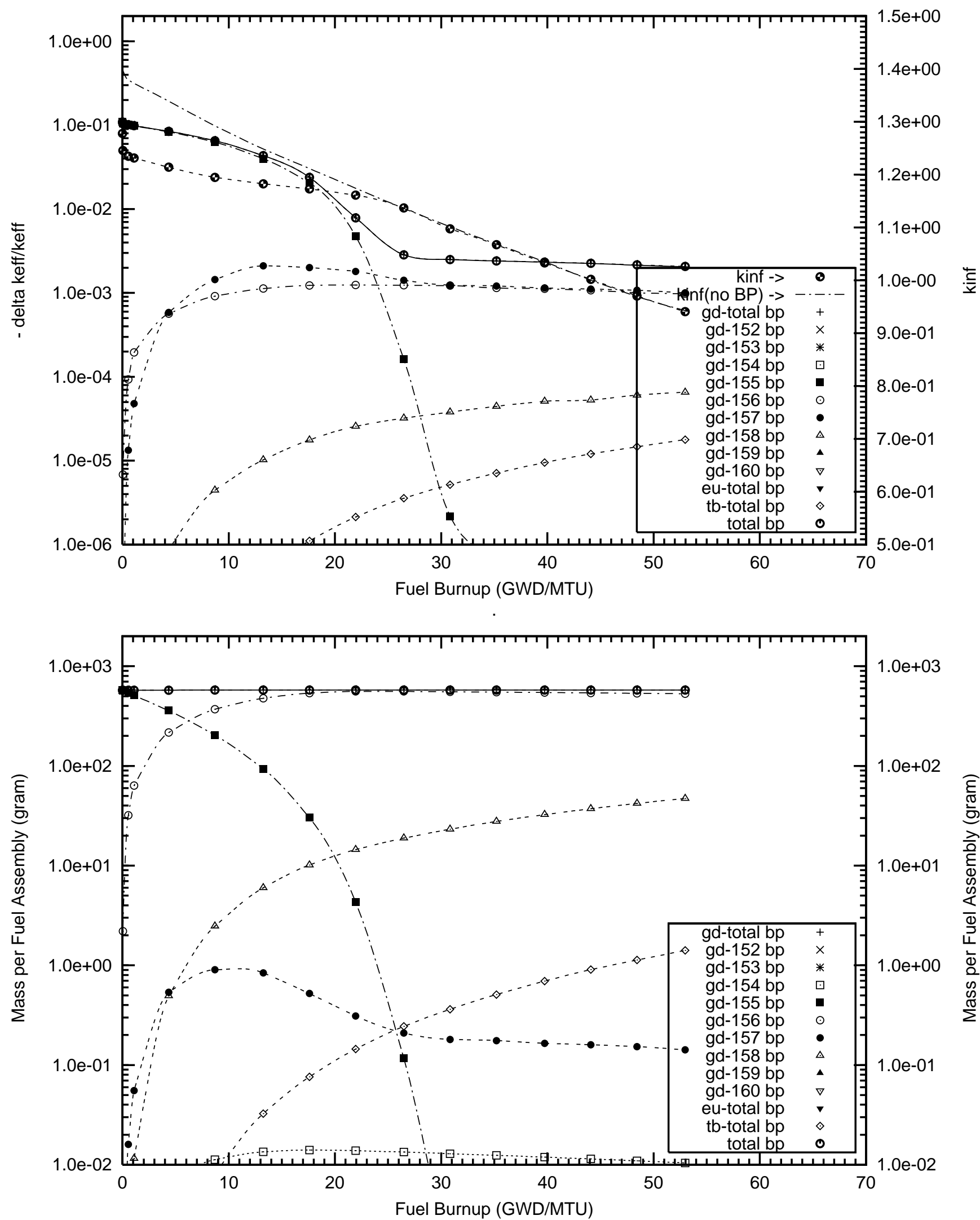
Figure 27. Negative Reactivity and Masses BP for Gd-157 and transmutation daughters as a function of fuel life for $17 \times 17$ fuel assemblies with 16 poison rods,

$0.5 \mathrm{wt} \% \mathrm{Gd} 2 \mathrm{O} 3$ poison homogeneously mixed in the UO2 pellets

Reactor power 3400 MWth, 193 fuel assemblies, initial enrichment 4.5wt\%U-235

(Case : gd157_0_100p_h2o_16_bp_fuel_2_o)
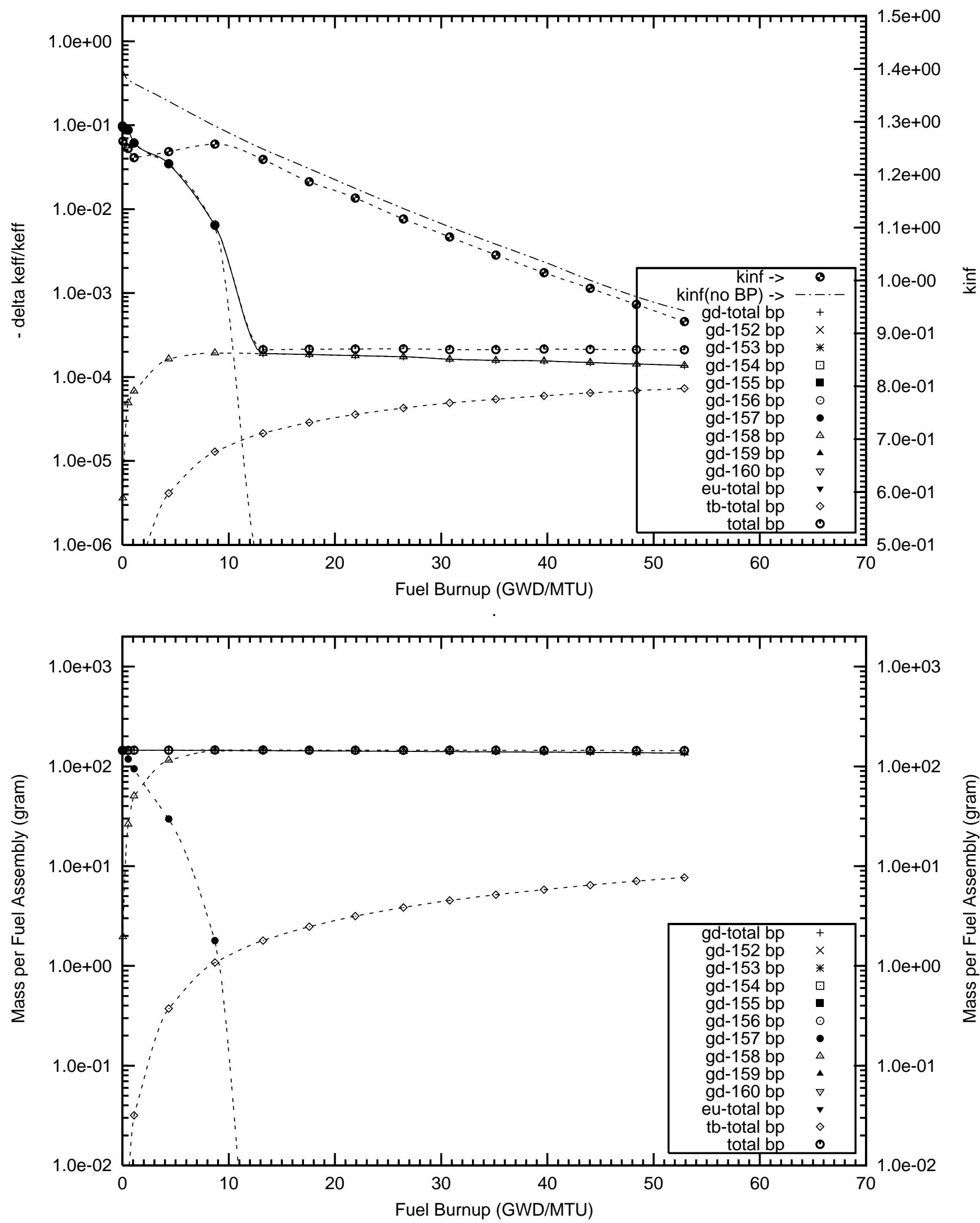
Figure 28. Negative Reactivity and Masses BP for Gd-157 and transmutation daughters as a function of fuel life for $17 \times 17$ fuel assemblies with 16 poison rods, $1.0 \mathrm{wt} \% \mathrm{Gd} 2 \mathrm{O} 3$ poison homogeneously mixed in the UO2 pellets

Reactor power 3400 MWth, 193 fuel assemblies, initial enrichment 4.5wt\%U-235

(Case : gd157_1_100p_h2o_16_bp_fuel_2_o)
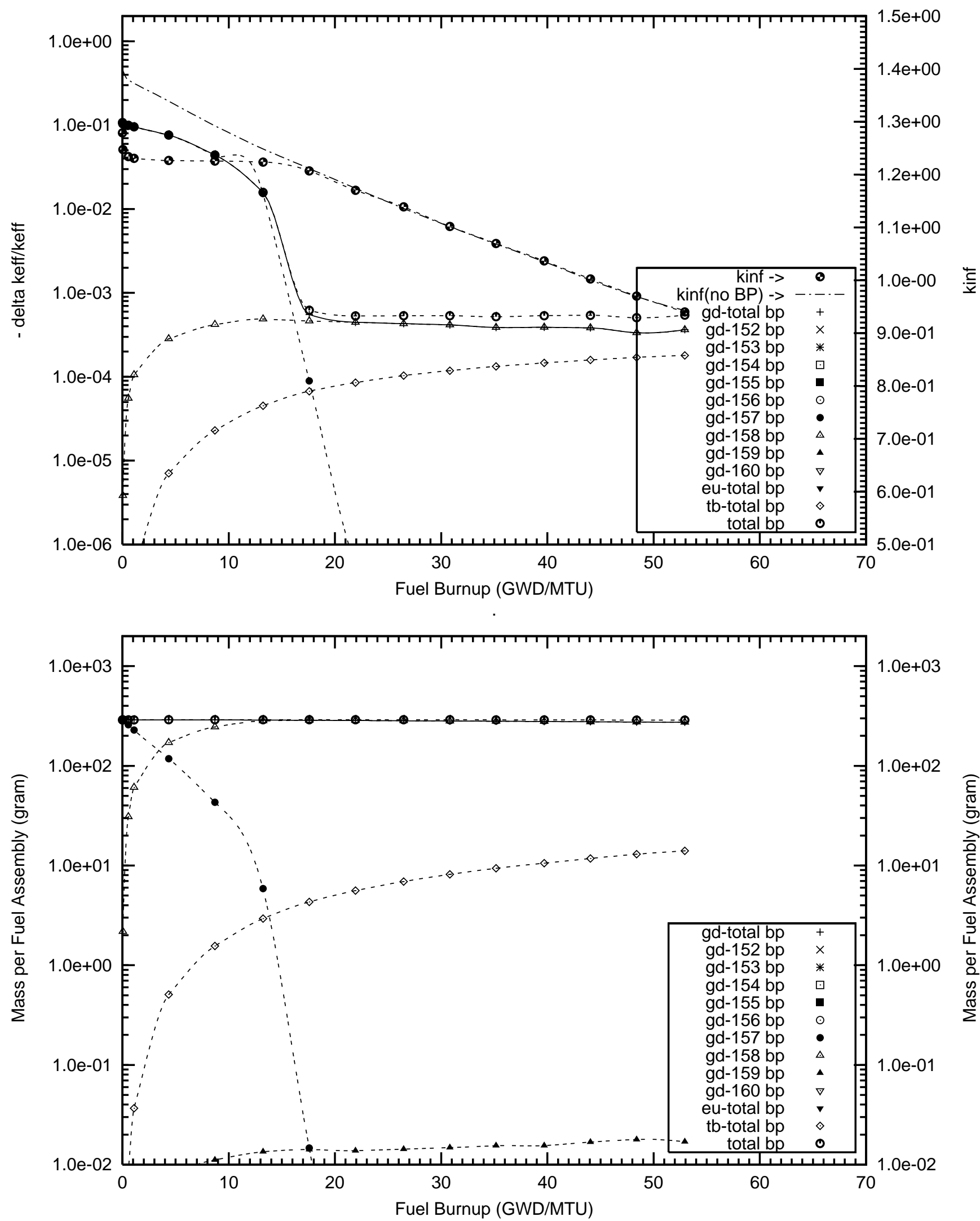
Figure 29. Negative Reactivity and Masses BP for Gd-157 and transmutation daughters as a function of fuel life for $17 \times 17$ fuel assemblies with 16 poison rods, $2.0 \mathrm{wt} \% \mathrm{Gd} 2 \mathrm{O} 3$ poison homogeneously mixed in the UO2 pellets

Reactor power 3400 MWth, 193 fuel assemblies, initial enrichment 4.5wt\%U-235

(Case : gd157_2_100p_h2o_16_bp_fuel_2_o)
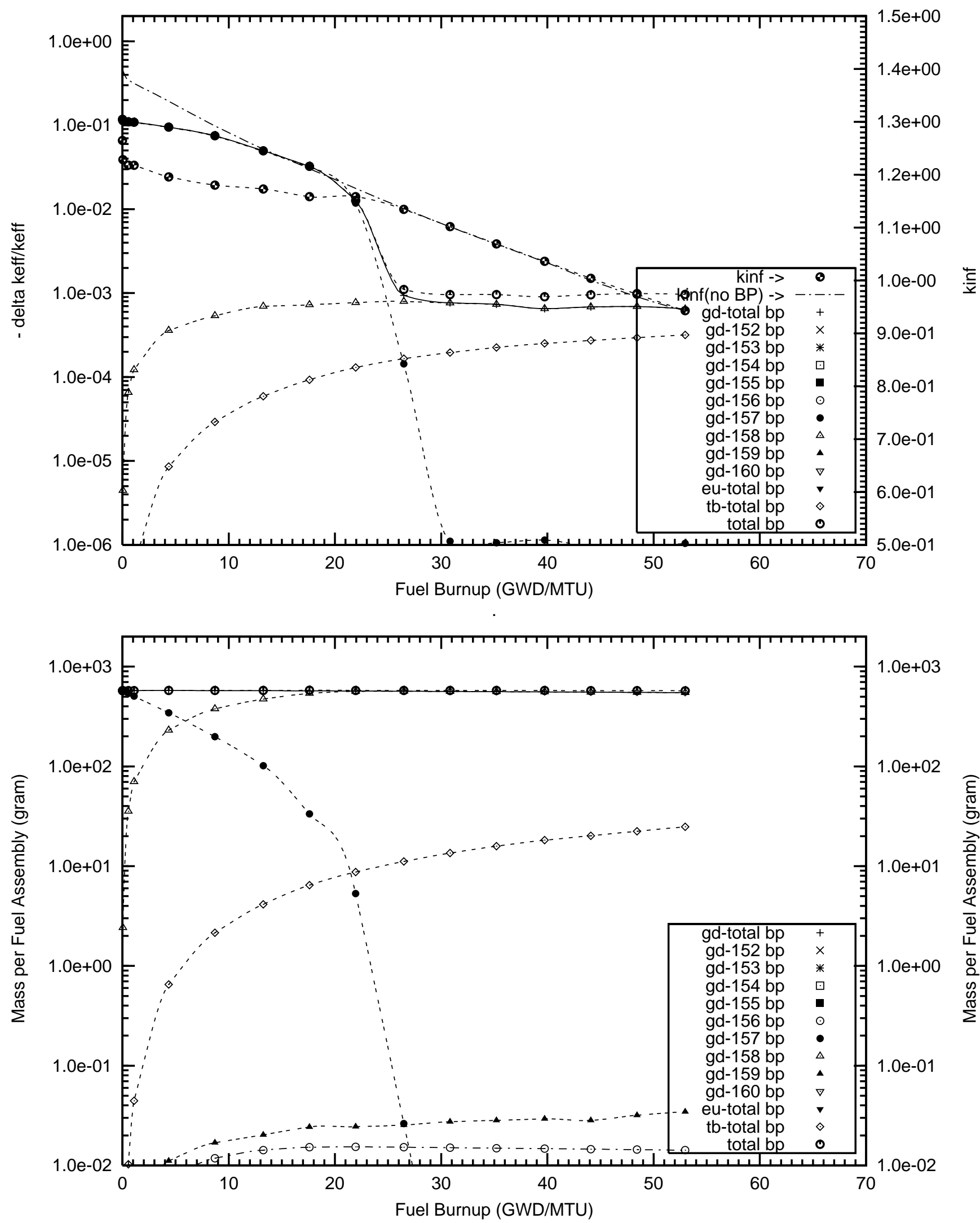
Figure 30. Negative Reactivity and Masses BP for Gd-157 and transmutation daughters as a function of fuel life for $17 \times 17$ fuel assemblies with 16 poison rods, $4.0 \mathrm{wt} \% \mathrm{Gd} 2 \mathrm{O} 3$ poison homogeneously mixed in the UO2 pellets

Reactor power 3400 MWth, 193 fuel assemblies, initial enrichment 4.5wt\%U-235

(Case : gd157_3_100p_h2o_16_bp_fuel_2_o)
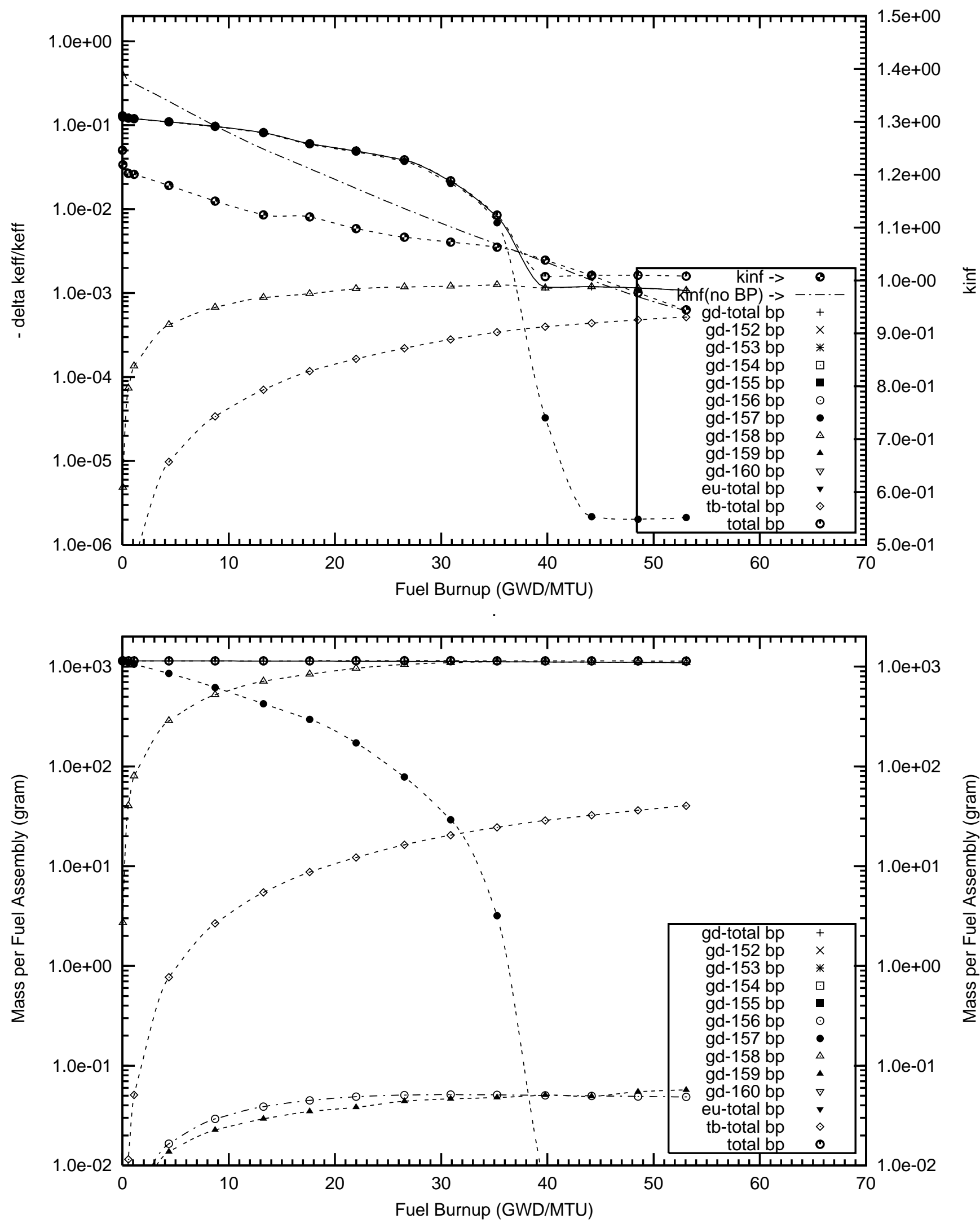


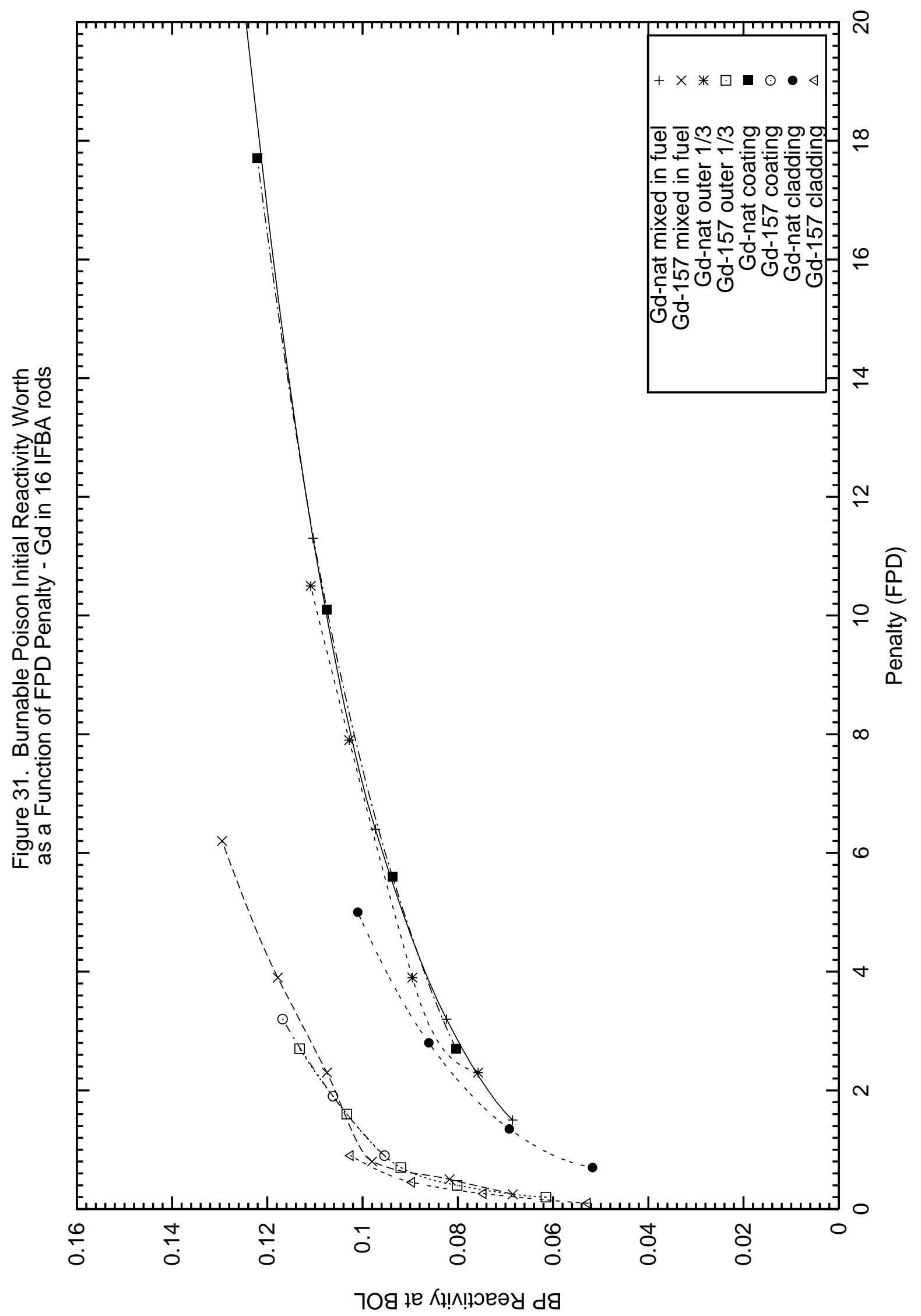




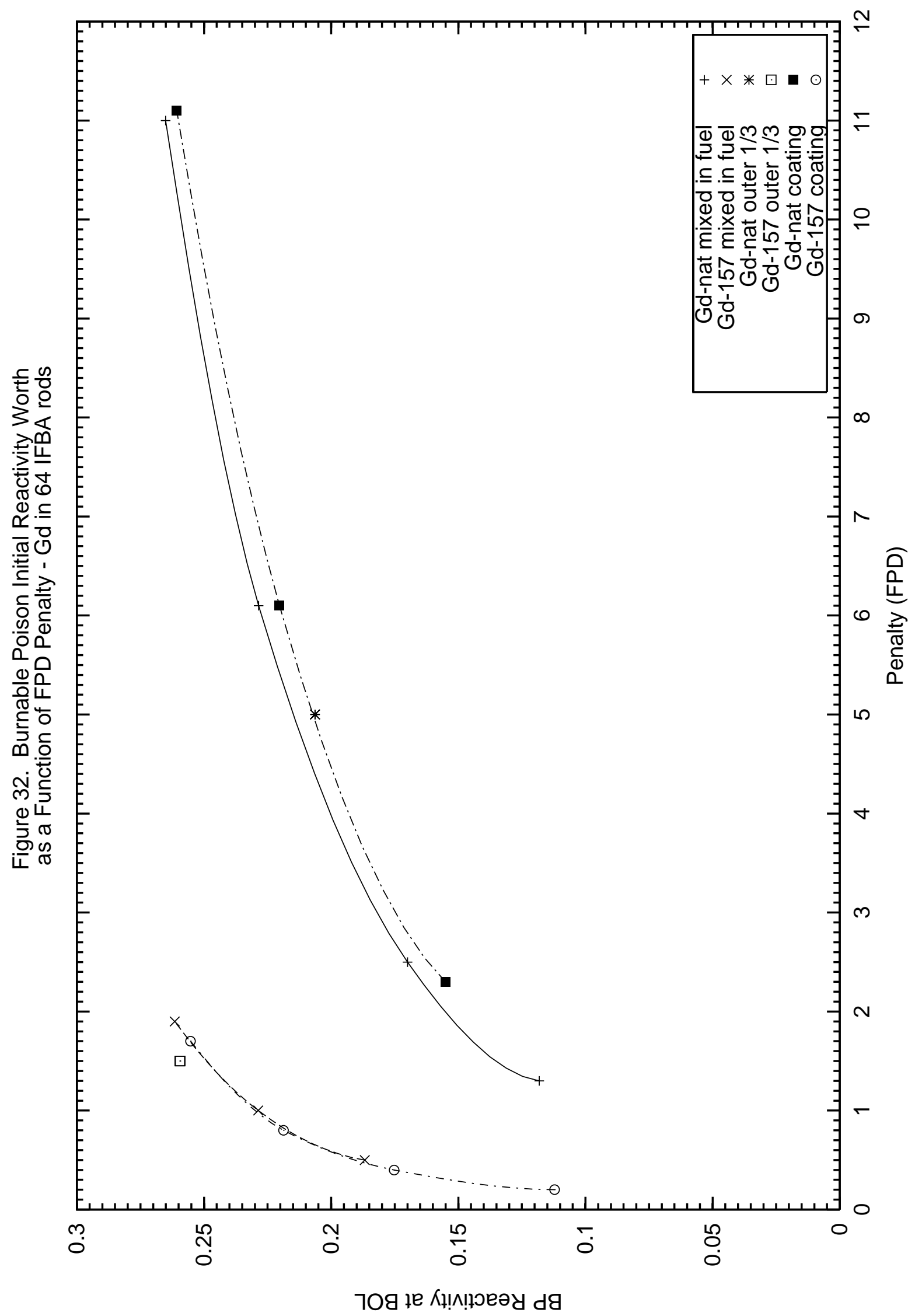




\section{NEUTRONICS PERFORMANCE RESULTS FOR SAMARIUM}

The use of samarium with natural isotopic abundances as a burnable poison has been previously discarded because of the large residual negative reactivity worth of the samarium burnable poison isotopes and their daughters at EOL of the fuel. The use of samarium enriched in ${ }^{149} \mathrm{Sm}$ greatly reduces this residual absorber burden.

In this section, we will investigate the potential benefits of using enriched samarium as a burnable absorber in PWRs. Four different configurations of the samarium burnable poison, similar to those of the case of $\mathrm{Gd}$, were investigated.

1. A homogeneous mixture of the burnable poison samaria $\left(\mathrm{Sm}_{2} \mathrm{O}_{3}\right)$ inside the fuel pellets of a limited number of fuel rods. A summary of the results is given in Table 9. The plots of the reactivity worths and the masses of the BP isotopes and their daughters are displayed in Appendix B1.

2. A homogeneous mixture of the burnable poison as an oxide (samaria) in the outer one-third volume of the fuel pellets. A summary of the results is given in Table 10. Plots of the reactivity worths and the masses of the BP isotopes and their daughters are displayed in Appendix B2.

3. An oxide burnable poison coating (samaria) on the surface of the fuel pellets in a limited number of fuel rods, similar to the $\mathrm{ZrB}_{2}$ coatings. A summary of the results is given in Table 11. Plots of the reactivity worths and the masses of the BP isotopes and their daughters are displayed in Appendix B3.

4. A homogeneous mixture of the burnable poison samarium in the form of a metal alloyed with the Zircaloy cladding of a number of fuel rods. Because of the low boiling point of samarium metal, it is conceived to first form a Sn-Sm alloy, which then would be mixed with zirconium to form a Zircaloy-samarium alloy. Feasibility experiments will be done during phase 3. A summary of the neutronics results is given in Table 12. Plots of the reactivity worths and the masses of the BP isotopes and their daughters are displayed in Appendix B4.

In all the neutronics calculations presented in this section, a theoretical density of $7.43 \mathrm{~g} / \mathrm{cm}^{3}$ was used for $\mathrm{Sm}_{2} \mathrm{O}_{3}$, and $7.7 \mathrm{~g} / \mathrm{cm}^{3}$ for metallic samarium. For $\mathrm{UO}_{2}$ a theoretical density of $10.97 \mathrm{~g} / \mathrm{cm}^{3}$ was used. The actual density fraction of the fuel pellets and of the burnable poison pellets was $95 \%$ of theoretical density.

The half-lives, natural isotopic abundances, and burnup chains related to the depletion and transmutation of samarium are given below. Note that the adjacent bands of isotopes such as the $\mathrm{Gd}$ isotopes (decay from $\mathrm{Eu}$ isotopes) and $\mathrm{Nd}$ isotopes (decay from $\mathrm{Pm}$ isotopes) are not shown. Also the isomeric states of certain isotopes are not shown. However, the generation of these daughters was taken into account in the BP depletion and decay calculations. 


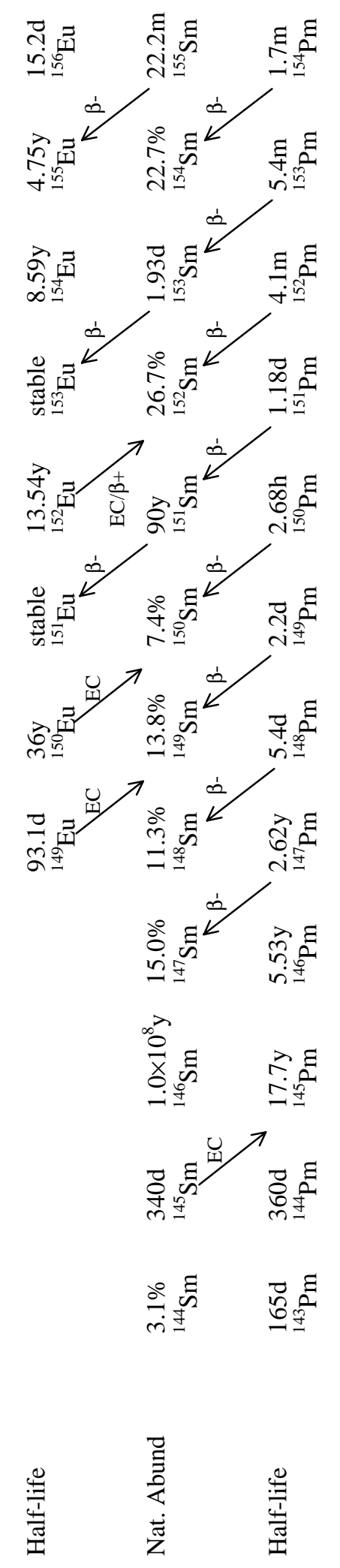


Figure 33 displays the neutron capture cross sections for the samarium isotopes, and Figure 34 displays the capture cross sections of several of the europium decay daughters from samarium. The ${ }^{149} \mathrm{Sm}$ stable isotope (13.8\% natural isotopic abundance) exhibits a very large neutron capture cross section (thermal cross section of 40,150 barns and a RI of 3,400 barns). The ${ }^{149} \mathrm{Sm}(\mathrm{n}, \gamma){ }^{150} \mathrm{Sm}$ reaction leads to ${ }^{150} \mathrm{Sm}$, which is stable and has a thermal capture cross section of 104 barns. ${ }^{144} \mathrm{Sm}$ (3\%) and ${ }^{148} \mathrm{Sm}(11 \%)$ have small capture cross sections (thermal values of 0.7 and 2.5 barns, respectively). ${ }^{154} \mathrm{Sm}(27 \%)$ has a capture cross section of approximately 10 barns at $0.0253 \mathrm{eV}$. The other stable isotopes, such as ${ }^{147} \mathrm{Sm}(15 \%)$ and ${ }^{150} \mathrm{Sm}(7 \%)$, have thermal capture cross sections of the order of 57 and 104 barns, respectively. The ${ }^{150} \mathrm{Sm}(\mathrm{n}, \gamma){ }^{151} \mathrm{Sm}$ reaction leads to ${ }^{151} \mathrm{Sm}$, which has a very large neutron capture cross section (thermal value of 15,200 barns and RI of 3,520 barns). ${ }^{151} \mathrm{Sm}$ decays to ${ }^{151} \mathrm{Eu}$ with a half-life of 93 years. Because of the long half-life, only very small amounts of ${ }^{151} \mathrm{Eu}$ will be generated. Note that ${ }^{151} \mathrm{Eu}$ exhibits a very large capture cross section. (thermal value of 9,200 barns and RI of 3,300 barns). Because of the long half-life of ${ }^{151} \mathrm{Sm}$ and the very large thermal capture cross section of ${ }^{151} \mathrm{Sm}$, the preferential path will be the transmutation of ${ }^{151} \mathrm{Sm}$ into ${ }^{152} \mathrm{Sm}$. The stable isotope ${ }^{152} \mathrm{Sm}(26.7 \%)$ has a large neutron capture cross section (thermal value of 206 barns and RI 2,970 barns). The ${ }^{152} \mathrm{Sm}(\mathrm{n}, \gamma){ }^{153} \mathrm{Sm}$ reaction leads to ${ }^{153} \mathrm{Sm}$ which decays to ${ }^{153} \mathrm{Eu}$ with a halflife of 46.7 hours. Because of the relatively short half-life of ${ }^{153} \mathrm{Sm}$, most of the generated ${ }^{153} \mathrm{Sm}$ will be converted into ${ }^{153} \mathrm{Eu} .{ }^{153} \mathrm{Eu}$ is stable and exhibits a very large capture cross section. Transmutation to ${ }^{154} \mathrm{Eu}$ (8.6 year half-life) and ${ }^{155} \mathrm{Eu}$ (4.76 year half-life) will still result in an appreciable combined residual capture. Because of the presence of isotopes with small to medium capture cross sections in samarium with natural isotopic abundances, and because of the generation of ${ }^{153} \mathrm{Eu}$, enriching samarium in ${ }^{149} \mathrm{Sm}$ should lead to a smaller residual neutron absorption of the BP.

Table 9 displays the results of using $\mathrm{Sm}_{2} \mathrm{O}_{3}$, mixed homogeneously with the fuel pellets of a number of fuel rods. Note that for the same initial reactivity worth of the BP, the residual negative reactivity worth of samarium enriched in ${ }^{149} \mathrm{Sm}$ is smaller than the residual worth of samarium with natural isotopic abundances. The relative contribution from the europium daughters is also smaller.

The reactivity worths of the $\mathrm{BP}$ isotopes, for a $4.0 \mathrm{wt} \%$ loading of $\mathrm{Sm}_{2} \mathrm{O}_{3}$ with natural isotopic abundances, homogeneously mixed inside the fuel pellets of 16 IFBA rods per fuel assembly, are shown in Figure 35. The initial reactivity worth of the BP is 0.112 and the residual reactivity ratio is $16.1 \%$. For a $1.0 \mathrm{wt} \%$ homogeneous loading in $16 \mathrm{IFBA}$ rods containing $\mathrm{Sm}_{2} \mathrm{O}_{3}$ fully enriched in ${ }^{149} \mathrm{Sm}$, the results are shown in Figure 37. The initial reactivity worth of the BP is 0.114 , and the residual reactivity ratio is $5.5 \%$. While for these two cases the initial reactivity worth of the BP is the same, the use of samarium enriched in ${ }^{149} \mathrm{Sm}$ requires a loading that is four times smaller than a BP containing samarium with natural isotopic abundances. If the weight percentage loading of the BP is a limiting factor due to material considerations, then the use of ${ }^{149} \mathrm{Sm}$ will produce an added benefit, such as increased allowable amounts of BP in each of the IFBA rods. Longer burnout times with the same initial reactivity worth of the BP can then be achieved, which are needed for long fuel cycles.

In Table 9 the residual reactivity worths, due to the Pm daughters of the BP containing samarium enriched in ${ }^{149} \mathrm{Sm}$, should essentially be zero, since only the decay of ${ }^{145} \mathrm{Sm}$ leads to ${ }^{145} \mathrm{Pm}$. There are essentially no paths from the transmutation of ${ }^{149} \mathrm{Sm}$ to any of the Pm isotopes. However, the table shows residual reactivity worths of the order of $1.0 \times 10^{-10}$. This is because for each case, all the BP isotopes and their daughters (which are not present in the initial loading of the BP) have been added as traces to the BP with a number density of $1.0 \times 10^{-15}$ instead of zero. This is to avoid problems at the $\mathrm{BOC}$ in the input to the MCNP4C code and to avoid computer exception errors (division by zero) when calculating spectrum-weighted cross sections in the TALLY code.

Comparison of the cases containing 64 IFBA rods with the cases containing 16 or 8 IFBA rods clearly show that, for a given initial reactivity worth, the burnout rates are greater for 64 IFBA rods 
than for 16 or 8 IFBA rods. This is clearly due to the self-shielding effect of the neutrons in the IFBA rods. Note that the residual BP reactivity worths are smaller for the 64 IFBA cases.

Figures 35 and 36 display the results of the reactivity worths for several cases with 16 IFBA rods per fuel assembly. The IFBA rods contained respectively 4 wt $\%$ and 8 wt $\% \mathrm{Sm}_{2} \mathrm{O}_{3}$ BP homogeneously mixed in the pellets. The $\mathrm{Sm}_{2} \mathrm{O}_{3}$ material contained samarium with natural isotopic abundances. The initial reactivity worth for the 4 wt \% case is 0.112 . Doubling the loading of $\mathrm{Sm}_{2} \mathrm{O}_{3}$ to 8 wt \% only slightly increases the initial worth by $14 \%$. This is due to the increased neutron self-shielding effect and the slightly lower uranium loading in the IFBA rods. Note that the combined $\mathrm{UO}_{2}-\mathrm{Sm}_{2} \mathrm{O}_{3}$ density was kept at $95 \%$ of the theoretical density of the mixture. The burnout time for the $8 \mathrm{wt} \%$ is also much longer than the $4 \mathrm{wt} \%$ case. Note also that the trend of the fuel assembly lattice $\mathrm{k}_{\mathrm{inf}}$ as a function of depletion time is fairly flat for much longer fuel depletion times. Figures 35 and 36 also display the masses of the burnable poison isotopes and their daughters as a function of depletion time.

The figures exhibit several interesting features. For example, the case with 16 IFBA rods whose fuel pellets contain 4.0 wt \% $\mathrm{Sm}_{2} \mathrm{O}_{3}$ with natural $\mathrm{Sm}$ (displayed in Figure 35) exhibit several interesting effects. The reactivity worth of the burnable poison isotope ${ }^{149} \mathrm{Sm}$ decreases rapidly with depletion time, and reaches a nearly constant value at approximately 350 FPDs (approximately 13 GWD/MTU burnup). The reactivity worth of ${ }^{148} \mathrm{Sm}$ increases slightly with depletion time, due the production of ${ }^{148} \mathrm{Sm}$ through the ${ }^{147} \mathrm{Sm}(\mathrm{n}, \gamma){ }^{148} \mathrm{Sm}$ reaction, and it reaches a nearly equilibrium value. Since the neutron capture cross section of ${ }^{149} \mathrm{Sm}$ is much greater than that of ${ }^{148} \mathrm{Sm}$, the capture reaction rate (and thus the reactivity worth) of ${ }^{149} \mathrm{Sm}$ reaches a secular equilibrium with the capture reaction rate of ${ }^{148} \mathrm{Sm}$. After most of the original ${ }^{149} \mathrm{Sm}$ nuclei have burned out, ${ }^{149} \mathrm{Sm}$ nuclei are produced through the ${ }^{148} \mathrm{Sm}(\mathrm{n}, \gamma){ }^{149} \mathrm{Sm}$ reaction. Since the neutron capture cross section of ${ }^{149} \mathrm{Sm}$ is much larger than that of ${ }^{148} \mathrm{Sm}$, these ${ }^{149} \mathrm{Sm}$ nuclei will burn away at a much higher rate than the ${ }^{148} \mathrm{Sm}$ nuclei, essentially immediately after they were created. This effect is similar to the secular equilibrium effect in the decay of a parent isotope that has a very long half-life together with its daughter, which has a very short half-life. In the case of ${ }^{148} \mathrm{Sm} /{ }^{149} \mathrm{Sm}$, we reach a secular equilibrium between a parent isotope that has a small neutron capture cross section $\left({ }^{148} \mathrm{Sm}\right)$, together with its daughter, which has a very large cross section $\left({ }^{149} \mathrm{Sm}\right)$.

Figures 37-39 show the reactivity worths of three different cases containing 16 IFBA rods per fuel assembly, when the samarium in the BP is fully enriched in ${ }^{149} \mathrm{Sm}$. The $\mathrm{Sm}_{2} \mathrm{O}_{3}$ loadings for these figures are $1.0 \mathrm{wt} \%, 2 \mathrm{wt} \%$, and $4 \mathrm{wt} \%$, respectively. The initial reactivity worths are $0.114,0.127$ and 0.141 . Doubling the loading from $1 \mathrm{wt} \%$ to $2 \mathrm{wt} \%$ increased the initial reactivity worth by $11 \%$, and by $24 \%$ when the $\mathrm{Sm}_{2} \mathrm{O}_{3}$ loading was quadrupled. However, the burnout times increase almost proportionally with the loadings. Note also that the trend of the fuel assembly lattice $\mathrm{k}_{\mathrm{inf}}$ as a function of depletion time remains relatively flat for longer depletion times. The flatness increases with the loading (compare figures 37, 38, and 39). Figures 37-39 also display the masses of the burnable poison isotopes and their daughters as a function of depletion time.

In Appendix B1, we have displayed the plots of the reactivity worths for each of the cases displayed in Table 9. In each plot the reactivity worths for each of the BP isotopes and their daughters are shown. Note that for a given initial reactivity worth of the BP, the burnout rate is higher when the BP is spread over 64 IFBA rods.

The MVCs were calculated by decreasing the water coolant density by $10 \%$. Note that for all the cases containing 8 and 16 IFBA rods, the MVCs remain negative. For the cases with 64 IFBA rods, the MVC becomes positive for the lattices containing identical standard $17 \times 17$ fuel assemblies with the same BP loadings. Calculations of the MVC were not performed for checkerboard loadings of selected fuel assemblies containing 64 IFBA rods. 
Figure 40 displays the initial reactivity worths of the samarium-based BP as a function of the residual BP penalty in FPDs for several configurations containing 16 IFBA rods. The trend for the four different forms of BP are very similar. Note also the clear advantage of using samarium enriched in ${ }^{149} \mathrm{Sm}$ over samarium with natural isotopic abundances. Figure 41 displays the initial reactivity worths of the BP vs FPD penalty for fuel assembly configurations containing 64 IFBA rods. 


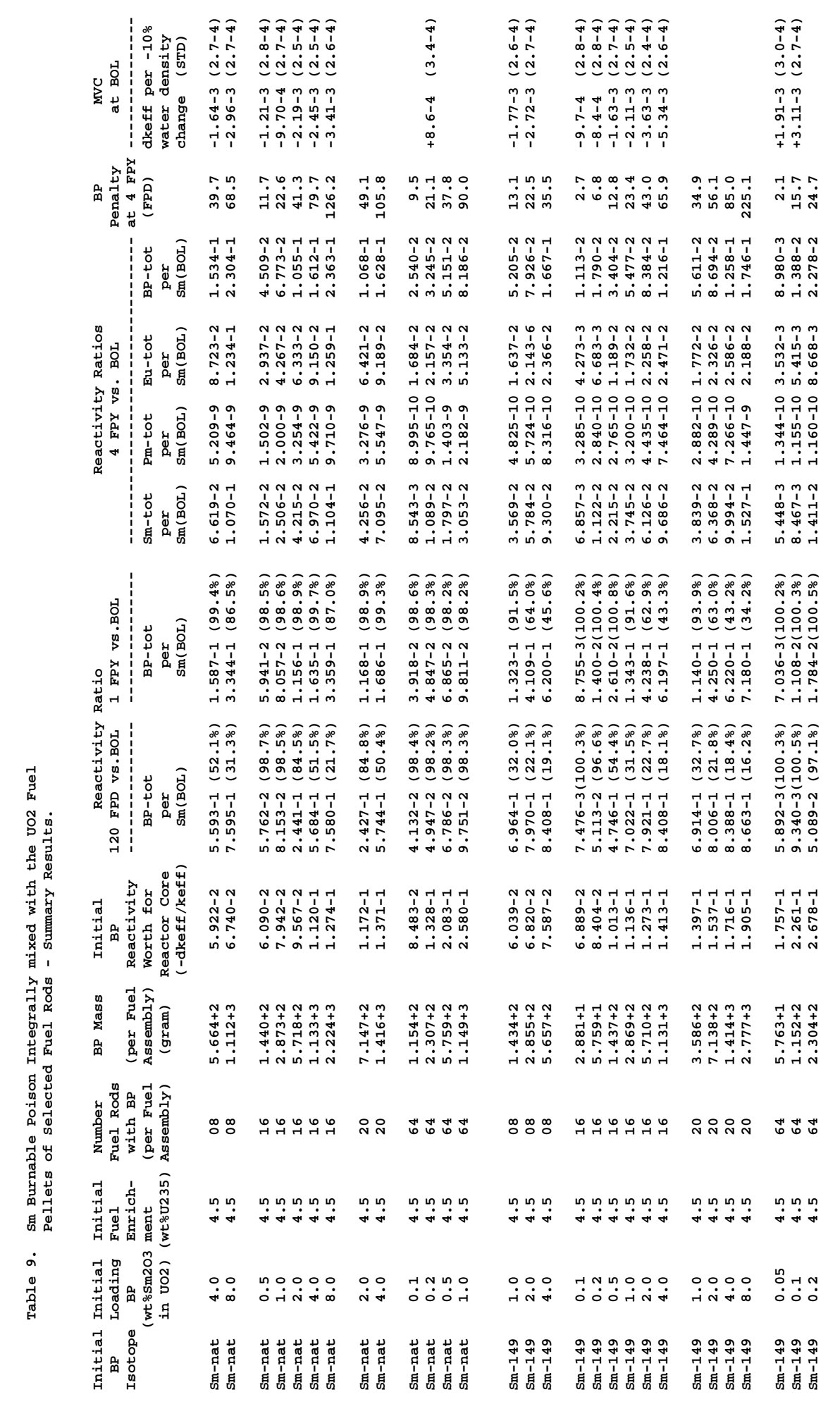




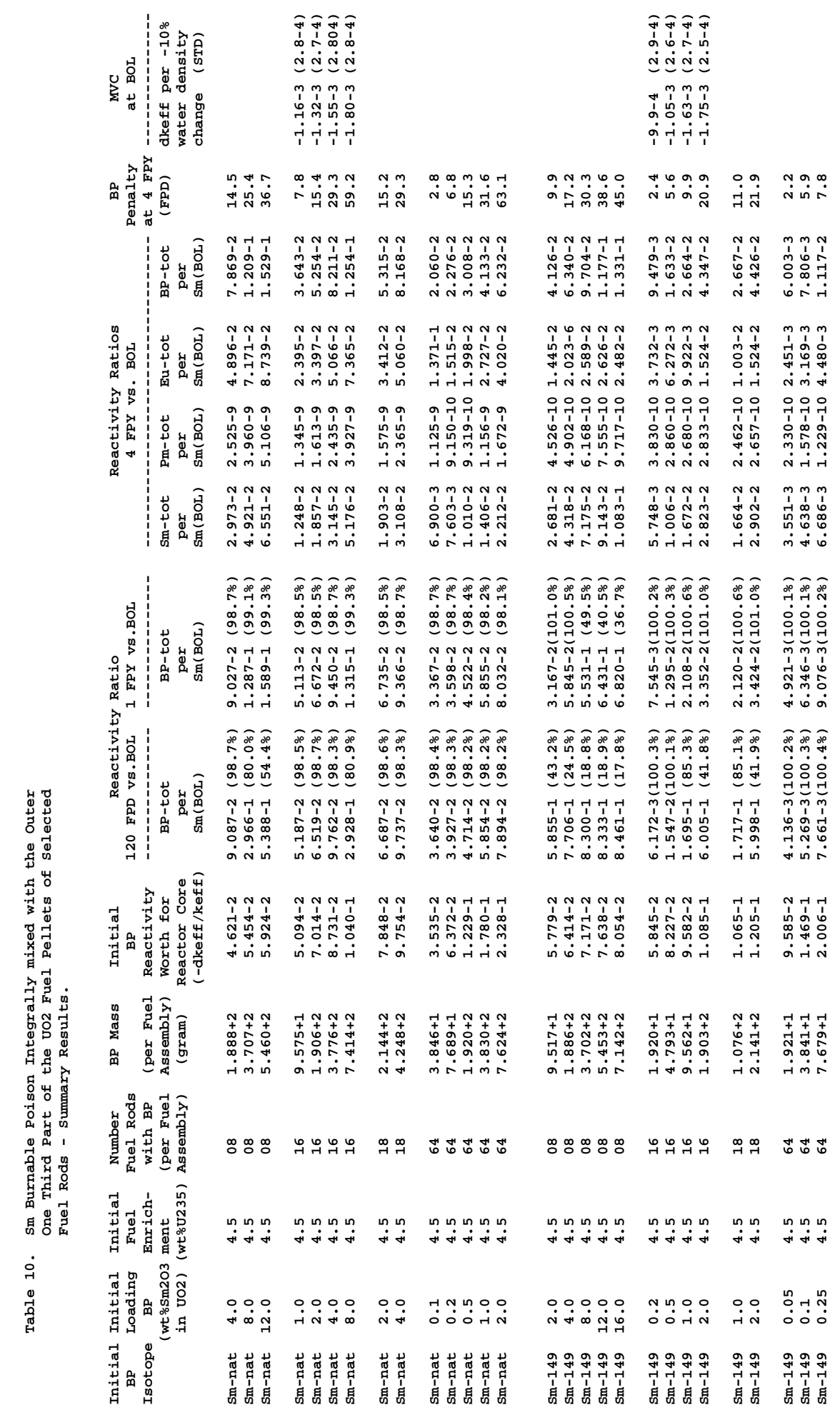




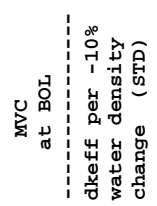<smiles></smiles>

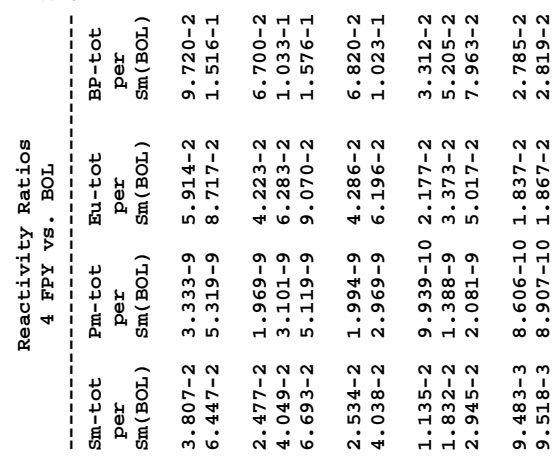

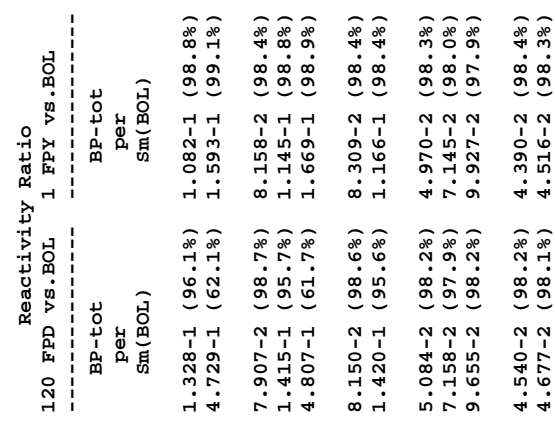

年

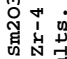

दू ॠ

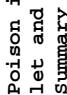

a d

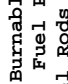

最要豆

章

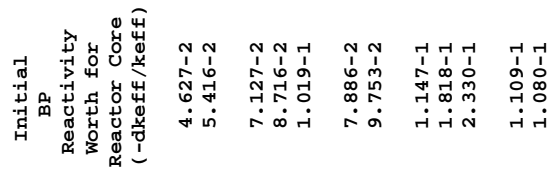

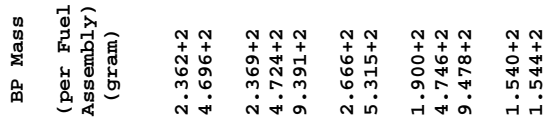

0
0

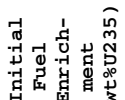

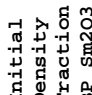

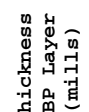

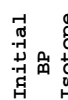

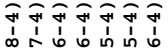

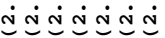

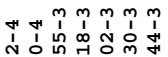

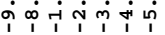

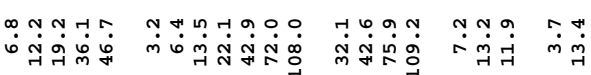

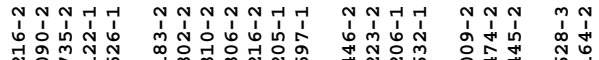

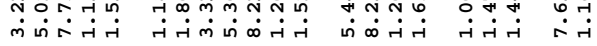

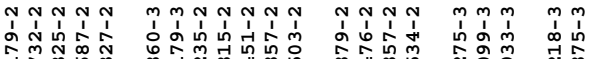
प.

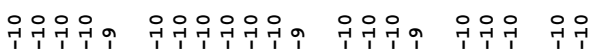

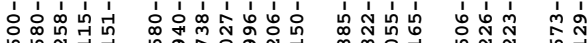

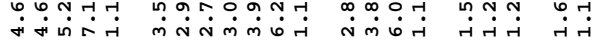

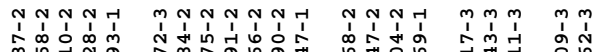

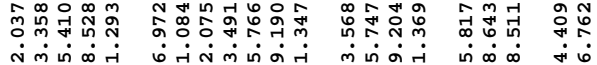

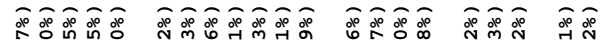

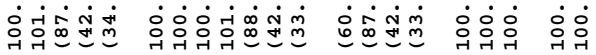
N

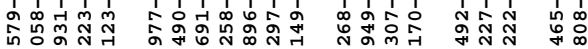

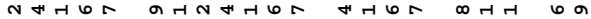

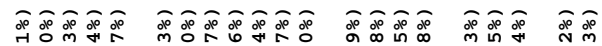

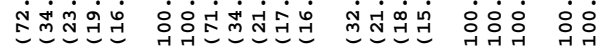

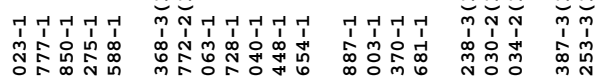
miri⿻上丨

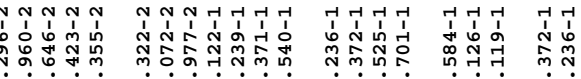

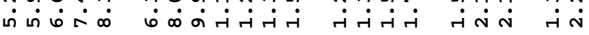

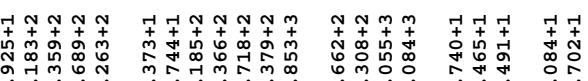

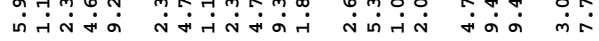

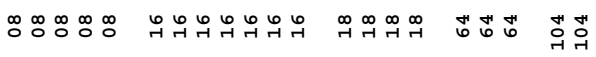

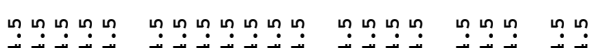

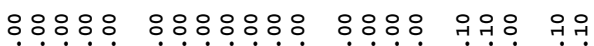

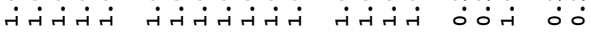

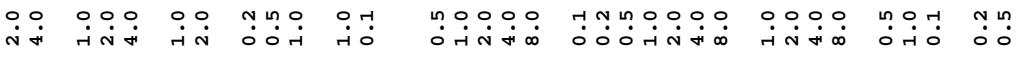

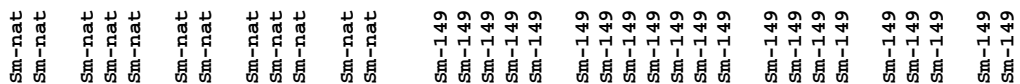




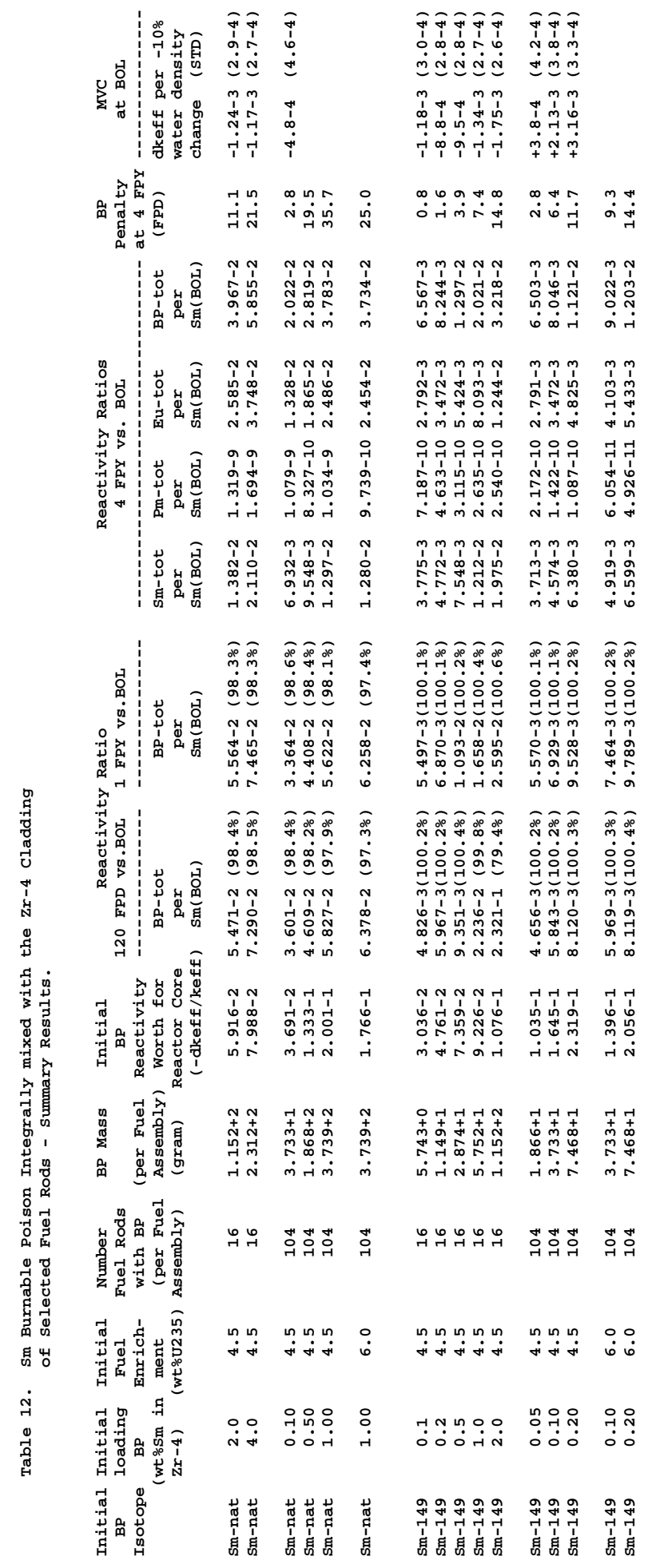


Figure 33. Neutron Cross-Sections as a Function of Energy

$\mathrm{Sm}-144$ (62144.40c)

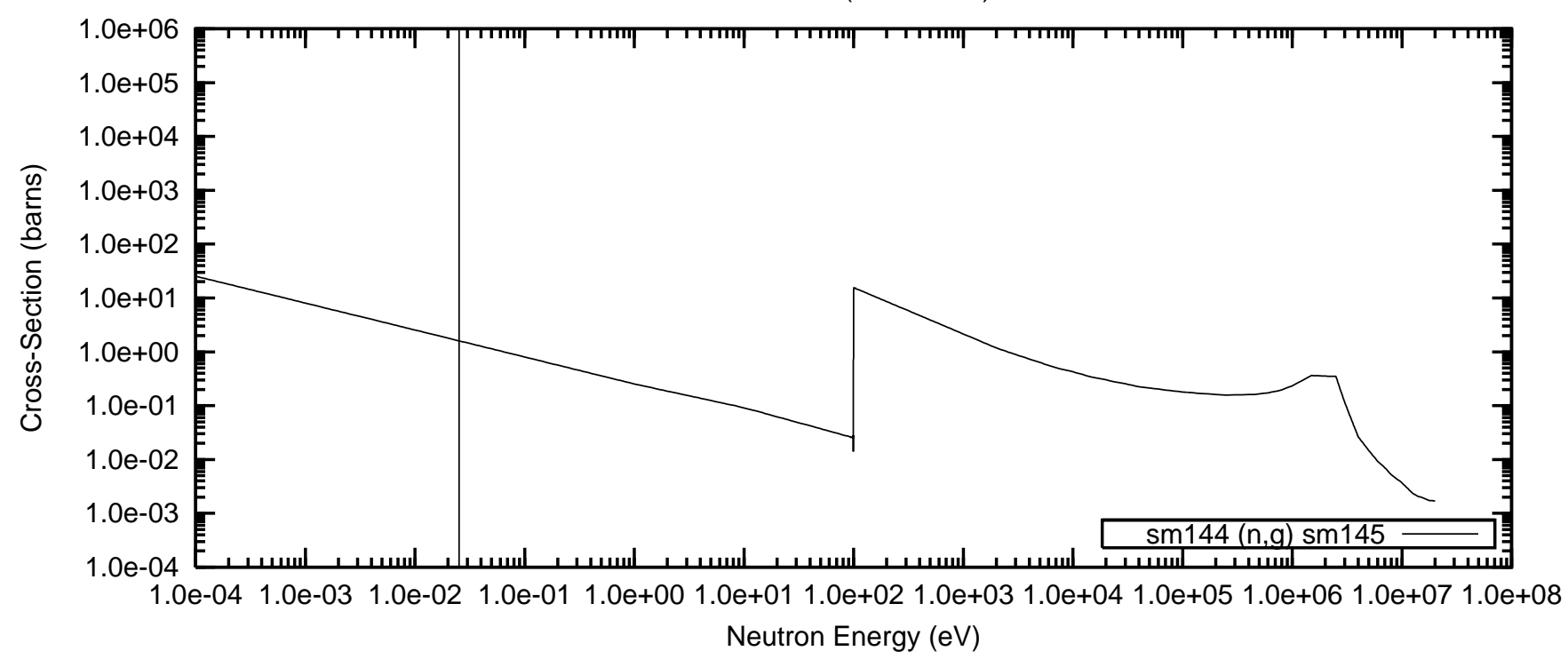

Sm-145 (62145.40c)

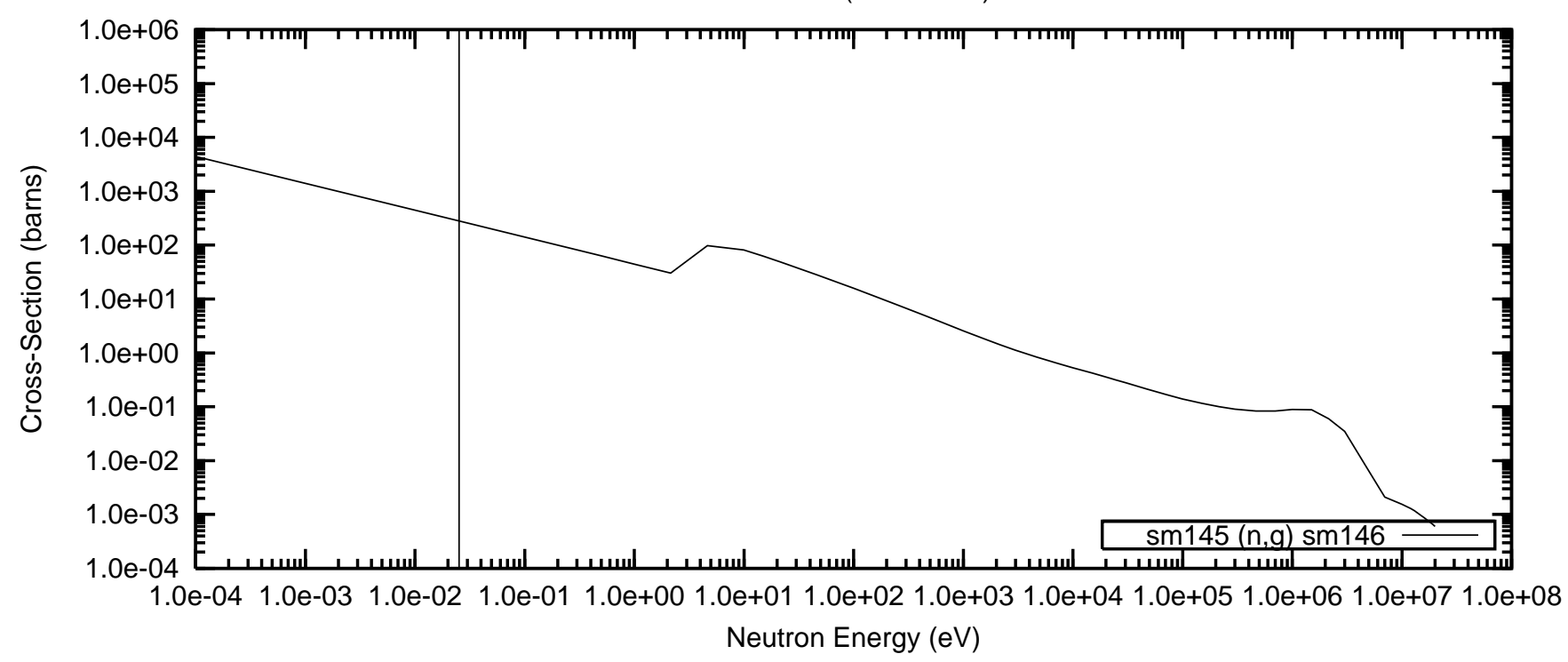

Sm-146 (62146.40c)

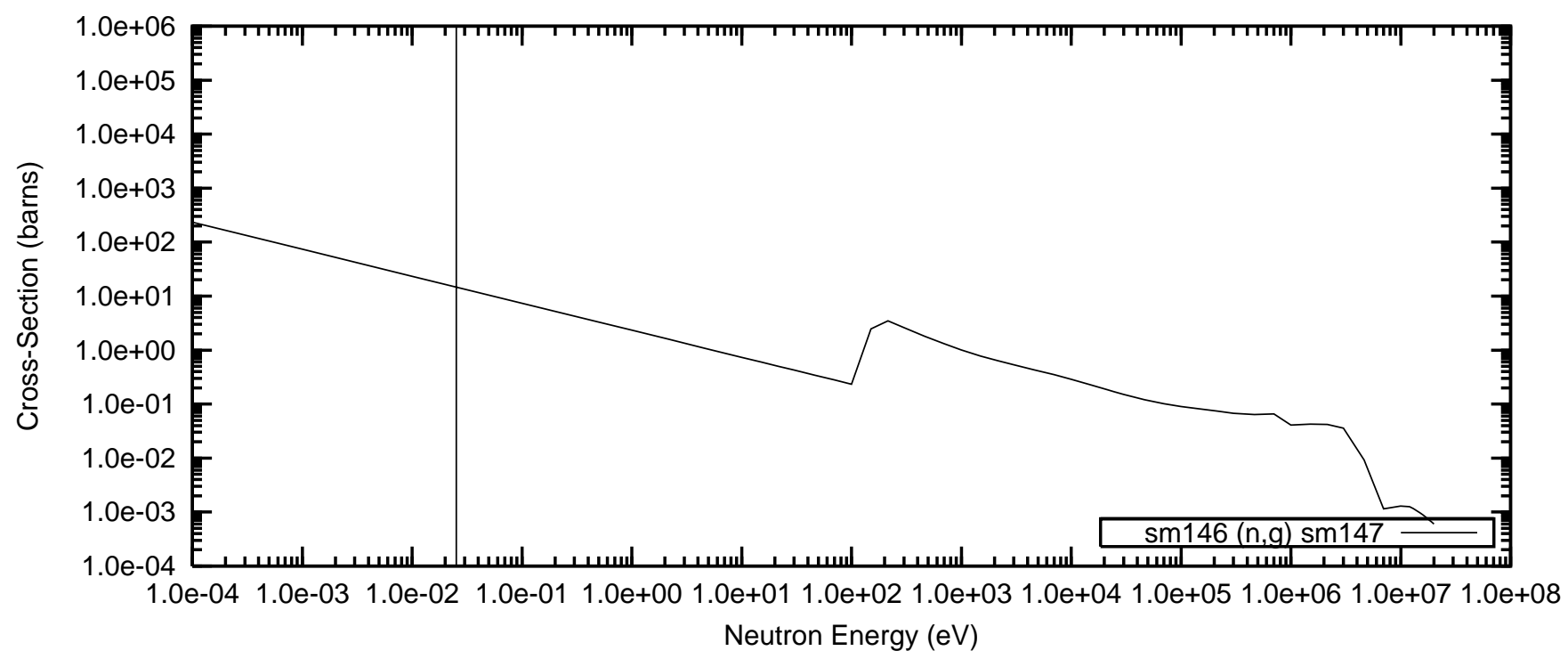


Figure 33(cont). Neutron Cross-Sections as a Function of Energy Sm-147 (62147.40c)

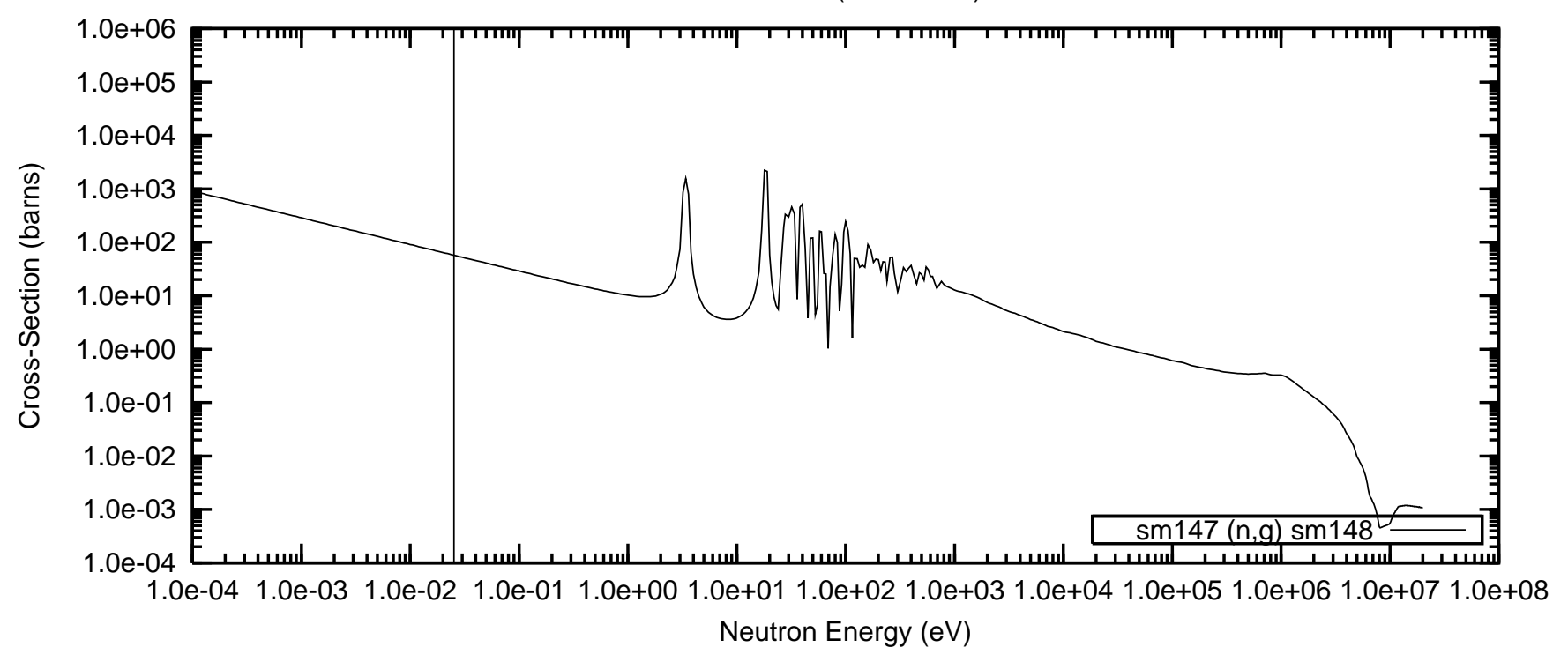

Sm-148 (62148.40c)

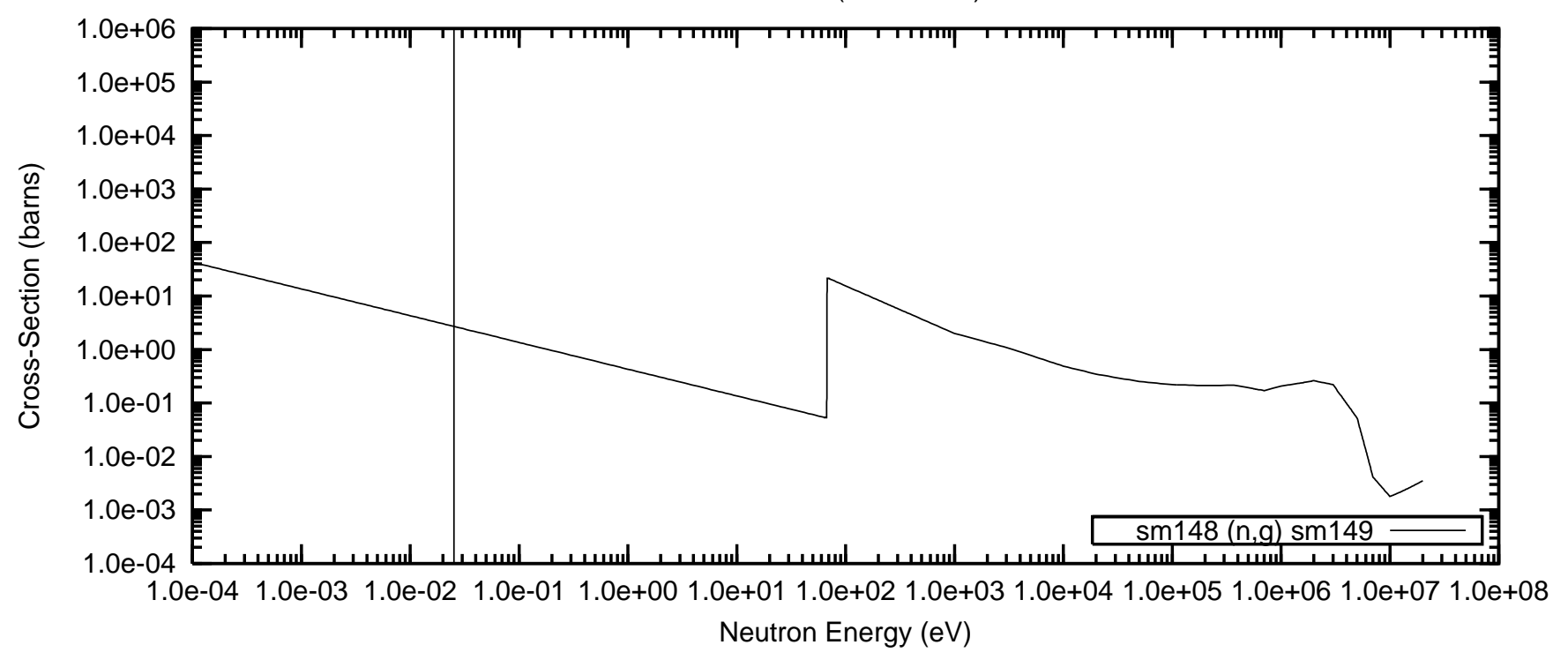

Sm-149 (62149.40c)

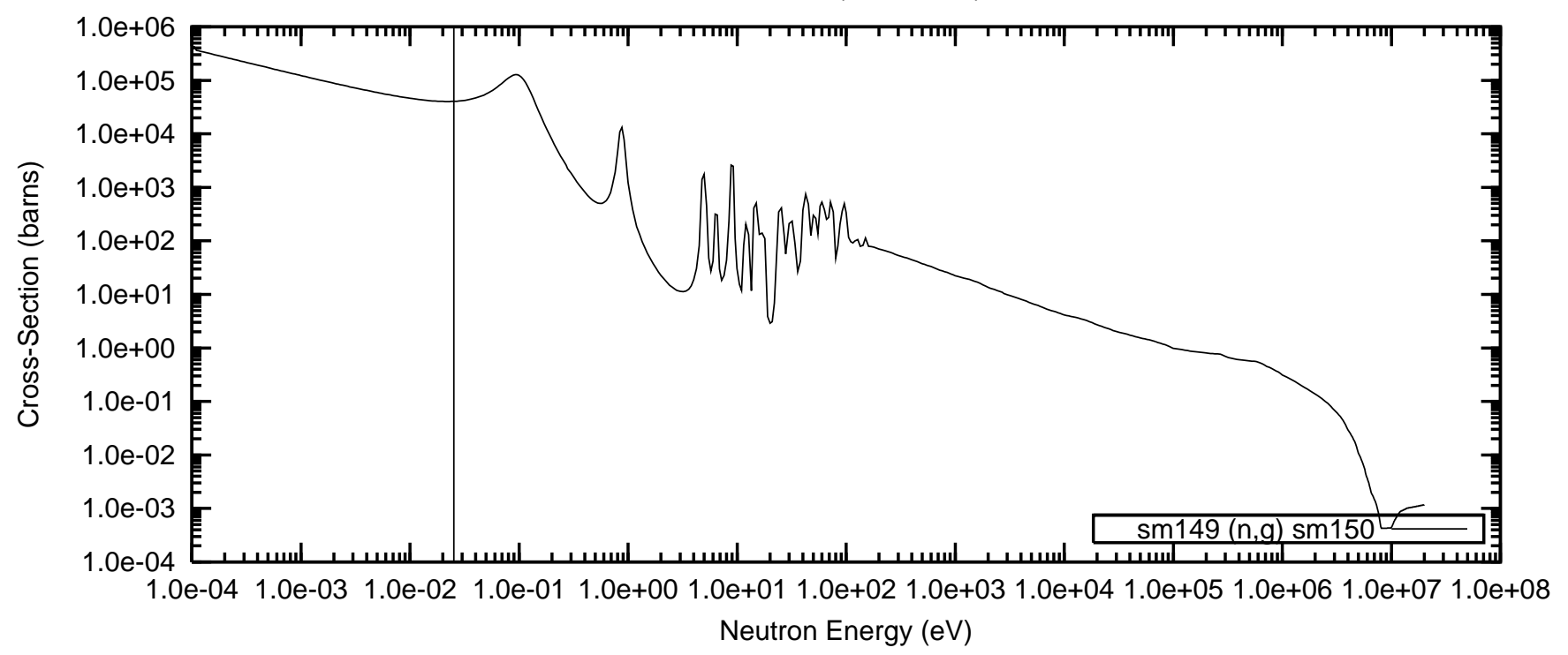


Figure 33(cont). Neutron Cross-Sections as a Function of Energy Sm-150 (62150.40c)

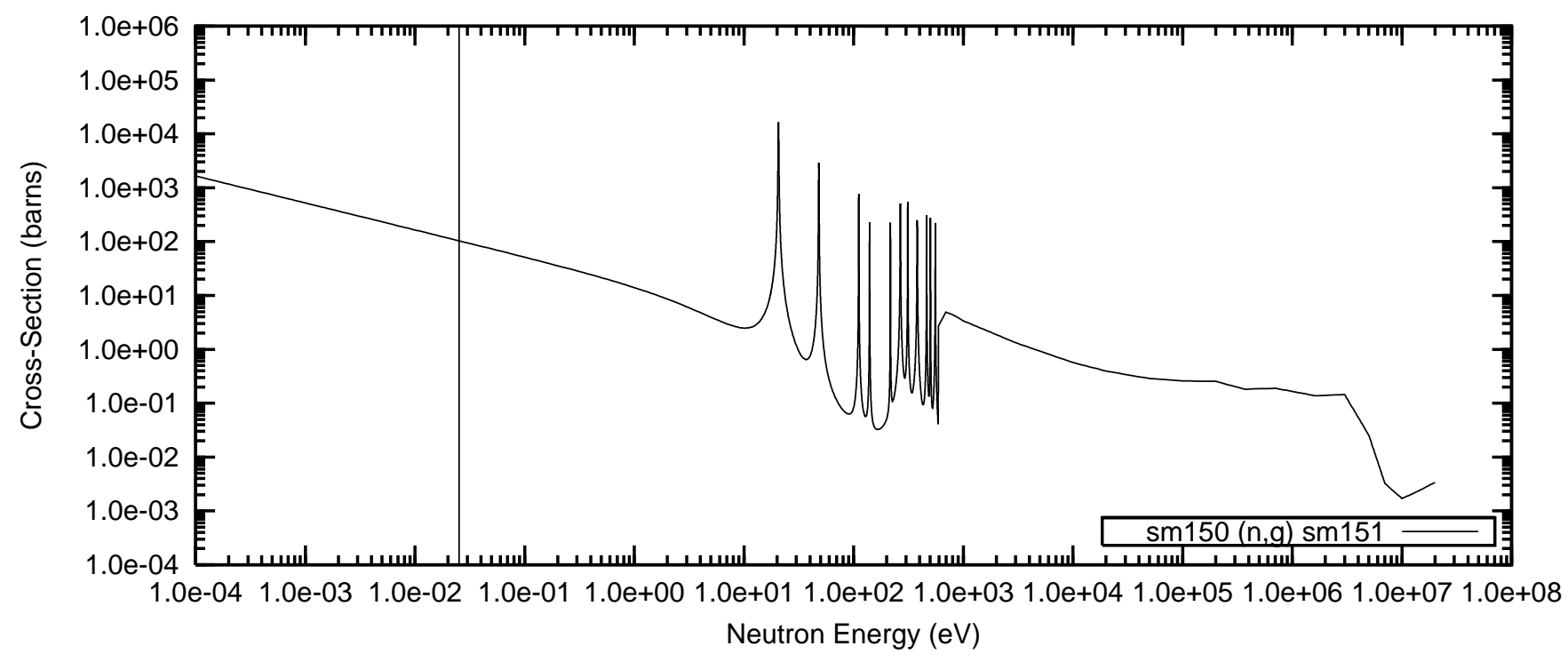

Sm-151 (62151.40c)

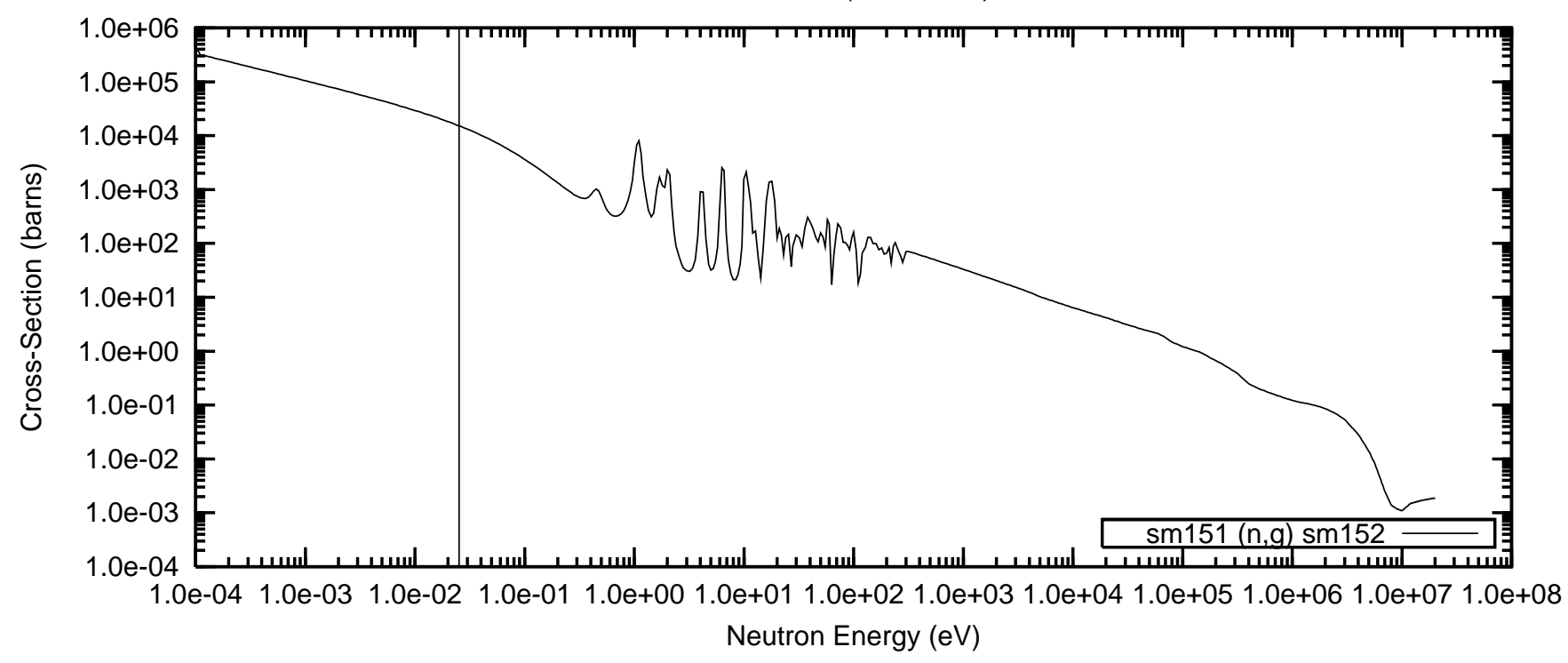

Sm-152 (62152.40c)

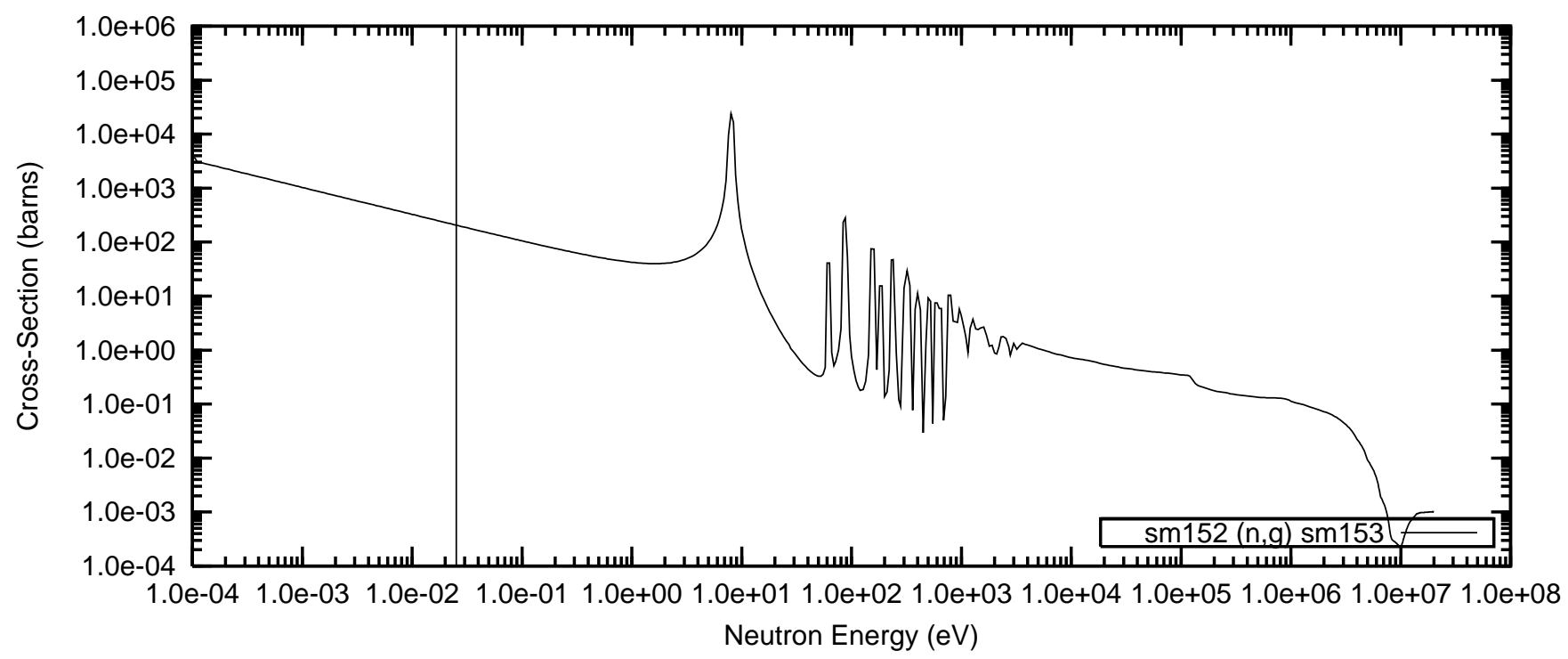


Figure 33(cont). Neutron Cross-Sections as a Function of Energy Sm-153 (62153.40c)

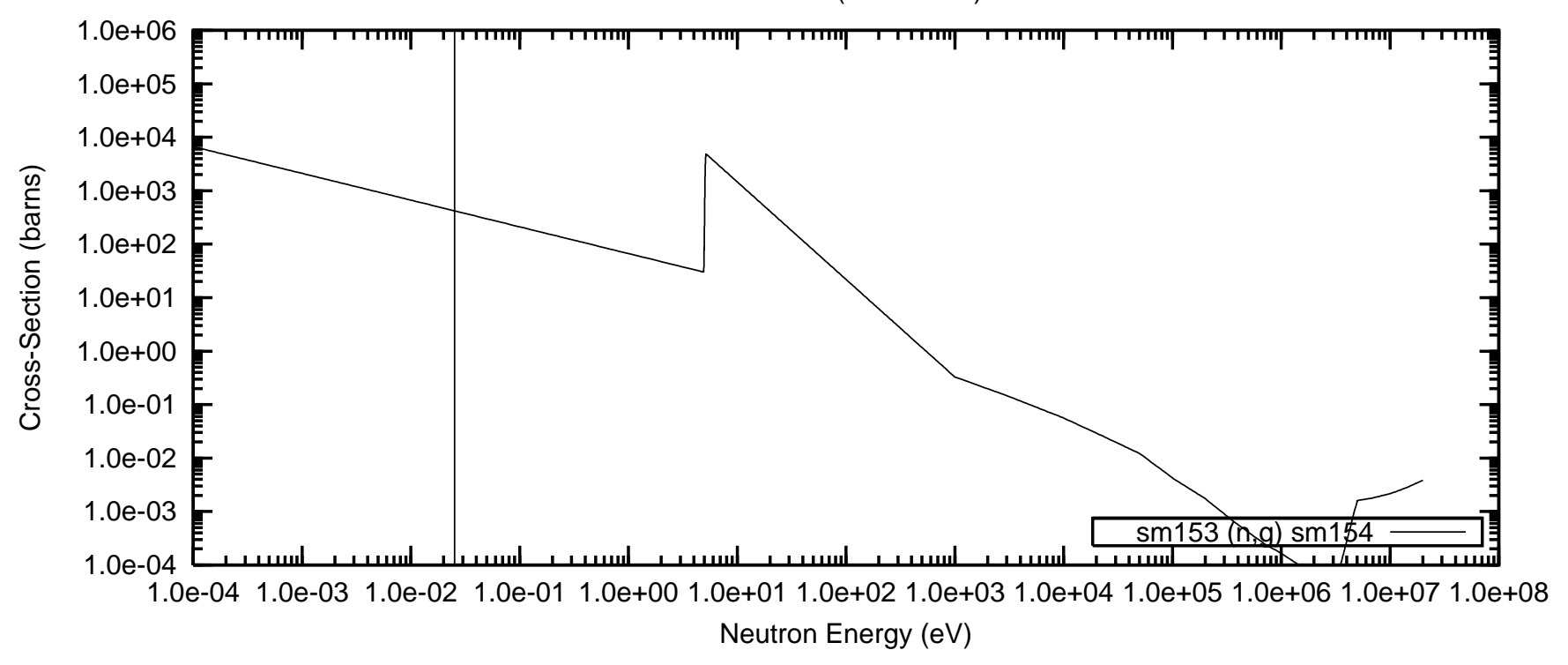

Sm-154 (62154.40c)

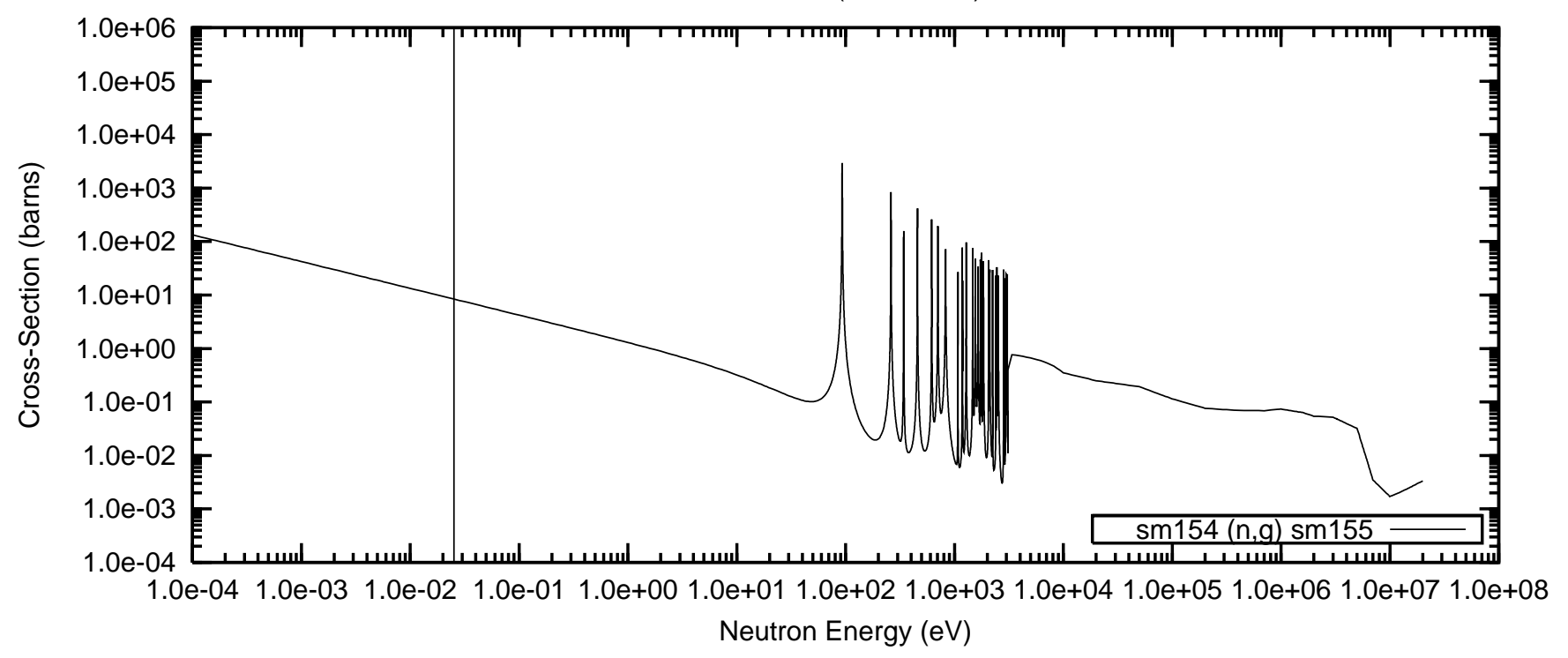


Figure 34. Neutron Cross-Sections as a Function of Energy

Eu-148 (63148.40c)

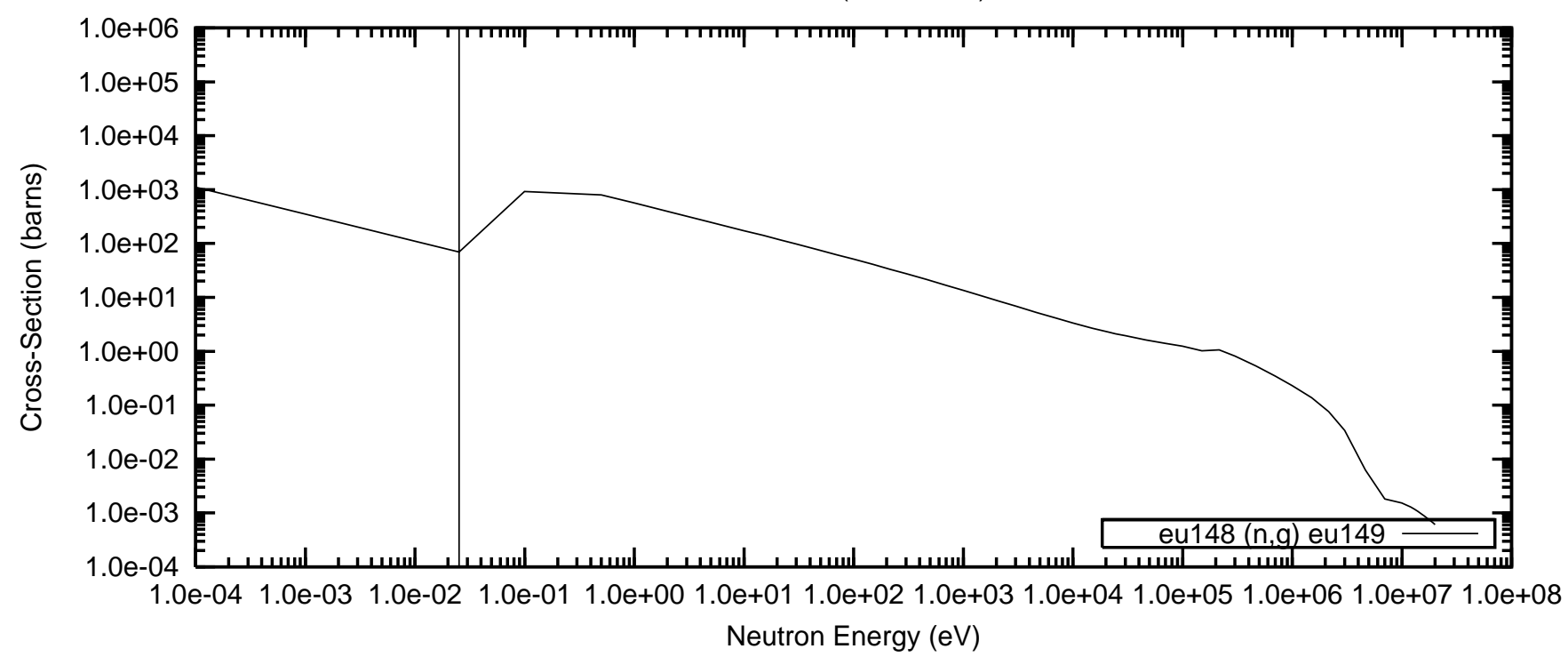

Eu-149 (63149.40c,46c)

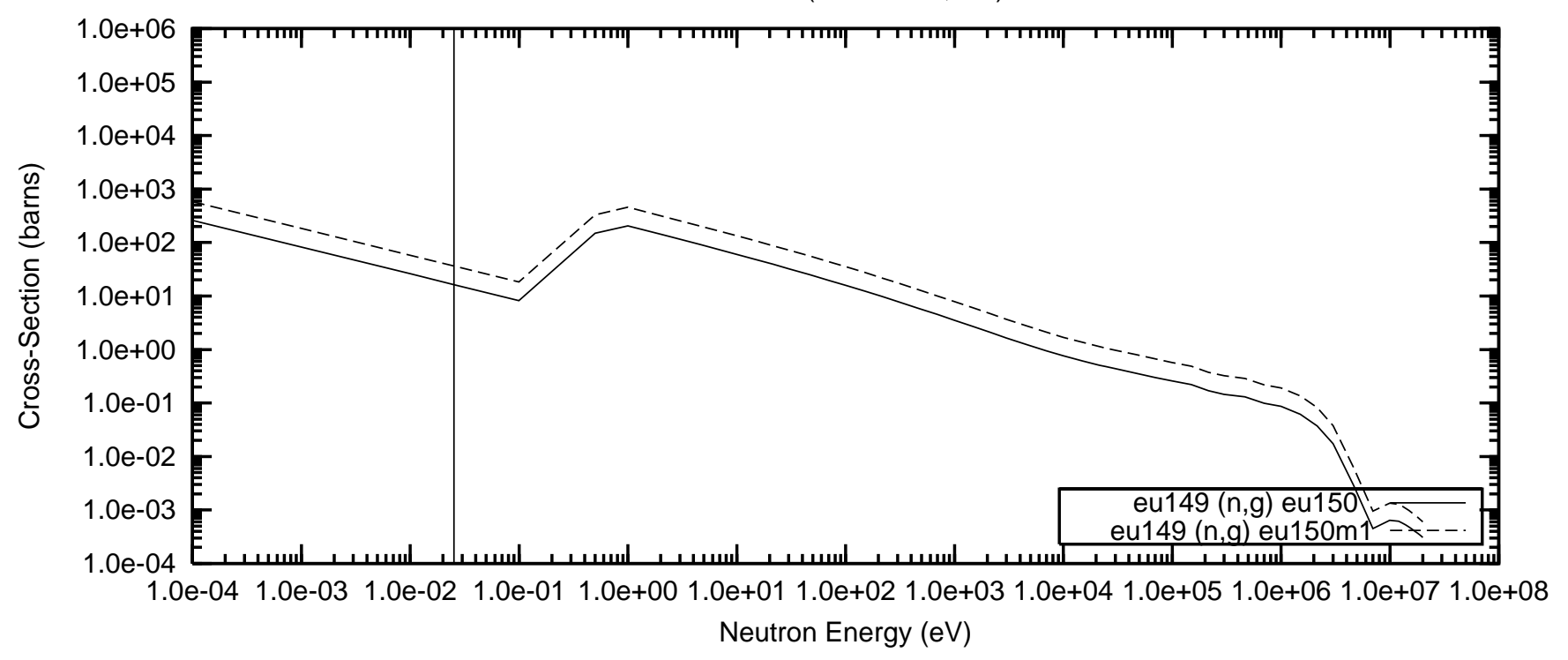

Eu-150 (63150.40c)

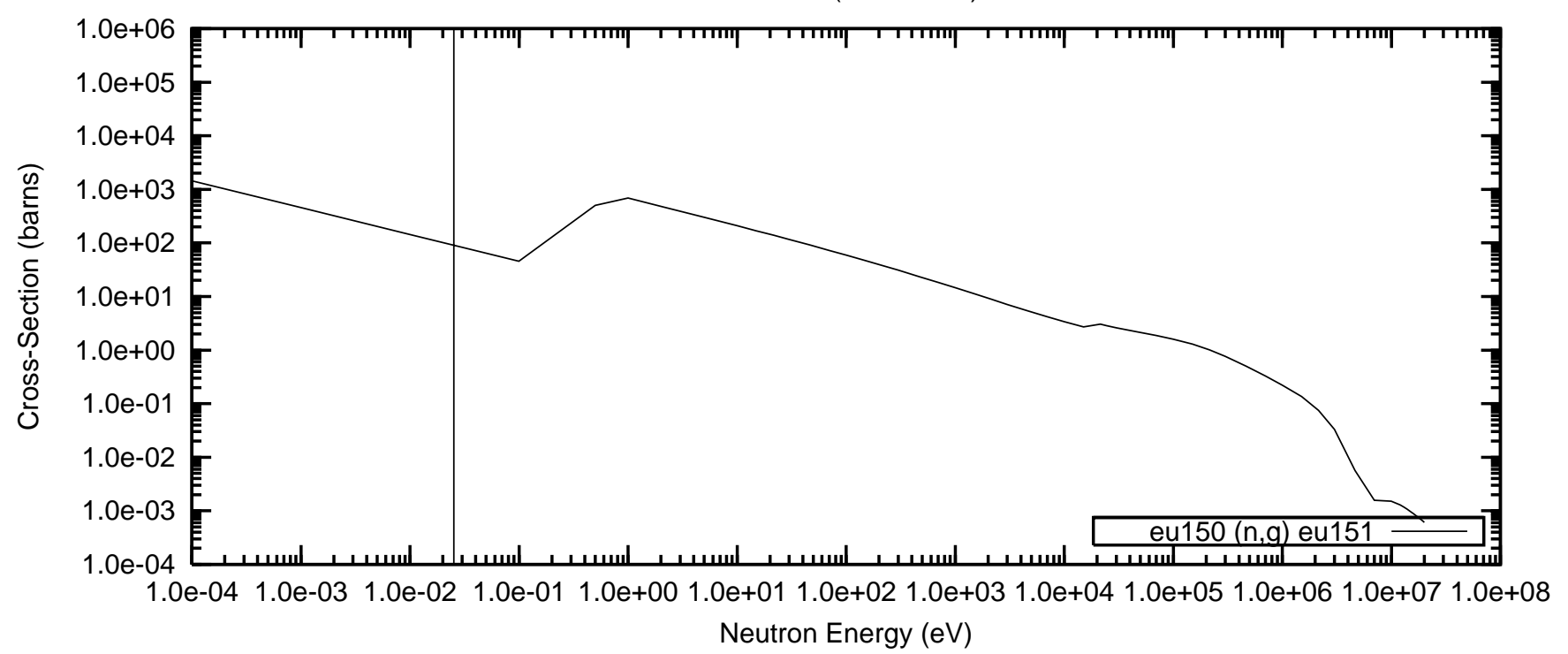


Figure 34(cont). Neutron Cross-Sections as a Function of Energy Eu-150m1 (63150.41c)

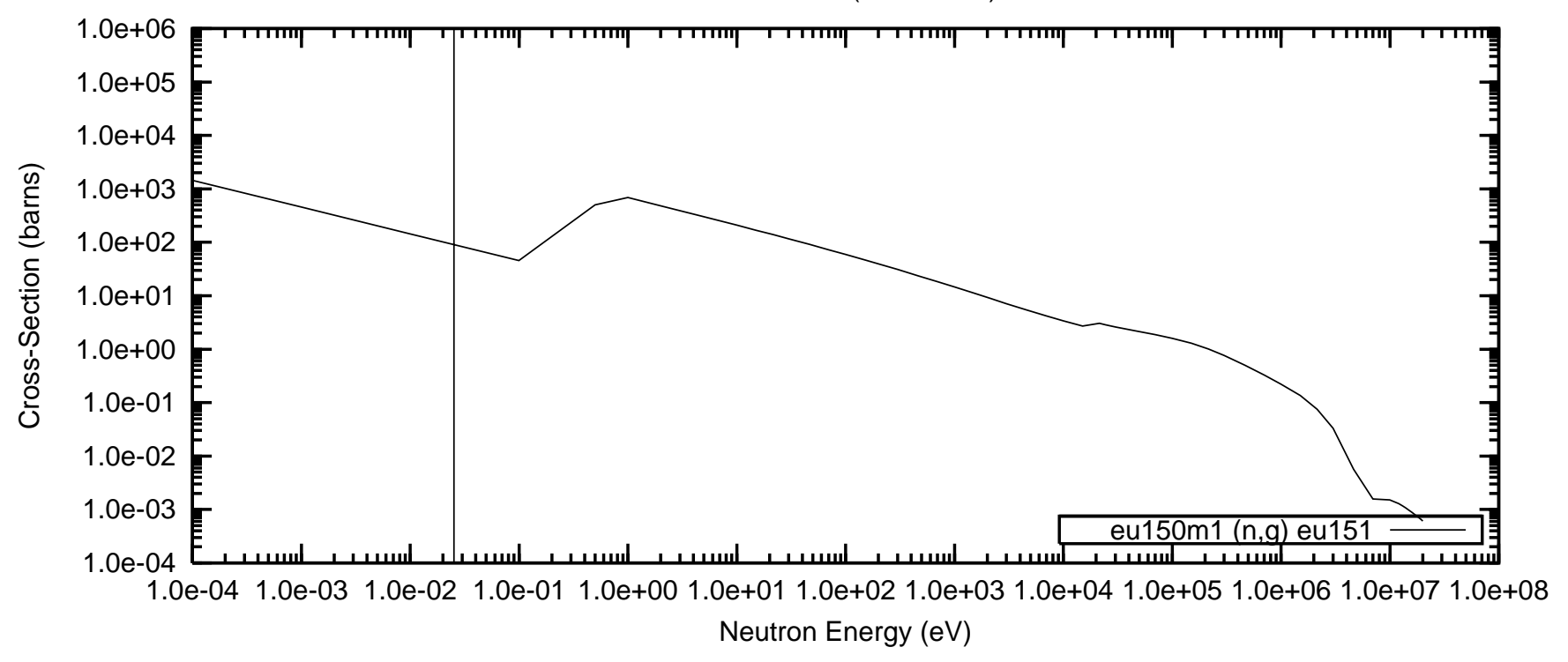

Eu-151 (63151.40c,46c,47c)

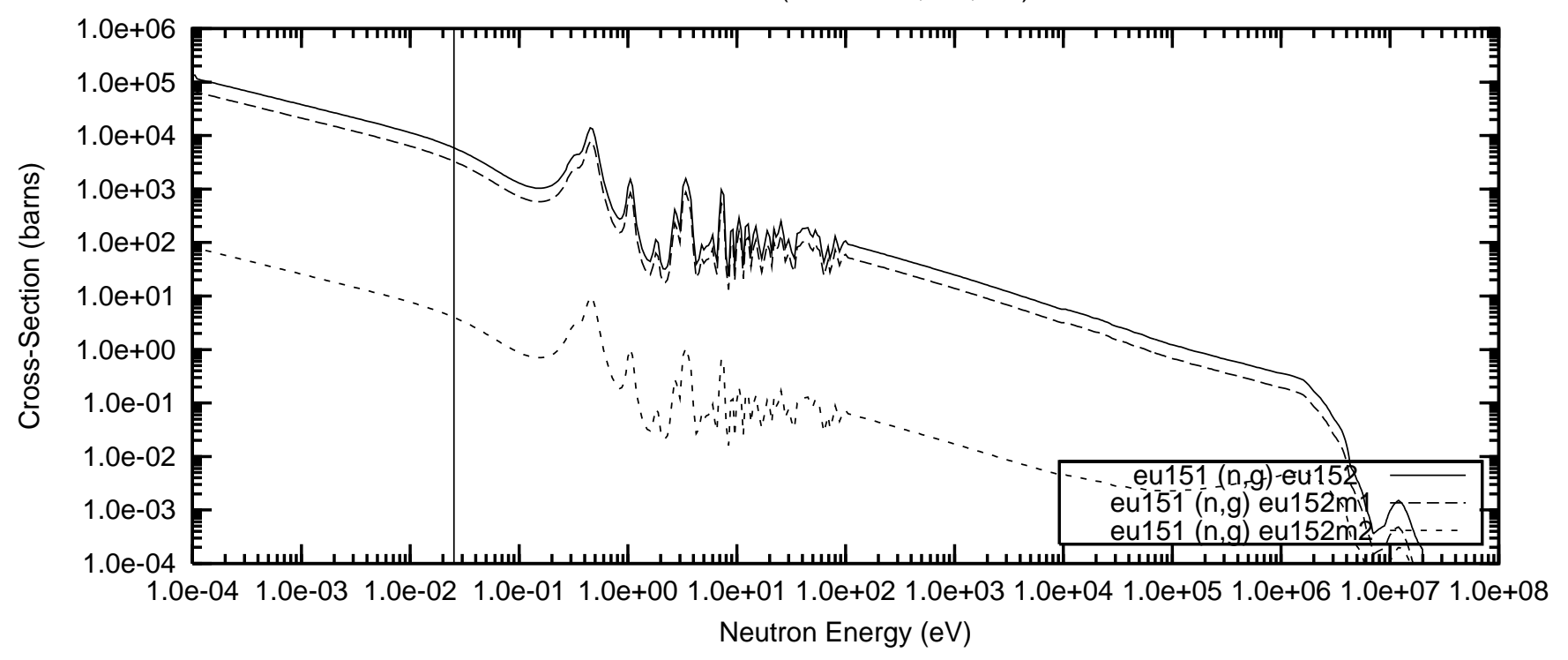

Eu-152 (63152.40c)

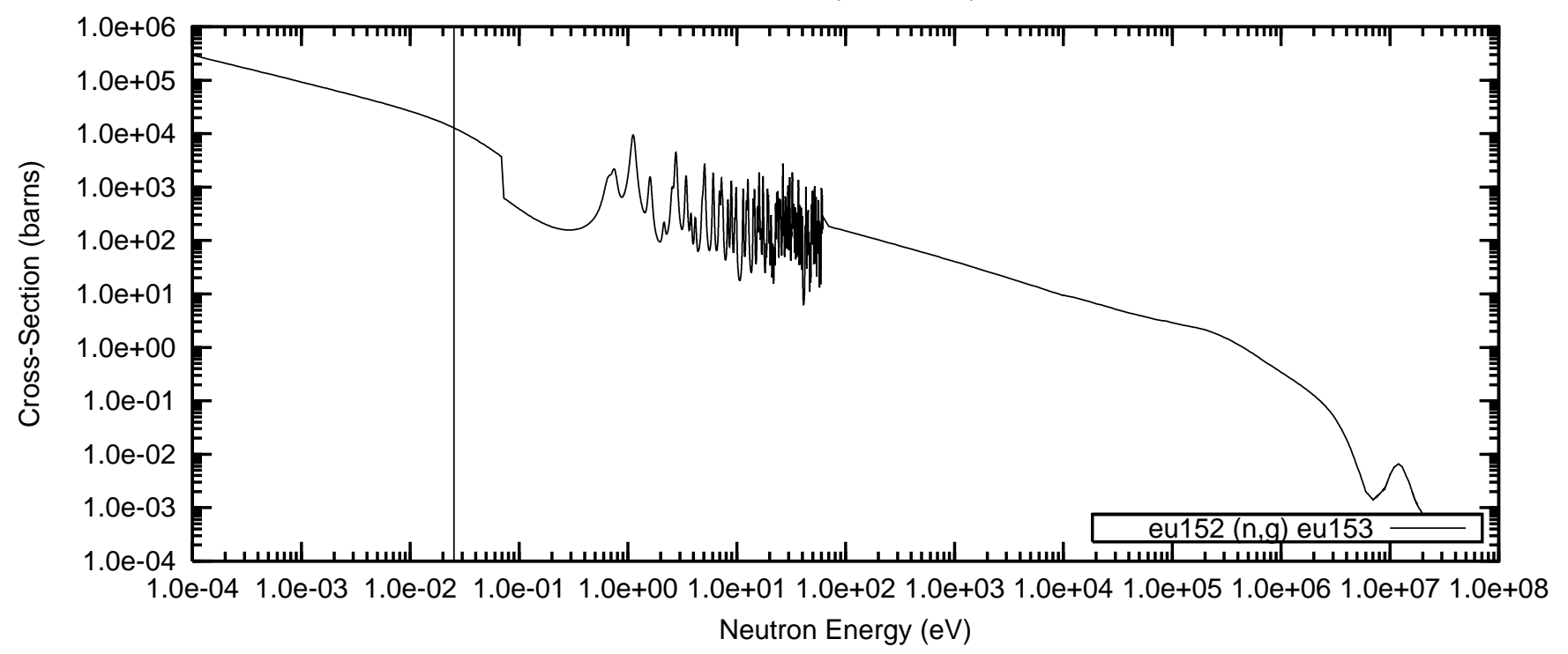


Figure 34(cont). Neutron Cross-Sections as a Function of Energy Eu-152m1 (63152.41c)

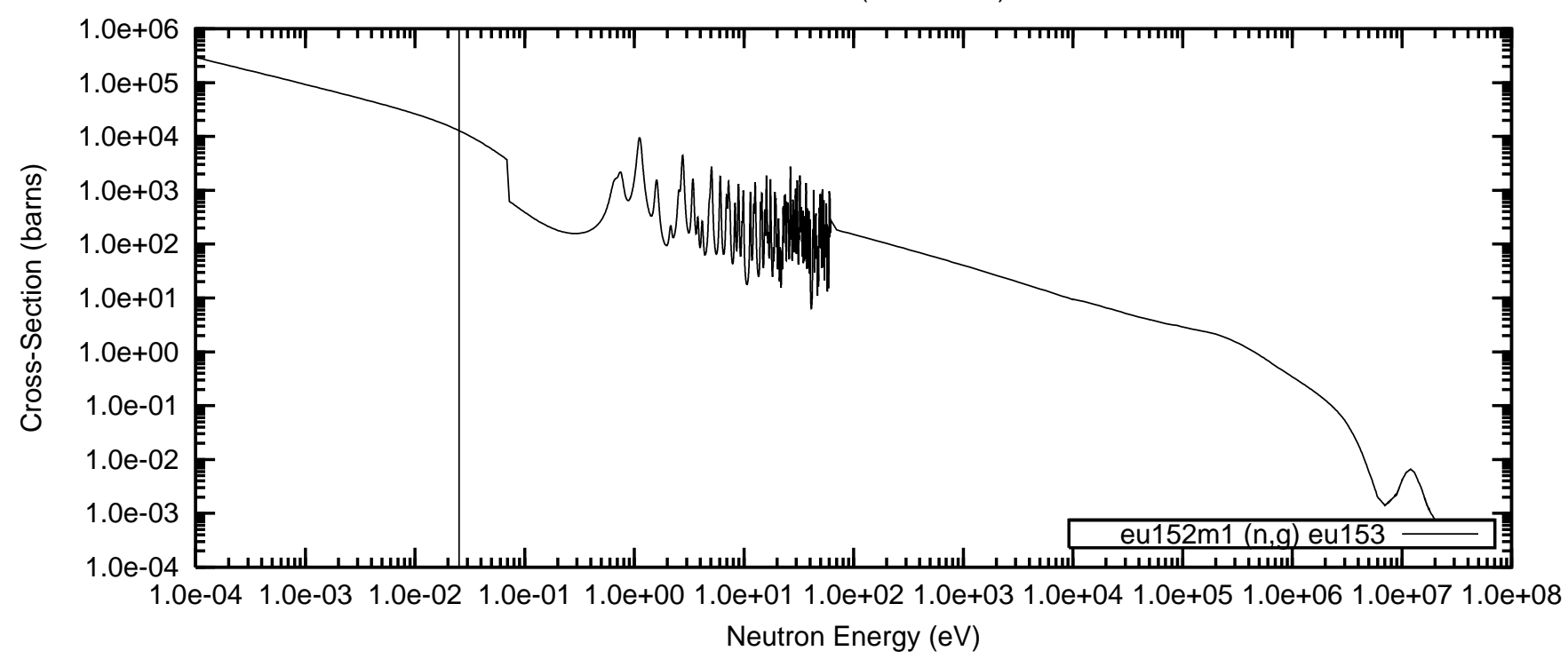

Eu-153 (63153.40c,46c)

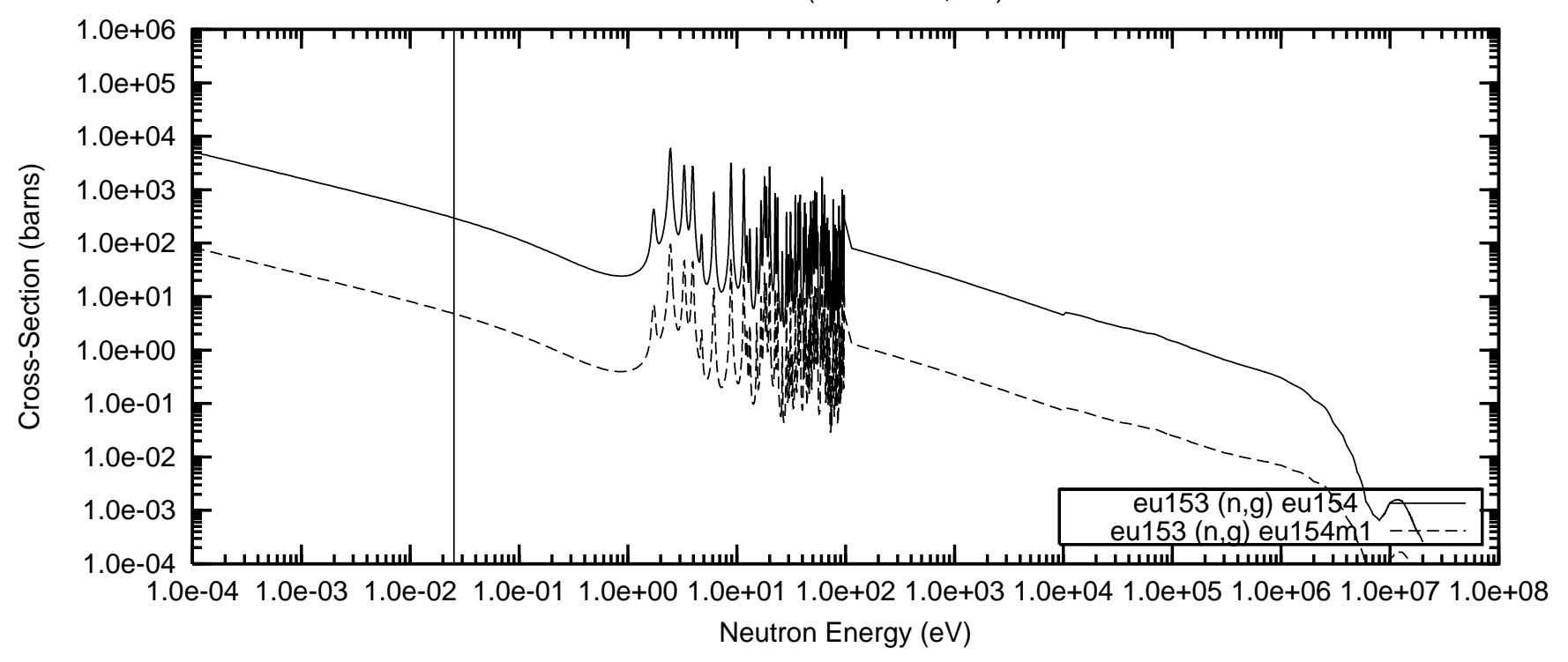

Eu-154 (63154.40c)

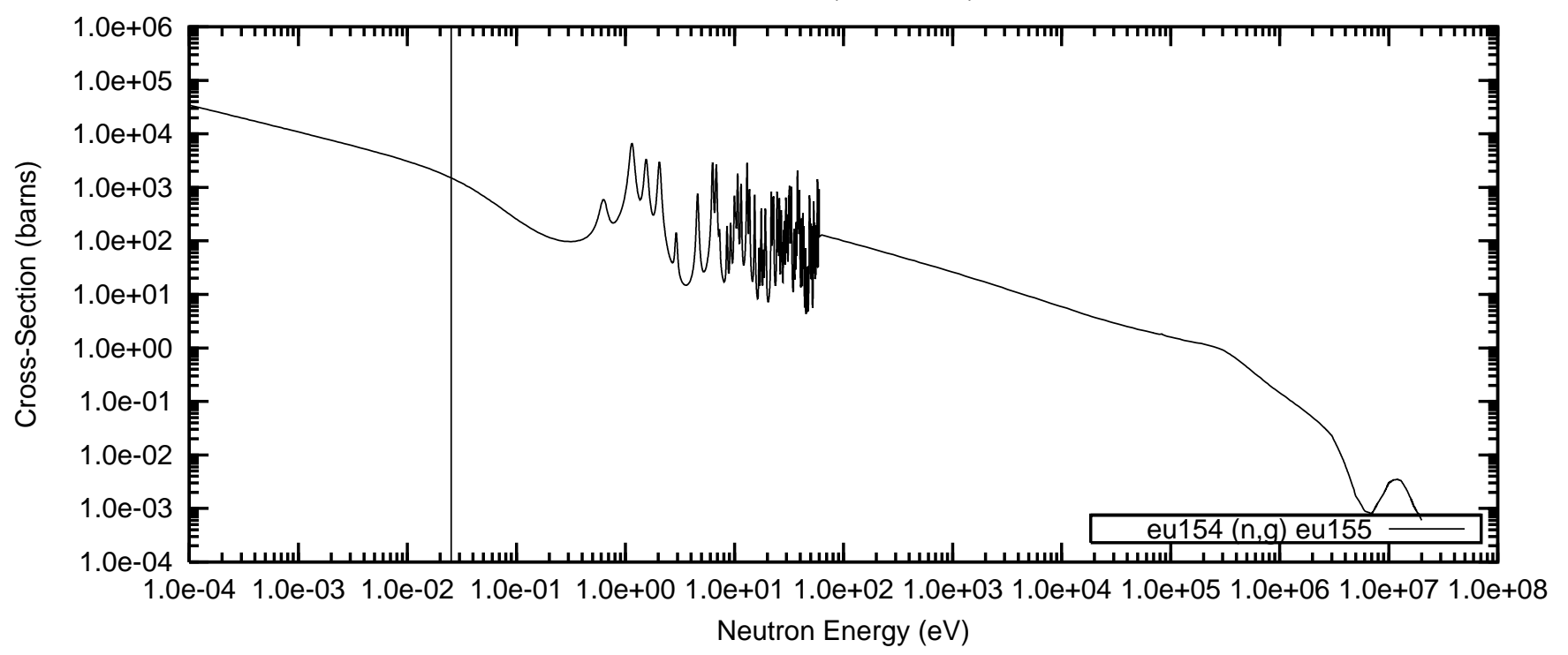


Figure 34(cont). Neutron Cross-Sections as a Function of Energy Eu-155 (63155.40c)

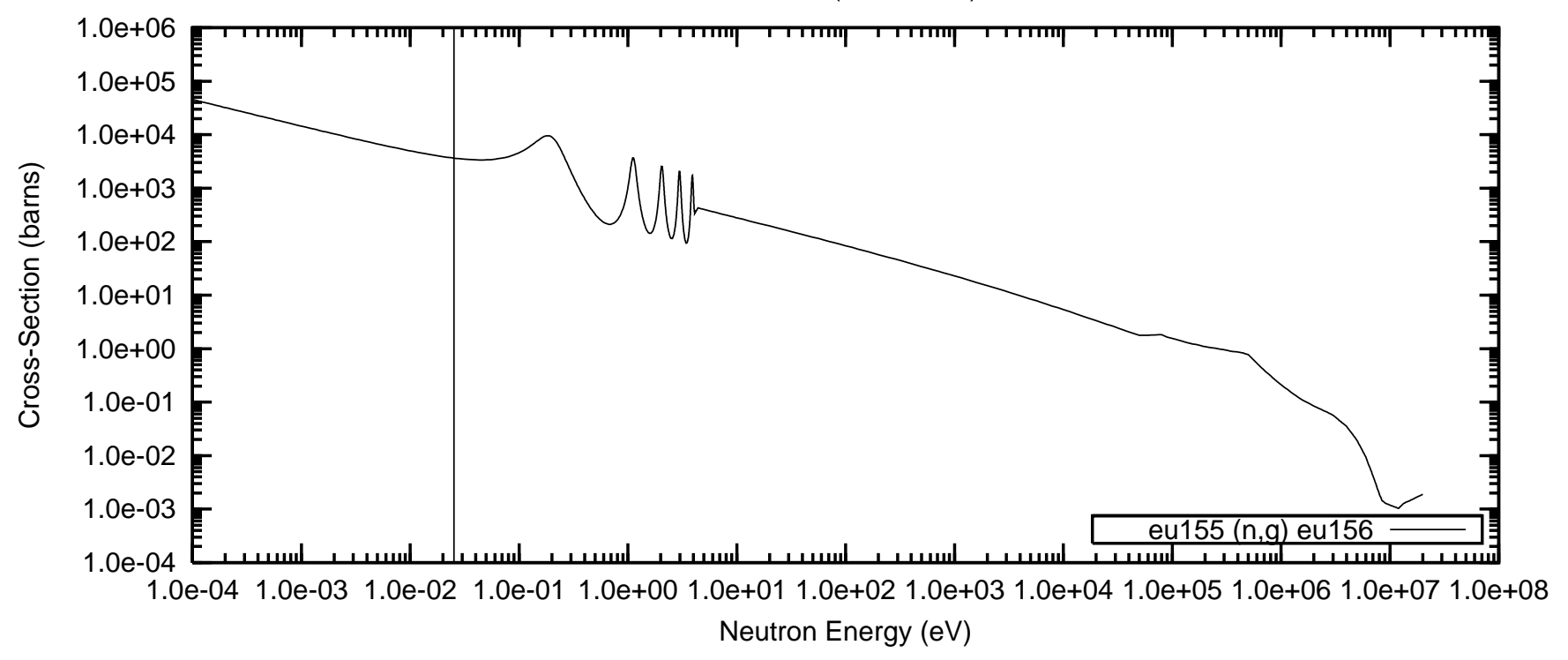

Eu-156 (63156.40c)

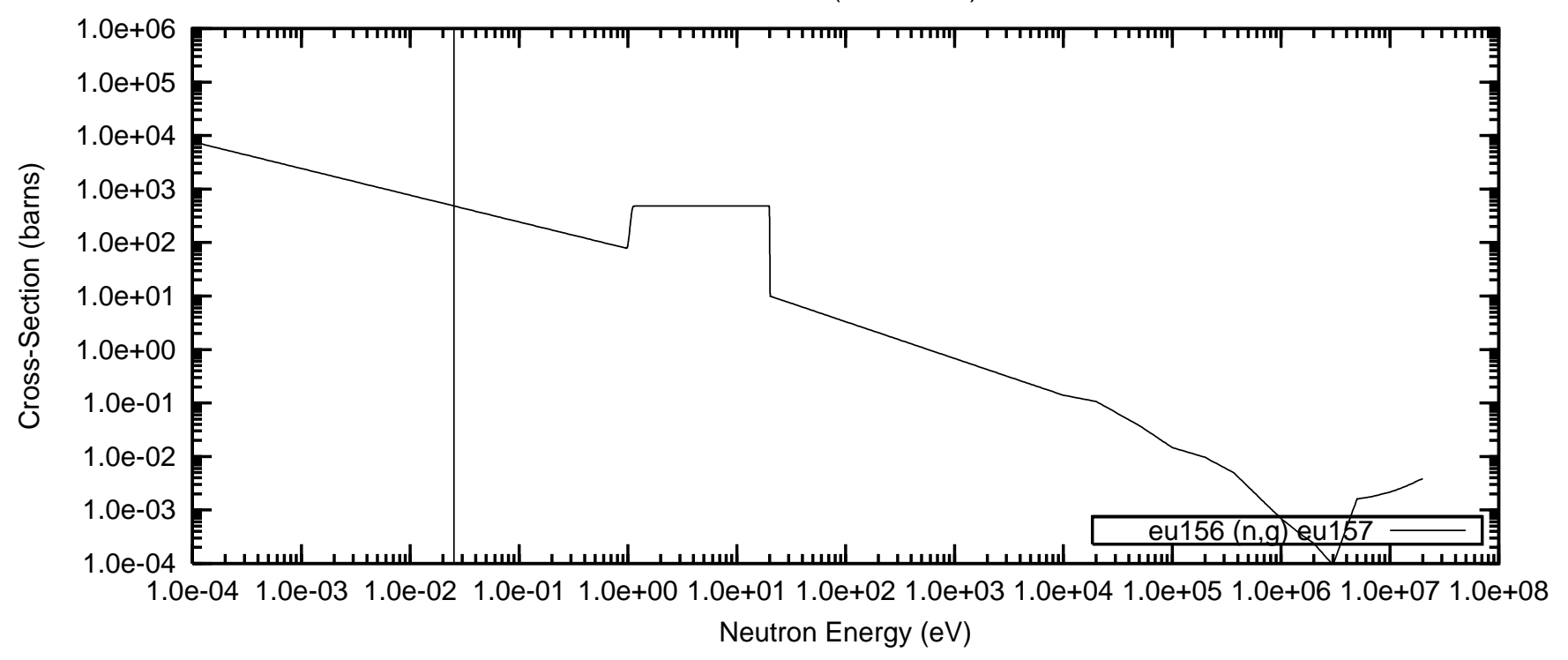

Eu-157 (63157.40c)

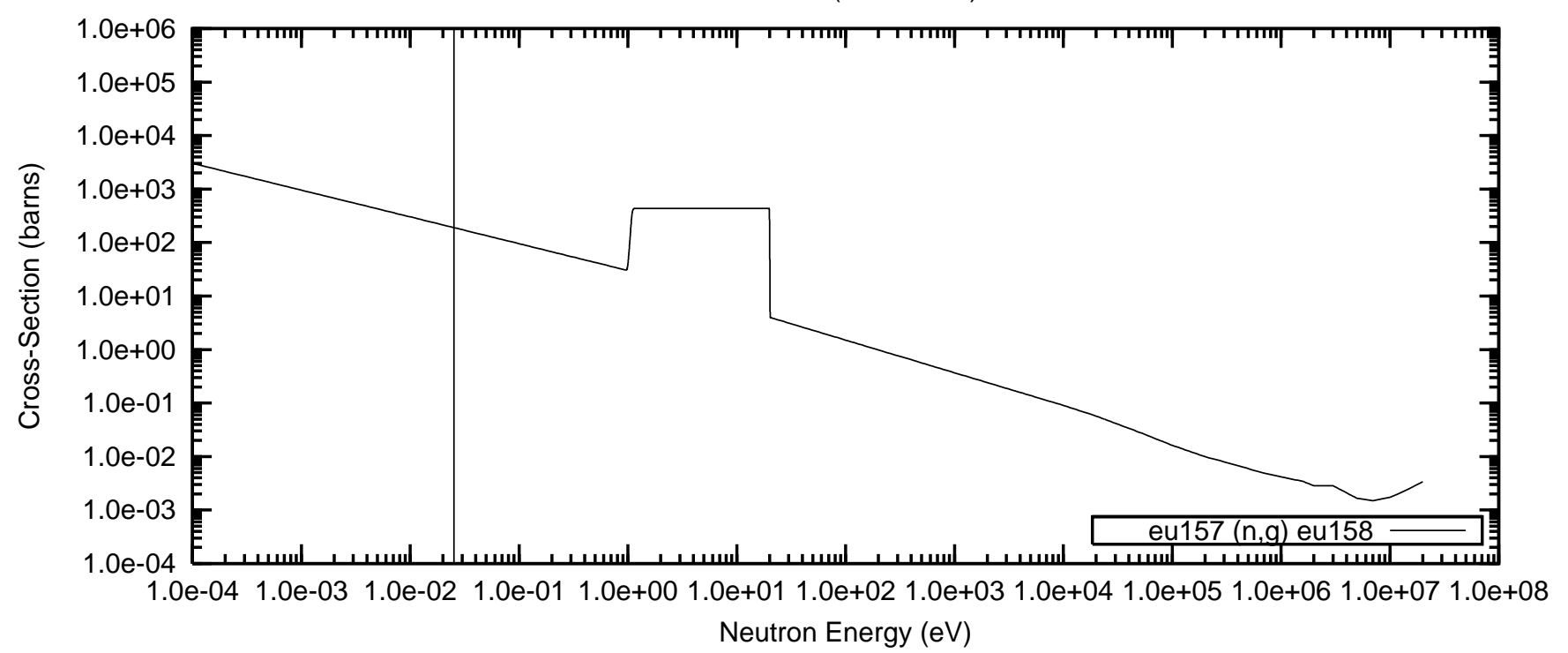


Figure 35. Negative Reactivity and Masses BP for Sm-nat and transmutation daughters as a function of fuel life for $17 \times 17$ fuel assemblies with 16 poison rods, $4.0 \mathrm{wt} \% \mathrm{Sm} 2 \mathrm{O} 3$ poison homogeneously mixed in the UO2 pellets

Reactor power $3400 \mathrm{MWth}, 193$ fuel assemblies, initial enrichment 4.5wt\%U-235

(Case : sm000_3_100p_h2o_16_bp_fuel_2_o)
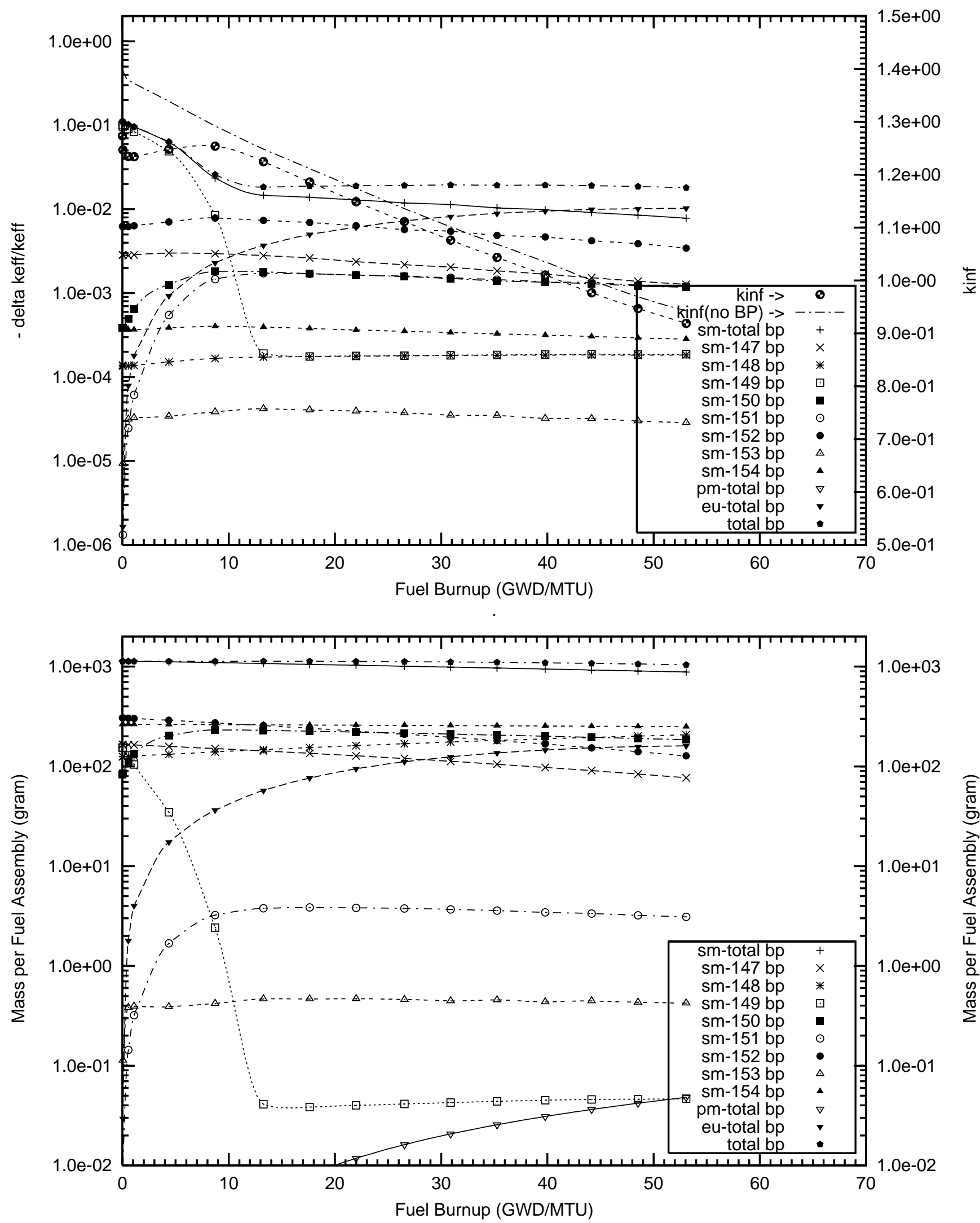
Figure 36. Negative Reactivity and Masses BP for Sm-nat and transmutation daughters as a function of fuel life for $17 \times 17$ fuel assemblies with 16 poison rods, $8.0 \mathrm{wt} \% \mathrm{Sm} 2 \mathrm{O} 3$ poison homogeneously mixed in the UO2 pellets

Reactor power $3400 \mathrm{MWth}, 193$ fuel assemblies, initial enrichment 4.5wt\%U-235

(Case : sm000_4_100p_h2o_16_bp_fuel_2_o)
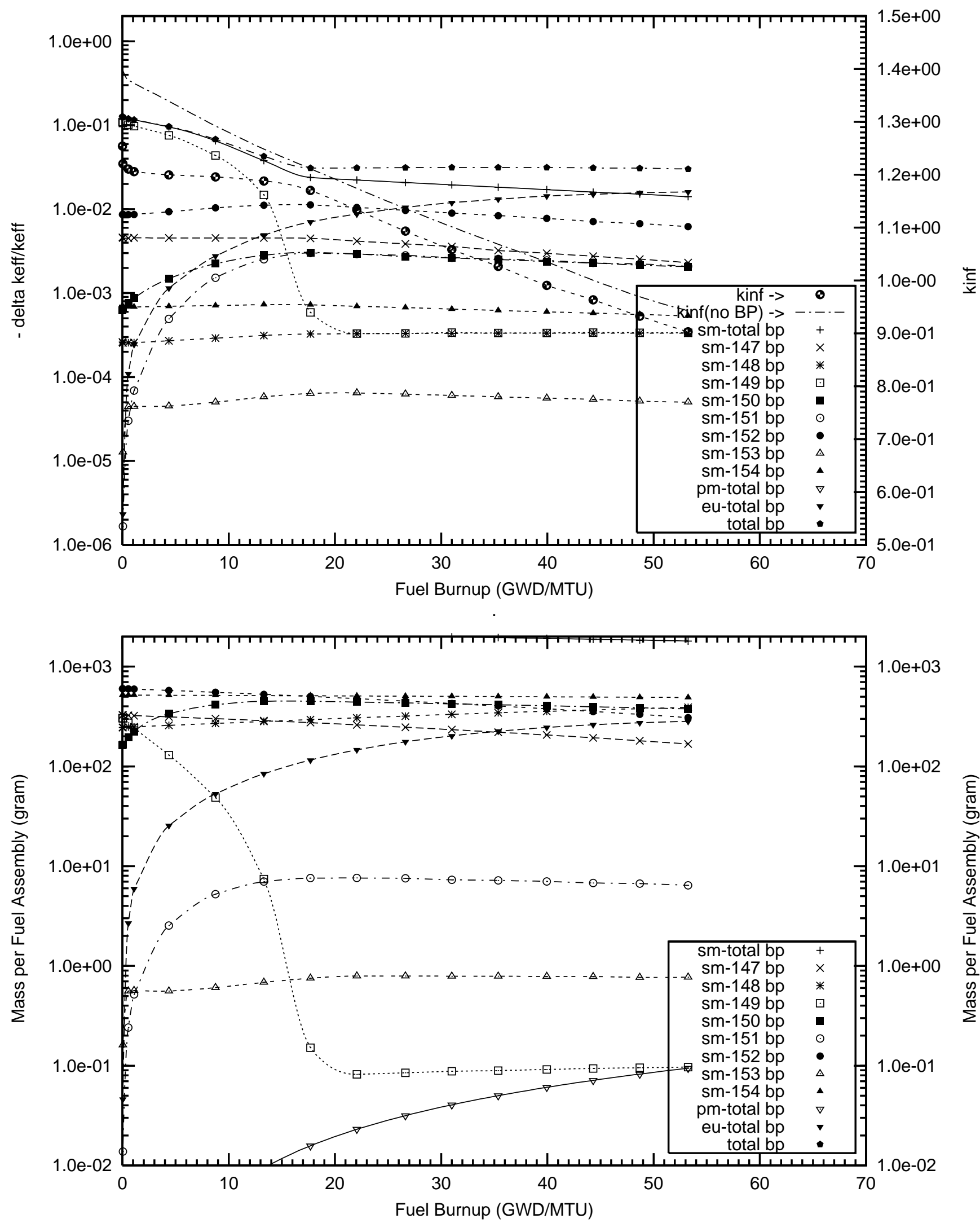
Figure 37. Negative Reactivity and Masses BP for Sm-149 and transmutation daughters as a function of fuel life for $17 \times 17$ fuel assemblies with 16 poison rods, $1.0 \mathrm{wt} \% \mathrm{Sm} 2 \mathrm{O} 3$ poison homogeneously mixed in the UO2 pellets

Reactor power $3400 \mathrm{MWth}, 193$ fuel assemblies, initial enrichment 4.5wt\%U-235

(Case : sm149_1_100p_h2o_16_bp_fuel_2_o)
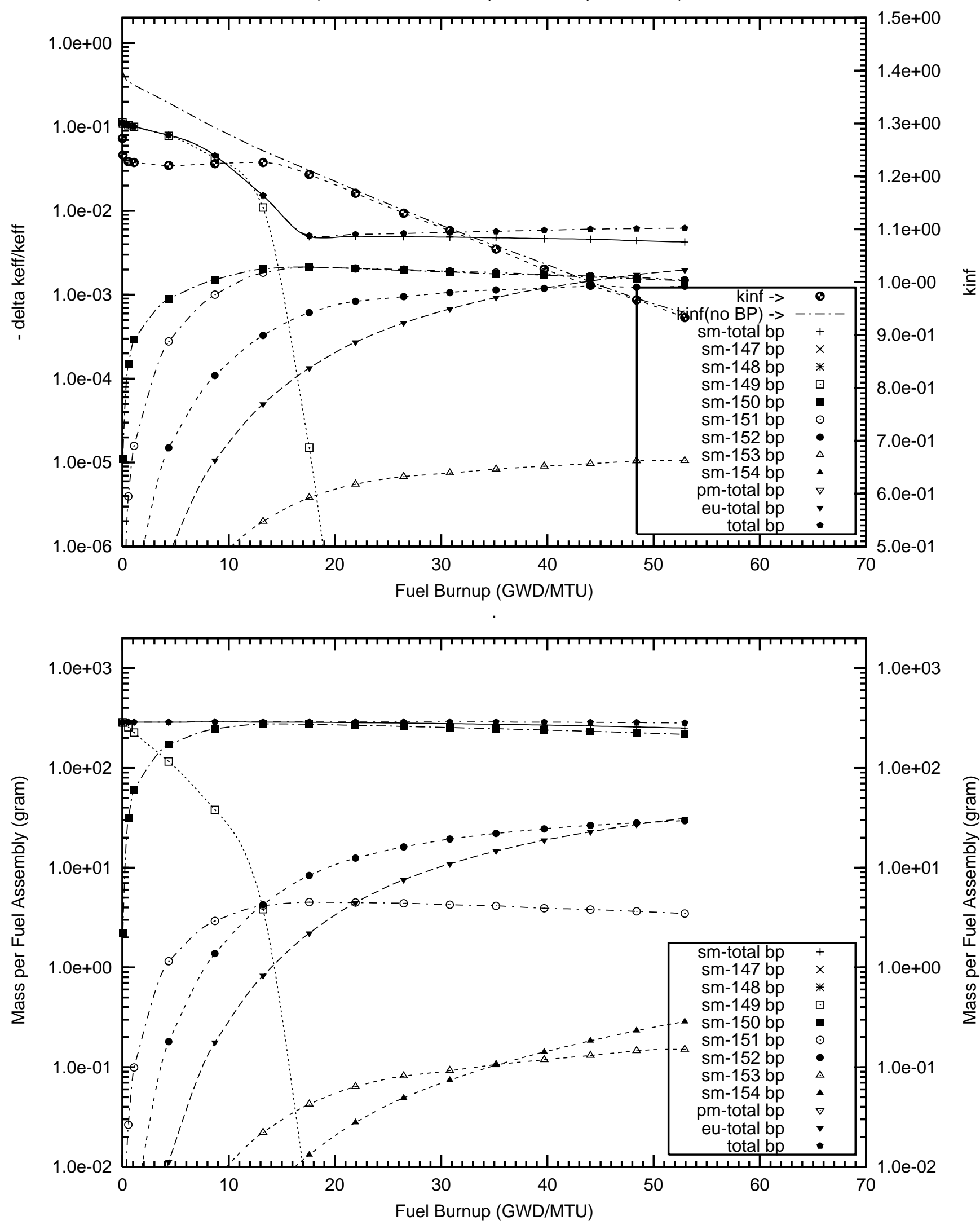
Figure 38. Negative Reactivity and Masses BP for Sm-149 and transmutation daughters as a function of fuel life for $17 \times 17$ fuel assemblies with 16 poison rods, $2.0 \mathrm{wt} \% \mathrm{Sm} 2 \mathrm{O} 3$ poison homogeneously mixed in the UO2 pellets

Reactor power $3400 \mathrm{MWth}, 193$ fuel assemblies, initial enrichment 4.5wt\%U-235

(Case : sm149_2_100p_h2o_16_bp_fuel_2_o)
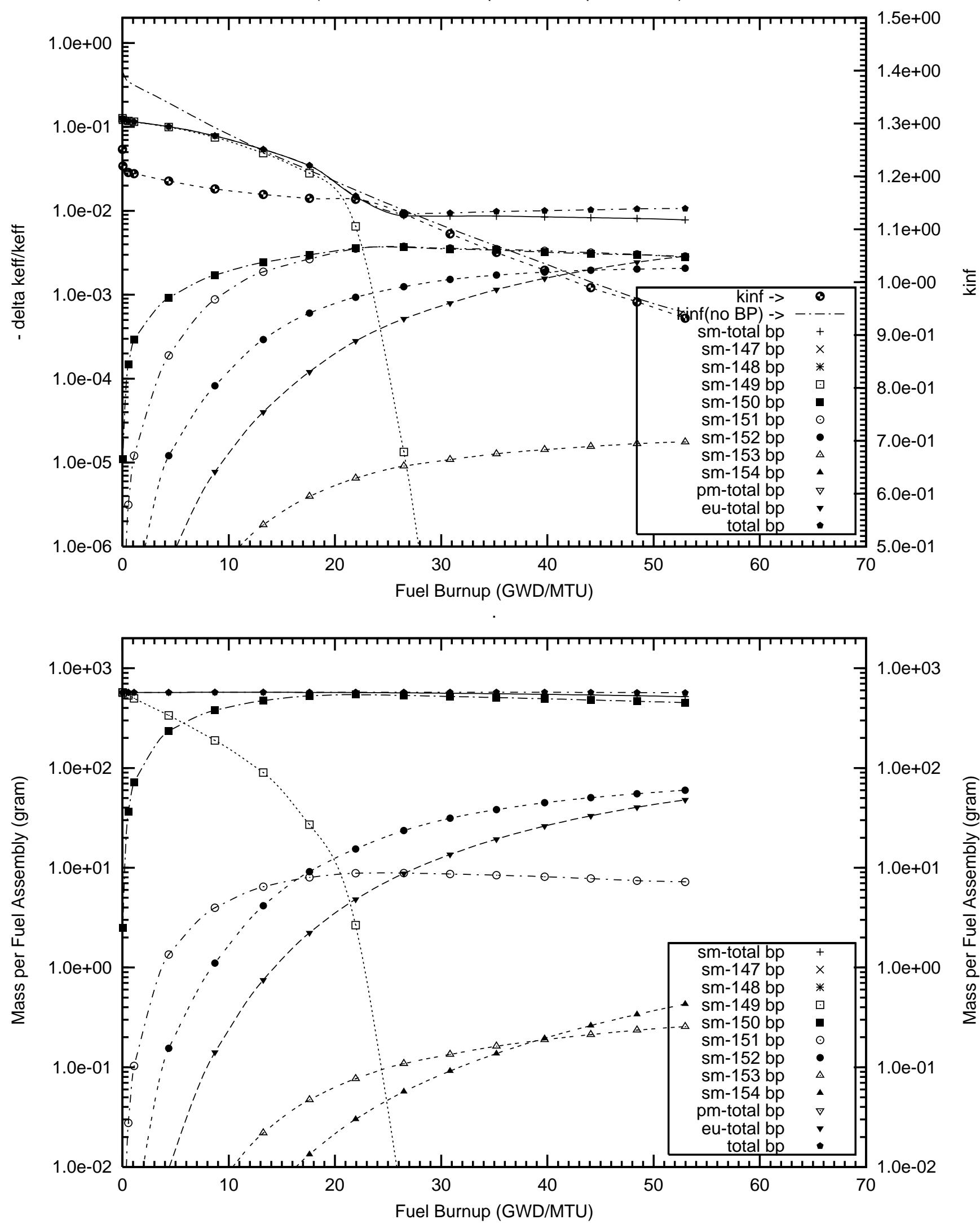
Figure 39. Negative Reactivity and Masses BP for Sm-149 and transmutation daughters as a function of fuel life for $17 \times 17$ fuel assemblies with 16 poison rods, $4.0 \mathrm{wt} \% \mathrm{Sm} 2 \mathrm{O} 3$ poison homogeneously mixed in the UO2 pellets

Reactor power $3400 \mathrm{MWth}, 193$ fuel assemblies, initial enrichment 4.5wt\%U-235

(Case : sm149_3_100p_h2o_16_bp_fuel_2_o)
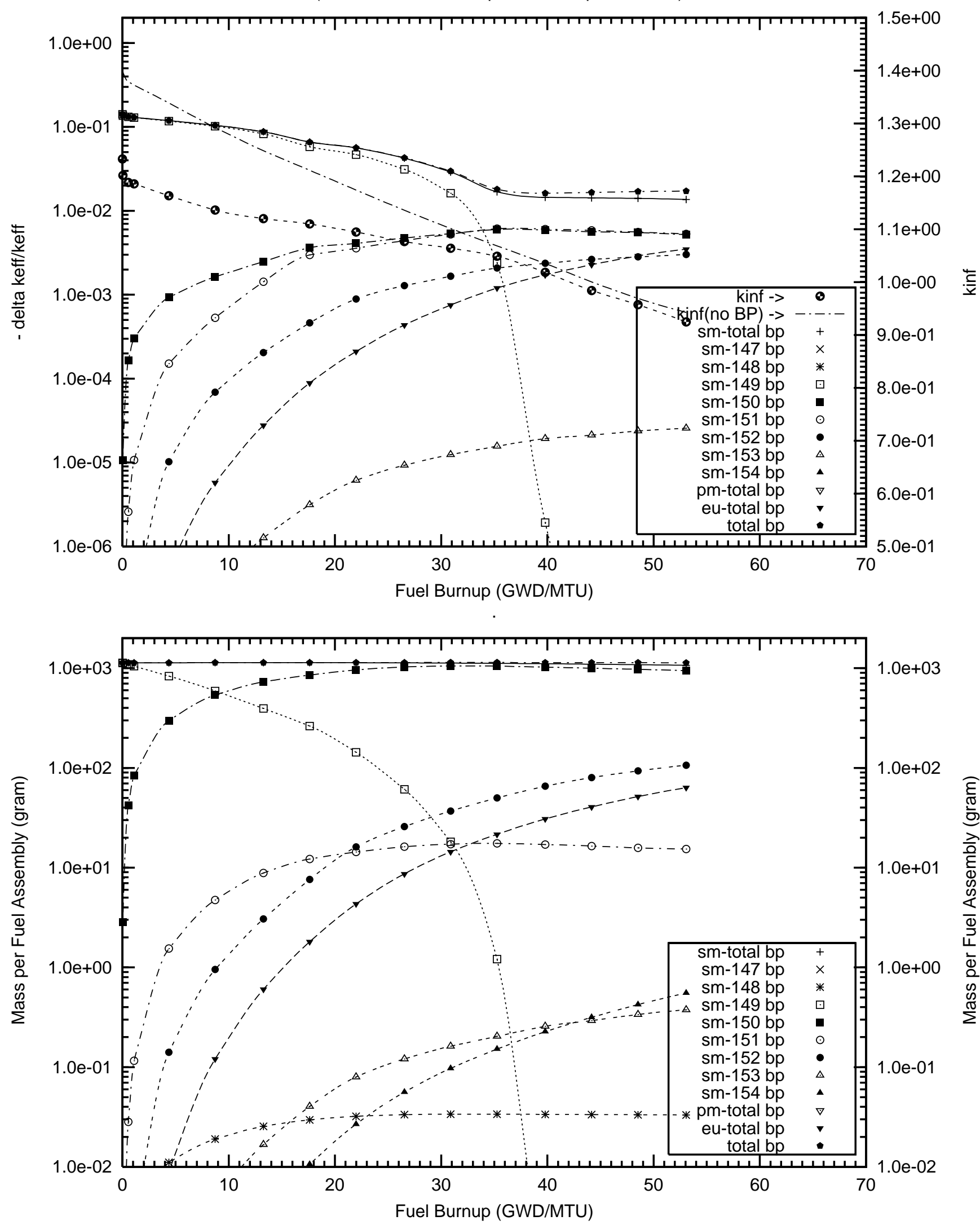


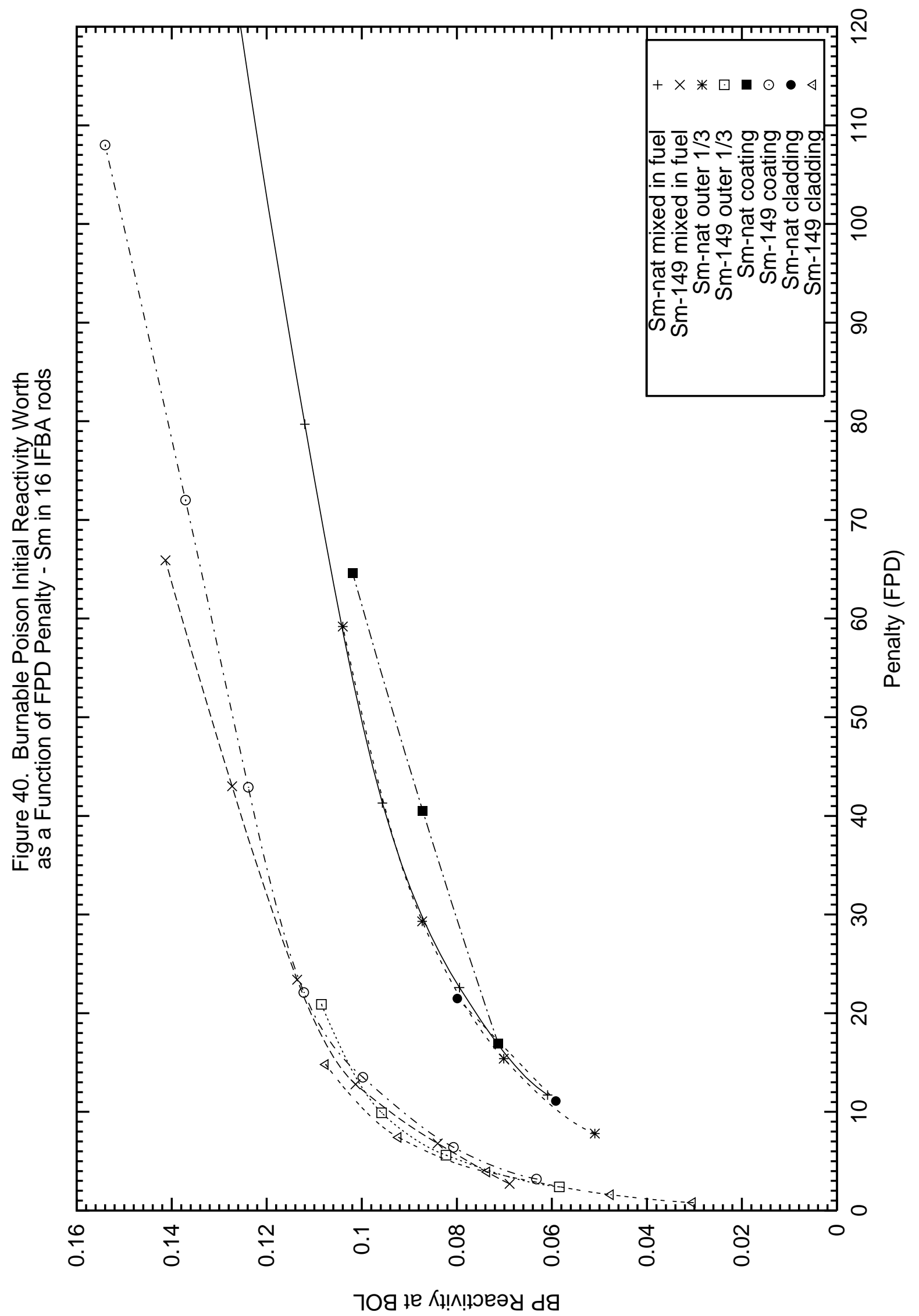




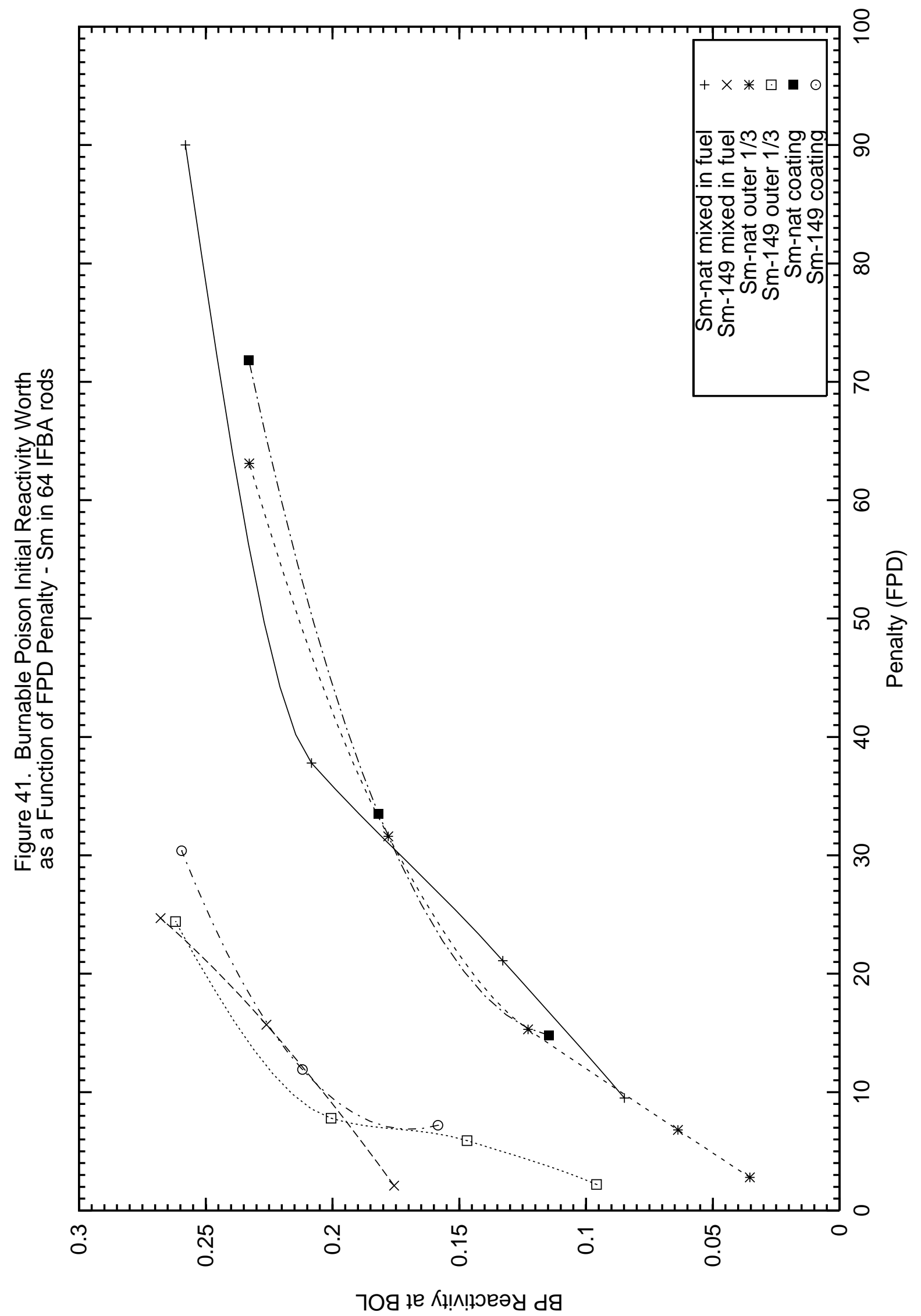




\section{NEUTRONICS PERFORMANCE RESULTS FOR ERBIUM}

Erbia $\left(\mathrm{Er}_{2} \mathrm{O}_{3}\right)$ as a burnable poison has been used in designs of the modular high temperature reactors (MHTRs). More recently $\mathrm{Er}_{2} \mathrm{O}_{3}$ has also been used as burnable poisons in System-80 PWRs. In this section, we will investigate the potential benefits of using enriched erbium as a burnable absorber in PWRs. Four different types of burnable poison rods containing erbium, similar to those of the cases of $\mathrm{Gd}$, were investigated.

1. A homogeneous mixture of the burnable poison $\mathrm{Er}_{2} \mathrm{O}_{3}$ inside the fuel pellets of a limited number of fuel rods. A summary of the results is given in Table 13. The plots of the reactivity worths and the masses of the BP isotopes and their daughters are displayed in Appendix C1.

2. A homogeneous mixture of the burnable poison as an oxide (erbia) in the outer one-third volume of the fuel pellets. A summary of the results is given in Table 14. Plots of the reactivity worths and the masses of the BP isotopes and their daughters are displayed in Appendix C2.

3. An oxide burnable poison coating (erbia) on the surface of the fuel pellets in a limited number of fuel rods, similar to the $\mathrm{ZrB}_{2}$ coatings. A summary of the results is given in Table 15. Plots of the reactivity worths and the masses of the BP isotopes and their daughters are displayed in Appendix C3.

4. A homogeneous mixture of the burnable poison erbium in the form of a metal alloyed with the Zircaloy cladding of a number of fuel rods. The summary results are given in Table 16. Plots of the reactivity worths and the masses of the BP isotopes and their daughters are displayed in Appendix C4.

In all the neutronics calculations presented in this section, a theoretical density of $8.64 \mathrm{~g} / \mathrm{cm}^{3}$ was used for $\mathrm{Er}_{2} \mathrm{O}_{3}$, and $9.00 \mathrm{~g} / \mathrm{cm}^{3}$ for metallic erbium. For $\mathrm{UO}_{2}$, a theoretical density of $10.97 \mathrm{~g} / \mathrm{cm}^{3}$ was used. The actual density fraction of the fuel pellets and of the burnable poison pellets was $95 \%$ of theoretical density.

The half-lives, natural isotopic abundances, and burnup chains related to the depletion and transmutation of erbium are given below. Note that the adjacent bands of isotopes such as the $\mathrm{Yb}$ isotopes (decay from Tm isotopes) and Dy isotopes (decay from Ho isotopes) are not shown. Also the isomeric states of certain isotopes are not shown. However, the generation of these daughters was taken into account in the BP depletion and decay calculations. 


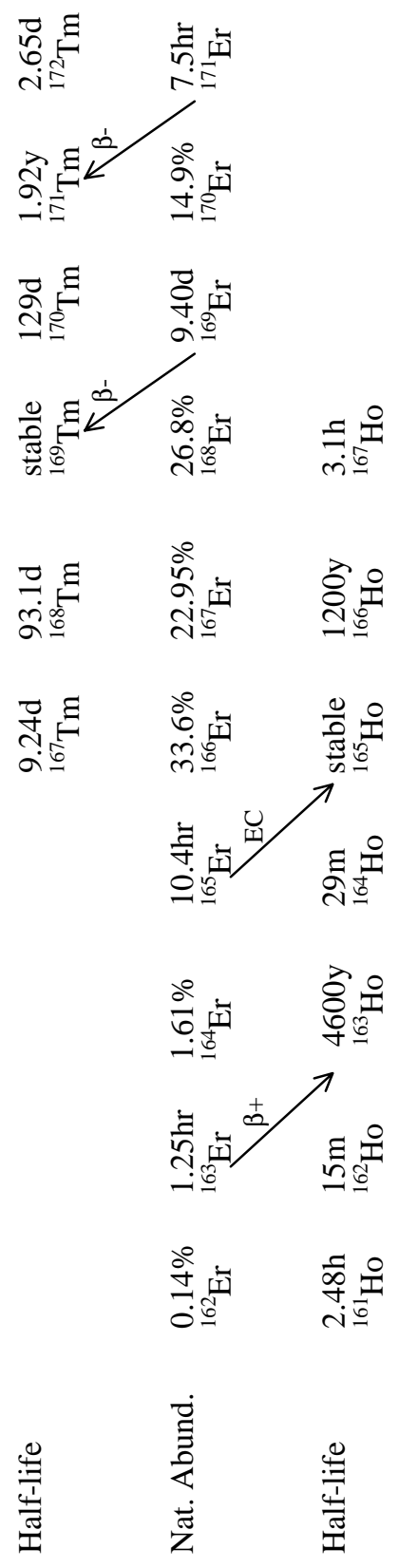


Figure 42 displays the capture cross sections for the erbium isotopes, Figure 43 displays the capture cross sections of several of the holmium decay daughters from erbium, and Figure 44 displays the cross sections of thulium decay daughters from erbium. The erbium stable isotope ${ }^{167} \mathrm{Er}(22.9 \%$ natural isotopic abundance) exhibits a large neutron capture cross section (thermal cross section of 660 barns and a RI of 2,970 barns). The isotopes ${ }^{162} \mathrm{Er}\left(0.14 \%\right.$ natural isotopic abundance), ${ }^{164} \mathrm{Er}$ (1.6\%), ${ }^{166} \mathrm{Er}(33.4 \%)$, and ${ }^{170} \mathrm{Er}(14.9 \%)$ have smaller capture cross sections: ${ }^{162} \mathrm{Er}$ (thermal 19 barns, RI 480 barns), ${ }^{164} \mathrm{Er}$ (thermal 13 barns, RI 105 barns), ${ }^{166} \mathrm{Er}$ (thermal 19.6 barns, RI 96 barns), and ${ }^{170} \mathrm{Er}$ (thermal 5.8 barns, RI 45 barns). Because of the small cross sections, these isotopes are difficult to burn out and they will thus leave a residual negative reactivity burden at EOL of the BP. ${ }^{168} \mathrm{Er}$ (27.1\% natural isotopic abundance) has a small neutron capture cross section (thermal 2.7 barns, RI 37 barns). The ${ }^{167} \mathrm{Er}(\mathrm{n}, \gamma){ }^{168} \mathrm{Er}$ reaction, together with the use of erbium fully enriched in ${ }^{167} \mathrm{Er}$, will thus mainly lead to ${ }^{168} \mathrm{Er}$. The residual burden of the BP enriched in ${ }^{167} \mathrm{Er}$ should thus be small. The ${ }^{168} \mathrm{Er}(\mathrm{n}, \gamma){ }^{169} \mathrm{Er}$ reaction leads to ${ }^{169} \mathrm{Er}$, which decays into ${ }^{169} \mathrm{Tm}$ with a half-life of 9.4 days. The ${ }^{169} \mathrm{Tm}$ capture cross section is large (thermal 105 barns, RI 1,720 barns). In erbium with natural isotopic abundances, ${ }^{168} \mathrm{Er}$ has an isotopic abundance of $26.8 \%$. Even though the capture cross section of ${ }^{168} \mathrm{Er}$ is small, the ${ }^{168} \mathrm{Er}(\mathrm{n}, \gamma){ }^{169} \mathrm{Er}$ reaction will lead to ${ }^{169} \mathrm{Tm}$. Also, ${ }^{170} \mathrm{Er}$ (14.9\% natural isotopic abundance) has a small neutron capture cross section (thermal 5.8 barns, RI 45 barns), but the ${ }^{170} \mathrm{Er}$ $(\mathrm{n}, \gamma){ }^{171} \mathrm{Er}$ reaction will lead to ${ }^{171} \mathrm{Er}$, which decays with a half-life of 7.5 hours to ${ }^{171} \mathrm{Tm}$. The capture cross section of ${ }^{171} \mathrm{Tm}$ is large (thermal 160 barns, RI 118 barns). Note that the use of ${ }^{167} \mathrm{Er}$ will not create any of the holmium isotopes. The use of erbium with natural isotopic abundances will lead to ${ }^{163} \mathrm{Ho}$ and ${ }^{165} \mathrm{Ho}$. These have large capture cross sections. However, since the natural isotopic abundances of ${ }^{162} \mathrm{Er}$ and ${ }^{164} \mathrm{Er}$ are small, they will only slightly contribute to the residual reactivity burden at EOL of the BP.

Table 13 displays the results of using $\mathrm{Er}_{2} \mathrm{O}_{3}$ mixed homogeneously with the fuel pellets of a number of fuel rods. Note that for the same initial reactivity worth of the BP, the residual negative reactivity worth of erbium fully enriched in ${ }^{167} \mathrm{Er}$ is smaller than for erbium with natural isotopic abundances. The relative contribution from the thulium daughters is also smaller.

The reactivity worths of the $\mathrm{BP}$ isotopes, for a $4.0 \mathrm{wt} \%$ loading of $\mathrm{Er}_{2} \mathrm{O}_{3}$ with natural isotopic abundances, homogeneously mixed inside the fuel pellets of 64 IFBA rods per fuel assembly, are shown in Figure 45 . The initial reactivity worth of the $\mathrm{BP}$ is 0.141 and the residual reactivity ratio is $9.0 \%$. For a $1.0 \mathrm{wt} \%$ homogeneous loading in $64 \mathrm{IFBA}$ rods of $\mathrm{Er}_{2} \mathrm{O}_{3}$ fully enriched in ${ }^{167} \mathrm{Er}$, the results are shown in Figure 46. The initial reactivity worth of the BP is 0.140 and the residual reactivity ratio is $1.6 \%$. The use of erbium enriched in ${ }^{167} \mathrm{Er}$, instead of with natural isotopic abundances, will thus lead to a substantial reduction of the residual reactivity burden at EOL of the fuel. Note that the trends of the fuel assembly lattice $\mathrm{k}_{\text {inf }}$ 's are fairly flat for almost 2 years of reactor operation. Figures 45 and 46 also display the masses of the burnable poison isotopes and their daughters as a function of depletion time. Figure 45 also displays a secular equilibrium between the ${ }^{166} \mathrm{Er}$ and ${ }^{167} \mathrm{Er}$ neutron capture reaction rates. However, since the cross section of ${ }^{167} \mathrm{Er}$ is smaller than in the case of gadolinium or samarium, the secular equilibrium happens much later (approximately 4 years).

In Tables 13 through 16 the residual reactivity worths, due to the Ho daughters of the BP containing erbium enriched in ${ }^{167} \mathrm{Er}$, should essentially be zero, since only the decay of ${ }^{163} \mathrm{Er}$ and ${ }^{165} \mathrm{Er}$ leads to ${ }^{163} \mathrm{Ho}$ and ${ }^{165} \mathrm{Ho}$, respectively. There are essentially no paths from the transmutation of ${ }^{167} \mathrm{Er}$ to any of the Ho isotopes. However, the tables show residual reactivity worths of the order of $1.0 \times 10^{-10}$. This is because for each case, all the BP isotopes and their daughters (which are not present in the initial loading of the BP) have been added as traces to the BP with a number density of $1.0 \times 10^{-15}$ instead of 
zero. This is to avoid problems at the BOC in the input to the MCNP4C code and to avoid computer exception errors (division by zero) when calculating spectrum-weighted cross sections.

The MVCs were calculated by decreasing the water coolant density by $10 \%$ (see Tables $13-16$ ). Note that for all the cases containing 8,16, and 64 IFBA rods, the MVCs remain negative. This is in contrast to the use of 64 IFBA rods containing gadolinium or samarium as the BP.

Figure 47 displays the initial reactivity worths of the erbium based BP as a function the residual BP penalty in FPDs for several configurations containing 16 IFBA rods. Figure 48 displays the initial reactivity worths of the BP vs FPD penalty for fuel assembly configurations containing 64 IFBA rods. The trends for the four different forms of BPs are very similar. Note also the clear advantage of using enriched erbium (enriched in ${ }^{167} \mathrm{Er}$ ) over erbium with natural isotopic abundances.

For the cases where the BP is mixed uniformly with the cladding of a number of fuel rods (see Table 16 and Appendix C4), the required loading using natural Er might be too high (e.g., $4.0 \mathrm{wt} \%$ ). In order to achieve a given initial reactivity worth of the BP, the use of erbium enriched in ${ }^{167} \mathrm{Er}$ substantially reduces the required loading in the cladding. Further studies of the metallurgical behavior of the Zircaloy cladding in the presence of erbium will be done during phase 3 of the project. 


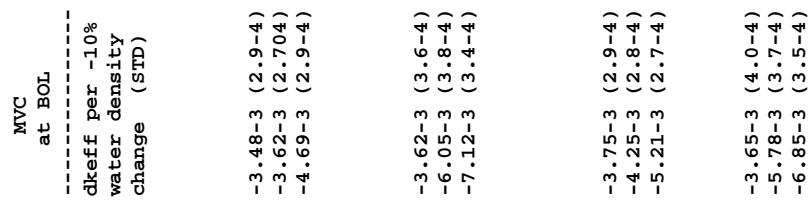

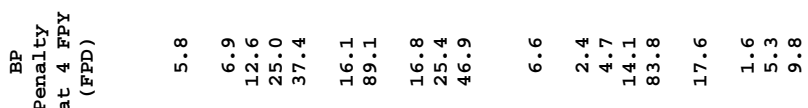

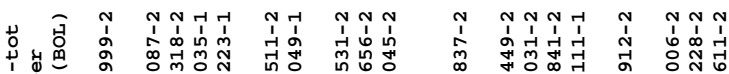

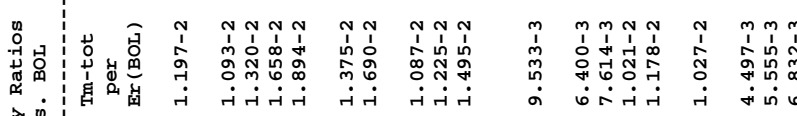
落

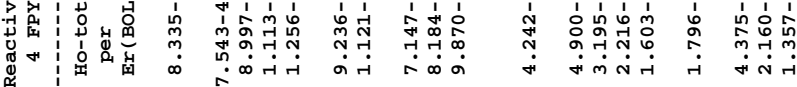
น

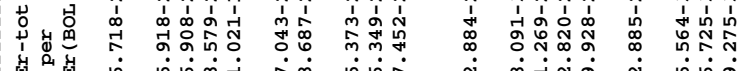

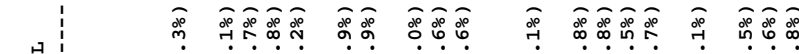

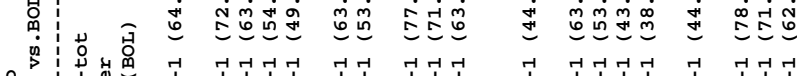

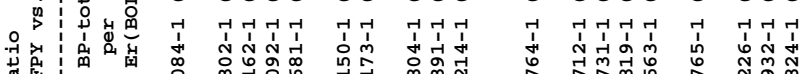

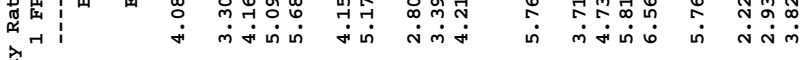

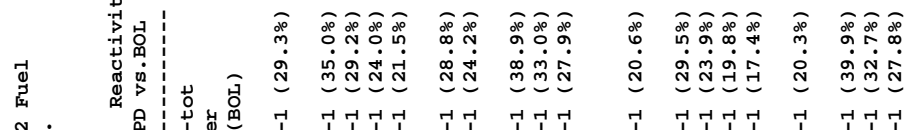

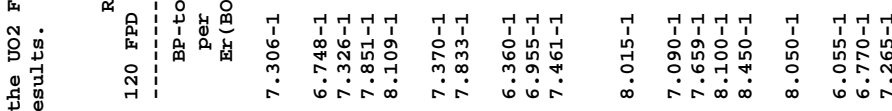

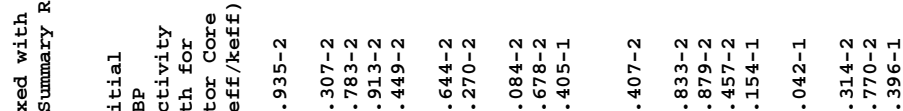

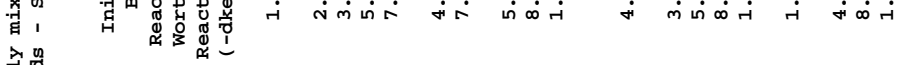

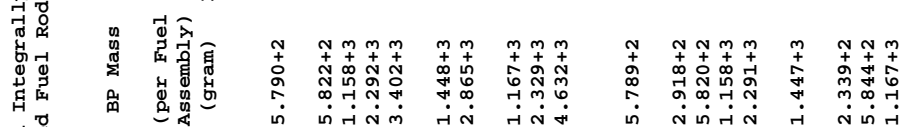

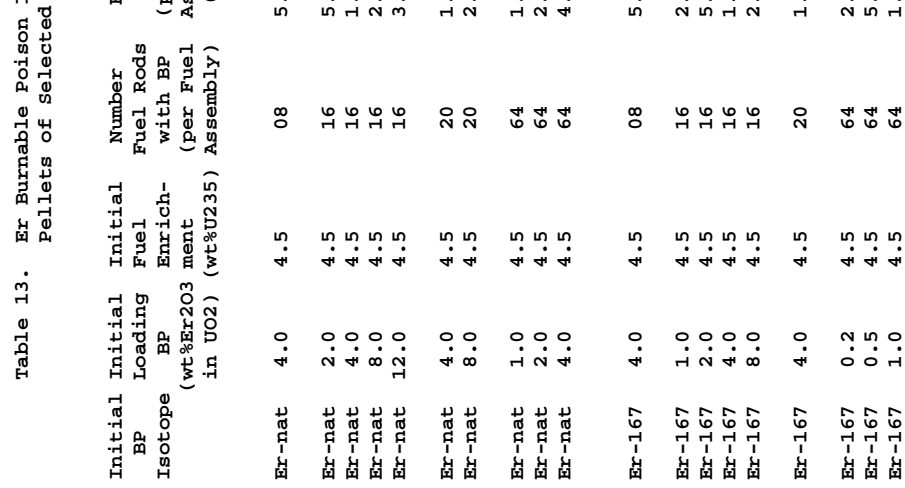




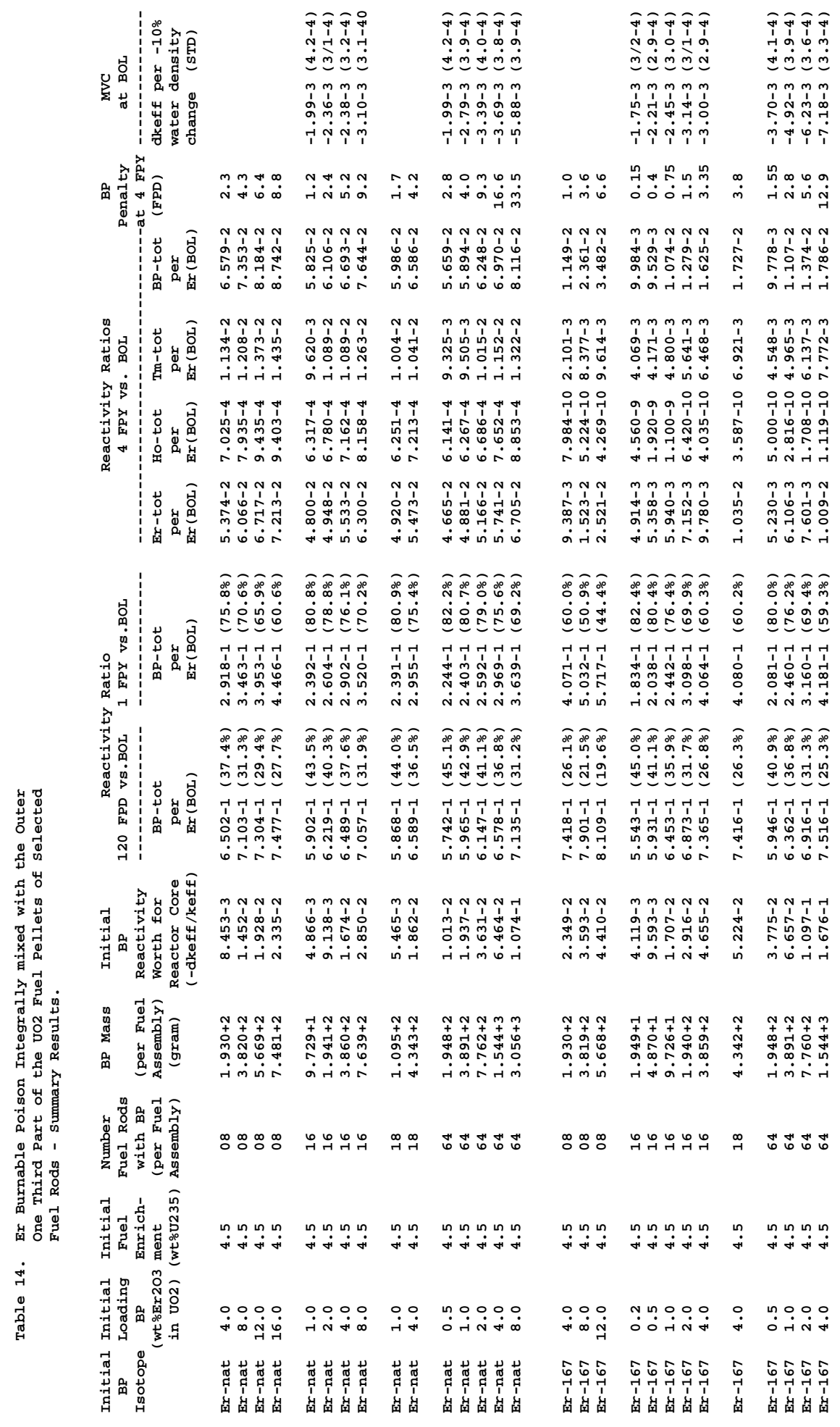




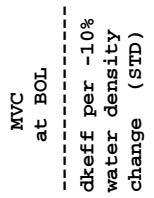
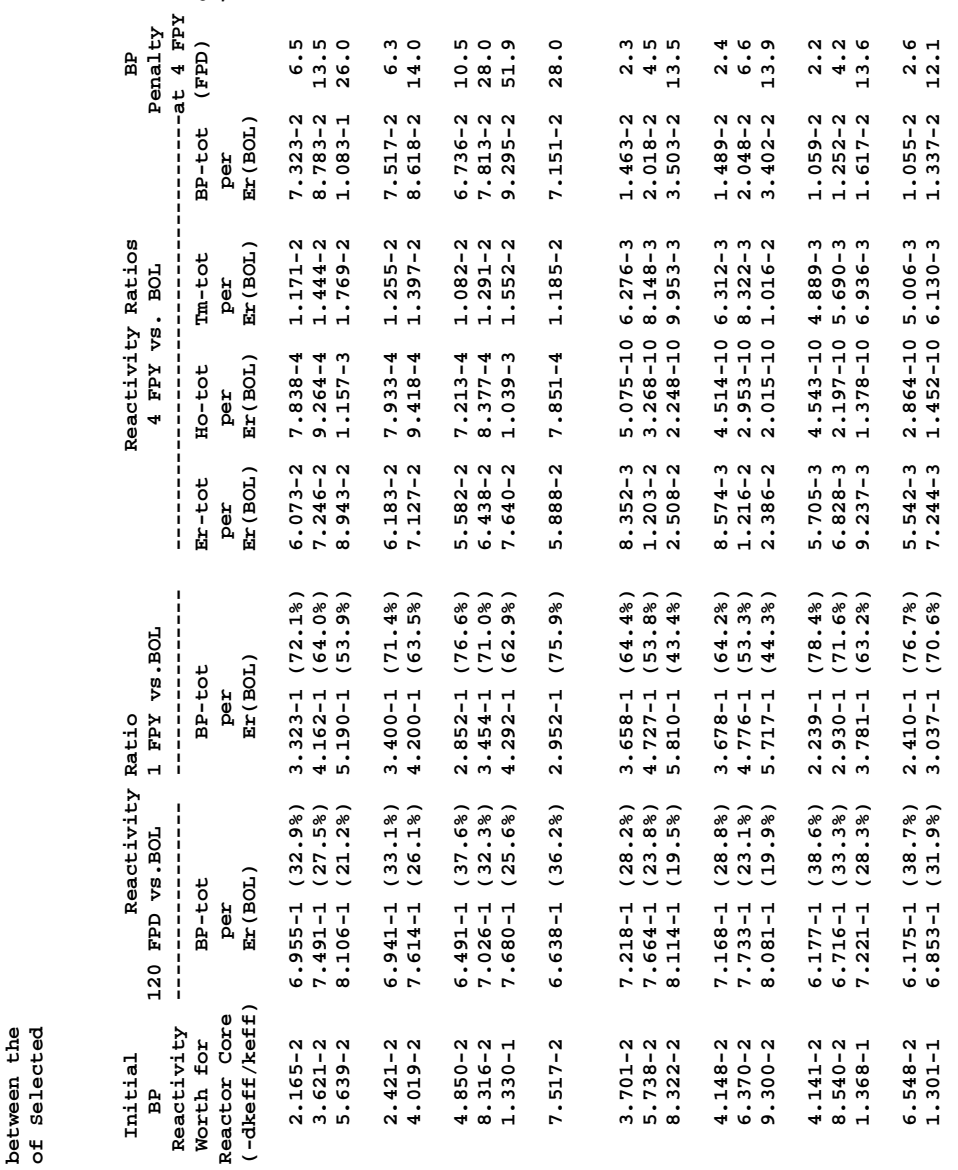

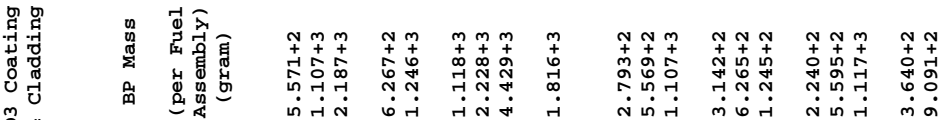

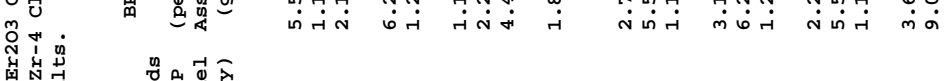

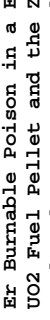

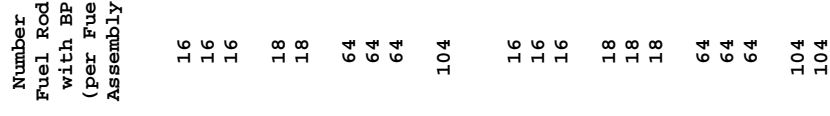

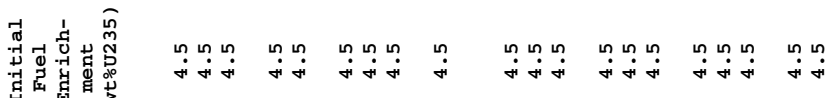

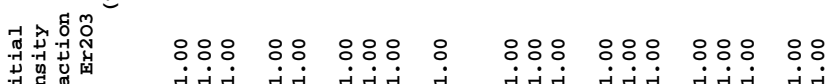
䒠器出

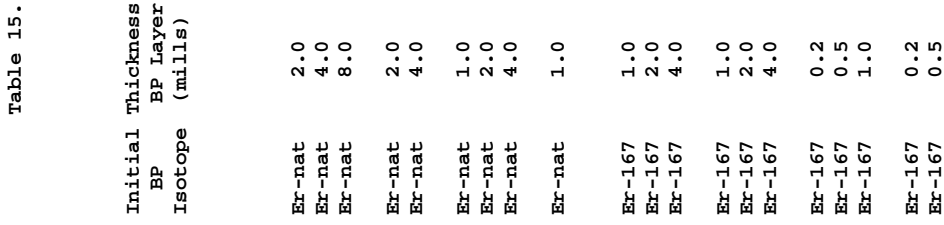




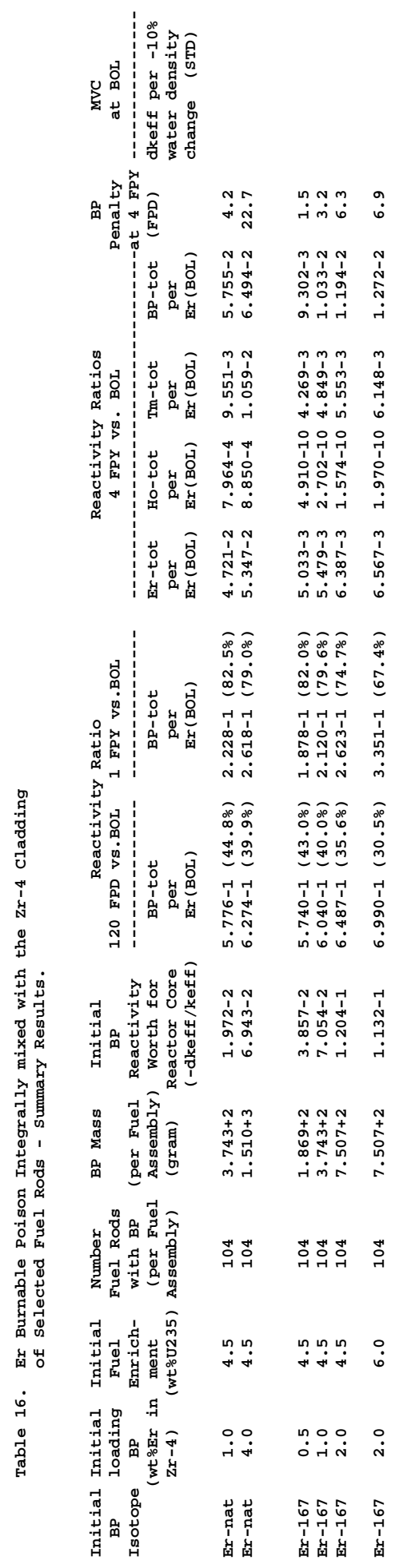


Figure 42. Neutron Cross-Sections as a Function of Energy Er-162 (68162.40c)

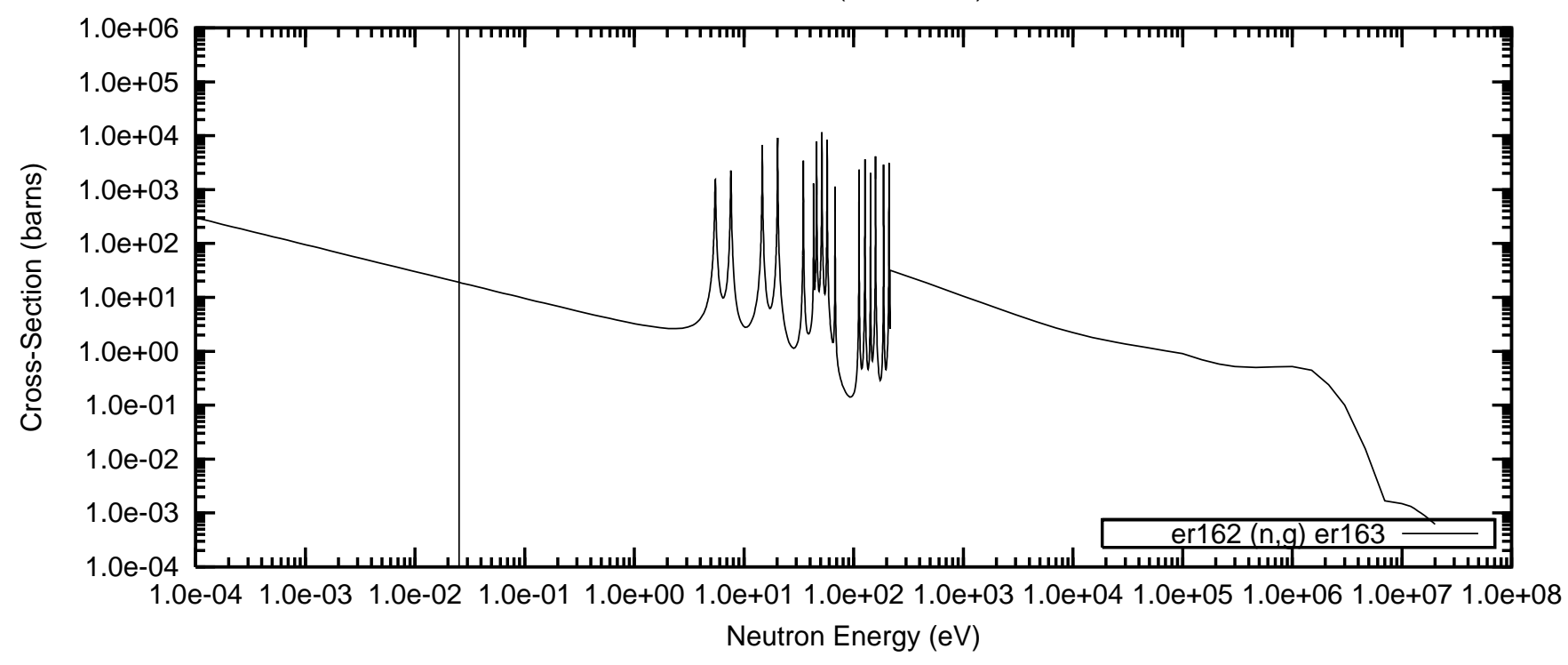

Er-164 (68164.40c)

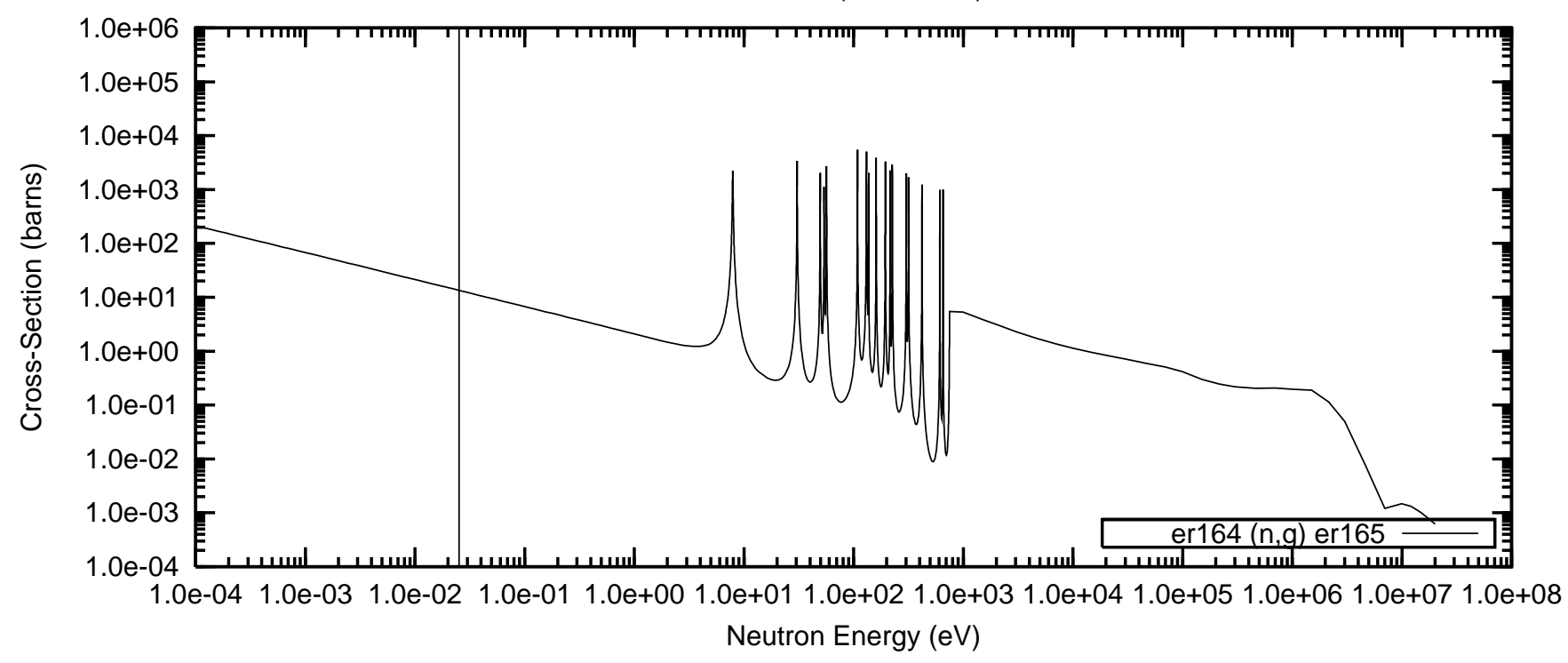

Er-165 (68165.40c)

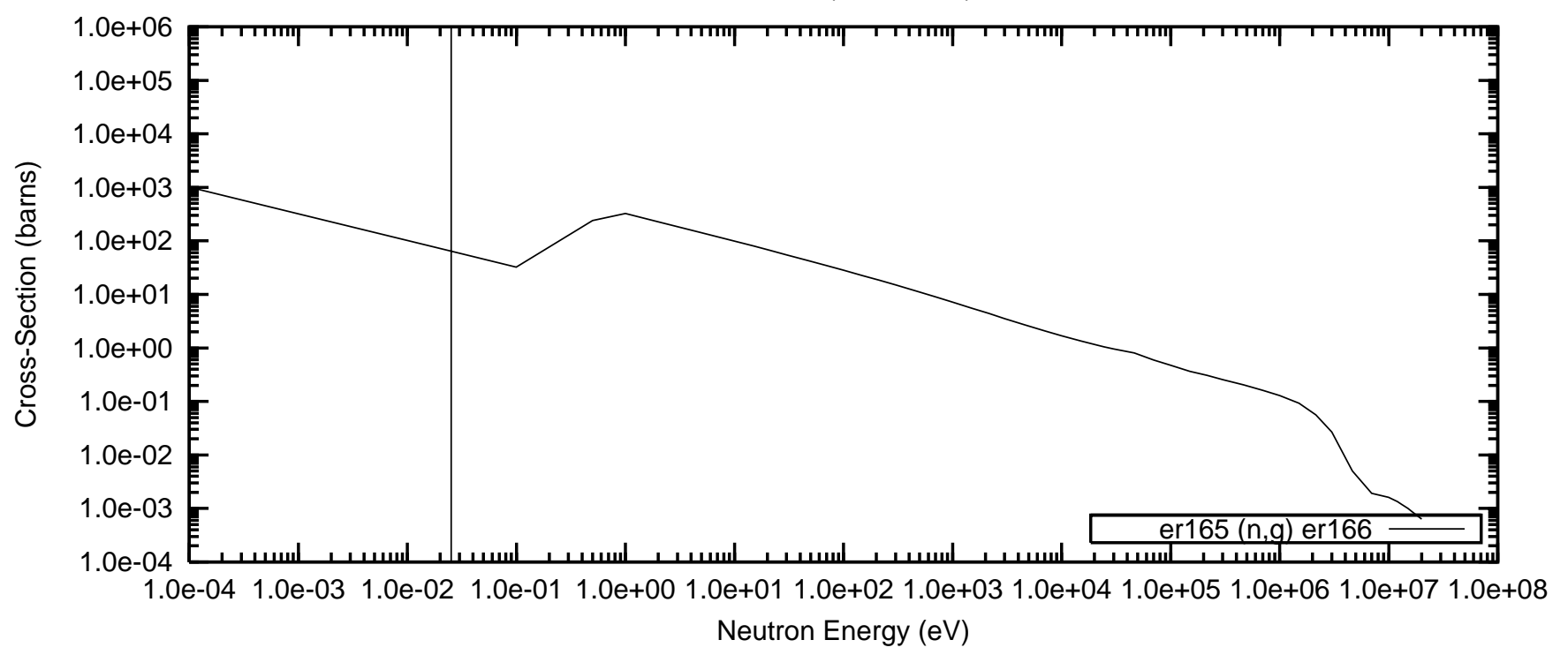


Figure 42(cont). Neutron Cross-Sections as a Function of Energy Er-166 (68166.40c,46c)

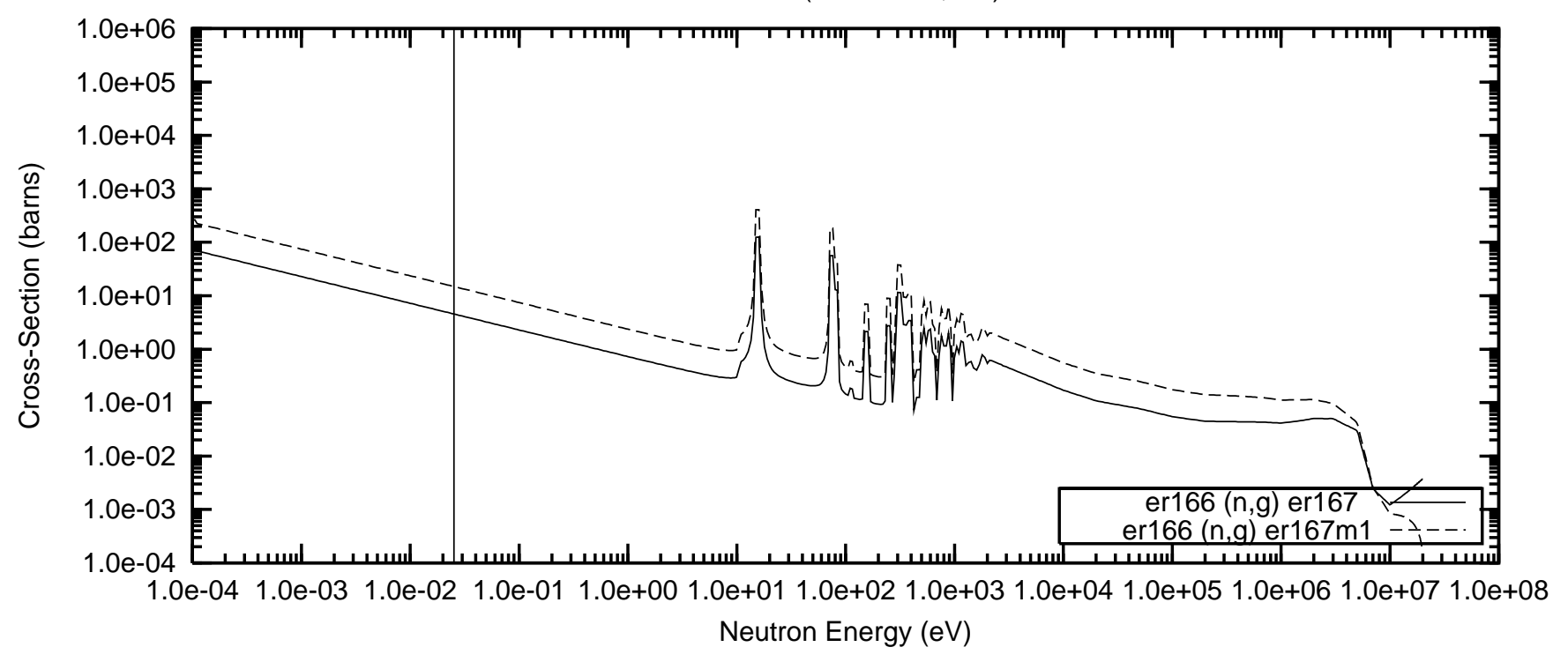

Er-167 (68167.40c)

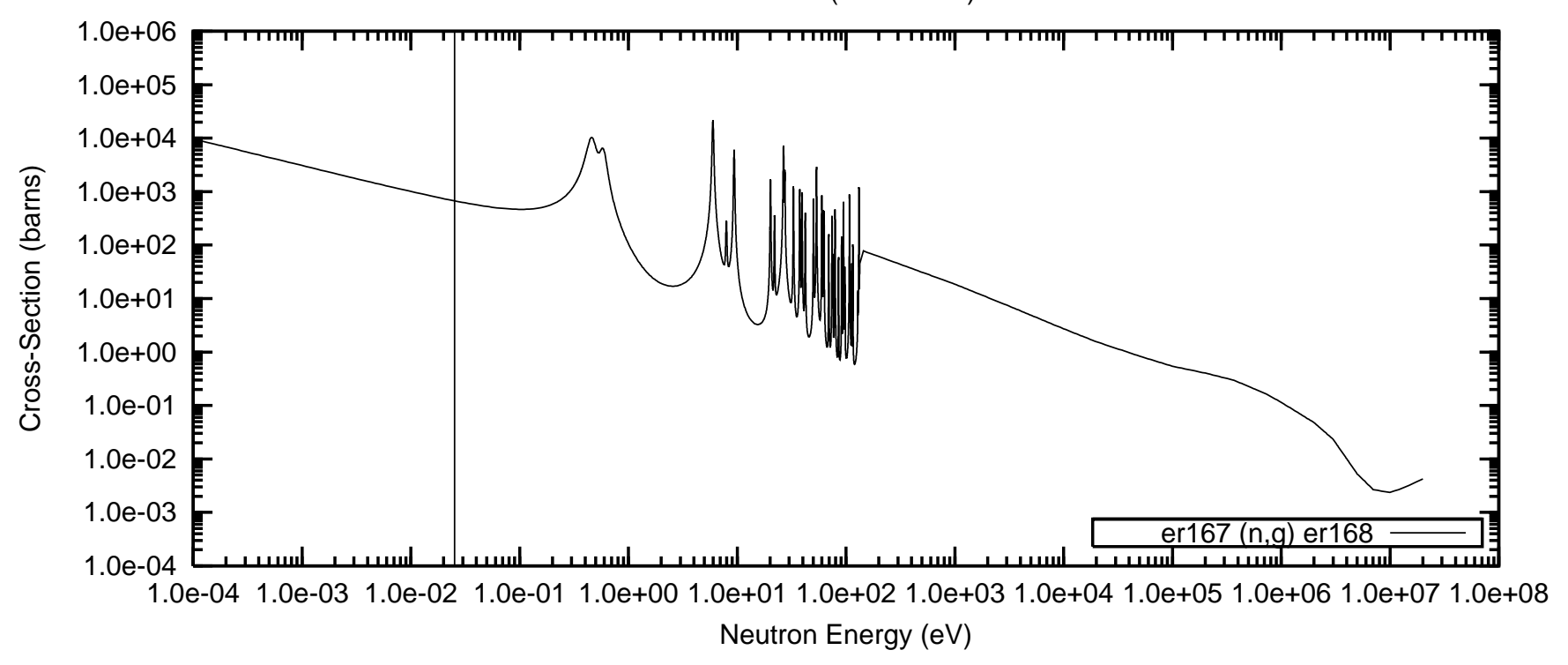

Er-168 (68168.40c)

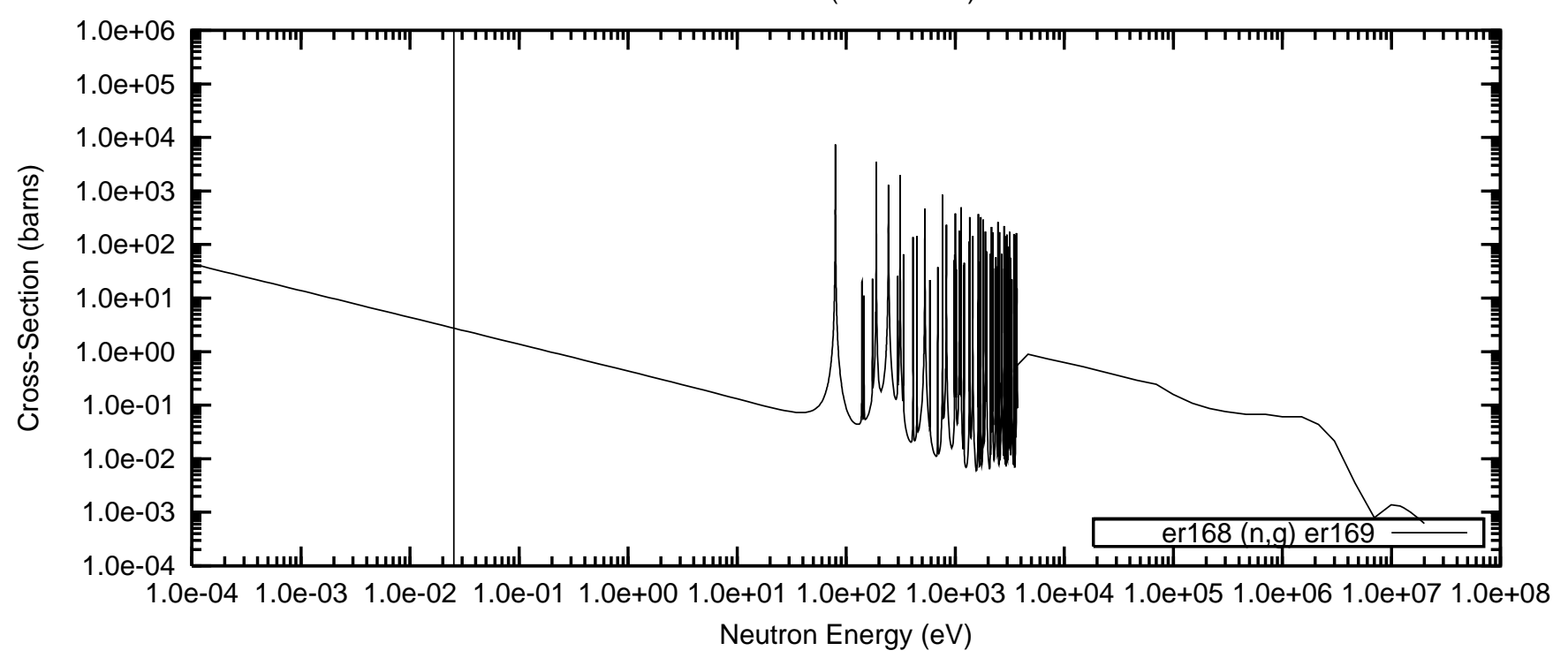


Figure 42(cont). Neutron Cross-Sections as a Function of Energy Er-169 (68169.40c)

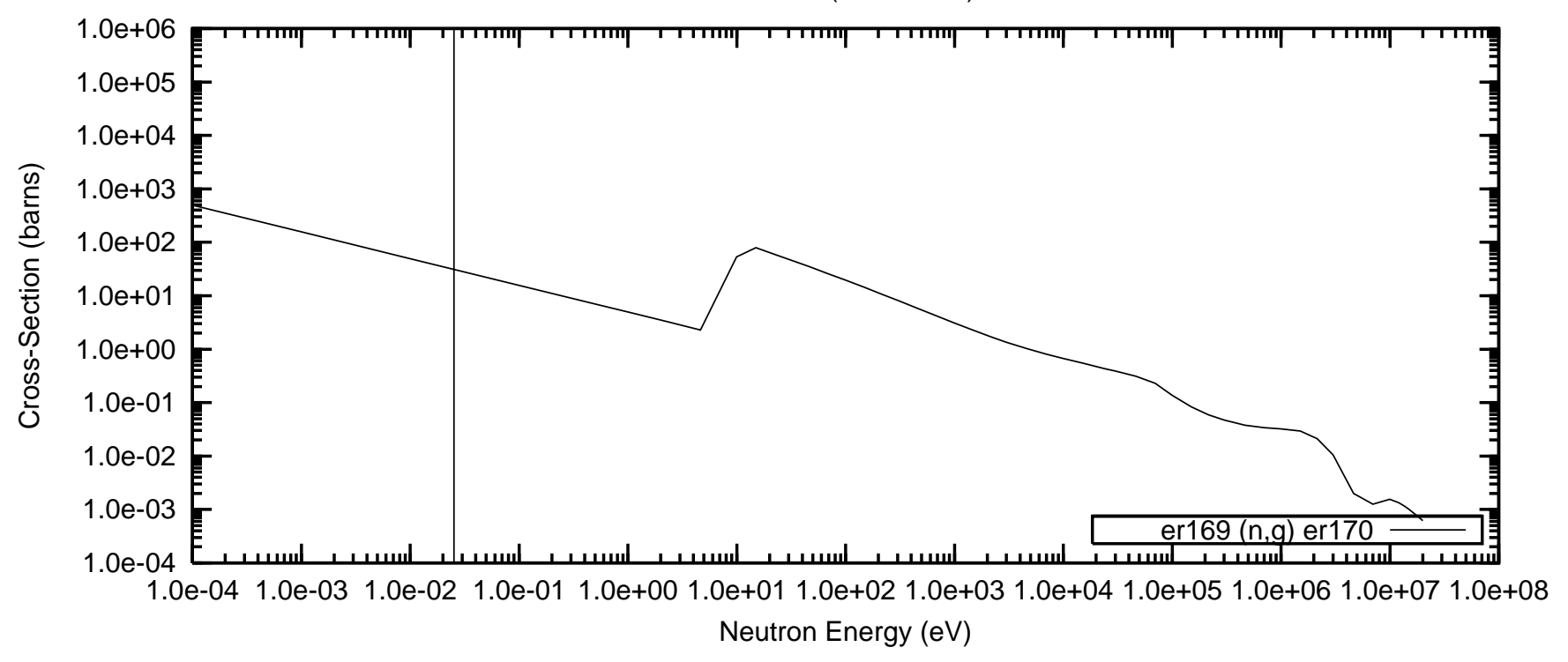

$\operatorname{Er}-170(68170.40 \mathrm{c})$

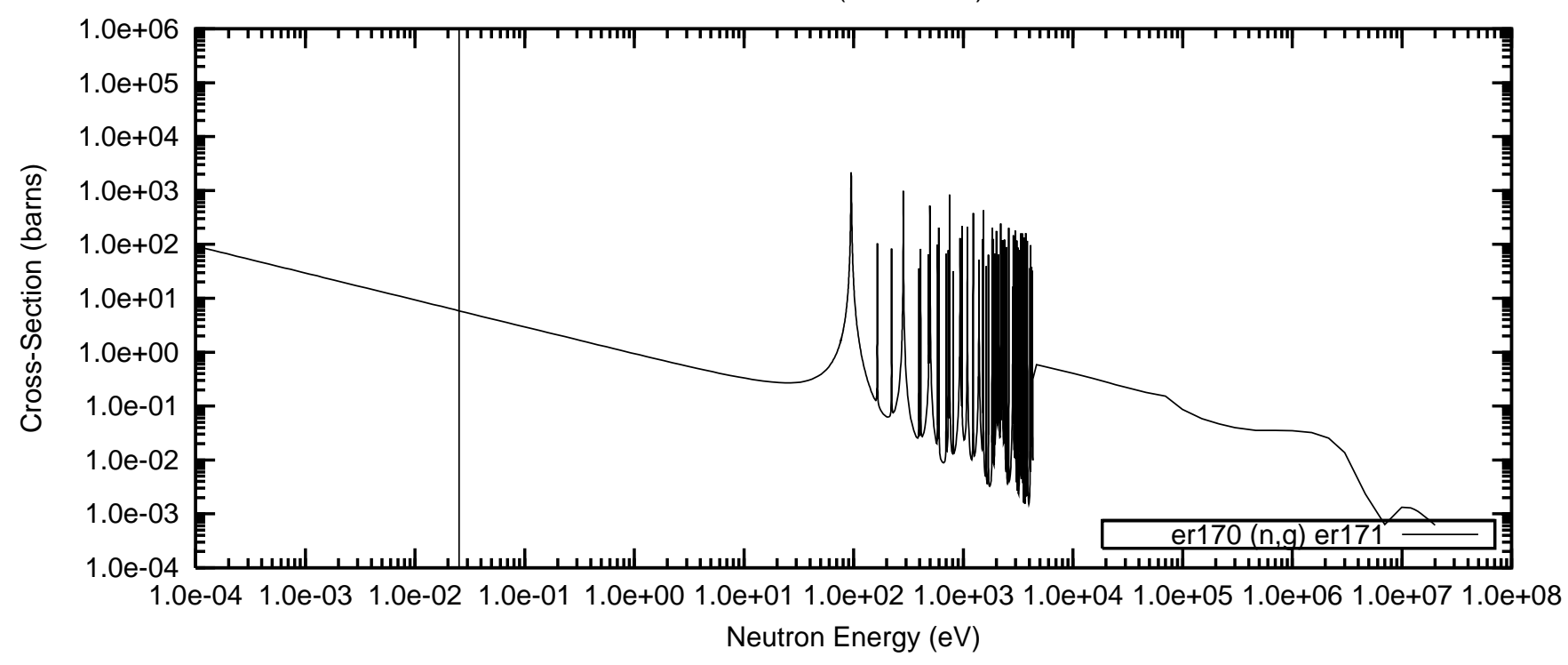

$\operatorname{Er}-171$ (68171.40c)

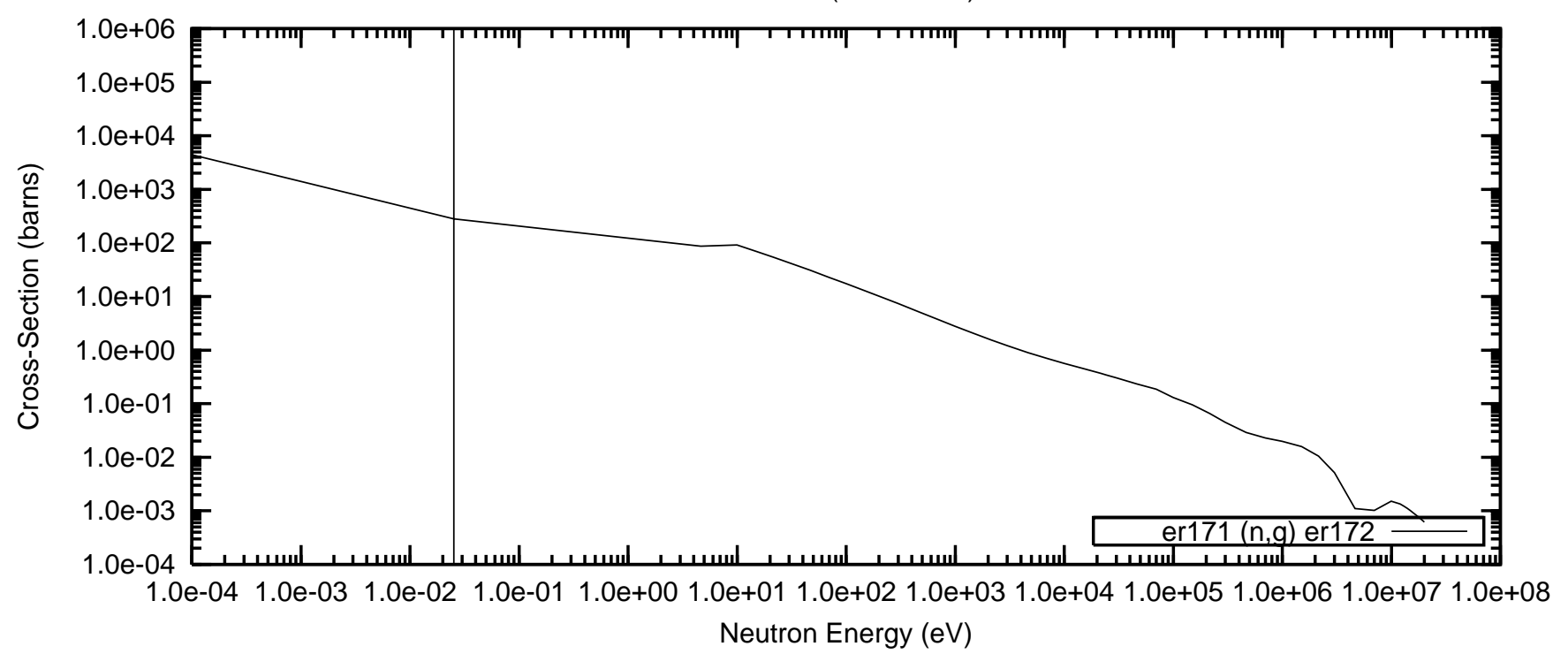


Figure 42(cont). Neutron Cross-Sections as a Function of Energy

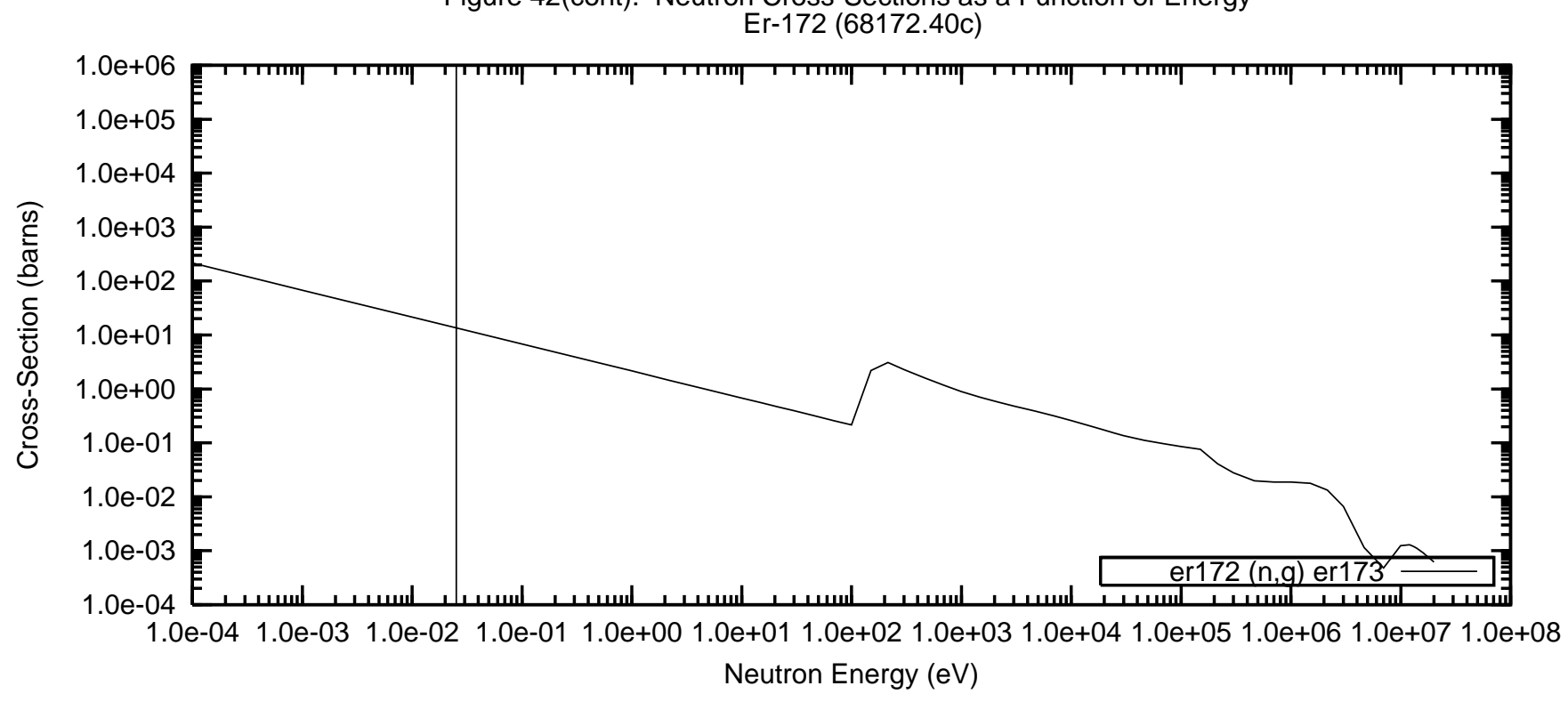


Figure 43. Neutron Cross-Sections as a Function of Energy

Ho-163 (67163.40c)

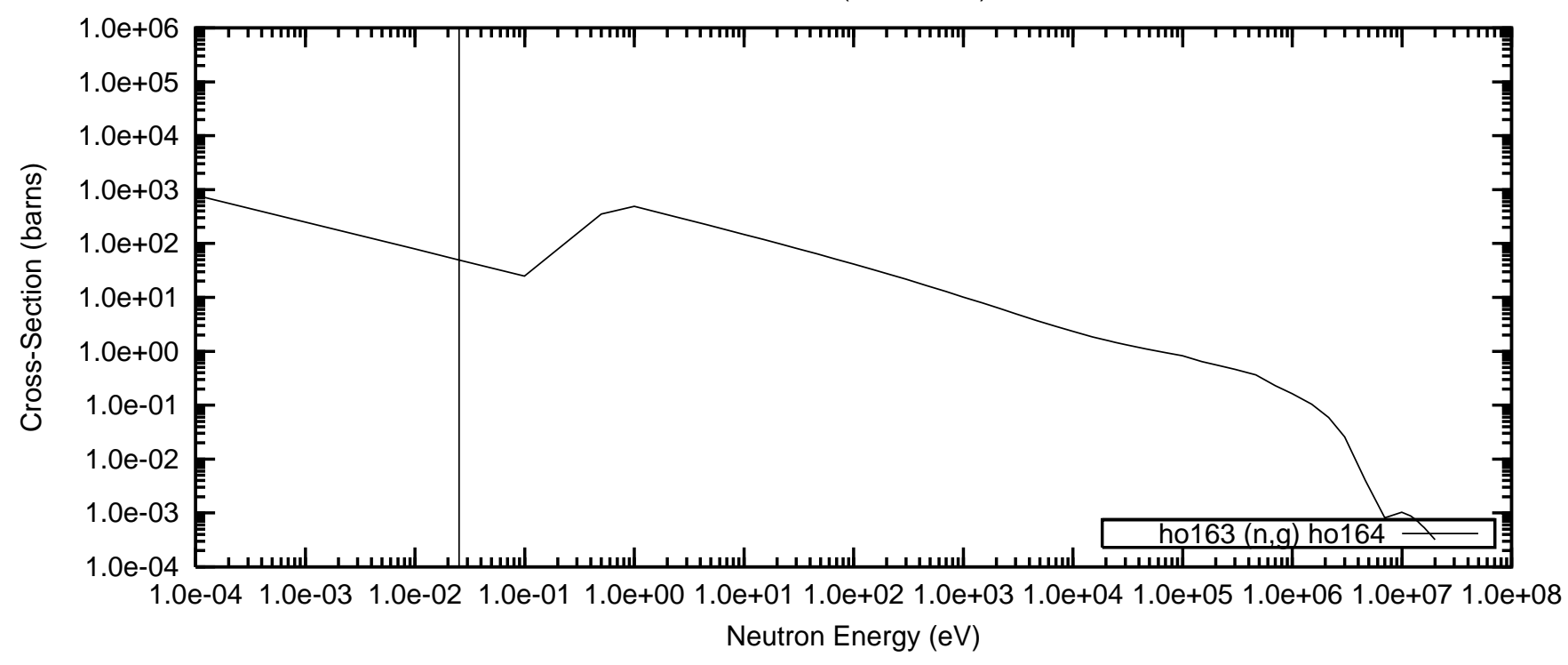

Ho-164 (67164.40c)

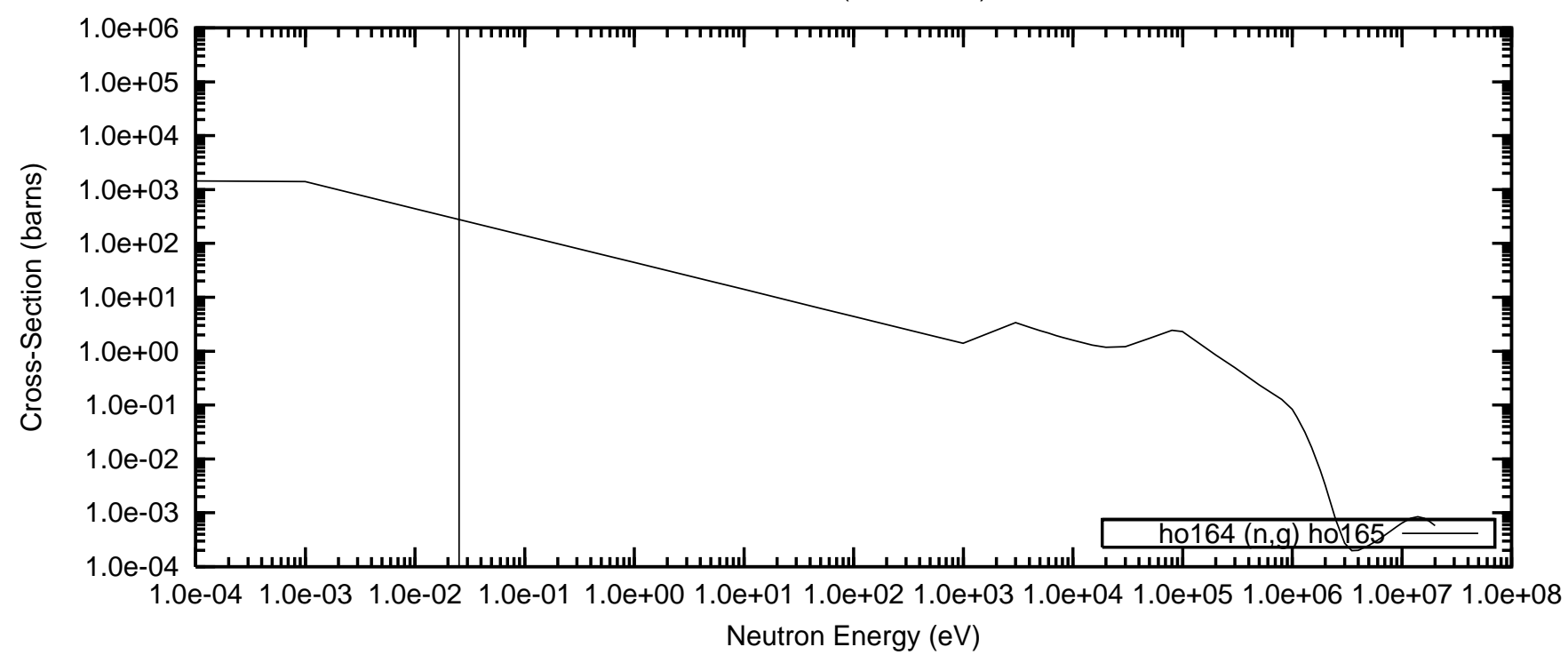

Ho-164mi (67164.41c)

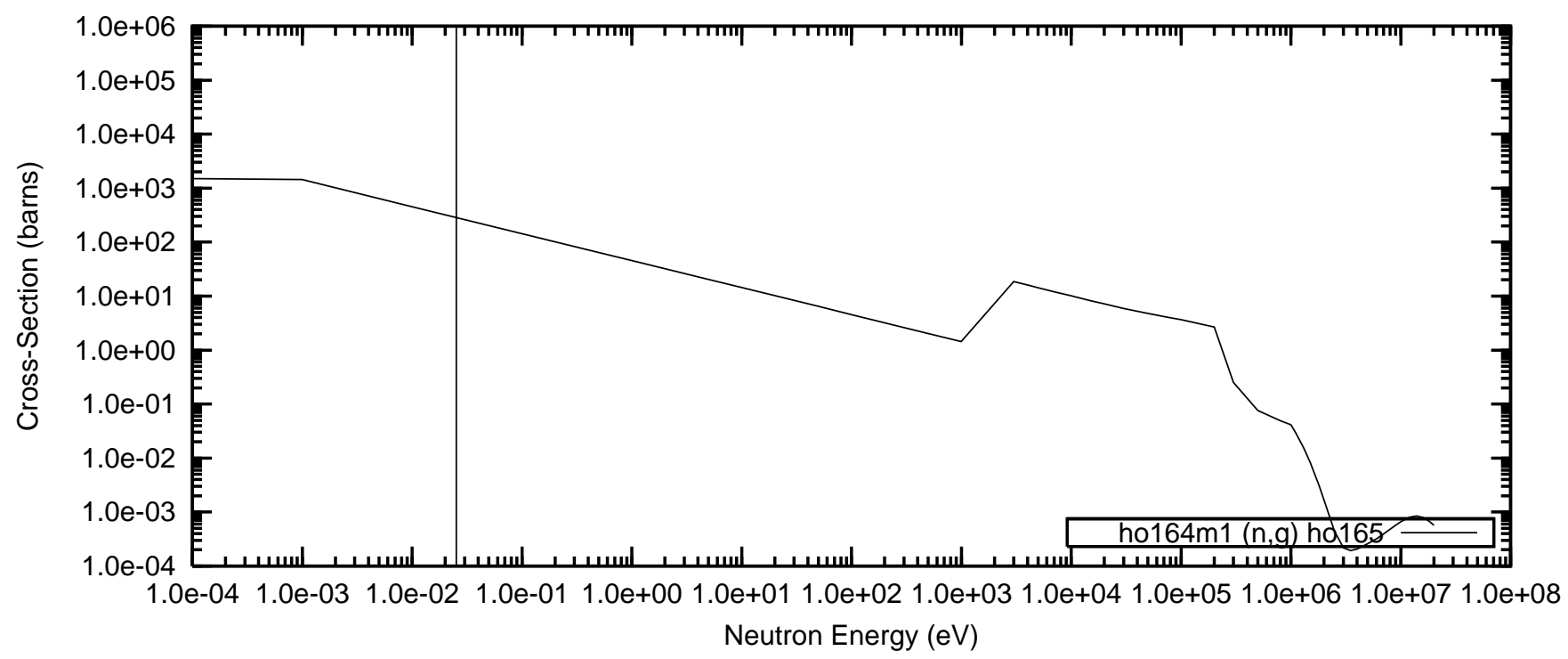


Figure 43(cont). Neutron Cross-Sections as a Function of Energy Ho-165 (67165.40c,46c)

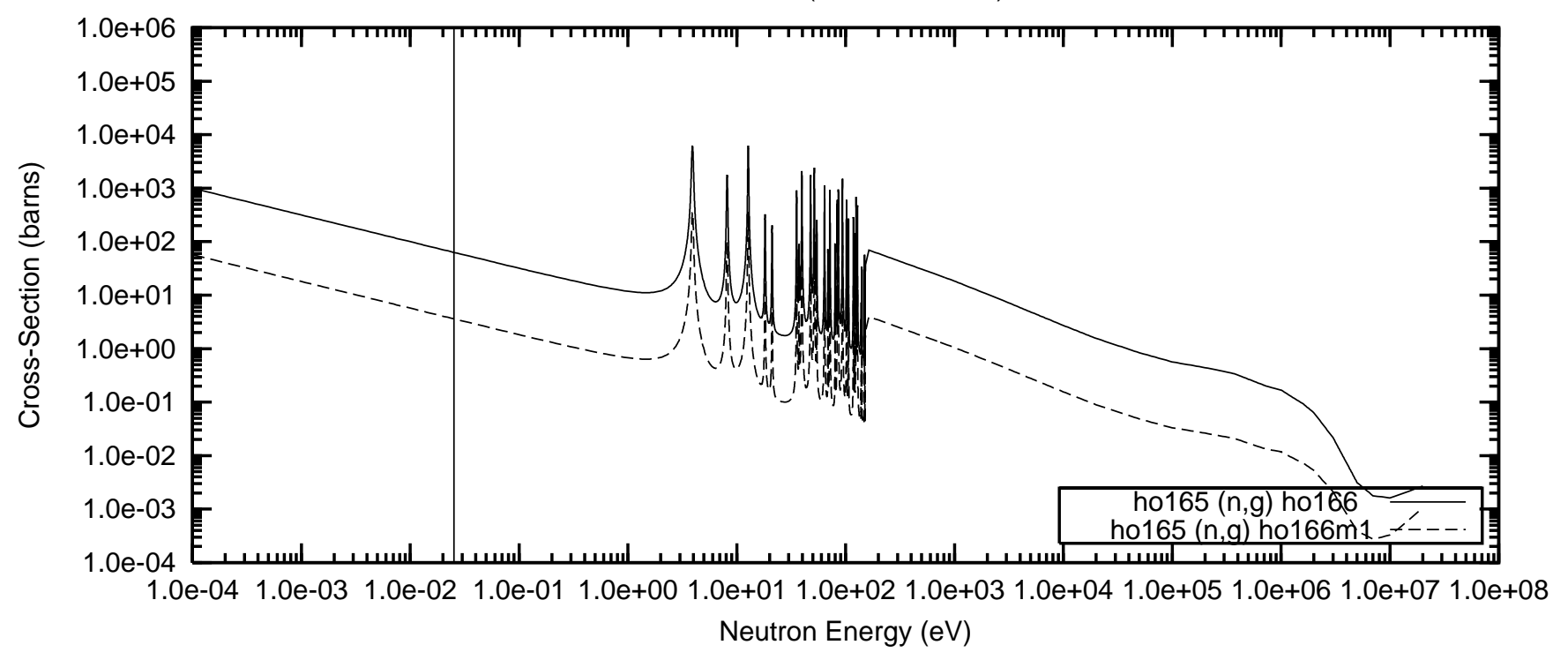

Ho-166 (67166.40c)

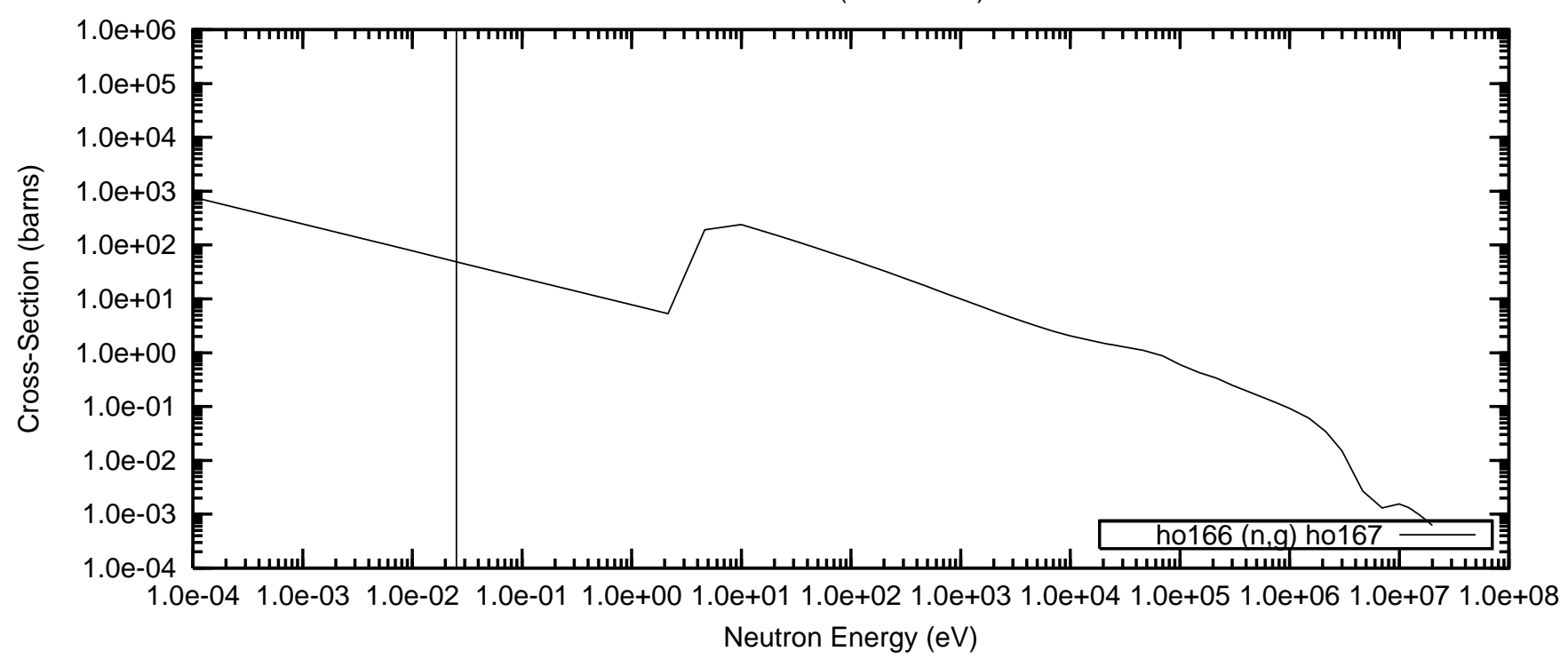

Ho-166mi (67166.41c)

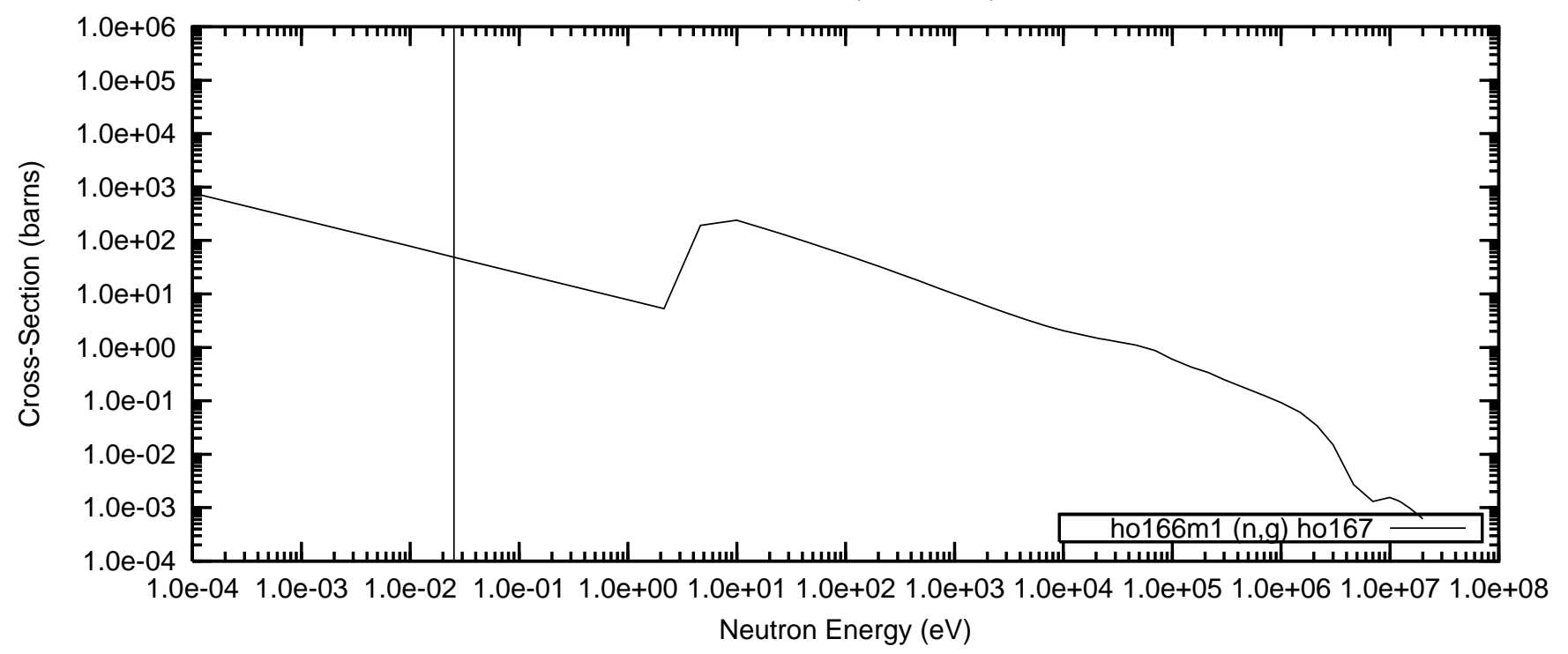


Figure 44. Neutron Cross-Sections as a Function of Energy

Tm-168 (69168.40c)

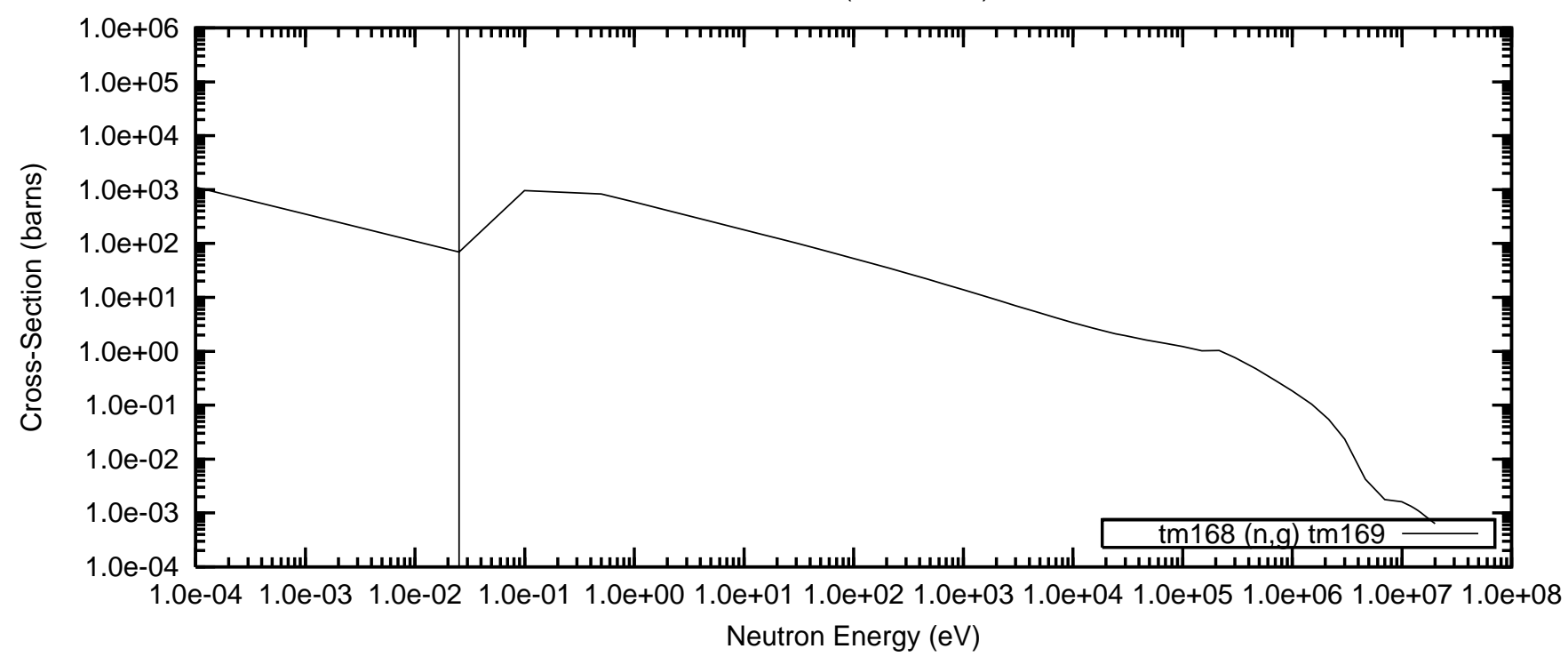

Tm-169 (69169.40c)

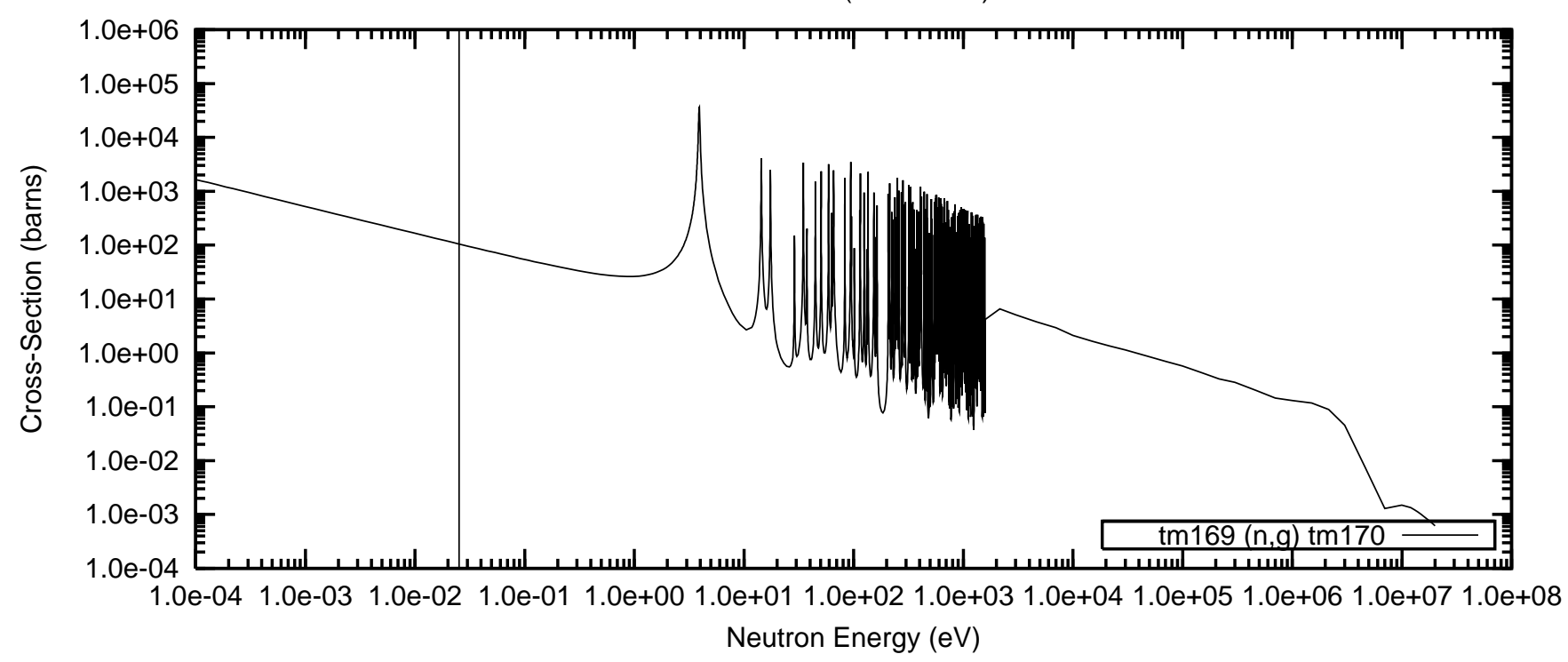

$\operatorname{Tm}-170$ (69170.40c)

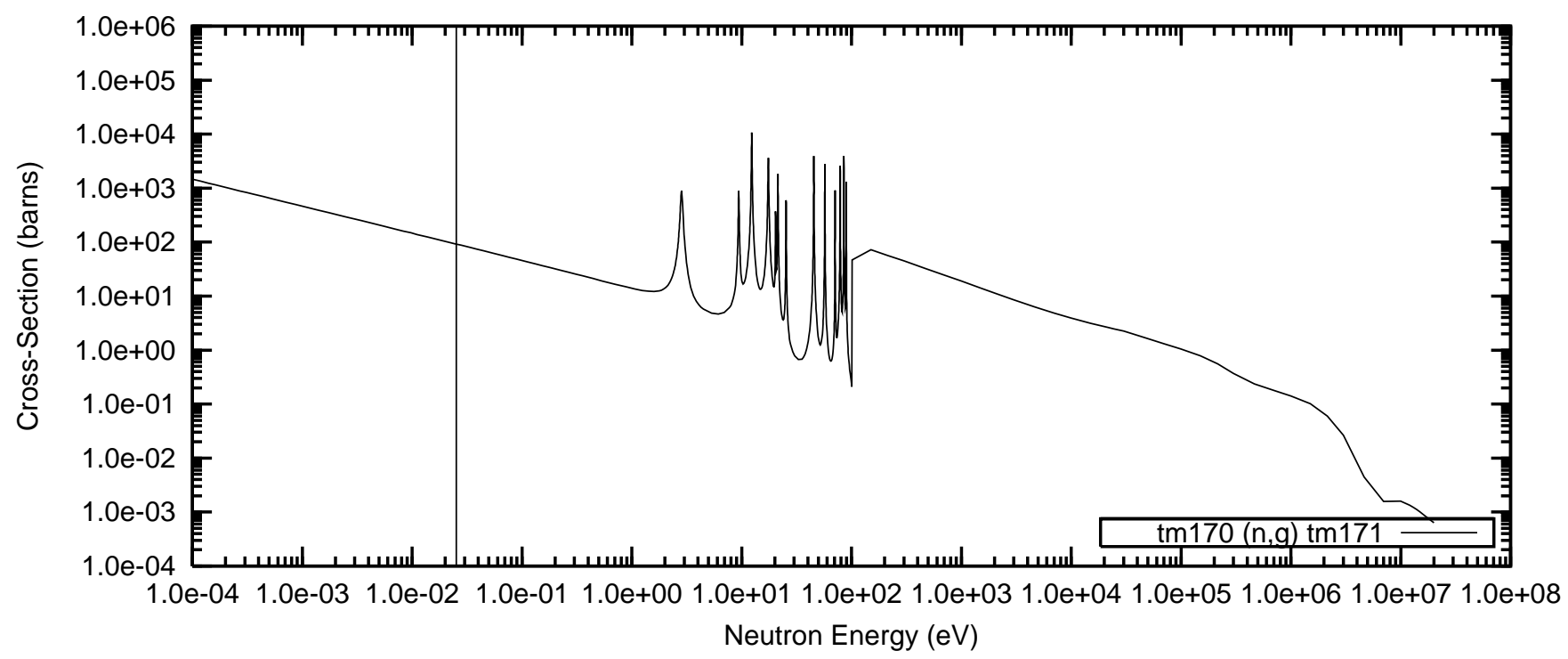


Figure 44(cont). Neutron Cross-Sections as a Function of Energy $\mathrm{Tm}-171$ (69171.40c)

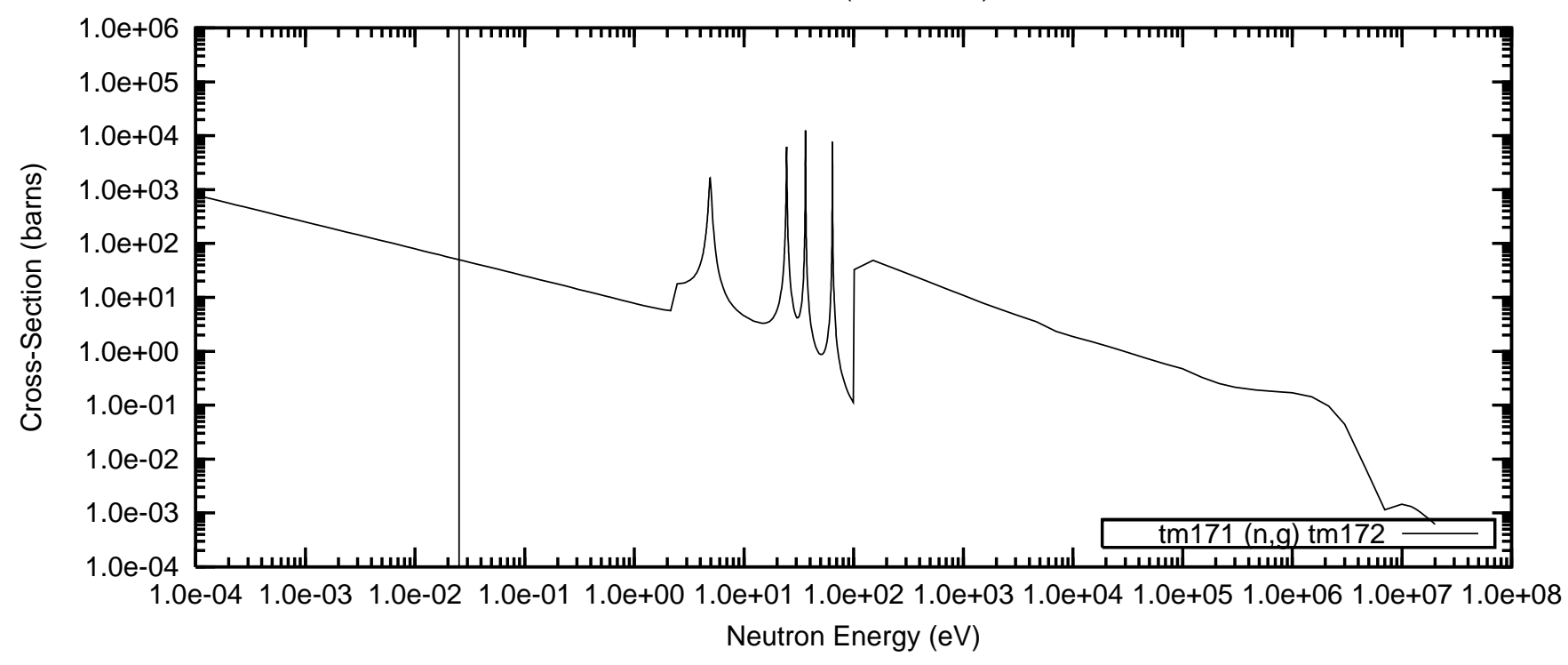

Tm-172 (69172.40c)

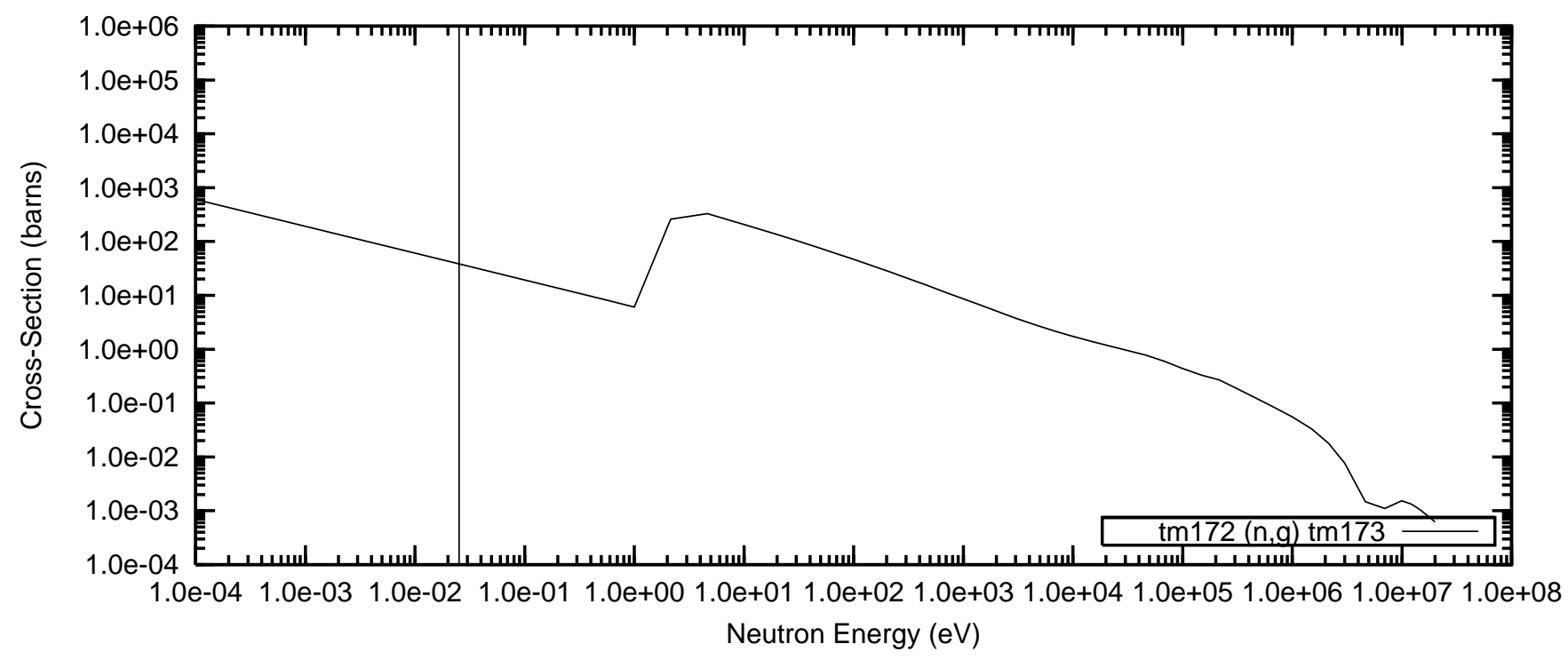


Figure 45. Negative Reactivity and Masses BP for Er-nat and transmutation daughters as a function of fuel life for $17 \times 17$ fuel assemblies with 64 poison rods, 4.0wt\% Er2O3 poison homogeneously mixed in the UO2 pellets

Reactor power $3400 \mathrm{MWth}, 193$ fuel assemblies, initial enrichment 4.5wt\%U-235

(Case : er000_3_100p_h2o_64_bp_fuel_2_o)
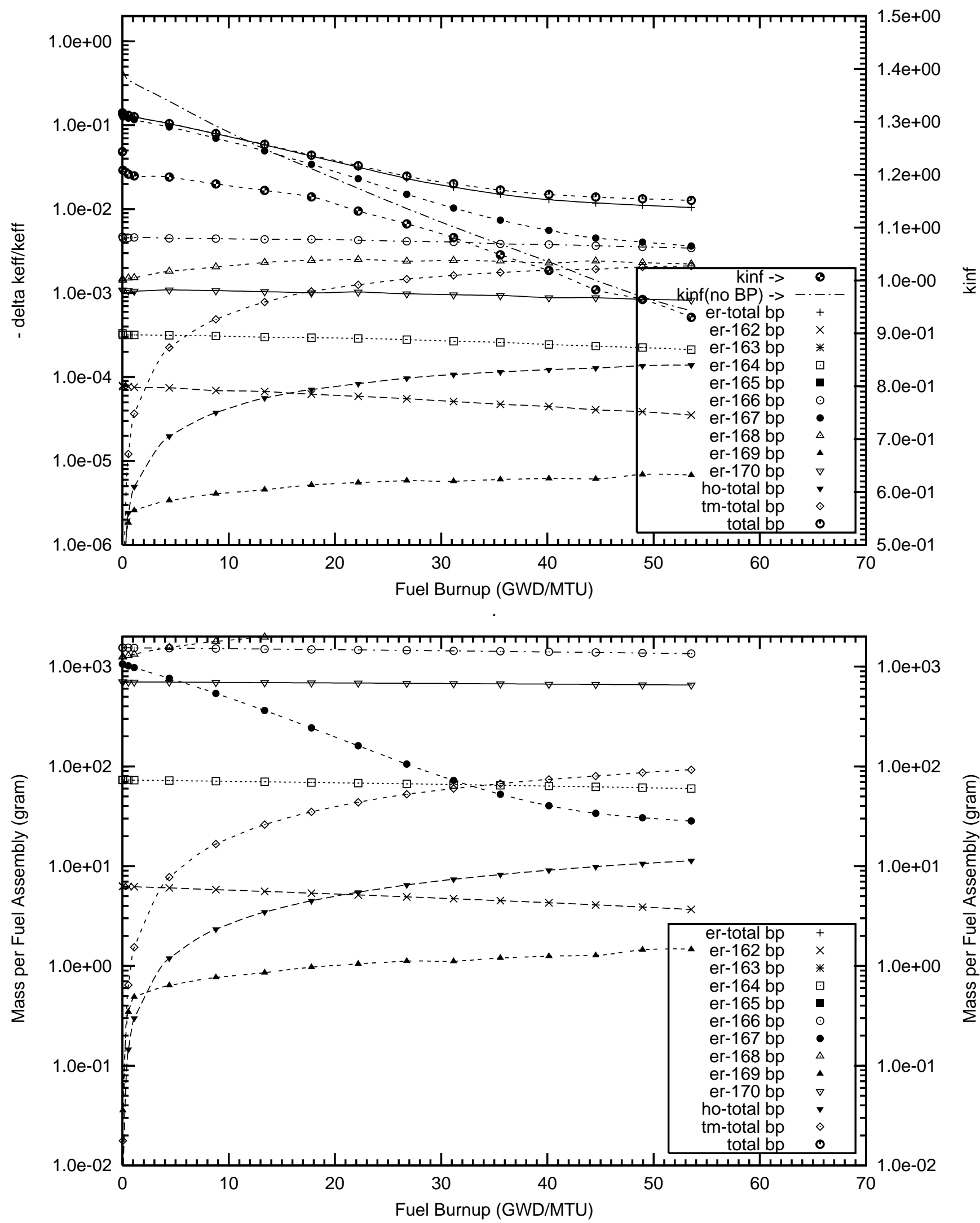
Figure 46. Negative Reactivity and Masses BP for Er-167 and transmutation daughters as a function of fuel life for $17 \times 17$ fuel assemblies with 64 poison rods, $1.0 \mathrm{wt} \%$ Er2O3 poison homogeneously mixed in the UO2 pellets

Reactor power $3400 \mathrm{MWth}, 193$ fuel assemblies, initial enrichment 4.5wt\%U-235

(Case : er167_1_64_bp_fuel o)
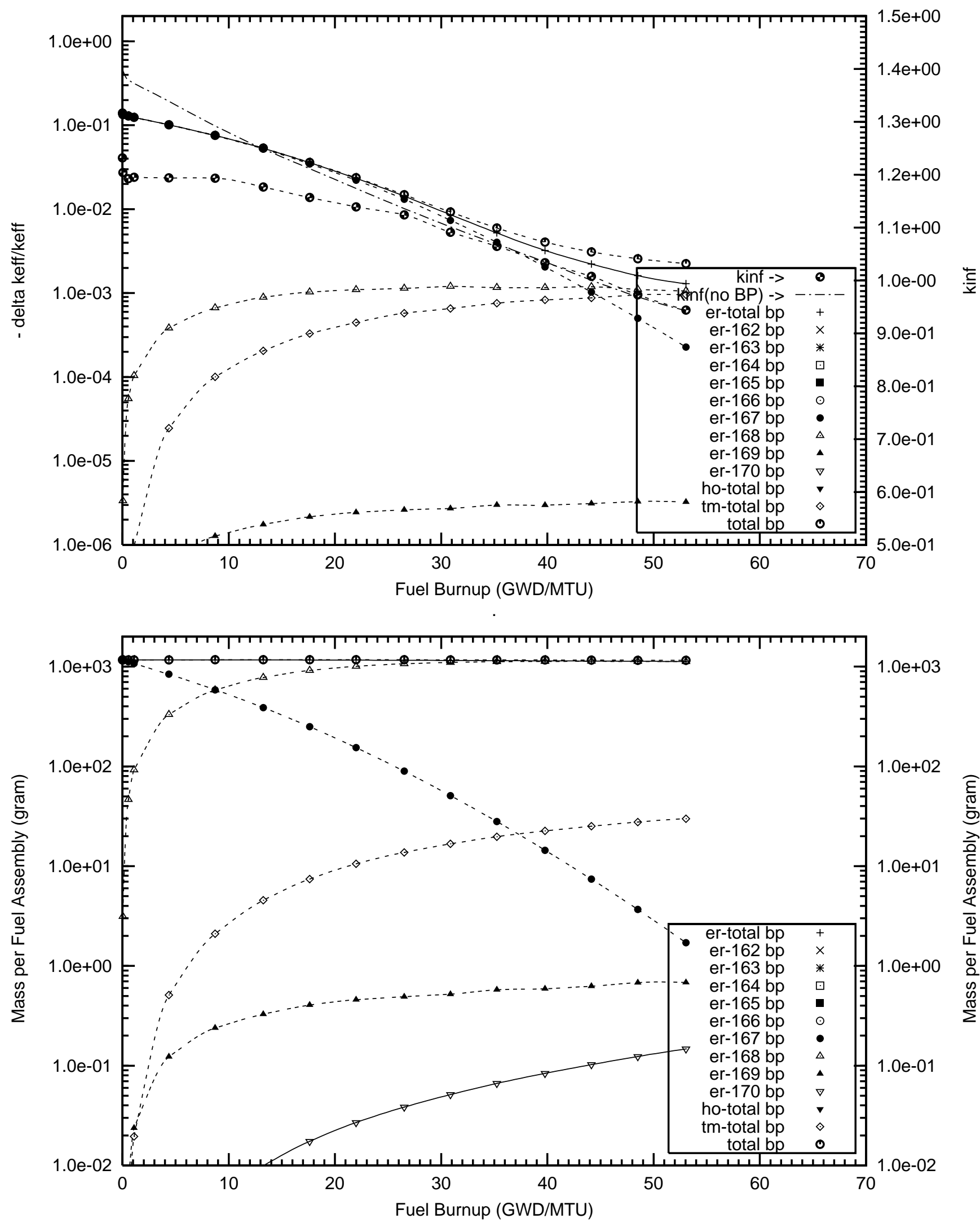


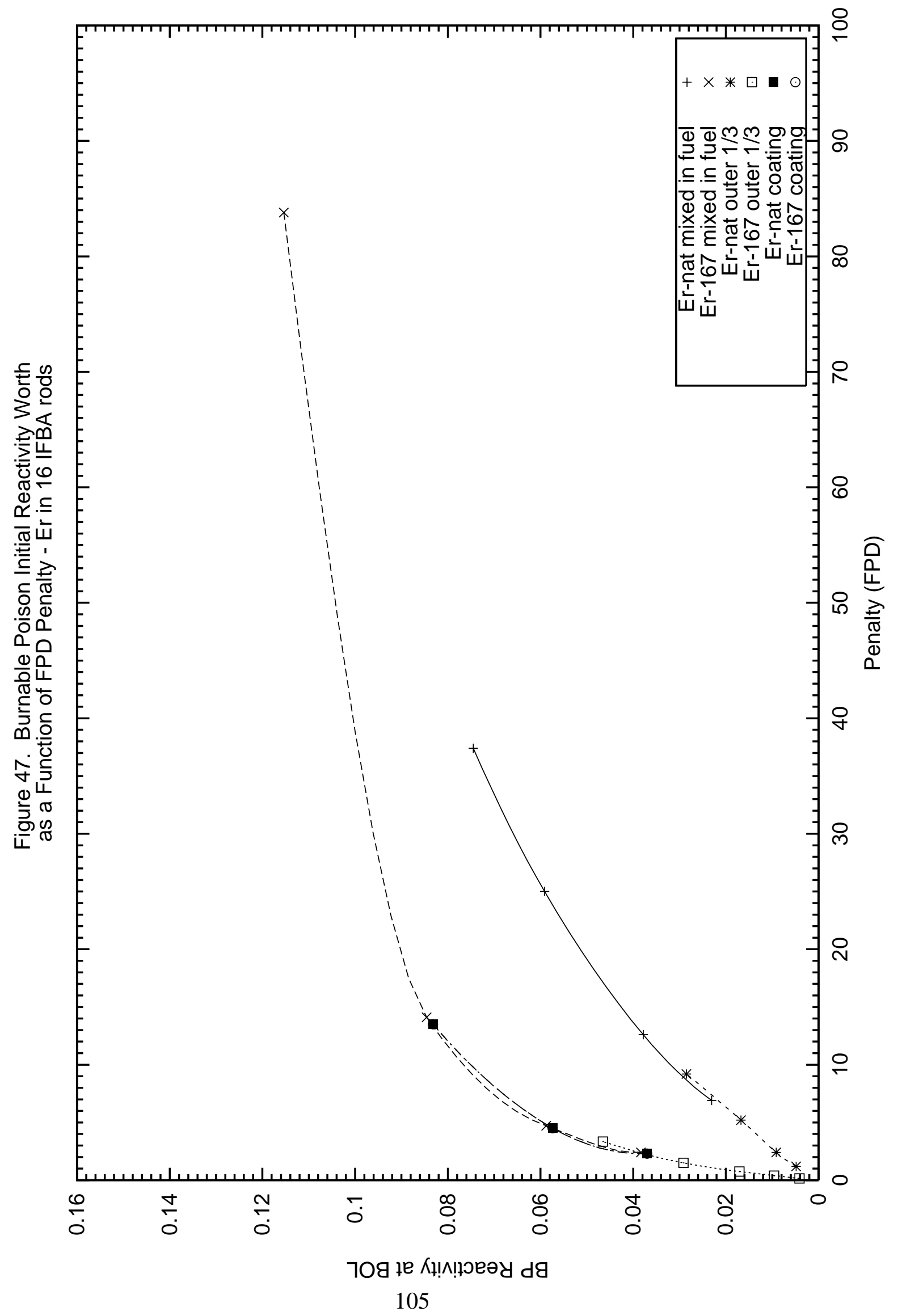




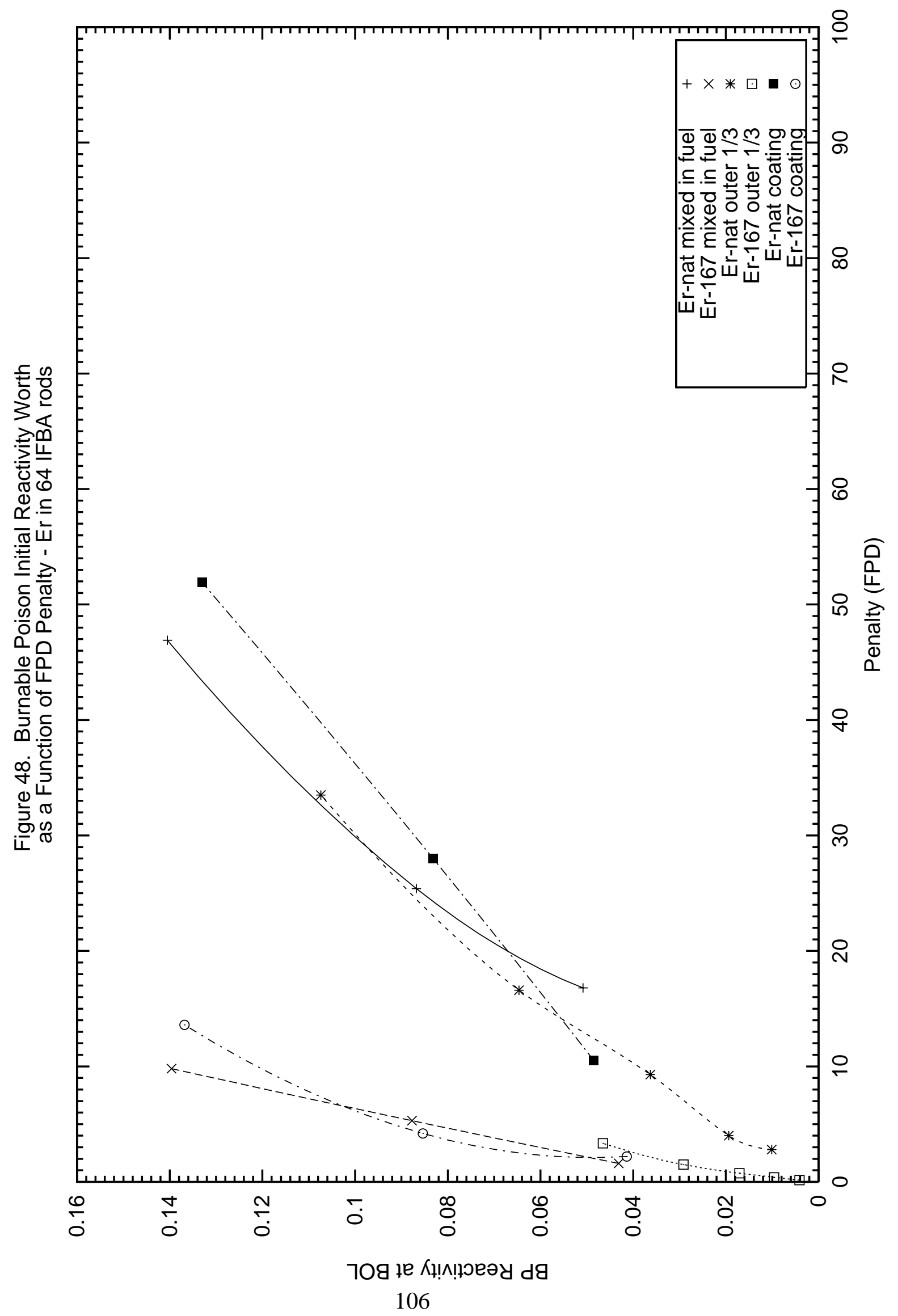




\section{NEUTRONICS PERFORMANCE RESULTS FOR DYSPROSIUM}

In the proposed use of the CANDU reactor for burning ex-weapons plutonium as MOX, depleted uranium is used as the matrix material throughout the fuel bundle (ref 1). In the central fuel rod and in the next ring of fuel rods, dysprosium burnable poison is mixed with the depleted uranium of those fuel rods not containing plutonium. This increases the amount of plutonium required to achieve a given burnup (and thus increases the Pu disposition rate), reduces the local peak power after refueling, and reduces the local positive MVC in the middle of the fuel bundle (and thus the dysprosium makes the overall bundle MVC more negative). In this case the residual reactivity worth should remain high throughout the life of the fuel.

In this section, we will show that the use of enriched dysprosium (fully enriched in ${ }^{164} \mathrm{Dy}$ ) will improve the performance of dysprosium-based BPs in PWRs. The residual reactivity worth ratio for BPs containing dysprosium with natural isotopic abundances is high, between 30 and $40 \%$, depending on the dysprosium initial loading and the number of IFBA rods per fuel assembly. The use of dysprosium fully enriched in ${ }^{164}$ Dy reduces the residual reactivity worth ratio of the BPs to between 8 and $18 \%$.

Four different configurations of the dysprosium burnable poison, similar to those of the cases of $\mathrm{Gd}$, were investigated.

1. A homogeneous mixture of the burnable poison dysprosia $\left(\mathrm{Dy}_{2} \mathrm{O}_{3}\right)$ inside the fuel pellets of a limited number of fuel rods. A summary of the results is given in Table 17. The plots of the reactivity worths and the masses of the $\mathrm{BP}$ isotopes and their daughters are displayed in Appendix D1.

2. A homogeneous mixture of the burnable poison as an oxide (dysprosia) in the outer radial onethird volume of the fuel pellets. A summary of the results is given in Table 18. Plots of the reactivity worths and the masses of the BP isotopes and their daughters are displayed in Appendix D2.

3. An oxide burnable poison coating (dysprosia) on the surface of the fuel pellets in a limited number of fuel rods, similar to the $\mathrm{ZrB}_{2}$ coatings. A summary of the results is given in Table 19. Plots of the reactivity worths and the masses of the BP isotopes and their daughters are displayed in Appendix D3.

4. A homogeneous mixture of the burnable poison dysprosium in the form of a metal alloyed with the Zircaloy cladding of a number of fuel rods. The summary results are given in Table 20. Plots of the reactivity worths and the masses of the BP isotopes and their daughters are displayed in Appendix D4.

In all the neutronics calculations presented in this section, a theoretical density of $7.81 \mathrm{~g} / \mathrm{cm}^{3}$ was used for $\mathrm{Dy}_{2} \mathrm{O}_{3}$, and $8.56 \mathrm{~g} / \mathrm{cm}^{3}$ for metallic dysprosium. For $\mathrm{UO}_{2}$ a theoretical density of $10.97 \mathrm{~g} / \mathrm{cm}^{3}$ was used. The actual density fraction of the fuel pellets and of the burnable poison pellets was $95 \%$ of theoretical density.

The half-lives, natural isotopic abundances and burnup chains related to the depletion and transmutation of dysprosium are given below. Note that the adjacent bands of isotopes such as the Er isotopes (decay from Ho isotopes) are not shown. Also, the isomeric states of certain isotopes are not shown. However, the generation of these daughters was taken into account in the BP depletion and decay calculations. 


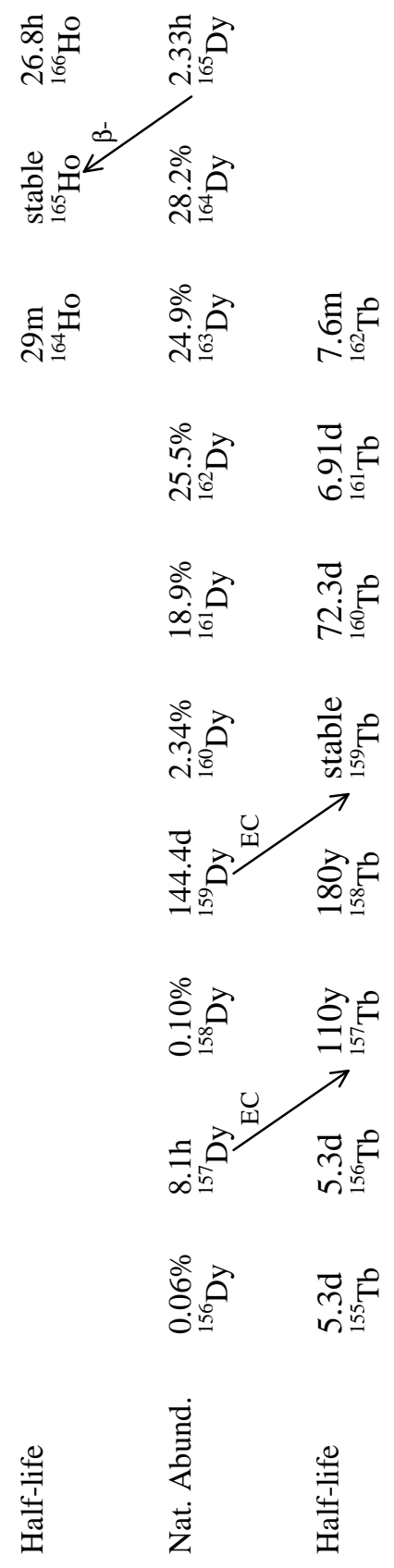


Figure 49 displays the capture cross sections for the dysprosium isotopes. Figure 50 displays the capture cross sections of several of the holmium decay daughters from dysprosium. The generation of $\mathrm{Tb}$ isotopes is essentially zero, since the natural isotopic abundances of ${ }^{156} \mathrm{Dy}(0.06 \%)$ and ${ }^{158} \mathrm{Dy}$ $(0.1 \%)$ are very small.

The stable isotopes ${ }^{156}$ Dy $(0.06 \%$ natural isotopic abundance, thermal cross section 33 barns, RI 884 barns), ${ }^{158}$ Dy (0.1\%, thermal 43 barns, RI 120 barns), ${ }^{160}$ Dy (2.3\%, thermal 56 barns, RI 1,160 barns), ${ }^{162}$ Dy (25.5\%, thermal 194 barns, RI 2,755 barns) and ${ }^{163} \mathrm{Dy}(24.9 \%$, thermal 124 barns, RI 1,470 barns) exhibit medium-sized capture cross sections, while ${ }^{161}$ Dy $(18.9 \%$, thermal 600 barns, RI 1,200 barns) and ${ }^{164}$ Dy (28.1\%, thermal 2,650 barns, RI 340 barns) have large capture cross sections (see Figure 49). Since the majority of the stable dysprosium isotopes $\left({ }^{160}\right.$ Dy through ${ }^{164} \mathrm{Dy}$ ) are adjacent to each other (in the chart of the nuclides), and since they have medium to large capture cross sections, the residual reactivity ratio of BPs containing dysprosium with natural isotopic abundances will be high. Also, the ${ }^{164} \mathrm{Dy}(\mathrm{n}, \gamma){ }^{165}$ Dy reaction leads to ${ }^{165} \mathrm{Dy}$, which decays to ${ }^{165} \mathrm{Ho}$ with a half-life of 2.33 hours. The thermal capture cross section of ${ }^{165} \mathrm{Ho}$ is approximately 65 barns, and the RI is 670 barns. Dysprosium with natural isotopic abundances is thus attractive if the burnout of the BP has to be minimized (such as for use in the Pu-burner CANDU). Since the thermal capture cross section of ${ }^{164} \mathrm{Dy}$ is much larger than that for ${ }^{165} \mathrm{Ho}$, enriching dysprosium in ${ }^{164} \mathrm{Dy}$ will thus reduce the residual reactivity ratio of the $\mathrm{BP}$.

Table 17 displays the results of using $\mathrm{Dy}_{2} \mathrm{O}_{3}$, mixed homogeneously with the fuel pellets of a number of fuel rods. For the same initial reactivity worth of the BP, the residual negative reactivity worth ratio of dysprosium, fully enriched in ${ }^{164} \mathrm{Dy}$, is more than two times smaller than the residual worth of dysprosium with natural isotopic abundances. The relative contribution to the residual reactivity ratio from ${ }^{165} \mathrm{Ho}$ is higher for BP containing enriched ${ }^{164} \mathrm{Dy}$.

The reactivity worths of the $\mathrm{BP}$ isotopes, for a $4.0 \mathrm{wt} \%$ loading of $\mathrm{Dy}_{2} \mathrm{O}_{3}$ with natural isotopic abundances, homogeneously mixed in the fuel pellets of 16 IFBA rods per fuel assembly, are shown in Figure 51. The initial reactivity worth of the BP is $6.08 \times 10^{-2}$ and the residual reactivity ratio is $38.5 \%$. Figure 51 also displays the masses of the burnable poison isotopes and their daughters. The residual reactivity worth of the $\mathrm{Tb}$ daughters is essentially zero, since the natural isotopic abundances of ${ }^{156}$ Dy and ${ }^{158}$ Dy are very small, $0.06 \%$ and $0.10 \%$, respectively.

For a 4.0 wt $\%$ homogeneous loading of $\mathrm{Dy}_{2} \mathrm{O}_{3}$ (fully enriched in ${ }^{164} \mathrm{Dy}$ ) in 16 IFBA rods, the results of the reactivity worths are shown in Figure 52 . The initial reactivity worth of the BP is $6.75 \times 10^{-2}$ and the residual reactivity ratio is $18.0 \%$. The total loading of ${ }^{164} \mathrm{Dy}$ per fuel assembly is 1,150 grams of ${ }^{164} \mathrm{Dy}$. Most of the residual reactivity burden of the enriched dysprosium BP comes from the buildup of ${ }^{165}$ Ho. Figure 52 also displays the masses of the burnable poison isotopes. The use of dysprosium as a BP, enriched in ${ }^{164} \mathrm{Dy}$ instead of with natural isotopic abundances, thus leads to a reduction of the residual reactivity burden at EOL of the fuel. Figure 53 displays the results of the reactivity worths for a $1.0 \mathrm{wt} \%$ loading of enriched $\mathrm{Dy}_{2} \mathrm{O}_{3}$ in 64 IFBA rods, and figure 53 also shows the masses of the burnable poison isotopes and daughters. The total loading of ${ }^{164} \mathrm{Dy}$ per fuel assembly is 1,163 grams of ${ }^{164} \mathrm{Dy}$, which is essentially the same as for the $4.0 \mathrm{wt} \%$ loading in 16 IFBA rods. The initial reactivity worth of the BP is 0.109 , which is larger, and the residual reactivity ratio is $10.0 \%$, instead of $18 \%$. Comparison between the fuel assembly lattice $\mathrm{k}_{\text {inf }}$ 's as a function of depletion time, for the cases represented in Figures 52 and 53, shows that the shape of the fuel assembly lattice $\mathrm{k}_{\mathrm{inf}}$ ' $\mathrm{s}$ is flatter for the case with 64 IFBA rods. The BP burnout rate is slower, which might be useful in applications requiring longer fuel cycles. 
Figure 54 displays the reactivity worths for an oxide burnable poison coating of 0.5 mils of dysprosia on the surface of the fuel pellets in 104 IFBA rods. Note that the BP burnout time is fairly long. The BP masses are also shown in Figure 54.

In Tables 17 through 20 the residual reactivity worths, due to the Tb daughters of the BP containing dysprosium enriched in ${ }^{164}$ Dy, should be zero, since there are essentially no paths from the transmutation of ${ }^{164} \mathrm{Dy}$ to any of the Tb isotopes. However, the tables show residual reactivity worths of the order of $1.0 \times 10^{-11}$. This is because for each case, all the BP isotopes and their daughters (which are not present in the initial loading of the BP) have been added as traces to the BP with a number density of $1.0 \times 10^{-15}$ instead of zero. This is to avoid problems at the BOC in the input to the MCNP4C code and to avoid computer exception errors (division by zero) when calculating spectrumweighted cross sections.

The MVCs were calculated by decreasing the water coolant density by $10 \%$. Note that for all the cases containing 8, 16, and 64 IFBA rods, the MVCs remain negative.

Figure 55 displays the initial reactivity worths of the BP containing dysprosium as a function of the residual BP penalty in FPDs for several configurations containing 64 IFBA rods. The trends for the different forms of the BPs are very similar. Note also the advantage of using dysprosium enriched in ${ }^{164}$ Dy over dysprosium with natural isotopic abundances. 


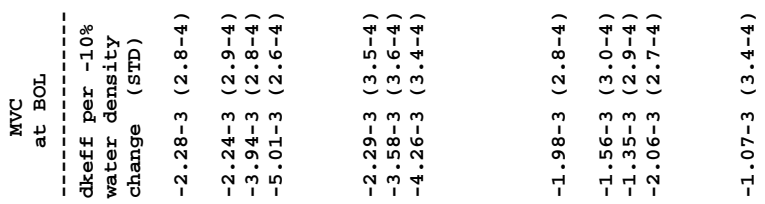

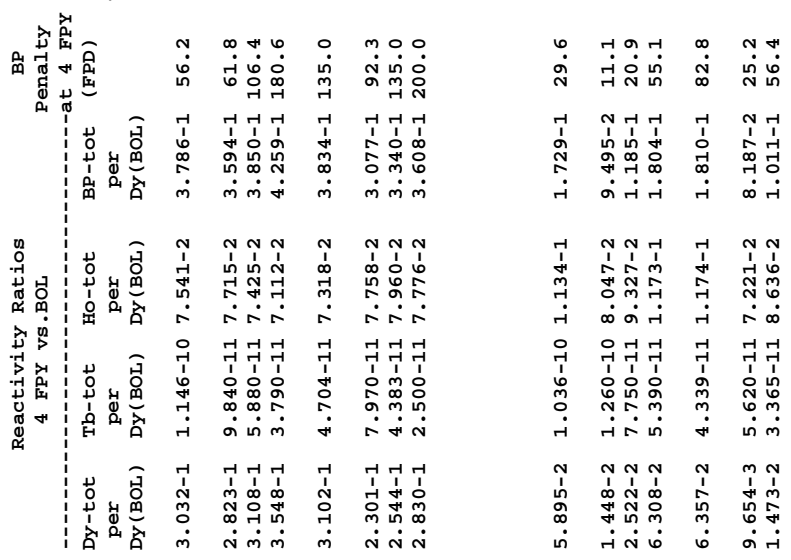

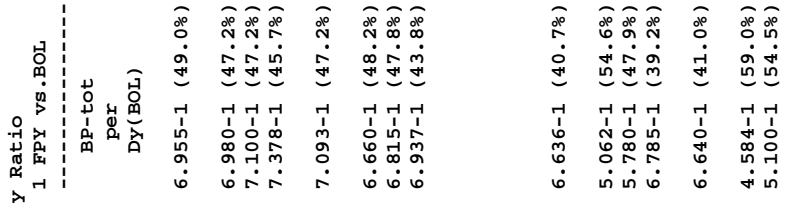

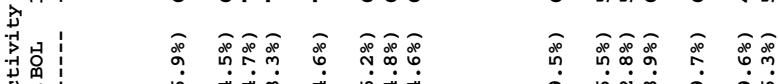

च

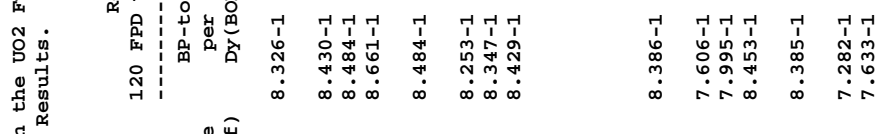

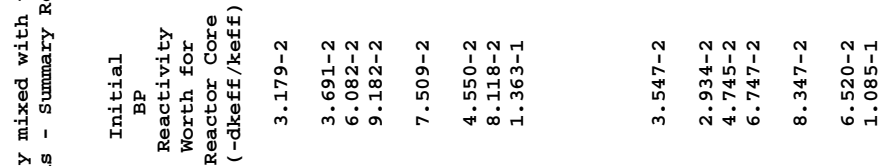

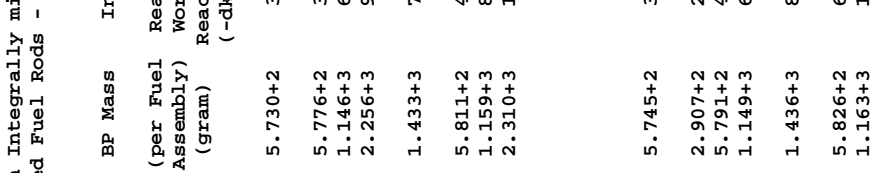

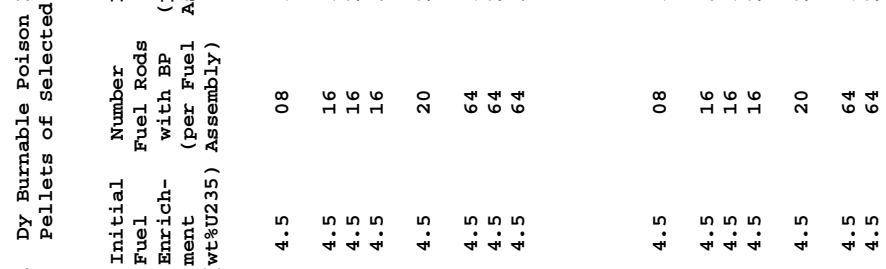

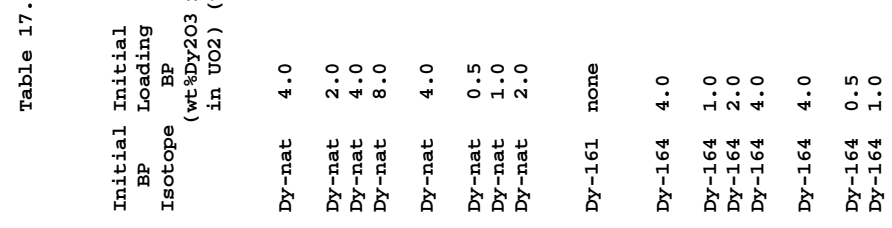




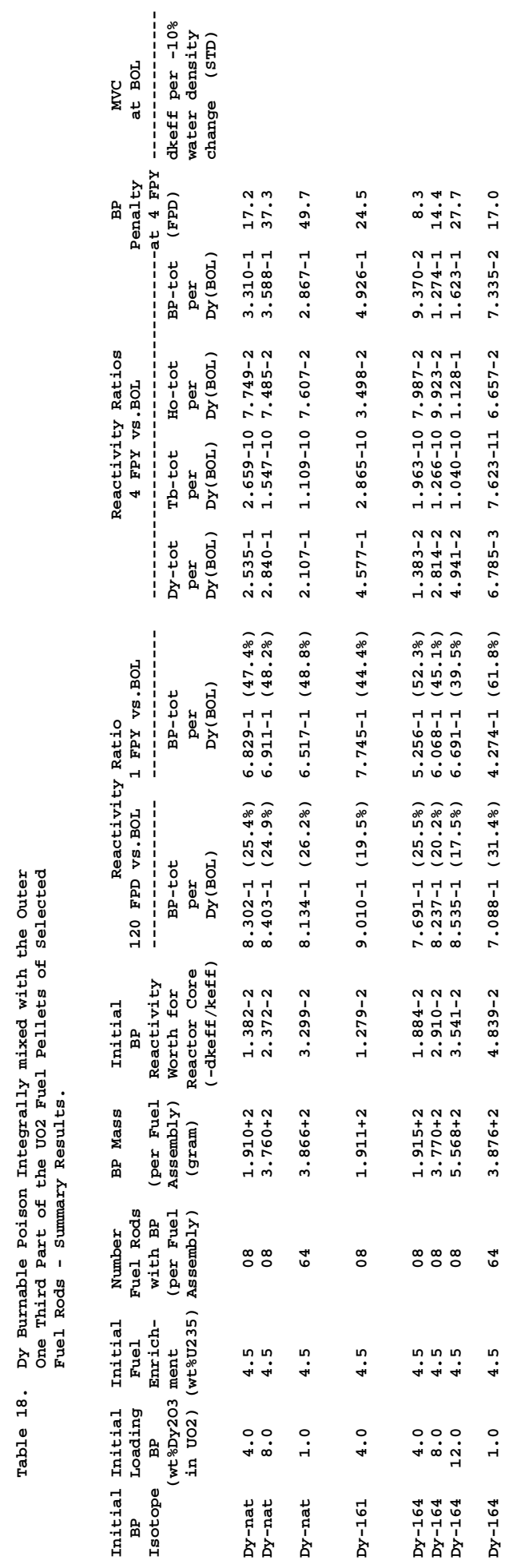




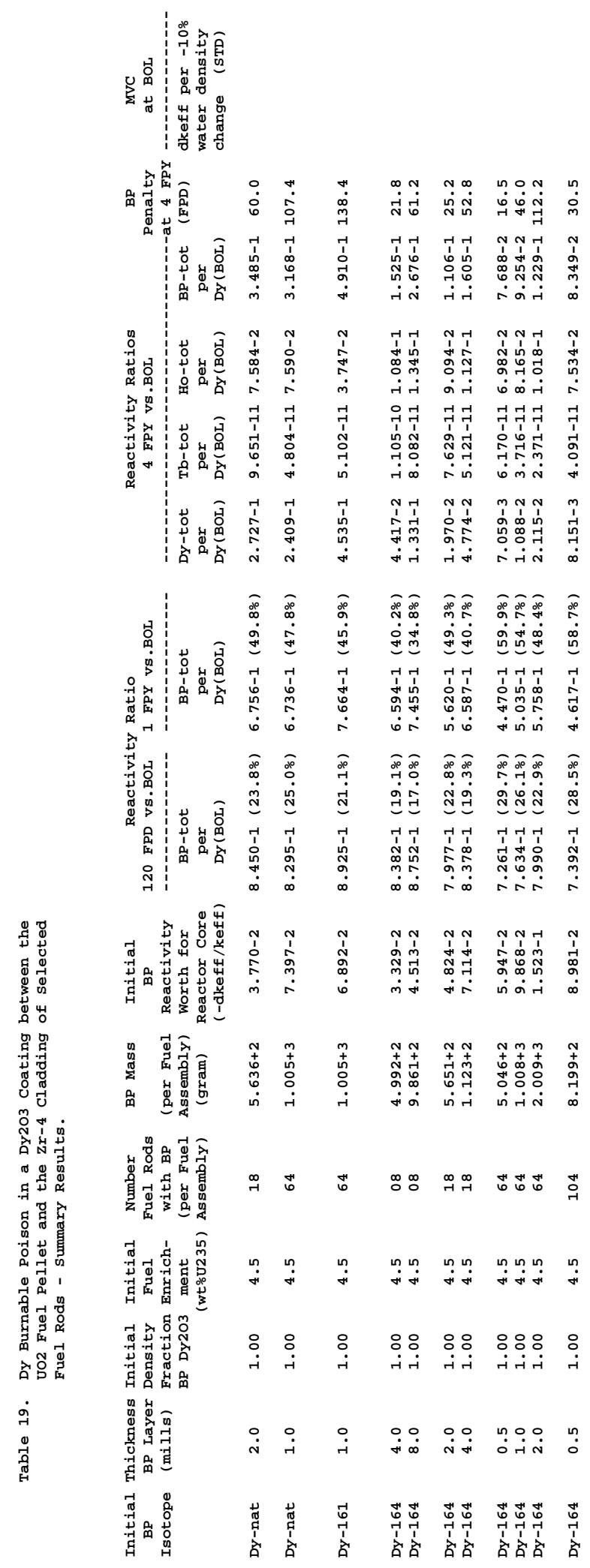




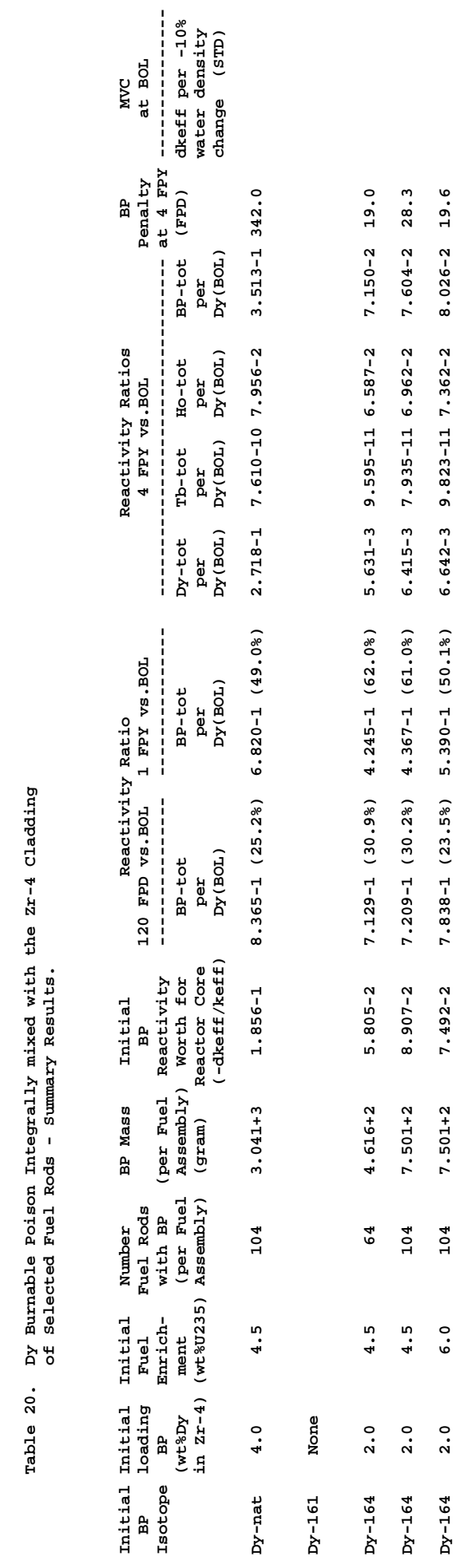


Figure 49. Neutron Cross-Sections as a Function of Energy

Dy-156 (66156.40c)

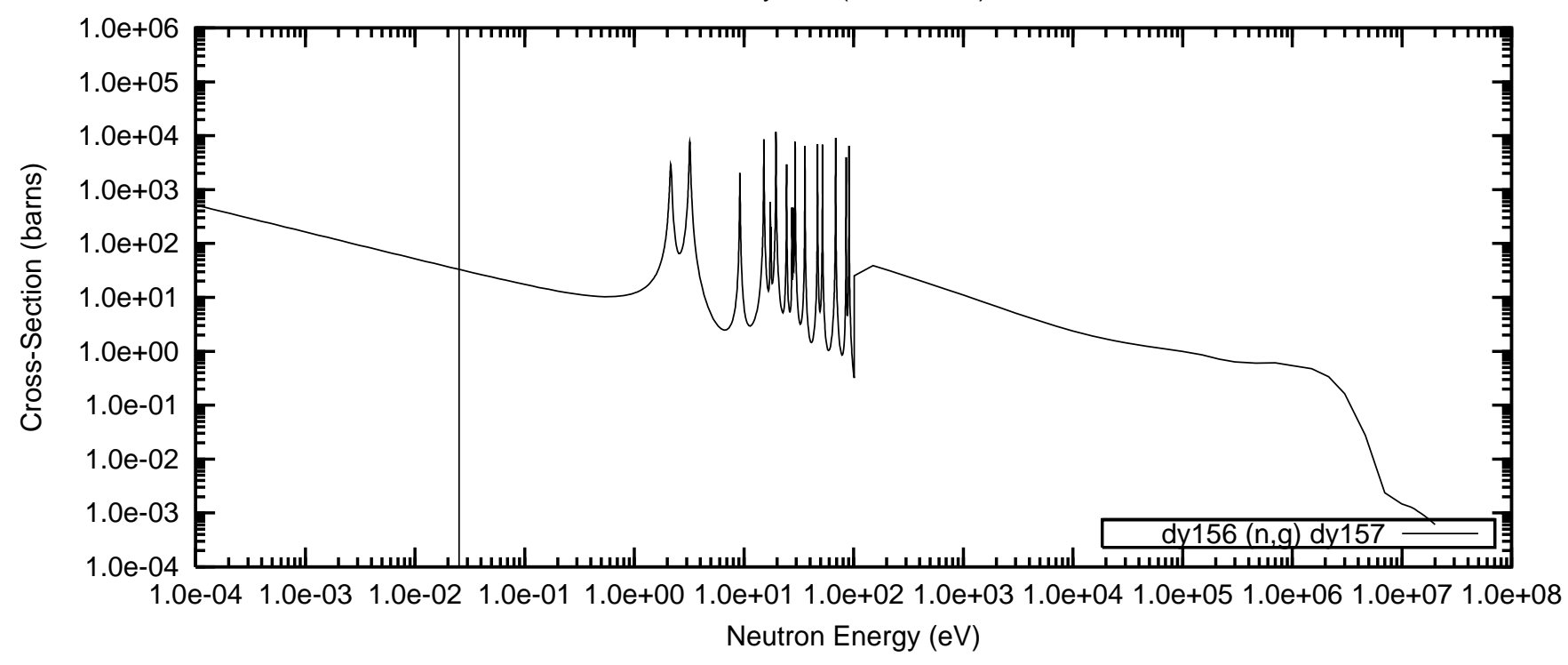

Dy-157 (66157.40c)

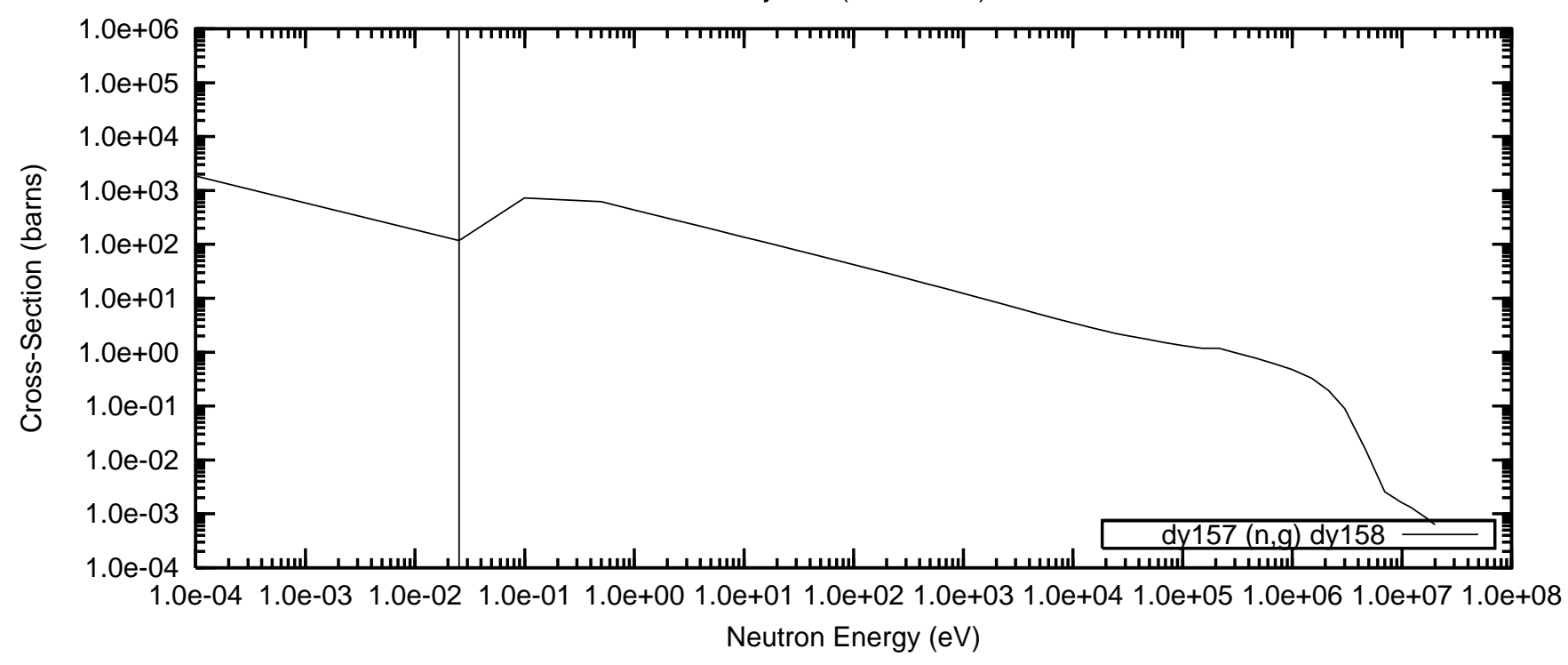

Dy-158 (66158.40c)

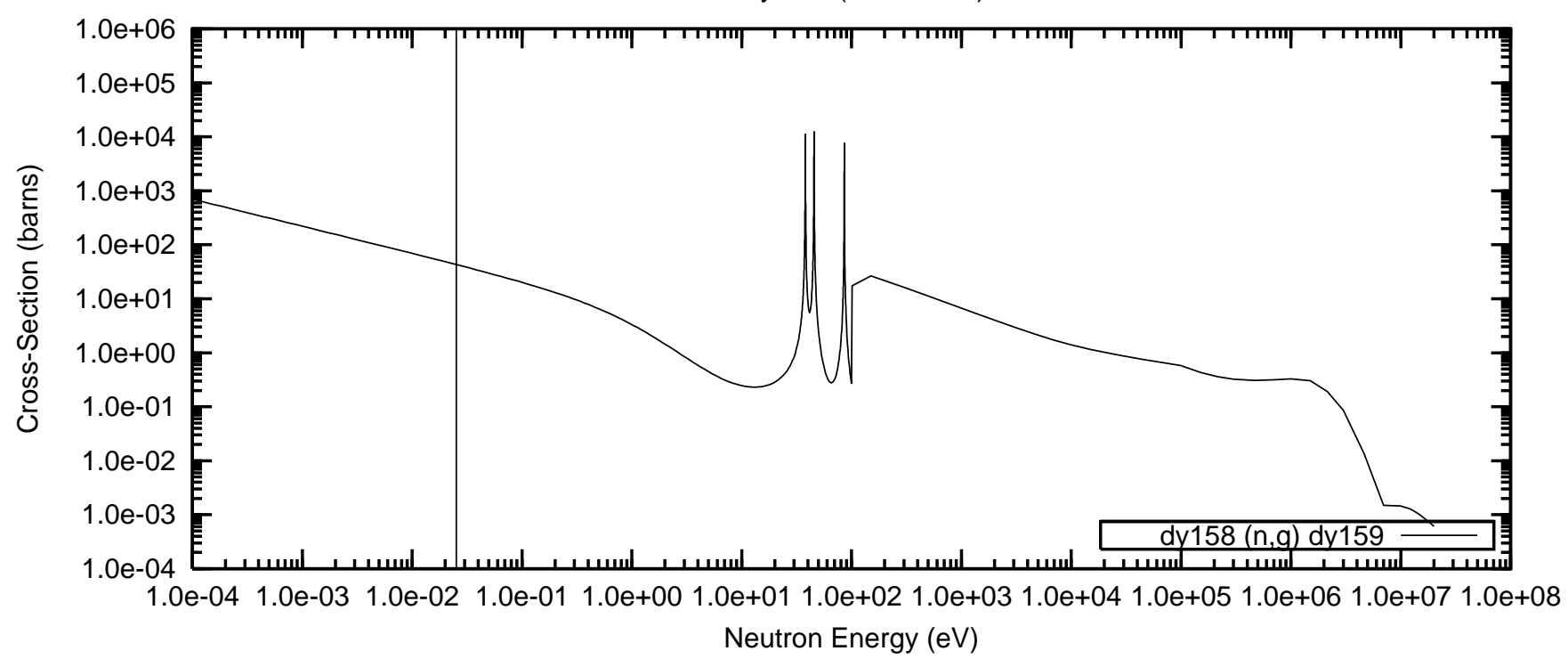


Figure 49(cont). Neutron Cross-Sections as a Function of Energy

Dy-159 (66159.40c)

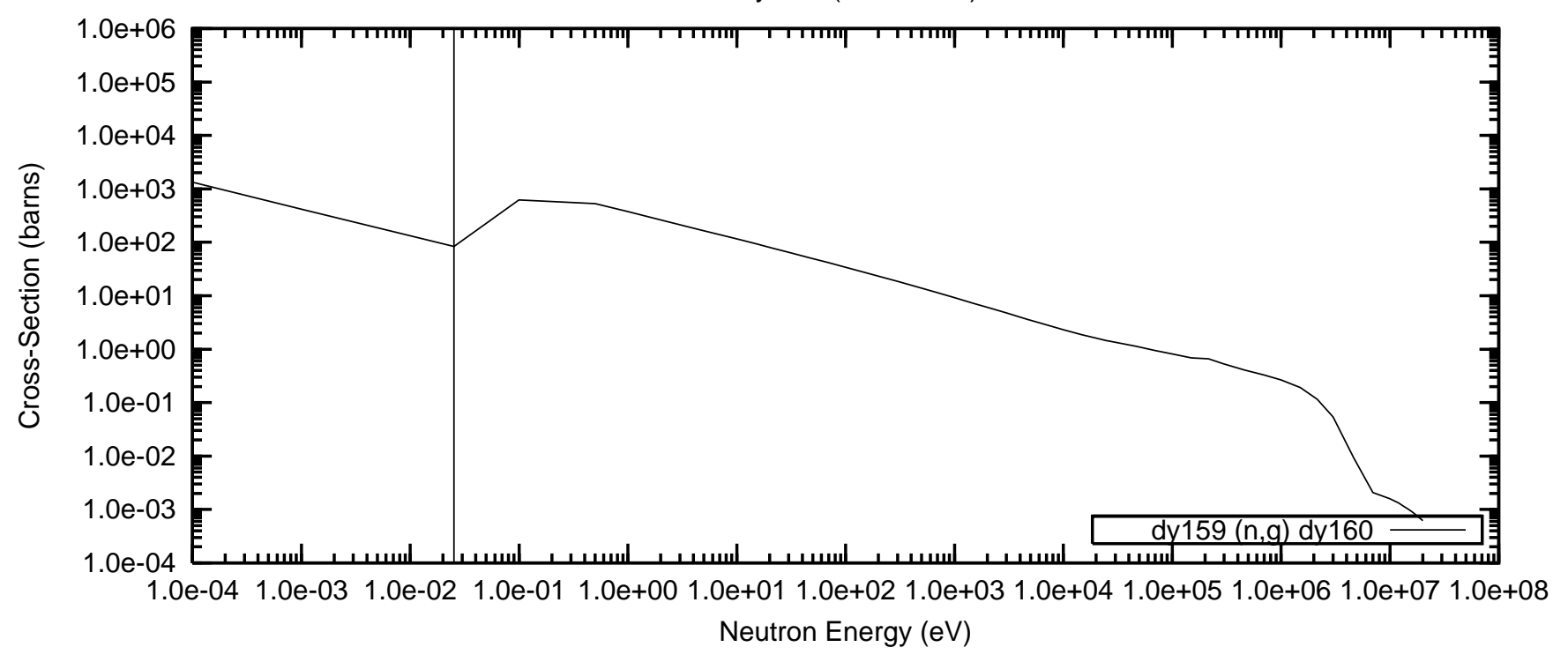

Dy-160 (66160.40c)

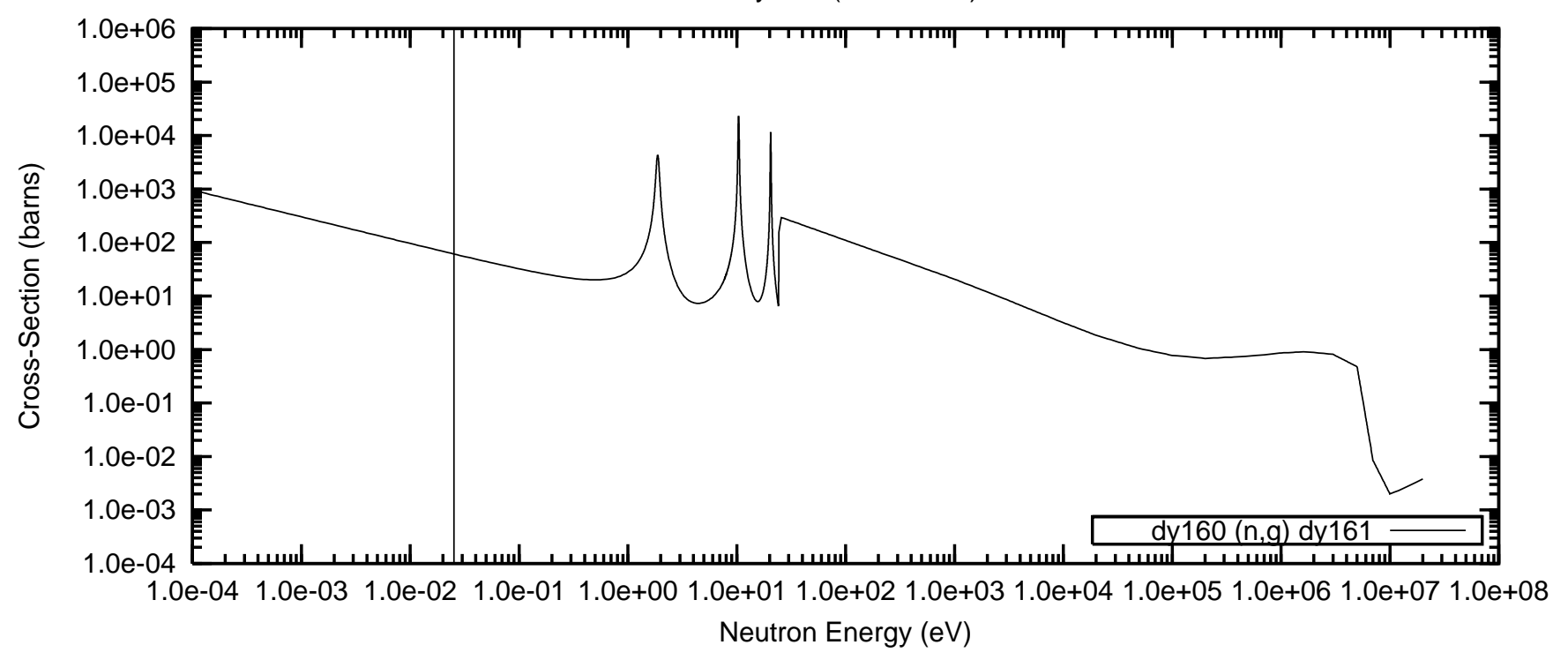

Dy-161 (66161.40c)

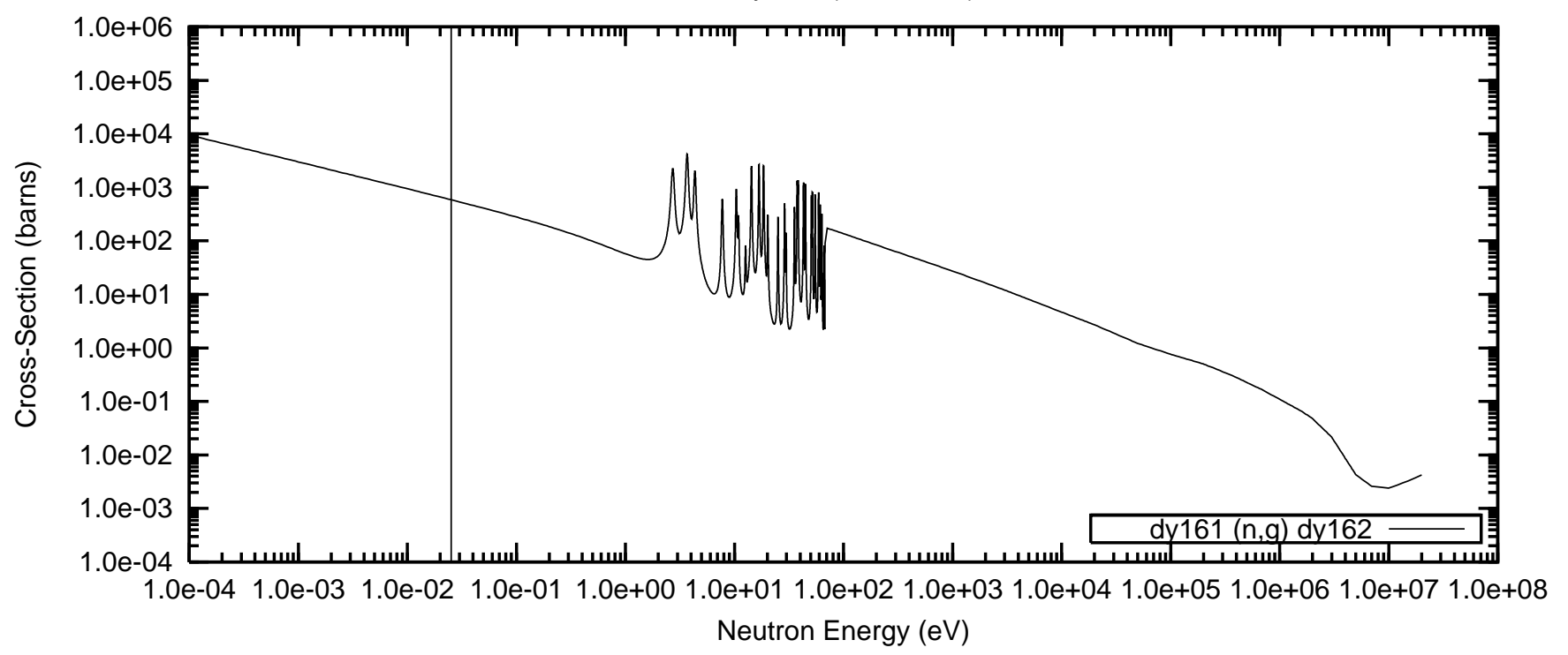


Figure 49(cont). Neutron Cross-Sections as a Function of Energy Dy-162 (66162.40c)

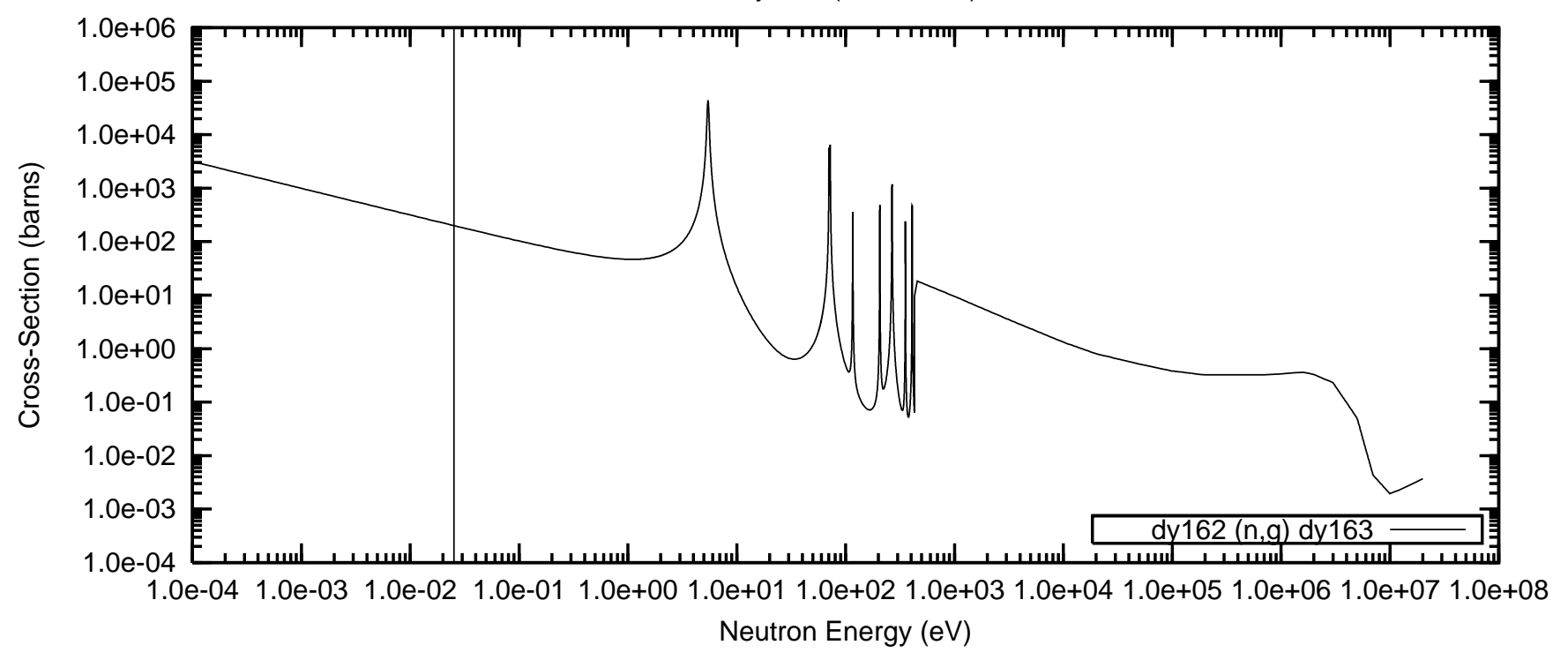

Dy-163 (66163.40c)

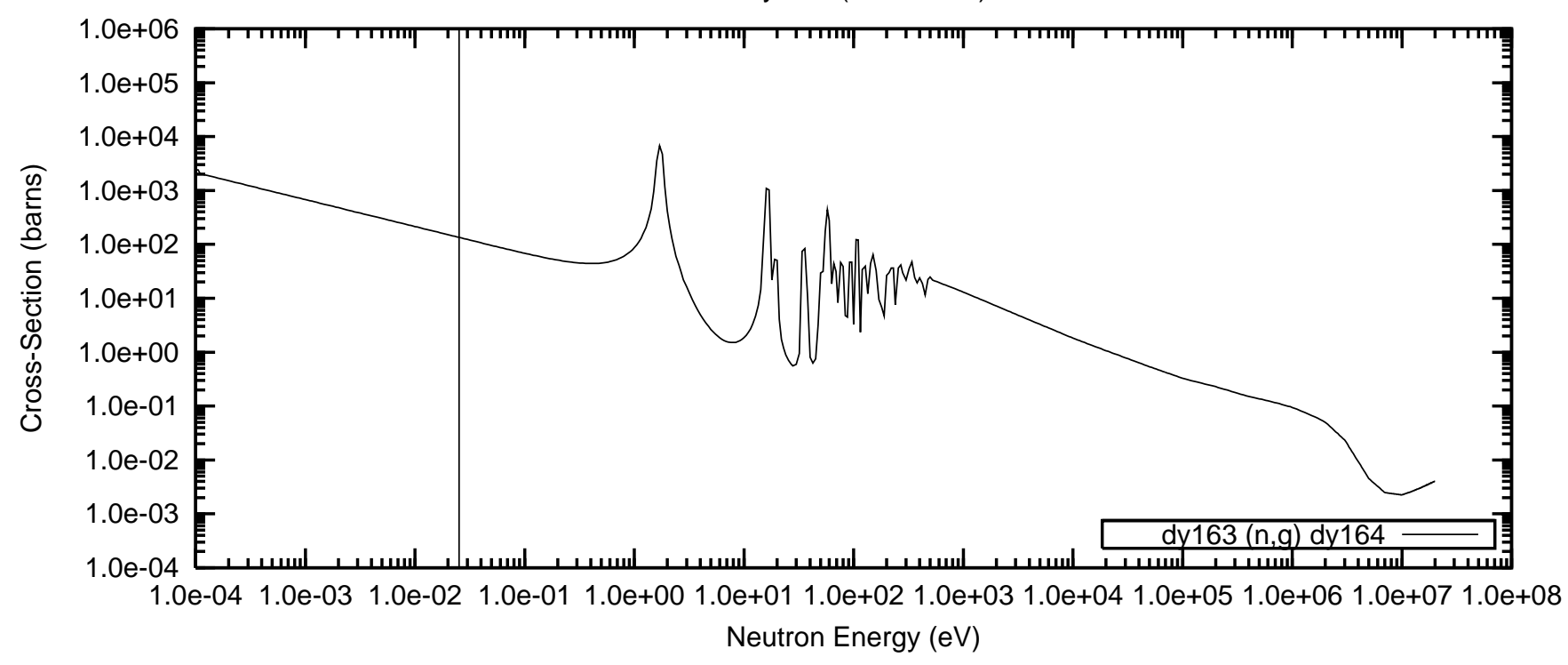

Dy-164 (66ं164.40c,46c)

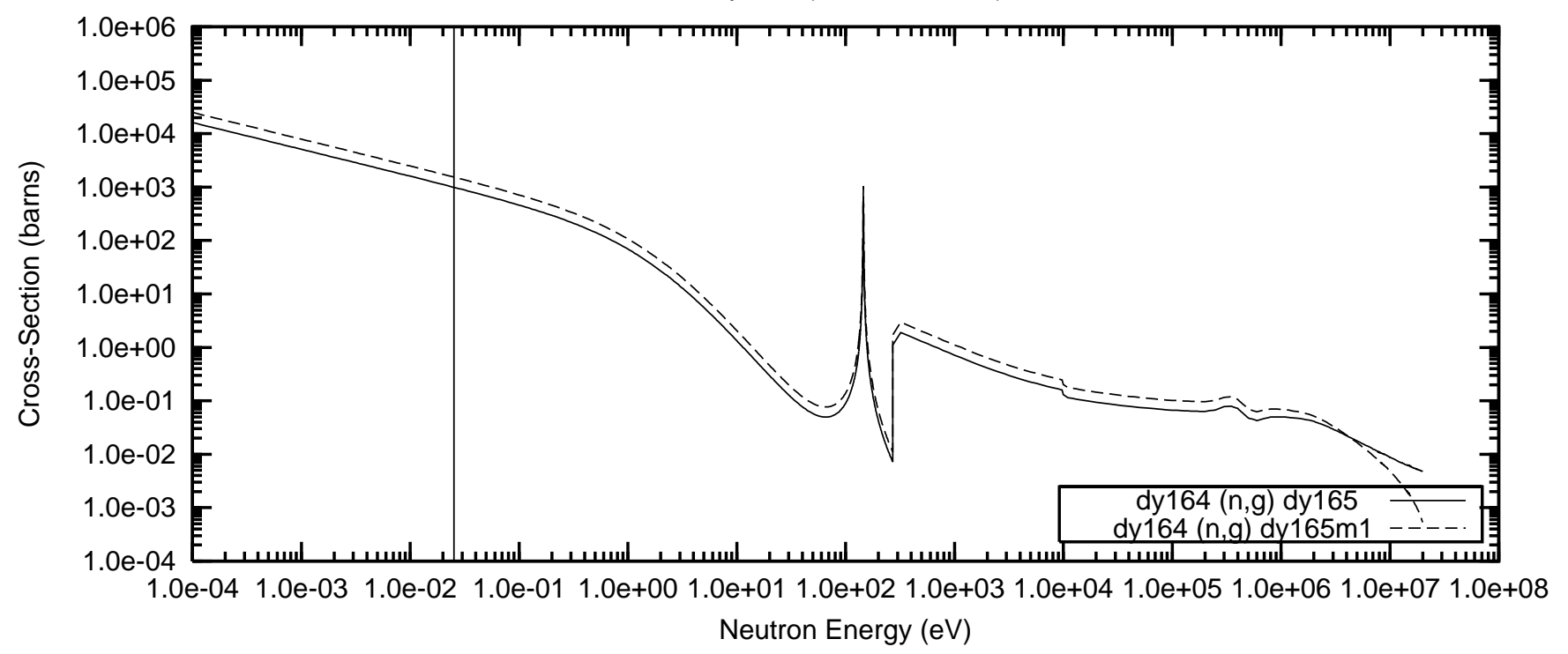


Figure 49(cont). Neutron Cross-Sections as a Function of Energy Dy-165 (66165.40c)

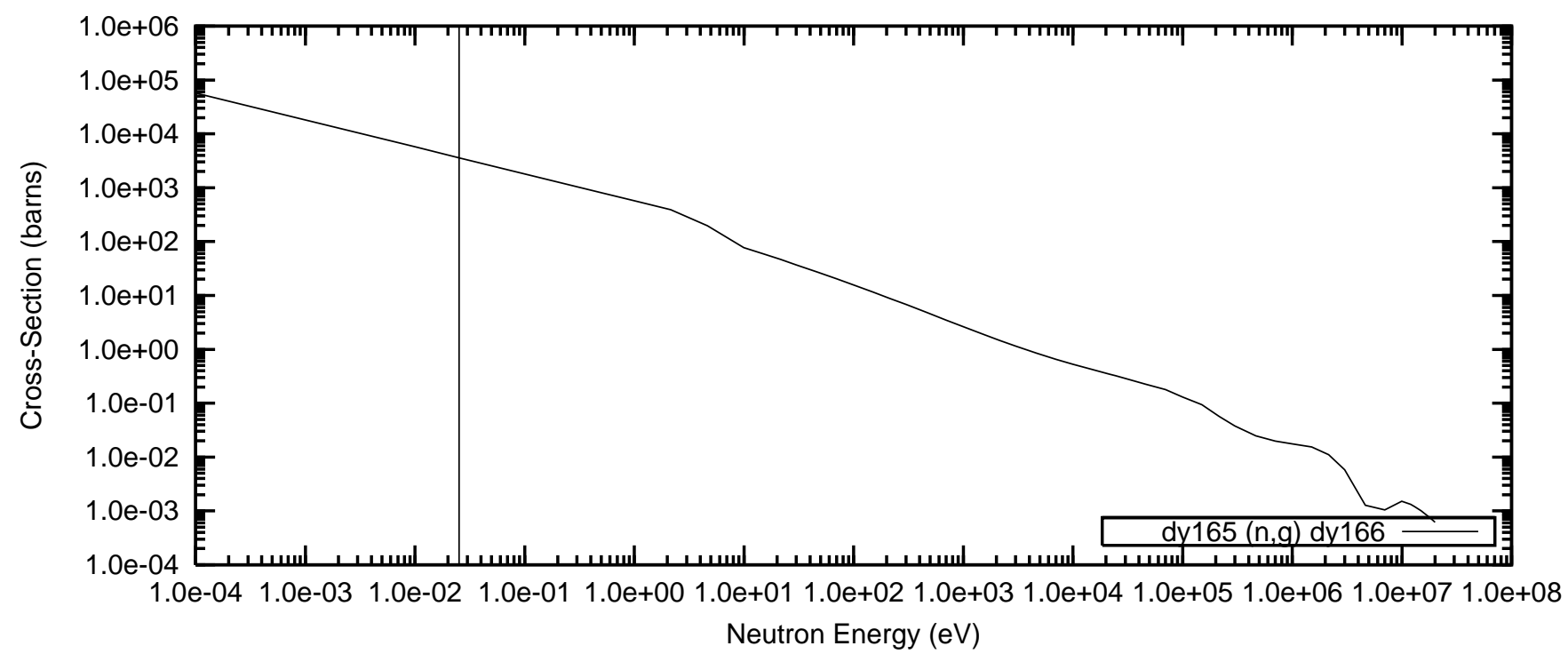


Figure 50. Neutron Cross-Sections as a Function of Energy Ho-165 (67165.40c,46c)

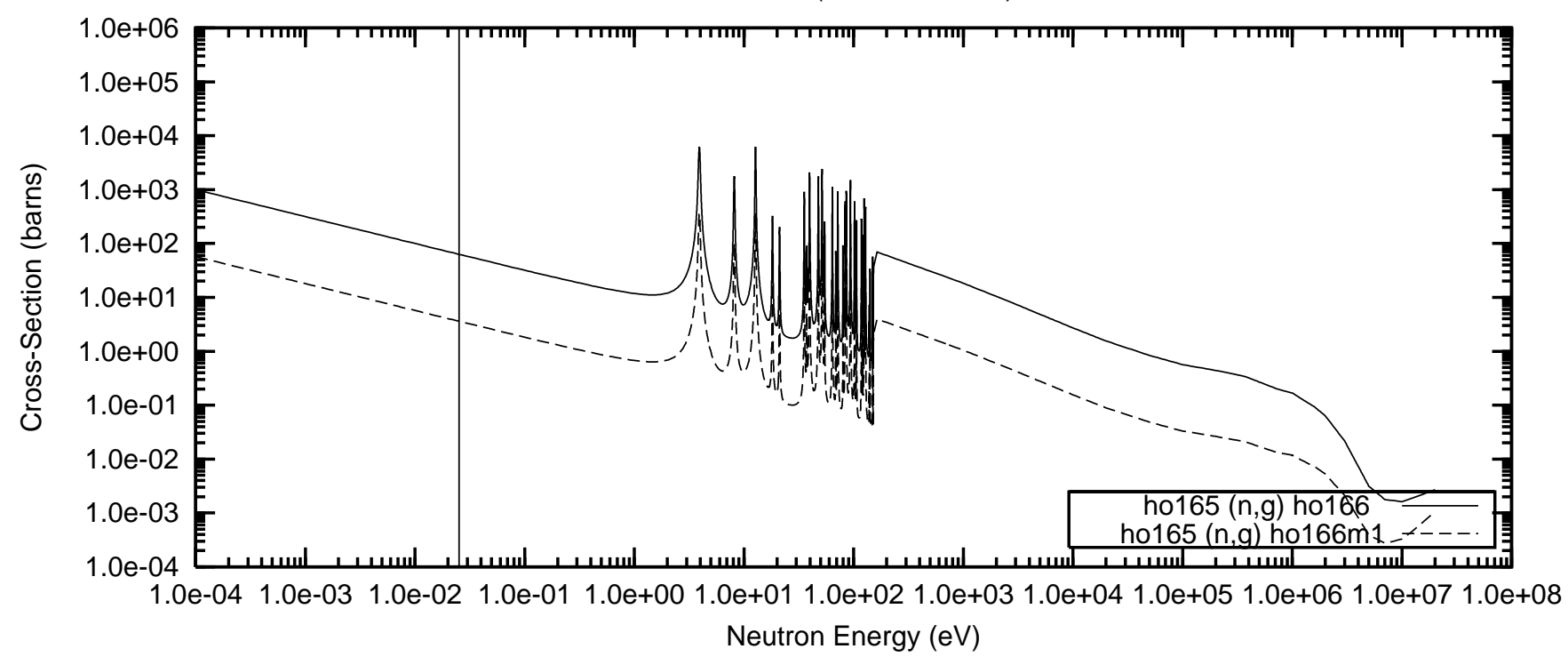

Ho-166 (67166.40c)

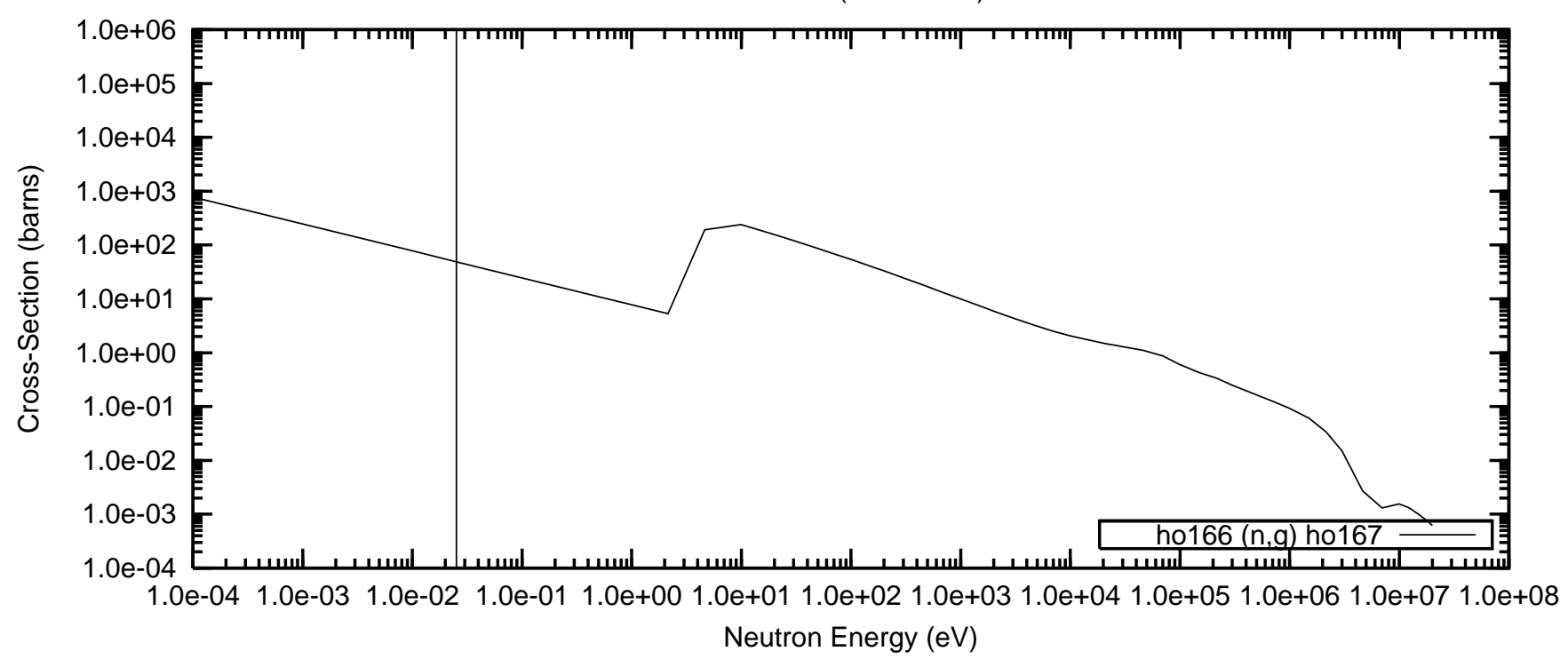

Ho-166mi (67166.41c)

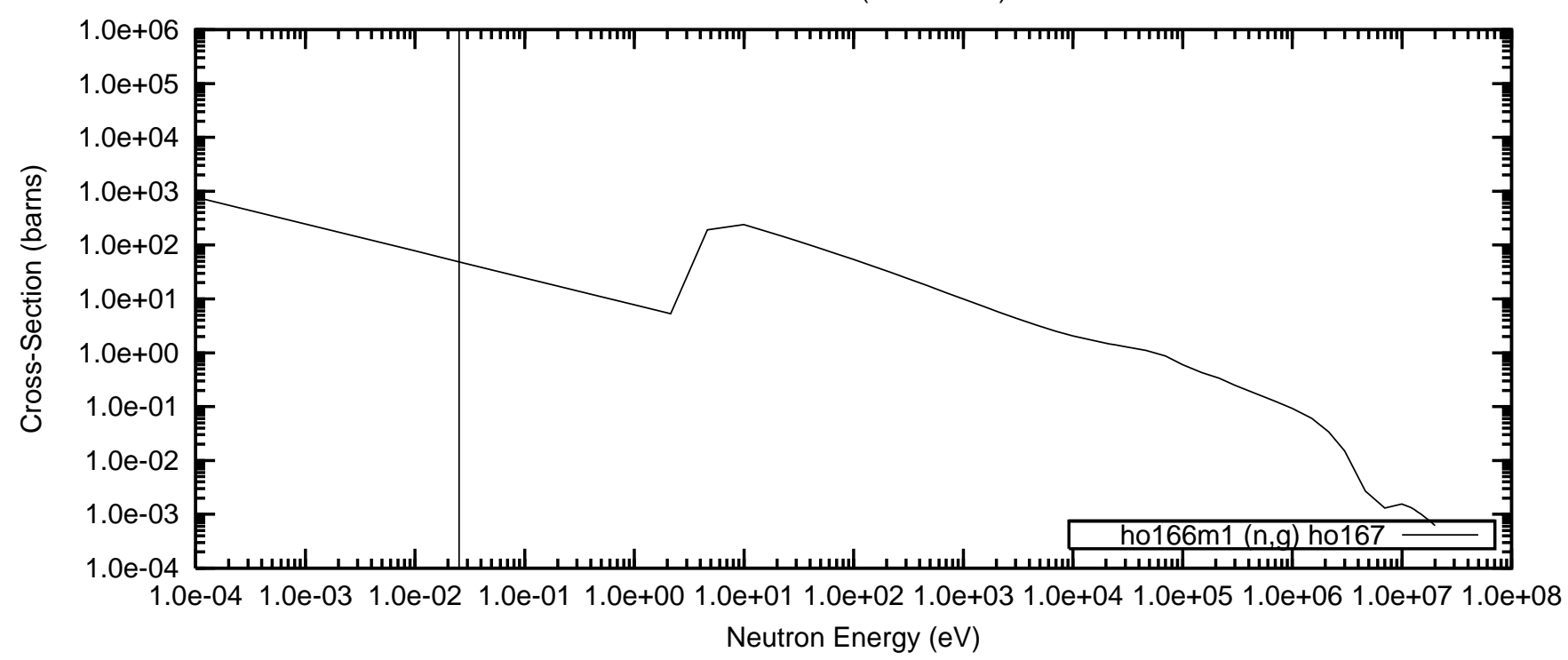


Figure 51. Negative Reactivity and Masses BP for Dy-nat and transmutation daughters as a function of fuel life for $17 \times 17$ fuel assemblies with 16 poison rods, $4.0 \mathrm{wt} \%$ Dy2O3 poison homogeneously mixed in the UO2 pellets

Reactor power $3400 \mathrm{MWth}, 193$ fuel assemblies, initial enrichment 4.5wt\%U-235

(Case : dy000_3_100p_h2o_16_bp_fuel_2_o)
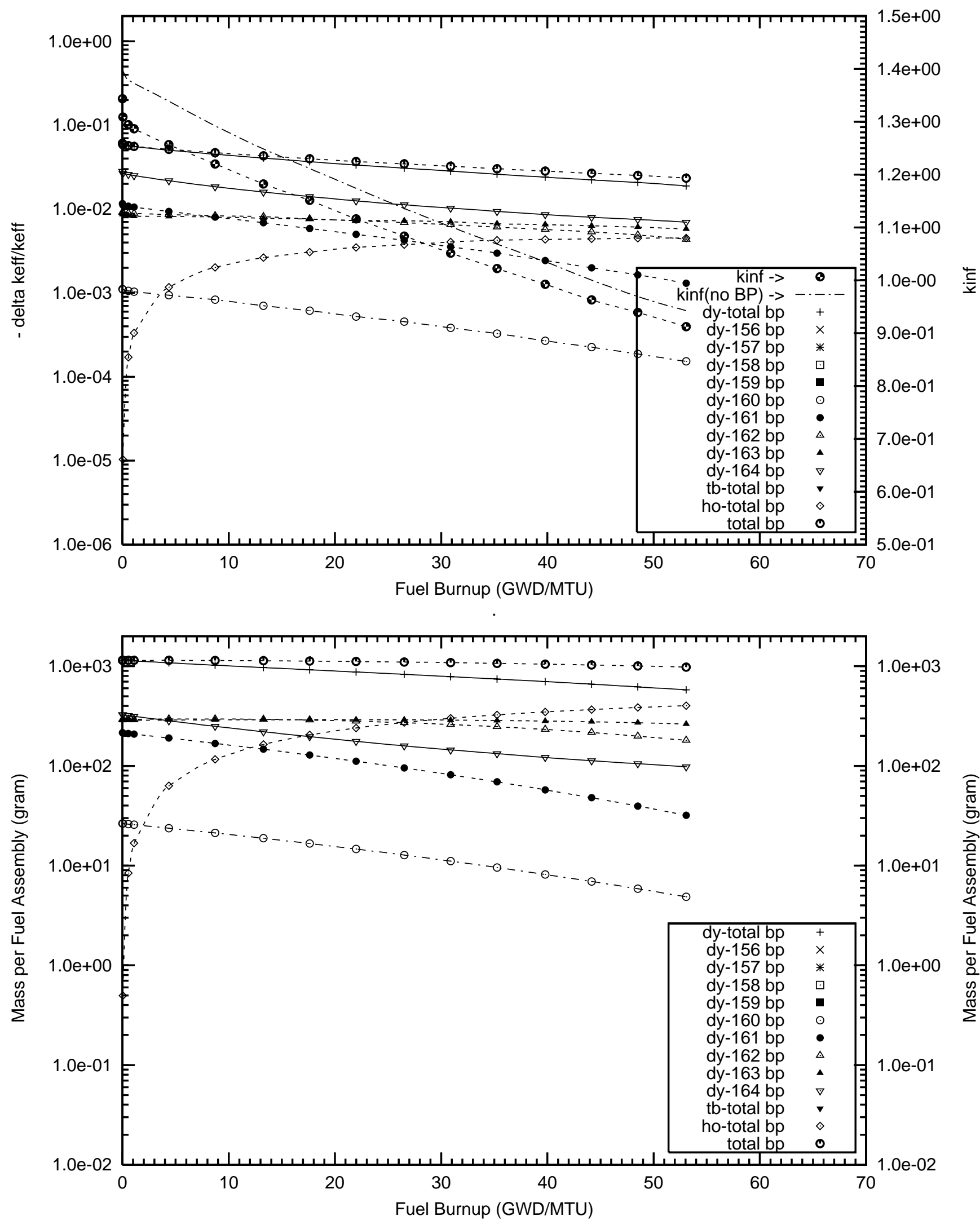
Figure 52. Negative Reactivity and Masses BP for Dy-164 and transmutation daughters as a function of fuel life for $17 \times 17$ fuel assemblies with 16 poison rods, $4.0 \mathrm{wt} \%$ Dy2O3 poison homogeneously mixed in the UO2 pellets

Reactor power $3400 \mathrm{MWth}, 193$ fuel assemblies, initial enrichment 4.5wt\%U-235

(Case : dy164_3_100p_h2o_16_bp_fuel_2_o)
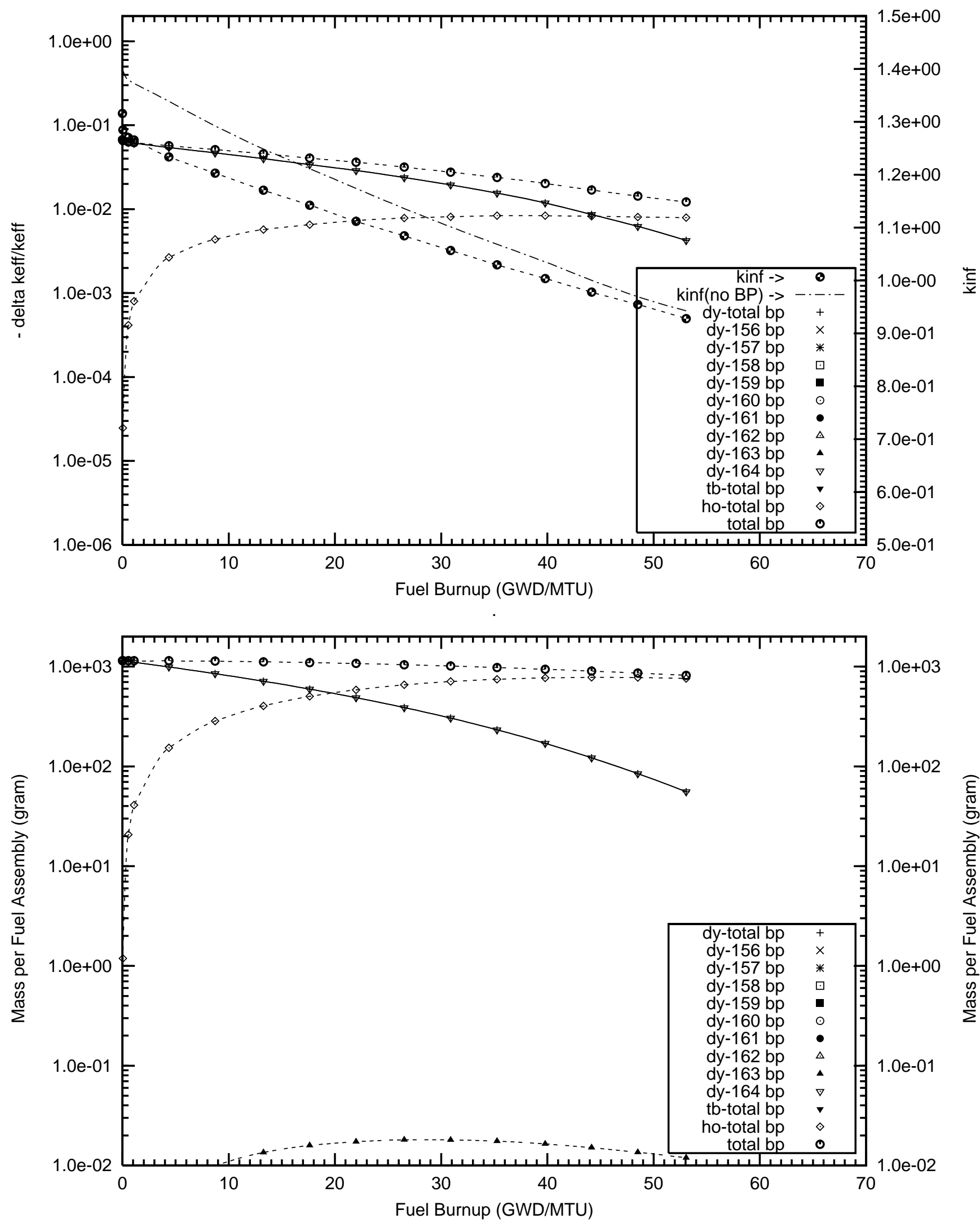
Figure 53. Negative Reactivity and Masses BP for Dy-164 and transmutation daughters as a function of fuel life for $17 \times 17$ fuel assemblies with 64 poison rods, $1.0 \mathrm{wt} \%$ Dy2O3 poison homogeneously mixed in the UO2 pellets

Reactor power 3400 MWth, 193 fuel assemblies, initial enrichment 4.5wt\%U-235

(Case : dy164_1_64_bp_fuel o)
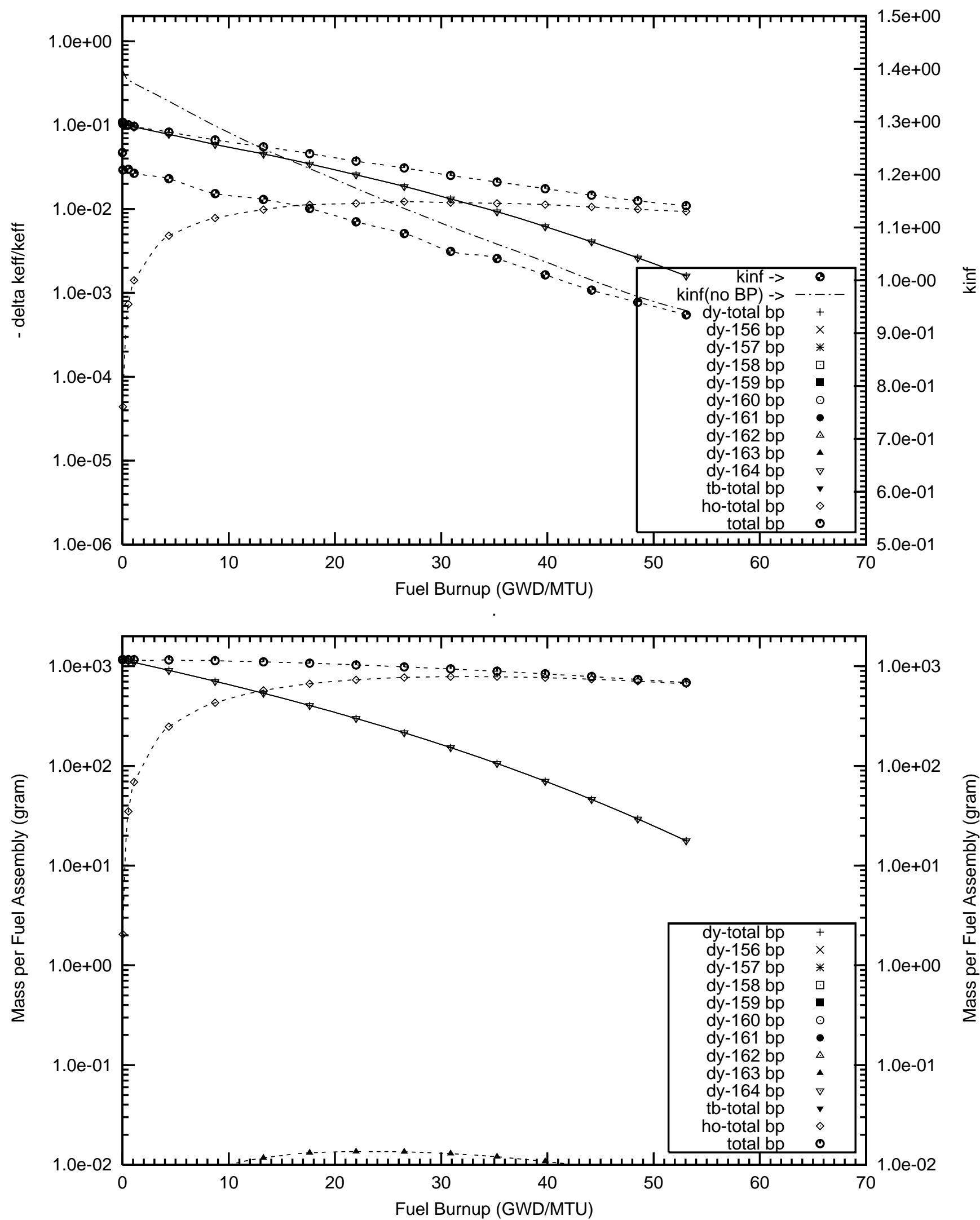
Figure 54. Negative Reactivity and Masses BP for Dy-164 and transmutation daughters as a function of fuel life for $17 \times 17$ fuel assemblies with 64 poison rods,

0.5 mill thick Dy2O3 poison coating on the outer radial surface of the UO2 pellets Reactor power 3400 MWth, 193 fuel assemblies, initial enrichment 4.5wt\%U-235

(Case : dy164_0f_64_bp_fuel o)
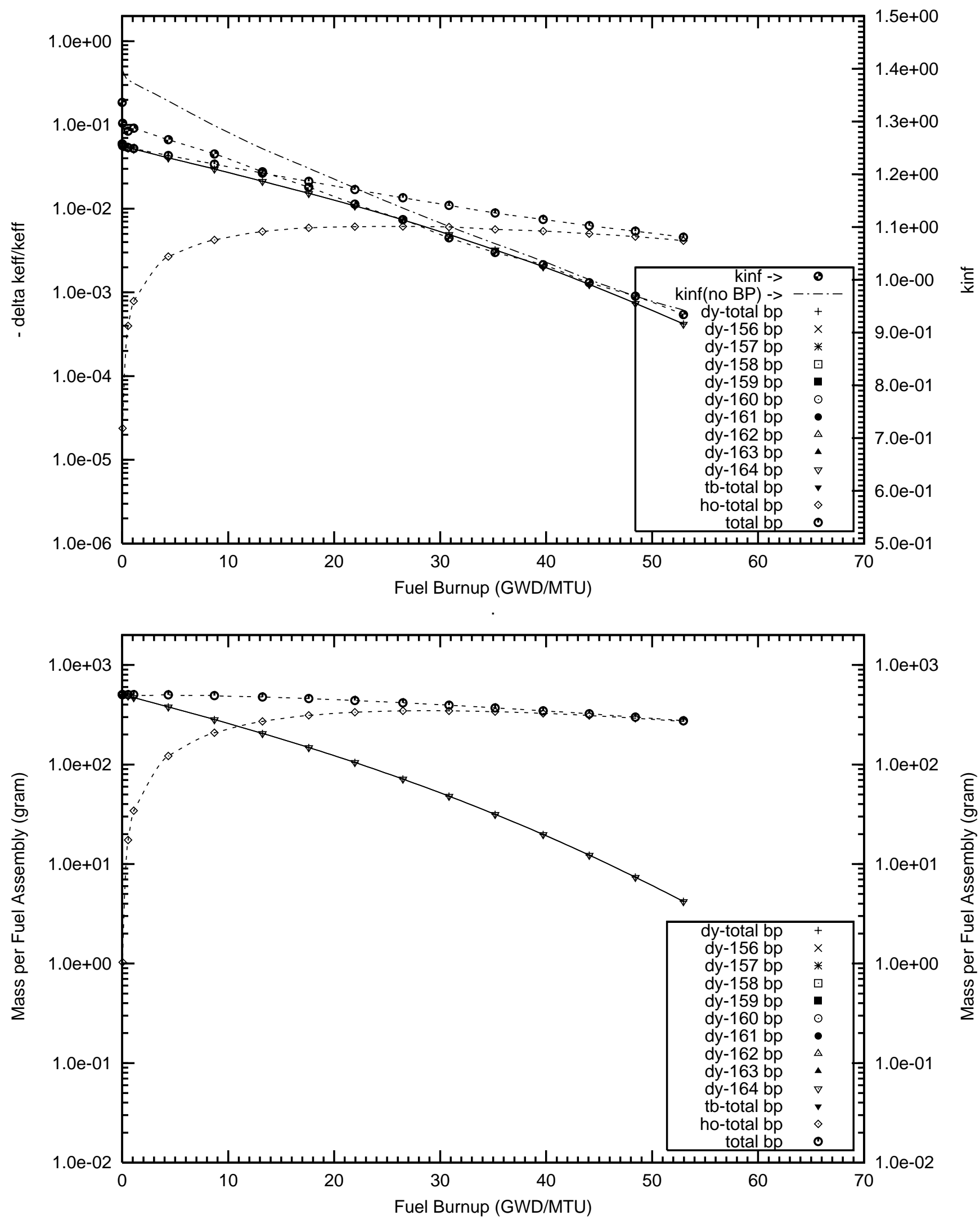


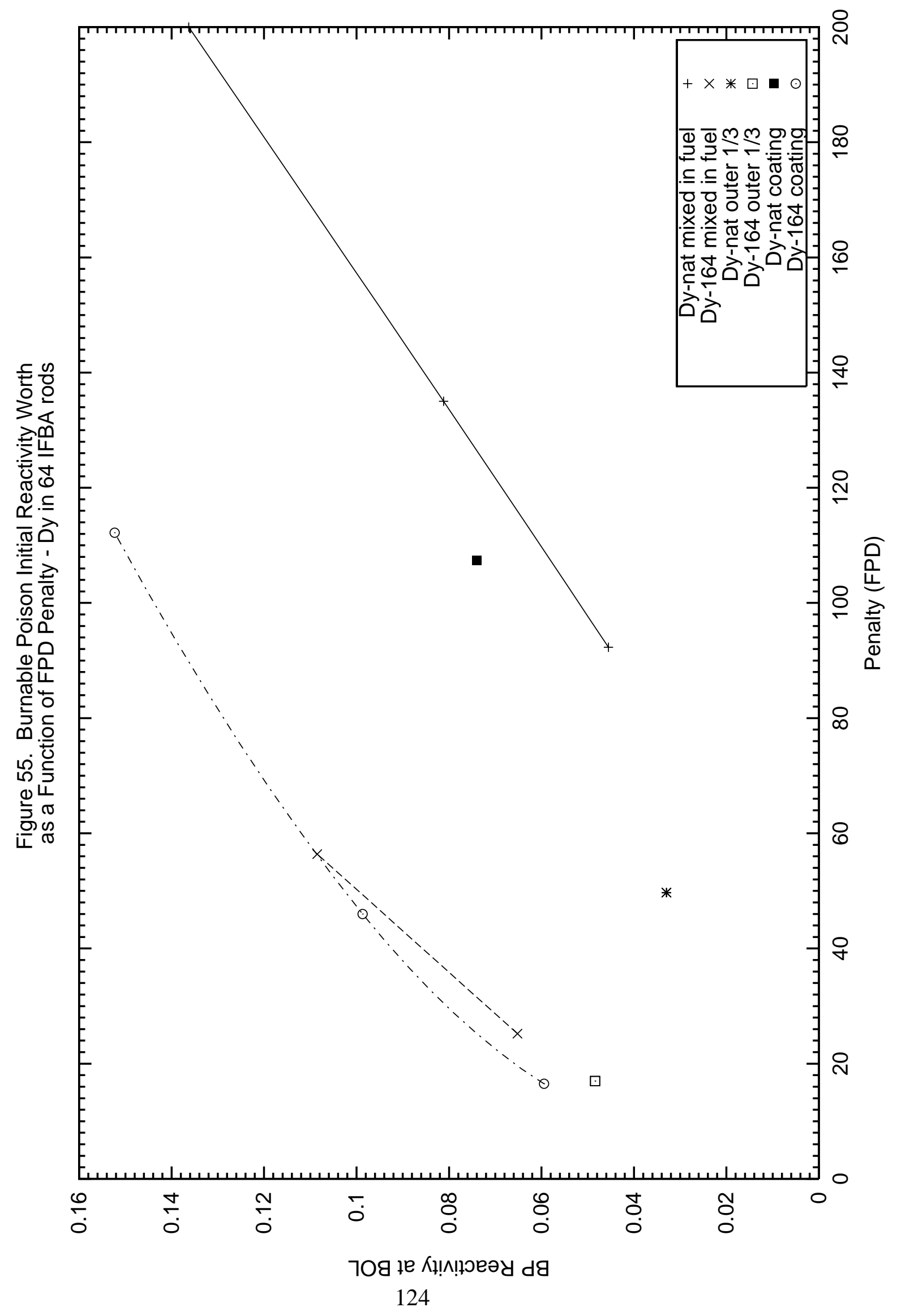




\section{NEUTRONICS PERFORMANCE RESULTS FOR EUROPIUM}

The stable isotopes of europium, ${ }^{151} \mathrm{Eu}\left(47.8 \%\right.$ natural isotopic abundance) and ${ }^{153} \mathrm{Eu}(52.2 \%)$, exhibit very large thermal neutron capture cross sections. The radioactive isotopes ${ }^{152} \mathrm{Eu},{ }^{154} \mathrm{Eu}$, and ${ }^{155} \mathrm{Eu}$, produced by neutron capture, have multi-year half-lives and exhibit large neutron capture cross sections. Because of these characteristics, europium with natural isotopic abundances will not burn out easily. It is thus an ideal control rod material and has been used in research reactors [e.g., the High Flux Isotope Reactor (HFIR)]. In this section, we will investigate whether europium, enriched in ${ }^{151} \mathrm{Eu}$, could be used as a burnable poison in PWRs.

The half-lives, natural isotopic abundances, and burnup chains related to the depletion and transmutation of europium are given below. The isomeric states of certain isotopes are not shown. However, the generation of these daughters was taken into account in the BP depletion and decay calculations.

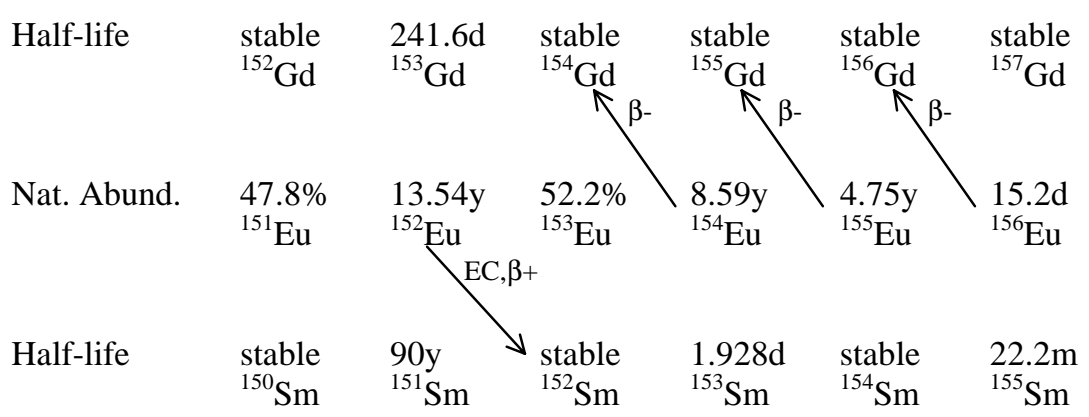

Figure 56 displays the neutron capture cross sections for the europium isotopes. The europium stable isotope ${ }^{151} \mathrm{Eu}$ (47.8\% natural isotopic abundance) exhibits a thermal capture cross section of 9,200 barns and a RI of 3,300 barns. The ${ }^{151} \mathrm{Eu}(\mathrm{n}, \gamma){ }^{152} \mathrm{Eu}$ reaction leads to ${ }^{152} \mathrm{Eu}$, which has a half-life of 13.54 years, a thermal neutron capture cross section of 12,800 barns, and a RI of 1,580 barns. The other stable europium isotope, ${ }^{153} \mathrm{Eu}(52.2 \%$ natural isotopic abundance) has a thermal neutron capture cross section of 310 barns and a RI of 1,420 barns. The ${ }^{153} \mathrm{Eu}(\mathrm{n}, \gamma){ }^{154} \mathrm{Eu}$ reaction leads to ${ }^{154} \mathrm{Eu}$, which has a half-life of 8.6 years, a thermal neutron capture cross section of 1,340 barns, and a RI of 800 barns. Because of the long half-life of ${ }^{154} \mathrm{Eu}$, the principal transmutation route for ${ }^{154} \mathrm{Eu}$ will be via the ${ }^{154} \mathrm{Eu}(\mathrm{n}, \gamma){ }^{155} \mathrm{Eu}$ reaction, leading to ${ }^{155} \mathrm{Eu}$, which has a half-life of 4.75 years, a capture cross section of 3,950 barns, and a RI of 23,200 barns. Again, because of the long half-life of ${ }^{155} \mathrm{Eu}$, the principal transmutation route for ${ }^{155} \mathrm{Eu}$ will be via the ${ }^{155} \mathrm{Eu}(\mathrm{n}, \gamma){ }^{156} \mathrm{Eu}$ reaction, leading to ${ }^{156} \mathrm{Eu}$, which decays to ${ }^{156} \mathrm{Gd}$ with a half-life of 15.2 days. ${ }^{156} \mathrm{Gd}$ has a capture cross section of 1.5 barns and a RI of 104 barns. The use of ${ }^{151} \mathrm{Eu}$ in a BP will lead by transmutation to ${ }^{152} \mathrm{Eu}$, which has a higher thermal neutron capture cross section than ${ }^{151} \mathrm{Eu}$. Transmutation of ${ }^{152} \mathrm{Eu}$ leads to ${ }^{153} \mathrm{Eu}$, which has a much smaller thermal cross section than either ${ }^{151} \mathrm{Eu}$ or ${ }^{152} \mathrm{Eu}$. The sequence of ${ }^{151} \mathrm{Eu}$ and ${ }^{152} \mathrm{Eu}$, exhibits a pair of very high thermal cross sections, followed by ${ }^{153} \mathrm{Eu}$ with a much smaller cross section. This will lead to a decrease of the burnout rate of the BP, together with a reduction of the residual reactivity fraction of the $\mathrm{BP}$. The use of ${ }^{153} \mathrm{Eu}$ as a $\mathrm{BP}$ will lead to ${ }^{154} \mathrm{Eu}$, which has a higher thermal neutron capture cross section than ${ }^{153} \mathrm{Eu}$. Further transmutation leads to ${ }^{155} \mathrm{Eu}$, which has an even higher thermal cross section than ${ }^{154} \mathrm{Eu}$. Transmutation to ${ }^{156} \mathrm{Eu}$ will lead to a 15.2-day decay of ${ }^{156} \mathrm{Eu}$ to ${ }^{156} \mathrm{Gd}$, which has a very small thermal cross section. Since the thermal cross section of ${ }^{153} \mathrm{Eu}$ is smaller than that of ${ }^{151} \mathrm{Eu}$, a larger amount of ${ }^{153} \mathrm{Eu}$ is needed than of ${ }^{151} \mathrm{Eu}$ in order to achieve a desired initial reactivity worth of the BP. In addition, the sequence ${ }^{153} \mathrm{Eu}^{154} \mathrm{Eu}^{155} \mathrm{Eu}$ exhibits an 
increasing thermal neutron capture cross section. This will thus give rise to a much higher residual reactivity fraction than the use of a BP fully enriched in ${ }^{151} \mathrm{Eu}$. Enriching europium in the ${ }^{151} \mathrm{Eu}$ isotope should thus reduce the residual negative reactivity fraction of the BP, together with decreasing the burnout rate of the $\mathrm{BP}$.

In all the neutronics calculations presented in this section, a theoretical density of $7.42 \mathrm{~g} / \mathrm{cm}^{3}$ was used for europia $\left(\mathrm{Eu}_{2} \mathrm{O}_{3}\right)$, and $5.22 \mathrm{~g} / \mathrm{cm}^{3}$ was used for metallic europium. For $\mathrm{UO}_{2}$, a theoretical density of $10.97 \mathrm{~g} / \mathrm{cm}^{3}$ was used. The actual density fraction of the fuel pellets and of the burnable poison pellets was $95 \%$ of theoretical density.

In this section, we will investigate the potential benefits of using enriched europium as a burnable absorber in PWRs. Four different configurations of the europium burnable poison, similar to those of the case of $\mathrm{Gd}$, were investigated.

1. A homogeneous mixture of the burnable poison europia inside the fuel pellets of a limited number of fuel rods. A summary of the results is given in Table 21. The plots of the reactivity worths and the masses of the BP isotopes and their daughters are displayed in Appendix E1.

2. A homogeneous mixture of the burnable poison as an oxide (europia) in the outer radial one-third volume of the fuel pellets. A summary of the results is given in Table 22. Plots of the reactivity worths and the masses of the BP isotopes and their daughters are displayed in Appendix E2.

3. An oxide burnable poison coating of europia on the surface of the fuel pellets in a limited number of fuel rods, similar to the $\mathrm{ZrB}_{2}$ coatings. A summary of the results is given in Table 23. Plots of the reactivity worths and the masses of the BP isotopes and their daughters are displayed in Appendix E3.

4. A homogeneous mixture of the burnable poison europium in the form of a metal alloyed with Zircaloy cladding of a number of fuel rods. The summary results are given in Table 24. Plots of the reactivity worths and the masses of the BP isotopes and their daughters are displayed in Appendix E4. Table 24 displays the results of using europium, mixed homogeneously and alloyed with the Zircaloy cladding of a number of fuel rods. Note that for a similar value of the initial reactivity worth of the $\mathrm{BP}$, the residual negative reactivity worth fraction for europium enriched in ${ }^{151} \mathrm{Eu}$ is smaller than for europium with natural isotopic abundances. The relative contribution from the gadolinium daughters is also smaller.

The reactivity worths of the $\mathrm{BP}$ isotopes, for a $2.0 \mathrm{wt} \%$ loading of europium with natural isotopic abundances, alloyed with the Zircaloy cladding of 64 fuel rods per fuel assembly, are shown in Figure 57. The initial reactivity worth of the BP is 0.124 , and the residual reactivity ratio was $11.4 \%$. Figure 57 also displays the masses of the burnable poison isotopes. The total initial mass of natural europium was 457 grams per fuel assembly containing BPs.

For a $1.0 \mathrm{wt} \%$ homogeneous loading of europium, fully enriched in ${ }^{151} \mathrm{Eu}$, alloyed in the Zircaloy cladding of 64 fuel rods, the reactivity worths and the masses of the BP isotopes are shown in Figure 58. The initial reactivity worth of the BP was 0.118 and the residual reactivity ratio was $6.8 \%$. The total initial mass of ${ }^{151} \mathrm{Eu}$ was 229 grams per fuel assembly. Figure 58 also shows that the reactivity worths of the BP transmutation daughters ${ }^{152} \mathrm{Eu}$ to ${ }^{155} \mathrm{Eu}$ initially increase with depletion time, then reach a maximum, followed by a decrease of the reactivity worth. The time at which the reactivity worth reaches its maximum increases with the isotope transmutation sequence chain from ${ }^{152} \mathrm{Eu}$ to ${ }^{155} \mathrm{Eu}$.

For a $0.5 \mathrm{wt} \%$ loading of ${ }^{151} \mathrm{Eu}$, alloyed with the Zircaloy cladding of 104 IFBA rods, the reactivity worths and the masses of the BP isotopes are shown in Figure 59. The initial reactivity worth of the $\mathrm{BP}$ was 0.108 , and the residual reactivity ratio was $6.1 \%$. The total initial mass of ${ }^{151} \mathrm{Eu}$ was 186 
grams per fuel assembly. Note that the shape of the fuel assembly lattice $\mathrm{k}_{\text {inf }}$ 's as a function of depletion time for this case are almost identical with the shape of the eigenvalues for the case of 104 IFBA rods per fuel assembly with $\mathrm{ZrB}_{2}$ coatings containing enriched boron $\left(30 \mathrm{wt} \%{ }^{10} \mathrm{~B}\right)$ (see Figure 16).

The reactivity worths for a 0.2 -mil coating of $\mathrm{Eu}_{2} \mathrm{O}_{3}$, fully enriched in ${ }^{151} \mathrm{Eu}$, on the surface of the fuel pellets in 64 IFBA rods, is shown in Figure 60. The BP masses are also shown in Figure 60. The initial reactivity worth was $9.80 \times 10^{-2}$, and the residual reactivity worth fraction was $7.0 \%$. The mass of ${ }^{151} \mathrm{Eu}$ was 190 grams per fuel assembly. The shape of the fuel assembly lattice $\mathrm{k}_{\mathrm{inf}}$ 's as a function of depletion time is very similar to the $\mathrm{ZrB}_{2}$ coatings.

The use of europium enriched in ${ }^{151} \mathrm{Eu}$, instead of with natural isotopic abundances, will thus lead to a reduction of the residual reactivity burden at EOL of the fuel.

The MVCs were calculated by decreasing the water coolant density by $10 \%$. The results are shown in Tables 21 through 24. For all the cases containing 64 and 104 IFBA rods, the MVCs remain negative. 


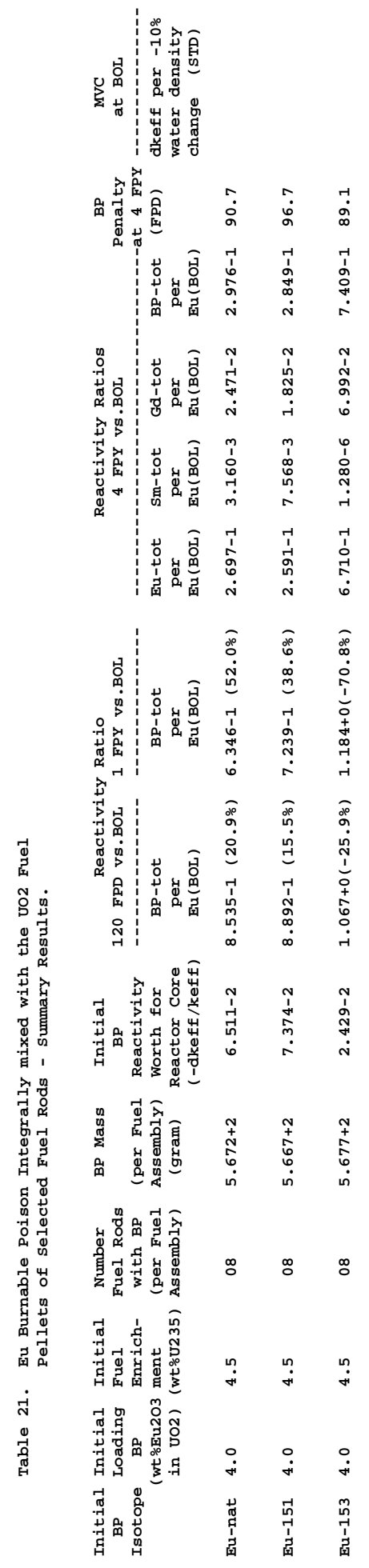




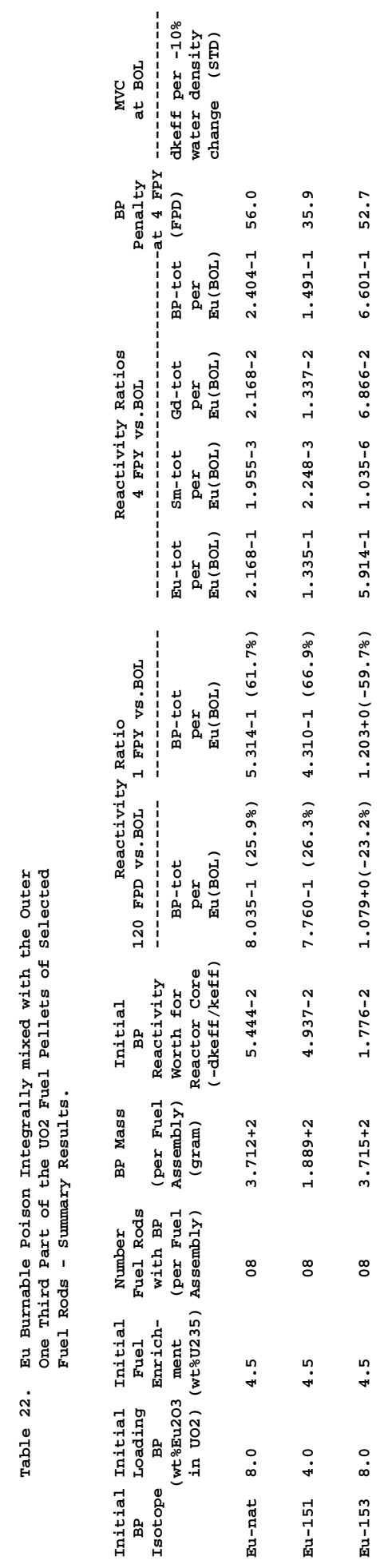




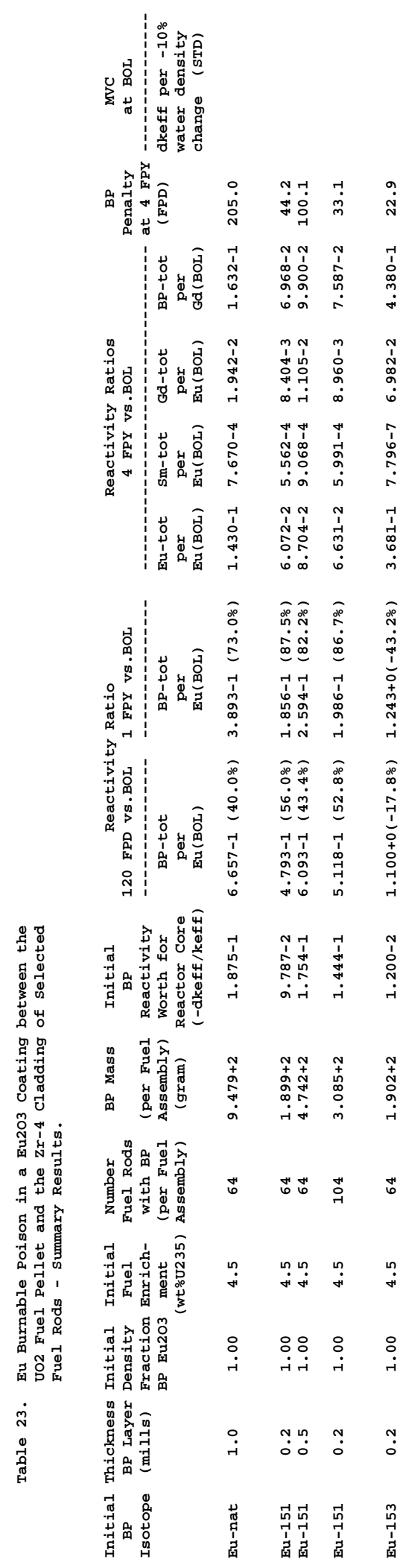




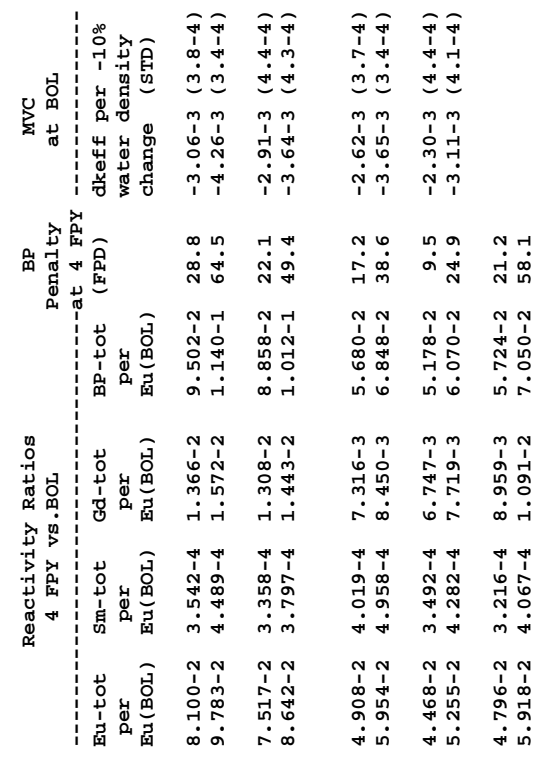

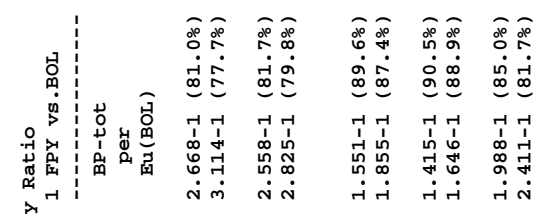

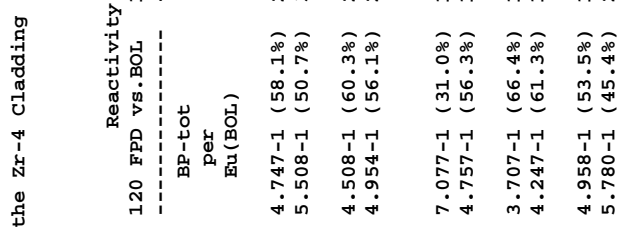

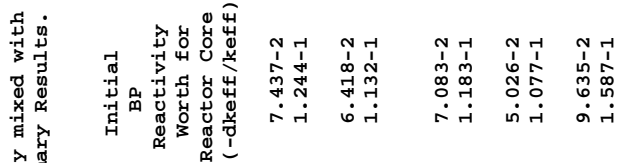

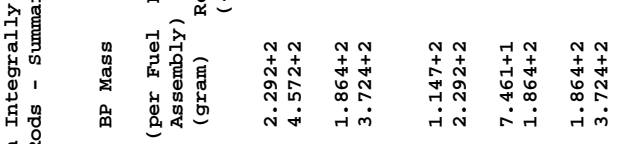

व

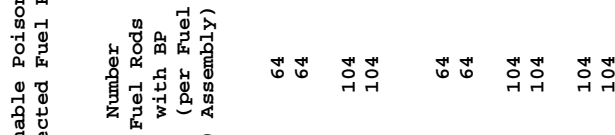

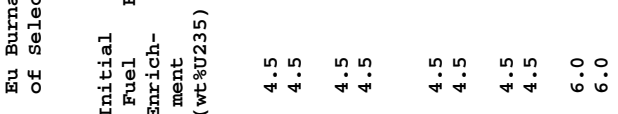

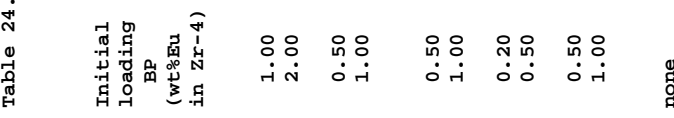

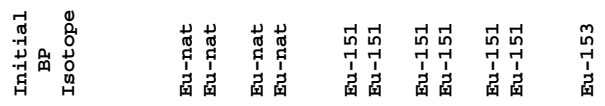


Figure 56. Neutron Cross-Sections as a Function of Energy

Eu-148 (63148.40c)

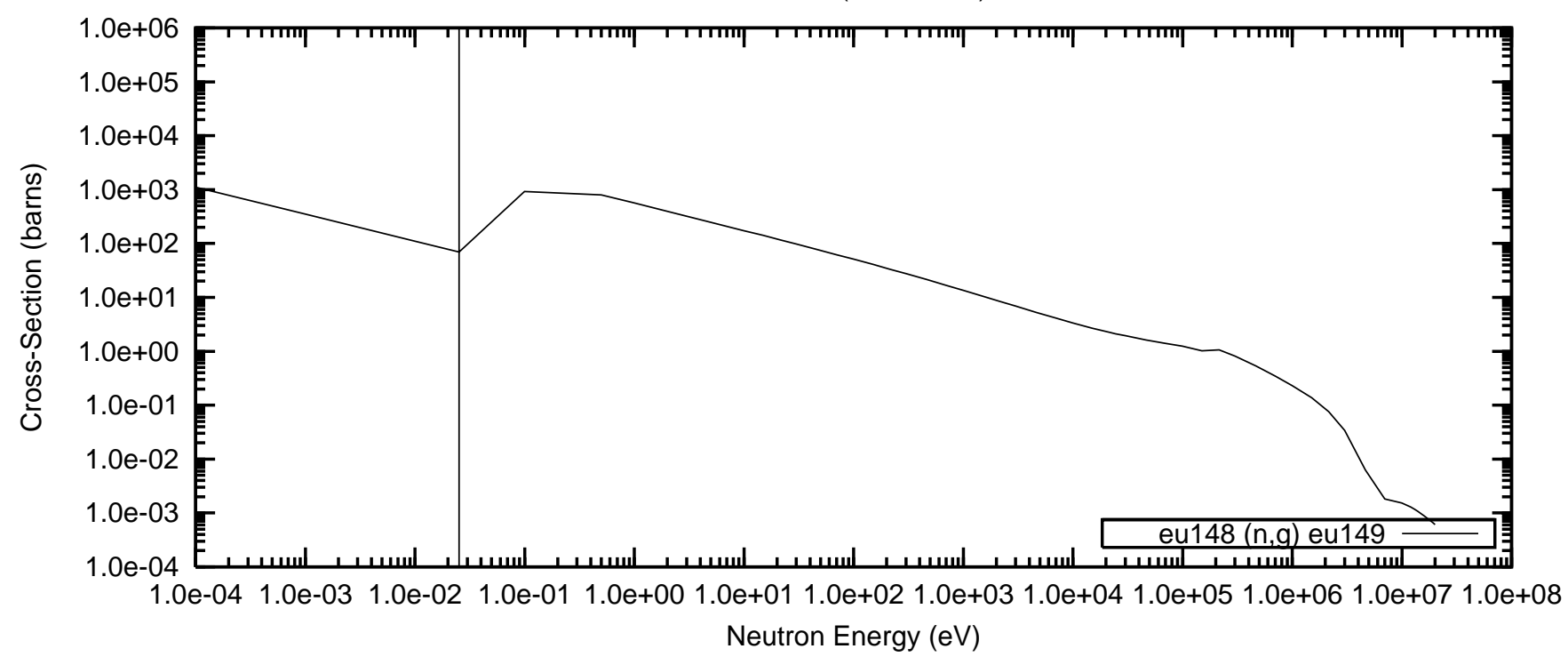

Eu-149 (63149.40c,46c)

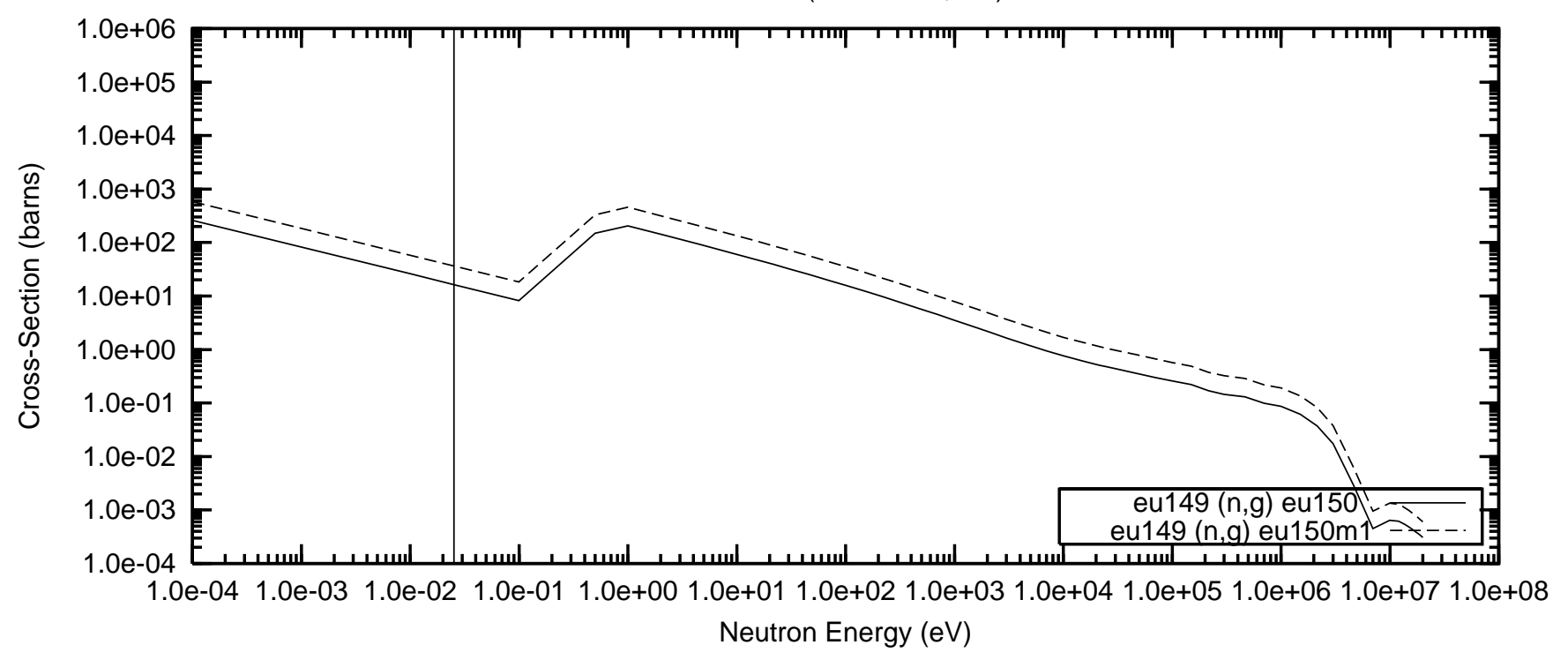

Eu-150 (63150.40c)

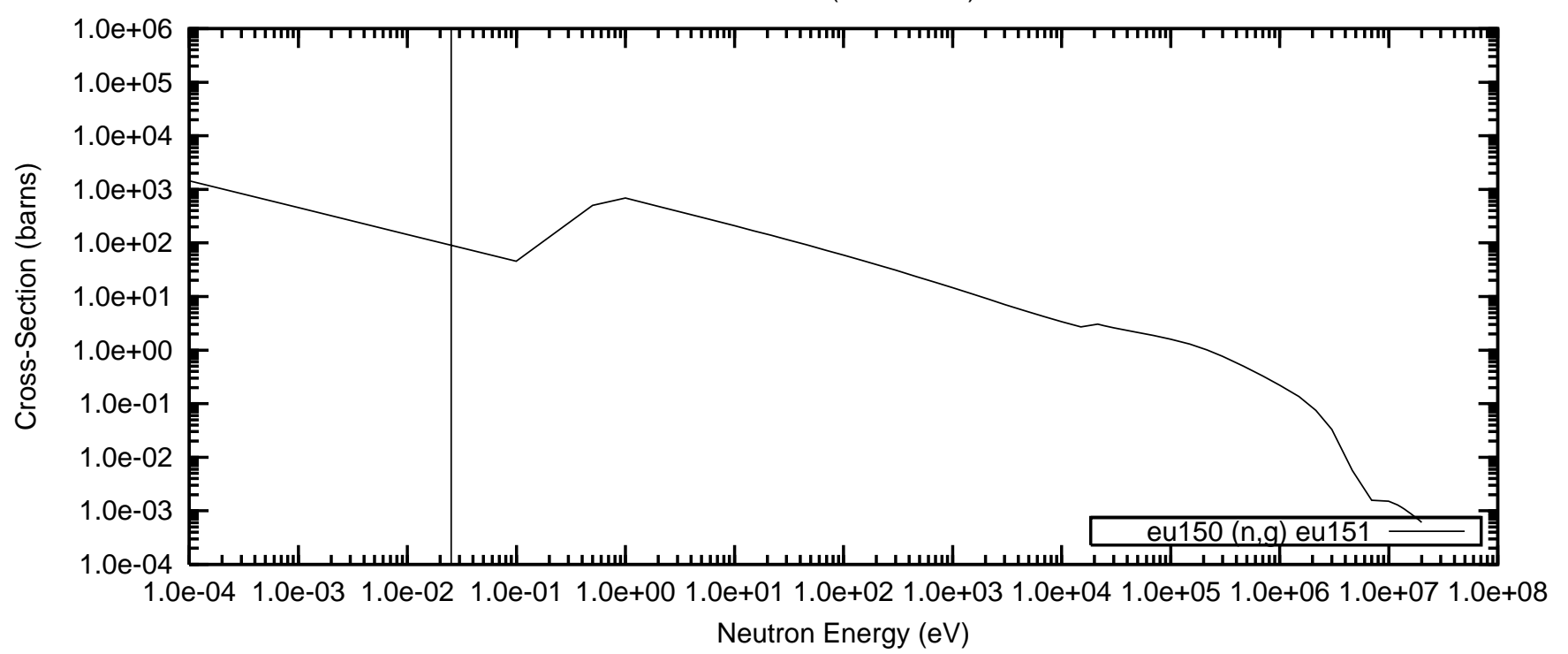


Figure 56(cont). Neutron Cross-Sections as a Function of Energy Eu-150m1 (63150.41c)

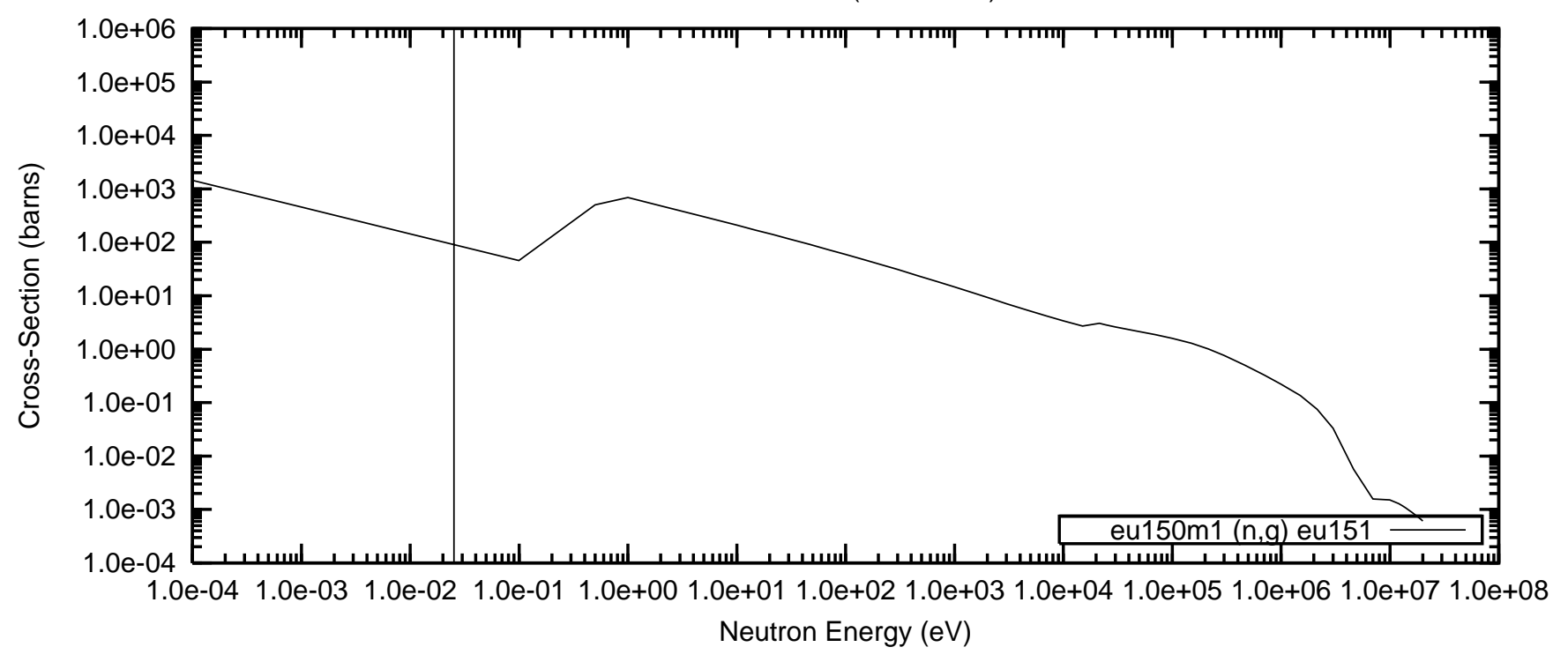

Eu-151 (63151.40c,46c,47c)

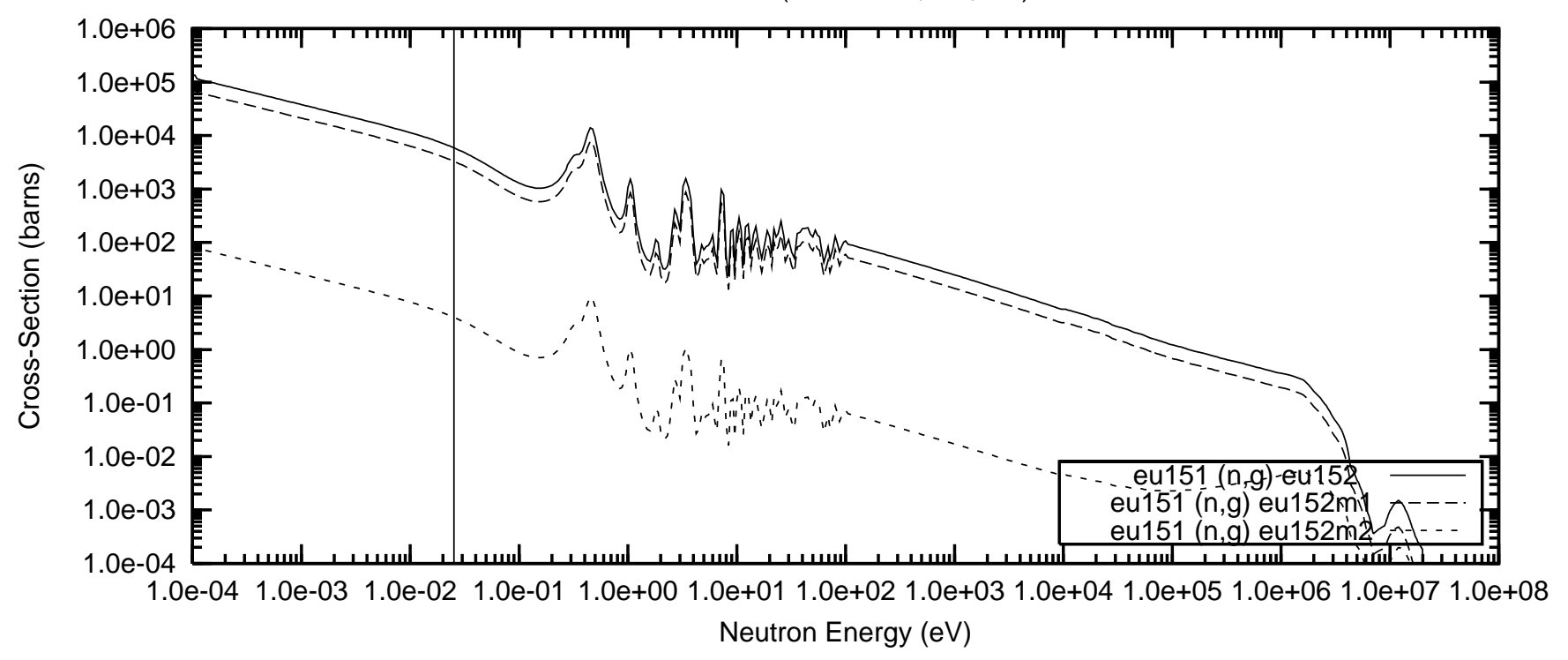

Eu-152 (63152.40c)

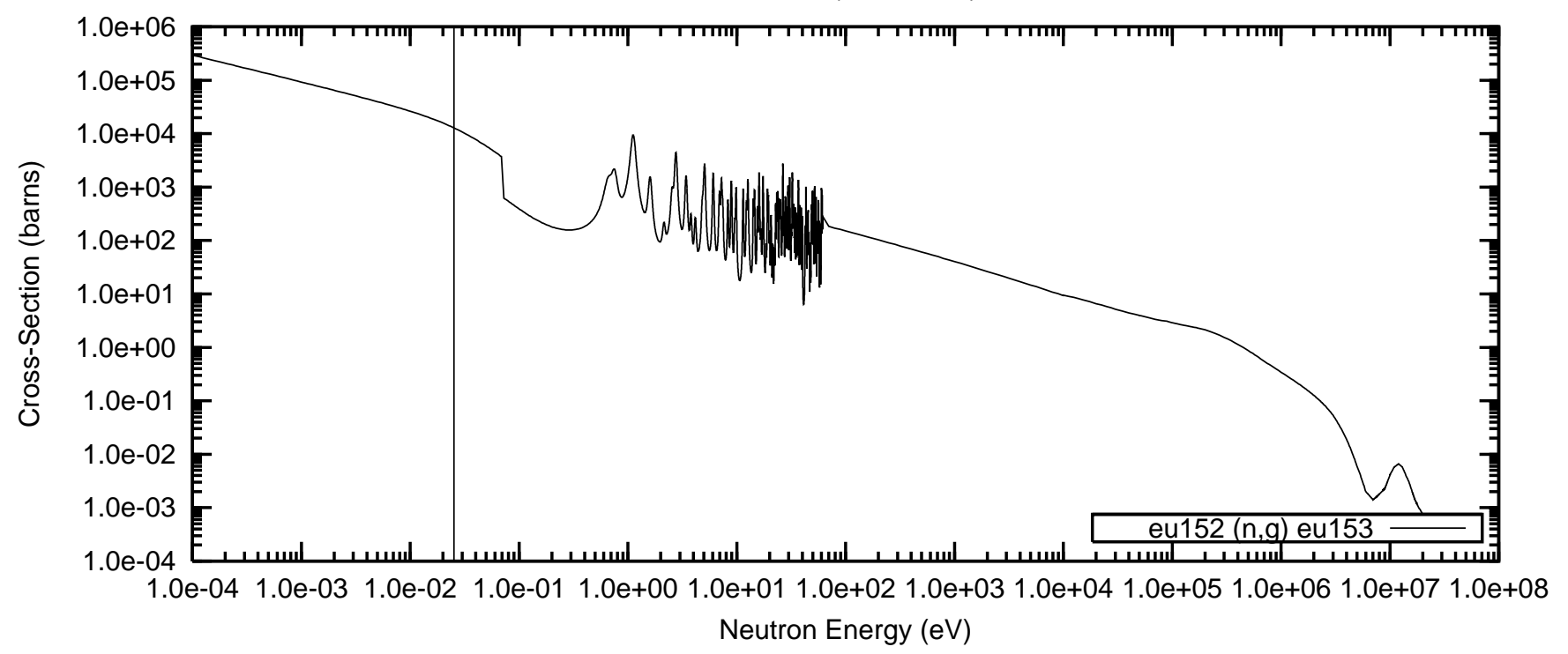


Figure 56(cont). Neutron Cross-Sections as a Function of Energy Eu-152m1 (63152.41c)

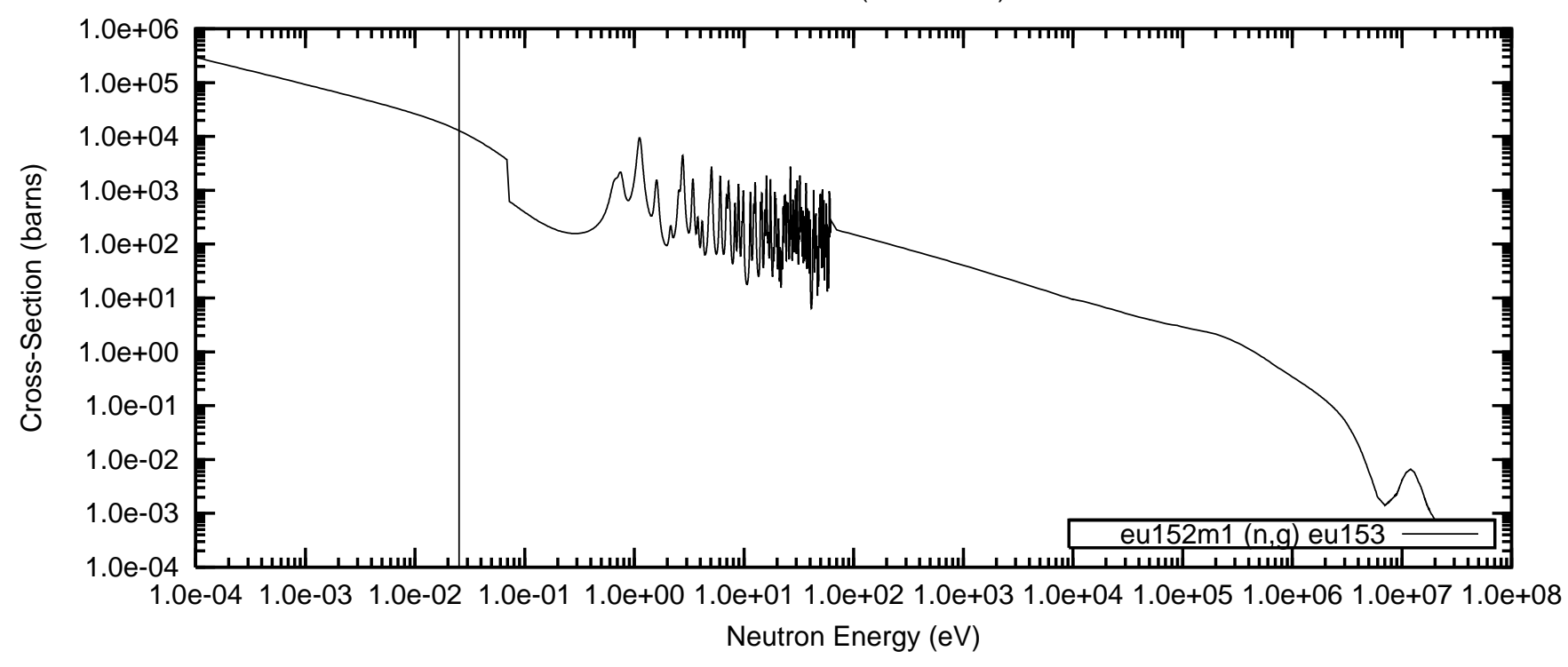

Eu-153 (63153.40c,46c)

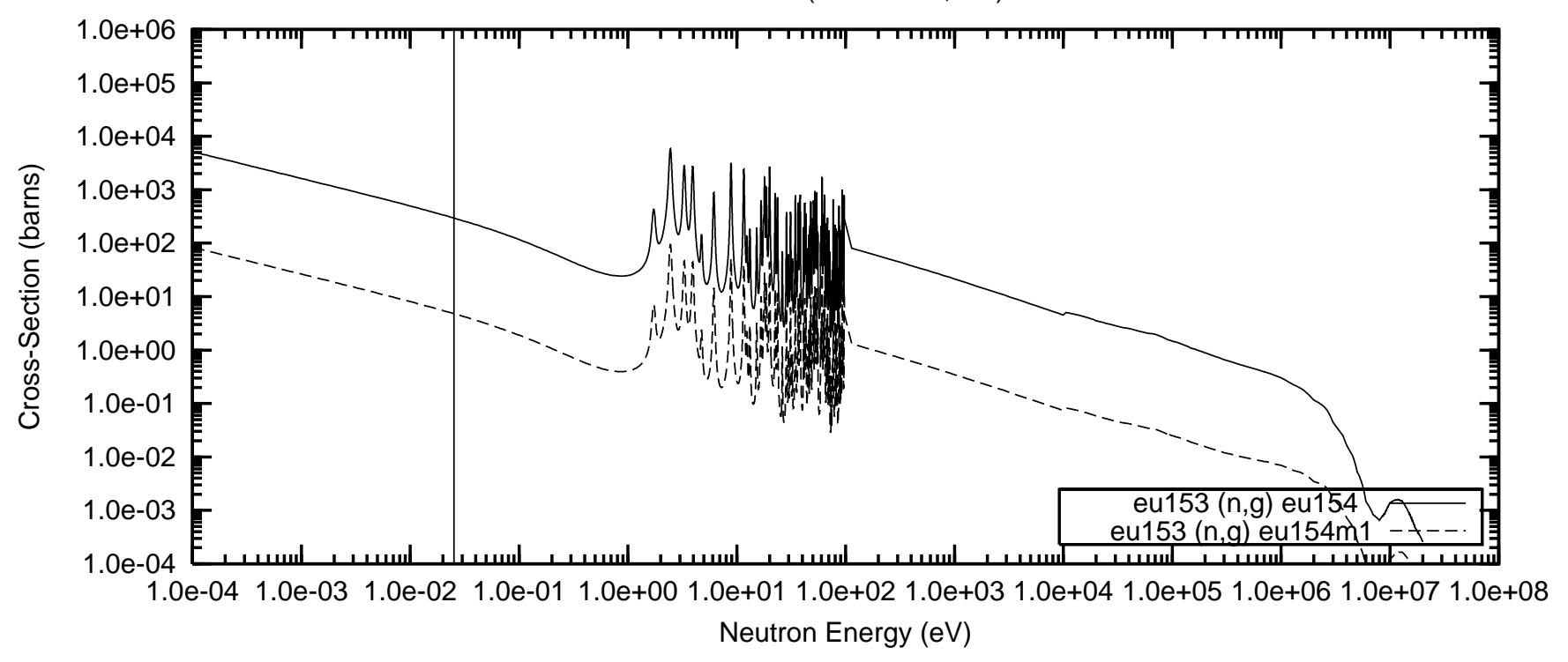

Eu-154 (63154.40c)

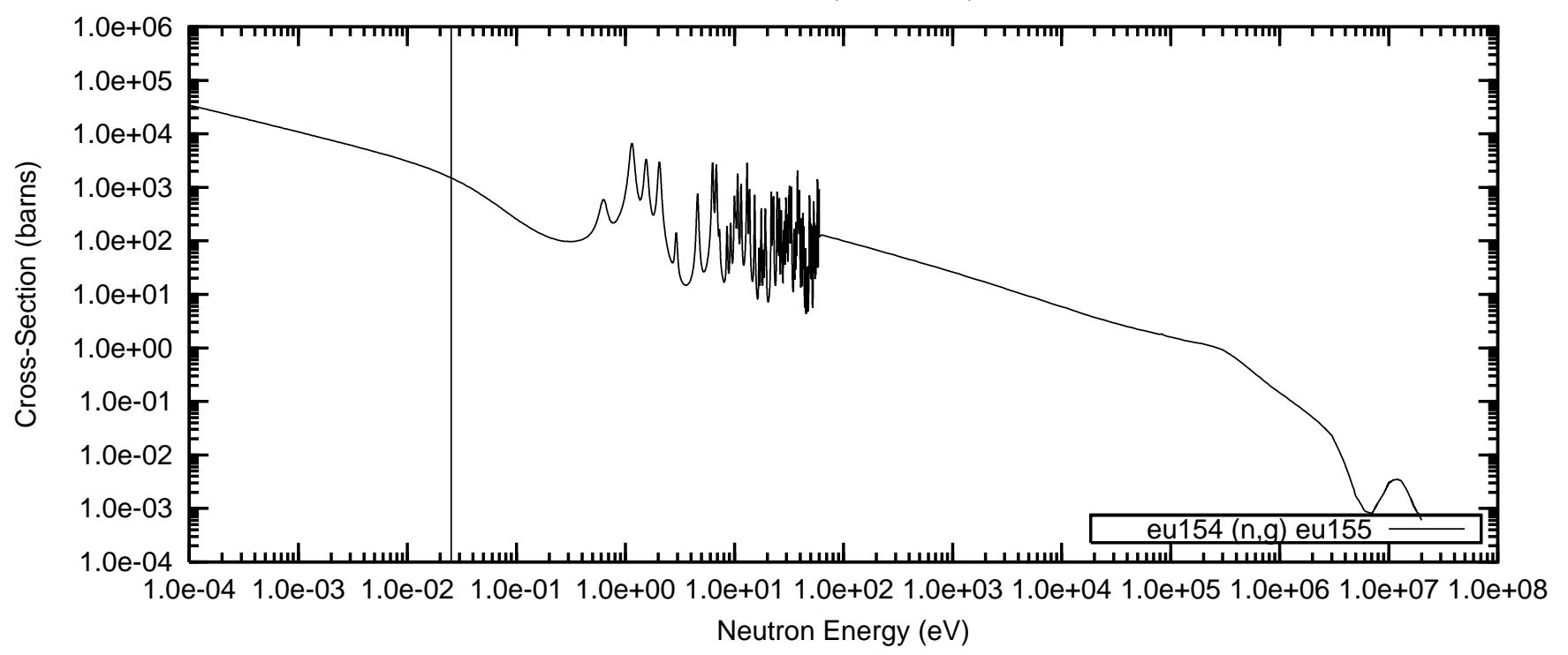


Figure 56(cont). Neutron Cross-Sections as a Function of Energy Eu-155 (63155.40c)

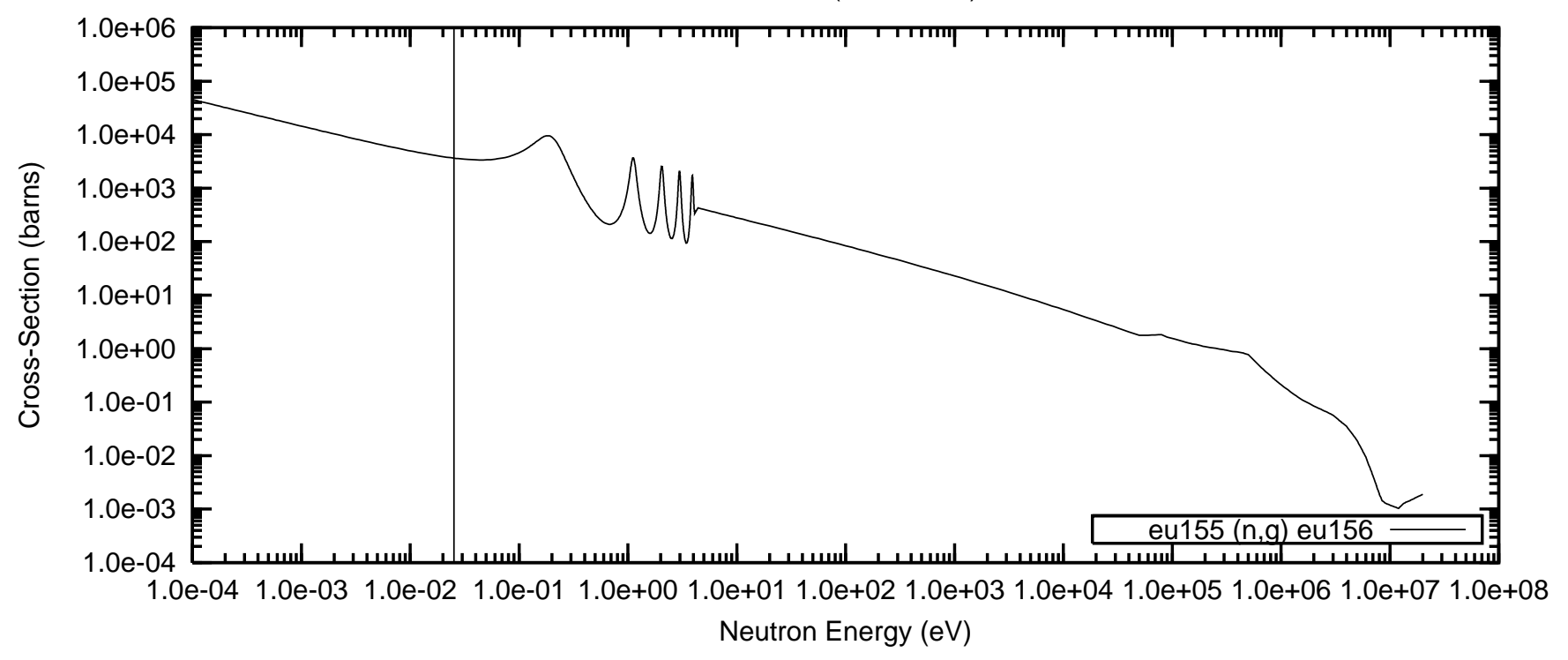

Eu-156 (63156.40c)

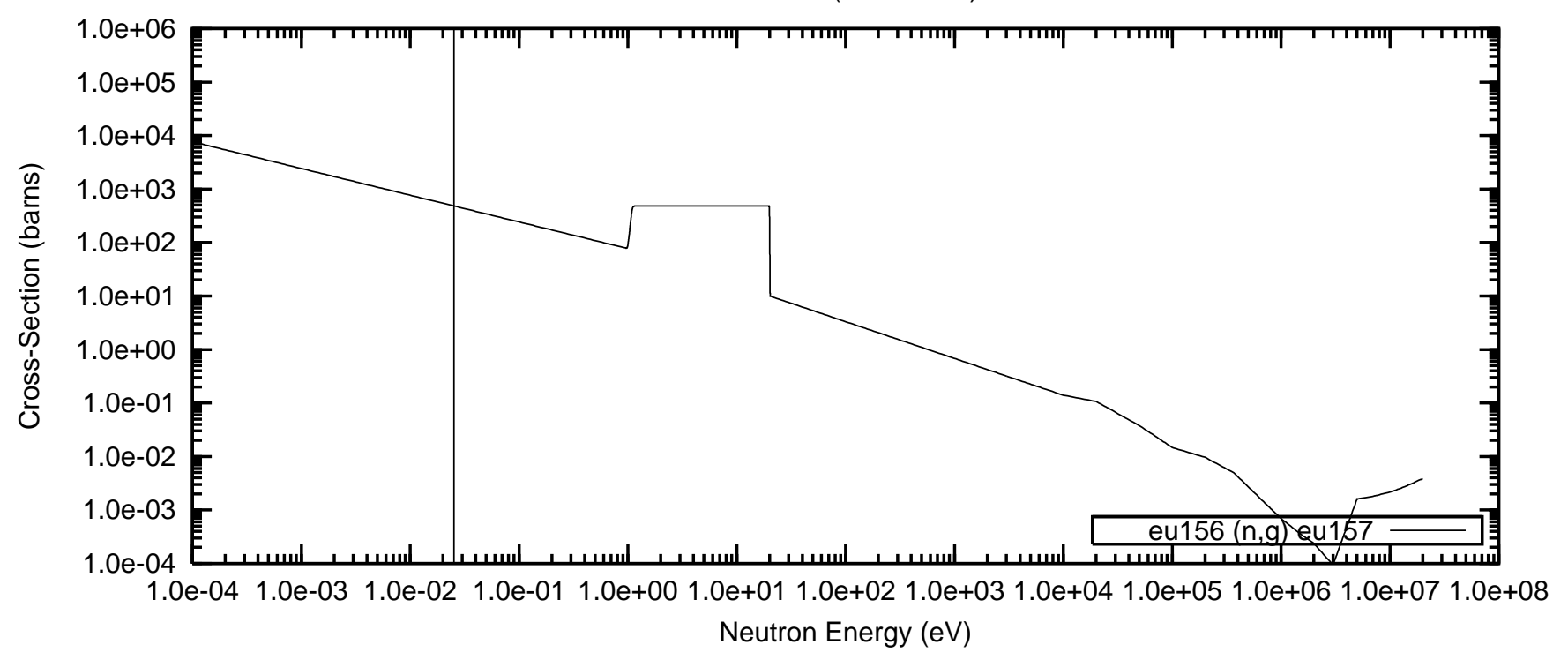

Eu-157 (63157.40c)

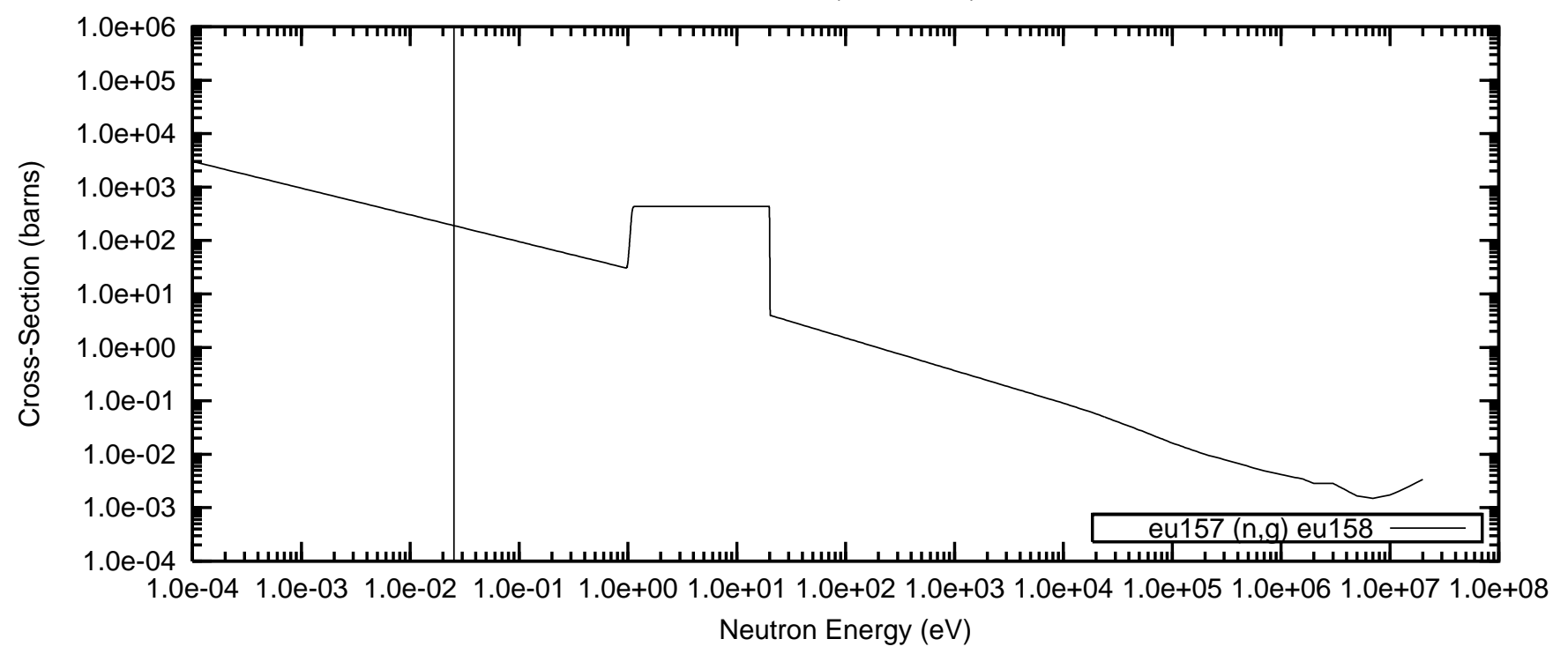


Figure 57. Negative Reactivity and Masses BP for Eu-nat and transmutation daughters as a function of fuel life for $17 \times 17$ fuel assemblies with 64 poison rods,

$2.0 \mathrm{wt} \%$ Eu poison homogeneously mixed in the fuel cladding,

Reactor power 3400 MWth, 193 fuel assemblies, initial enrichment 4.5wt\%U-235

(Case : eu000_2c_100p_h2o_64_bp_fuel_2_o)
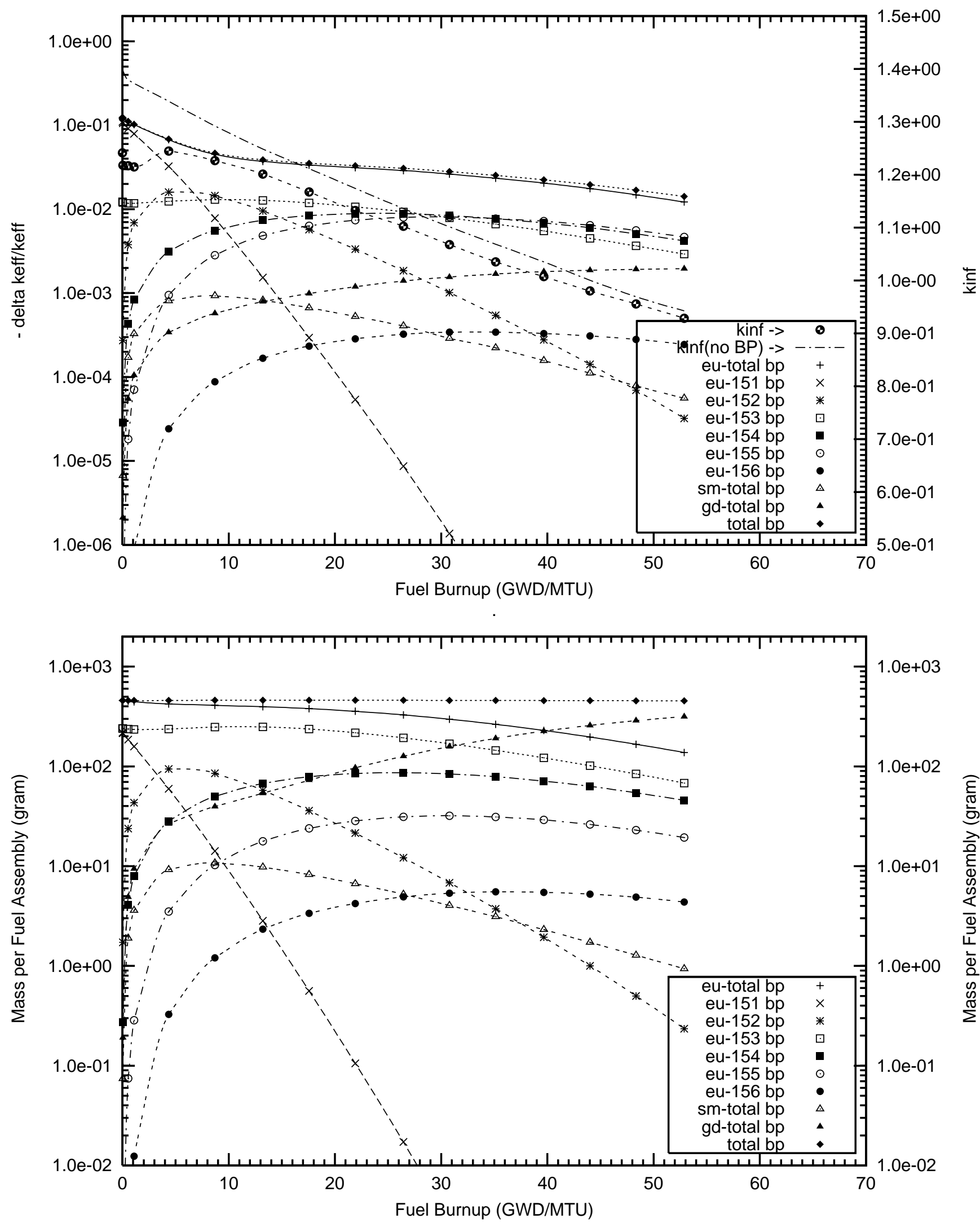
Figure 58. Negative Reactivity and Masses BP for Eu-151 and transmutation daughters as a function of fuel life for $17 \times 17$ fuel assemblies with 64 poison rods,

$1.0 w t \%$ Eu poison homogeneously mixed in the fuel cladding,

Reactor power 3400 MWth, 193 fuel assemblies, initial enrichment 4.5wt\%U-235

(Case : eu151_1c_100p_h2o_64_bp_fuel_2_o)
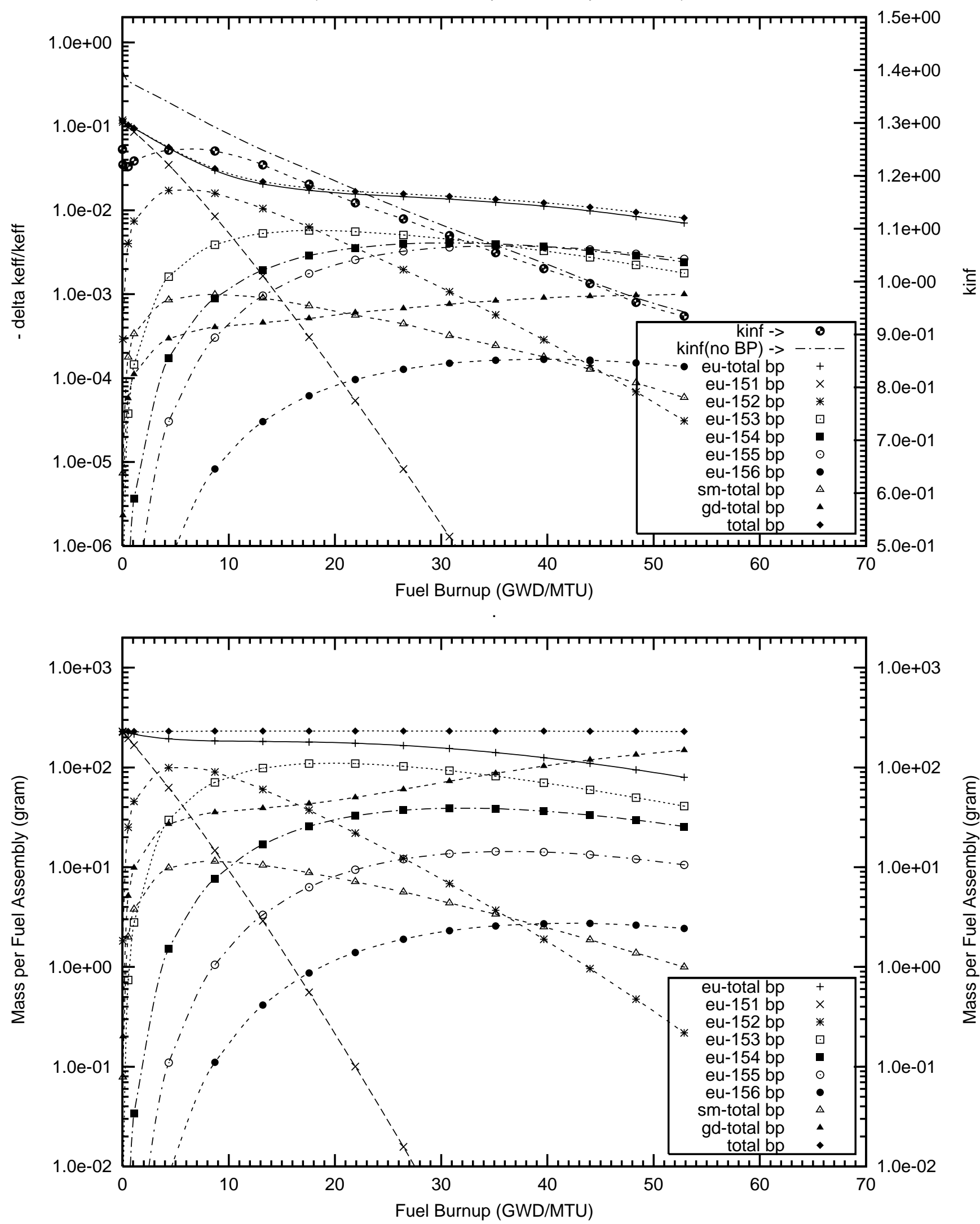
Figure 59. Negative Reactivity and Masses BP for Eu-151 and transmutation daughters as a function of fuel life for $17 \times 17$ fuel assemblies with 104 poison rods,

$0.5 w t \%$ Eu poison homogeneously mixed in the fuel cladding,

Reactor power 3400 MWth, 193 fuel assemblies, initial enrichment 4.5wt\%U-235

(Case : eu151_0c_104_bp_fuel o)
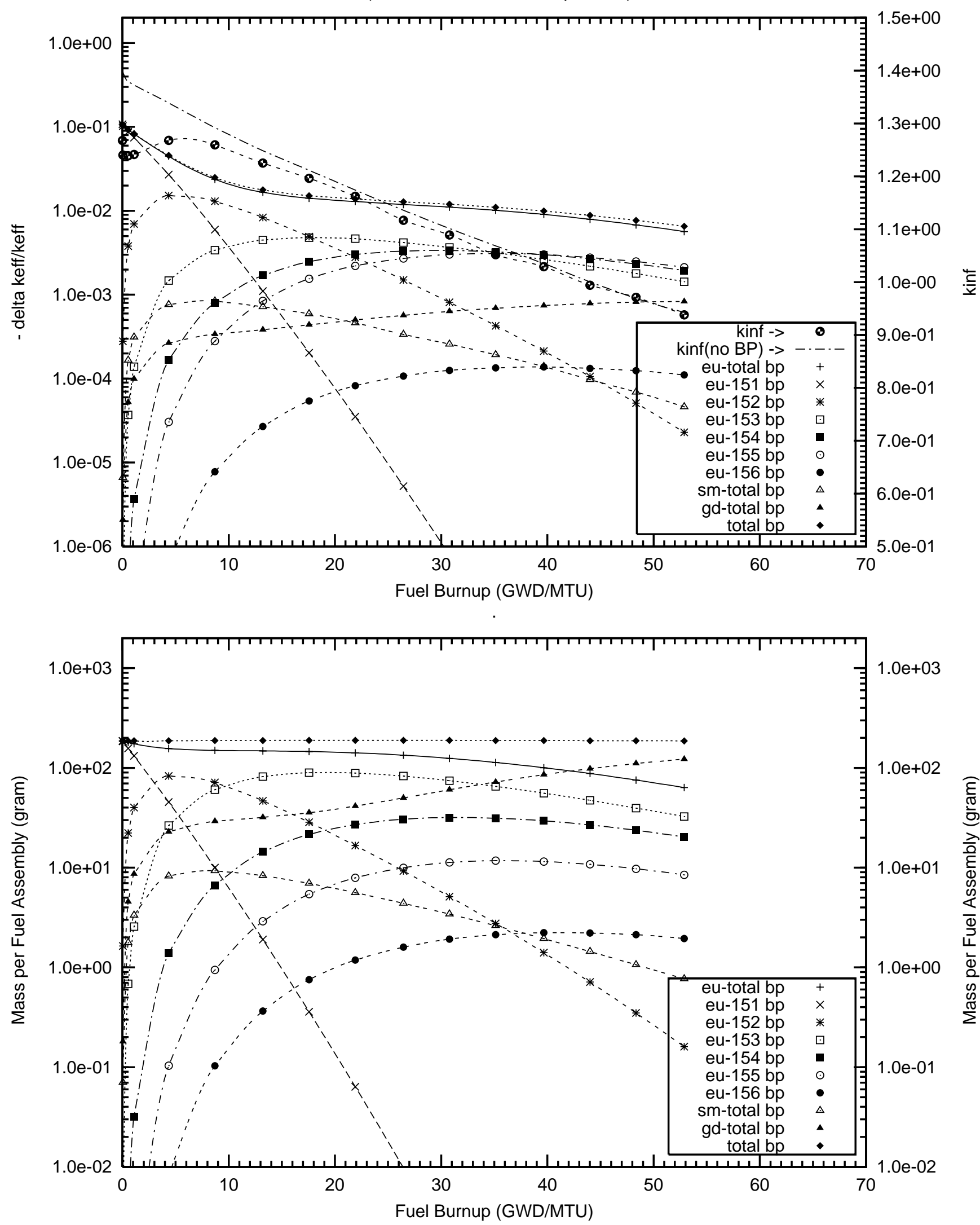
Figure 60. Negative Reactivity and Masses BP for Eu-151 and transmutation daughters as a function of fuel life for $17 \times 17$ fuel assemblies with 64 poison rods,

0.2 mill thick Eu2O3 poison coating on the outer radial surface of the UO2 pellets Reactor power 3400 MWth, 193 fuel assemblies, initial enrichment 4.5wt\%U-235

(Case : eu151_8f_64_bp_fuel o)
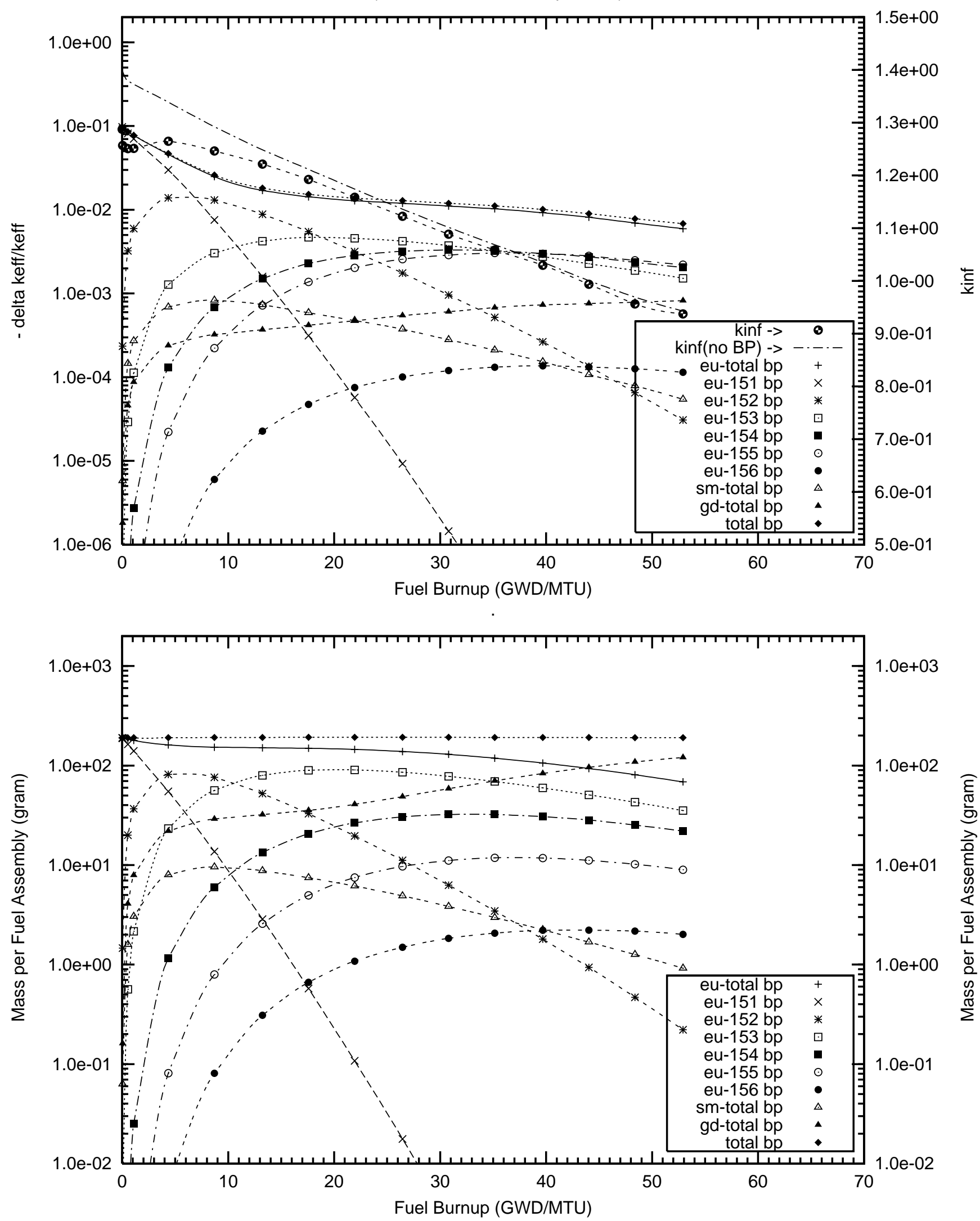


\section{NEUTRONICS PERFORMANCE RESULTS FOR HAFNIUM}

The half-lives, natural isotopic abundances, and burnup chains related to the depletion and transmutation of hafnium are given below. The isomeric states of certain isotopes are not shown. However, the generation of these daughters was taken into account in the BP depletion and decay calculations.

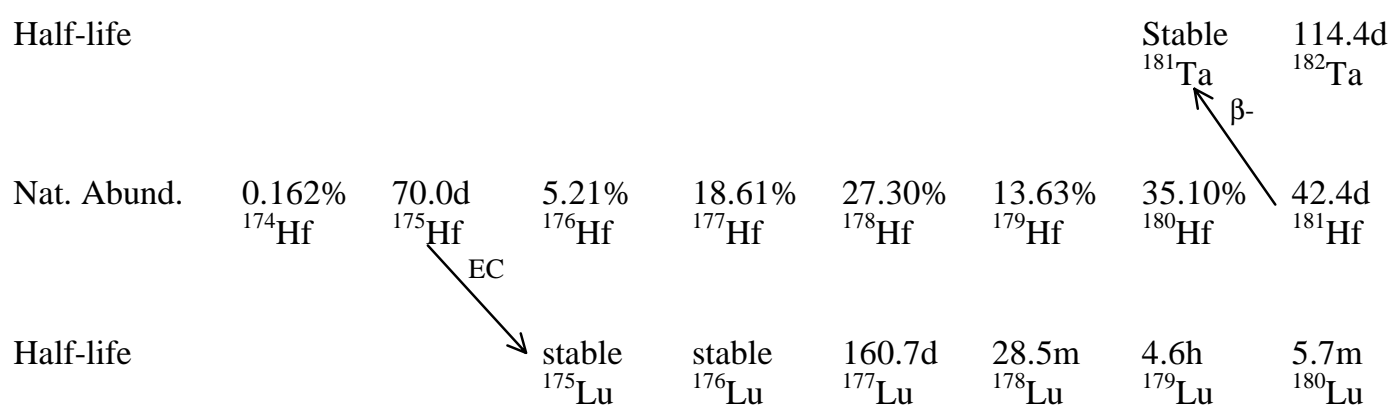

Figure 61 displays the neutron capture cross sections as a function of energy for the hafnium isotopes. The stable hafnium isotopes are ${ }^{174} \mathrm{Hf}(0.16 \%$ natural isotopic abundance, thermal capture cross section 560 barns, RI 436 barns), ${ }^{176} \mathrm{Hf}\left(5.2 \%, 23\right.$ barns, RI 880 barns), ${ }^{177} \mathrm{Hf}(18,6 \%, 373$ barns, RI 7,173 barns), ${ }^{178} \mathrm{Hf}\left(27.1 \%, 84\right.$ barns, RI 1,950 barns), ${ }^{179} \mathrm{Hf}(13.7 \%, 41$ barns, RI 630 barns), and ${ }^{180} \mathrm{Hf}(35.2 \%, 13$ barns, RI 35 barns). Transmutation reactions of the hafnium isotopes through the $(\mathrm{n}, \gamma)$ reaction lead to other hafnium isotopes with relatively high capture cross sections. The ${ }^{180} \mathrm{Hf}$ $(\mathrm{n}, \gamma){ }^{181} \mathrm{Hf}$ reaction leads to ${ }^{181} \mathrm{Hf}$, which decays to ${ }^{181} \mathrm{Ta}$ with a half-life of 42.4 days. ${ }^{181} \mathrm{Ta}$ has a thermal cross section of 20 barns and a RI of 660 barns. The relatively high capture cross sections of the transmutant hafnium isotopes will lead to a substantial residual negative reactivity worth ratio of the BP. However, the ${ }^{177} \mathrm{Hf}(\mathrm{n}, \gamma){ }^{178} \mathrm{Hf}$ reaction leads to ${ }^{178} \mathrm{Hf}$, which has a lower capture cross section than that of ${ }^{177} \mathrm{Hf}$. Further transmutation to ${ }^{179} \mathrm{Hf}$ and ${ }^{180} \mathrm{Hf}$ will lead to progressively smaller thermal neutron capture cross sections. Enriching hafnium in ${ }^{177} \mathrm{Hf}$ should thus lead to a lower residual reactivity burden than that of hafnium with natural isotopic abundances.

In all the neutronics calculations presented in this section, a theoretical density of $9.69 \mathrm{~g} / \mathrm{cm}^{3}$ was used for hafnia $\left(\mathrm{HfO}_{2}\right)$, and $13.11 \mathrm{~g} / \mathrm{cm}^{3}$ for metallic hafnium. For $\mathrm{UO}_{2}$ a theoretical density of $10.97 \mathrm{~g} / \mathrm{cm}^{3}$ was used. The actual density fraction of the fuel pellets and of the burnable poison pellets was $95 \%$ of theoretical density.

In this section, we will investigate the potential benefits of using enriched hafnium as a burnable absorber. Four different configurations of the hafnium burnable poison, similar to those of the case of $\mathrm{Gd}$, were investigated.

1. A homogeneous mixture of the burnable poison hafnia inside the fuel pellets of a limited number of fuel rods. A summary of the results is given in Table 25. The plots of the reactivity worths and the masses of the BP isotopes and their daughters are displayed in Appendix F1.

2. A homogeneous mixture of the burnable poison as an oxide (hafnia) in the outer radial one-third volume of the fuel pellets. A summary of the results is given in Table 26. Plots of the reactivity worths and the masses of the BP isotopes and their daughters are displayed in Appendix F2.

3. An oxide burnable poison coating of hafnia on the surface of the fuel pellets in a limited number of fuel rods, similar to the $\mathrm{ZrB}_{2}$ coatings. A summary of the results is given in Table 27. Plots of the reactivity worths and the masses of the BP isotopes and their daughters are displayed in Appendix F3. 
4. A homogeneous mixture of the burnable poison hafnium in the form of a metal alloyed with the Zircaloy cladding of a number of fuel rods. The summary results are given in Table 28. Plots of the reactivity worths and the masses of the BP isotopes and their daughters are displayed in Appendix F4.

The results, given in Tables 25 through 28 , show that a reduction of the residual reactivity ratio by a factor of two can be achieved by enriching hafnium in ${ }^{177} \mathrm{Hf}$.

The reactivity worths of the $\mathrm{BP}$ isotopes for a 1.0-mil coating of the fuel pellets with $\mathrm{HfO}_{2}$ (fully enriched in ${ }^{177} \mathrm{Hf}$ ) inside 64 IFBA rods are shown in Figure 62 . The initial reactivity worth of the BP is 0.112 , and the residual reactivity ratio is approximately $8.8 \%$. Figure 62 also displays the masses of the burnable poison isotopes. The total initial mass of ${ }^{177} \mathrm{Hf}$ was 1,212 grams per fuel assembly containing BPs.

The use of hafnium enriched in ${ }^{177} \mathrm{Hf}$ instead of with natural isotopic abundances will thus lead to a reduction of the residual reactivity burden at EOL of the fuel. However, the savings is small.

The MVCs were calculated by decreasing the water coolant density by $10 \%$. Note that for the cases containing 64 and 104 IFBA rods, the MVCs remain negative. 


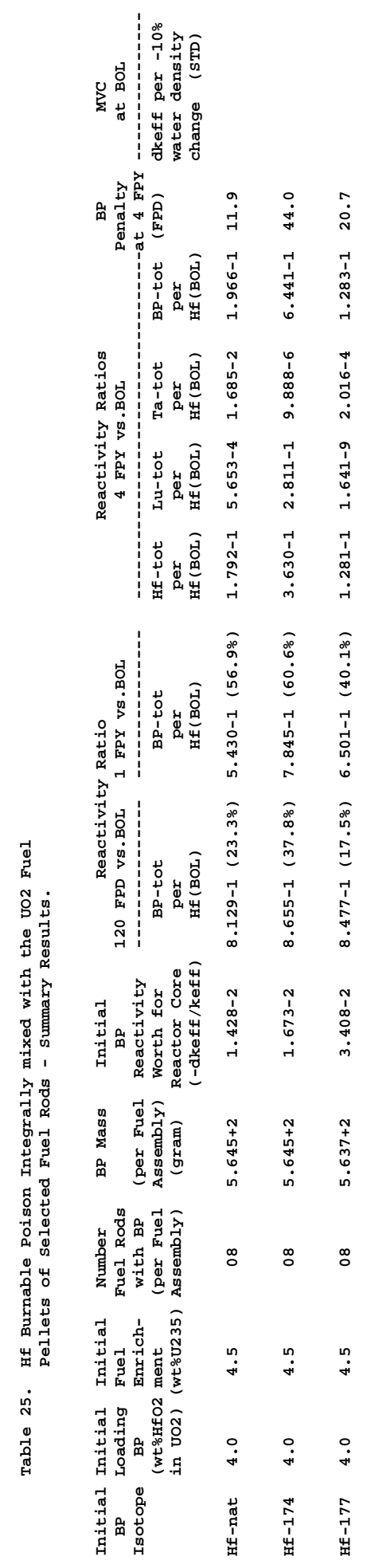




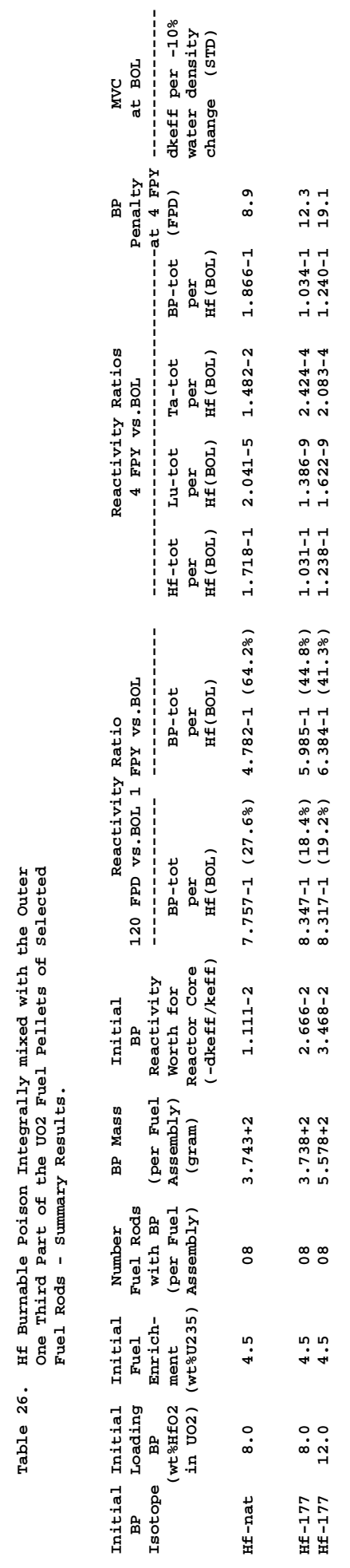



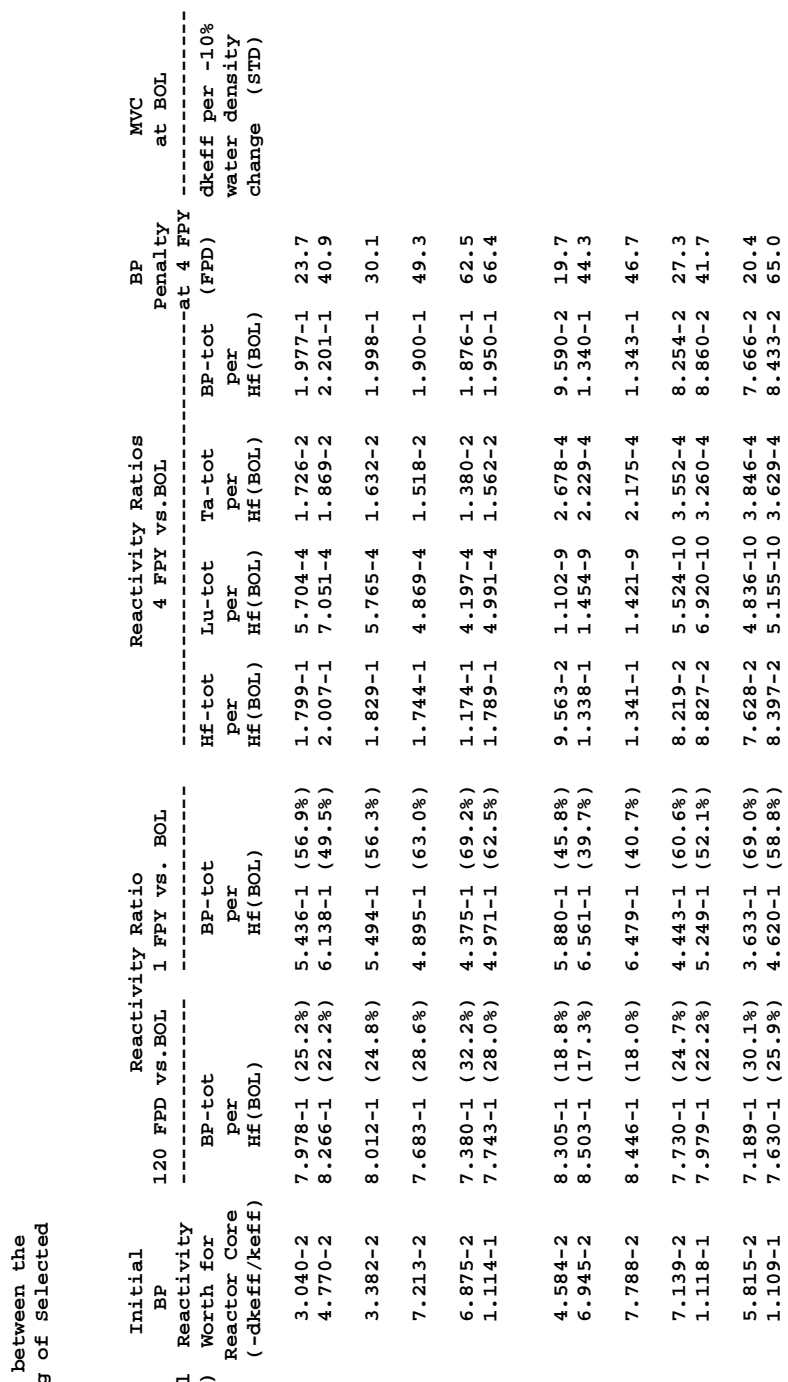

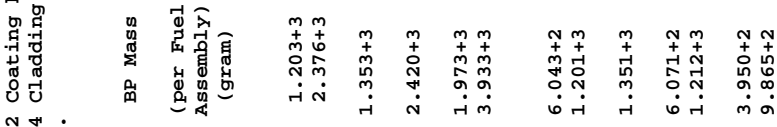

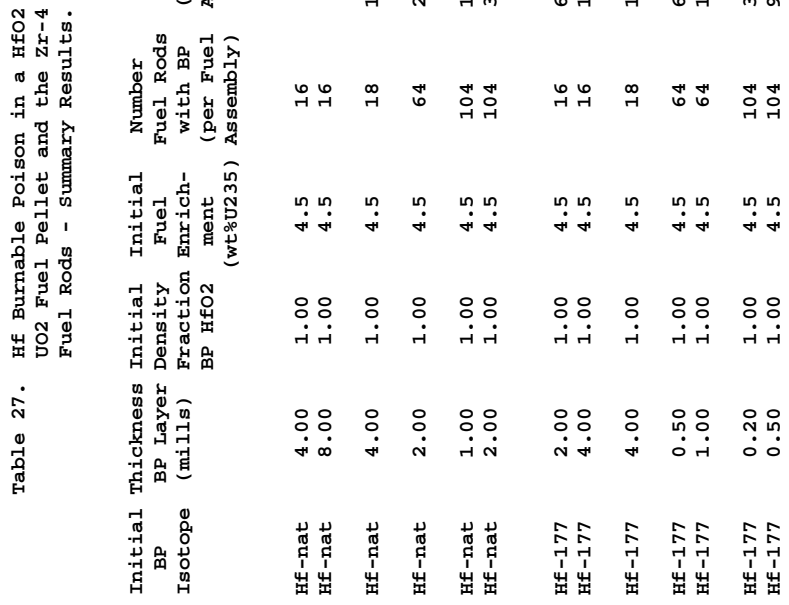




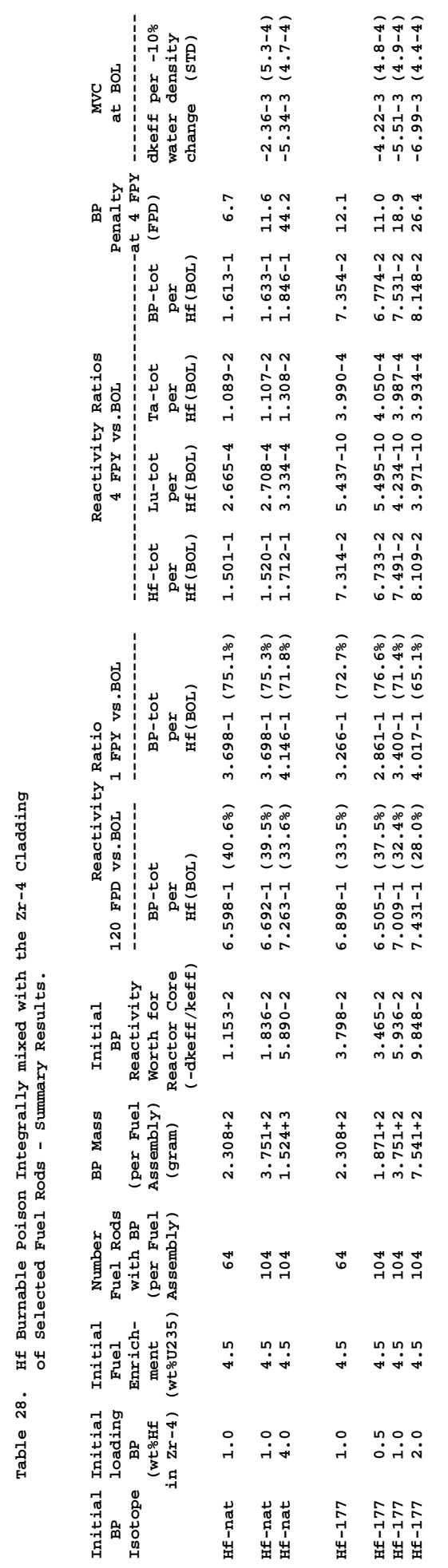


Figure 61. Neutron Cross-Sections as a Function of Energy

Hf-173 (72173.40c)

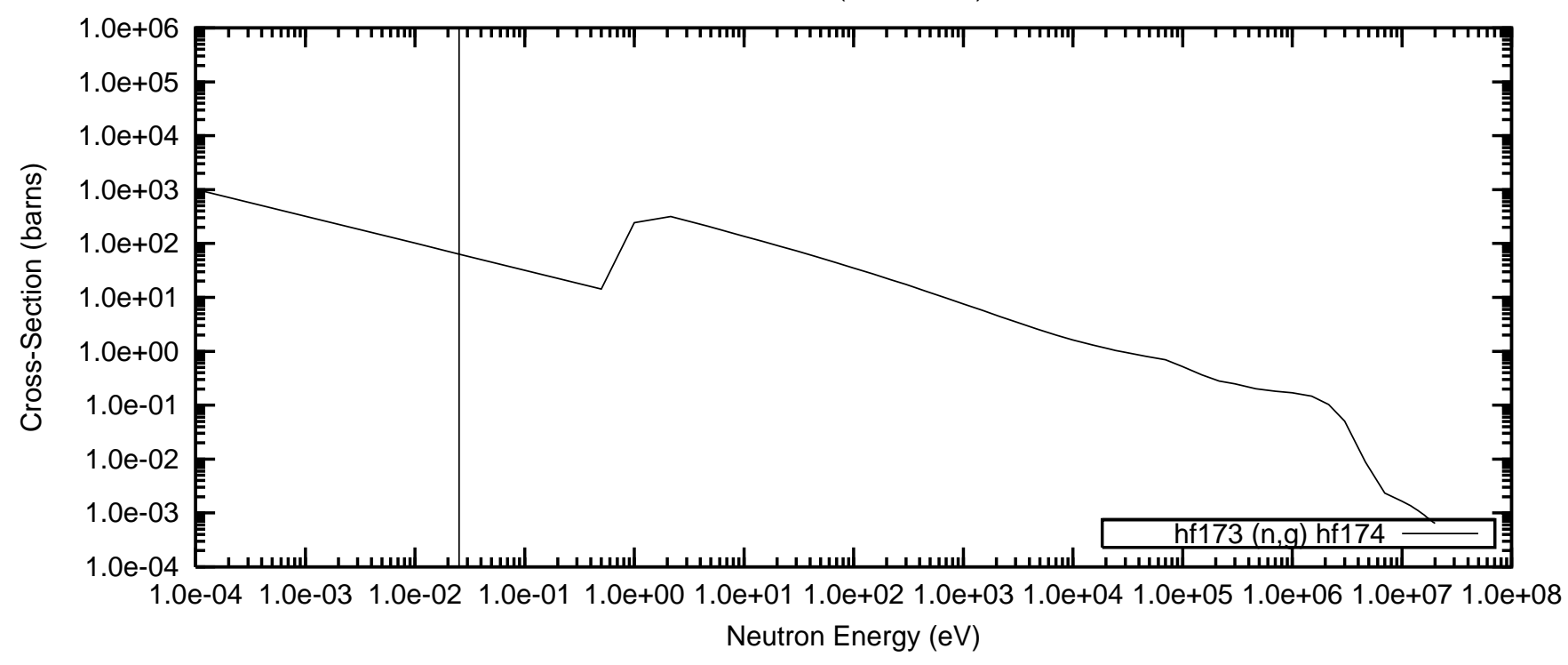

Hf-174 (72174.40c)

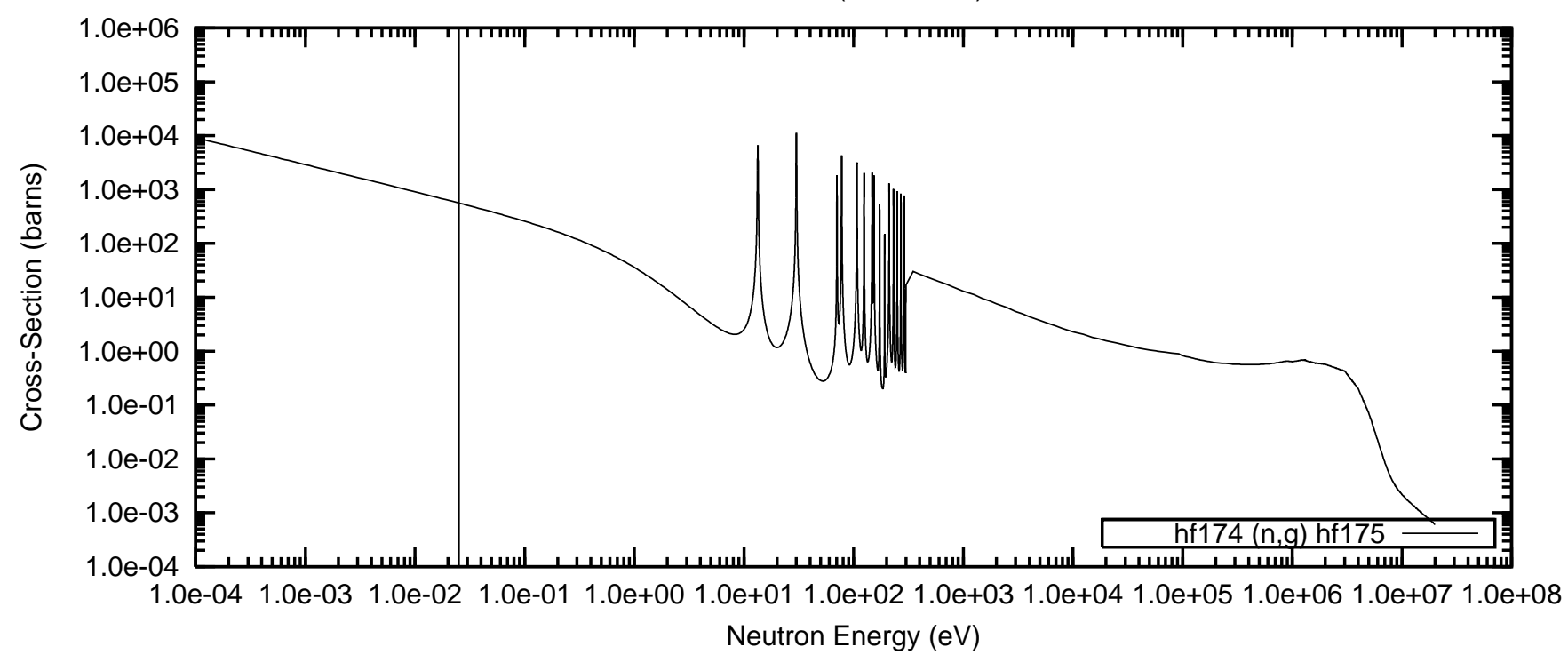

Hf-175 (72175.40c)

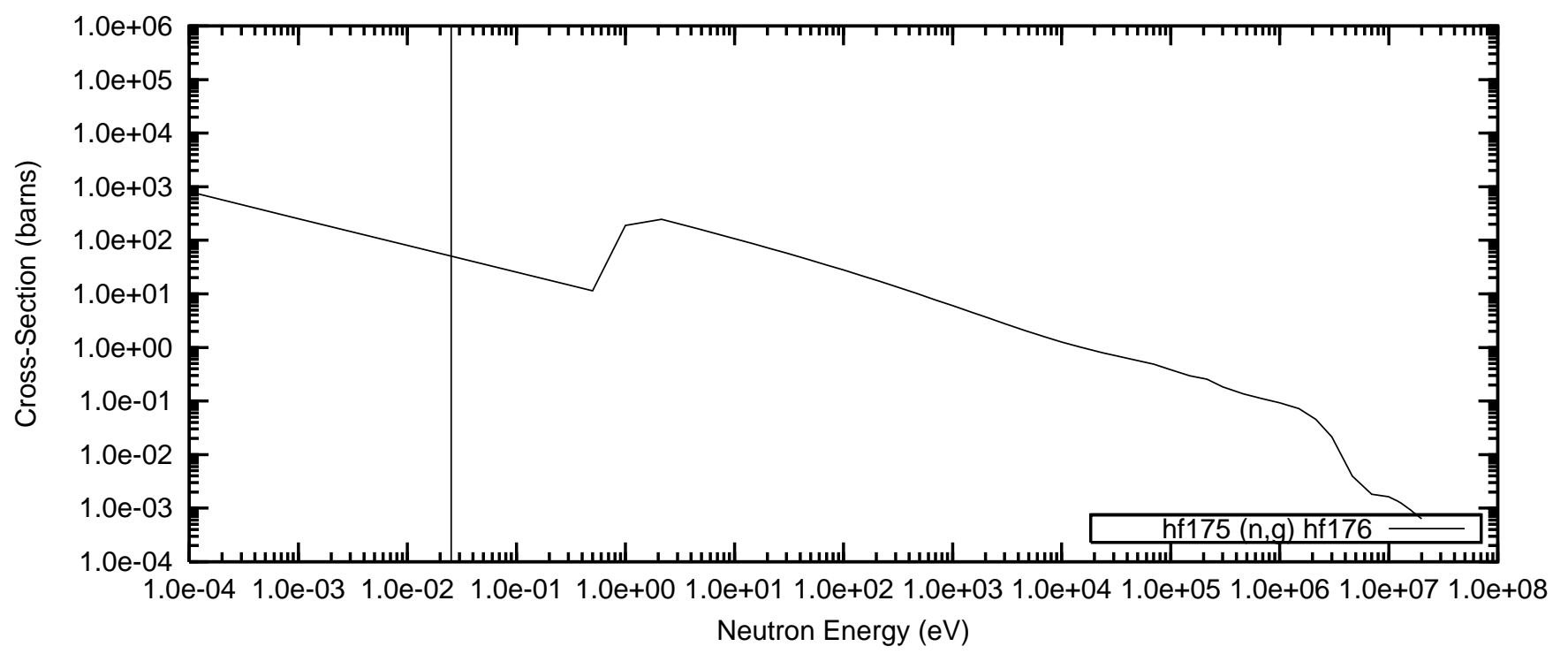


Figure 61(cont). Neutron Cross-Sections as a Function of Energy Hf-176 (72176.40c,46c,47c)

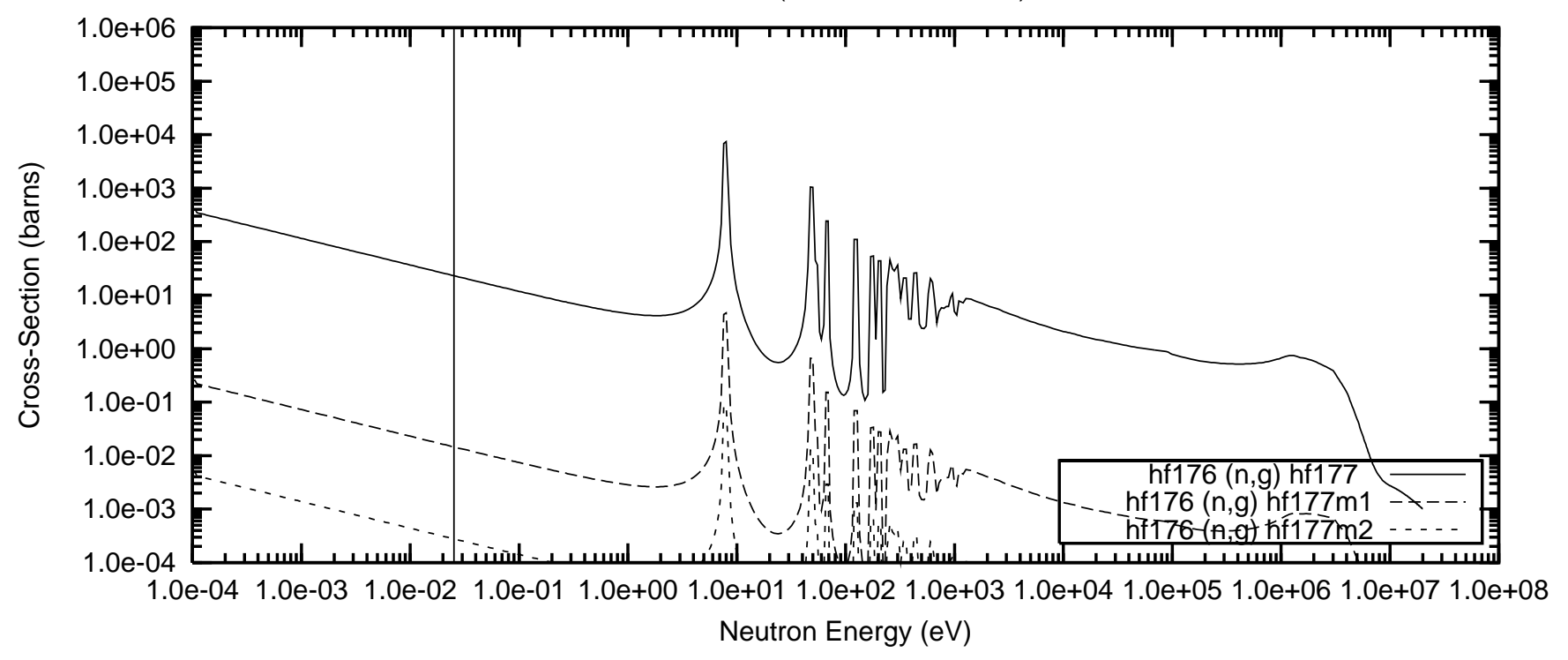

$\mathrm{Hf}-177$ (72177.40c,46c,47c)

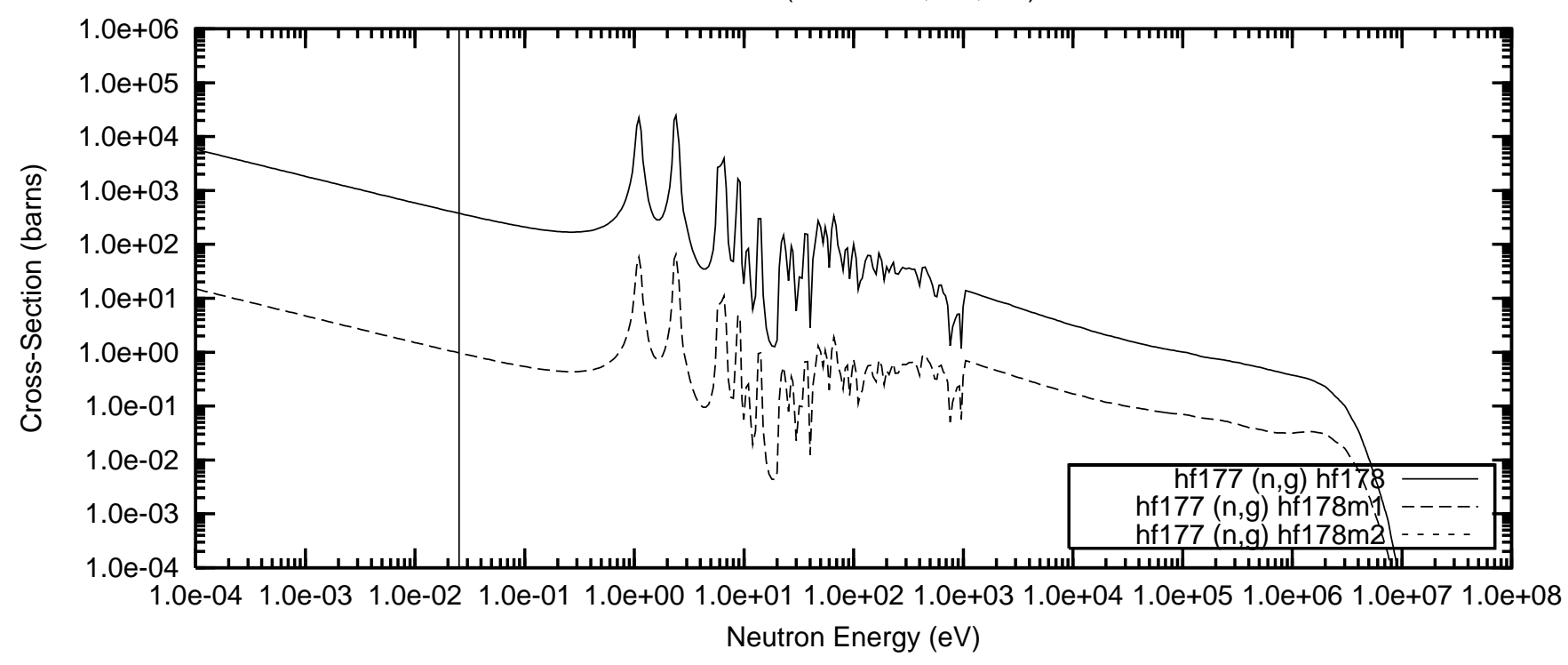

Hf-178 (72178.40c,46c,47c)

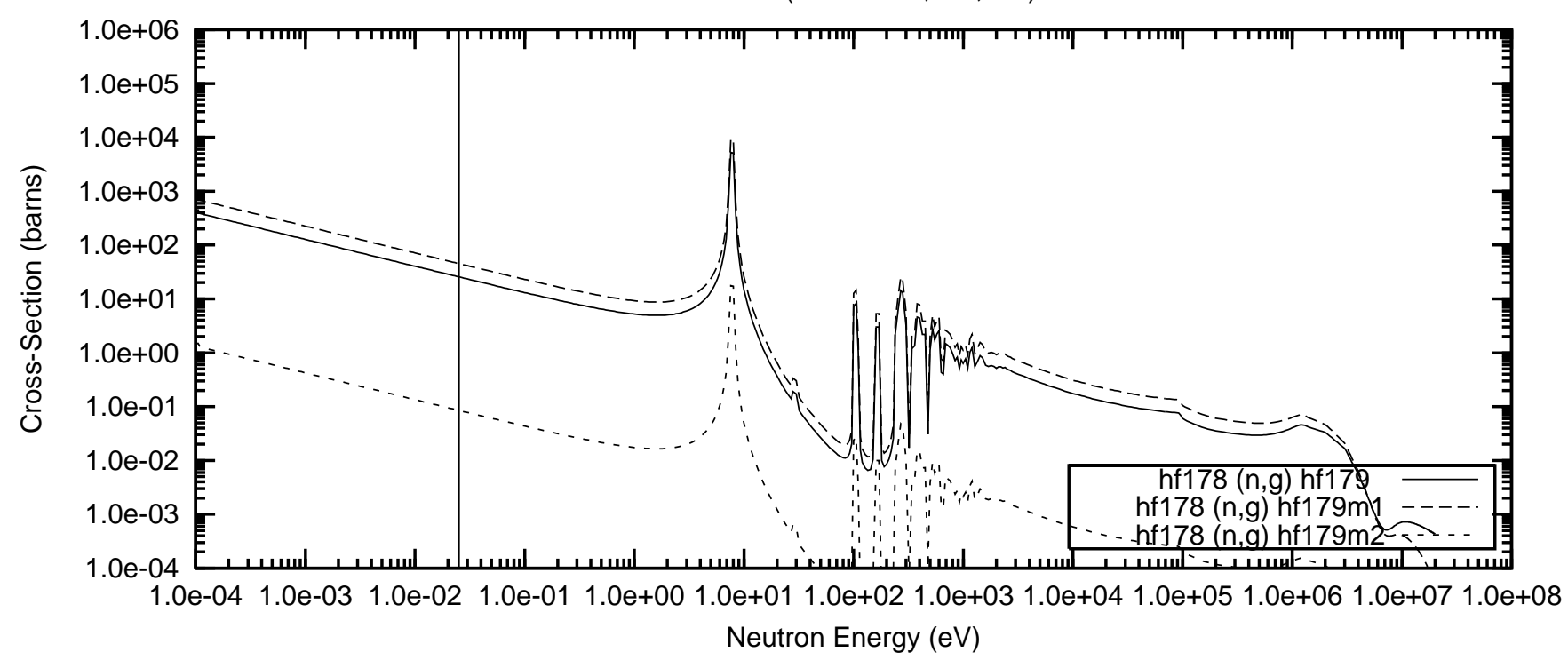


Figure 61(cont). Neutron Cross-Sections as a Function of Energy Hf-178m2 (72178.42c,48c,49c)

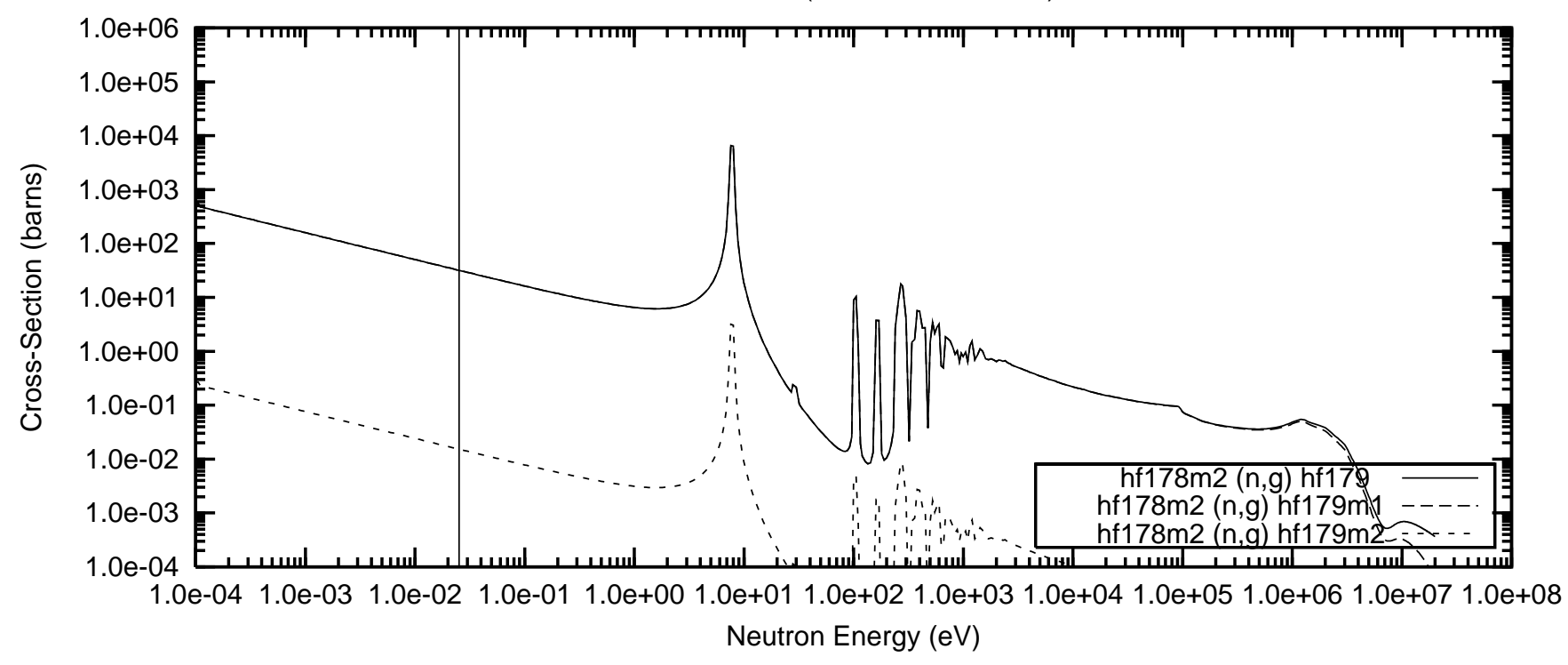

Hf-179 (72179.40c,46c)

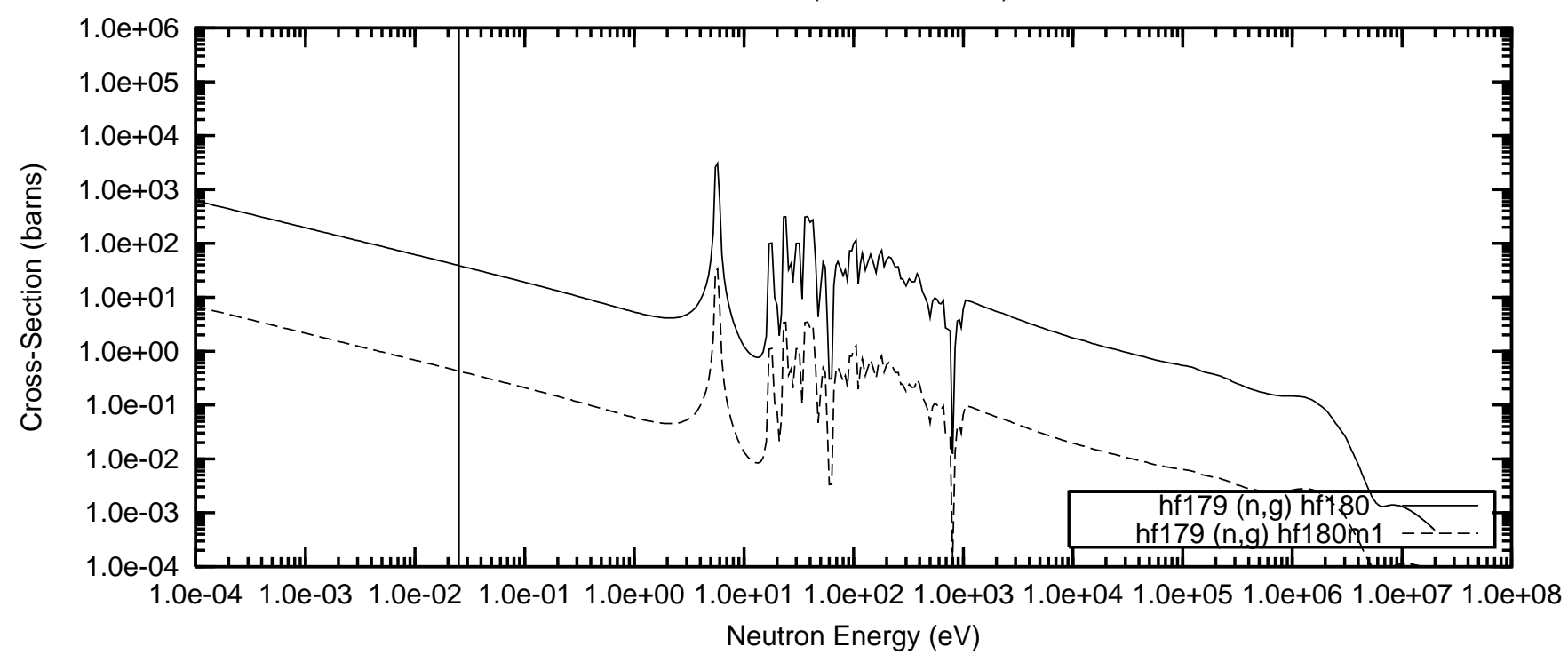

$\mathrm{Hf}-179 \mathrm{~m} 2(\mathbf{7} 2179.42 \mathrm{c}, 48 \mathrm{c})$

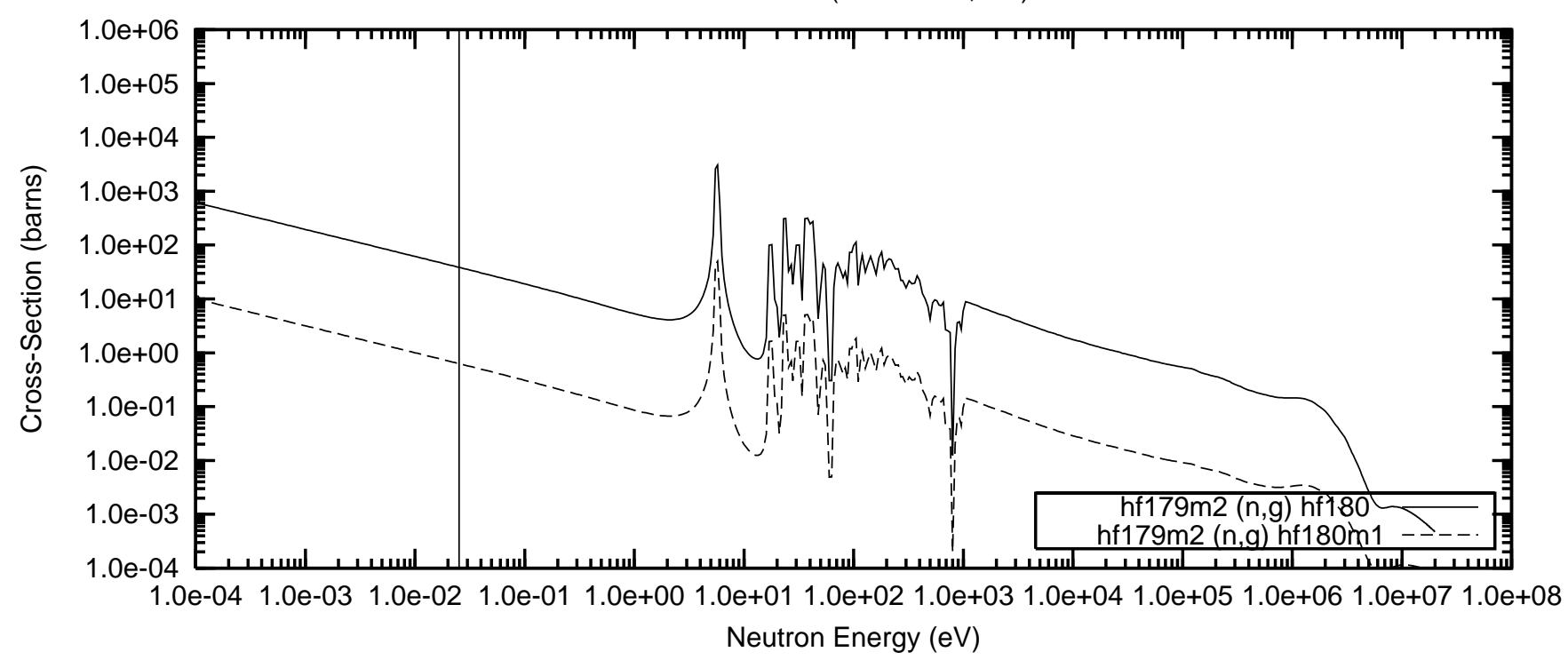


Figure 61(cont). Neutron Cross-Sections as a Function of Energy $\mathrm{Hf}-180$ (72180.40c)

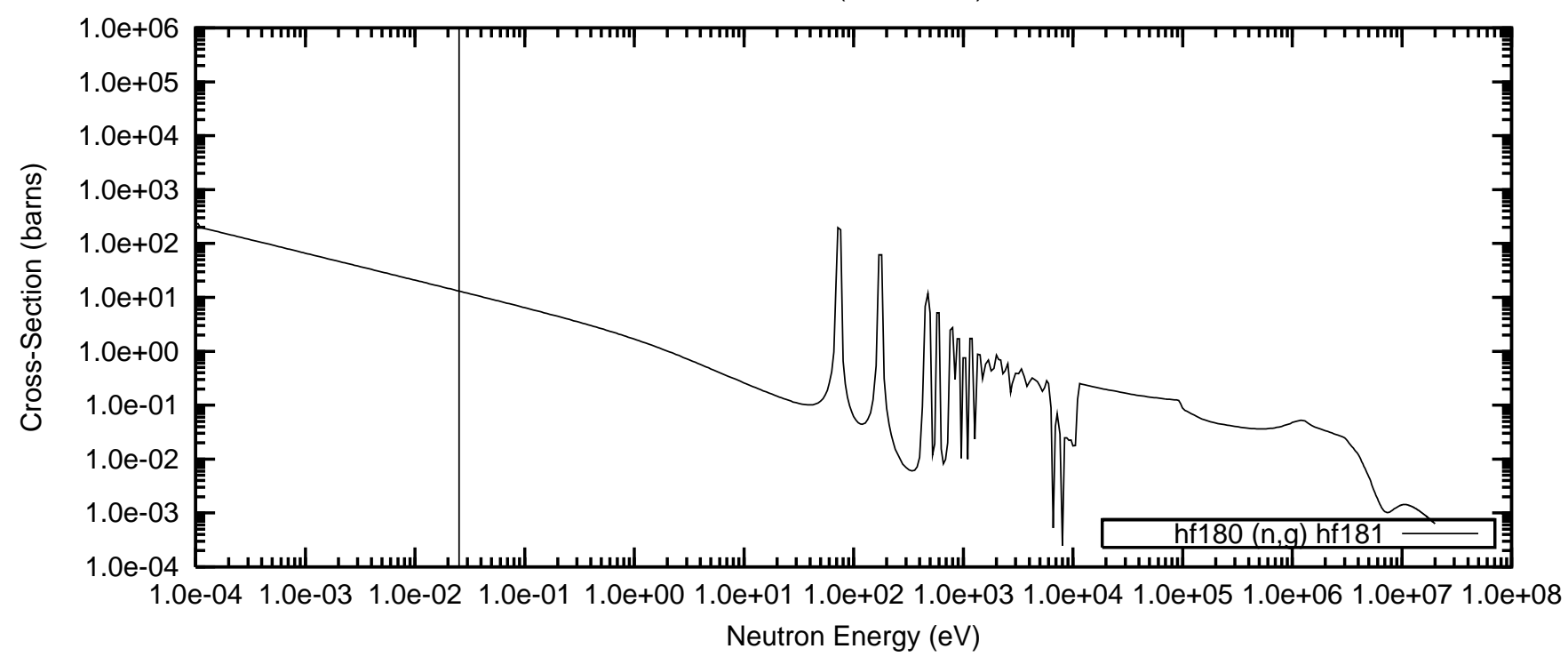

Hf-180m1' (72180.41c)

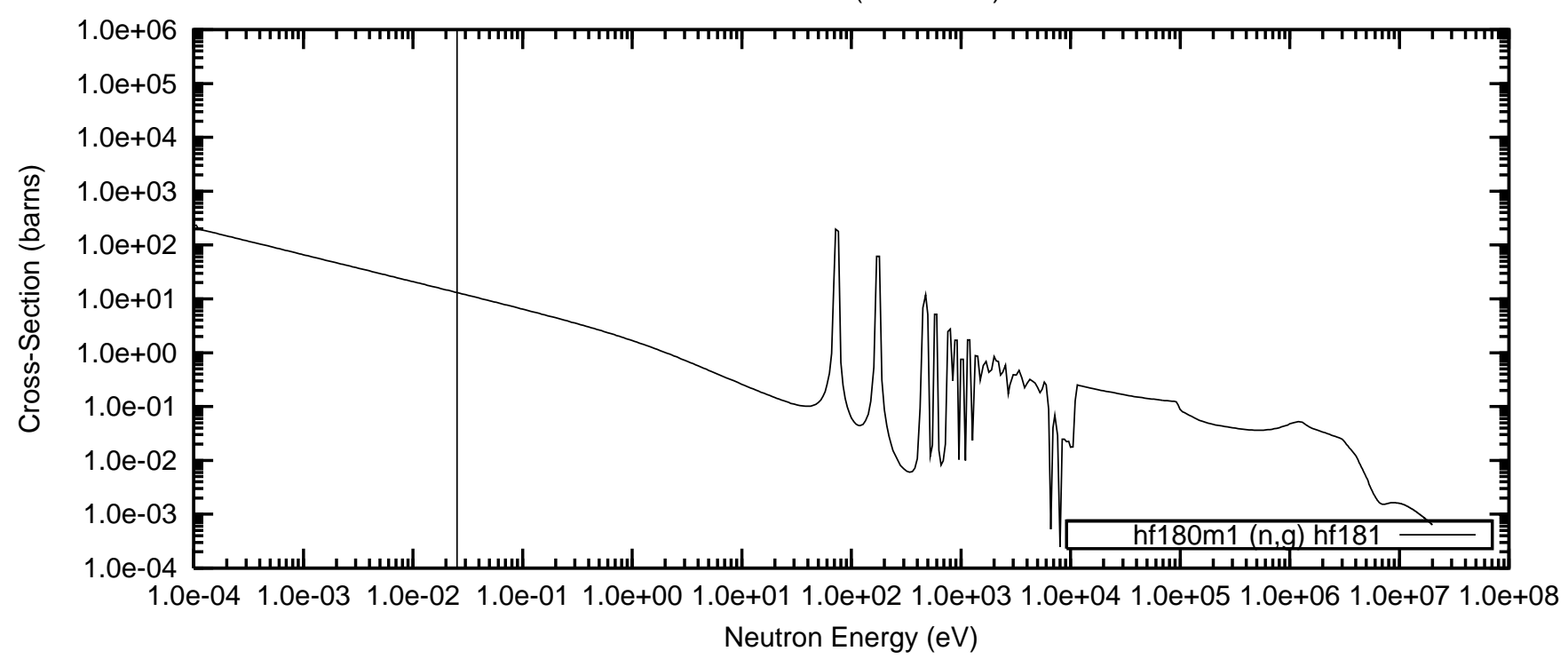

Hf-181 (72181.40c,46c)

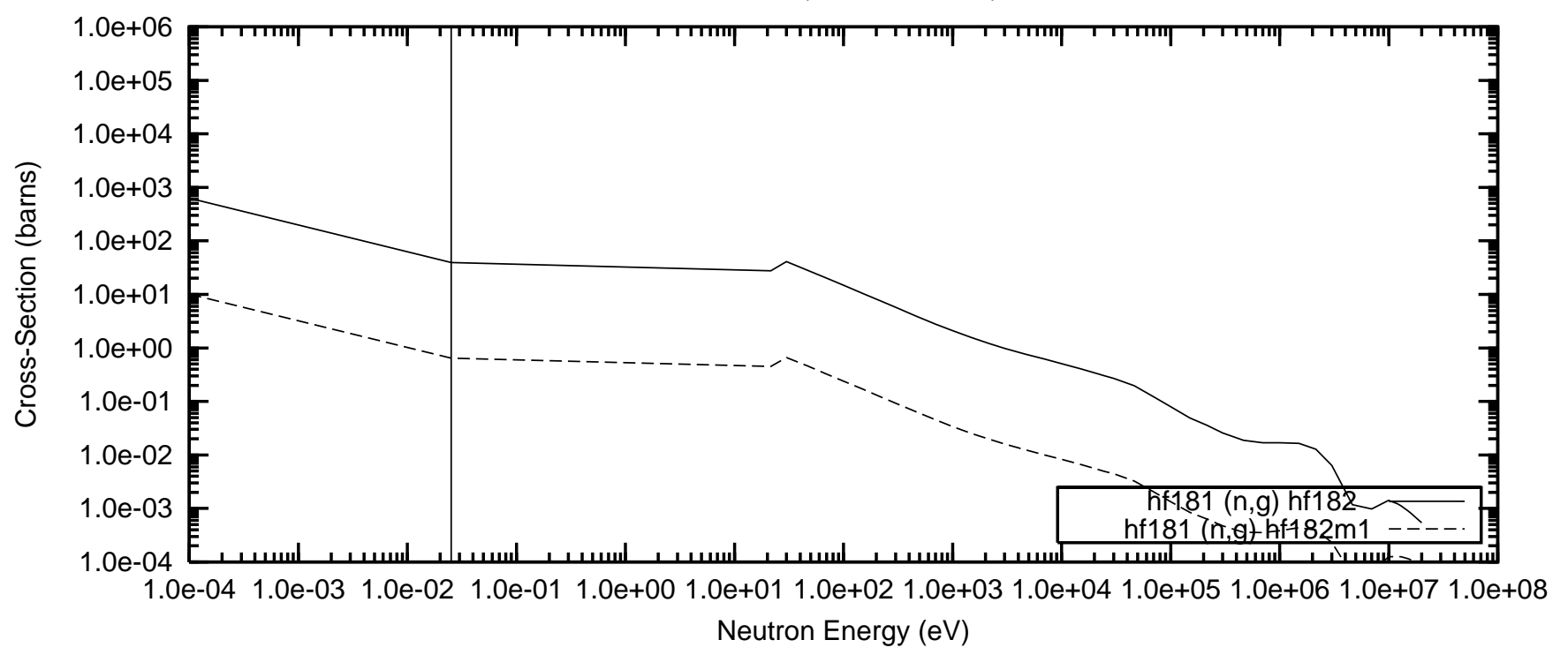


Figure 61 (cont). Neutron Cross-Sections as a Function of Energy

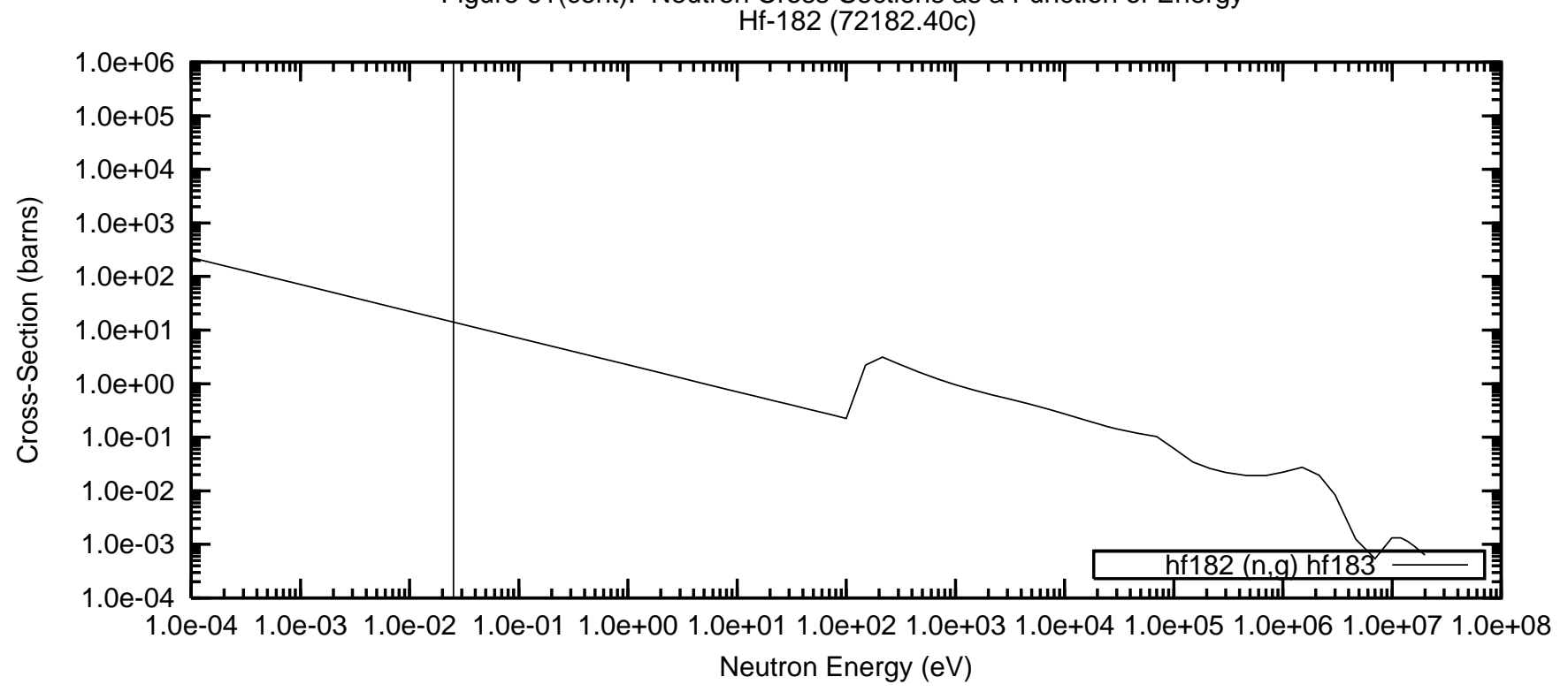


Figure 62. Negative Reactivity and Masses BP for $\mathrm{Hf}-177$ and transmutation daughters as a function of fuel life for $17 \times 17$ fuel assemblies with 64 poison rods,

1.0 mill thick $\mathrm{HfO} 2$ poison coating on the outer radial surface of the UO2 pellets

Reactor power 3400 MWth, 193 fuel assemblies, initial enrichment 4.5wt\%U-235

(Case : hf177_1f_64_bp_fuel o)
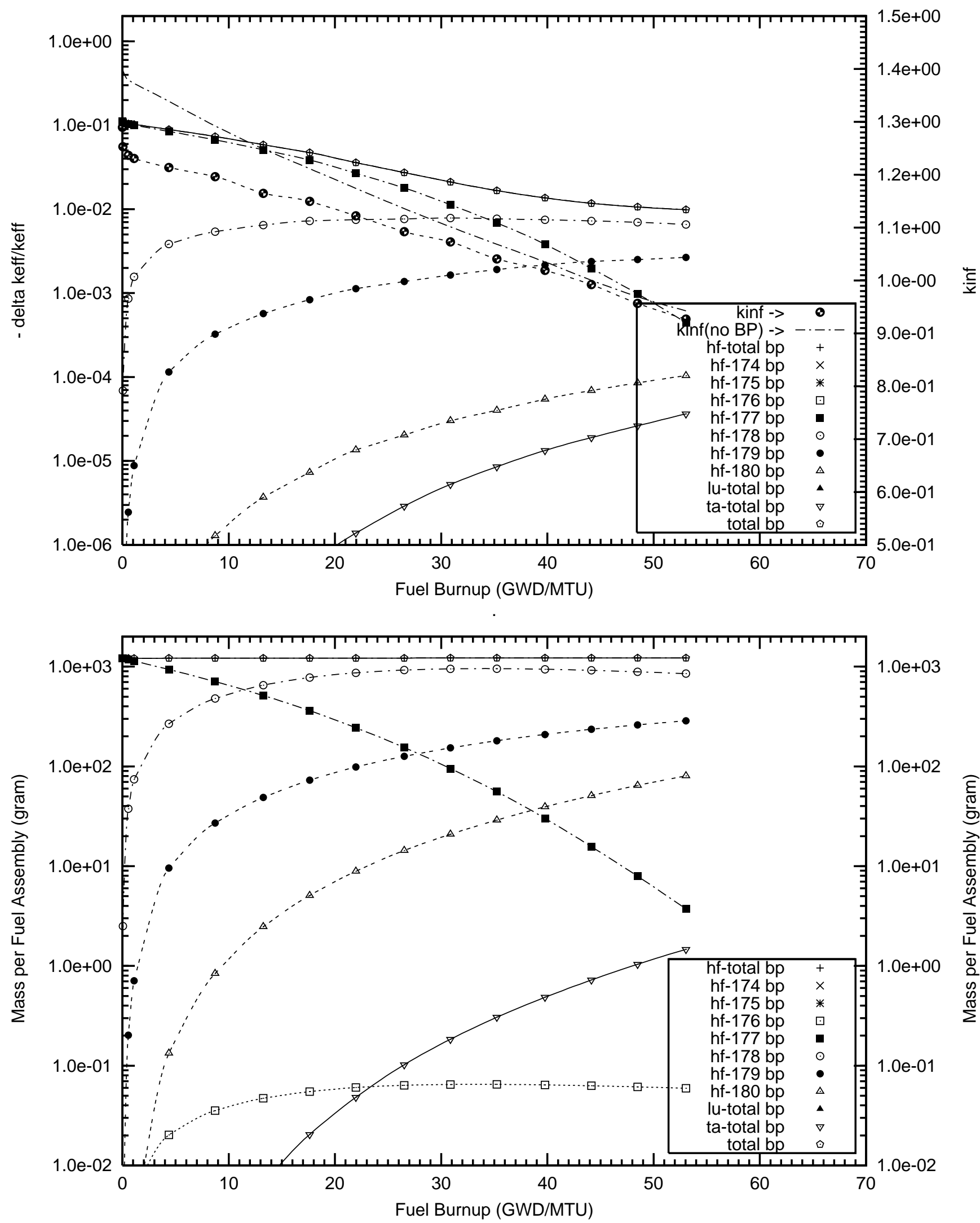


\section{NEUTRONICS PERFORMANCE RESULTS FOR OTHER ELEMENTS}

During this project, we investigated the potential of enriching other elements for use as burnable poisons. We have discarded them, however, mostly for economic reasons, with the exception of lutetium, which, although costly, was considered worth evaluation.

The use of lutetium as a burnable poison was investigated. The lutetium stable isotopes are ${ }^{175} \mathrm{Lu}$ and

${ }^{176} \mathrm{Lu}$, with natural isotopic abundances of $97.4 \%$ and $2.6 \%$, respectively. ${ }^{175} \mathrm{Lu}$ has a thermal neutron capture cross section of 30 barns and a RI of 610 barns. The (n, $\gamma)$ reaction on ${ }^{176} \mathrm{Lu}$ leads to ${ }^{177} \mathrm{Lu}$ and ${ }^{177 \mathrm{ml}} \mathrm{Lu}$. The ${ }^{176} \mathrm{Lu}(\mathrm{n}, \gamma){ }^{177} \mathrm{Lu}$ reaction has a thermal neutron capture cross section of 2,090 barns and a RI of 1,090 barns. ${ }^{177} \mathrm{Lu}$ decays to ${ }^{177} \mathrm{Hf}$ with a half-life of 6.71 days. Another branch in the $(\mathrm{n}, \gamma)$ reaction on ${ }^{176} \mathrm{Lu}$ leads to ${ }^{177 \mathrm{ml}} \mathrm{Lu}$ with a thermal capture cross section of 7.0 barns. ${ }^{177 \mathrm{~m} 1} \mathrm{Lu}$ decays to ${ }^{177} \mathrm{Hf}$ with a half-life of 160.7 days. The ${ }^{177} \mathrm{Hf}(\mathrm{n}, \gamma){ }^{178} \mathrm{Hf}$ reaction leads to ${ }^{178} \mathrm{Hf}$ with a thermal neutron capture cross section of 373 barns and a RI of 7,173 barns. The ${ }^{178} \mathrm{Hf}(\mathrm{n}, \gamma){ }^{179} \mathrm{Hf}$ reaction leads to ${ }^{179} \mathrm{Hf}$ with a thermal capture cross section of 84 barns and a RI of 1,950 barns. The sequence ${ }^{176} \mathrm{Lu} \rightarrow{ }^{177} \mathrm{Lu} \rightarrow{ }^{177} \mathrm{Hf} \rightarrow{ }^{178} \mathrm{Hf} \rightarrow{ }^{179} \mathrm{Hf}$ will lead via neutron transmutations and decay to nuclides with smaller and smaller thermal neutron capture cross sections. The ${ }^{175} \mathrm{Lu}(\mathrm{n}, \gamma){ }^{176} \mathrm{Lu}$ reaction will thus transmute ${ }^{175} \mathrm{Lu}$, which has a small thermal cross section, into ${ }^{176} \mathrm{Lu}$, which has a much larger capture cross section. In order to achieve a desired initial reactivity worth of the BP, the use of natural lutetium will require a much larger amount of lutetium than lutetium fully enriched in ${ }^{176} \mathrm{Lu}$. The transmutation from ${ }^{175} \mathrm{Lu}$ into the ${ }^{176} \mathrm{Lu} \rightarrow{ }^{177} \mathrm{Lu} \rightarrow{ }^{177} \mathrm{Hf} \rightarrow{ }^{178} \mathrm{Hf} \rightarrow{ }^{179} \mathrm{Hf}$ sequence will thus lead to a substantially greater residual reactivity fraction of the BP. Enriching lutetium in ${ }^{176} \mathrm{Lu}$ will thus reduce substantially the residual reactivity worth ratio of the BP.

A summary of the results for lutetium is shown in Table 29. The table shows that the decrease of the residual reactivity ratio of lutetium fully enriched in ${ }^{176} \mathrm{Lu}$ is substantial when compared with the use of lutetium with natural isotopic abundances. Plots for the reactivity worths and the masses of the BP isotopes and their daughters are displayed in Appendix G1.

The reactivity worths of the BP isotopes, for a 0.5 -mil thick coating with $\mathrm{Lu}_{2} \mathrm{O}_{3}$ (fully enriched in ${ }^{176} \mathrm{Lu}$ ) of the fuel pellets in 64 IFBA rods, are shown in Figure 63. The masses of the BP isotopes are also displayed in Figure 63. The initial reactivity worth of the BP was 0.171, and the residual reactivity ratio is approximately $3.8 \%$. The total mass of ${ }^{176} \mathrm{Lu}$ per fuel assembly was 614 grams. Figure 64 displays the reactivity worths and the masses of the BP for a 1.0-mil coating of enriched $\mathrm{Lu}_{2} \mathrm{O}_{3}$ in 64 IFBA rods. The initial reactivity worth of the BP was 0.241 , and the residual reactivity ratio is approximately $4.4 \%$. The total mass of ${ }^{176} \mathrm{Lu}$ per fuel assembly was 1,226 grams.

Note that the shapes of the fuel assembly lattice $\mathrm{k}_{\mathrm{inf}}$ 's for theses cases are fairly flat. However, the natural isotopic abundance of ${ }^{176} \mathrm{Lu}$ is only $2.6 \%$. Enriching lutetium in ${ }^{176} \mathrm{Lu}$ will probably be expensive.

Other elements and isotopes, such as $\mathrm{Nd}$ and ${ }^{143} \mathrm{Nd}$ and $\mathrm{Os}$ and ${ }^{184} \mathrm{Os}$, were also investigated. We have discarded them because of the very low natural abundance or the very small reduction in the residual reactivity worth ratios of the $\mathrm{BP}$. 


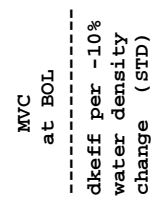

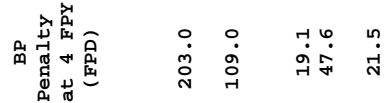

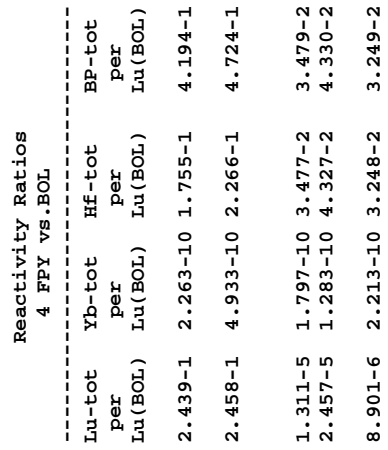

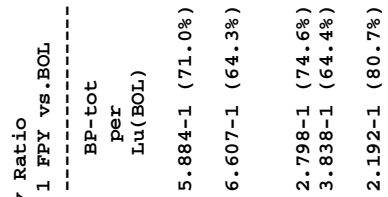

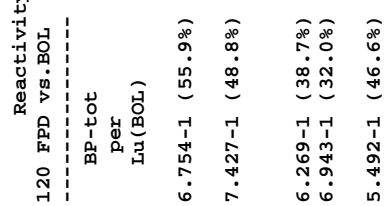

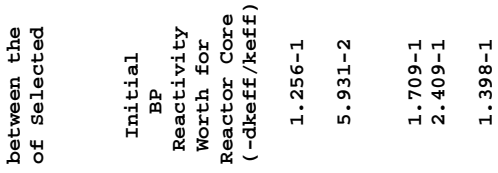

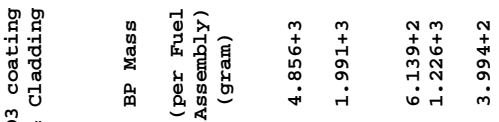

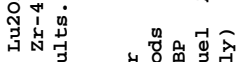

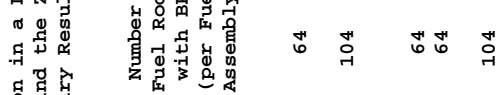

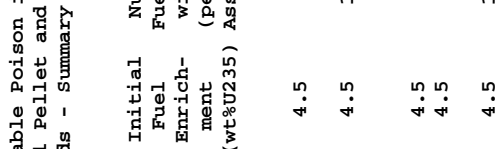

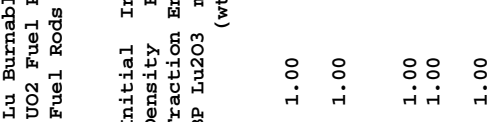

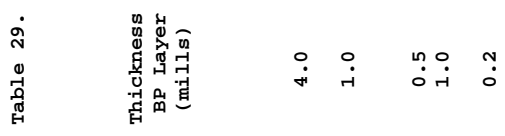

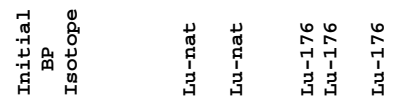


Figure 63. Negative Reactivity and Masses BP for Lu-176 and transmutation daughters as a function of fuel life for $17 \times 17$ fuel assemblies with 64 poison rods,

0.5 mill thick Lu2O3 poison coating on the outer radial surface of the UO2 pellets Reactor power 3400 MWth, 193 fuel assemblies, initial enrichment 4.5wt\%U-235

(Case : lu176_0f_64_bp_fuel o)
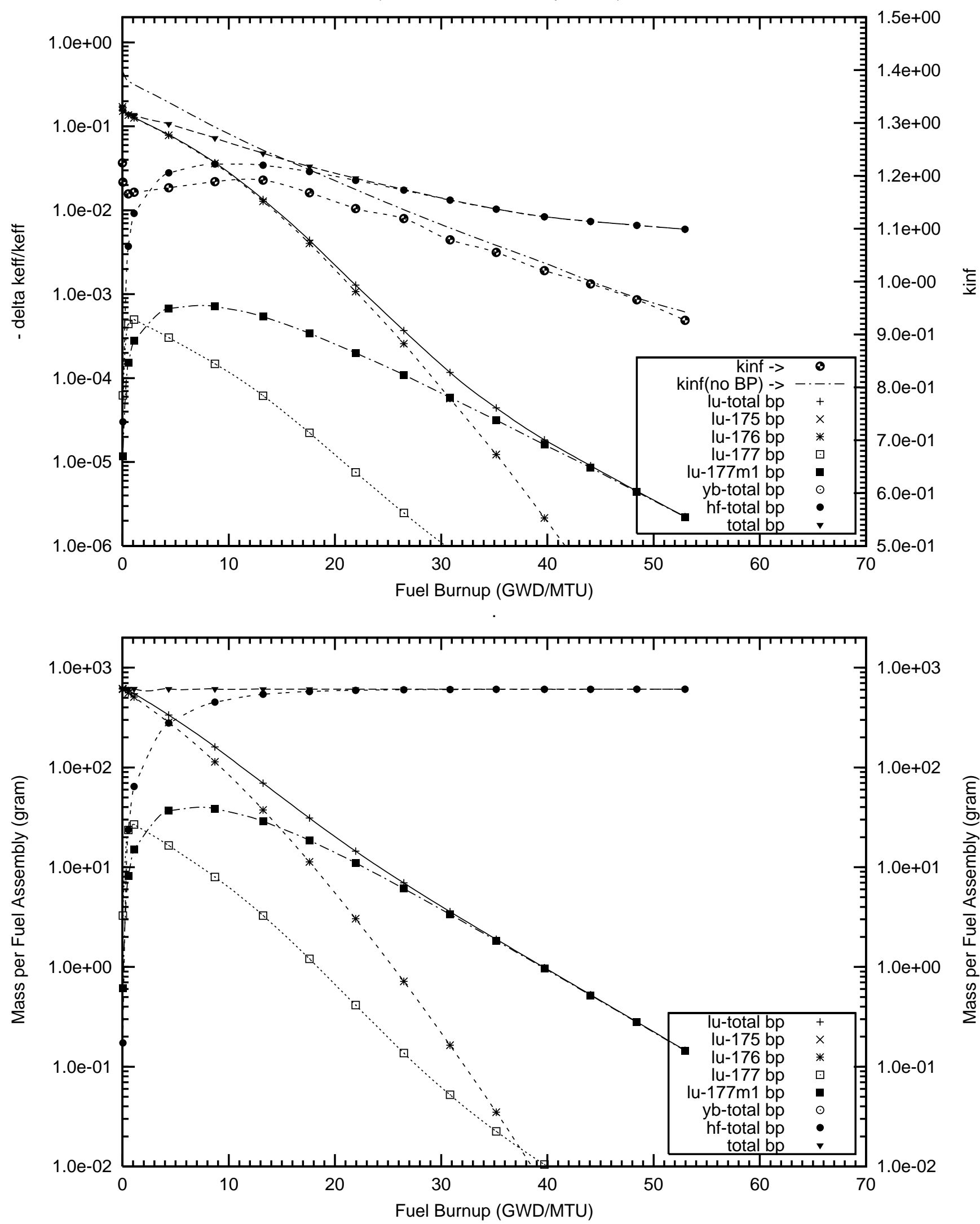
Figure 64. Negative Reactivity and Masses BP for Lu-176 and transmutation daughters as a function of fuel life for $17 \times 17$ fuel assemblies with 64 poison rods,

1.0 mill thick Lu2O3 poison coating on the outer radial surface of the UO2 pellets Reactor power 3400 MWth, 193 fuel assemblies, initial enrichment 4.5wt\%U-235

(Case : lu176_1f_64_bp_fuel o)
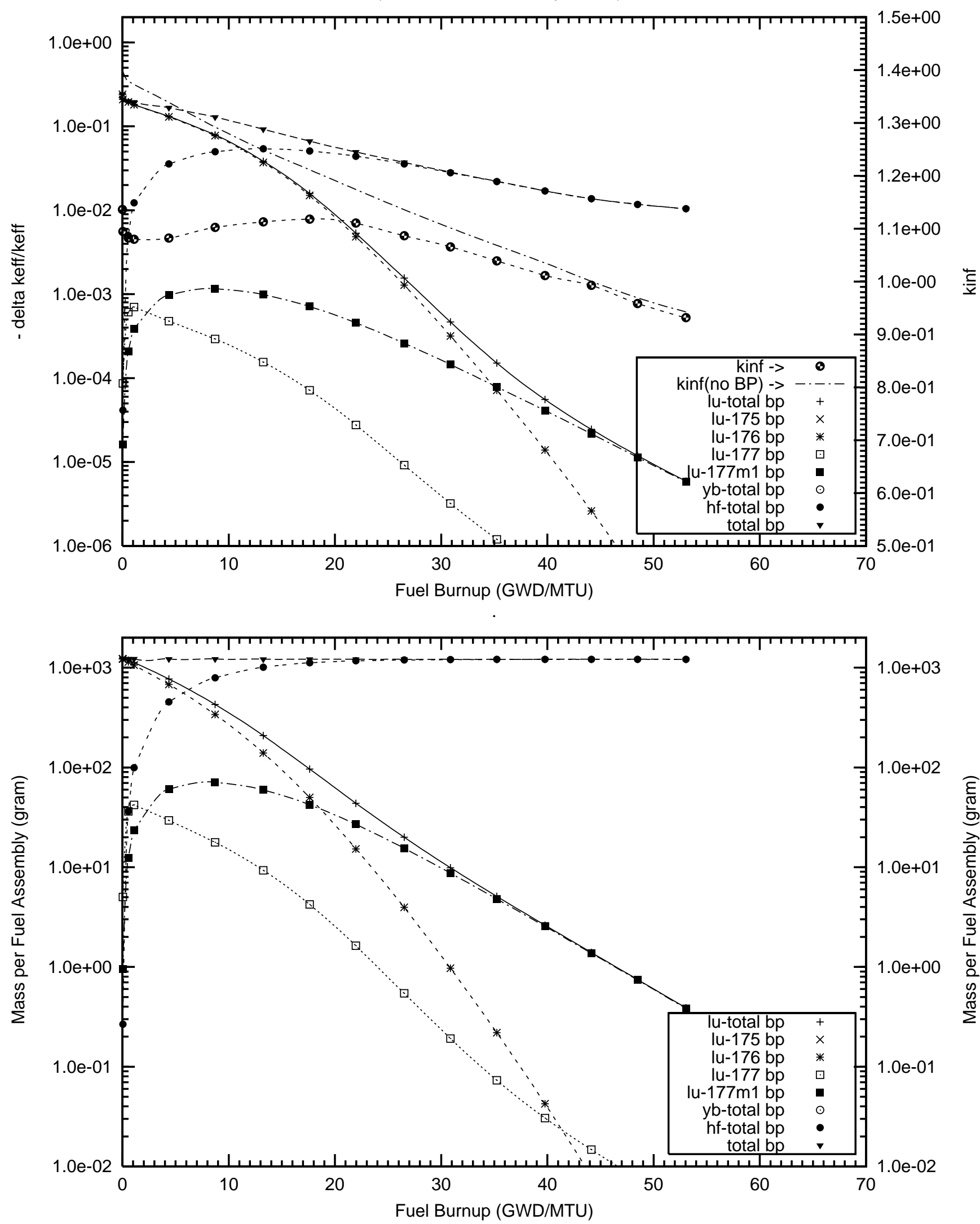



\section{DISCUSSION}

The primary objective of the project was to evaluate separated isotopes of candidate burnable poisons. This was achieved with pleasing results. In addition, much was learned about configurations of burnable poisons. This is especially evident in the suggestion and analysis of incorporating burnable poisons in the cladding. It is also noteworthy that the fuel life used was 4 years with a limited number of cases explored with $6 \%$ enrichment to achieve a 5-year fuel life.

Tables 30 through 48 show a summary of input parameters and results for the four different configurations of the natural elements and separated isotopes of ${ }^{157} \mathrm{Gd},{ }^{149} \mathrm{Sm},{ }^{167} \mathrm{Er},{ }^{164} \mathrm{Dy},{ }^{177} \mathrm{Hf}$, and

${ }^{151} \mathrm{Eu}$. A measure of the isotope separation benefit is the ratio of the residual negative reactivity worth at EOL to that at BOL for a separated isotope divided by a similar ratio for the naturally occurring element. From these tables it can be observed that the residual reactivity worth ratio changes slightly with BP loading and that it is dependent on the number of IFBA rods and on the nature of the BP isotopes. The ratio decreases when the poison is spread over more IFBA rods. When the number of fuel pins containing BP increases, the BP is spread thinner, and this reduces the rod self-shielding.

Another important parameter that must be considered in selecting a burnable poison is the reactivity worth burnout rate of the BP (including the BP daughters). The rate of burnup is considered by displaying the ratio of the negative reactivity worth of the BP at three different burnup time intervals of interest (e.g., 120 days, 1 year, and 4 years) to the reactivity worth of the BP at BOL. The ratio depends on the BP loading, the number of IFBA rods per fuel assembly, the BP loading type, and the $\mathrm{BP}$ element or isotopes. 
Table 30. The effect of isotope separation for the case of burnable poison in the form of gadolinium oxide homogeneously mixed with the fuel.

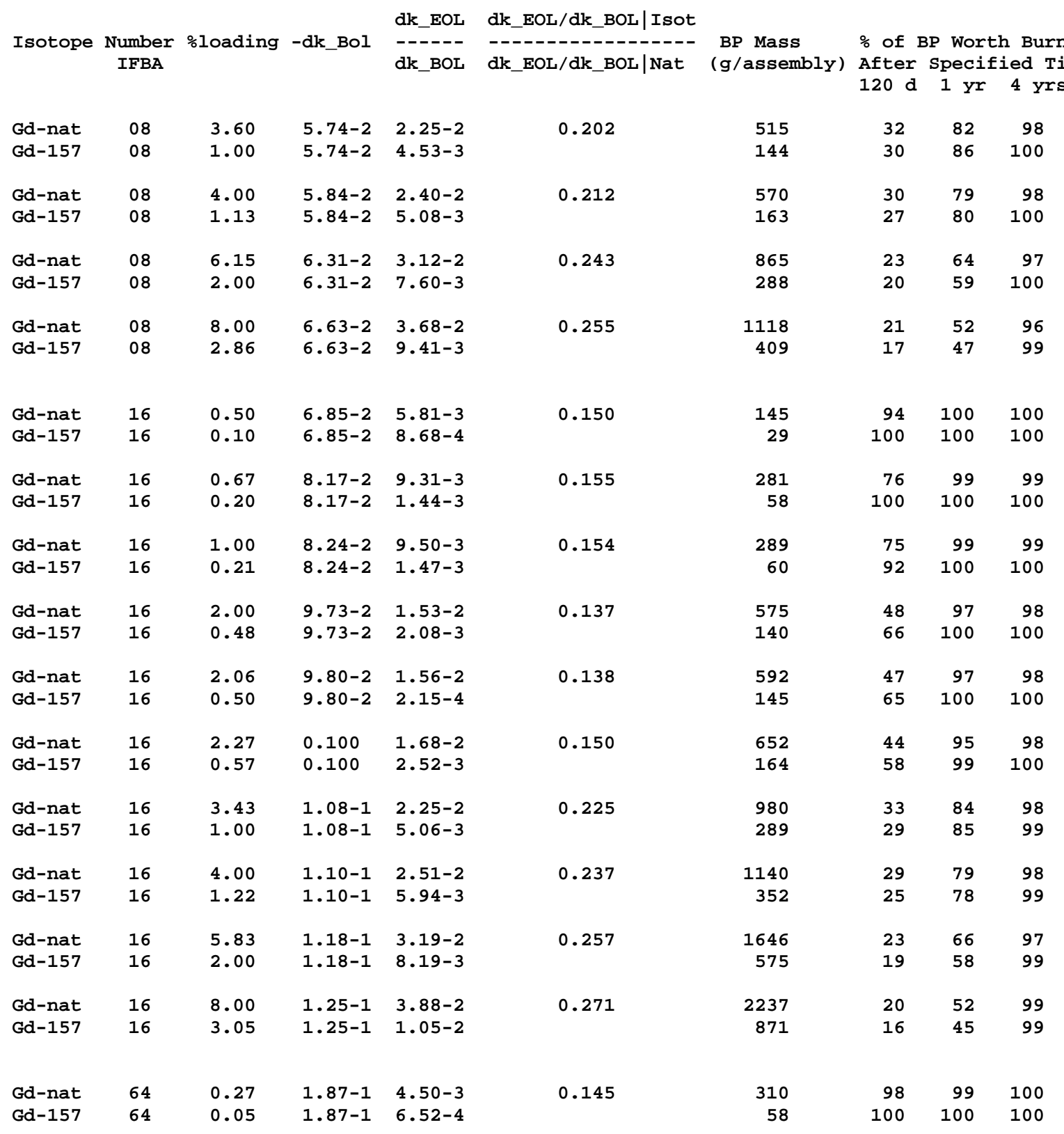


Table 31. The effect of isotope separation for the case of burnable poison in the form of samarium oxide homogeneously mixed with the fuel.

\begin{tabular}{|c|c|c|c|c|c|c|c|c|c|}
\hline Isotope & $\begin{array}{l}\text { Number } \\
\text { IFBA }\end{array}$ & oloading & -dk_Bol & $\begin{array}{l}\text { dk_EOL } \\
------ \\
\text { dk_BOL }\end{array}$ & $\begin{array}{l}\text { dk_EOL/dk_BOL|Isot } \\
\text { dk_EOL/dk_BOL/Nat }\end{array}$ & $\begin{array}{l}\text { BP Mass } \\
(g / a s s e m b l y)\end{array}$ & $\begin{array}{l}\text { o of } B \\
\text { After }\end{array}$ & $\begin{array}{l}\text { BP Worth } \\
\text { Specifi }\end{array}$ & $\begin{array}{l}\text { Bur } \\
\text { Led } T\end{array}$ \\
\hline & & & & & & & $120 \mathrm{~d}$ & $1 y^{r}$ & $4 y^{r}$ \\
\hline Sm-nat & 08 & 4.57 & $6.04-2$ & $1.64-1$ & 0.317 & 644 & 41 & 82 & 84 \\
\hline Sm-149 & 08 & 1.00 & $6.04-2$ & $5.21-2$ & & 144 & 30 & 87 & 95 \\
\hline Sm-nat & 08 & 8.00 & $6.74-2$ & $2.30-1$ & 0.320 & 1112 & 24 & 67 & 77 \\
\hline $\mathrm{Sm}-149$ & 08 & 1.85 & $6.74-2$ & $7.36-2$ & & 264 & 21 & 61 & 93 \\
\hline Sm-nat & 16 & 0.65 & $6.89-2$ & $5.23-1$ & 0.213 & 186 & 95 & 93 & 95 \\
\hline $\mathrm{Sm}-149$ & 16 & 0.10 & $6.89-2$ & $1.11-2$ & & 29 & 99 & 99 & 99 \\
\hline Sm-nat & 16 & 1.00 & $7.94-2$ & $6.77-1$ & 0.224 & 287 & 92 & 92 & 93 \\
\hline $\mathrm{Sm}-149$ & 16 & 0.16 & $7.94-2$ & $1.52-2$ & & 45 & 99 & 99 & 99 \\
\hline Sm-nat & 16 & 1.22 & $8.40-2$ & $7.67-2$ & 0.233 & 350 & 89 & 91 & 92 \\
\hline $\mathrm{Sm}-149$ & 16 & 0.20 & $8.40-2$ & $1.79-2$ & & 58 & 95 & 99 & 99 \\
\hline Sm-nat & 16 & 2.00 & $9.57-2$ & $1.06-1$ & 0.260 & 572 & 76 & 88 & 89 \\
\hline Sm-149 & 16 & 0.38 & $9.57-2$ & $2.74-2$ & & 109 & 66 & 98 & 97 \\
\hline Sm-nat & 16 & 2.38 & $1.0-1$ & $1.18-1$ & 0.274 & 680 & 68 & 87 & 88 \\
\hline Sm-149 & 16 & 0.47 & $1 \cdot 0-1$ & $3.24-2$ & & 135 & 55 & 97 & 97 \\
\hline Sm-nat & 16 & 2.52 & $1.013-1$ & $1 \cdot 22-1$ & 0.278 & 718 & 65 & 87 & 88 \\
\hline Sm-149 & 16 & 0.50 & $1.013-1$ & $3.40-2$ & & 144 & 53 & 97 & 97 \\
\hline Sm-nat & 16 & 4.00 & $1.12-1$ & $1.61-1$ & 0.321 & 1133 & 43 & 84 & 84 \\
\hline Sm-149 & 16 & 0.92 & $1.12-1$ & $5.17-2$ & & 265 & 32 & 89 & 95 \\
\hline Sm-nat & 16 & 4.28 & $1.14-1$ & $1.68-1$ & 0.326 & 1212 & 41 & 83 & 83 \\
\hline $\mathrm{Sm}-149$ & 16 & 1.00 & $1.14-1$ & $5.48-2$ & & 287 & 30 & 87 & 95 \\
\hline Sm-nat & 16 & 7.97 & $1.27-1$ & $2.36-1$ & 0.356 & 2215 & 24 & 67 & 76 \\
\hline $\mathrm{Sm}-149$ & 16 & 2.00 & 1. 27-1 & $8.38-2$ & & 571 & 21 & 58 & 92 \\
\hline Sm-nat & 16 & 8.00 & $1.27-1$ & $2.36-1$ & 0.356 & 2224 & 24 & 66 & 76 \\
\hline Sm-149 & 16 & 2.01 & $1.27-1$ & $8.41-2$ & & 574 & 21 & 57 & 92 \\
\hline
\end{tabular}


Table 32. The effect of isotope separation for the case of burnable poison in the form of erbium oxide homogeneously mixed with the fuel.

\begin{tabular}{|c|c|c|c|c|c|c|c|c|c|}
\hline Isotope & $\begin{array}{l}\text { Number } \\
\text { IFBA }\end{array}$ & loading & -dk_Bol & \begin{tabular}{l} 
dk_EOL \\
\hdashline---- \\
$d k \_B O L$
\end{tabular} & 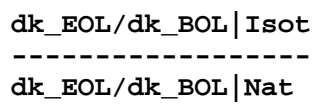 & $\begin{array}{l}\text { BP Mass } \\
(g / a s s e m b l y)\end{array}$ & $\begin{array}{l}\text { \% of } B I \\
\text { After }\end{array}$ & $\begin{array}{l}\text { 3P Worth } \\
\text { Specifi }\end{array}$ & $\begin{array}{l}\text { Bur } \\
\text { ied } T\end{array}$ \\
\hline & & & & & & & $120 \mathrm{~d}$ & $1 y^{x}$ & $4 y$ \\
\hline Er-nat & 16 & 4.08 & $3.83-2$ & $8 \cdot 36-2$ & 0.173 & 1181 & 27 & 58 & 92 \\
\hline Er-167 & 16 & 1.00 & $3.83-2$ & $1.45-2$ & & 292 & 29 & 63 & 99 \\
\hline Er-nat & 16 & 7.93 & $5.88-2$ & $1.03-1$ & 0.197 & 2271 & 22 & 49 & 90 \\
\hline Er-167 & 16 & 2.00 & $5.88-2$ & $2.03-2$ & & 582 & 23 & 53 & 98 \\
\hline Er-nat & 16 & 8.00 & $5.91-2$ & $1.04-1$ & 0.198 & 2292 & 21 & 49 & 90 \\
\hline Er-167 & 16 & 2.02 & $5.91-2$ & $2.04-2$ & & 588 & 23 & 53 & 98 \\
\hline Er-nat & 16 & 12.00 & $7.45-2$ & $1.22-1$ & 0.240 & 3402 & 19 & 43 & 88 \\
\hline Er-167 & 16 & 3.10 & $7.45-2$ & $2.93-2$ & & 901 & 21 & 46 & 97 \\
\hline Er-nat & 64 & 1.00 & $5.08-2$ & $6.53-2$ & 0.159 & 1167 & 36 & 72 & 93 \\
\hline Er-167 & 64 & 0.24 & $5.08-2$ & $1.04-2$ & & 284 & 38 & 77 & 99 \\
\hline Er-nat & 64 & 2.00 & $8.68-2$ & $7.66-2$ & 0.160 & 2329 & 30 & 66 & 92 \\
\hline Er-167 & 64 & 0.49 & $8.68-2$ & $1.22-2$ & & 576 & 32 & 71 & 99 \\
\hline Er-nat & 64 & 2.03 & $8.77-2$ & $7.68-2$ & 0.160 & 2364 & 30 & 66 & 92 \\
\hline Er-167 & 64 & 0.50 & $8.77-2$ & $1.23-2$ & & 584 & 32 & 71 & 99 \\
\hline Er-nat & 64 & 2.44 & 0.100 & $8.03-2$ & 0.163 & 2843 & 29 & 64 & 92 \\
\hline Er-167 & 64 & 0.61 & 0.100 & $1.31-2$ & & 707 & 31 & 69 & 99 \\
\hline Er-nat & 64 & 3.96 & $1.40-1$ & $9.02-2$ & 0.179 & 4589 & 25 & 58 & 91 \\
\hline Er-167 & 64 & 1.00 & $1.40-1$ & $1.61-2$ & & 1167 & 27 & 62 & 98 \\
\hline
\end{tabular}


Table 33. The effect of isotope separation for the case of burnable poison in the form of dysprosium oxide homogeneously mixed with the fuel.

\begin{tabular}{|c|c|c|c|c|c|c|c|c|c|}
\hline Isotope & $\begin{array}{l}\text { Number } \\
\text { IFBA }\end{array}$ & \%loading & -dk_Bol & $\begin{array}{l}\text { dk_EOL } \\
-\because---- \\
\text { dk_BOL }\end{array}$ & $\begin{array}{l}\text { dk_EOL/dk_BOL | Isot } \\
\text { dk_EOL/dk_BOL | Nat }\end{array}$ & $\begin{array}{l}\text { BP Mass } \\
(g / a s s e m b l y)\end{array}$ & $\begin{array}{l}\% \text { of } B \\
\text { After }\end{array}$ & $\begin{array}{l}\text { BP Worth } \\
\text { Specifi }\end{array}$ & $\begin{array}{l}\text { h Bur } \\
\text { ied } \mathrm{T}\end{array}$ \\
\hline & & & & & & & $120 \mathrm{~d}$ & $1 \mathrm{yr}$ & $4 y$ \\
\hline Dy-nat & 16 & 2.00 & $3.69-2$ & $3.59-1$ & 0.281 & 578 & 16 & 30 & 64 \\
\hline$D y-164$ & 16 & 1.32 & $3.69-2$ & $1.01-1$ & & 384 & 22 & 47 & 90 \\
\hline Dy-nat & 16 & 2.75 & $4.75-2$ & $3.70-1$ & 0.320 & 793 & 16 & 30 & 63 \\
\hline$D y-164$ & 16 & 2.00 & $4.75-2$ & $1.19-1$ & & 579 & 20 & 42 & 88 \\
\hline Dy-nat & 16 & 4.00 & $6.08-2$ & $3.85-1$ & 0.405 & 1146 & 15 & 29 & 62 \\
\hline Dy-164 & 16 & 3.24 & $6.08-2$ & $1.56-1$ & & 932 & 17 & 36 & 84 \\
\hline Dy-nat & 16 & 4.74 & $6.75-2$ & $3.93-1$ & 0.459 & 1353 & 15 & 29 & 61 \\
\hline$D y-164$ & 16 & 4.00 & $6.75-2$ & $1.80-1$ & & 1149 & 15 & 32 & 82 \\
\hline Dy-nat & 64 & 0.76 & $6.52-2$ & $3.23-1$ & 0.253 & 880 & 17 & 32 & 68 \\
\hline Dy -164 & 64 & 0.50 & $6.52-2$ & $8.19-2$ & & 583 & 27 & 54 & 92 \\
\hline Dy-nat & 64 & 1.00 & $8.12-2$ & $3 \cdot 34-1$ & 0.266 & 1159 & 17 & 32 & 67 \\
\hline Dy-164 & 64 & 0.68 & $8.12-2$ & $8.90-2$ & & 797 & 26 & 52 & 91 \\
\hline Dy-nat & 64 & 1.32 & 0.100 & $3.45-1$ & 0.282 & 1523 & 16 & 31 & 66 \\
\hline Dy-164 & 64 & 0.90 & 0.100 & $9.73-2$ & & 1049 & 24 & 50 & 90 \\
\hline Dy-nat & 64 & 1.47 & $1.09-2$ & 3. 49-1 & 0.290 & 1697 & 16 & 31 & 65 \\
\hline Dy -164 & 64 & 1.00 & $1.09-2$ & $1.01-1$ & & 1163 & 24 & 49 & 90 \\
\hline
\end{tabular}




\begin{tabular}{|c|c|c|c|c|c|c|c|c|c|}
\hline \multirow{2}{*}{ Isotope } & \multirow{2}{*}{$\begin{array}{l}\text { Number } \\
\text { IFBA }\end{array}$} & \multirow{2}{*}{ ஃloading } & \multirow{2}{*}{-dk_Bol } & \multirow{2}{*}{$\begin{array}{l}\text { dk_EOL } \\
----- \\
d k \_B O L\end{array}$} & \multirow{2}{*}{$\begin{array}{l}\text { dk_EOL/dk_BOL } \mid \text { Isot } \\
-d k \_E O L / d k \_B O L \mid N a t\end{array}$} & \multirow[b]{2}{*}{$\begin{array}{l}\text { BP Mass } \\
(\mathrm{g} / \text { assembly) }\end{array}$} & \multirow{2}{*}{\multicolumn{3}{|c|}{$\begin{array}{l}\text { \% of BP Worth Burned } \\
\text { After Specified Time } \\
120 \text { d } 1 \text { yr } 4 \text { yrs }\end{array}$}} \\
\hline & & & & & & & & & \\
\hline Gd-nat & 08 & 12.00 & $5.90-2$ & $2.29-2$ & 0.249 & 549 & 29 & 89 & 98 \\
\hline Gd-157 & 08 & 4.00 & $6.08-2$ & $5.70-3$ & & 190 & 23 & 98 & 99 \\
\hline Gd-nat & 16 & 2.00 & $7.58-2$ & $6.83-3$ & 0.151 & 192 & 90 & 99 & 99 \\
\hline Gd-157 & 16 & 0.39 & $7.58-2$ & $1.03-3$ & & 38 & 99 & 100 & 100 \\
\hline Gd-nat & 16 & 2.41 & $8.02-2$ & $7.95-3$ & 0.153 & 231 & 84 & 99 & 99 \\
\hline Gd-157 & 16 & 0.50 & $8.02-2$ & $1.22-3$ & & 48 & 99 & 100 & 100 \\
\hline Gd-nat & 16 & 4.00 & $8.96-2$ & $1.13-2$ & 0.161 & 380 & 68 & 98 & 99 \\
\hline Gd-157 & 16 & 0.88 & $8.96-2$ & $1.83-3$ & & 84 & 85 & 100 & 100 \\
\hline Gd-nat & 16 & 4.56 & $9.20-2$ & $1.25-2$ & 0.164 & 432 & 63 & 98 & 99 \\
\hline Gd-157 & 16 & 1.00 & $9.20-2$ & $2.04-3$ & & 96 & 80 & 100 & 100 \\
\hline Gd-nat & 16 & 6.96 & $1.00-1$ & $1.67-2$ & 0.185 & 652 & 45 & 97 & 98 \\
\hline Gd-157 & 16 & 1.63 & $1.00-1$ & $3.08-3$ & & 156 & 49 & 100 & 100 \\
\hline Gd-nat & 16 & 8.00 & $1.028-1$ & $1.84-2$ & 0.194 & 746 & 39 & 97 & 98 \\
\hline Gd-157 & 16 & 1.94 & $1.028-1$ & $3.56-2$ & & 186 & 39 & 100 & 100 \\
\hline Gd-nat & 16 & 8.20 & $1.033-1$ & $1.87-2$ & 0.195 & 763 & 38 & 96 & 98 \\
\hline Gd-157 & 16 & 2.00 & $1.033-1$ & $3.65-3$ & & 192 & 38 & 100 & 100 \\
\hline Gd-nat & 16 & 12.00 & $1.11-1$ & $2.38-2$ & 0.222 & 1098 & 28 & 89 & 98 \\
\hline Gd-157 & 16 & 3.43 & 1. 11-1 & $5.28-3$ & & 326 & 24 & 98 & 99 \\
\hline
\end{tabular}


Table 35. The effect of isotope separation for the case of burnable poison in the form of samarium oxide homogeneously mixed into the outer one-third volume of fuel pellets.

\begin{tabular}{|c|c|c|c|c|c|c|c|c|c|}
\hline \multirow[t]{2}{*}{ Isotope } & \multirow[t]{2}{*}{$\begin{array}{l}\text { Number } \\
\text { IFBA }\end{array}$} & \multirow[t]{2}{*}{ loading } & \multirow[t]{2}{*}{-dk_Bol } & \multirow[t]{2}{*}{$\begin{array}{l}\text { dk_EOL } \\
------ \\
d k \_B O L\end{array}$} & $\begin{array}{l}\text { dk_EOL/dk_BOL } \mid \text { Isot } \\
-d k \text { sOL/dk_BOL } \mid \text { Nat }\end{array}$ & \multirow[t]{2}{*}{$\begin{array}{l}\text { BP Mass } \\
(g / \text { assembly) }\end{array}$} & $\begin{array}{l}\text { o of } B \\
\text { After }\end{array}$ & 3P Wort & $\begin{array}{l}\text { h Burned } \\
\text { ied Time }\end{array}$ \\
\hline & & & & & & & $120 d$ & $1 \mathrm{yr}$ & 4 yrs \\
\hline Sm-nat & 08 & 10.58 & $5.78-2$ & $1.42-1$ & 0.290 & 484 & 55 & 85 & 86 \\
\hline Sm-149 & 08 & 2.00 & $5.78-2$ & $4.13-2$ & & 95 & 41 & 97 & 96 \\
\hline Sm-nat & 08 & 12.00 & $5.92-2$ & $1.53-1$ & 0.299 & 546 & 46 & 84 & 85 \\
\hline $\mathrm{Sm}-149$ & 08 & 2.34 & $5.92-2$ & $4.57-2$ & & 112 & 36 & 95 & 96 \\
\hline Sm-nat & 16 & 1.26 & $5.85-2$ & $4.07-2$ & 0.233 & 121 & 94 & 94 & 96 \\
\hline $\mathrm{Sm}-149$ & 16 & 0.20 & $5.85-2$ & $9.48-3$ & & 19 & 99 & 99 & 99 \\
\hline Sm-nat & 16 & 2.00 & $7.01-2$ & $5.25-2$ & 0.219 & 191 & 93 & 93 & 95 \\
\hline Sm-149 & 16 & 0.28 & $7.01-2$ & $1.15-2$ & & 27 & 99 & 99 & 99 \\
\hline Sm-nat & 16 & 3.26 & $8.23-2$ & $7.19-2$ & 0.227 & 309 & 92 & 92 & 93 \\
\hline $\mathrm{Sm}-149$ & 16 & 0.50 & $8.23-2$ & $1.63-2$ & & 48 & 99 & 99 & 99 \\
\hline Sm-nat & 16 & 4.00 & $8.73-2$ & $8.21-2$ & 0.236 & 378 & 90 & 91 & 92 \\
\hline $\mathrm{Sm}-149$ & 16 & 0.64 & $8.73-2$ & $1.93-2$ & & 61 & 95 & 98 & 98 \\
\hline Sm-nat & 16 & 5.74 & $9.58-2$ & $1.02-1$ & 0.260 & 537 & 83 & 89 & 90 \\
\hline $\mathrm{Sm}-149$ & 16 & 1.00 & $9.58-2$ & $2.66-2$ & & 96 & 83 & 98 & 98 \\
\hline Sm-nat & 16 & 6.84 & $1.00-1$ & $1.14-1$ & 0.276 & 636 & 77 & 88 & 89 \\
\hline $\mathrm{Sm}-149$ & 16 & 1.26 & $1.00-1$ & $3.13-2$ & & 120 & 73 & 98 & 97 \\
\hline Sm-nat & 16 & 8.00 & $1.04-1$ & $1.25-1$ & 0.292 & 741 & 71 & 87 & 87 \\
\hline $\mathrm{Sm}-149$ & 16 & 1.58 & $1.04-1$ & $3.66-2$ & & 150 & 59 & 97 & 97 \\
\hline Sm-nat & 64 & 0.35 & $9.59-2$ & $2.64-2$ & 0.227 & 135 & 96 & 96 & 97 \\
\hline Sm-149 & 64 & 0.05 & $9.59-2$ & $6.00-3$ & & 19 & 99 & 99 & 100 \\
\hline Sm-nat & 64 & 0.37 & 0.100 & $2.69-2$ & 0.226 & 143 & 96 & 96 & 97 \\
\hline $\mathrm{Sm}-149$ & 64 & 0.05 & 0.100 & $6.10-3$ & & 20 & 100 & 100 & 100 \\
\hline Sm-nat & 64 & 0.50 & $1.23-1$ & $3.00-2$ & 0.226 & 192 & 95 & 95 & 97 \\
\hline $\mathrm{Sm}-149$ & 64 & 0.065 & $1.23-1$ & $6.78-3$ & & 27 & 100 & 100 & 100 \\
\hline Sm-nat & 64 & 1.00 & 1. 78-1 & $4.13-2$ & 0.232 & 383 & 94 & 94 & 96 \\
\hline $\mathrm{Sm}-149$ & 64 & 0.18 & 1. 78-1 & $9.58-3$ & & 58 & 99 & 99 & 99 \\
\hline Sm-nat & 64 & 1.34 & $2.00-1$ & $4.85-2$ & 0.230 & 511 & 93 & 93 & 95 \\
\hline $\mathrm{Sm}-149$ & 64 & 0.25 & $2.00-1$ & $1.12-2$ & & 77 & 99 & 99 & 99 \\
\hline
\end{tabular}


Table 36. The effect of isotope separation for the case of burnable poison in the form of erbium oxide homogeneously mixed onto the outer one-third volume of fuel pellets.

\begin{tabular}{|c|c|c|c|c|c|c|c|c|c|}
\hline \multirow[t]{2}{*}{ Isotope } & \multirow[t]{2}{*}{$\begin{array}{l}\text { Number } \\
\text { IFBA }\end{array}$} & \multirow[t]{2}{*}{ \%loading } & \multirow[t]{2}{*}{-dk_Bol } & \multirow[t]{2}{*}{$\begin{array}{l}\text { dk_EOL } \\
------ \\
\text { dk_BOL }\end{array}$} & \multirow[t]{2}{*}{$\begin{array}{l}\text { dk_EOL/dk_BOL | Isot } \\
\text { dk_EOL/dk_BOL/Nat }\end{array}$} & \multirow[t]{2}{*}{$\begin{array}{l}\text { BP Mass } \\
(g / a s s e m b l y)\end{array}$} & $\begin{array}{l}\text { o of } B \\
\text { After }\end{array}$ & 3P Worth & \multirow{2}{*}{$\begin{array}{l}\text { h Burned } \\
\text { ied Time } \\
4 \text { yrs }\end{array}$} \\
\hline & & & & & & & $120 \mathrm{~d}$ & $1 y^{r}$ & \\
\hline Er-nat & 16 & 1.00 & $4.87-3$ & $5.83-2$ & 0.169 & 97 & 41 & 76 & 94 \\
\hline Er-167 & 16 & 0.24 & $4.87-3$ & $9.83-3$ & & 23 & 44 & 81 & 99 \\
\hline Er-nat & 16 & 2.00 & $9.14-3$ & $6.11-2$ & 0.155 & 194 & 38 & 74 & 94 \\
\hline Er-167 & 16 & 0.47 & $9.14-3$ & $9.47-3$ & & 46 & 41 & 80 & 99 \\
\hline Er-nat & 16 & 2.11 & $9.60-3$ & $6.14-2$ & 0.155 & 205 & 38 & 74 & 94 \\
\hline Er-167 & 16 & 0.50 & $9.60-3$ & $9.53-3$ & & 49 & 41 & 80 & 99 \\
\hline Er-nat & 16 & 4.00 & $1.67-2$ & $6.69-2$ & 0.160 & 386 & 35 & 71 & 93 \\
\hline Er-167 & 16 & 0.98 & $1.67-2$ & $1.07-2$ & & 95 & 36 & 76 & 99 \\
\hline Er-nat & 16 & 4.09 & $4.71-2$ & $6.72-2$ & 0.160 & 395 & 35 & 71 & 94 \\
\hline Er-167 & 16 & 1.00 & $4.71-2$ & $1.07-2$ & & 97 & 35 & 76 & 99 \\
\hline Er-nat & 16 & 8.00 & $2.85-2$ & $7.64-2$ & 0.166 & 764 & 29 & 65 & 92 \\
\hline Er-167 & 16 & 1.94 & $2.85-2$ & $1.27-2$ & & 188 & 31 & 69 & 99 \\
\hline Er-nat & 64 & 2.09 & $3.78-2$ & $6.28-2$ & 0.156 & 811 & 38 & 74 & 94 \\
\hline Er-167 & 64 & 0.50 & $3.78-2$ & $9.78-3$ & & 195 & 41 & 79 & 99 \\
\hline Er-nat & 64 & 4.00 & $6.46-2$ & $6.97-2$ & 0.157 & 1544 & 34 & 70 & 94 \\
\hline Er-167 & 64 & 0.96 & $6.46-2$ & $1.10-2$ & & 375 & 37 & 76 & 99 \\
\hline Er-nat & 64 & 4.15 & $6.66-2$ & $7.02-2$ & 0.158 & 1602 & 34 & 70 & 93 \\
\hline Er-167 & 64 & 1.00 & $6.66-2$ & $1.11-2$ & & 389 & 36 & 75 & 99 \\
\hline Er-nat & 64 & 7.23 & $1.00-1$ & $7.91-2$ & 0.166 & 2768 & 30 & 65 & 92 \\
\hline Er-167 & 64 & 1.75 & $1.00-1$ & $1.31-2$ & & 678 & 32 & 70 & 99 \\
\hline Er-nat & 64 & 8.00 & $1.07-1$ & $8.12-2$ & 0.167 & 3056 & 29 & 64 & 92 \\
\hline Er-167 & 64 & 1.94 & $1.07-1$ & $1.36-2$ & & 752 & 31 & 69 & 99 \\
\hline
\end{tabular}


Table 37. The effect of isotope separation for the case of burnable poison in the form of dysprosium oxide homogeneously mixed into the outer one-third volume of fuel pellets.

\begin{tabular}{|c|c|c|c|c|c|c|c|c|c|}
\hline Isotope & $\begin{array}{l}\text { Number } \\
\text { IFBA }\end{array}$ & loading & -dk_Bol & $\begin{array}{l}\text { dk_EOL } \\
-\because---- \\
\text { dk_BOL }\end{array}$ & $\begin{array}{l}\text { dk_EOL/dk_BOL } \mid \text { Isot } \\
- \text { dk_EOL/dk_BOL } \mid \text { Nat }\end{array}$ & $\begin{array}{l}\text { BP Mass } \\
(g / a s s e m b l y)\end{array}$ & $\begin{array}{l}\text { \% of } B \\
\text { After }\end{array}$ & $\begin{array}{l}\text { WP Worth } \\
\text { Specifi }\end{array}$ & $\begin{array}{l}\text { Burned } \\
\text { Led Time }\end{array}$ \\
\hline & & & & & & & $120 \mathrm{~d}$ & $1 \mathrm{yr}$ & 4 yrs \\
\hline Dy-nat & 08 & 6.03 & $1.88-2$ & $3.45-1$ & 0.272 & 285 & 16 & 31 & 65 \\
\hline Dy-164 & 08 & 4.00 & $1.88-2$ & $9.37-2$ & & 192 & 23 & 47 & 91 \\
\hline Dy-nat & 08 & 8.00 & $2.37-2$ & $3.59-1$ & 0.298 & 376 & 16 & 31 & 64 \\
\hline Dy-164 & 08 & 5.59 & $2.37-2$ & $1.07-1$ & & 266 & 20 & 44 & 89 \\
\hline Dy-nat & 64 & 1.00 & $3.29-2$ & $2.87-1$ & 0.256 & 387 & 19 & 35 & 71 \\
\hline Dy -164 & 64 & 1.00 & $4.84-2$ & $7.34-2$ & & 387 & 29 & 57 & 93 \\
\hline
\end{tabular}

Table 38. The effect of isotope separation for the case of burnable poison in the form of gadolinium oxide deposited in a thin coating on the lateral surface of the fuel pellets.

\begin{tabular}{|c|c|c|c|c|c|c|c|c|c|c|}
\hline \multirow[t]{2}{*}{ Isotope } & \multirow[t]{2}{*}{$\begin{array}{c}\text { Number } \\
\text { IFBA }\end{array}$} & $\begin{array}{l}\text { Coating } \\
\text { Thickness }\end{array}$ & \multirow[t]{2}{*}{$\begin{array}{l}\text { Relative } \\
\text { Density }\end{array}$} & \multirow[t]{2}{*}{-dk_Bol } & \multirow[t]{2}{*}{$\begin{array}{l}\text { dk_EOL } \\
------ \\
d k \_B O L\end{array}$} & \multirow[t]{2}{*}{\begin{tabular}{l} 
dk_EOL/dk_BOL | Isot \\
\hdashline$-=--$ \\
dk_EOL/dk_BOL | Nat
\end{tabular}} & \multirow[t]{2}{*}{$\begin{array}{c}\text { BP Mass } \\
(g / a s b l)\end{array}$} & \multicolumn{3}{|c|}{$\begin{array}{l}\circ \text { of BP Worth } \\
\text { Burned After } \\
\text { Specified Time }\end{array}$} \\
\hline & & $(0.001$ in $)$ & & & & & & $120 \mathrm{~d}$ & $1 \mathrm{yr}^{\mathrm{r}}$ & 4 yrs \\
\hline Gd-nat & 16 & 2.18 & 1.00 & $9.54-2$ & $1.45-2$ & 0.176 & 516 & 55 & 98 & 99 \\
\hline Gd-157 & 16 & 0.50 & 1.00 & $9.54-2$ & $2.54-3$ & & 119 & 65 & 100 & 100 \\
\hline Gd-nat & 16 & 2.76 & 1.00 & $1.00-1$ & $1.69-2$ & 0.184 & 652 & 45 & 97 & 98 \\
\hline Gd-157 & 16 & 0.650 & 1.00 & $1.00-1$ & $3.12-3$ & & 155 & 47 & 98 & 100 \\
\hline Gd-nat & 16 & 3.76 & 1.00 & $1.06-1$ & $2.07-2$ & 0.203 & 885 & 34 & 94 & 98 \\
\hline Gd-157 & 16 & 1.00 & 1.00 & $1.06-1$ & $4 \cdot 20-3$ & & 238 & 31 & 99 & 100 \\
\hline Gd-nat & 16 & 4.00 & 1.00 & $1.08-1$ & $2.16-2$ & 0.207 & 942 & 32 & 93 & 98 \\
\hline Gd-157 & 16 & 1.10 & 1.00 & $1.08-1$ & $4 \cdot 47-3$ & & 260 & 28 & 98 & 100 \\
\hline Gd-nat & 16 & 6.31 & 1.00 & $1.17-1$ & $2.88-2$ & 0.239 & 1473 & 23 & 77 & 97 \\
\hline Gd-157 & 16 & 2.00 & 1.00 & $1.17-1$ & $6.87-3$ & & 474 & 20 & 79 & 99 \\
\hline Gd-nat & 64 & 0.20 & 1.00 & $1.55-1$ & $3.49-3$ & 0.137 & 191 & 99 & 100 & 100 \\
\hline Gd-157 & 64 & 0.36 & 0.10 & $1.55-1$ & $4.80-4$ & & 35 & 100 & 100 & 100 \\
\hline Gd-nat & 64 & 0.24 & 1.00 & $1.75-1$ & $3.96-3$ & 0.145 & 227 & 82 & 99 & 100 \\
\hline Gd-157 & 64 & 0.50 & 0.10 & $1.75-1$ & $5.72-4$ & & 48 & 100 & 100 & 100 \\
\hline Gd-nat & 104 & 0.50 & 0.10 & $1.05-1$ & $2.13-3$ & 0.189 & 74 & 100 & 100 & 100 \\
\hline Gd-157 & 104 & 0.20 & 0.10 & $1.64-1$ & $4.02-4$ & & 31 & 100 & 100 & 100 \\
\hline
\end{tabular}




\begin{tabular}{|c|c|c|c|c|c|c|c|c|c|c|}
\hline \multirow{3}{*}{ Isotope } & \multirow{3}{*}{$\begin{array}{l}\text { Number } \\
\text { IFBA }\end{array}$} & \multirow[b]{2}{*}{$\begin{array}{l}\text { Coating } \\
\text { Thickness }\end{array}$} & \multirow{3}{*}{$\begin{array}{l}\text { Relative } \\
\text { Density }\end{array}$} & \multirow{3}{*}{-dk_Bol } & \multirow{3}{*}{$\begin{array}{l}\text { dk_EOL } \\
-\because---- \\
\text { dk_BOL }\end{array}$} & \multirow{3}{*}{ 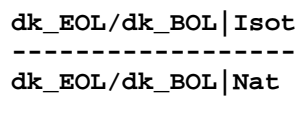 } & \multirow{3}{*}{$\begin{array}{l}\text { BP Mass } \\
(g / a s b l)\end{array}$} & \multirow{2}{*}{\multicolumn{3}{|c|}{$\begin{array}{l}\% \text { of BP Worth } \\
\text { Burned After } \\
\text { Specified Time }\end{array}$}} \\
\hline & & & & & & & & & & \\
\hline & & $(0.001$ in $)$ & & & & & & $120 \mathrm{~d}$ & $1 \mathrm{yr}$ & 4 yrs \\
\hline Sm-nat & 08 & 3.70 & 1.00 & $5.30-2$ & $1.43-1$ & 0.224 & 434 & 58 & 85 & 86 \\
\hline $\mathrm{Sm}-149$ & 08 & 0.50 & 1.00 & $5.30-2$ & $3.22-2$ & & 59 & 70 & 97 & 97 \\
\hline Sm-nat & 08 & 4.00 & 1.00 & $5.42-2$ & $1.52-1$ & 0.231 & 470 & 53 & 84 & 85 \\
\hline $\mathrm{Sm}-149$ & 08 & 0.56 & 1.00 & $5.42-2$ & $3.50-2$ & & 66 & 61 & 98 & 97 \\
\hline Sm-nat & 16 & 1.00 & 1.00 & $7.13-2$ & $6.70-2$ & 0.207 & 237 & 92 & 92 & 93 \\
\hline Sm-149 & 16 & 0.13 & 1.00 & $7.13-2$ & $1.39-2$ & & 30 & 99 & 99 & 99 \\
\hline Sm-nat & 16 & 1.46 & 1.00 & $8.07-2$ & $8.59-2$ & 0.210 & 345 & 92 & 92 & 91 \\
\hline Sm-149 & 16 & 0.20 & 1.00 & $8.07-2$ & $1.80-2$ & & 47 & 98 & 98 & 98 \\
\hline Sm-nat & 16 & 2.00 & 1.00 & $8.72-2$ & $1.03-1$ & 0.212 & 472 & 86 & 89 & 90 \\
\hline $\mathrm{Sm}-149$ & 16 & 0.27 & 1.00 & $8.72-2$ & $2.19-2$ & & 65 & 91 & 98 & 98 \\
\hline Sm-nat & 16 & 3.64 & 1.00 & $9.98-2$ & $1.48-1$ & 0.223 & 856 & 59 & 84 & 85 \\
\hline Sm-149 & 16 & 0.50 & 1.00 & $9.98-2$ & $3.31-2$ & & 119 & 69 & 97 & 97 \\
\hline Sm-nat & 16 & 3.68 & 1.00 & $1.00-1$ & $1.49-1$ & 0.223 & 865 & 58 & 84 & 85 \\
\hline $\mathrm{Sm}-149$ & 16 & 0.50 & 1.00 & $1.00-1$ & $3.33-2$ & & 120 & 69 & 97 & 97 \\
\hline Sm-nat & 16 & 4.00 & 1.00 & $1.02-1$ & $1.58-1$ & 0.226 & 939 & 52 & 83 & 84 \\
\hline $\mathrm{Sm}-149$ & 16 & 0.55 & 1.00 & $1.02-1$ & $3.55-2$ & & 131 & 64 & 97 & 97 \\
\hline Sm-nat & 64 & 0.36 & 1.00 & $1.58-1$ & $4.35-2$ & 0.232 & 337 & 94 & 94 & 96 \\
\hline Sm-149 & 64 & 0.50 & 0.10 & $1.58-1$ & $1.01-2$ & & 47 & 99 & 99 & 99 \\
\hline Sm-nat & 64 & 0.50 & 1.00 & $1.82-1$ & $5.21-2$ & 0.232 & 475 & 93 & 93 & 95 \\
\hline $\mathrm{Sm}-149$ & 64 & 0.50 & 0.10 & $1.82-1$ & $1.21-2$ & & 47 & 99 & 99 & 99 \\
\hline
\end{tabular}




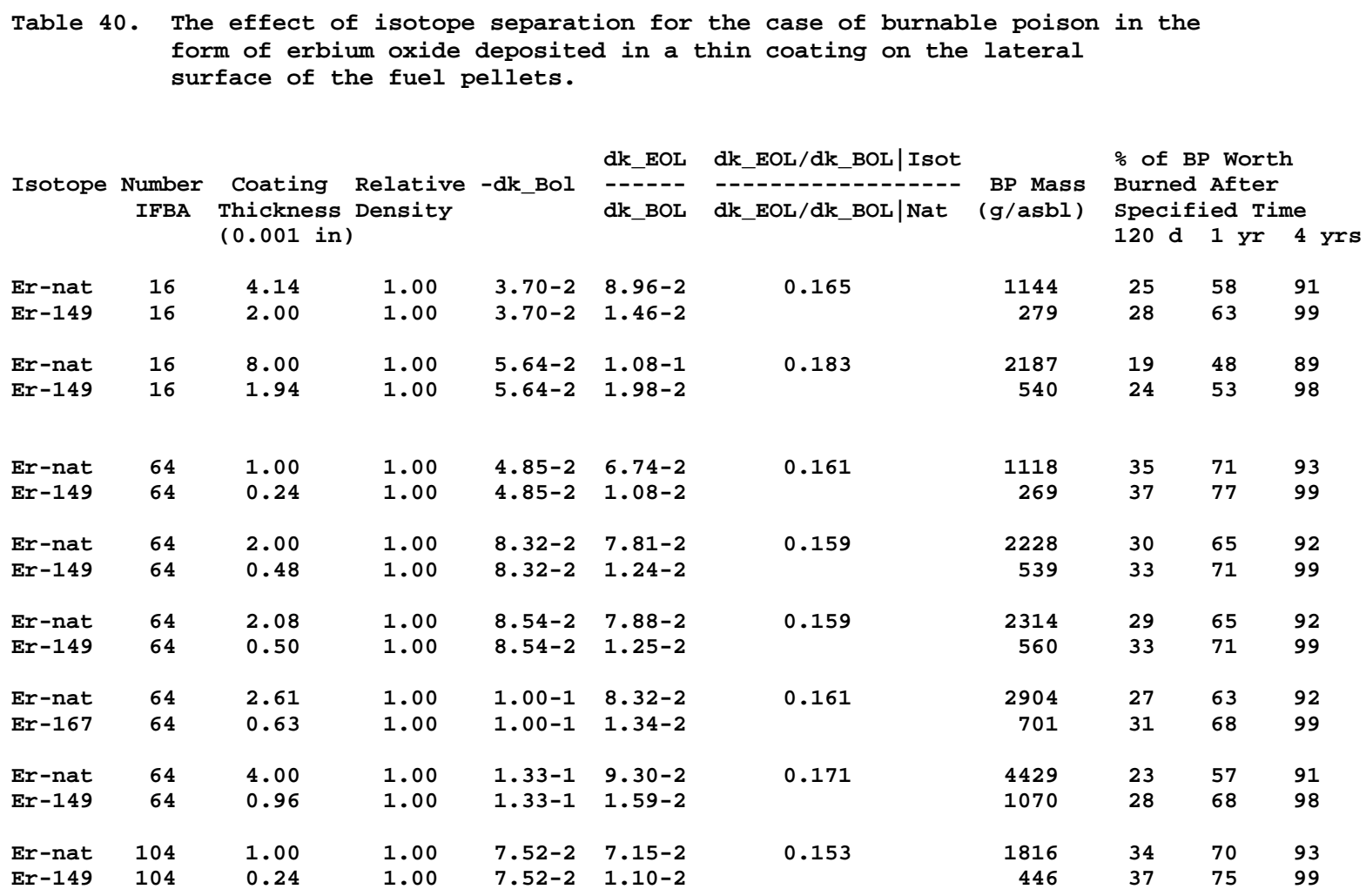

Table 41. The effect of isotope separation for the case of burnable poison in the form of dysprosium oxide deposited in a thin coating on the lateral surface of the fuel pellets.

\begin{tabular}{|c|c|c|c|c|c|c|c|c|c|c|}
\hline \multirow[t]{2}{*}{ Isotope } & \multirow[t]{2}{*}{$\begin{array}{l}\text { Number } \\
\text { IFBA }\end{array}$} & \multirow{2}{*}{$\begin{array}{l}\text { Coating } \\
\text { Thickness } \\
(0.001 \text { in) }\end{array}$} & \multirow{2}{*}{$\begin{array}{l}\text { Relative } \\
\text { Density }\end{array}$} & \multirow[t]{2}{*}{-dk_Bol } & \multirow[t]{2}{*}{$\begin{array}{l}\text { dk_EOL } \\
------ \\
\text { dk_BOL }\end{array}$} & \multirow[t]{2}{*}{$\begin{array}{l}\text { dk_EOL/dk_BOL | Isot } \\
- \text { dk_EOL/dk_BOL | Nat }\end{array}$} & \multirow[t]{2}{*}{$\begin{array}{l}\text { BP Mass } \\
(g / a s b l)\end{array}$} & \multicolumn{3}{|c|}{$\begin{array}{l}\text { of } \text { BP Worth } \\
\text { Burned After } \\
\text { Specified Time }\end{array}$} \\
\hline & & & & & & & & $120 \mathrm{~d}$ & $1 \mathrm{yr}^{\mathrm{r}}$ & 4 yrs \\
\hline Dy-nat & 64 & 1.00 & 1.00 & $7.40-2$ & $3.17-1$ & 0.259 & 1005 & 17 & 33 & 68 \\
\hline Dy -164 & 64 & 0.66 & 1.00 & $7.40-2$ & $8.19-2$ & & 664 & 26 & 53 & 92 \\
\hline Dy-nat & 64 & 1.00 & 1.00 & $7.40-2$ & $3.17-1$ & 0.292 & 1005 & 17 & 33 & 68 \\
\hline$D y-164$ & 64 & 1.00 & 1.00 & $9.87-2$ & $9.25-2$ & & 1008 & 24 & 50 & 91 \\
\hline
\end{tabular}




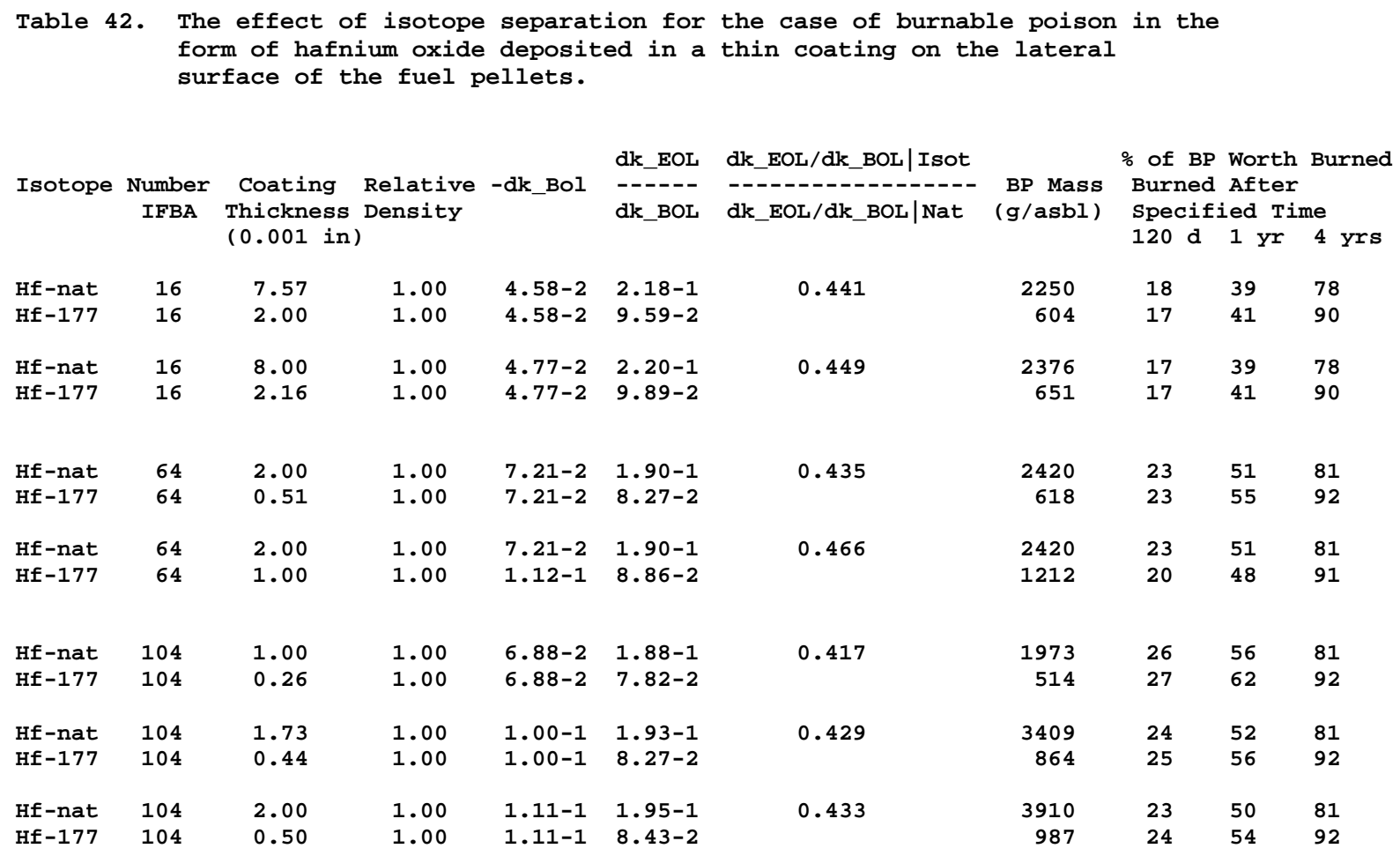

Table 43. The effect of isotope separation for the case of burnable poison in the form of gadolinium alloyed in the zircaloy cladding.

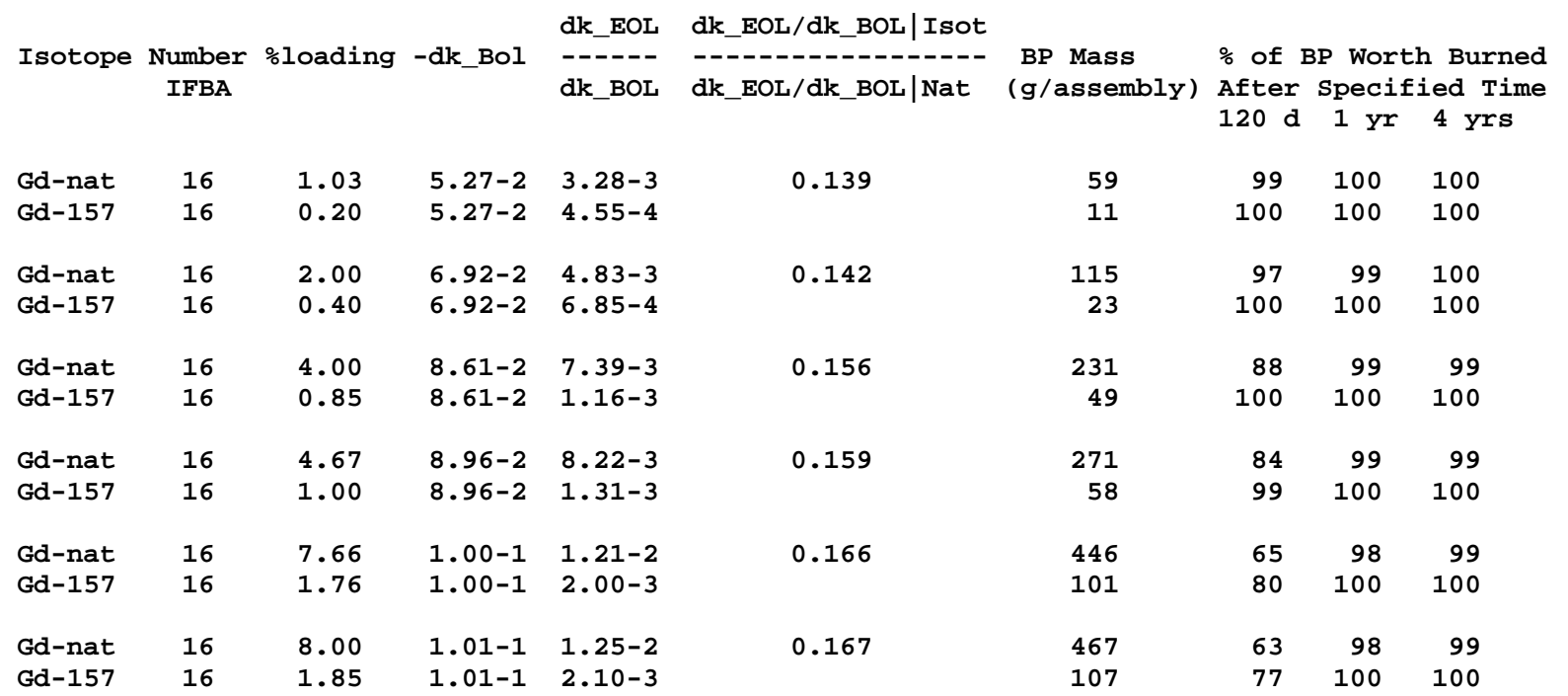


Table 44. The effect of isotope separation for the case of burnable poison in the form of samarium alloyed in the zircaloy cladding.

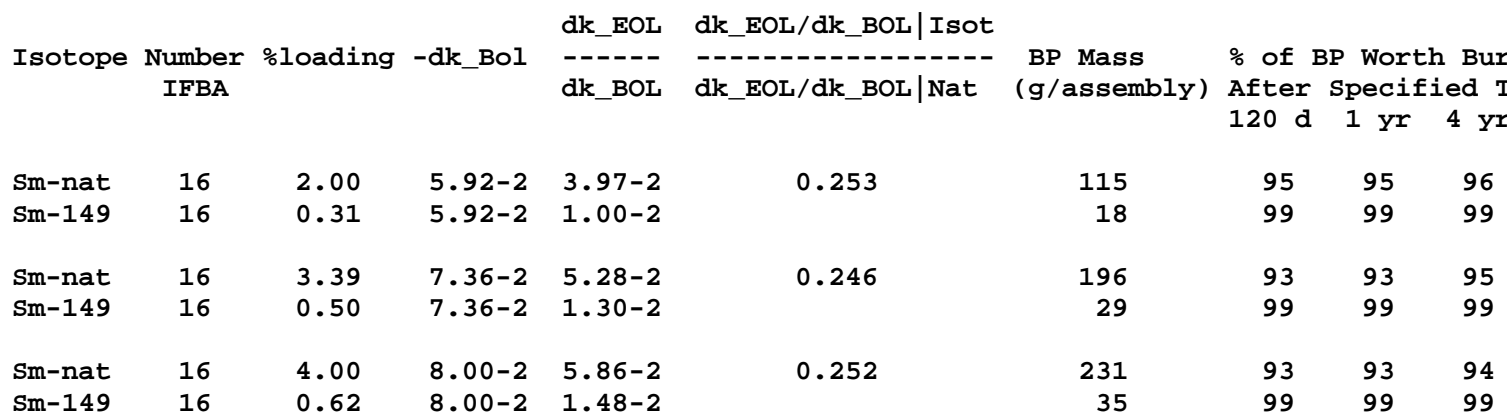

Table 45. The effect of isotope separation for the case of burnable poison in the form of erbium alloyed in the zircaloy cladding.

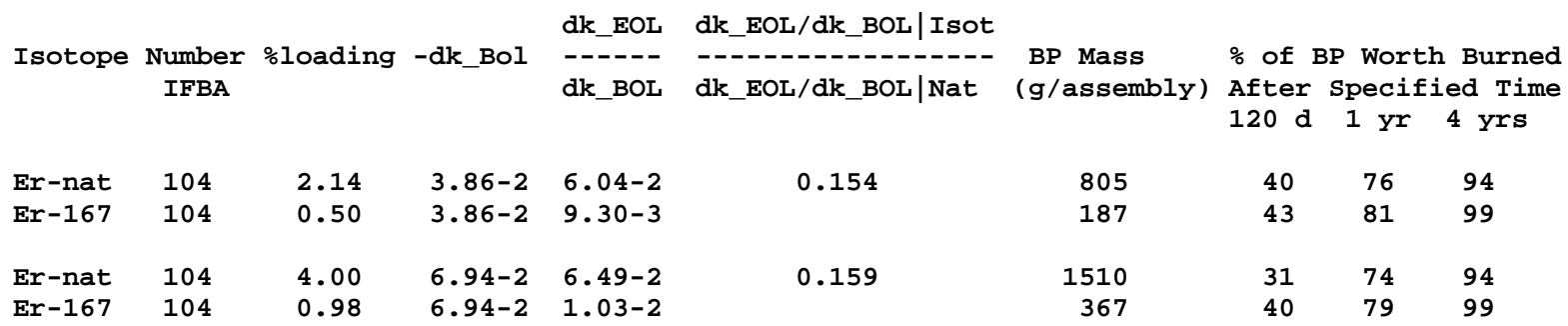

Table 46. The effect of isotope separation for the case of burnable poison in the form of europium alloyed in the zircaloy cladding.

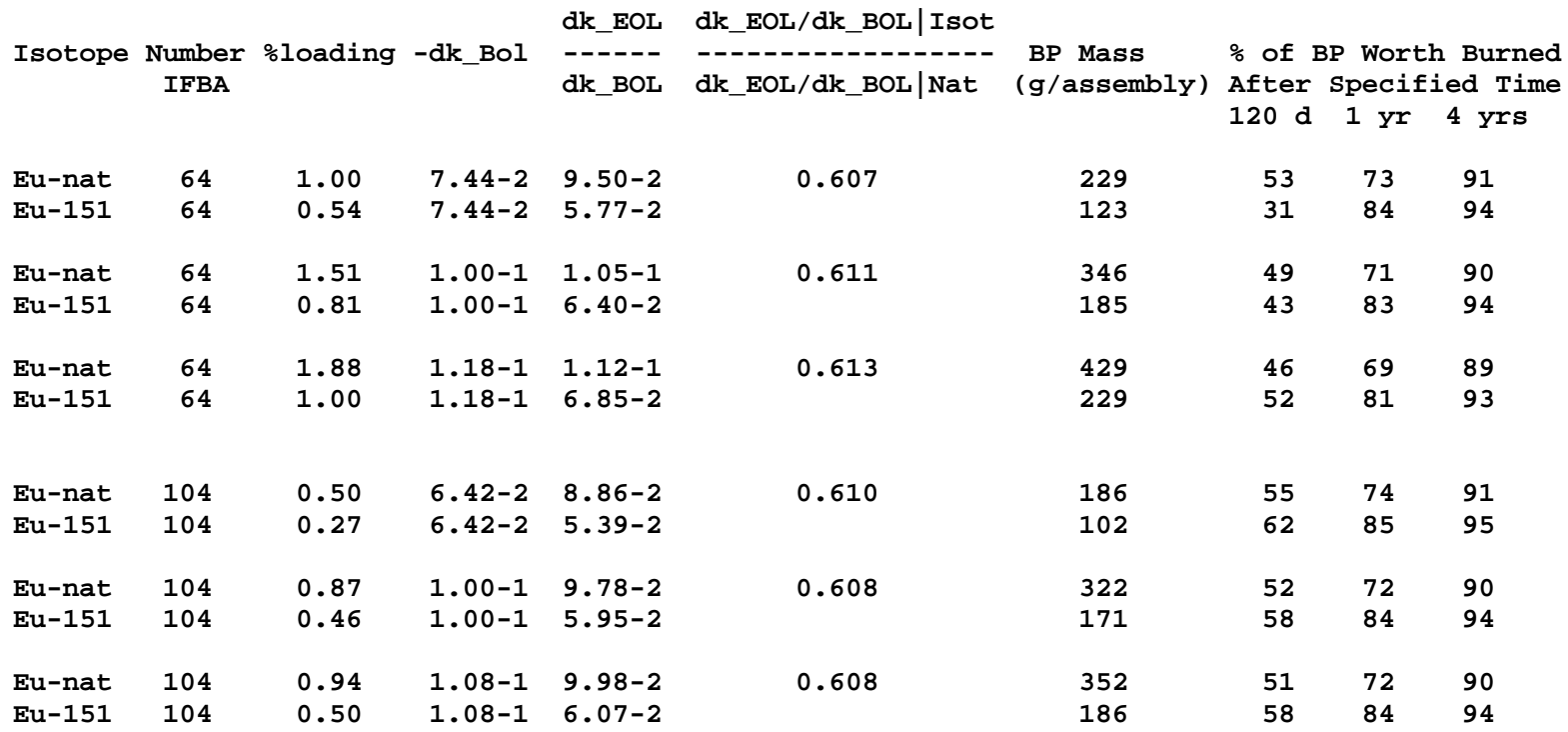




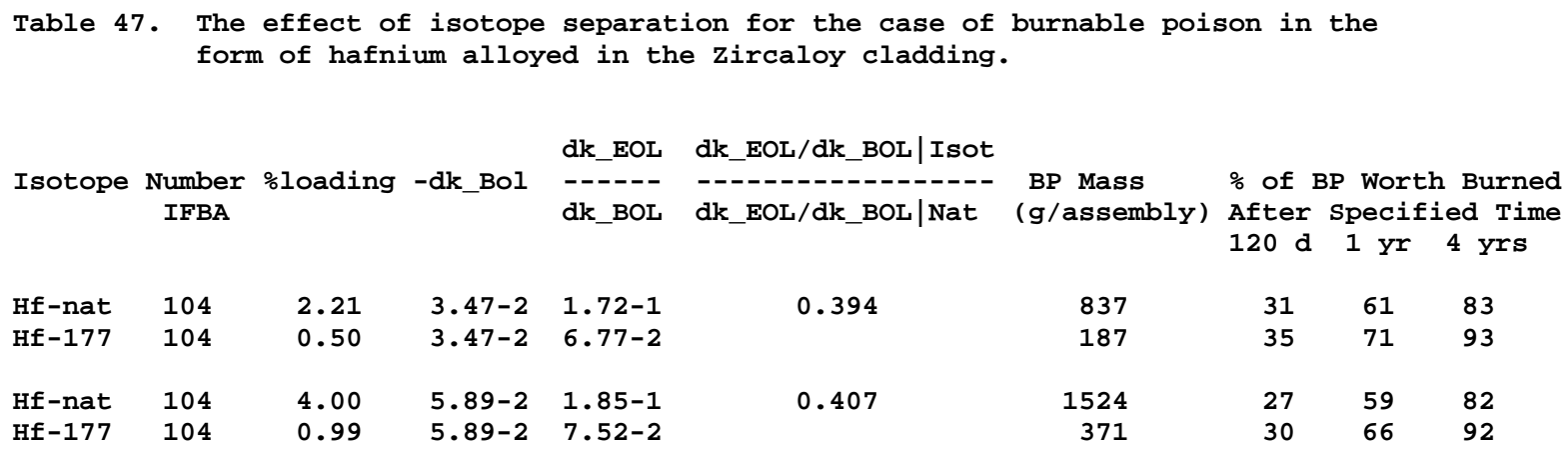

\begin{tabular}{|c|c|c|c|c|c|c|c|c|c|}
\hline \multirow{3}{*}{ Isotope } & \multirow{3}{*}{$\begin{array}{l}\text { Number } \\
\text { IFBA }\end{array}$} & \multirow{3}{*}{ oloading } & \multirow{3}{*}{-dk_Bol } & \multirow{3}{*}{\begin{tabular}{l} 
dk_EOL \\
\hdashline----- \\
$d k \_B O L$
\end{tabular}} & \multirow{3}{*}{$\begin{array}{l}\text { dk_EOL/dk_BOL } \mid \text { Isot } \\
- \text { dk_EOL/dk_BOL } \mid \text { Nat }\end{array}$} & \multirow{3}{*}{$\begin{array}{l}\text { BP Mass } \\
(g / a s s e m b l y)\end{array}$} & \multirow{2}{*}{$\begin{array}{l}\text { \% of } \\
\text { After }\end{array}$} & \multirow{2}{*}{$\begin{array}{l}\text { BP Wort } \\
\text { Specif }\end{array}$} & \multirow{3}{*}{$\begin{array}{l}\text { h Burned } \\
\text { ied Time } \\
4 \text { yrs }\end{array}$} \\
\hline & & & & & & & & & \\
\hline & & & & & & & $120 \mathrm{~d}$ & $1 y^{r}$ & \\
\hline Os-nat & 264 & 15.00 & $8.96-2$ & $5.43-1$ & 0.109 & 19620 & 11 & 17 & 46 \\
\hline Os-184 & 264 & 0.35 & $8.96-2$ & $5.92-2$ & & 378 & 47 & 77 & 94 \\
\hline Os-nat & 264 & 17.50 & $1.00-1$ & $5.44-1$ & 0.111 & 22291 & 10 & 17 & 46 \\
\hline Os-184 & 264 & 0.40 & $1.00-1$ & $6.02-2$ & & 432 & 46 & 77 & 94 \\
\hline Os-nat & 264 & 17.67 & $1.01-1$ & $5.45-1$ & 0.111 & 22474 & 10 & 17 & 46 \\
\hline Os-184 & 264 & 0.40 & $1.01-1$ & $6.03-2$ & & 436 & 46 & 77 & 94 \\
\hline Os-nat & 264 & 20.00 & $1.12-1$ & $5.46-1$ & 0.112 & 25340 & 10 & 17 & 45 \\
\hline Os-184 & 264 & 0.45 & $1.12-1$ & $6.13-2$ & & 494 & 46 & 77 & 94 \\
\hline Os-nat & 264 & 25.00 & $1.39-1$ & $5.48-1$ & 0.117 & 33050 & 11 & 18 & 45 \\
\hline Os -184 & 264 & 0.60 & $1.39-1$ & $6.41-2$ & & 651 & 45 & 76 & 94 \\
\hline
\end{tabular}

The results have been further arranged in a set of four summary tables, one for each configuration. Tables 49 through 52 show input parameters and results from a representative number of runs to compare natural elements with separated isotopes. As much as possible, the initial negative reactivity has been held near $10 \%$ in these tables. Since the long run time for each case prohibited many iterations for fine tuning, there is sometimes a greater variation from 10\% than desired; however, the effect of this variation has been minimized by taking appropriate ratios.

The calculations for the first phase of the project have assumed that the isotopes have been separated completely. In reality, the enrichment achievable depends upon the particular isotope, its abundance, and the number of passes in the enrichment process. It is estimated that a single pass in the PSP process to obtain ${ }^{157} \mathrm{Gd}$ would result in 46 to $72 \%$ enrichment, depending upon the magnetic field strength used. Higher enrichments of ${ }^{157} \mathrm{Gd}$ (and other isotopes) would require additional passes through the PSP process, which would increase cost. The use of single-pass burnable poison enrichments, including the performance and further fine tuning of the burnable poisons, will be done in the second phase of the project. 
Table 49. The effect of isotope separation for the case of burnable poison in the form of oxide homogeneously mixed with the fuel.

\begin{tabular}{|c|c|c|c|c|c|c|c|c|c|}
\hline Isotope & $\begin{array}{l}\text { Number } \\
\text { IFBA }\end{array}$ & \%loading & -dk_Bol & $\begin{array}{l}\text { dk_EOL } \\
-\overline{d k \_B O L}\end{array}$ & $\begin{array}{l}\text { dk_EOL/dk_BOL } / \text { Isot } \\
\text { dk_EOL/dk_BOL | Nat }\end{array}$ & $\begin{array}{l}\text { BP Mass } \\
(g / a s s e m b l y)\end{array}$ & $\begin{array}{l}\circ \text { of } B \\
\text { After }\end{array}$ & $\begin{array}{l}\text { BP Wortl } \\
\text { Specif }\end{array}$ & $\begin{array}{l}\text { h Bur } \\
\text { ied T }\end{array}$ \\
\hline & & & & & & & $120 \mathrm{~d}$ & $1 y^{r}$ & $4 y^{r}$ \\
\hline Gd-nat & 16 & 2.27 & 0.100 & $1.68-2$ & 0.15 & 652 & 44 & 95 & 98 \\
\hline Gd-157 & 16 & 0.57 & 0.100 & $2.52-3$ & & 164 & 58 & 99 & 100 \\
\hline Sm-nat & 16 & 2.38 & 0.100 & $1.18-1$ & 0.27 & 680 & 68 & 87 & 88 \\
\hline $\mathrm{Sm}-149$ & 16 & 0.47 & 0.100 & $3.24-2$ & & 135 & 55 & 97 & 97 \\
\hline Er-nat & 16 & 12.00 & $7.45-2$ & $1.22-1$ & 0.24 & 3402 & 19 & 43 & 88 \\
\hline Er-167 & 16 & 3.10 & $7.45-2$ & $2.93-2$ & & 901 & 21 & 46 & 97 \\
\hline Er-nat & 64 & 2.44 & 0.100 & $8.03-2$ & 0.16 & 2843 & 29 & 64 & 92 \\
\hline Er-167 & 64 & 0.60 & 0.100 & $1.31-2$ & & 707 & 31 & 69 & 99 \\
\hline Dy-nat & 16 & 4.74 & $6.75-2$ & $3.93-1$ & 0.46 & 1353 & 15 & 29 & 61 \\
\hline Dy -164 & 16 & 4.00 & $6.75-2$ & $1.80-1$ & & 1149 & 15 & 32 & 82 \\
\hline Dy-nat & 64 & 1.32 & 0.100 & $3.45-1$ & 0.28 & 1523 & 16 & 31 & 66 \\
\hline$D y-164$ & 64 & 0.90 & 0.100 & $9.73-2$ & & 1049 & 24 & 50 & 90 \\
\hline Eu-nat & 08 & 4.00 & $6.51-2$ & 2. $98-1$ & 0.96 & 567 & 15 & 37 & 70 \\
\hline Eu-151 & 08 & 4.00 & $7.37-2$ & $2.85-1$ & & 567 & 11 & 28 & 72 \\
\hline Hf-nat & 08 & 4.00 & $1.43-2$ & $1.97-1$ & 0.65 & 565 & 19 & 46 & 80 \\
\hline Hf-177 & 08 & 4.00 & $3.41-2$ & $1.28-1$ & & 564 & 15 & 35 & 87 \\
\hline Lu-nat & 08 & 4.00 & $1.54-2$ & $4.39-1$ & 0.25 & 585 & 29 & 39 & 56 \\
\hline $\mathrm{Lu}-176$ & 08 & 4.00 & $6.26-2$ & $1.08-1$ & & 585 & 18 & 32 & 89 \\
\hline Yb-nat & 08 & 4.00 & $3.01-3$ & $5.56-1$ & 0.25 & 583 & 14 & 26 & 44 \\
\hline$Y b-169$ & 08 & 4.00 & $4.80-2$ & $1.42-1$ & & 581 & 11 & 30 & 86 \\
\hline
\end{tabular}




\begin{tabular}{|c|c|c|c|c|c|c|c|c|c|}
\hline \multirow[t]{2}{*}{ Isotope } & \multirow[t]{2}{*}{$\begin{array}{l}\text { Number } \\
\text { IFBA }\end{array}$} & \multirow[t]{2}{*}{ loading } & \multirow[t]{2}{*}{-dk_Bol } & \multirow[t]{2}{*}{$\begin{array}{l}\text { dk_EOL } \\
------ \\
\text { dk_BOL }\end{array}$} & \multirow[t]{2}{*}{$\begin{array}{l}\text { dk_EOL/dk_BOL } \mid \text { Isot } \\
-d k \_E O L / d k \_B O L \mid N a t\end{array}$} & \multirow[t]{2}{*}{$\begin{array}{l}\text { BP Mass } \\
(\mathrm{g} / \text { assembly) }\end{array}$} & \multirow{2}{*}{\multicolumn{3}{|c|}{$\begin{array}{l}\text { \% of BP Worth Burned } \\
\text { After Specified Time } \\
120 \text { d } 1 \text { yr } 4 \text { yrs }\end{array}$}} \\
\hline & & & & & & & & & \\
\hline Gd-nat & 16 & 6.96 & 0.100 & $1.67-2$ & 0.18 & 652 & 45 & 98 & 98 \\
\hline Gd-157 & 16 & 1.63 & 0.100 & $3.08-3$ & & 156 & 49 & 100 & 100 \\
\hline Sm-nat & 16 & 6.84 & 0.100 & $1.14-1$ & 0.28 & 636 & 77 & 88 & 89 \\
\hline $\mathrm{Sm}-149$ & 16 & 1.26 & 0.100 & $3.13-2$ & & 120 & 73 & 97 & 97 \\
\hline Sm-nat & 64 & $3.73-1$ & 0.100 & $2.69-2$ & 0.23 & 143 & 96 & 96 & 97 \\
\hline Sm-149 & 64 & $5.06-2$ & 0.100 & $6.10-3$ & & 20 & 100 & 100 & 100 \\
\hline Er-nat & 64 & 7.23 & 0.100 & $7.91-2$ & 0.17 & 2768 & 30 & 65 & 92 \\
\hline Er-167 & 64 & 1.75 & 0.100 & $1.31-2$ & & 678 & 32 & 70 & 99 \\
\hline Dy-nat & 64 & 1.00 & $3.30-2$ & $2.87-1$ & 0.26 & 287 & 19 & 35 & 71 \\
\hline$D y-164$ & 64 & 1.00 & $4.84-2$ & $7.34-2$ & & 388 & 29 & 57 & 92 \\
\hline Eu-nat & 08 & 8.00 & $5.44-2$ & $2.40-1$ & 0.62 & 371 & 20 & 42 & 76 \\
\hline Eu-151 & 08 & 4.00 & $4.94-2$ & $1.49-1$ & & 189 & 22 & 57 & 85 \\
\hline Hf-nat & 08 & 8.00 & $1.11-2$ & $1.87-1$ & 0.55 & 374 & 22 & 52 & 82 \\
\hline Hf-177 & 08 & 8.00 & $2.67-2$ & $1.03-1$ & & 374 & 17 & 40 & 90 \\
\hline Yb-nat & 08 & 16.00 & $3.82-3$ & $5.75-1$ & 0.15 & 760 & 14 & 23 & 43 \\
\hline$Y b-168$ & 08 & 4.00 & $2.74-2$ & $8.59-2$ & & 194 & 14 & 45 & 91 \\
\hline
\end{tabular}




\begin{tabular}{|c|c|c|c|c|c|c|c|c|c|}
\hline \multirow{3}{*}{ Isotope } & \multirow{3}{*}{$\begin{array}{l}\text { Number } \\
\text { IFBA }\end{array}$} & \multirow{3}{*}{\multicolumn{2}{|c|}{$\begin{array}{l}\text { Coating -dk_Bol } \\
\text { Thickness }\end{array}$}} & \multirow{3}{*}{$\begin{array}{l}\text { dk_EOL } \\
-\because---- \\
\text { dk_BOL }\end{array}$} & \multirow{3}{*}{ 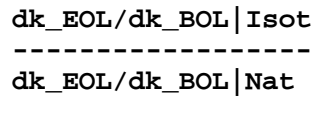 } & \multirow{3}{*}{$\begin{array}{l}\text { BP Mass } \\
(g / a s b l)\end{array}$} & \multirow{2}{*}{\multicolumn{3}{|c|}{$\begin{array}{l}\% \text { of BP Worth } \\
\text { Burned After } \\
\text { Specified Time }\end{array}$}} \\
\hline & & & & & & & & & \\
\hline & & & & & & & $120 \mathrm{~d}$ & $1 y$ & 4 yrs \\
\hline Gd-nat & 16 & 2.76 & 0.100 & $1.69-2$ & 0.18 & 652 & 45 & 97 & 98 \\
\hline Gd-157 & 16 & 0.65 & 0.100 & $3.12-3$ & & 155 & 47 & 98 & 100 \\
\hline Sm-nat & 16 & 3.68 & 0.100 & $1.49-1$ & 0.22 & 865 & 58 & 84 & 85 \\
\hline $\mathrm{Sm}-149$ & 16 & 0.51 & 0.100 & $3.33-2$ & & 120 & 69 & 97 & 97 \\
\hline Er-nat & 16 & 8.0 & $5.64-2$ & $1.08-1$ & 0.18 & 2187 & 19 & 48 & 89 \\
\hline Er-167 & 16 & 1.94 & $5.64-2$ & $1.98-2$ & & 540 & 24 & 53 & 98 \\
\hline Er-nat & 64 & 2.61 & 0.100 & $8.32-2$ & 0.16 & 2904 & 27 & 63 & 92 \\
\hline Er-167 & 64 & 0.63 & 0.100 & $1.34-2$ & & 701 & 31 & 68 & 99 \\
\hline Dy-nat & 64 & 1.00 & $7.40-2$ & $3.17-1$ & 0.26 & 1005 & 17 & 33 & 68 \\
\hline Dy -164 & 64 & 0.66 & $7.40-2$ & $8.19-2$ & & 664 & 26 & 53 & 92 \\
\hline Eu-nat & 64 & 1.00 & $1.88-1$ & $1.63-1$ & 0.61 & 948 & 33 & 61 & 84 \\
\hline Eu-151 & 64 & 0.50 & $1.75-1$ & $5.90-2$ & & 474 & 39 & 74 & 90 \\
\hline Hf-nat & 16 & 8.00 & $4.77-2$ & $2.20-1$ & 0.45 & 2376 & 17 & 39 & 78 \\
\hline Hf-177 & 16 & 2.16 & $4.77-2$ & $9.89-2$ & & 651 & 17 & 41 & 90 \\
\hline Hf-nat & 64 & 2.00 & $7.21-2$ & $1.90-1$ & 0.43 & 2420 & 23 & 51 & 81 \\
\hline Hf-177 & 64 & 0.51 & $7.21-2$ & $8.27-2$ & & 618 & 23 & 55 & 92 \\
\hline Hf-nat & 104 & 1.73 & 0.100 & $1.93-1$ & 0.43 & 3409 & 24 & 52 & 81 \\
\hline $\mathrm{Hf}-177$ & 104 & 0.44 & 0.100 & $8.27-2$ & & 864 & 25 & 56 & 92 \\
\hline
\end{tabular}




\begin{tabular}{|c|c|c|c|c|c|c|c|c|c|}
\hline \multirow{3}{*}{ Isotope } & \multirow{3}{*}{$\begin{array}{l}\text { Number } \\
\text { IFBA }\end{array}$} & \multirow{3}{*}{ ㅇoading } & \multirow{3}{*}{-dk_Bol } & \multirow{3}{*}{$\begin{array}{l}\text { dk_EOL } \\
------ \\
\text { dk_BOL }\end{array}$} & \multirow{3}{*}{$\begin{array}{l}\text { dk_EOL/dk_BOL | Isot } \\
\text { dk_EOL/dk_BOL/Nat }\end{array}$} & \multirow{3}{*}{$\begin{array}{l}\text { BP Mass } \\
(g / a s s e m b l y)\end{array}$} & \multirow{3}{*}{$\begin{array}{l}\text { o of } B \\
\text { After } \\
120 \mathrm{~d}\end{array}$} & \multirow{3}{*}{\multicolumn{2}{|c|}{$\begin{array}{l}\text { 3P Worth Burned } \\
\text { Specified Time } \\
1 \text { yr } 4 \text { yrs }\end{array}$}} \\
\hline & & & & & & & & & \\
\hline & & & & & & & & & \\
\hline Gd-nat & 16 & 7.66 & 0.100 & $1.20-2$ & 0.17 & 446 & 65 & 98 & 99 \\
\hline Gd-157 & 16 & 1.76 & 0.100 & $2.00-3$ & & 101 & 80 & 100 & 100 \\
\hline Sm-nat & 16 & 4.00 & $8.00-2$ & $5.86-2$ & 0.25 & 231 & 93 & 93 & 94 \\
\hline $\mathrm{Sm}-149$ & 16 & 0.62 & $8.00-2$ & $1.48-2$ & & 35 & 99 & 99 & 99 \\
\hline Er-nat & 104 & 4.00 & $6.94-2$ & $6.49-2$ & 0.16 & 1510 & 31 & 74 & 94 \\
\hline Er-167 & 104 & 1.00 & $7.05-2$ & $1.03-2$ & & 37 & 40 & 79 & 99 \\
\hline Dy-nat & 104 & 8.00 & $1.86-1$ & $3.51-1$ & 0.22 & 3041 & 16 & 32 & 65 \\
\hline$D y-164$ & 104 & 2.00 & $8.91-2$ & $7.60-2$ & & 750 & 28 & 56 & 92 \\
\hline Eu-nat & 64 & 1.51 & 0.100 & $1.05-1$ & 0.61 & 346 & 49 & 71 & 90 \\
\hline $\mathrm{Eu}-151$ & 64 & 0.81 & 0.100 & $6.40-2$ & & 185 & 43 & 83 & 94 \\
\hline Eu-nat & 104 & 0.87 & 0.100 & $9.78-2$ & 0.61 & 322 & 52 & 72 & 90 \\
\hline Eu-151 & 104 & 0.46 & 0.100 & $5.95-2$ & & 171 & 58 & 84 & 94 \\
\hline Hf-nat & 104 & 4.00 & $5.89-1$ & $1.85-1$ & 0.41 & 1524 & 27 & 59 & 82 \\
\hline Hf -177 & 104 & 1.00 & $5.89-1$ & $7.52-2$ & & 371 & 30 & 66 & 92 \\
\hline Os-nat & 264 & 17.50 & 0.100 & $5.44-1$ & 0.11 & 22290 & 10 & 17 & 46 \\
\hline Os -184 & 264 & 0.40 & 0.100 & $6.02-2$ & & 432 & 46 & 77 & 94 \\
\hline
\end{tabular}

\section{RESIDUAL REACTIVITY}

Detailed results of the residual reactivity worth of the BPs are displayed in Tables 30 through 48 . The results are further summarized by graphing the data in Tables 49 through 52 in several forms. The first plot is shown in Figure 65, where the residual reactivity ratio, the ratio of negative reactivity due to the burnable poison at EOL to the negative reactivity due to the burnable poison at BOL, $\mathrm{dk}_{\mathrm{EOL}} / \mathrm{dk}_{\mathrm{BOL}}$, is plotted for each of the four configurations of each of six isotopes. Two observations may be made from Figure 65. The first is that the residual reactivity ratios are best for ${ }^{157} \mathrm{Gd}$ and worsen in the order of ${ }^{167} \mathrm{Er},{ }^{149} \mathrm{Sm},{ }^{164} \mathrm{Dy},{ }^{177} \mathrm{Hf}$, and ${ }^{151} \mathrm{Eu}$. The second observation is that, for the same initial reactivity worth of the BP, the ratio decreases when the poison is spread over more IFBA rods. When the number of fuel pins containing BP increases, the BP is spread thinner and is placed in a higher thermal flux position, and this reduces self-shielding.

Another observation that may be made directly from Tables 49 through 52 is that the loading of separated isotopes to achieve the same negative reactivity at BOL is much smaller than for naturally occurring elements. This is also an intuitive result and is often not a great benefit. However, in cases where the amount of material added is limited, there is a definite benefit. This is particularly true in advanced reactors, where it is desired to extend the fuel cycle. Thus more fuel may be added in a limited core volume. In the case of coatings, time of deposition, impediment of heat transfer, and cracking of thick coatings due to thermal expansion all limit the thickness of the coatings. In the case of BP in the cladding, high concentrations of rare earths might alter the structure of the cladding or cause degradation by segregation at grain boundaries in the metal. In all these cases, there is a decided benefit to isotope separation. 
A measure of the isotope separation benefit is the ratio of the residual negative reactivity at EOL to that at BOL for a separated isotope divided by a similar ratio for the naturally occurring element. This compound ratio is shown in Tables 49 through 52 and is plotted in Figure 66. Here it is seen that the benefit ratio, defined above, varies with configuration and isotope in the range of 0.15 to 0.96 , which shows a reactivity ratio benefit of nearly an order of magnitude for ${ }^{157} \mathrm{Gd},{ }^{149} \mathrm{Sm}$, and ${ }^{167} \mathrm{Er}$ to almost no benefit for ${ }^{151} \mathrm{Eu}$ homogeneously mixed with the fuel in eight IFBAs per fuel assembly. As expected, the ratio improves when the self-shielding is minimized by the configuration. Exceptions exist in the figure, largely because of differences in initial reactivity due to running a limited number of cases.

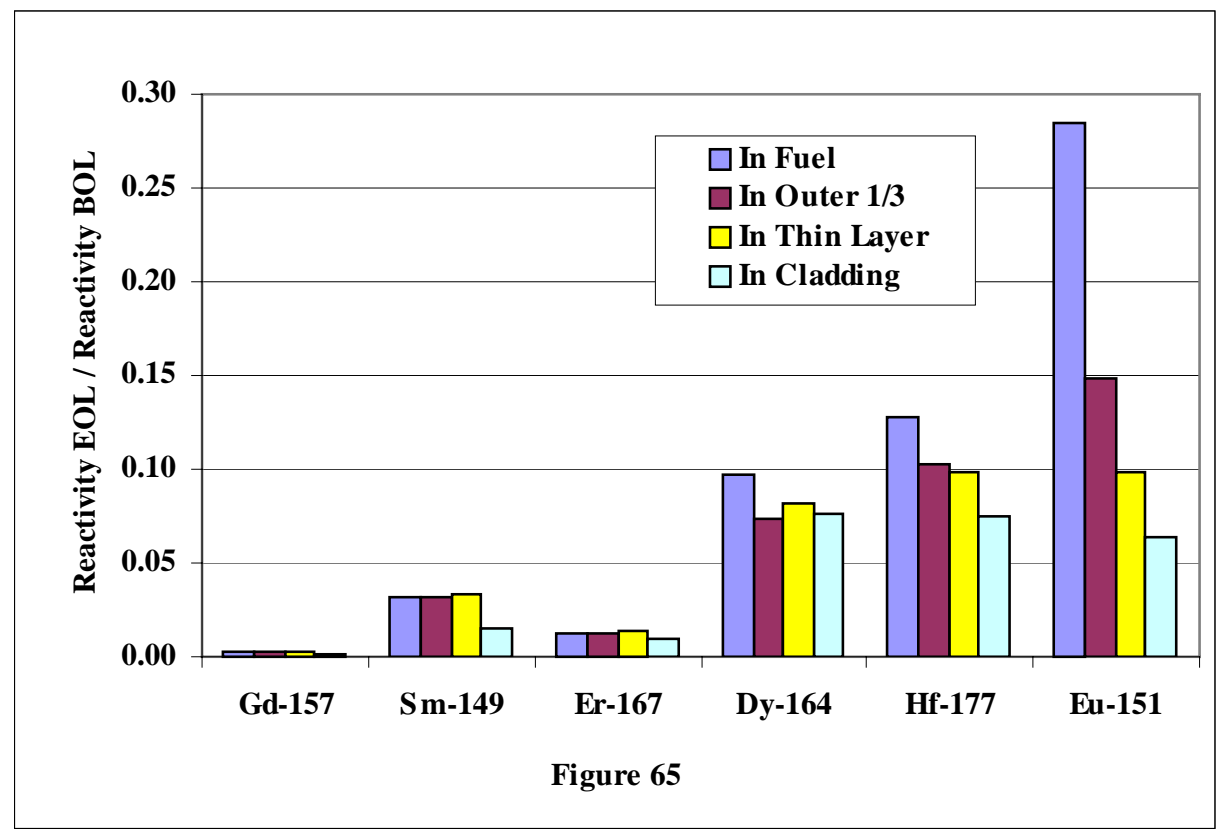

Figure 65. Ratio of negative reactivity due to burnable poison (BP) at the end of fuel life to that at the beginning of fuel life for various configurations of the BP. 


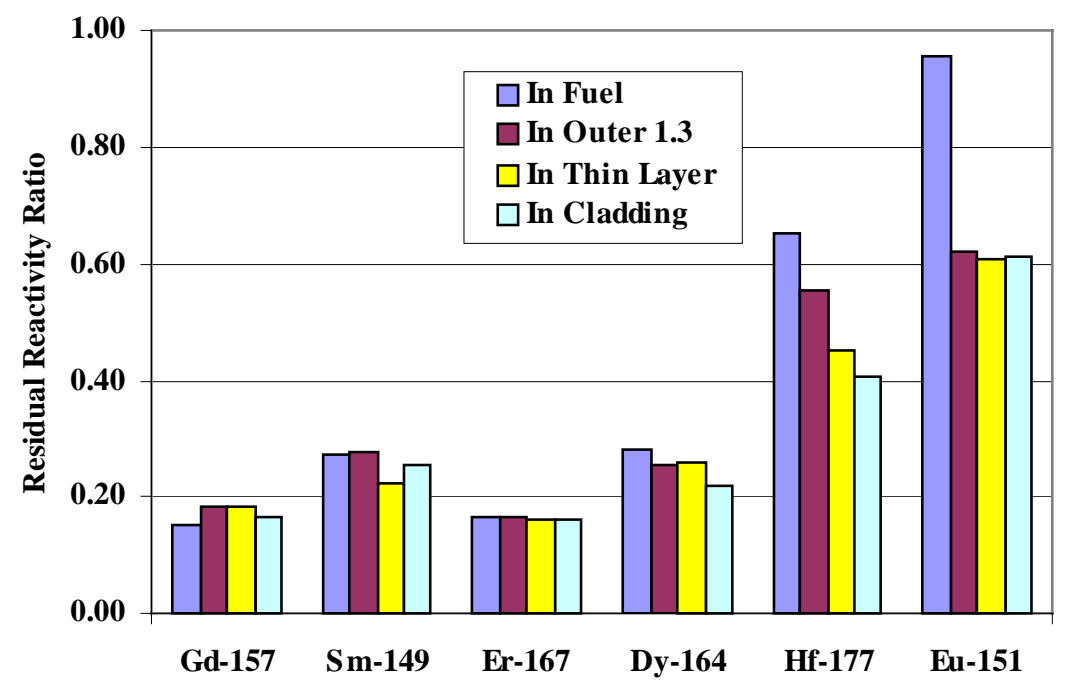

Figure 66

Figure 66. Isotope separation benefit shown by the compound ratio of negative reactivity due to the burnable poison (BP) at end of life to that at beginning of life for a single isotope to a similar ratio for the corresponding natural element.

\section{TIME DEPENDENCE}

Residual reactivity is not the only parameter that must be considered in selecting a burnable poison. Another important consideration is the time dependence of burnup. The residual reactivity ratio can be minimized by choosing very high absorbers, but if the BP burns too fast, it will be useless. The rate of burnup is considered by displaying the ratio of the negative reactivity worth of the BP at each of three time intervals of interest to the reactivity worth of the BP at BOL. The times chosen were 120 days, 1 year, and 4 years.

The results are tabulated as a percentage of BOL reactivity worth in Tables 49 through 52 and are plotted in Figures 67 through 74. From Figure 67, showing the time dependence of burnup of burnable poisons mixed in the fuel, it can be seen that, except for $\mathrm{Gd}$ and $\mathrm{Sm}$, which burn rather rapidly, the time dependence is uniform, but the burnup is incomplete with the exception of $\mathrm{Gd}, \mathrm{Sm}$, and Er. From Figure 68, for separated isotopes, it can be seen that the ${ }^{157} \mathrm{Gd}$ as well as ${ }^{149} \mathrm{Sm}$ burn too rapidly. However, at the end of 4 years, the burnup is greater than $90 \%$ for ${ }^{157} \mathrm{Gd},{ }^{149} \mathrm{Sm},{ }^{167} \mathrm{Er}$, and ${ }^{164}$ Dy, and almost $90 \%$ for ${ }^{177} \mathrm{Hf}$.

For the case of mixing the BP in the outer one-third of the fuel, Figures 69 and 70 show that both natural Gd and ${ }^{157} \mathrm{Gd}$ as well as ${ }^{149} \mathrm{Sm}$ burn too rapidly. However, ${ }^{167} \mathrm{Er}$ and ${ }^{164} \mathrm{Dy}$ now become uniformly burning poisons with over $90 \%$ burnup at the end of 4 years. Figures 71 and 72 illustrate the case of a coating on the fuel pellets. Here it is apparent that Gd and Sm burn too fast to be useful. However, ${ }^{167} \mathrm{Er}$ with $96 \%$ burnup at the end of 4 years becomes very promising, and ${ }^{164} \mathrm{Dy},{ }^{177} \mathrm{Hf}$, and ${ }^{151} \mathrm{Eu}$ all become candidates. For the case of BP in the cladding, separation of isotopes becomes more advantageous. As shown in Figures 73 and 74, the burnup at 4 years is improved for most candidate isotopes. For ${ }^{157} \mathrm{Gd}$ and ${ }^{149} \mathrm{Sm}$, the burnup rates are clearly too high, but natural $\mathrm{Eu},{ }^{167} \mathrm{Er},{ }^{164} \mathrm{Dy},{ }^{177} \mathrm{Hf}$, 
${ }^{151} \mathrm{Eu}$, and ${ }^{184} \mathrm{Os}$ all become good candidates on the basis of burnup. However, cost and availability are also major factors and will almost certainly eliminate Os and possibly Eu.

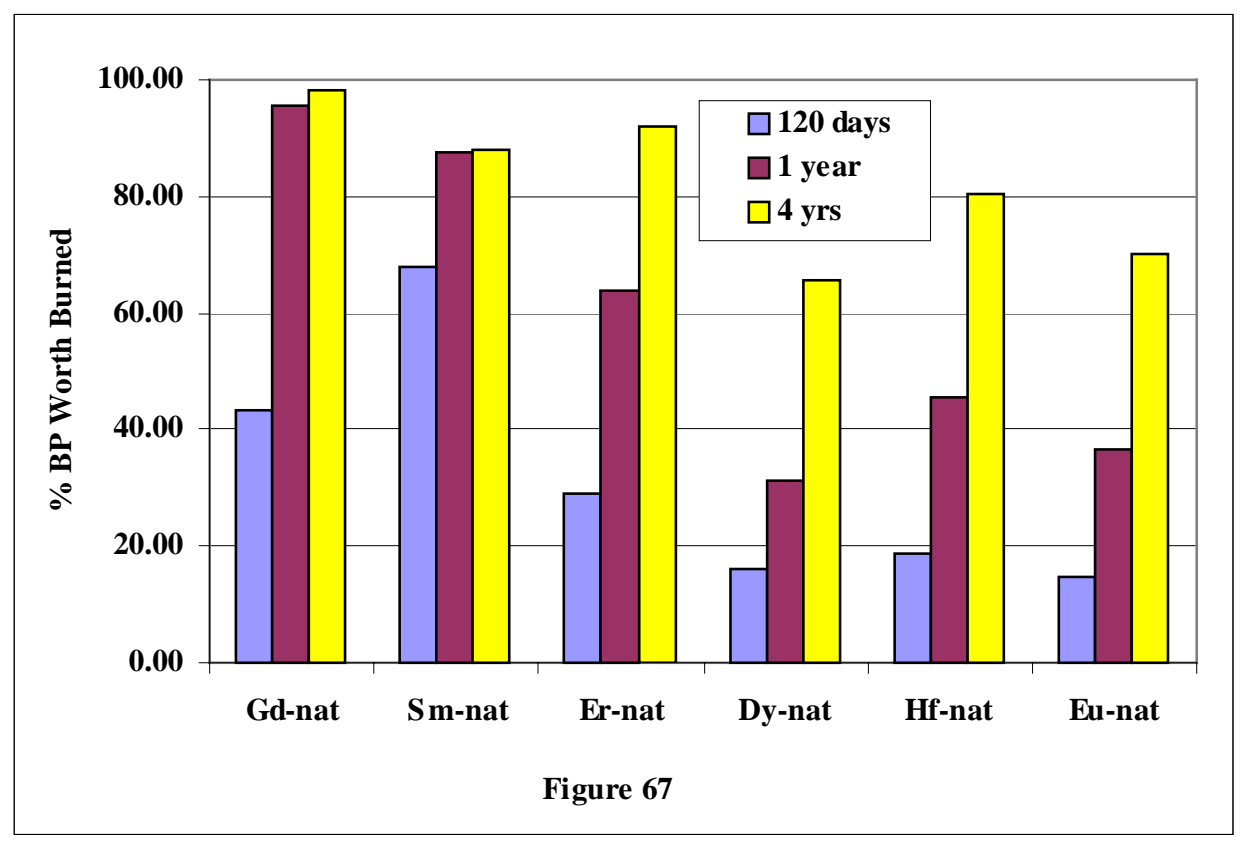

Figure 67. Burn rate for naturally occurring element burnable poisons homogeneously mixed in the fuel as oxides shown as a percentage of the initial negative reactivity remaining at a given time.

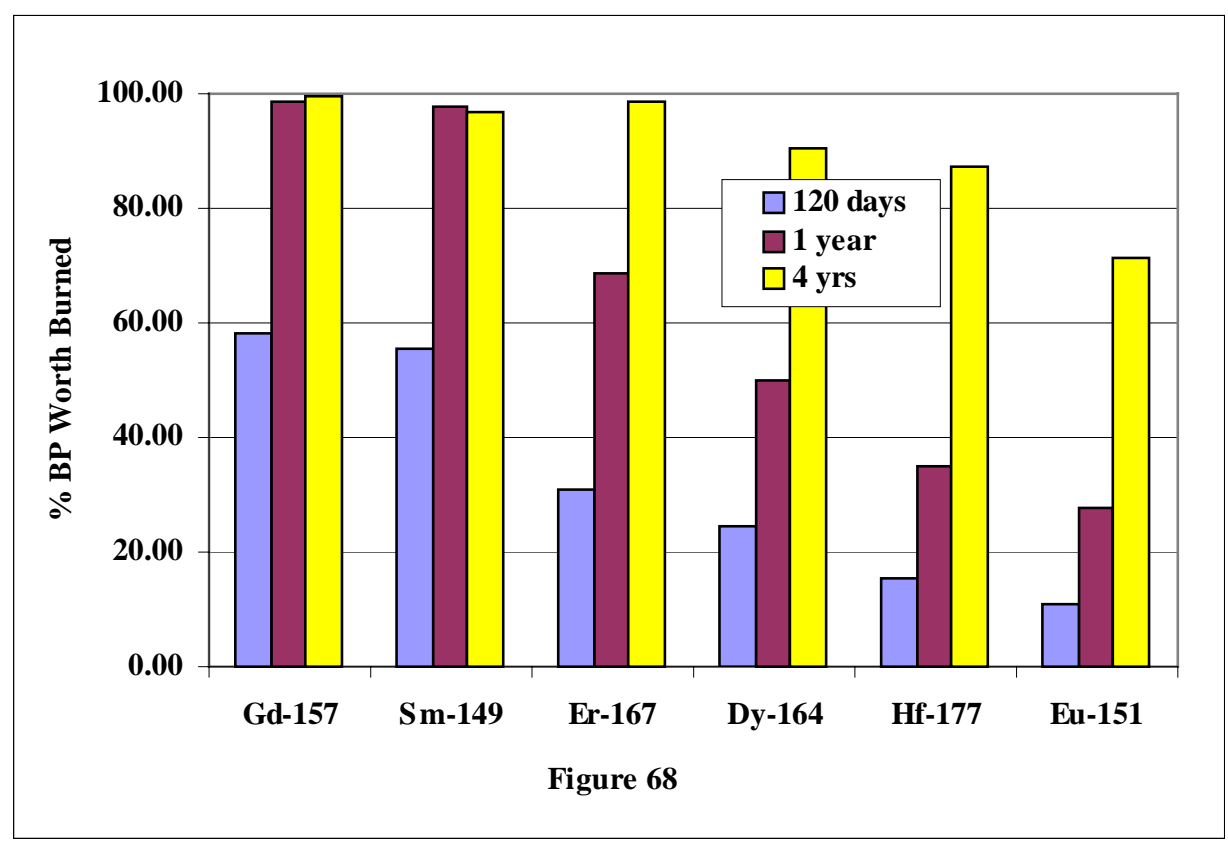

Figure 68. Burn rate for single burnable poisons homogeneously mixed in the fuel as oxides shown as a percentage of the initial negative reactivity remaining at a given time. 


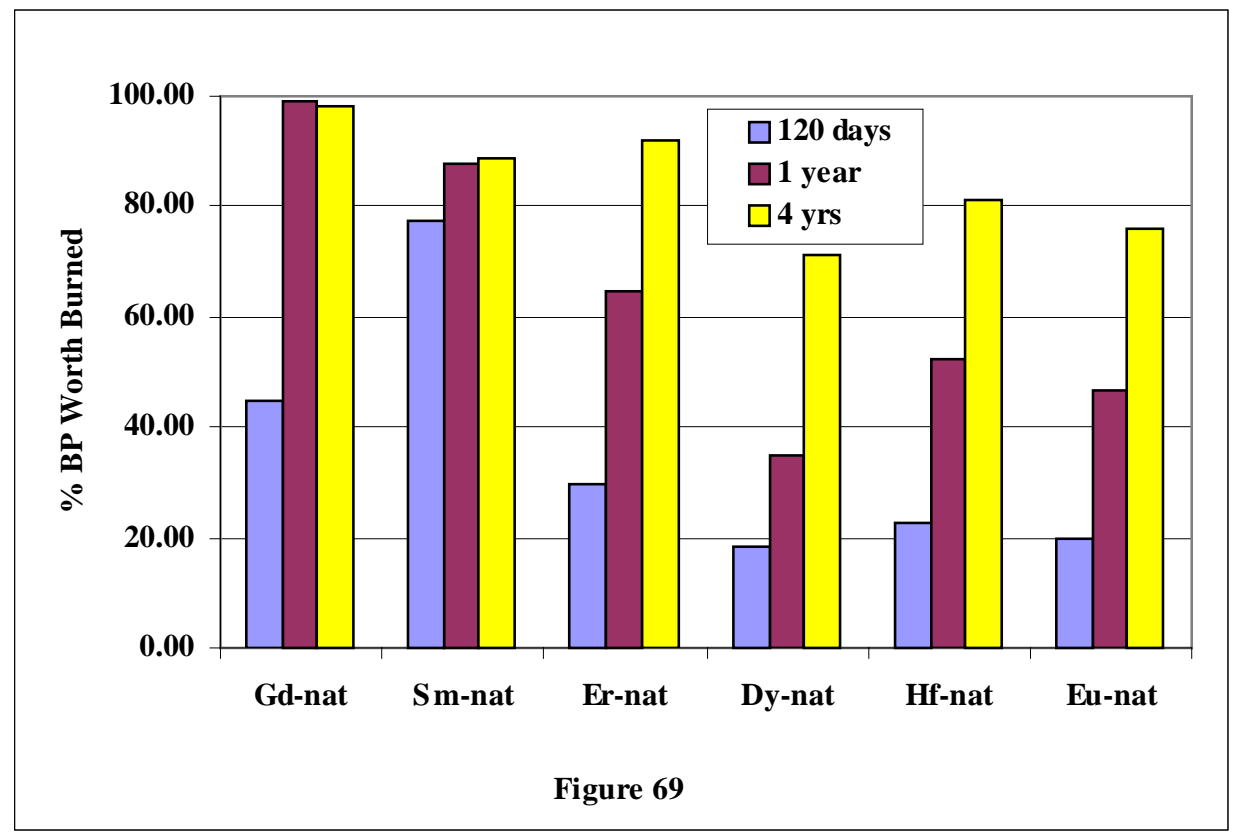

Figure 69. Burn rate for naturally occurring element burnable poisons homogeneously mixed into the outer one-third of the fuel pellets shown as a percentage of the initial negative reactivity remaining at a given time.

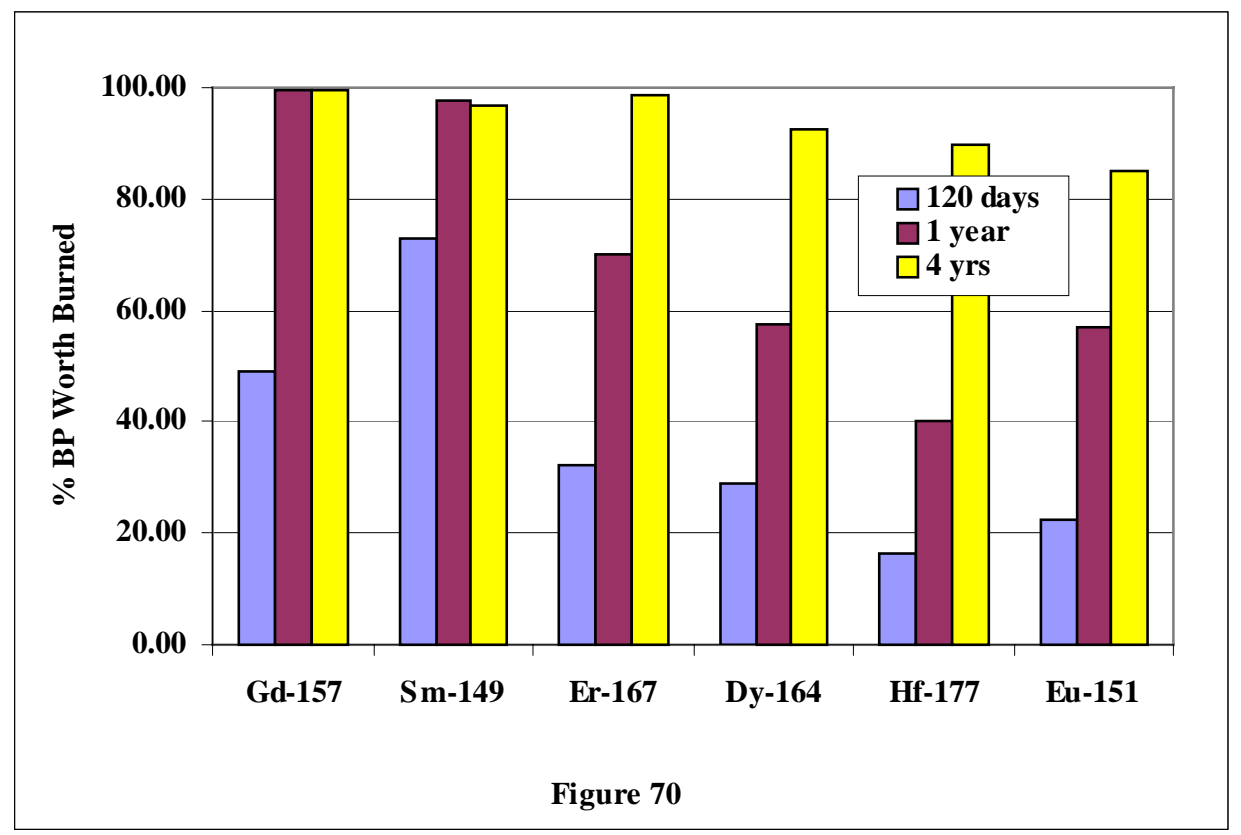

Figure 70. Burn rate for single burnable poisons homogeneously mixed into the outer one-third of the fuel pellets shown as a percentage of the initial negative reactivity remaining at a given time. 


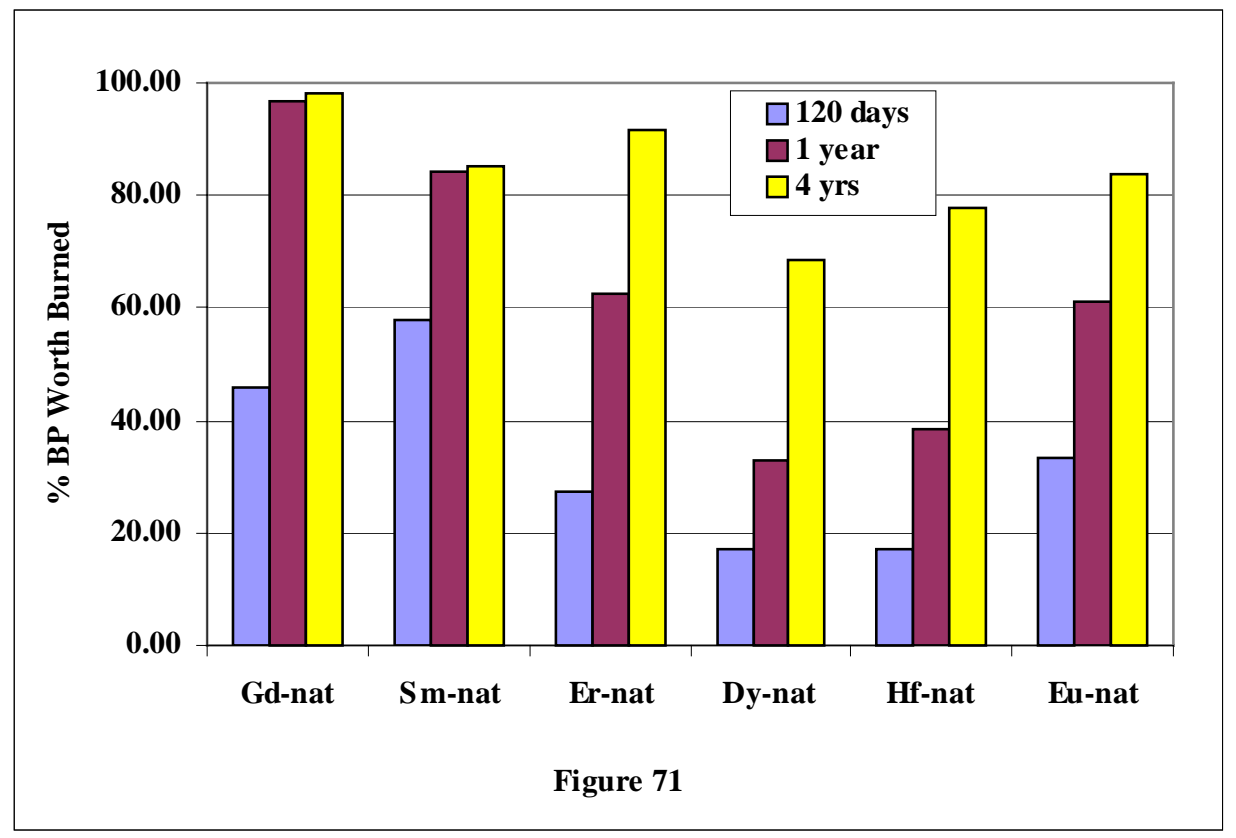

Figure 71. Burn rate for naturally occurring element burnable poisons coated on the outer surface of the fuel pellets as oxides, shown as a percentage of the initial negative reactivity remaining at a given time.

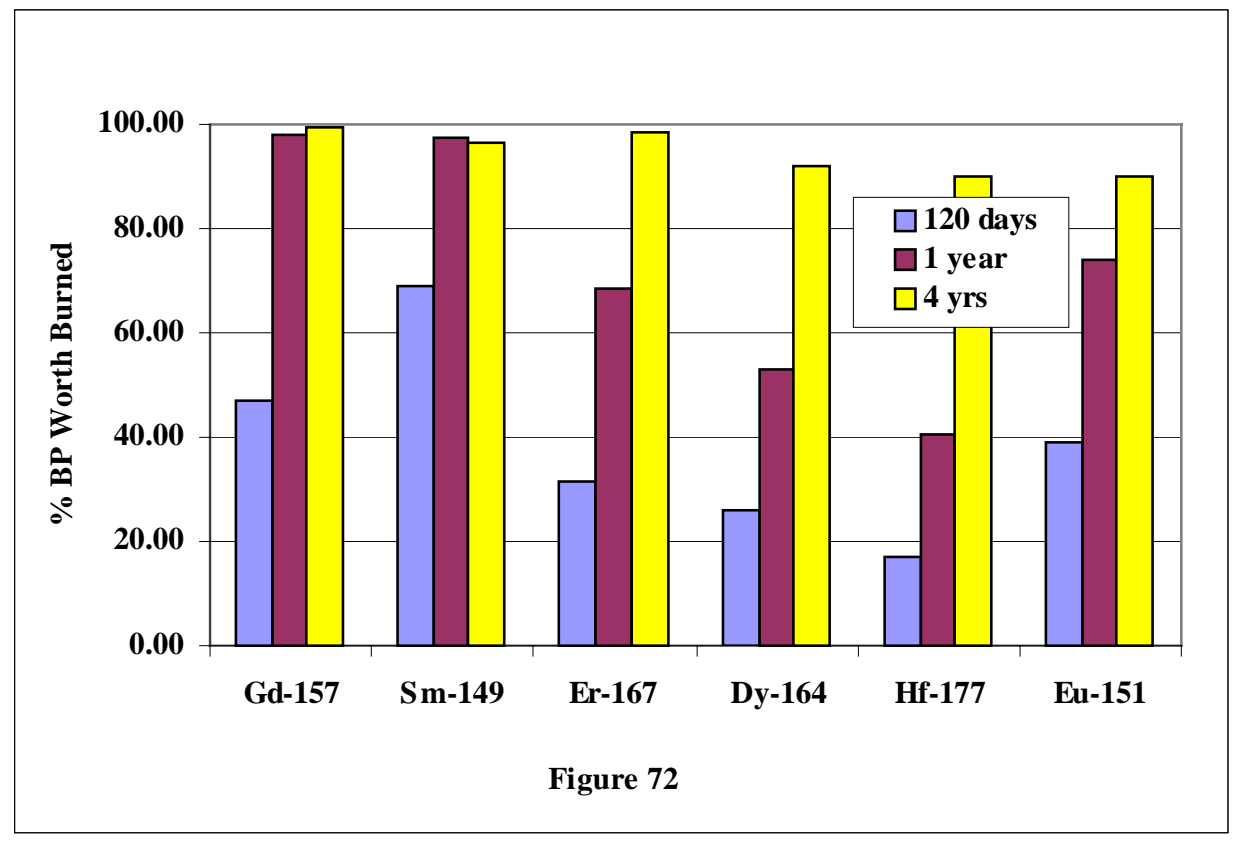

Figure 72. Burn rate for single burnable poisons coated on the outer surface of the fuel pellets as oxides, shown as a percentage of the initial negative reactivity remaining at a given time. 


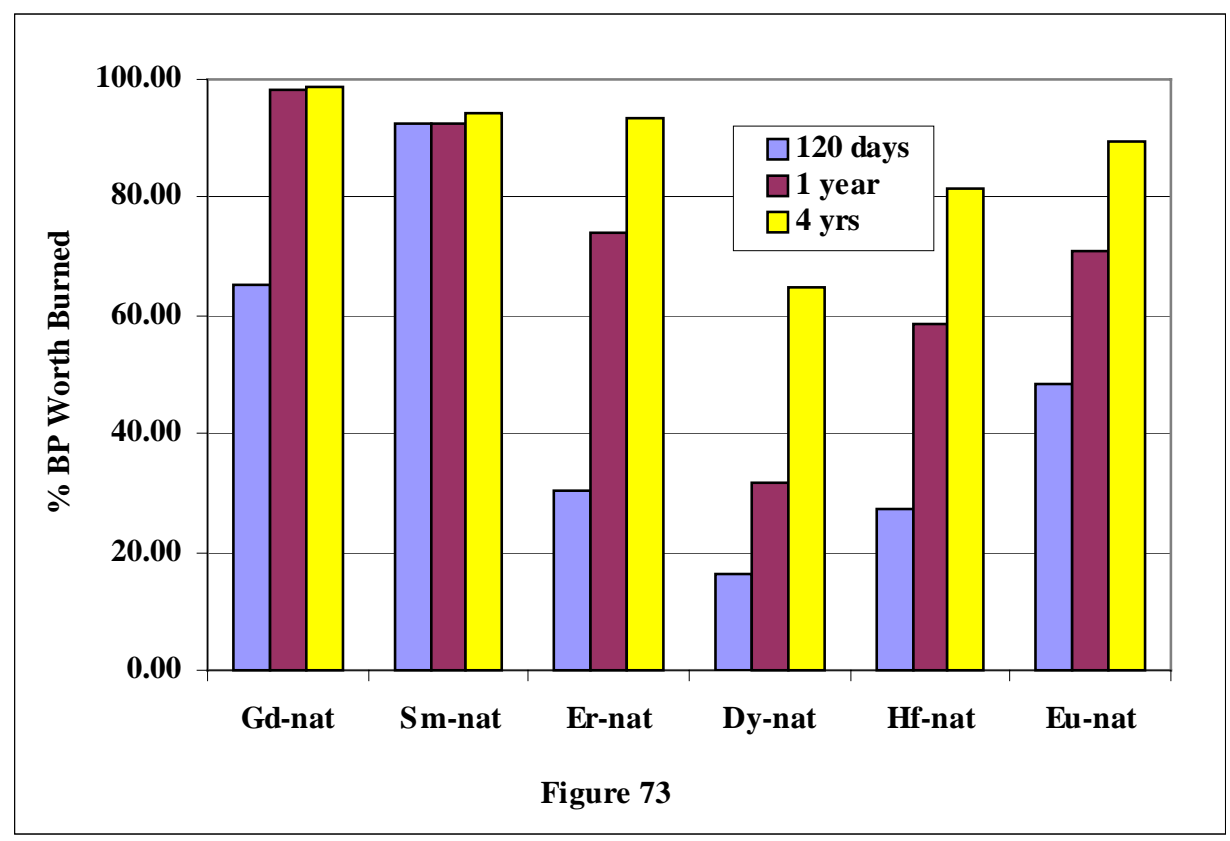

Figure 73. Burn rate for naturally occurring element burnable poisons in the form of a metal alloyed in the Zircaloy cladding, shown as a percentage of the initial negative reactivity remaining at a given time.

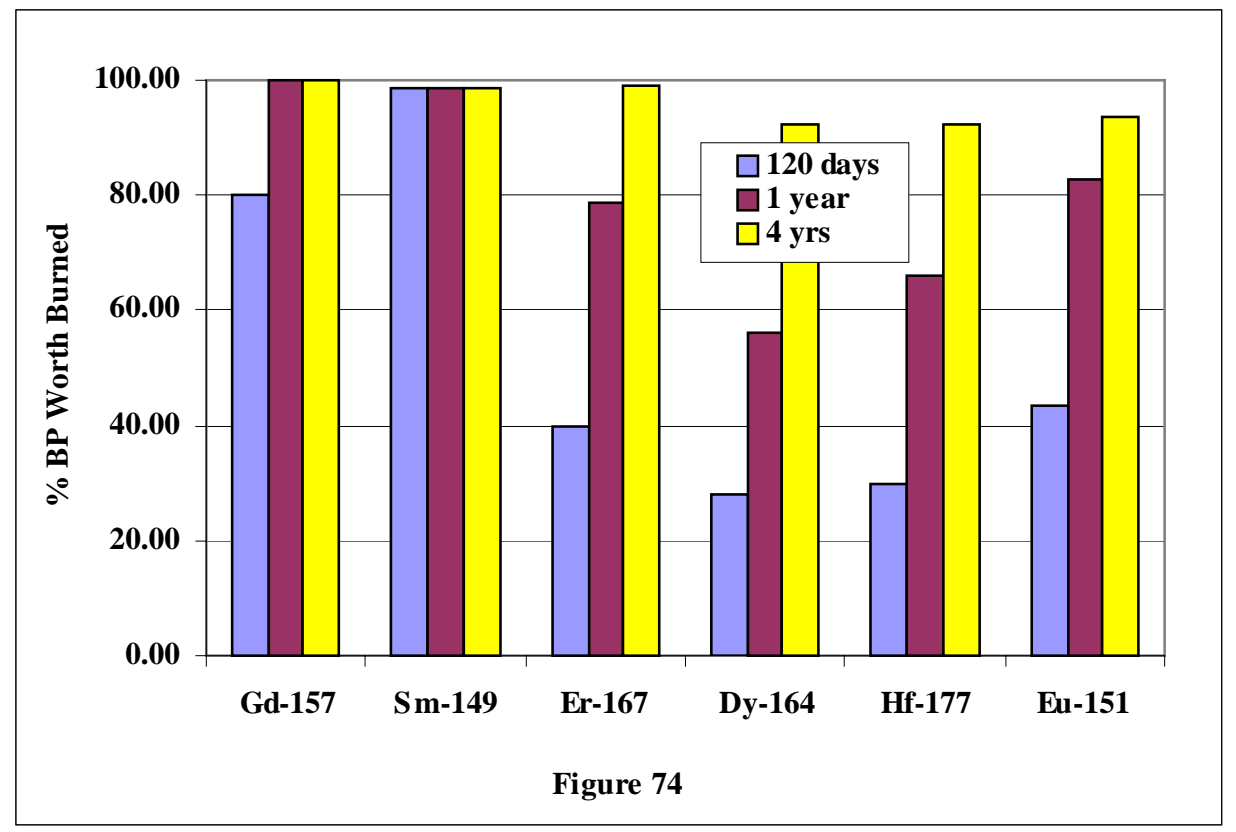

Figure 74. Burn rate for single burnable poisons in the form of a metal alloyed in the Zircaloy cladding, shown as a percentage of the initial negative reactivity remaining at a given time. 


\section{RESIDUAL REACTIVITY PENALTY}

A useful method to evaluate the isotope separation advantage is to plot the initial negative reactivity as a function of the number of days lost due to residual BP at the end of 4 years, which we shall call the residual absorber penalty (RAP). These parameters are plotted for the both naturally occurring elements and separated isotopes. The difference in the RAP in FPDs between the natural element and the single isotope is the bonus for using separated isotopes. The initial negative reactivity as a function of the RAP (all configurations of the BP), has been previously plotted in Figures 31, 40, and 47 for the case of 16 rods and in Figures 32, 41, 48, and 55 for the case of 64 rods. Table 53 summarizes the results. The RAP for the naturally occurring element is given for the cases of 16 and 64 fuel rods as well as the savings achieved by using the specified separated isotope. It can be seen that using ${ }^{157} \mathrm{Gd}$ in 16 rods results in a savings of $6.3 \mathrm{FPD}$, leaving only one day RAP. The isotope ${ }^{167} \mathrm{Er}$ results in a savings of 32 days for 16 fuel rods, but a RAP of 33 days remains. On the basis of RAP alone, Gd is the most beneficial isotope, but there are other considerations, as discussed previously. In order to achieve a uniform burnout over several years, lower absorbing isotopes must be used, either alone or in conjunction with Gd. However, in all cases, separation of isotopes results in a significant savings, considering that the revenue from a 1000-MW plant is about $\$ 1$ million per day.

Table 53. Savings in full-power days achieved by the use of single isotopes over the same naturally occurring elements for $10 \%$ initial negative reactivity

\begin{tabular}{|l|c|c|c|c|}
\hline \multicolumn{1}{|l|}{ Isotope } & $\begin{array}{c}\text { RAP (natural) } \\
16 \text { rods }\end{array}$ & $\begin{array}{c}\text { Savings } \\
16 \text { rods }\end{array}$ & $\begin{array}{c}\text { RAP (natural) } \\
64 \text { rods }\end{array}$ & $\begin{array}{c}\text { Savings } \\
64 \text { rods }\end{array}$ \\
\hline${ }^{157} \mathrm{Gd}$ & 7 & 6.3 & 1 & 1 \\
\hline${ }^{149} \mathrm{Sm}$ & 50 & 41 & 10 & 10 \\
\hline${ }^{167} \mathrm{Er}$ & 65 & 32 & 30 & 28 \\
\hline${ }^{164} \mathrm{Dy}$ & 200 & 64 & 160 & 110 \\
\hline${ }^{177} \mathrm{Hf}$ & $\sim 100$ & 20 & & \\
\hline${ }^{151} \mathrm{Eu}$ & & & 45 & 30 \\
\hline
\end{tabular}

An interesting phenomenon is that the curves are similar for each configuration of the BP. The initial reactivity is strongly dependent upon where the BP is placed, but for a given initial negative reactivity, the penalty at 4 years is the same. This is not the case when the number of fuel rods containing BP is changed. When Sm is distributed in 64 fuel rods, the new set of curves shown in Figure 41 is obtained. The savings from isotope separation is nonetheless apparent.

\section{ISOTOPE SEPARATION}

Preliminary calculations have been performed by Theragenics Corporation to assess the feasibility of isotope enrichment of candidate BP materials. Table 54 presents results from the preliminary calculations of enrichment fraction and annual production. Feasibility will be tested as soon as the equipment is available to obtain an actual separation run to permit cost estimation.

These are calculated values and remain to be demonstrated. However, the fact that production rates are given in tens of kilograms per year is encouraging. A loading of an entire fuel assembly requires quantities of BP in the range of tens of grams to kilograms. The scale of production is appropriate. 
Table 54. Enrichment percentage and annual production for candidate isotopes by plasma separation.

$\begin{array}{cc}\text { Isotope } & \begin{array}{c}\text { Natural Ab } \\ \left(\frac{\circ}{0}\right)\end{array} \\ { }^{157} \mathrm{Gd} & 15.65 \\ { }^{149} \mathrm{Sm} & 13.8 \\ { }^{167} \mathrm{Er} & 22.9 \\ { }^{164} \mathrm{Dy} & 28.2 \\ { }^{177} \mathrm{Hf} & 18.6 \\ { }^{184} \mathrm{Os} & 0.02\end{array}$

\begin{tabular}{|c|c|}
\hline \multicolumn{2}{|c|}{ Enrichment } \\
\hline (\% per & pass) \\
\hline 2-Tesla & 6-Tesl \\
\hline Device & Device \\
\hline 45.8 & 72.5 \\
\hline 45.8 & 71.7 \\
\hline 55.5 & 78.9 \\
\hline 63.1 & 83.6 \\
\hline 46.0 & 71.9 \\
\hline 0.14 & 0.41 \\
\hline
\end{tabular}

$\begin{array}{cc}\begin{array}{c}\text { Production Rate } \\ (\mathrm{kg} / \mathrm{y} r)\end{array} \\ \begin{array}{c}\text { 2-Tesla } \\ \text { Device }\end{array} & \begin{array}{l}\text { 6-Tesla } \\ \text { Device }\end{array} \\ 51 & 130 \\ 43 & 109 \\ 79 & 203 \\ 96 & 245 \\ 68 & 175 \\ 76 & 195\end{array}$




\section{CONCLUSIONS}

1. For a PWR, ${ }^{157} \mathrm{Gd},{ }^{167} \mathrm{Er},{ }^{149} \mathrm{Sm},{ }^{164} \mathrm{Dy}$, and ${ }^{177} \mathrm{Hf}$ have been identified as candidates for isotope separation study.

2. Introduction of burnable poison into the fuel cladding is a new method that permits more rapid and complete burnup.

3. An understanding of burnable poison behavior in various configurations has been achieved that can be applied to advanced reactors with extended fuel cycles.

4. Calculations have demonstrated that there is a potential savings of several million dollars over a 4 year period; thus further studies in isotope separation are justified.

5. Following isotope separation studies, calculations will be performed with the new data to be obtained on isotope enrichment.

The isotopes ${ }^{157} \mathrm{Gd},{ }^{149} \mathrm{Sm},{ }^{167} \mathrm{Er},{ }^{164} \mathrm{Dy}$, and ${ }^{177} \mathrm{Hf}$ were identified as potential candidates based on nuclear performance. All of them result in a significant cost saving over the naturally occurring elements. The cost of separation of the isotopes must now be evaluated. It is planned to use plasma separation to prepare small amounts of isotopes to assess the feasibility and cost of separation. This process typically produces isotopes in kilogram quantities, resulting in a substantial reduction in cost over previously used processes such as calutrons. In addition to cost savings, absorbers can be used to increase fuel enrichment and extend the fuel cycle. Fuel cycle lengths of several years without refueling are possible. 



\section{REFERENCES}

1. P. G. Boczar, "CANDU Fuel-Cycle Vision," presented at IAEA Technical Committee Meeting on "Fuel Cycle Options for LWRs and HWRs, Victoria, B.C., April 28-May 1, 1998.

2. P. Blanpain, D. Haas, and F. Motte, "High Rated and High Burnup Gadolinia Fuel Irradiated in the BR3 $17 \times 17$ PWR," Proc. Symposium on Improvements in Water Reactor Fuel Technology and Utilization, Vienna, IAEA, 1987, p. 305.

3. A. Radkowsky, Naval Reactors Physics Handbook, Vol.1, U.S. Atomic Energy Commission, Washington, D.C., 1964.

4. IAEA, Design and Performance of WWER Fuel, Technical Reports Series No.379, Vienna, International Atomic Energy Agency, 1996, p. 44.

5. F. J. Rahn, A. G. Adamantiades, J. E. Kenton, and C. Braun, A Guide to Nuclear Power Technology: A Resource for Decision Making, New York, John Wiley \& Sons, 1984, 436-38.

6. R. G. Cochran and N. Tsoulfanidis, The Nuclear Fuel Cycle: Analysis and Management American Nuclear Society, La Grange Park, Ill., 1990, p. 84.

7. C. Collette, G.Francillon, F. Abadia, and A. Darraud, "Operating Experience of the Fragema Gadolinia Bearing Fuel Assembly," Proc. Symposium on Improvements in Water Reactor Fuel Technology and Utilization, Vienna, IAEA, 1987, p. 255.

8. L. Goldstein and A. A. Strasser, "A Comparison of Gadolinia and Boron for Burnable Poison Applications in Pressurized Water Reactors," Nucl.Tech. 60352 (1983).

9. C. M. Hove and S. W. Spetz, "Improvement of Gadolinia Fuel Cycle Economics by Isotopic Enrichment of ${ }^{157} \mathrm{Gd}, "$ Trans. Am. Nucl. Soc. 50, 102 (1985).

10. Private communication, W. S. Aaron, Oak Ridge National Laboratory, November, 1998.

11. A. G. Croff, ORIGEN2.1-A Revised and updated Version of the Oak Ridge Isotope Generation and Depletion Code, ORNL-5621, July 1980.

12. E. A. Villarino, R. J. Stamm'ler, A. A. Ferri, and J. J. Casal, Nucl Sci. Eng. 112, 16 (1992).

13. HELIOS-General Two-Dimensional Neutron and Gamma Transport with Depletion Code, Studvik-Scandpower, Inc.

14. R. E. MacFarlane and D. W. Muir, The NJOY Nuclear Data Processing System, LA-12740-M, 1994.

15. J. F. Briesmeister, ed., MCNP-A General Monte Carlo N-Particle Transport Code 4C, LA13709-M, April 2000.

16. J. P. Renier and C. W. Alexander, An Advanced Version of the Oak Ridge Isotope Generation and Depletion Code with Fission Product Yield for 34 Actinides, ORNL-TM (to be published 2001). 


\section{Appendix A1}

Reactivity Worths and Masses of $\mathrm{Gd}_{2} \mathrm{O}_{3}$ Burnable Poison Homogeneously Mixed in the Fuel Pellets

\section{Table 5 Cases}



A1 - Gadolinium with Natural Isotopic Abundances 

Figure A1.1. Negative Reactivity and Masses BP for Gd-nat and transmutation daughters as a function of fuel life for $17 \times 17$ fuel assemblies with 08 poison rods, $2.0 \mathrm{w} \% \mathrm{Gd} 2 \mathrm{O} 3$ poison homogeneously mixed in the UO2 pellets

Reactor power $3400 \mathrm{MWth}, 193$ fuel assemblies, initial enrichment 4.5w\%U-235

(Case : gd000_2_100p_h2o_08_bp_fuel_2_o)
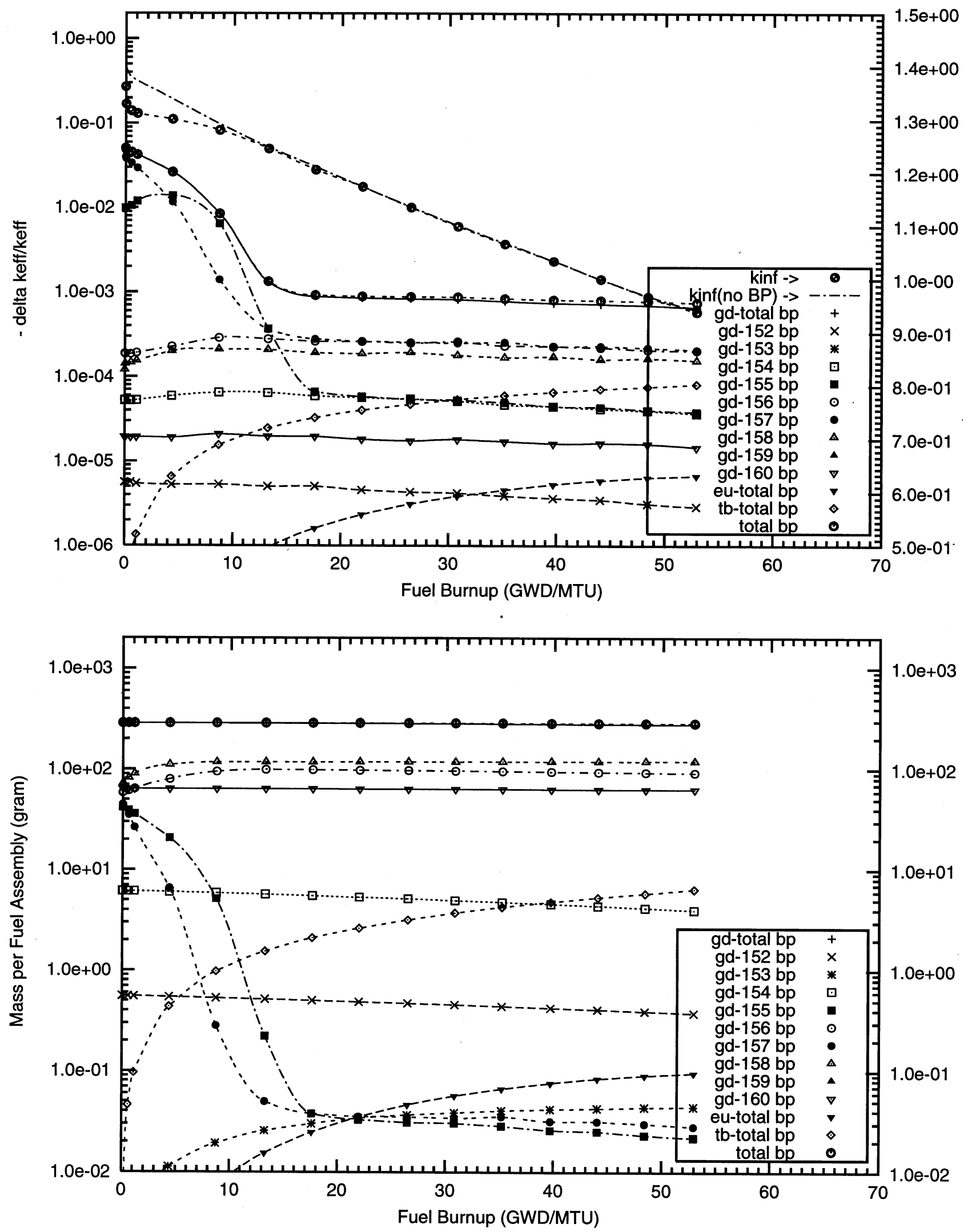
Figure A1.2. Negative Reactivity and Masses BP for Gd-nat and transmutation daughters as a function of fuel life for $17 \times 17$ fuel assemblies with 08 poison rods, $4.0 \mathrm{w} \% \mathrm{Gd} 2 \mathrm{O} 3$ poison homogeneously mixed in the UO2 pellets

Reactor power $3400 \mathrm{MWth}, 193$ fuel assemblies, initial enrichment 4.5w\%U-235 (Case : gd000_3_08_bp_fuel o)

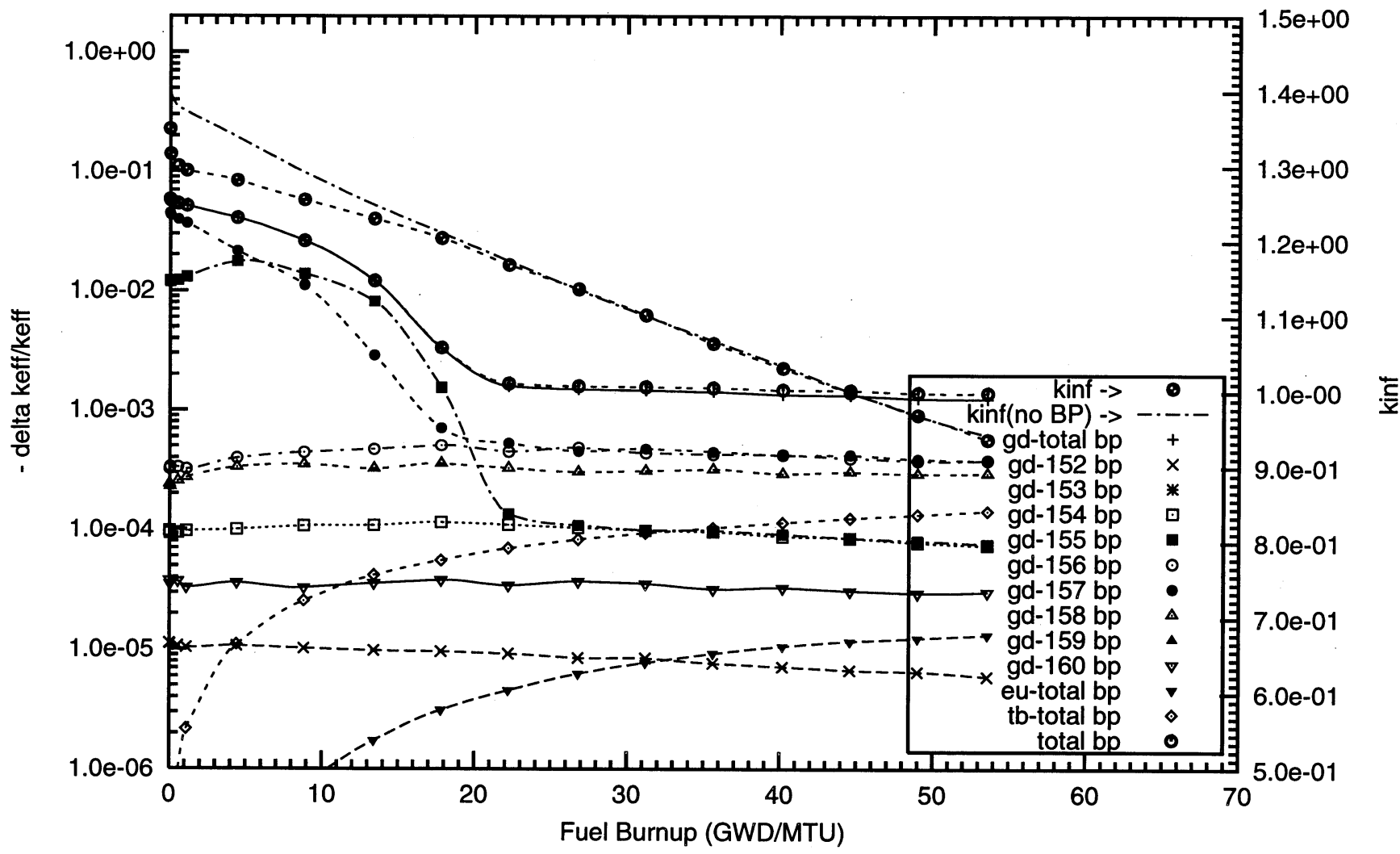


Figure A1.3. Negative Reactivity and Masses BP for Gd-nat and transmutation daughters as a function of fuel life for $17 \times 17$ fuel assemblies with 08 poison rods, $8.0 \mathrm{w} \% \mathrm{Gd} 2 \mathrm{O} 3$ poison homogeneously mixed in the UO2 pellets

Reactor power $3400 \mathrm{MWth}, 193$ fuel assemblies, initial enrichment $4.5 \mathrm{w} \% \mathrm{U}-235$

(Case : gd000_4_100p_h2o_08_bp_fuel_2_0)
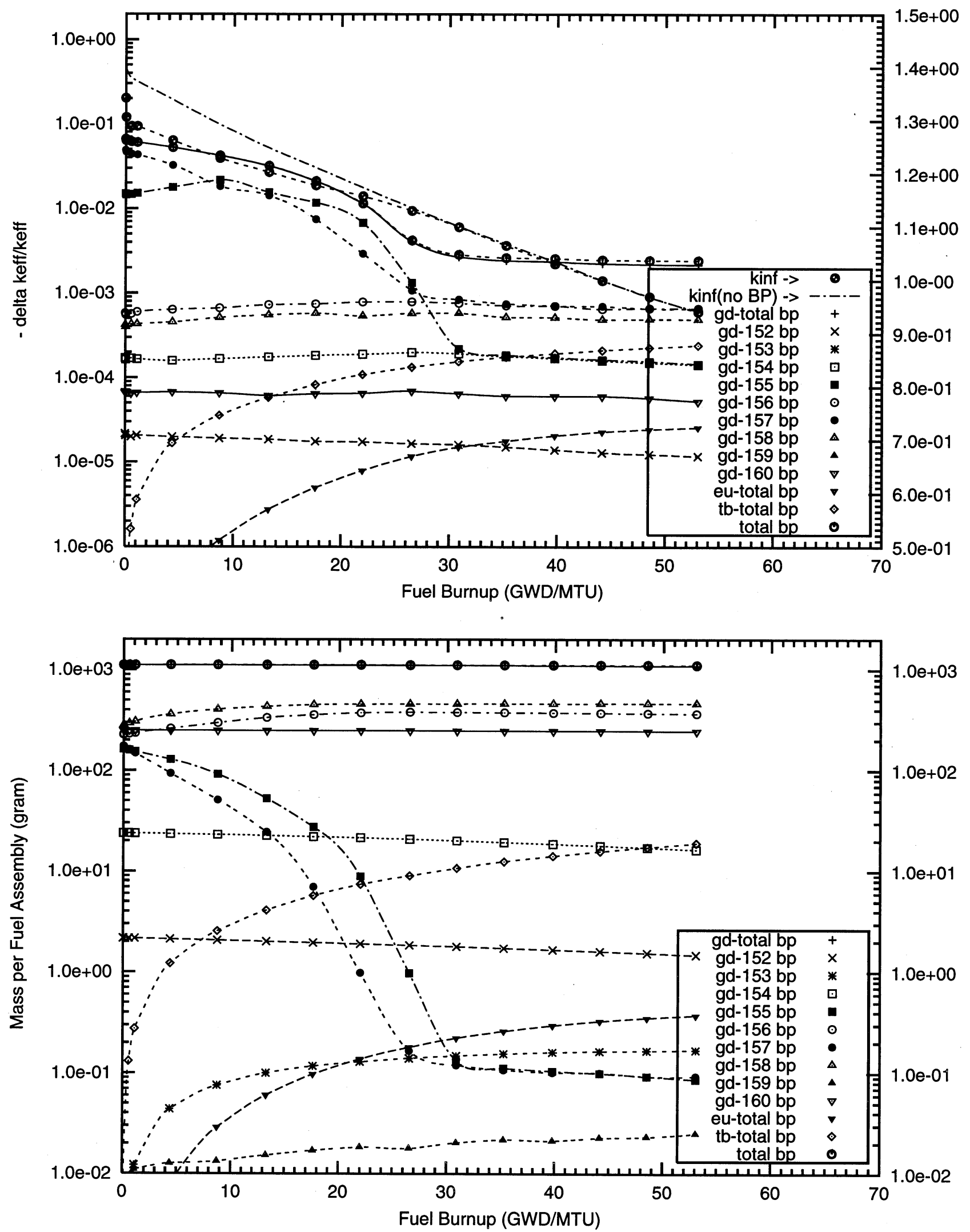
Figure A1.4. Negative Reactivity and Masses BP for Gd-nat and transmutation daughters as a function of fuel life for $17 \times 17$ fuel assemblies with 16 poison rods,

$0.5 \mathrm{w} \% \mathrm{Gd} 2 \mathrm{O} 3$ poison homogeneously mixed in the UO2 pellets

Reactor power $3400 \mathrm{MWth}, 193$ fuel assemblies, initial enrichment $4.5 \mathrm{w} \% \mathrm{U}-235$

(Case : gd000_0_100p_h20_16_bp_fuel_2_o)
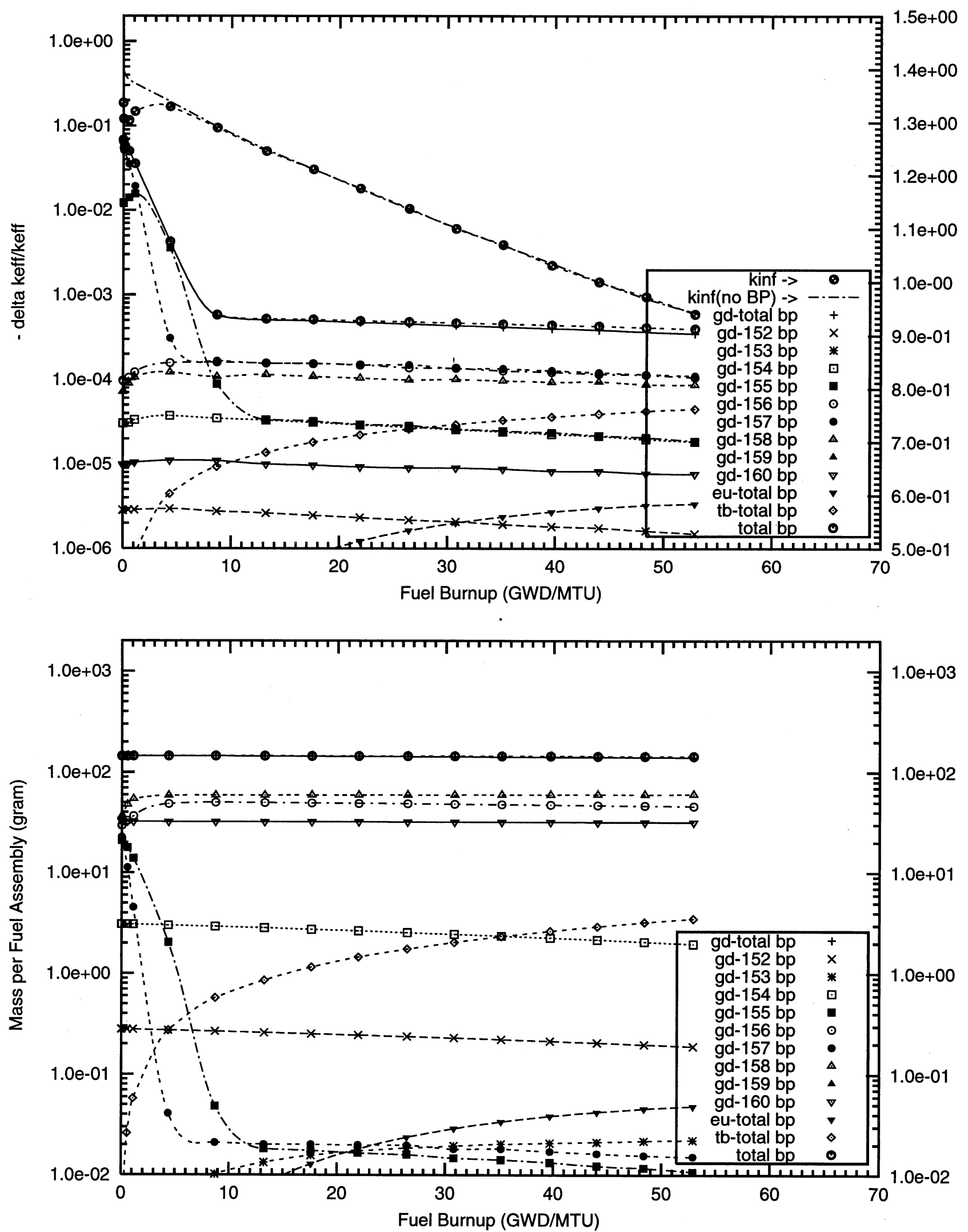
Figure A1.5. Negative Reactivity and Masses BP for Gd-nat and transmutation daughters as a function of fuel life for $17 \times 17$ fuel assemblies with 16 poison rods, $1.0 \mathrm{w} \% \mathrm{Gd} 2 \mathrm{O} 3$ poison homogeneously mixed in the UO2 pellets

Reactor power $3400 \mathrm{MWth}, 193$ fuel assemblies, initial enrichment 4.5w\%U-235

(Case : gd000_1_100p_h2o_16_bp_fuel_2_o)
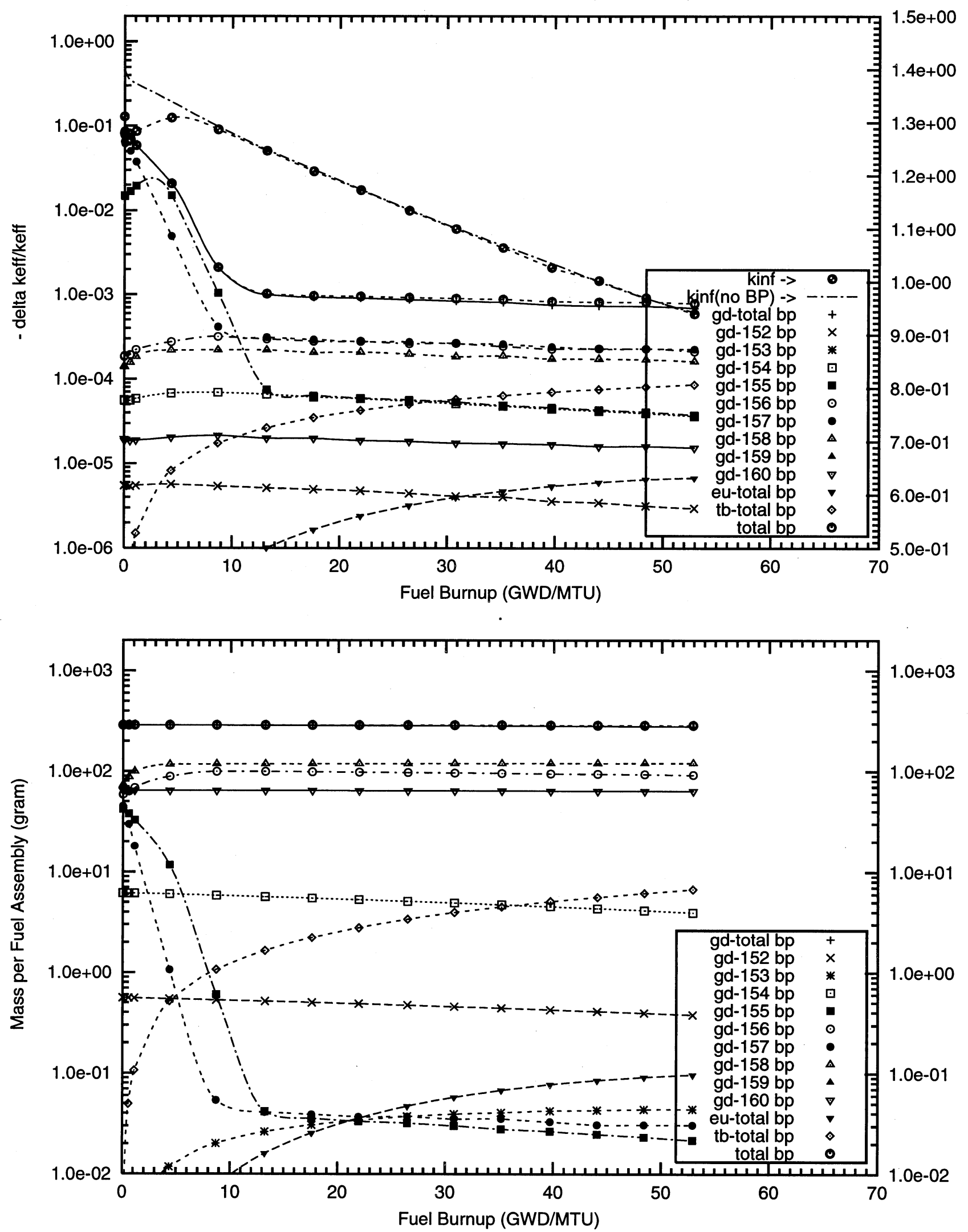
Figure A1.6. Negative Reactivity and Masses BP for Gd-nat and transmutation daughters as a function of fuel life for $17 \times 17$ fuel assemblies with 16 poison rods, $2.0 \mathrm{w} \% \mathrm{Gd} 2 \mathrm{O} 3$ poison homogeneously mixed in the UO2 pellets

Reactor power $3400 \mathrm{MWth}, 193$ fuel assemblies, initial enrichment $4.5 \mathrm{w} \% \mathrm{U}-235$

(Case : gd000_2_100p_h2o_16_bp_fuel_2_o)
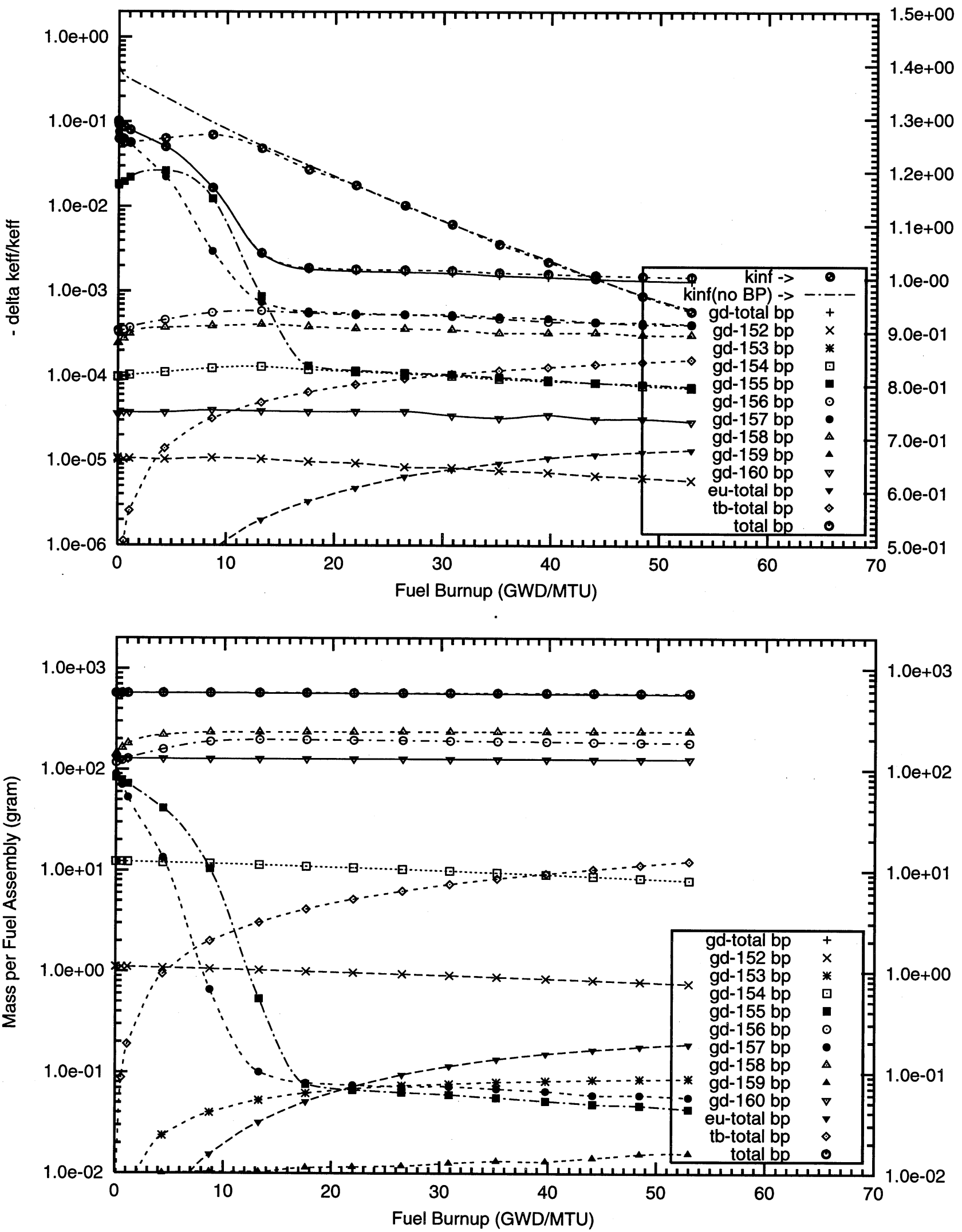
Figure A1.7. Negative Reactivity and Masses BP for Gd-nat and transmutation daughters as a function of fuel life for $17 \times 17$ fuel assemblies with 16 poison rods, $4.0 \mathrm{w} \% \mathrm{Gd} 2 \mathrm{O} 3$ poison homogeneously mixed in the UO2 pellets

Reactor power $3400 \mathrm{MWth}, 193$ fuel assemblies, initial enrichment $4.5 \mathrm{w} \% \mathrm{U}-235$

(Case : gd000_3_100p_h2o_16_bp_fuel_2_o)
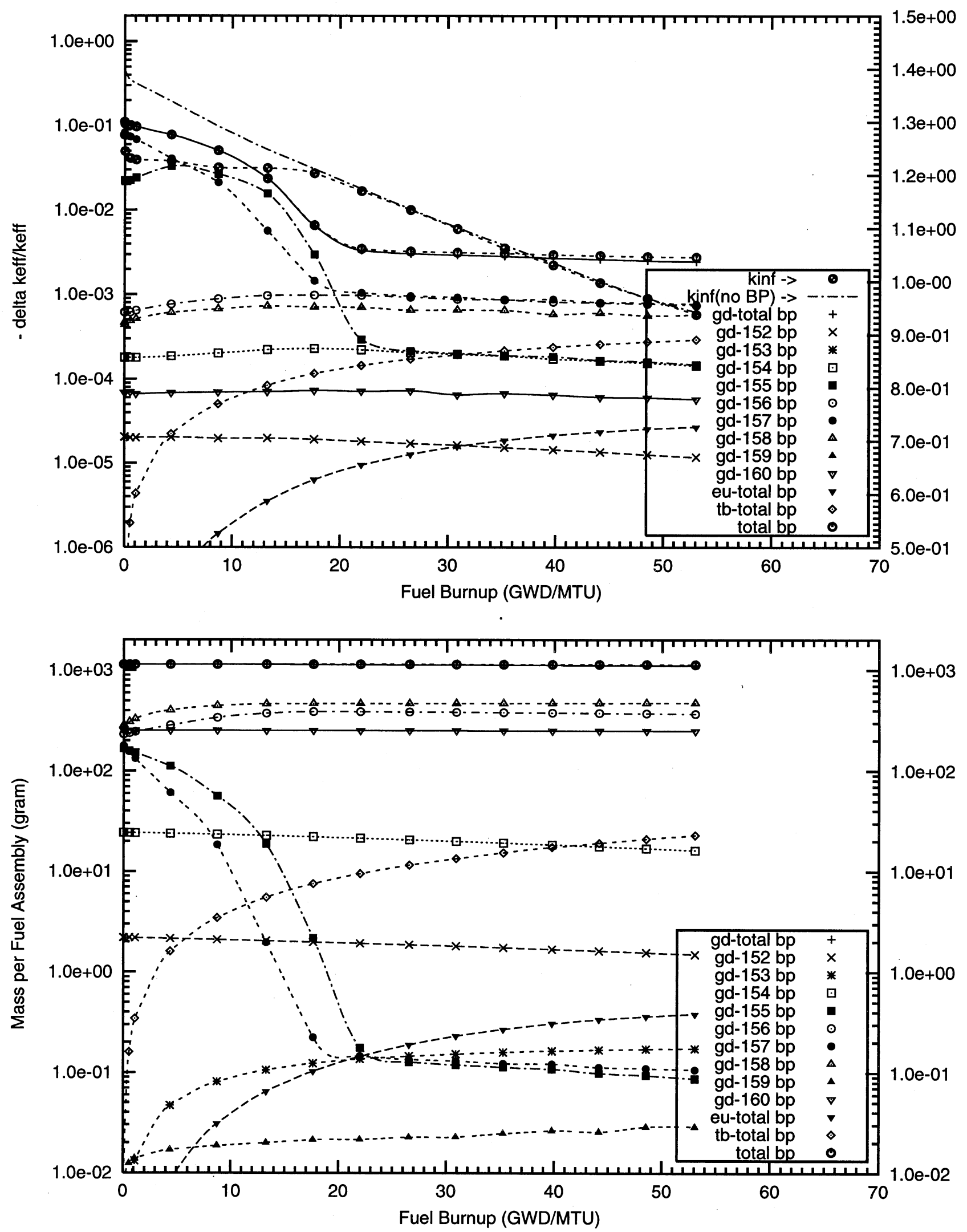
Figure A1.8. Negative Reactivity and Masses BP for Gd-nat and transmutation daughters as a function of fuel life for $17 \times 17$ fuel assemblies with 16 poison rods,

$8.0 \mathrm{w} \% \mathrm{Gd} 2 \mathrm{O} 3$ poison homogeneously mixed in the UO2 pellets

Reactor power $3400 \mathrm{MWth}, 193$ fuel assemblies, initial enrichment $4.5 \mathrm{w} \% \mathrm{U}-235$

(Case : gd000_4_100p_h20_16_bp_fuel_2_o)
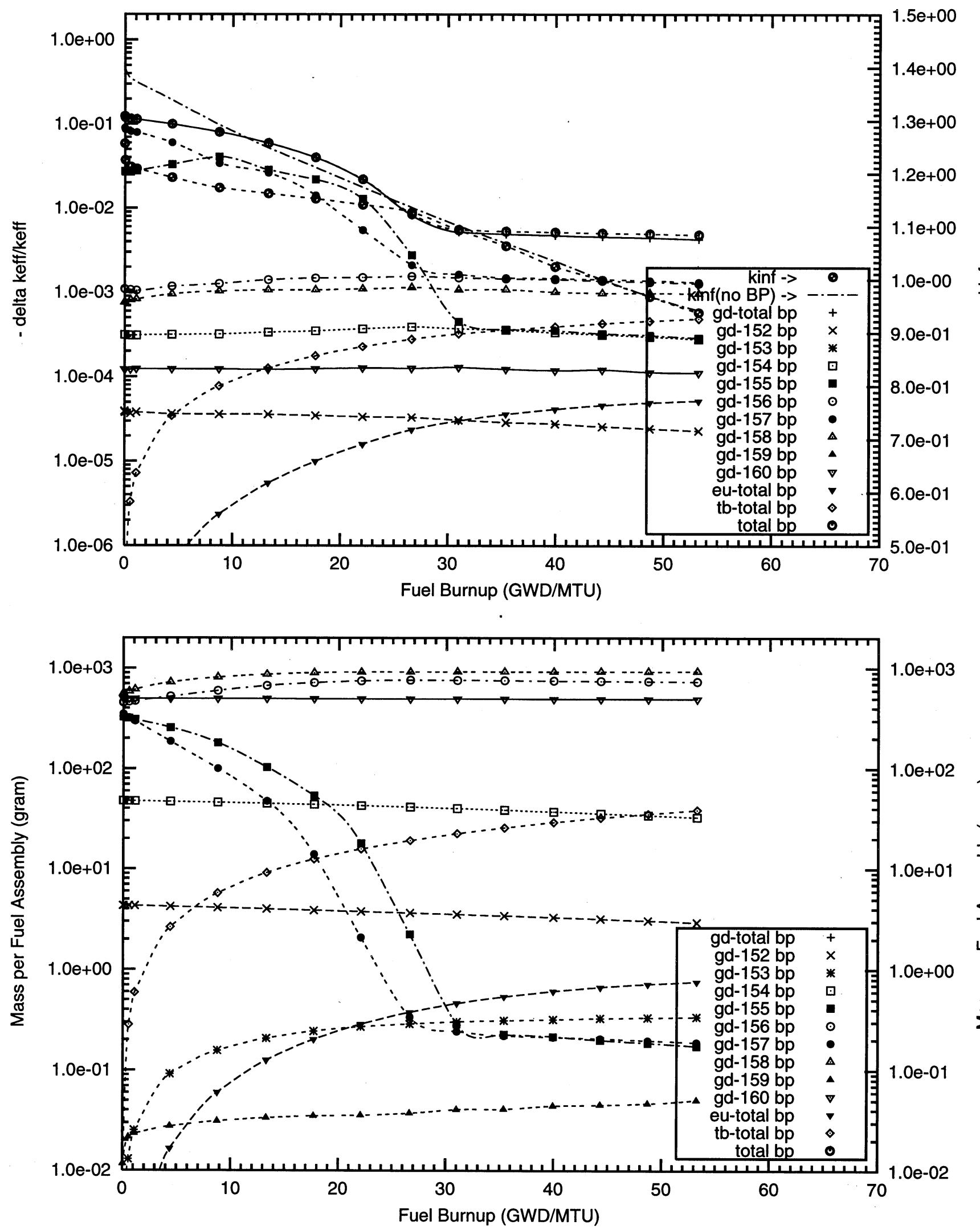
Figure A1.9. Negative Reactivity and Masses BP for Gd-nat and transmutation daughters as a function of fuel life for $17 \times 17$ fuel assemblies with 20 poison rods, $4.0 \mathrm{w} \% \mathrm{Gd} 2 \mathrm{O} 3$ poison homogeneously mixed in the UO2 pellets

Reactor power $3400 \mathrm{MWth}, 193$ fuel assemblies, initial enrichment 4.5w\%U-235

(Case : gd000_3_16_bp_fuel (old) o)
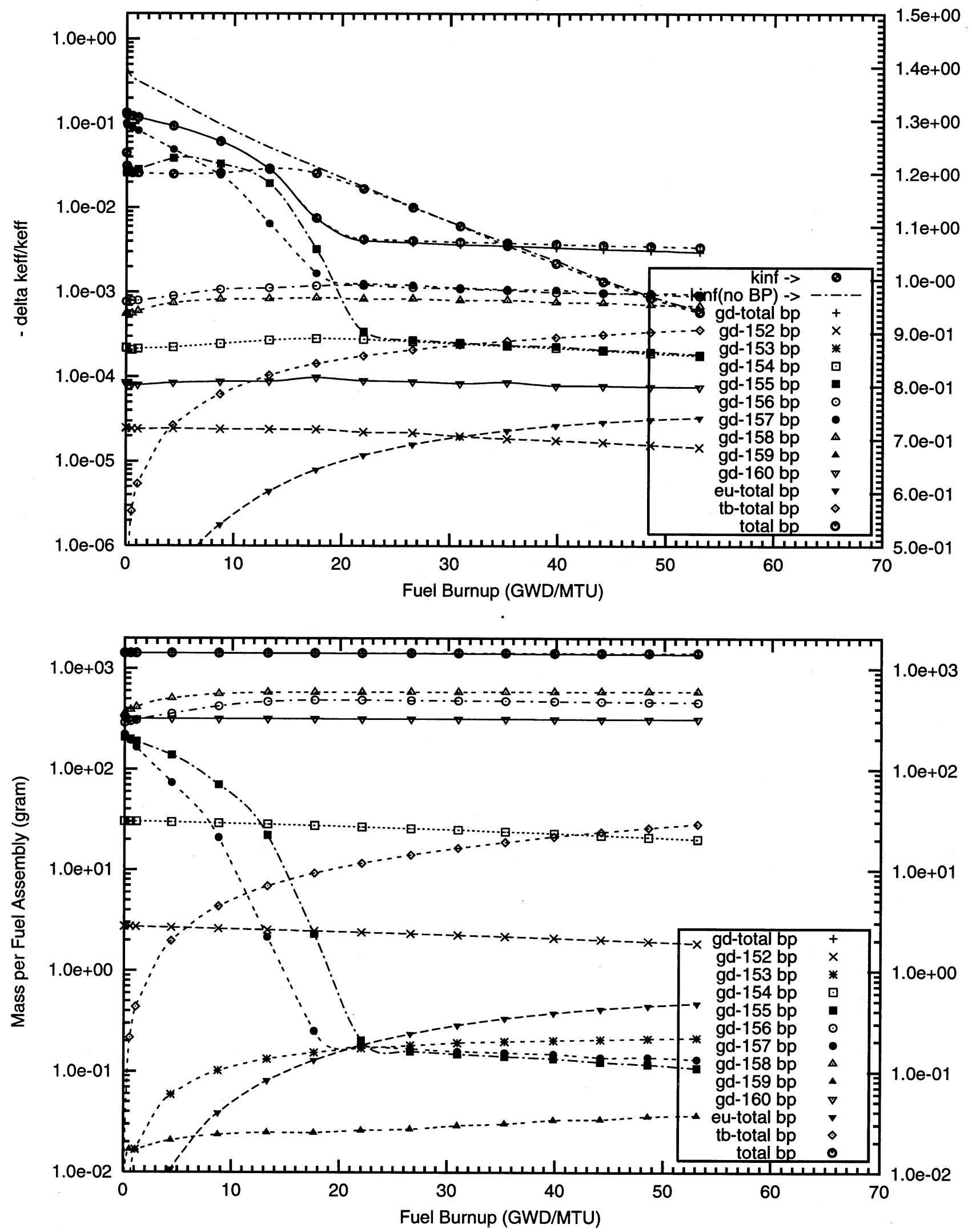
Figure A1.10. Negative Reactivity and Masses BP for Gd-nat and transmutation daughters as a function of fuel life for $17 \times 17$ fuel assemblies with 64 poison rods, $0.2 \mathrm{w} \% \mathrm{Gd} 2 \mathrm{O} 3$ poison homogeneously mixed in the UO2 pellets

Reactor power $3400 \mathrm{MWth}, 193$ fuel assemblies, initial enrichment $4.5 \mathrm{w} \% \mathrm{U}-235$

(Case : gd000_8_64_bp_fuel o)
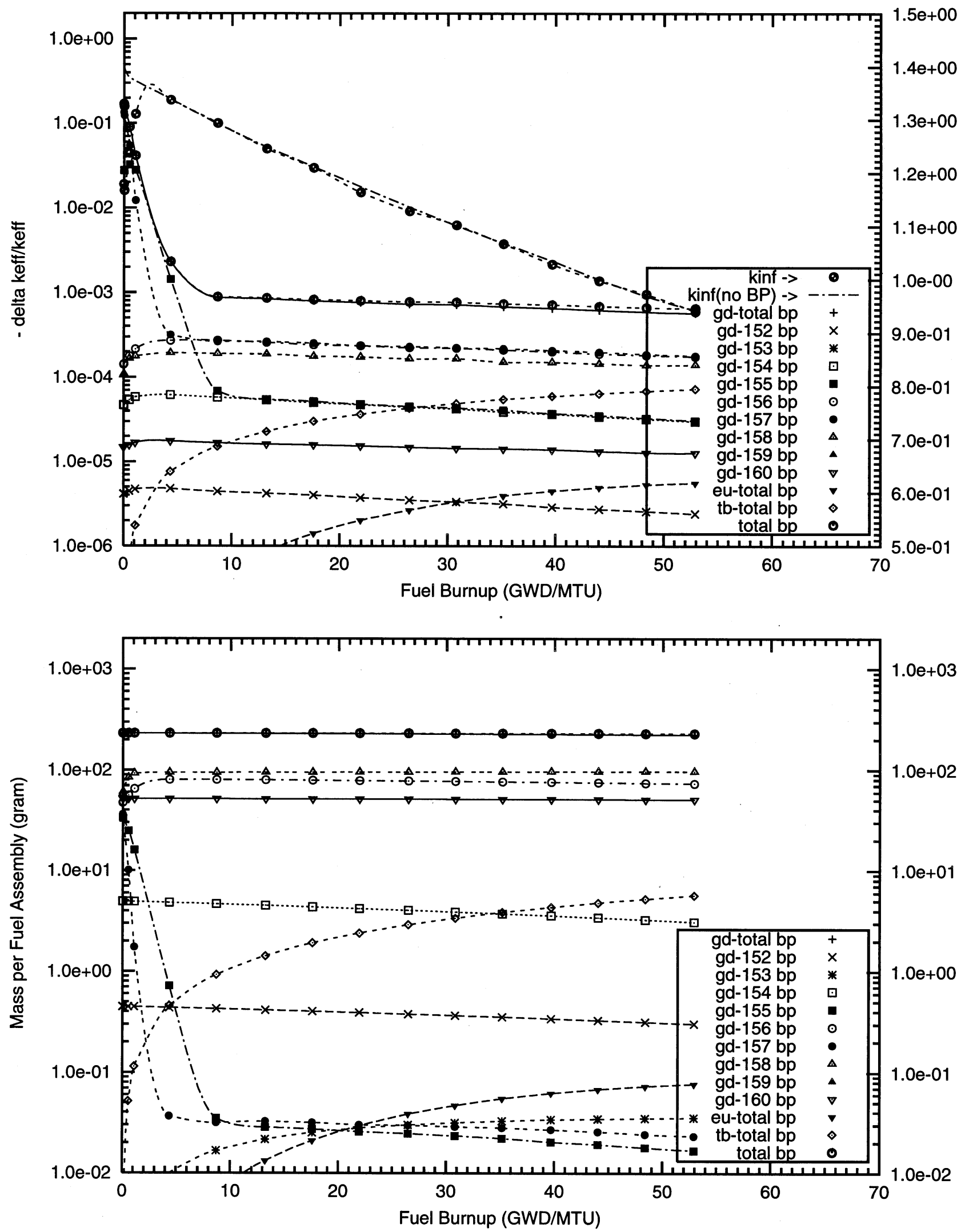
Figure A1.11. Negative Reactivity and Masses BP for Gd-nat and transmutation daughters as a function of fuel life for $17 \times 17$ fuel assemblies with 64 poison rods, $0.5 \mathrm{w} \% \mathrm{Gd} 2 \mathrm{O} 3$ poison homogeneously mixed in the UO2 pellets

Reactor power $3400 \mathrm{MWth}, 193$ fuel assemblies, initial enrichment $4.5 \mathrm{w} \% \mathrm{U}-235$

(Case : gd000_0_64_bp_fuel o)
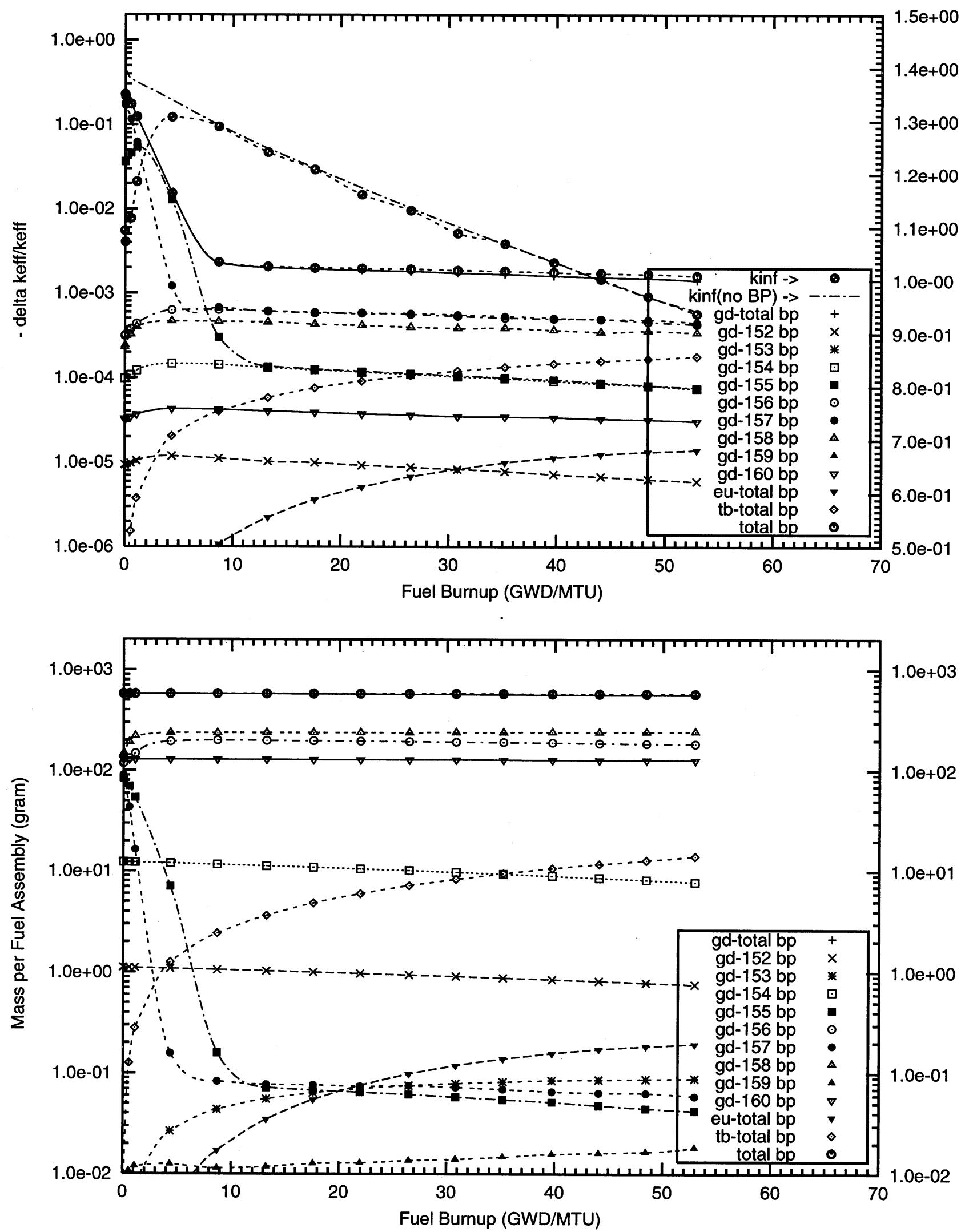

A1 - Gadolinium Fully Enriched in ${ }^{155}$ Gd 

Figure A1.12. Negative Reactivity and Masses BP for Gd-155 and transmutation daughters as a function of fuel life for $17 \times 17$ fuel assemblies with 08 poison rods, $1.0 \mathrm{w} \% \mathrm{Gd} 2 \mathrm{O} 3$ poison homogeneously mixed in the UO2 pellets

Reactor power $3400 \mathrm{MWth}, 193$ fuel assemblies, initial enrichment $4.5 \mathrm{w} \% \mathrm{U}-235$

(Case : gd155_1_100p_h20_08_bp_fuel_2_o)
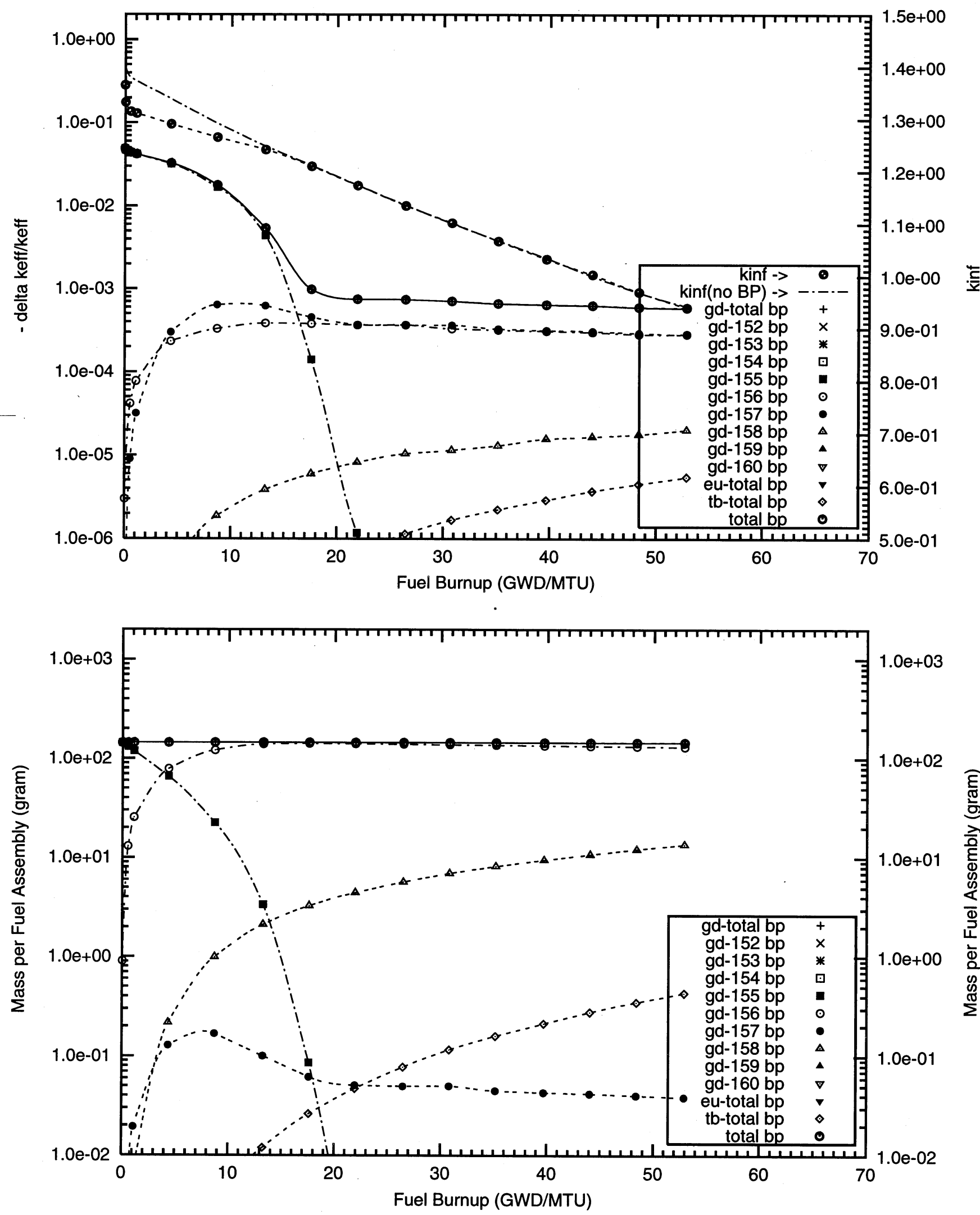
Figure A1.13. Negative Reactivity and Masses BP for Gd-155 and transmutation daughters as a function of fuel life for $17 \times 17$ fuel assemblies with 08 poison rods, $2.0 \mathrm{w} \% \mathrm{Gd} 2 \mathrm{O} 3$ poison homogeneously mixed in the UO2 pellets

Reactor power $3400 \mathrm{MWth}, 193$ fuel assemblies, initial enrichment $4.5 \mathrm{w} \% \mathrm{U}-235$

(Case : gd155_2_100p_h2o_08_bp_fuel_2_o)
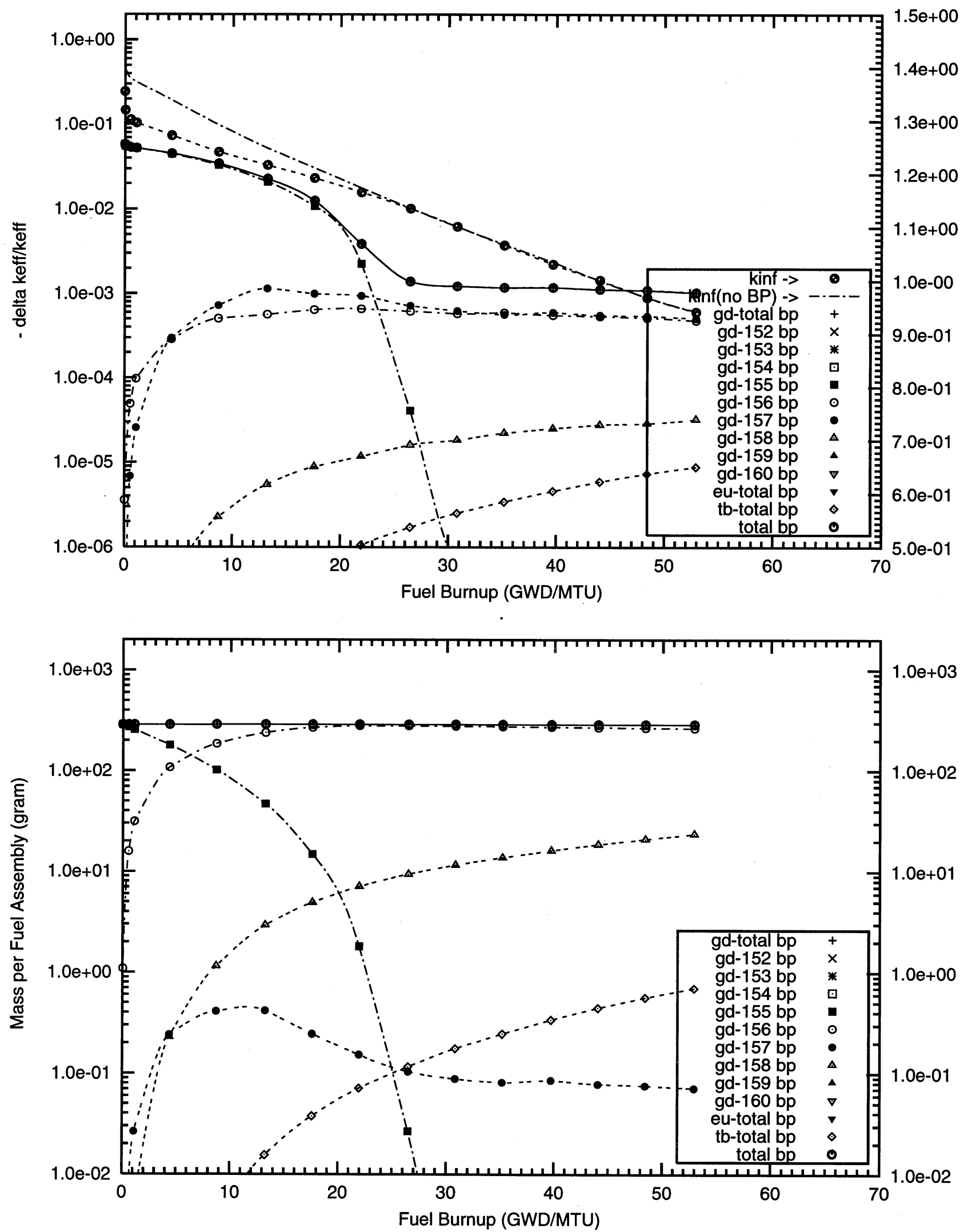
Figure A1.14. Negative Reactivity and Masses BP for Gd-155 and transmutation daughters as a function of fuel life for $17 \times 17$ fuel assemblies with 08 poison rods, $4.0 \mathrm{w} \% \mathrm{Gd} 2 \mathrm{O} 3$ poison homogeneously mixed in the UO2 pellets

Reactor power $3400 \mathrm{MWth}, 193$ fuel assemblies, initial enrichment $4.5 \mathrm{w} \% \mathrm{U}-235$ (Case : gd155_3_08_bp_fuel o)

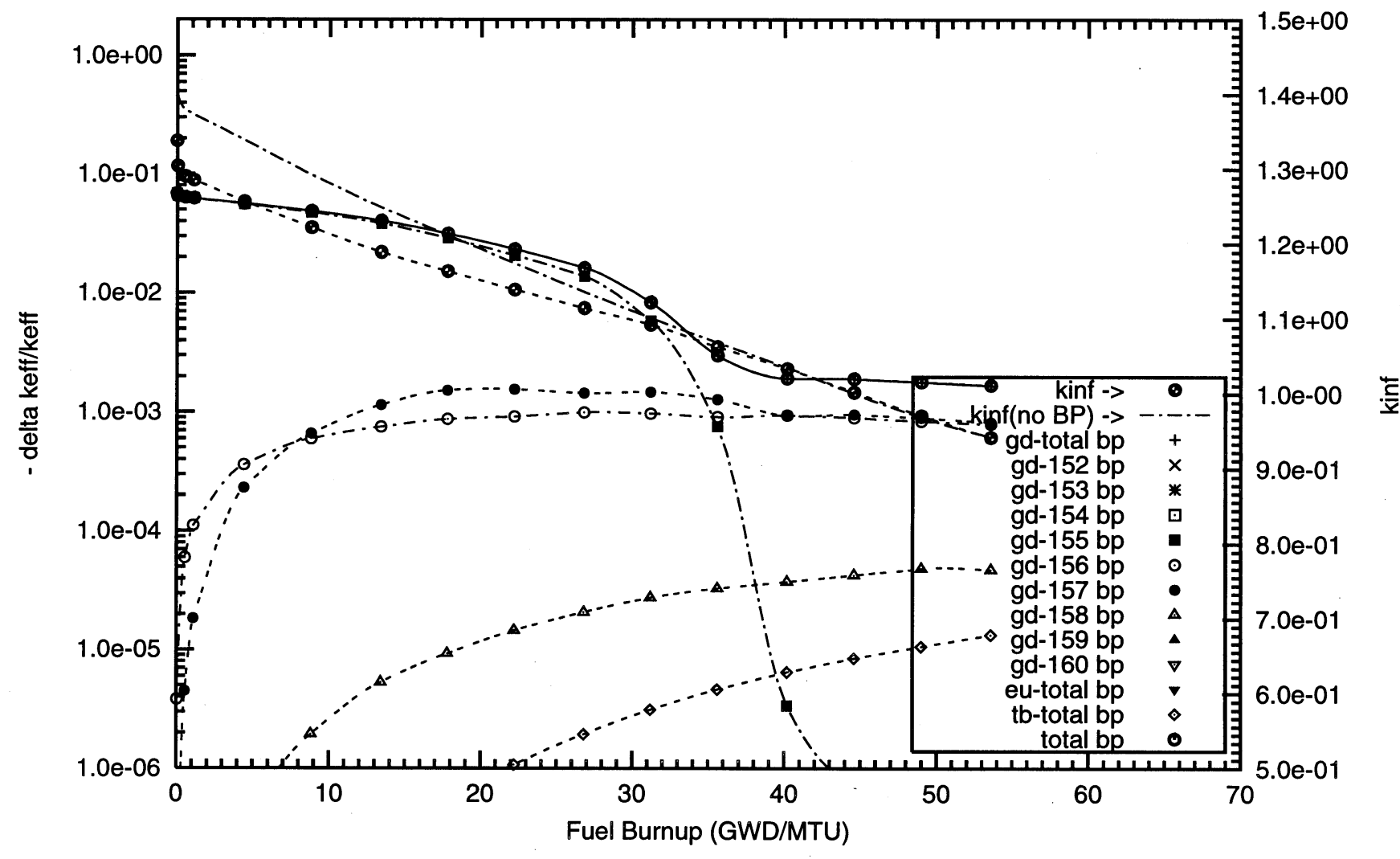


Figure A1.15. Negative Reactivity and Masses BP for Gd-155 and transmutation daughters as a function of fuel life for $17 \times 17$ fuel assemblies with 16 poison rods, $1.0 \mathrm{w} \% \mathrm{Gd} 2 \mathrm{O} 3$ poison homogeneously mixed in the UO2 pellets

Reactor power $3400 \mathrm{MWth}, 193$ fuel assemblies, initial enrichment 4.5w\%U-235

(Case : gd155_1_100p_h20_16_bp_fuel_2_o)
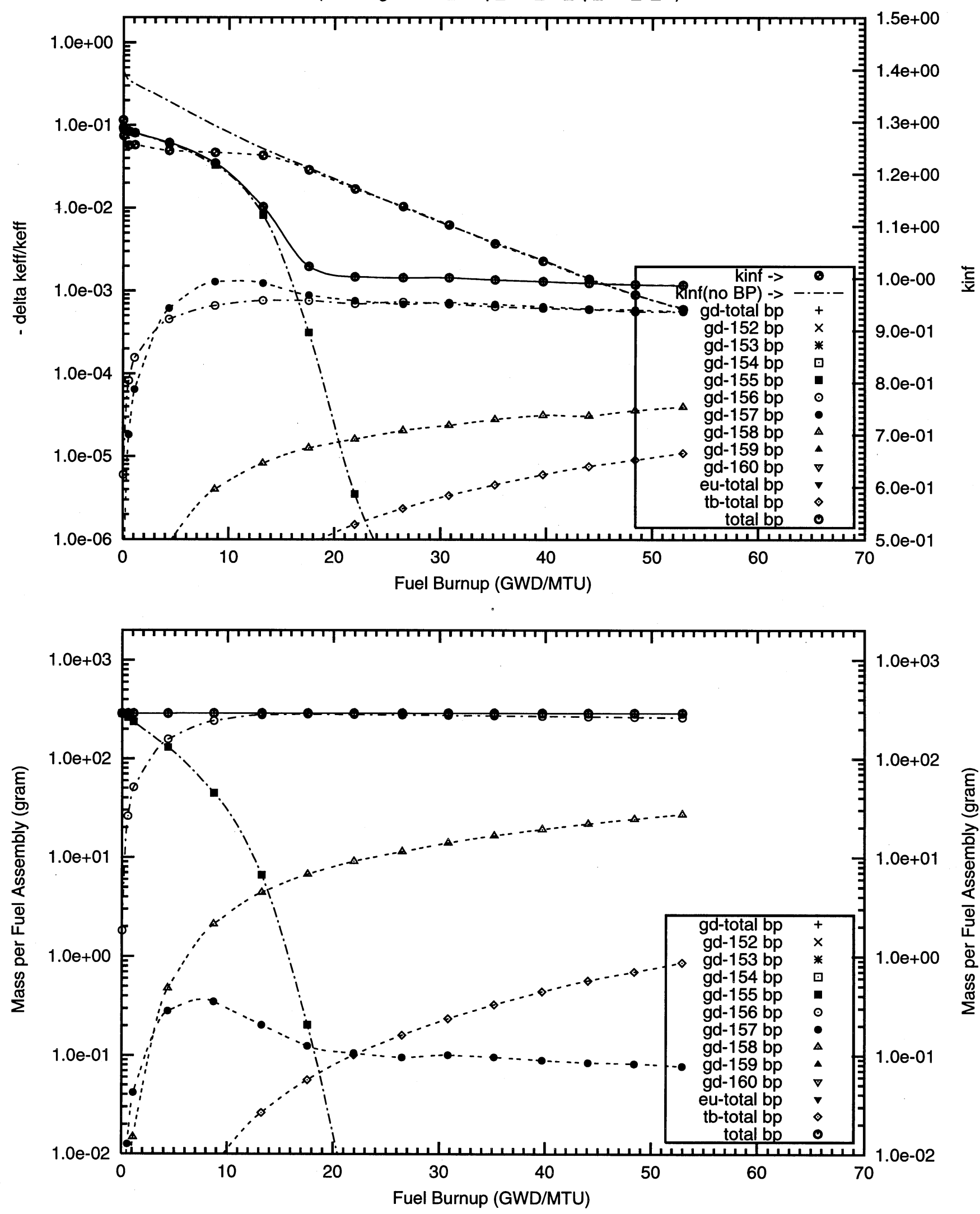
Figure A1.16. Negative Reactivity and Masses BP for Gd-155 and transmutation daughters as a function of fuel life for $17 \times 17$ fuel assemblies with 16 poison rods, $2.0 \mathrm{w} \% \mathrm{Gd} 2 \mathrm{O} 3$ poison homogeneously mixed in the $\mathrm{UO} 2$ pellets

Reactor power $3400 \mathrm{MWth}, 193$ fuel assemblies, initial enrichment 4.5w\%U-235

(Case : gd155_2_100p_h2o_16_bp_fuel_2_o)
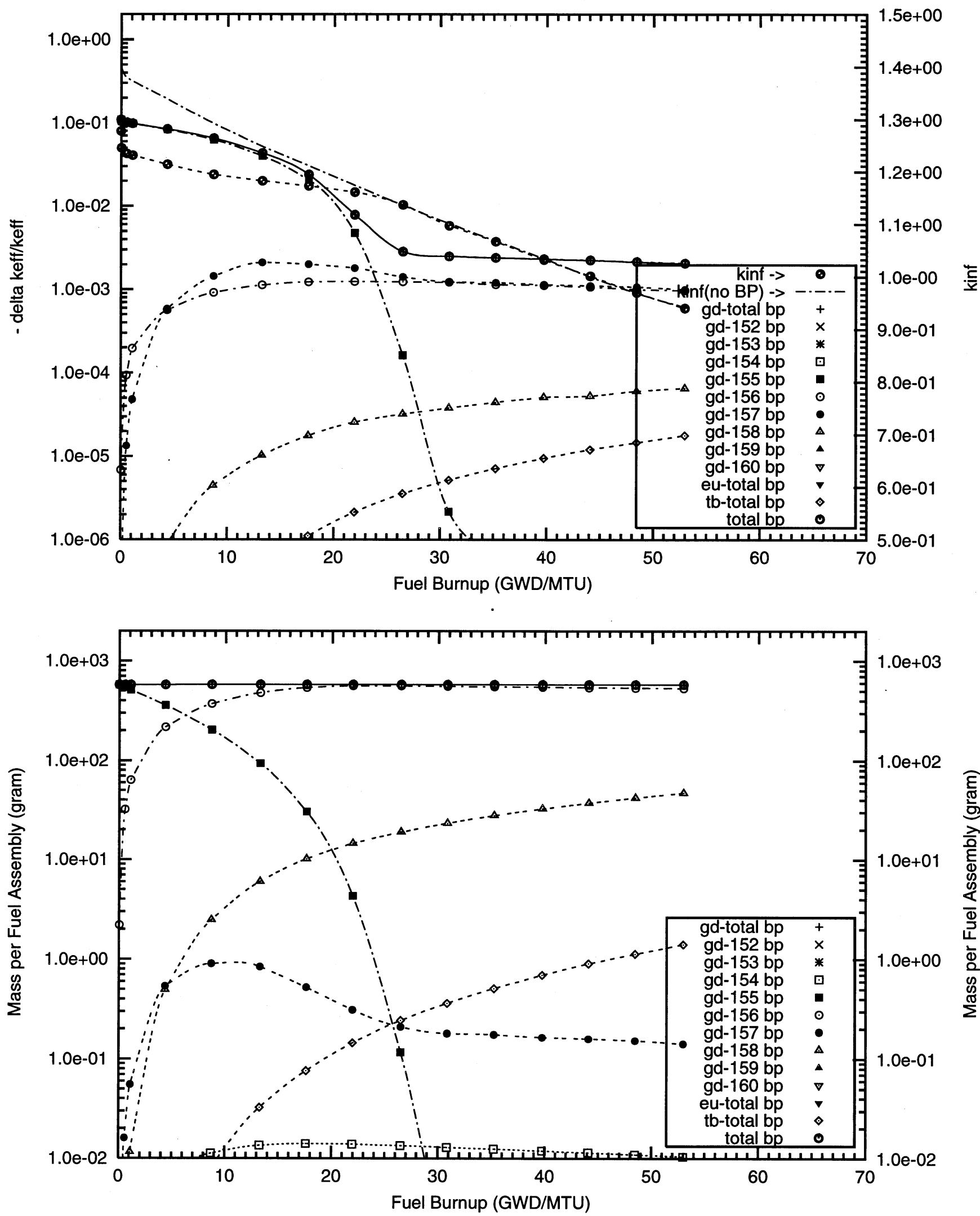
Figure A1.17. Negative Reactivity and Masses BP for Gd-155 and transmutation daughters as a function of fuel life for $17 \times 17$ fuel assemblies with 20 poison rods, $2.0 \mathrm{w} \% \mathrm{Gd} 2 \mathrm{O} 3$ poison homogeneously mixed in the UO2 pellets

Reactor power $3400 \mathrm{MWth}, 193$ fuel assemblies, initial enrichment 4.5W\%U-235

(Case : gd155_2_16_bp_fuel (old) o)
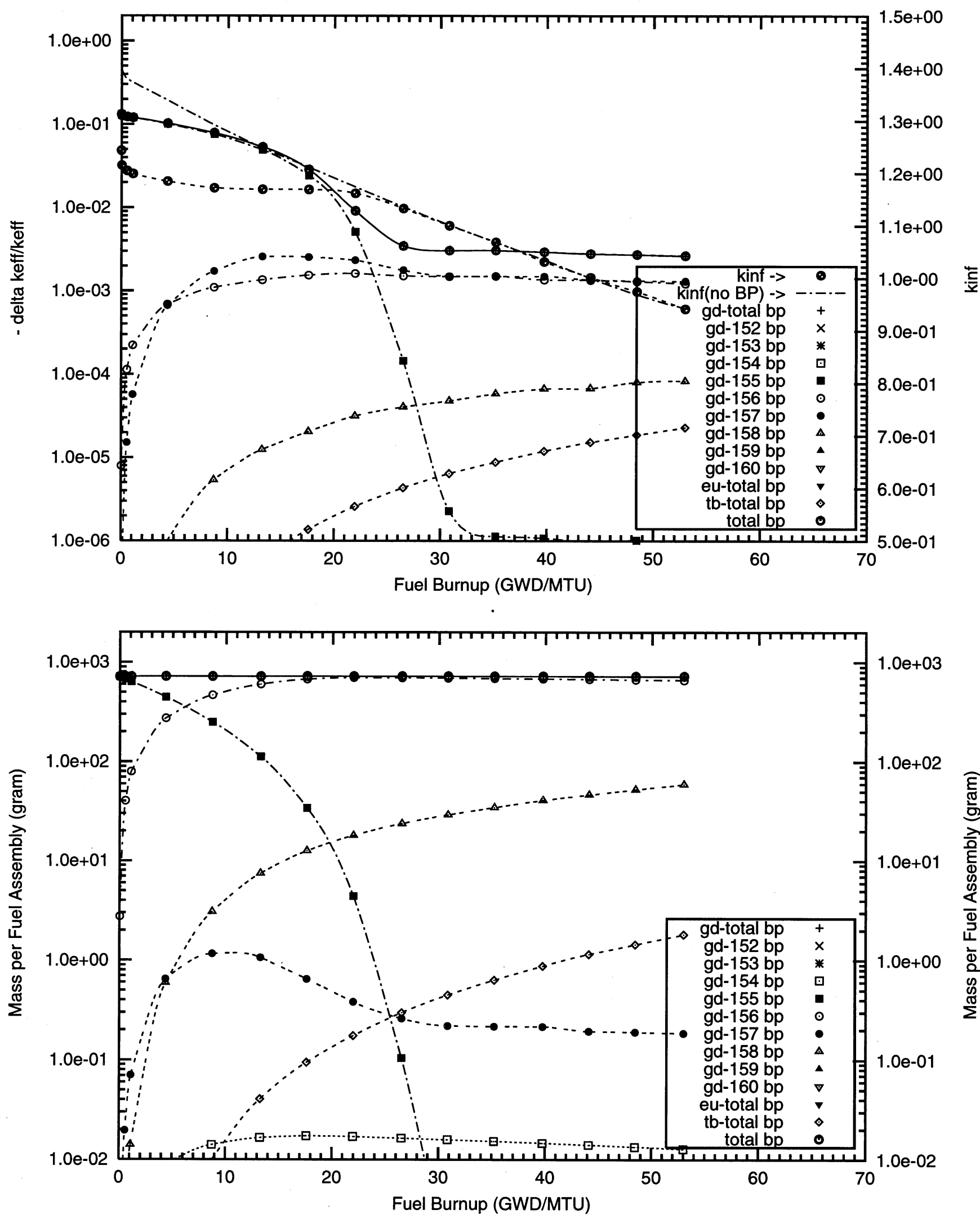
A1 - Gadolinium Fully Enriched in ${ }^{157}$ Gd 
Figure A1.18. Negative Reactivity and Masses BP for Gd-157 and transmutation daughters as a function of fuel life for $17 \times 17$ fuel assemblies with 08 poison rods, $1.0 \mathrm{w} \% \mathrm{Gd} 2 \mathrm{O} 3$ poison homogeneously mixed in the UO2 pellets

Reactor power $3400 \mathrm{MWth}, 193$ fuel assemblies, initial enrichment 4.5w\%U-235

(Case : gd157_1_100p_h2o_08_bp_fuel_2_o)
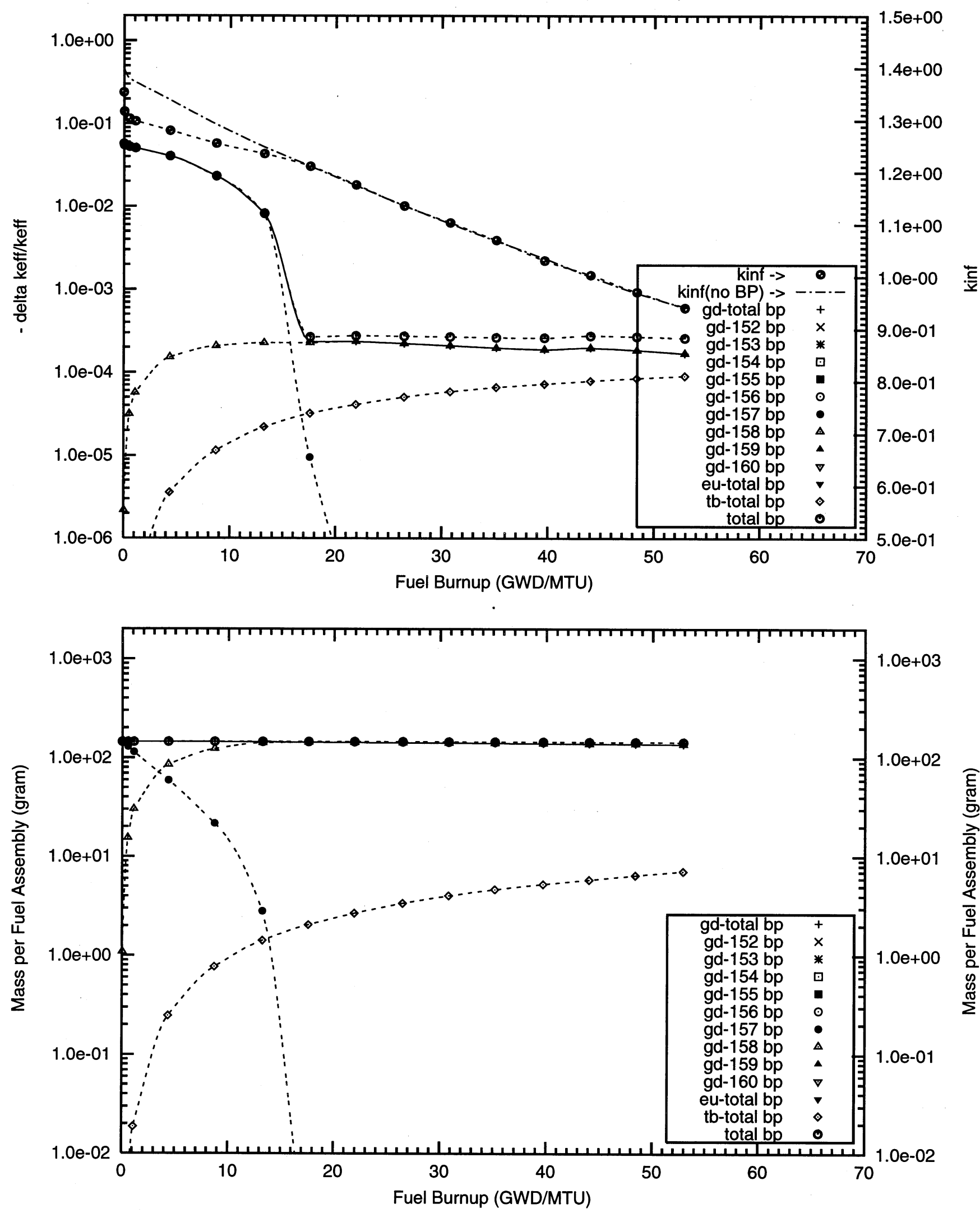
Figure A1.19. Negative Reactivity and Masses BP for Gd-157 and transmutation daughters as a function of fuel life for $17 \times 17$ fuel assemblies with 08 poison rods, $2.0 \mathrm{w} \% \mathrm{Gd} 2 \mathrm{O} 3$ poison homogeneously mixed in the UO2 pellets

Reactor power $3400 \mathrm{MWth}, 193$ fuel assemblies, initial enrichment $4.5 \mathrm{w} \% \mathrm{U}-235$

(Case : gd157_2_100p_h20_08_bp_fuel_2_o)
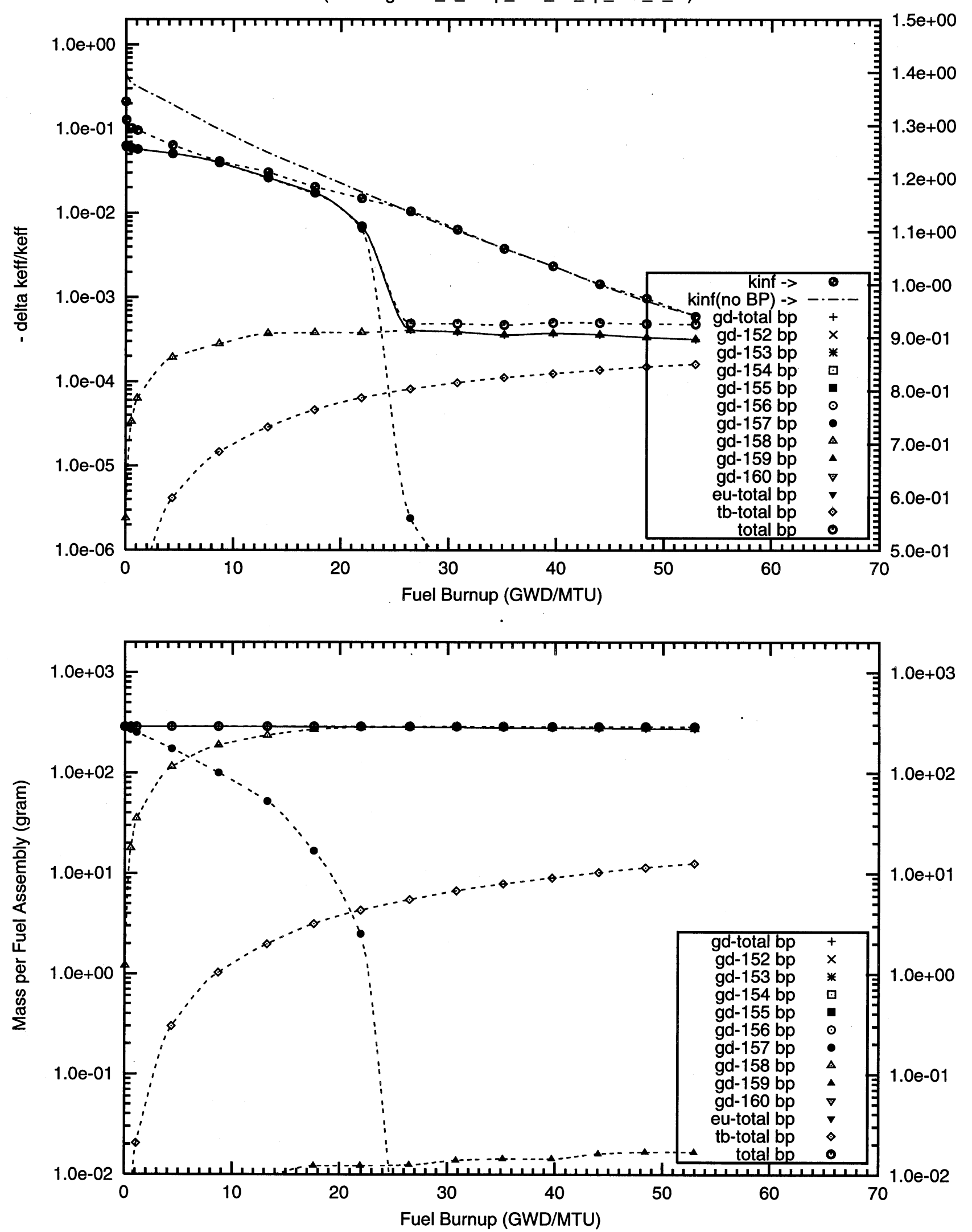
Figure A1.20. Negative Reactivity and Masses BP for Gd-157and transmutation daughters as a function of fuel life for $17 \times 17$ fuel assemblies with 08 poison rods,

$4.0 \mathrm{w} \% \mathrm{Gd} 2 \mathrm{O} 3$ poison homogeneously mixed in the UO2 pellets

Reactor power $3400 \mathrm{MWth}, 193$ fuel assemblies, initial enrichment $4.5 \mathrm{w} \% \mathrm{U}-235$

(Case : gd157_3_08_bp_fuel o)

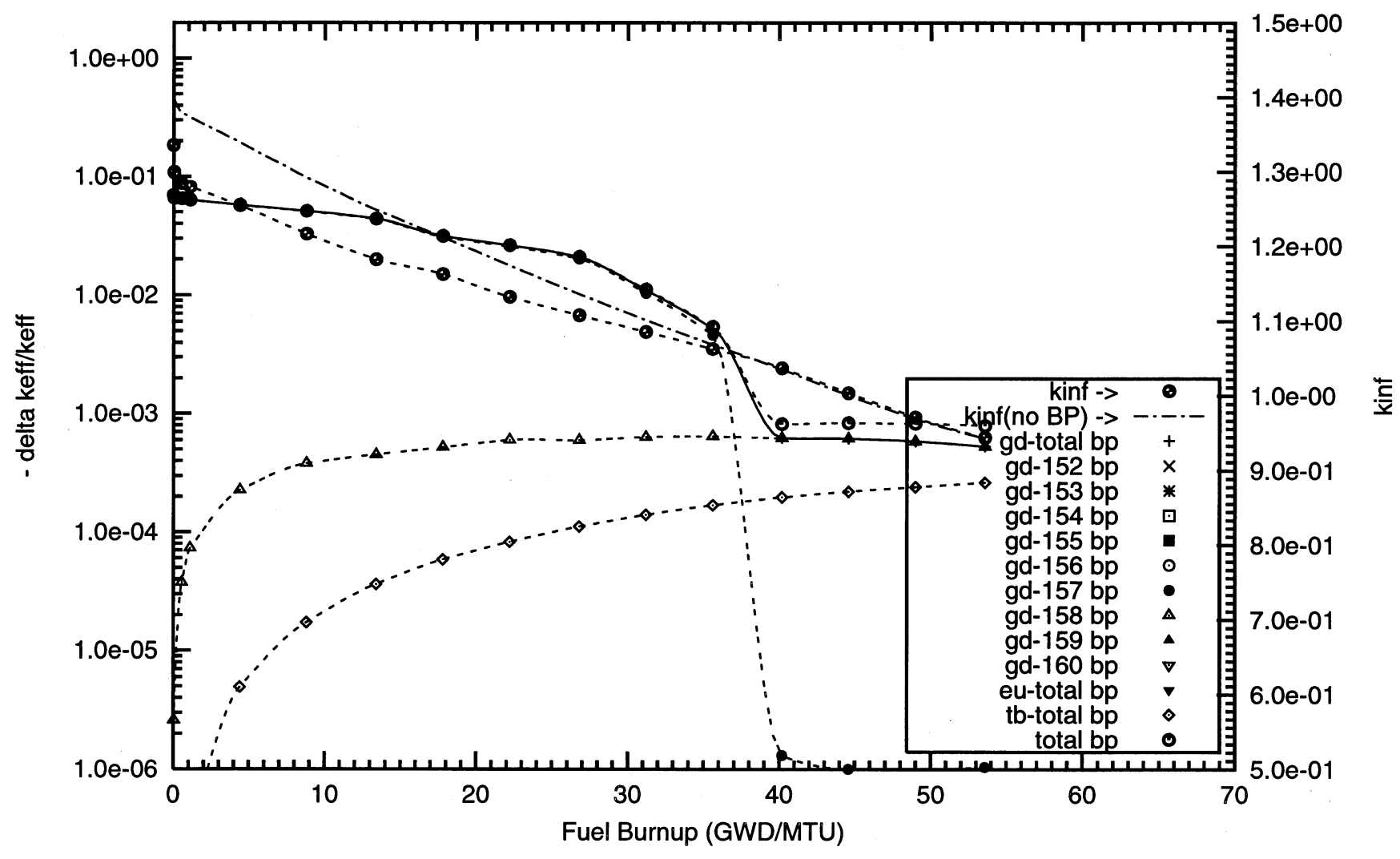


Figure A1.21. Negative Reactivity and Masses BP for Gd-157 and transmutation daughters as a function of fuel life for $17 \times 17$ fuel assemblies with 16 poison rods,

$0.1 \mathrm{w} \% \mathrm{Gd} 2 \mathrm{O} 3$ poison homogeneously mixed in the UO2 pellets

Reactor power $3400 \mathrm{MWth}, 193$ fuel assemblies, initial enrichment 4.5w\%U-235

(Case : gd157_9_100p_h20_16_bp_fuel_2_o)
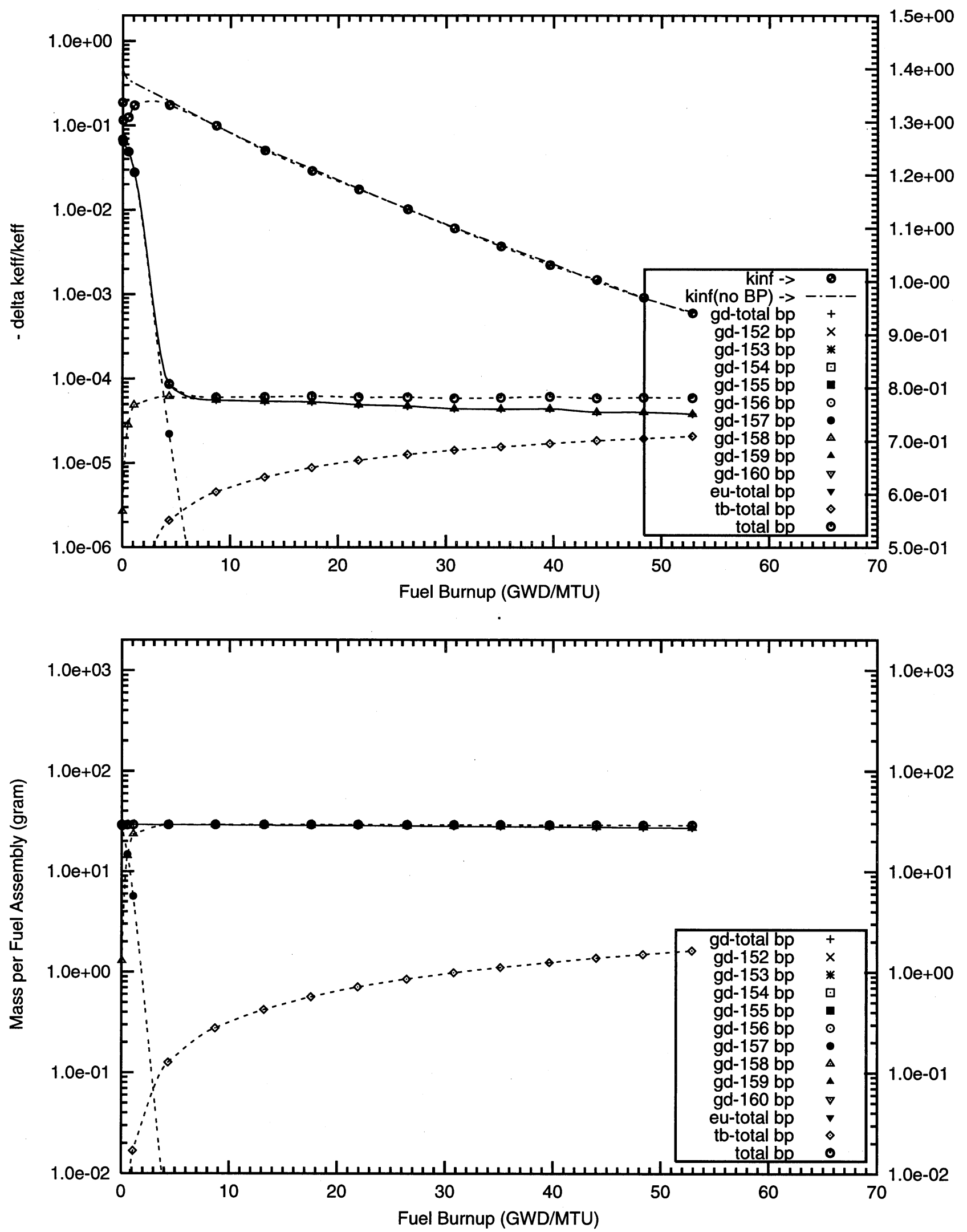
Figure A1.22. Negative Reactivity and Masses BP for Gd-157 and transmutation daughters as a function of fuel life for $17 \times 17$ fuel assemblies with 16 poison rods,

$0.2 \mathrm{w} \% \mathrm{Gd} 2 \mathrm{O} 3$ poison homogeneously mixed in the UO2 pellets

Reactor power $3400 \mathrm{MWth}, 193$ fuel assemblies, initial enrichment 4.5w\%U-235

(Case : gd157_8_100p_h2o_16_bp_fuel_2_o)

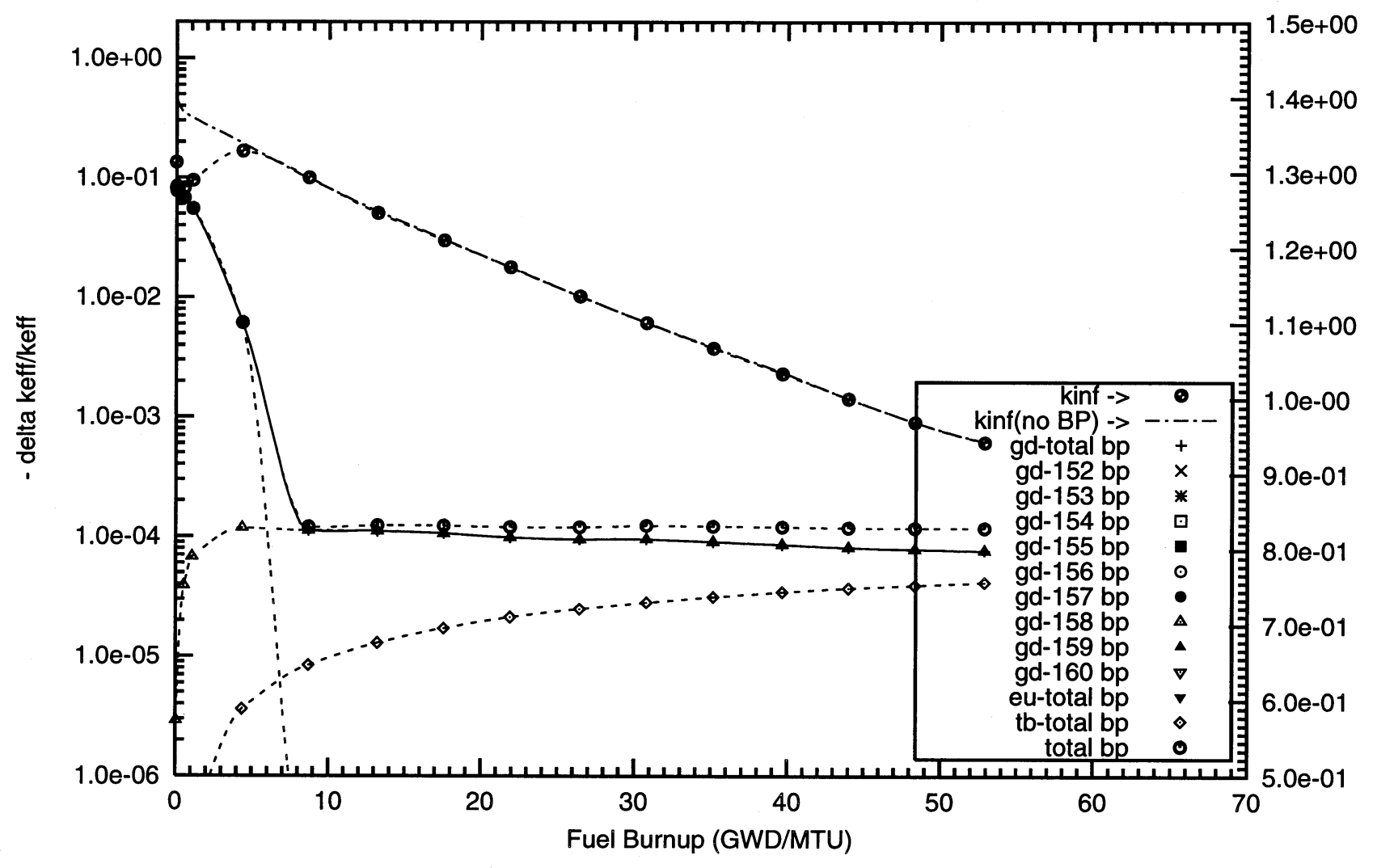

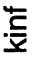

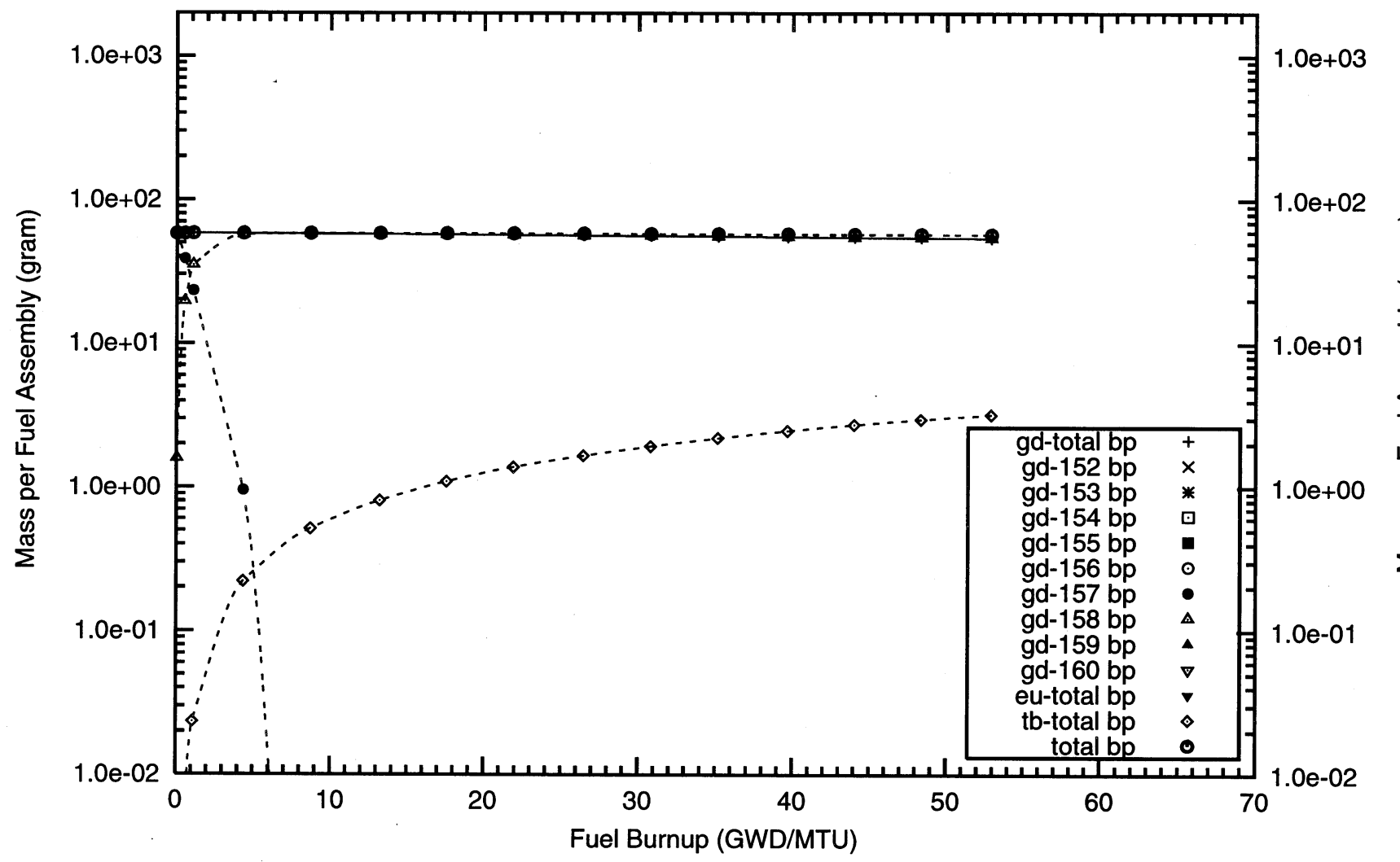


Figure A1.23. Negative Reactivity and Masses BP for Gd-157 and transmutation daughters as a tunction of fuel lite for $17 \times 17$ fuel assemblies with 16 poison rods,

$0.5 \mathrm{w} \% \mathrm{Gd} 2 \mathrm{O} 3$ poison homogeneously mixed in the UO2 pellets

Reactor power $3400 \mathrm{MWth}, 193$ fuel assemblies, initial enrichment 4.5w\%U-235

(Case : gd157_0_100p_h20_16_bp_fuel_2_o)
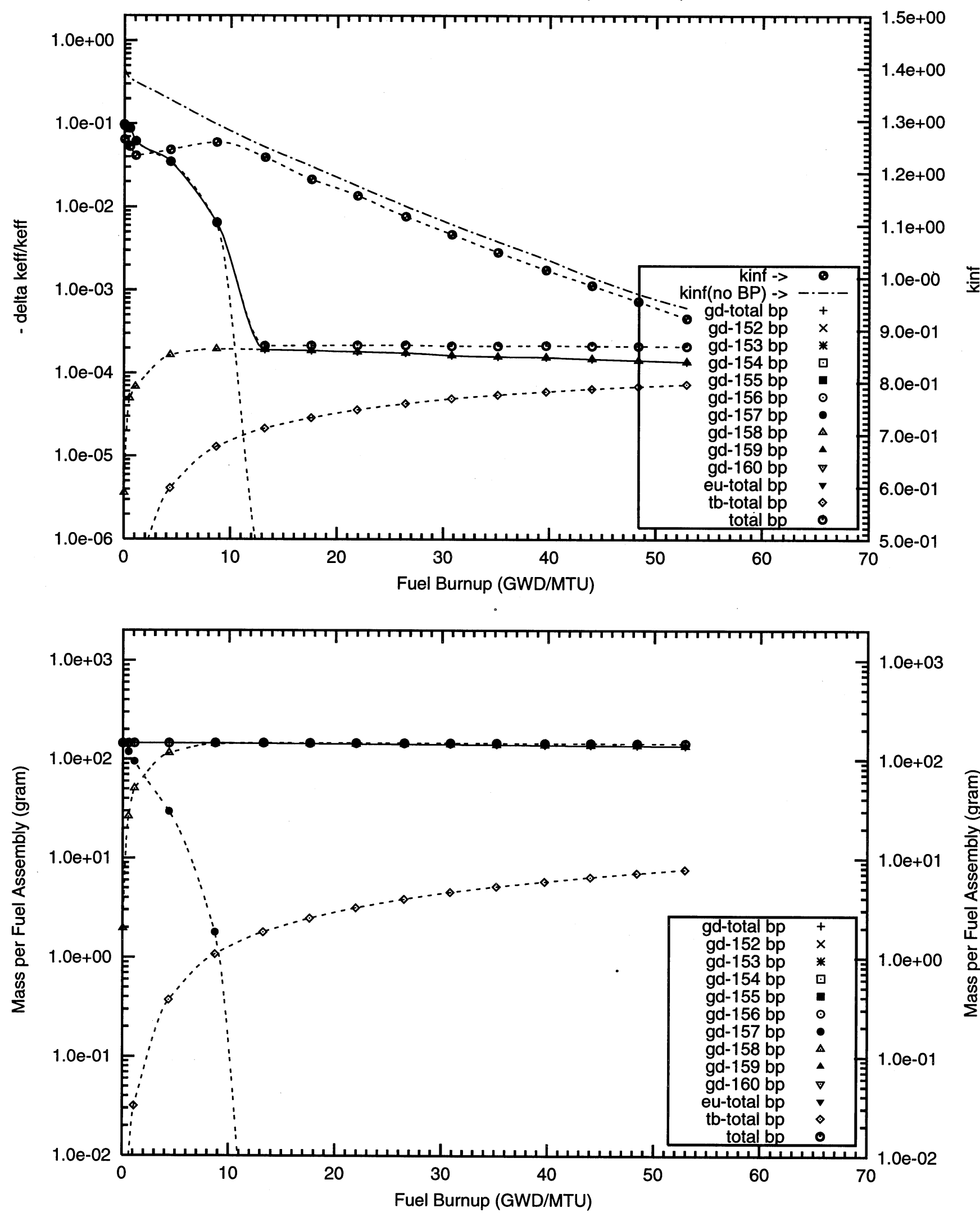
Figure A1.24. Negative Reactivity and Masses BP for Gd-157 and transmutation daughters

as a function of fuel life for $17 \times 17$ fuel assemblies with 16 poison rods,

$1.0 \mathrm{w} \% \mathrm{Gd} 2 \mathrm{O} 3$ poison homogeneously mixed in the UO2 pellets

Reactor power $3400 \mathrm{MWth}, 193$ fuel assemblies, initial enrichment 4.5w\%U-235

(Case : gd157_1_100p_h20_16_bp_fuel_2_o)
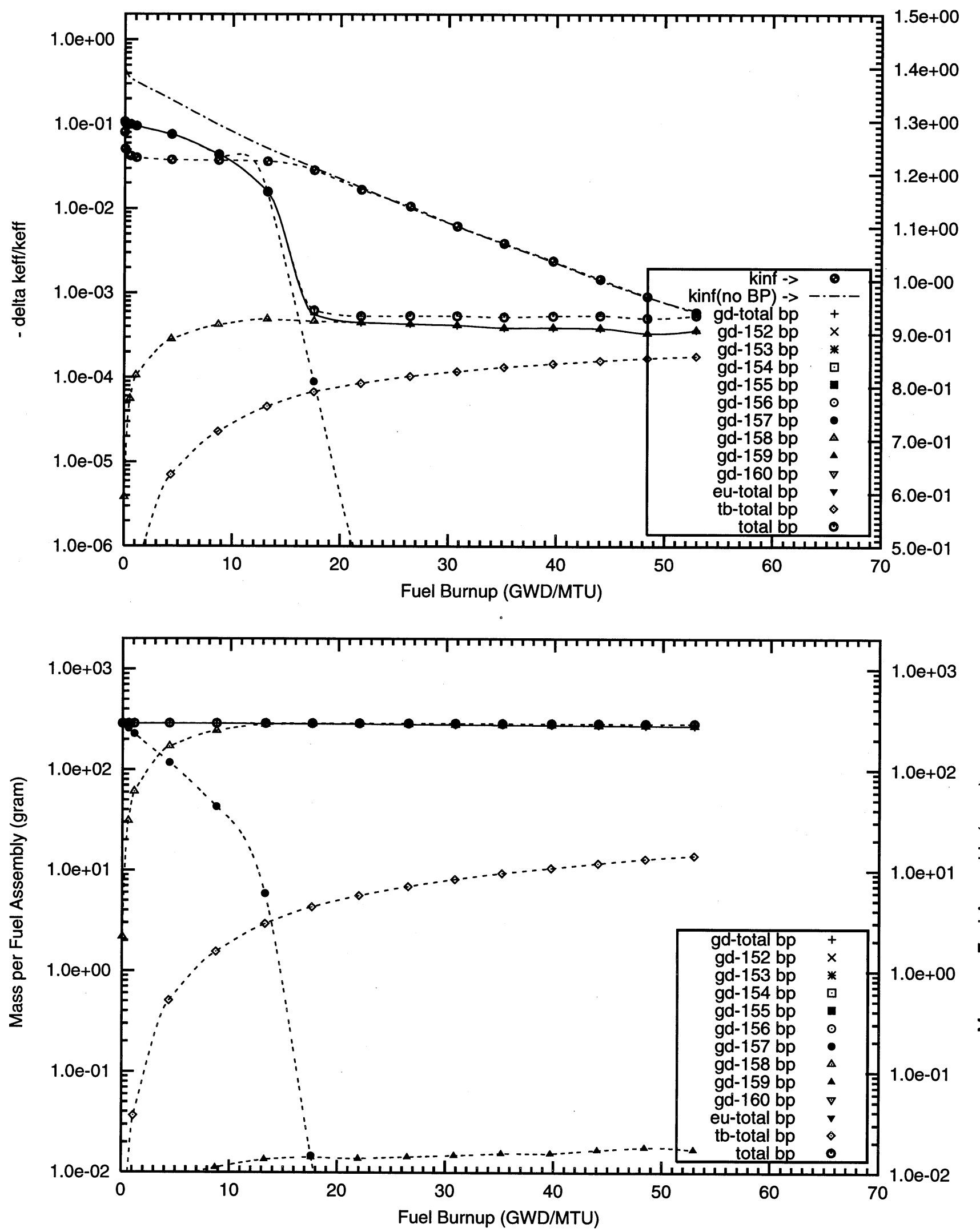
Figure A1.25. Negative Reactivity and Masses BP for Gd-157 and transmutation daughters as a function of fuel life for $17 \times 17$ fuel assemblies with 16 poison rods, $2.0 \mathrm{w} \% \mathrm{Gd} 2 \mathrm{O} 3$ poison homogeneously mixed in the UO2 pellets

Reactor power $3400 \mathrm{MWth}, 193$ fuel assemblies, initial enrichment $4.5 \mathrm{w} \% \mathrm{U}-235$

(Case : gd157_2_100p_h2o_16_bp_fuel_2_o)
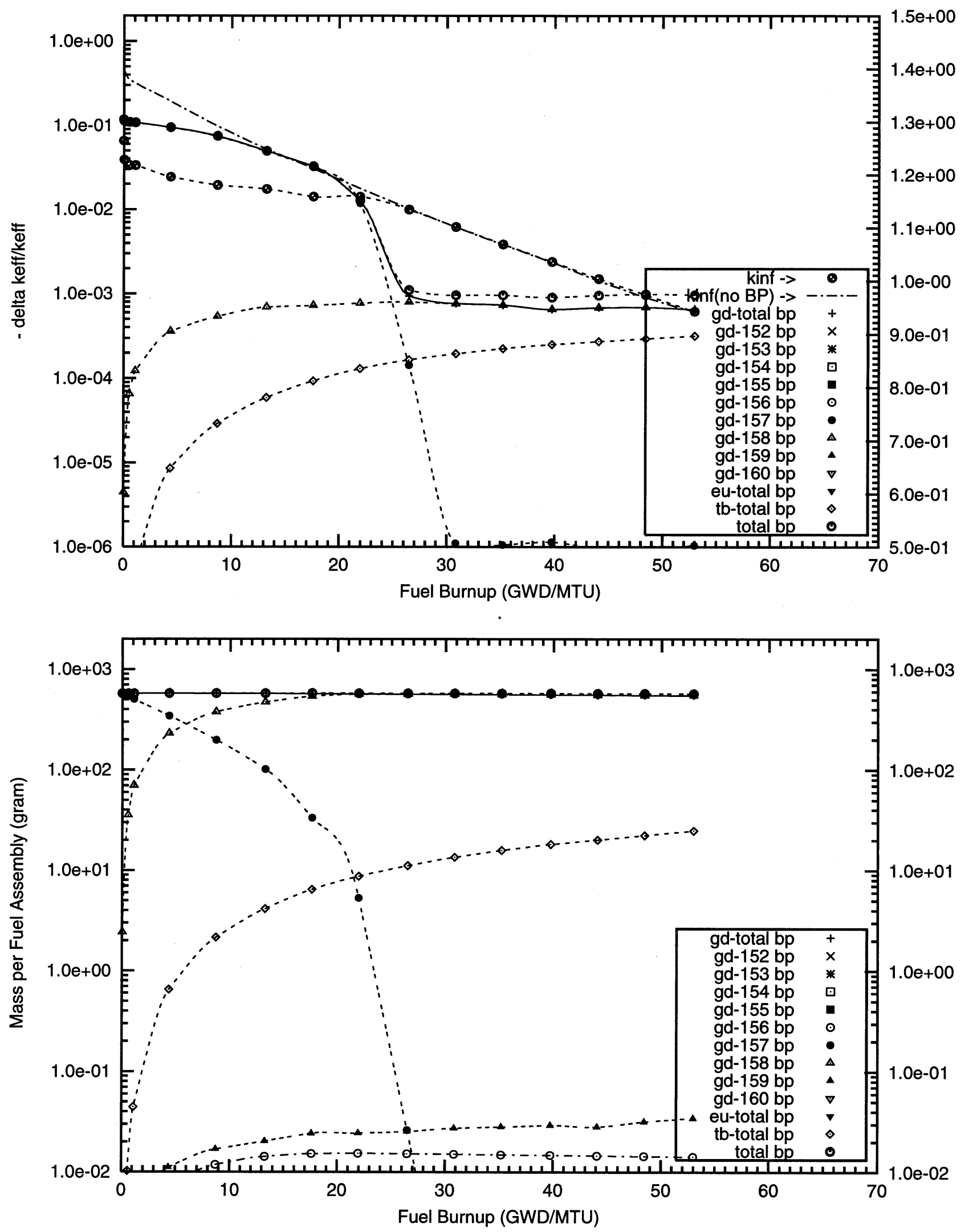
Figure A1.26. Negative Reactivity and Masses BP for Gd-157 and transmutation daughters as a function of fuel life for $17 \times 17$ fuel assemblies with 16 poison rods, $4.0 \mathrm{w} \% \mathrm{Gd} 2 \mathrm{O} 3$ poison homogeneously mixed in the UO2 pellets

Reactor power $3400 \mathrm{MWth}, 193$ fuel assemblies, initial enrichment $4.5 \mathrm{w} \% \mathrm{U}-235$

(Case : gd157_3_100p_h20_16_bp_fuel_2_o)
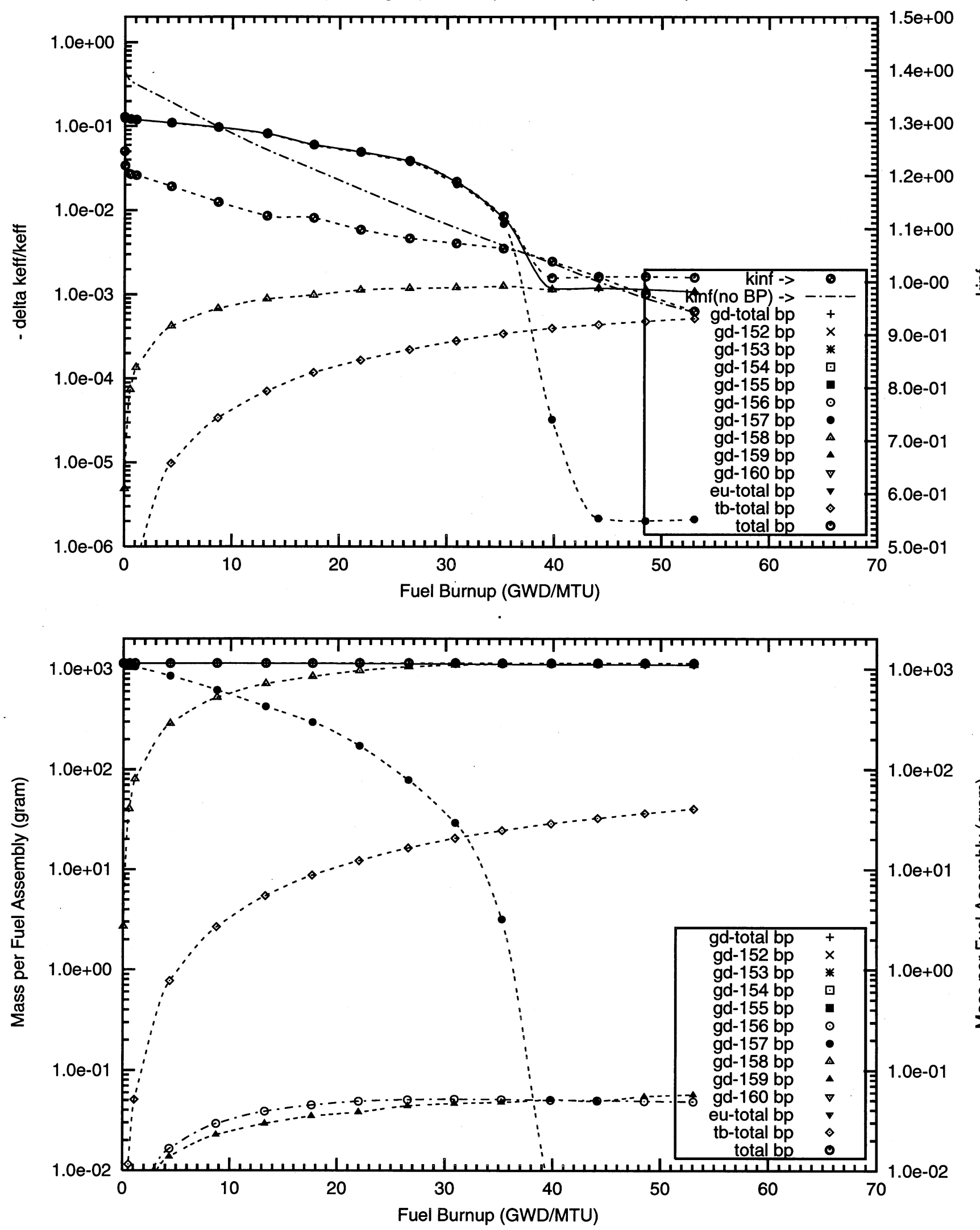
Figure A1.27. Negative Reactivity and Masses BP for Gd-157 and transmutation daughters

as a function of fuel life for $17 \times 17$ fuel assemblies with 20 poison rods,

$1.0 \mathrm{w} \% \mathrm{Gd} 2 \mathrm{O} 3$ poison homogeneously mixed in the UO2 pellets

Reactor power $3400 \mathrm{MWth}, 193$ fuel assemblies, initial enrichment 4.5w\%U-235

(Case : gd157_1_16_bp_fuel (old) o)

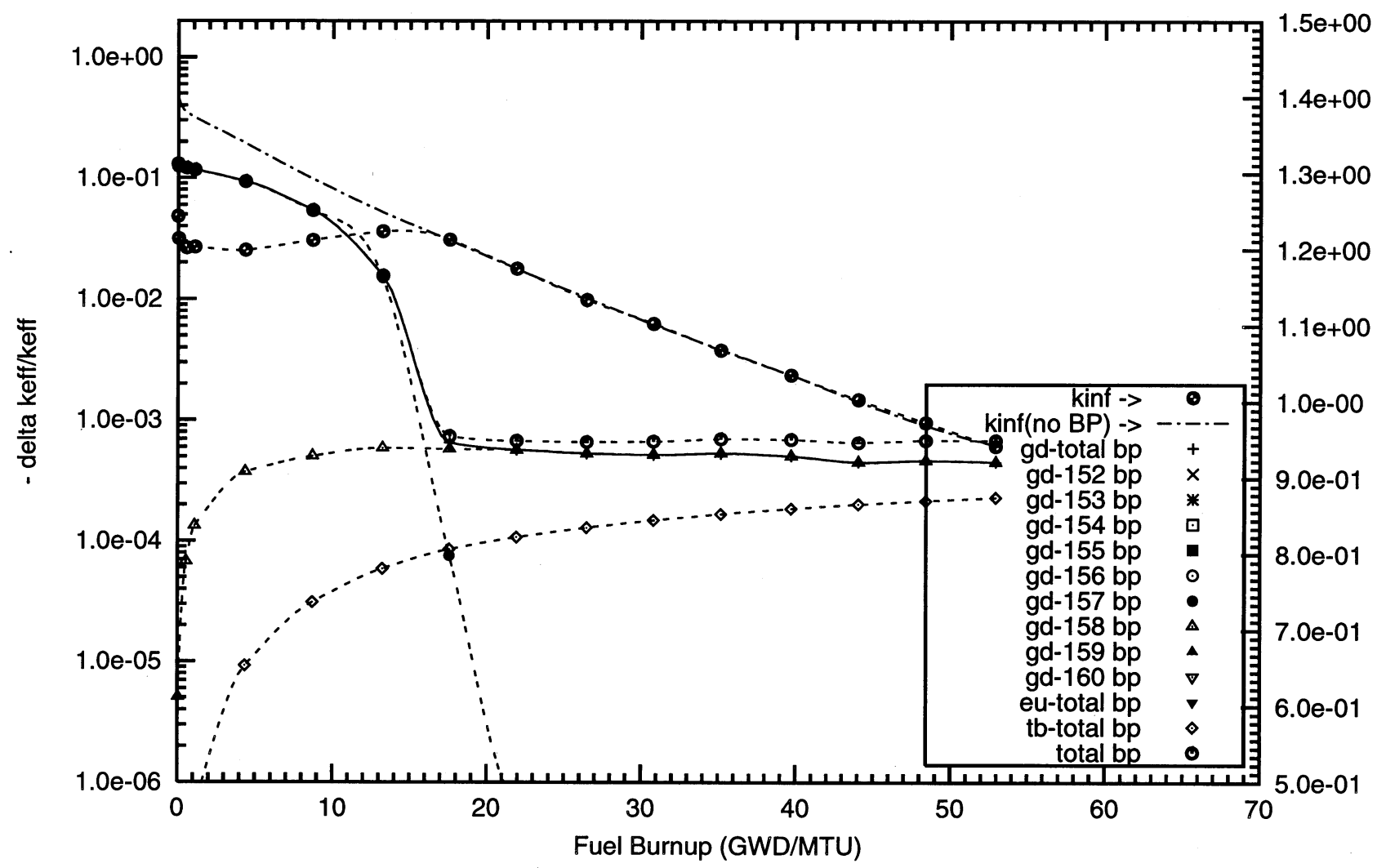

$\frac{.}{\frac{1}{x}}$

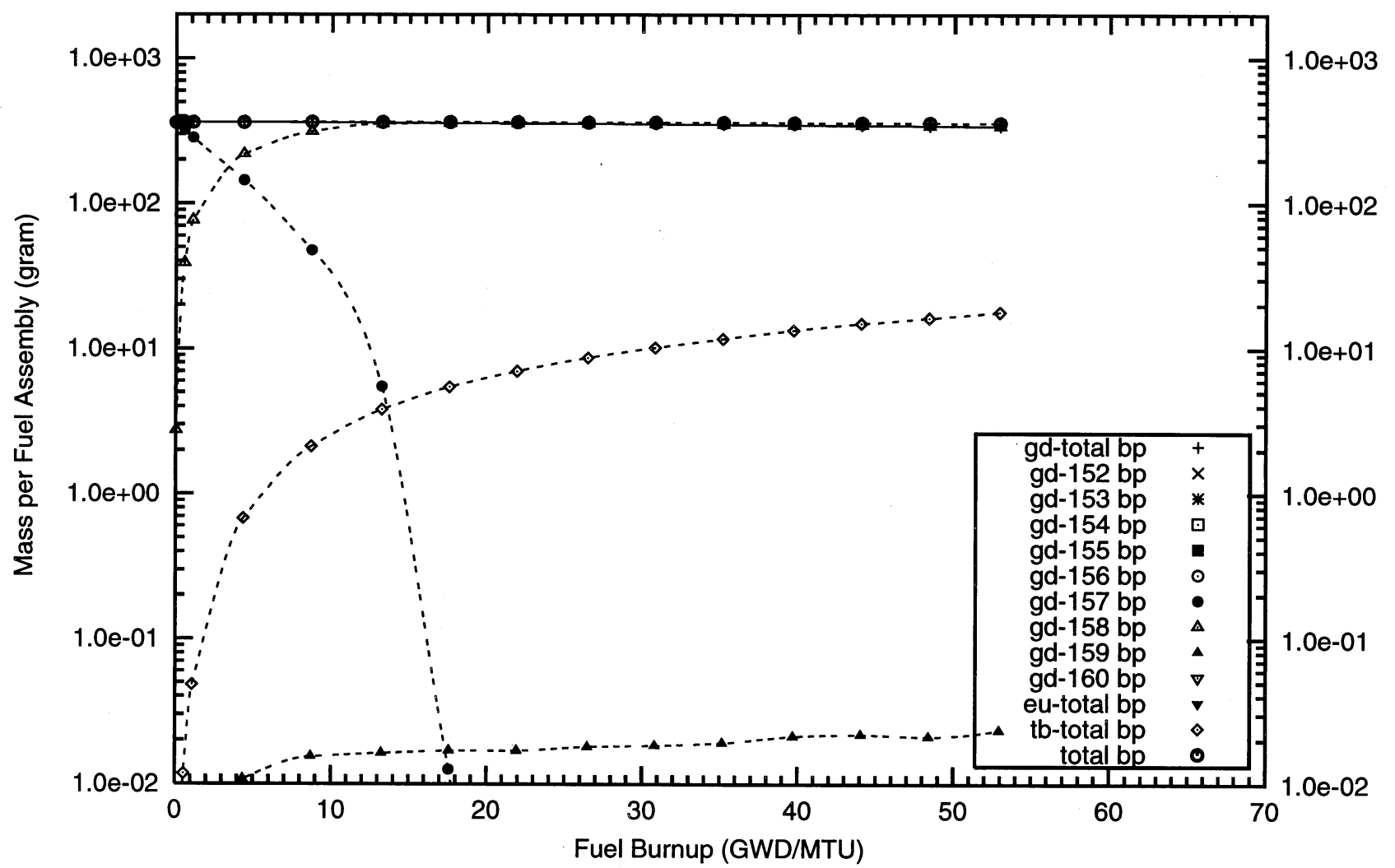


Figure A1.28. Negative Reactivity and Masses BP for Gd-157 and transmutation daughters as a function of fuel life for $17 \times 17$ fuel assemblies with 64 poison rods,

$0.05 \mathrm{w} \% \mathrm{Gd} 2 \mathrm{O} 3$ poison homogeneously mixed in the UO2 pellets

Reactor power $3400 \mathrm{MWth}, 193$ fuel assemblies, initial enrichment 4.5w\%U-235

(Case : gd157_10_100p_h2o_64_bp_fuel_2_o)
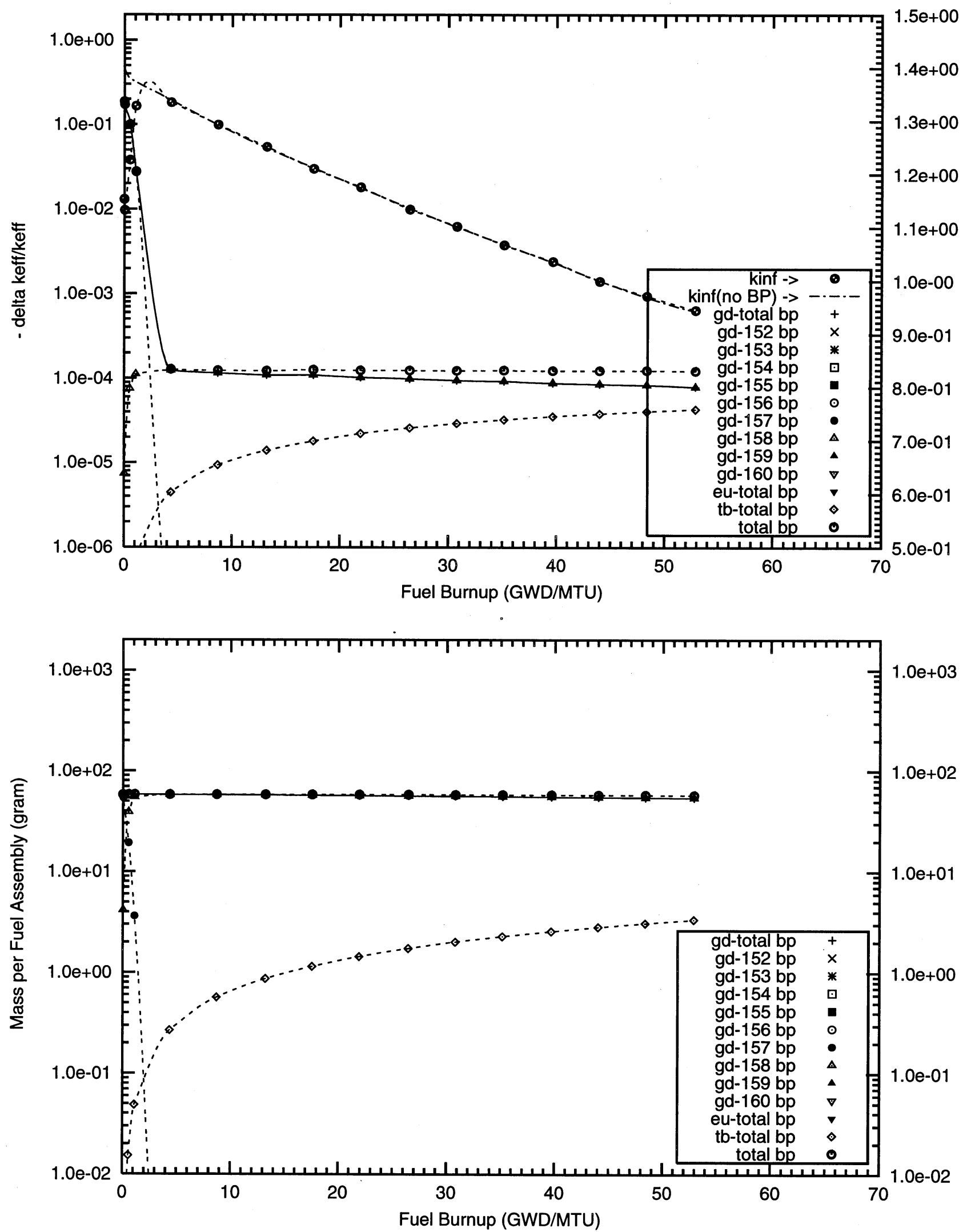
Figure A1.29. Negative Reactivity and Masses BP for Gd-157 and transmutation daughters as a function of fuel life for $17 \times 17$ fuel assemblies with 64 poison rods,

$0.1 \mathrm{w} \% \mathrm{Gd} 2 \mathrm{O} 3$ poison homogeneously mixed in the UO2 pellets

Reactor power $3400 \mathrm{MWth}, 193$ fuel assemblies, initial enrichment $4.5 \mathrm{w} \% \mathrm{U}-235$

(Case : gd157_9_100p_h2o_64_bp_fuel_2_o)

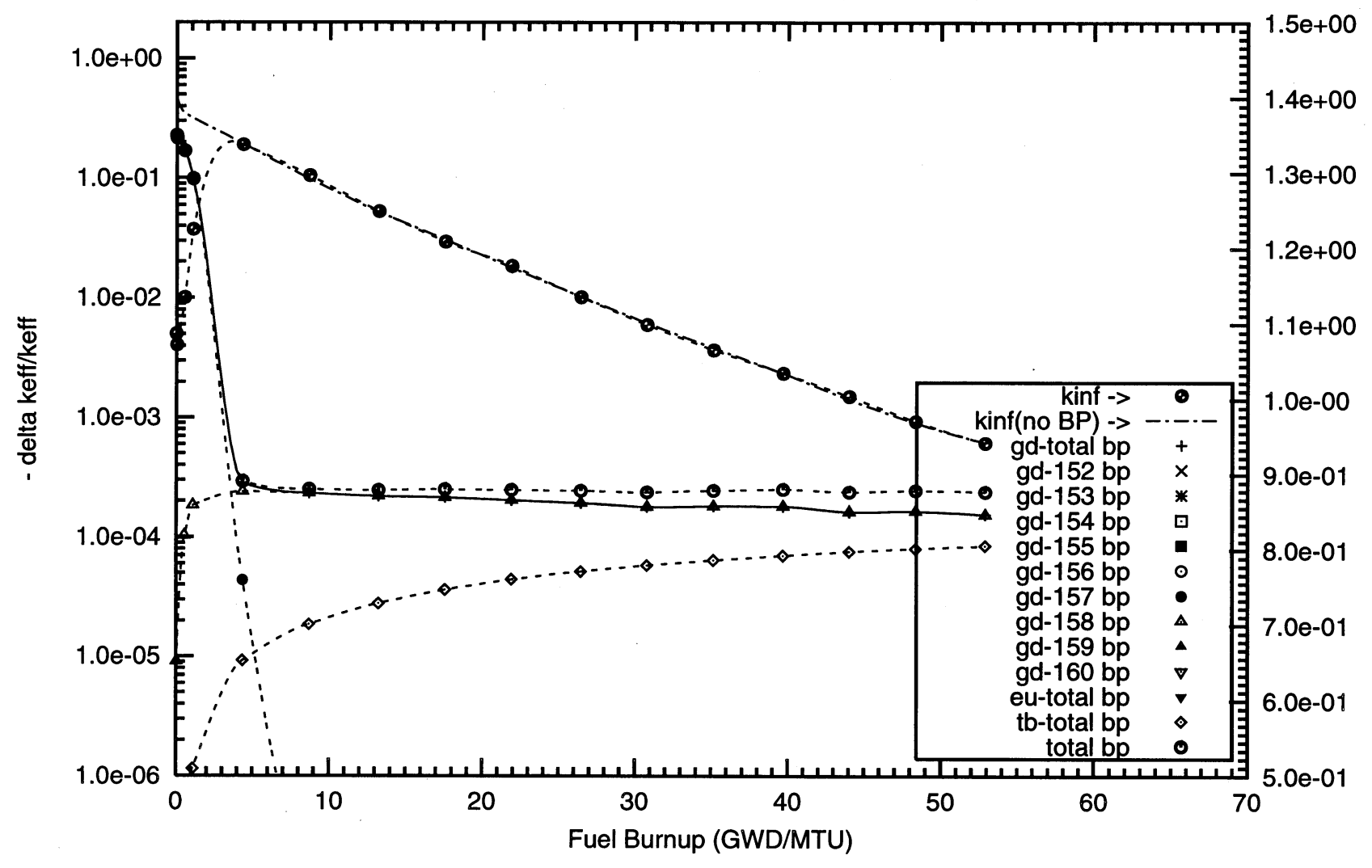

$\underline{\frac{E}{y}}$

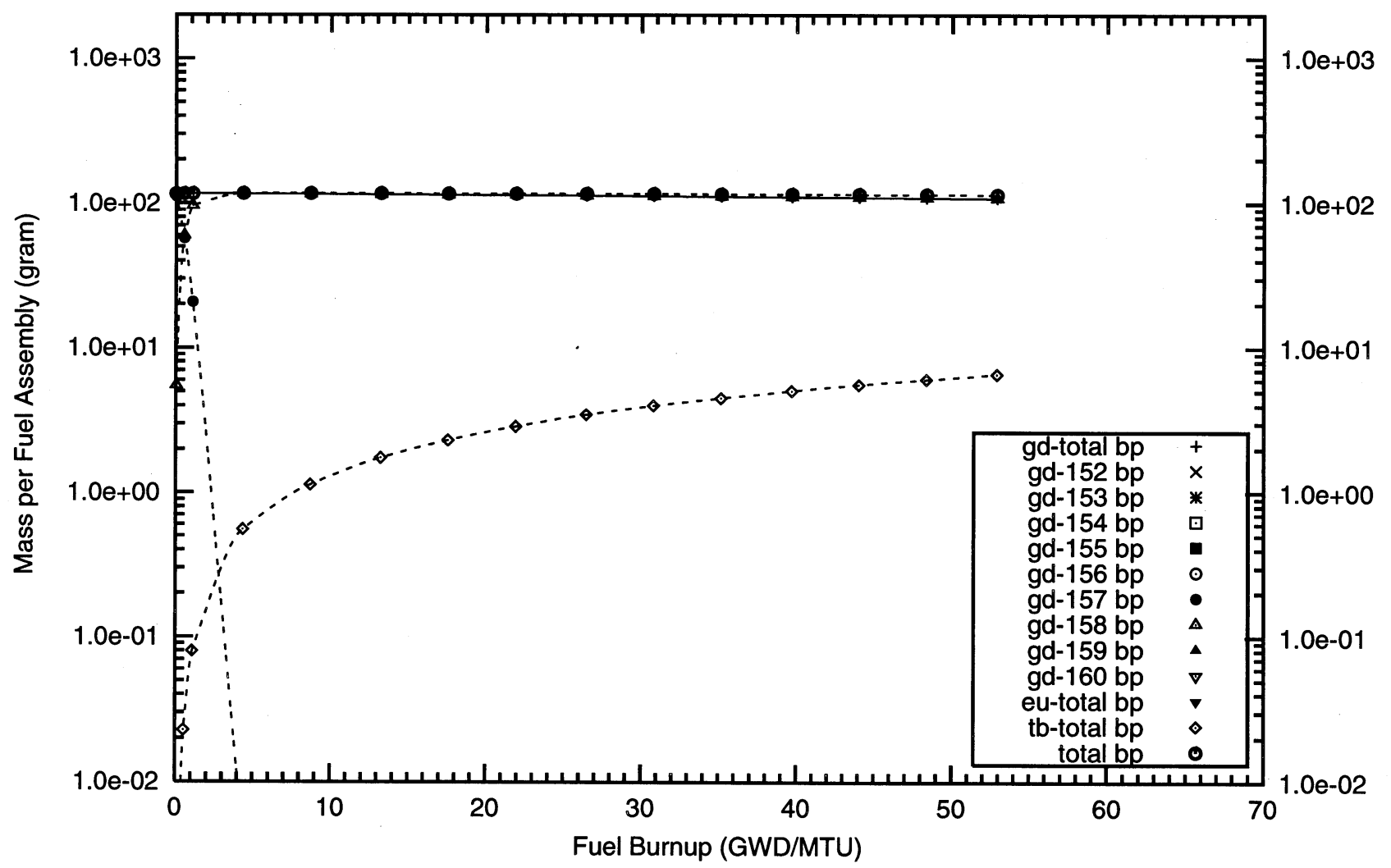




\section{Appendix A2}

Reactivity Worths and Masses of $\mathrm{Gd}_{2} \mathrm{O}_{3}$ Burnable Poison Homogeneously Mixed in the Outer One-Third Volume of the Fuel Pellets

\section{Table 6 Cases}



A2 - Gadolinium with Natural Isotopic Abundances 
Figure A2.1. Negative Reactivity and Masses BP for Gd-nat and transmutation daughters as a function of fuel life for $17 \times 17$ fuel assemblies with 08 poison rods,

$4.0 \mathrm{w} \% \mathrm{Gd} 2 \mathrm{O} 3$ poison homogeneously mixed in the outer one-third volume of the UO2 pellets

Reactor power $3400 \mathrm{MWth}, 193$ fuel assemblies, initial enrichment 4.5W\%U-235

(Case : gd000_3b_08_bp_fuel o)

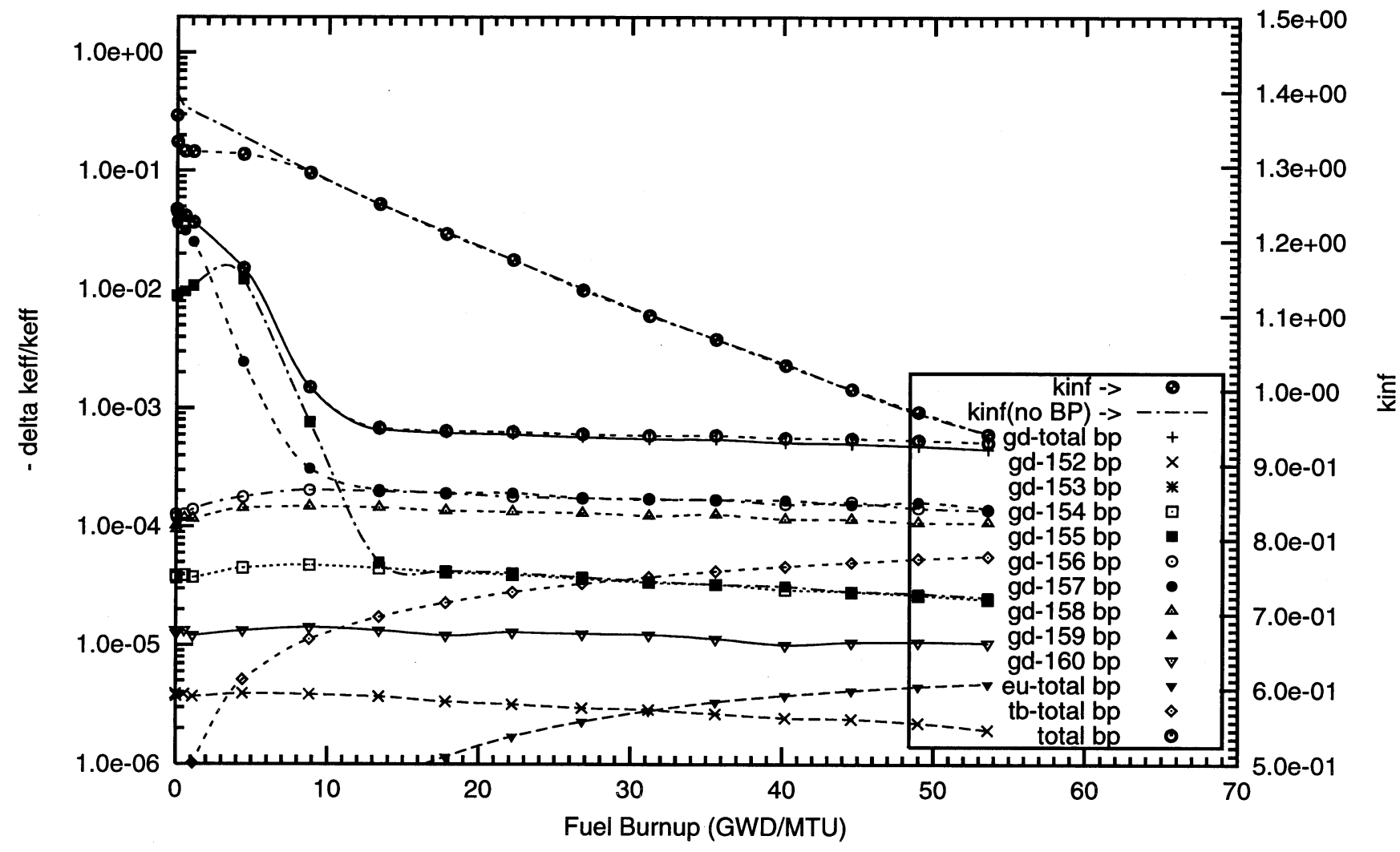


Figure A2.2. Negative Reactivity and Masses BP for Gd-nat and transmutation daughters as a function of fuel life for $17 \times 17$ fuel assemblies with 08 poison rods,

$8.0 w \%$ Gd2O3 poison homogeneously mixed in the outer one-third volume of the UO2 pellets

Reactor power $3400 \mathrm{MW}$ th, 193 fuel assemblies, initial enrichment $4.5 \mathrm{w} \% \mathrm{U}-235$

(Case : gd000_4b_100p_h2o_08_bp_fuel_2_o)

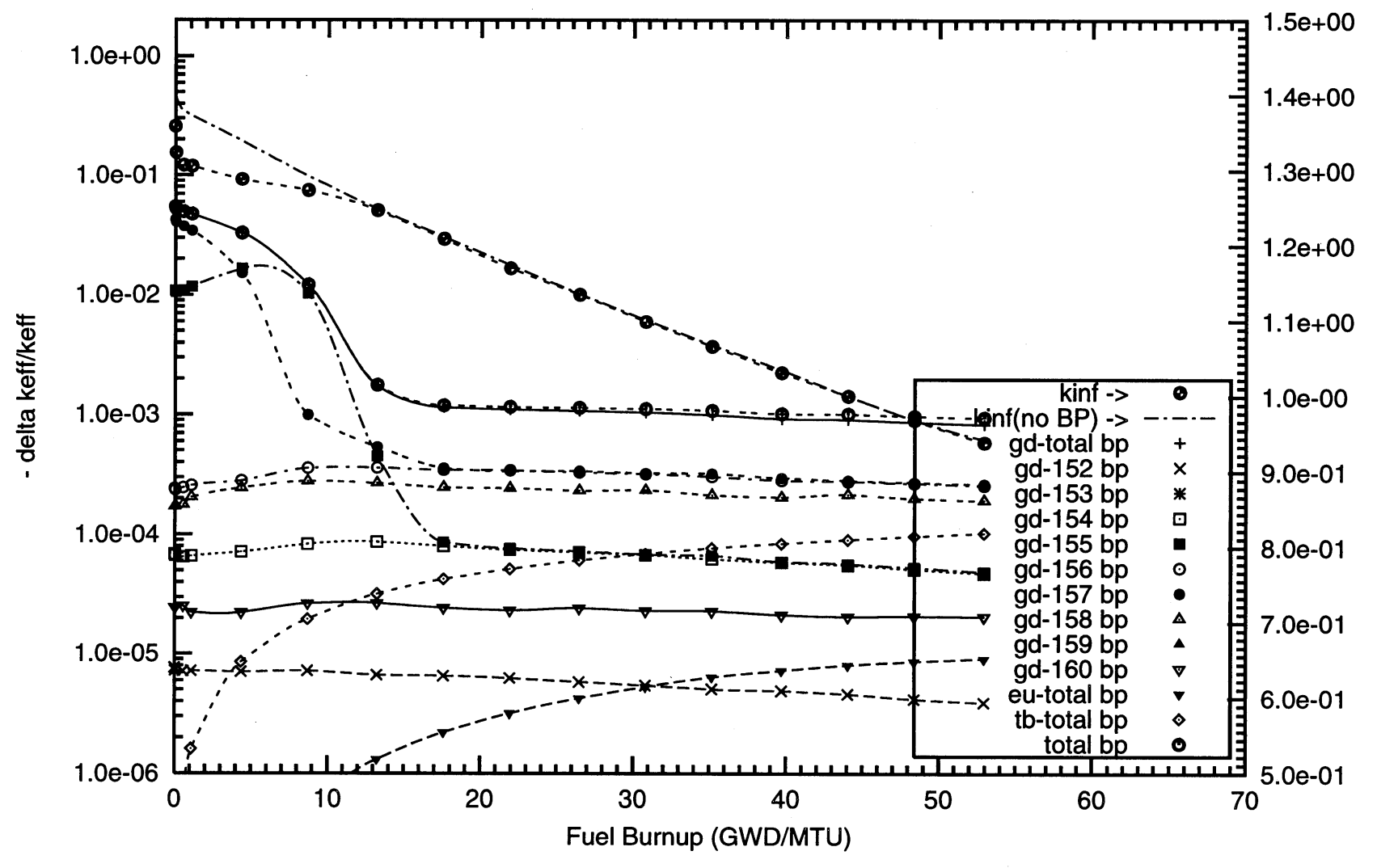

$\frac{E}{\bar{x}}$

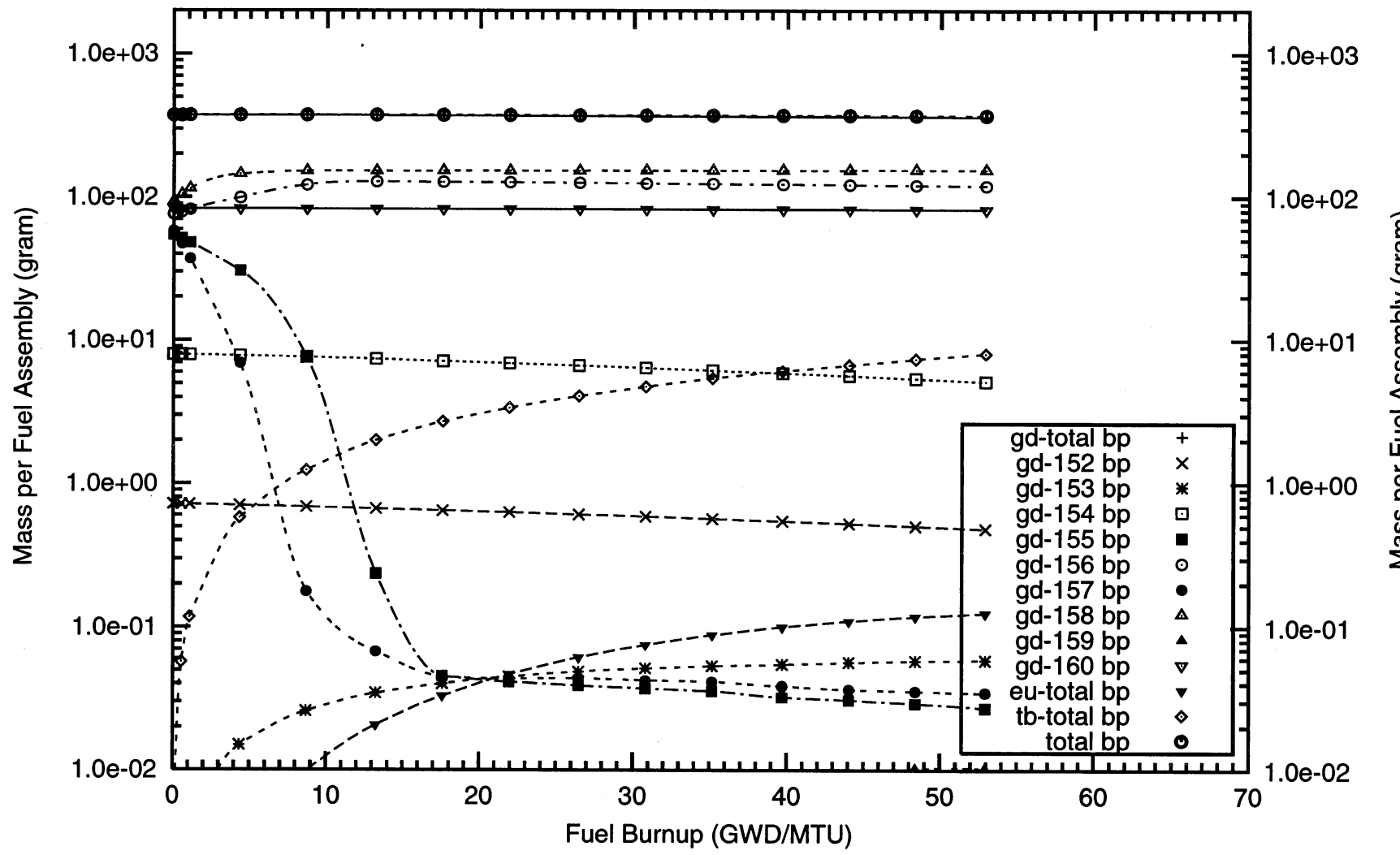


Figure A2.3. Negative Reactivity and Masses BP for Gd-nat and transmutation daughters as a tunction of tuel lite for $17 \times 17$ fuel assemblies with 08 poison rods,

$12.0 \mathrm{w} \% \mathrm{Gd} 2 \mathrm{O} 3$ poison homogeneously mixed in the outer one-third volume of the UO2 pellets

Reactor power $3400 \mathrm{MW}$ th, 193 fuel assemblies, initial enrichment $4.5 \mathrm{w} \% \mathrm{U}-235$

(Case : gd000_5b_100p_h20_08_bp_fuel_2_o)
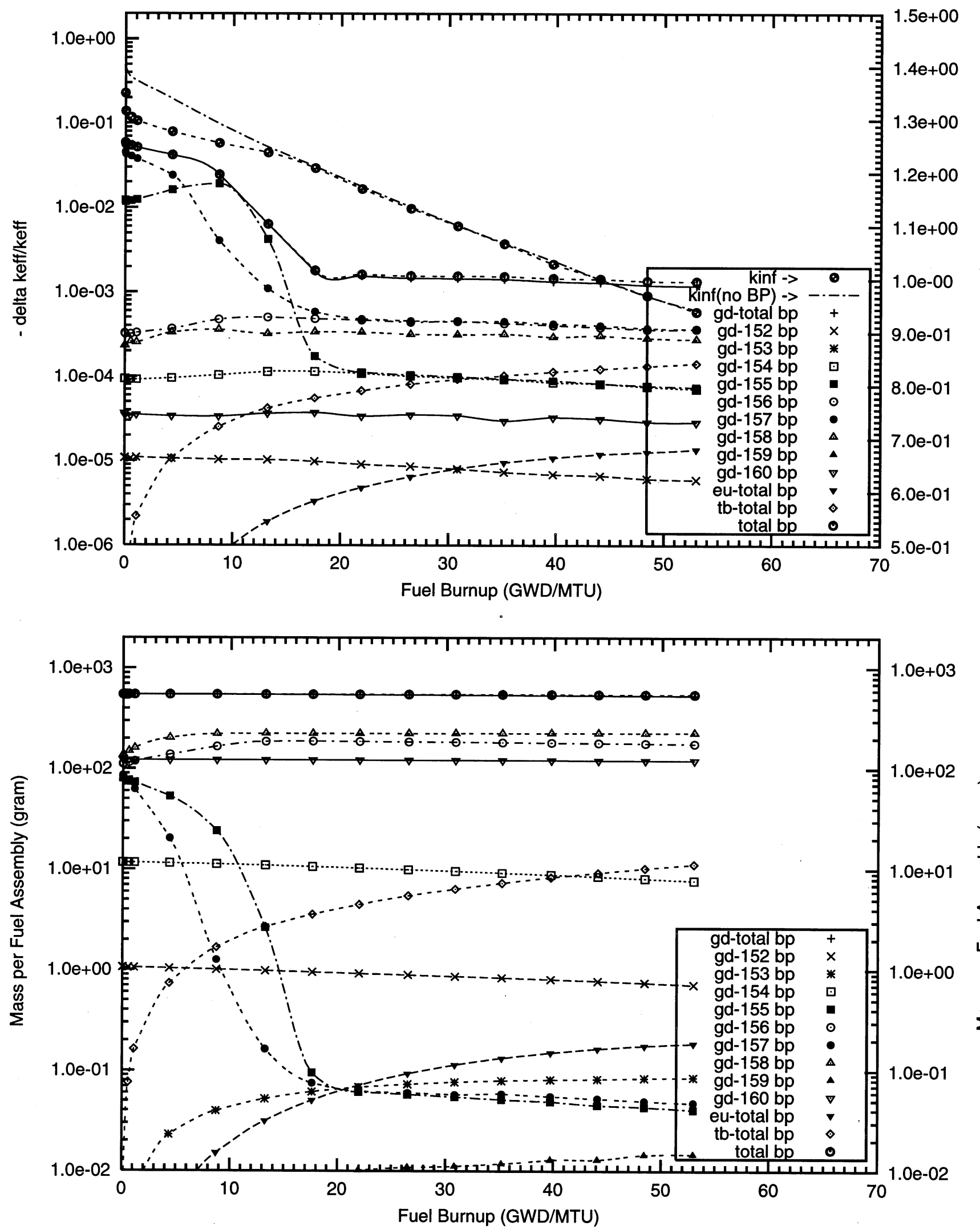
Figure A2.4. Negative Reactivity and Masses BP for Gd-nat and transmutation daughters as a function of fuel lite for $17 \times 17$ fuel assemblies with 16 poison rods,

$2.0 \mathrm{w} \% \mathrm{Gd} 2 \mathrm{O} 3$ poison homogeneously mixed in the outer one-third volume of the $\mathrm{UO} 2$ pellets

Reactor power $3400 \mathrm{MW}$ th, 193 fuel assemblies, initial enrichment $4.5 \mathrm{w} \% \mathrm{U}-235$

(Case : gd000_2b_100p_h20_16_bp_fuel_2_o)
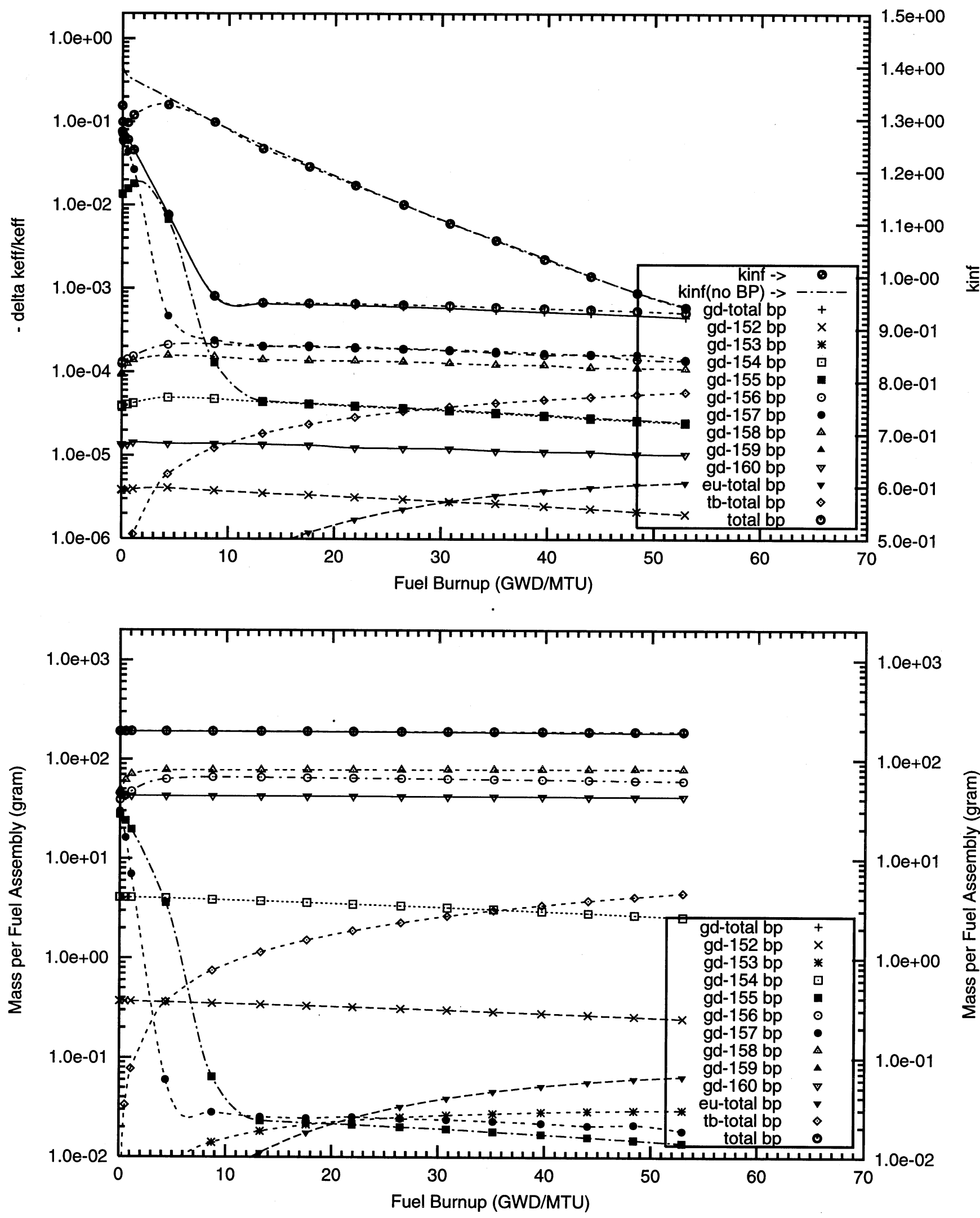
Figure A2.5. Negative Reactivity and Masses BP for Gd-nat and transmutation daughters as a function of fuel life for $17 \times 17$ fuel assemblies with 16 poison rods;

$4.0 \mathrm{w} \% \mathrm{Gd} 2 \mathrm{O} 3$ poison homogeneously mixed in the outer one-third volume of the UO2 pellets

Reactor power $3400 \mathrm{MWth}, 193$ fuel assemblies, initial enrichment 4.5w\%U-235

(Case : gd000_3b_100p_h20_16_bp_fuel_2_o)
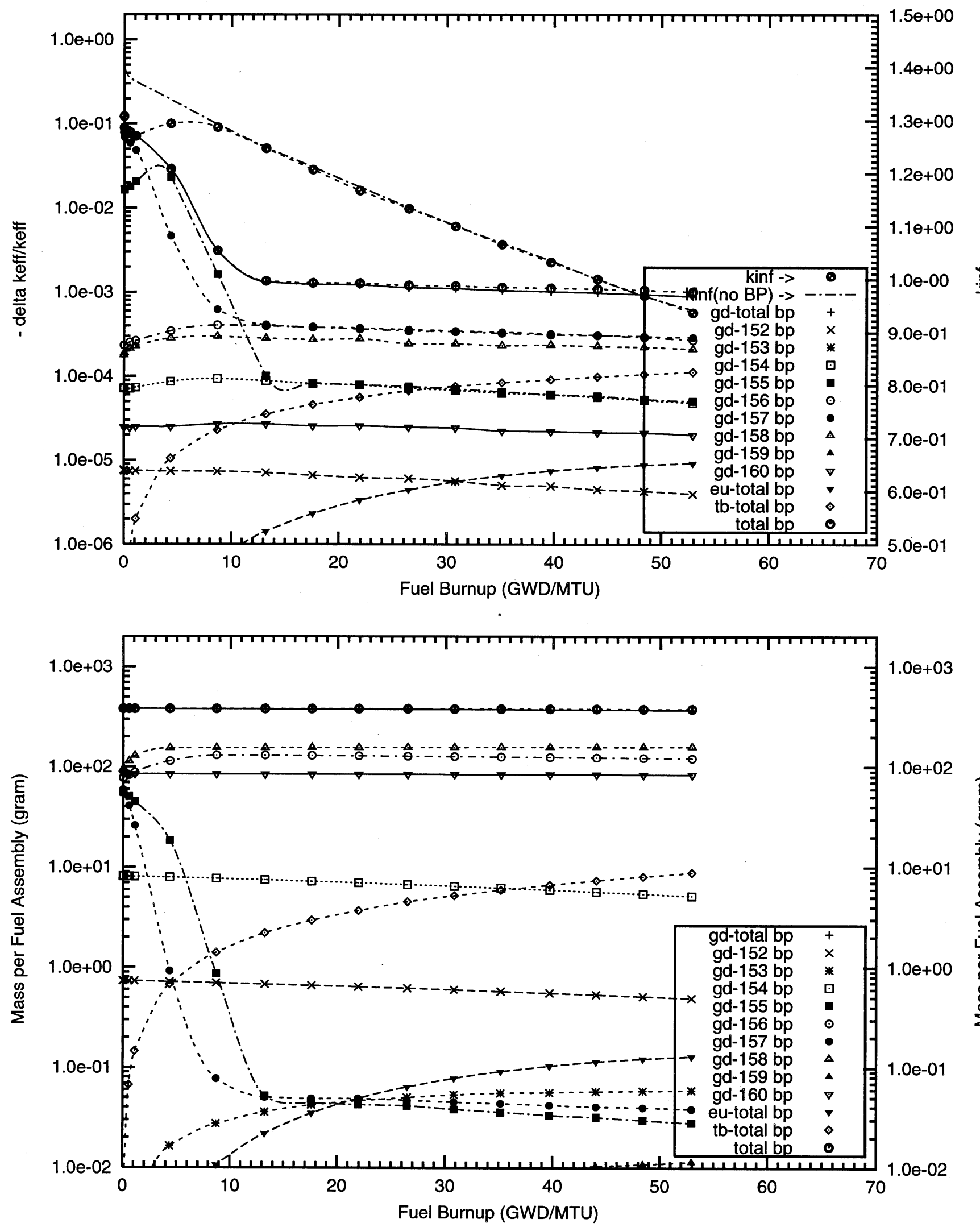
Figure A2.6. Negative Reactivity and Masses BP for Gd-nat and transmutation daughters as a function of fuel life for $17 \times 17$ fuel assemblies with 16 poison rods,

$8.0 \mathrm{w} \% \mathrm{Gd} 2 \mathrm{O} 3$ poison homogeneously mixed in the outer one-third volume of the UO2 pellets

Reactor power $3400 \mathrm{MWth}, 193$ fuel assemblies, initial enrichment 4.5w\%U-235

(Case : gd000_4b_100p_h2o_16_bp_fuel_2_o)
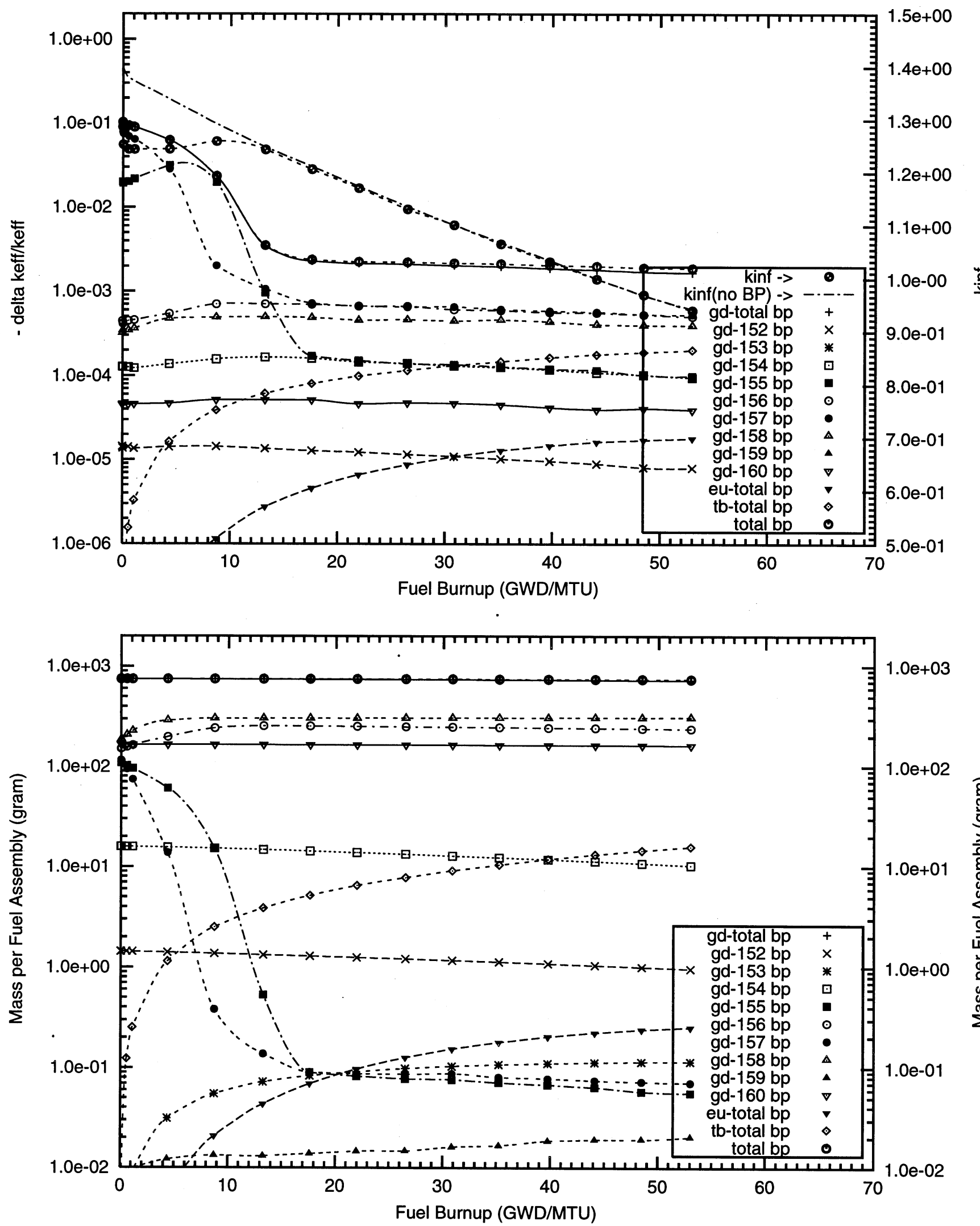
Figure A2.7. Negative Reactivity and Masses BP for Gd-nat and transmutation daughters as a function of tuel lite for $17 \times 17$ fuel assemblies with 16 poison rods,

$12.0 \mathrm{w} \% \mathrm{Gd} 2 \mathrm{O} 3$ poison homogeneously mixed in the outer one-third volume of the UO2 pellets

Reactor power $3400 \mathrm{MW}$ th, 193 fuel assemblies, initial enrichment $4.5 \mathrm{w} \% \mathrm{U}-235$

(Case : gd000_5b_100p_h20_16_bp_fuel_2_o)
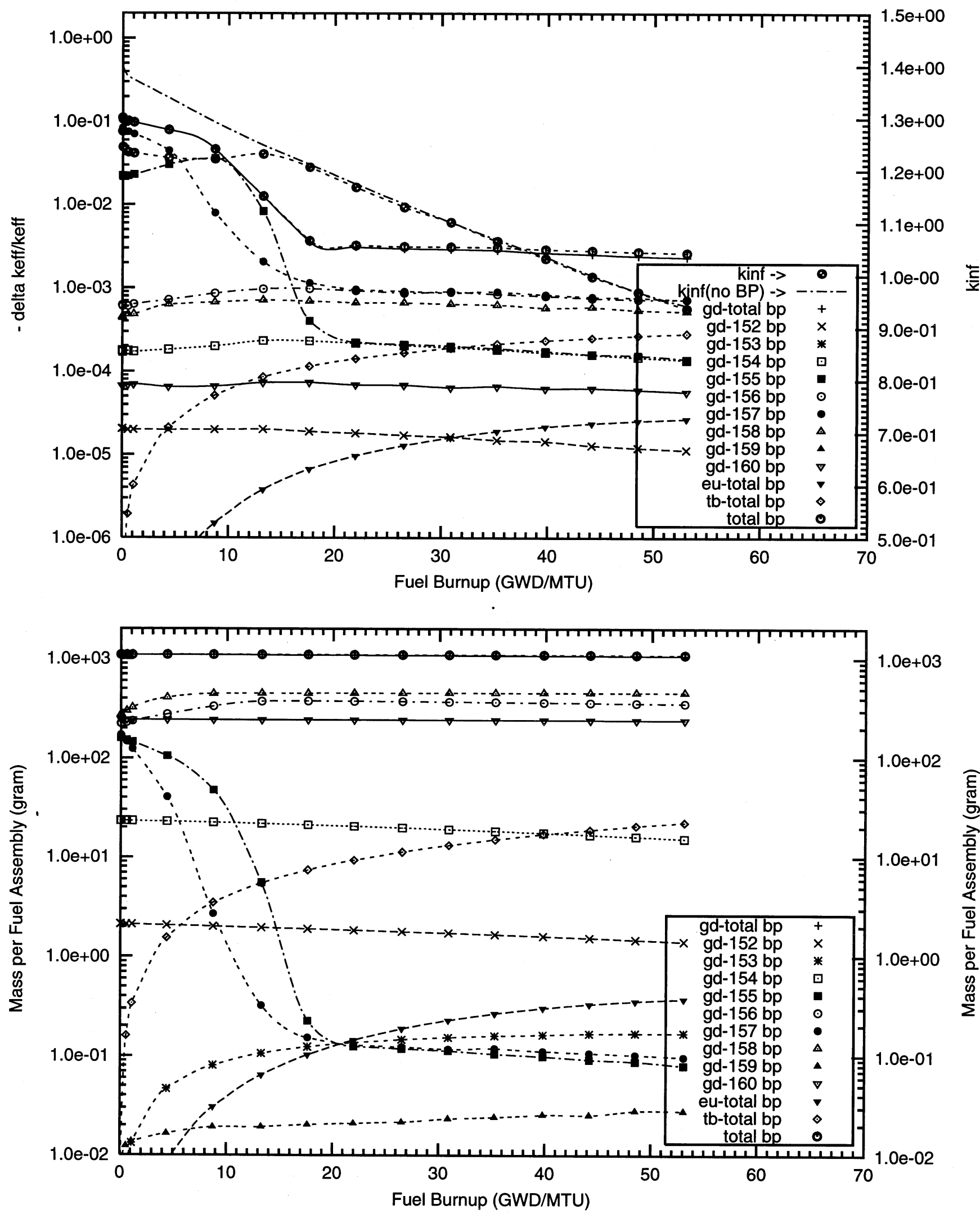
Figure A2.8. Negative Reactivity and Masses BP for Gd-nat and transmutation daughters as a function of fuel life for $17 \times 17$ fuel assemblies with 18 poison rods,

$4.0 \mathrm{w} \% \mathrm{Gd} 2 \mathrm{O} 3$ poison homogeneously mixed in the outer one-third volume of the $\mathrm{UO} 2$ pellets

Reactor power $3400 \mathrm{MWth}, 193$ fuel assemblies, initial enrichment 4.5w\%U-235

(Case : gd000_3b_16_bp_fuel (old) o)

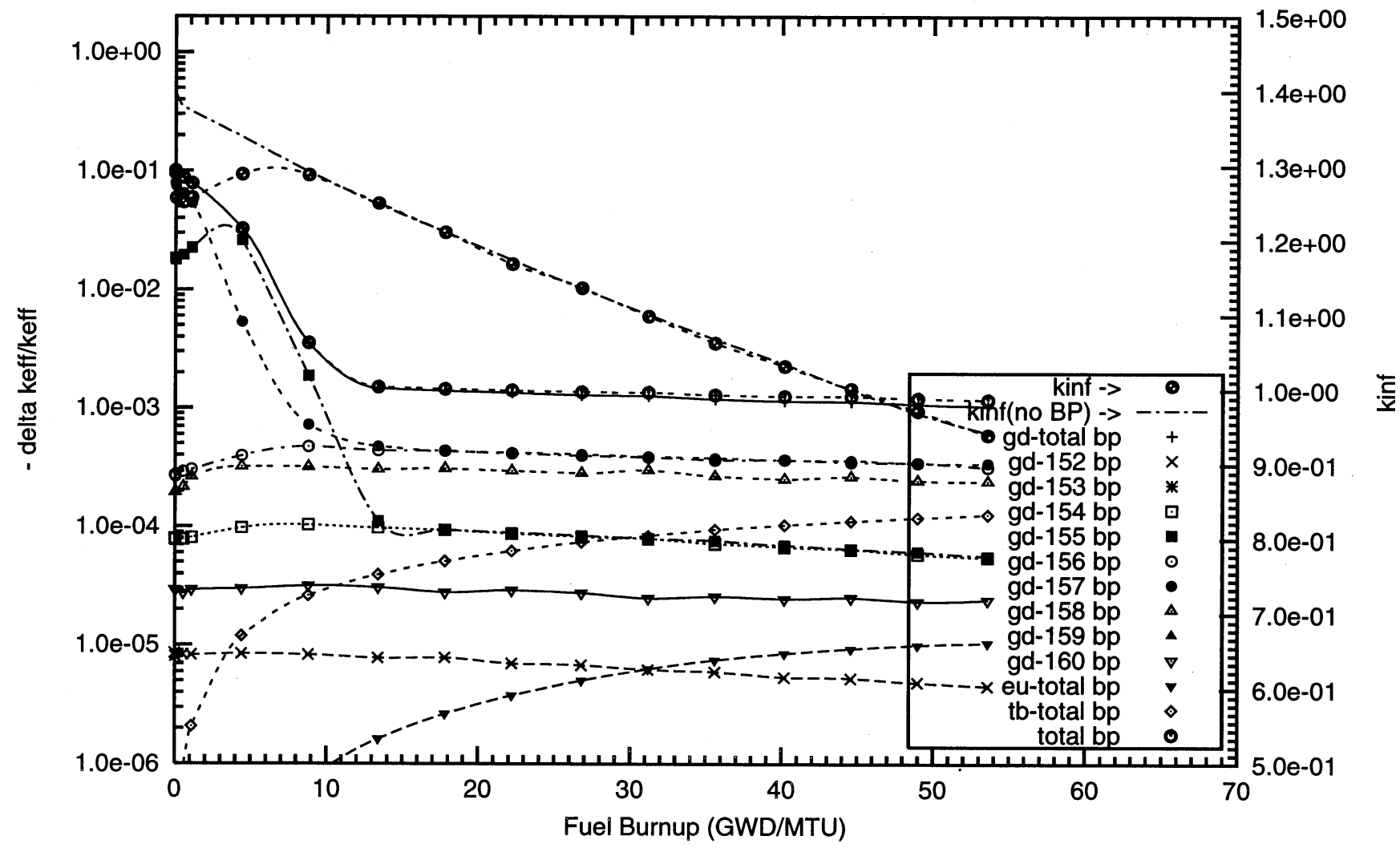


Figure A2.9. Negative Reactivity and Masses BP for Gd-nat and transmutation daughters as a function of fuel life for $17 \times 17$ fuel assemblies with 64 poison rods,

$1.0 \mathrm{w} \% \mathrm{Gd} 2 \mathrm{O} 3$ poison homogeneously mixed in the outer one-third volume of the UO2 pellets

Reactor power $3400 \mathrm{MW}$ th, 193 fuel assemblies, initial enrichment $4.5 \mathrm{w} \% \mathrm{U}-235$

(Case : gd000_1b_64_bp_fuel o)
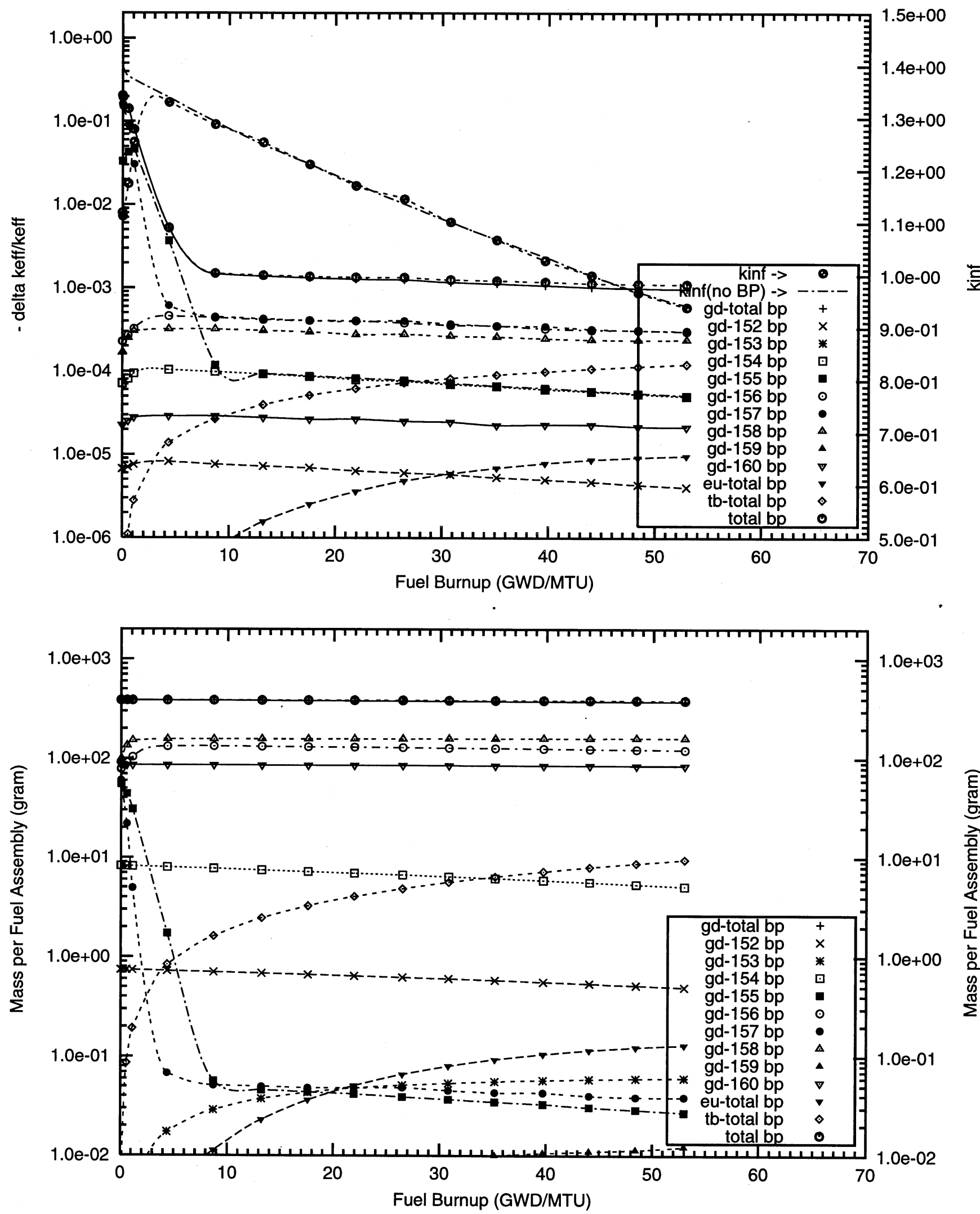
A2 - Gadolinium Fully Enriched in ${ }^{155}$ Gd 
Figure A2.10. Negative Reactivity and Masses BP for Gd-155 and transmutation daughters as a function of fuel life for $17 \times 17$ fuel assemblies with 08 poison rods,

$4.0 \mathrm{w} \% \mathrm{Gd} 2 \mathrm{O} 3$ poison homogeneously mixed in the outer one-third volume of the UO2 pellets

Reactor power $3400 \mathrm{MWth}, 193$ fuel assemblies, initial enrichment 4.5w\%U-235

(Case : gd155_3b_08_bp_fuel o)

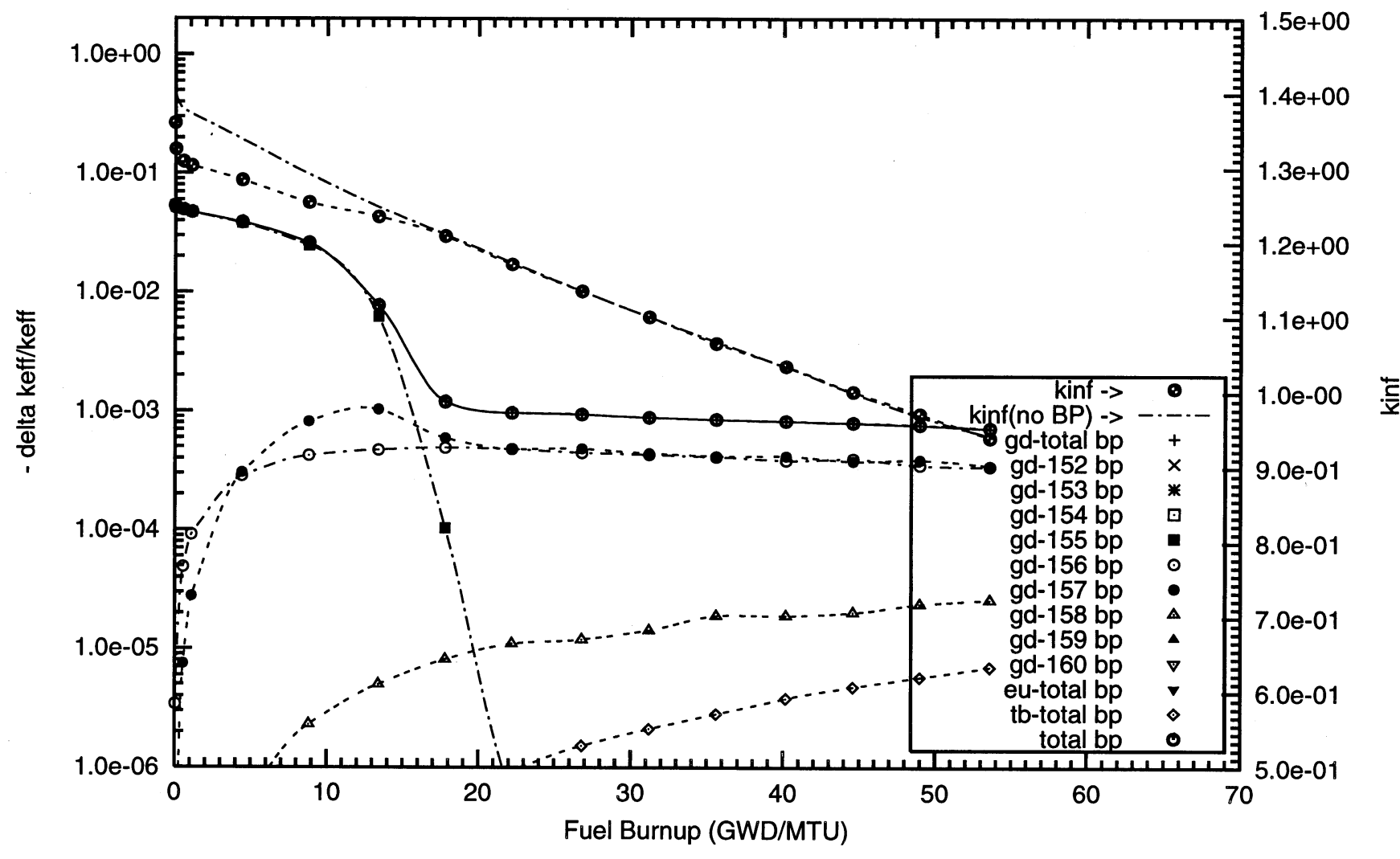


Figure A2.11. Negative Reactivity and Masses BP for Gd-155 and transmutation daughters as a function of fuel life for $17 \times 17$ fuel assemblies with 16 poison rods,

$1.0 \mathrm{w} \% \mathrm{Gd} 2 \mathrm{O} 3$ poison homogeneously mixed in the outer one-third volume of the UO2 pellets

Reactor power $3400 \mathrm{MWth}, 193$ fuel assemblies, initial enrichment 4.5w\%U-235

(Case : gd155_1b_100p_h2o_16_bp_fuel_2_o)

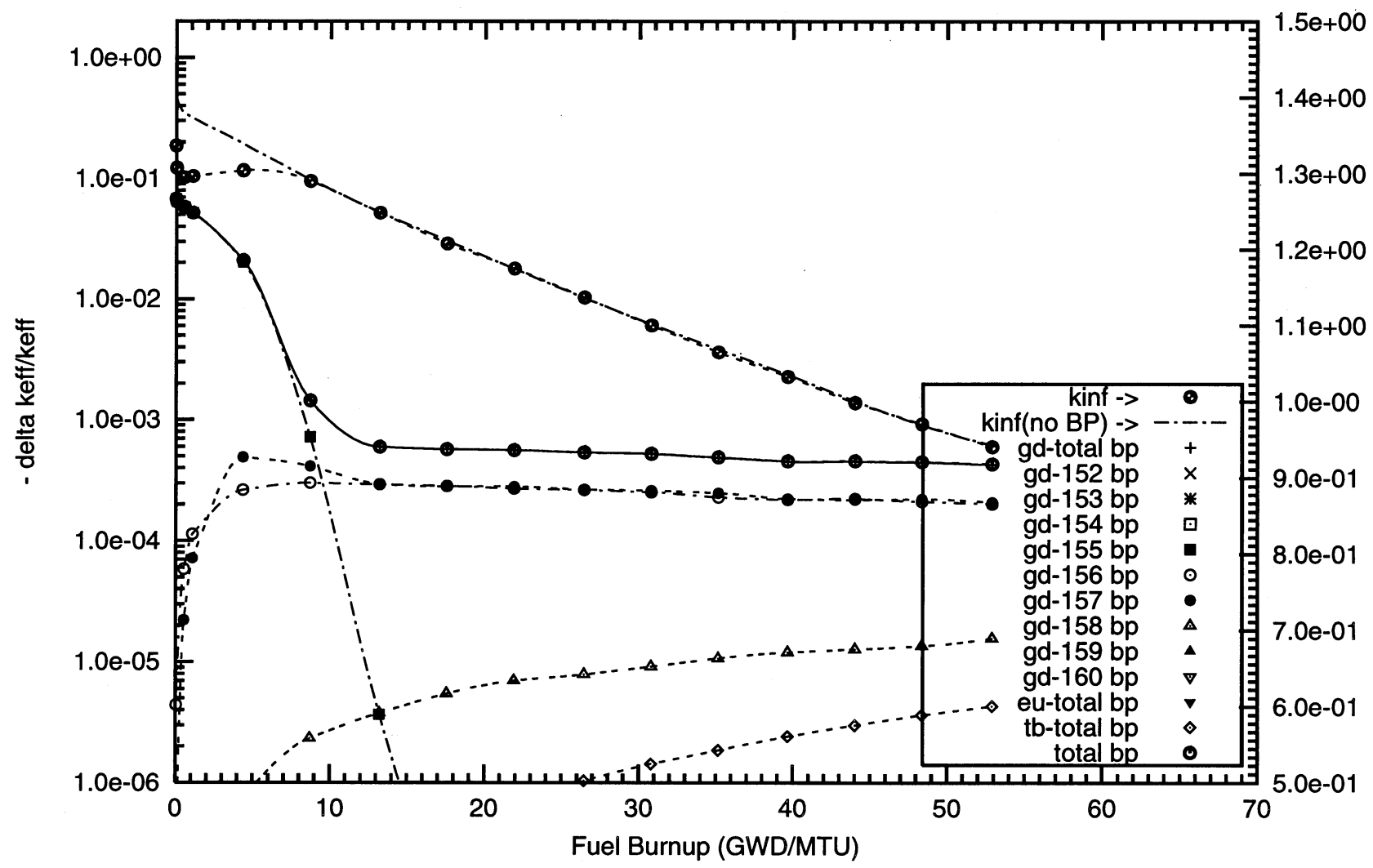

$\frac{\bar{E}}{\bar{x}}$

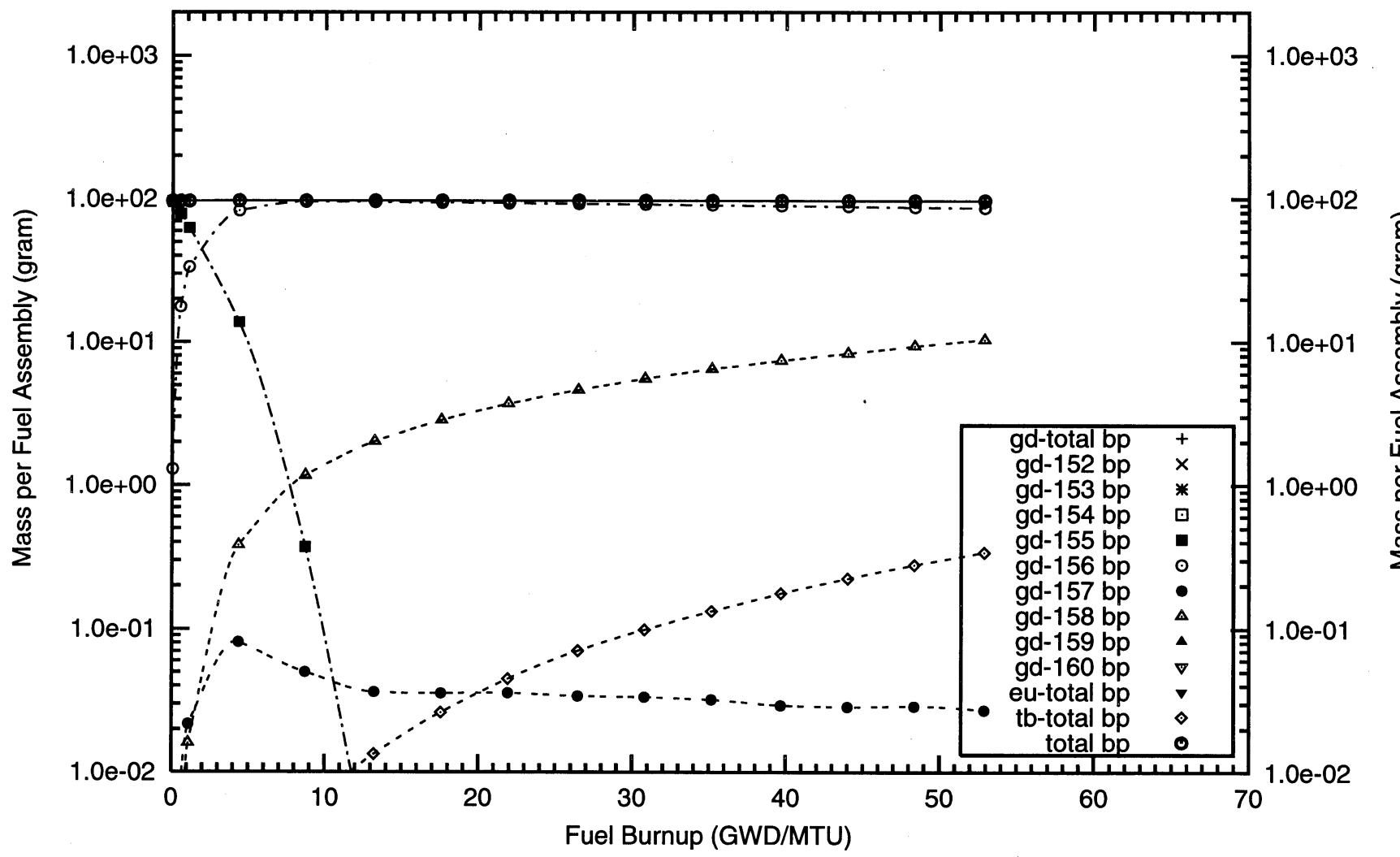


Figure A2.12. Negative Reactivity and Masses BP for Gd-155 and transmutation daughters as a function of fuel life for $17 \times 17$ fuel assemblies with 16 poison rods,

$2.0 \mathrm{w} \% \mathrm{Gd} 2 \mathrm{O} 3$ poison homogeneously mixed in the outer one-third volume of the UO2 pellets

Reactor power $3400 \mathrm{MWth}, 193$ fuel assemblies, initial enrichment 4.5W\%U-235

(Case : gd155_2b_100p_h2o_16_bp_fuel_2_o)

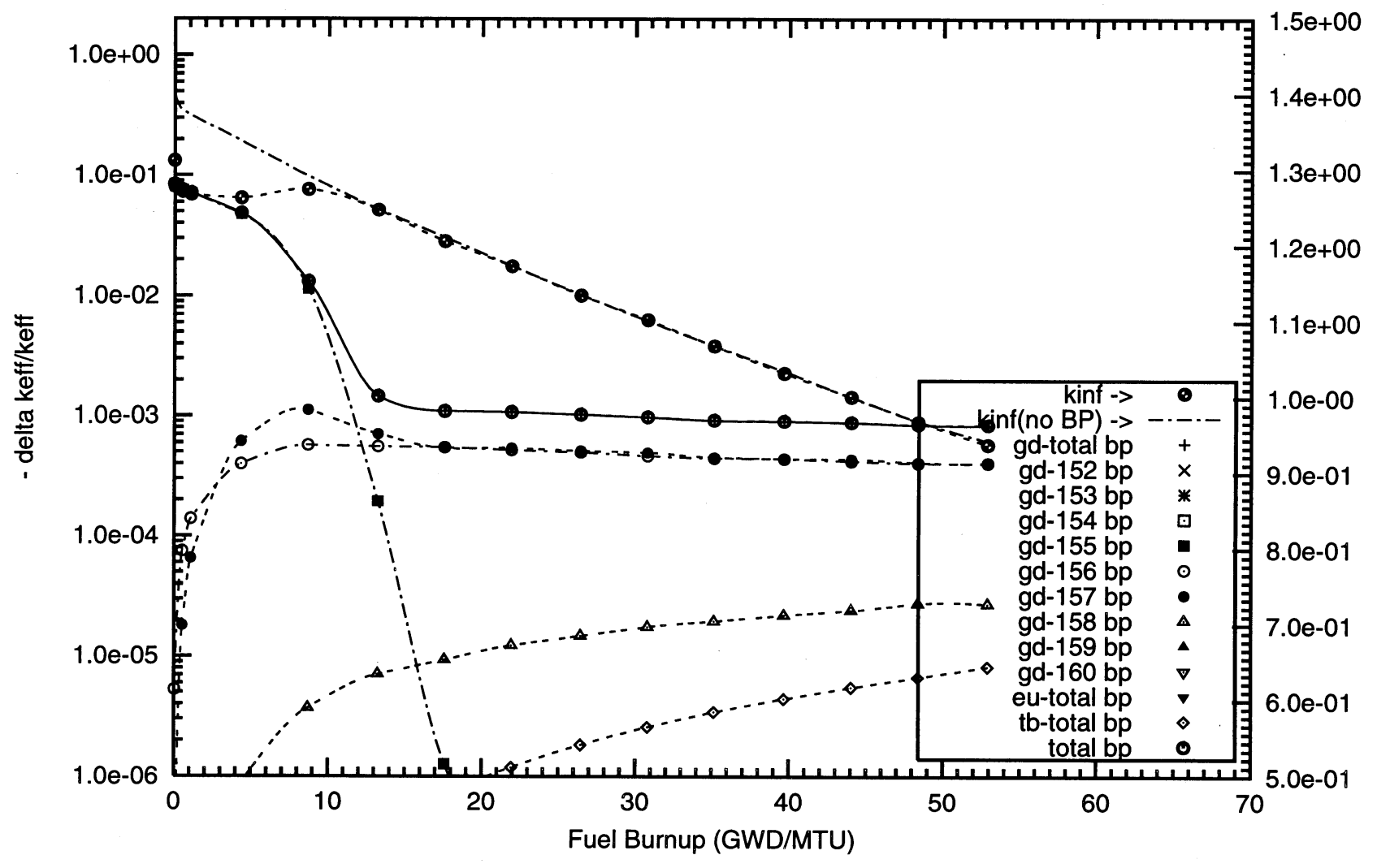

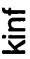

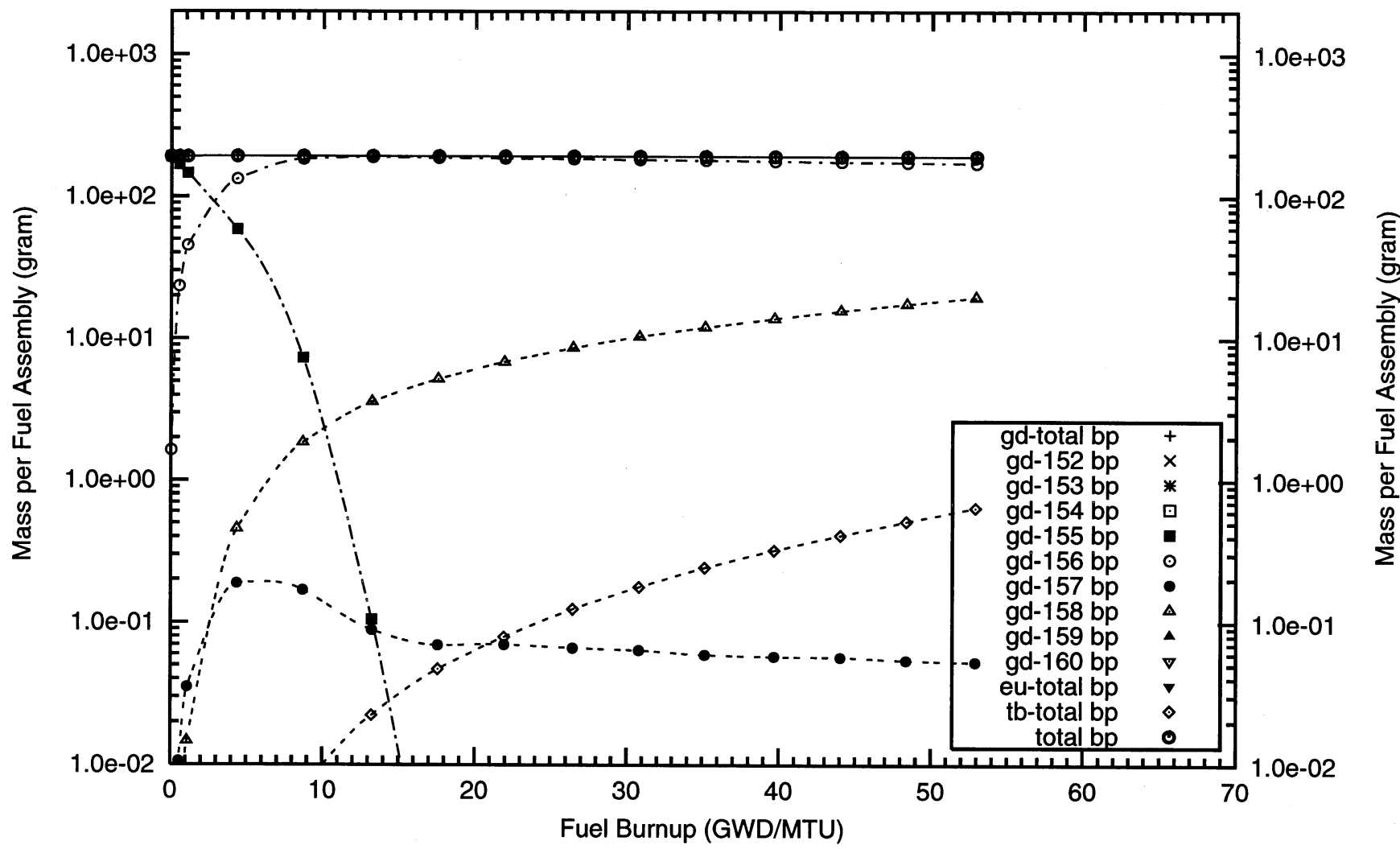


Figure A2.13. Negative Reactivity and Masses BP for Gd-155 and transmutation daughters as a function of fuel life for $17 \times 17$ fuel assemblies with 16 poison rods,

$4.0 \mathrm{w} \% \mathrm{Gd} 2 \mathrm{O} 3$ poison homogeneously mixed in the outer one-third volume of the $\mathrm{UO} 2$ pellets

Reactor power $3400 \mathrm{MW}$ th, 193 fuel assemblies, initial enrichment 4.5w\%U-235

(Case : gd155_3b_100p_h2o_16_bp_fuel_2_o)
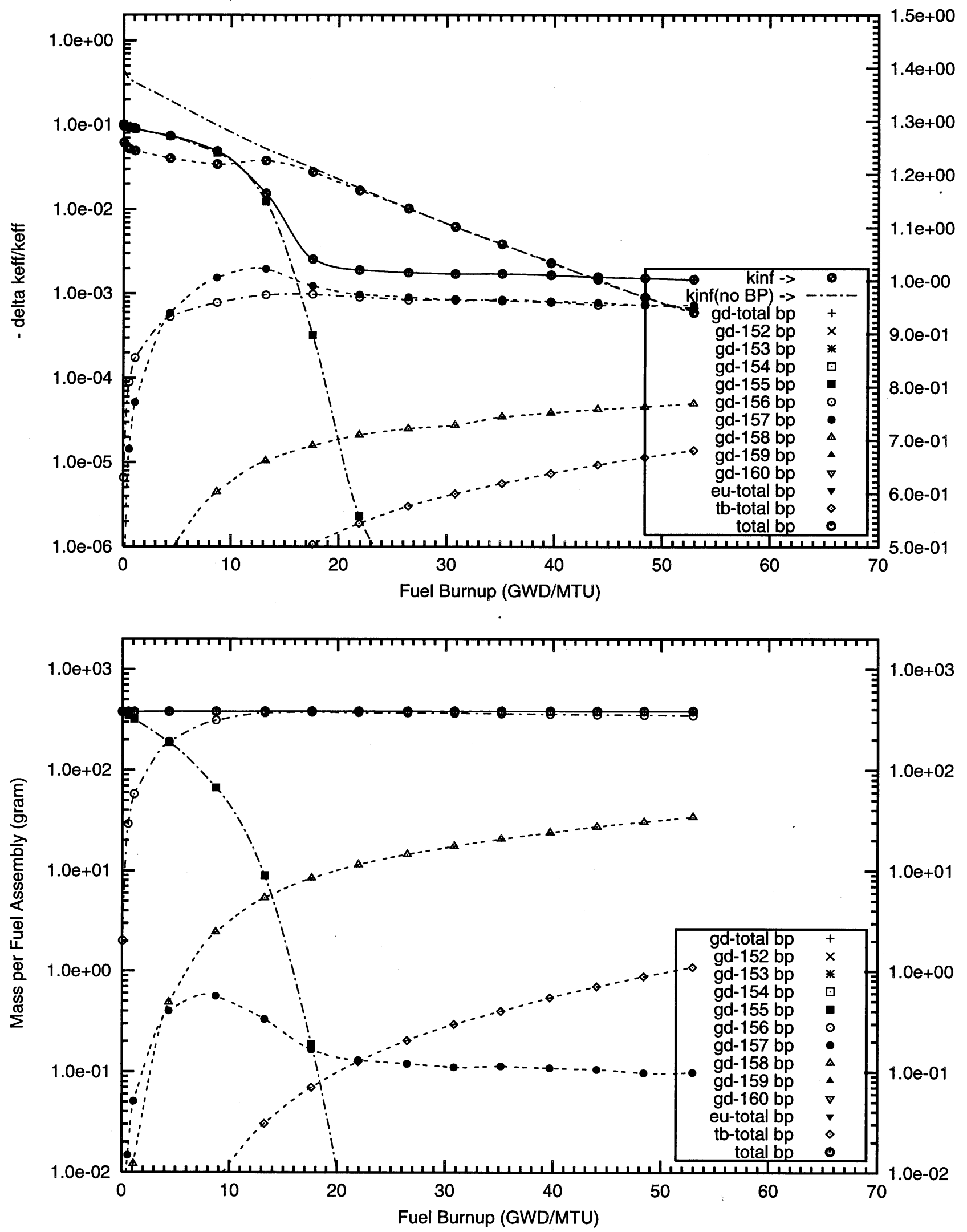
Figure A2.14. Negative Reactivity and Masses BP for Gd-155 and transmutation daughters as a function of tuel lite for $17 \times 17$ tuel assemblies with 16 poison rods,

$8.0 \mathrm{w} \% \mathrm{Gd} 2 \mathrm{O} 3$ poison homogeneously mixed in the outer one-third volume of the UO2 pellets Reactor power $3400 \mathrm{MWth}, 193$ fuel assemblies, initial enrichment 4.5w\%U-235

(Case : gd155_4b_100p_h2o_16_bp_fuel_2_o)
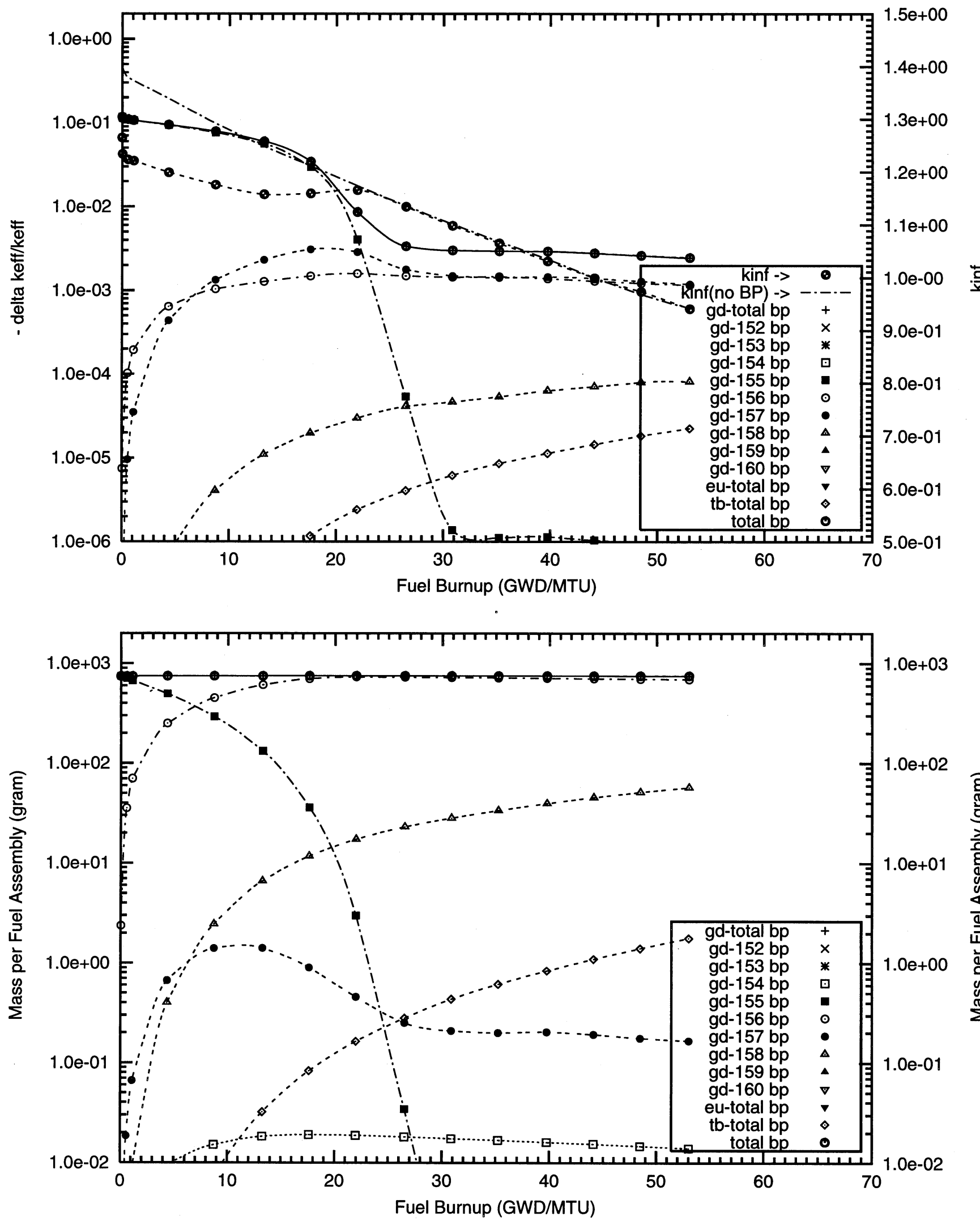
Figure A2.15. Negative Reactivity and Masses BP for Gd-155 and transmutation daughters as a function of fuel life for $17 \times 17$ fuel assemblies with 18 poison rods,

$2.0 \mathrm{w} \% \mathrm{Gd} 2 \mathrm{O} 3$ poison homogeneously mixed in the outer one-third volume of the $\mathrm{UO} 2$ pellets

Reactor power $3400 \mathrm{MWth}, 193$ fuel assemblies, initial enrichment 4.5W\%U-235

(Case : gd155_2b_16_bp_fuel (old) o)

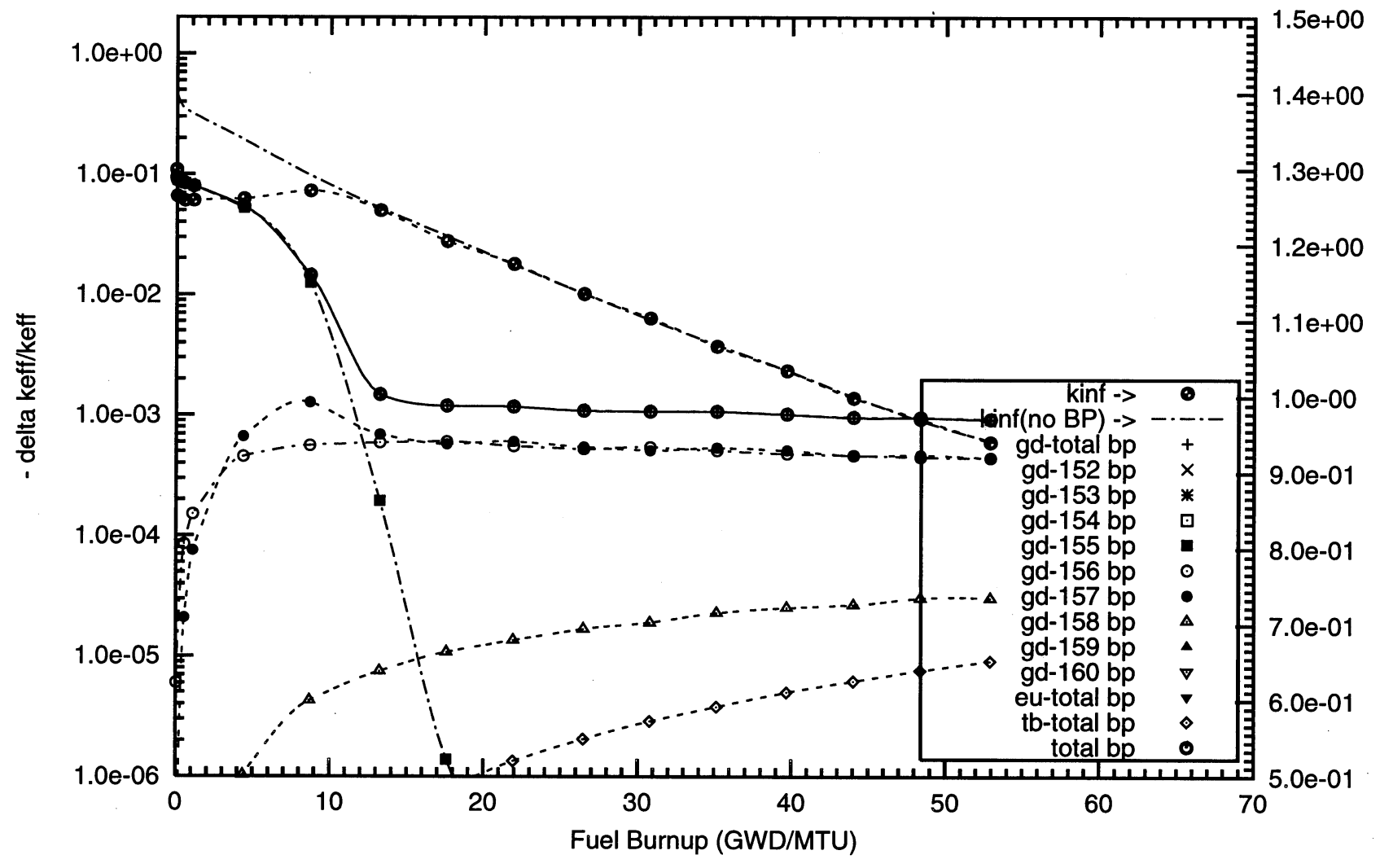

言

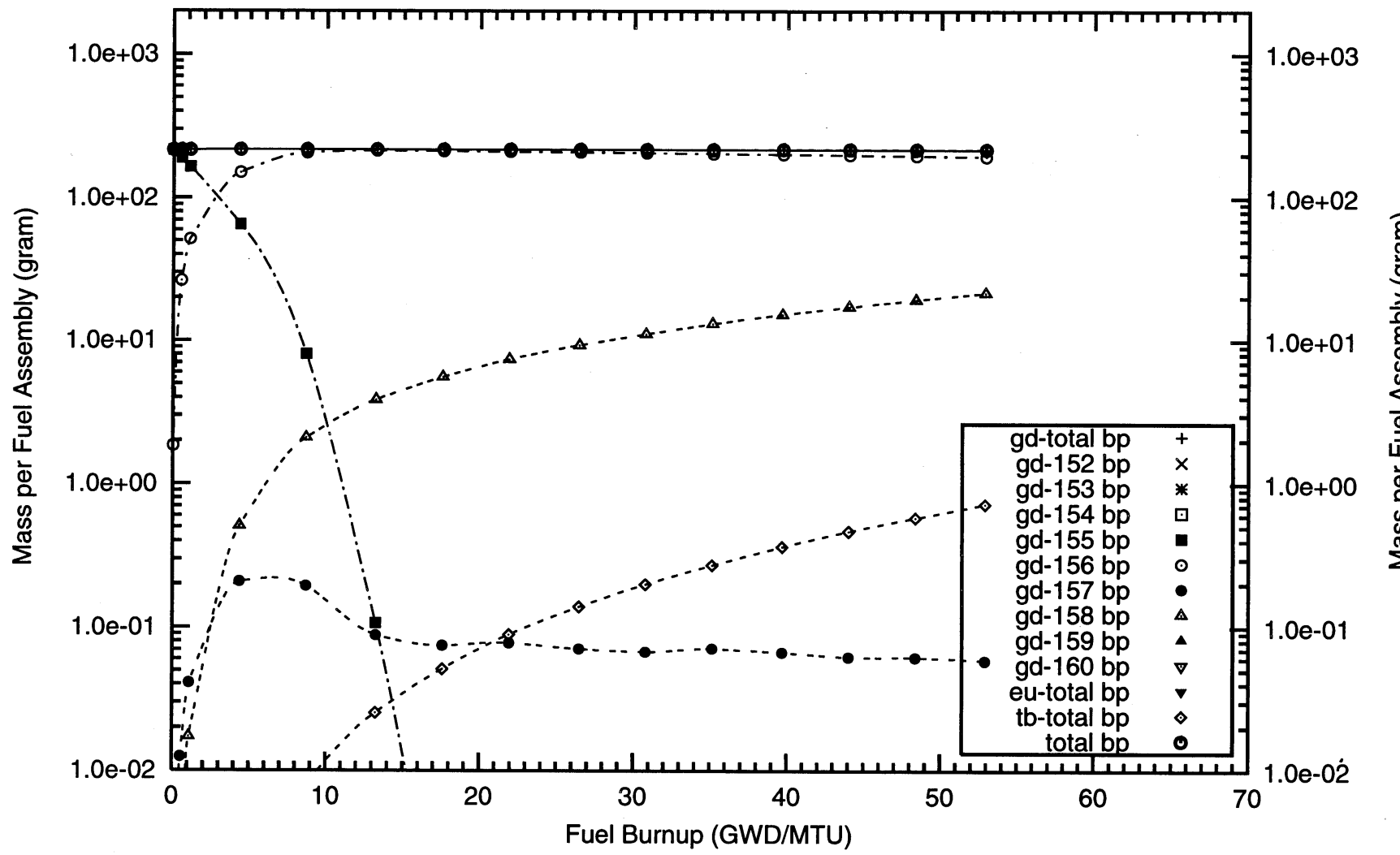


Figure A2.16. Negative Reactivity and Masses BP for Gd-155 and transmutation daughters as a function of fuel life for $17 \times 17$ fuel assemblies with 64 poison rods,

$1.0 \mathrm{w} \% \mathrm{Gd} 2 \mathrm{O} 3$ poison homogeneously mixed in the outer one-third volume of the UO2 pellets Reactor power $3400 \mathrm{MW}$ th, 193 fuel assemblies, initial enrichment $4.5 \mathrm{w} \% \mathrm{U}-235$

(Case : gd155_1b_64_bp_fuel o)
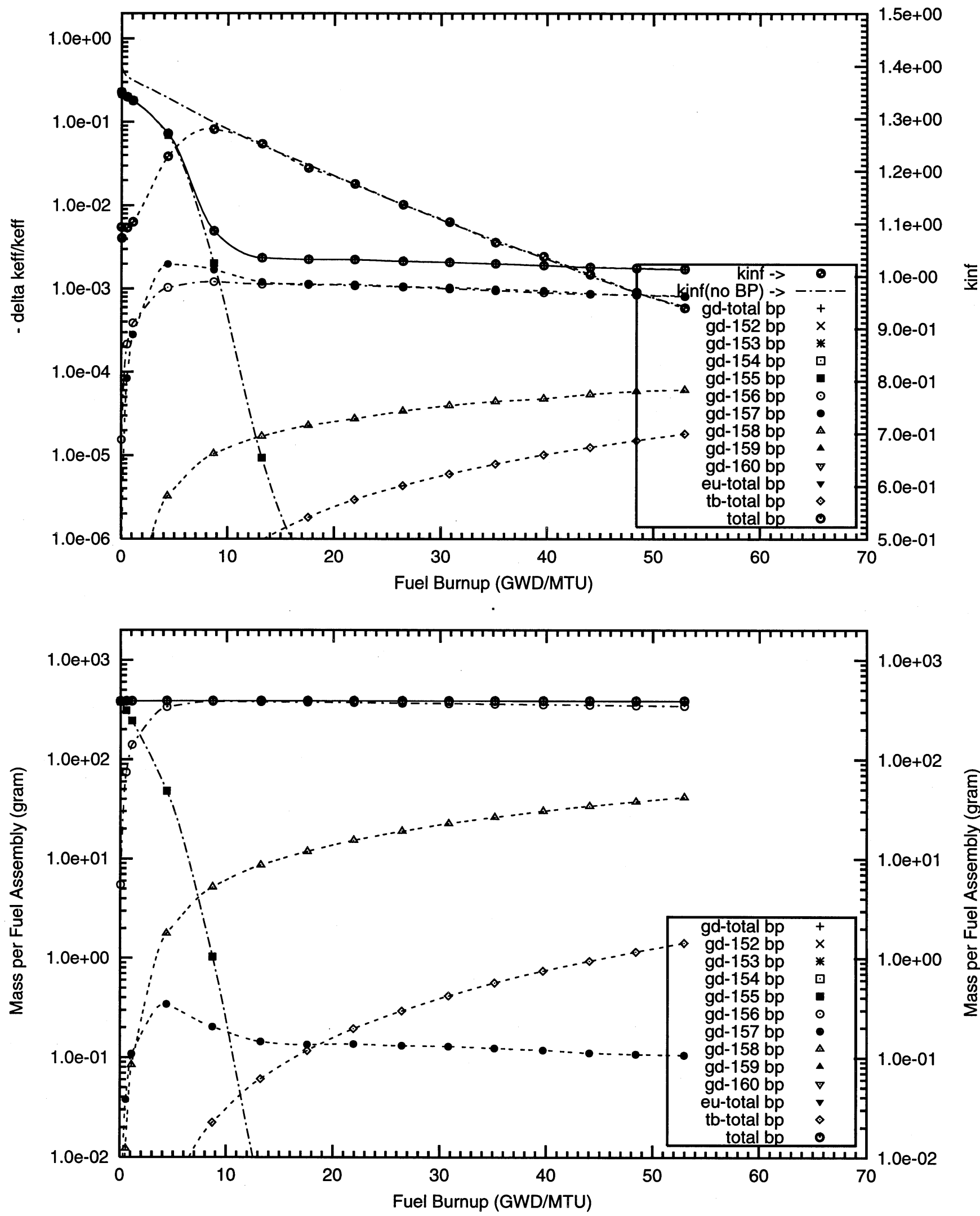

A2 - Gadolinium Fully Enriched in ${ }^{157}$ Gd 

Figure A2.17. Negative Reactivity and Masses BP for Gd-157 and transmutation daughters as a function of fuel life for $17 \times 17$ fuel assemblies with 08 poison rods,

$4.0 \mathrm{w} \% \mathrm{Gd} 2 \mathrm{O} 3$ poison homogeneously mixed in the outer one-third volume of the UO2 pellets Reactor power $3400 \mathrm{MWth}, 193$ fuel assemblies, initial enrichment $4.5 \mathrm{w} \% \mathrm{U}-235$

(Case : gd157_3b_08_bp_fuel o)

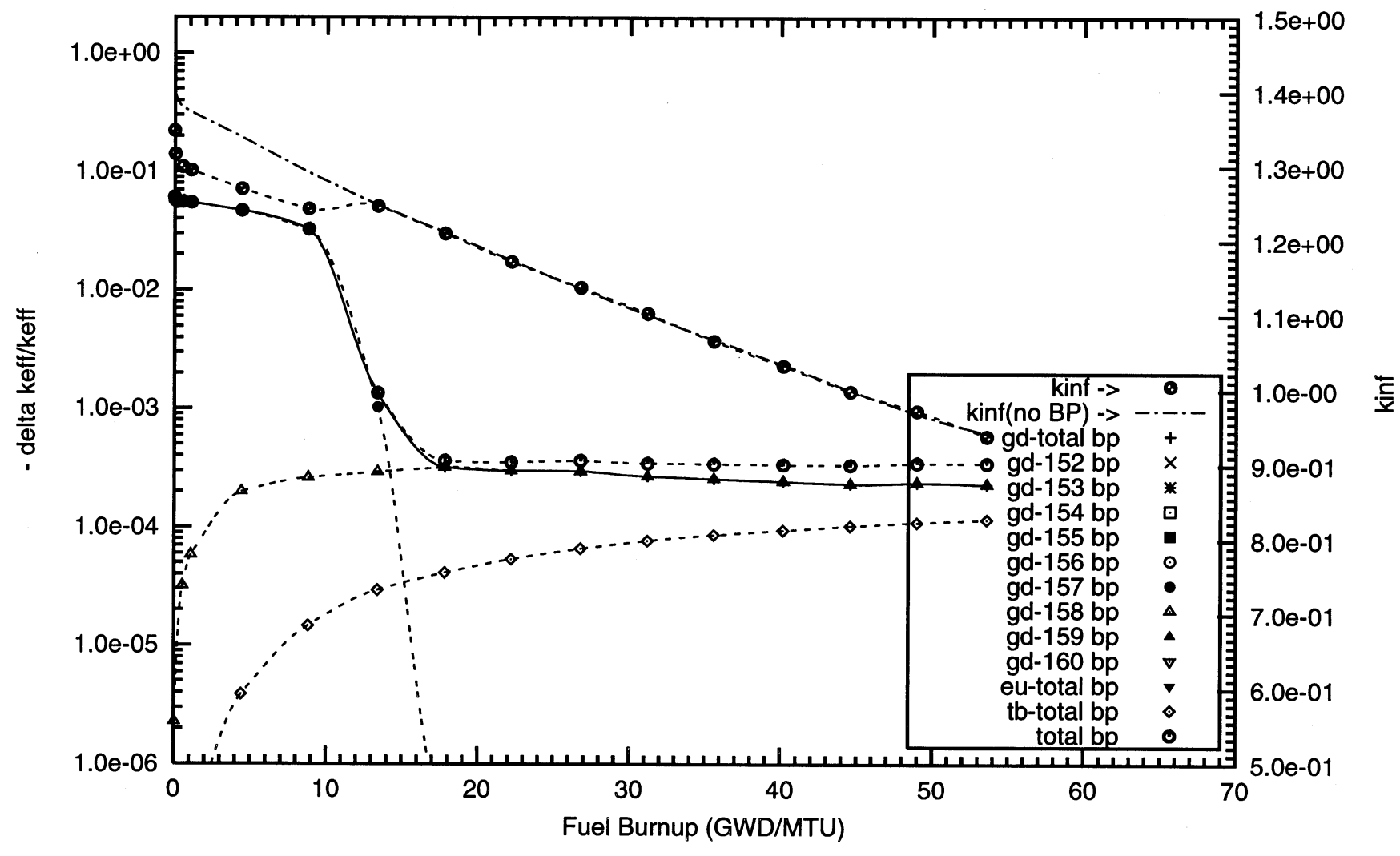


Figure A2.18. Negative Reactivity and Masses BP for Gd-157 and transmutation daughters as a function of fuel life for $17 \times 17$ fuel assemblies with 16 poison rods,

$0.2 \mathrm{w} \% \mathrm{Gd} 2 \mathrm{O} 3$ poison homogeneously mixed in the outer one-third volume of the $\mathrm{UO} 2$ pellets

Reactor power $3400 \mathrm{MWth}, 193$ fuel assemblies, initial enrichment $4.5 \mathrm{w} \% \mathrm{U}-235$

(Case : gd157_8b_100p_h2o_16_bp_fuel_2_o)

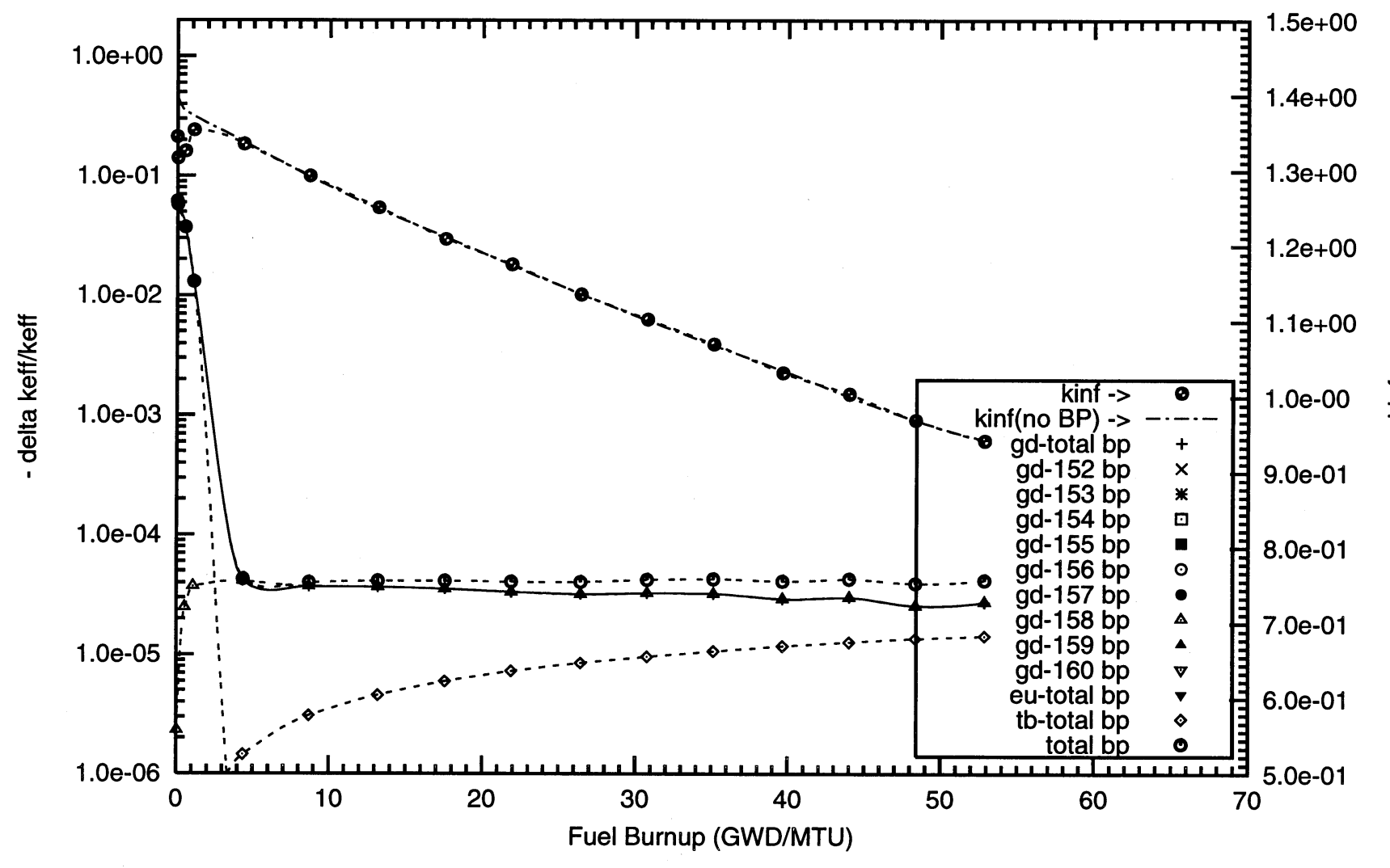

$\underline{\frac{E}{x}}$

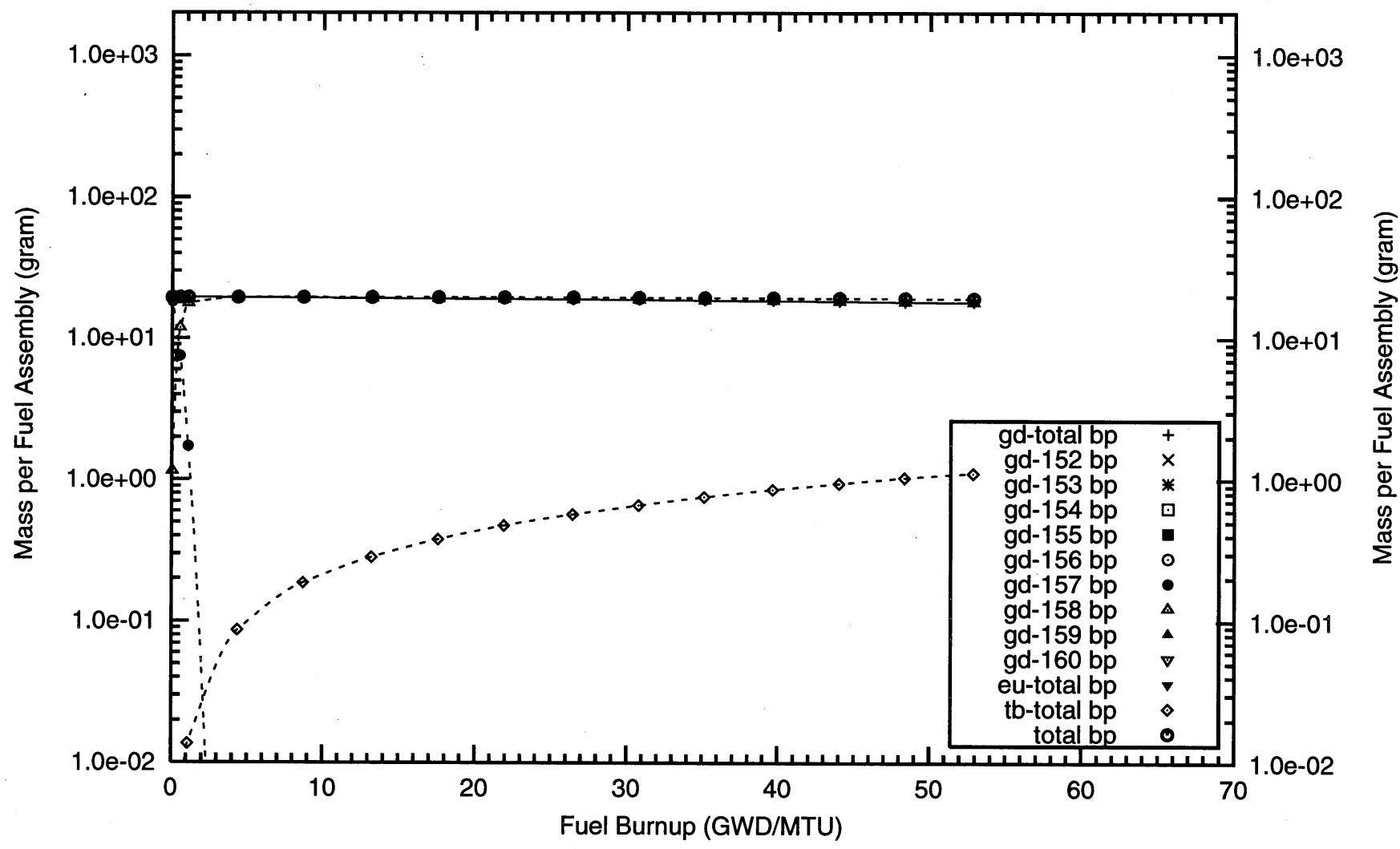


Figure A2.19. Negative Reactivity and Masses BP for Gd-157and transmutation daughters as a function of fuel life for $17 \times 17$ fuel assemblies with 16 poison rods,

$0.5 \mathrm{w} \% \mathrm{Gd} 2 \mathrm{O} 3$ poison homogeneously mixed in the outer one-third volume of the UO2 pellets

Reactor power $3400 \mathrm{MWth}, 193$ fuel assemblies, initial enrichment 4.5w\%U-235

(Case : gd157_0b_100p_h2o_16_bp_fuel_2_o)
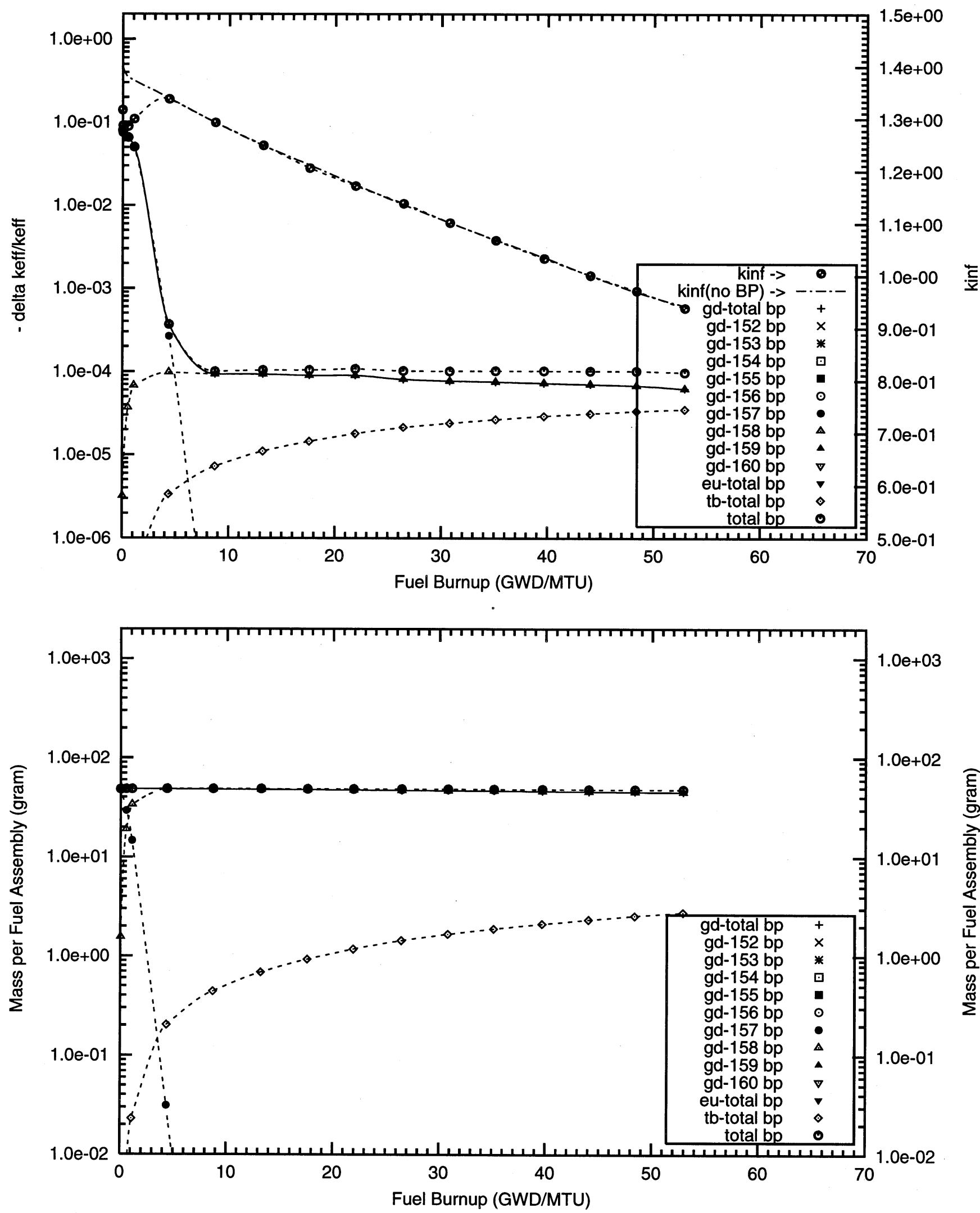
Figure A2.20. Negative Reactivity and Masses BP for Gd-157 and transmutation daughters as a function of fuel life for $17 \times 17$ fuel assemblies with 16 poison rods,

$1.0 \mathrm{w} \% \mathrm{Gd} 2 \mathrm{O} 3$ poison homogeneously mixed in the outer one-third volume of the $\mathrm{UO} 2$ pellets

Reactor power $3400 \mathrm{MW}$ th, 193 fuel assemblies, initial enrichment $4.5 \mathrm{w} \% \mathrm{U}-235$

(Case : gd157_1b_100p_h2o_16_bp_fuel_2_o)

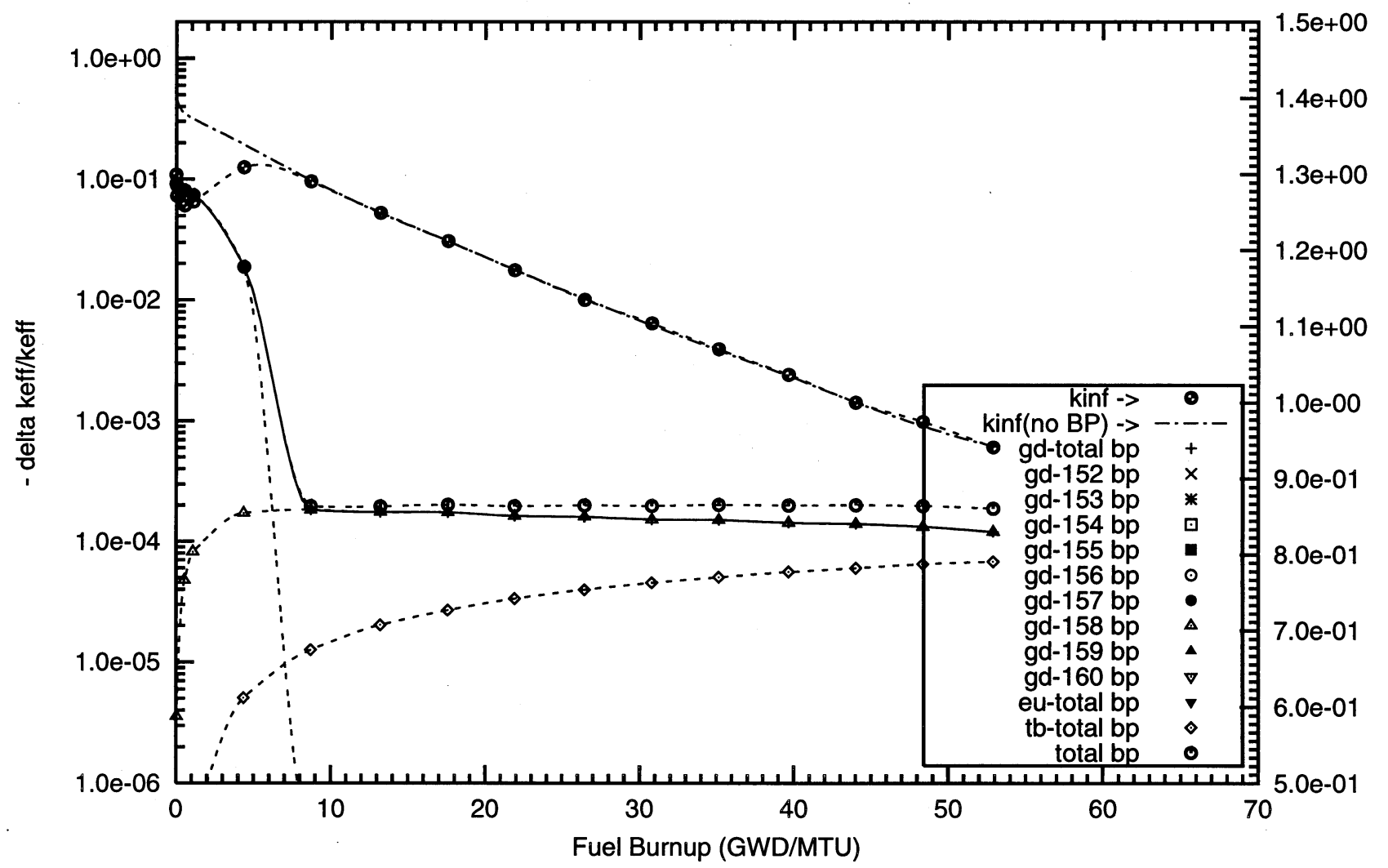

$\frac{\bar{x}}{\bar{x}}$

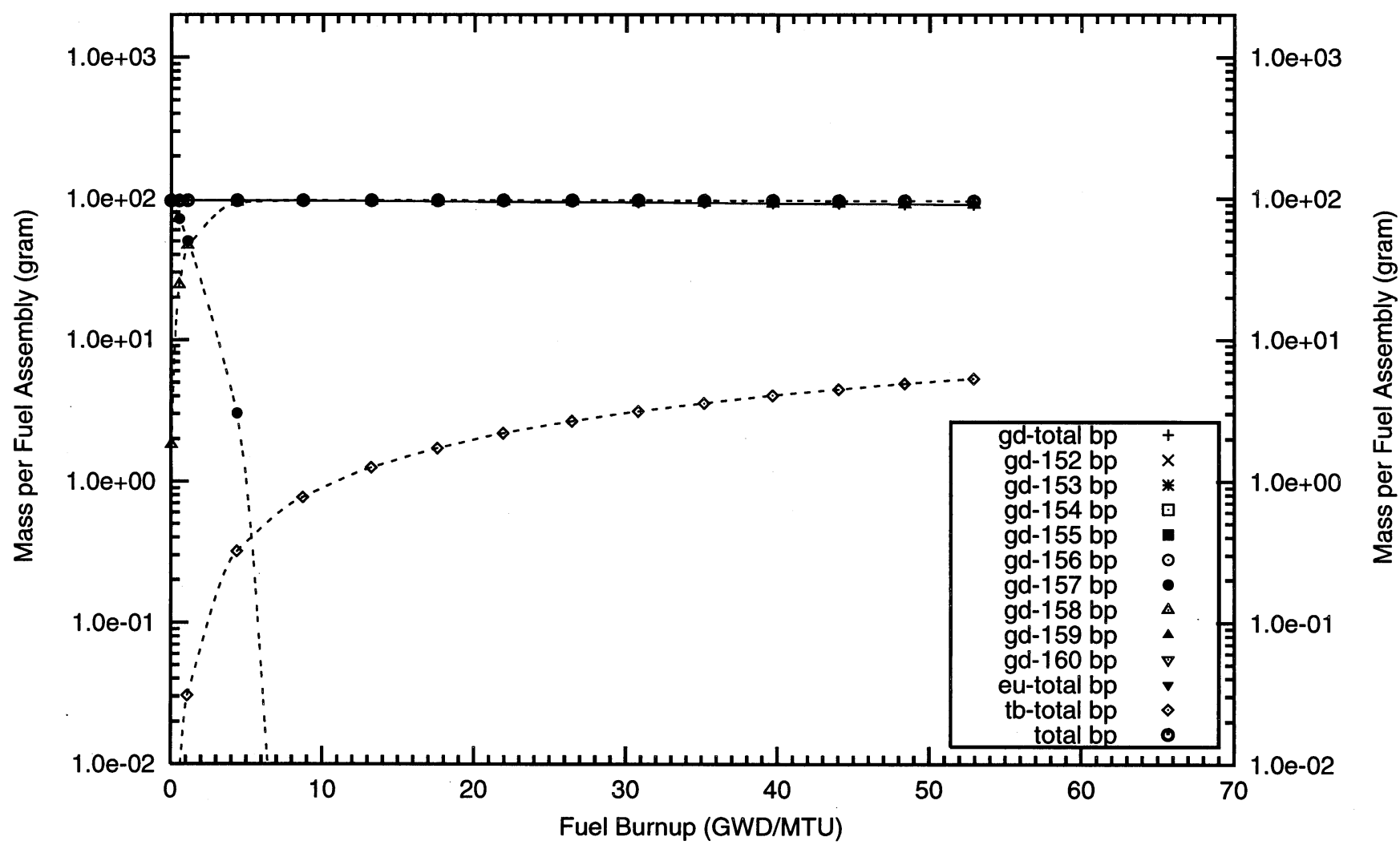


Figure A2.21. Negative Reactivity and Masses BP for Gd-157 and transmutation daughters as a tunction of tuel lite for $17 \times 17$ fuel assemblies with 16 poison rods,

$2.0 \mathrm{w} \% \mathrm{Gd} 2 \mathrm{O} 3$ poison homogeneously mixed in the outer one-third volume of the $\mathrm{UO} 2$ pellets

Reactor power $3400 \mathrm{MW}$ th, 193 fuel assemblies, initial enrichment $4.5 \mathrm{w} \% \mathrm{U}-235$

(Case : gd157_2b_100p_h2o_16_bp_fuel_2_o)
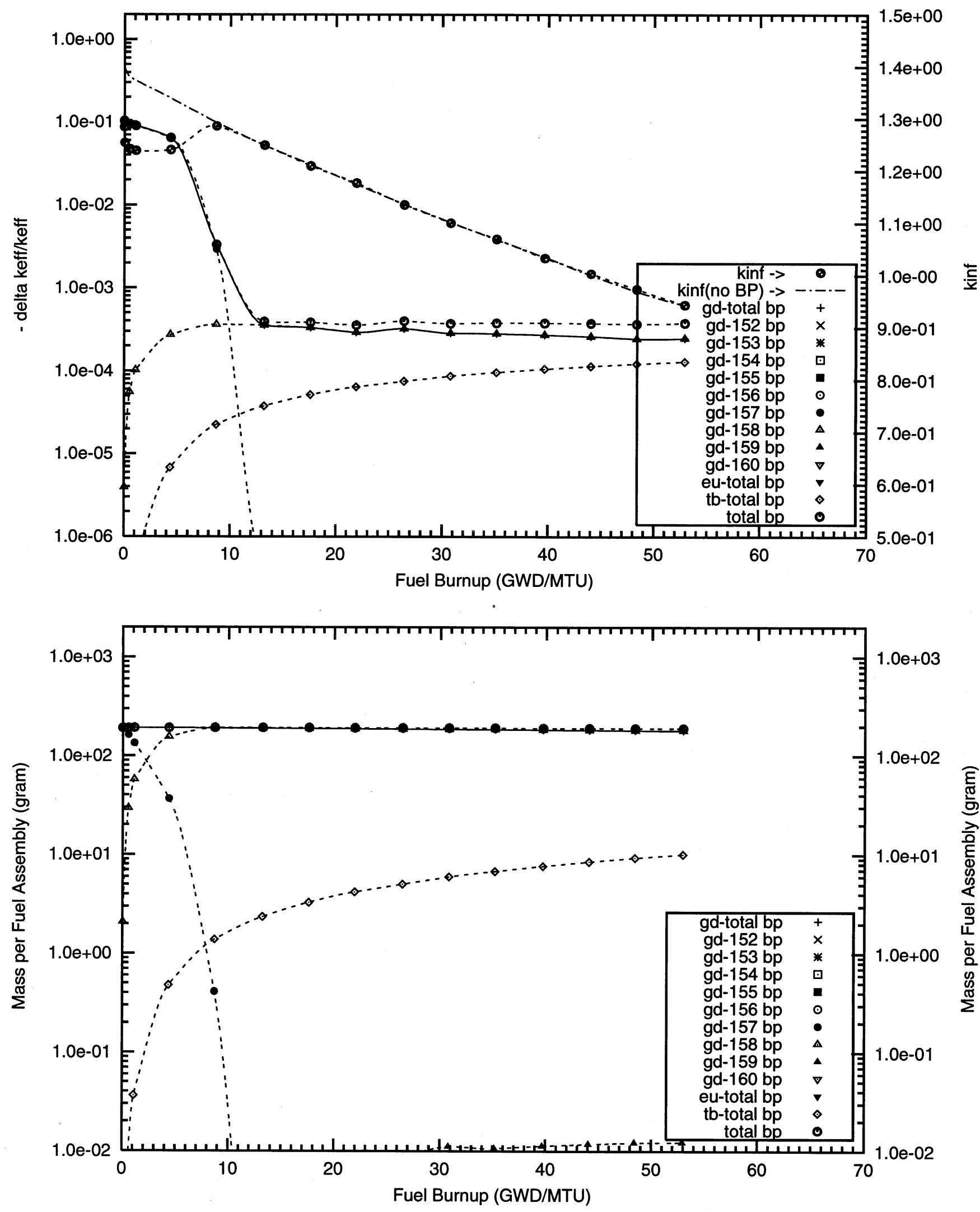
Figure A2.22. Negative Reactivity and Masses BP for Gd-157 and transmutation daughters as a function of fuel life for $17 \times 17$ fuel assemblies with 16 poison rods,

$4.0 \mathrm{w} \% \mathrm{Gd} 2 \mathrm{O} 3$ poison homogeneously mixed in the outer one-third volume of the UO2 pellets

Reactor power $3400 \mathrm{MWth}, 193$ fuel assemblies, initial enrichment 4.5w\%U-235

(Case : gd157_3b_100p_h2o_16_bp_fuel_2_o)
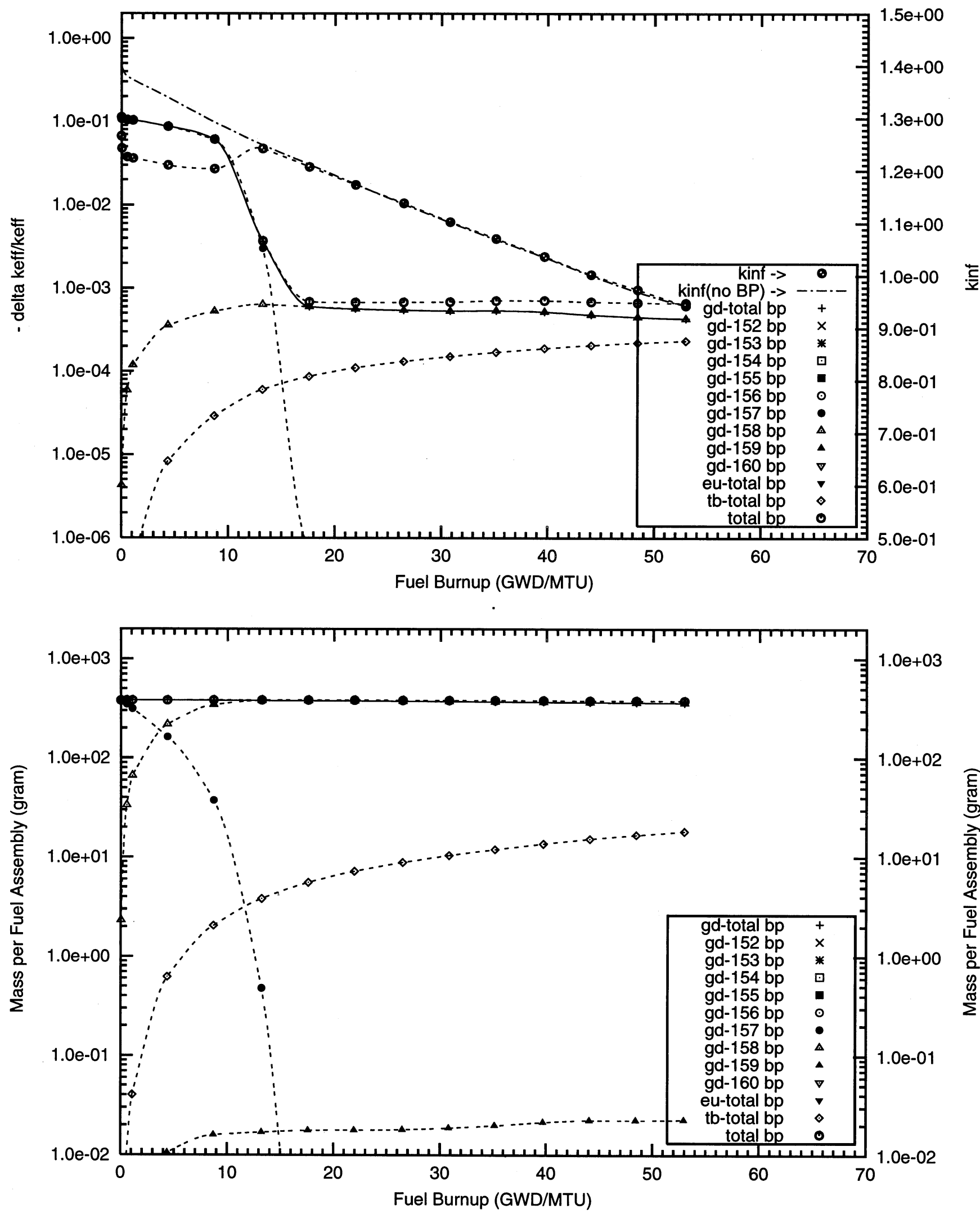
Figure A2.23. Negative Reactivity and Masses BP for Gd-157 and transmutation daughters as a function of fuel life for $17 \times 17$ fuel assemblies with 64 poison rods,

$0.5 \mathrm{w} \% \mathrm{Gd} 2 \mathrm{O} 3$ poison homogeneously mixed in the outer one-third volume of the UO2 pellets Reactor power $3400 \mathrm{MWth}, 193$ fuel assemblies, initial enrichment 4.5w\%U-235

(Case : gd157_0b_64_bp_fuel o)
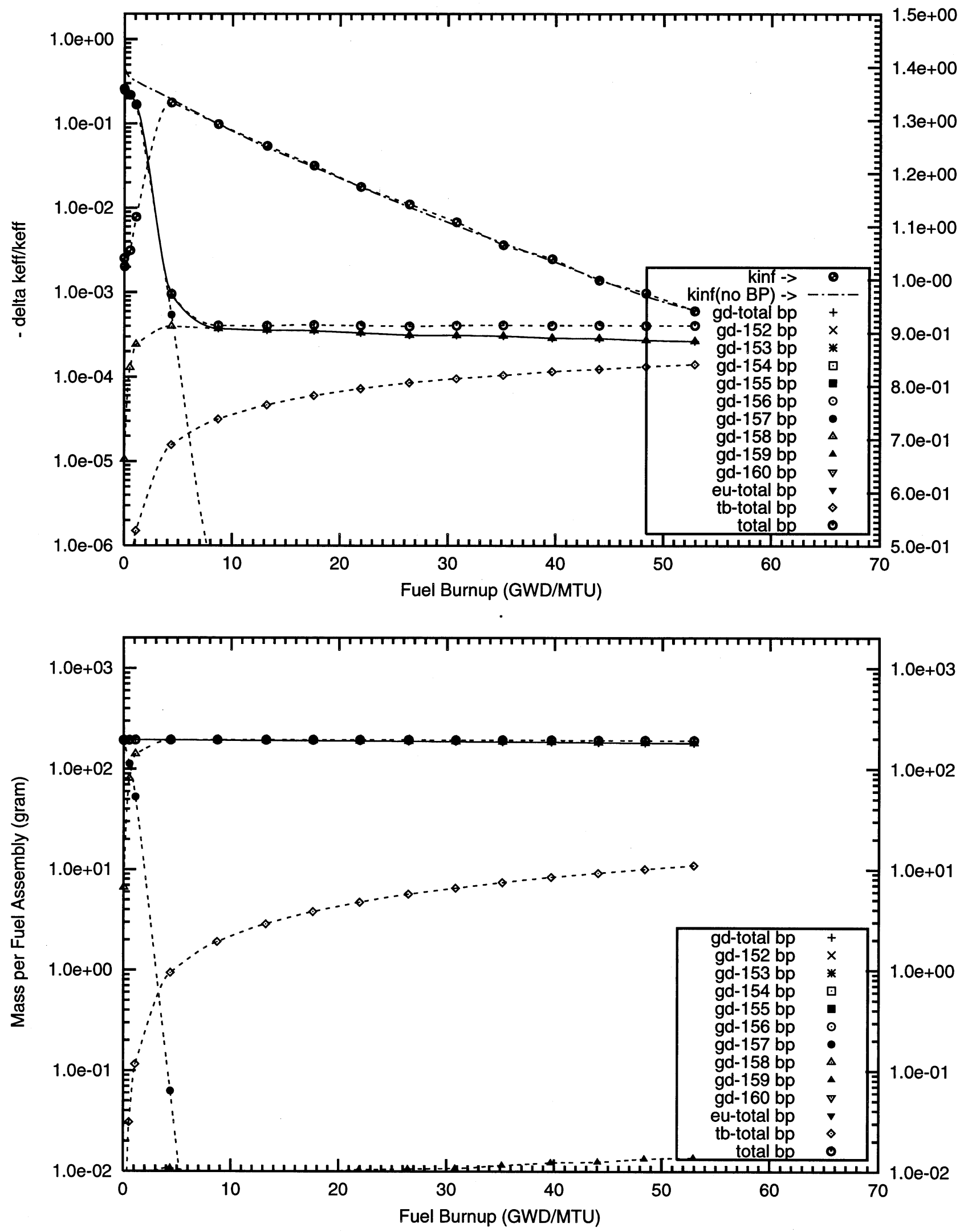


\section{Appendix A3}

Reactivity Worths and Masses of $\mathrm{Gd}_{2} \mathrm{O}_{3}$ Coating on the Surface of the Fuel Pellets

\section{Table 7 Cases}



A3 - Gadolinium with Natural Isotopic Abundances 
Figure A3.1. Negative Reactivity and Masses BP for Gd-nat and transmutation daughters as a function of fuel life for $17 \times 17$ fuel assemblies with 08 poison rods,

4.0 mill thick Gd2O3 poison coating on the outer radial surface of the UO2 pellets

Reactor power $3400 \mathrm{MWth}, 193$ fuel assemblies, initial enrichment $4.5 \mathrm{w} \% \mathrm{U}-235$

(Case : gd000_3f_100p_h20_08_bp_fuel_2_o)
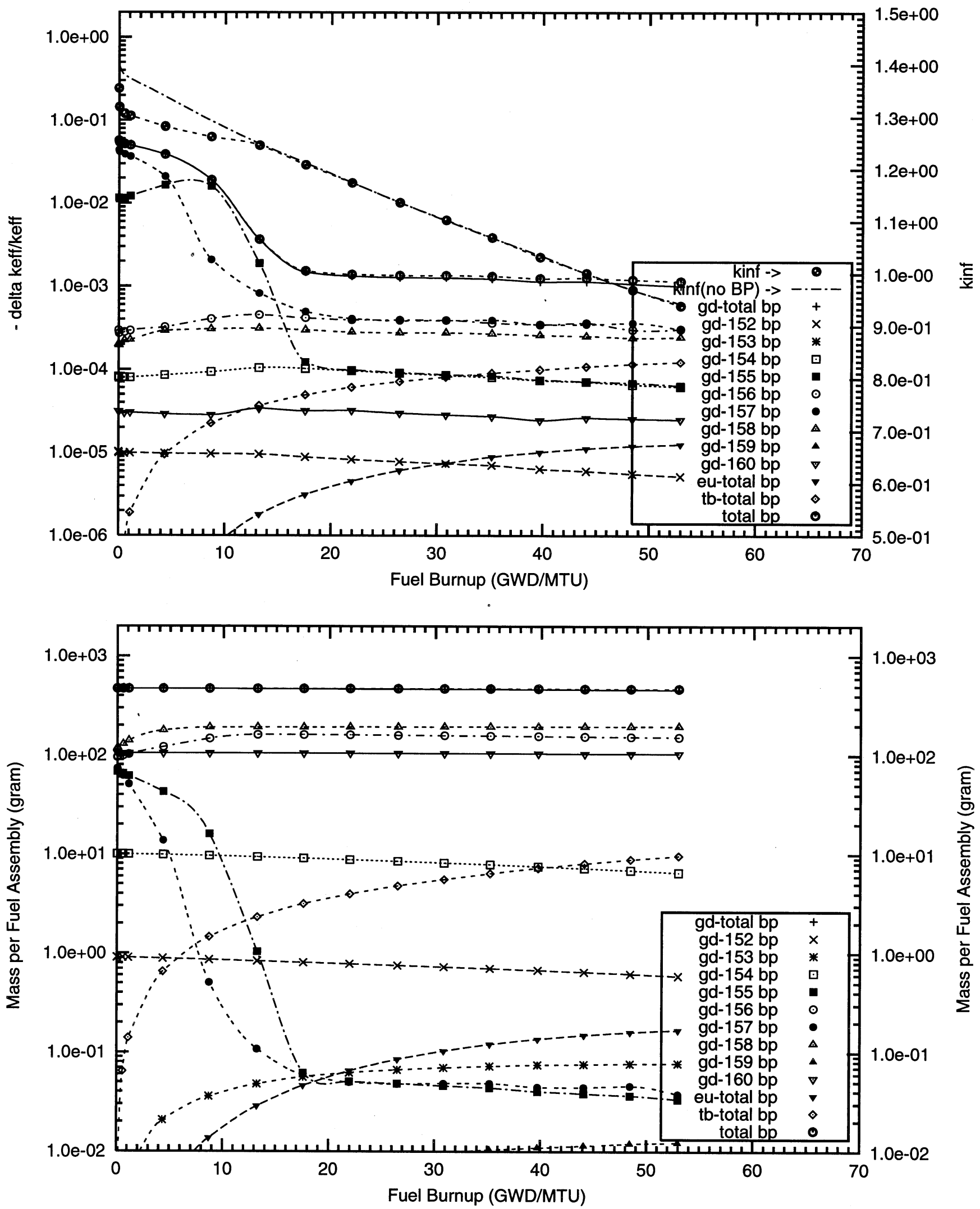
Figure A3.2. Negative Reactivity and Masses BP for Gd-nat and transmutation daughters as a function of fuel life for $17 \times 17$ fuel assemblies with 16 poison rods,

1.0 mill thick Gd2O3 poison coating on the outer radial surface of the UO2 pellets

Reactor power $3400 \mathrm{MWth}, 193$ fuel assemblies, initial enrichment 4.5W\%U-235

(Case : gd000_1f_100p_h2o_16_bp_fuel_2_o)
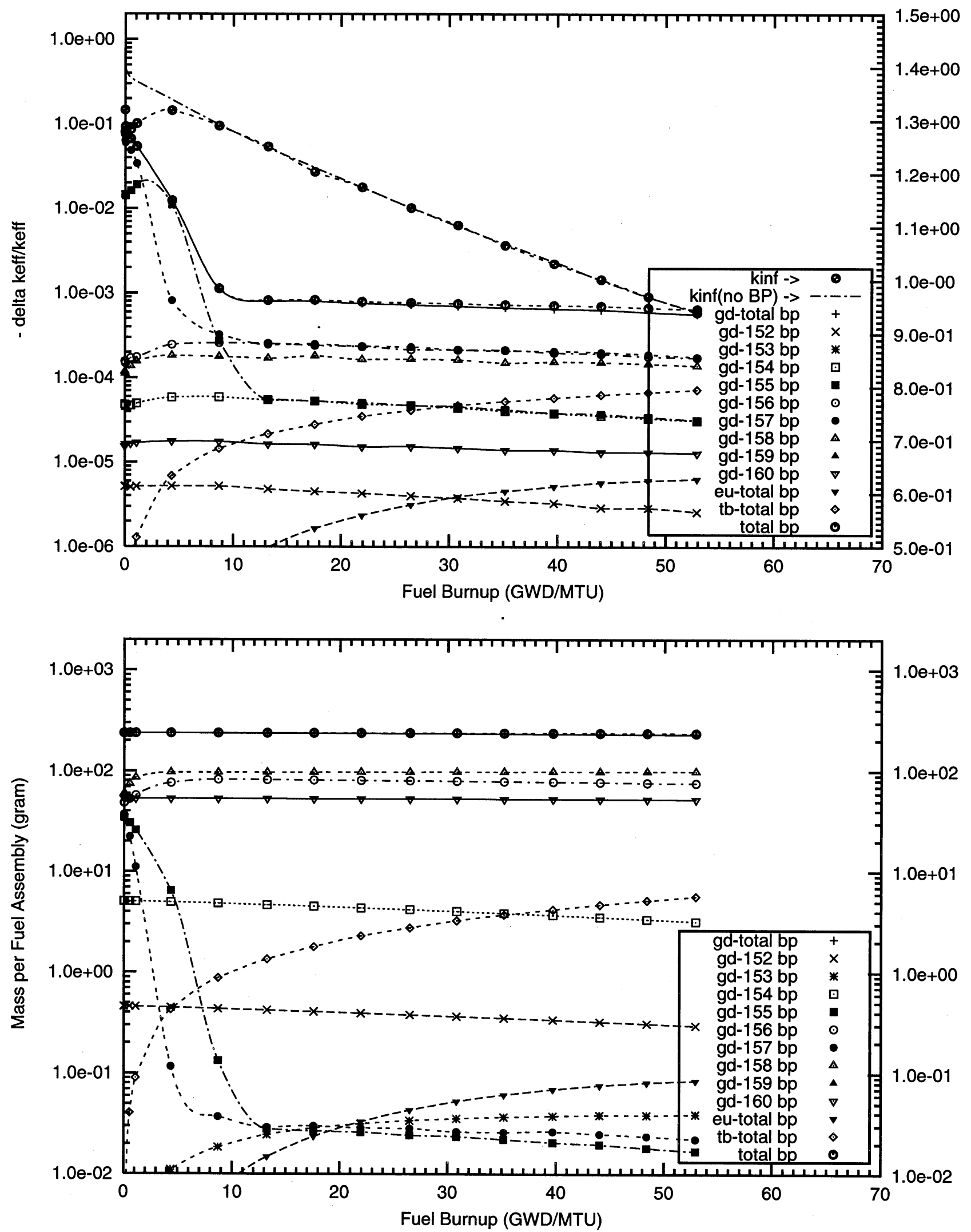
Figure A3.3. Negative Reactivity and Masses BP for Gd-nat and transmutation daughters as a function of fuel life for $17 \times 17$ fuel assemblies with 16 poison rods, 2.0 mill thick Gd2O3 poison coating on the outer radial surface of the $\mathrm{UO} 2$ pellets Reactor power $3400 \mathrm{MWth}, 193$ fuel assemblies, initial enrichment 4.5w\%U-235

(Case : gd000_2f_100p_h20_16_bp_fuel_2_o)
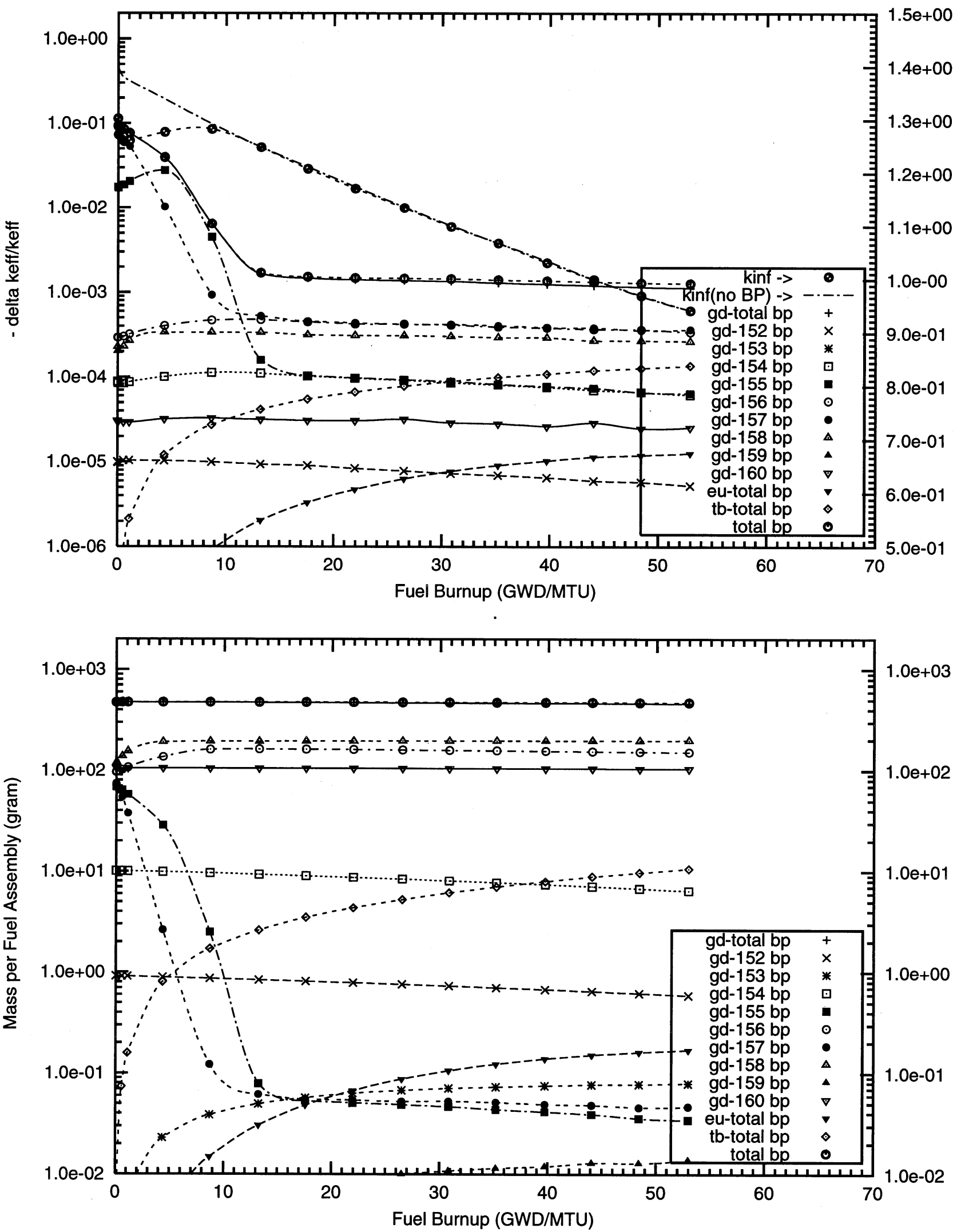
Figure A3.4. Negative Reactivity and Masses BP for Gd-nat and transmutation daughters as a function of fuel life for $17 \times 17$ fuel assemblies with 16 poison rods,

4.0 mill thick Gd2O3 poison coating on the outer radial surface of the UO2 pellets

Reactor power $3400 \mathrm{MWth}, 193$ fuel assemblies, initial enrichment 4.5w\%U-235

(Case : gd000_3f_100p_h20_16_bp_fuel_2_o)
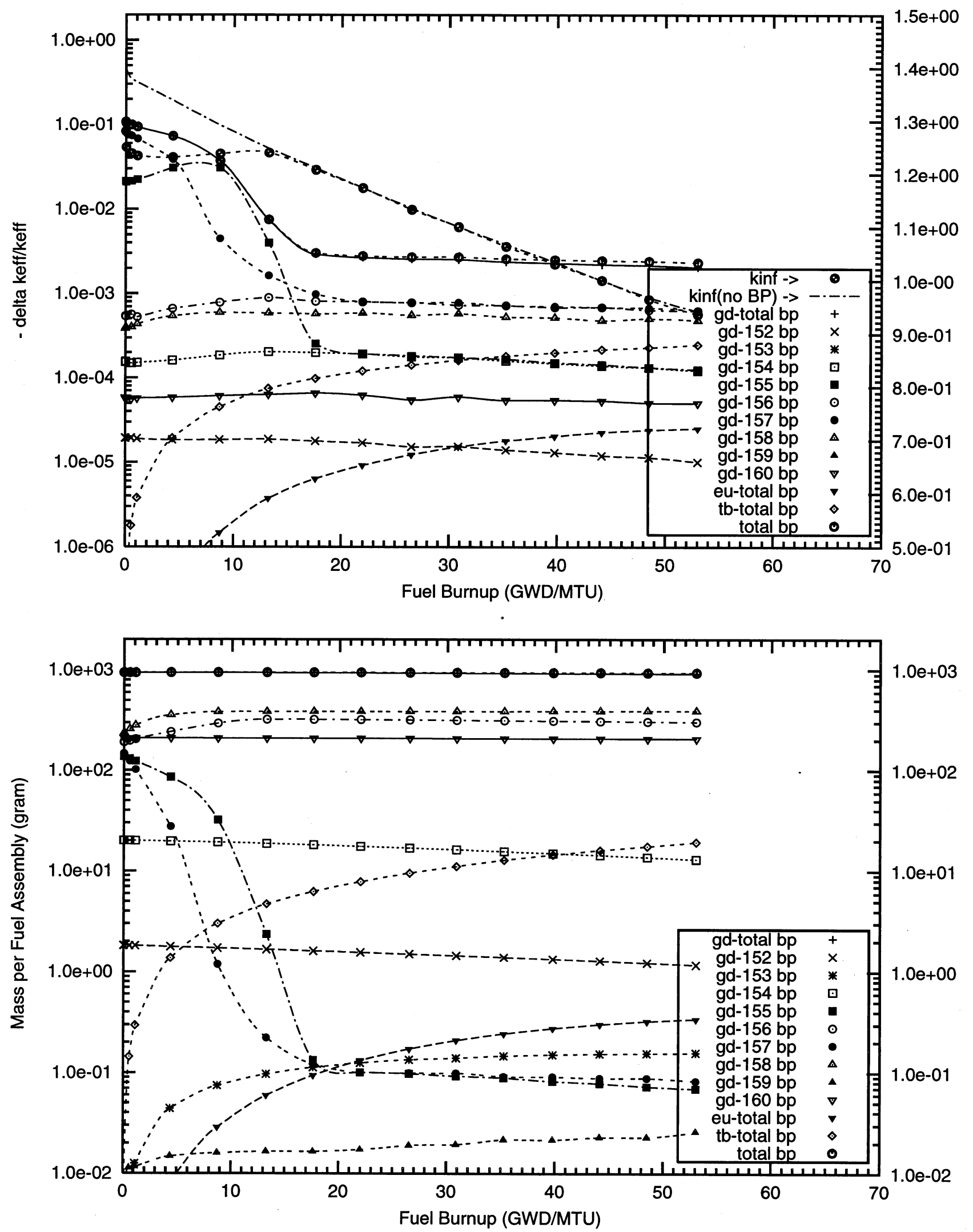
Figure A3.5. Negative Reactivity and Masses BP for Gd-nat and transmutation daughters as a function of fuel life for $17 \times 17$ fuel assemblies with 16 poison rods,

8.0 mill thick Gd2O3 poison coating on the outer radial surface of the $\mathrm{UO} 2$ pellets

Reactor power $3400 \mathrm{MWth}, 193$ fuel assemblies, initial enrichment 4.5w\%U-235

(Case : gd000_4f_100p_h2o_16_bp_fuel_2_o)
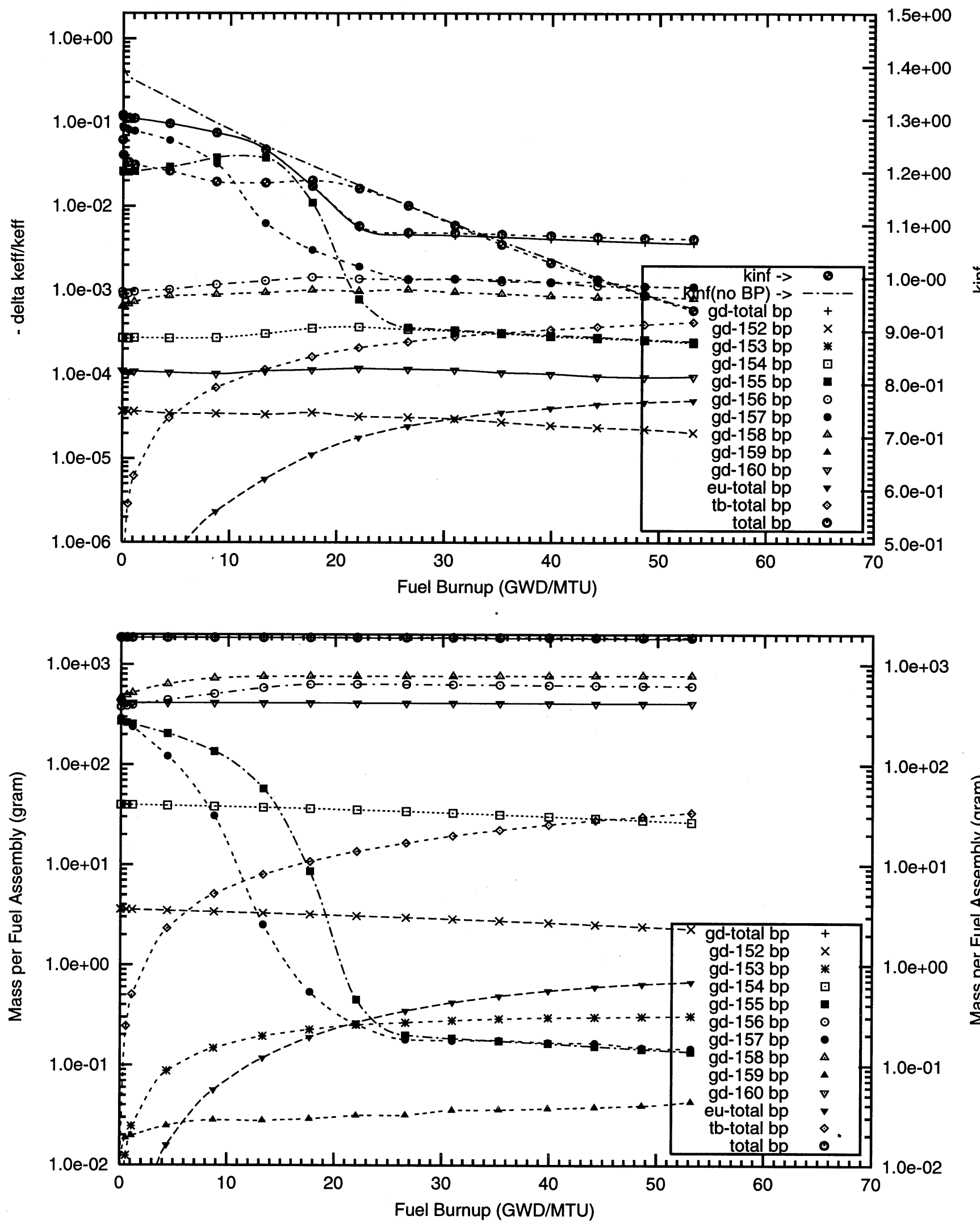
Figure A3.6. Negative Reactivity and Masses BP for Gd-nat and transmutation daughters as a function of fuel life for $17 \times 17$ fuel assemblies with 64 poison rods,

0.2 mill thick $\mathrm{Gd} 2 \mathrm{O} 3$ poison coating on the outer radial surface of the UO2 pellets

Reactor power $3400 \mathrm{MW}$ th, 193 fuel assemblies, initial enrichment 4.5w\%U-235

(Case : gd000_8f_64_bp_fuel o)
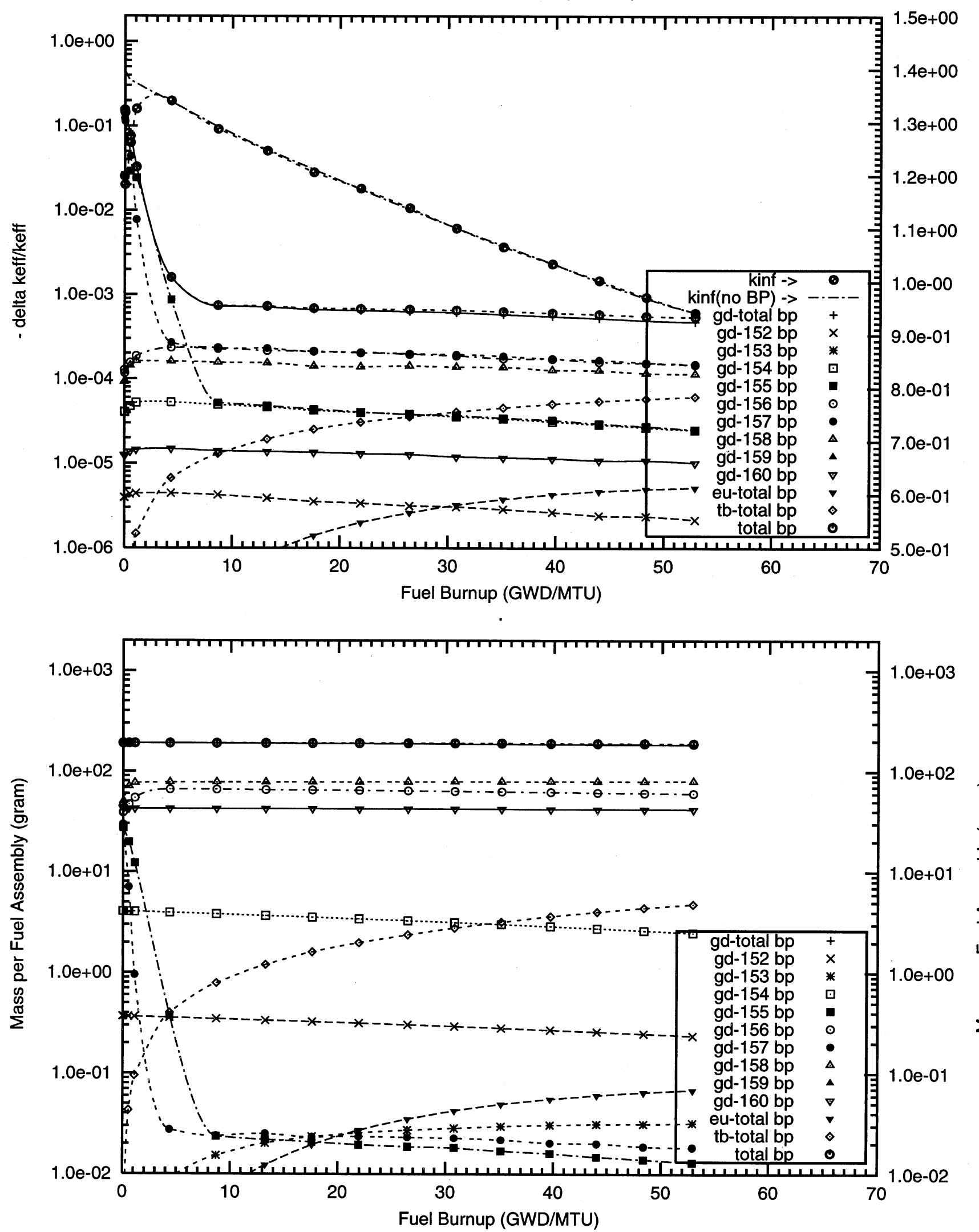
Figure A3.7. Negative Reactivity and Masses BP for Gd-nat and transmutation daughters as a function of fuel life for $17 \times 17$ fuel assemblies with 64 poison rods,

0.5 mill thick Gd2O3 poison coating on the outer radial surface of the UO2 pellets

Reactor power $3400 \mathrm{MWth}, 193$ fuel assemblies, initial enrichment $4.5 \mathrm{w} \% \mathrm{U}-235$

(Case : gd000_0f_64_bp_fuel o)
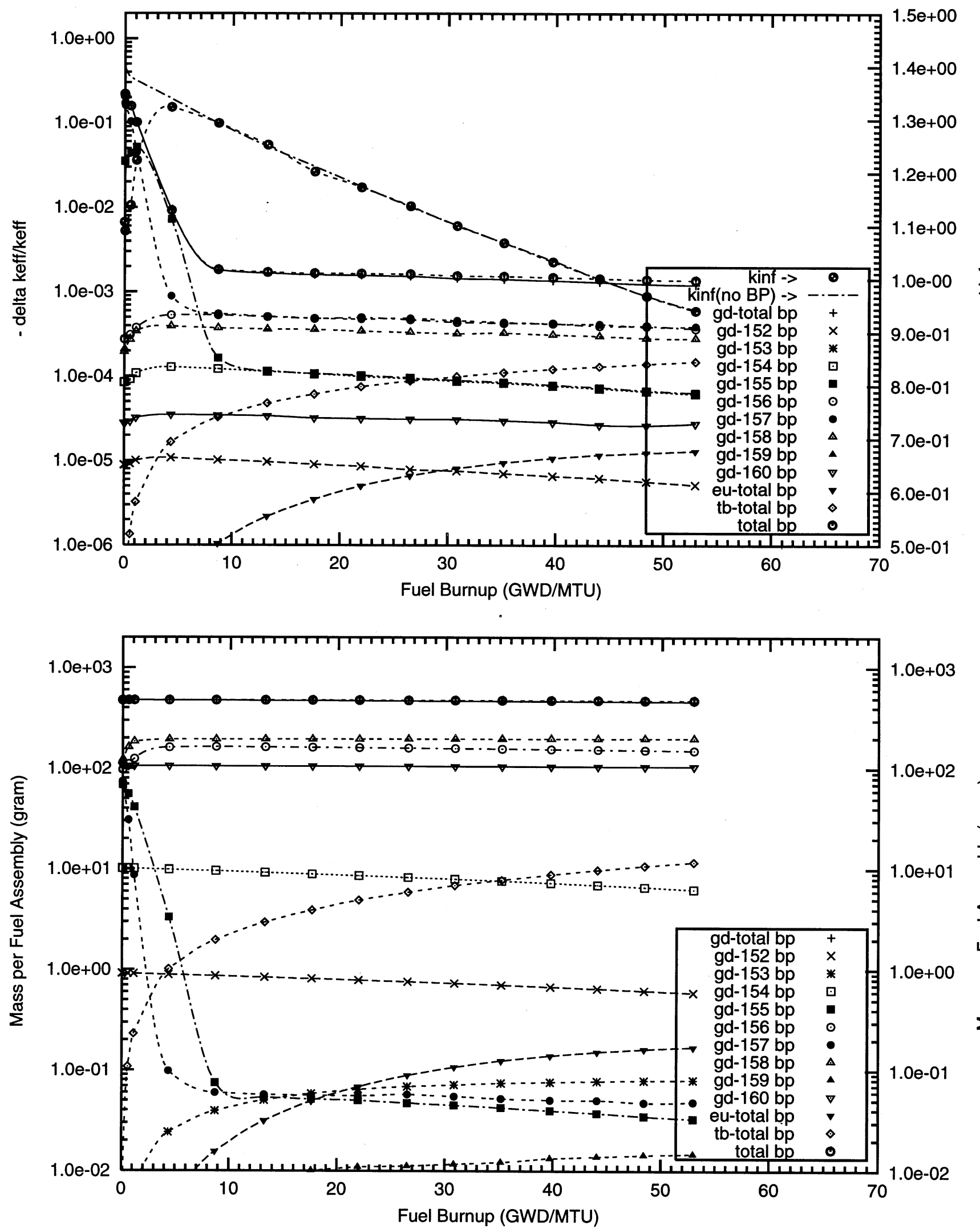
Figure A3.8. Negative Reactivity and Masses BP for Gd-nat and transmutation daughters as a function of fuel life for $17 \times 17$ fuel assemblies with 64 poison rods,

1.0 mill thick Gd2O3 poison coating on the outer radial surface of the UO2 pellets

Reactor power 3400 MWth, 193 fuel assemblies, initial enrichment 4.5w\%U-235

(Case : gd000_1f_64_bp_fuel o)
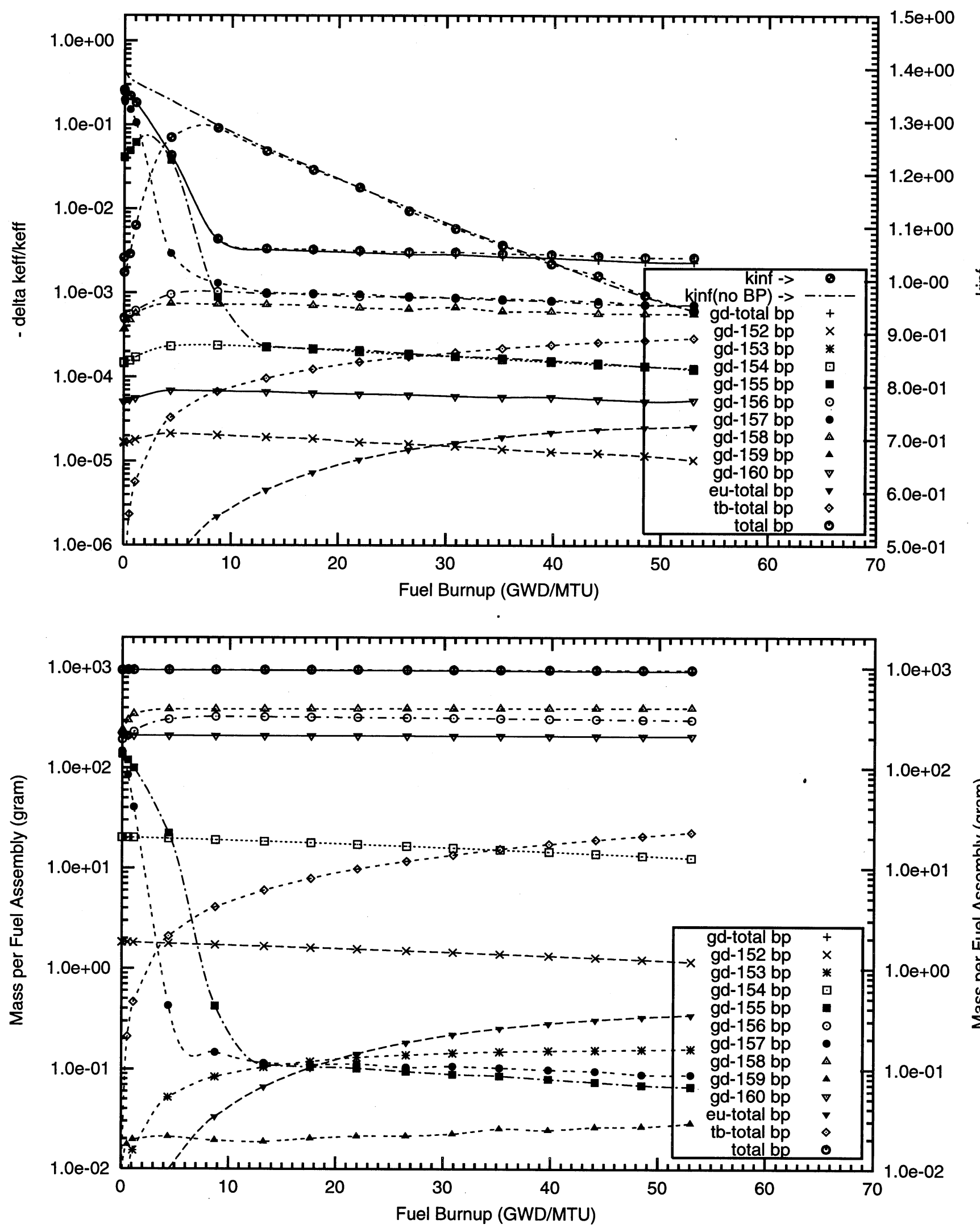
Figure A3.9. Negative Reactivity and Masses BP for Gd-nat and transmutation daughters as a function of fuel life for $17 \times 17$ fuel assemblies with 104 poison rods,

0.5 mill thick (0.10TD) Gd2O3 poison coating on the outer radial surface of the UO2 pellets

Reactor power $3400 \mathrm{MWth}, 193$ fuel assemblies, initial enrichment $4.5 \mathrm{w} \% \mathrm{U}-235$

(Case : gd000_12f_104_bp_fuel o)
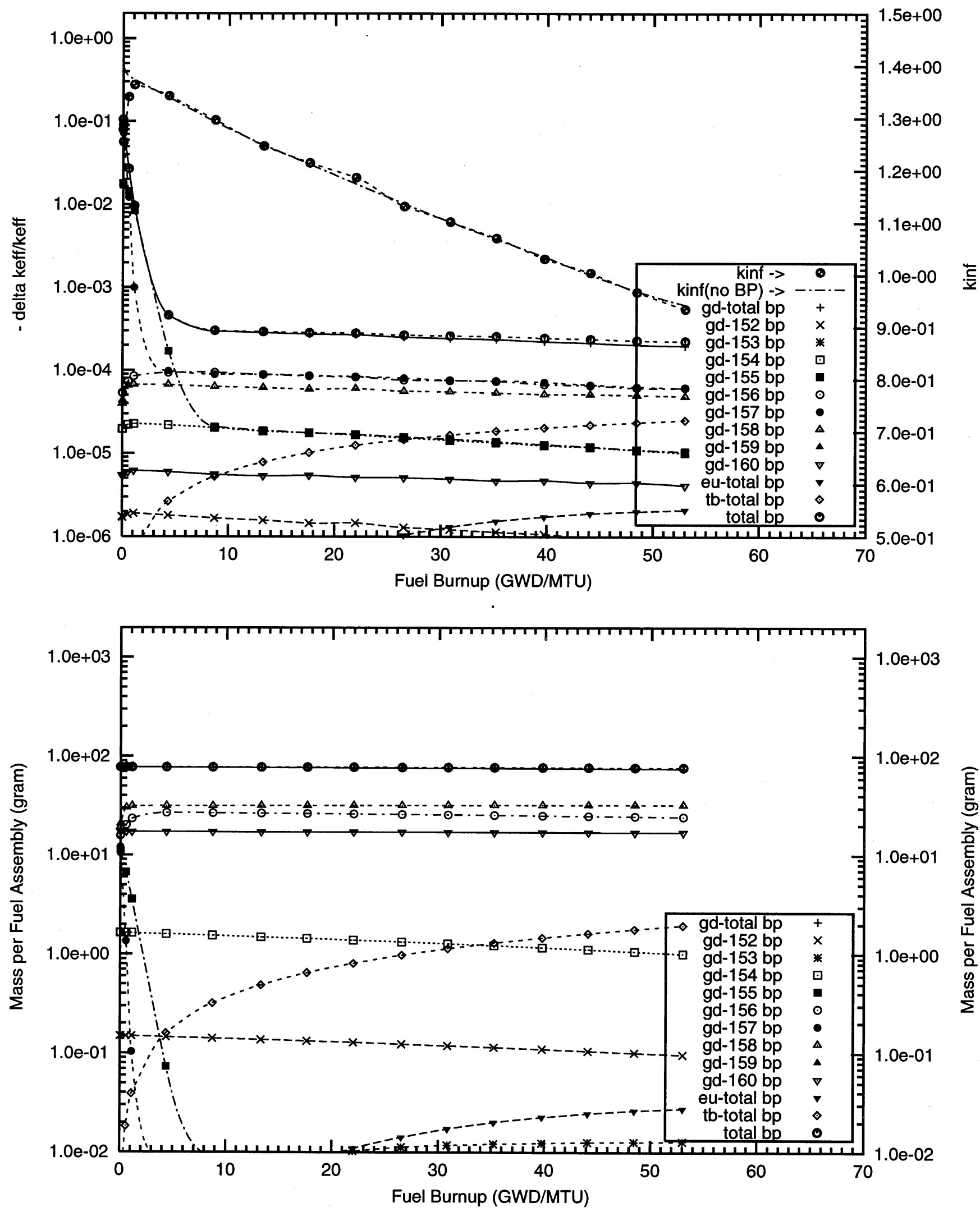
A3 - Gadolinium Fully Enriched in ${ }^{155}$ Gd 

Figure A3.10. Negative Reactivity and Masses BP for Gd-155 and transmutation daughters as a function of fuel life for $17 \times 17$ fuel assemblies with 08 poison rods,

4.0 mill thick Gd2O3 poison coating on the outer radial surface of the UO2 pellets

Reactor power $3400 \mathrm{MWth}, 193$ fuel assemblies, initial enrichment 4.5w\%U-235

(Case : gd155_3f_08_bp_fuel o)

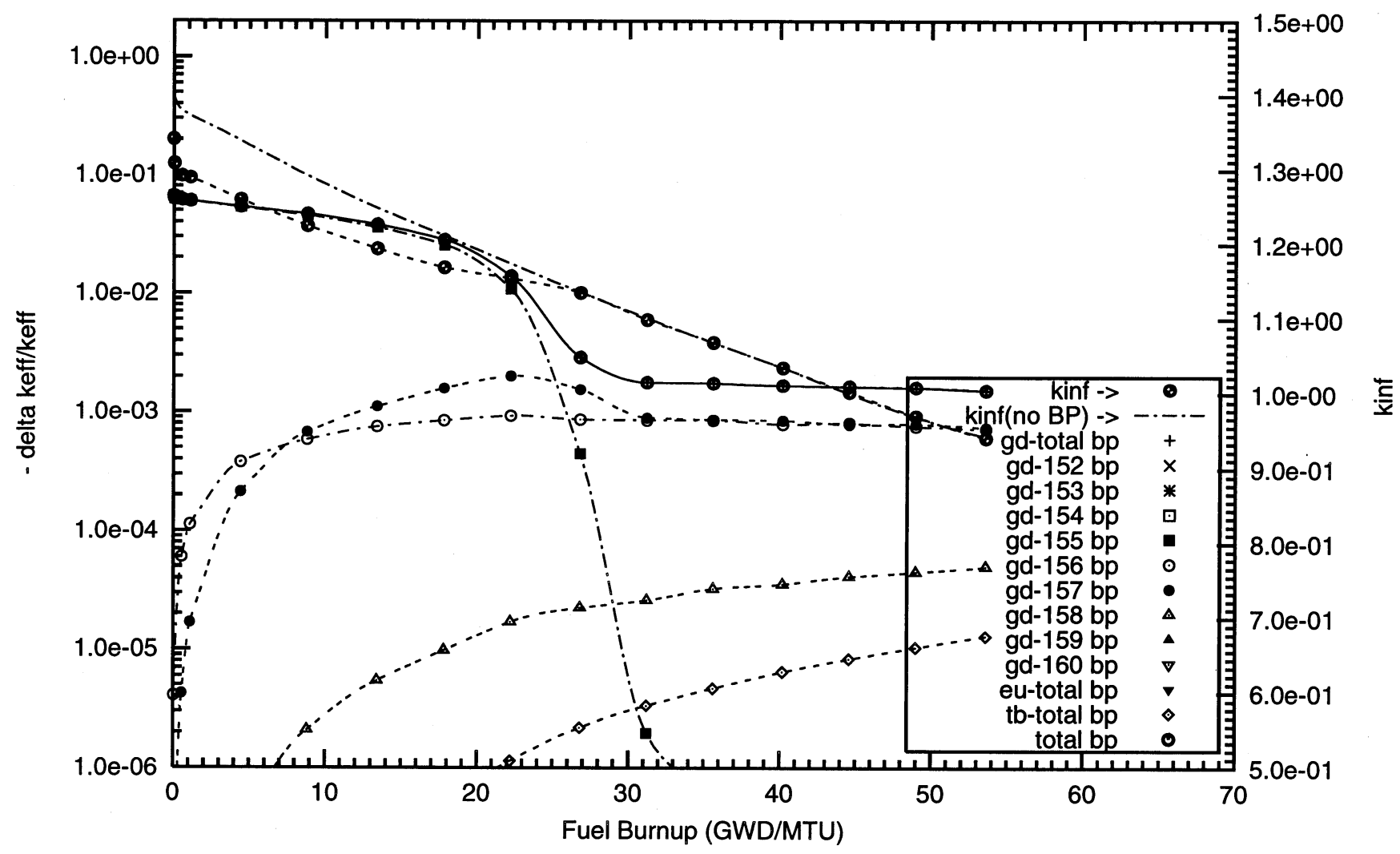


Figure A3.11. Negative Reactivity and Masses BP for Gd-155 and transmutation daughters as a function of fuel life for $17 \times 17$ fuel assemblies with 16 poison rods,

$1.0 \mathrm{w} \% \mathrm{Gd} 2 \mathrm{O} 3$ poison homogeneously mixed in the outer one-third volume of the UO2 pellets

Reactor power $3400 \mathrm{MWth}, 193$ fuel assemblies, initial enrichment $4.5 \mathrm{~W} \% \mathrm{U}-235$

(Case : gd155_1f_100p_h20_16_bp_fuel_2_o)

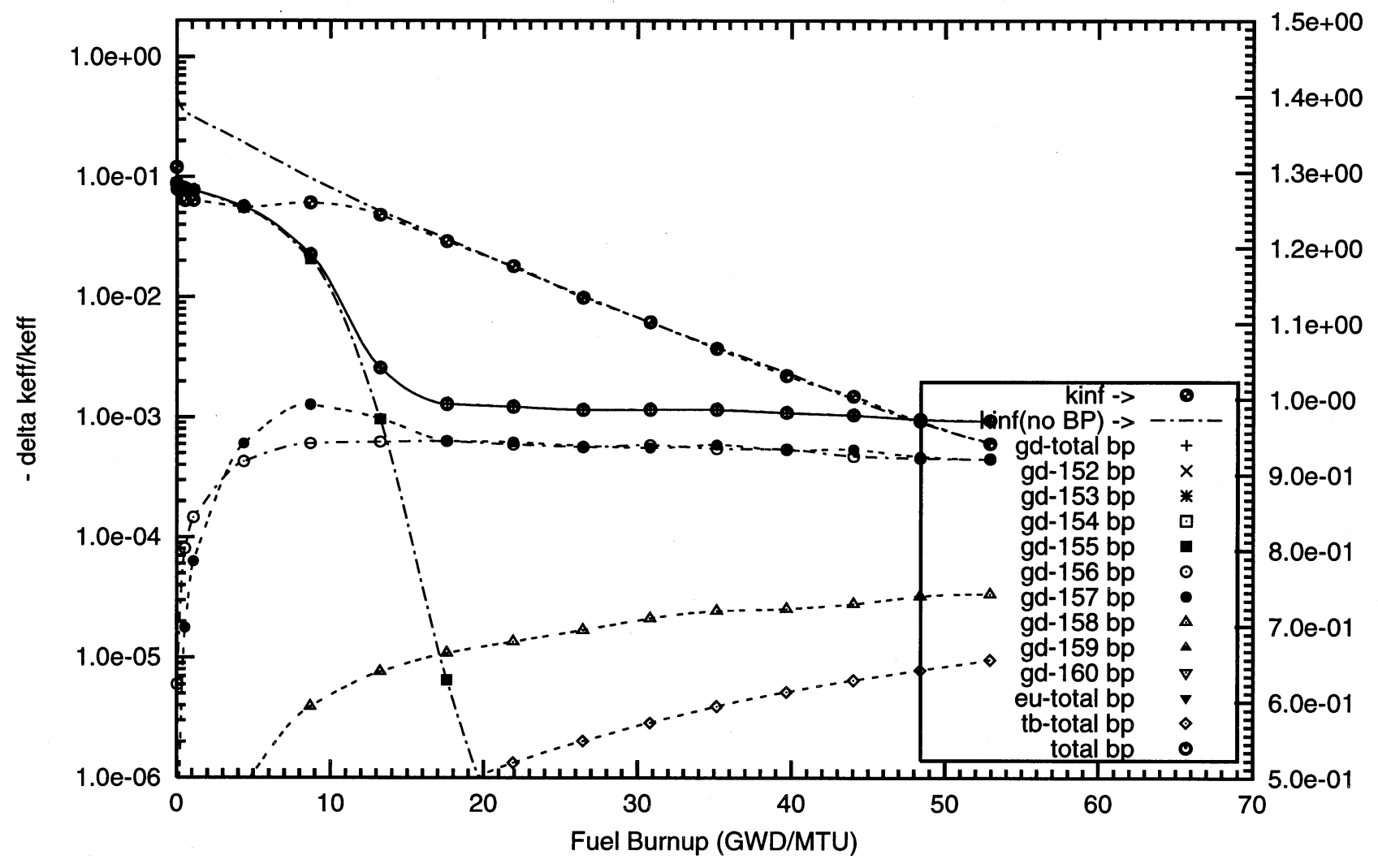

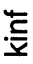

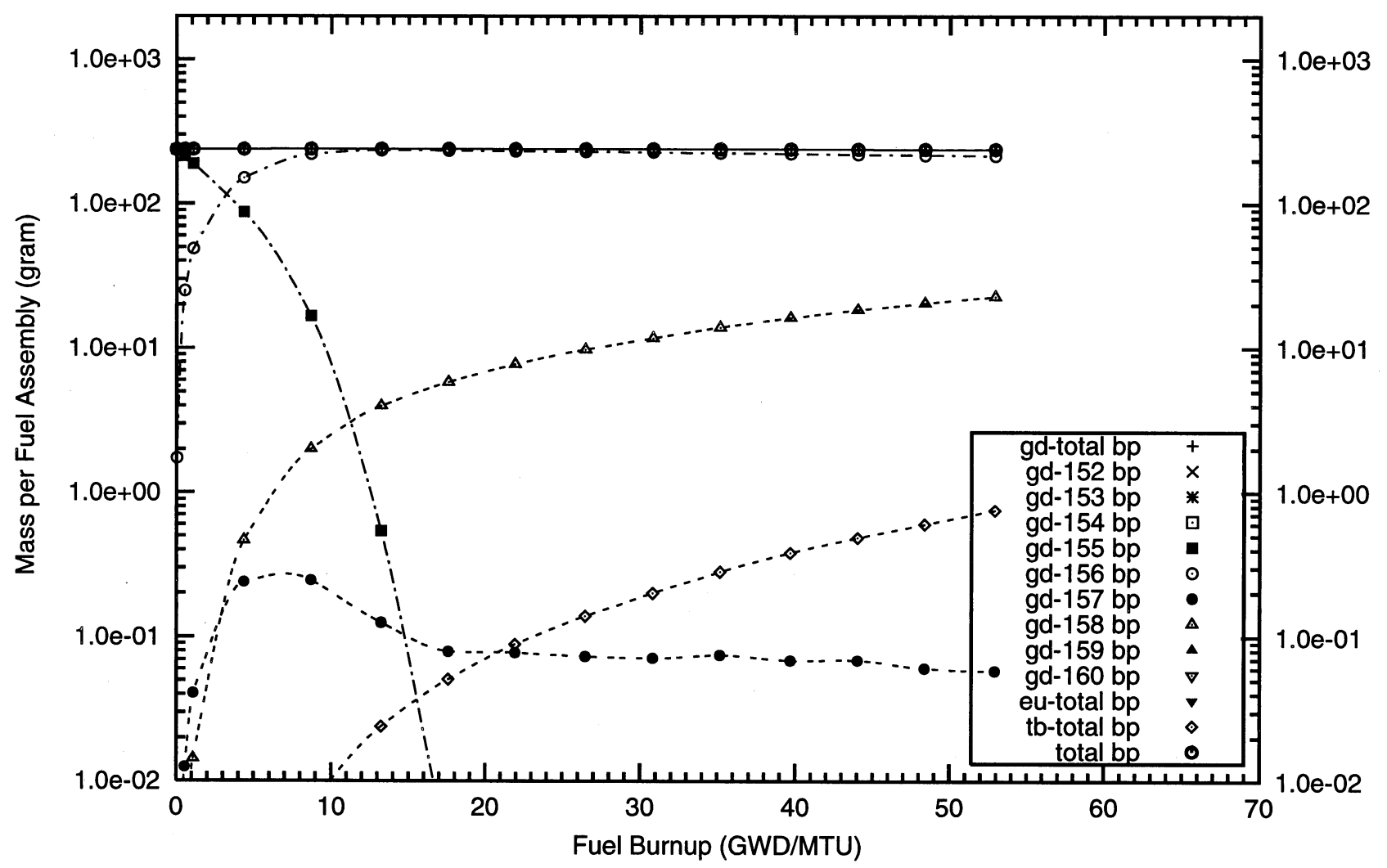


Figure A3.12. Negative Reactivity and Masses BP for Gd-155 and transmutation daughters as a function of fuel life for $17 \times 17$ fuel assemblies with 16 poison rods,

$2.0 \mathrm{w} \% \mathrm{Gd} 2 \mathrm{O} 3$ poison homogeneously mixed in the outer one-third volume of the $\mathrm{UO} 2$ pellets

Reactor power $3400 \mathrm{MW}$ th, 193 fuel assemblies, initial enrichment $4.5 \mathrm{w} \% \mathrm{U}-235$

(Case : gd155_2f_100p_h2o_16_bp_fuel_2_o)
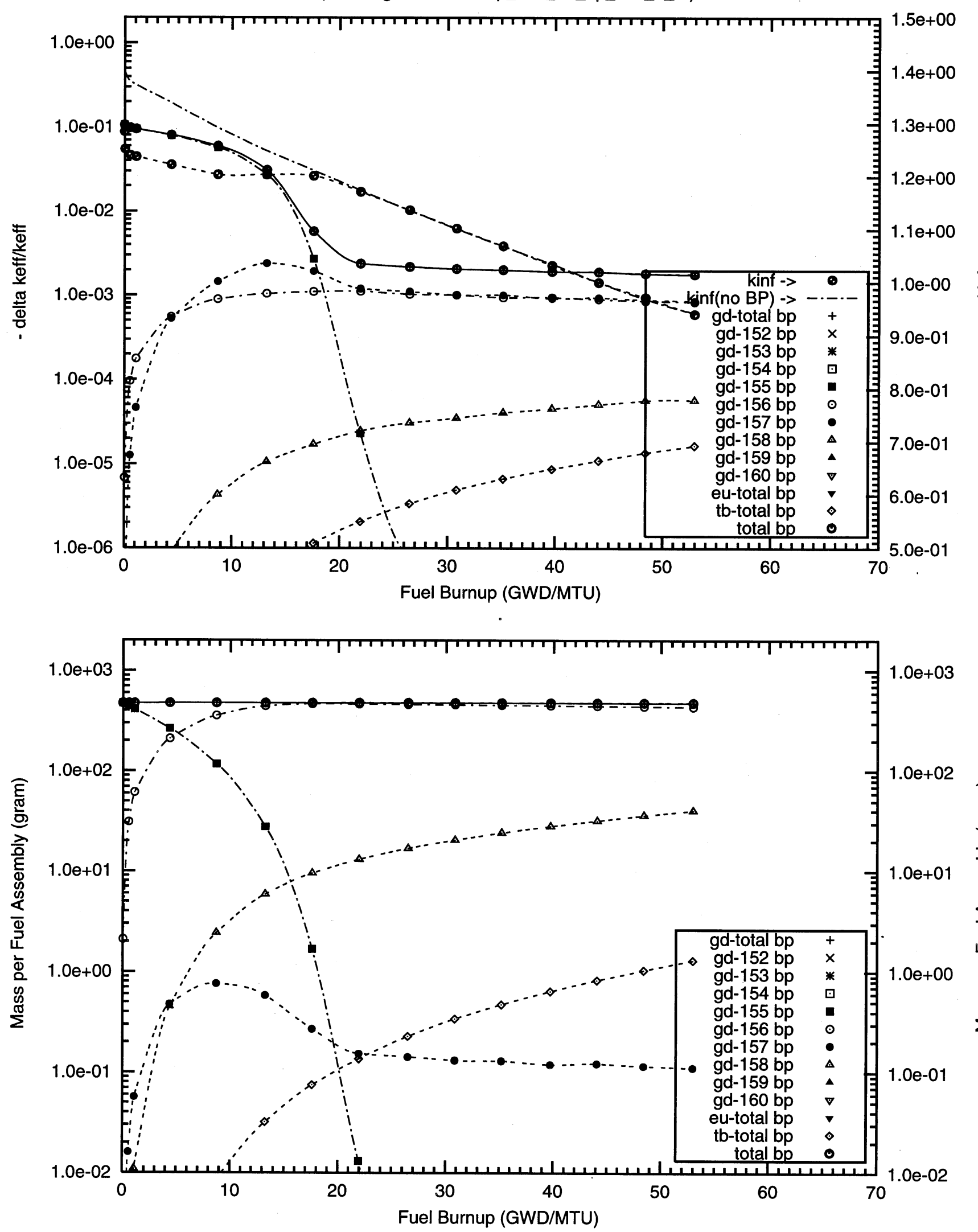
Figure A3.13. Negative Reactivity and Masses BP for Gd-155 and transmutation daughters as a function of fuel life for $17 \times 17$ fuel assemblies with 16 poison rods,

$4.0 \mathrm{w} \% \mathrm{Gd} 2 \mathrm{O} 3$ poison homogeneously mixed in the outer one-third volume of the UO2 pellets

Reactor power $3400 \mathrm{MWth}, 193$ fuel assemblies, initial enrichment 4.5w\%U-235

(Case : gd155_3f_100p_h2o_16_bp_fuel_2_o)
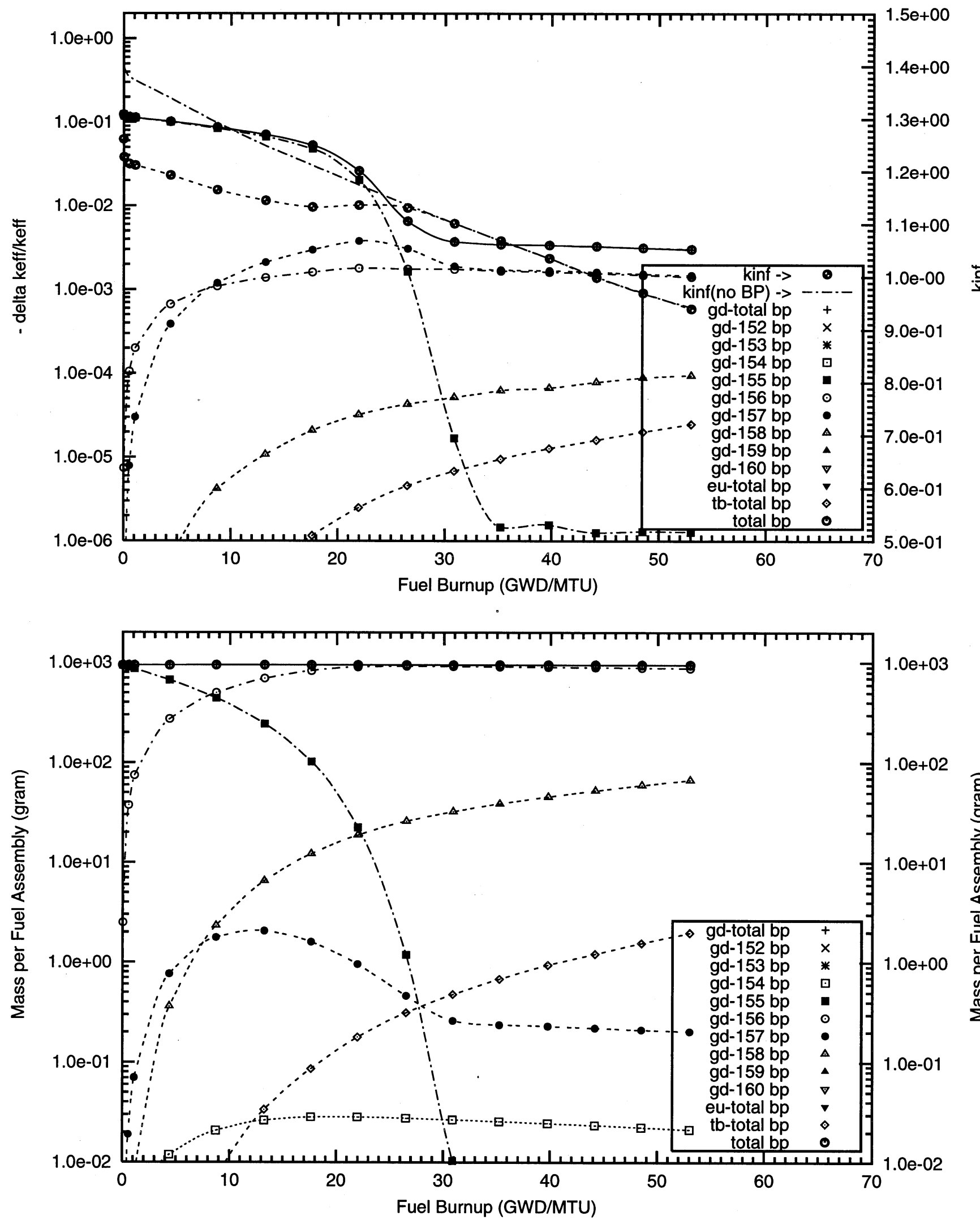
Figure A3.14. Negative Reactivity and Masses BP for Gd-155 and transmutation daughters as a function of fuel life for $17 \times 17$ fuel assemblies with 18 poison rods,

$2.0 \mathrm{w} \% \mathrm{Gd} 2 \mathrm{O} 3$ poison homogeneously mixed in the outer one-third volume of the $\mathrm{UO} 2$ pellets

Reactor power $3400 \mathrm{MWth}, 193$ fuel assemblies, initial enrichment 4.5W\%U-235

(Case : gd155_2f_16_bp_fuel (old) o)
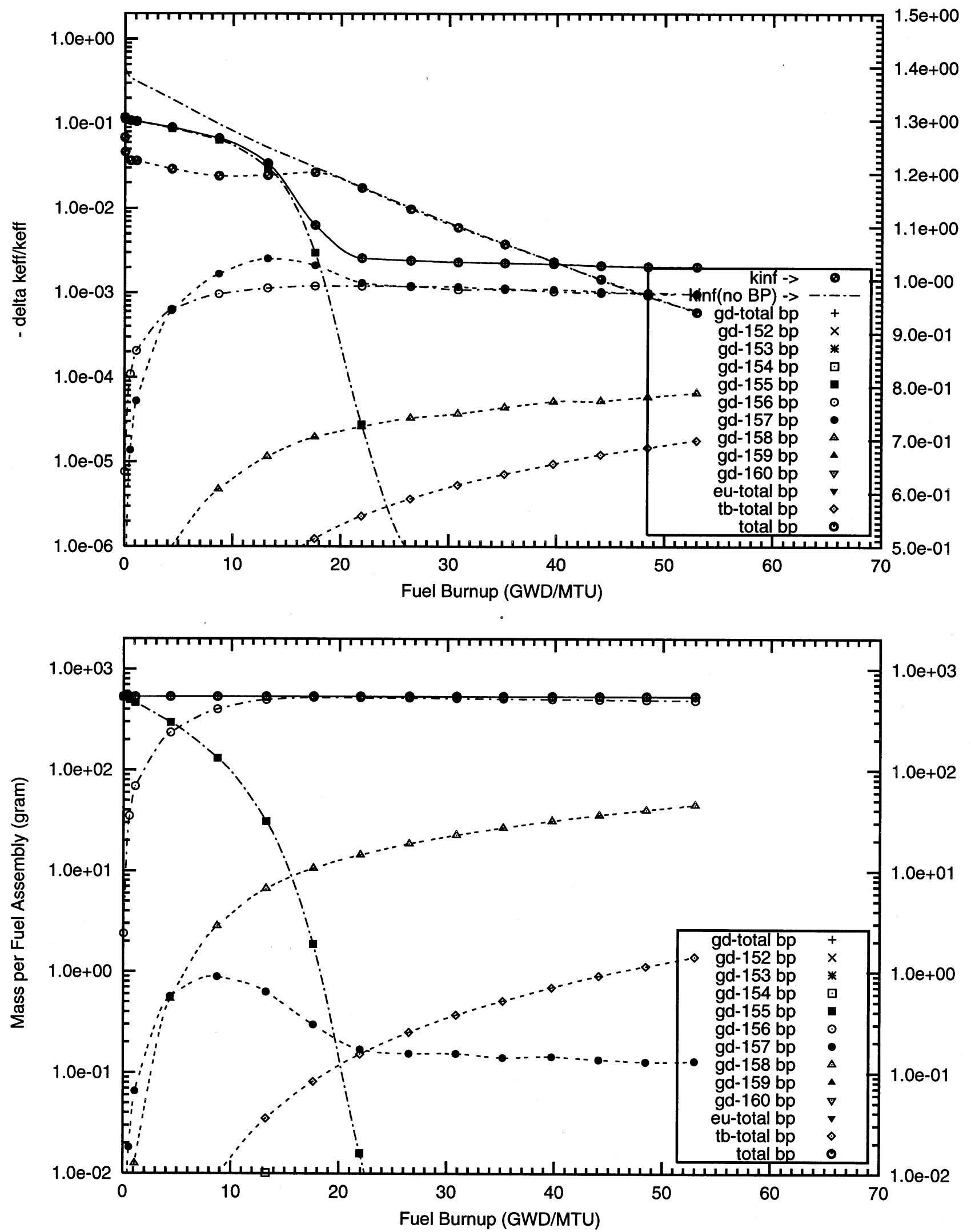
Figure A3.15. Negative Reactivity and Masses BP for Gd-155 and transmutation daughters as a function of fuel life for $17 \times 17$ fuel assemblies with 18 poison rods,

4.0 mill thick Gd2O3 poison coating on the outer radial surface of the UO2 pellets

Reactor power $3400 \mathrm{MWth}, 193$ fuel assemblies, initial enrichment 4.5w\%U-235

(Case : gd155_3f_16_bp_fuel (old) o)

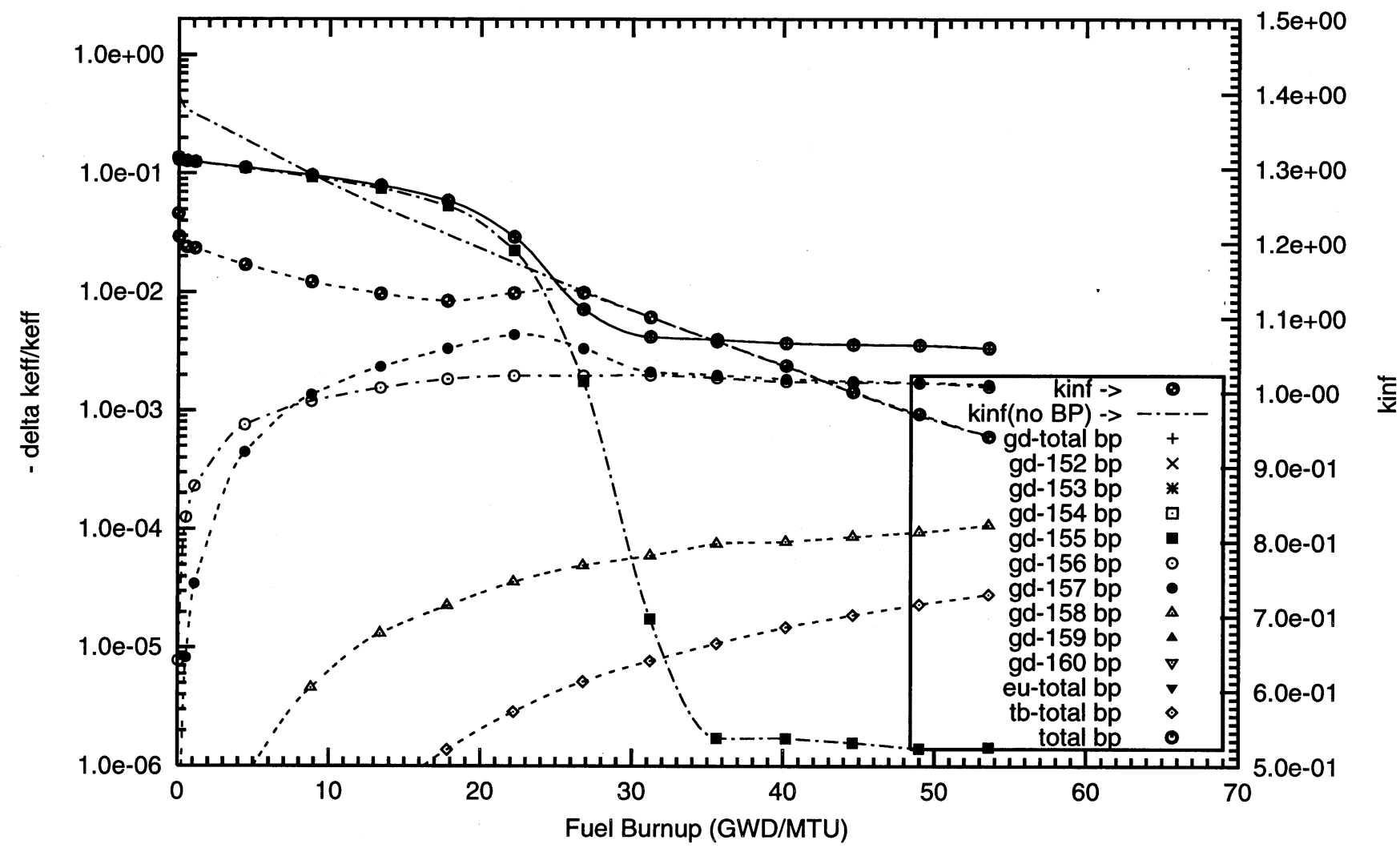


Figure A3.16. Negative Reactivity and Masses BP for Gd-155 and transmutation daughters as a function of fuel life for $17 \times 17$ fuel assemblies with 64 poison rods, 0.2 mill thick Gd2O3 poison coating on the outer radial surface of the $\mathrm{UO} 2$ pellets Reactor power 3400 MWth, 193 fuel assemblies, initial enrichment 4.5w\%U-235 (Case : gd155_8f_64_bp_fuel o)
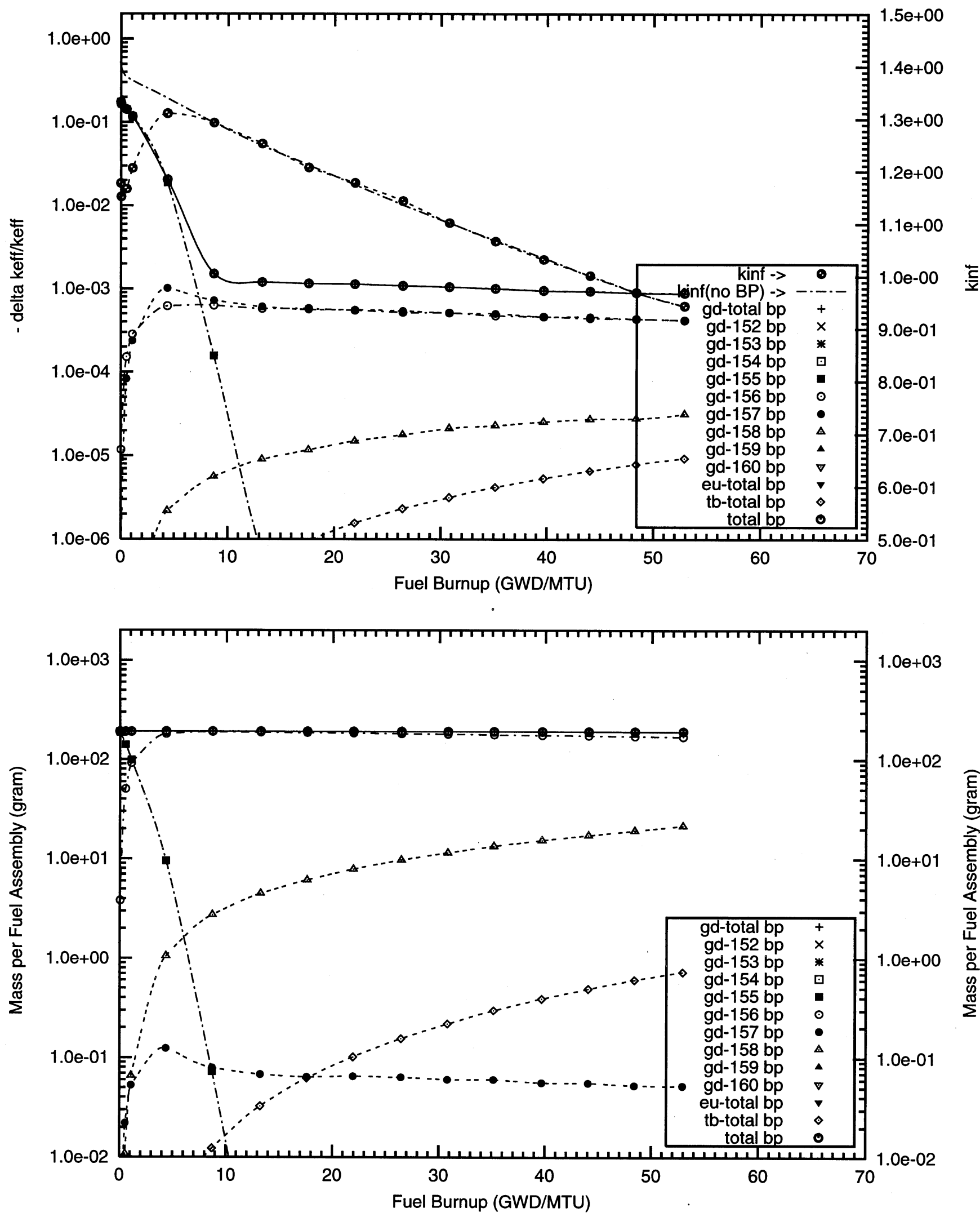
Figure A3.17. Negative Reactivity and Masses BP for Gd-15 and transmutation,daughters as a function of fuel life for $17 \times 17$ fuel assemblies with 104 poison rods,

0.5 mill thick (0.10TD) Gd2O3 poison coating on the outer radial surface of the UO2 pellets

Reactor power $3400 \mathrm{MWth}, 193$ fuel assemblies, initial enrichment 4.5W\%U-235

(Case : gd155_12f_104_bp_fuel o)
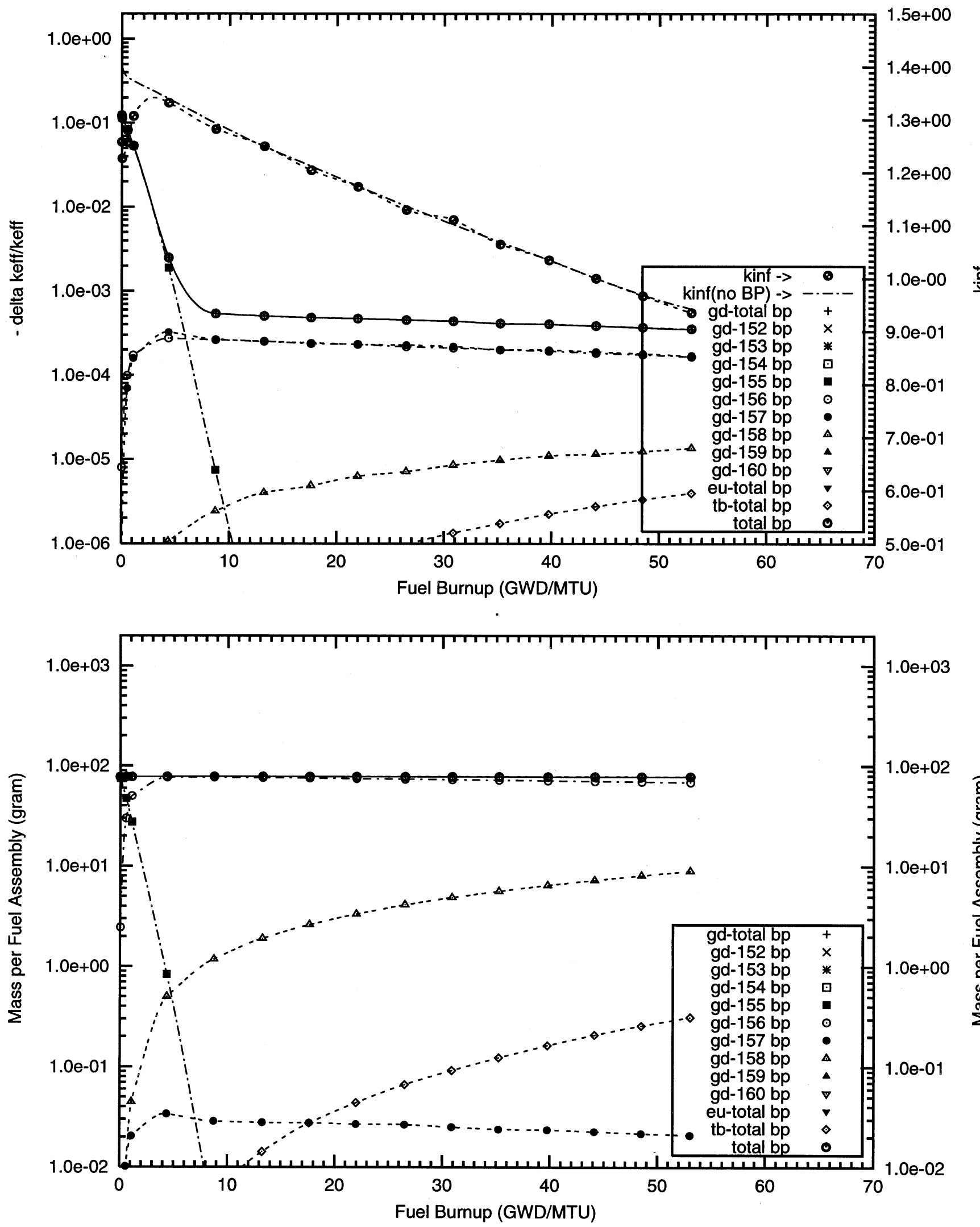
Figure A3.18. Negative Reactivity and Masses BP for Gd-155 and transmutation daughters as a function of fuel life for $17 \times 17$ fuel assemblies with 104 poison rods,

1.0 mill thick (0.10TD) Gd2O3 poison coating on the outer radial surface of the UO2 pellets

Reactor power $3400 \mathrm{MWth}, 193$ fuel assemblies, initial enrichment 4.5W\%U-235

(Case : gd155_11f_104_bp_fuel o)
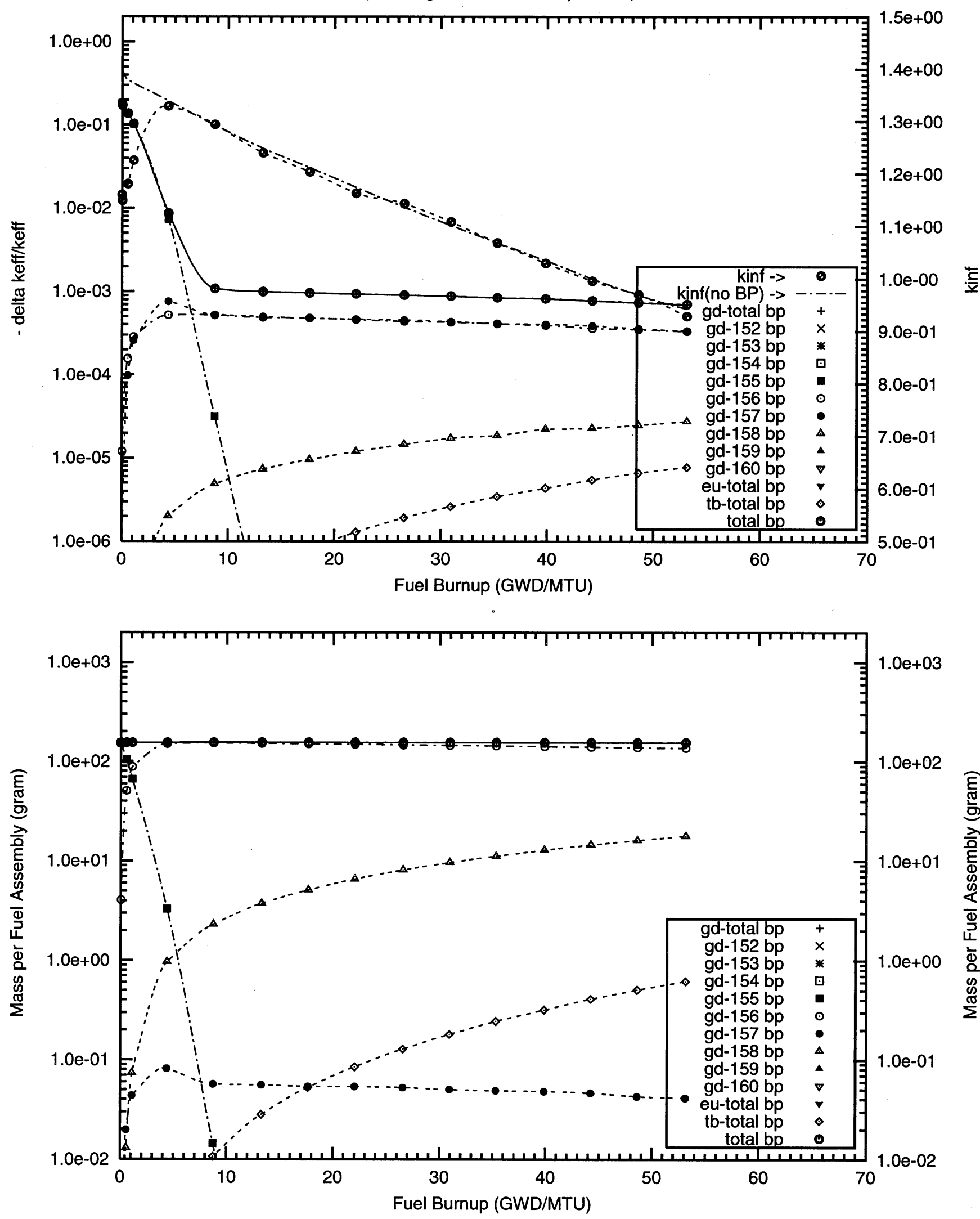
A3 - Gadolinium Fully Enriched in ${ }^{157}$ Gd 
Figure A3.19. Negative Reactivity and Masses BP for Gd-157 and transmutation daughters as a function of fuel life for $17 \times 17$ fuel assemblies with 08 poison rods, 4.0 mill thick $\mathrm{Gd} 2 \mathrm{O} 3$ poison coating on the outer radial surface of the $\mathrm{UO} 2$ pellets Reactor power $3400 \mathrm{MWth}, 193$ fuel assemblies, initial enrichment 4.5w\%U-235 (Case : gd157_3f_08_bp_fuel o)

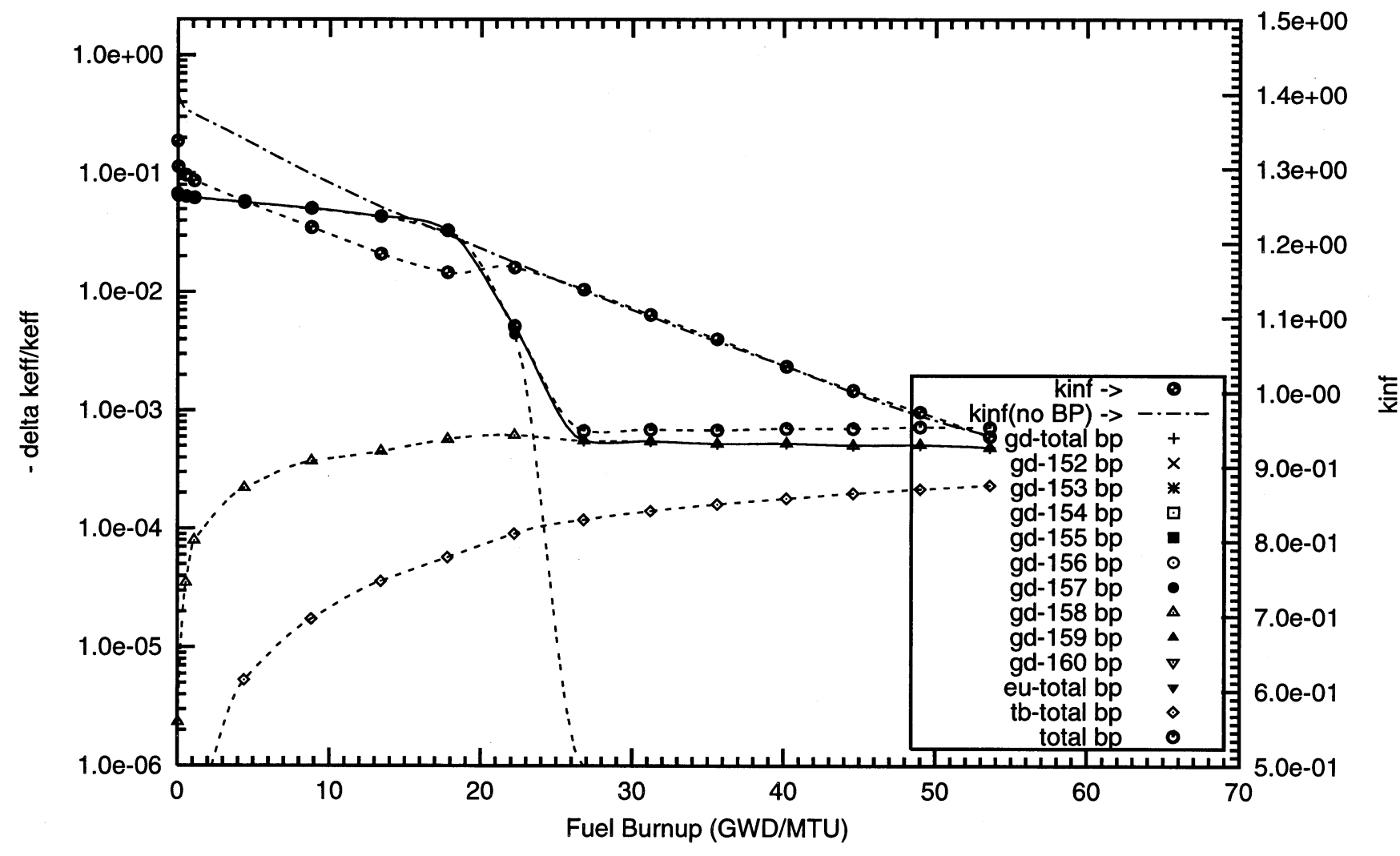


Figure A3.20. Negative Reactivity and Masses BP for Gd-157 and transmutation daughters as a function of fuel life for $17 \times 17$ fuel assemblies with 16 poison rods,

0.5 mill thick $\mathrm{Gd} 2 \mathrm{O} 3$ poison coating on the outer radial surface of the UO2 pellets

Reactor power $3400 \mathrm{MWth}, 193$ fuel assemblies, initial enrichment 4.5w\%U-235

(Case : gd157_of_100p_h20_16_bp_fuel_2_o)
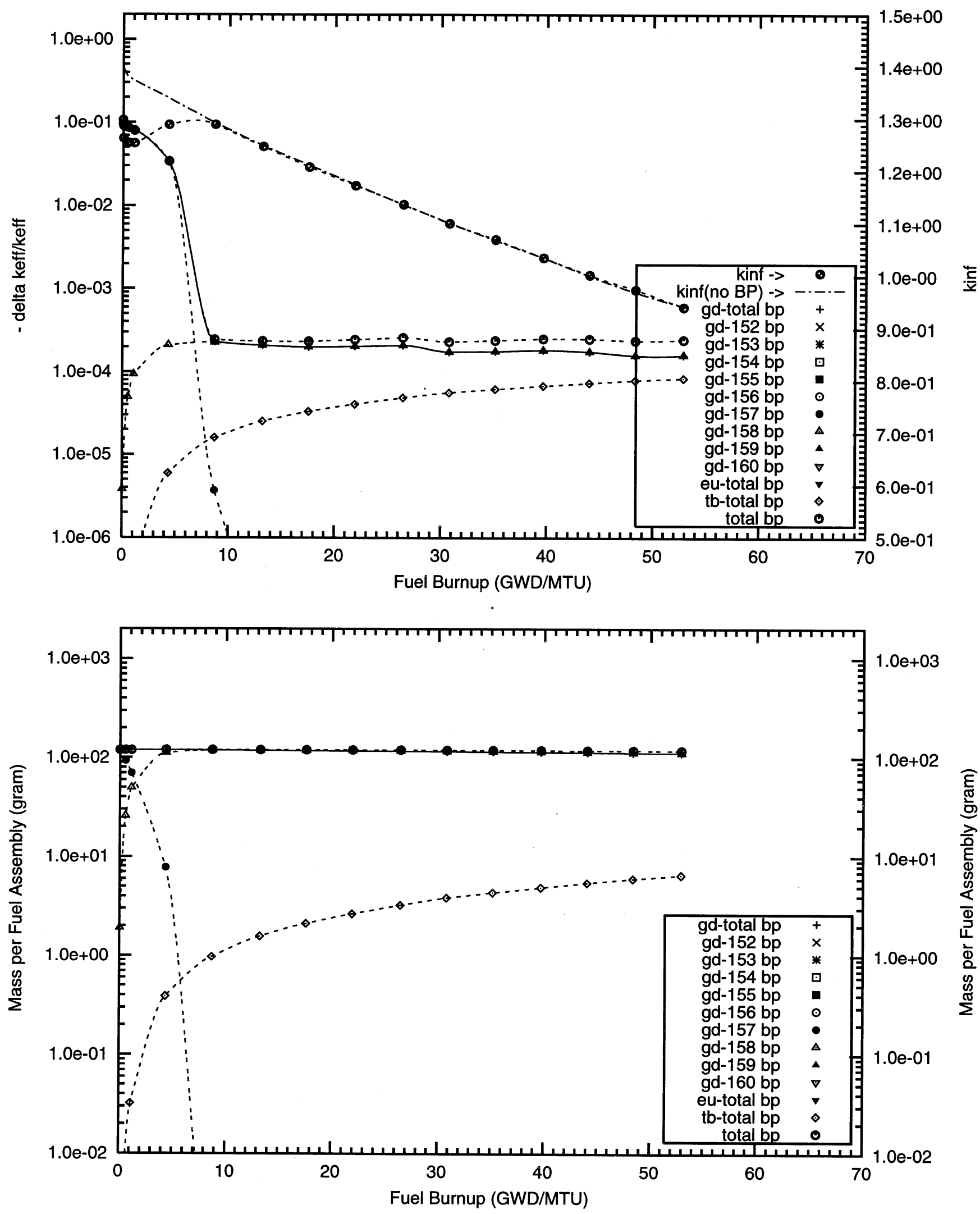
Figure A3.21. Negative Reactivity and Masses BP for Gd-157 and transmutation daughters as a function of fuel life for $17 \times 17$ fuel assemblies with 16 poison rods, 1.0 mill thick $\mathrm{Gd} 2 \mathrm{O} 3$ poison coating on the outer radial surface of the UO2 pellets Reactor power $3400 \mathrm{MWth}, 193$ fuel assemblies, initial enrichment $4.5 \mathrm{w} \% \mathrm{U}-235$

(Case : gd157_1f_100p_h20_16_bp_fuel_2_o)
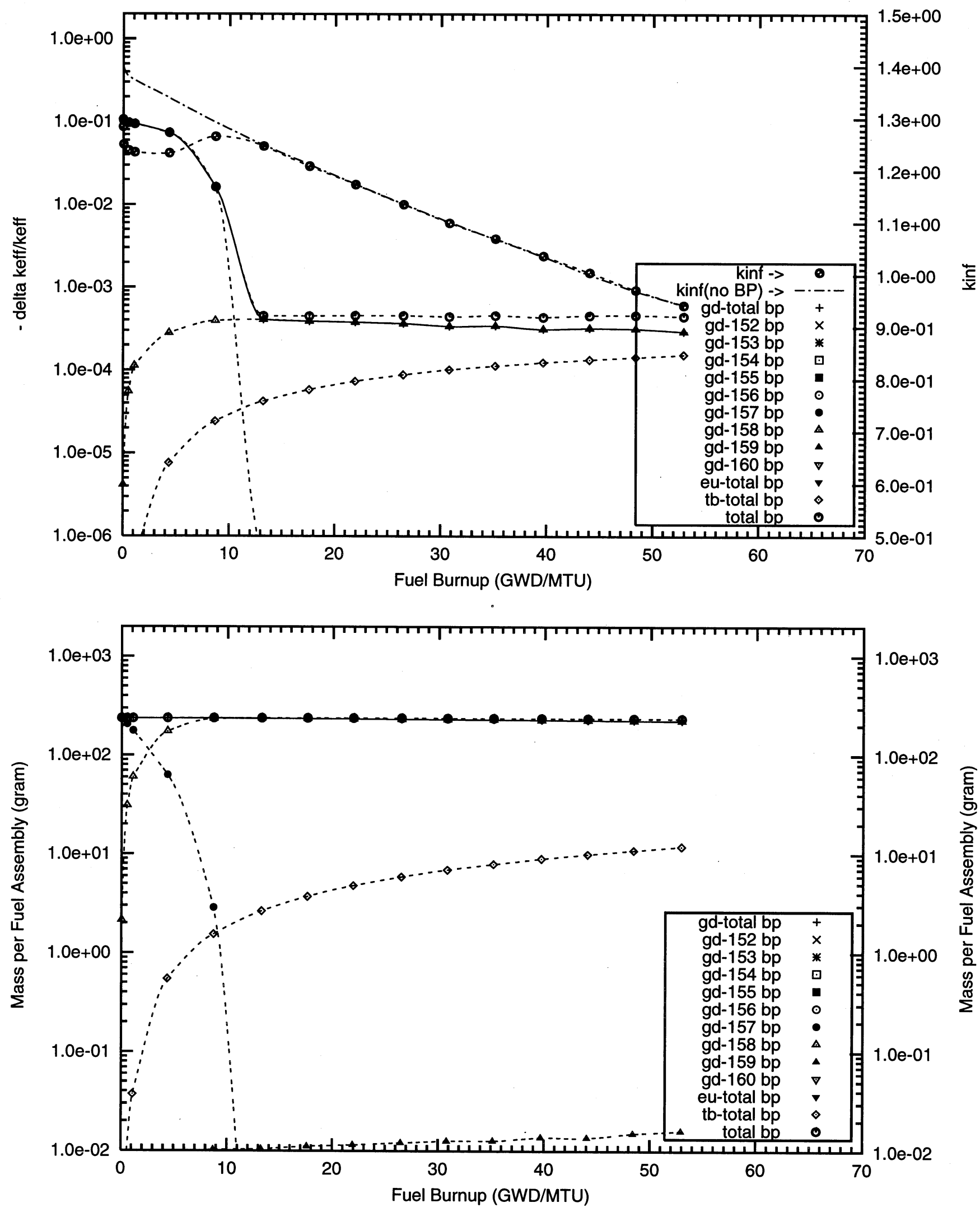
Figure A3.22. Negative Reactivity and Masses BP for Gd-157 and transmutation daughters as a function of fuel life for $17 \times 17$ fuel assemblies with 16 poison rods,

2.0 mill thick Gd2O3 poison coating on the outer radial surface of the UO2 pellets

Reactor power 3400 MWth, 193 fuel assemblies, initial enrichment 4.5w\%U-235

(Case : gd157_2f_100p_h2o_16_bp_fuel_2_o)
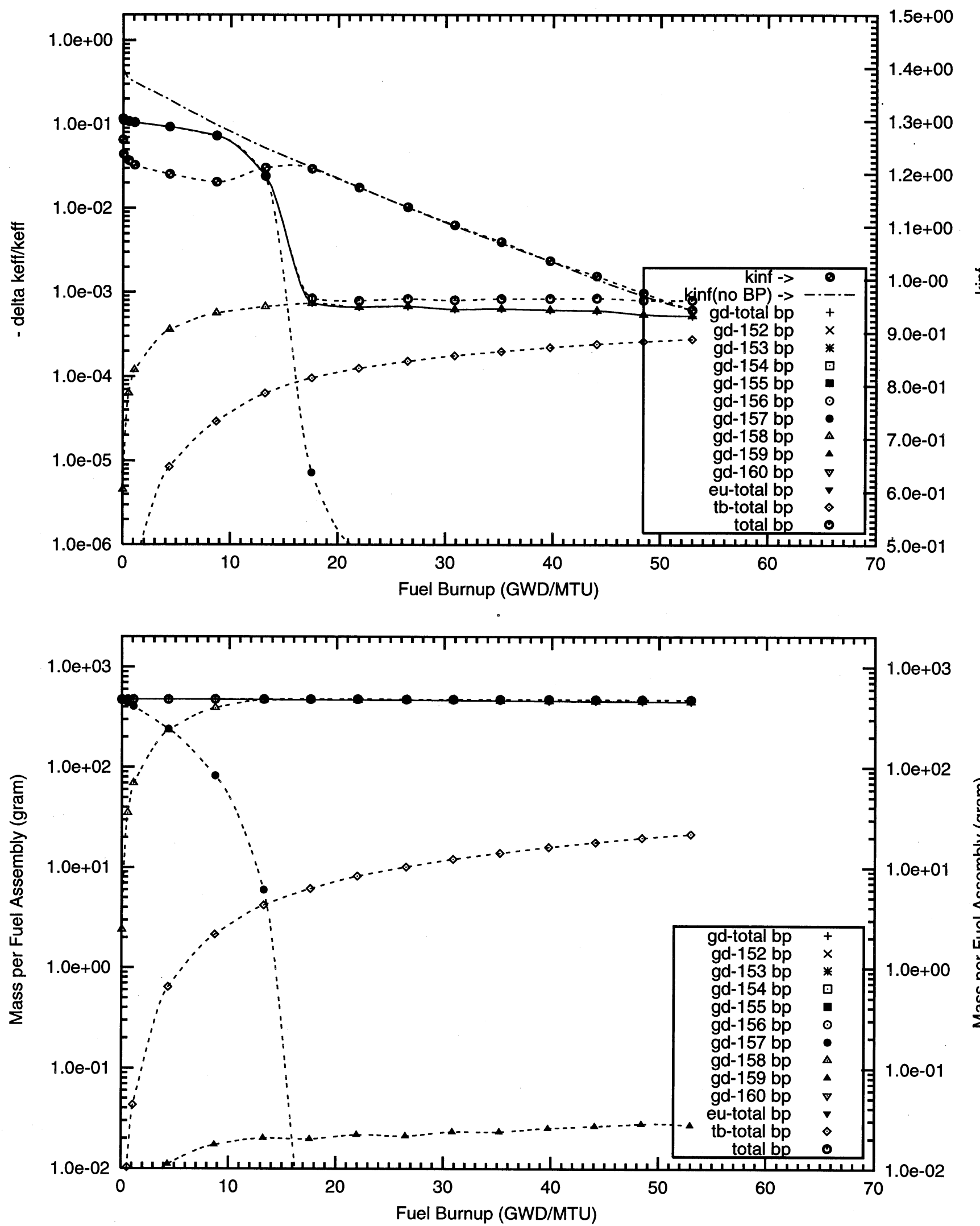
Figure A3.23. Negative Reactivity and Masses BP for Gd-157 and transmutation daughters as a function of fuel life for $17 \times 17$ fuel assemblies with 18 poison rods, 1.0 mill thick Gd2O3 poison coating on the outer radial surface of the UO2 pellets Reactor power $3400 \mathrm{MWth}, 193$ fuel assemblies, initial enrichment $4.5 \mathrm{w} \% \mathrm{U}-235$

(Case : gd157_1f_16_bp_fuel (old) o)
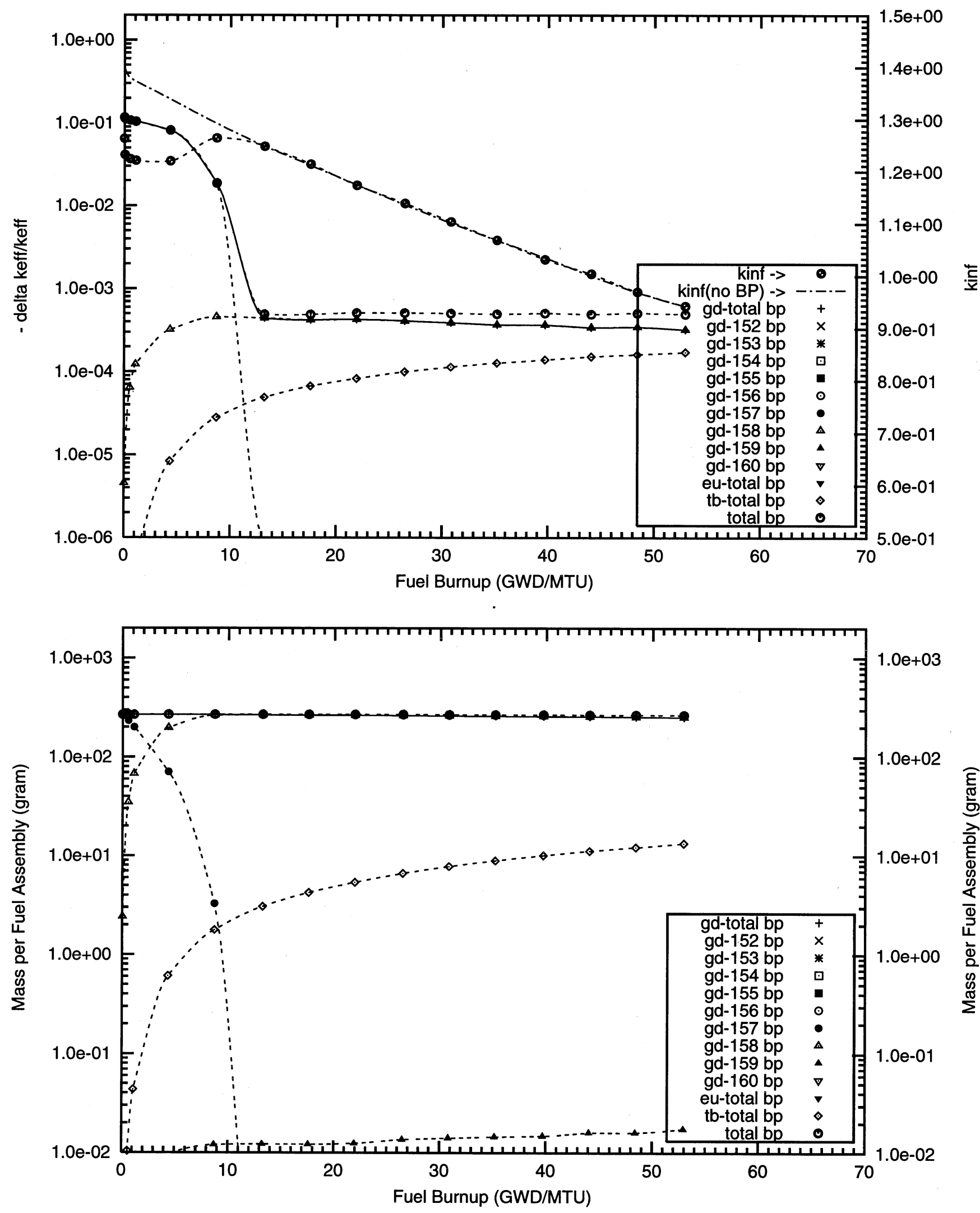
Figure A3.24. Negative Reactivity and Masses BP for Gd-157 and transmutation daughters as a function of fuel life for $17 \times 17$ fuel assemblies with 18 poison rods,

2.0 mill thick $\mathrm{Gd} 2 \mathrm{O} 3$ poison coating on the outer radial surface of the $\mathrm{UO} 2$ pellets

Reactor power $3400 \mathrm{MWth}, 193$ fuel assemblies, initial enrichment $4.5 \mathrm{w} \% \mathrm{U}-235$

(Case : gd157_2f_16_bp_fuel (old) o)
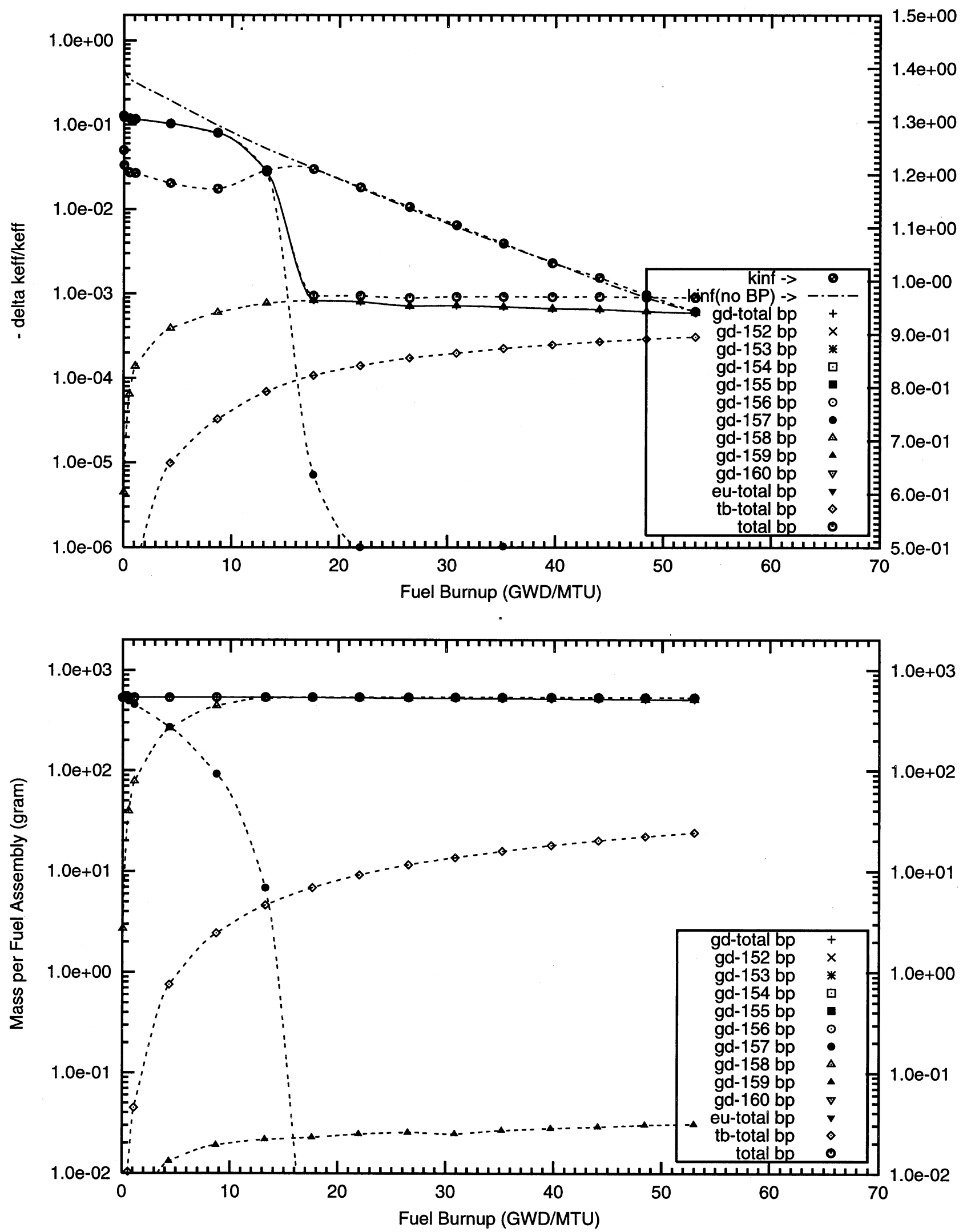
Figure A3.25. Negative Reactivity and Masses BP for Gd-157 and transmutation daughters as a function of fuel life for $17 \times 17$ fuel assemblies with 64 poison rods,

0.2 mill thick (0.10TD) Gd2O3 poison coating on the outer radial surface of the UO2 pellets

Reactor power $3400 \mathrm{MWth}, 193$ fuel assemblies, initial enrichment 4.5w\%U-235

(Case : gd157_14f_64_bp_fuel o)
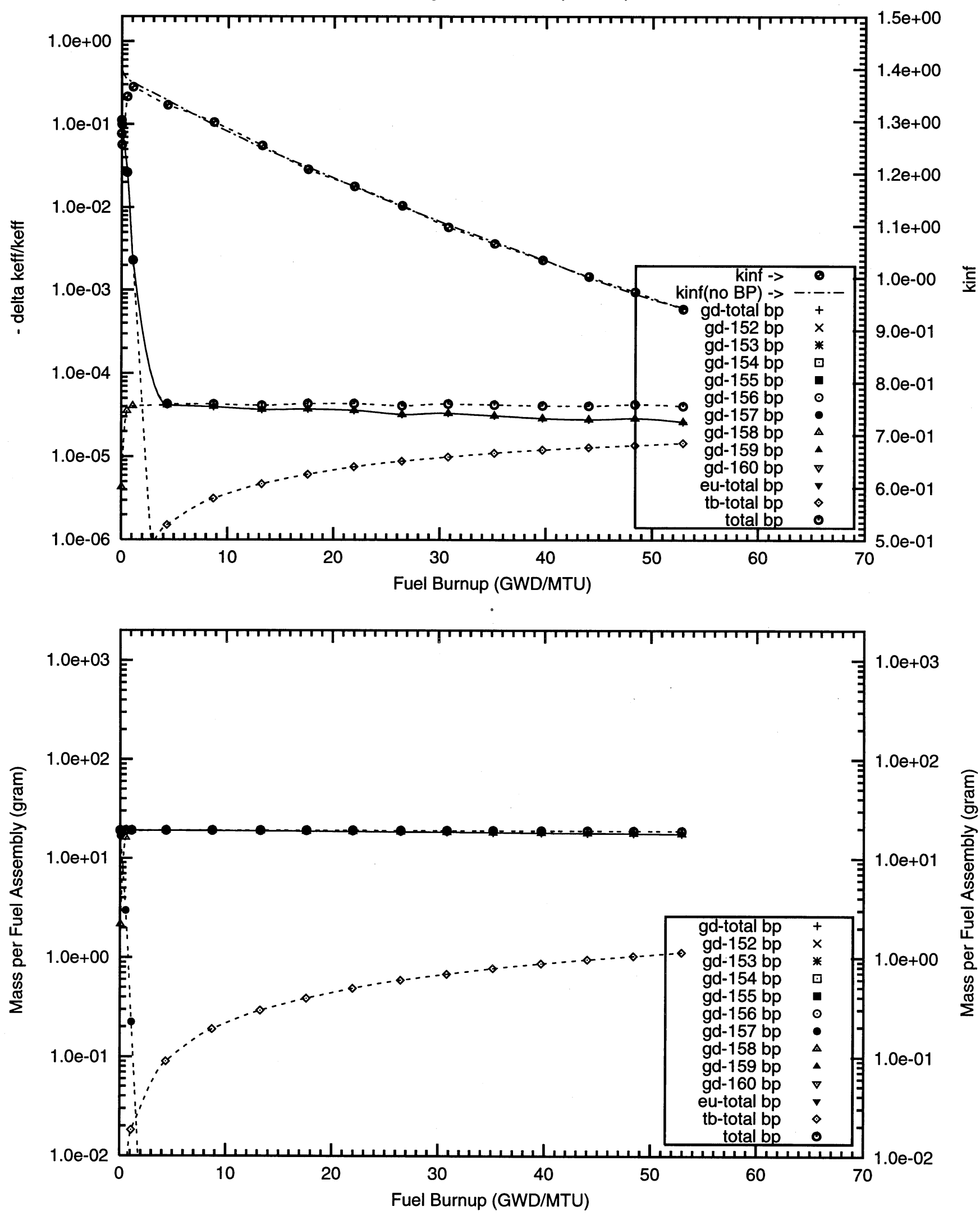
Figure A3.26. Negative Reactivity and Masses BP for Gd-157 and transmutation daughters as a tunction of tuel lite tor $17 \times 17$ tuel assemblies with 64 poison rods,

0.5 mill thick (0.10TD) Gd2O3 poison coating on the outer radial surface of the UO2 pellets

Reactor power $3400 \mathrm{MW}$ th, 193 fuel assemblies, initial enrichment $4.5 \mathrm{w} \% \mathrm{U}-235$

(Case : gd157_12f_64_bp_fuel o)
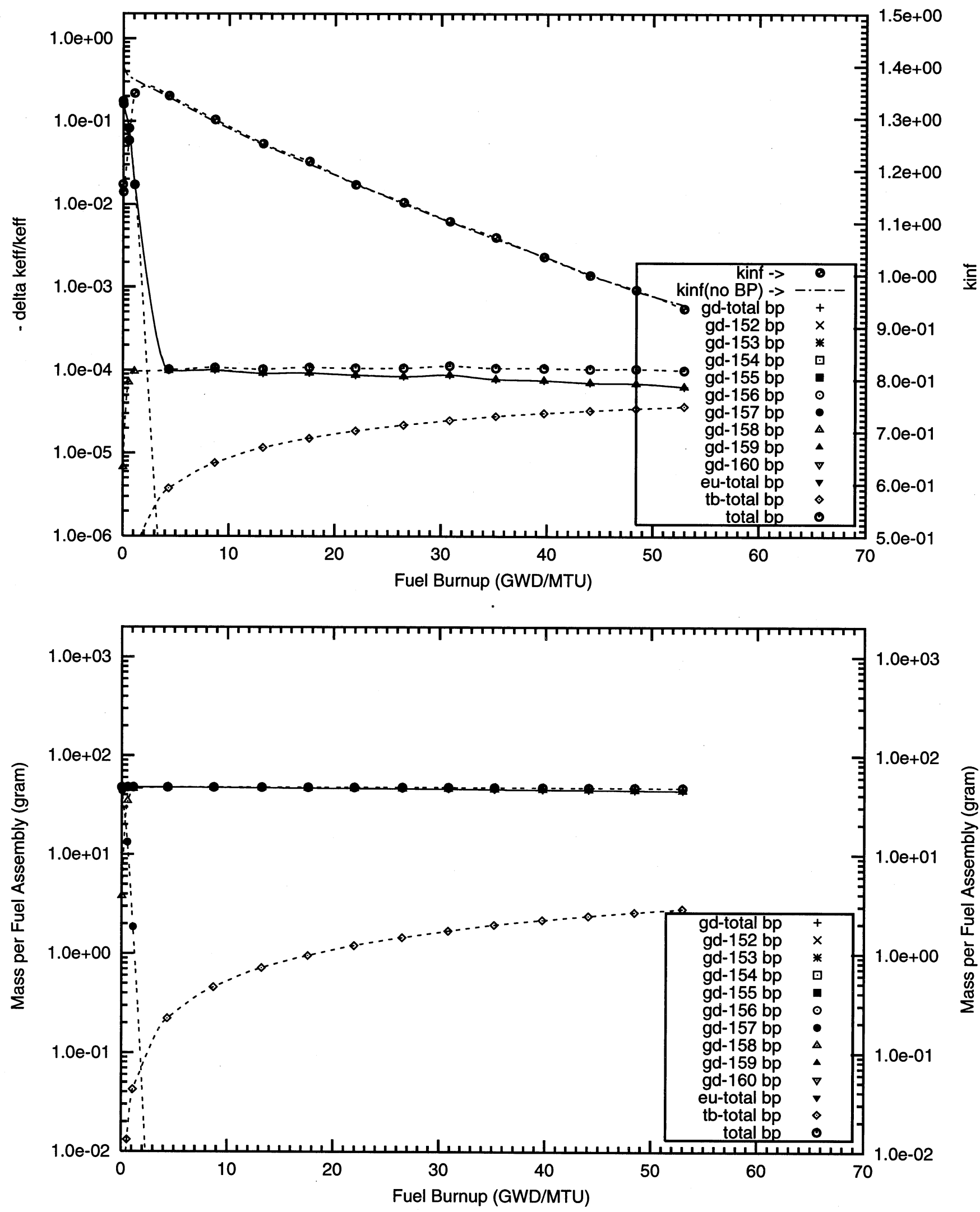
Figure A3.27. Negative Reactivity and Masses BP for Gd-157 and transmutation daughters as a function of fuel life for $17 \times 17$ fuel assemblies with 64 poison rods,

1.0 mill thick (0.10TD) Gd2O3 poison coating on the outer radial surface of the UO2 pellets Reactor power $3400 \mathrm{MWth}, 193$ fuel assemblies, initial enrichment 4.5w\%U-235

(Case : gd157_11f_64_bp_fuel o)
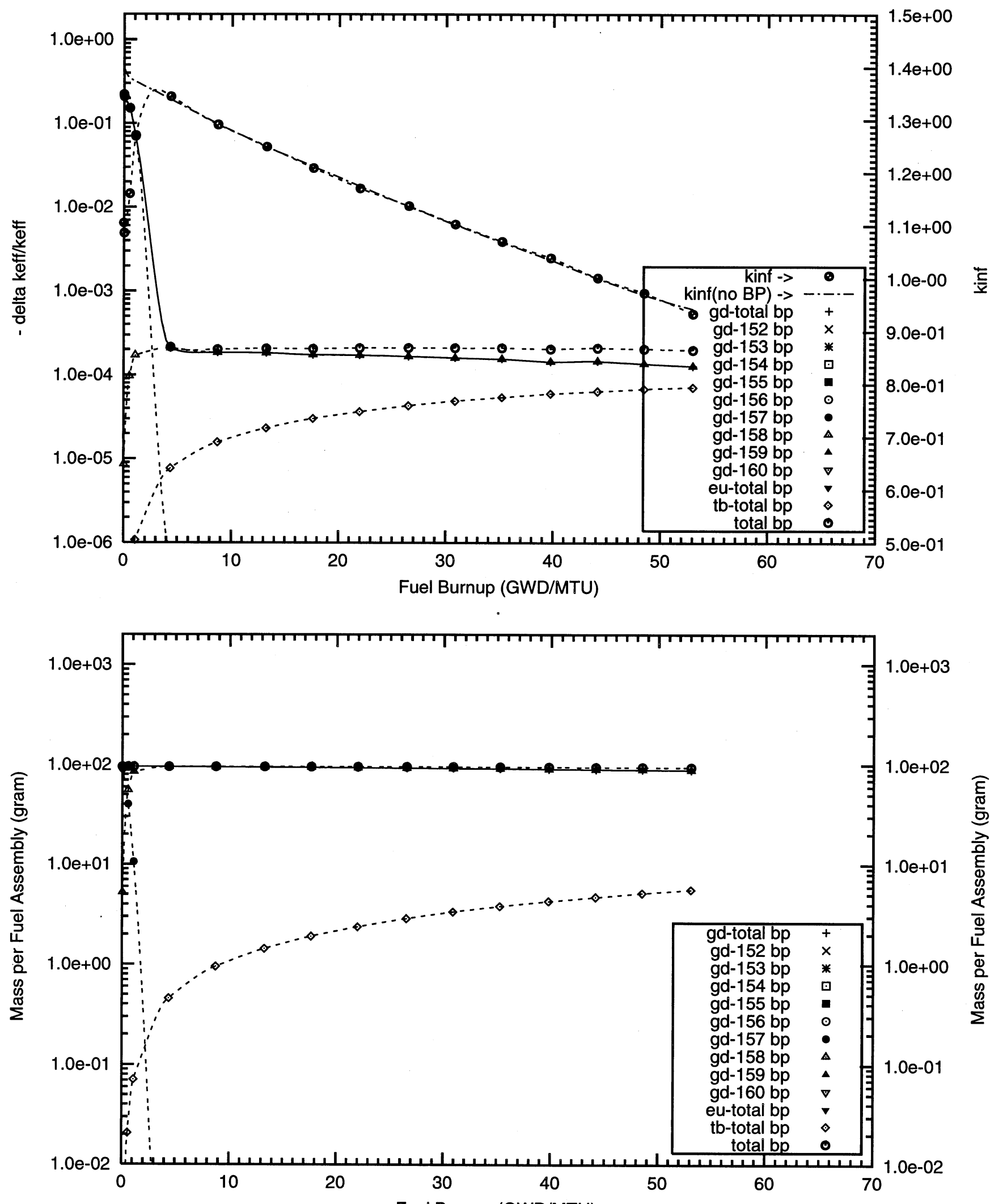

Fuel Burnup (GWD/MTU) 
Figure A3.28. Negative Reactivity and Masses BP for Gd-157 and transmutation daughters as a function of tuel lite for $17 \times 17$ tuel assemblies with 64 poison rods,

0.1 mill thick $\mathrm{Gd} 2 \mathrm{O} 3$ poison coating on the outer radial surface of the UO2 pellets

Reactor power $3400 \mathrm{MWth}, 193$ fuel assemblies, initial enrichment $4.5 \mathrm{w} \% \mathrm{U}-235$

(Case : gd157_9f_64_bp_fuel o)
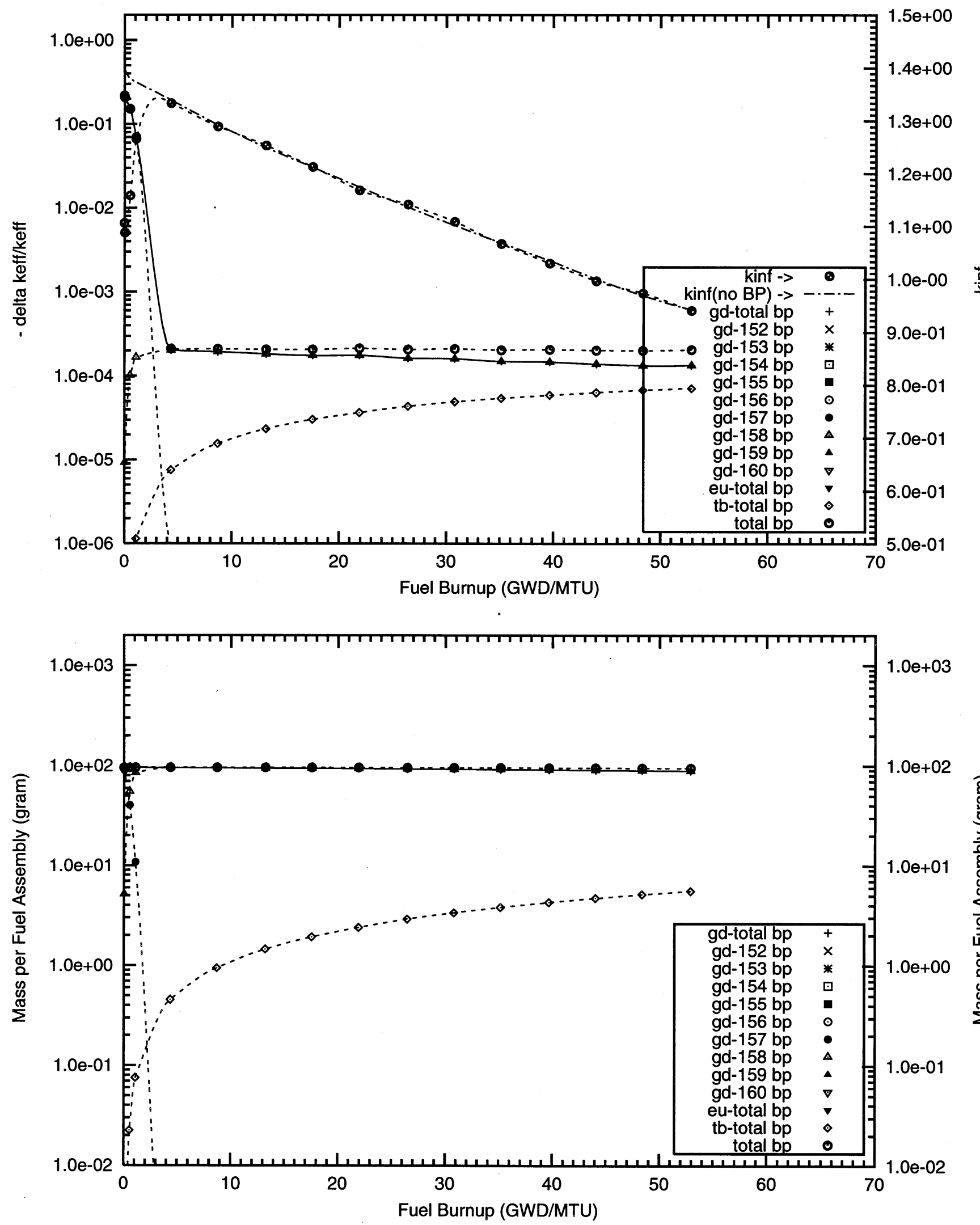
Figure A3.29. Negative Reactivity and Masses BP for Gd-157 and transmutation daughters as a function of fuel life for $17 \times 17$ fuel assemblies with 104 poison rods,

0.2 mill thick (0.10TD) Gd2O3 poison coating on the outer radial surface of the UO2 pellets

Reactor power $3400 \mathrm{MWth}, 193$ fuel assemblies, initial enrichment 4.5w\%U-235

(Case : gd157_14f_104_bp_fuel o)
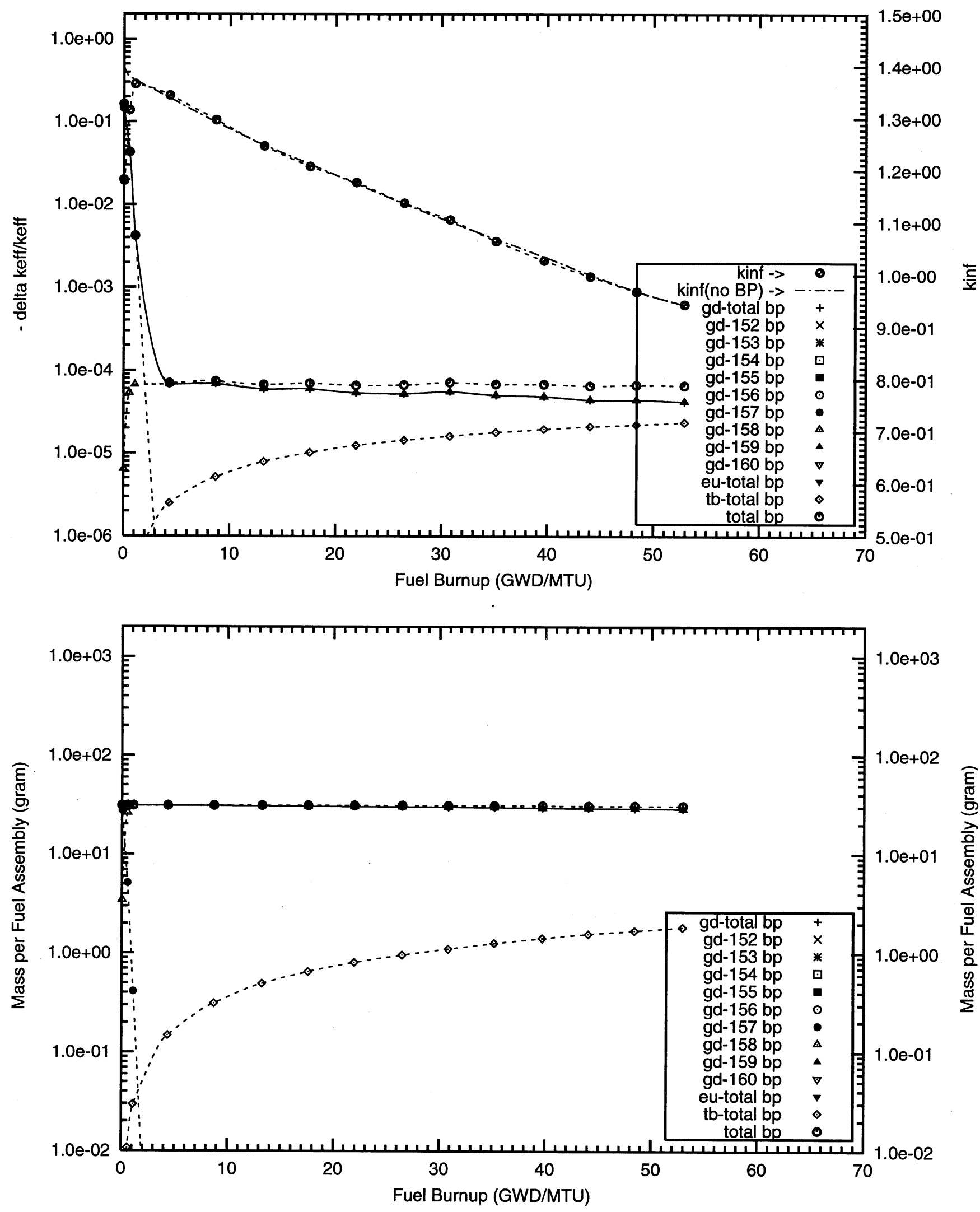
Figure A3.30. Negative Reactivity and Masses BP for Gd-157 and transmutation daughters as a function of fuel life for $17 \times 17$ fuel assemblies with 104 poison rods,

0.5 mill thick (0.10TD) Gd2O3 poison coating on the outer radial surface of the UO2 pellets

Reactor power $3400 \mathrm{MWth}, 193$ fuel assemblies, initial enrichment 4.5W\%U-235

(Case : gd157_12f_104_bp_fuel o)
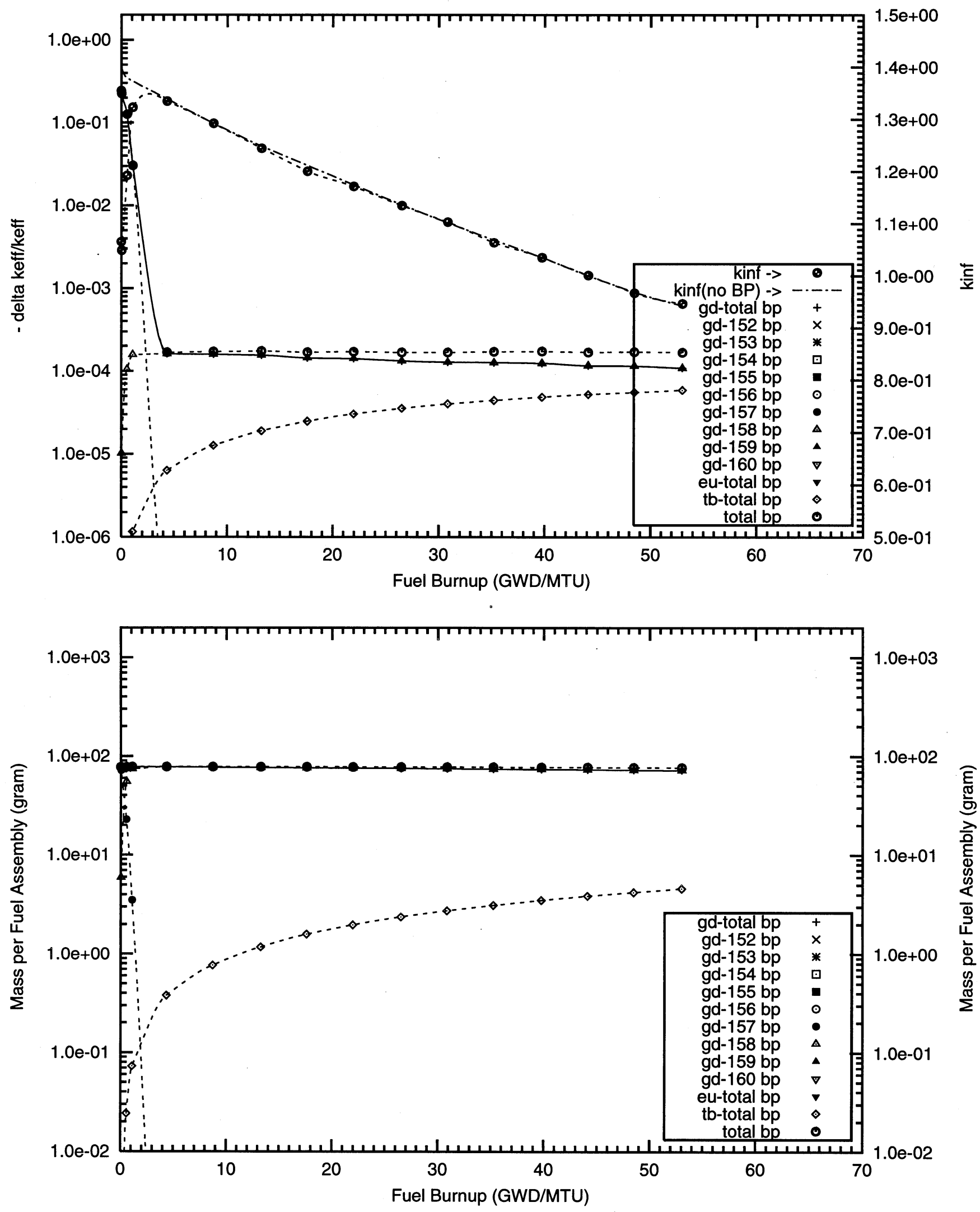


\section{Appendix A4}

Reactivity Worths and Masses of Gadolinium Alloyed with the Zircaloy Cladding of Fuel Rods

\section{Table 8 Cases}


A4 - Gadolinium with Natural Isotopic Abundances 
Figure A4.1. Negative Reactivity and Masses BP for Gd-nat and transmutation daughters as a function of fuel life for $17 \times 17$ fuel assemblies with 16 poison rods,

$1.0 \mathrm{w} \% \mathrm{Gd}$ poison homogeneously mixed in the fuel cladding,

Reactor power $3400 \mathrm{MWth}, 193$ fuel assemblies, initial enrichment $4.5 \mathrm{w} \% \mathrm{U}-235$

(Case : gd000_1c_100p_h20_16_bp_fuel_2_o)

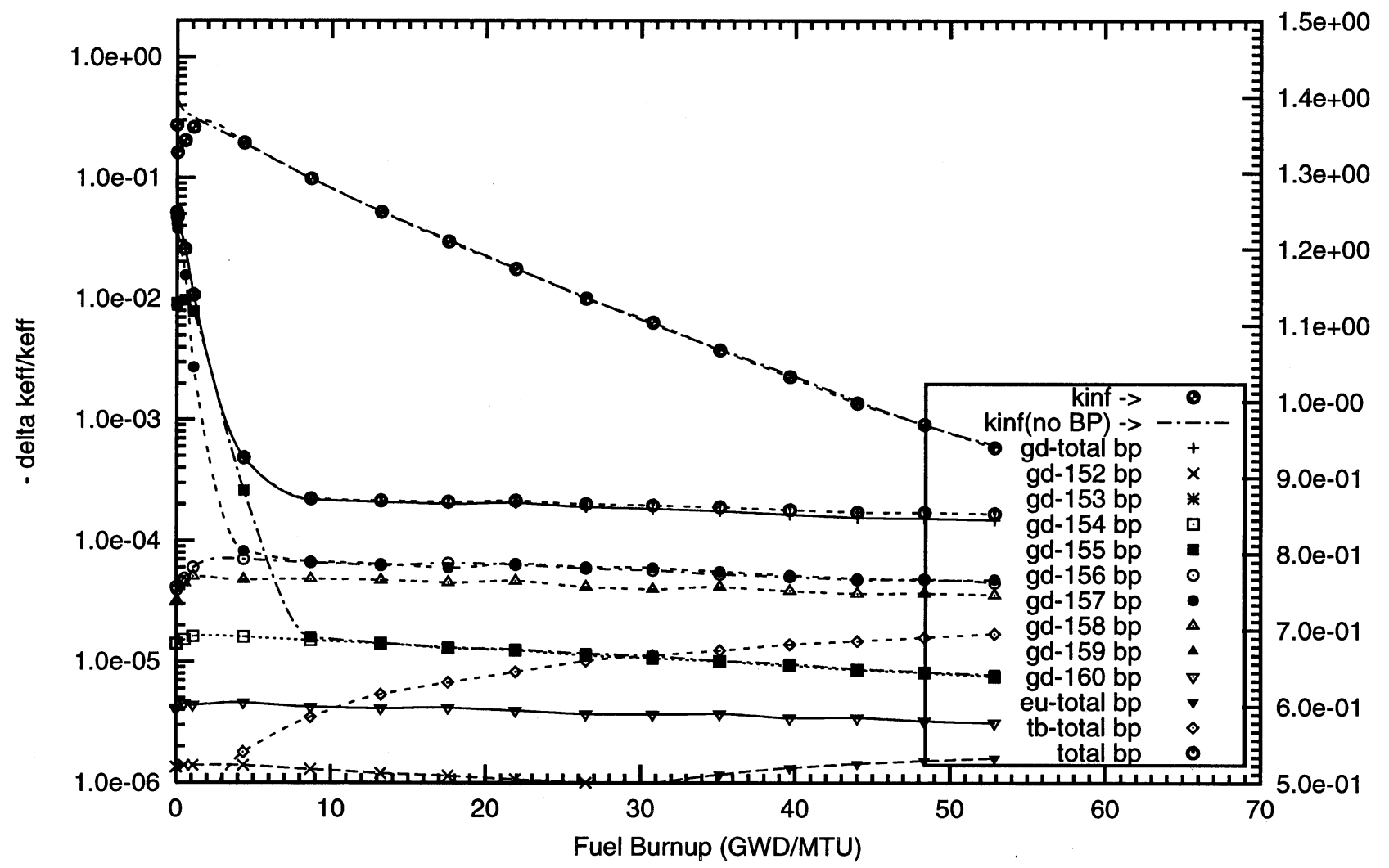

$\overline{\frac{1}{x}}$

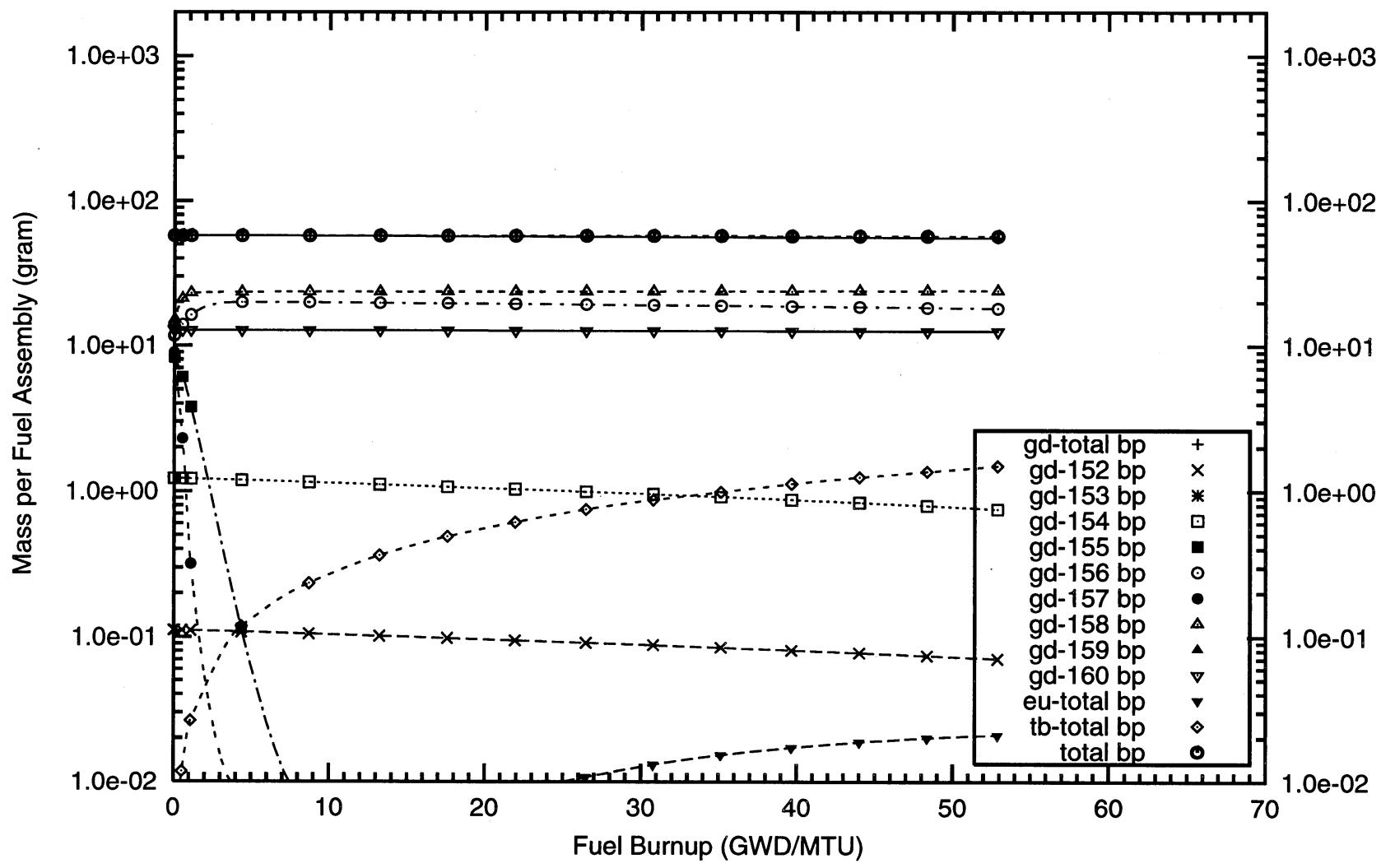


Figure A4.2. Negative Reactivity and Masses BP for Gd-nat and transmutation daughters as a function of fuel life for $17 \times 17$ fuel assemblies with 16 poison rods,

$2.0 \mathrm{w} \% \mathrm{Gd}$ poison homogeneously mixed in the fuel cladding,

Reactor power $3400 \mathrm{MWth}, 193$ fuel assemblies, initial enrichment $4.5 \mathrm{w} \% \mathrm{U}-235$

(Case : gd000_2c_100p_h2o_16_bp_fuel_2_o)
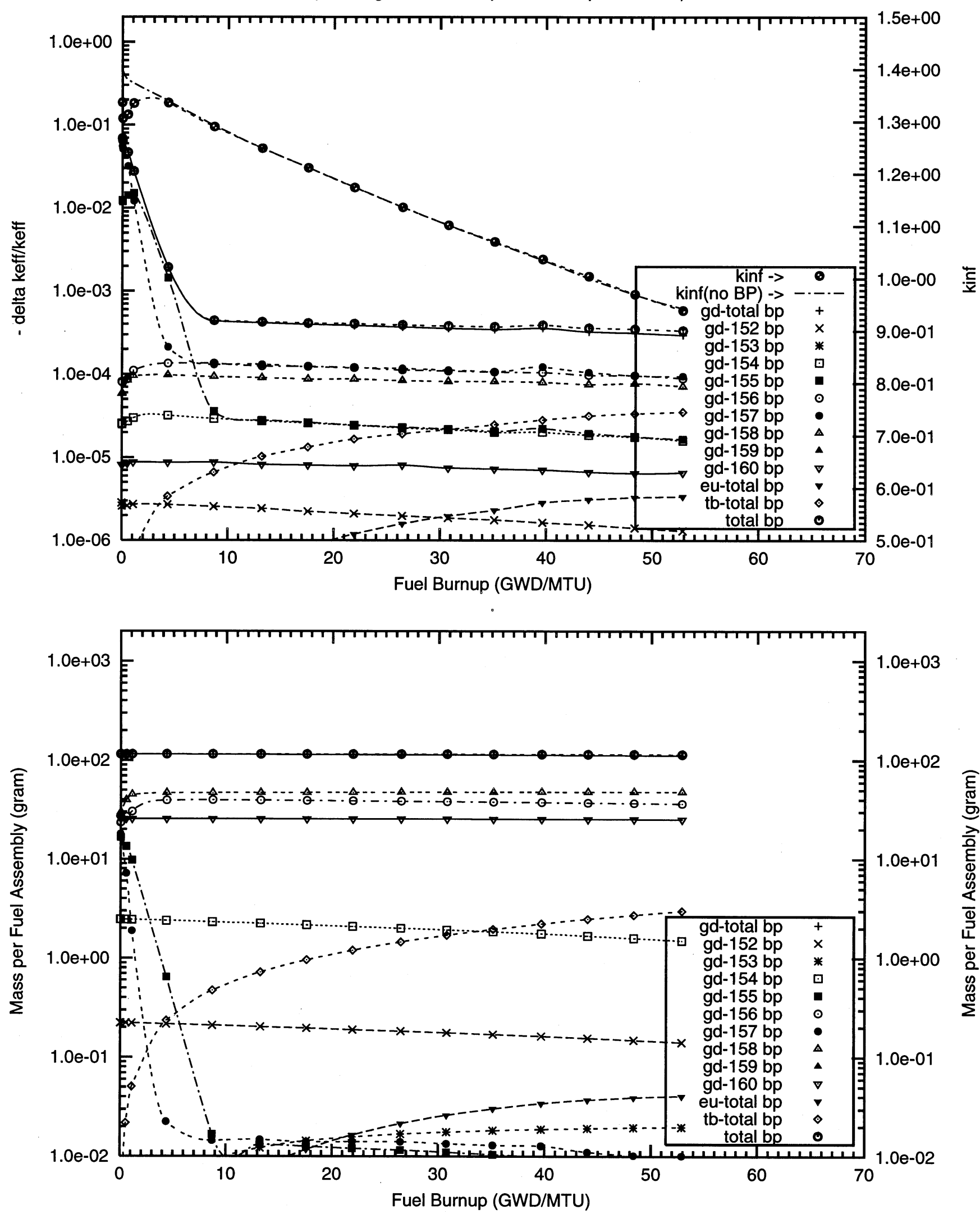
Figure A4.3. Negative Reactivity and Masses BP for Gd-nat and transmutation daughters as a function of fuel life for $17 \times 17$ fuel assemblies with 16 poison rods,

$4.0 \mathrm{w} \% \mathrm{Gd}$ poison homogeneously mixed in the fuel cladding,

Reactor power $3400 \mathrm{MWth}, 193$ fuel assemblies, initial enrichment $4.5 \mathrm{w} \% \mathrm{U}-235$

(Case : gd000_3c_100p_h2o_16_bp_fuel_2_o)
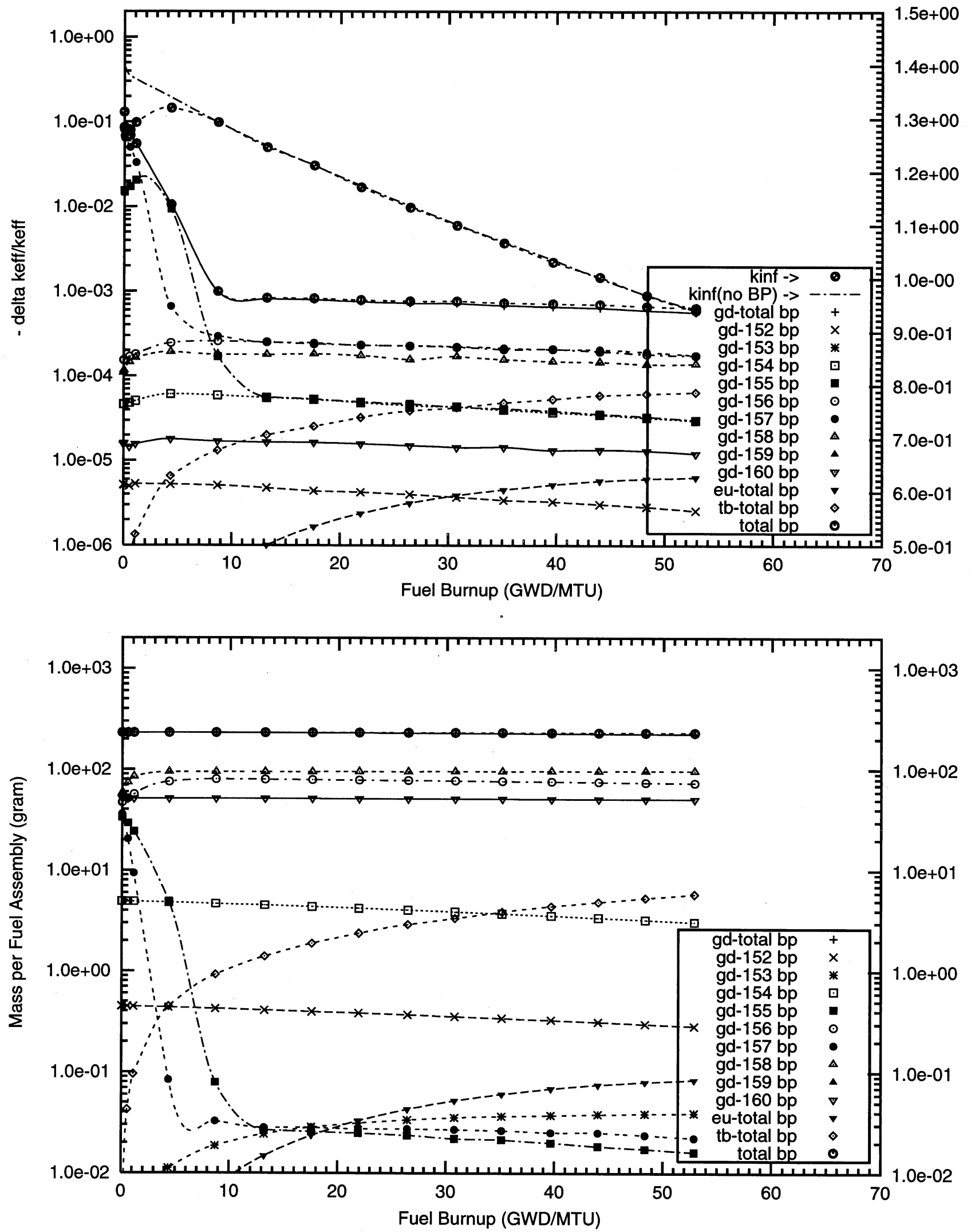
Figure A4.4. Negative Reactivity and Masses BP for Gd-nat and transmutation daughters as a function of fuel life for $17 \times 17$ fuel assemblies with 16 poison rods,

$8.0 \mathrm{w} \% \mathrm{Gd}$ poison homogeneously mixed in the fuel cladding,

Reactor power $3400 \mathrm{MWth}, 193$ fuel assemblies, initial enrichment $4.5 \mathrm{w} \% \mathrm{U}-235$

(Case : gd000_4c_100p_h2o_16_bp_fuel_2_o)
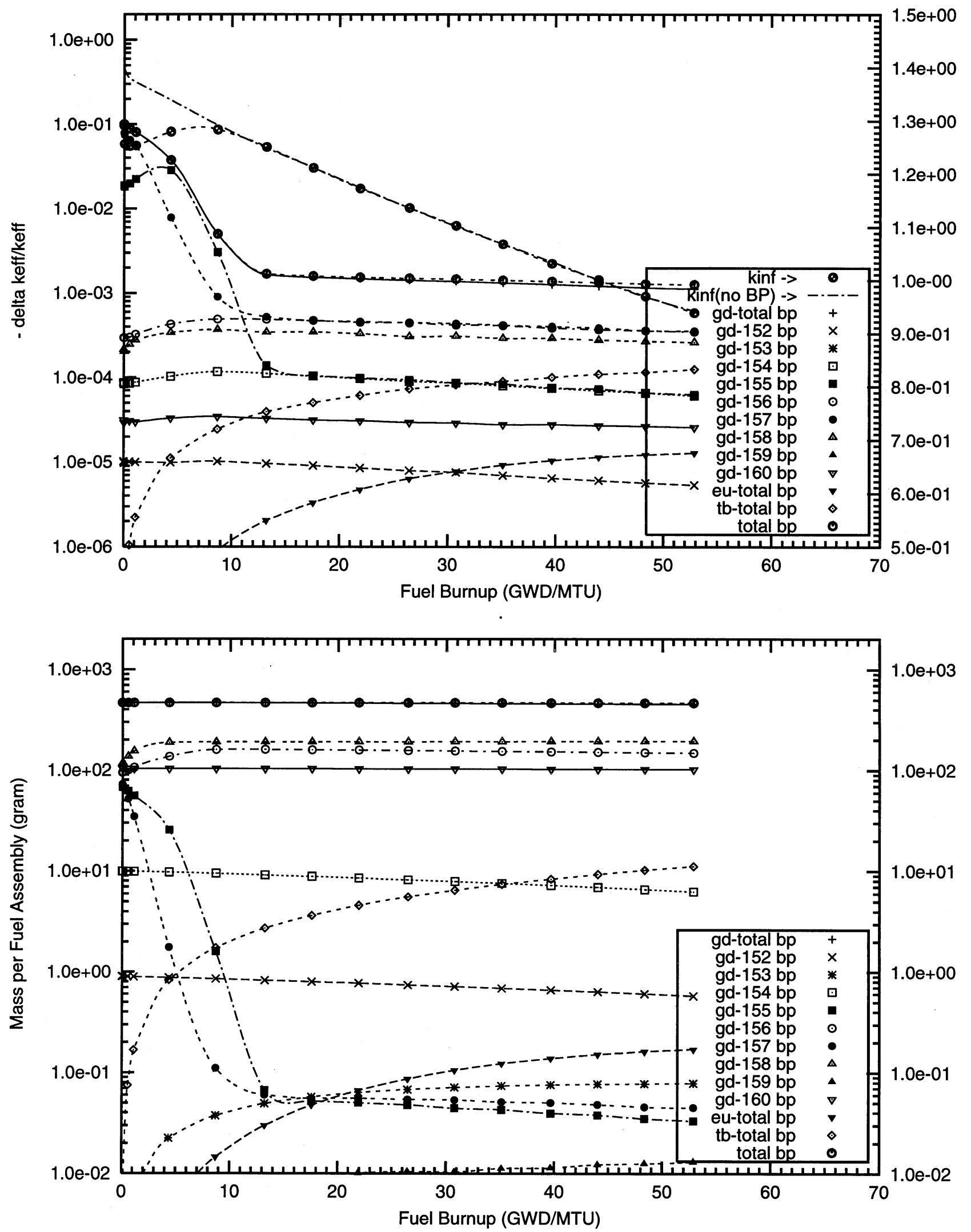
Figure A4.5. Negative Reactivity and Masses BP for Gd-nat and transmutation daughters as a function of fuel life for $17 \times 17$ fuel assemblies with 104 poison rods, $0.2 w \%$ Gd poison homogeneously mixed in the fuel cladding,

Reactor power $3400 \mathrm{MWth}, 193$ fuel assemblies, initial enrichment $4.5 \mathrm{w} \% \mathrm{U}-235$ (Case : gd000_8c_104_bp_fuel o)
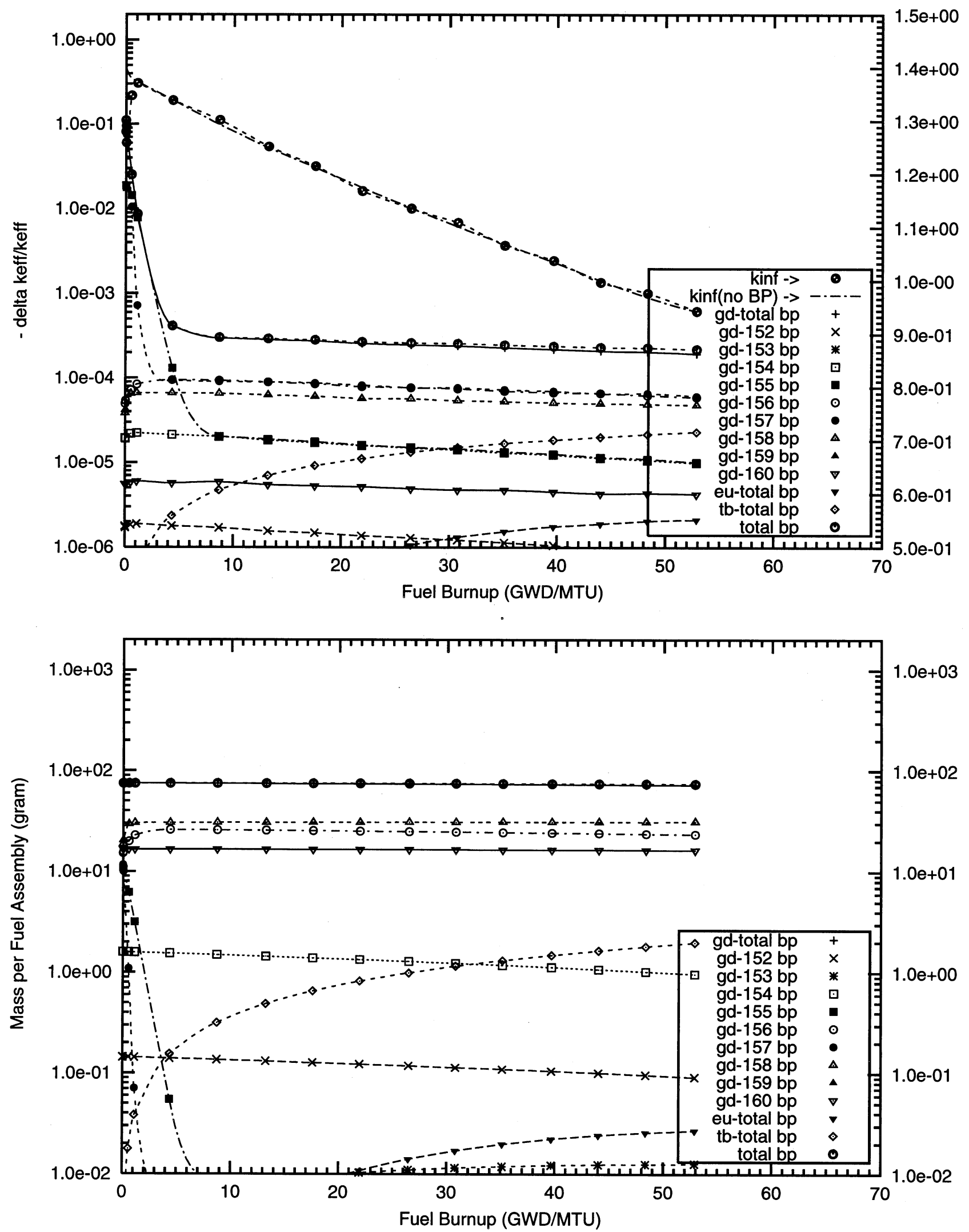
A4 - Gadolinium Fully Enriched in ${ }^{155}$ Gd 

Figure A4.6. Negative Reactivity and Masses BP for Gd-155 and transmutation daughters as a function of fuel life for $17 \times 17$ fuel assemblies with 16 poison rods,

$1.0 \mathrm{w} \% \mathrm{Gd}$ poison homogeneously mixed in the fuel cladding,

Reactor power $3400 \mathrm{MWth}, 193$ fuel assemblies, initial enrichment 4.5w\%U-235

(Case : gd155_1c_100p_h2o_16_bp_fuel_2_o)
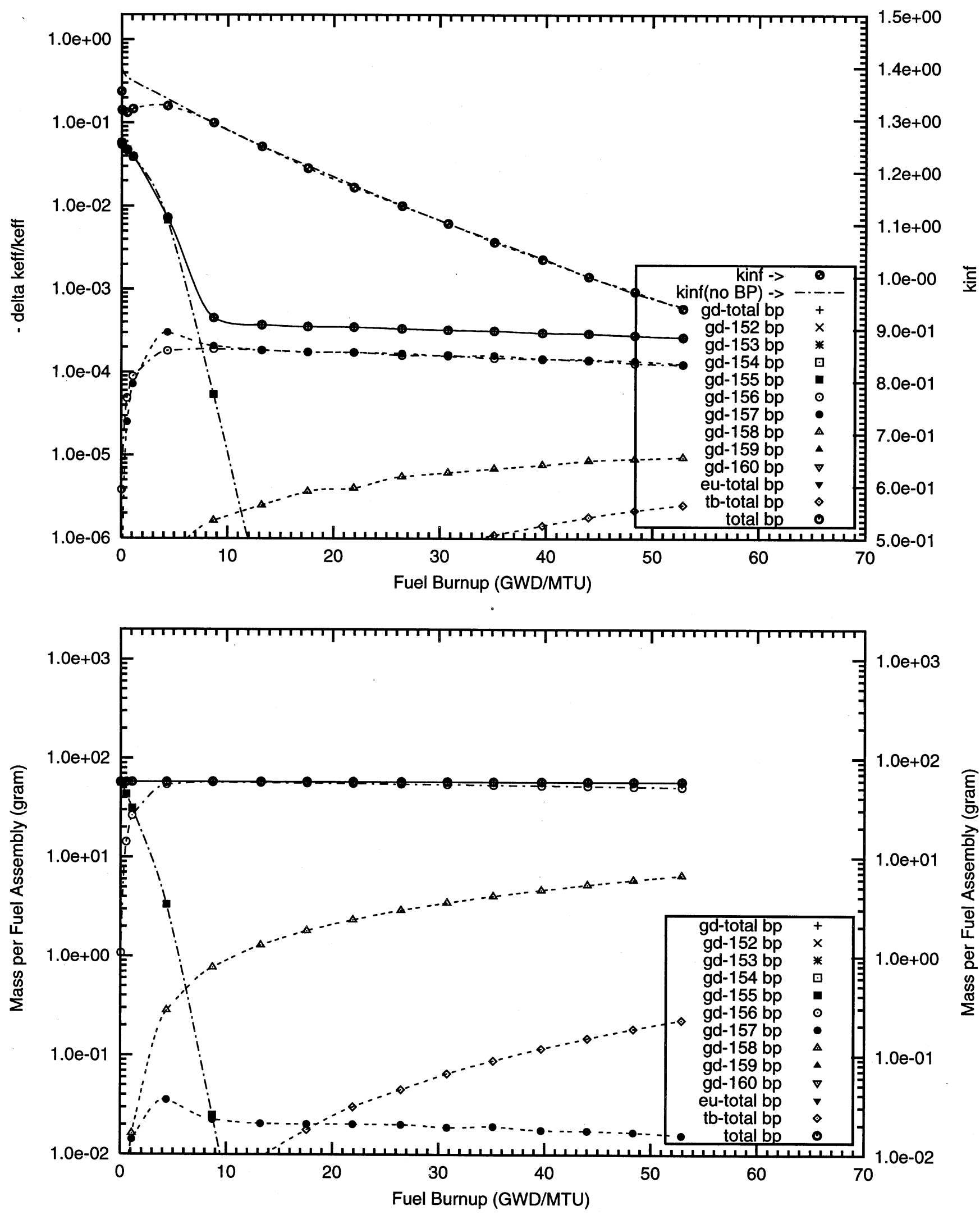
Figure A4.7. Negative Reactivity and Masses BP for Gd-155 and transmutation daughters as a function of fuel life for $17 \times 17$ fuel assemblies with 16 poison rods,

$2.0 \mathrm{w} \% \mathrm{Gd}$ poison homogeneously mixed in the fuel cladding,

Reactor power $3400 \mathrm{MWth}, 193$ fuel assemblies, initial enrichment $4.5 \mathrm{w} \% \mathrm{U}-235$

(Case : gd155_2c_100p_h20_16_bp_fuel_2_o)
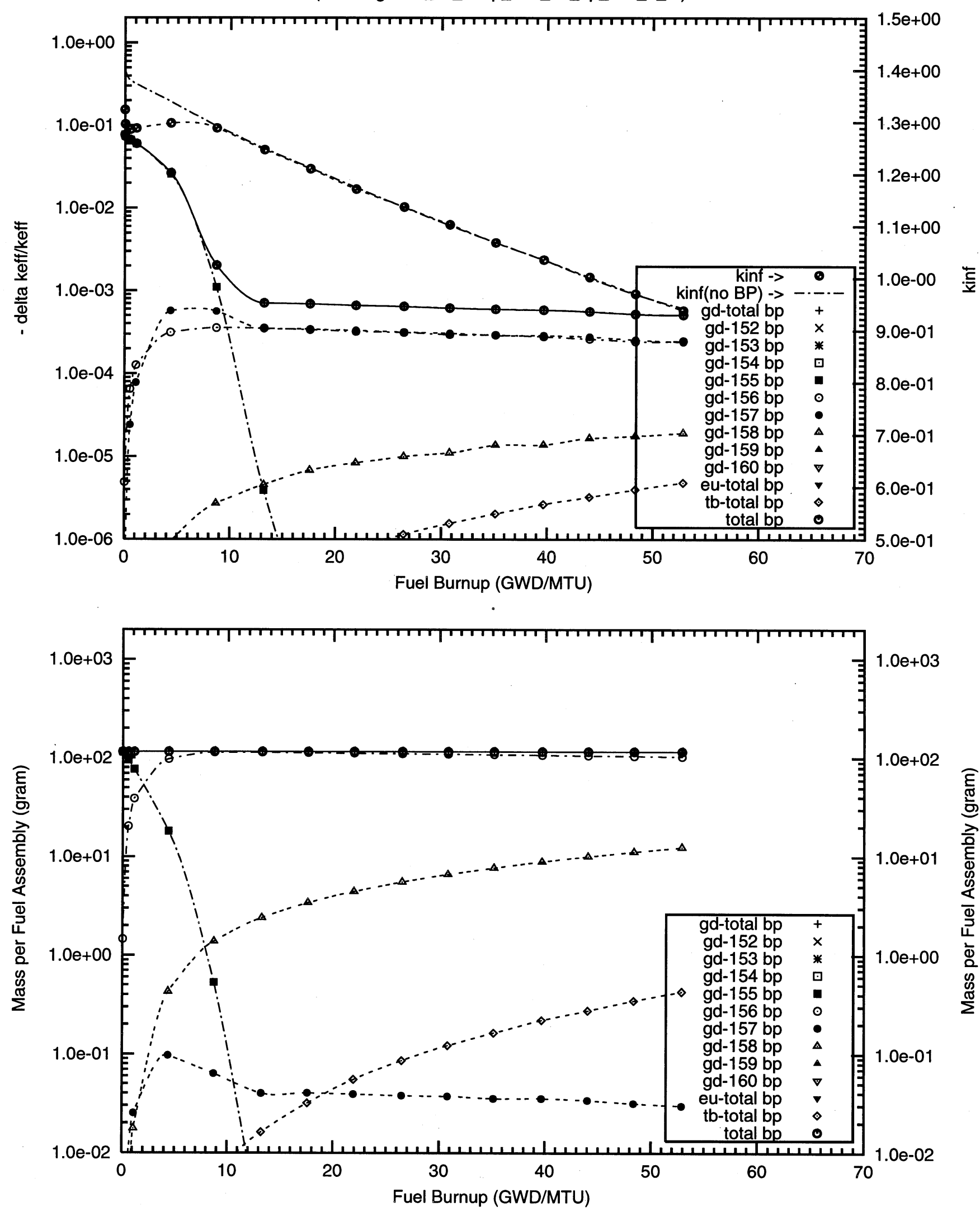
Figure A4.8. Negative Reactivity and Masses BP for Gd-155 and transmutation daughters as a function of fuel life for $17 \times 17$ fuel assemblies with 16 poison rods,

$4.0 \mathrm{w} \% \mathrm{Gd}$ poison homogeneously mixed in the fuel cladding,

Reactor power $3400 \mathrm{MWth}, 193$ fuel assemblies, initial enrichment 4.5w\%U-235

(Case : gd155_3c_100p_h20_16_bp_fuel_2_o)
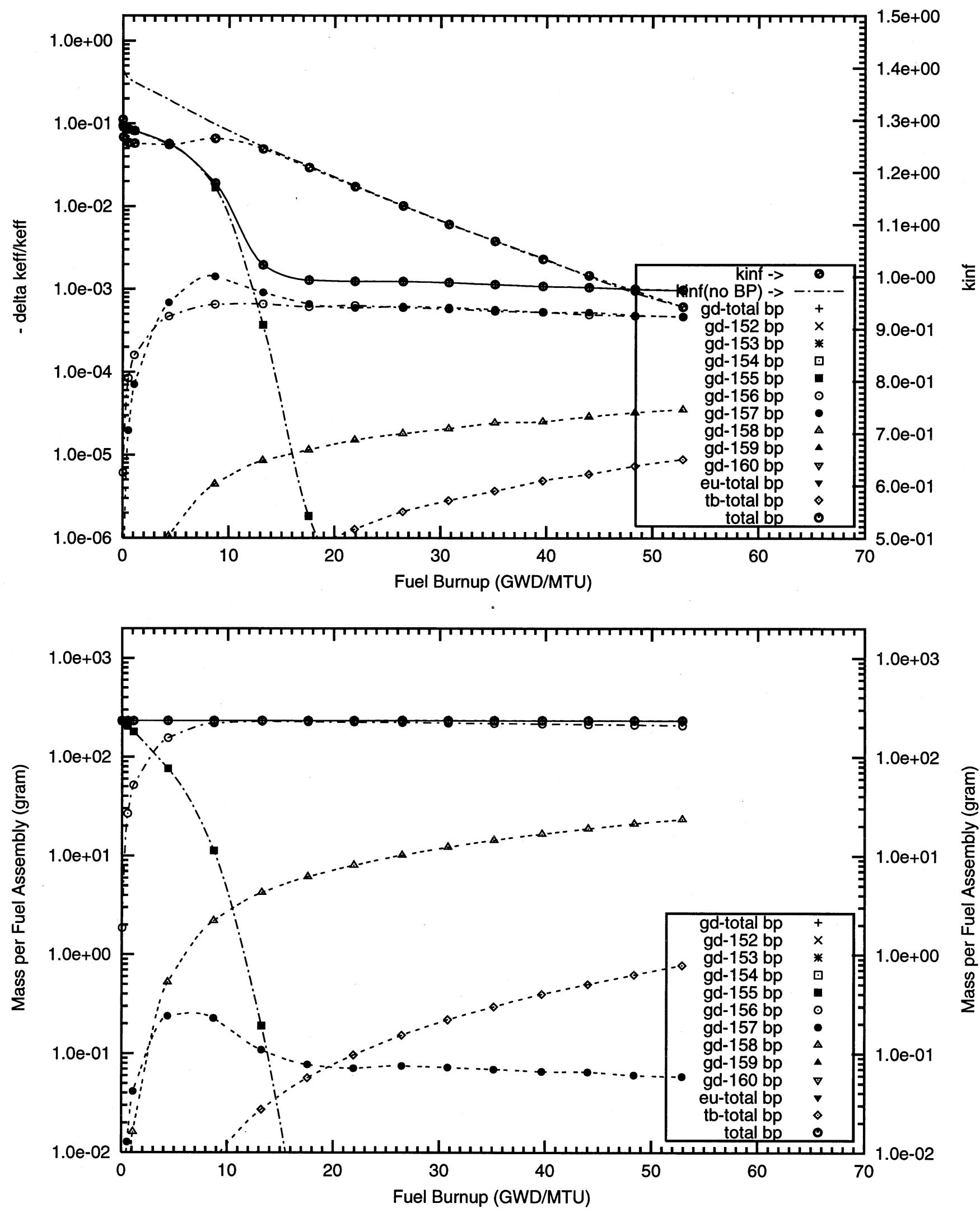
Figure A4.9. Negative Reactivity and Masses BP for Gd-155 and transmutation daughters as a function of fuel life for $17 \times 17$ fuel assemblies with 16 poison rods,

$8.0 \mathrm{w} \% \mathrm{Gd}$ poison homogeneously mixed in the fuel cladding,

Reactor power $3400 \mathrm{MWth}, 193$ fuel assemblies, initial enrichment $4.5 \mathrm{w} \% \mathrm{U}-235$

(Case : gd155_4c_100p_h2o_16_bp_fuel_2_o)
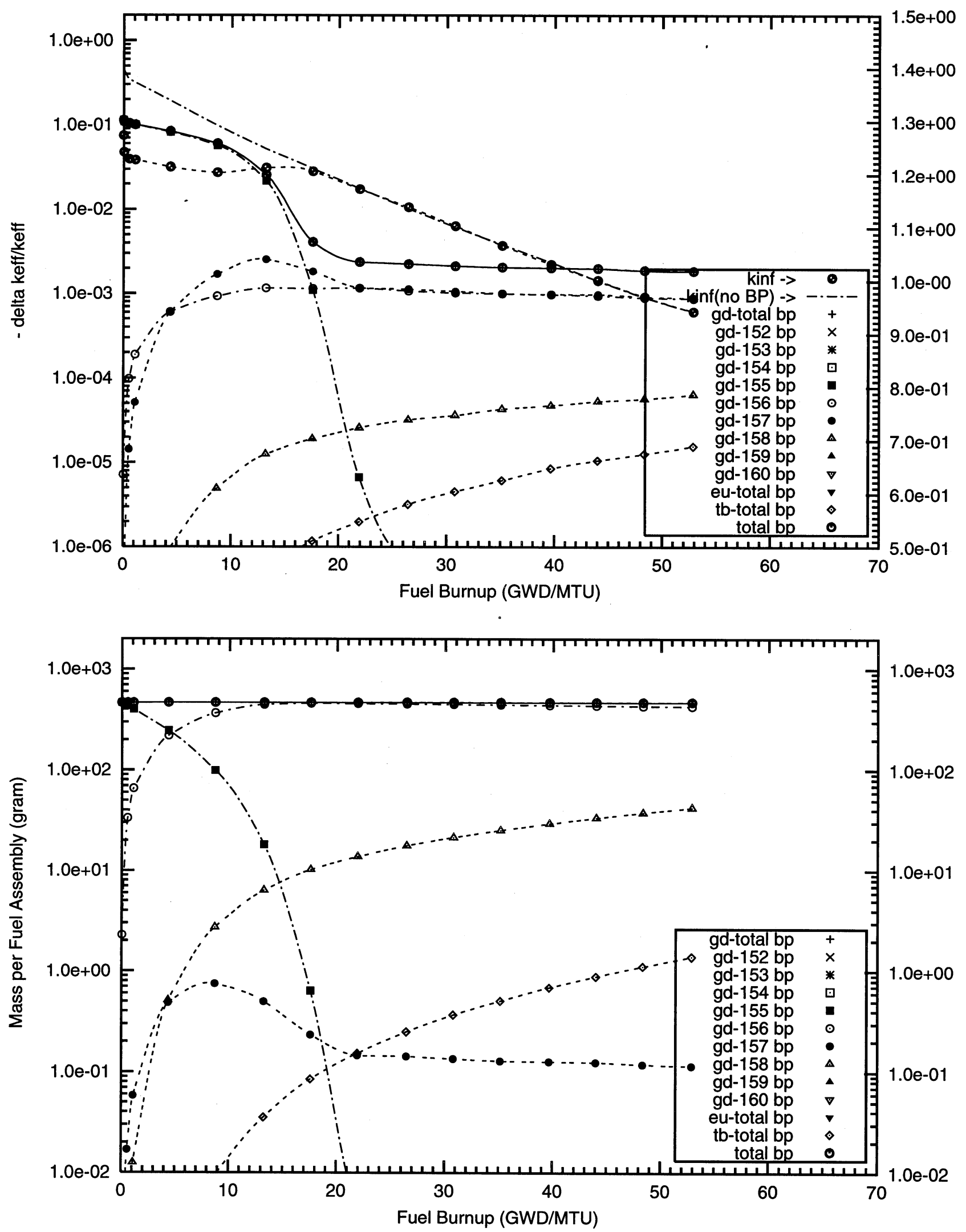
Figure A4.10. Negative Reactivity and Masses BP for Gd-155 and transmutation daughters as a function of fuel life for $17 \times 17$ fuel assemblies with 104 poison rods,

$0.05 \mathrm{w} \% \mathrm{Gd}$ poison homogeneously mixed in the fuel cladding

Reactor power $3400 \mathrm{MWth}, 193$ fuel assemblies, initial enrichment $4.5 \mathrm{w} \% \mathrm{U}-235$

(Case : gd155_10c_104_bp_fuel o)
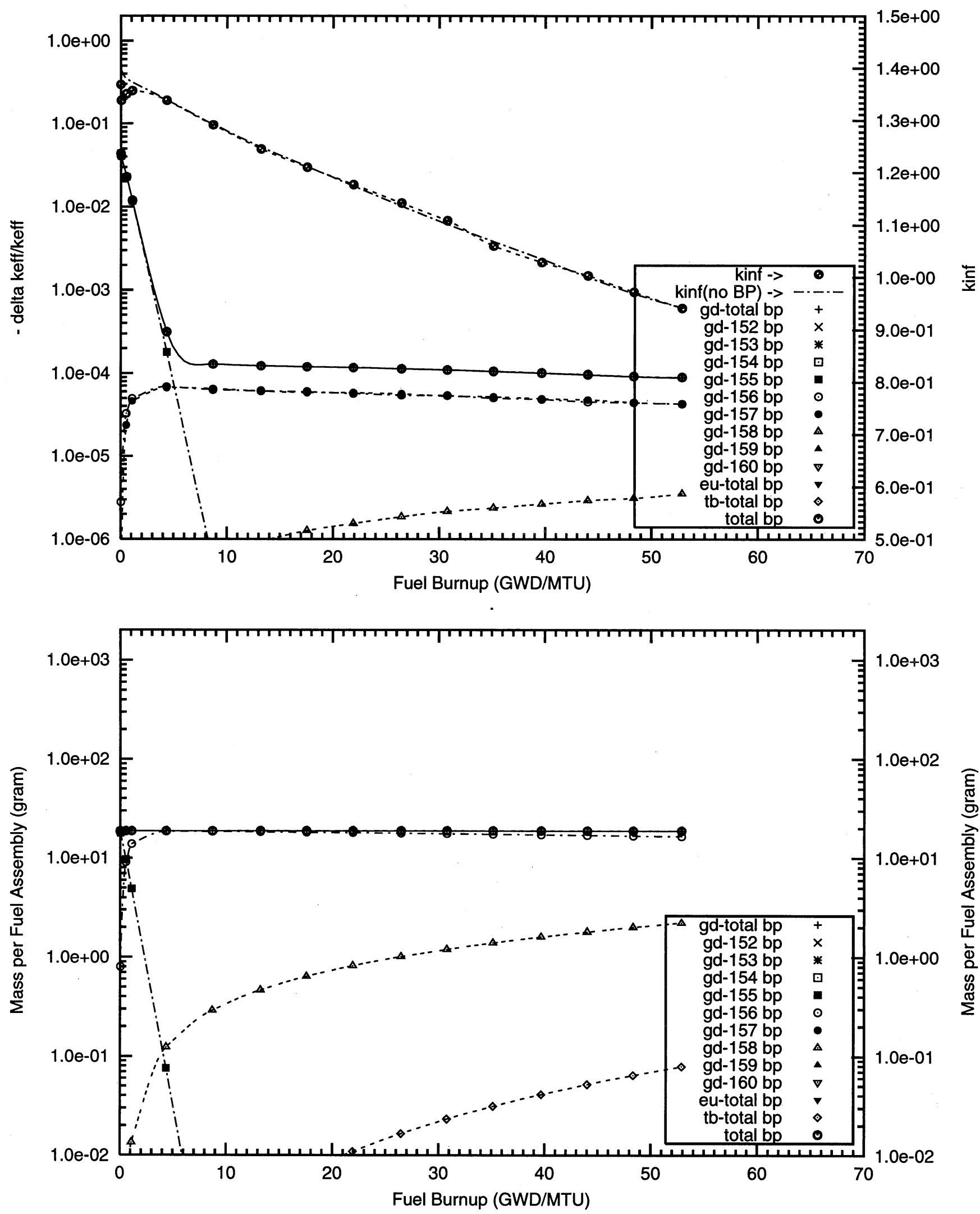
Figure A4.11. Negative Reactivity and Masses BP for Gd-155 and transmutation daughters as a function of fuel life for $17 \times 17$ fuel assemblies with 104 poison rods,

$0.2 \mathrm{w} \% \mathrm{Gd}$ poison homogeneously mixed in the fuel cladding,

Reactor power $3400 \mathrm{MWth}, 193$ fuel assemblies, initial enrichment $4.5 \mathrm{w} \% \mathrm{U}-235$

(Case : gd155_8c_104_bp_fuel o)
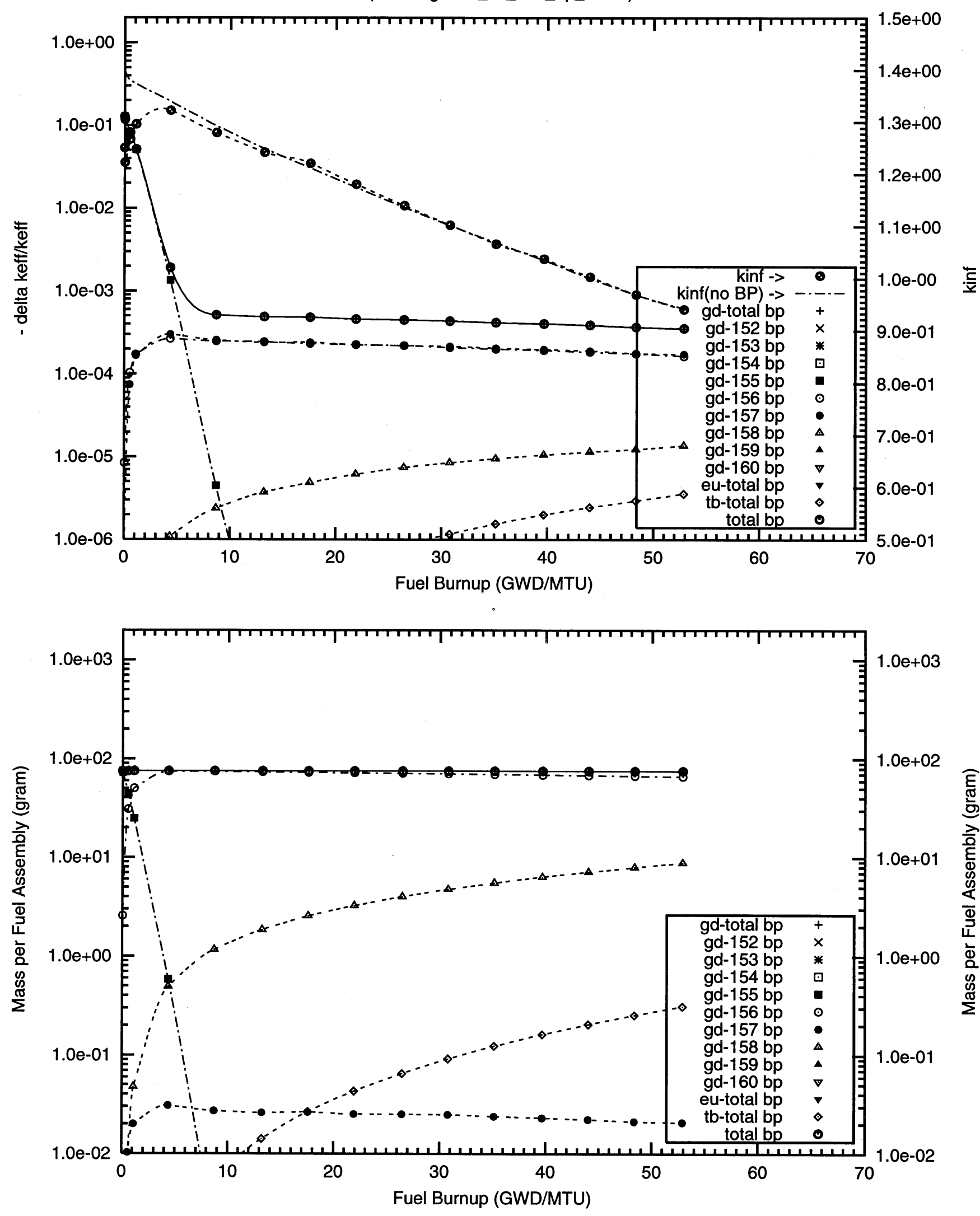
A4 - Gadolinium Fully Enriched in ${ }^{157}$ Gd 

Figure A4.12. Negative Reactivity and Masses BP for Gd-157 and transmutation daughters as a function of fuel life for $17 \times 17$ fuel assemblies with 08 poison rods,

$1.0 \mathrm{w} \% \mathrm{Gd}$ poison homogeneously mixed in the fuel cladding,

Reactor power $3400 \mathrm{MWth}, 193$ fuel assemblies, initial enrichment $4.5 \mathrm{w} \% \mathrm{U}-235$

(Case : gd157_1c_100p_h20_08_bp_fuel_2_o)
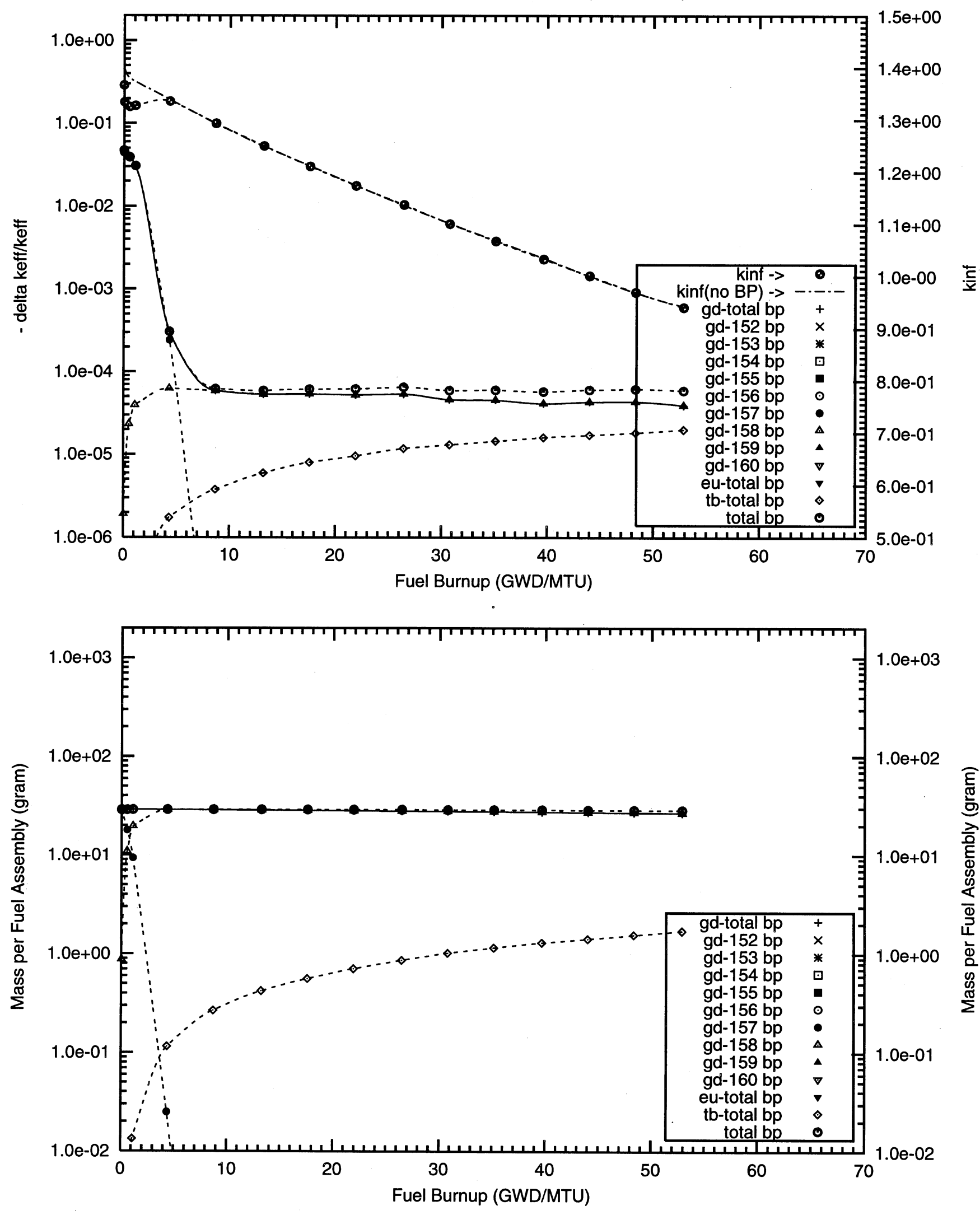
Figure A4.13. Negative Reactivity and Masses BP for Gd-157 and transmutation daughters as a function of fuel life for $17 \times 17$ fuel assemblies with 08 poison rods,

$2.0 \mathrm{w} \% \mathrm{Gd}$ poison homogeneously mixed in the fuel cladding,

Reactor power $3400 \mathrm{MWth}, 193$ fuel assemblies, initial enrichment $4.5 \mathrm{w} \% \mathrm{U}-235$

(Case : gd157_2c_100p_h2o_08_bp_fuel_2_o)
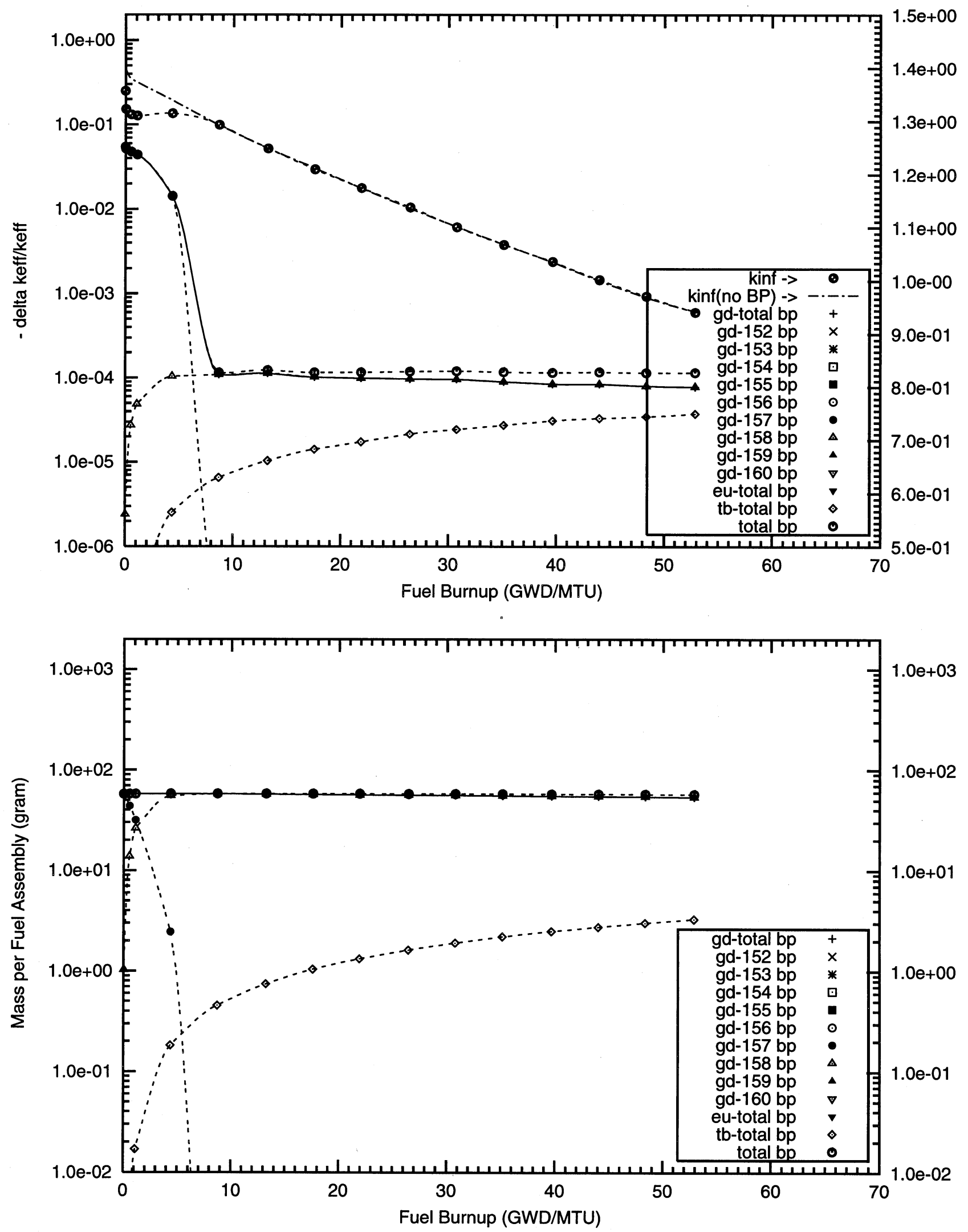
Figure A4.14. Negative Reactivity and Masses BP for Gd-157 and transmutation daughters as a function of fuel life for $17 \times 17$ fuel assemblies with 16 poison rods,

$0.2 \mathrm{w} \% \mathrm{Gd}$ poison homogeneously mixed in the fuel cladding,

Reactor power $3400 \mathrm{MWth}, 193$ fuel assemblies, initial enrichment $4.5 \mathrm{w} \% \mathrm{U}-235$

(Case : gd157_8c_100p_h2o_16_bp_fuel_2_o)
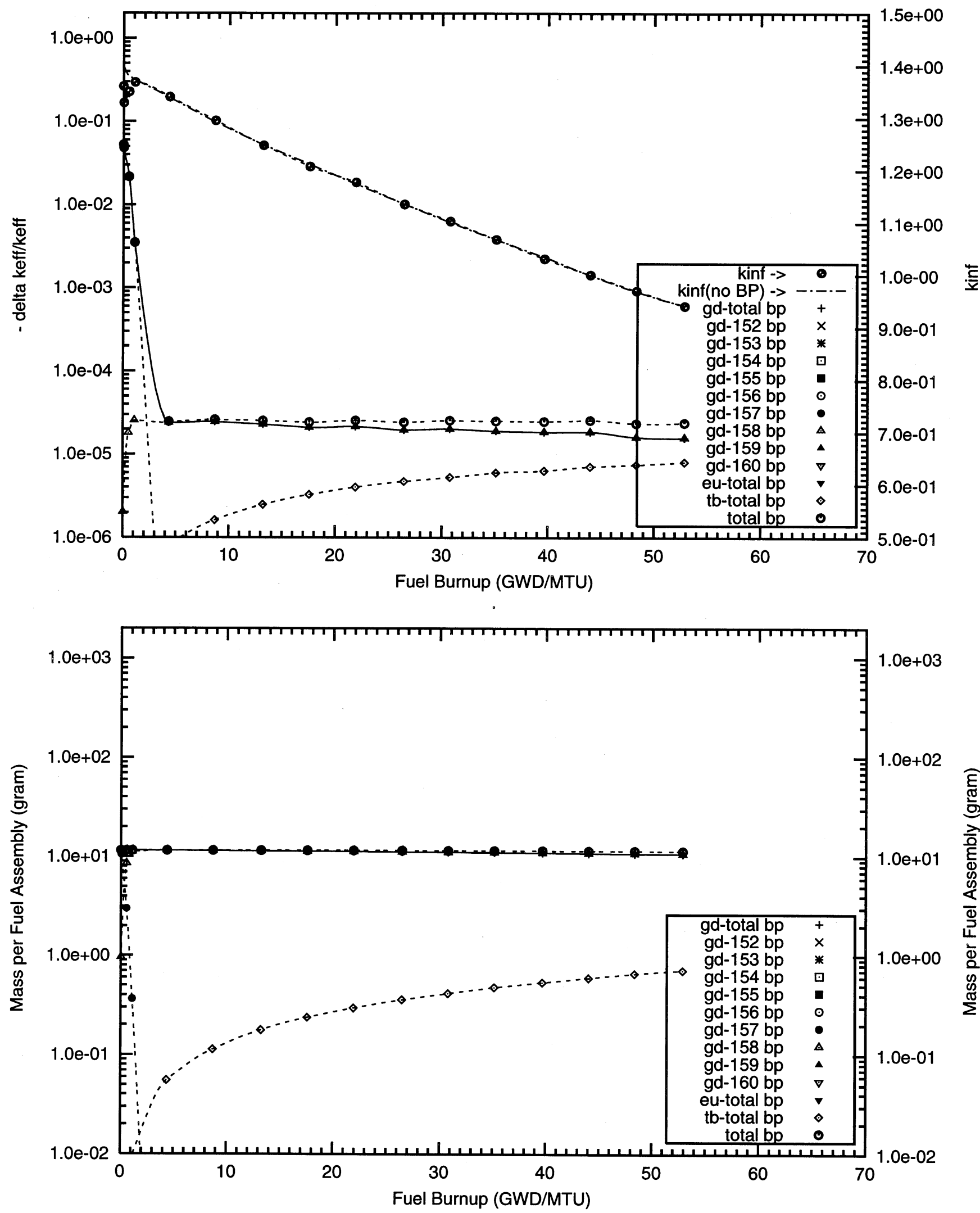
Figure A4.15. Negative Reactivity and Masses BP for Gd-157 and transmutation daughters as a function of fuel life for $17 \times 17$ fuel assemblies with 16 poison rods,

$0.5 \mathrm{w} \% \mathrm{Gd}$ poison homogeneously mixed in the fuel cladding,

Reactor power $3400 \mathrm{MWth}, 193$ fuel assemblies, initial enrichment $4.5 \mathrm{w} \% \mathrm{U}-235$

(Case : gd157_0c_100p_h2o_16_bp_fuel_2_0)
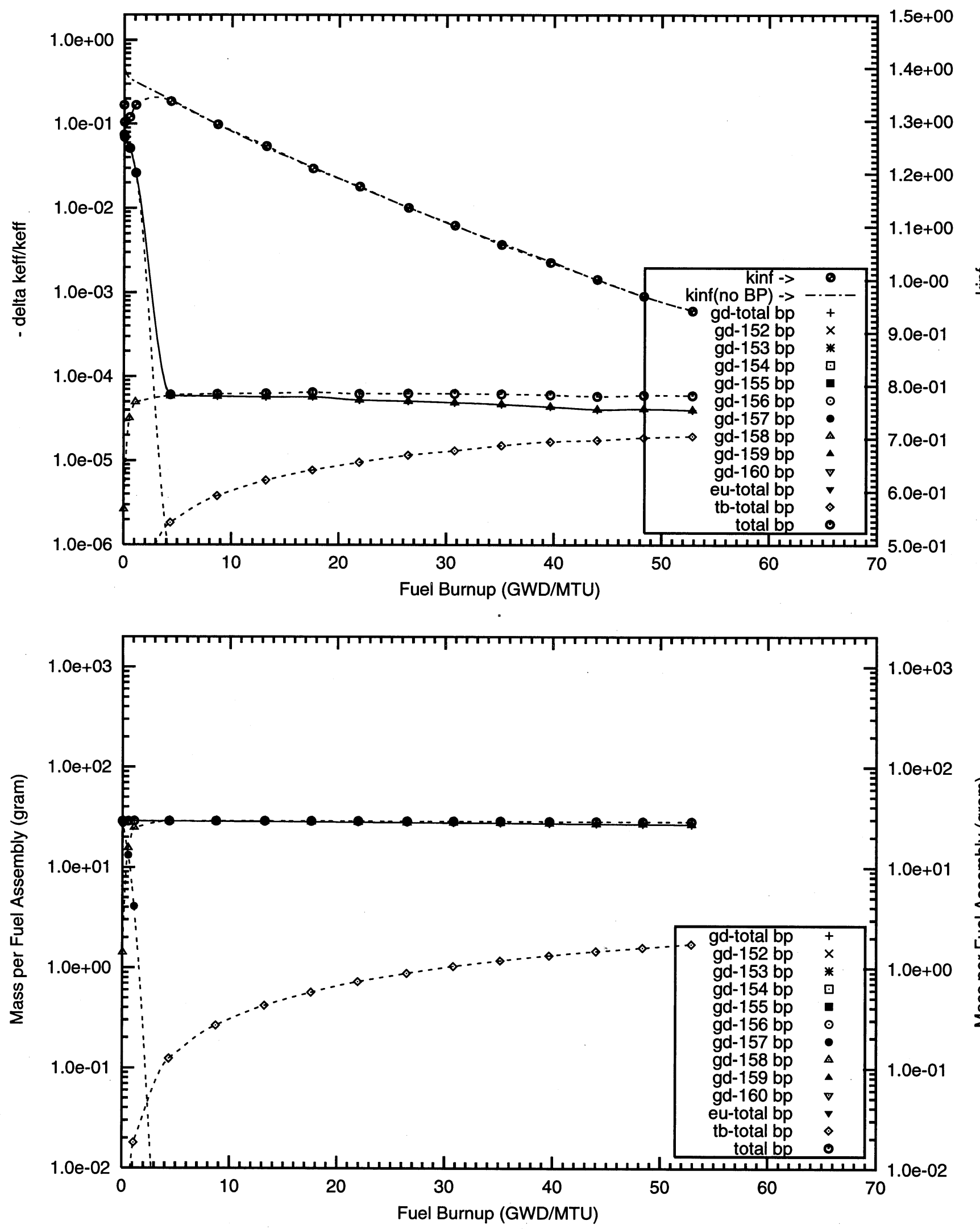
Figure A4.16. Negative Reactivity and Masses BP for Gd-157 and transmutation daughters as a function of fuel life for $17 \times 17$ fuel assemblies with 16 poison rods,

$1.0 \mathrm{w} \% \mathrm{Gd}$ poison homogeneously mixed in the fuel cladding,

Reactor power $3400 \mathrm{MWth}, 193$ fuel assemblies, initial enrichment $4.5 \mathrm{~W} \% \mathrm{U}-235$

(Case : gd157_1c_100p_h2o_16_bp_fuel_2_o)
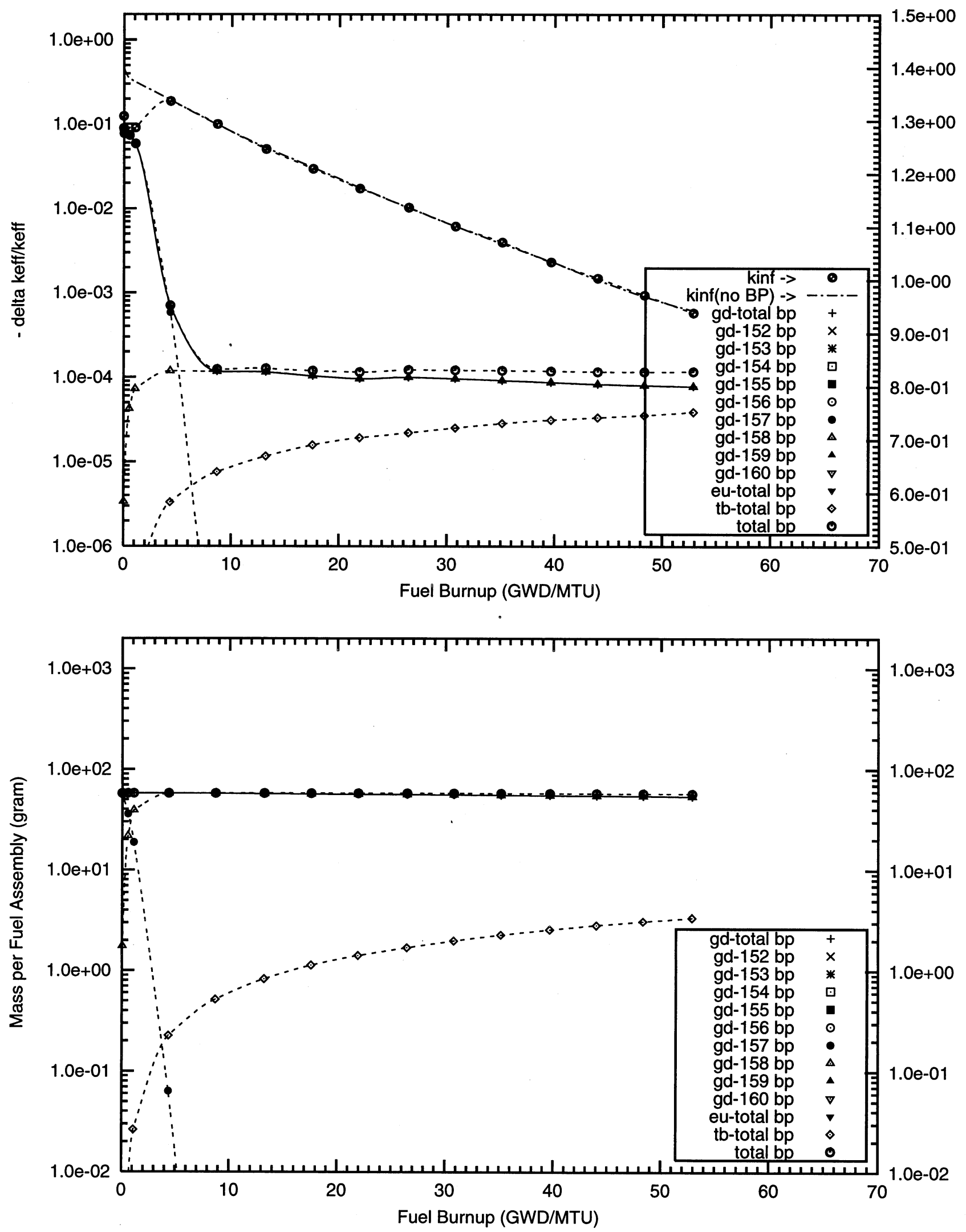
Figure A4.17. Negative Reactivity and Masses BP for Gd-157 and transmutation daughters as a function of fuel life for $17 \times 17$ fuel assemblies with 16 poison rods,

$2.0 \mathrm{w} \% \mathrm{Gd}$ poison homogeneously mixed in the fuel cladding,

Reactor power $3400 \mathrm{MWth}, 193$ fuel assemblies, initial enrichment $4.5 \mathrm{w} \% \mathrm{U}-235$

(Case : gd157_2c_100p_h2o_16_bp_fuel_2_o)
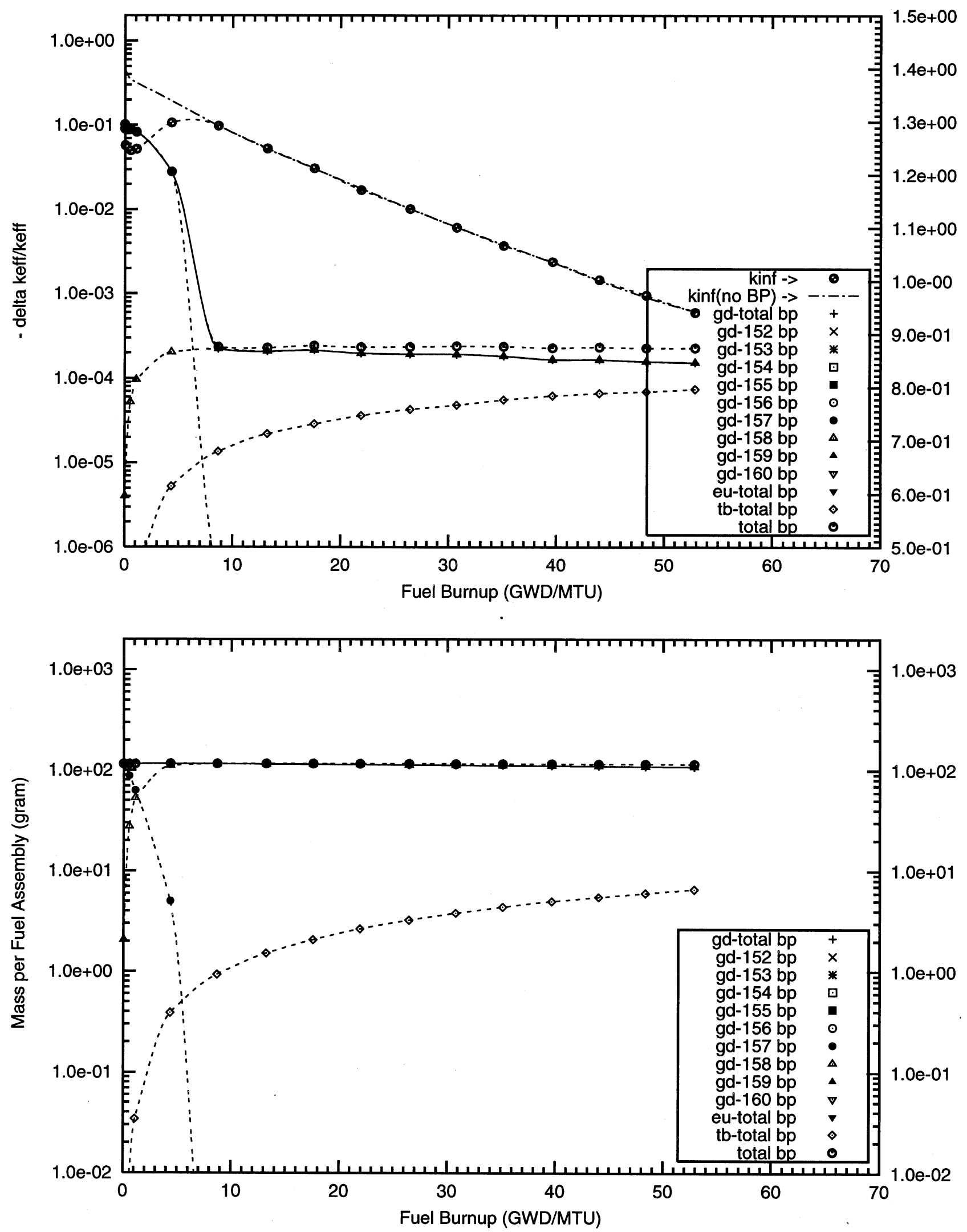
Figure A4.18. Negative Reactivity and Masses BP for Gd-157 and transmutation daughters as a function of fuel life for $17 \times 17$ fuel assemblies with 104 poison rods,

$0.05 \mathrm{w} \% \mathrm{Gd}$ poison homogeneously mixed in the fuel cladding,

Reactor power $3400 \mathrm{MWth}, 193$ fuel assemblies, initial enrichment $4.5 \mathrm{w} \% \mathrm{U}-235$

(Case : gd157_10c_104_bp_fuel o)
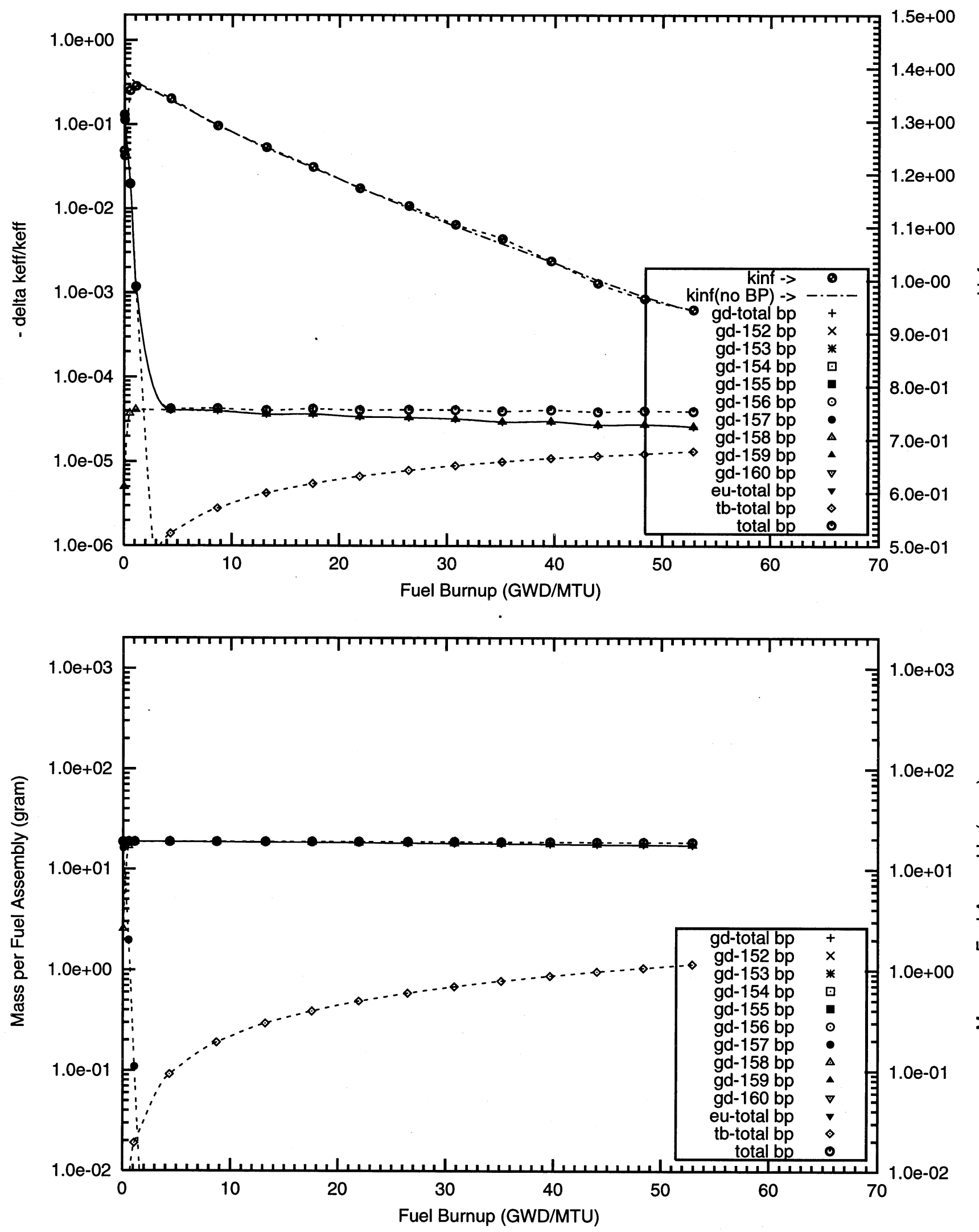
Figure A4.19. Negative Reactivity and Masses BP for Gd-157 and transmutation daughters as a function of fuel life for $17 \times 17$ fuel assemblies with 104 poison rods,

$0.1 \mathrm{w} \% \mathrm{Gd}$ poison homogeneously mixed in the fuel cladding

Reactor power $3400 \mathrm{MWth}, 193$ fuel assemblies, initial enrichment 4.5w\%U-235

(Case : gd157_9c_104_bp_fuel o)
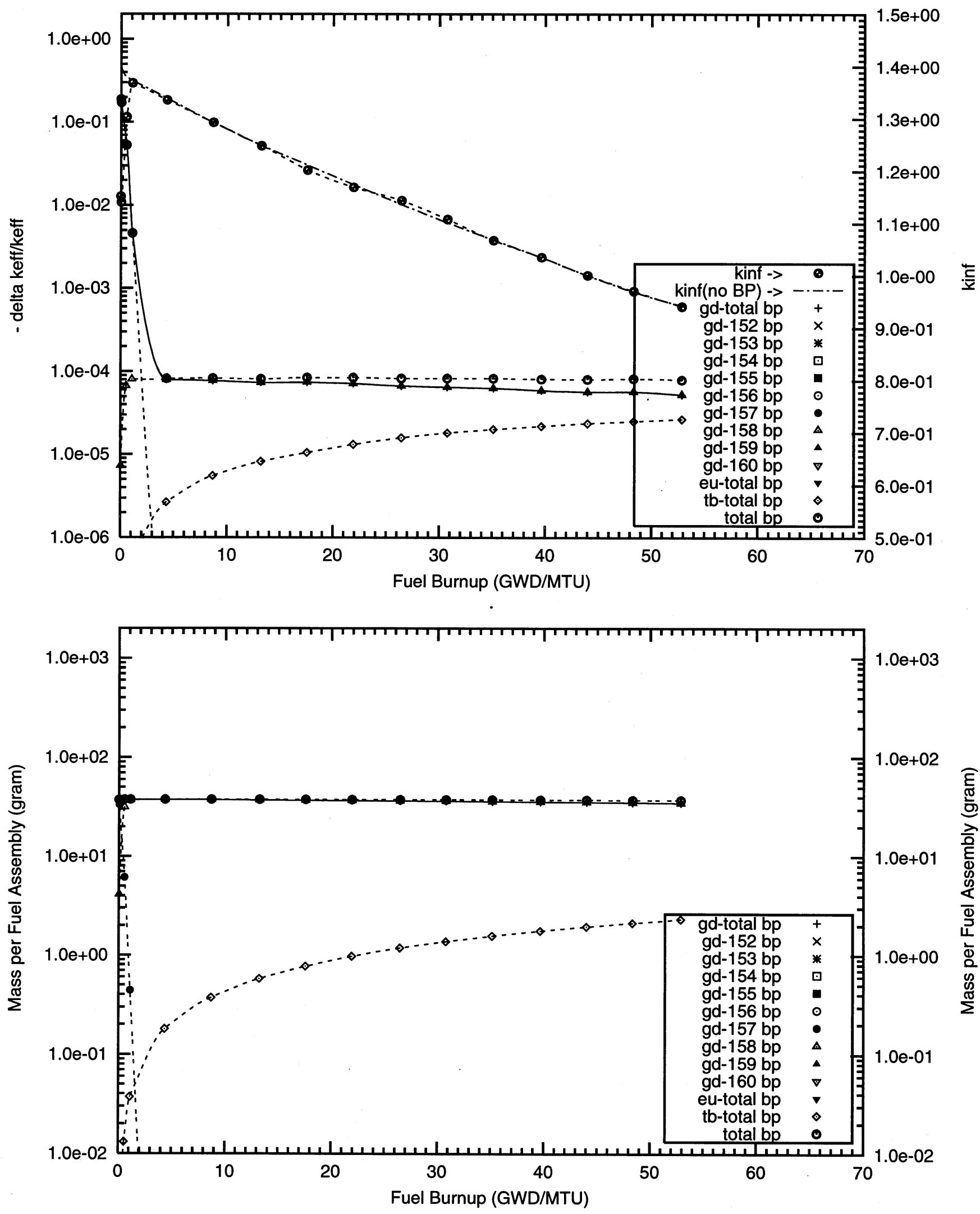


\title{
Appendix B1
}

Reactivity Worths and Masses of $\mathrm{Sm}_{2} \mathrm{O}_{3}$ Burnable Poison Homogeneously Mixed in the Fuel Pellets

\author{
Table 9 Cases
}





\section{B1 - Samarium with Natural Isotopic Abundances}



Figure B1.1. Negative Reactivity and Masses BP for Sm-nat and transmutation daughters as a function of fuel life for $17 \times 17$ fuel assemblies with 08 poison rods,

$4.0 \mathrm{w} \% \mathrm{Sm} 2 \mathrm{O} 3$ poison homogeneously mixed in the UO2 pellets

Reactor power $3400 \mathrm{MWth}, 193$ fuel assemblies, initial enrichment $4.5 \mathrm{w} \% \mathrm{U}-235$

(Case : sm000_3_08_bp_fuel o)

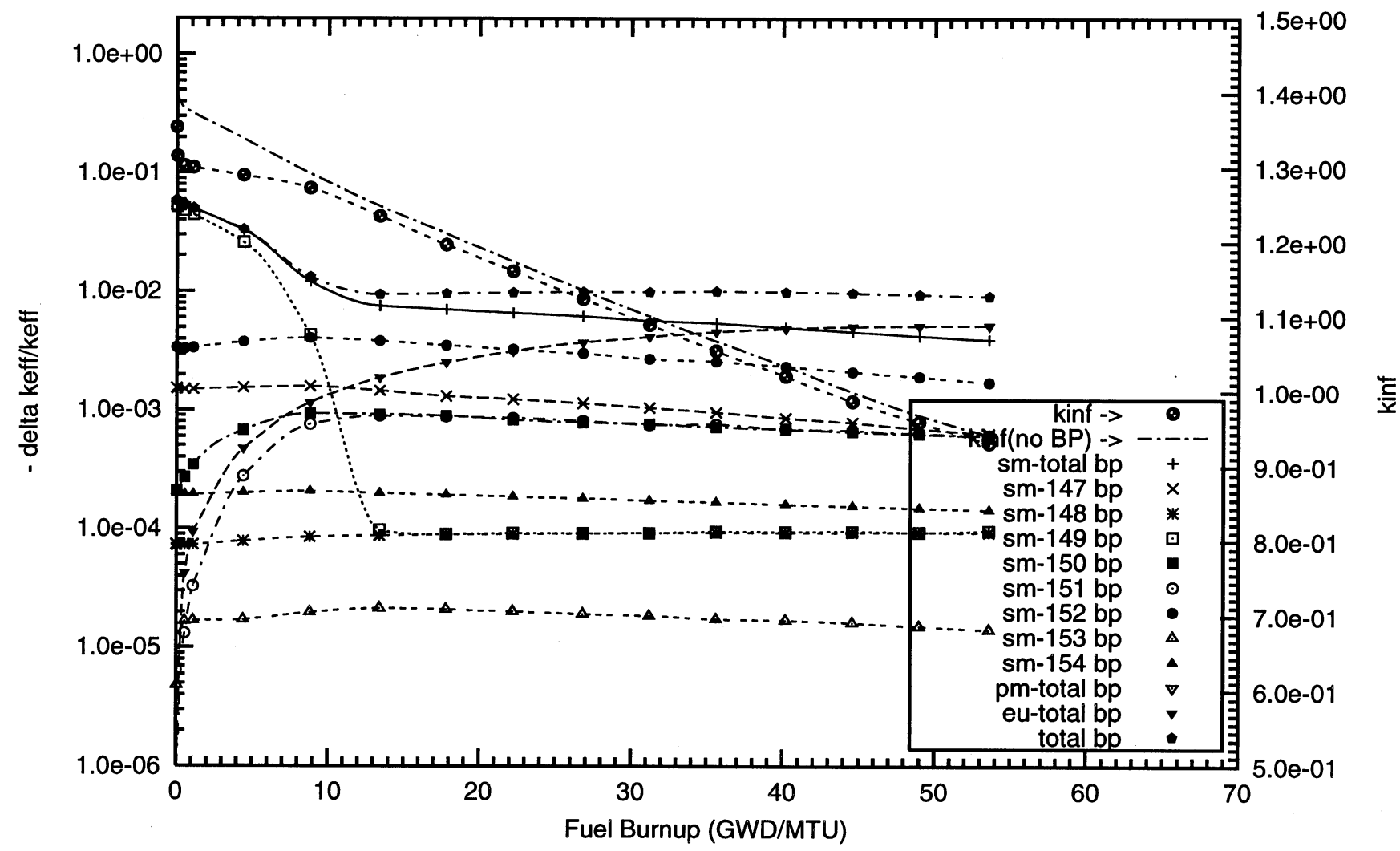


Figure B1.2. Negative Reactivity and Masses BP for Sm-nat and transmutation daughters as a function of fuel life for $17 \times 17$ fuel assemblies with 08 poison rods,

$8.0 \mathrm{w} \% \mathrm{Sm} 2 \mathrm{O} 3$ poison homogeneously mixed in the UO2 pellets

Reactor power $3400 \mathrm{MWth}, 193$ fuel assemblies, initial enrichment $4.5 \mathrm{w} \% \mathrm{U}-235$

(Case : sm000_4_100p_h20_08_bp_fuel_2_o)
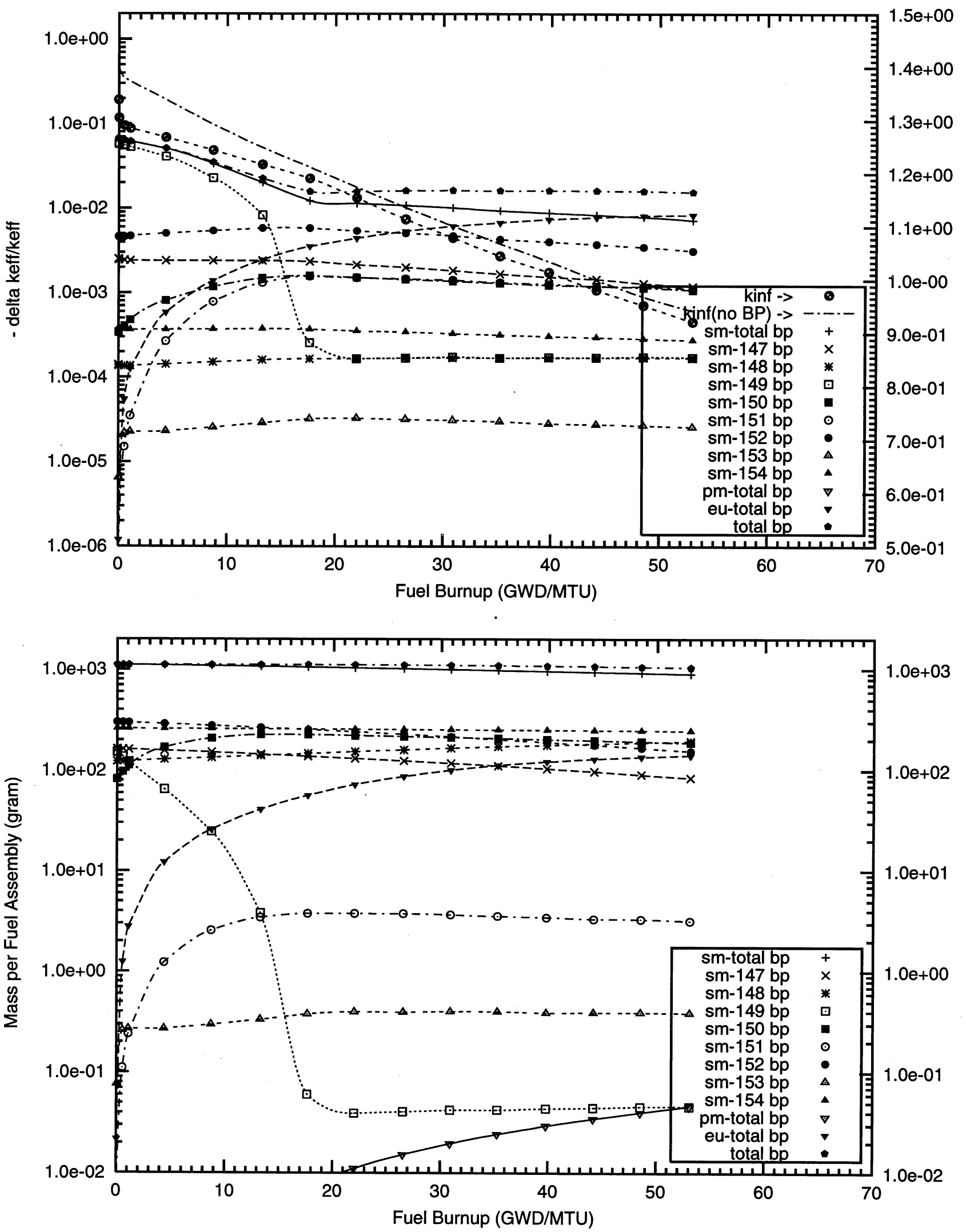
Figure B1.3. Negative Reactivity and Masses BP for Sm-nat and transmutation daughters

as a function of fuel life for $17 \times 17$ fuel assemblies with 16 poison rods,

$0.5 \mathrm{w} \%$ Sm2O3 poison homogeneously mixed in the UO2 pellets

Reactor power $3400 \mathrm{MW}$ th, 193 fuel assemblies, initial enrichment $4.5 \mathrm{w} \% \mathrm{U}-235$

(Case : sm000_0_100p_h2o_16_bp_fuel_2_o)
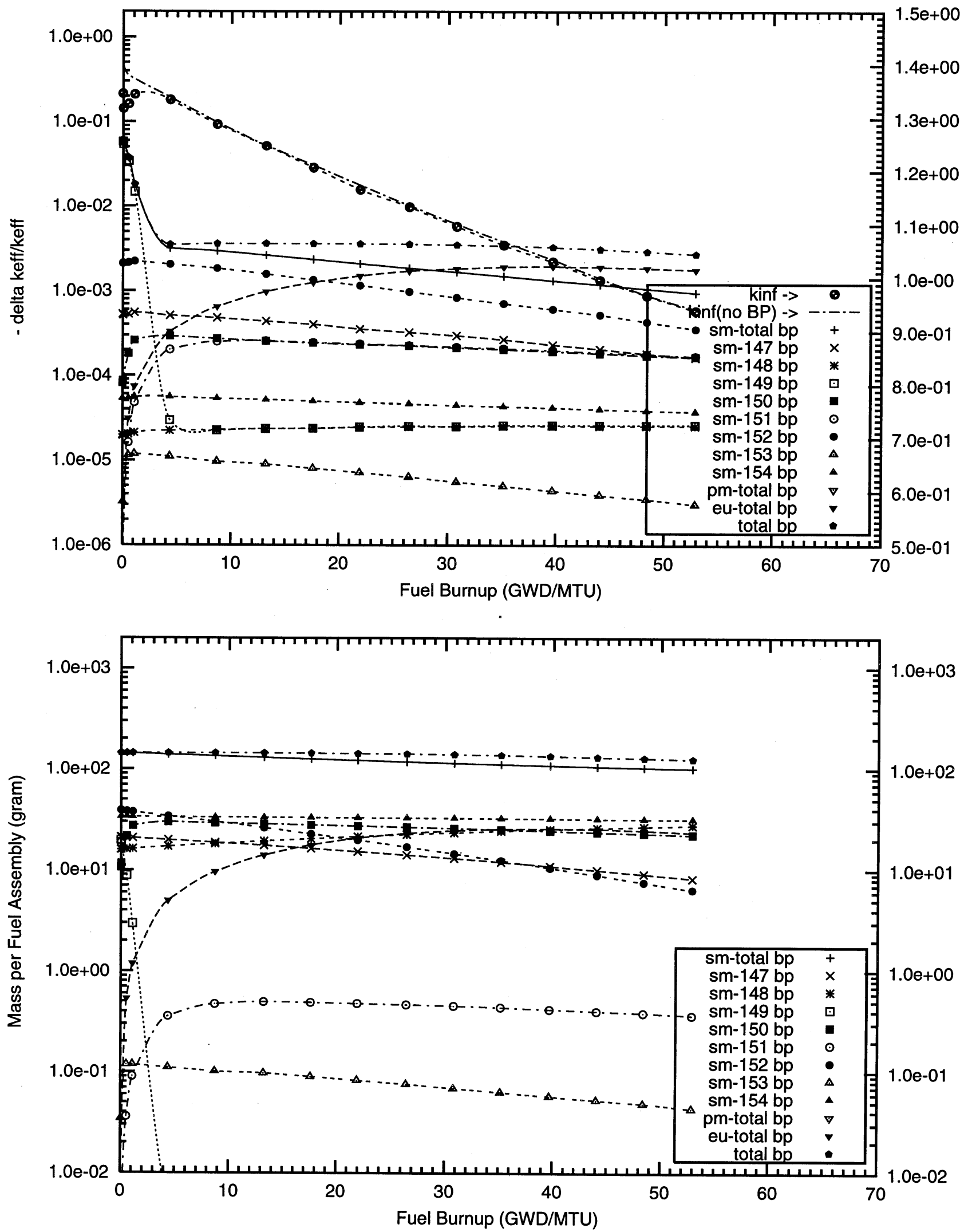
Figure B1.4. Negative Reactivity and Masses BP for Sm-nat and transmutation daughters as a function of fuel life for $17 \times 17$ fuel assemblies with 16 poison rods,

$1.0 \mathrm{w} \% \mathrm{Sm} 2 \mathrm{O} 3$ poison homogeneously mixed in the UO2 pellets

Reactor power $3400 \mathrm{MWth}, 193$ fuel assemblies, initial enrichment $4.5 \mathrm{w} \% \mathrm{U}-235$

(Case : sm000_1_100p_h2o_16_bp_fuel_2_o)
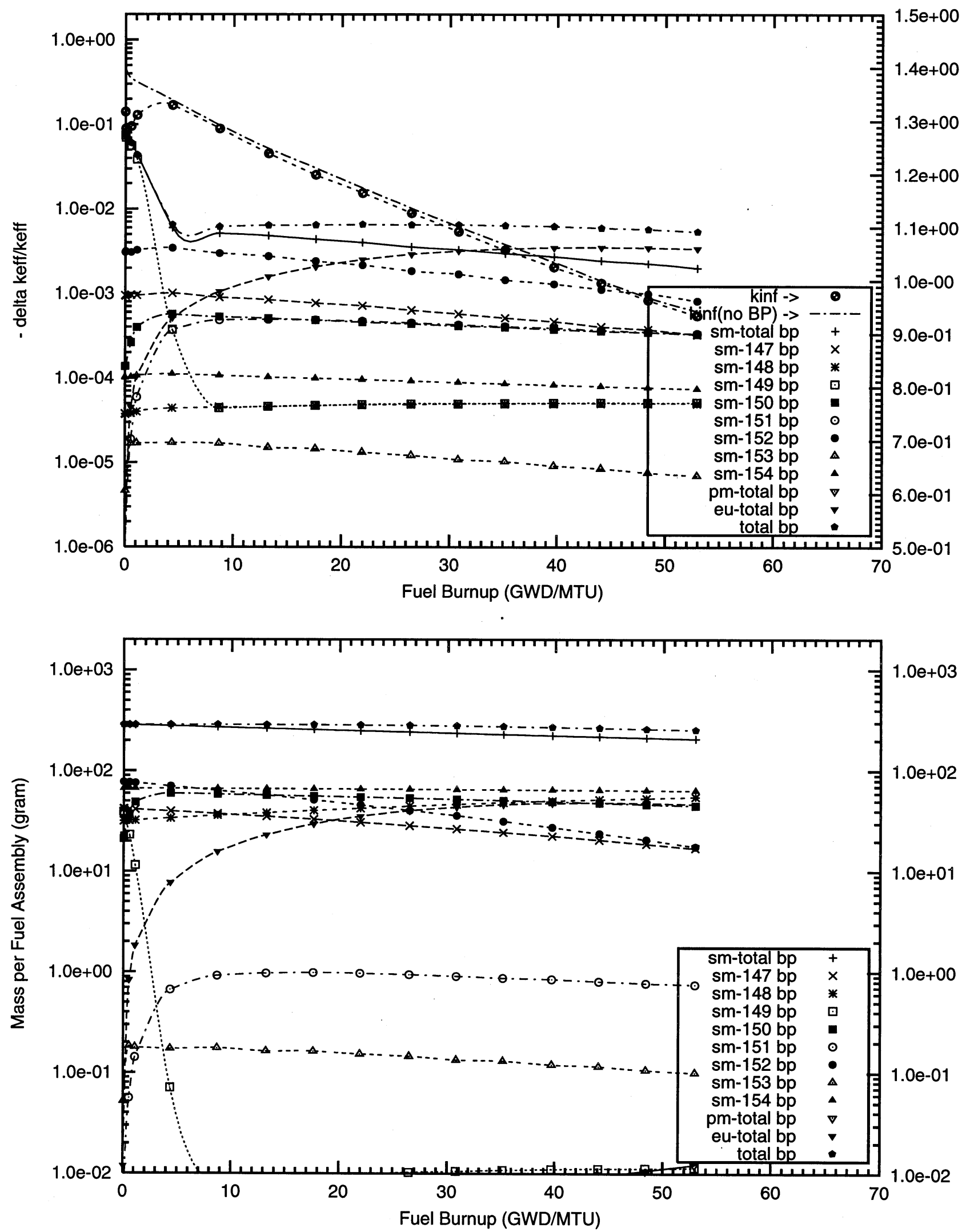
Figure B1.5. Negative Reactivity and Masses BP for Sm-nat and transmutation daughters as a function of fuel life for $17 \times 17$ fuel assemblies with 16 poison rods,

$2.0 \mathrm{w} \% \mathrm{Sm} 2 \mathrm{O} 3$ poison homogeneously mixed in the UO2 pellets

Reactor power $3400 \mathrm{MWth}, 193$ fuel assemblies, initial enrichment 4.5w\%U-235

(Case : sm000_2_100p_h2o_16_bp_fuel_2_o)
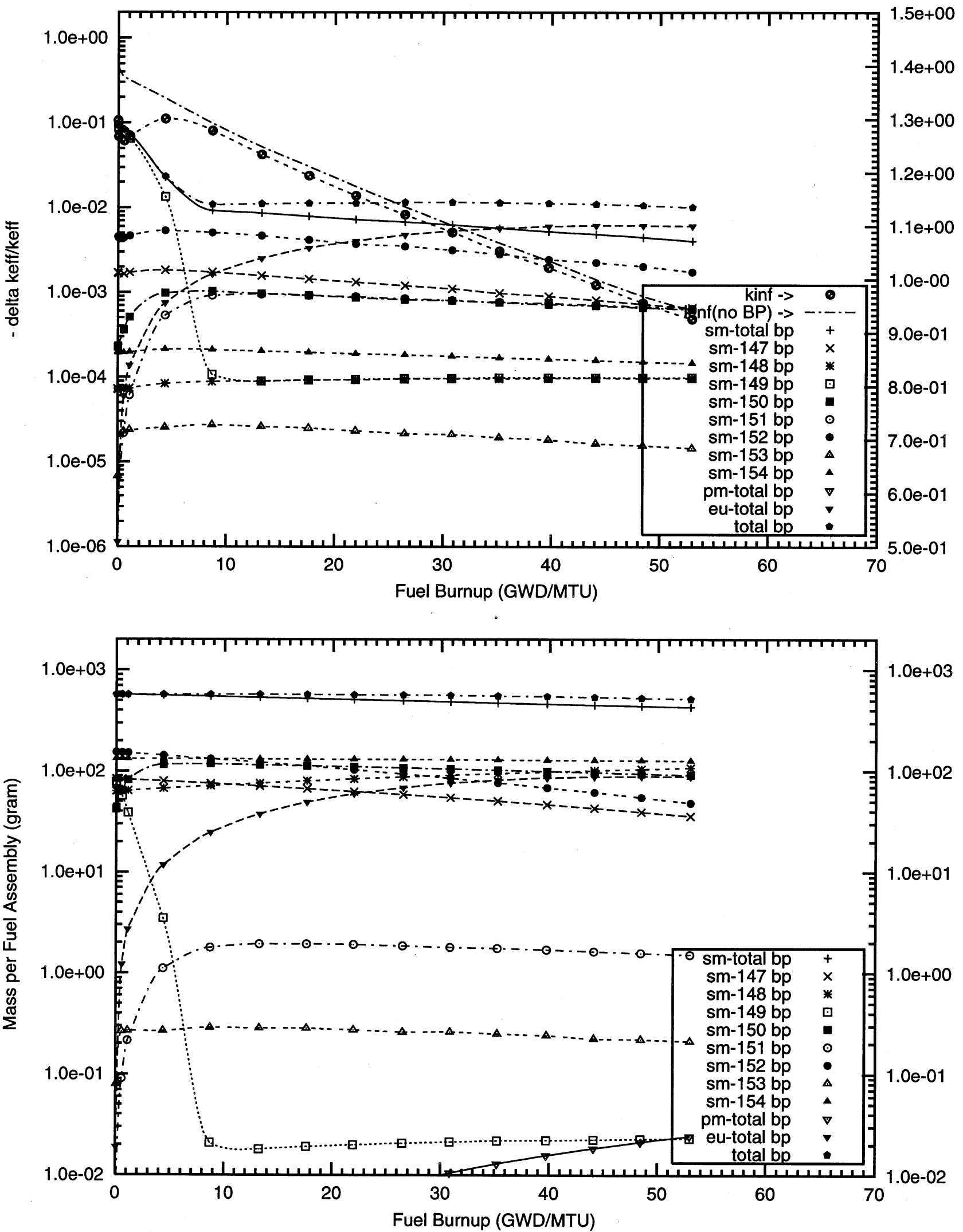
Figure B1.6. Negative Reactivity and Masses BP for Sm-nat and transmutation daughters as a function of fuel life for $17 \times 17$ fuel assemblies with 16 poison rods,

$4.0 \mathrm{w} \% \mathrm{Sm} 2 \mathrm{O} 3$ poison homogeneously mixed in the UO2 pellets

Reactor power $3400 \mathrm{MWth}, 193$ fuel assemblies, initial enrichment 4.5w\%U-235

(Case : sm000_3_100p_h2o_16_bp_fuel_2_o)
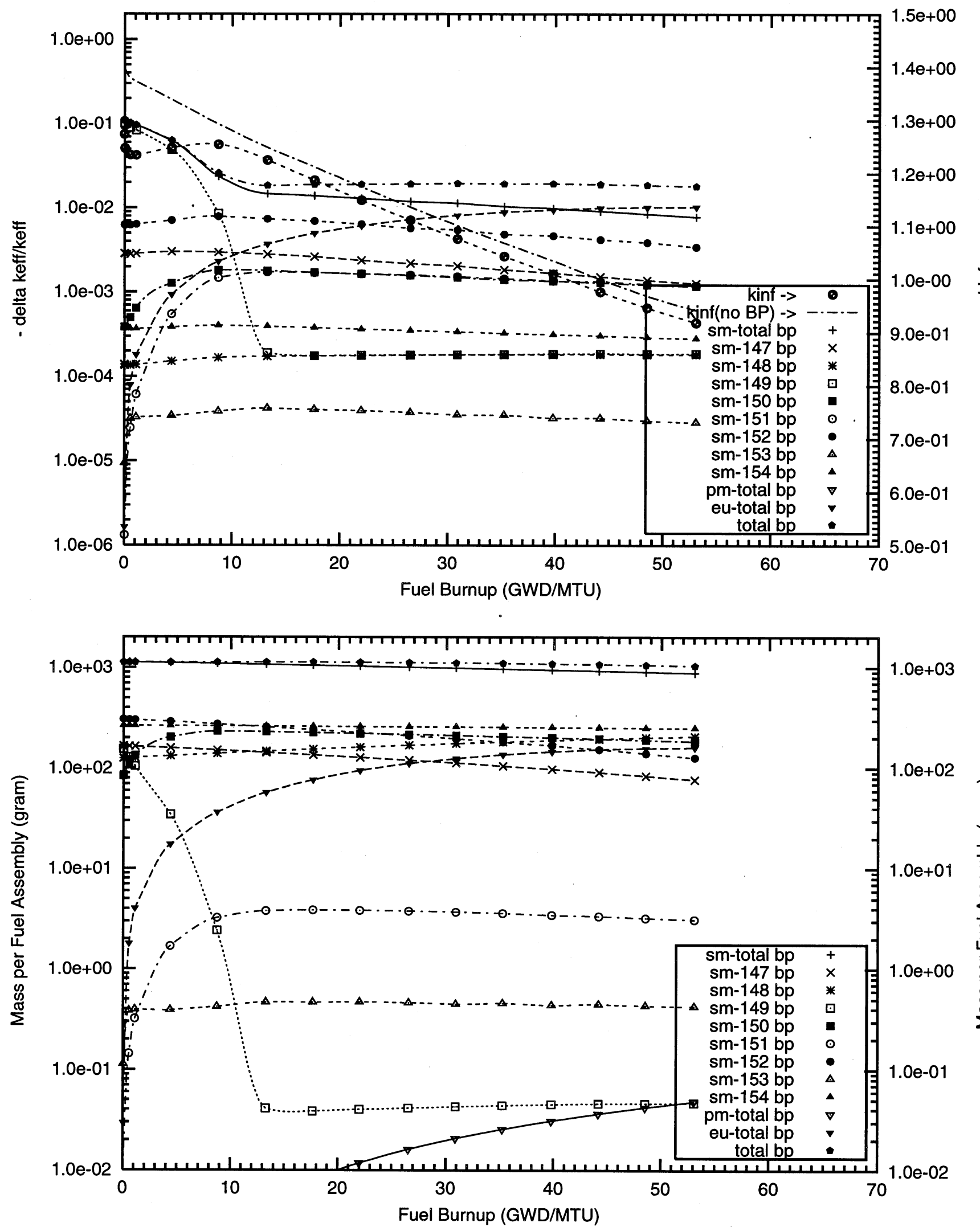
Figure B1.7. Negative Reactivity and Masses BP for Sm-nat and transmutation daughters as a function of fuel life for $17 \times 17$ fuel assemblies with 16 poison rods,

$8.0 \mathrm{w} \% \mathrm{Sm} 2 \mathrm{O} 3$ poison homogeneously mixed in the UO2 pellets

Reactor power $3400 \mathrm{MWth}, 193$ fuel assemblies, initial enrichment $4.5 \mathrm{w} \% \mathrm{U}-235$

(Case : sm000_4_100p_h2o_16_bp_fuel_2_o)
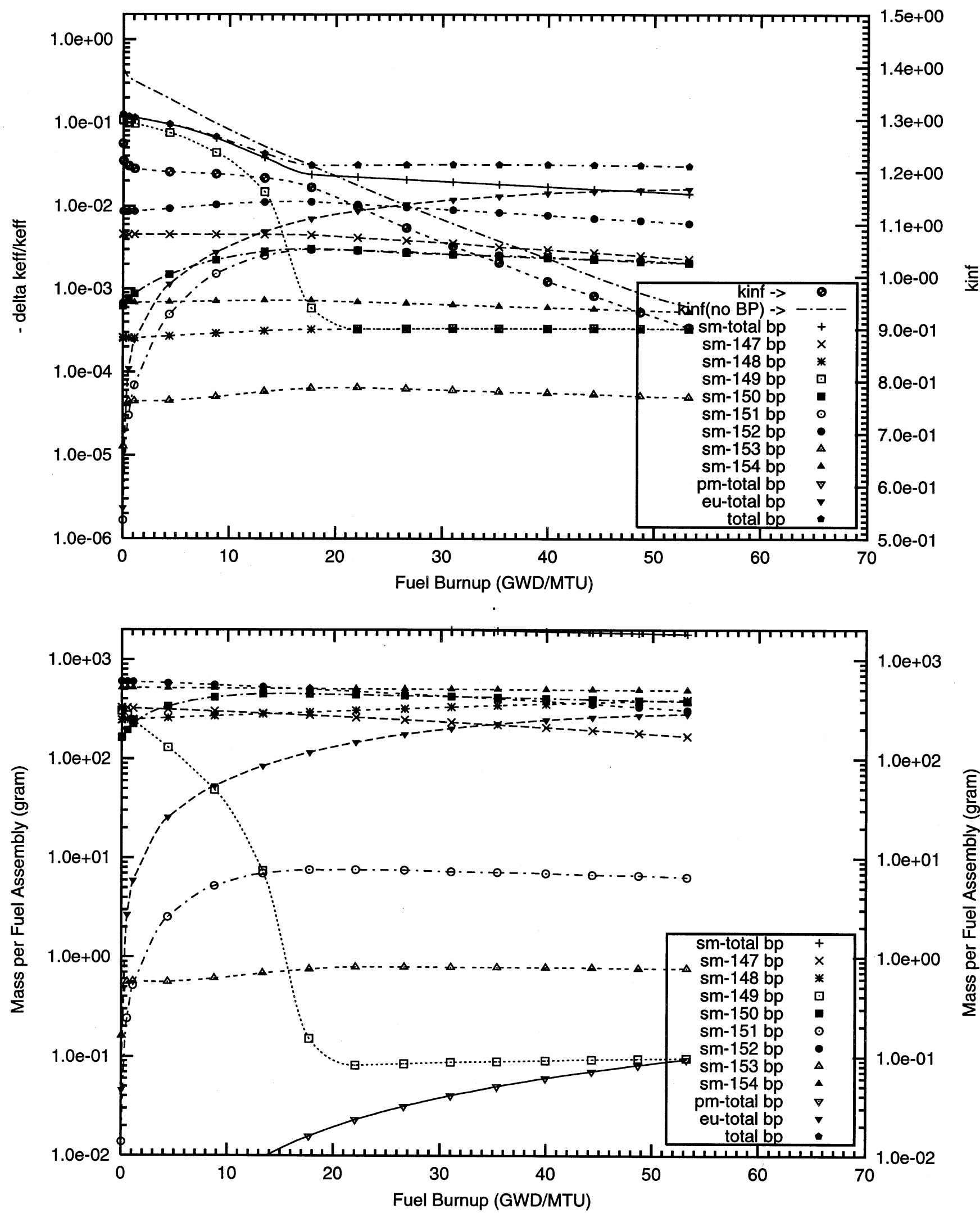
Figure B1.8. Negative Reactivity and Masses BP for Sm-nat and transmutation daughters as a function of fuel life for $17 \times 17$ fuel assemblies with 20 poison rods, $2.0 \mathrm{w} \% \mathrm{Sm} 2 \mathrm{O} 3$ poison homogeneously mixed in the UO2 pellets

Reactor power $3400 \mathrm{MWth}, 193$ fuel assemblies, initial enrichment $4.5 \mathrm{w} \% \mathrm{U}-235$

(Case : sm000_2_16_bp_fuel (old) o)

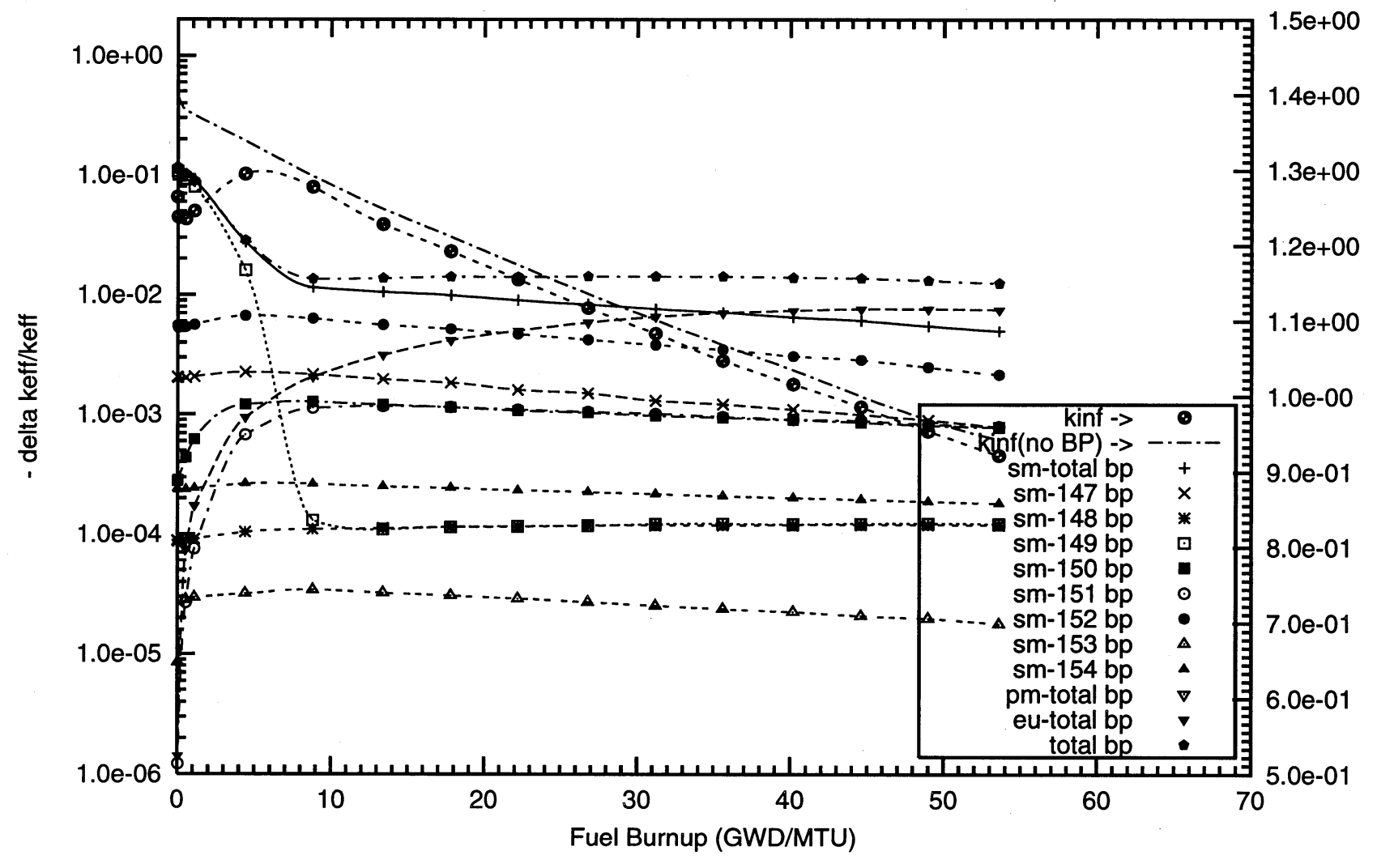


Figure B1.9. Negative Reactivity and Masses BP for Sm-nat and transmutation daughters as a function of fuel life for $17 \times 17$ fuel assemblies with 20 poison rods,

$4.0 \mathrm{w} \% \mathrm{Sm} 2 \mathrm{O} 3$ poison homogeneously mixed in the UO2 pellets

Reactor power $3400 \mathrm{MWth}, 193$ fuel assemblies, initial enrichment 4.5w\%U-235

(Case : sm000_3_16_bp_fuel o)

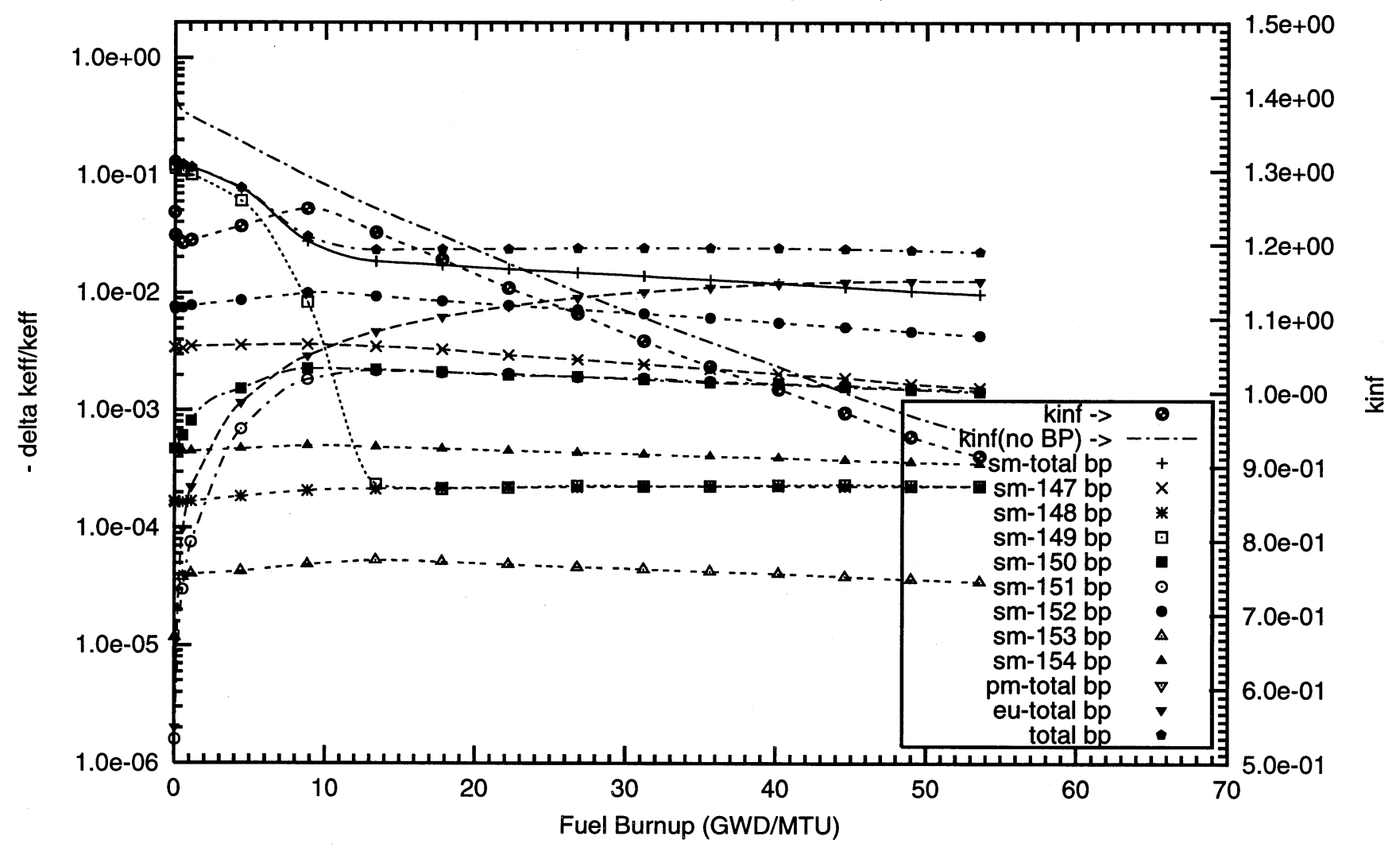


Figure B1.10. Negative Reactivity and Masses BP for Sm-nat and transmutation daughters as a function of fuel life for $17 \times 17$ fuel assemblies with 64 poison rods,

$0.1 \mathrm{w} \% \mathrm{Sm} 2 \mathrm{O} 3$ poison homogeneously mixed in the UO2 pellets

Reactor power $3400 \mathrm{MWth}, 193$ fuel assemblies, initial enrichment $4.5 \mathrm{~W} \% \mathrm{U}-235$

(Case : sm000_9_100p_h2o_64_bp_fuel_2_o)

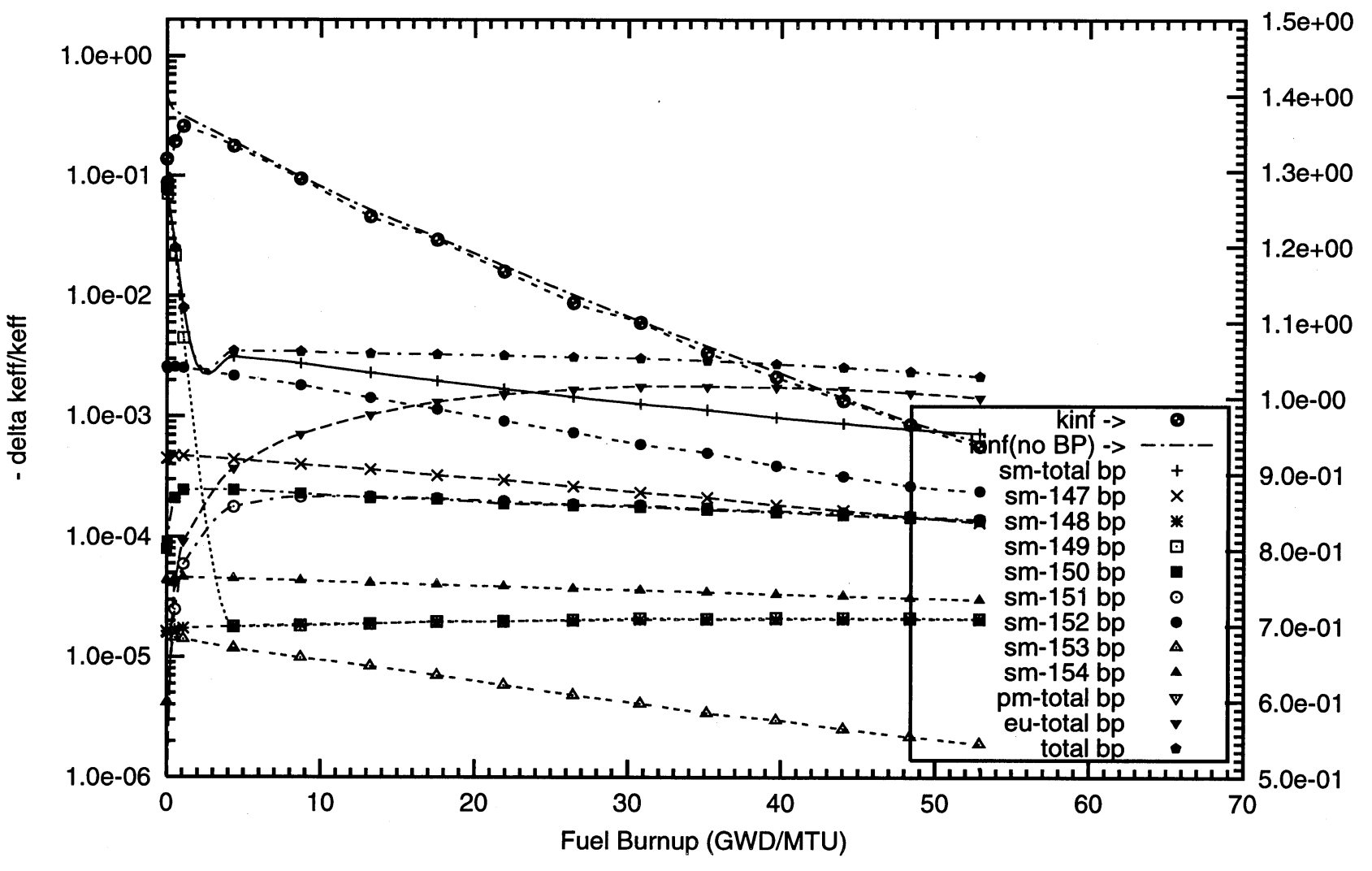

言

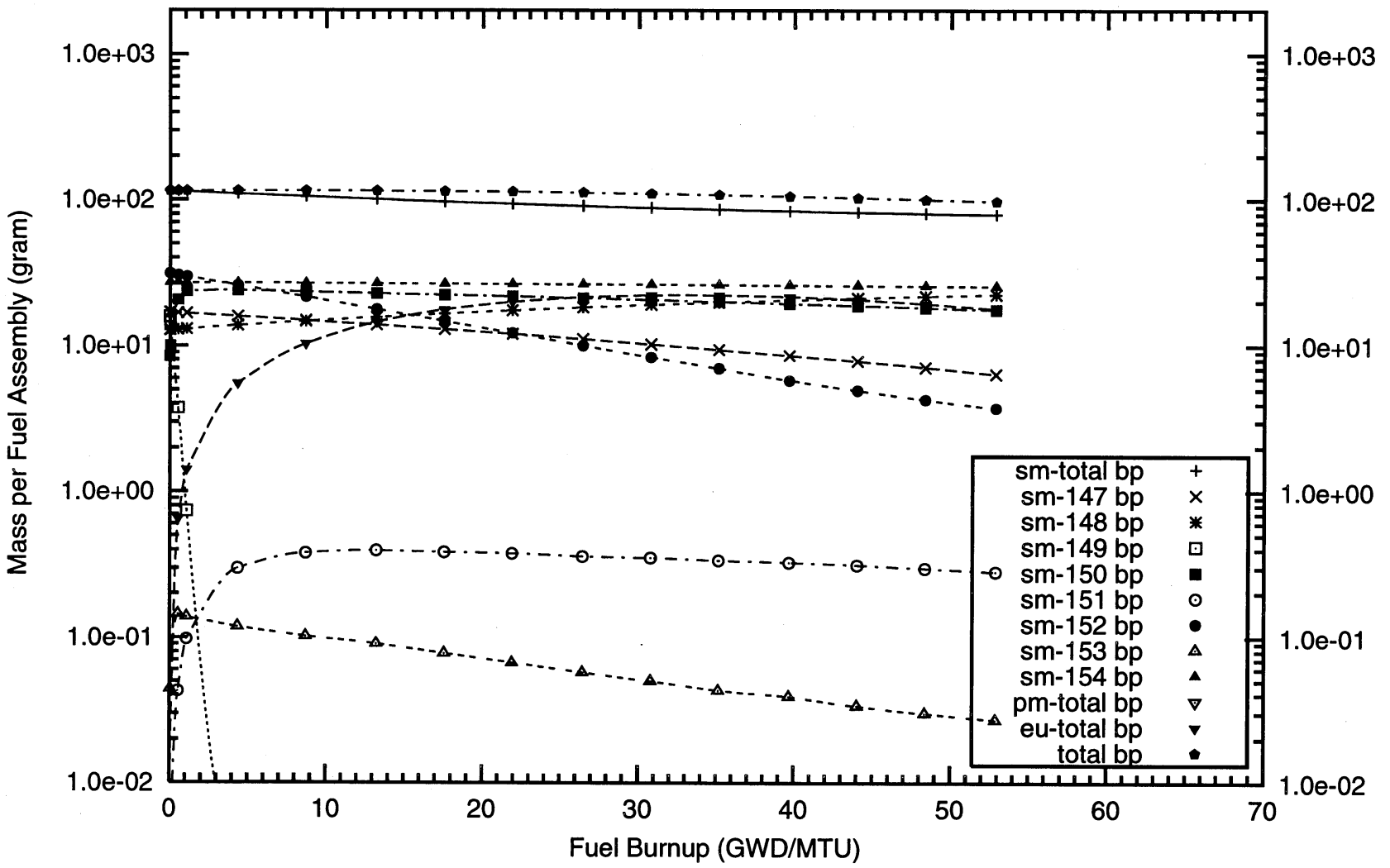


Figure B1.11. Negative Reactivity and Masses BP for Sm-nat and transmutation daughters as a function of fuel life for $17 \times 17$ fuel assemblies with 64 poison rods, $0.2 \mathrm{w} \% \mathrm{Sm} 2 \mathrm{O} 3$ poison homogeneously mixed in the UO2 pellets

Reactor power $3400 \mathrm{MWth}, 193$ fuel assemblies, initial enrichment $4.5 \mathrm{w} \% \mathrm{U}-235$

(Case : sm000_8_64_bp_fuel o)
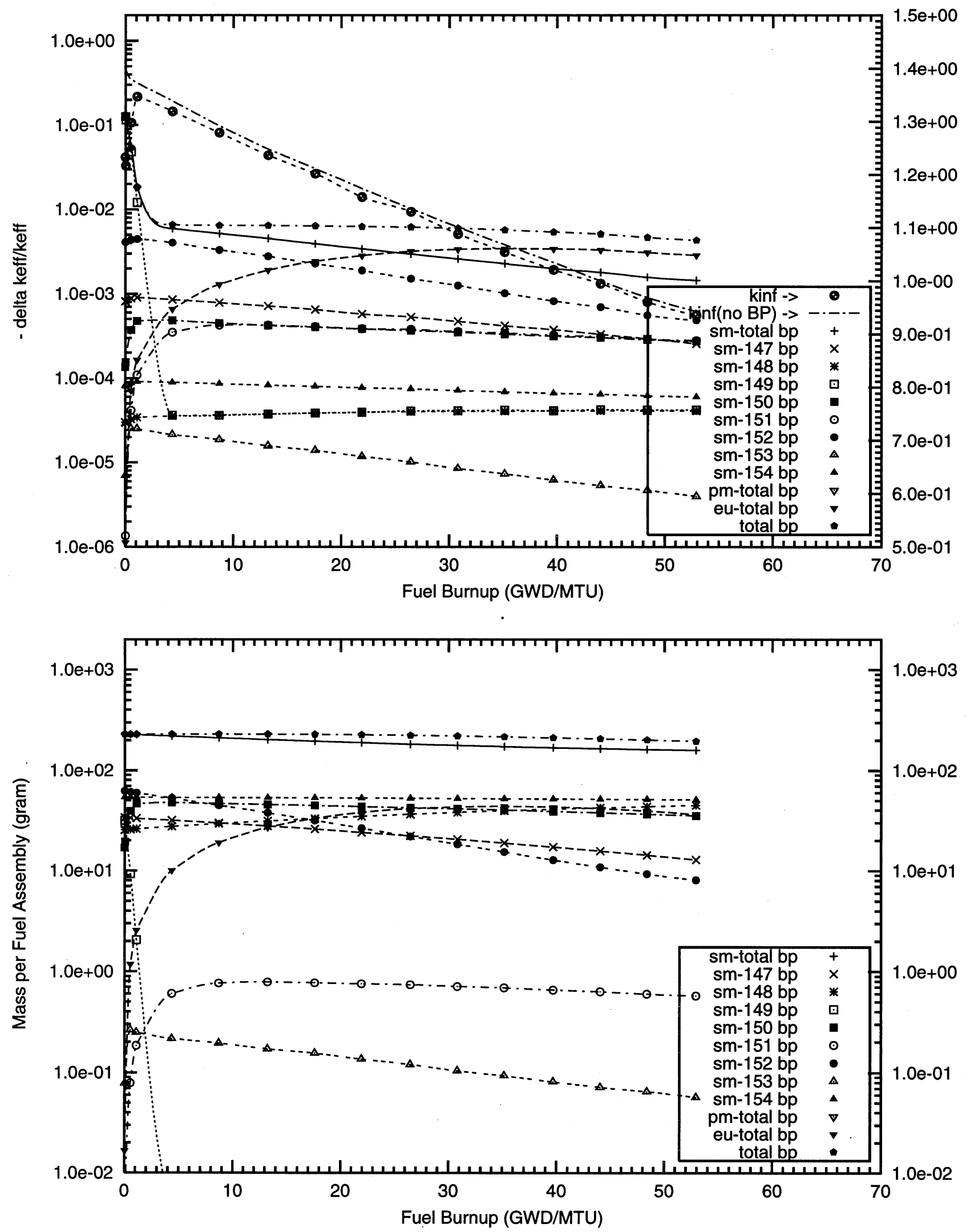
Figure B1.12. Negative Reactivity and Masses BP for Sm-nat and transmutation daughters as a function of fuel life for $17 \times 17$ fuel assemblies with 64 poison rods,

$0.5 \mathrm{w} \% \mathrm{Sm} 2 \mathrm{O} 3$ poison homogeneously mixed in the UO2 pellets

Reactor power $3400 \mathrm{MWth}, 193$ fuel assemblies, initial enrichment $4.5 \mathrm{w} \% \mathrm{U}-235$

(Case : sm000_0_64_bp_fuel o)
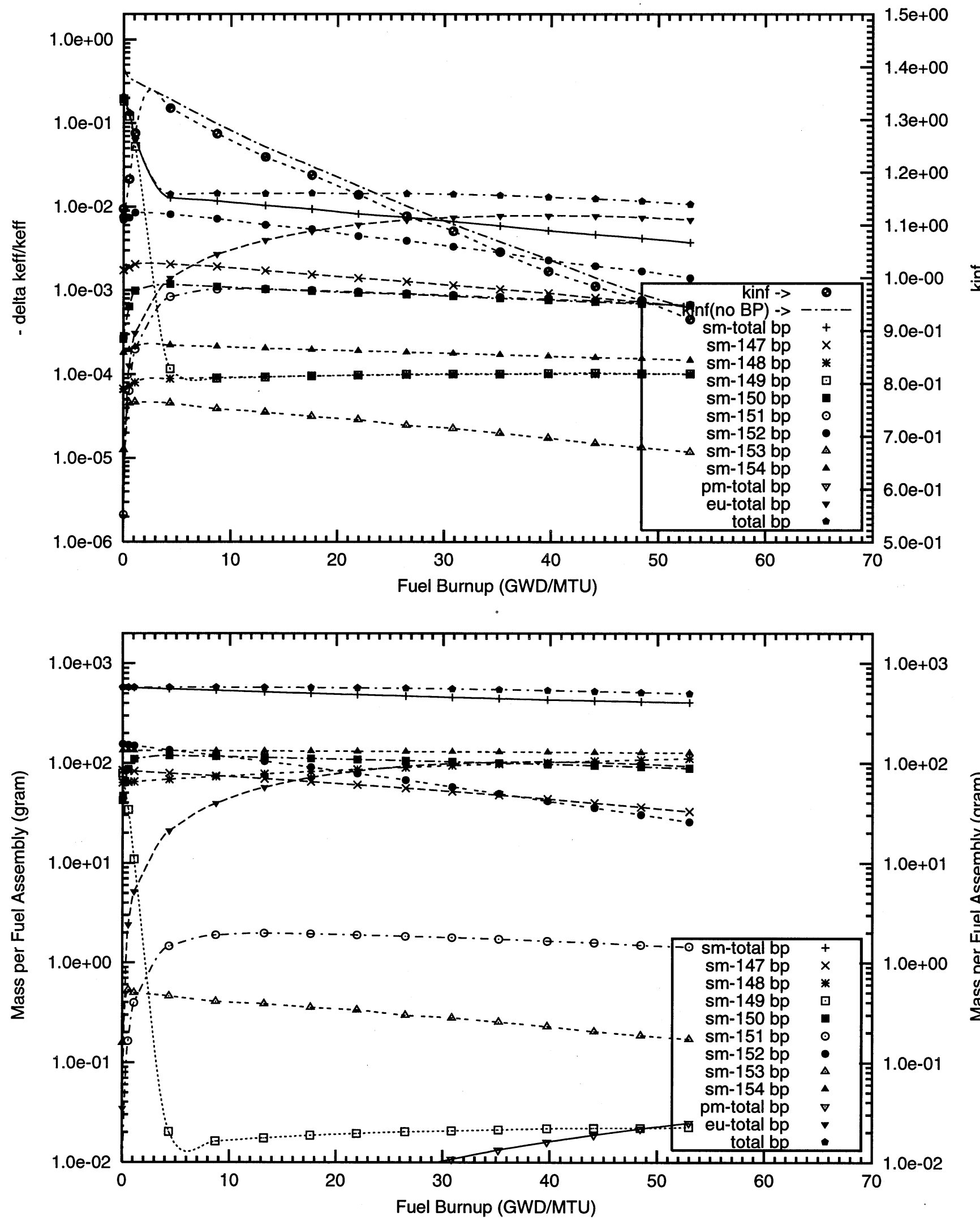
Figure B1.13. Negative Reactivity and Masses BP for Sm-nat and transmutation daughters as a function of fuel life for $17 \times 17$ fuel assemblies with 64 poison rods, $1.0 \mathrm{w} \% \mathrm{Sm} 2 \mathrm{O} 3$ poison homogeneously mixed in the UO2 pellets

Reactor power $3400 \mathrm{MW}$ th, 193 fuel assemblies, initial enrichment $4.5 \mathrm{w} \% \mathrm{U}-235$

(Case : sm000_1_64_bp_fuel o)
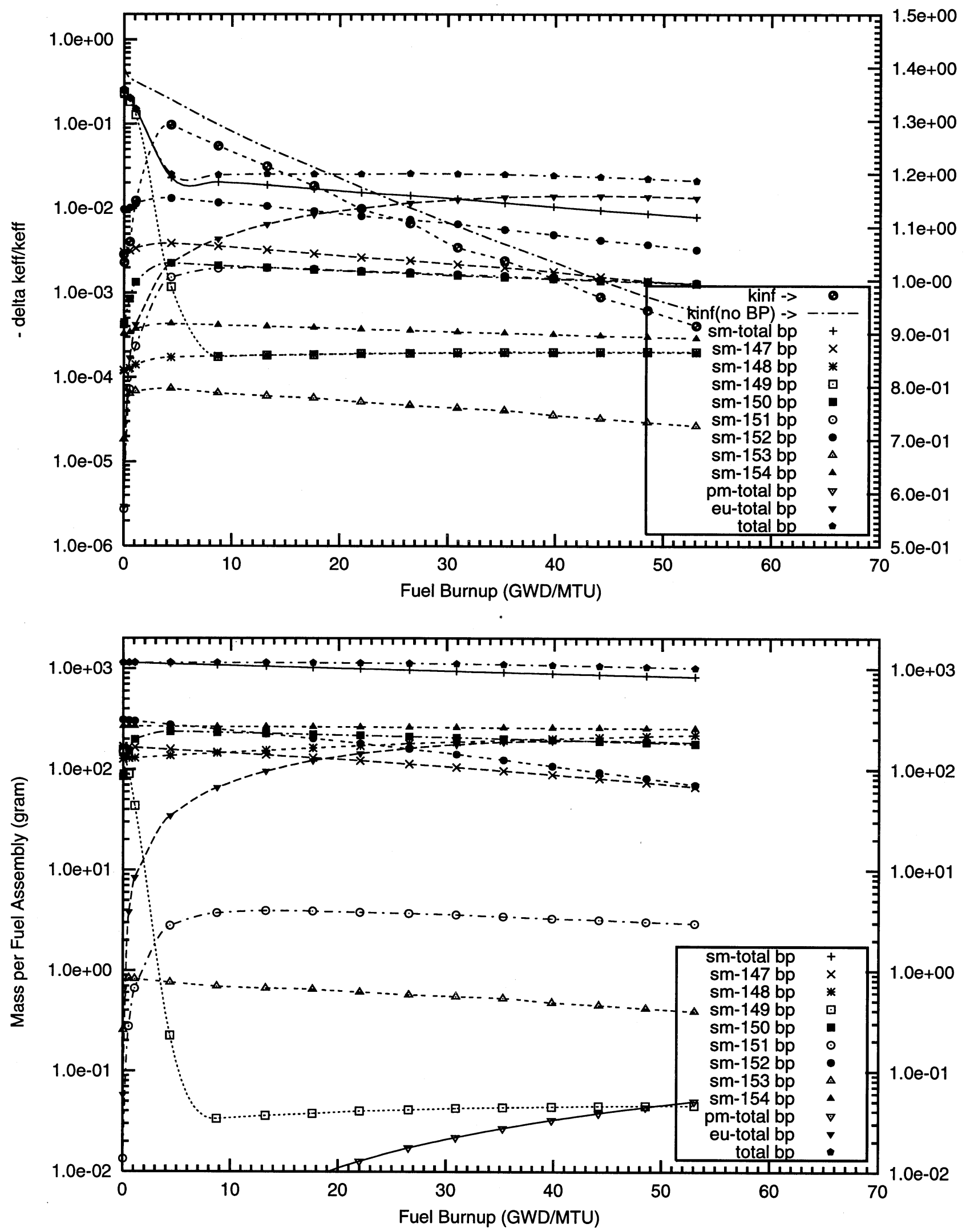



\section{B1 - Samarium Fully Enriched in ${ }^{149} \mathrm{Sm}$}



Figure B1.14. Negative Reactivity and Masses BP for Sm-149 and transmutation daughters as a function of fuel life for $17 \times 17$ fuel assemblies with 08 poison rods,

$1.0 \mathrm{w} \% \mathrm{Sm} 2 \mathrm{O} 3$ poison homogeneously mixed in the UO2 pellets

Reactor power $3400 \mathrm{MWth}, 193$ fuel assemblies, initial enrichment $4.5 \mathrm{w} \% \mathrm{U}-235$

(Case : sm149_1_100p_h2o_08_bp_fuel_2_o)
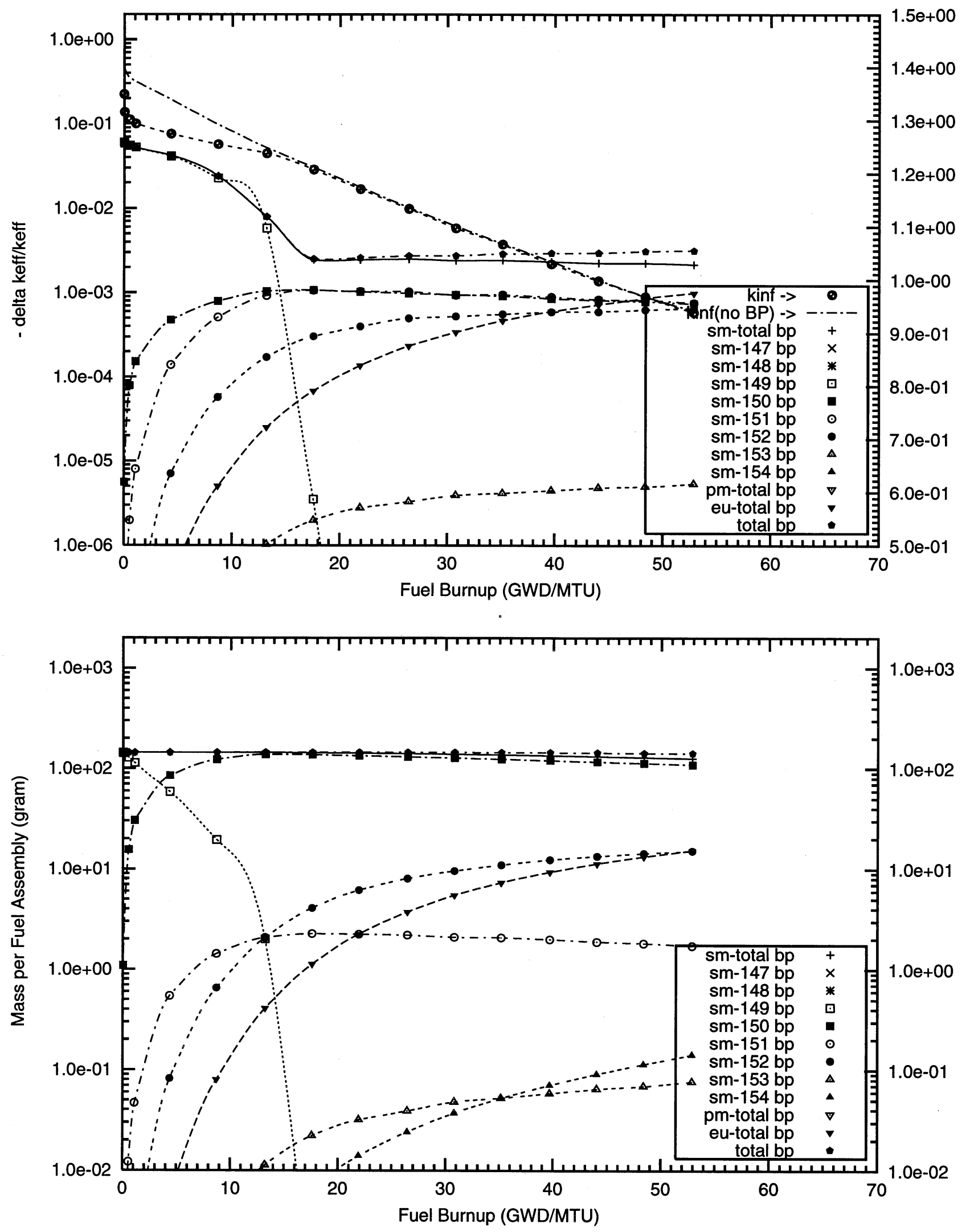
Figure B1.15. Negative Reactivity and Masses BP for Sm-149 and transmutation daughters as a function of fuel life for $17 \times 17$ fuel assemblies with 08 poison rods, $2.0 \mathrm{w} \% \mathrm{Sm} 2 \mathrm{O} 3$ poison homogeneously mixed in the UO2 pellets

Reactor power 3400 MWth, 193 fuel assemblies, initial enrichment 4.5w\%U-235

(Case : sm149_2_08_bp_fuel o)
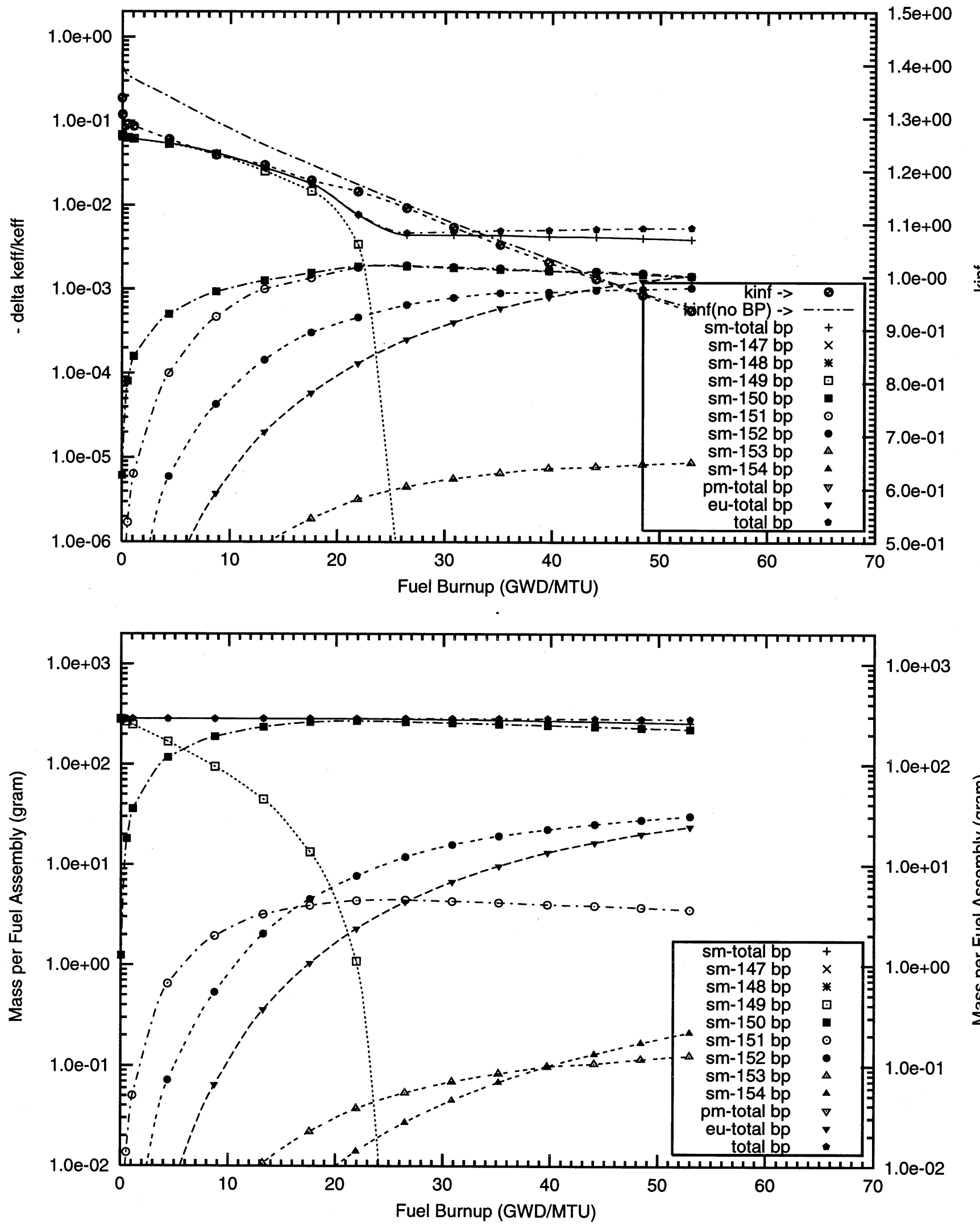
Figure B1.16. Negative Reactivity and Masses BP for Sm-149 and transmutation daughters

as a function of fuel life for $17 \times 17$ fuel assemblies with 08 poison rods,

$4.0 \mathrm{w} \% \mathrm{Sm} 2 \mathrm{O} 3$ poison homogeneously mixed in the UO2 pellets

Reactor power $3400 \mathrm{MWth}, 193$ fuel assemblies, initial enrichment 4.5W\%U-235

(Case : sm149_3_08_bp_fuel o)
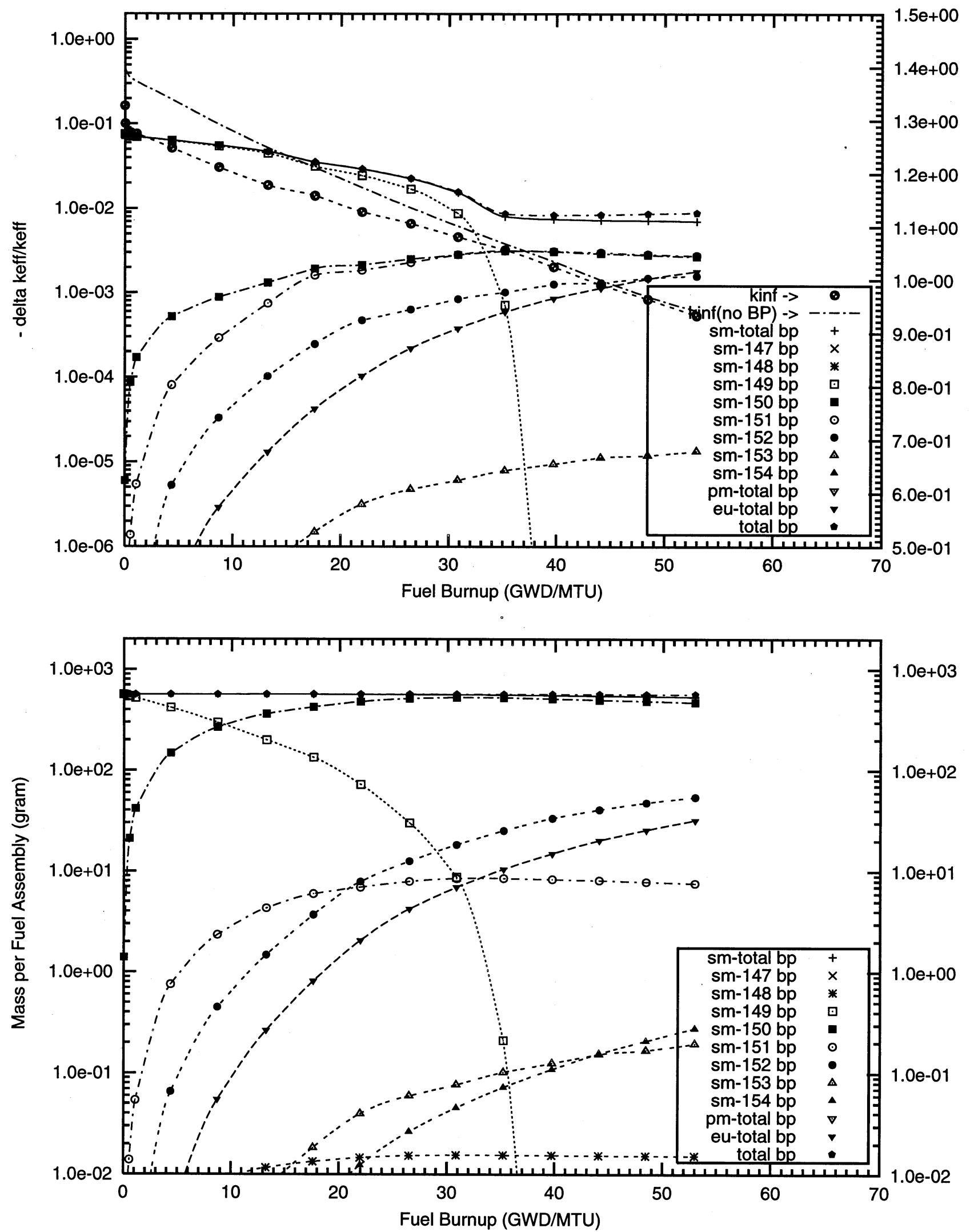
Figure B1.17. Negative Reactivity and Masses BP for Sm-149 and transmutation daughters

as a function of fuel life for $17 \times 17$ fuel assemblies with 16 poison rods,

$0.1 \mathrm{w} \% \mathrm{Sm} 2 \mathrm{O} 3$ poison homogeneously mixed in the UO2 pellets

Reactor power $3400 \mathrm{MWth}, 193$ fuel assemblies, initial enrichment $4.5 \mathrm{w} \% \mathrm{U}-235$

(Case : sm149_9_100p_h2o_16_bp_fuel_2_o)
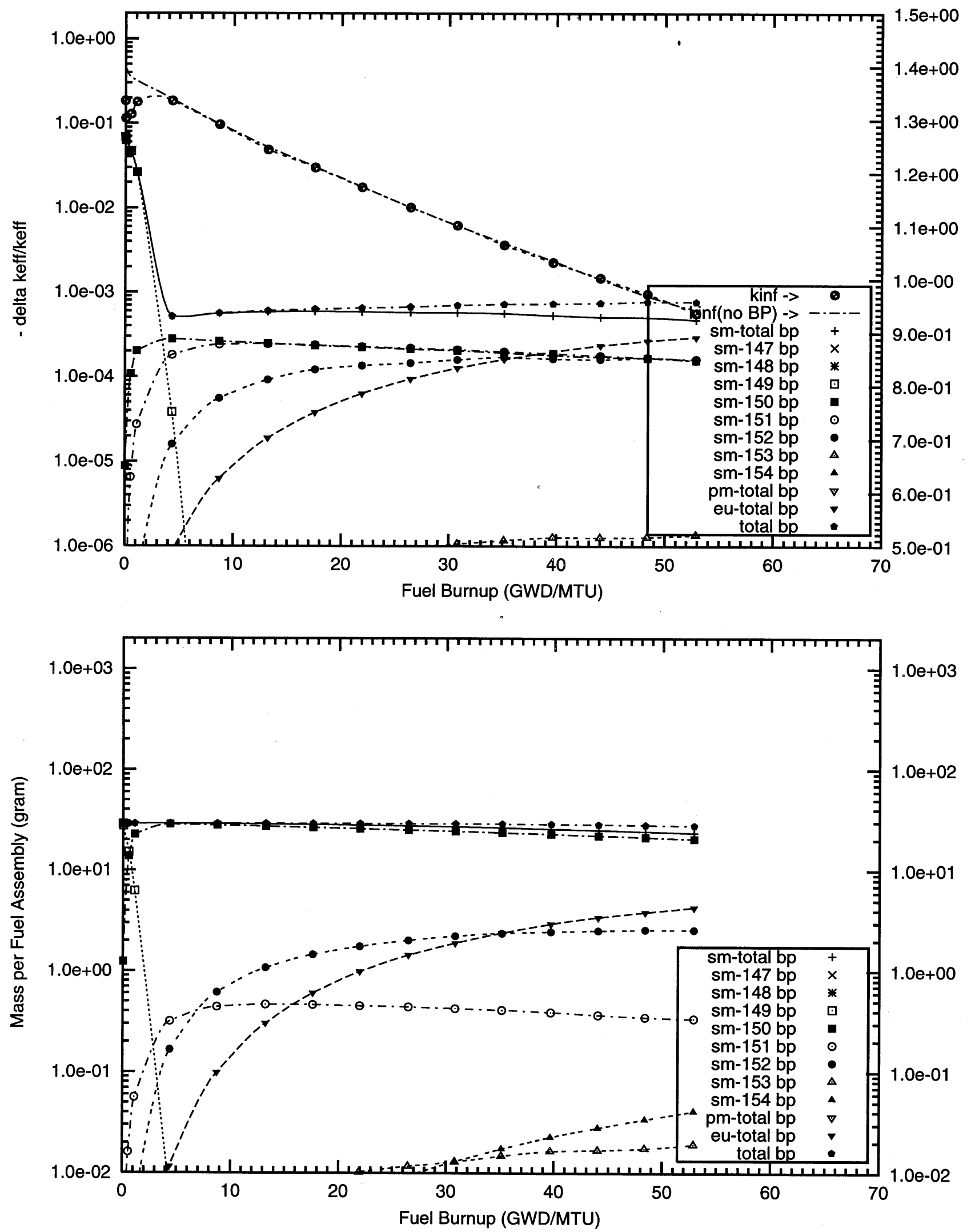
Figure B1.18. Negative Reactivity and Masses BP for Sm-149 and transmutation daughters as a function of fuel life for $17 \times 17$ fuel assemblies with 16 poison rods, $0.2 \mathrm{w} \% \mathrm{Sm} 2 \mathrm{O} 3$ poison homogeneously mixed in the UO2 pellets

Reactor power $3400 \mathrm{MWth}, 193$ fuel assemblies, initial enrichment 4.5w\%U-235

(Case : sm149_8_100p_h2o_16_bp_fuel_2_o)
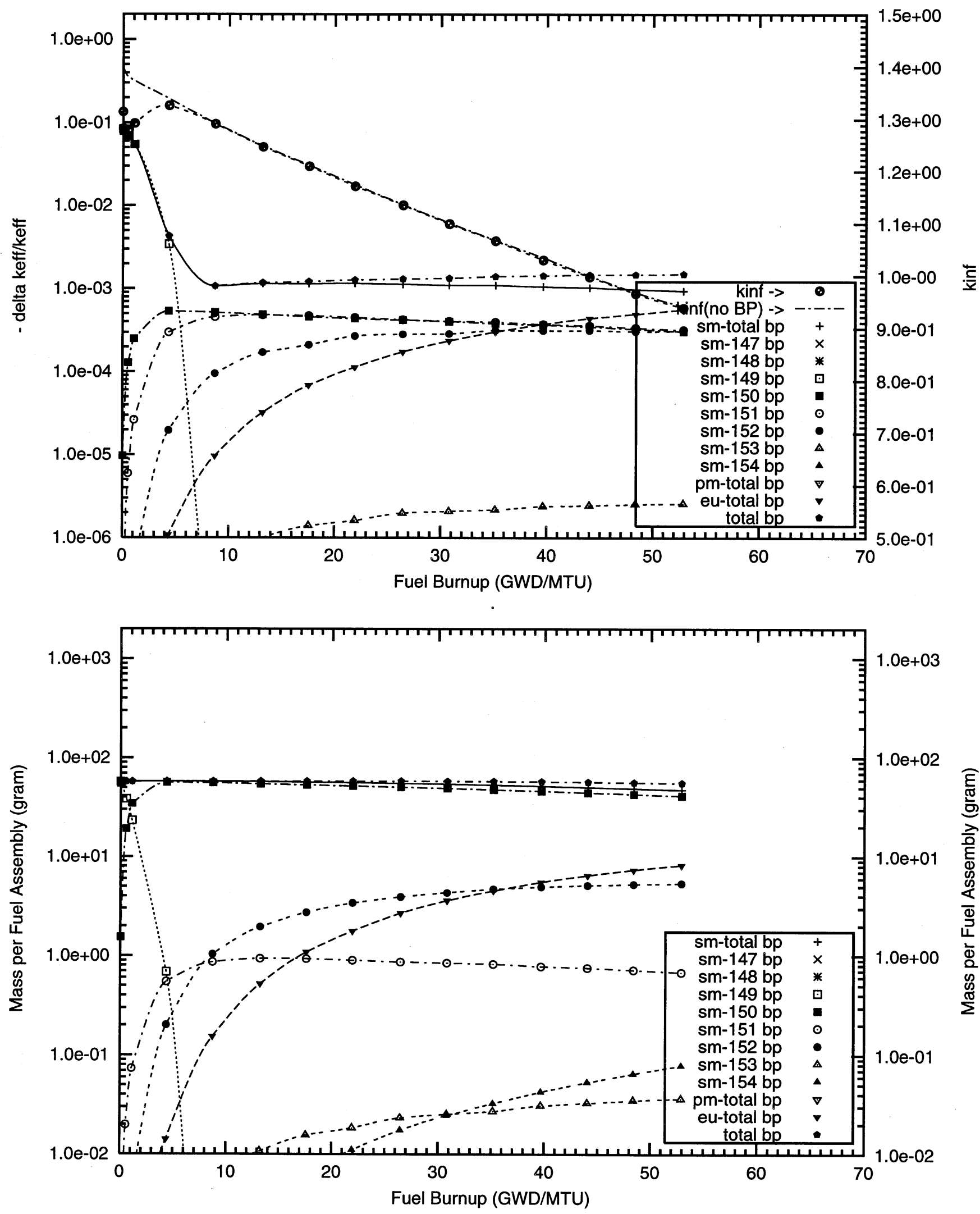
Figure B1.19. Negative Reactivity and Masses BP for Sm-149 and transmutation daughters

as a function of fuel life for $17 \times 17$ fuel assemblies with 16 poison rods,

$0.5 \mathrm{w} \% \mathrm{Sm} 2 \mathrm{O} 3$ poison homogeneously mixed in the UO2 pellets

Reactor power $3400 \mathrm{MWth}, 193$ fuel assemblies, initial enrichment $4.5 \mathrm{w} \% \mathrm{U}-235$

(Case : sm149_0_100p_h2o_16_bp_fuel_2_o)
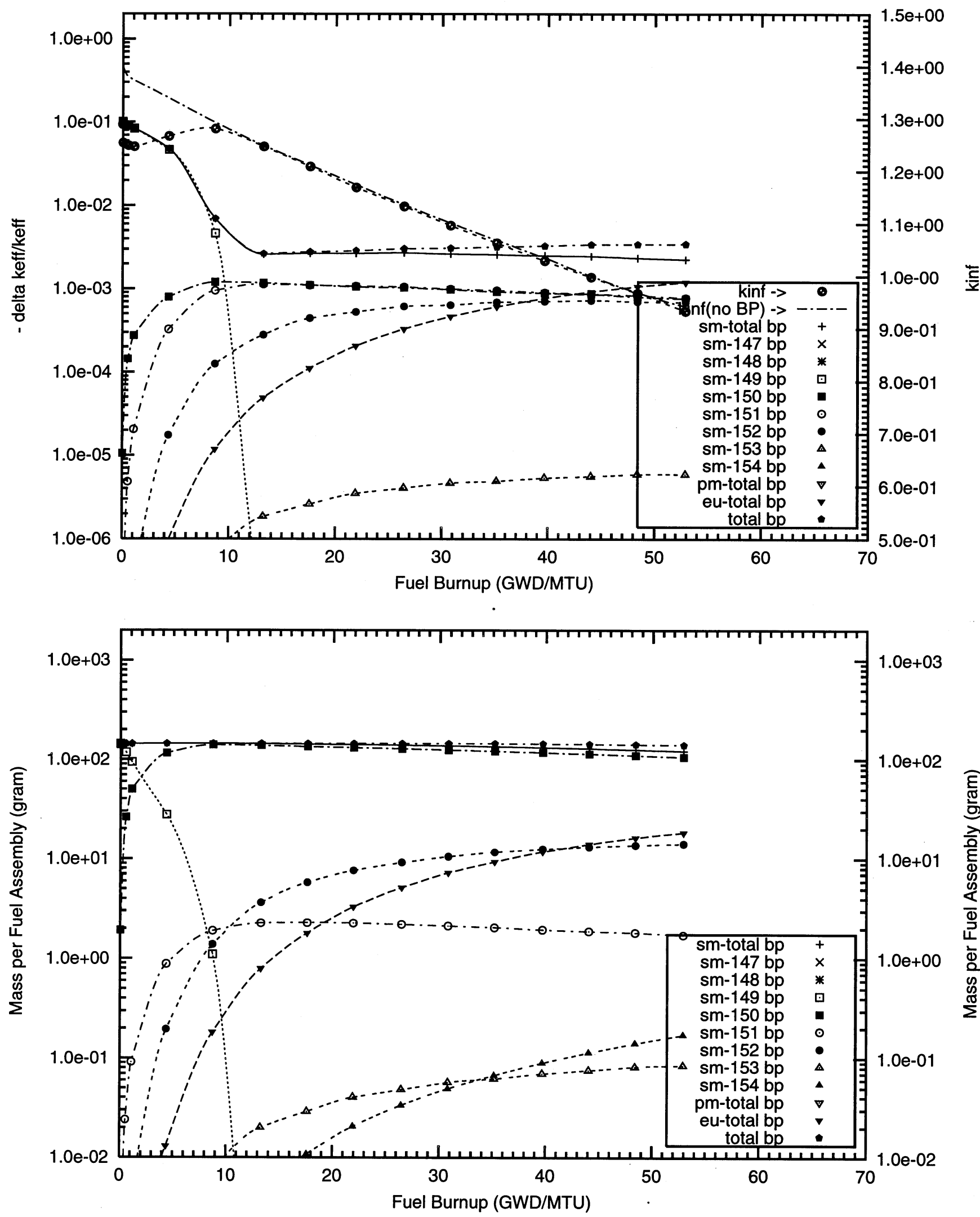
Figure B1.20. Negative Reactivity and Masses BP for Sm-149 and transmutation daughters as a function of fuel life for $17 \times 17$ fuel assemblies with 16 poison rods,

$1.0 \mathrm{w} \% \mathrm{Sm} 2 \mathrm{O} 3$ poison homogeneously mixed in the UO2 pellets

Reactor power $3400 \mathrm{MW}$ th, 193 fuel assemblies, initial enrichment $4.5 \mathrm{w} \% \mathrm{U}-235$

(Case : sm149_1_100p_h20_16_bp_fuel_2_o)
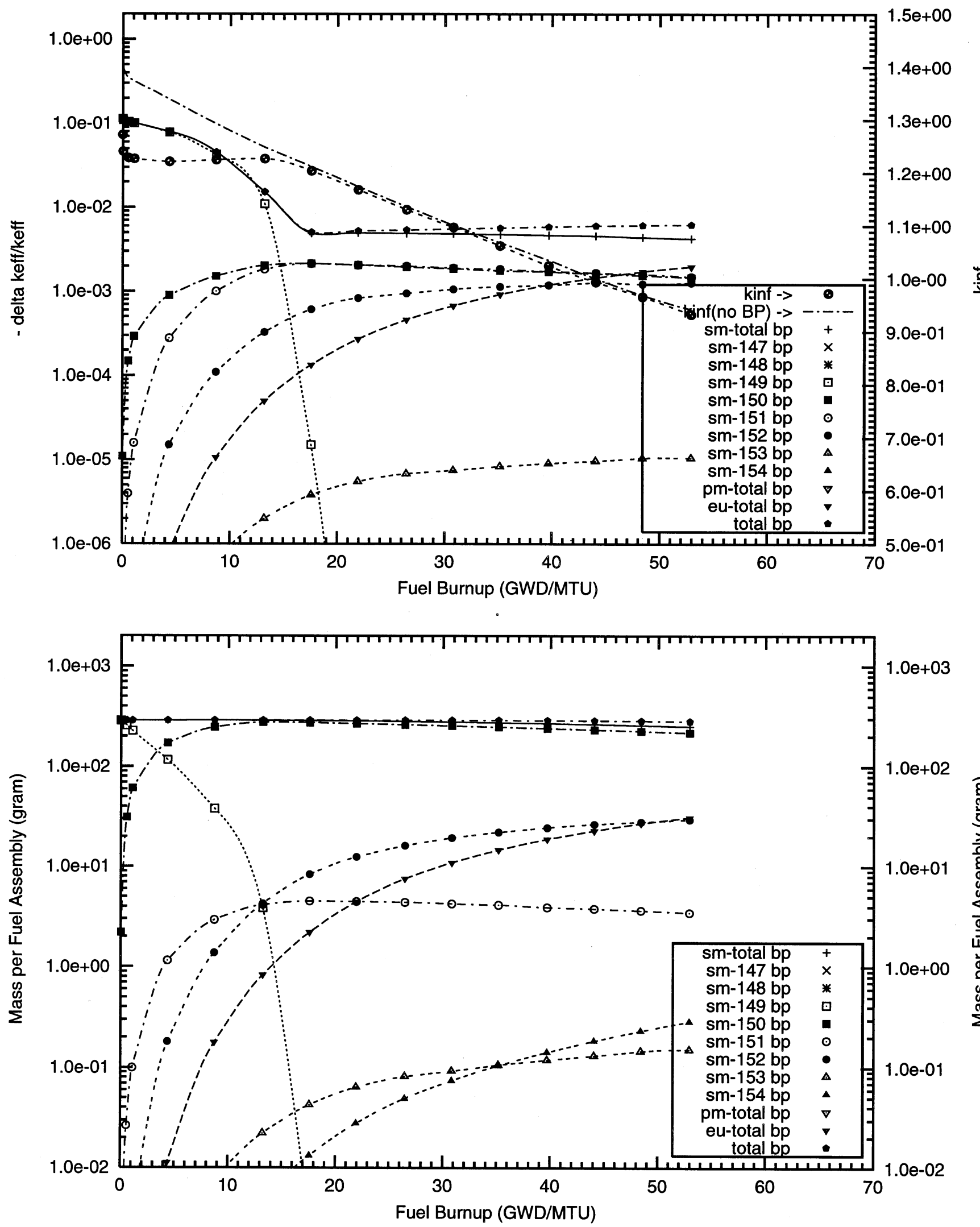
Figure B1.21. Negative Reactivity and Masses BP for Sm-149 and transmutation daughters

as a function of fuel life for $17 \times 17$ fuel assemblies with 16 poison rods,

$2.0 \mathrm{w} \% \mathrm{Sm} 2 \mathrm{O} 3$ poison homogeneously mixed in the UO2 pellets

Reactor power 3400 MWth, 193 fuel assemblies, initial enrichment $4.5 \mathrm{w} \% \mathrm{U}-235$

(Case : sm149_2_100p_h20_16_bp_fuel_2_o)

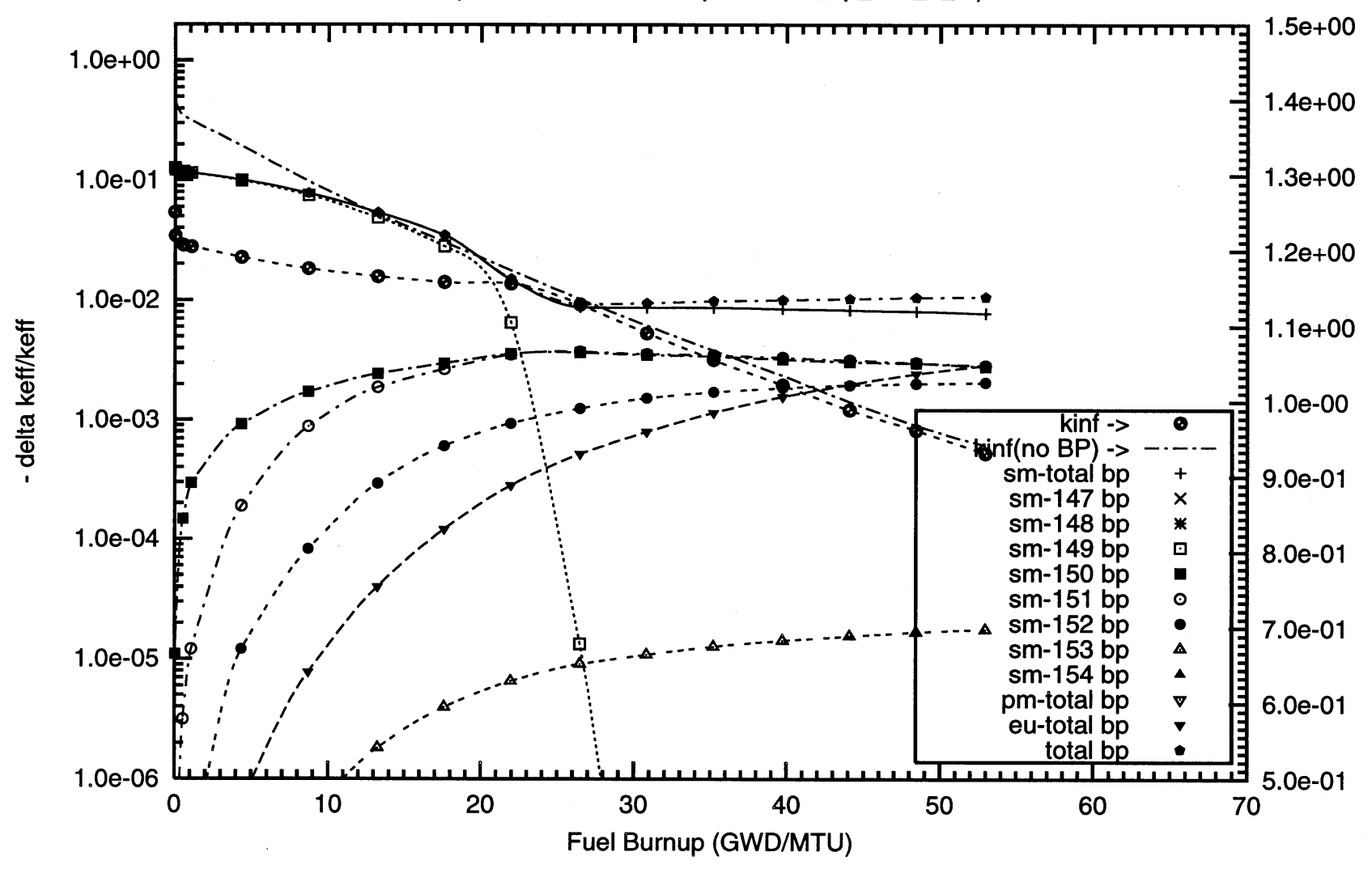

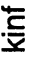

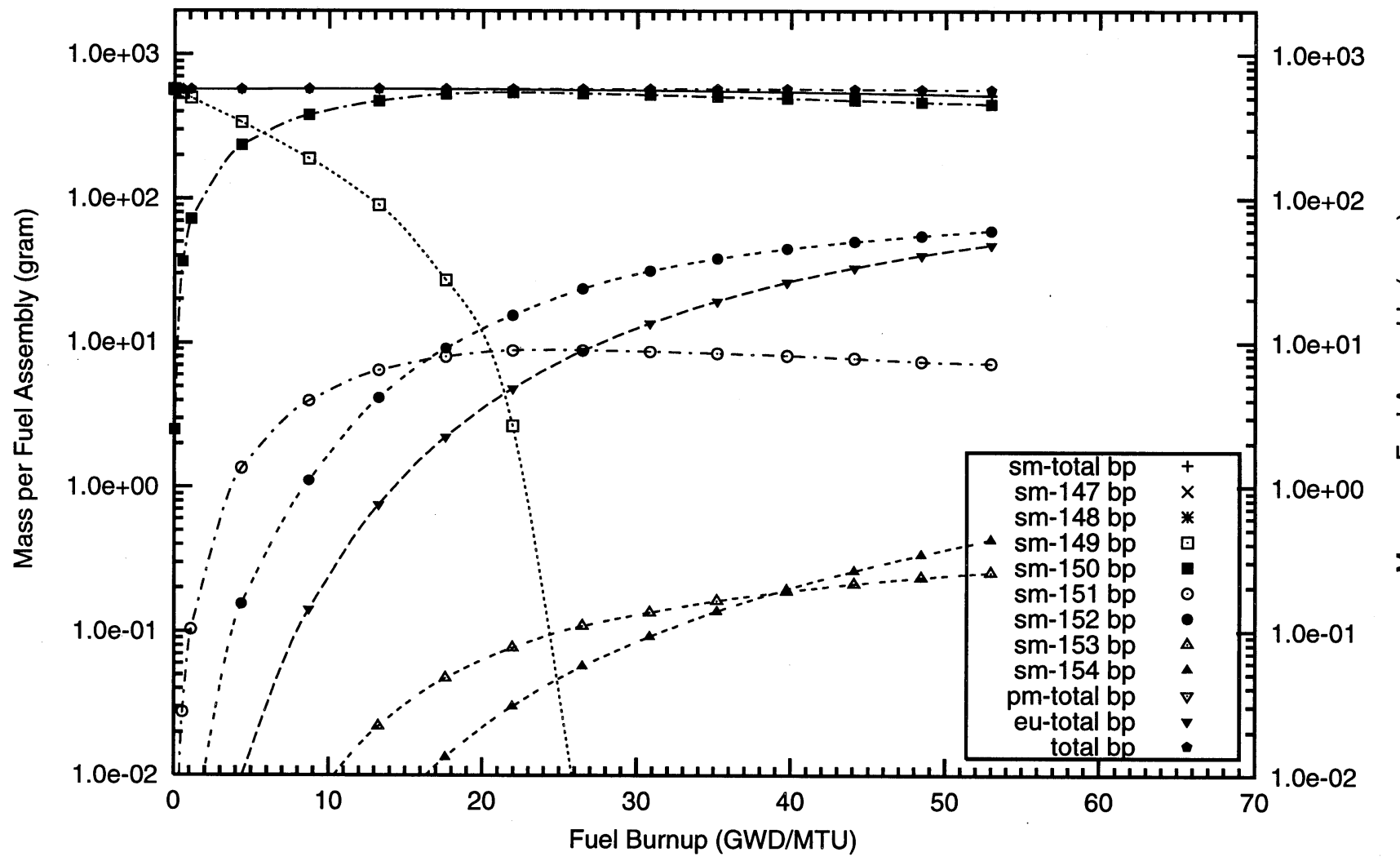


Figure B1.22. Negative Reactivity and Masses BP for Sm-149 and transmutation daughters

as a function of fuel life for $17 \times 17$ fuel assemblies with 16 poison rods,

$4.0 \mathrm{w} \% \mathrm{Sm} 2 \mathrm{O} 3$ poison homogeneously mixed in the UO2 pellets

Reactor power $3400 \mathrm{MWth}, 193$ fuel assemblies, initial enrichment 4.5w\%U-235

(Case : sm149_3_100p_h20_16_bp_fuel_2_o)
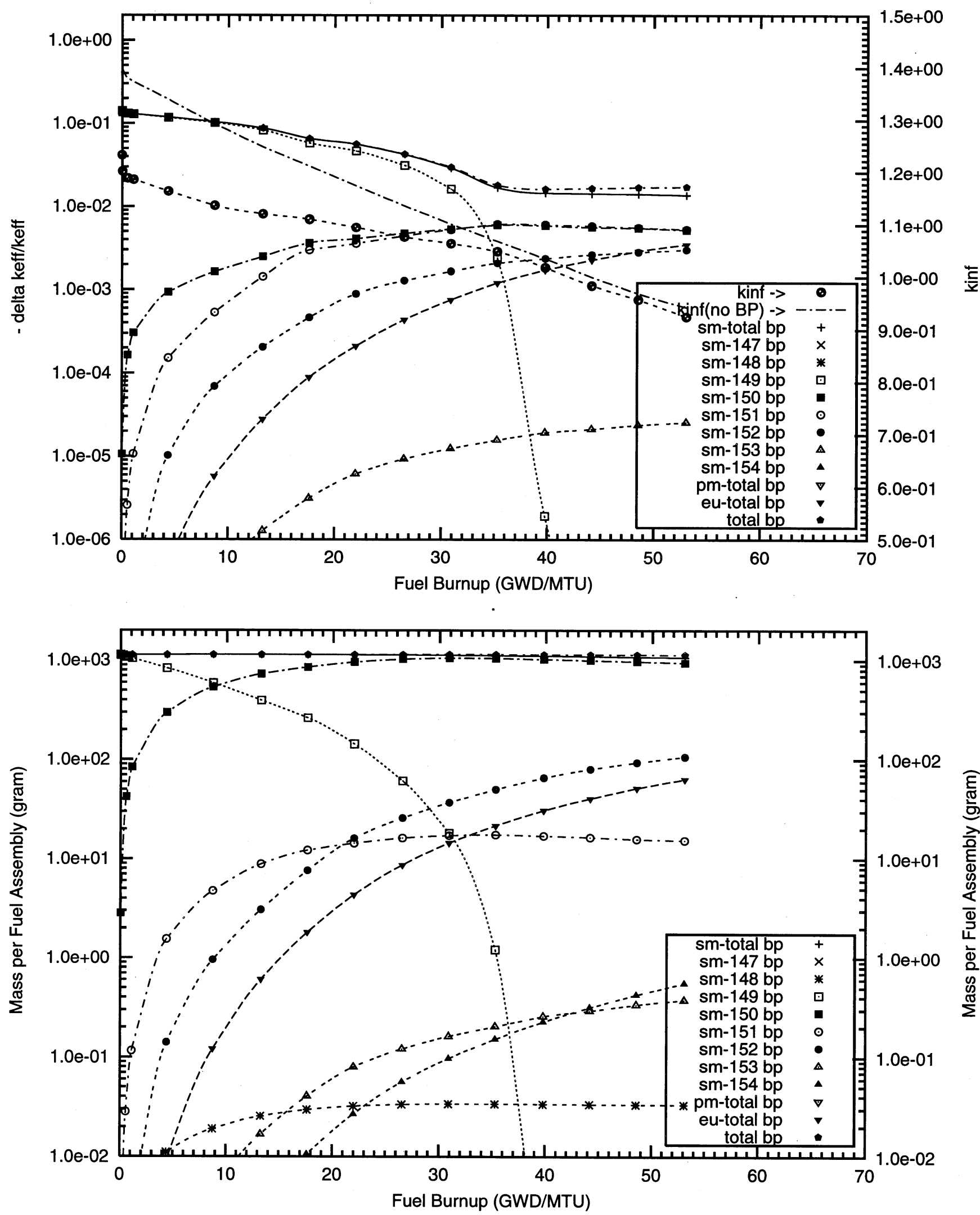
Figure B1.23. Negative Reactivity and Masses BP for Sm-149 and transmutation daughters as a function of fuel life for $17 \times 17$ fuel assemblies with 20 poison rods,

$1.0 \mathrm{w} \% \mathrm{Sm} 2 \mathrm{O} 3$ poison homogeneously mixed in the $\mathrm{UO} 2$ pellets

Reactor power $3400 \mathrm{MWth}, 193$ fuel assemblies, initial enrichment $4.5 \mathrm{w} \% \mathrm{U}-235$

(Case : sm149_1_16_bp_fuel (old) o)
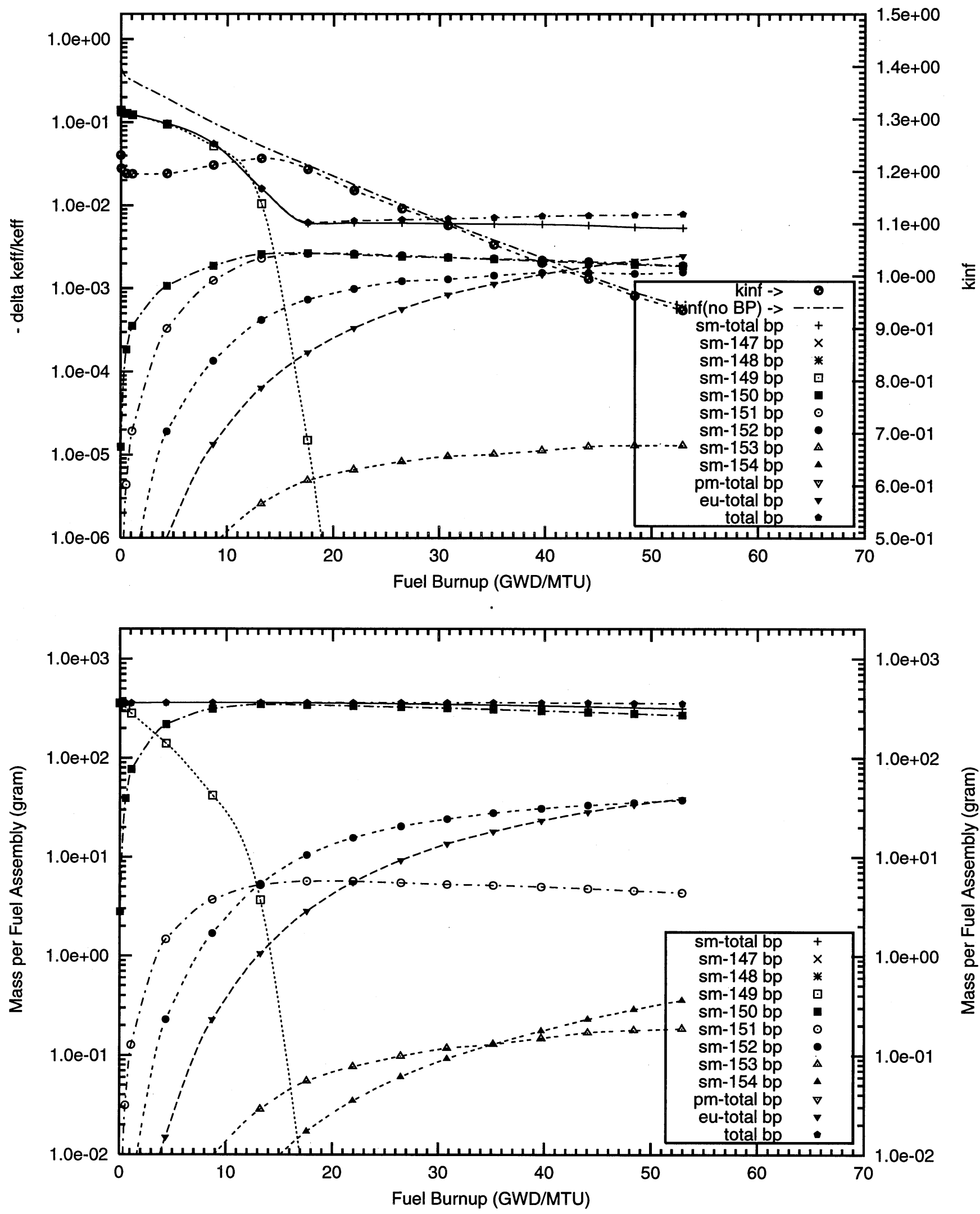
Figure B1.24. Negative Reactivity and Masses BP for Sm-149 and transmutation daughters as a function of fuel life for $17 \times 17$ fuel assemblies with 20 poison rods, $2.0 \mathrm{w} \% \mathrm{Sm} 2 \mathrm{O} 3$ poison homogeneously mixed in the UO2 pellets

Reactor power $3400 \mathrm{MWth}, 193$ fuel assemblies, initial enrichment $4.5 \mathrm{w} \% \mathrm{U}-235$ (Case : sm149_2_16_bp_fuel (old) o)

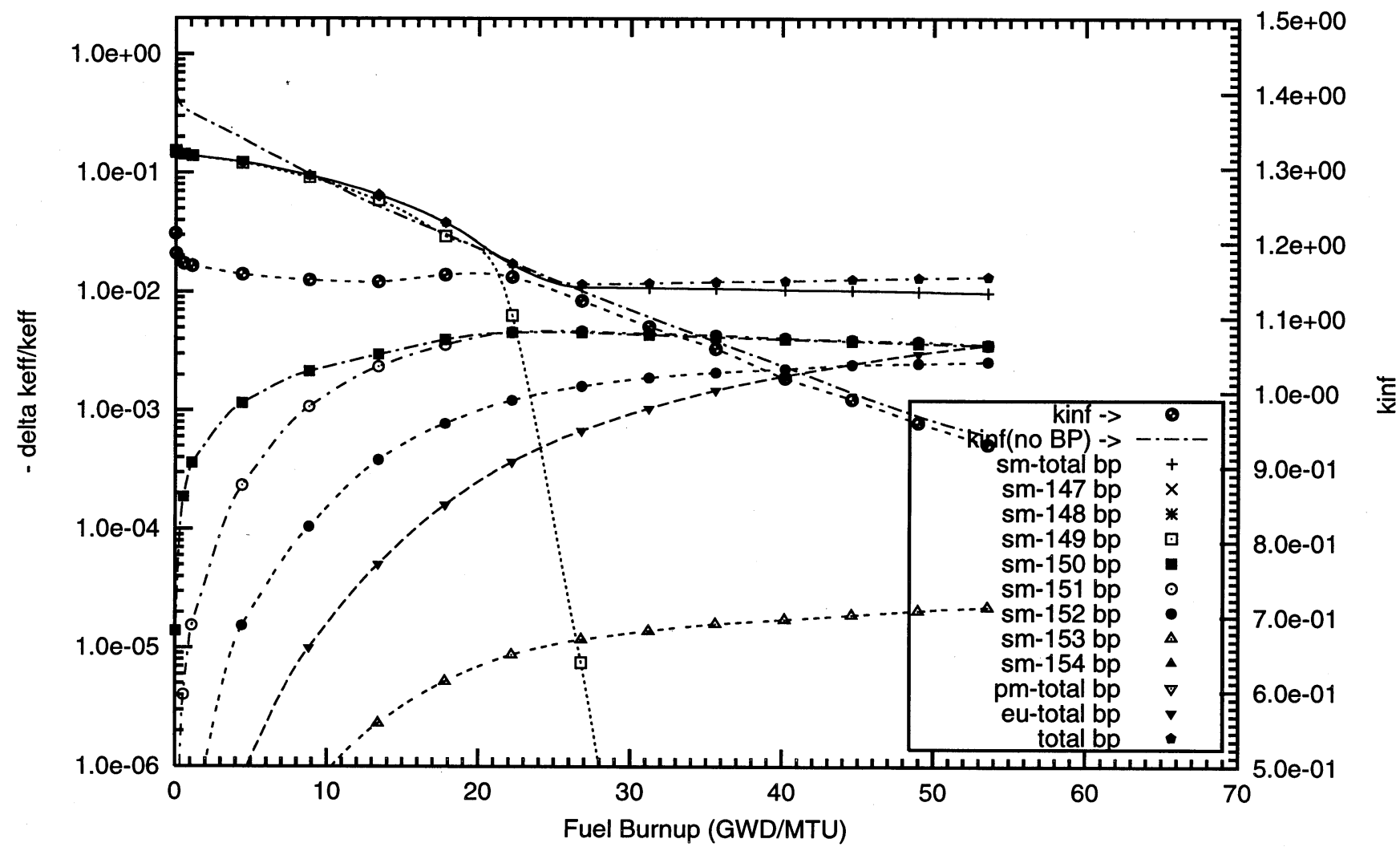


Figure B1.25. Negative Reactivity and Masses BP for Sm-149 and transmutation daughters as a function of fuel life for $17 \times 17$ fuel assemblies with 20 poison rods,

$4.0 \mathrm{w} \% \mathrm{Sm} 2 \mathrm{O} 3$ poison homogeneously mixed in the UO2 pellets

Reactor power $3400 \mathrm{MWth}, 193$ fuel assemblies, initial enrichment $4.5 \mathrm{w} \% \mathrm{U}-235$

(Case : sm149_3_16_bp_fuel (old) o)

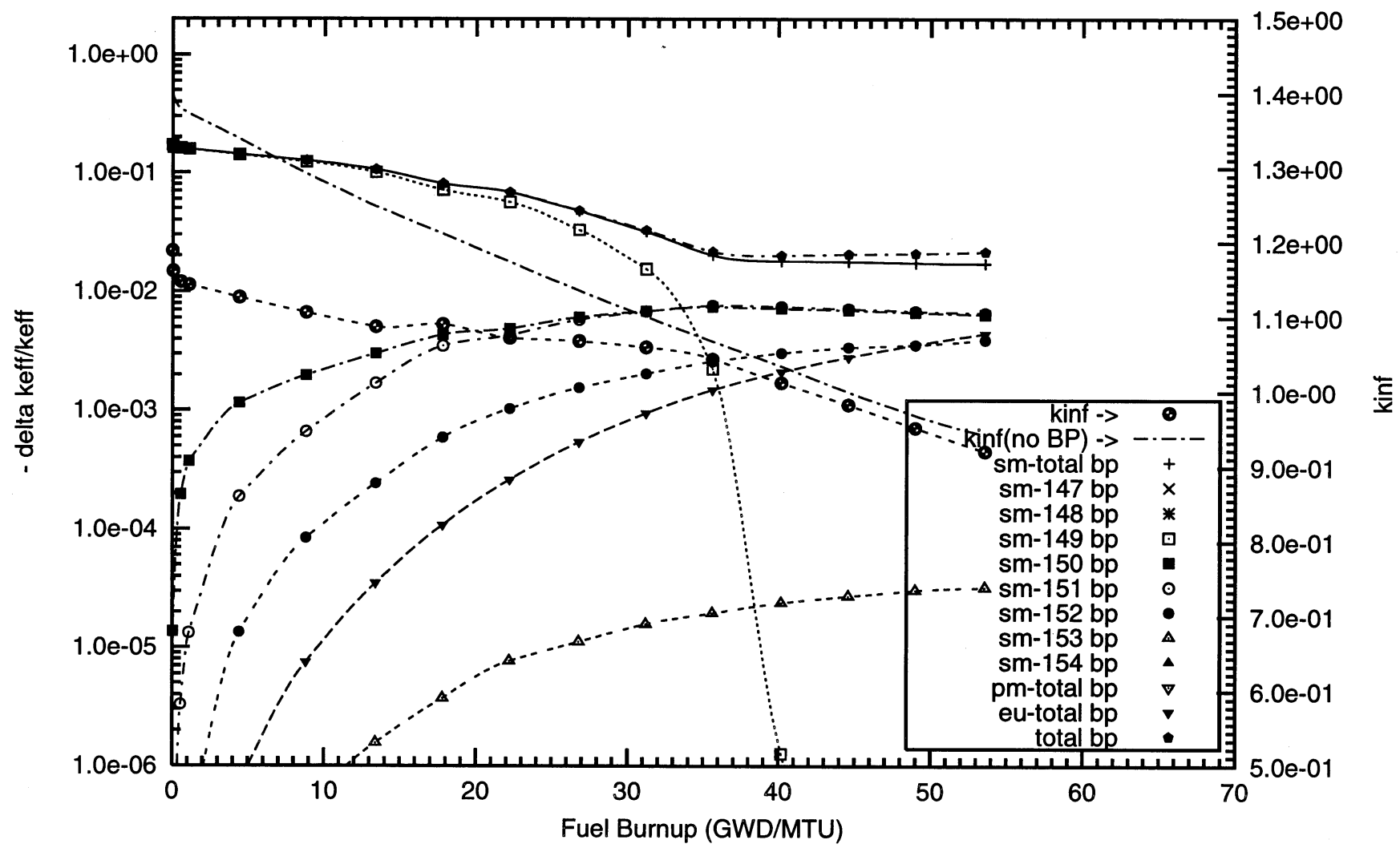


Figure B1.26. Negative Reactivity and Masses BP for Sm-149 and transmutation daughters as a function of fuel life for $17 \times 17$ fuel assemblies with 20 poison rods,

$8.0 \mathrm{w} \% \mathrm{Sm} 2 \mathrm{O} 3$ poison homogeneously mixed in the UO2 pellets

Reactor power $3400 \mathrm{MWth}, 193$ fuel assemblies, initial enrichment $4.5 \mathrm{w} \% \mathrm{U}-235$

(Case : sm149_4_16_bp_fuel (old) o)

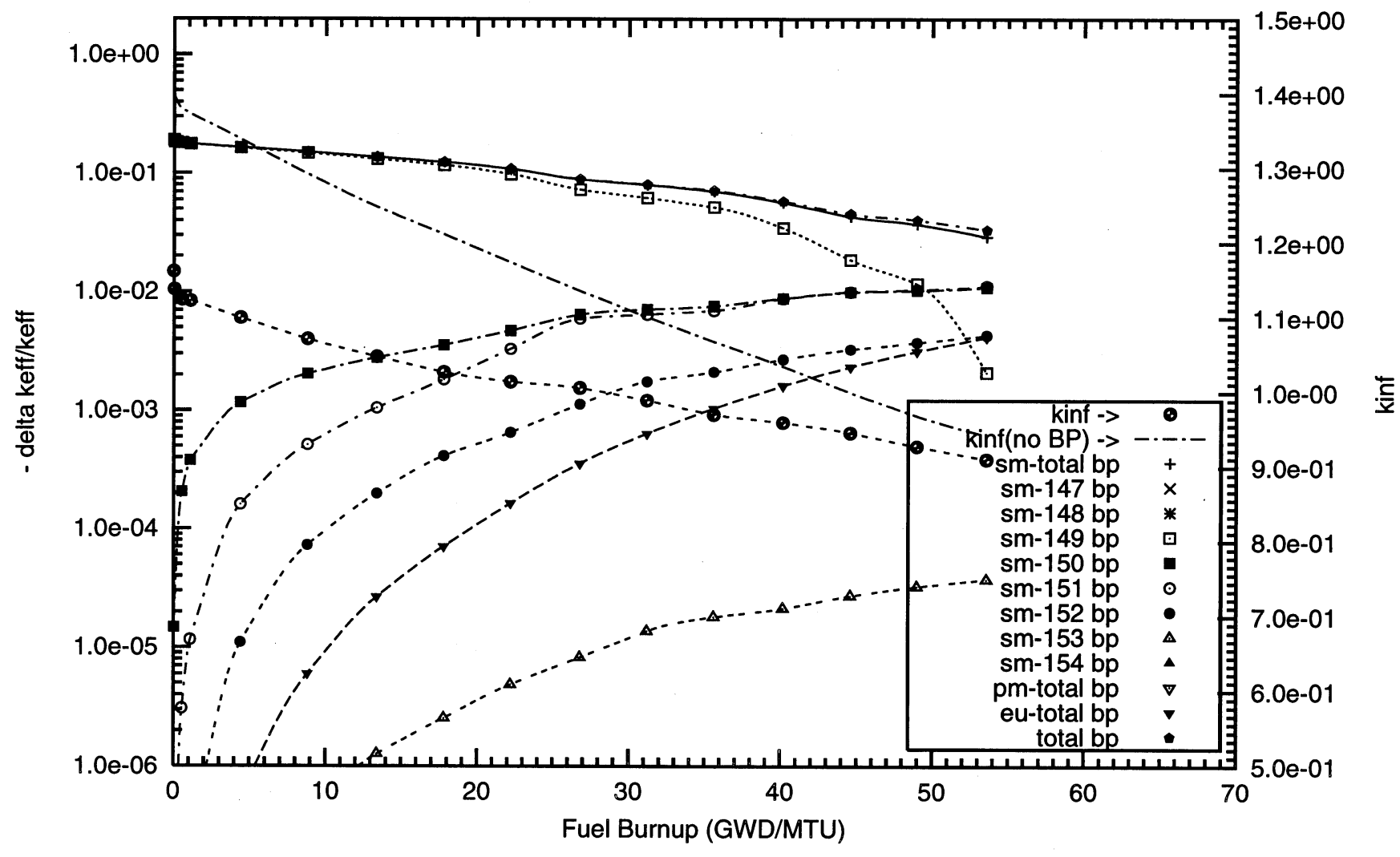


Figure B1.27. Negative Reactivity and Masses BP for Sm-149 and transmutation daughters as a function of fuel life for $17 \times 17$ fuel assemblies with 64 poison rods,

$0.05 \mathrm{w} \% \mathrm{Sm} 2 \mathrm{O} 3$ poison homogeneously mixed in the UO2 pellets

Reactor power $3400 \mathrm{MWth}, 193$ fuel assemblies, initial enrichment $4.5 \mathrm{w} \% \mathrm{U}-235$

(Case : sm149_10_64_bp_fuel o)
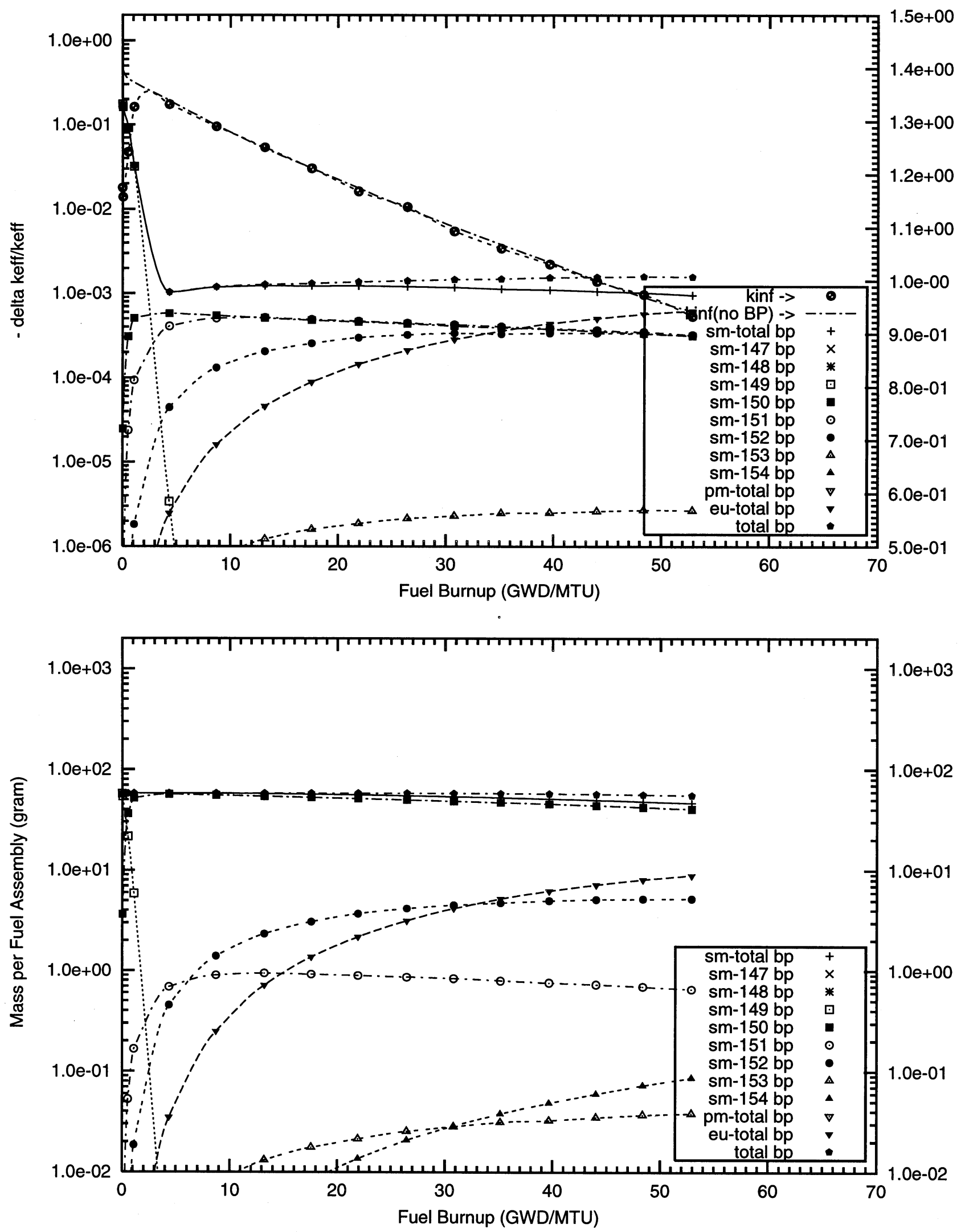
Figure B1.28. Negative Reactivity and Masses BP for Sm-149 and transmutation daughters

as a function of fuel life for $17 \times 17$ fuel assemblies with 64 poison rods,

$0.1 \mathrm{w} \% \mathrm{Sm} 2 \mathrm{O} 3$ poison homogeneously mixed in the UO2 pellets

Reactor power $3400 \mathrm{MWth}, 193$ fuel assemblies, initial enrichment $4.5 \mathrm{w} \% \mathrm{U}-235$

(Case : sm149_9_64_bp_fuel o)
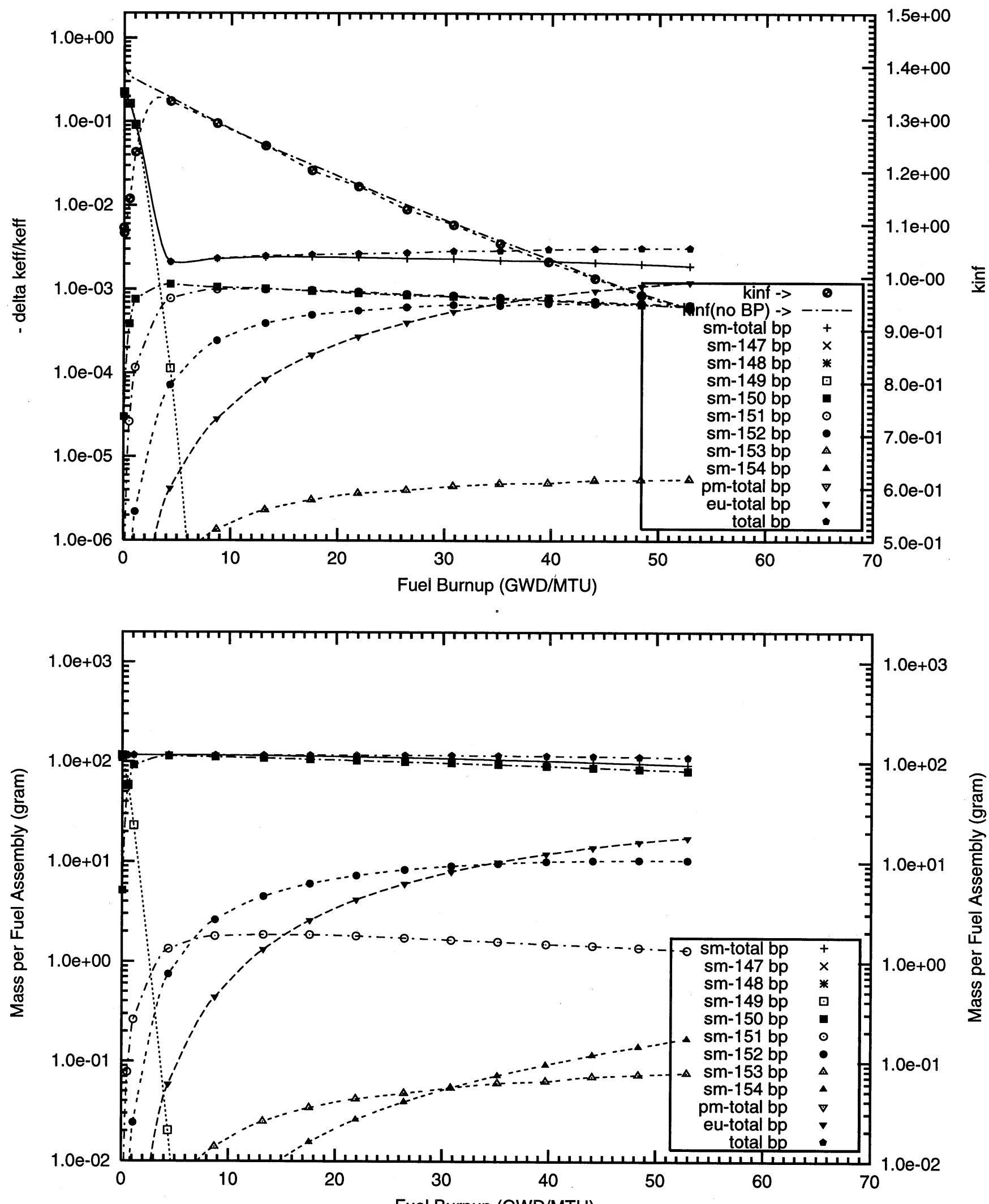

Fuel Burnup (GWD/MTU) 
Figure B1.29. Negative Reactivity and Masses BP for Sm-149 and transmutation daughters

as a function of fuel life for $17 \times 17$ fuel assemblies with 64 poison rods,

$0.2 \mathrm{w} \% \mathrm{Sm} 2 \mathrm{O} 3$ poison homogeneously mixed in the UO2 pellets

Reactor power $3400 \mathrm{MWth}, 193$ fuel assemblies, initial enrichment $4.5 \mathrm{w} \% \mathrm{U}-235$

(Case : sm149_8_64_bp_fuel o)

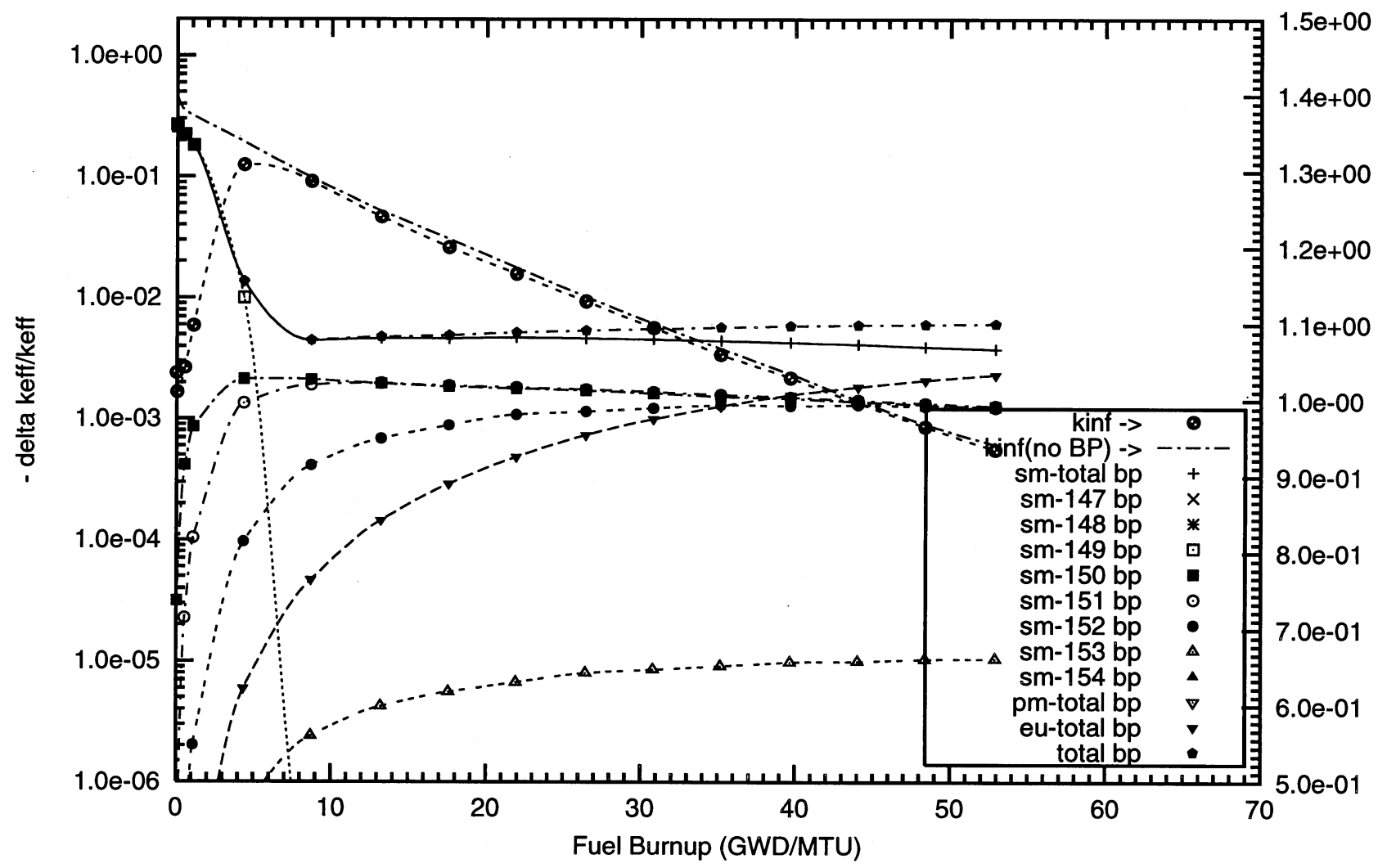

$\ddot{\bar{x}}$

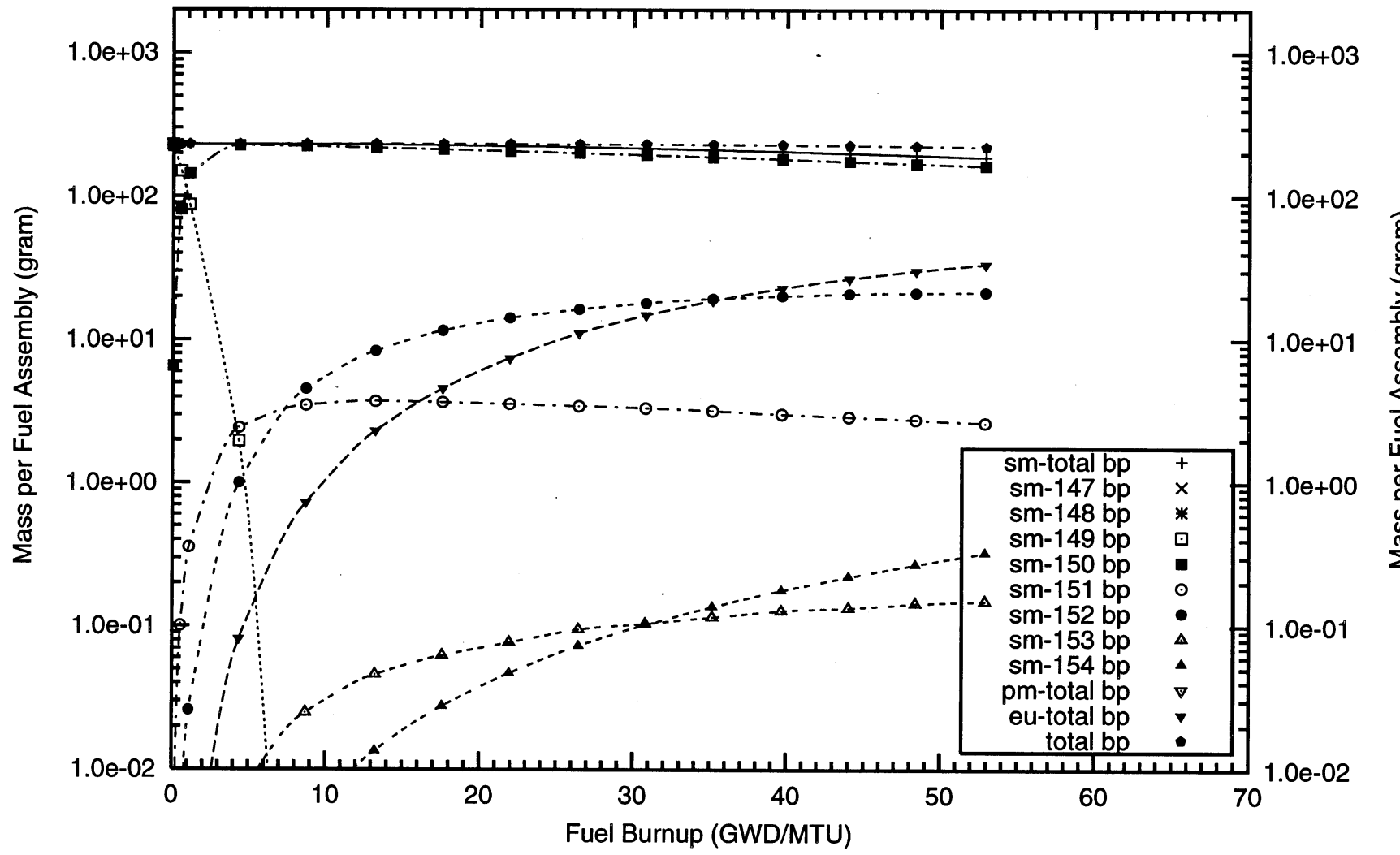




\section{Appendix B2}

Reactivity Worths and Masses of $\mathrm{Sm}_{2} \mathrm{O}_{3}$ Burnable Poison Homogeneously Mixed in the Outer One-Third Volume of the Fuel Pellets

\section{Table 10 Cases}





\section{B2 - Samarium with Natural Isotopic Abundances}



Figure B2.1. Negative Reactivity and Masses BP for Sm-nat and transmutation daughters as a function of fuel life for $17 \times 17$ fuel assemblies with 08 poison rods,

4.0w\% Sm2O3 poison homogeneously mixed in the outer one-third volume of the UO2 pellets Reactor power $3400 \mathrm{MWth}, 193$ fuel assemblies, initial enrichment 4.5w\%U-235

(Case : sm000_3b_08_bp_fuel o)

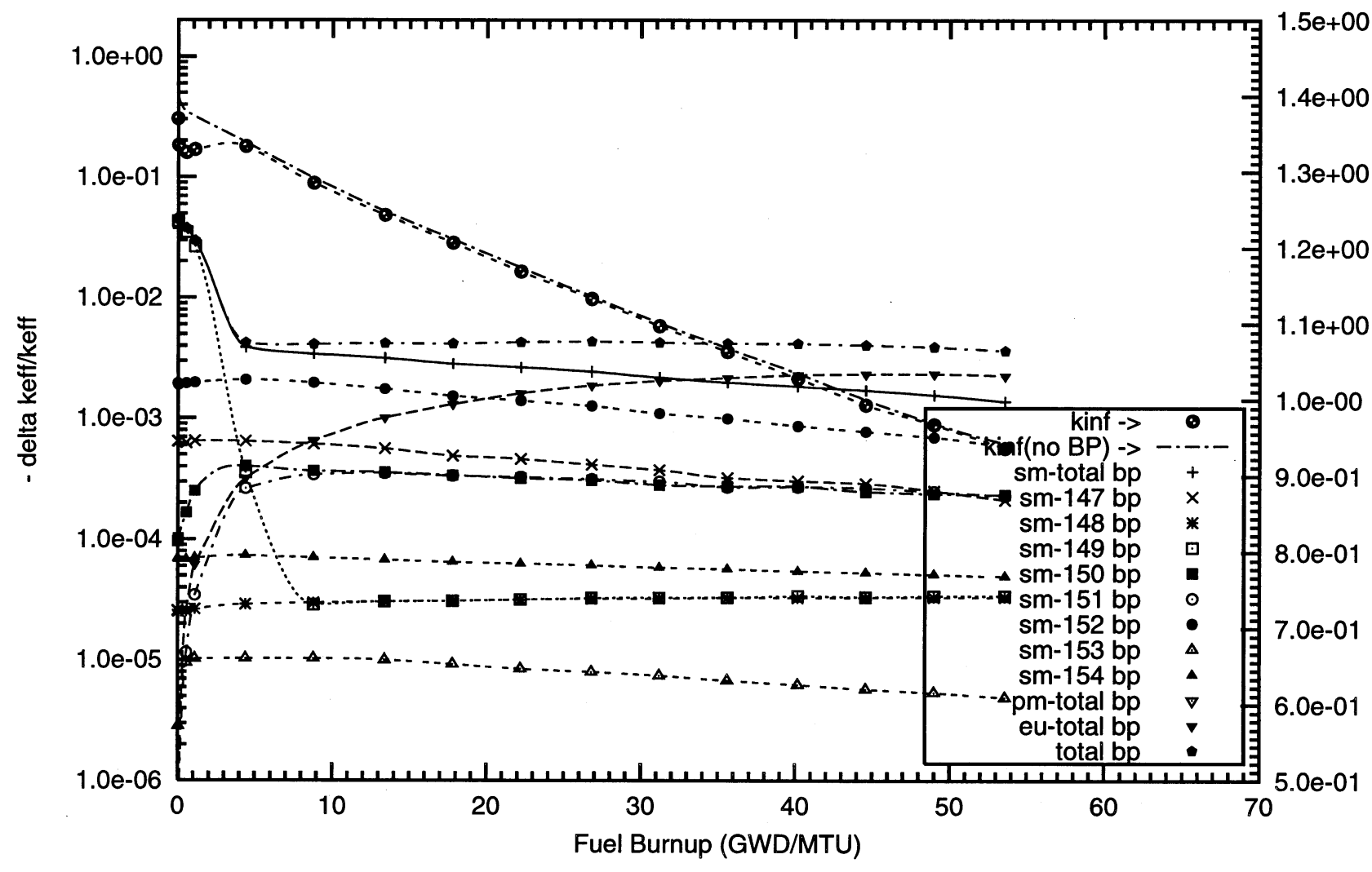


Figure B2.2. Negative Reactivity and Masses BP for Sm-nat and transmutation daughters as a function of fuel life for $17 \times 17$ fuel assemblies with 08 poison rods,

$8.0 \mathrm{w} \% \mathrm{Sm} 2 \mathrm{O} 3$ poison homogeneously mixed in the outer one-third volume of the $\mathrm{UO} 2$ pellets Reactor power $3400 \mathrm{MWth}, 193$ fuel assemblies, initial enrichment 4.5W\%U-235 (Case : sm000_4b_08_bp_fuel o)

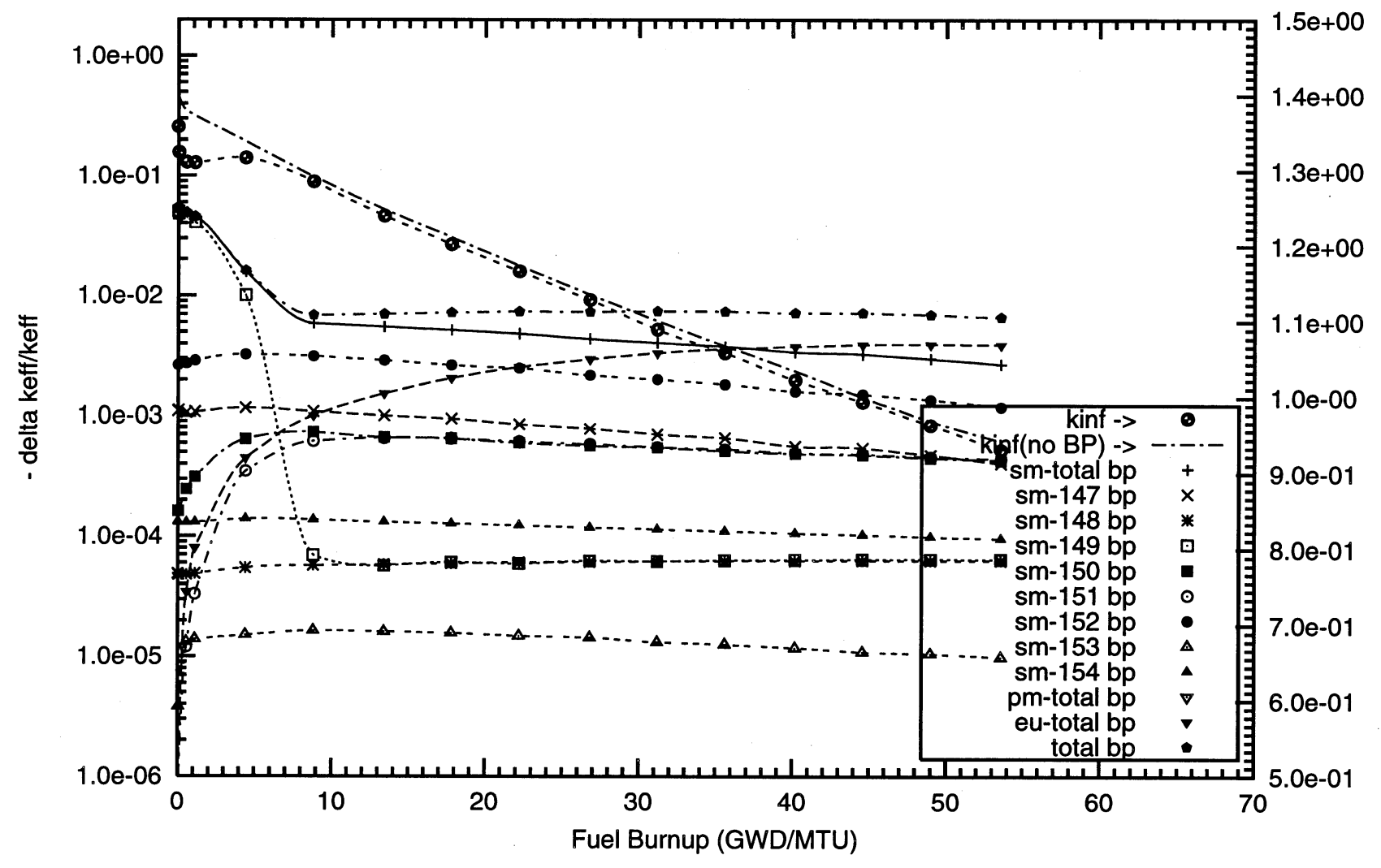


Figure B2.3. Negative Reactivity and Masses BP for Sm-nat and transmutation daughters as a function of fuel life for $17 \times 17$ fuel assemblies with 08 poison rods,

$12.0 \mathrm{w} \% \mathrm{Sm} 2 \mathrm{O} 3$ poison homogeneously mixed in the outer one-third volume of the UO2 pellets Reactor power $3400 \mathrm{MWth}, 193$ fuel assemblies, initial enrichment 4.5w\%U-235

(Case : sm000_5b_08_bp_fuel o)

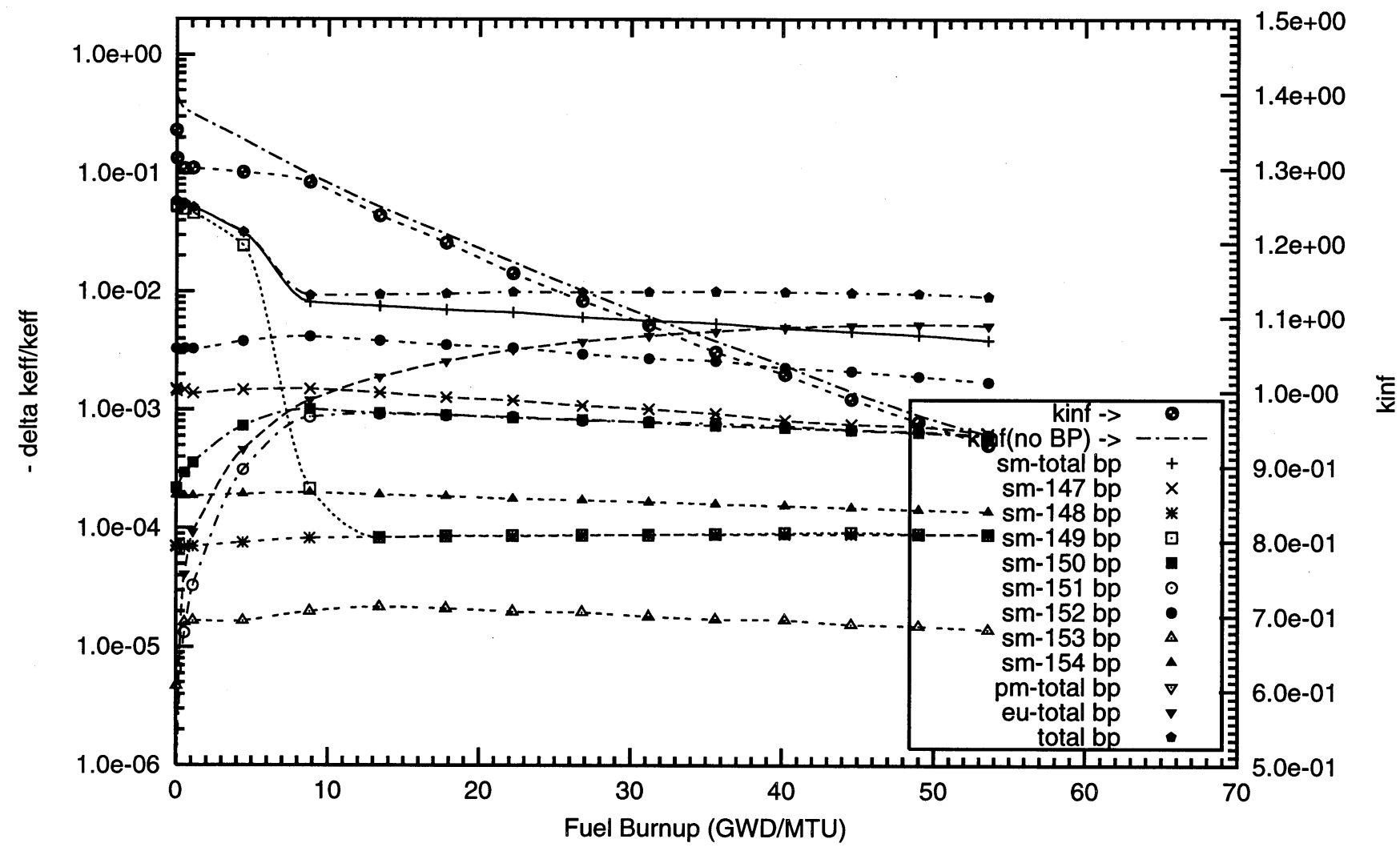


Figure B2.4. Negative Reactivity and Masses BP for Sm-nat and transmutation daughters as a function of fuel life for $17 \times 17$ fuel assemblies with 16 poison rods,

$1.0 \mathrm{w} \% \mathrm{Sm} 2 \mathrm{O} 3$ poison homogeneously mixed in the outer one-third volume of the UO2 pellets

Reactor power $3400 \mathrm{MWth}, 193$ fuel assemblies, initial enrichment 4.5w\%U-235

(Case : sm000_1b_100p_h20_16_bp_fuel_2_o)
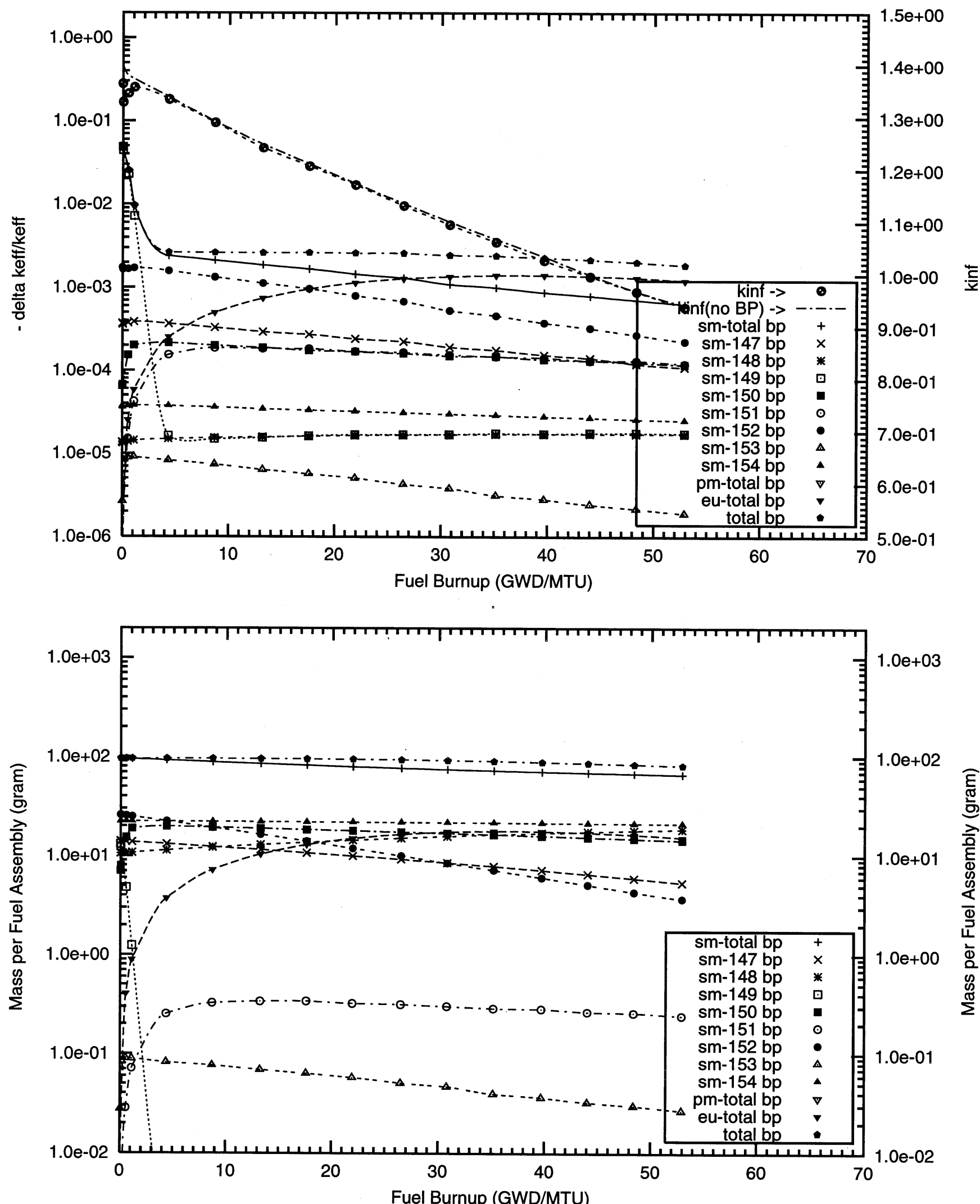
Figure B2.5. Negative Reactivity and Masses BP for Sm-nat and transmutation daughters as a function of fuel life for $17 \times 17$ fuel assemblies with 16 poison rods,

$2.0 \mathrm{w} \% \mathrm{Sm} 2 \mathrm{O} 3$ poison homogeneously mixed in the outer one-third volume of the UO2 pellets Reactor power $3400 \mathrm{MWth}, 193$ fuel assemblies, initial enrichment $4.5 \mathrm{w} \% \mathrm{U}-235$

(Case : sm000_2b_100p_h2o_16_bp_fuel_2_o)
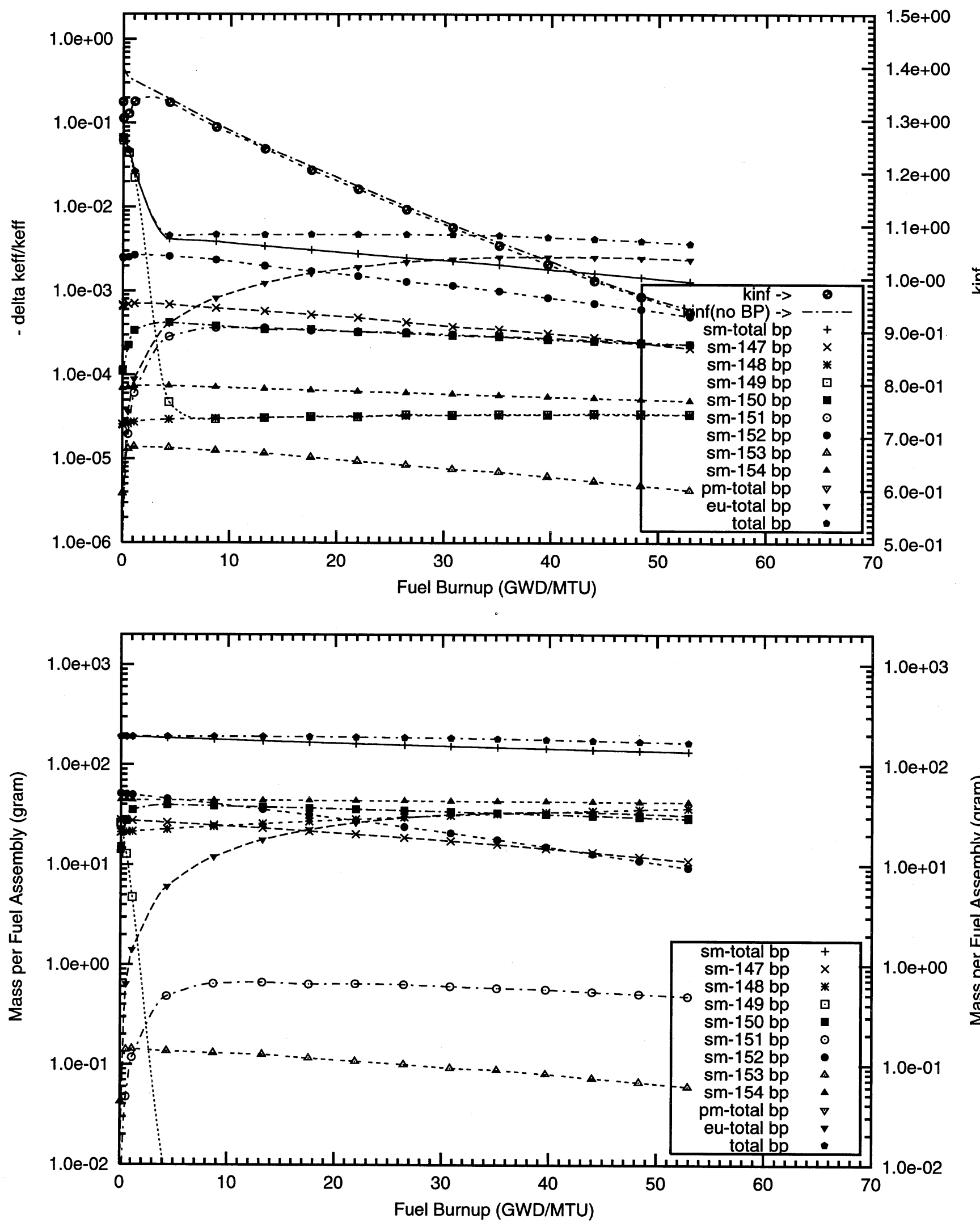
Figure B2.6. Negative Reactivity and Masses BP for Sm-nat and transmutation daughters as a function of fuel life for $17 \times 17$ fuel assemblies with 16 poison rods,

$4.0 \mathrm{w} \% \mathrm{Sm} 2 \mathrm{O} 3$ poison homogeneously mixed in the outer one-third volume of the UO2 pellets Reactor power $3400 \mathrm{MWth}, 193$ fuel assemblies, initial enrichment $4.5 \mathrm{w} \% \mathrm{U}-235$

(Case : sm000_3b_100p_h20_16_bp_fuel_2_o)
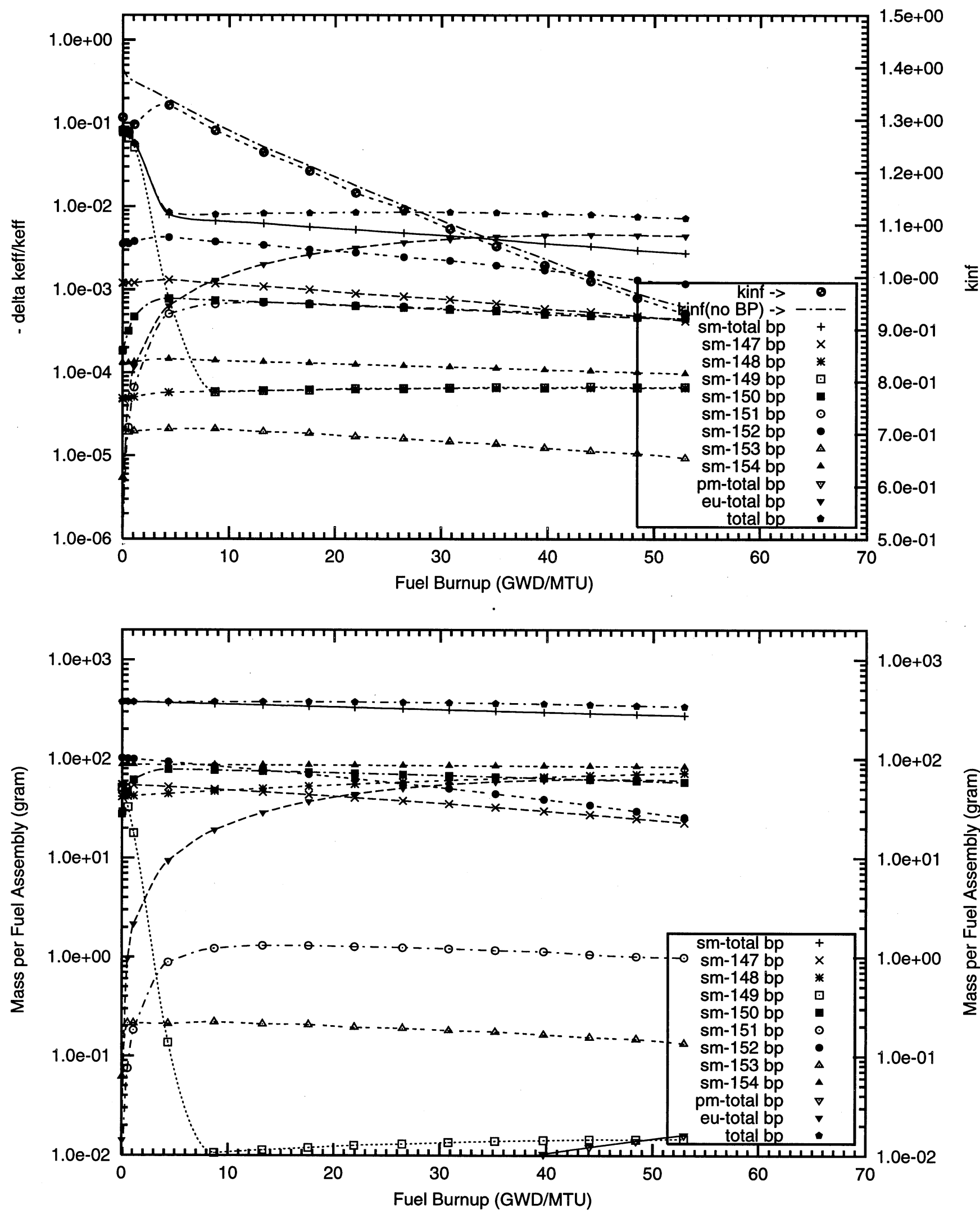
Figure B2.7. Negative Reactivity and Masses BP for Sm-nat and transmutation daughters as a function of fuel life for $17 \times 17$ fuel assemblies with 16 poison rods,

$8.0 \mathrm{w} \% \mathrm{Sm} 2 \mathrm{O} 3$ poison homogeneously mixed in the outer one-third volume of the UO2 pellets

Reactor power $3400 \mathrm{MW}$ th, 193 fuel assemblies, initial enrichment $4.5 \mathrm{w} \% \mathrm{U}-235$

(Case : sm000_4b_100p_h2o_16_bp_fuel_2_o)
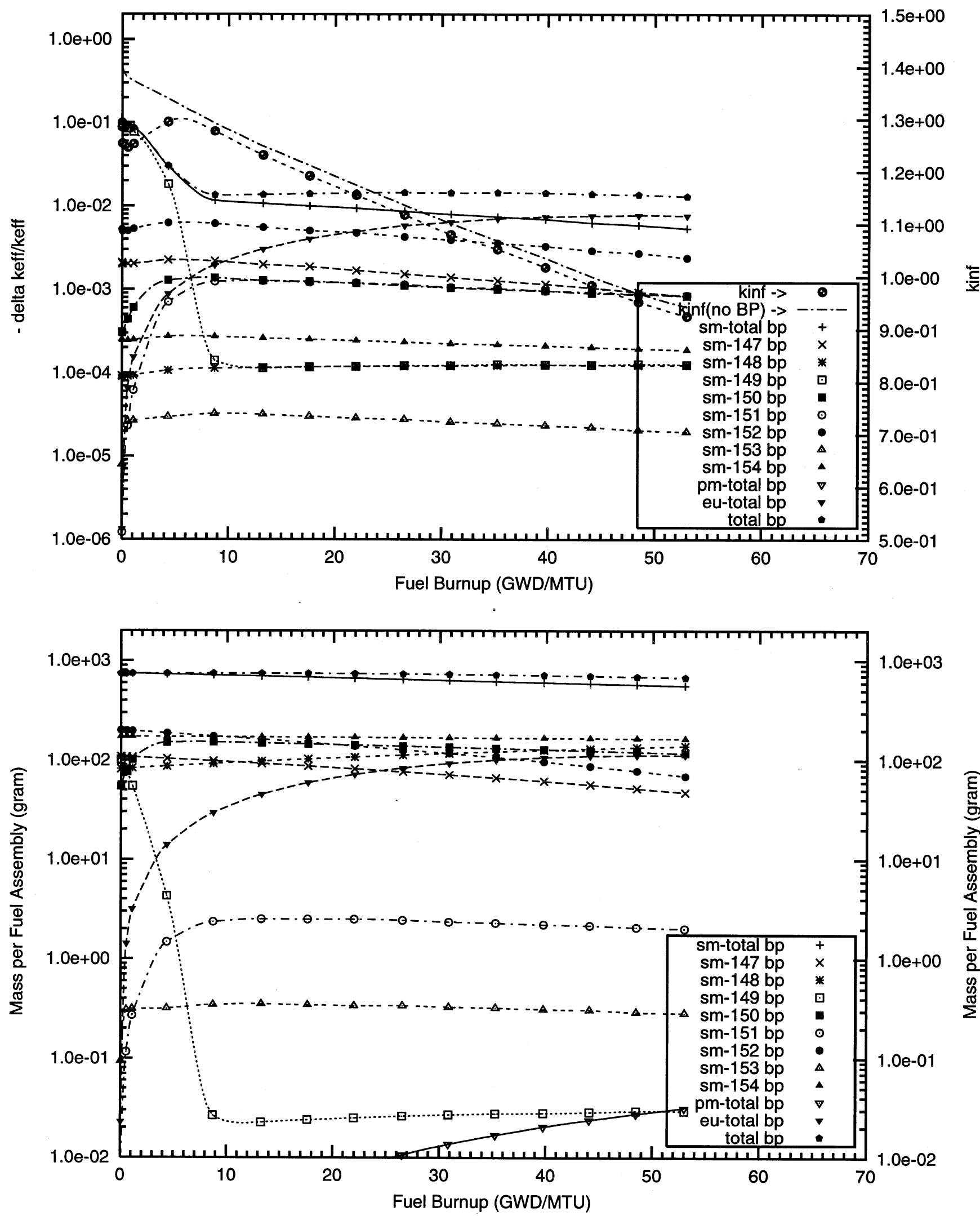
Figure B2.8. Negative Reactivity and Masses BP for Sm-nat and transmutation daughters as a function of fuel life for $17 \times 17$ fuel assemblies with 18 poison rods,

$2.0 \mathrm{w} \% \mathrm{Sm} 2 \mathrm{O} 3$ poison homogeneously mixed in the outer one-third volume of the $\mathrm{UO} 2$ pellets

Reactor power $3400 \mathrm{MW}$ th, 193 fuel assemblies, initial enrichment 4.5W\%U-235

(Case : sm000_2b_16_bp_fuel (old) o)
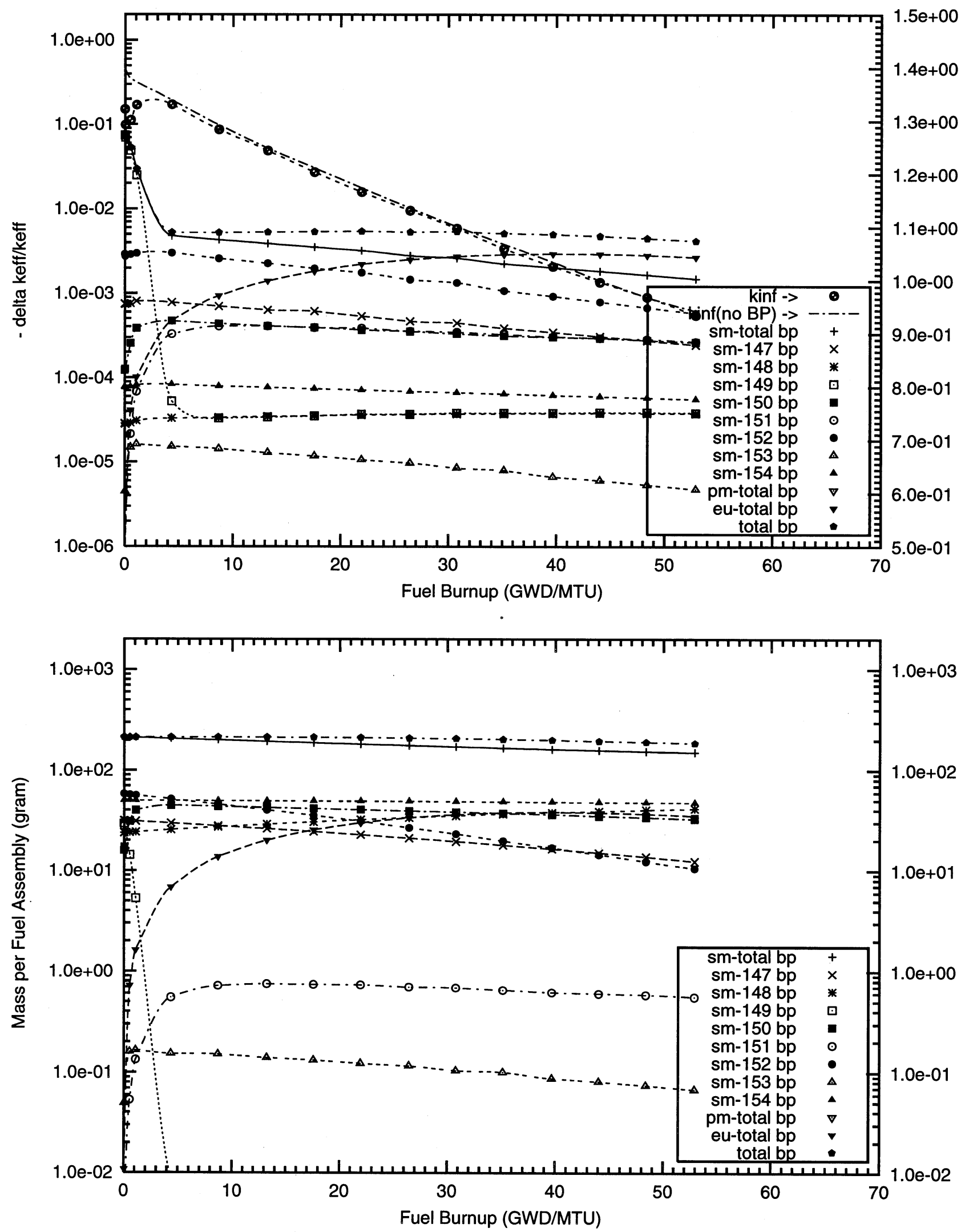
Figure B2.9. Negative Reactivity and Masses BP for Sm-nat and transmutation daughters as a function of fuel life for $17 \times 17$ fuel assemblies with 18 poison rods,

$4.0 \mathrm{w} \% \mathrm{Sm} 2 \mathrm{O} 3$ poison homogeneously mixed in the outer one-third volume of the UO2 pellets

Reactor power $3400 \mathrm{MWth}, 193$ fuel assemblies, initial enrichment $4.5 \mathrm{w} \% \mathrm{U}-235$

(Case : sm000_3b_16_bp_fuel (old) o)
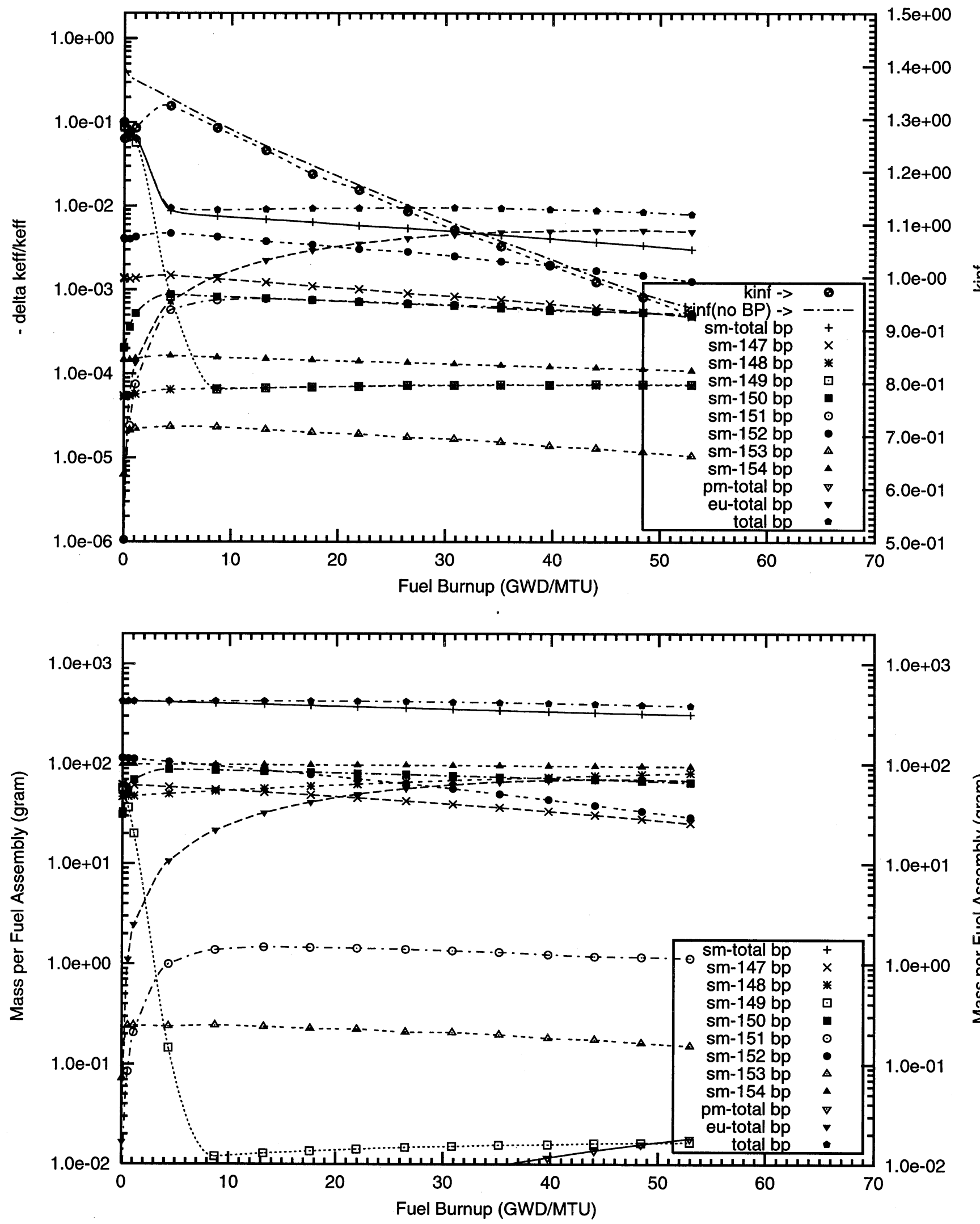
Figure B2.10. Negative Reactivity and Masses BP for Sm-nat and transmutation daughters as a function of fuel life for $17 \times 17$ fuel assemblies with 64 poison rods,

$0.1 \mathrm{w} \% \mathrm{Sm} 2 \mathrm{O} 3$ poison homogeneously mixed in the outer one-third volume of the $\mathrm{UO} 2$ pellets Reactor power $3400 \mathrm{MWth}, 193$ fuel assemblies, initial enrichment 4.5w\%U-235

(Case : sm000_9b_100p_h2o_64_bp_fuel_2_o)
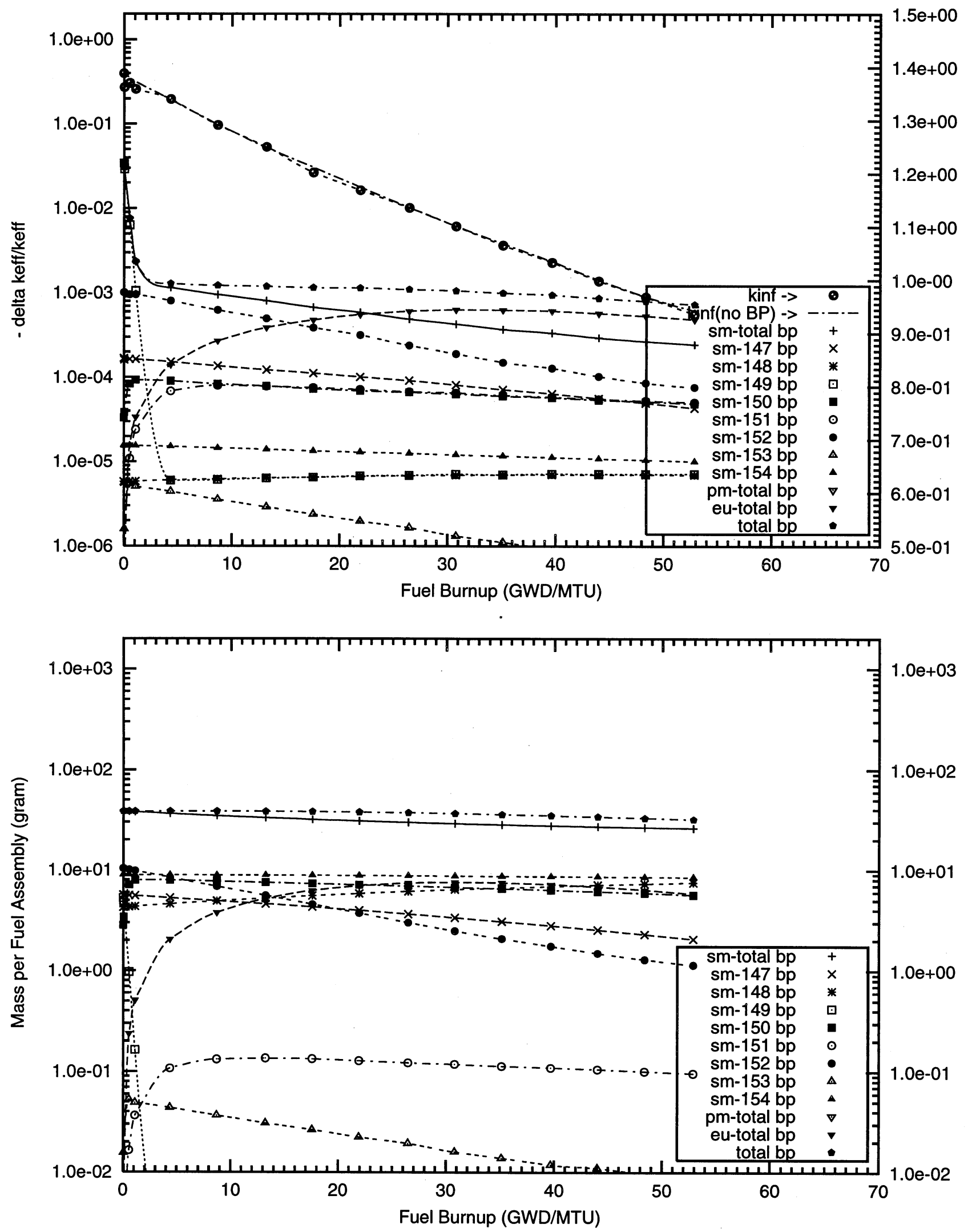
Figure B2.11. Negative Reactivity and Masses BP for Sm-nat and transmutation daughters as a function of fuel life for $17 \times 17$ fuel assemblies with 64 poison rods,

$0.2 \mathrm{w} \% \mathrm{Sm} 2 \mathrm{O} 3$ poison homogeneously mixed in the outer one-third volume of the UO2 pellets Reactor power $3400 \mathrm{MWth}, 193$ fuel assemblies, initial enrichment $4.5 \mathrm{w} \% \mathrm{U}-235$

(Case : sm000_8b_100p_h20_64_bp_fuel_2_o)
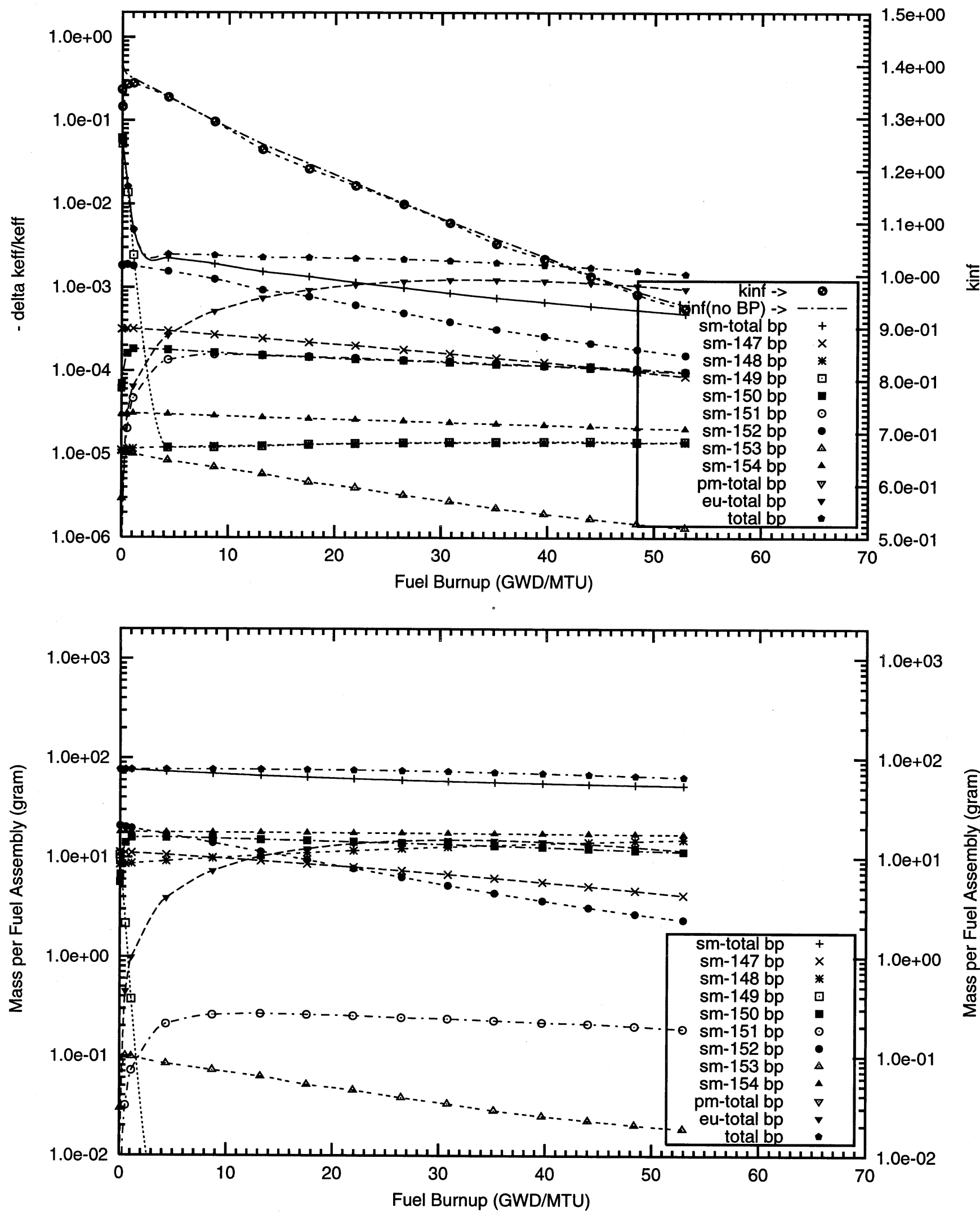
Figure B2.12. Negative Reactivity and Masses BP for Sm-nat and transmutation daughters as a function of fuel life for $17 \times 17$ fuel assemblies with 64 poison rods,

$0.5 \mathrm{w} \% \mathrm{Sm} 2 \mathrm{O} 3$ poison homogeneously mixed in the outer one-third volume of the UO2 pellets Reactor power $3400 \mathrm{MWth}, 193$ fuel assemblies, initial enrichment 4.5w\%U-235

(Case : sm000_0b_64_bp_fuel o)
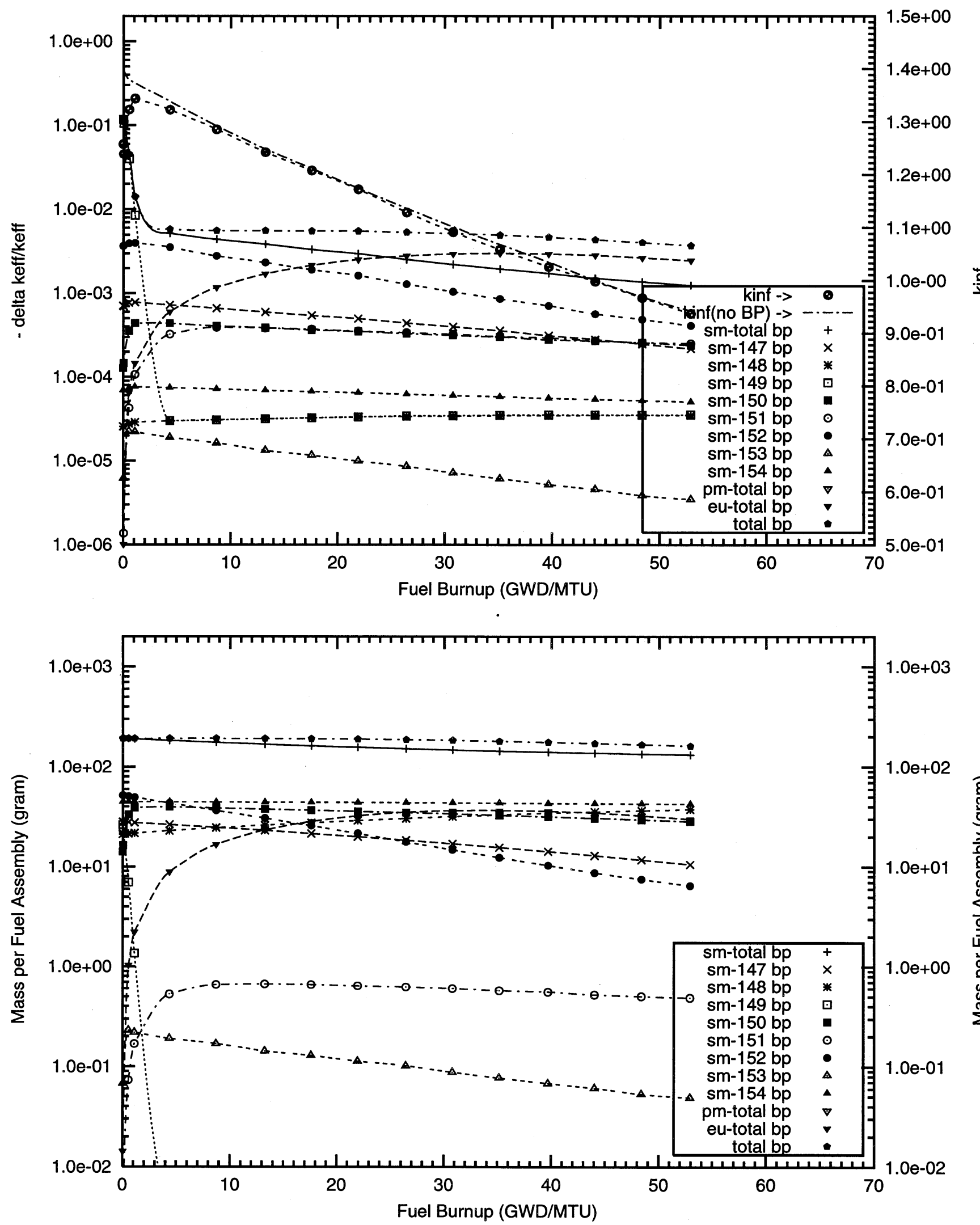
Figure B2.13. Negative Reactivity and Masses BP for Sm-nat and transmutation daughters as a function of fuel life for $17 \times 17$ fuel assemblies with 64 poison rods,

$1.0 \mathrm{w} \% \mathrm{Sm} 2 \mathrm{O} 3$ poison homogeneously mixed in the outer one-third volume of the UO2 pellets Reactor power $3400 \mathrm{MWth}, 193$ fuel assemblies, initial enrichment 4.5w\%U-235 (Case : sm000_1b_64_bp_fuel o)
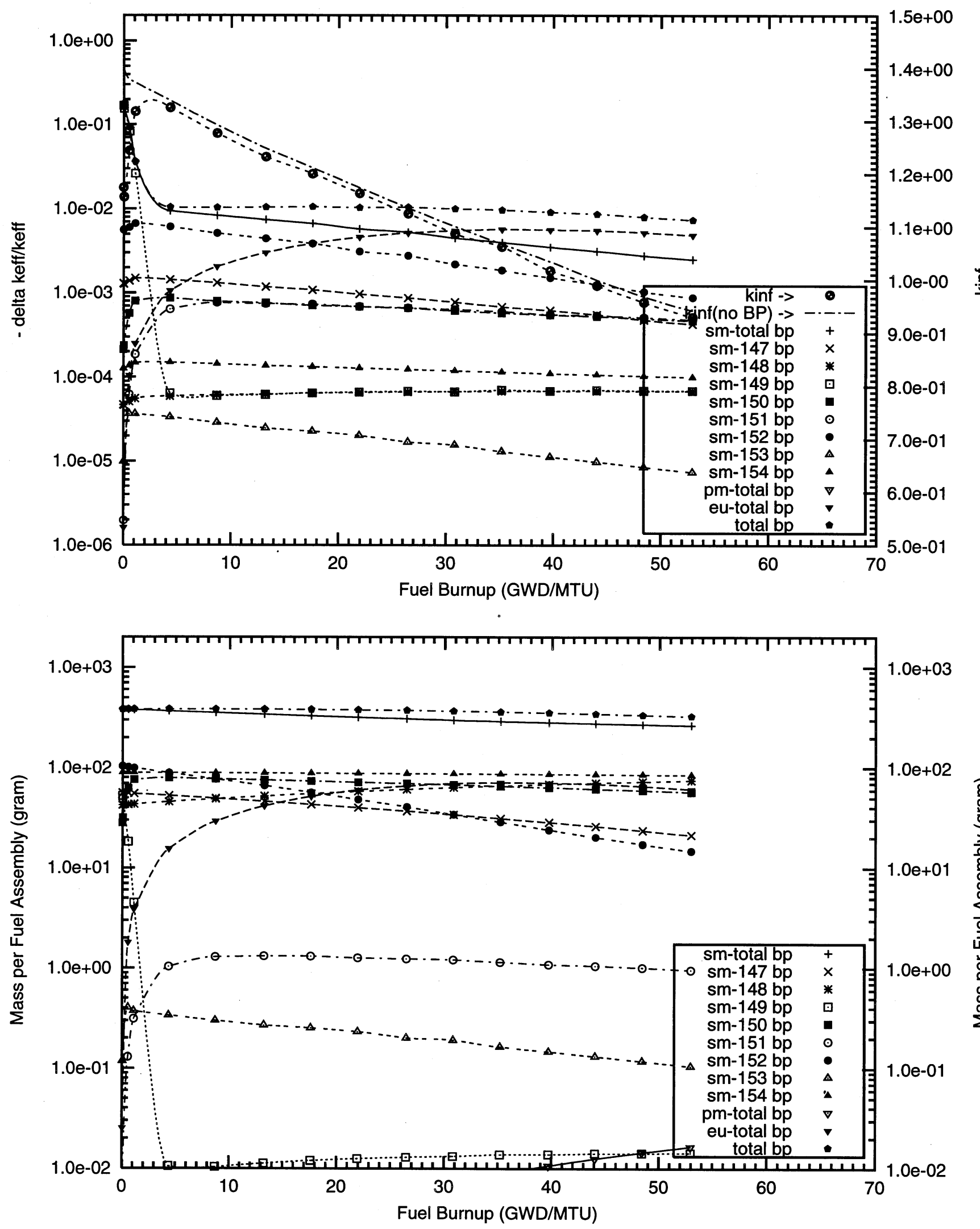
Figure B2.14. Negative Reactivity and Masses BP for Sm-nat and transmutation daughters as a function of fuel life for $17 \times 17$ fuel assemblies with 64 poison rods,

$2.0 \mathrm{w} \% \mathrm{Sm} 2 \mathrm{O} 3$ poison homogeneously mixed in the outer one-third volume of the UO2 pellets Reactor power $3400 \mathrm{MWth}, 193$ fuel assemblies, initial enrichment $4.5 \mathrm{w} \% \mathrm{U}-235$

(Case : sm000_2b_64_bp_fuel o)
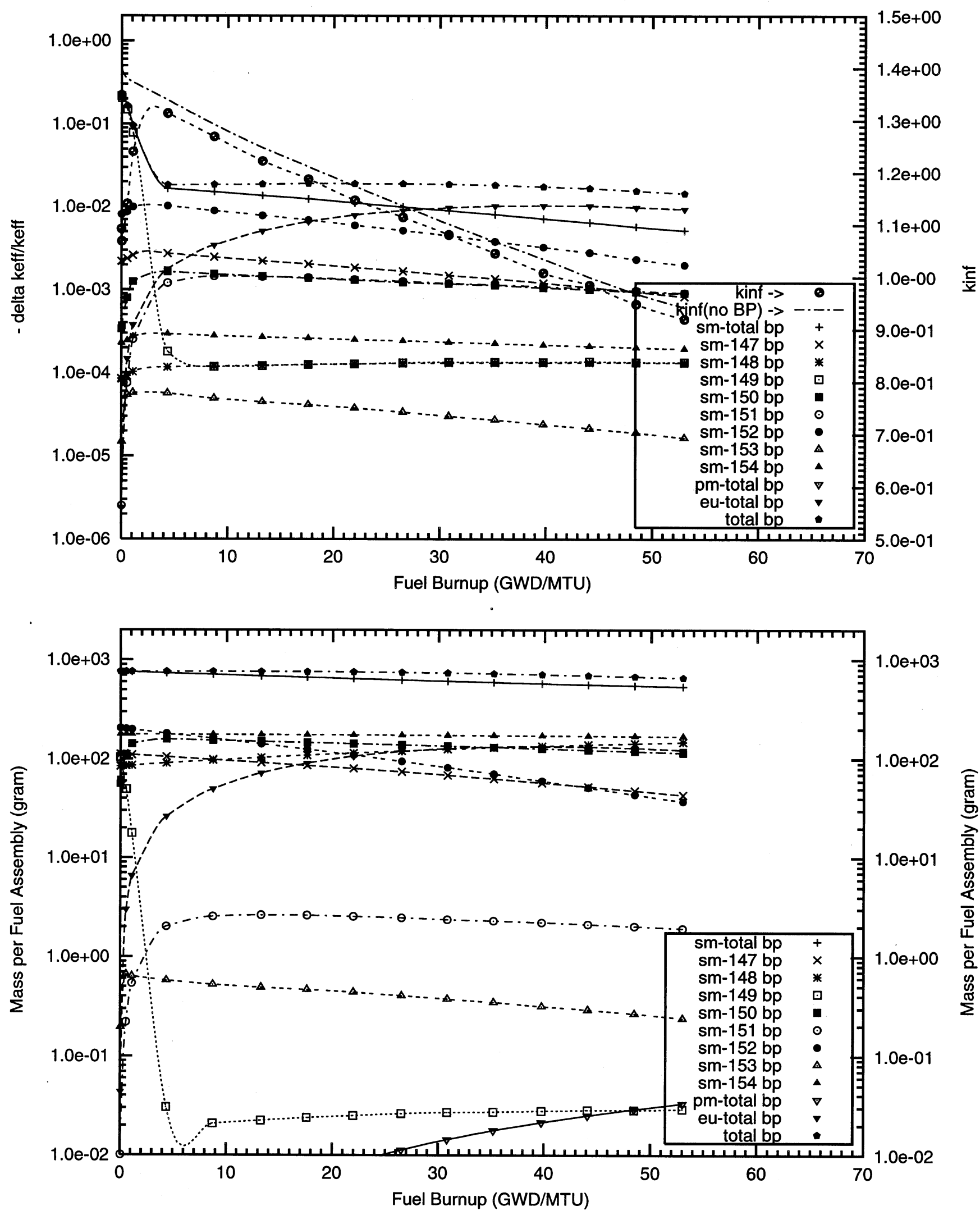


\section{B2 - Samarium Fully Enriched in ${ }^{149} \mathrm{Sm}$}


Figure B2.15. Negative Reactivity and Masses BP for Sm-149 and transmutation daughters as a function of fuel life for $17 \times 17$ fuel assemblies with 08 poison rods,

$2.0 \mathrm{w} \% \mathrm{Sm} 2 \mathrm{O} 3$ poison homogeneously mixed in the outer one-third volume of the UO2 pellets Reactor power $3400 \mathrm{MWth}, 193$ fuel assemblies, initial enrichment 4.5w\%U-235

(Case : sm149_2b_08_bp_fuel o)

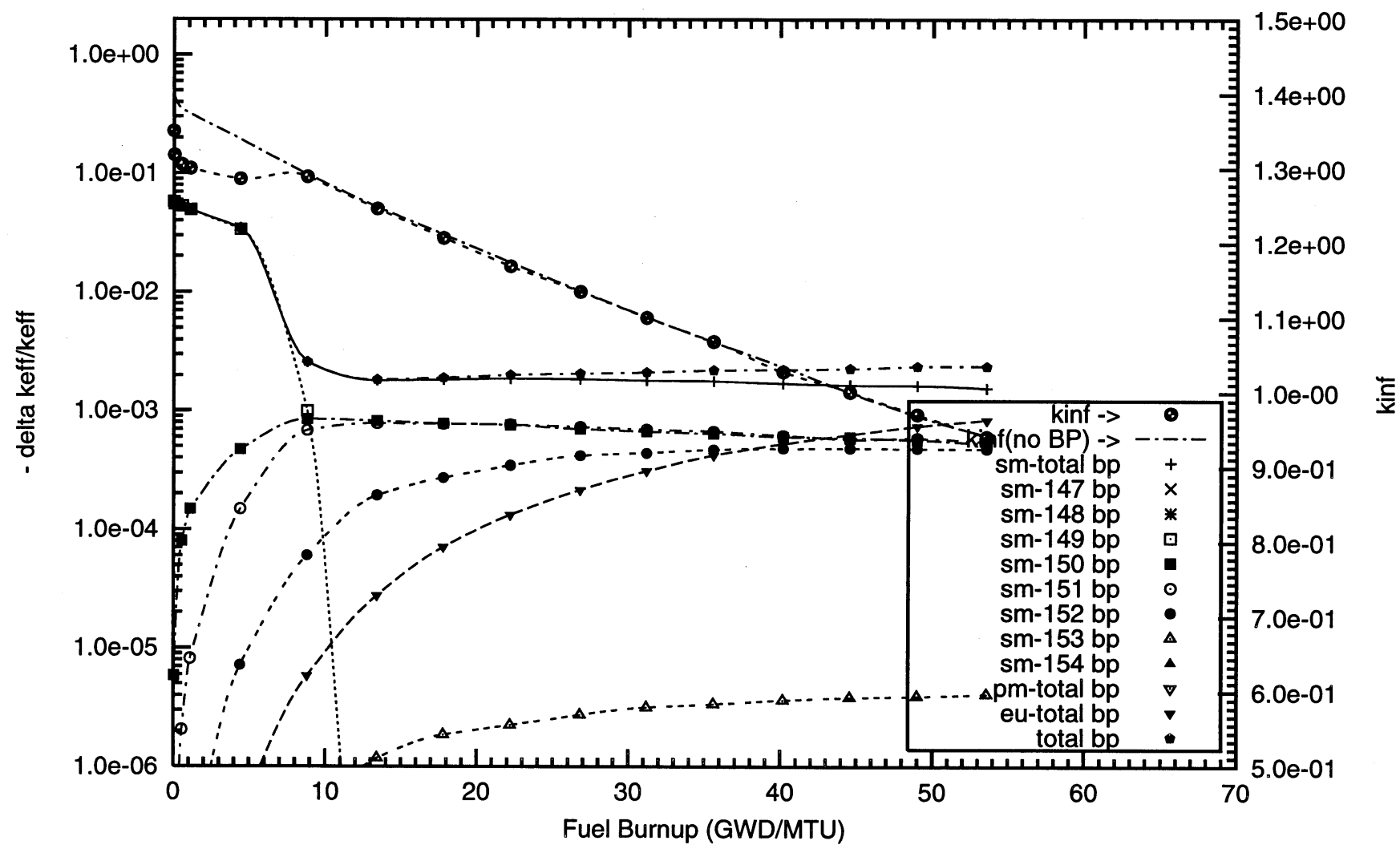


Figure B2.16. Negative Reactivity and Masses BP for Sm-149 and transmutation daughters as a function of fuel life for $17 \times 17$ fuel assemblies with 08 poison rods,

$4.0 \mathrm{w} \% \mathrm{Sm} 2 \mathrm{O} 3$ poison homogeneously mixed in the outer one-third volume of the UO2 pellets Reactor power $3400 \mathrm{MW}$ th, 193 fuel assemblies, initial enrichment $4.5 \mathrm{w} \% \mathrm{U}-235$

(Case : sm149_3b_08_bp_fuel o)

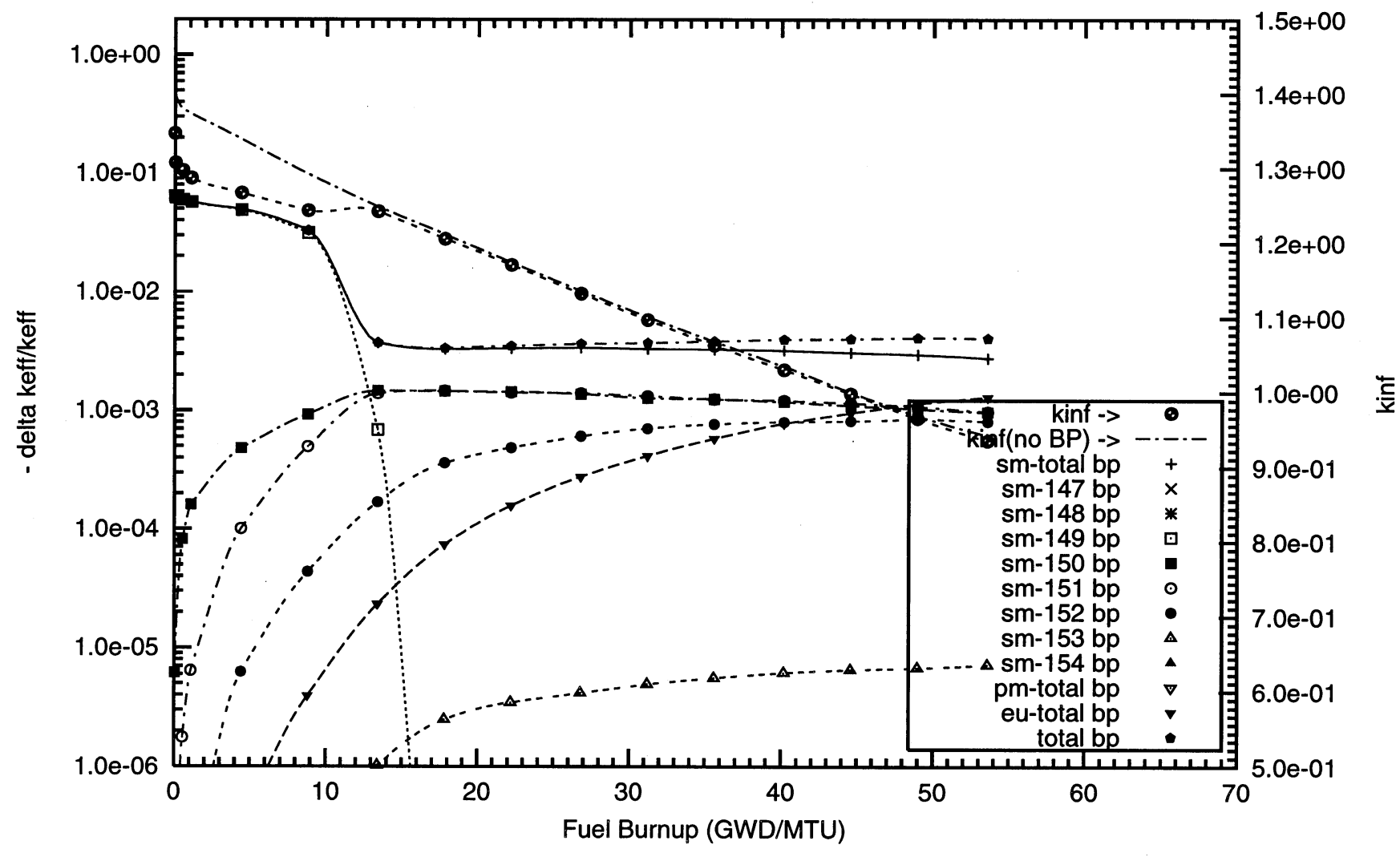


Figure B2.17. Negative Reactivity and Masses BP for Sm-149 and transmutation daughters as a function of fuel life for $17 \times 17$ fuel assemblies with 08 poison rods,

$8.0 \mathrm{w} \% \mathrm{Sm} 2 \mathrm{O} 3$ poison homogeneously mixed in the outer one-third volume of the $\mathrm{UO} 2$ pellets Reactor power $3400 \mathrm{MW}$ th, 193 fuel assemblies, initial enrichment $4.5 \mathrm{w} \% \mathrm{U}-235$

(Case : sm149_4b_08_bp_fuel o)

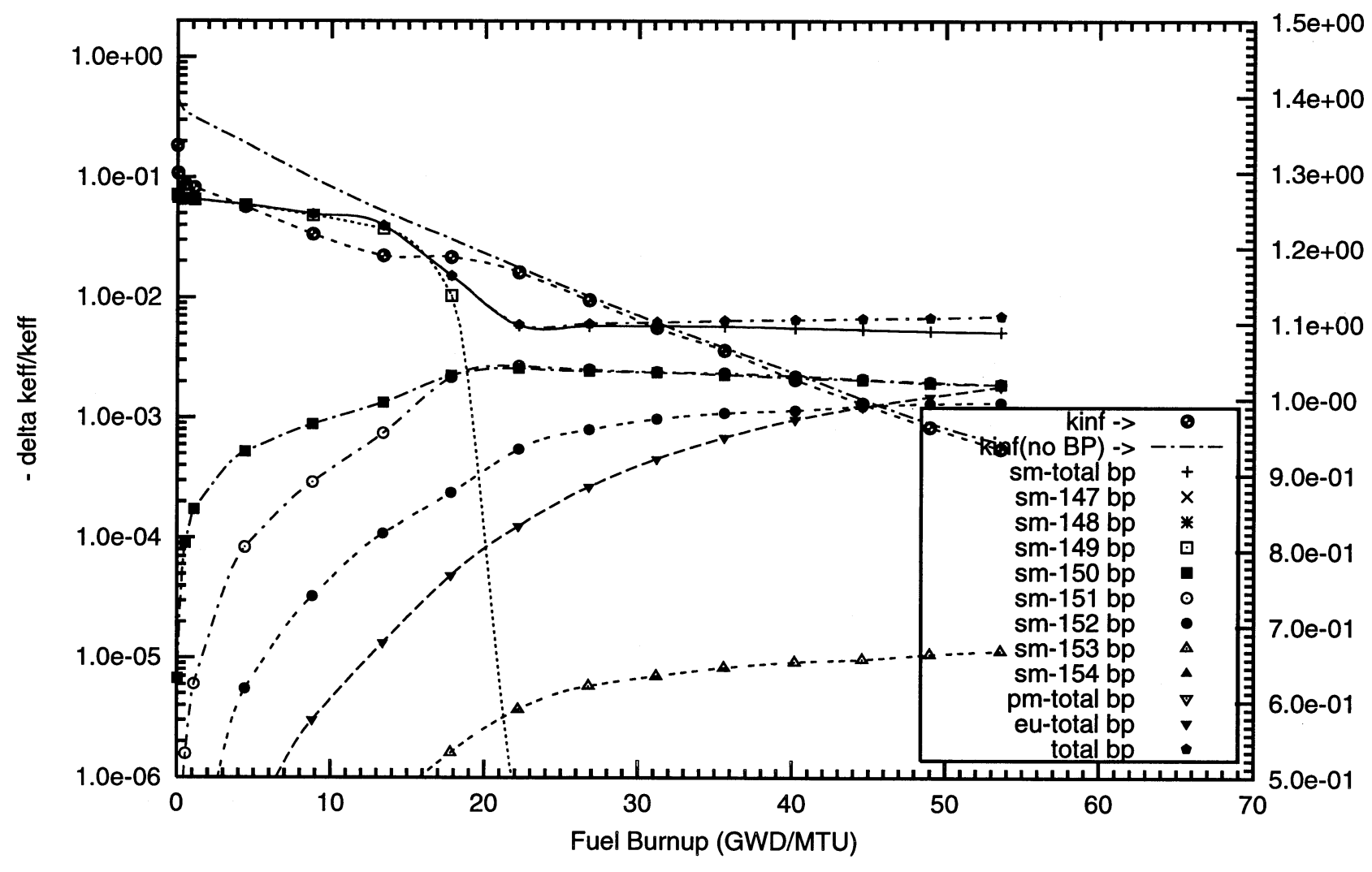

$\frac{\bar{E}}{\bar{x}}$ 
Figure B2.18. Negative Reactivity and Masses BP for Sm-149 and transmutation daughters as a function of fuel life for $17 \times 17$ fuel assemblies with 08 poison rods,

$12.0 \mathrm{w} \% \mathrm{Sm} 2 \mathrm{O} 3$ poison homogeneously mixed in the outer one-third volume of the UO2 pellets

Reactor power $3400 \mathrm{MWth}, 193$ fuel assemblies, initial enrichment $4.5 \mathrm{w} \% \mathrm{U}-235$

(Case : sm149_5b_08_bp_fuel o)

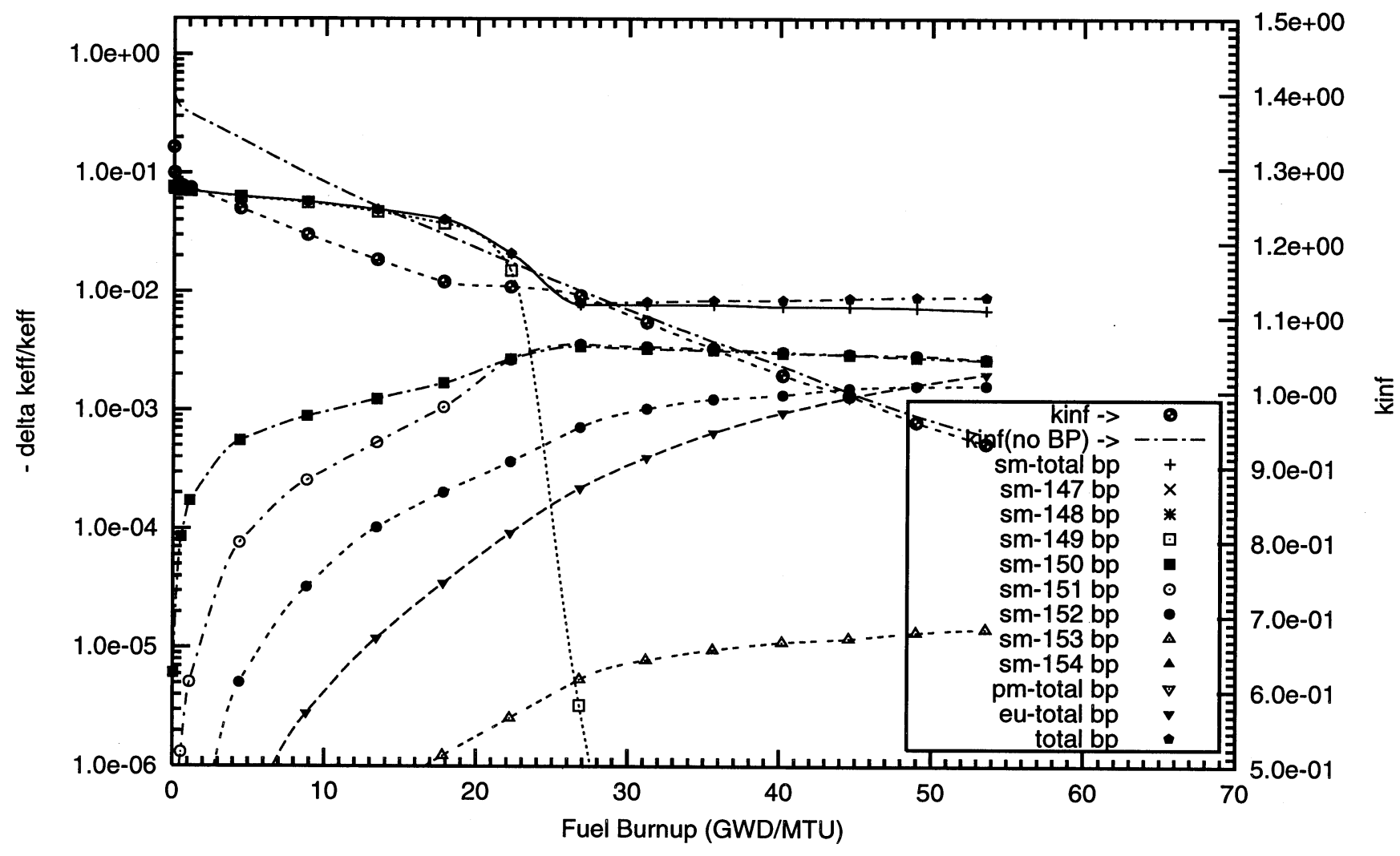


Figure B2.19. Negative Reactivity and Masses BP for Sm-149 and transmutation daughters as a function of fuel life for $17 \times 17$ fuel assemblies with 08 poison rods,

$16.0 \mathrm{w} \% \mathrm{Sm} 2 \mathrm{O} 3$ poison homogeneously mixed in the outer one-third volume of the UO2 pellets

Reactor power $3400 \mathrm{MWth}, 193$ fuel assemblies, initial enrichment 4.5w\%U-235 (Case : sm149_6b_08_bp_fuel o)

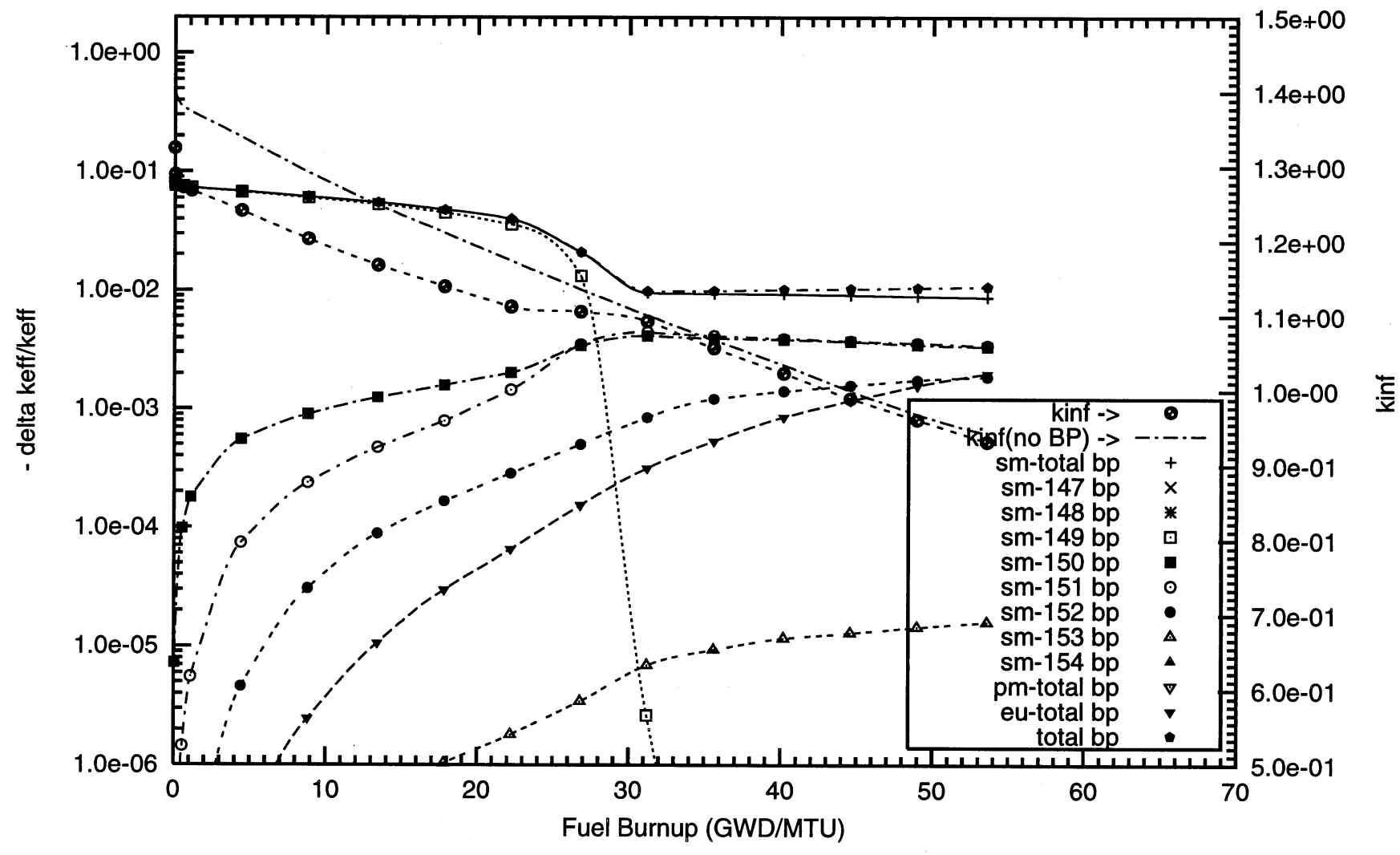


Figure B2.20. Negative Reactivity and Masses BP for Sm-149 and transmutation daughters as a function of fuel life for $17 \times 17$ fuel assemblies with 16 poison rods,

$0.2 \mathrm{w} \% \mathrm{Sm} 2 \mathrm{O} 3$ poison homogeneously mixed in the outer one-third volume of the UO2 pellets

Reactor power $3400 \mathrm{MWth}, 193$ fuel assemblies, initial enrichment 4.5W\%U-235

(Case : sm149_8b_100p_h2o_16_bp_fuel_2_o)
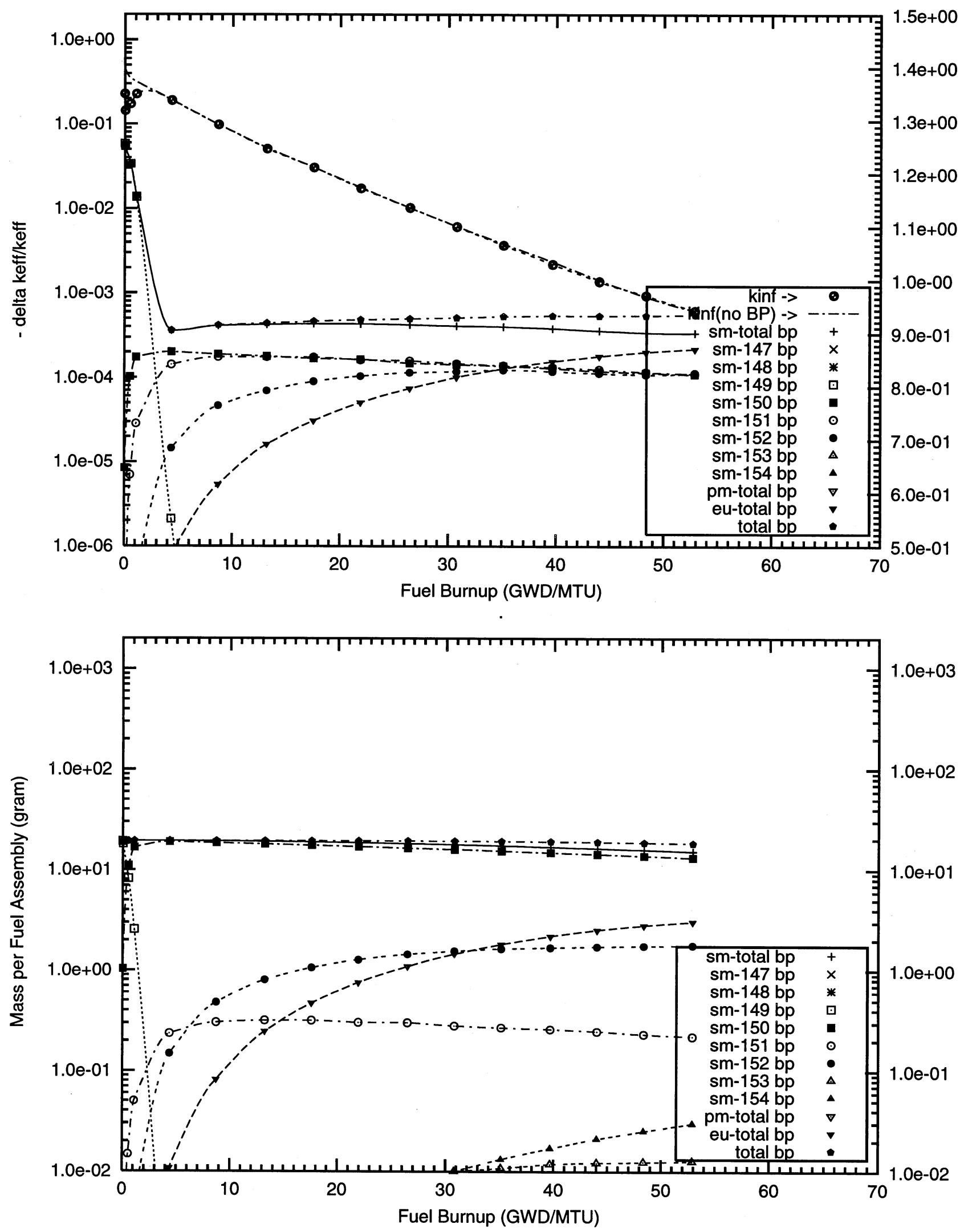
Figure B2.21. Negative Reactivity and Masses BP for Sm-149 and transmutation daughters as a function of fuel life for $17 \times 17$ fuel assemblies with 16 poison rods,

$0.5 \mathrm{w} \% \mathrm{Sm} 2 \mathrm{O} 3$ poison homogeneously mixed in the outer one-third volume of the $\mathrm{UO} 2$ pellets Reactor power $3400 \mathrm{MWth}, 193$ fuel assemblies, initial enrichment 4.5w\%U-235

(Case : sm149_0b_100p_h2o_16_bp_fuel_2_o)
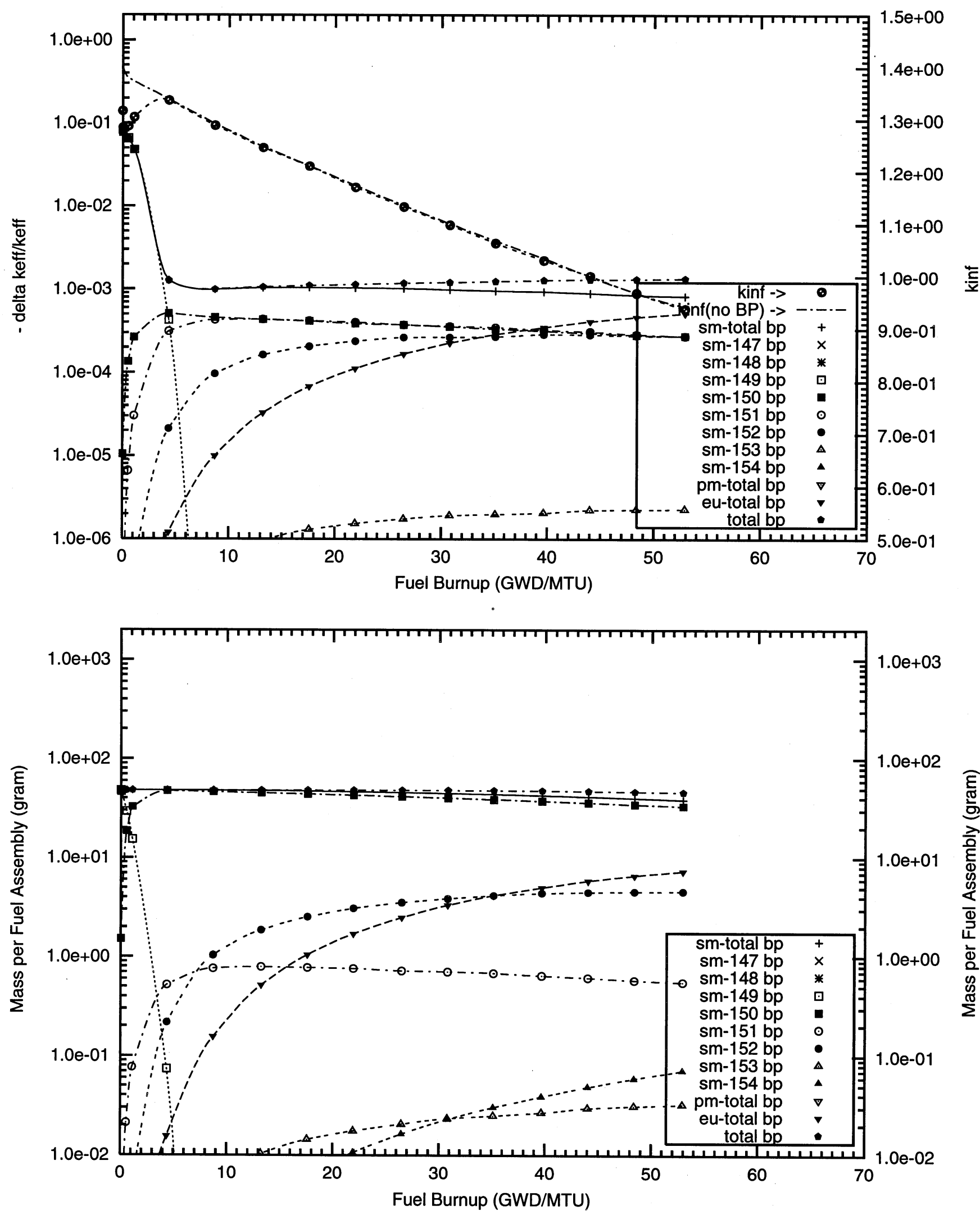
Figure B2.22. Negative Reactivity and Masses BP for Sm-149 and transmutation daughters as a function of fuel life for $17 \times 17$ fuel assemblies with 16 poison rods,

$1.0 \mathrm{w} \% \mathrm{Sm} 2 \mathrm{O} 3$ poison homogeneously mixed in the outer one-third volume of the UO2 pellets Reactor power $3400 \mathrm{MW}$ th, 193 fuel assemblies, initial enrichment 4.5w\%U-235 (Case : sm149_1b_100p_h20_16_bp_fuel_2_o)
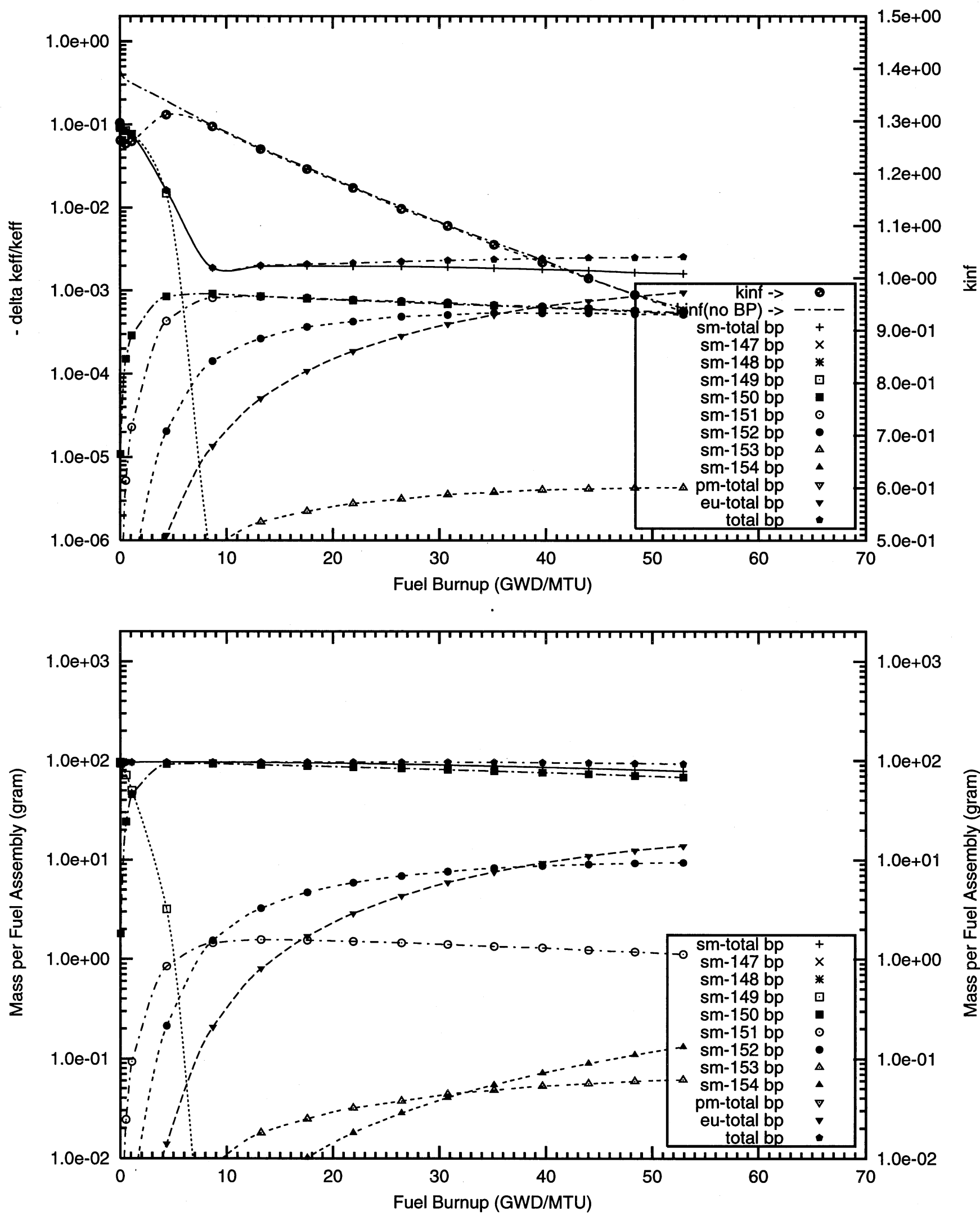
Figure B2.23. Negative Reactivity and Masses BP for Sm-149 and transmutation daughters as a function of fuel life for $17 \times 17$ fuel assemblies with 16 poison rods,

$2.0 \mathrm{w} \% \mathrm{Sm} 2 \mathrm{O} 3$ poison homogeneously mixed in the outer one-third volume of the UO2 pellets Reactor power $3400 \mathrm{MWth}, 193$ fuel assemblies, initial enrichment 4.5w\%U-235

(Case : sm149_2b_100p_h2o_16_bp_fuel_2_o)
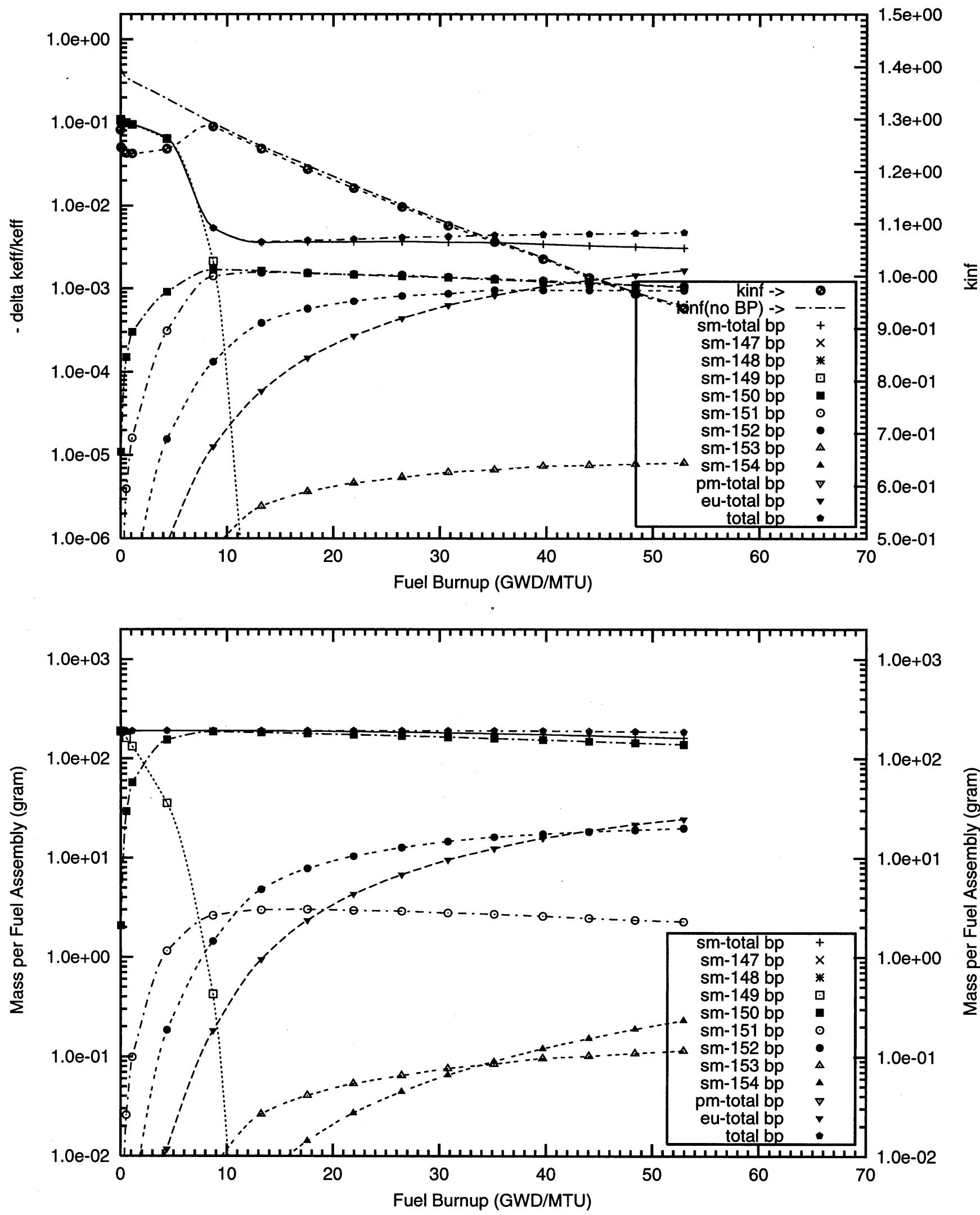
Figure B2.24. Negative Reactivity and Masses BP for Sm-149 and transmutation daughters as a function of fuel life for $17 \times 17$ fuel assemblies with 18 poison rods,

$1.0 \mathrm{w} \% \mathrm{Sm} 2 \mathrm{O} 3$ poison homogeneously mixed in the outer one-third volume of the $\mathrm{UO} 2$ pellets Reactor power $3400 \mathrm{MWth}, 193$ fuel assemblies, initial enrichment $4.5 \mathrm{w} \% \mathrm{U}-235$

(Case : sm149_1b_16_bp_fuel o)
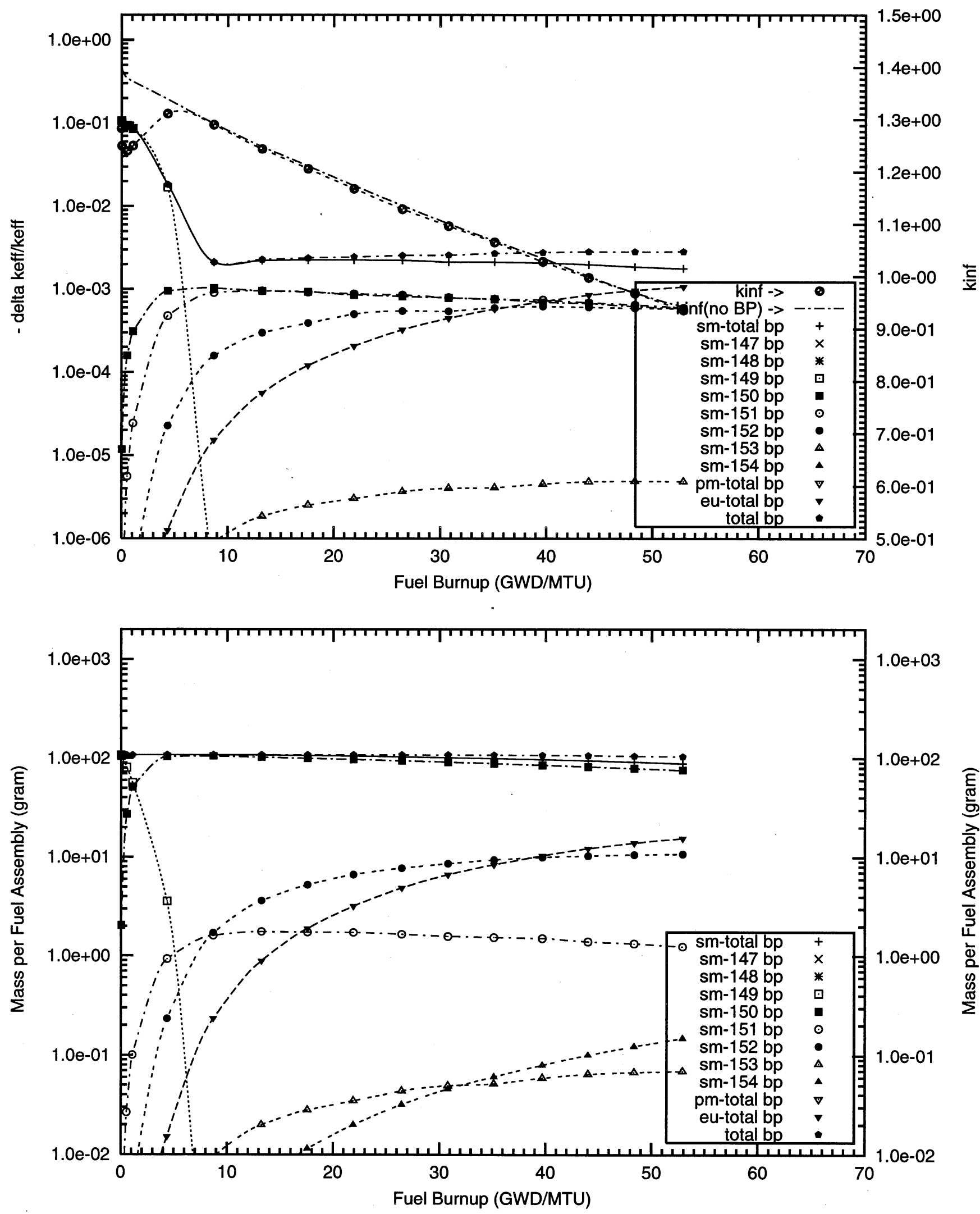
Figure B2.25. Negative Reactivity and Masses BP for Sm-149 and transmutation daughters as a function of fuel life for $17 \times 17$ fuel assemblies with 18 poison rods,

$2.0 \mathrm{w} \% \mathrm{Sm} 2 \mathrm{O} 3$ poison homogeneously mixed in the outer one-third volume of the $\mathrm{UO} 2$ pellets Reactor power $3400 \mathrm{MWth}, 193$ fuel assemblies, initial enrichment $4.5 \mathrm{w} \% \mathrm{U}-235$

(Case : sm149_2b_16_bp_fuel (old) o)
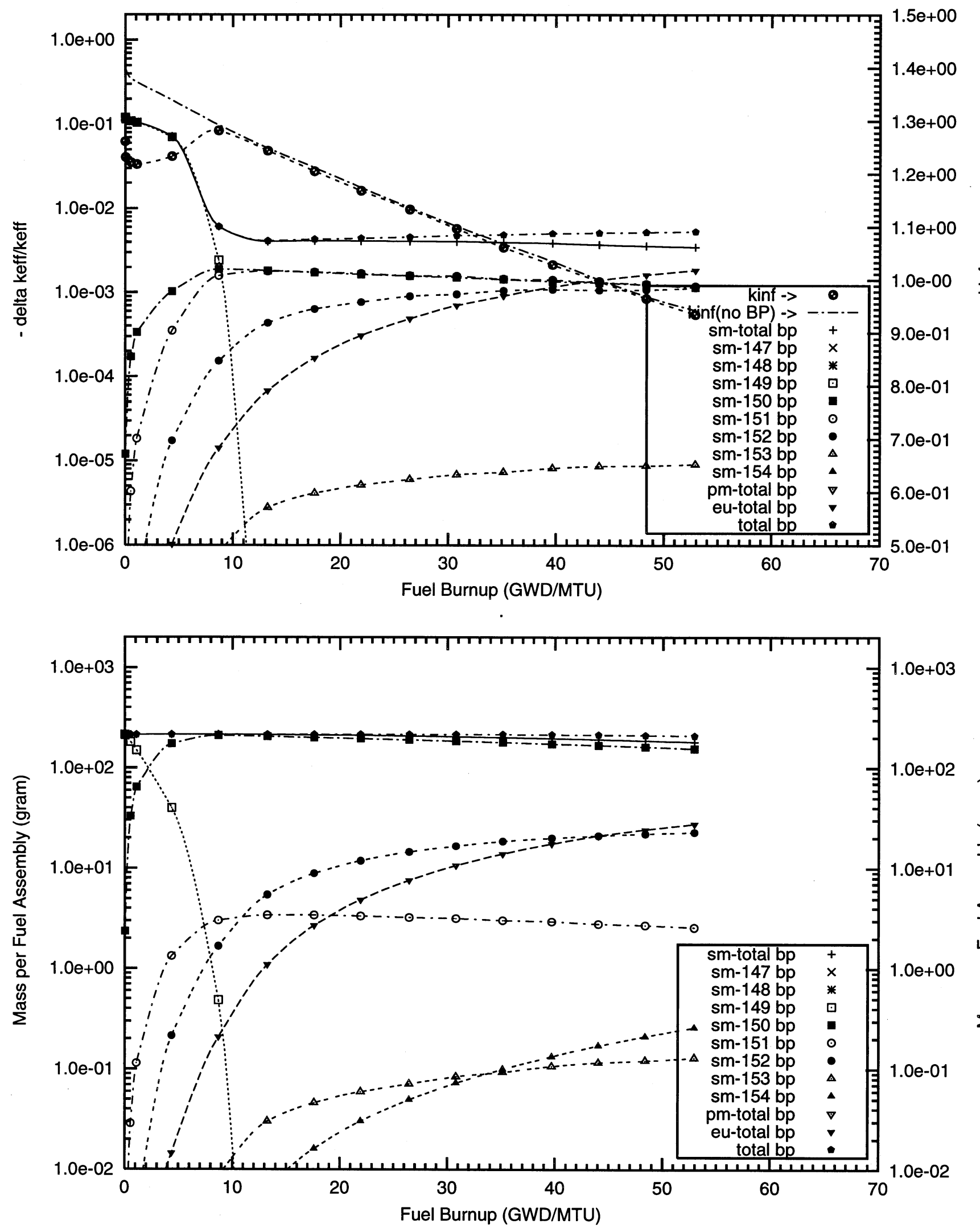
Figure B2.26. Negative Reactivity and Masses BP for Sm-149 and transmutation daughters as a function of fuel life for $17 \times 17$ fuel assemblies with 64 poison rods,

$0.05 \mathrm{w} \%$ Sm2O3 poison homogeneously mixed in the outer one-third volume of the UO2 pellets

Reactor power $3400 \mathrm{MWth}, 193$ fuel assemblies, initial enrichment $4.5 \mathrm{w} \% \mathrm{U}-235$

(Case : sm149_10b_100p_h2o_64_bp_fuel_2_o)
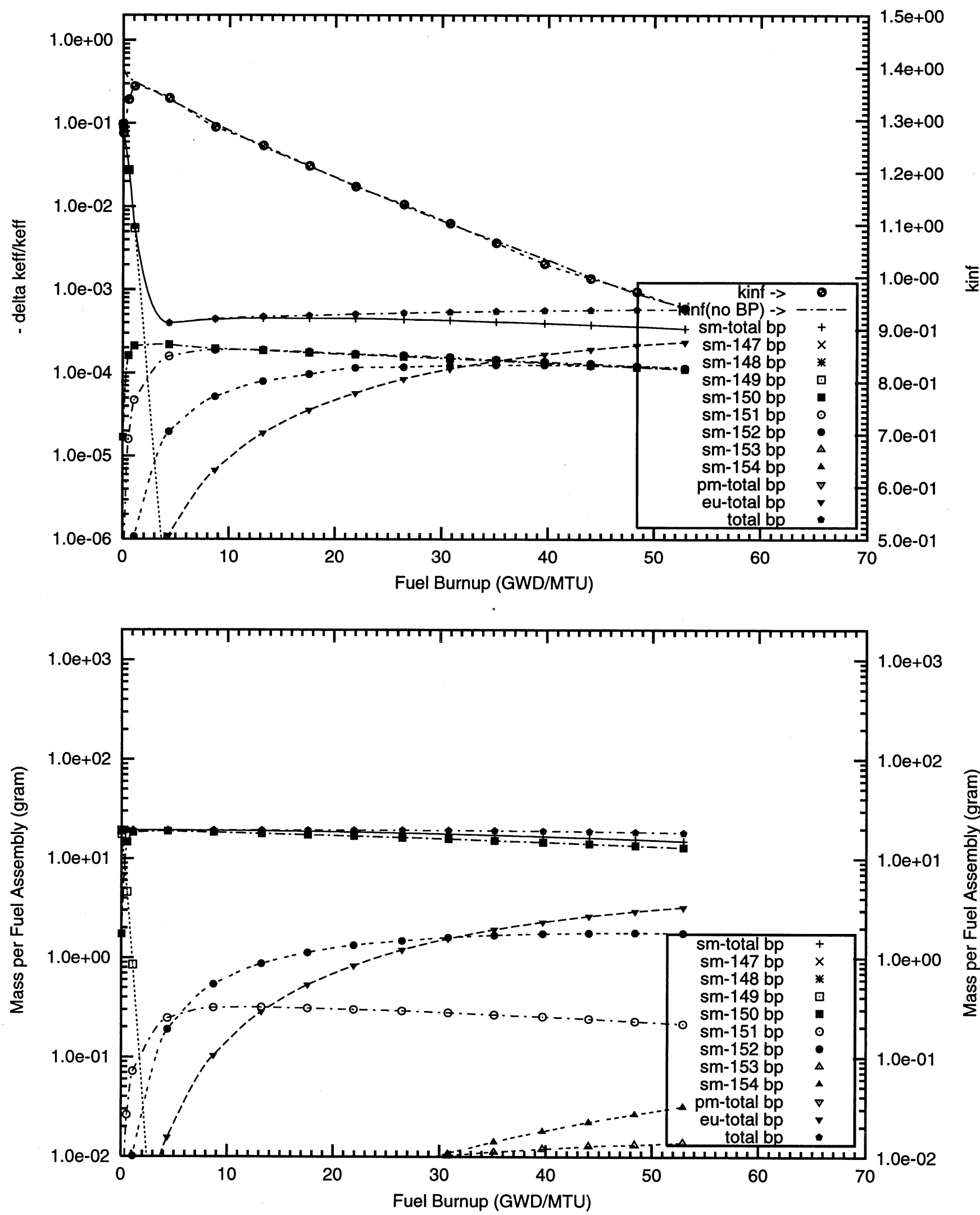
Figure B2.27. Negative Reactivity and Masses BP for Sm-149 and transmutation daughters as a function of fuel life for $17 \times 17$ fuel assemblies with 64 poison rods,

$0.1 \mathrm{w} \% \mathrm{Sm} 2 \mathrm{O} 3$ poison homogeneously mixed in the outer one-third volume of the UO2 pellets

Reactor power $3400 \mathrm{MW}$ th, 193 fuel assemblies, initial enrichment $4.5 \mathrm{w} \% \mathrm{U}-235$

(Case : sm149_9b_100p_h2o_64_bp_fuel_2_o)
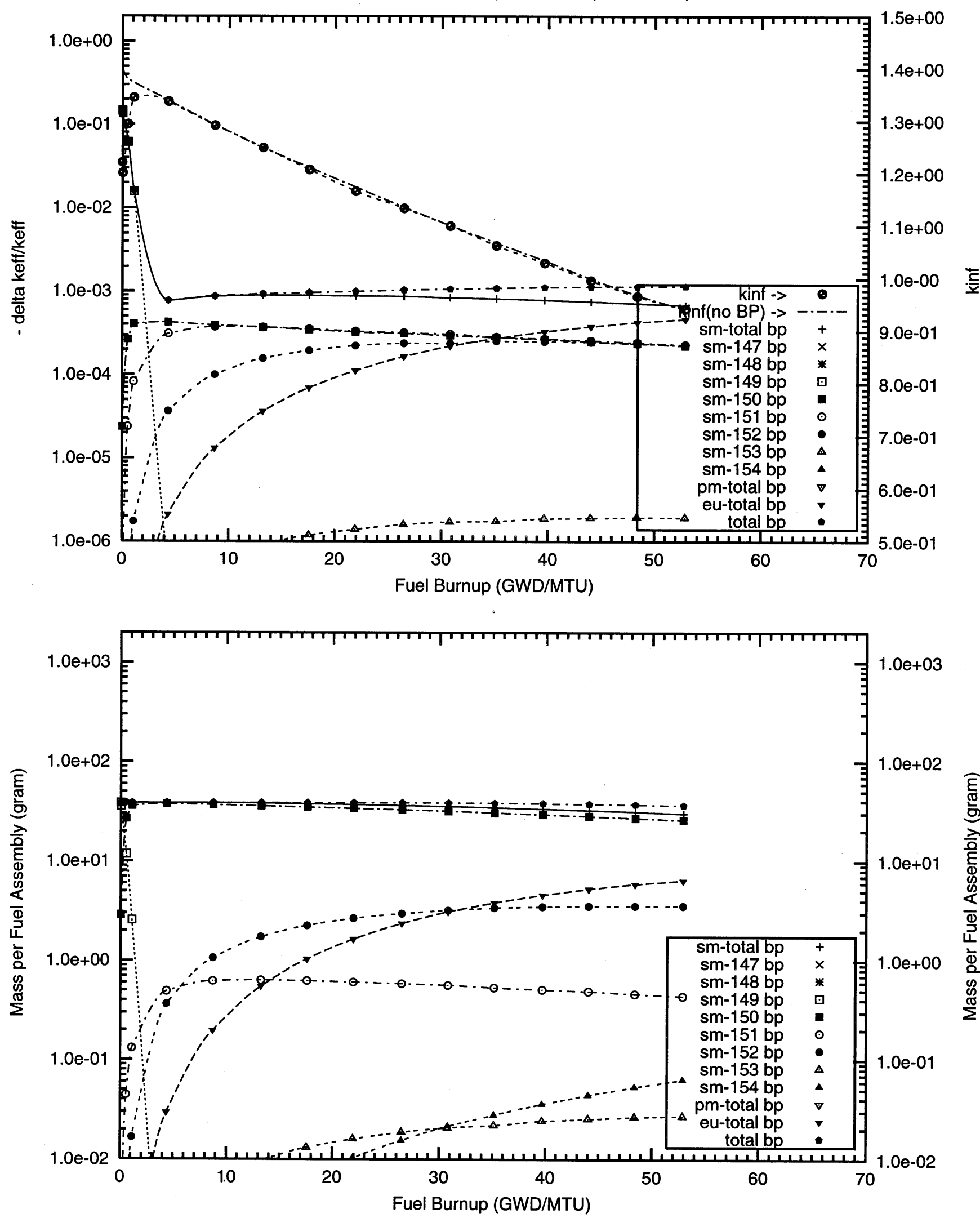
Figure B2.28. Negative Reactivity and Masses BP for Sm-149 and transmutation daughters as a function of fuel life for $17 \times 17$ fuel assemblies with 64 poison rods,

$0.2 \mathrm{w} \% \mathrm{Sm} 2 \mathrm{O} 3$ poison homogeneously mixed in the outer one-third volume of the UO2 pellets

Reactor power $3400 \mathrm{MW}$ th, 193 fuel assemblies, initial enrichment $4.5 \mathrm{w} \% \mathrm{U}-235$

(Case : sm149_8b_64_bp_fuel o)
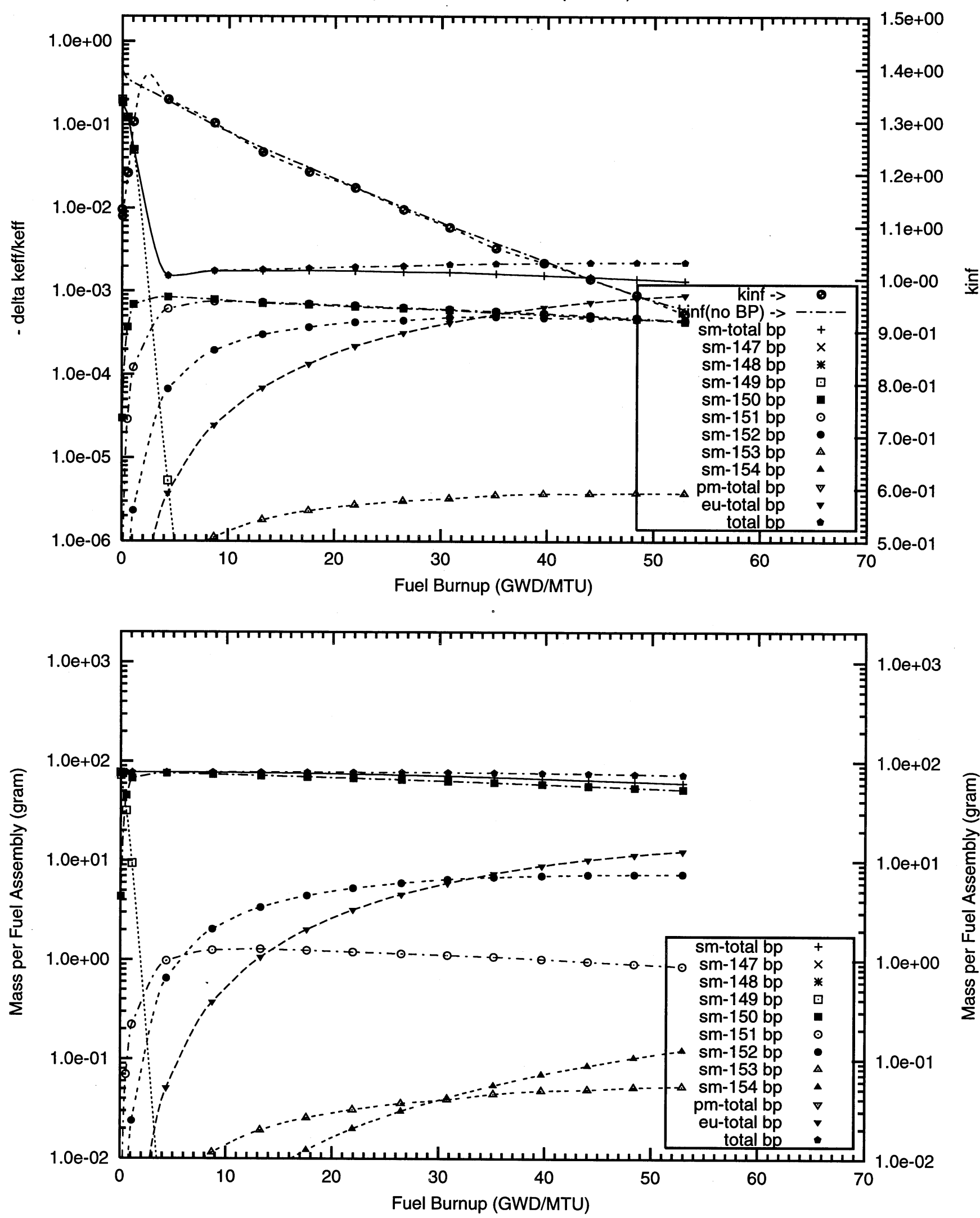
Appendix B3

Reactivity Worths and Masses of $\mathrm{Sm}_{2} \mathrm{O}_{3}$ Coating on the Surface of the Fuel Pellets

\section{Table 11 Cases}





\section{B3 - Samarium with Natural Isotopic Abundances}


Figure B3.1. Negative Reactivity and Masses BP for Sm-nat and transmutation daughters as a function of fuel life for $17 \times 17$ fuel assemblies with 08 poison rods,

2.0 mill thick Sm2O3 poison coating on the outer radial surface of the $\mathrm{UO} 2$ pellets Reactor power $3400 \mathrm{MWth}, 193$ fuel assemblies, initial enrichment 4.5W\%U-235 (Case : sm000_2f_08_bp_fuel o)
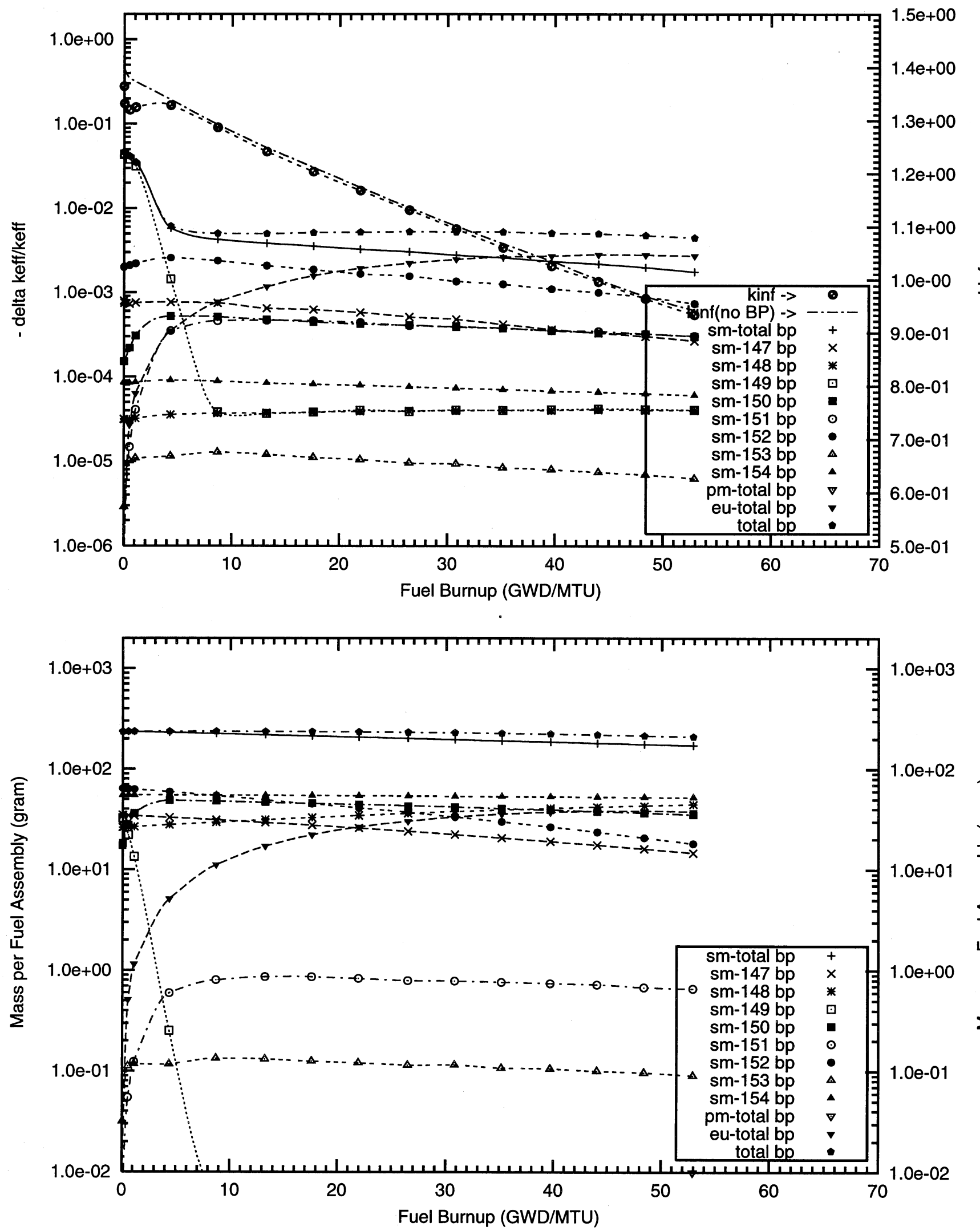
Figure B3.2. Negative Reactivity and Masses BP for Sm-nat and transmutation daughters as a function of fuel life for $17 \times 17$ fuel assemblies with 08 poison rods, 4.0 mill thick Sm2O3 poison coating on the outer radial surface of the UO2 pellets Reactor power $3400 \mathrm{MWth}, 193$ fuel assemblies, initial enrichment 4.5wt\%U-235 - (Case : sm000_3f_100p_h2o_08_bp_fuel_2_o)
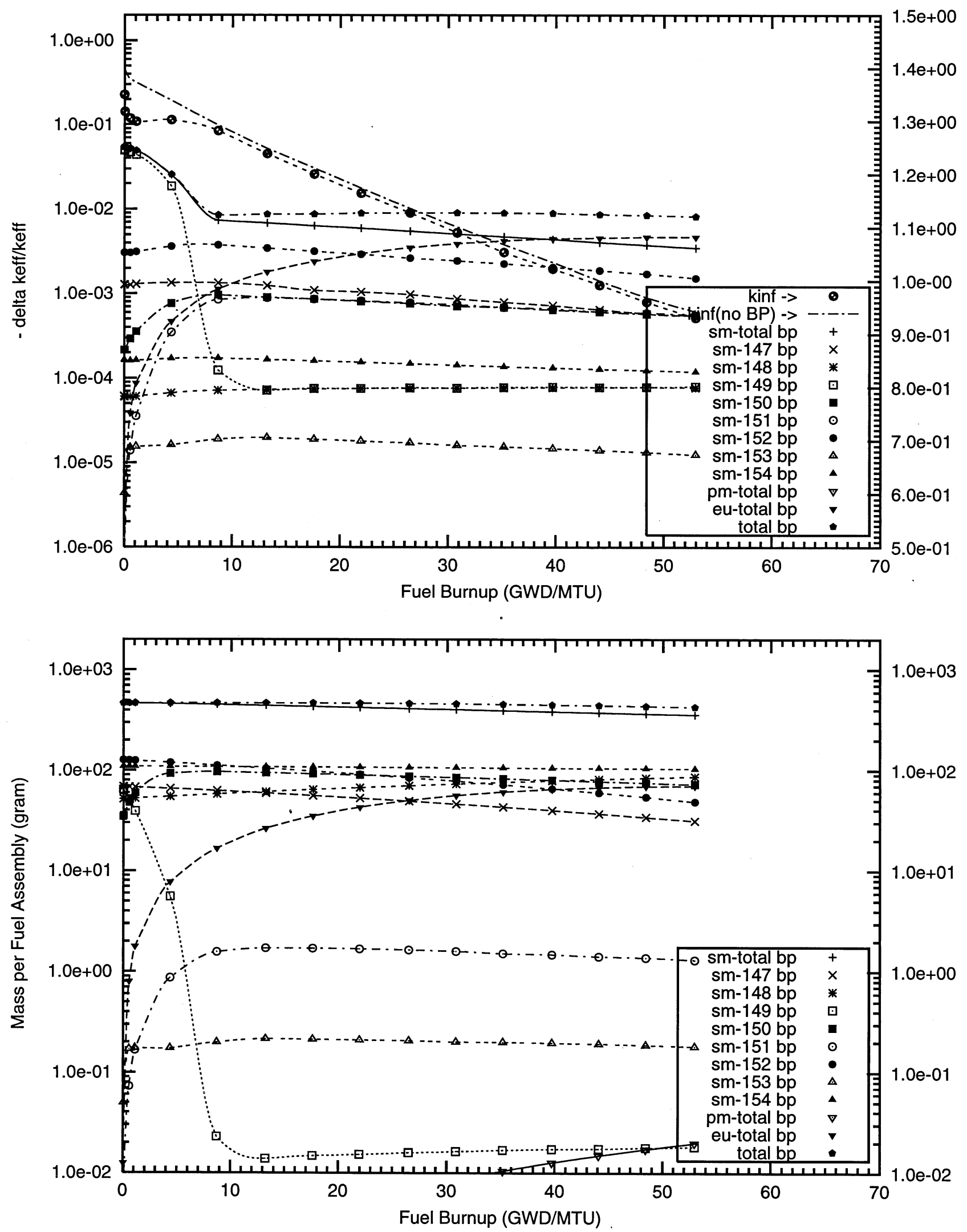
Figure B3.3. Negative Reactivity and Masses BP for Sm-nat and transmutation daughters as a function of fuel life for $17 \times 17$ fuel assemblies with 16 poison rods,

1.0 mill thick Sm2O3 poison coating on the outer radial surface of the UO2 pellets Reactor power $3400 \mathrm{MWth}, 193$ fuel assemblies, initial enrichment 4.5w\%U-235

(Case : sm000_1f_100p_h20_16_bp_fuel_2_o)
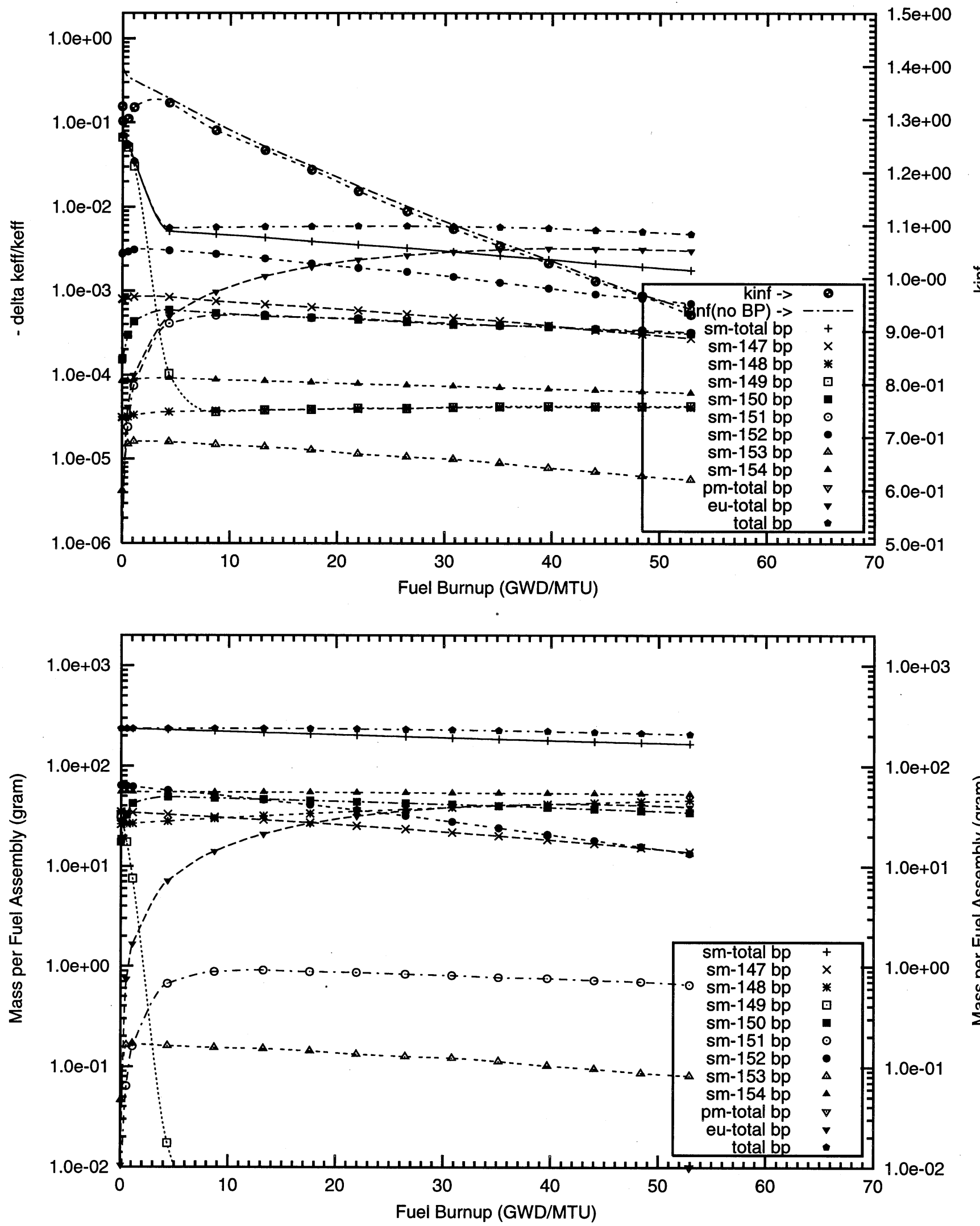
Figure B3.4. Negative Reactivity and Masses BP for Sm-nat and transmutation daughters as a function of fuel life for $17 \times 17$ fuel assemblies with 16 poison rods,

2.0 mill thick Sm2O3 poison coating on the outer radial surface of the UO2 pellets

Reactor power $3400 \mathrm{MWth}, 193$ fuel assemblies, initial enrichment $4.5 \mathrm{w} \% \mathrm{U}-235$

(Case : sm000_2f_100p_h20_16_bp_fuel_2_o)
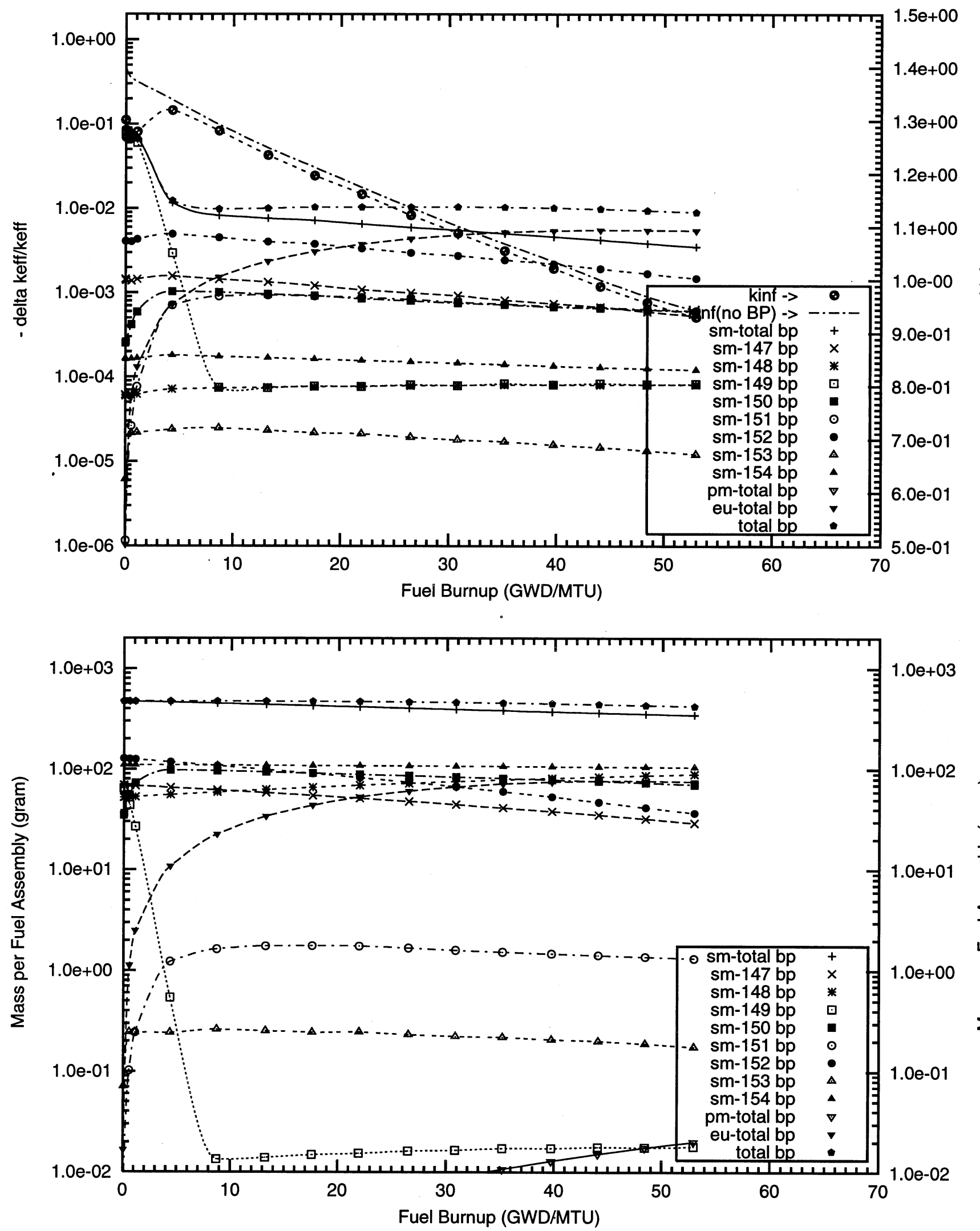
Figure B3.5. Negative Reactivity and Masses BP for Sm-nat and transmutation daughters as a function of fuel life for $17 \times 17$ fuel assemblies with 16 poison rods,

4.0 mill thick Sm2O3 poison coating on the outer radial surface of the UO2 pellets

Reactor power $3400 \mathrm{MWth}, 193$ fuel assemblies, initial enrichment 4.5w\%U-235

(Case : sm000_3f_100p_h20_16_bp_fuel_2_o)
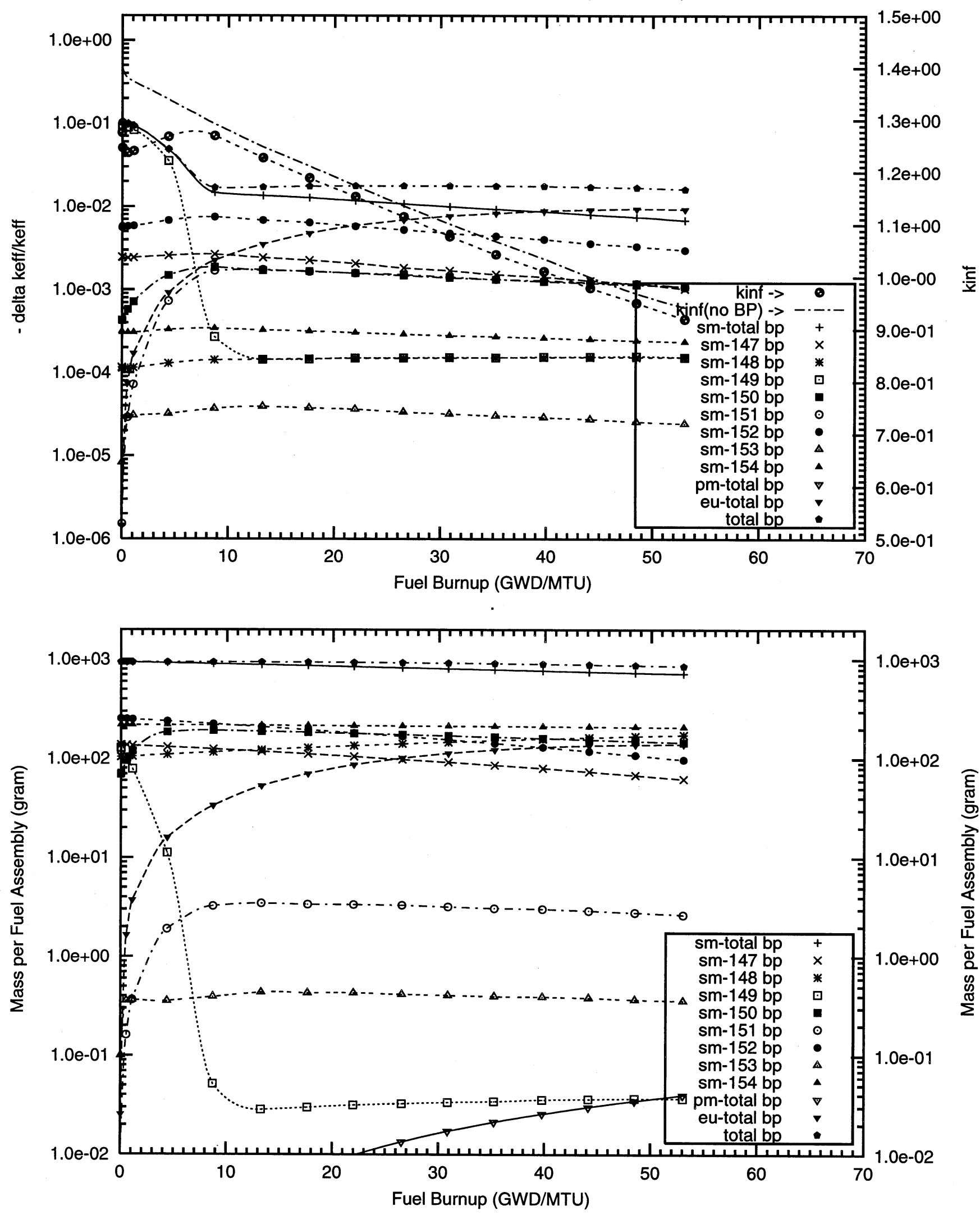
Figure B3.6. Negative Reactivity and Masses BP for Sm-nat and transmutation daughters as a function of fuel life for $17 \times 17$ fuel assemblies with 18 poison rods,

1.0 mill thick $\mathrm{Sm} 2 \mathrm{O} 3$ poison coating on the outer radial surface of the UO2 pellets Reactor power $3400 \mathrm{MWth}, 193$ fuel assemblies, initial enrichment 4.5w\%U-235

(Case : sm000_1f_16_bp_fuel (old) o)
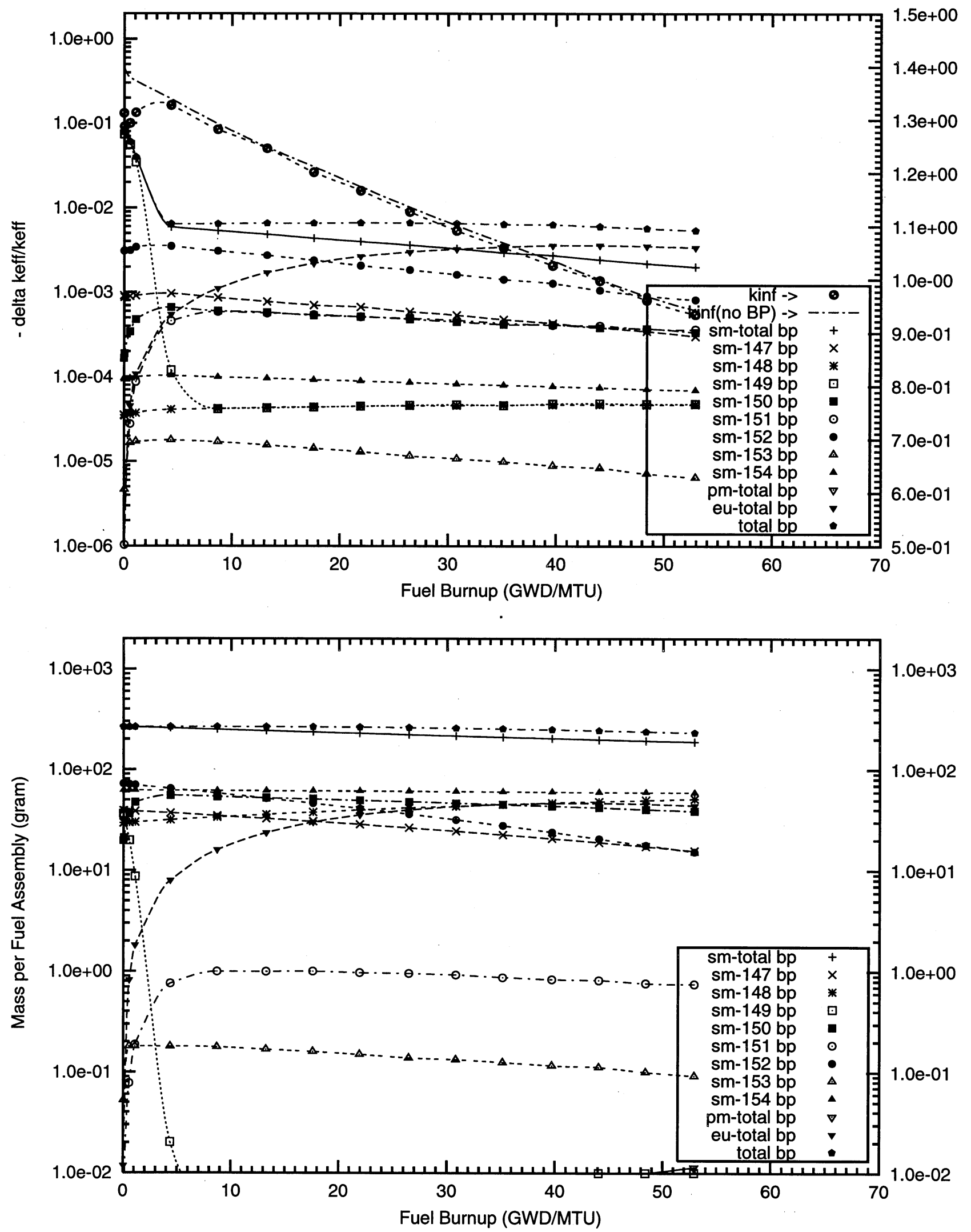
Figure B3.7. Negative Reactivity and Masses BP for Sm-nat and transmutation daughters as a function of fuel life for $17 \times 17$ fuel assemblies with 18 poison rods,

2.0 mill thick Sm2O3 poison coating on the outer radial surface of the UO2 pellets

Reactor power 3400 MWth, 193 fuel assemblies, initial enrichment 4.5w\%U-235

(Case : sm000_2f_16_bp_fuel (old) o)
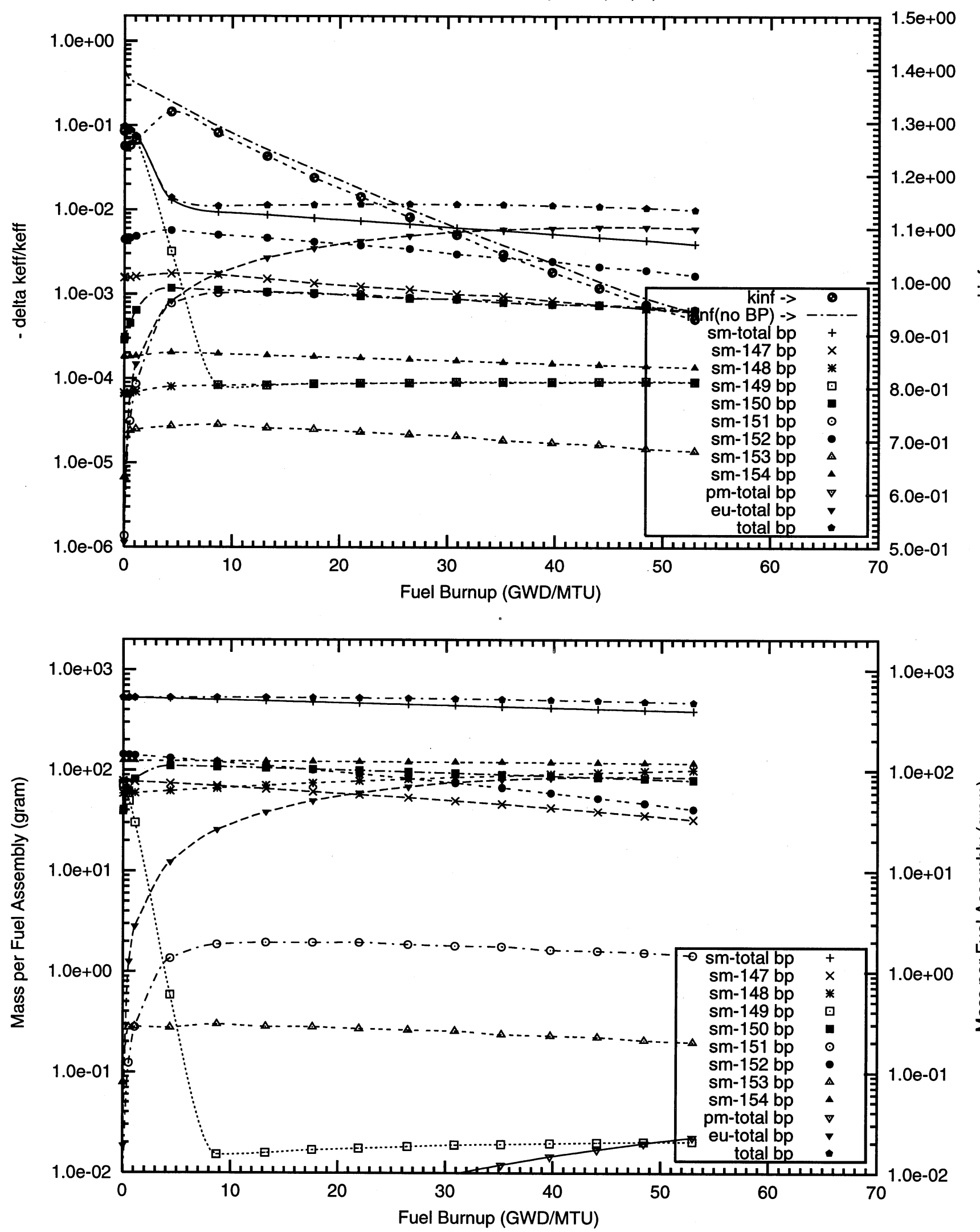
Figure B3.8. Negative Reactivity and Masses BP for Sm-nat and transmutation daughters as a function of fuel life for $17 \times 17$ fuel assemblies with 64 poison rods, 0.2 mill thick Sm2O3 poison coating on the outer radial surface of the $\mathrm{UO} 2$ pellets Reactor power $3400 \mathrm{MWth}, 193$ fuel assemblies, initial enrichment 4.5w\%U-235 (Case : sm000_8f_64_bp_fuel o)
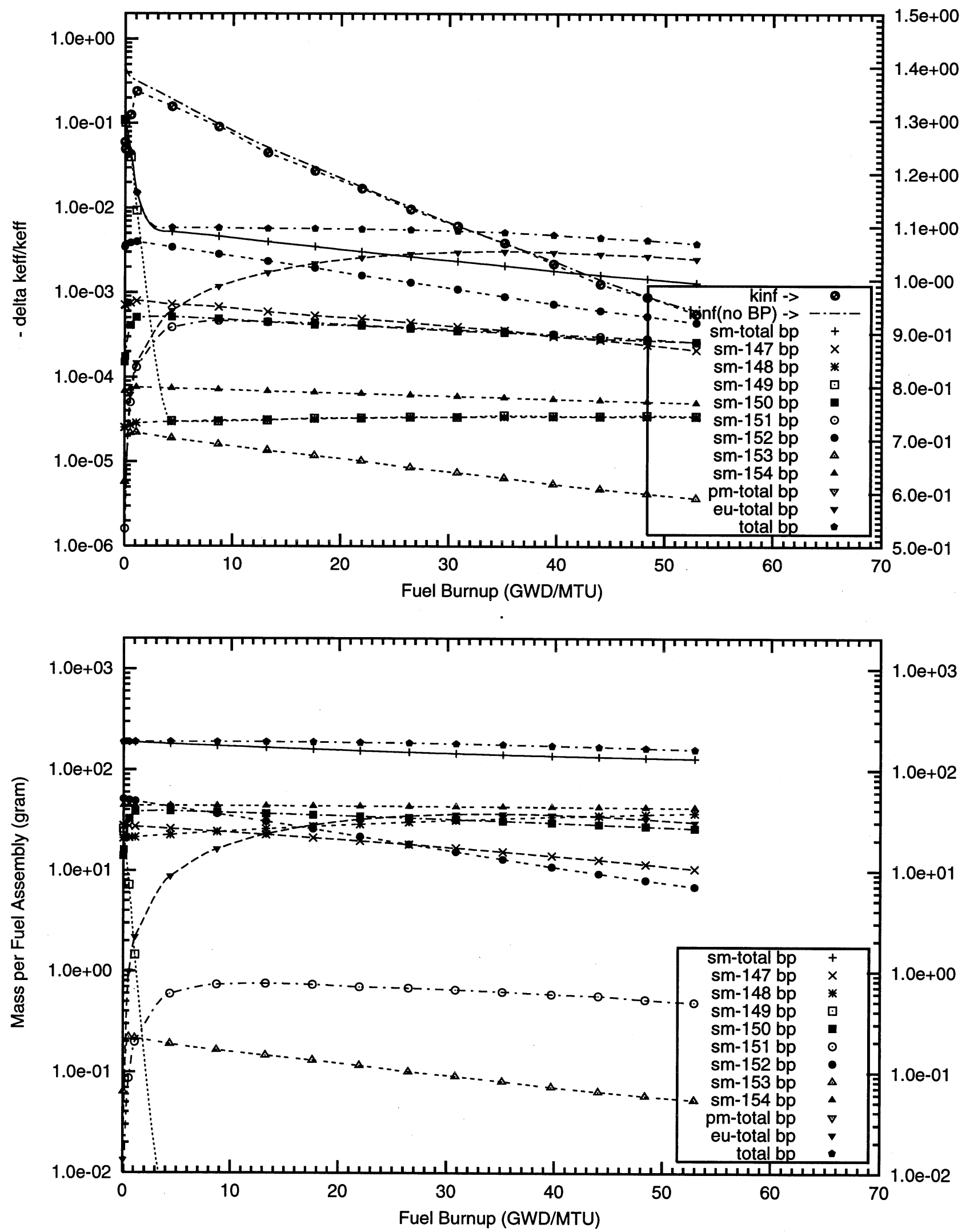
Figure B3.9. Negative Reactivity and Masses BP for Sm-nat and transmutation daughters as a function of fuel life for $17 \times 17$ fuel assemblies with 64 poison rods,

0.5 mill thick Sm2O3 poison coating on the outer radial surface of the UO2 pellets

Reactor power $3400 \mathrm{MWth}, 193$ fuel assemblies, initial enrichment $4.5 \mathrm{w} \% \mathrm{U}-235$

(Case : sm000_0f_64_bp_fuel o)

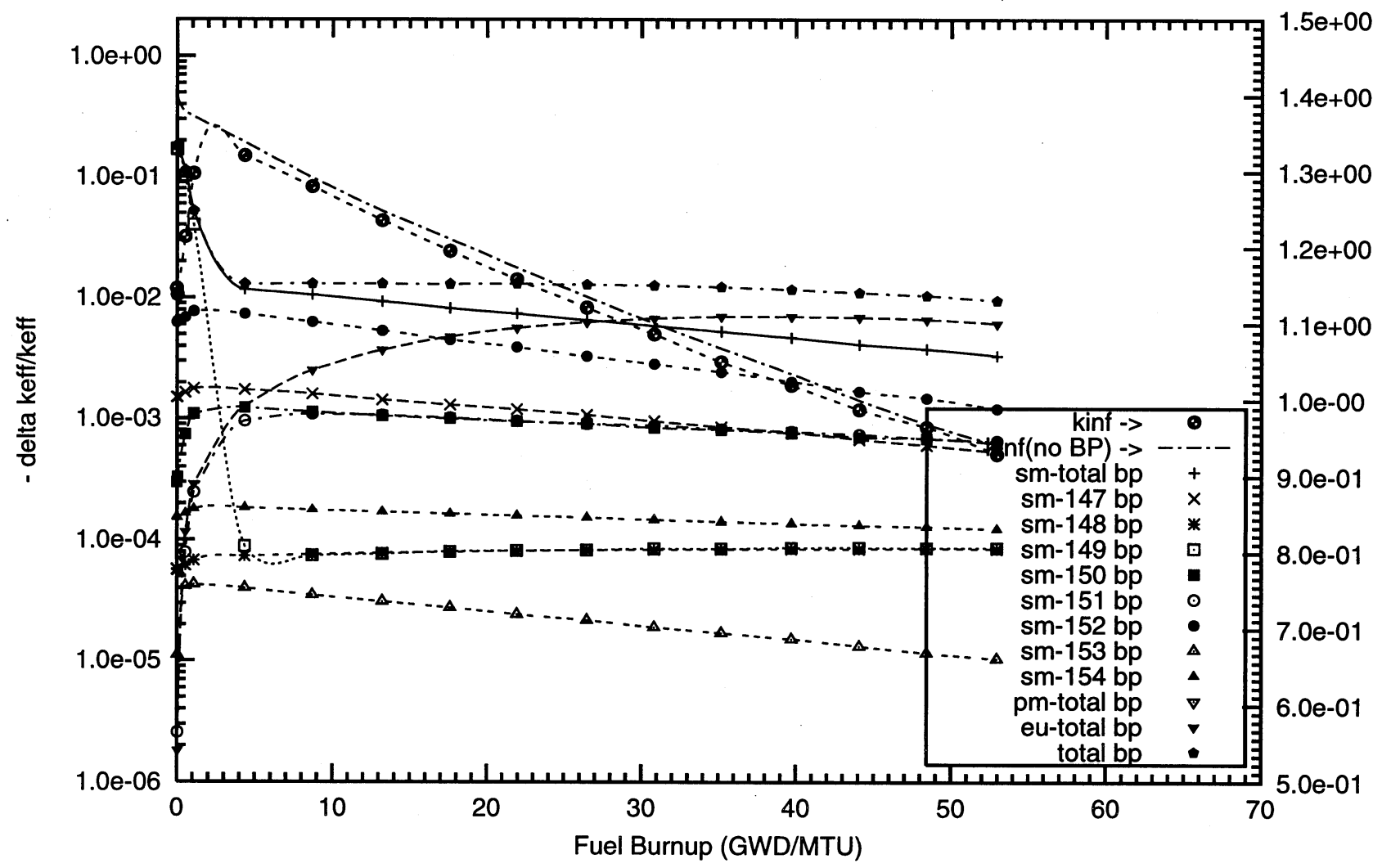

言

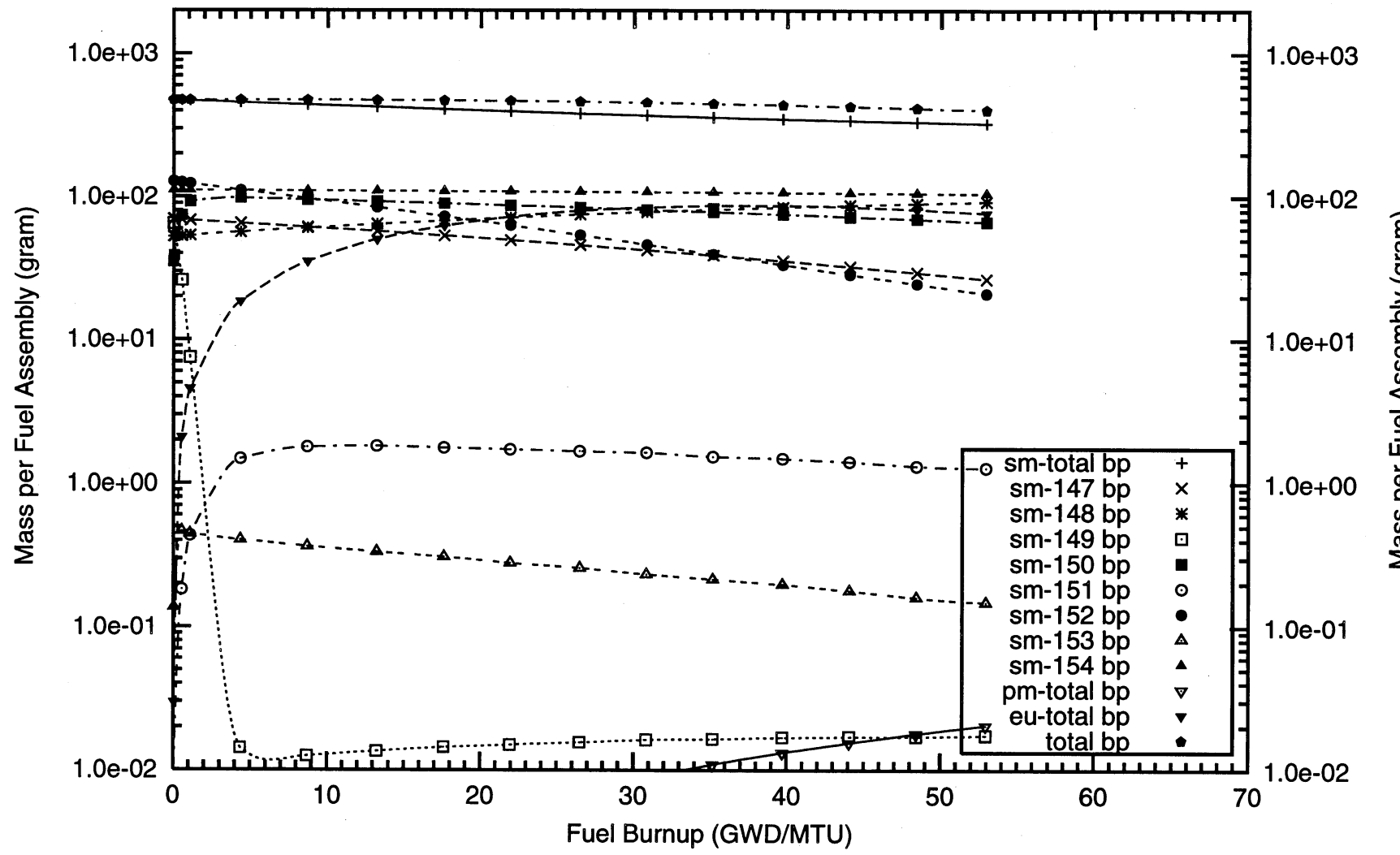


Figure B3.10. Negative Reactivity and Masses BP for Sm-nat and transmutation daughters as a function of fuel life for $17 \times 17$ fuel assemblies with 64 poison rods, 1.0 mill thick Sm2O3 poison coating on the outer radial surface of the UO2 pellets Reactor power $3400 \mathrm{MWth}, 193$ fuel assemblies, initial enrichment 4.5w\%U-235 (Case : sm000_1f_64_bp_fuel o)
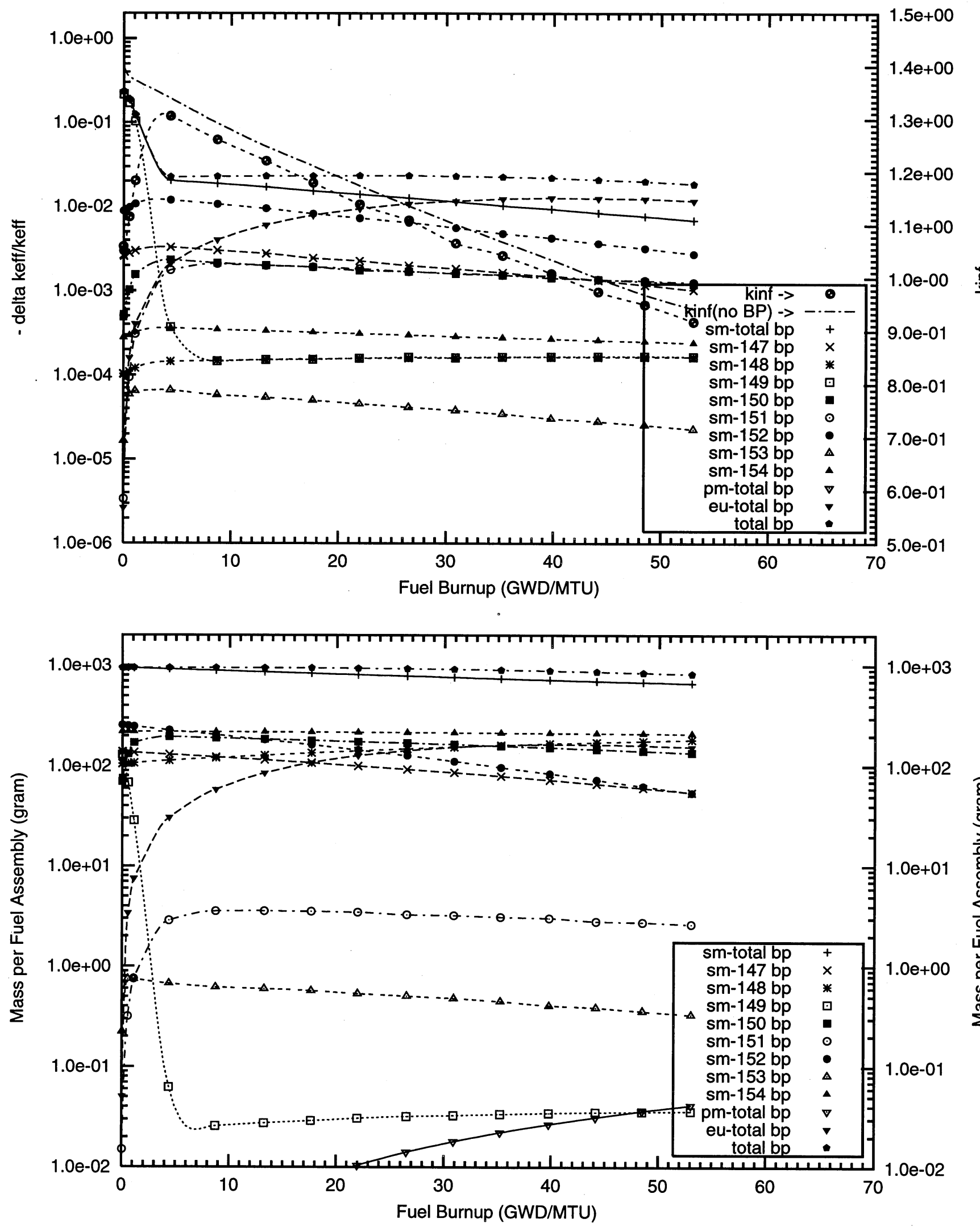
Figure B3.11. Negative Reactivity and Masses BP for Sm-nat and transmutation daughters as a function of fuel life for $17 \times 17$ fuel assemblies with 104 poison rods,

1.0 mill thick (0.10TD) Sm2O3 poison coating on the outer radial surface of the UOO2 pellets

Reactor power $3400 \mathrm{MWth}, 193$ fuel assemblies, initial enrichment 4.5w\%U-235

(Case : sm000_11f_104_bp_fuel o)
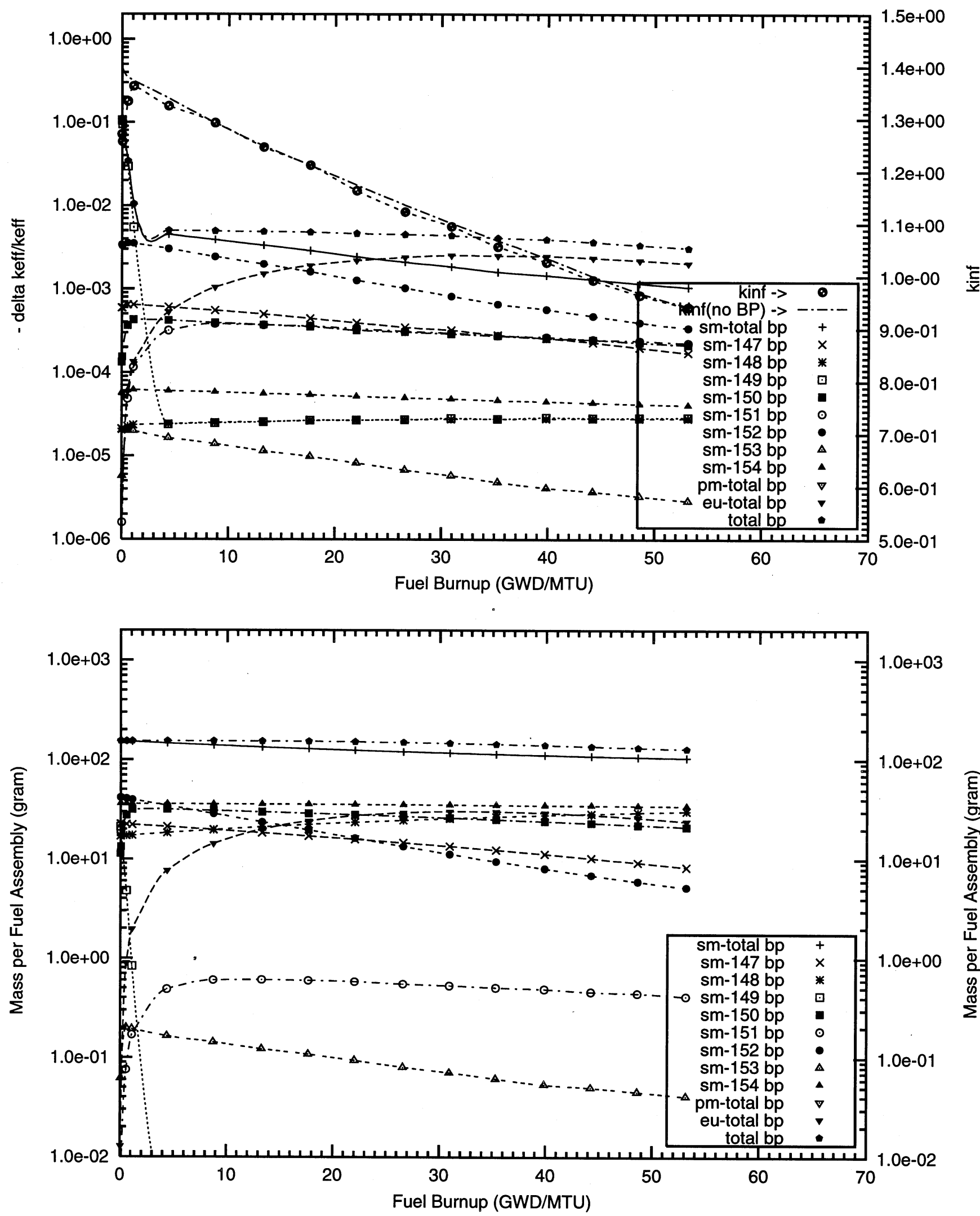
Figure B3.12. Negative Reactivity and Masses BP for Sm-nat and transmutation daughters as a function of fuel life for $17 \times 17$ fuel assemblies with 104 poison rods,

0.1 mill thick Sm2O3 poison coating on the outer radial surface of the UO2 pellets

Reactor power $3400 \mathrm{MWth}, 193$ fuel assemblies, initial enrichment $4.5 \mathrm{w} \% \mathrm{U}-235$

(Case : sm000_9f_104_bp_fuel o)
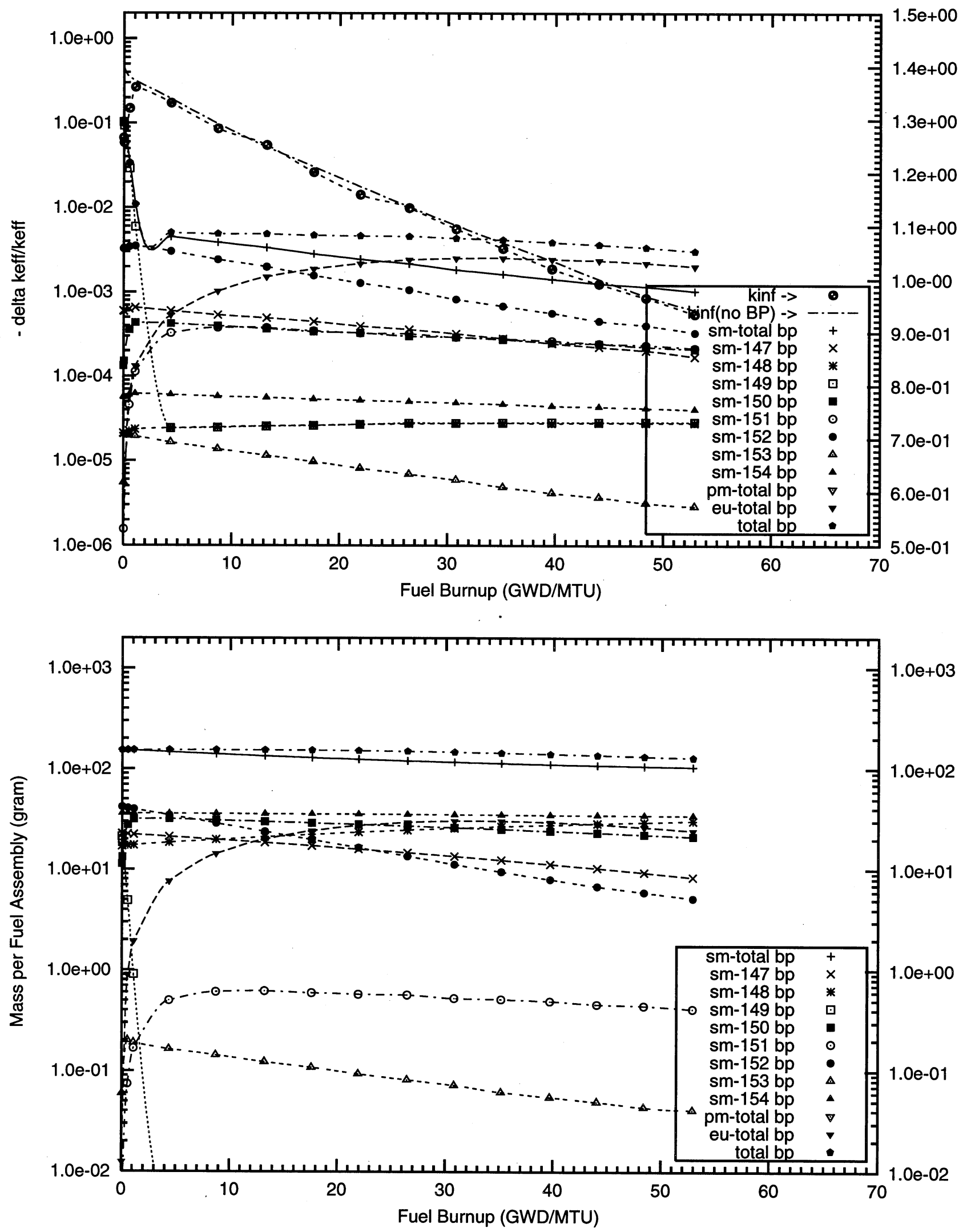


\section{B3 - Samarium Fully Enriched in ${ }^{149} \mathrm{Sm}$}


Figure B3.13. Negative Reactivity and Masses BP for Sm-149 and transmutation daughters as a function of fuel life for $17 \times 17$ fuel assemblies with 08 poison rods,

0.5 mill thick Sm2O3 poison coating on the outer radial surface of the UO2 pellets

Reactor power $3400 \mathrm{MWth}, 193$ fuel assemblies, initial enrichment 4.5w\%U-235

(Case : sm149_of_100p_h20_08_bp_fuel_2_o)
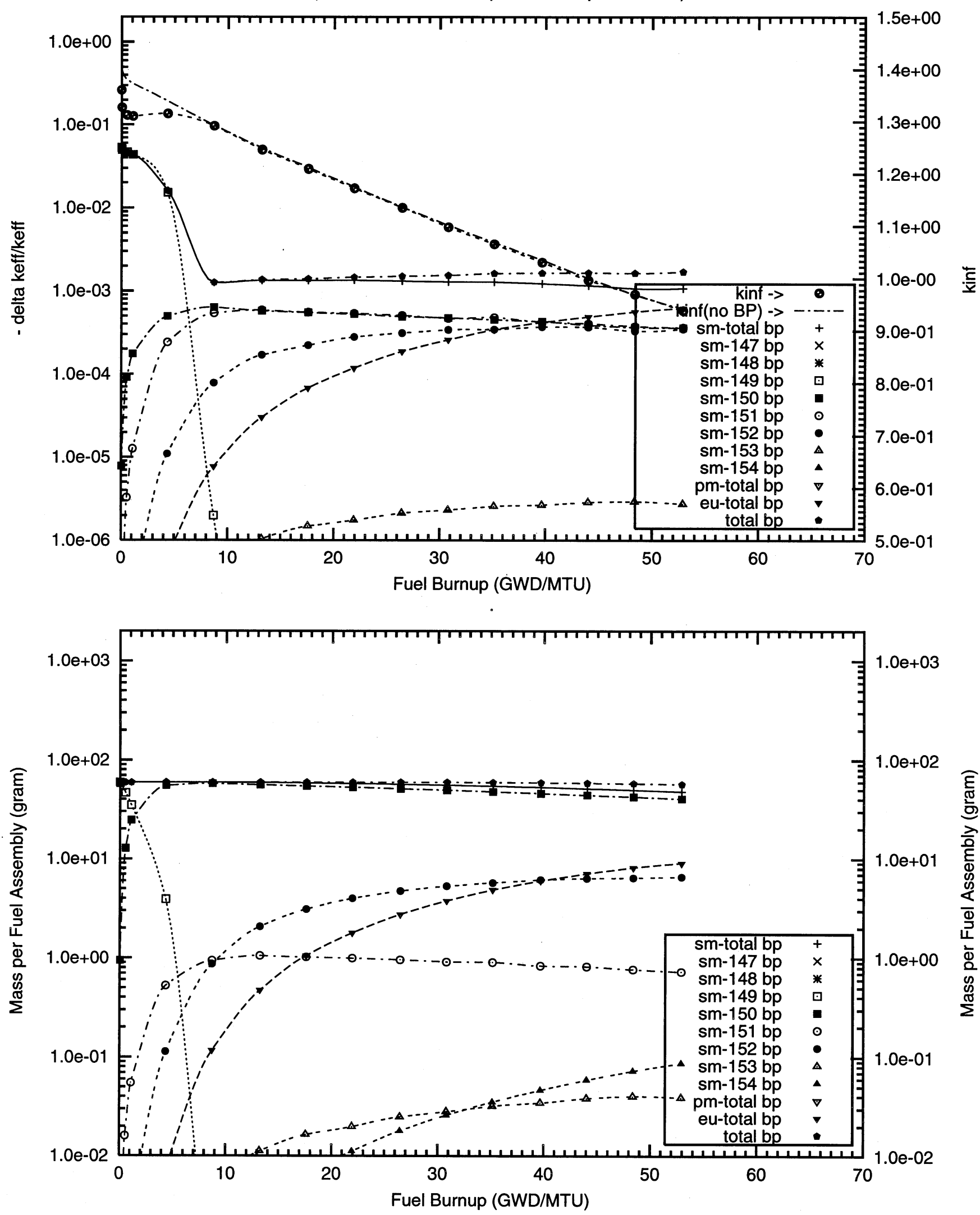
Figure B3.14. Negative Reactivity and Masses BP for Sm-149 and transmutation daughters as a function of fuel life for $17 \times 17$ fuel assemblies with 08 poison rods,

1.0 mill thick Sm2O3 poison coating on the outer radial surface of the UO2 pellets

Reactor power 3400 MWth, 193 fuel assemblies, initial enrichment 4.5w\%U-235

(Case : sm149_1f_100p_h2o_08_bp_fuel_2_o)
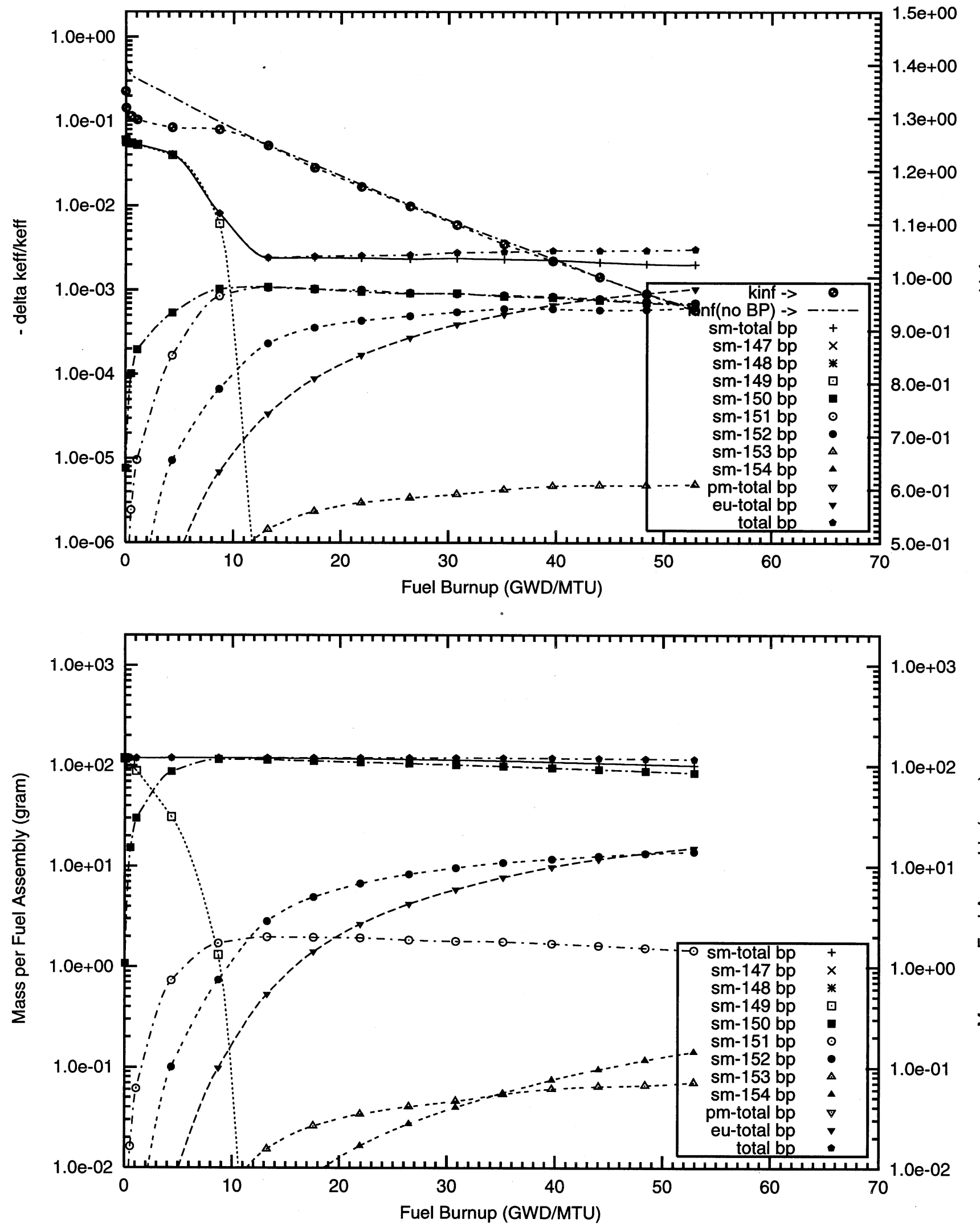
Figure B3.15. Negative Reactivity and Masses BP for Sm-149 and transmutation daughters as a function of fuel life for $17 \times 17$ fuel assemblies with 08 poison rods, 2.0 mill thick $\mathrm{Sm} 2 \mathrm{O} 3$ poison coating on the outer radial surface of the UO2 pellets Reactor power $3400 \mathrm{MWth}, 193$ fuel assemblies, initial enrichment 4.5w\%U-235 (Case : sm149_2f_08_bp_fuel o)
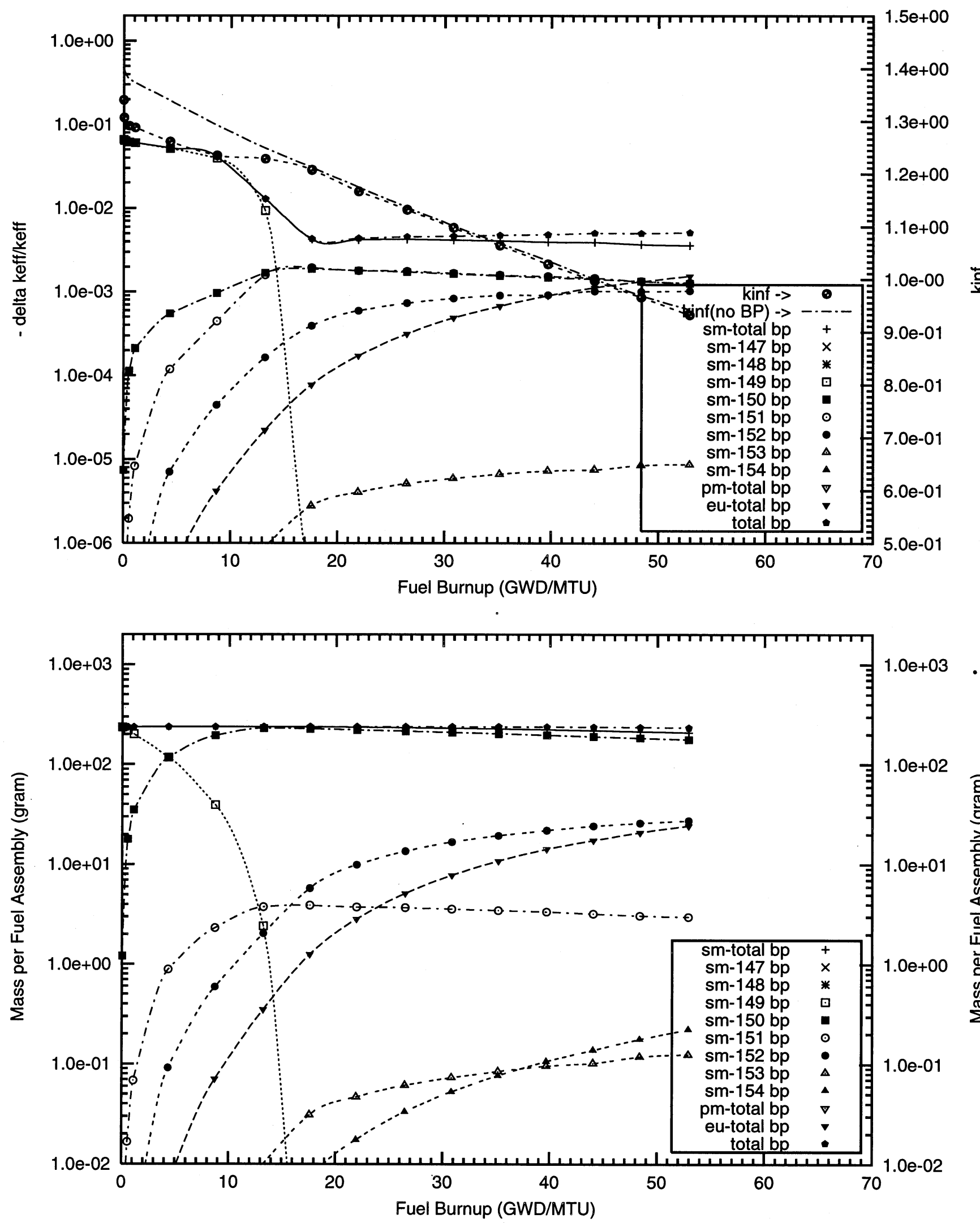
Figure B3.16. Negative Reactivity and Masses BP for Sm-149 and transmutation daughters as a function of fuel life for $17 \times 17$ fuel assemblies with 08 poison rods,

4.0 mill thick Sm2O3 poison coating on the outer radial surface of the UO2 pellets Reactor power $3400 \mathrm{MWth}, 193$ fuel assemblies, initial enrichment $4.5 \mathrm{w} \% \mathrm{U}-235$ (Case : sm149_3f_08_bp_fuel o)

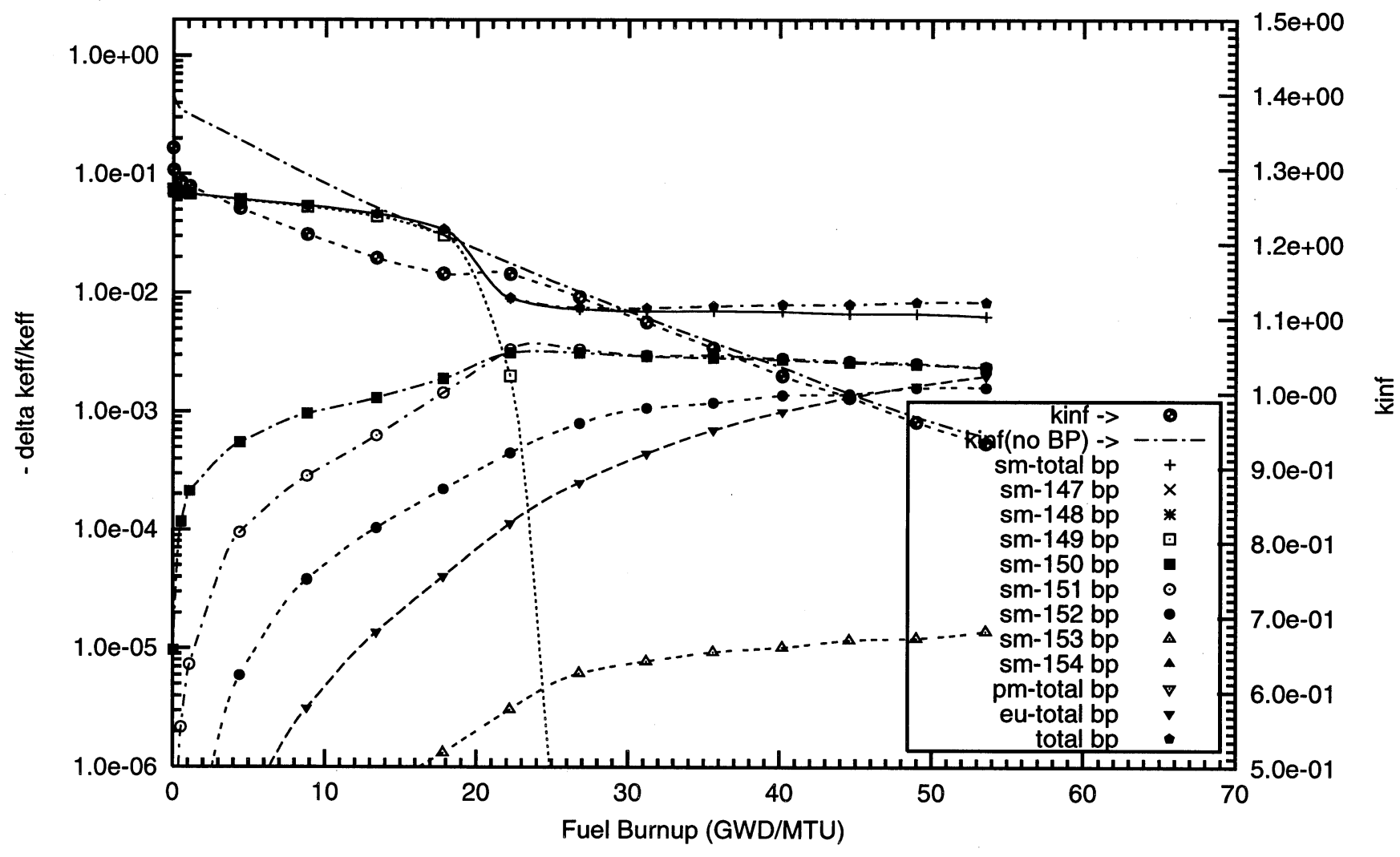


Figure B3.17. Negative Reactivity and Masses BP for Sm-149 and transmutation daughters as a function of fuel life for $17 \times 17$ fuel assemblies with 08 poison rods,

8.0 mill thick Sm2O3 poison coating on the outer radial surface of the UO2 pellets

Reactor power $3400 \mathrm{MWth}, 193$ fuel assemblies, initial enrichment 4.5w\%U-235

(Case : sm149_4f_08_bp_fuel o)

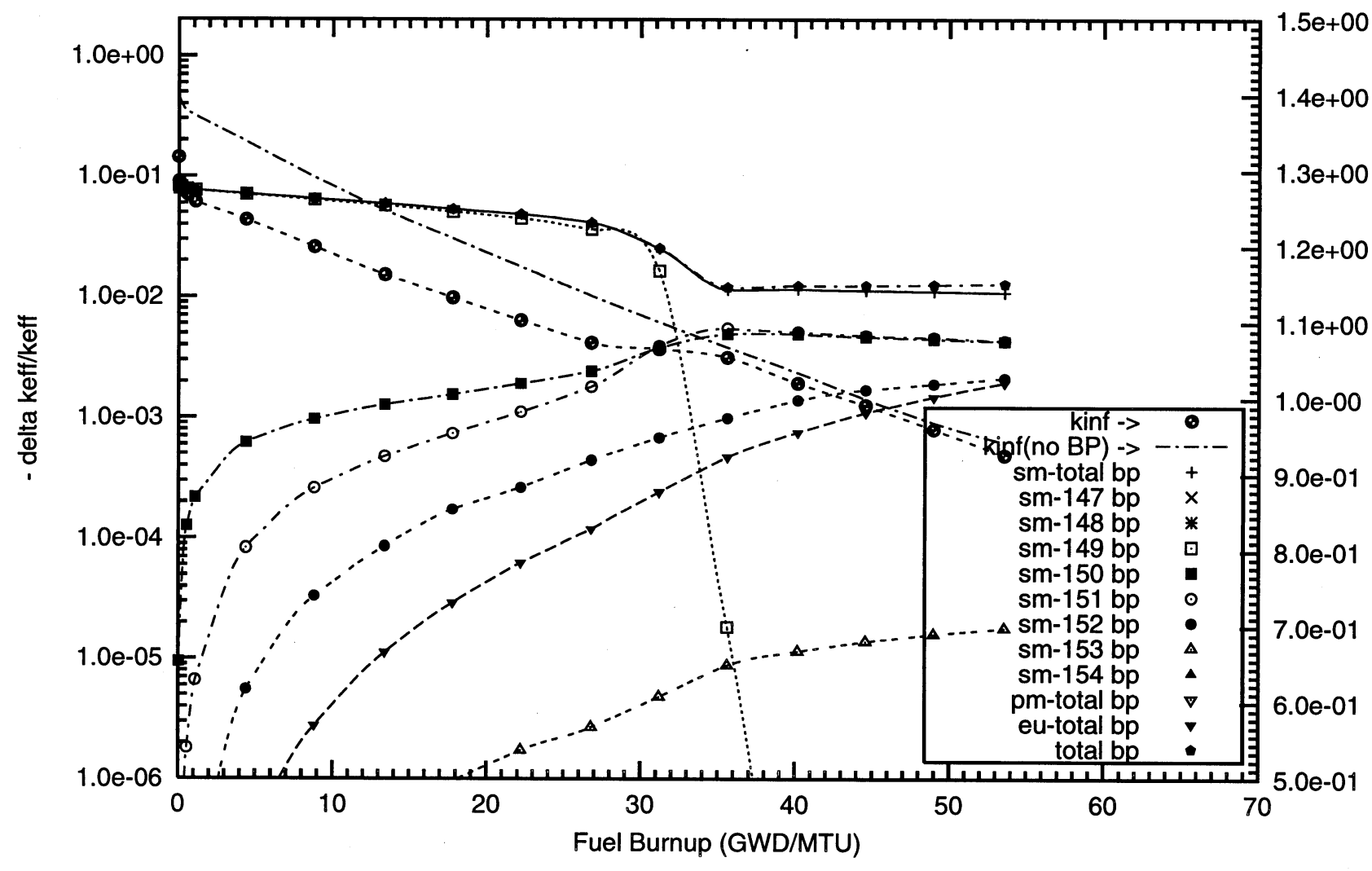

$\frac{4}{x}$ 
Figure B3.18. Negative Reactivity and Masses BP for Sm-149 and transmutation daughters

as a function of fuel life for $17 \times 17$ fuel assemblies with 16 poison rods,

0.1 mill thick Sm2O3 poison coating on the outer radial surface of the UO2 pellets

Reactor power $3400 \mathrm{MWth}, 193$ fuel assemblies, initial enrichment $4.5 \mathrm{w} \% \mathrm{U}-235$

(Case : sm149_9f_100p_h20_16_bp_fuel_2_o)
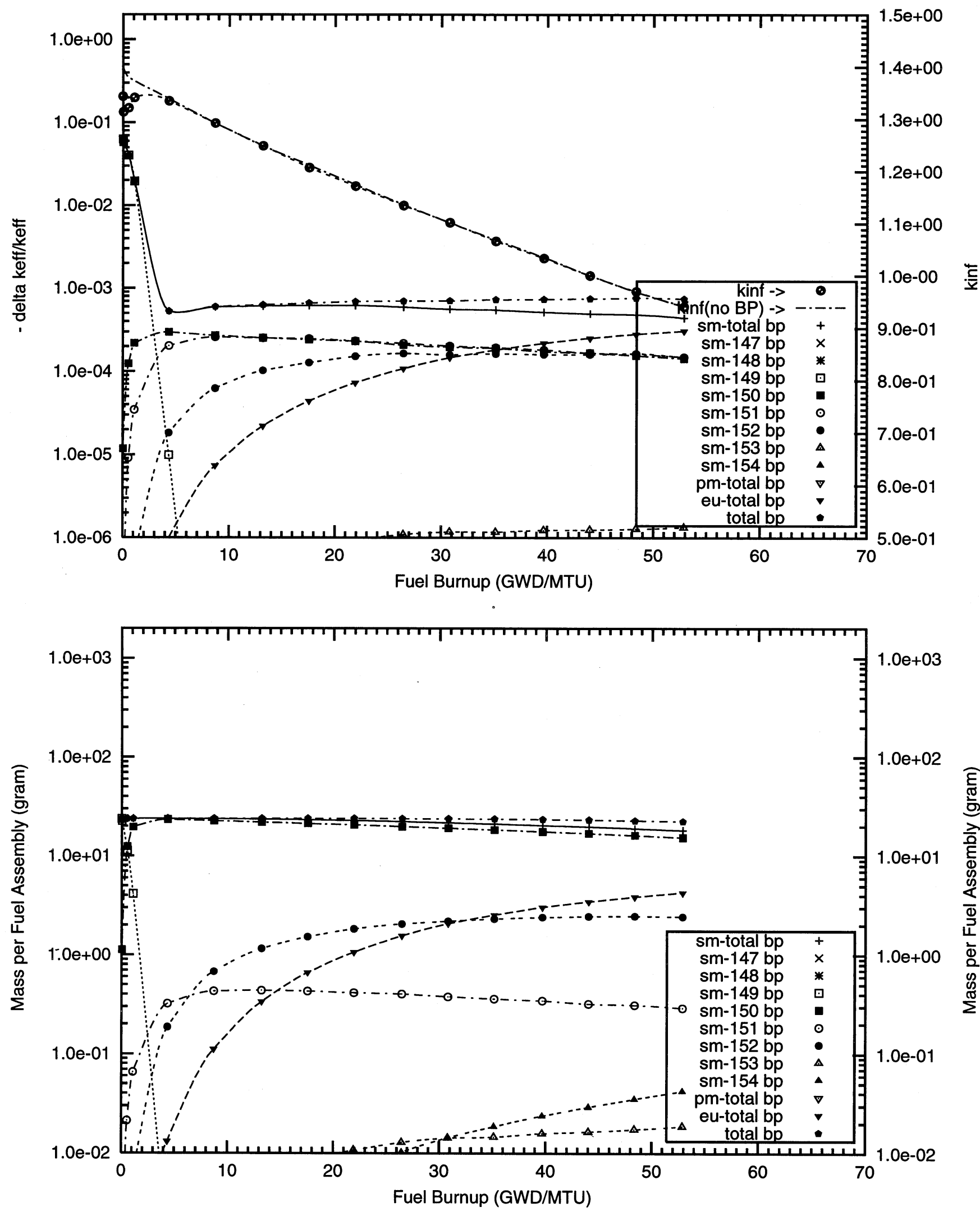
Figure B3.19. Negative Reactivity and Masses BP for Sm-149 and transmutation daughters as a function of fuel life for $17 \times 17$ fuel assemblies with 16 poison rods, 0.2 mill thick Sm2O3 poison coating on the outer radial surface of the UO2 pellets Reactor power $3400 \mathrm{MWth}, 193$ fuel assemblies, initial enrichment $4.5 \mathrm{w} \% \mathrm{U}-235$

(Case : sm149_8f_100p_h2o_16_bp_fuel_2_o)
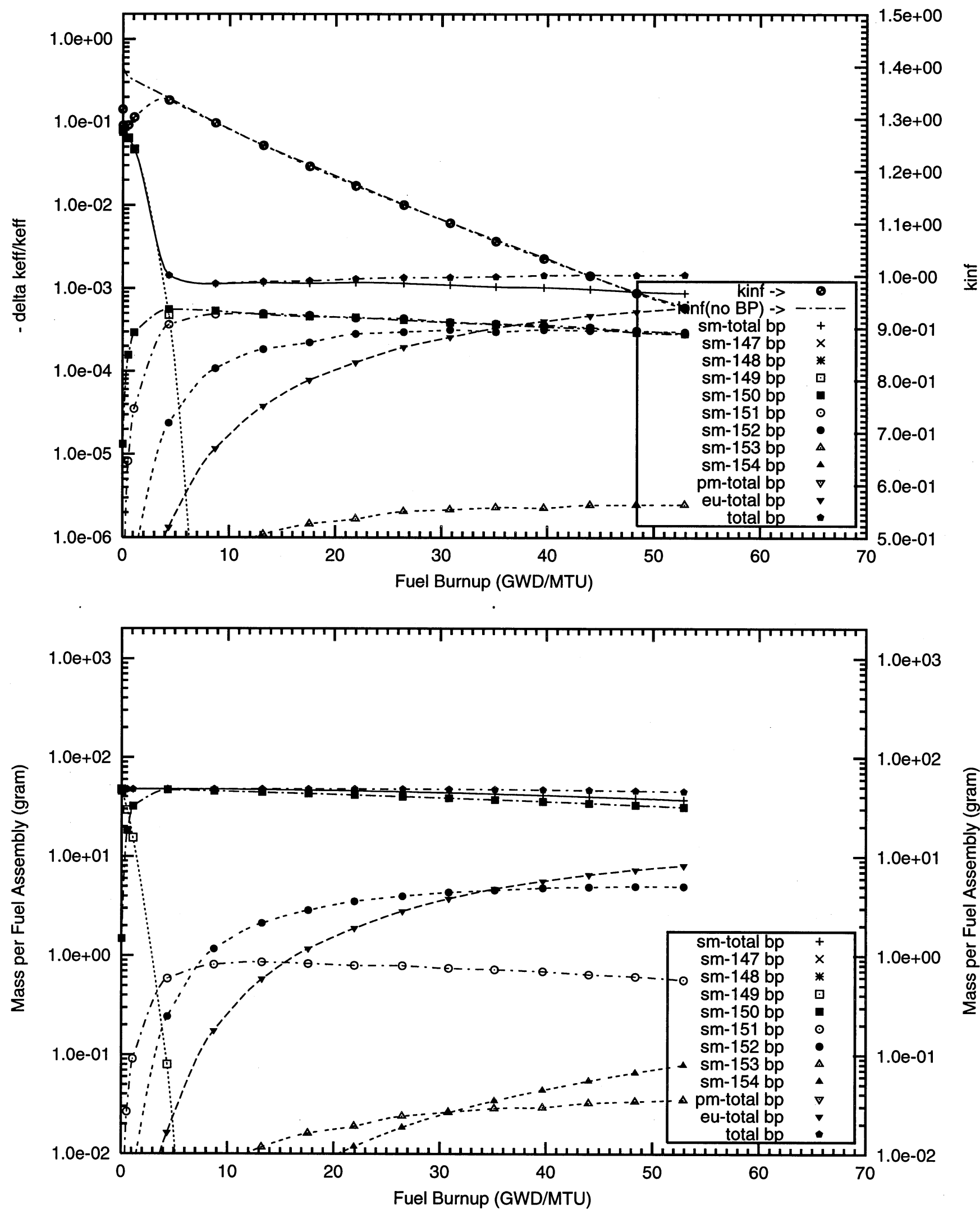
Figure B3.20. Negative Reactivity and Masses BP for Sm-149 and transmutation daughters

as a function of fuel life for $17 \times 17$ fuel assemblies with 16 poison rods,

0.5 mill thick Sm2O3 poison coating on the outer radial surface of the UO2 pellets

Reactor power $3400 \mathrm{MWth}, 193$ fuel assemblies, initial enrichment 4.5W\%U-235

(Case : sm149_of_100p_h2o_16_bp_fuel_2_o)
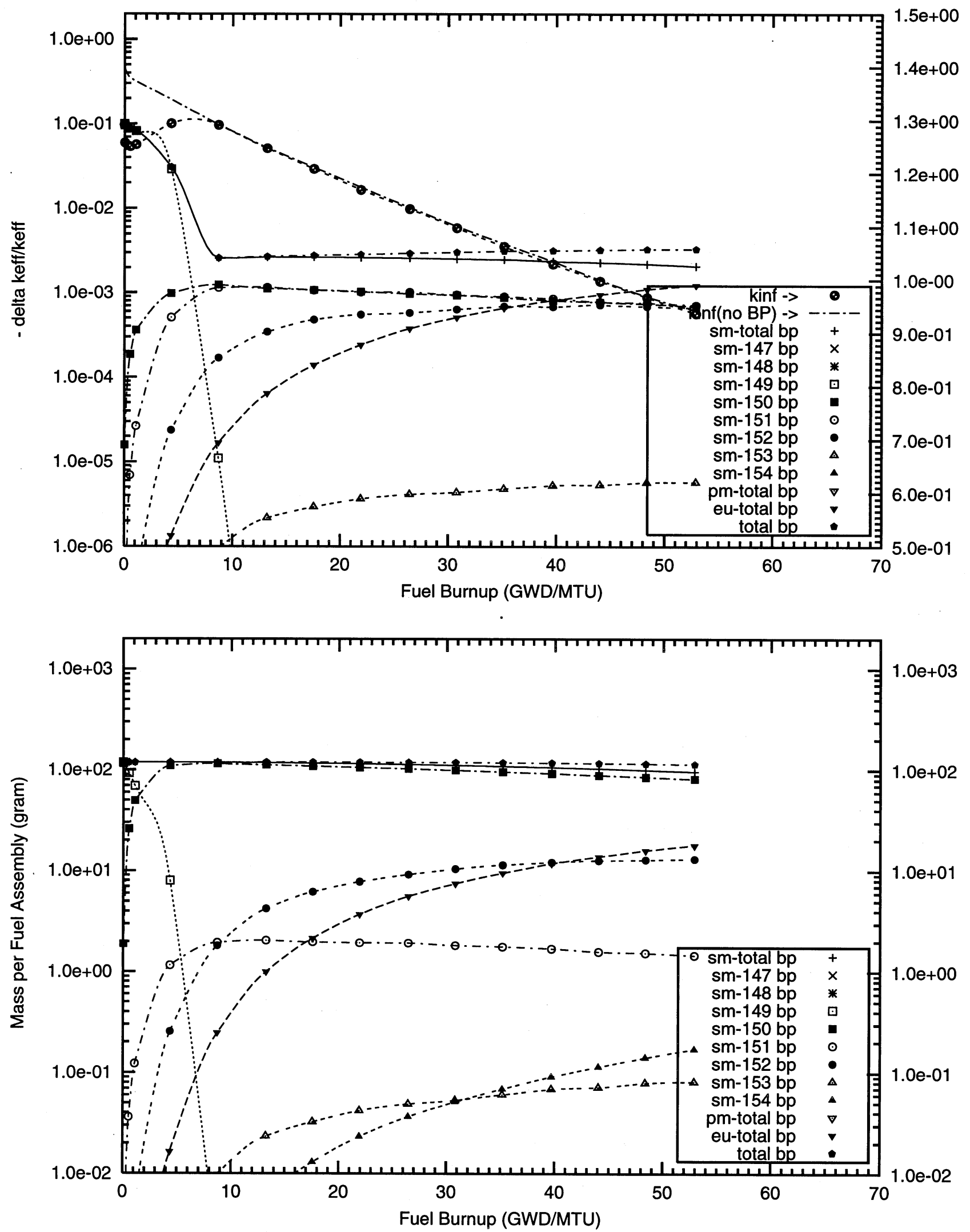
Figure B3.21. Negative Reactivity and Masses BP for Sm-149 and transmutation daughters as a function of fuel life for $17 \times 17$ fuel assemblies with 16 poison rods,

1.0 mill thick Sm2O3 poison coating on the outer radial surface of the UO2 pellets Reactor power $3400 \mathrm{MWth}, 193$ fuel assemblies, initial enrichment $4.5 \mathrm{w} \% \mathrm{U}-235$

(Case : sm149_1f_100p_h2o_16_bp_fuel_2_o)
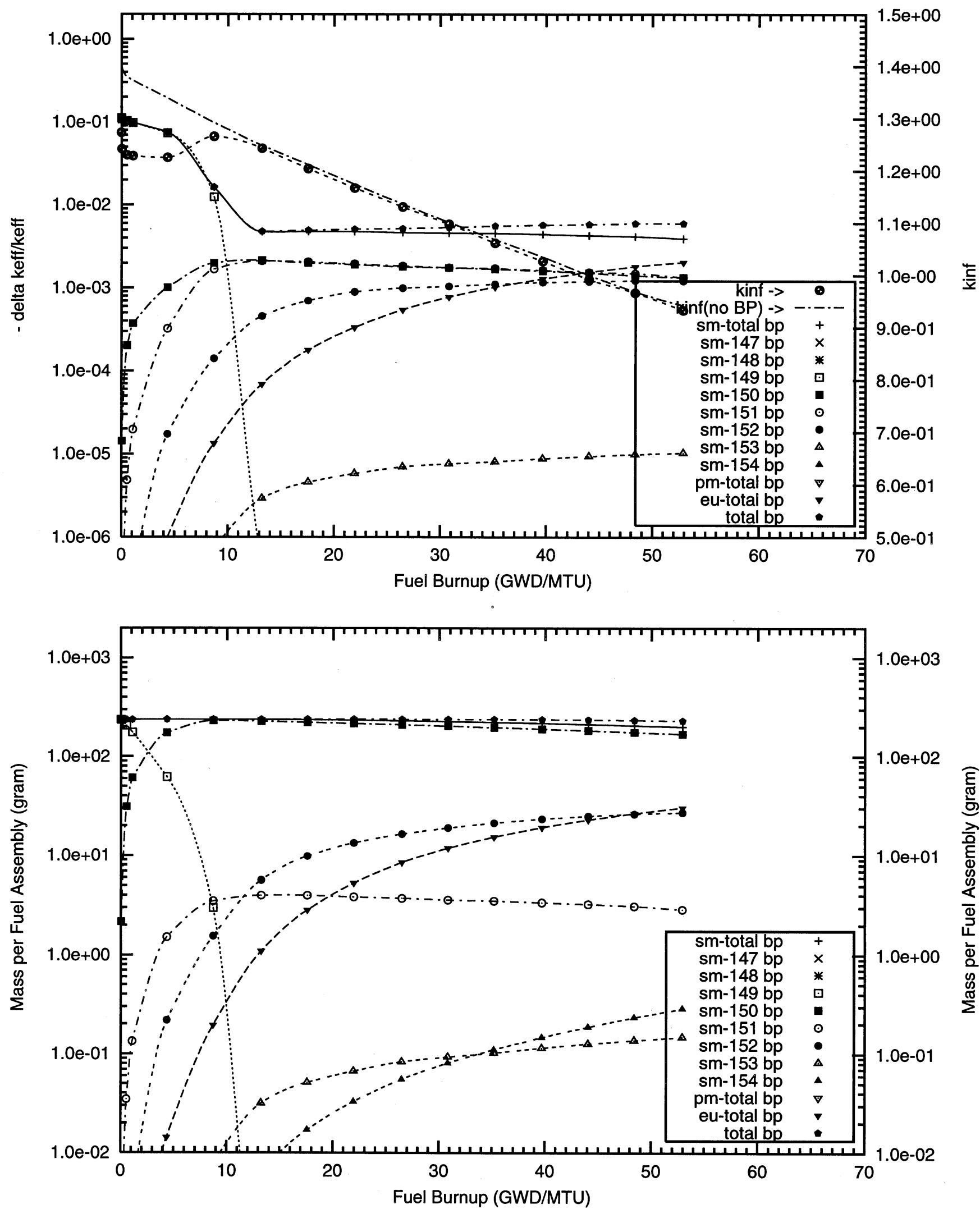
Figure B3.22. Negative Reactivity and Masses BP for Sm-149 and transmutation daughters as a function of fuel life for $17 \times 17$ fuel assemblies with 16 poison rods,

2.0 mill thick Sm2O3 poison coating on the outer radial surface of the UO2 pellets

Reactor power $3400 \mathrm{MWth}, 193$ fuel assemblies, initial enrichment 4.5w\%U-235

(Case : sm149_2f_100p_h2o_16_bp_fuel_2_o)

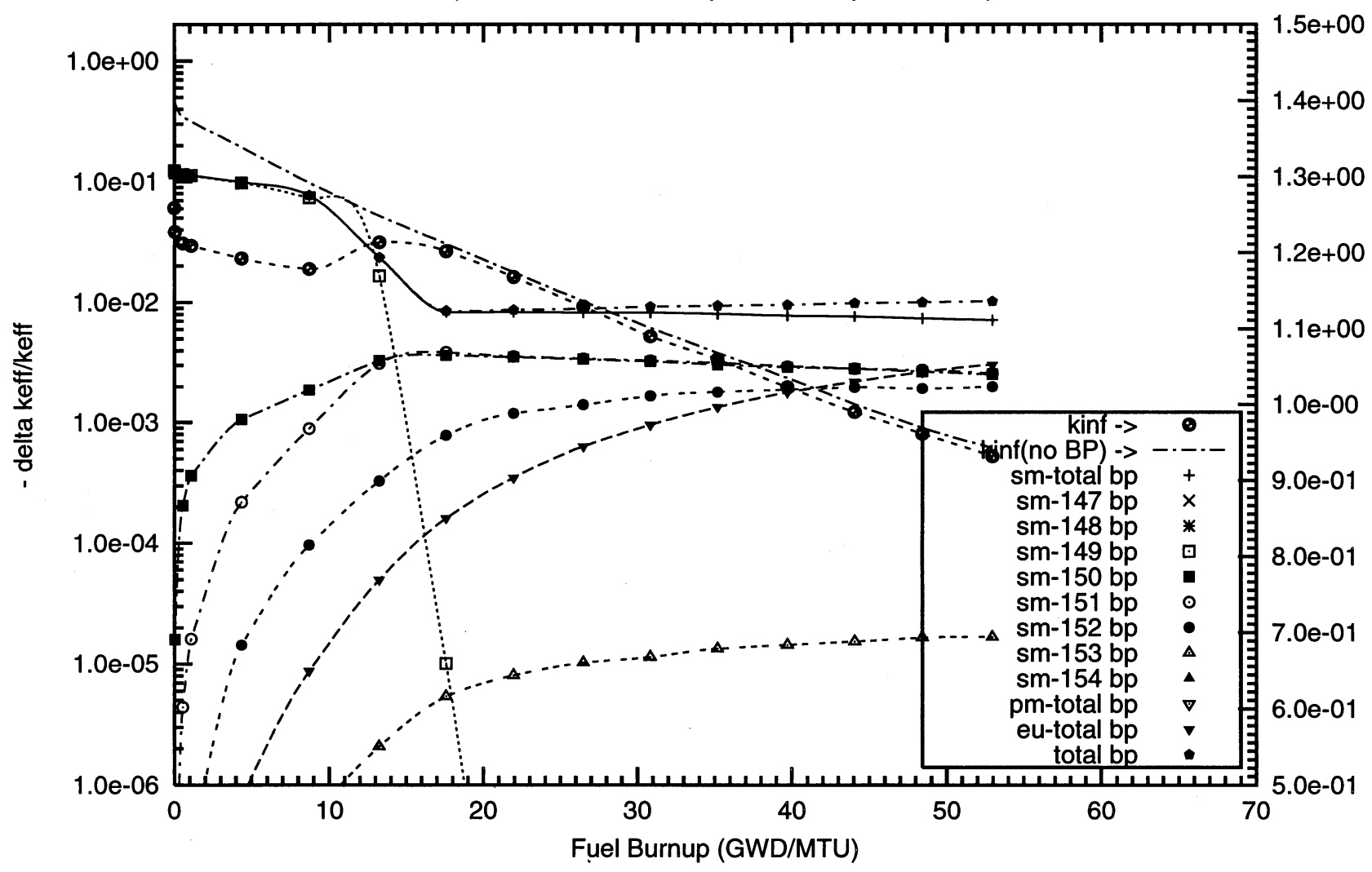

$\overline{\frac{\bar{C}}{x}}$

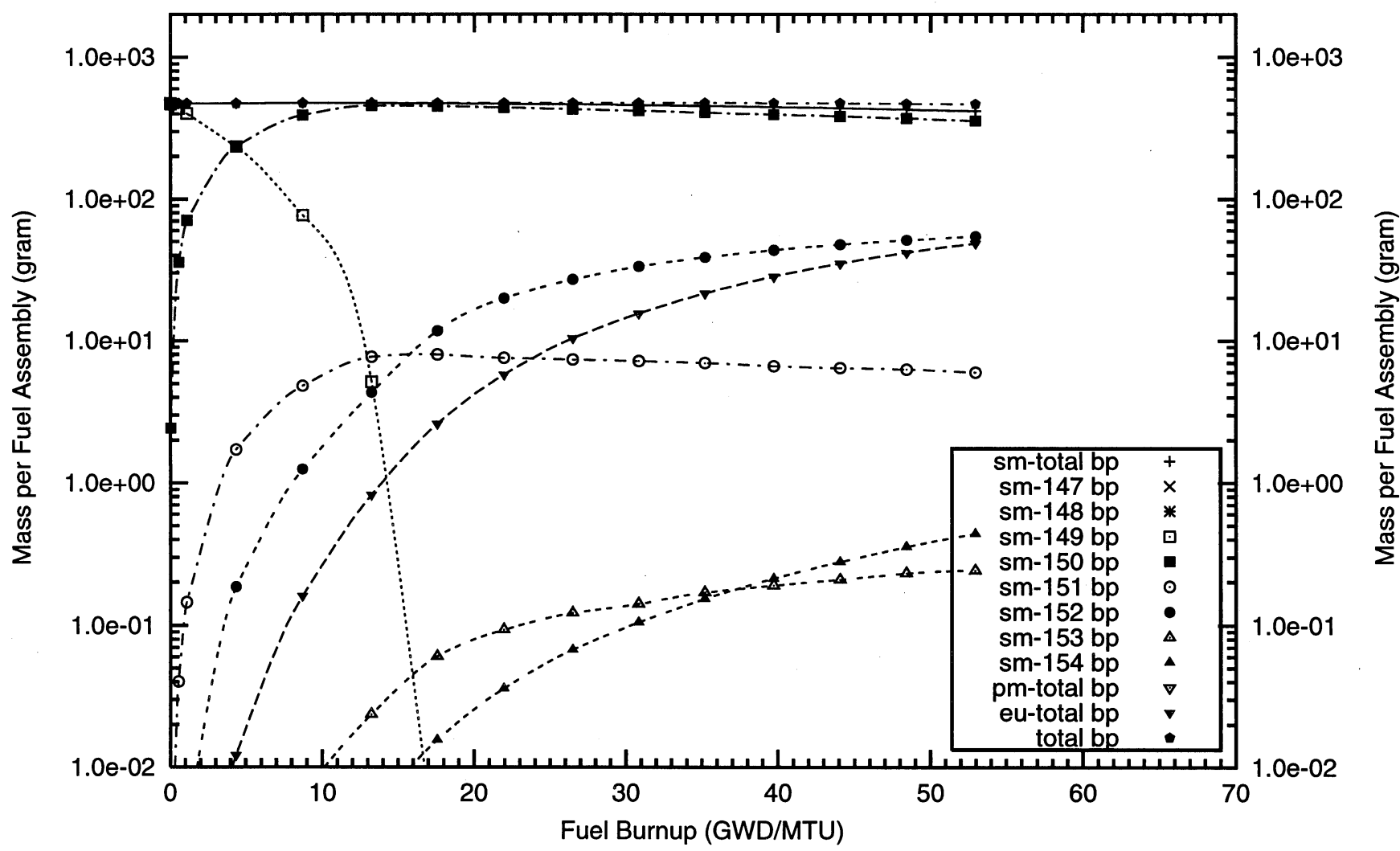


Figure B3.23. Negative Reactivity and Masses BP for Sm-149 and transmutation daughters

as a function of fuel life for $17 \times 17$ fuel assemblies with 16 poison rods,

4.0 mill thick $\mathrm{Sm} 2 \mathrm{O} 3$ poison coating on the outer radial surface of the $\mathrm{UO} 2$ pellets

Reactor power $3400 \mathrm{MWth}, 193$ fuel assemblies, initial enrichment $4.5 \mathrm{w} \% \mathrm{U}-235$

(Case : sm149_3f_100p_h2o_16_bp_fuel_2_o)
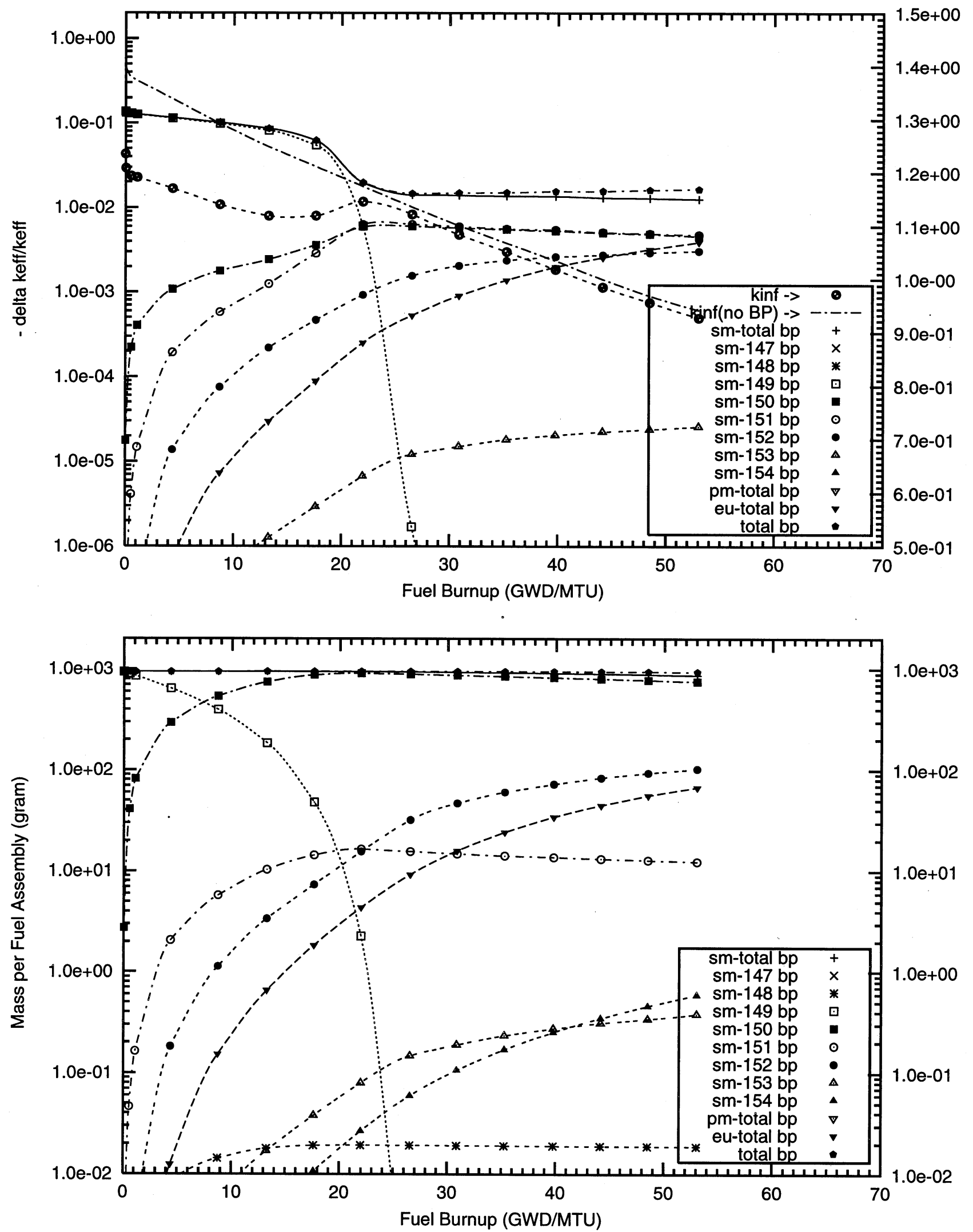
Figure B3.24. Negative Reactivity and Masses BP for Sm-149 and transmutation daughters

as a function of fuel life for $17 \times 17$ fuel assemblies with 16 poison rods,

8.0 mill thick Sm2O3 poison coating on the outer radial surface of the $\mathrm{UO} 2$ pellets

Reactor power $3400 \mathrm{MWth}, 193$ fuel assemblies, initial enrichment 4.5w\%U-235

(Case : sm149_4f_100p_h2o_16_bp_fuel_2_o)
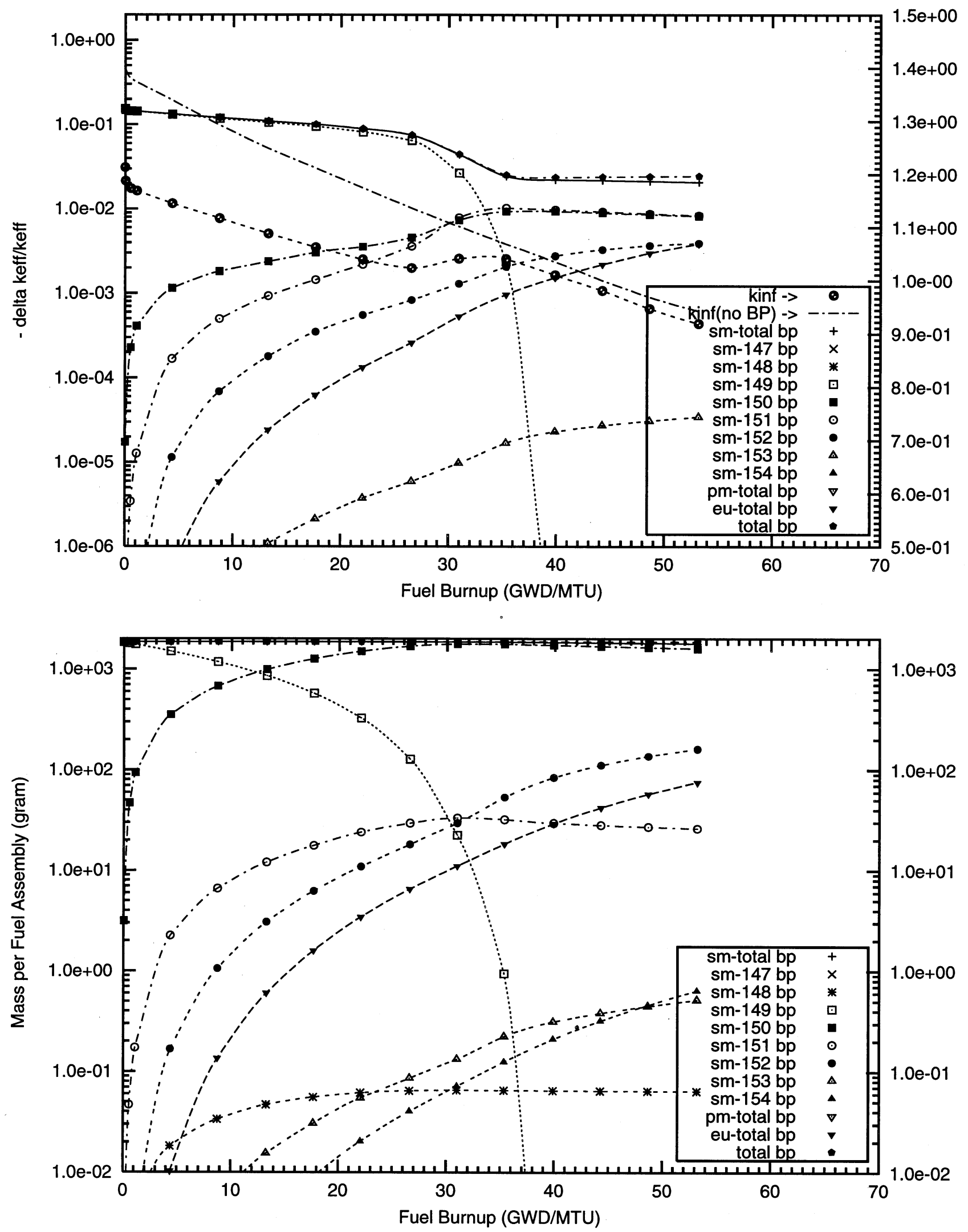
Figure B3.25. Negative Reactivity and Masses BP for Sm-149 and transmutation daughters as a function of fuel life for $17 \times 17$ fuel assemblies with 18 poison rods, 1.0 mill thick Sm2O3 poison coating on the outer radial surface of the UO2 pellets Reactor power $3400 \mathrm{MWth}, 193$ fuel assemblies, initial enrichment 4.5w\%U-235

(Case : sm149_1f_16_bp_fuel (old) o)
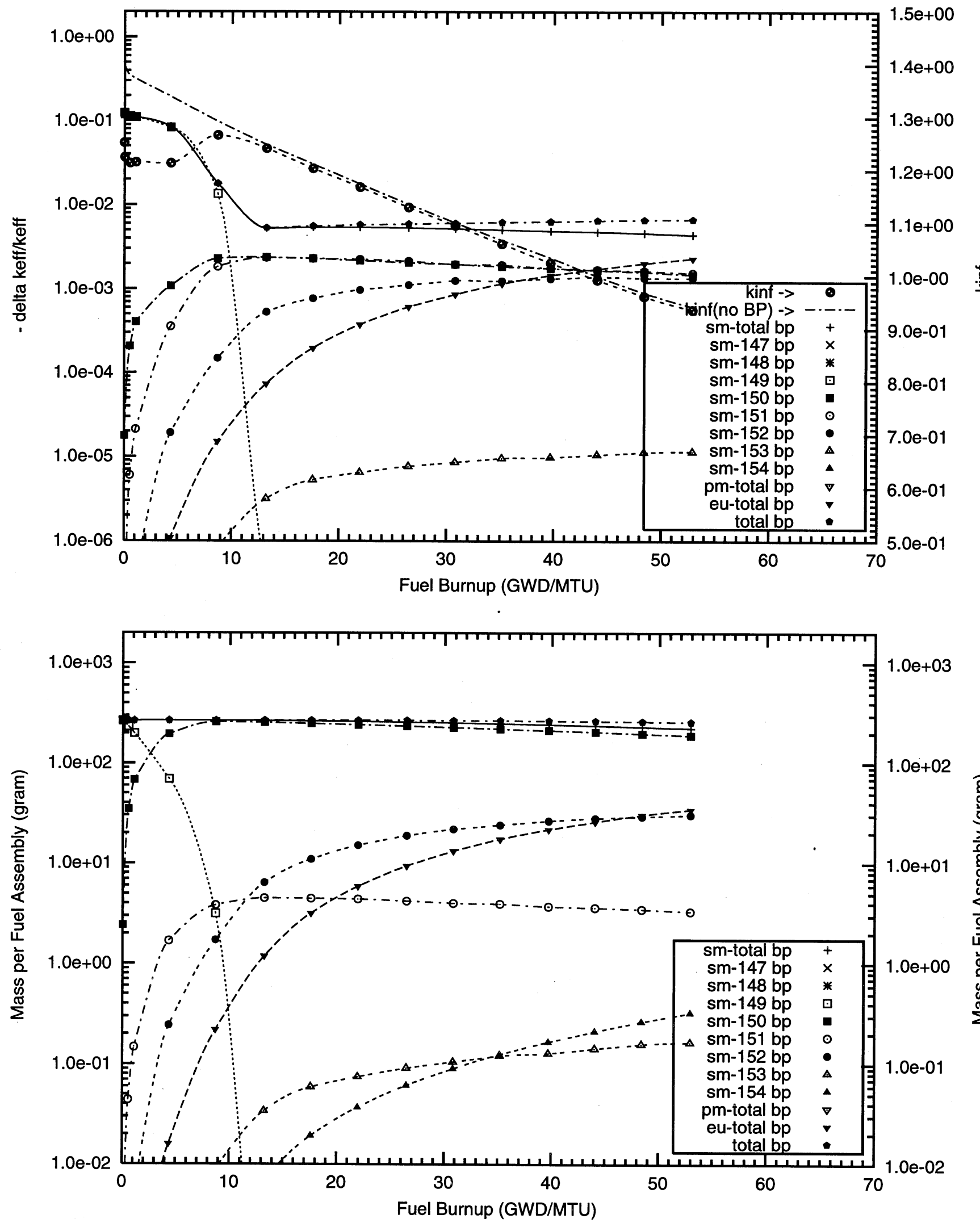
Figure B3.26. Negative Reactivity and Masses BP for Sm-149 and transmutation daughters as a function of fuel life for $17 \times 17$ fuel assemblies with 18 poison rods,

2.0 mill thick Sm2O3 poison coating on the outer radial surface of the $\mathrm{UO} 2$ pellets

Reactor power $3400 \mathrm{MWth}, 193$ fuel assemblies, initial enrichment 4.5w\%U-235

(Case : sm149_2f_16_bp_fuel (old) o)

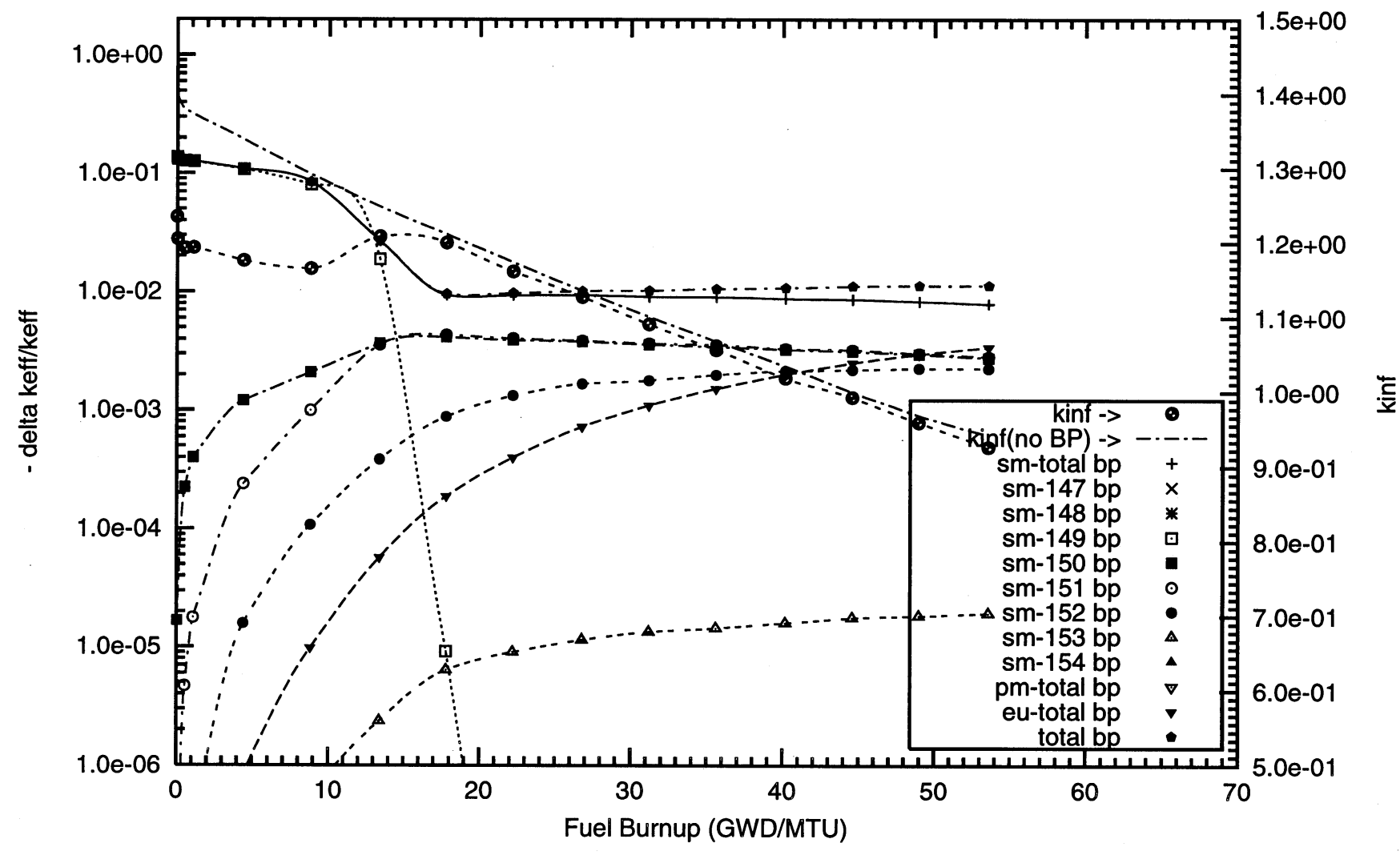


Figure B3.27. Negative Reactivity and Masses BP for Sm-149 and transmutation daughters as a function of fuel life for $17 \times 17$ fuel assemblies with 18 poison rods, 4.0 mill thick Sm2O3 poison coating on the outer radial surface of the UO2 pellets Reactor power $3400 \mathrm{MWth}, 193$ fuel assemblies, initial enrichment 4.5w\%U-235

(Case : sm149_3f_16_bp_fuel (old) o)

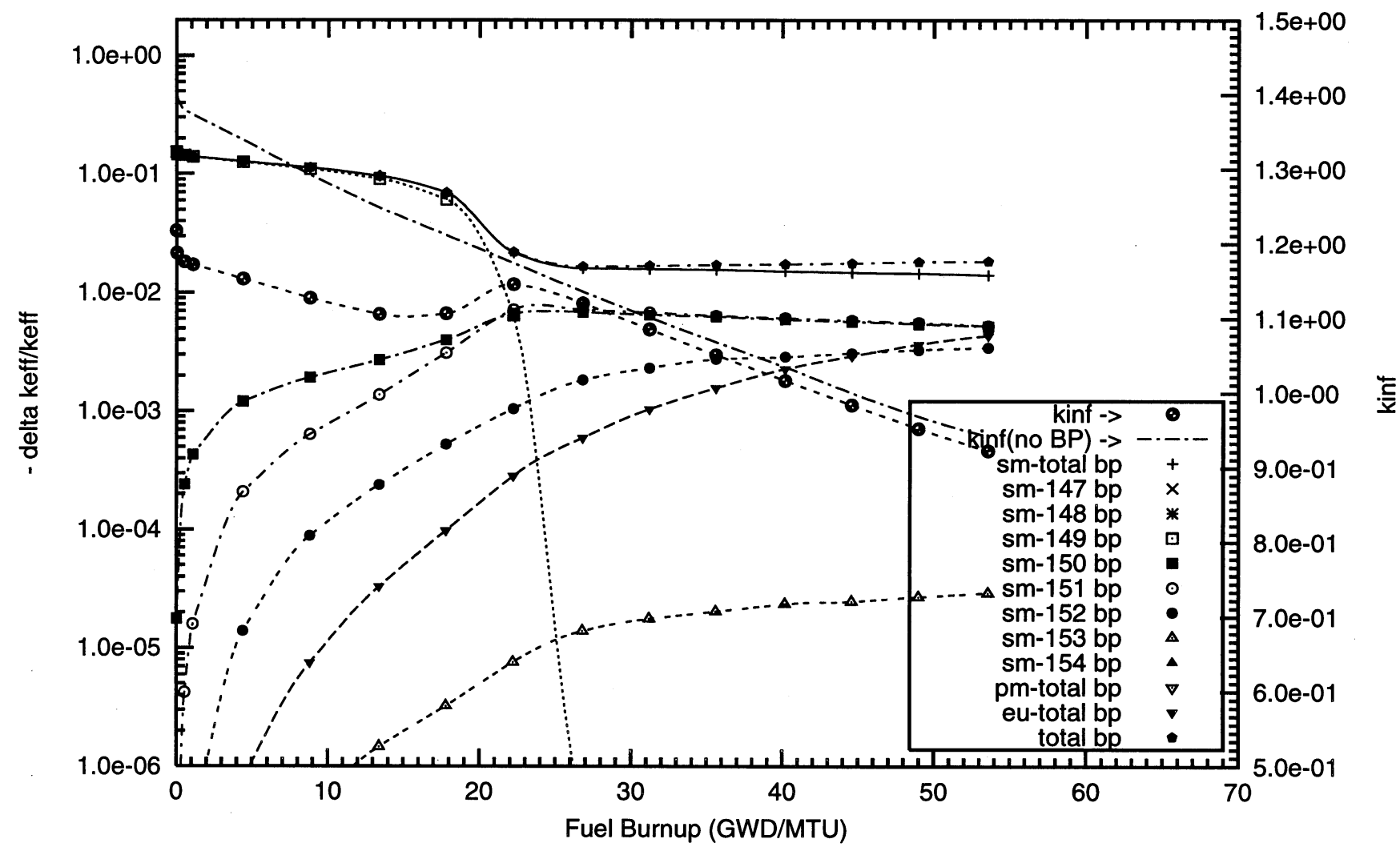


Figure B3.28. Negative Reactivity and Masses BP for Sm-149 and transmutation daughters as a function of fuel life for $17 \times 17$ fuel assemblies with 18 poison rods,

8.0 mill thick Sm2O3 poison coating on the outer radial surface of the UO2 pellets

Reactor power $3400 \mathrm{MWth}, 193$ fuel assemblies, initial enrichment 4.5w\%U-235

(Case : sm149_4f_16_bp_fuel (old) o)

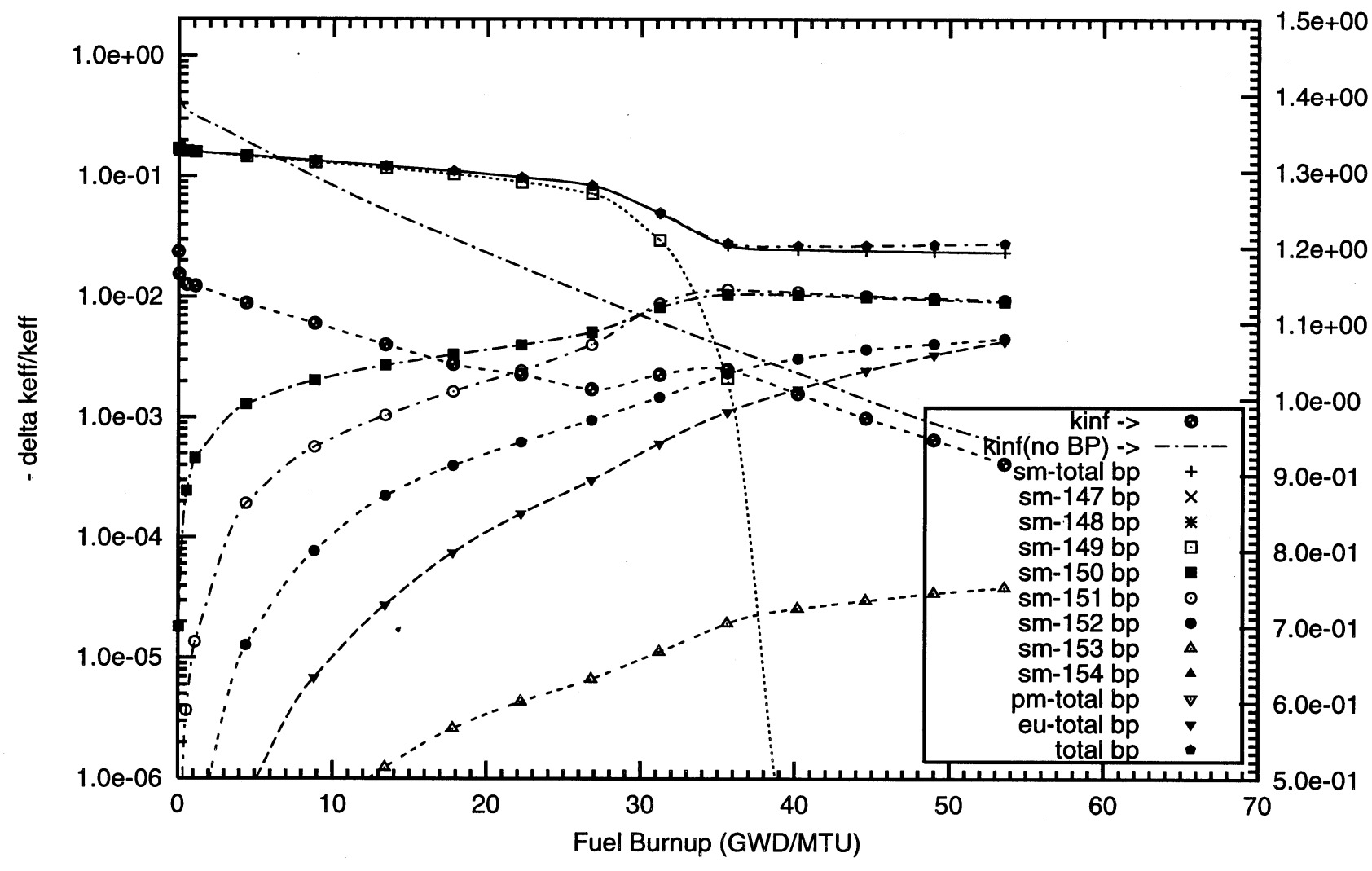

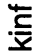


Figure B3.29. Negative Reactivity and Masses BP for Sm-149 and transmutation daughters as a function of fuel life for $17 \times 17$ fuel assemblies with 64 poison rods,

0.5 mill thick (0.10TD) Sm2O3 poison coating on the outer radial surface of the UO2 pellets

Reactor power $3400 \mathrm{MWth}, 193$ fuel assemblies, initial enrichment 4.5w\%U-235

(Case : sm149_12f_64_bp_fuel o)
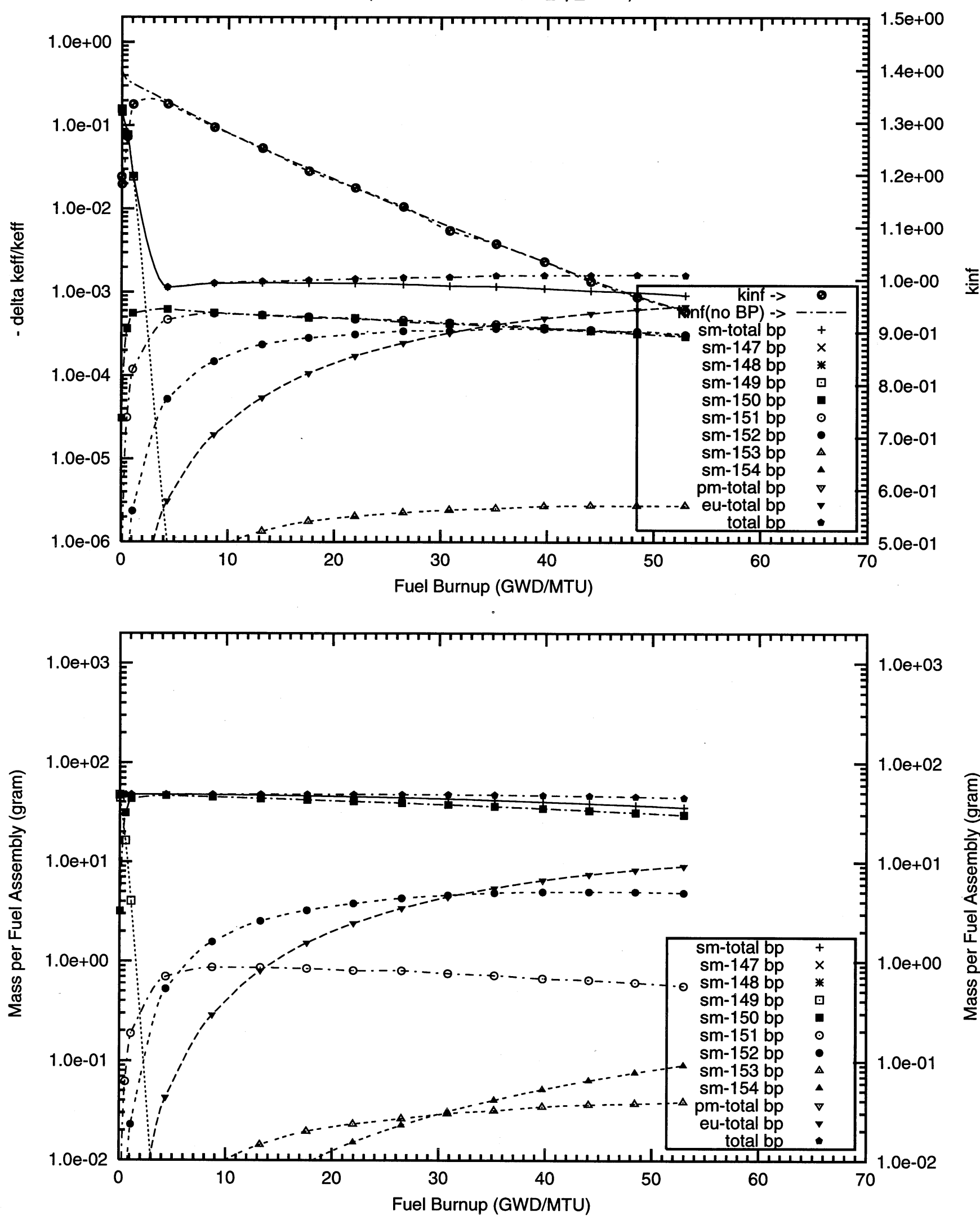
Figure B3.30. Negative Reactivity and Masses BP for Sm-149 and transmutation daughters as a function of fuel life for $17 \times 17$ fuel assemblies with 64 poison rods,

1.0 mill thick (0.10TD) Sm2O3 poison coating on the outer radial surface of the UO2 pellets

Reactor power $3400 \mathrm{MWth}, 193$ fuel assemblies, initial enrichment 4.5w\%U-235

(Case : sm149_11f_64_bp_fuel o)
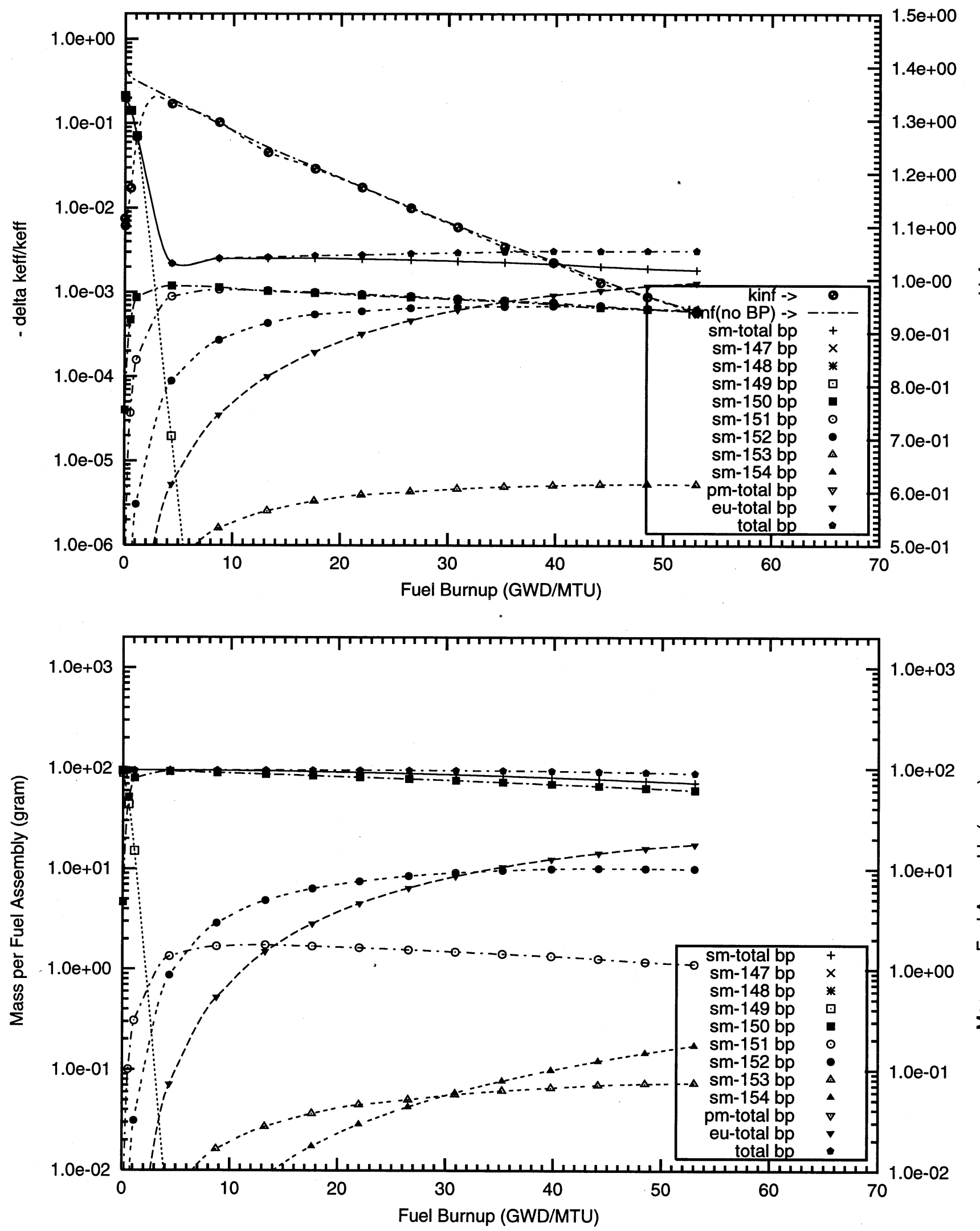
Figure B3.31. Negative Reactivity and Masses BP for Sm-149 and transmutation daughters as a function of fuel life for $17 \times 17$ fuel assemblies with 64 poison rods,

0.1 mill thick Sm2O3 poison coating on the outer radial surface of the UO2 pellets

Reactor power $3400 \mathrm{MWth}, 193$ fuel assemblies, initial enrichment $4.5 \mathrm{w} \% \mathrm{U}-235$

(Case : sm149_9f_64_bp_fuel o)

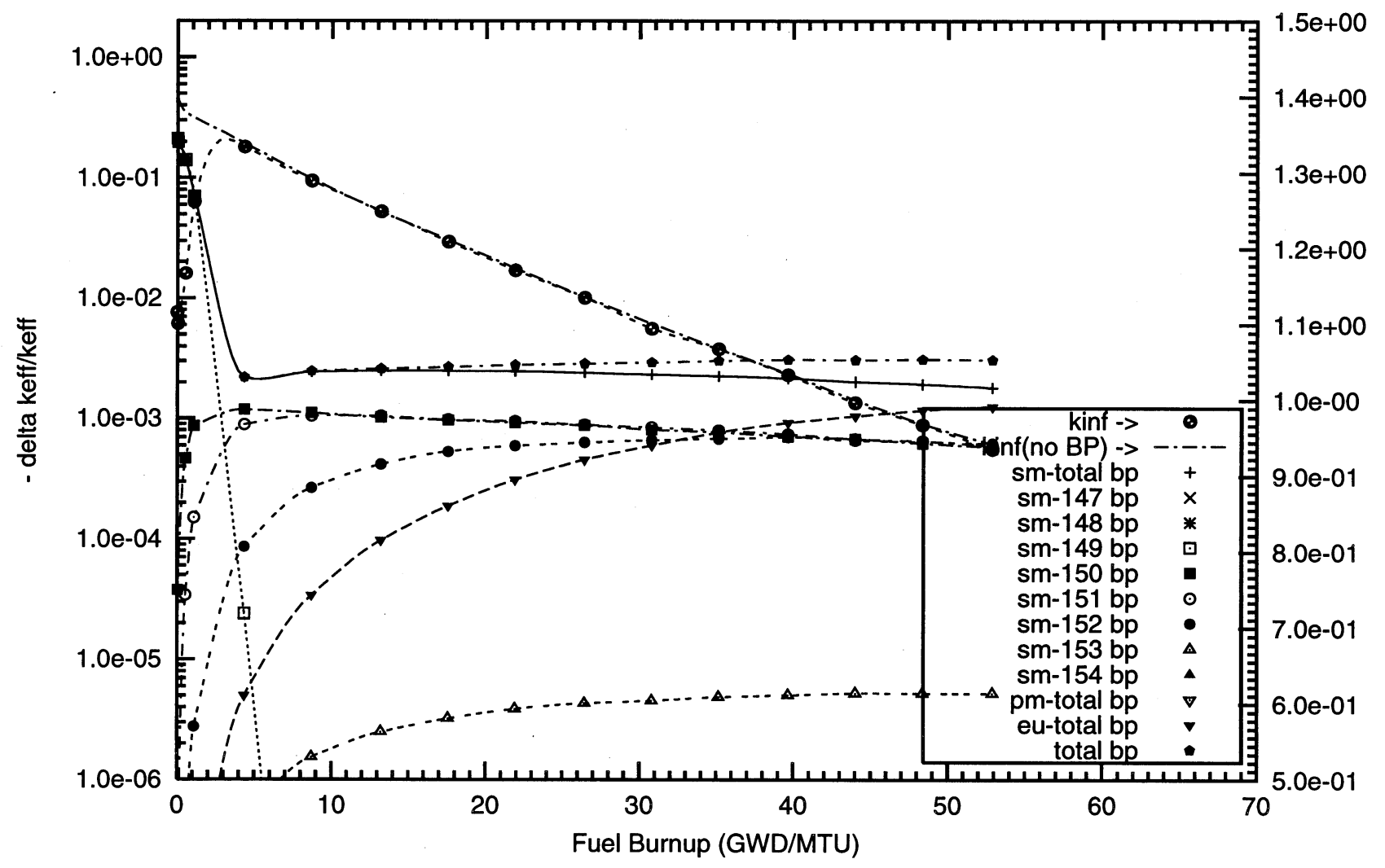

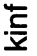

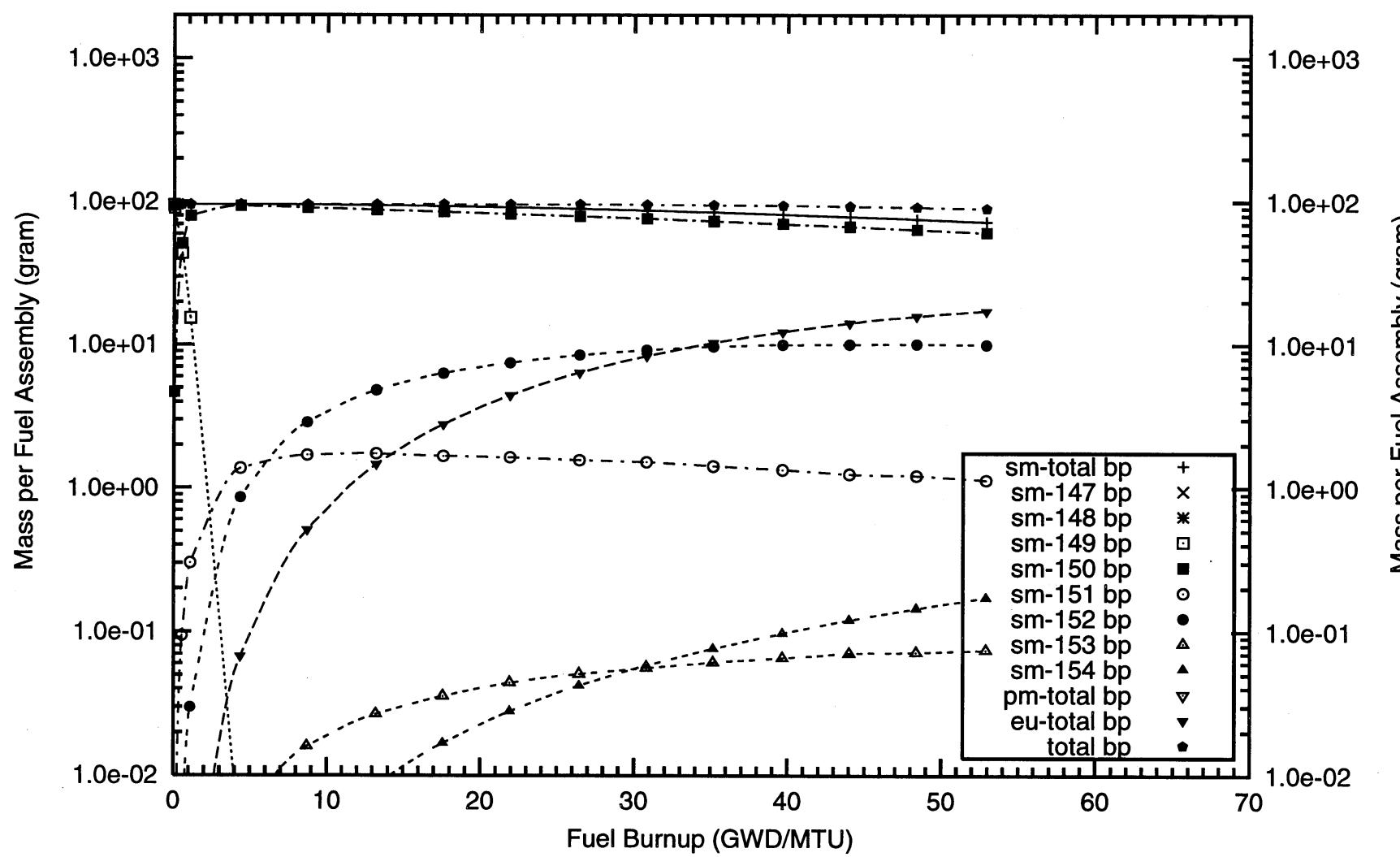


Figure B3.32. Negative Reactivity and Masses BP for Sm-149 and transmutation daughters as a function of fuel life for $17 \times 17$ fuel assemblies with 104 poison rods,

0.2 mill thick (0.10TD) Sm2O3 poison coating on the outer radial surface of the UO2 pellets

Reactor power $3400 \mathrm{MWth}, 193$ fuel assemblies, initial enrichment 4.5W\%U-235

(Case : sm149_14f_104_bp_fuel o)
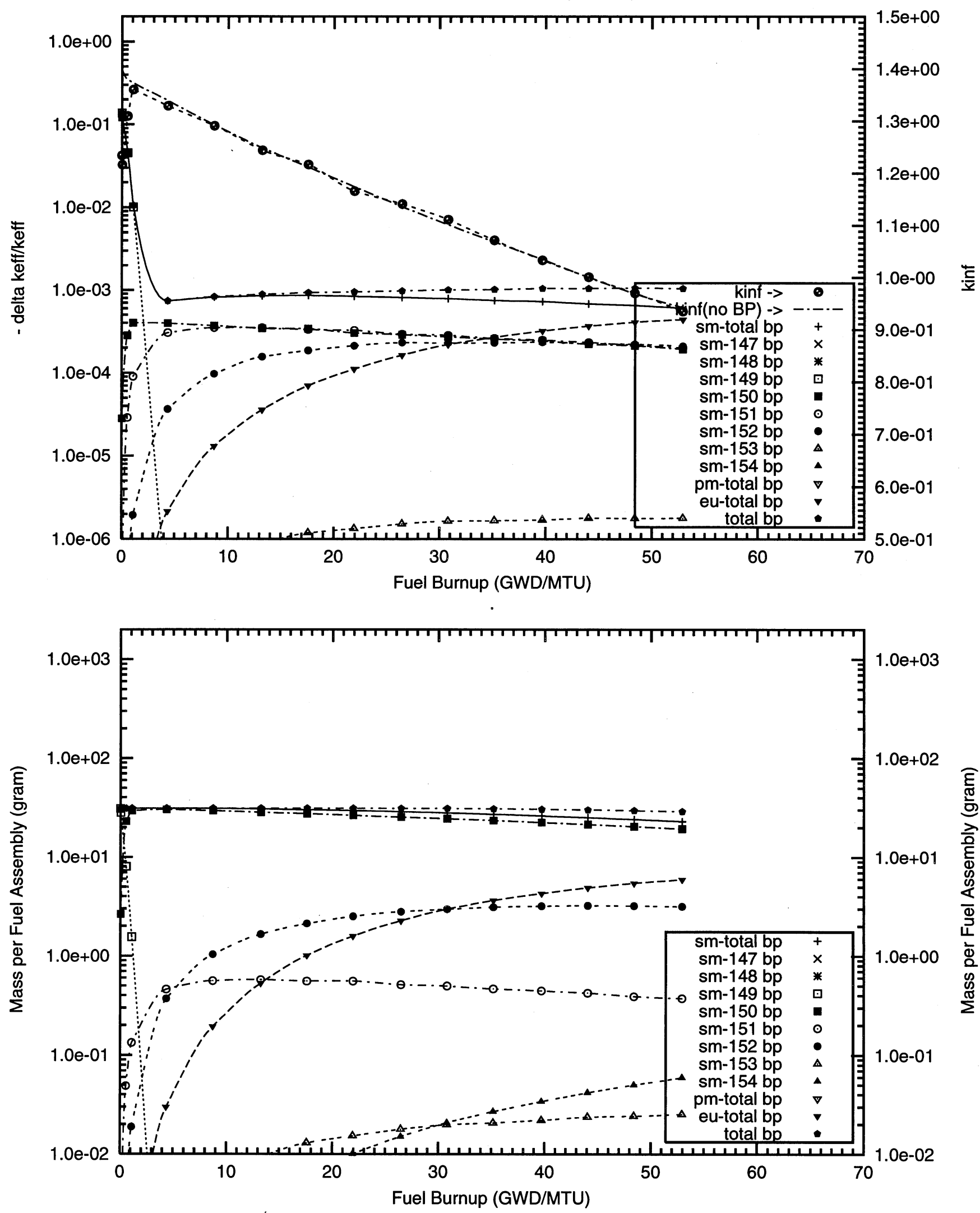
Figure B3.33. Negative Reactivity and Masses BP for Sm-149 and transmutation daughters as a function of fuel life for $17 \times 17$ fuel assemblies with 104 poison rods,

0.5 mill thick (0.10TD) Sm2O3 poison coating on the outer radial surface of the UO2 pellets

Reactor power $3400 \mathrm{MWth}, 193$ fuel assemblies, initial enrichment 4.5w\%U-235

(Case : sm149_12f_104_bp_fuel o)
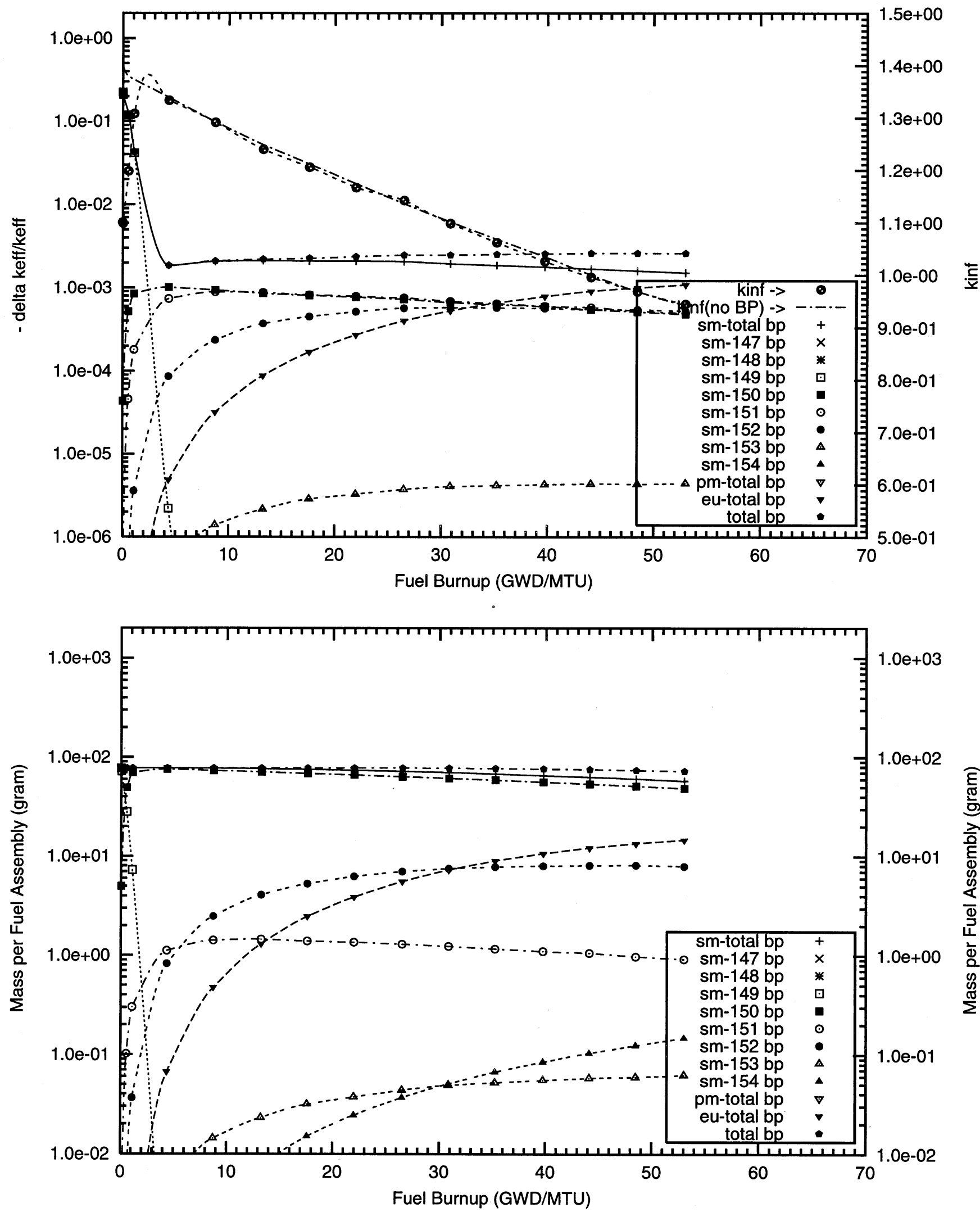


\section{Appendix B4}

Reactivity Worths and Masses of Samarium Alloyed with the Zircaloy Cladding of Fuel Rods

\section{Table 12 Cases}





\section{B4 - Samarium with Natural Isotopic Abundances}



Figure B4.1. Negative Reactivity and Masses BP for Sm-nat and transmutation daughters

as a function of fuel life for $17 \times 17$ fuel assemblies with 16 poison rods,

$2.0 \mathrm{w} \% \mathrm{Sm}$ poison homogeneously mixed in the fuel cladding,

Reactor power $3400 \mathrm{MWth}, 193$ fuel assemblies initial enrichment 4.5w\%U-235

(Case : sm000_2c_100p_h20_16_bp_fuel_2_o)
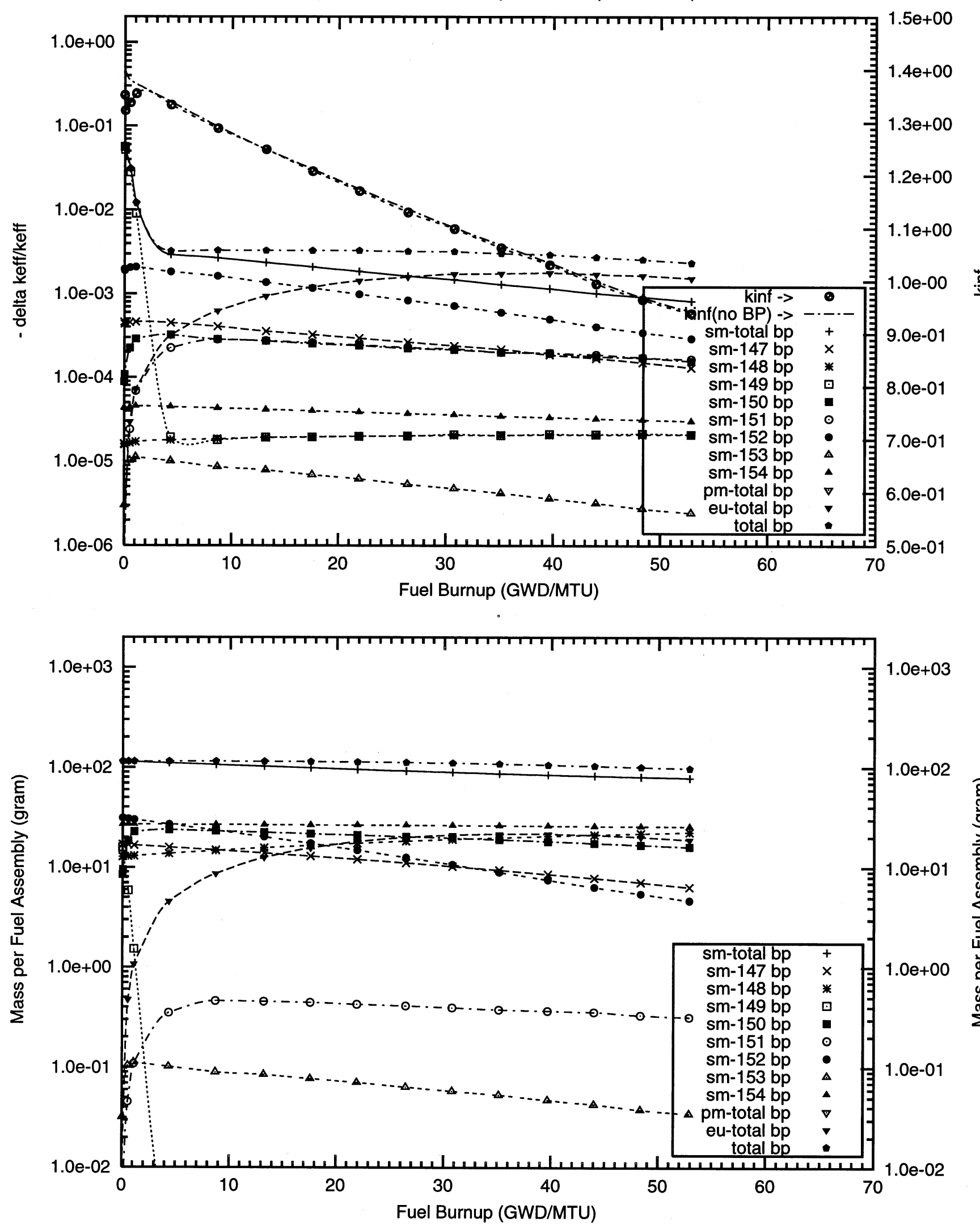
Figure B4.2. Negative Reactivity and Masses BP for Sm-nat and transmutation daughters as a function of fuel life for $17 \times 17$ fuel assemblies with 16 poison rods,

$4.0 \mathrm{w} \% \mathrm{Sm}$ poison homogeneously mixed in the fuel cladding,

Reactor power $3400 \mathrm{MWth}, 193$ fuel assemblies initial enrichment $4.5 \mathrm{w} \% \mathrm{U}-235$

(Case : sm000_3c_100p_h20_16_bp_fuel_2_o)
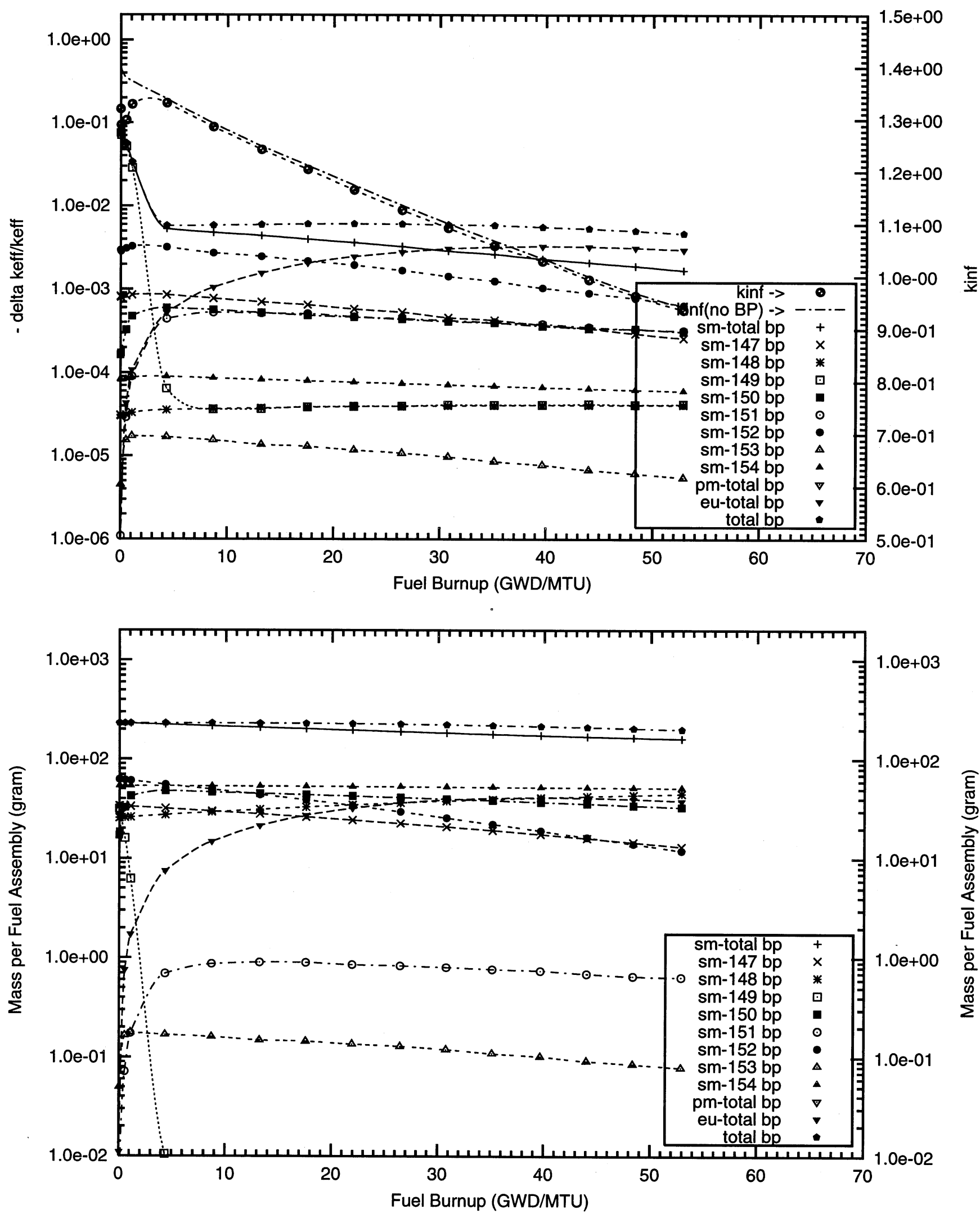
Figure B4.3. Negative Reactivity and Masses BP for Sm-nat and transmutation daughters as a function of fuel life for $17 \times 17$ fuel assemblies with 104 poison rods,

$0.1 \mathrm{w} \% \mathrm{Sm}$ poison homogeneously mixed in the fuel cladding,

Reactor power $3400 \mathrm{MWth}, 193$ fuel assemblies initial enrichment 4.5w\%U-235

(Case : sm000_9c_104_bp_fuel o)
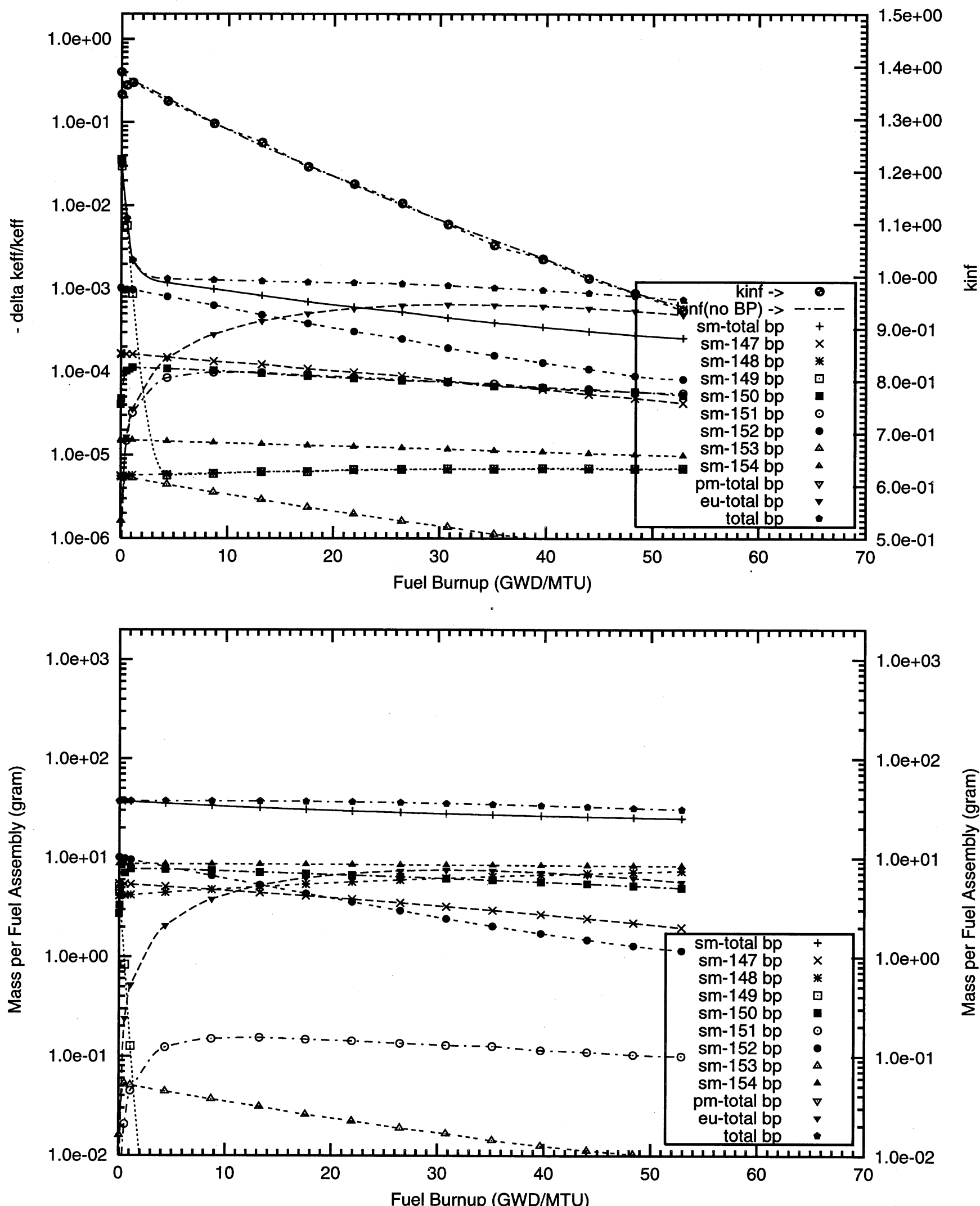
Figure B4.4. Negative Reactivity and Masses BP for Sm-nat and transmutation daughters as a function of fuel life for $17 \times 17$ fuel assemblies with 104 poison rods,

$0.5 \mathrm{w} \% \mathrm{Sm}$ poison homogeneously mixed in the fuel cladding,

Reactor power $3400 \mathrm{MWth}, 193$ fuel assemblies, initial enrichment $4.5 \mathrm{w} \% \mathrm{U}-235$

(Case : sm000_0c_104_bp_fuel o)
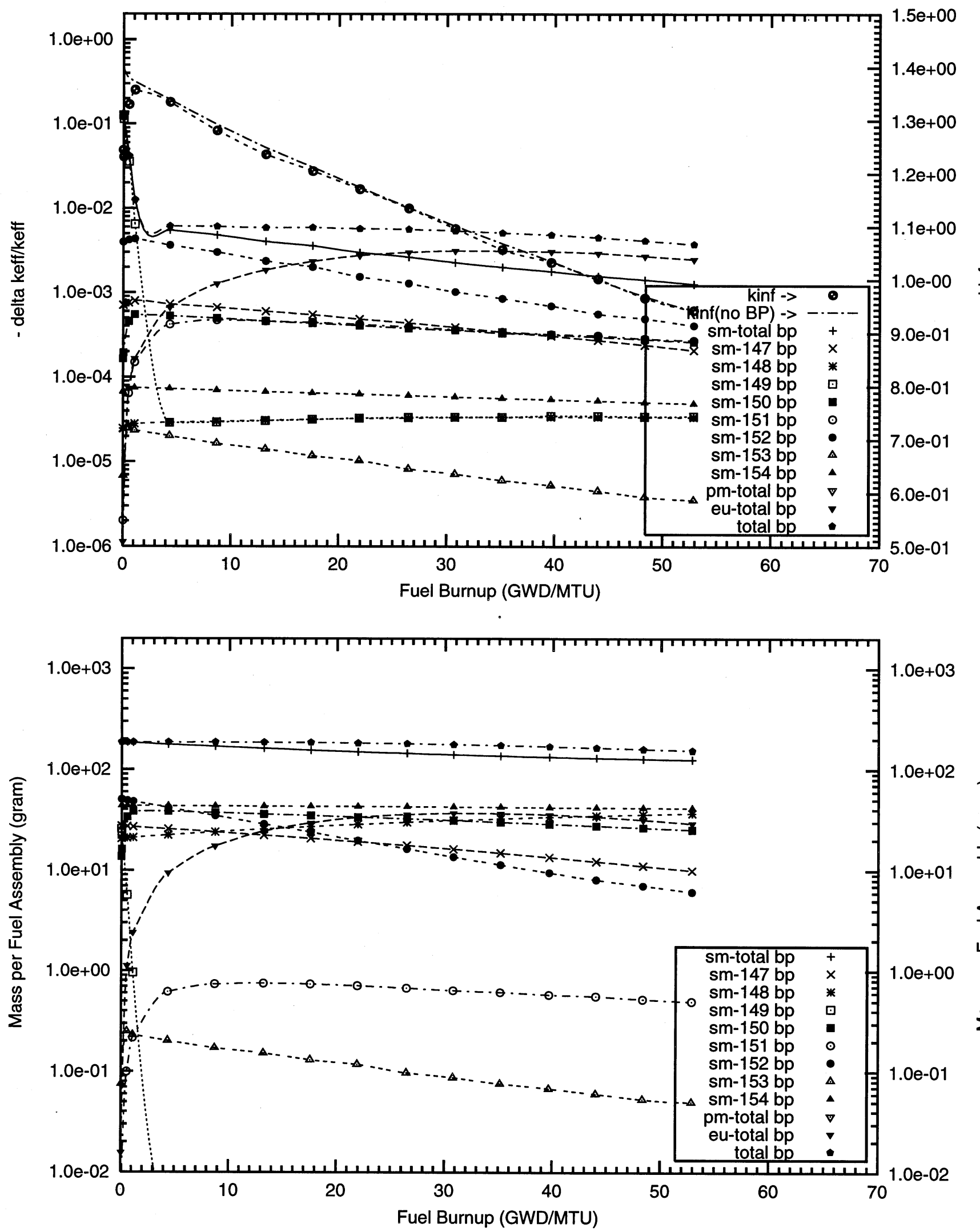
Figure B4.5. Negative Reactivity and Masses BP for Sm-nat and transmutation daughters as a function of fuel life for $17 \times 17$ fuel assemblies with 104 poison rods,

$1.0 \mathrm{w} \% \mathrm{Sm}$ poison homogeneously mixed in the fuel cladding,

Reactor power $3400 \mathrm{MWth}, 193$ fuel assemblies, initial enrichment 4.5w\%U-235

(Case : sm000_1c_104_bp_fuel o)
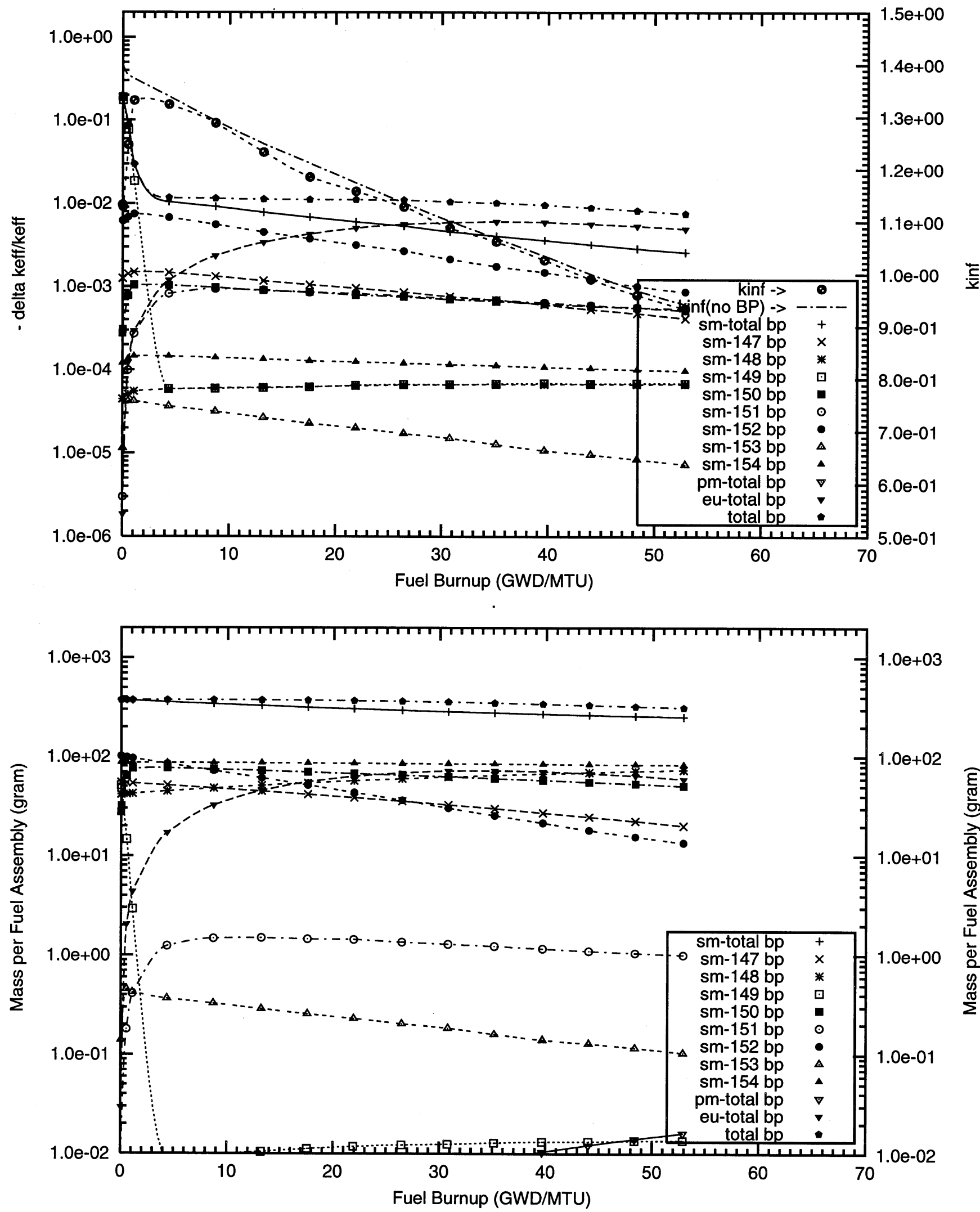
Figure B4.6. Negative Reactivity and Masses BP for Sm-nat and transmutation daughters as a function of fuel life for $17 \times 17$ fuel assemblies with 104 poison rods, $1.0 \mathrm{w} \% \mathrm{Sm}$ poison homogeneously mixed in the fuel cladding,

Reactor power $3400 \mathrm{MWth}, 193$ fuel assemblies, initial enrichment $6.0 \mathrm{w} \% \mathrm{U}-235$

(Case : sm000_1c_enru_6p0_104_bp_fuel o)
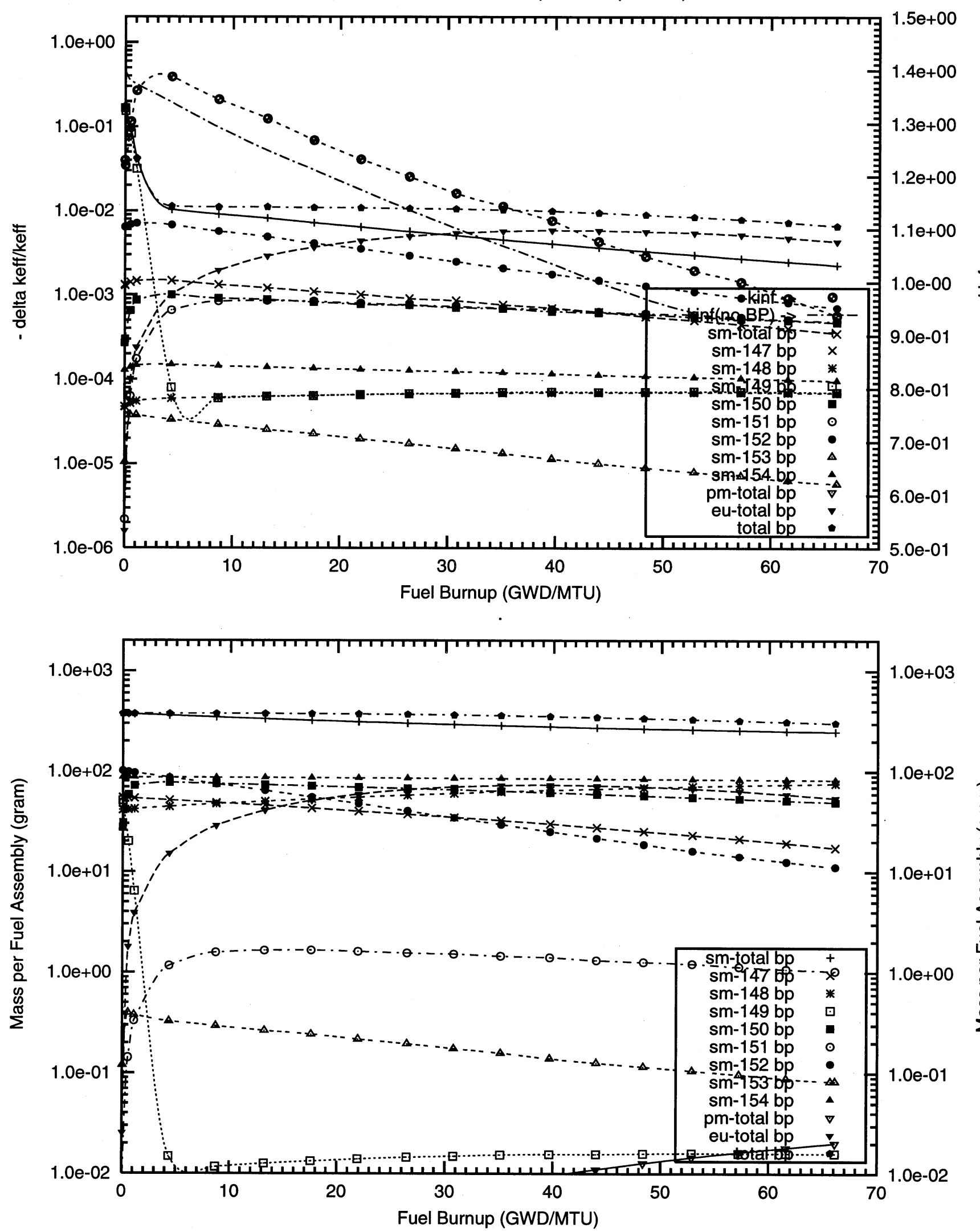


\section{B4 - Samarium Fully Enriched in ${ }^{149} \mathrm{Sm}$}



Figure B4.7. Negative Reactivity and Masses BP for Sm-149 and transmutation daughters as a function of fuel life for $17 \times 17$ fuel assemblies with 16 poison rods,

$0.1 \mathrm{w} \% \mathrm{Sm}$ poison homogeneously mixed in the fuel cladding,

Reactor power $3400 \mathrm{MWth}, 193$ fuel assemblies, initial enrichment 4.5W\%U-235

(Case : sm149_9c_100p_h2o_16_bp_fuel_2_o)

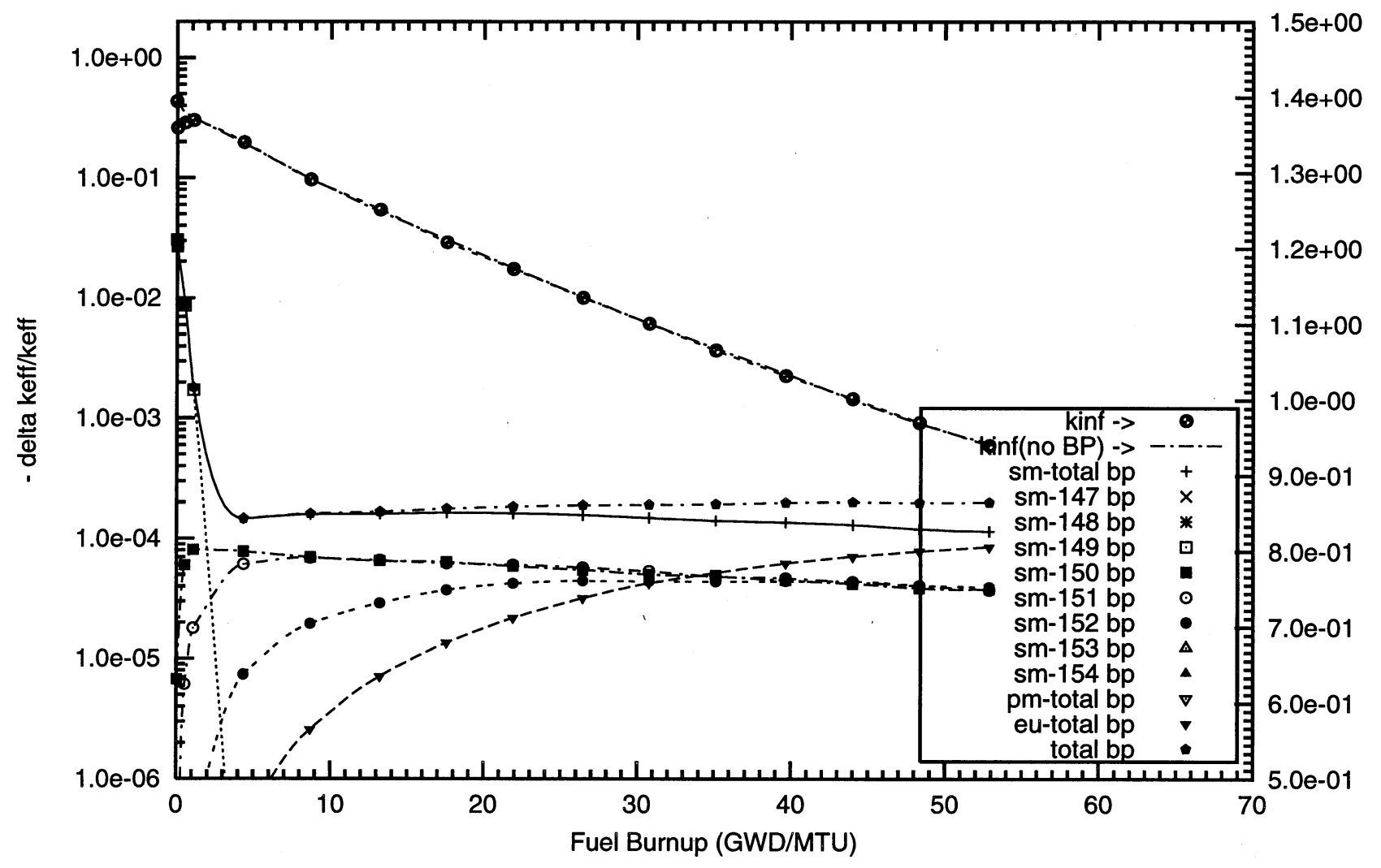

$\frac{\bar{E}}{\bar{x}}$

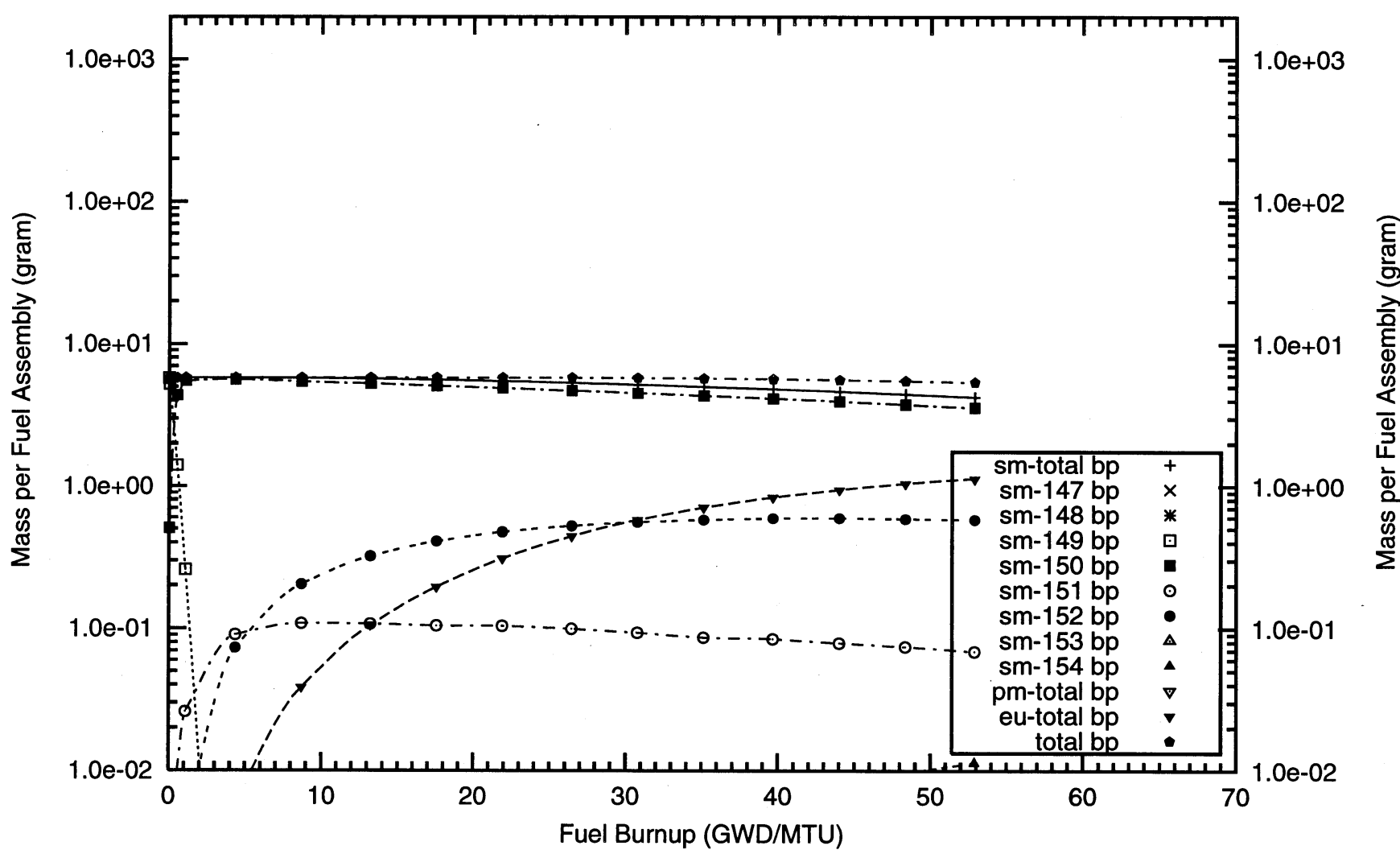


Figure B4.8. Negative Reactivity and Masses BP for Sm-149 and transmutation daughters as a function of fuel life for $17 \times 17$ fuel assemblies with 16 poison rods,

$0.2 \mathrm{w} \% \mathrm{Sm}$ poison homogeneously mixed in the fuel cladding,

Reactor power $3400 \mathrm{MWth}, 193$ fuel assemblies, initial enrichment 4.5w\%U-235

(Case : sm149_8c_100p_h20_16_bp_fuel_2_o)
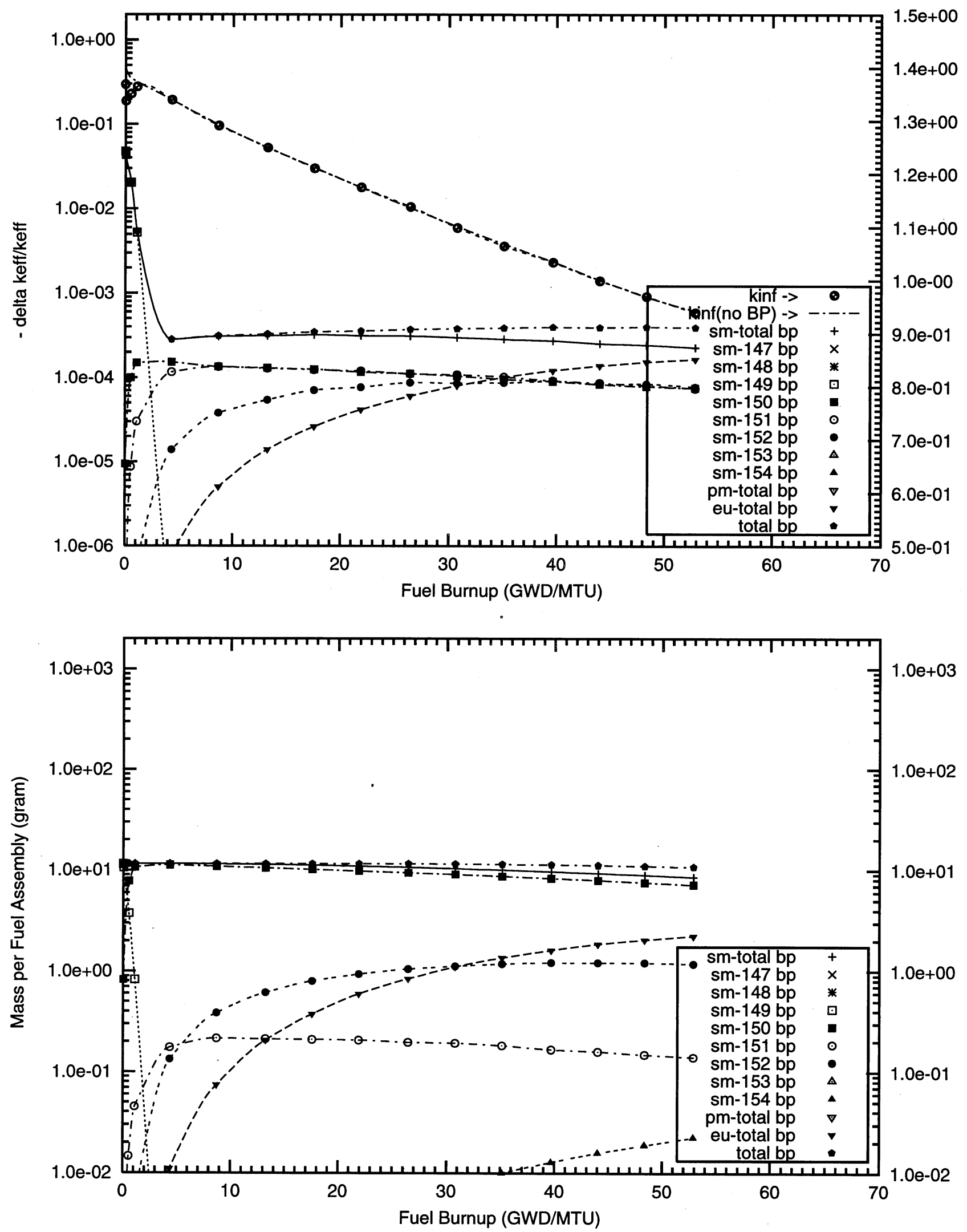
Figure B4.9. Negative Reactivity and Masses BP for Sm-149 and transmutation daughters as a function of fuel life for $17 \times 17$ fuel assemblies with 16 poison rods,

$0.5 \mathrm{w} \% \mathrm{Sm}$ poison homogeneously mixed in the fuel cladding,

Reactor power $3400 \mathrm{MWth}, 193$ fuel assemblies, initial enrichment 4.5w\%U-235

(Case : sm149_0c_100p_h20_16_bp_fuel_2_o)
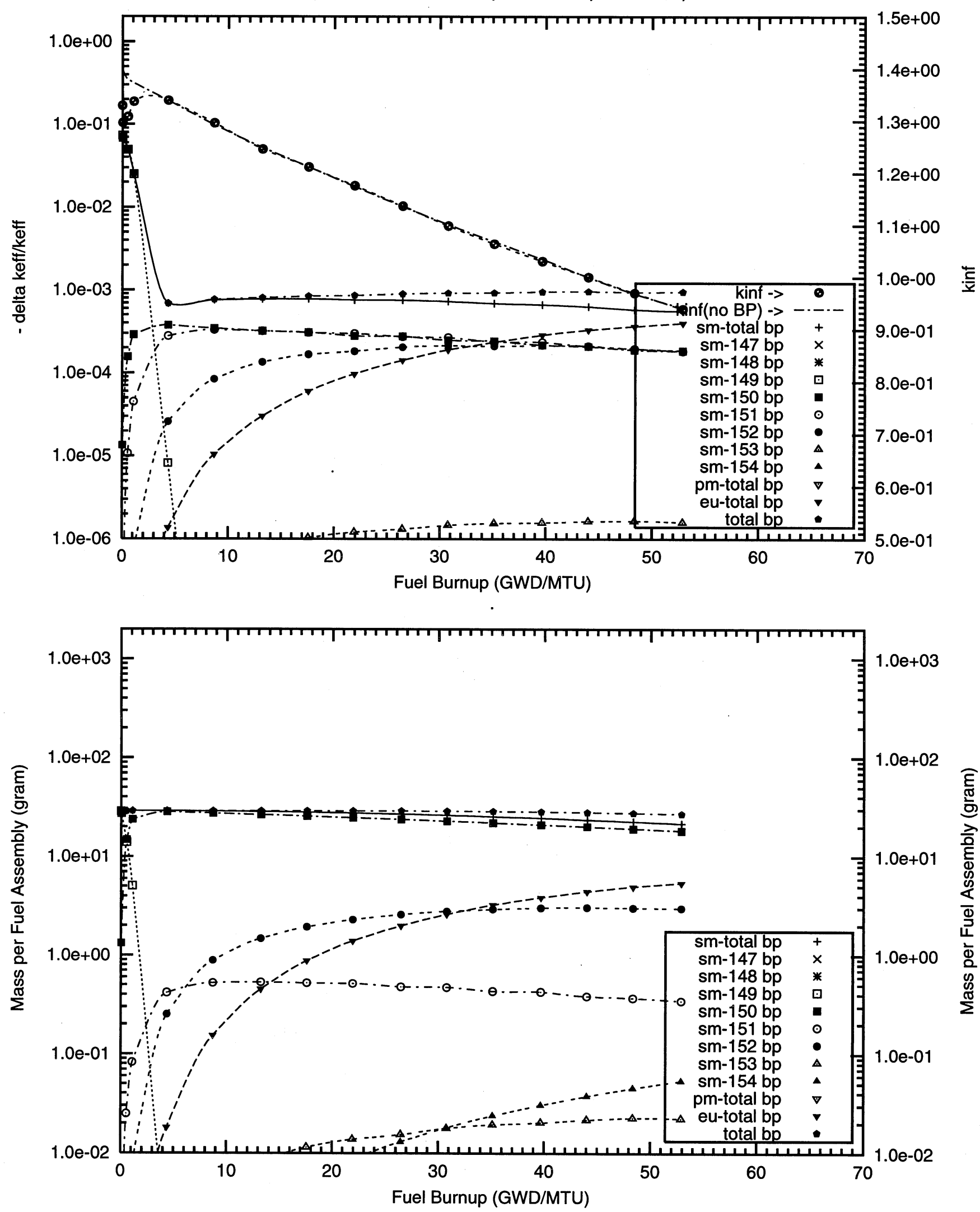
Figure B4.10. Negative Reactivity and Masses BP for Sm-149 and transmutation daughters as a function of fuel life for $17 \times 17$ fuel assemblies with 16 poison rods,

$1.0 \mathrm{w} \% \mathrm{Sm}$ poison homogeneously mixed in the fuel cladding,

Reactor power $3400 \mathrm{MWth}, 193$ fuel assemblies, initial enrichment $4.5 \mathrm{w} \% \mathrm{U}-235$

(Case : sm149_1c_100p_h2o_16_bp_fuel_2_o)

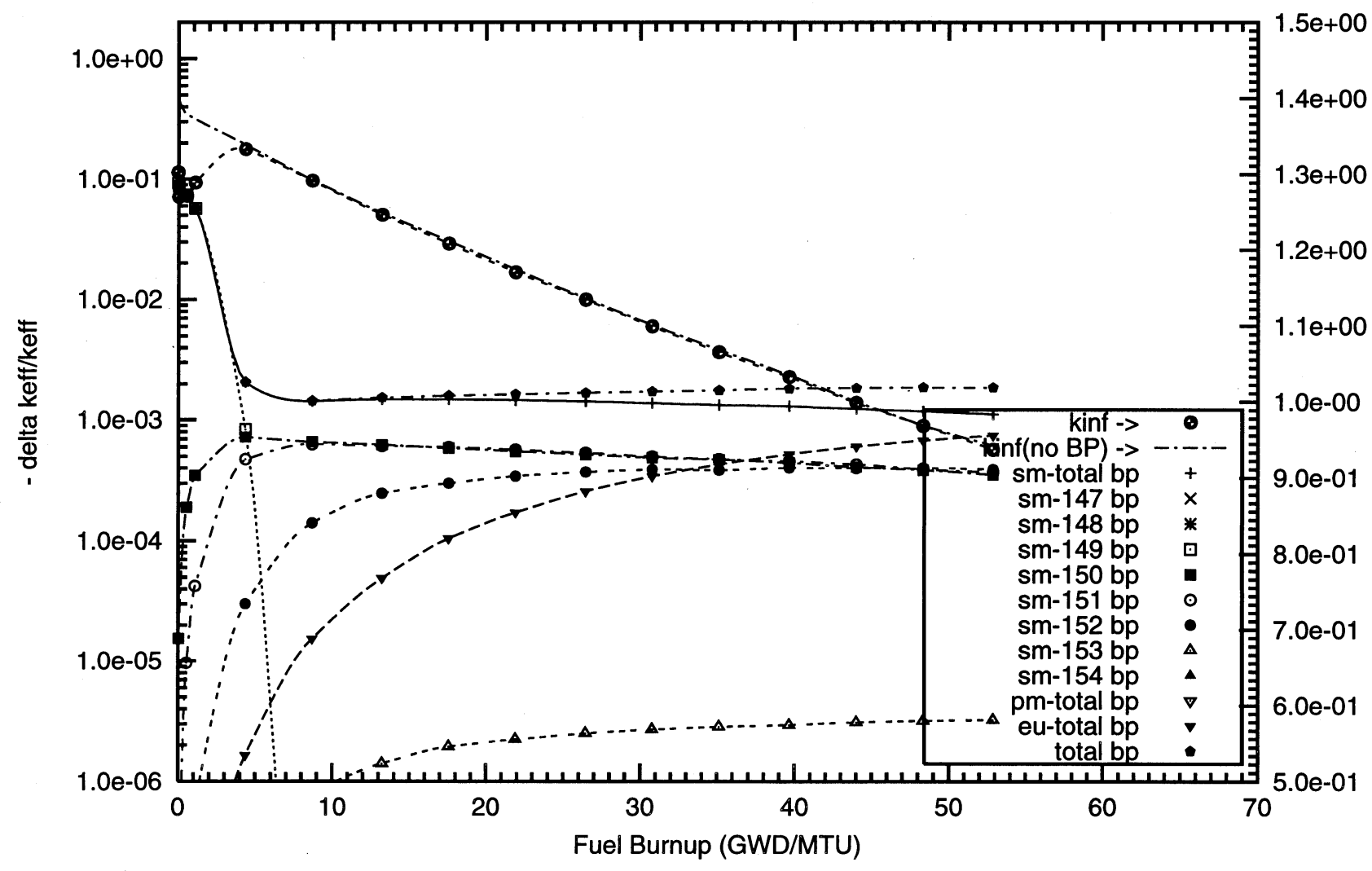

$\overline{\frac{E}{x}}$

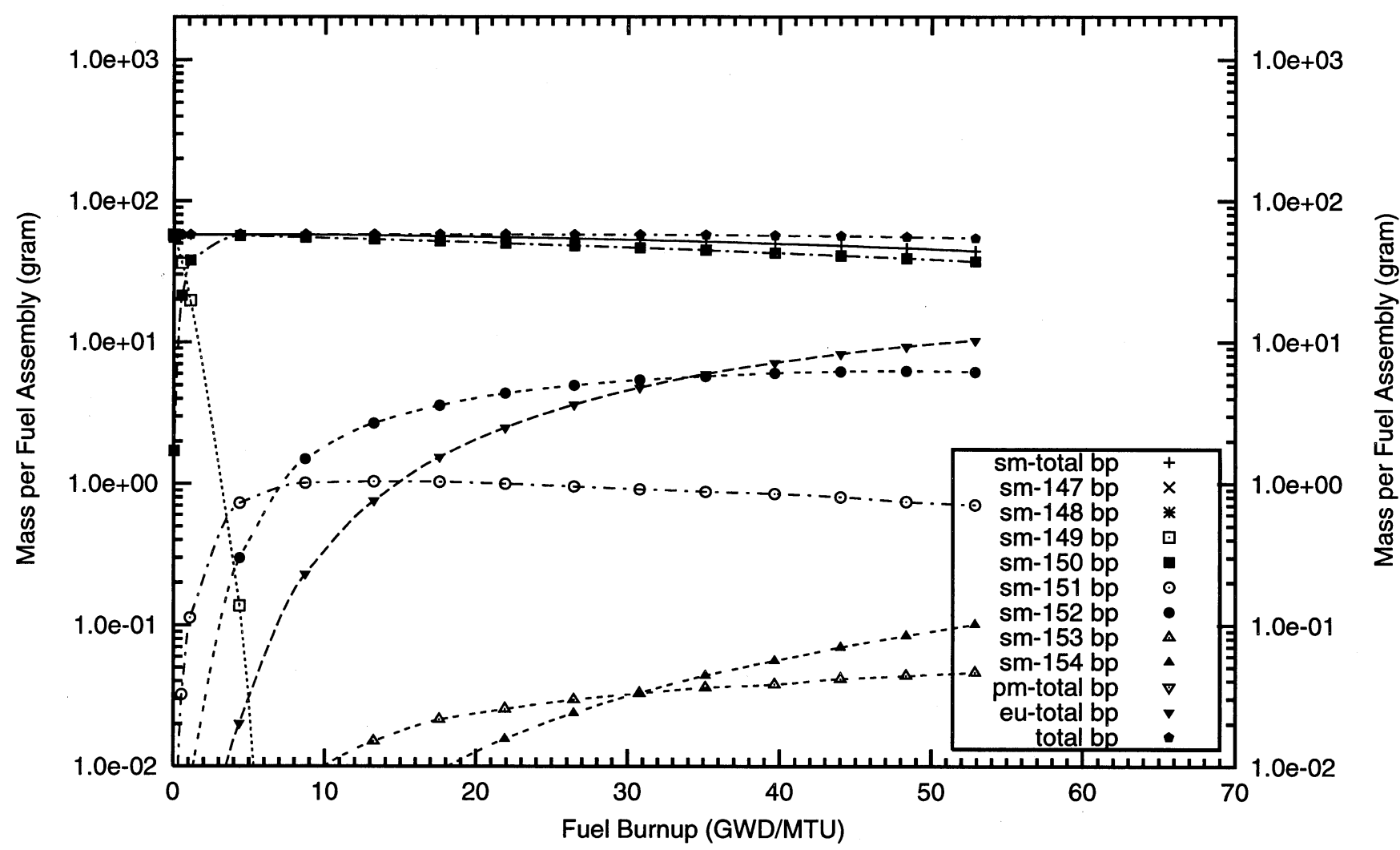


Figure B4.11. Negative Reactivity and Masses BP for Sm-149 and transmutation daughters as a function of fuel life for $17 \times 17$ fuel assemblies with 16 poison rods,

$2.0 \mathrm{w} \% \mathrm{Sm}$ poison homogeneously mixed in the fuel cladding,

Reactor power $3400 \mathrm{MWth}, 193$ fuel assemblies, initial enrichment $4.5 \mathrm{w} \% \mathrm{U}-235$

(Case : sm149_2c_100p_h2o_16_bp_fuel_2_o)
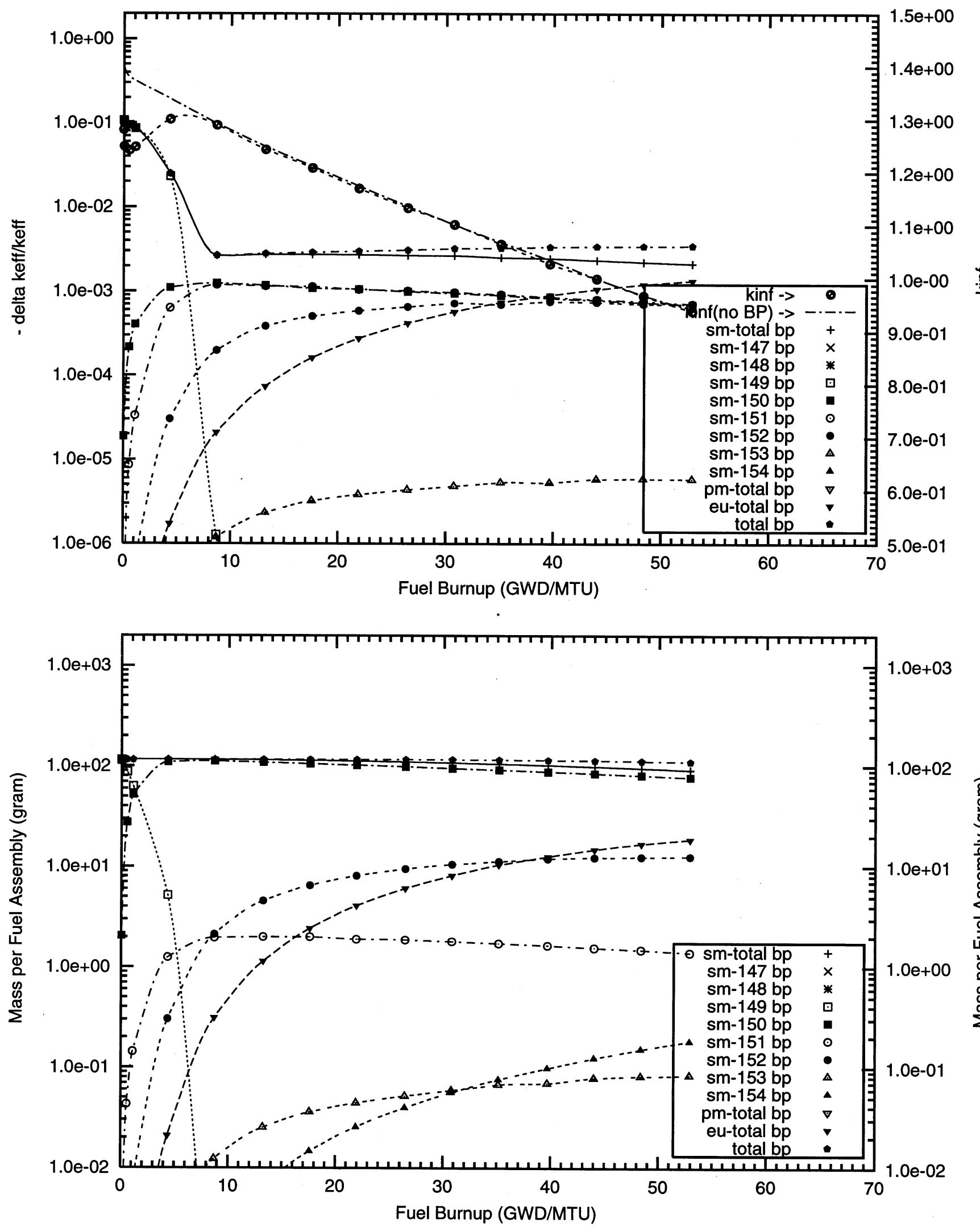
Figure B4.12. Negative Reactivity and Masses BP for Sm-149 and transmutation daughters as a function of fuel life for $17 \times 17$ fuel assemblies with 104 poison rods,

$0.05 \mathrm{w} \% \mathrm{Sm}$ poison homogeneously mixed in the fuel cladding,

Reactor power 3400 MWth, 193 fuel assemblies, initial enrichment 4.5W\%U-235

(Case : sm149_10c_104_bp_fuel o)
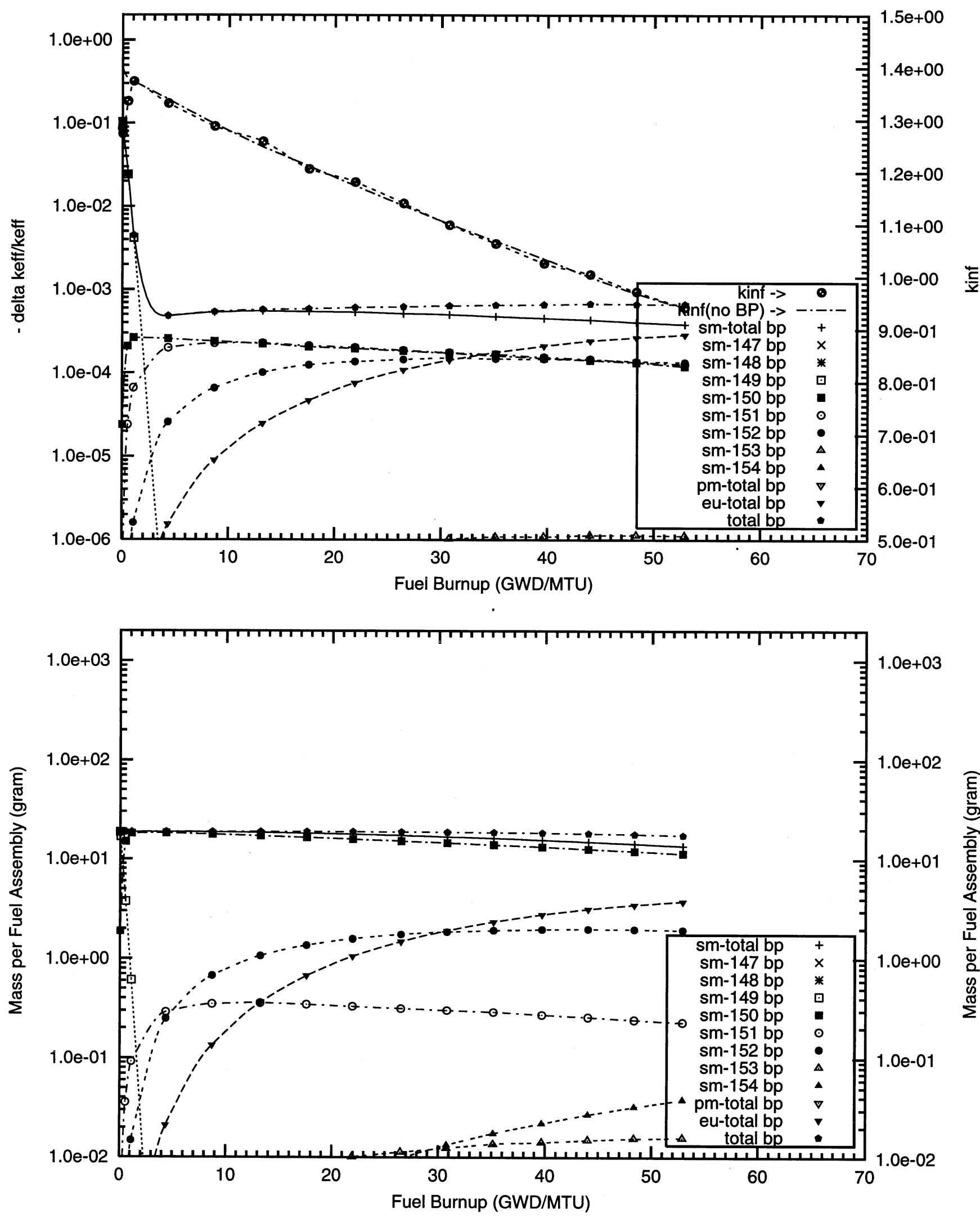
Figure B4.13. Negative Reactivity and Masses BP for Sm-149 and transmutation daughters as a function of fuel life for $17 \times 17$ fuel assemblies with 104 poison rods,

$0.1 \mathrm{w} \% \mathrm{Sm}$ poison homogeneously mixed in the fuel cladding,

Reactor power $3400 \mathrm{MWth}, 193$ fuel assemblies, initial enrichment $4.5 \mathrm{w} \% \mathrm{U}-235$

(Case : sm149_9c_104_bp_fuel o)
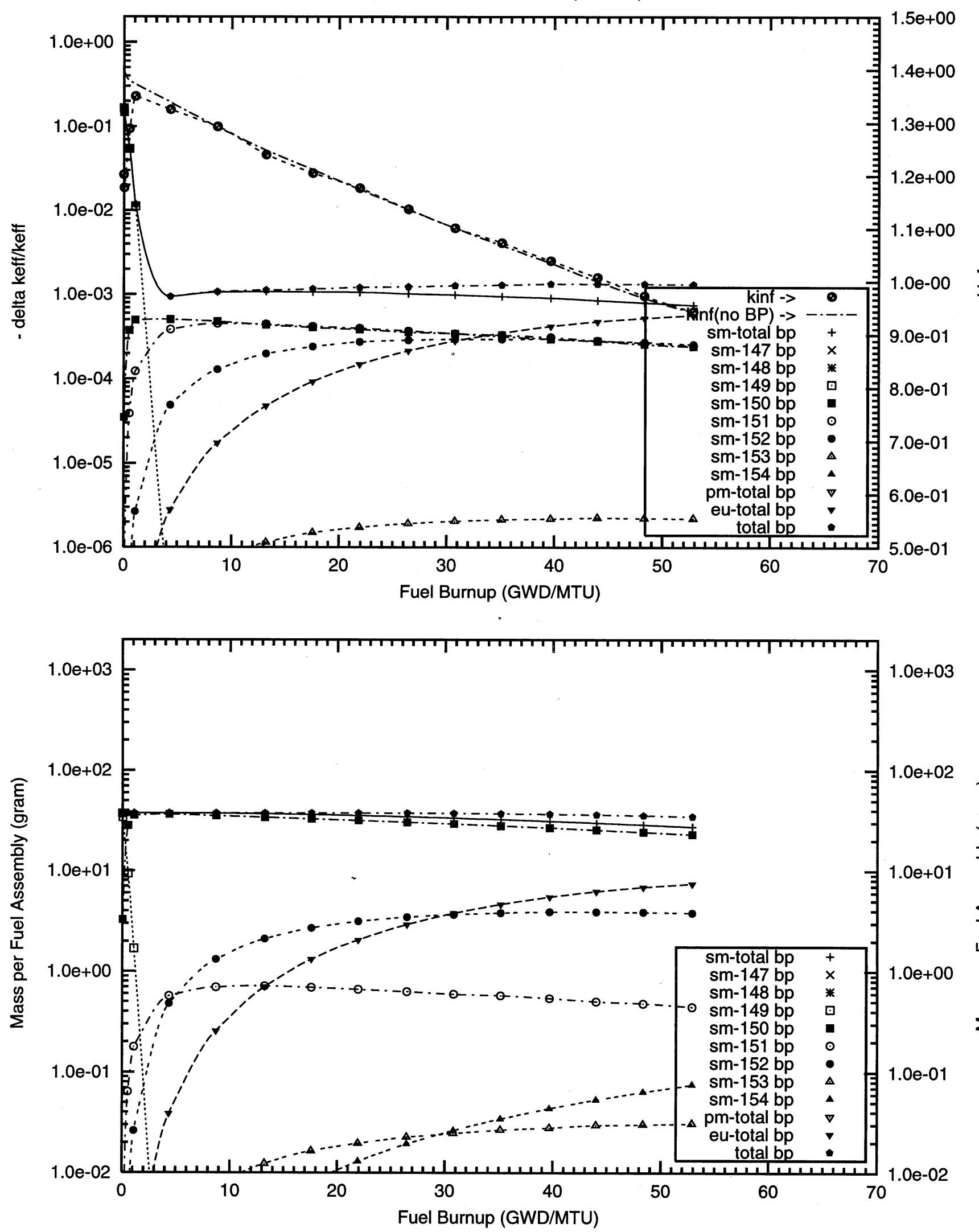
Figure B4.14. Negative Reactivity and Masses BP for Sm-149 and transmutation daughters as a function of fuel life for $17 \times 17$ fuel assemblies with 104 poison rods,

$0.2 \mathrm{w} \% \mathrm{Sm}$ poison homogeneously mixed in the fuel cladding

Reactor power $3400 \mathrm{MWth}, 193$ fuel assemblies, initial enrichment $4.5 \mathrm{w} \% \mathrm{U}-235$

(Case : sm149_8c_104_bp_fuel o)
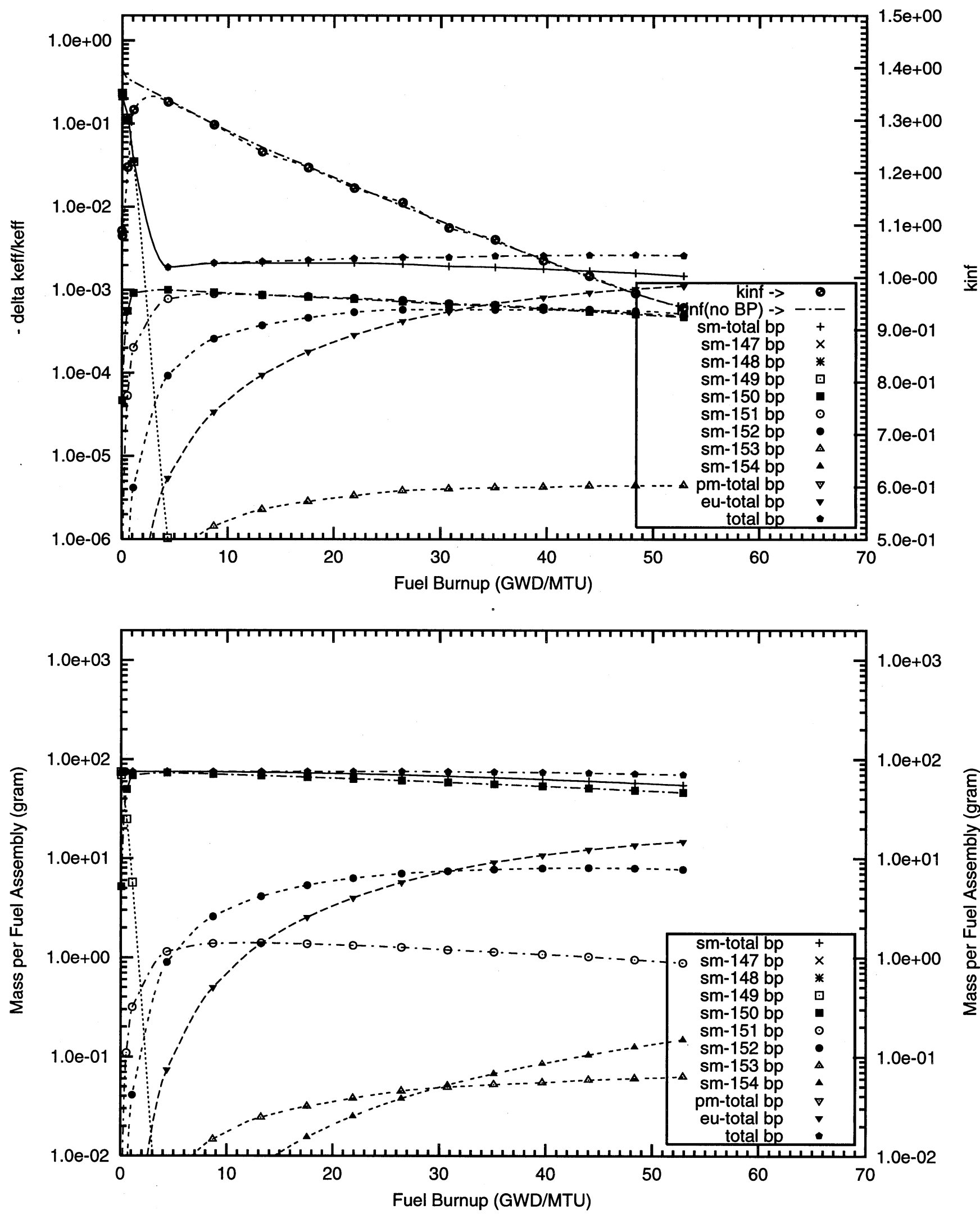
Figure B4.15. Negative Reactivity and Masses BP for Sm-149 and transmutation daughters as a function of fuel life for $17 \times 17$ fuel assemblies with 104 poison rods,

$0.1 \mathrm{w} \% \mathrm{Sm}$ poison homogeneously mixed in the fuel cladding,

Reactor power $3400 \mathrm{MWth}, 193$ fuel assemblies, initial fuel enrichment 6.0w\%U-235

(Case : sm149_9c_enru_6p0_104_bp_fuel o)
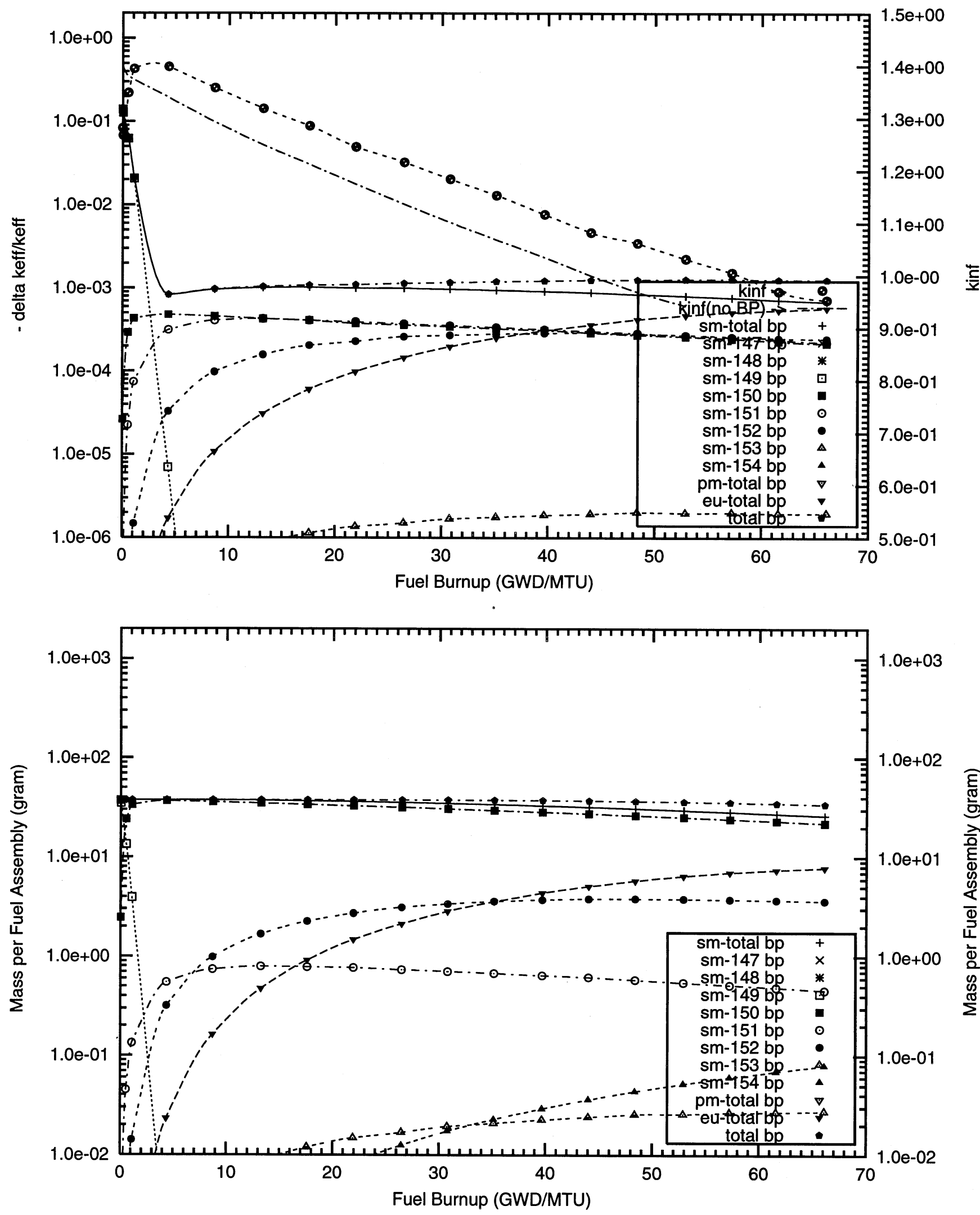
Figure B4.16. Negative Reactivity and Masses BP for Sm-149 and transmutation daughters as a function of fuel life for $17 \times 17$ fuel assemblies with 104 poison rods, $0.2 \mathrm{w} \% \mathrm{Sm}$ poison homogeneously mixed in the fuel cladding,

Reactor power $3400 \mathrm{MWth}, 193$ fuel assemblies, initial fuel enrichment $6.0 \mathrm{w} \% \mathrm{U}-235$ (Case : sm149_8c_enru_6p0_104_bp_fuel o)
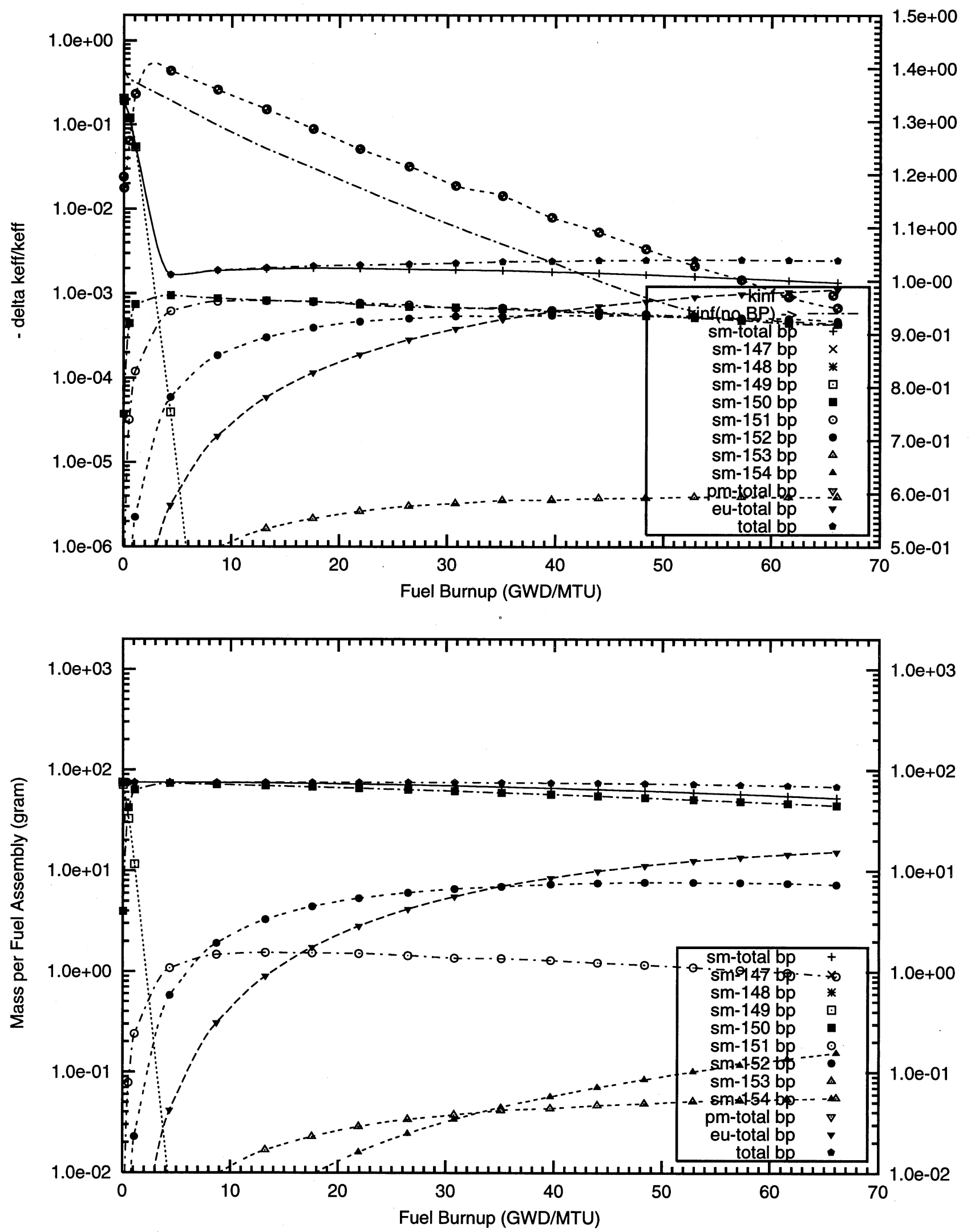


\title{
Appendix C1
}

Reactivity Worths and Masses of $\mathrm{Er}_{2} \mathrm{O}_{3}$ Burnable Poison Homogeneously Mixed in the Fuel Pellets

\author{
Table 13 Cases
}



C1 - Erbium with Natural Isotopic Abundances 

Figure C1.1. Negative Reactivity and Masses BP for Er-nat and transmutation daughters as a function of fuel life for $17 \times 17$ fuel assemblies with 08 poison rods, $4.0 \mathrm{w} \%$ Er2O3 poison homogeneously mixed in the UO2 pellets

Reactor power $3400 \mathrm{MWth}, 193$ fuel assemblies, initial enrichment 4.5w\%U-235 (Case : er000_3_08_bp_fuel o)

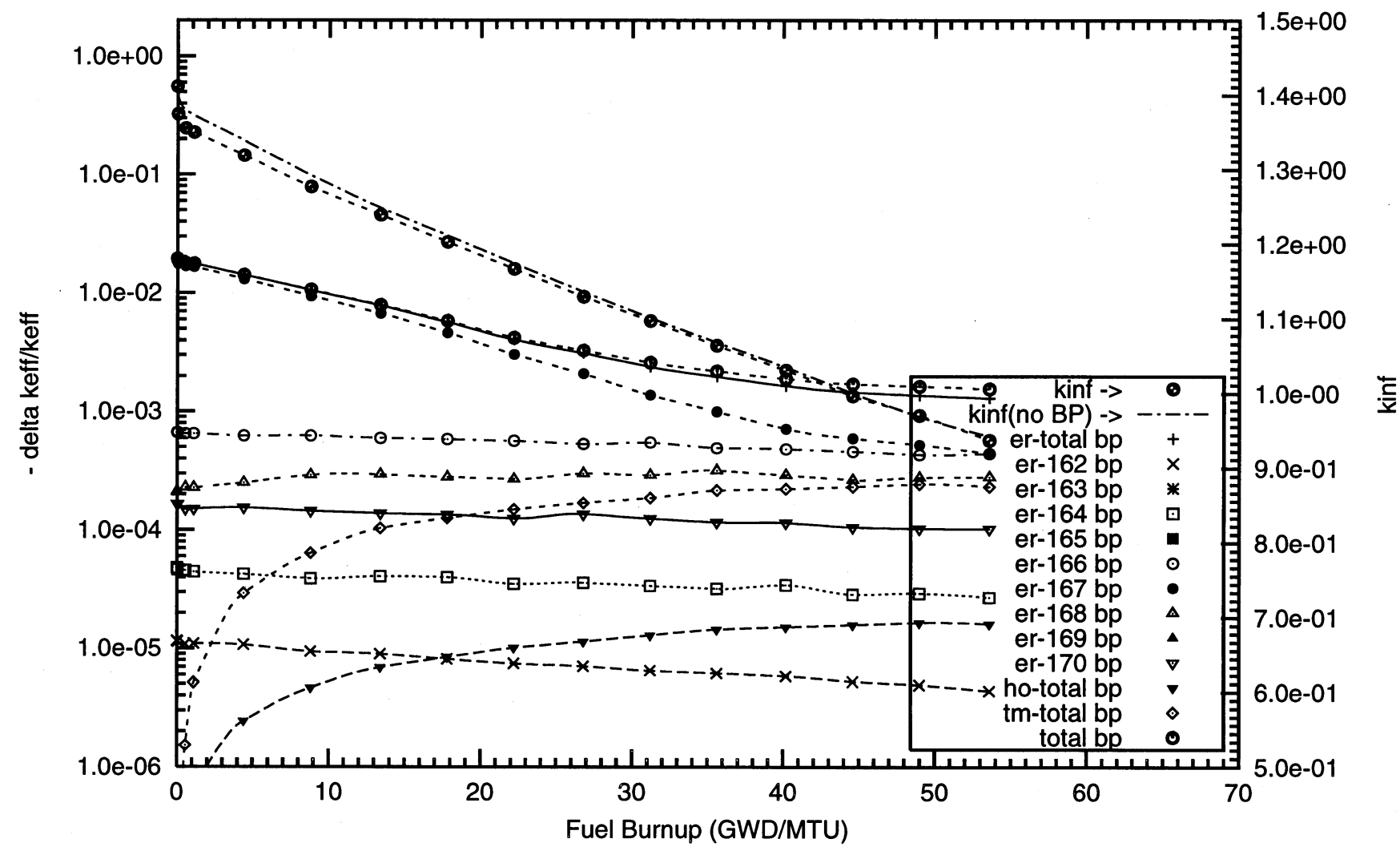


Figure C1.2. Negative Reactivity and Masses BP for Er-nat and transmutation daughters as a function of fuel life for $17 \times 17$ fuel assemblies with 16 poison rods,

$2.0 \mathrm{w} \%$ Er2O3 poison homogeneously mixed in the UO2 pellets

Reactor power $3400 \mathrm{MWth}, 193$ fuel assemblies, initial enrichment 4.5W\%U-235

(Case : er000_2_100p_h20_16_bp_fuel_2_o)
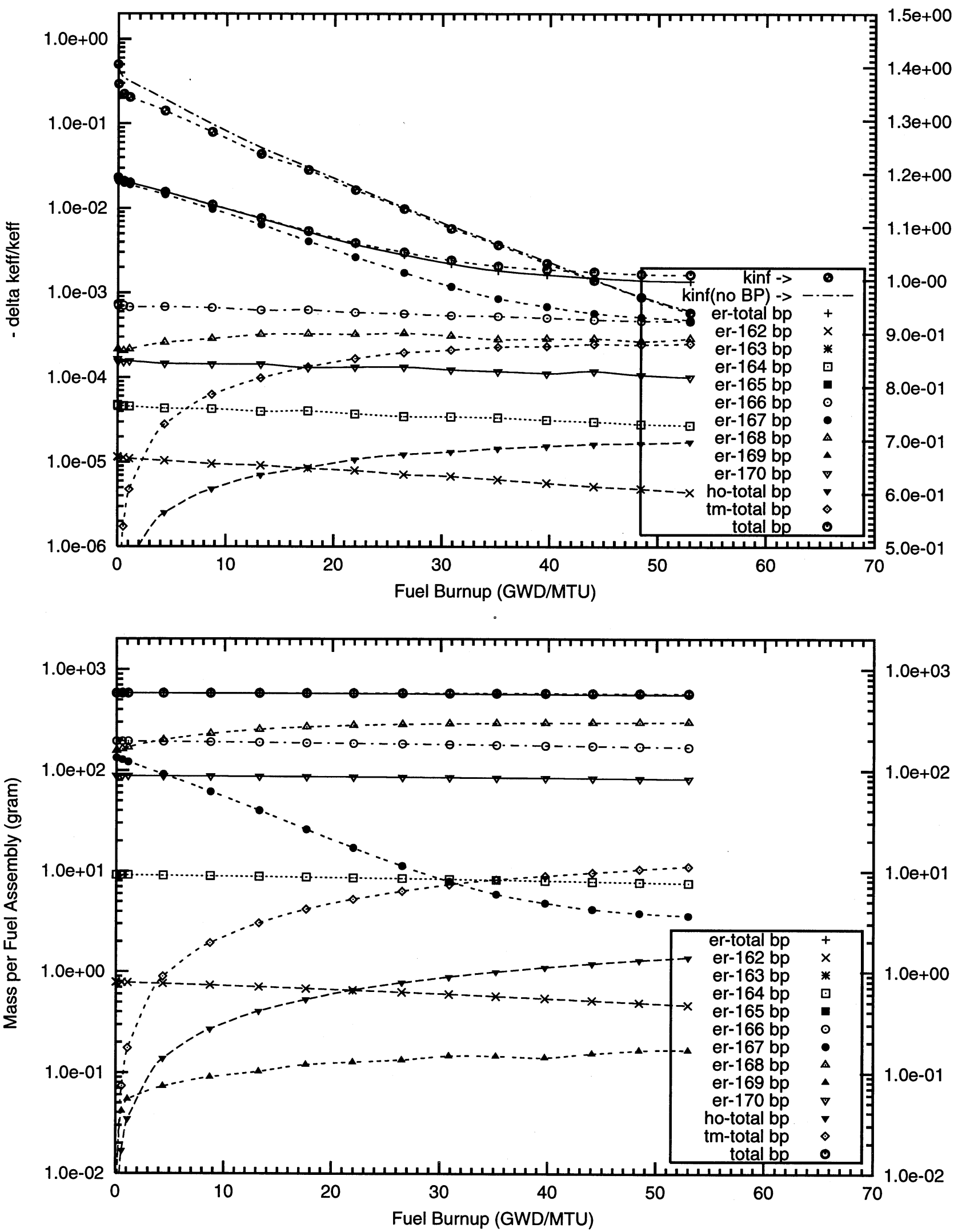
Figure C1.3. Negative Reactivity and Masses BP for Er-nat and transmutation daughters

as a function of fuel life for $17 \times 17$ fuel assemblies with 16 poison rods,

$4.0 \mathrm{w} \%$ Er2O3 poison homogeneously mixed in the UO2 pellets

Reactor power $3400 \mathrm{MWth}, 193$ fuel assemblies, initial enrichment 4.5w\%U-235

(Case : er000_3_100p_h20_16_bp_fuel_2_o)
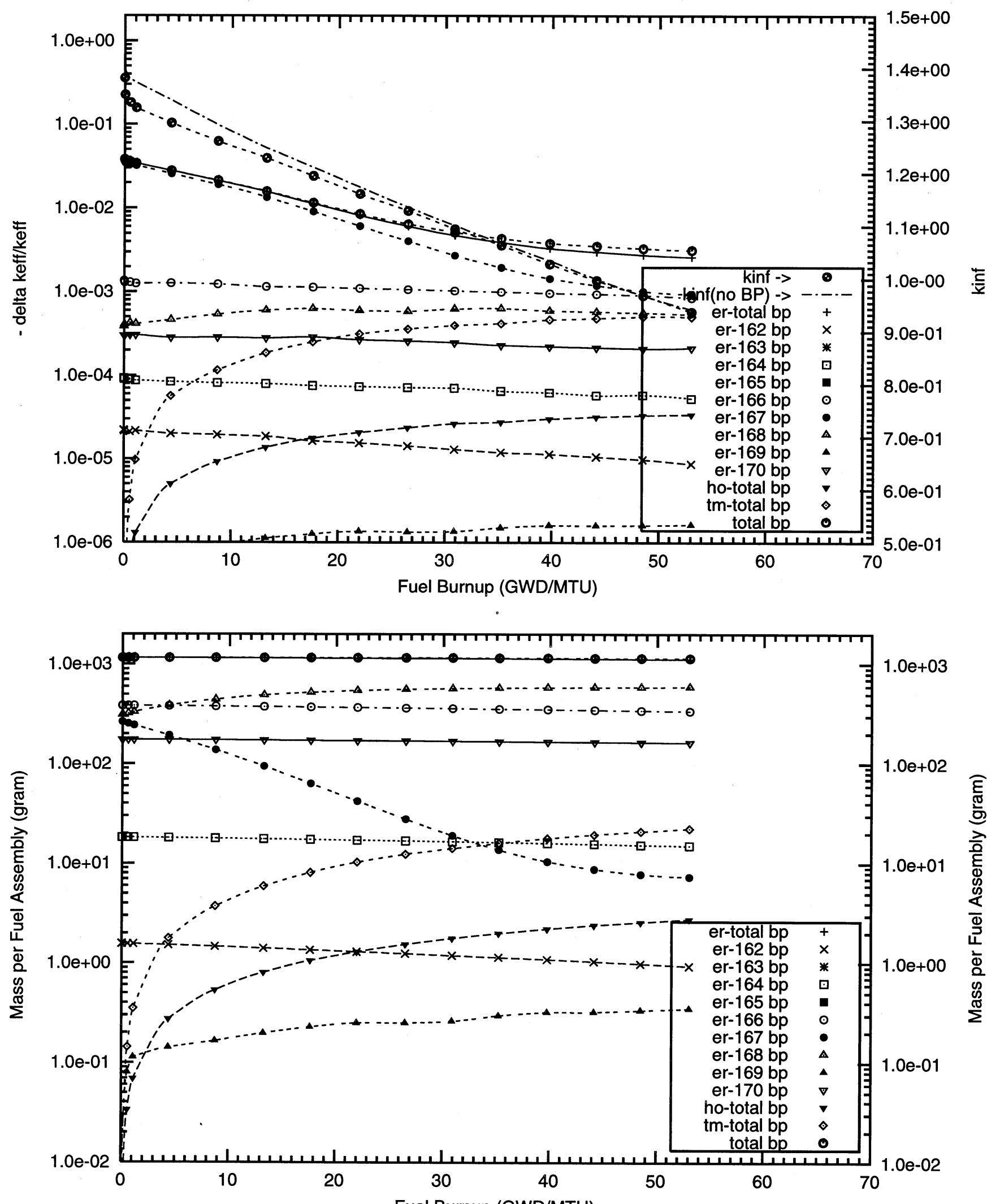

Fuel Burnup (GWD/MTU) 
Figure C1.4. Negative Reactivity and Masses BP for Er-nat and transmutation daughters as a function of fuel life for $17 \times 17$ fuel assemblies with 16 poison rods, $8.0 \mathrm{w} \%$ Er2O3 poison homogeneously mixed in the UO2 pellets

Reactor power $3400 \mathrm{MWth}, 193$ fuel assemblies, initial enrichment 4.5w\%U-235 (Case : er000_4_100p_h20_16_bp_fuel_2_o)
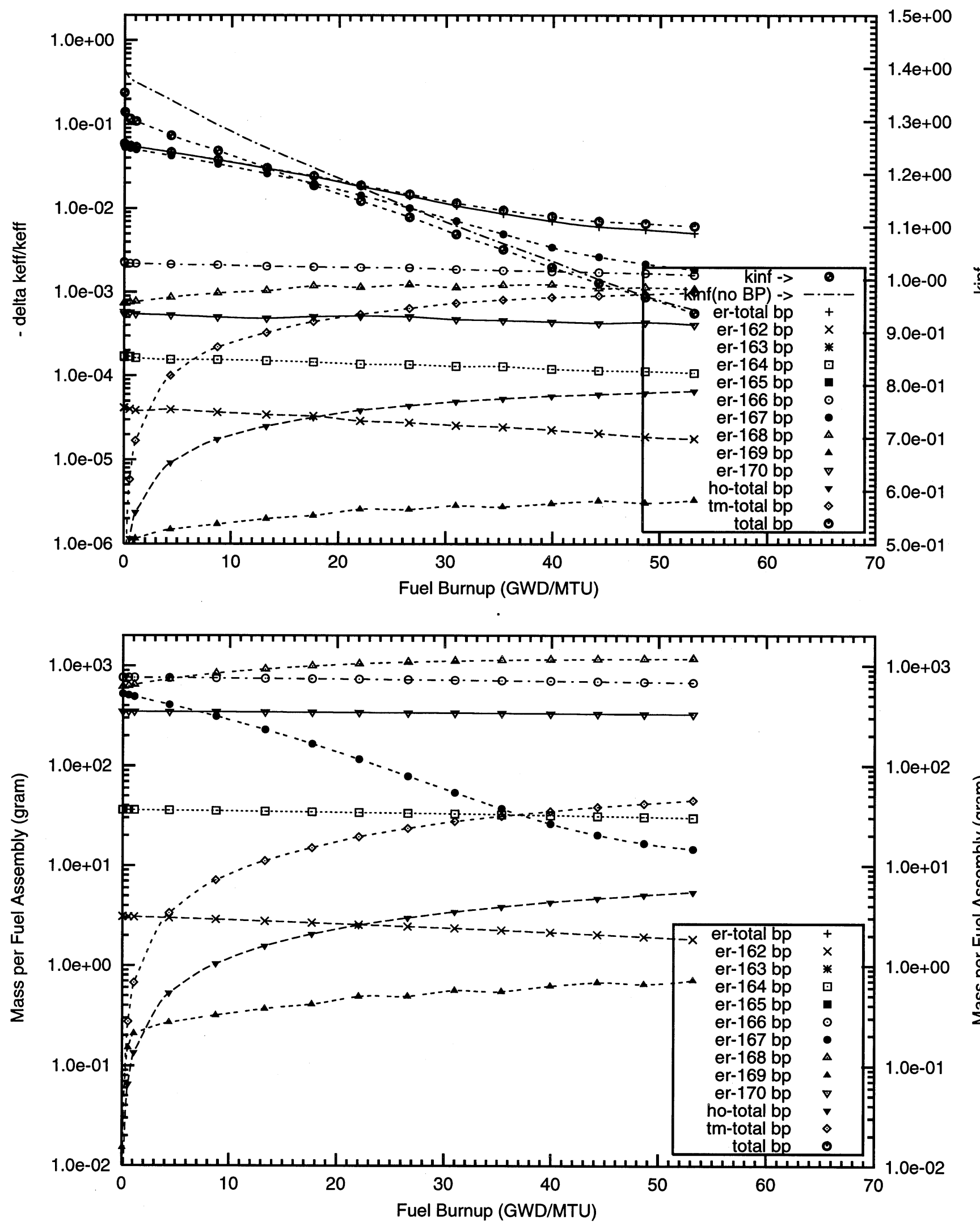
Figure C1.5. Negative Reactivity and Masses BP for Er-nat and transmutation daughters as a function of fuel life for $17 \times 17$ fuel assemblies with 16 poison rods,

$12.0 \mathrm{w} \%$ Er2O3 poison homogeneously mixed in the UO2 pellets

Reactor power $3400 \mathrm{MWth}, 193$ fuel assemblies, initial enrichment 4.5w\%U-235

(Case : er000_5_100p_h20_16_bp_fuel_2_o)
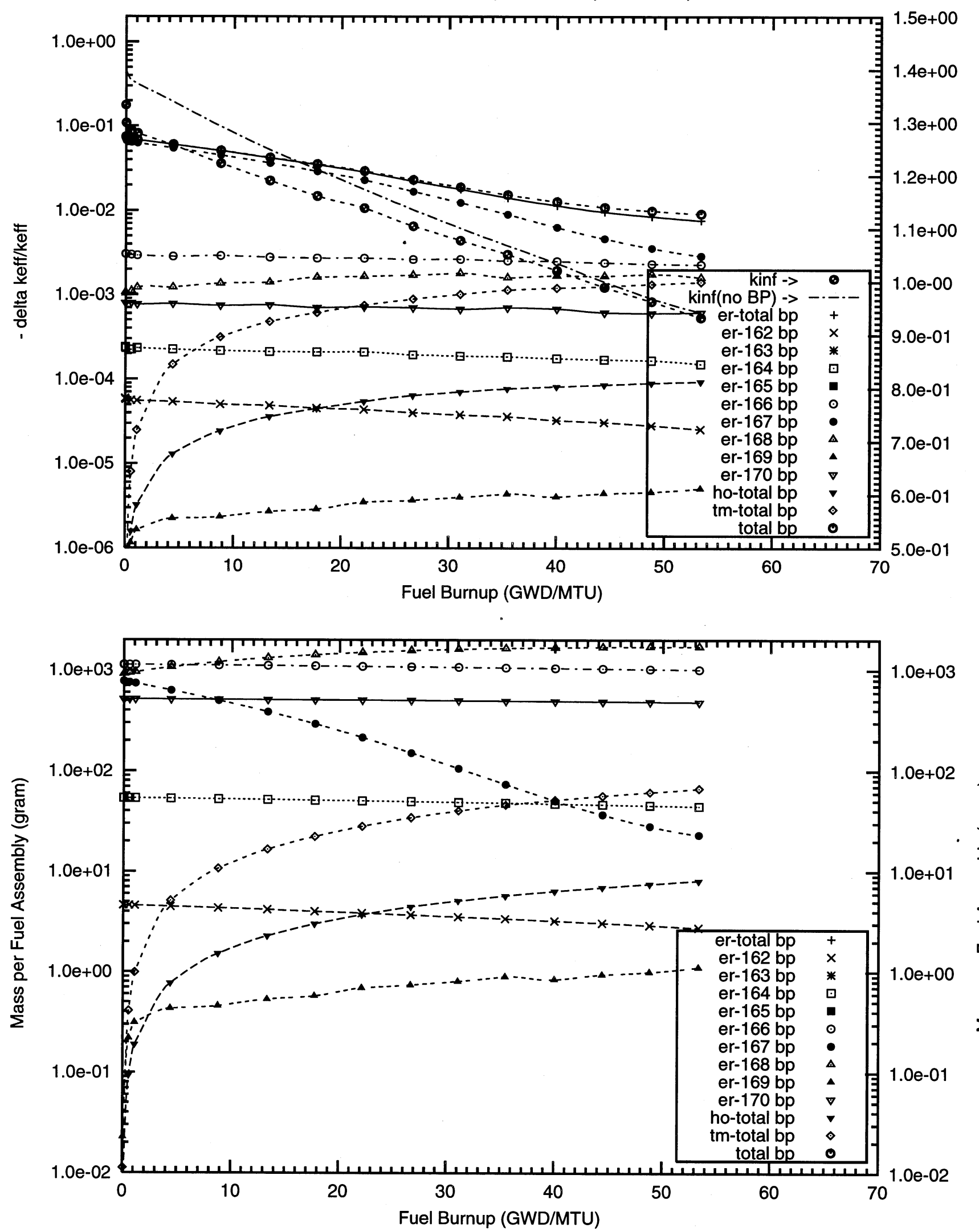
Figure C1.6. Negative Reactivity and Masses BP for Er-nat and transmutation daughters as a function of fuel life for $17 \times 17$ fuel assemblies with 20 poison rods, 4.0w\% Er2O3 poison homogeneously mixed in the UO2 pellets

Reactor power $3400 \mathrm{MWth}, 193$ fuel assemblies, initial enrichment 4.5w\%U-235

(Case : er000_3_16_bp_fuel (old) o)

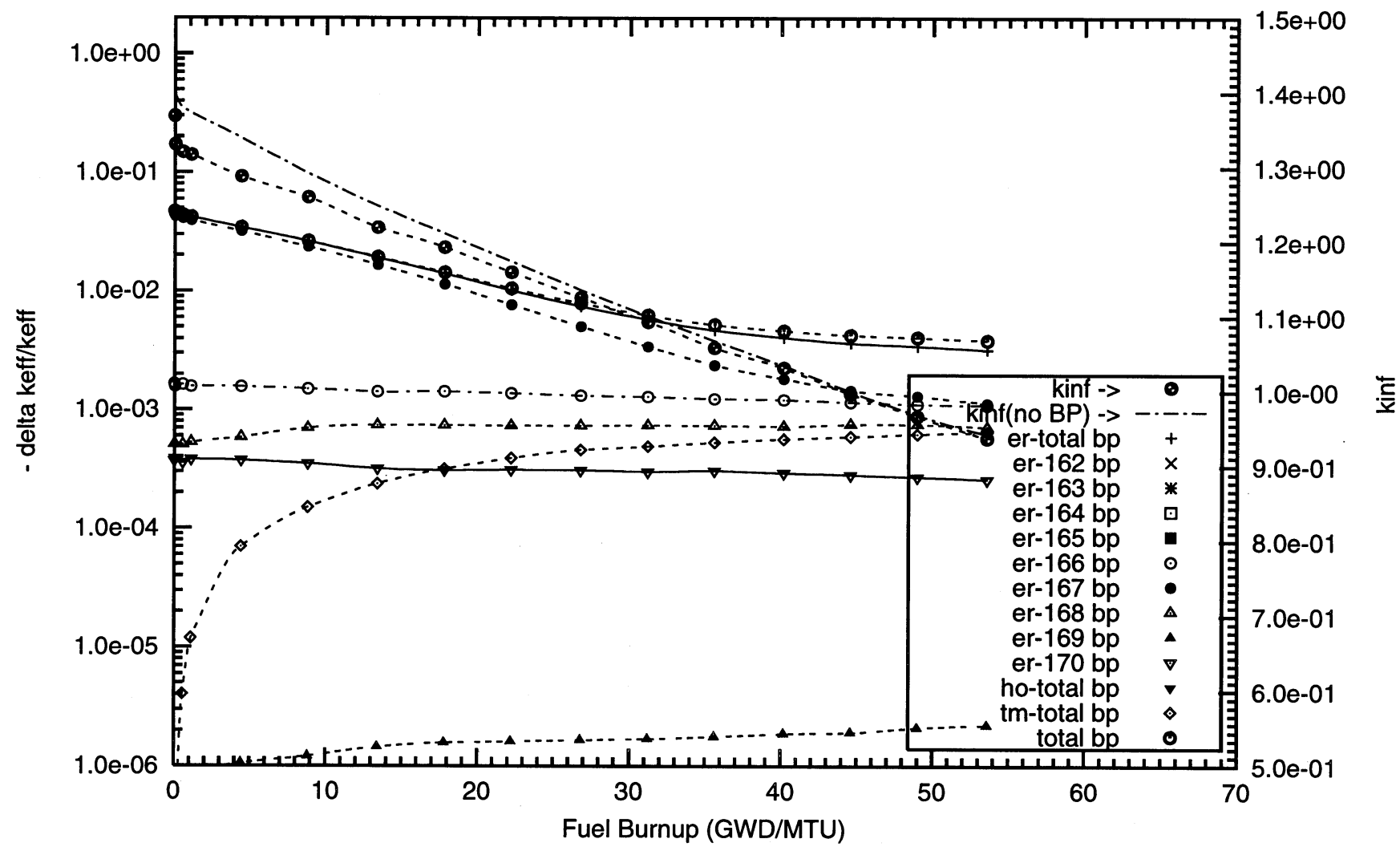


Figure C1.7. Negative Reactivity and Masses BP for Er-nat and transmutation daughters as a function of fuel life for $17 \times 17$ fuel assemblies with 20 poison rods, $8.0 \mathrm{w} \%$ Er2O3 poison homogeneously mixed in the UO2 pellets

Reactor power $3400 \mathrm{MWth}, 193$ fuel assemblies, initial enrichment 4.5w\%U-235

(Case : er000_4_16_bp_fuel (old) o)
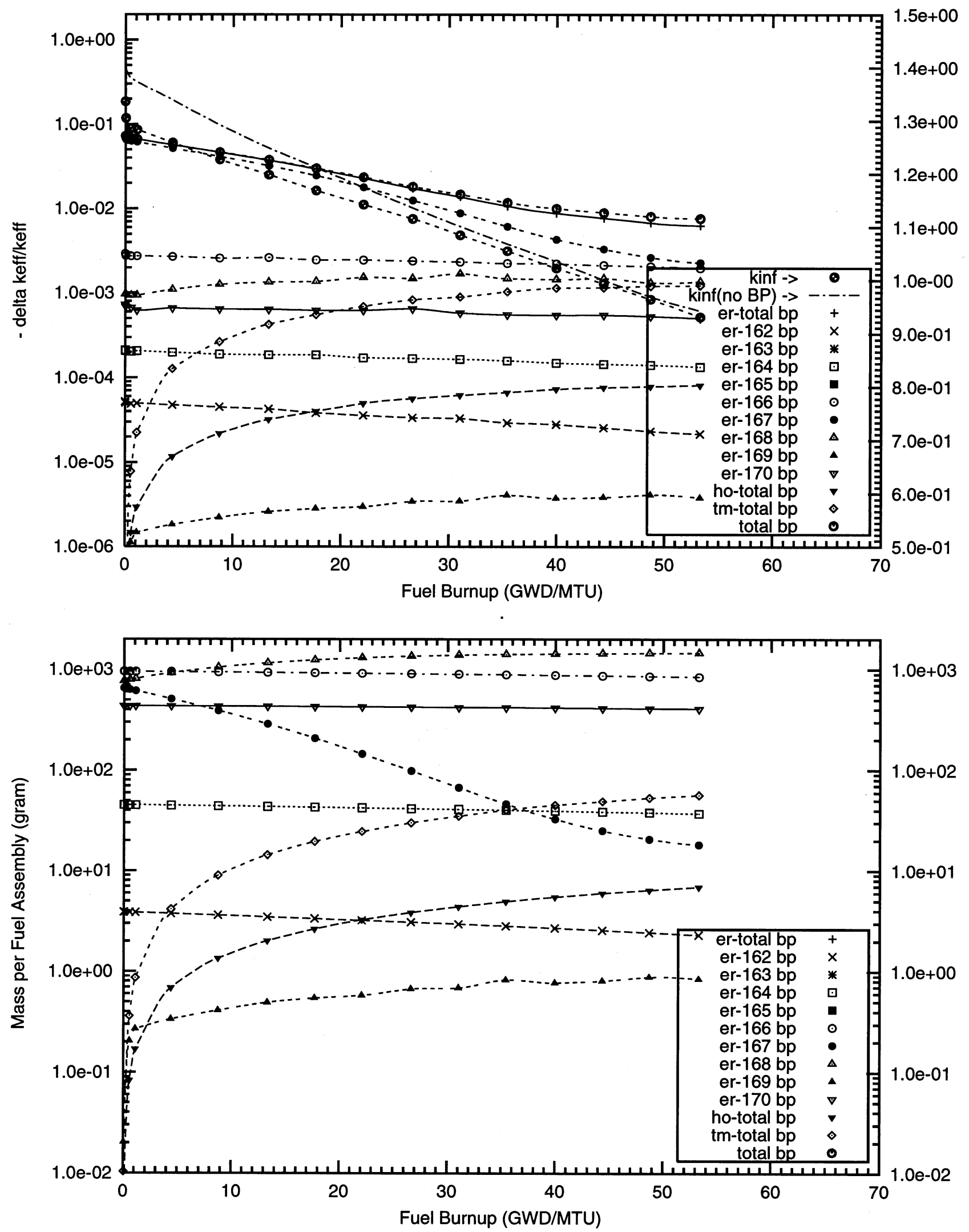
Figure C1.8. Negative Reactivity and Masses BP for Er-nat and transmutation daughters

as a function of fuel life for $17 \times 17$ fuel assemblies with 64 poison rods,

$1.0 \mathrm{w} \%$ Er2O3 poison homogeneously mixed in the UO2 pellets

Reactor power 3400 MWth, 193 fuel assemblies, initial enrichment 4.5w\%U-235

(Case : er000_1_64_bp_fuel o)
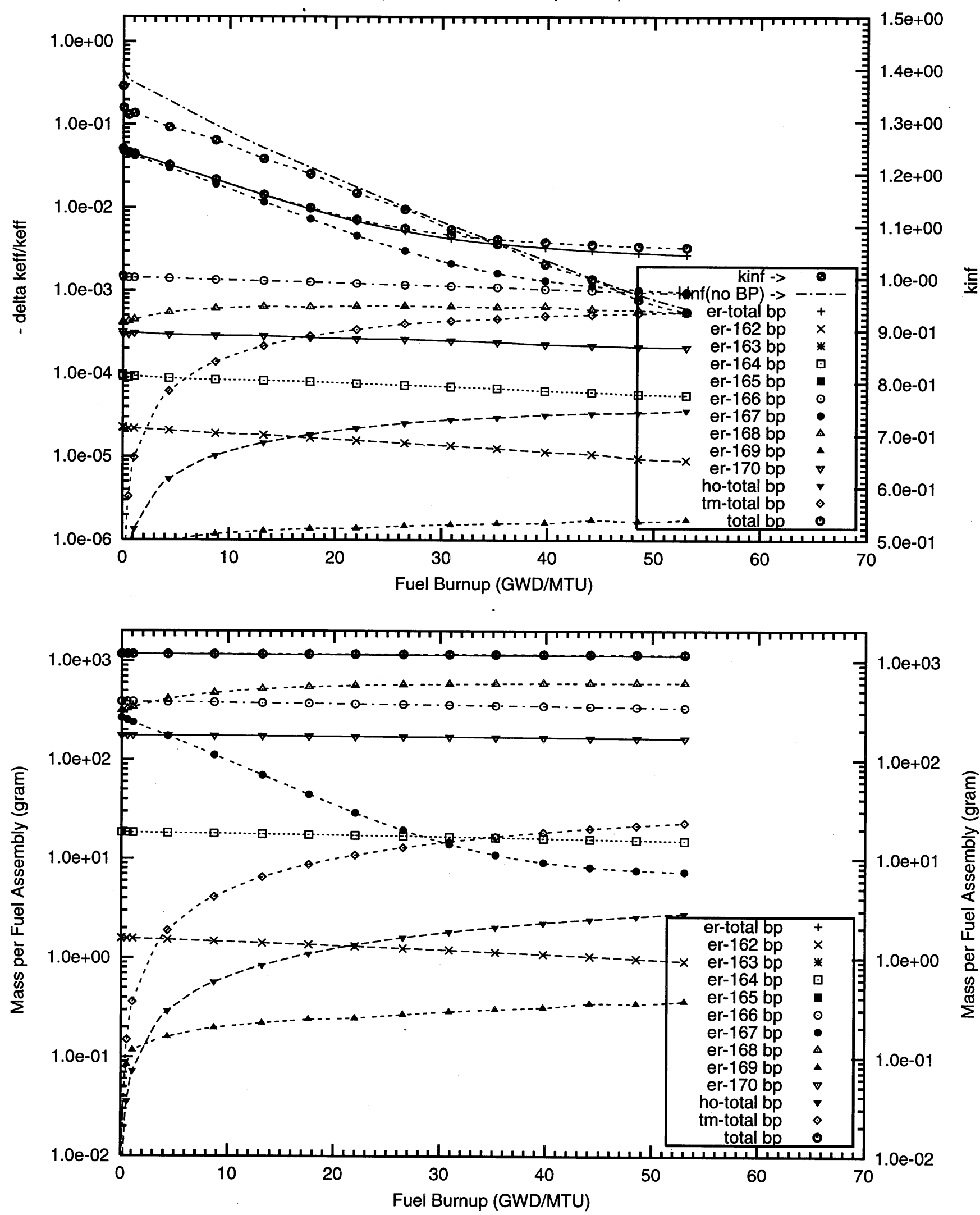
Figure C1.9. Negative Reactivity and Masses BP for Er-nat and transmutation daughters

as a function of fuel life for $17 \times 17$ fuel assemblies with 64 poison rods,

$2.0 \mathrm{w} \%$ Er2O3 poison homogeneously mixed in the UO2 pellets

Reactor power $3400 \mathrm{MWth}, 193$ fuel assemblies, initial enrichment 4.5w\%U-235

(Case : er000_2_64_bp_fuel o)
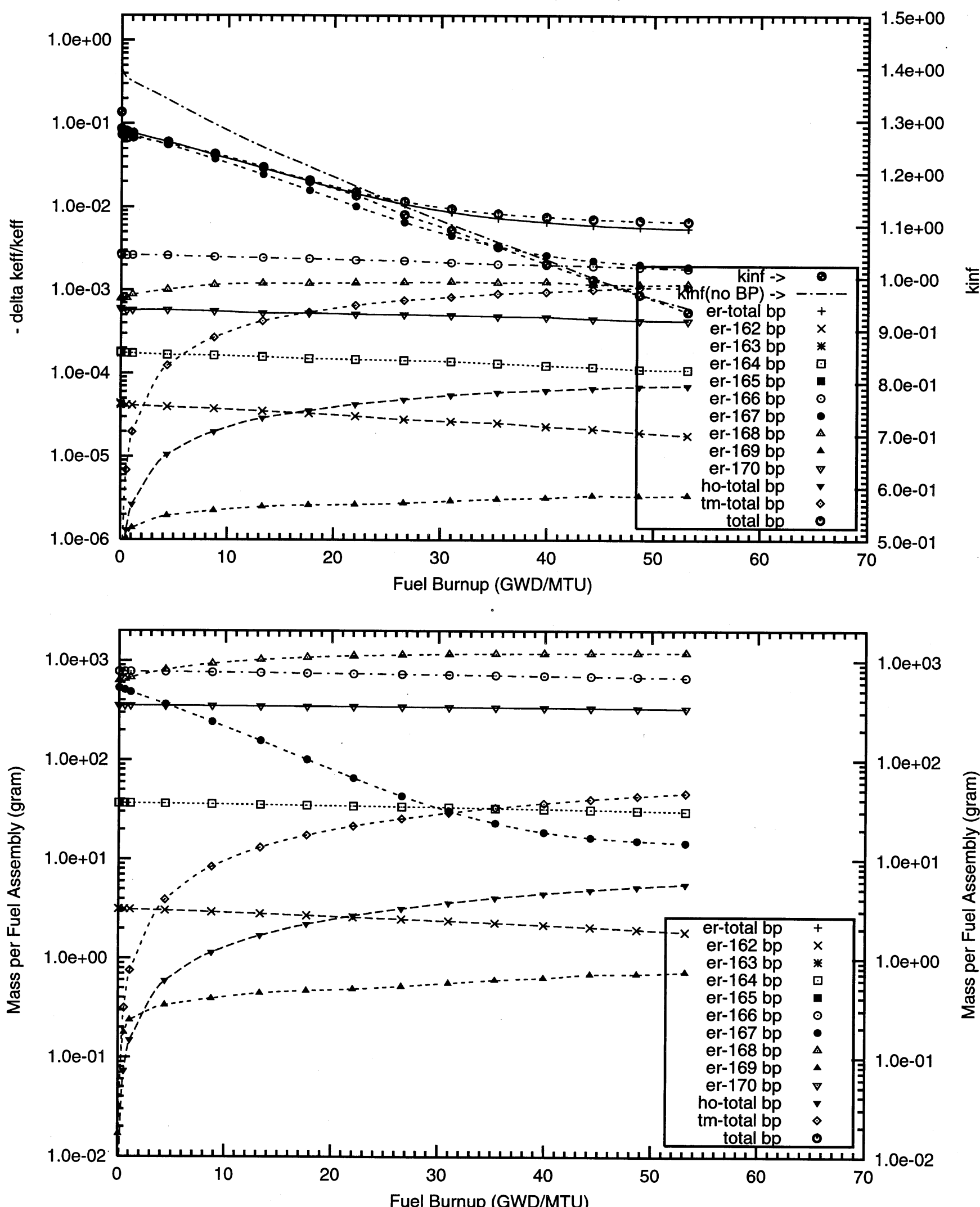
Figure C1.10. Negative Reactivity and Masses BP for Er-nat and transmutation daughters as a function of fuel life for $17 \times 17$ fuel assemblies with 64 poison rods,

4.0w\% Er2O3 poison homogeneously mixed in the UO2 pellets

Reactor power $3400 \mathrm{MWth}, 193$ fuel assemblies, initial enrichment 4.5w\%U-235

(Case : er000_3_100p_h20_64_bp_fuel_2_o)

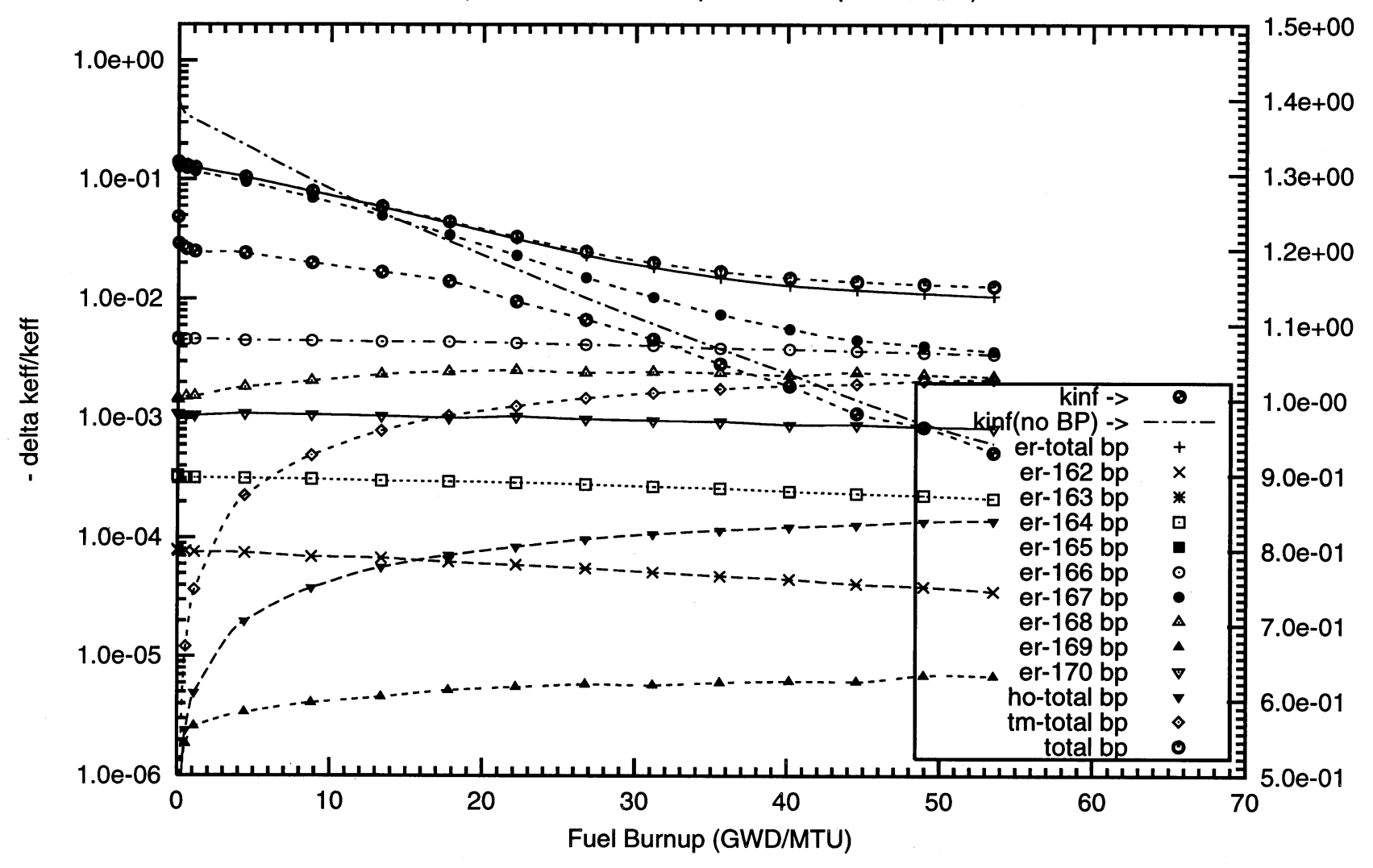

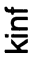

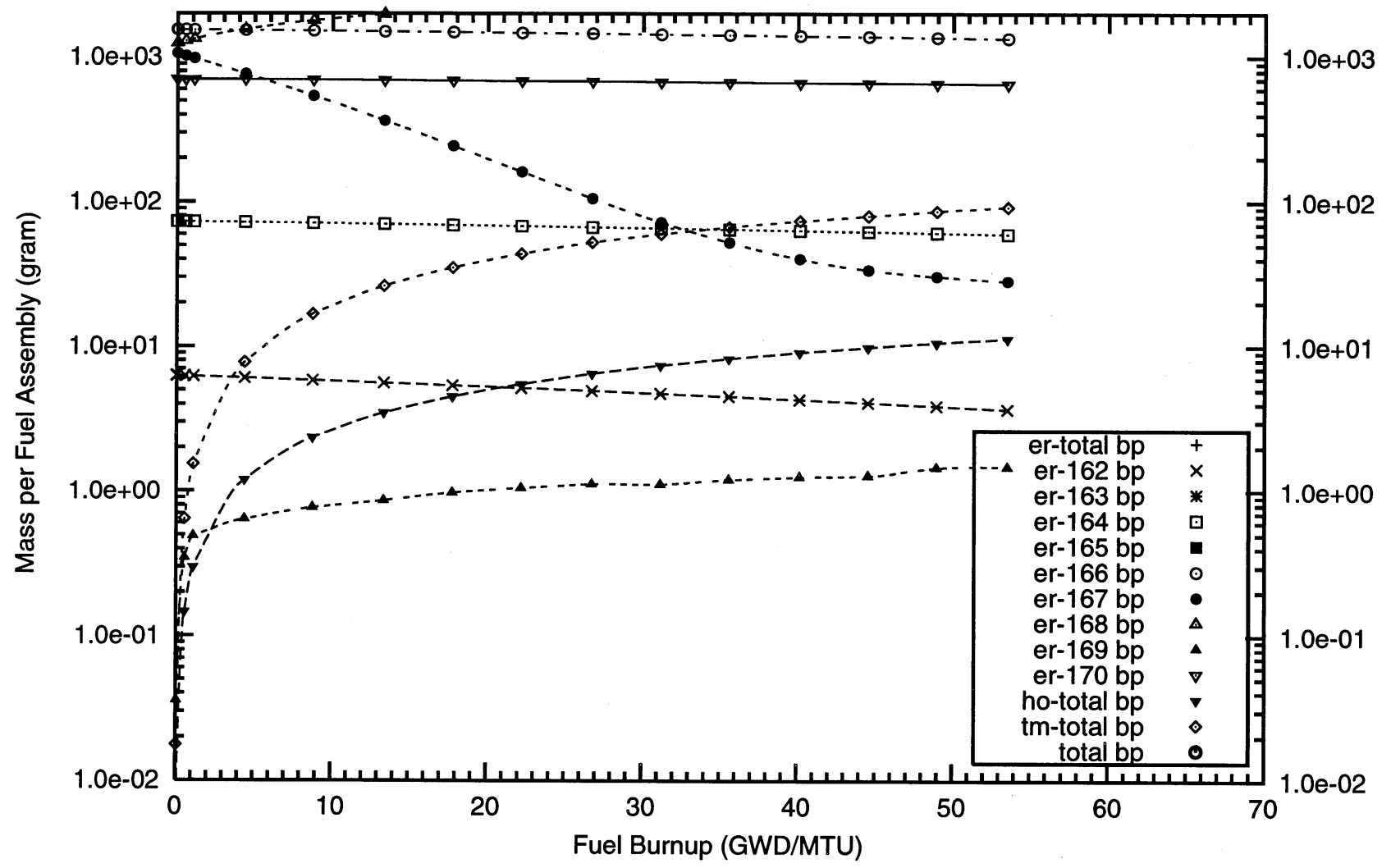


C1 - Erbium Fully Enriched in ${ }^{167} \mathrm{Er}$ 

Figure C1.11. Negative Reactivity and Masses BP for Er-167 and transmutation daughters as a function of fuel life for $17 \times 17$ fuel assemblies with 08 poison rods,

$4.0 \mathrm{w} \% \mathrm{Er} 2 \mathrm{O} 3$ poison homogeneously mixed in the UO2 pellets

Reactor power $3400 \mathrm{MWth}, 193$ fuel assemblies, initial enrichment $4.5 \mathrm{w} \% \mathrm{U}-235$ (Case : er167_3_08_bp_fuel o)

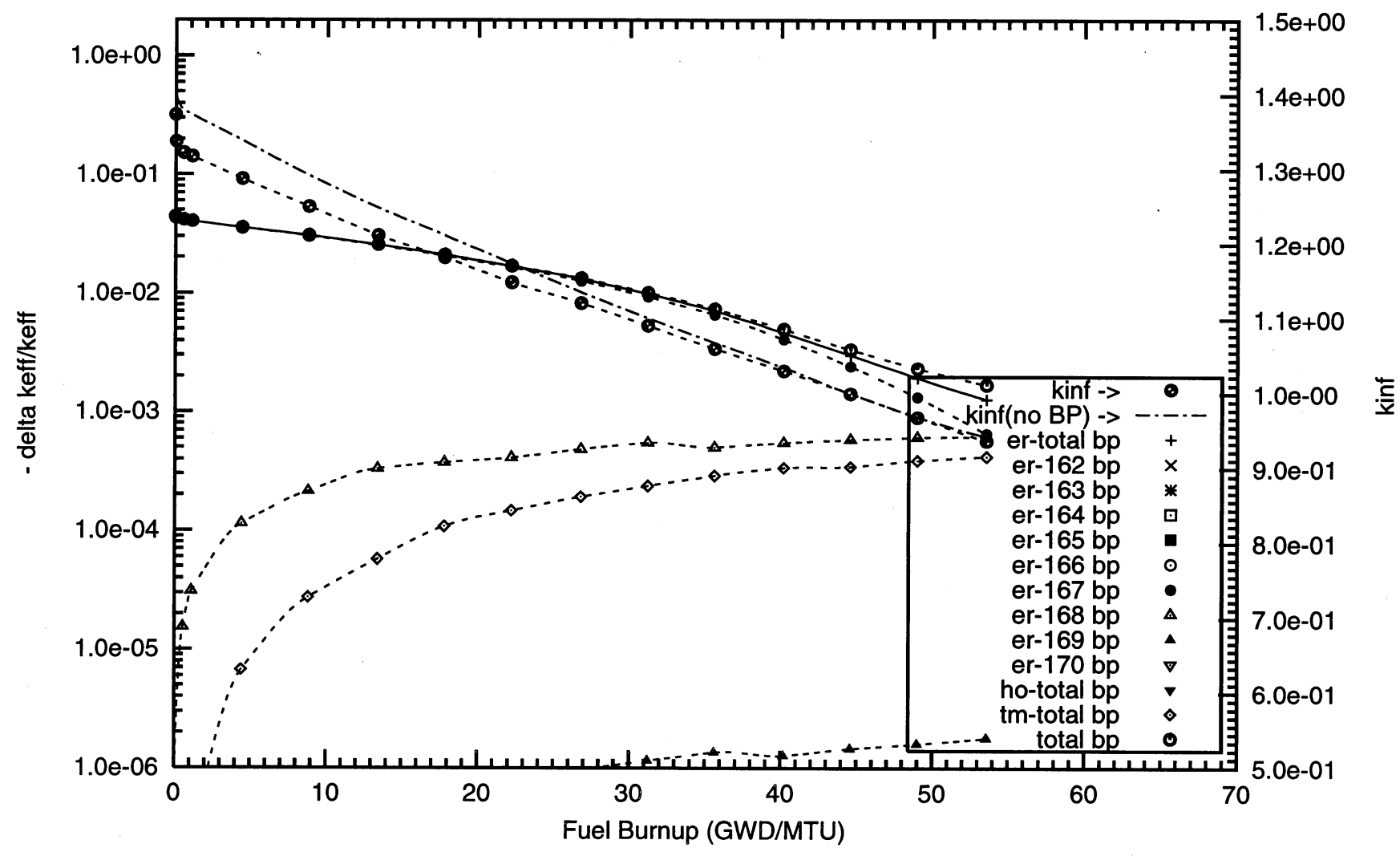


Figure C1.12. Negative Reactivity and Masses BP for Er-167 and transmutation daughters as a function of fuel life for $17 \times 17$ fuel assemblies with 16 poison rods,

$1.0 w 1 \%$ Er2O3 poison homogeneously mixed in the UO2 pellets

Reactor power $3400 \mathrm{MWth}, 193$ fuel assemblies, initial enrichment 4.5wt\%U-235

(Case : er167_1_100p_h2o_16_bp_fuel_2_o)
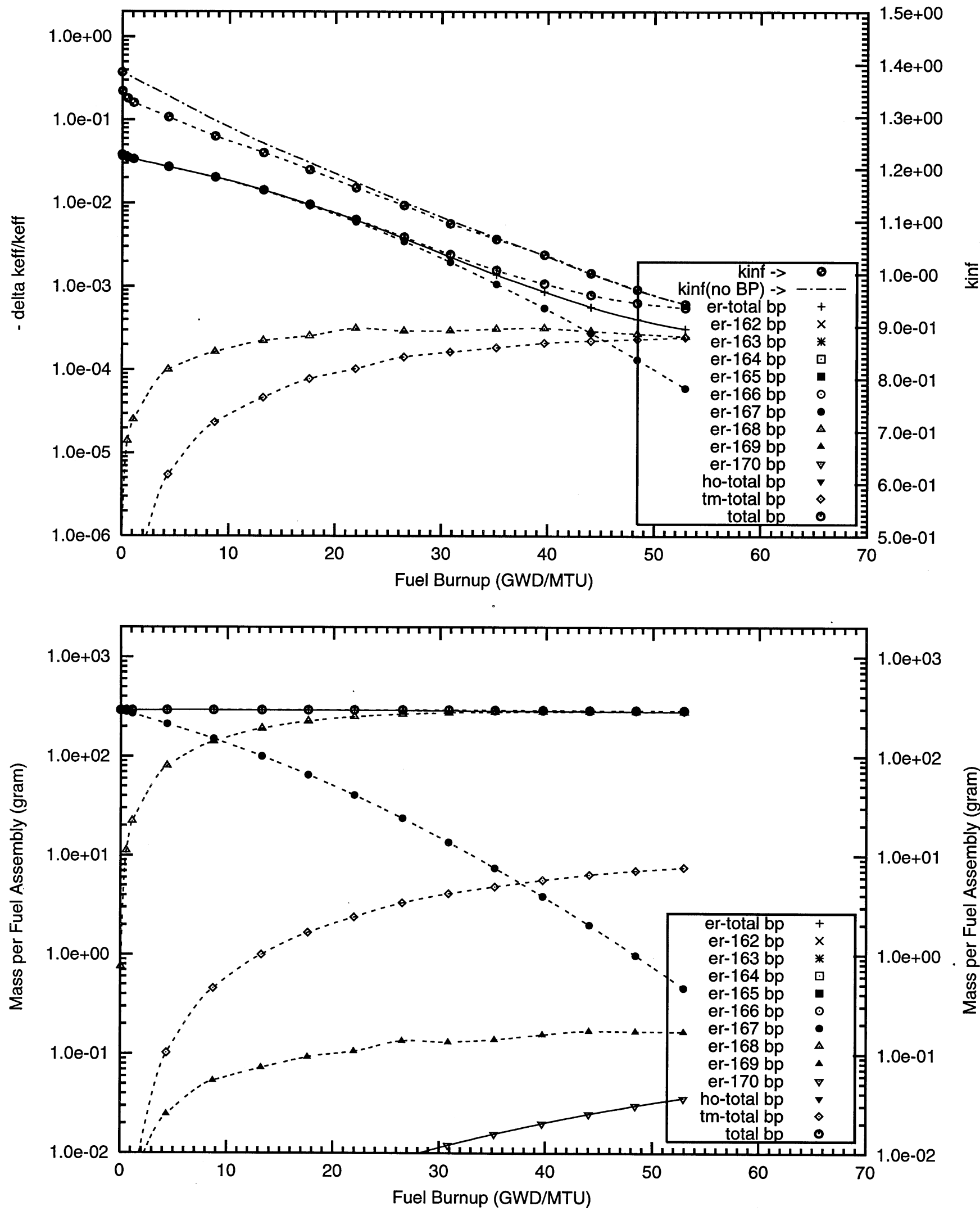
Figure C1.13. Negative Reactivity and Masses BP for Er-167 and transmutation daughters as a function of fuel life for $17 \times 17$ fuel assemblies with 16 poison rods,

$2.0 w 1 \%$ Er2O3 poison homogeneously mixed in the UO2 pellets

Reactor power $3400 \mathrm{MWth}, 193$ fuel assemblies, initial enrichment 4.5wt\%U-235

(Case : er167_2_100p_h2o_16_bp_fuel_2_o)
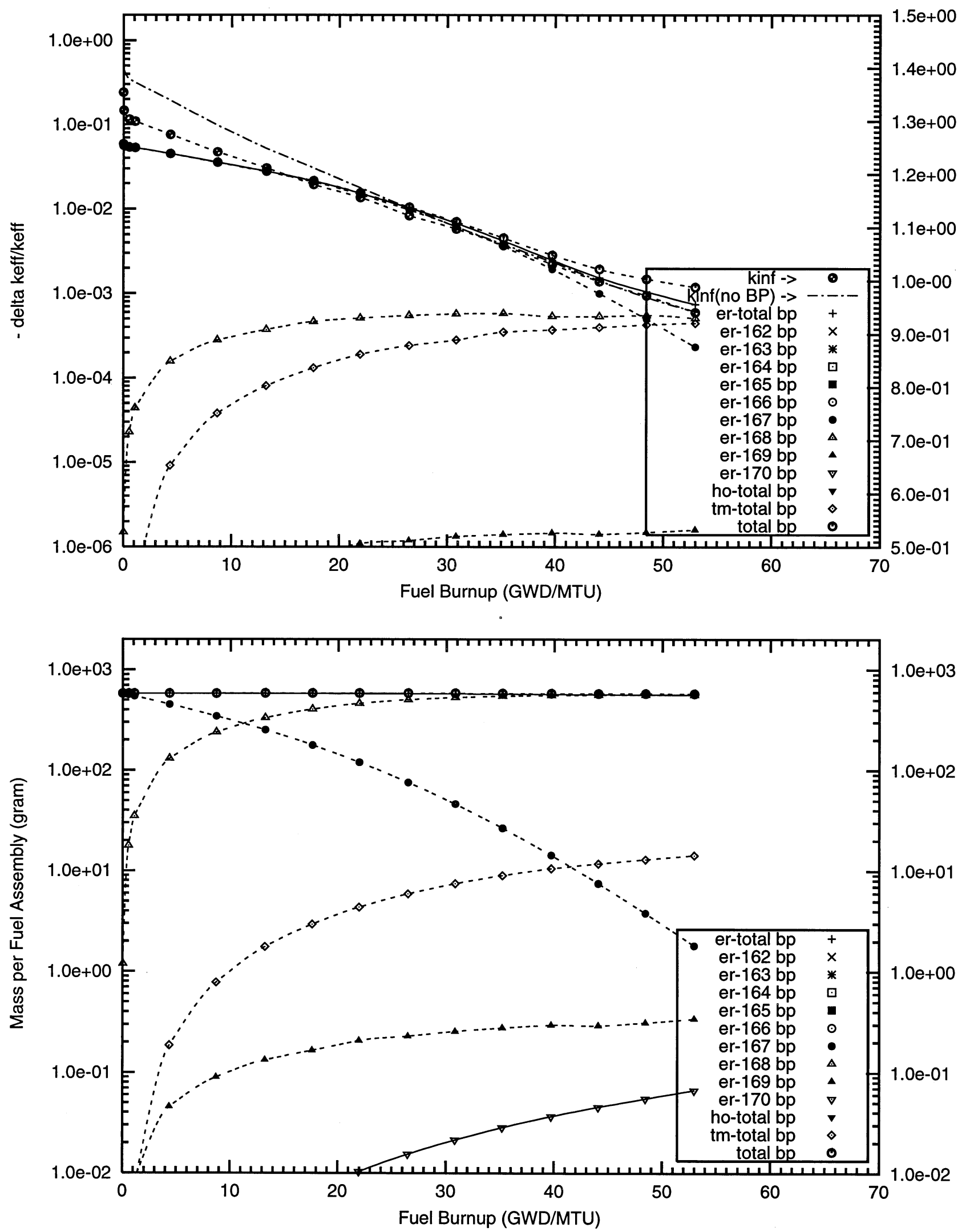
Figure C1.14. Negative Reactivity and Masses BP for Er-167 and transmutation daughters as a function of fuel life for $17 \times 17$ fuel assemblies with 16 poison rods, $4.0 \mathrm{w} \% \mathrm{Er} 2 \mathrm{O} 3$ poison homogeneously mixed in the UO2 pellets

Reactor power $3400 \mathrm{MWth}, 193$ fuel assemblies, initial enrichment 4.5w\%U-235

(Case : er167_3_100p_h20_16_bp_fuel_2_o)
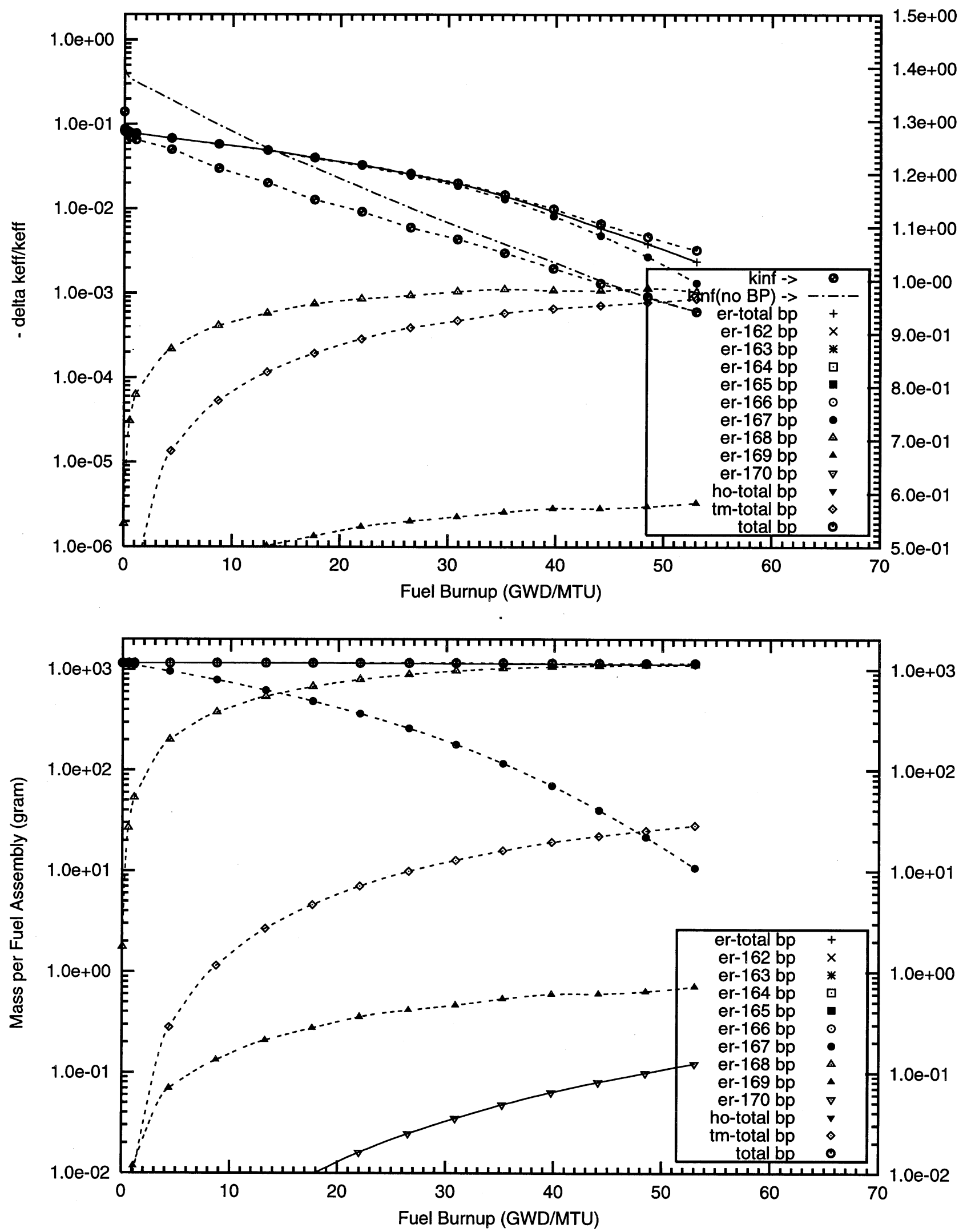
Figure C1.15. Negative Reactivity and Masses BP for Er-167 and transmutation daughters as a function of fuel life for $17 \times 17$ fuel assemblies with 16 poison rods,

$8.0 \mathrm{w} \%$ Er2O3 poison homogeneously mixed in the UO2 pellets

Reactor power $3400 \mathrm{MWth}, 193$ fuel assemblies, initial enrichment 4.5w\%U-235 (Case : er167_4_100p_h2o_16_bp_fuel_2_o)

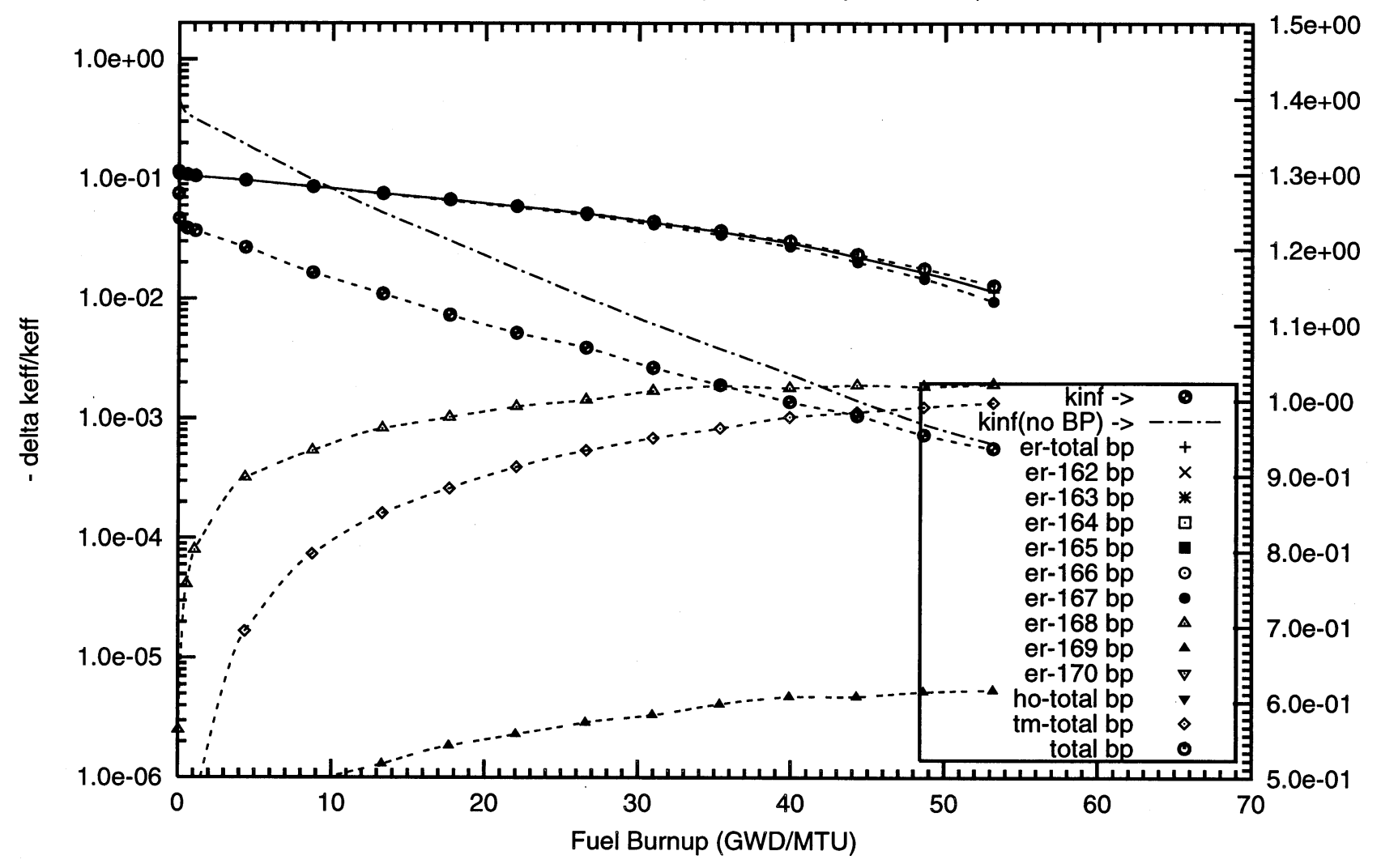

\section{言}

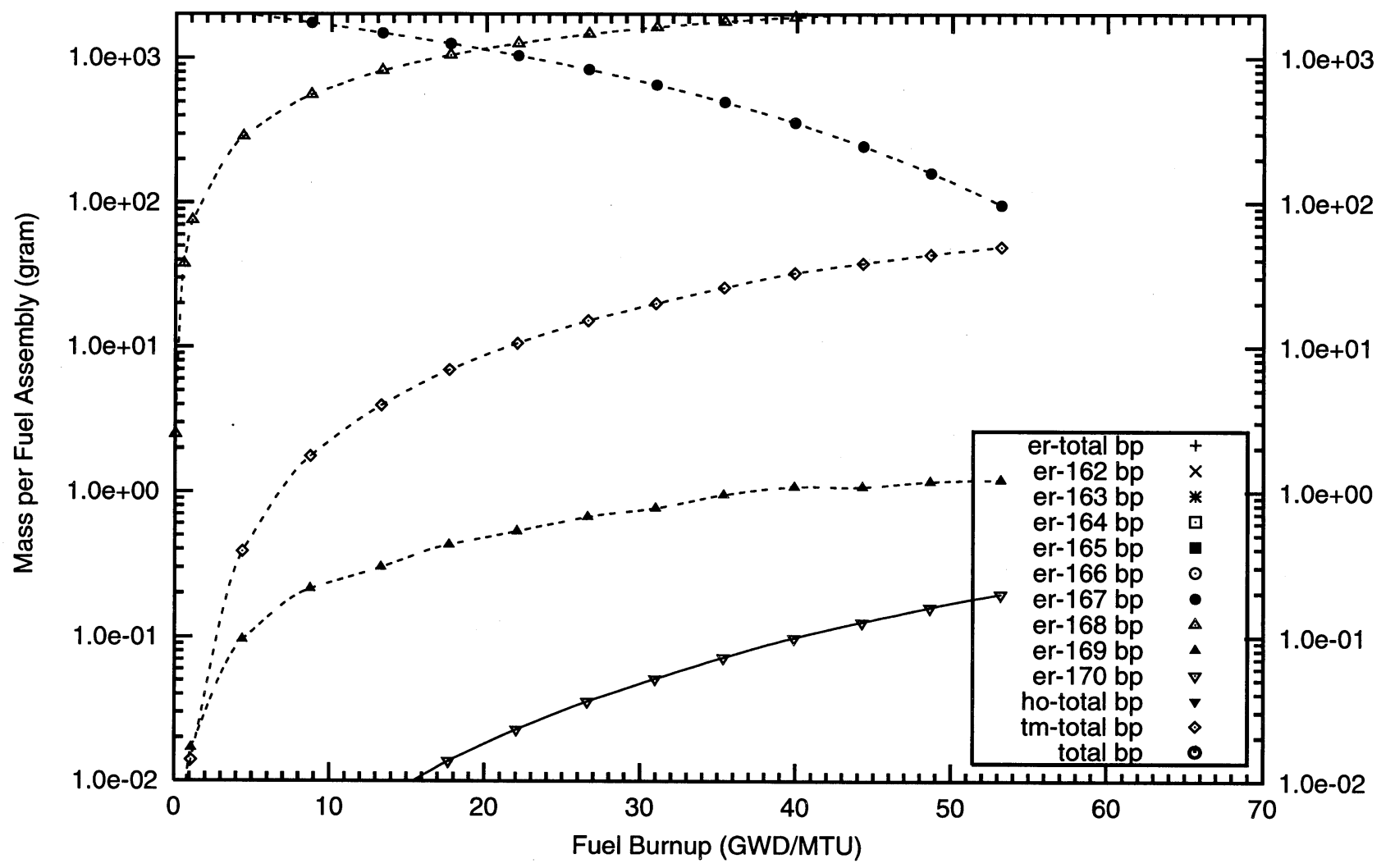


Figure C1.16. Negative Reactivity and Masses BP for Er-167 and transmutation daughters as a function of fuel life for $17 \times 17$ fuel assemblies with 20 poison rods,

$4.0 \mathrm{w} \%$ Er2O3 poison homogeneously mixed in the UO2 pellets

Reactor power $3400 \mathrm{MWth}, 193$ fuel assemblies, initial enrichment 4.5w\%U-235

(Case : er167_3_16_bp_fuel (old) o)

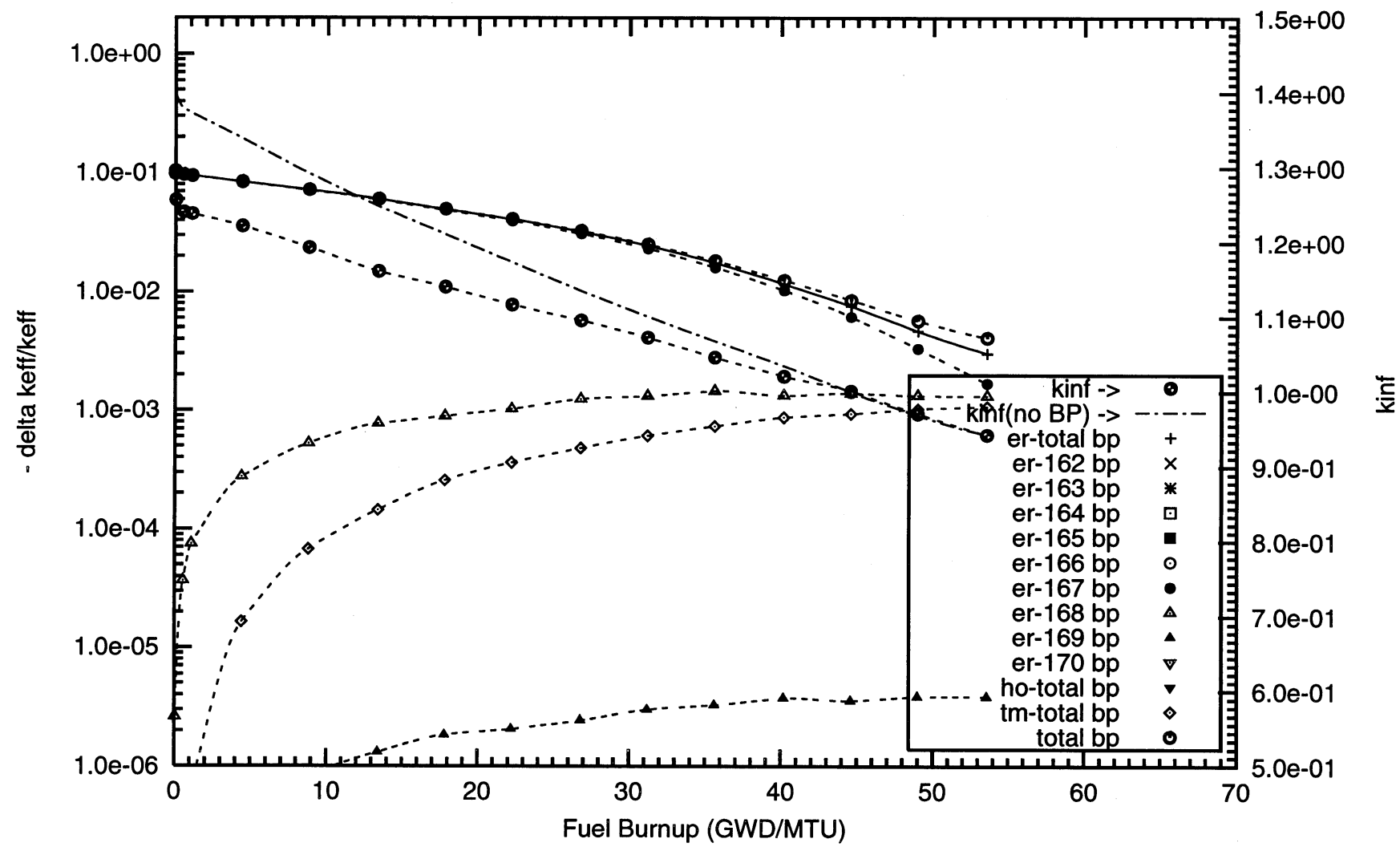


Figure C1.17. Negative Reactivity and Masses BP for Er-167 and transmutation daughters as a function of fuel life for $17 \times 17$ fuel assemblies with 64 poison rods,

$0.2 \mathrm{w} \%$ Er2O3 poison homogeneously mixed in the UO2 pellets

Reactor power $3400 \mathrm{MWth}, 193$ fuel assemblies, initial enrichment 4.5w\%U-235

(Case : er167_8_100p_h2o_64_bp_fuel_2_o)
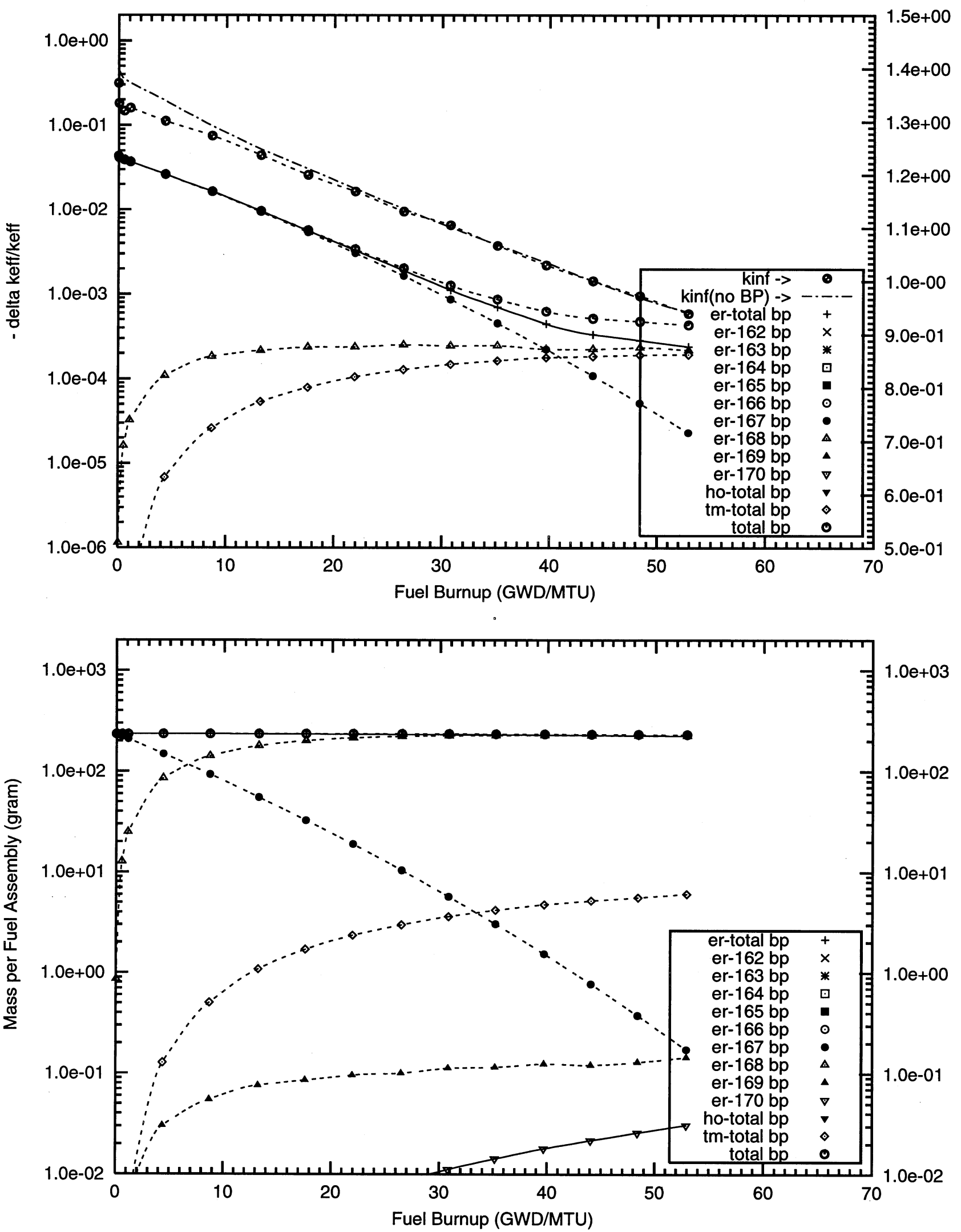
Figure C1.18. Negative Reactivity and Masses BP for Er-167 and transmutation daughters as a function of fuel life for $17 \times 17$ fuel assemblies with 64 poison rods,

$0.5 \mathrm{w} \%$ Er2O3 poison homogeneously mixed in the UO2 pellets

Reactor power $3400 \mathrm{MWth}, 193$ fuel assemblies, initial enrichment 4.5w\%U-235

(Case : er167_0_100p_h20_64_bp_fuel_2_o)
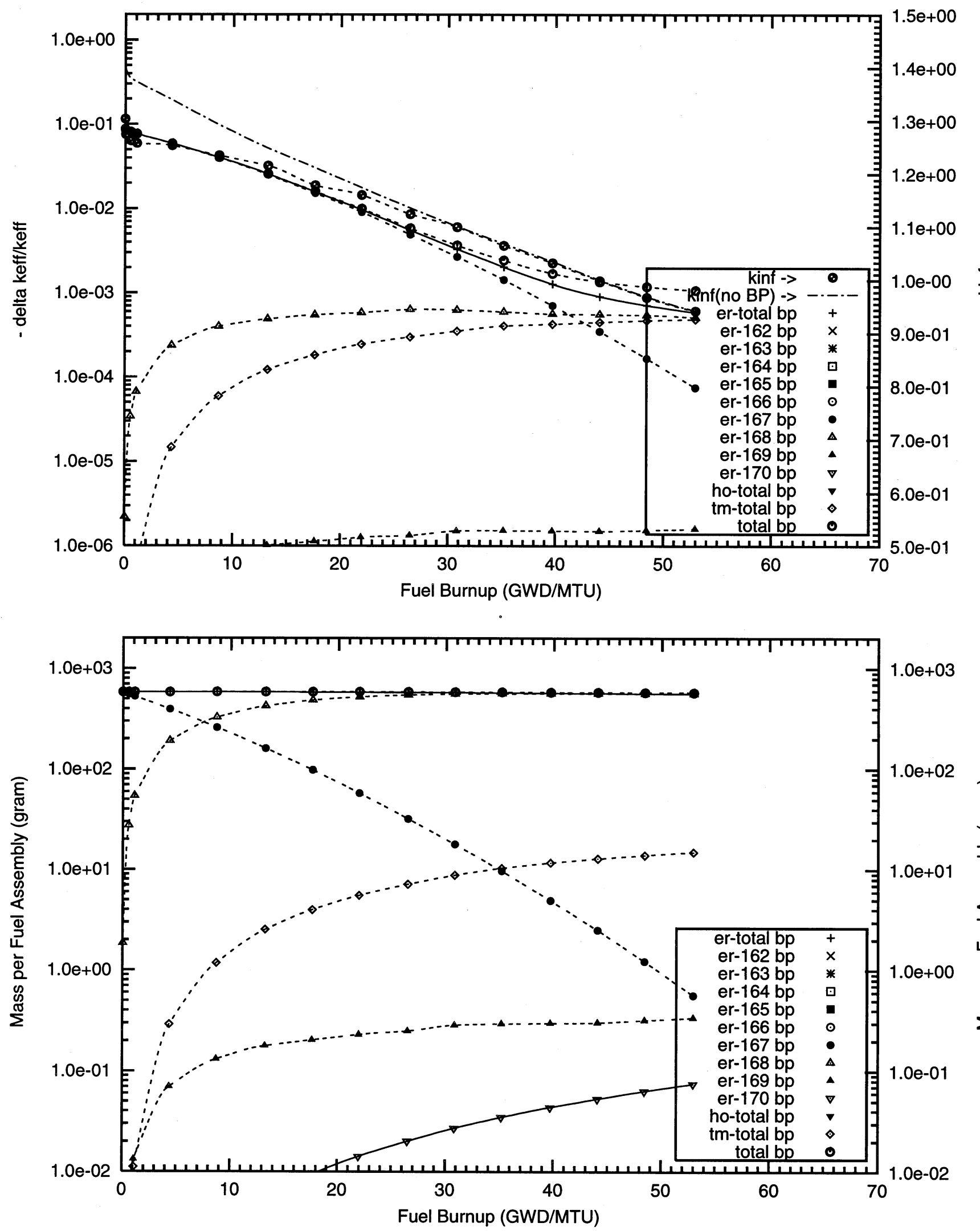
Figure C1.19. Negative Reactivity and Masses BP for Er-167 and transmutation daughters as a function of fuel life for $17 \times 17$ fuel assemblies with 64 poison rods,

$1.0 \mathrm{w} \% \mathrm{Er} 2 \mathrm{O} 3$ poison homogeneously mixed in the UO2 pellets

Reactor power $3400 \mathrm{MWth}, 193$ fuel assemblies, initial enrichment 4.5w\%U-235

(Case : er167_1_64_bp_fuel o)
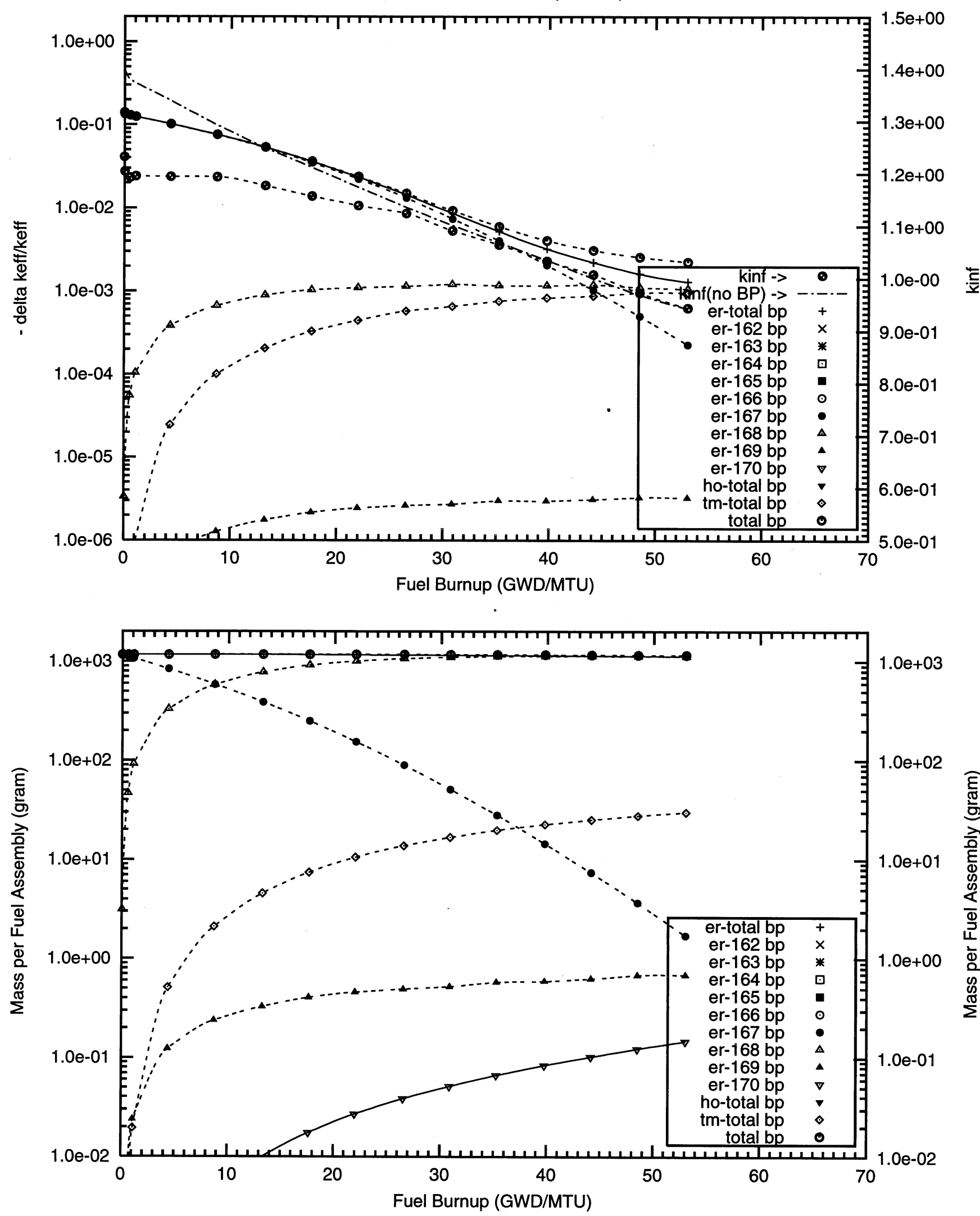


\section{Appendix C2}

Reactivity Worths and Masses of $\mathrm{Er}_{2} \mathrm{O}_{3}$ Burnable Poison Homogeneously Mixed in the Outer One-Third Volume of the Fuel Pellets

\section{Table 14 Cases}


C2 - Erbium with Natural Isotopic Abundances 

Figure C2.1. Negative Reactivity and Masses BP for Er-nat and transmutation daughters as a function of fuel life for $17 \times 17$ fuel assemblies with 08 poison rods,

4.0w\% Er2O3 poison homogeneously mixed in the outer one-third volume of the UO2 pellets Reactor power $3400 \mathrm{MWth}, 193$ fuel assemblies, initial enrichment 4.5w\%U-235

(Case : er000_3b_08_bp_fuel o)
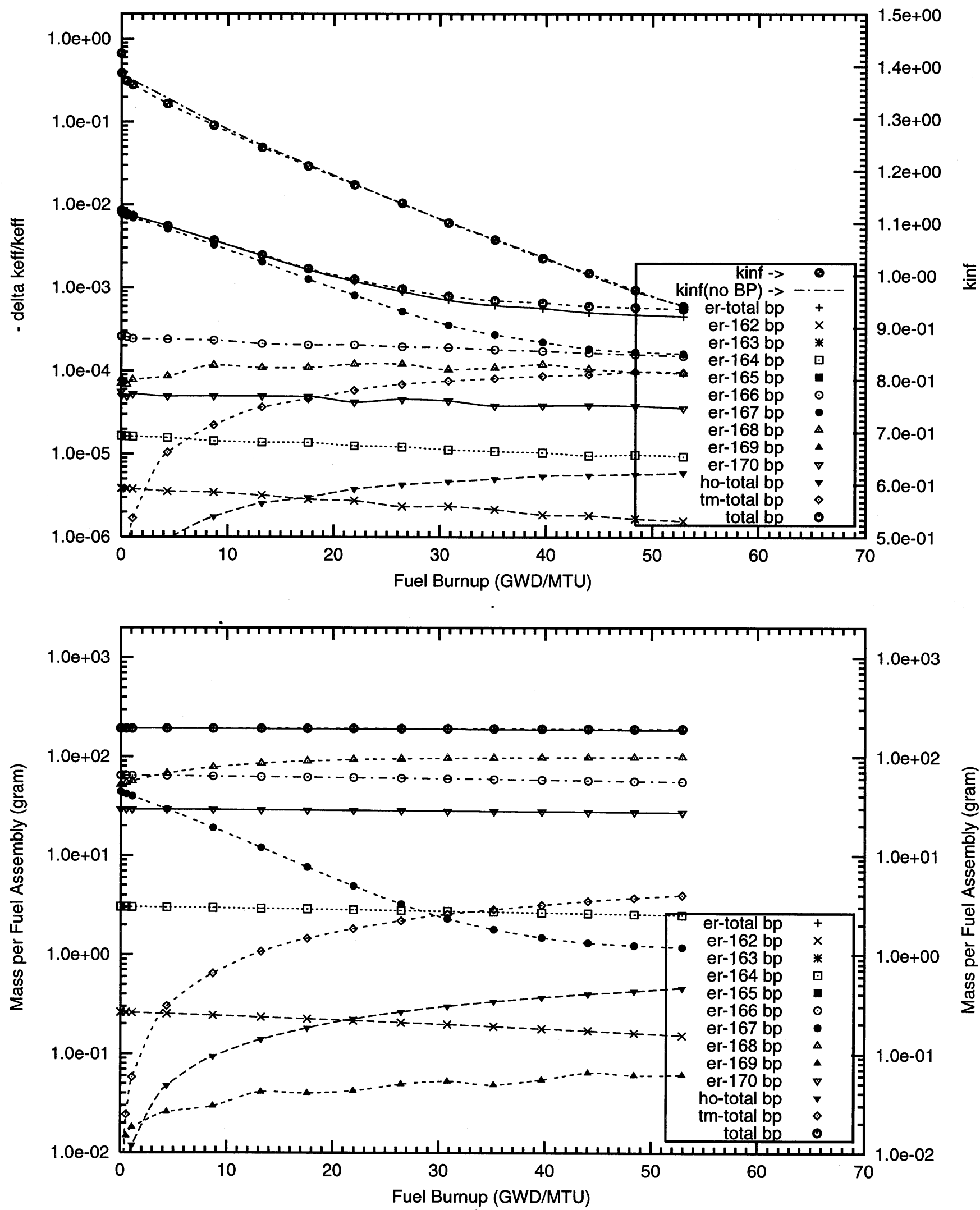
Figure C2.2. Negative Reactivity and Masses BP for Er-nat and transmutation daughters as a function of fuel life for $17 \times 17$ fuel assemblies with 08 poison rods,

$8.0 \mathrm{w} \%$ Er2O3 poison homogeneously mixed in the outer one-third volume of the UO2 pellets

Reactor power $3400 \mathrm{MWth}, 193$ fuel assemblies, initial enrichment 4.5w\%U-235

(Case : er000_4b_100p_h2o_08_bp_fuel_2_o)
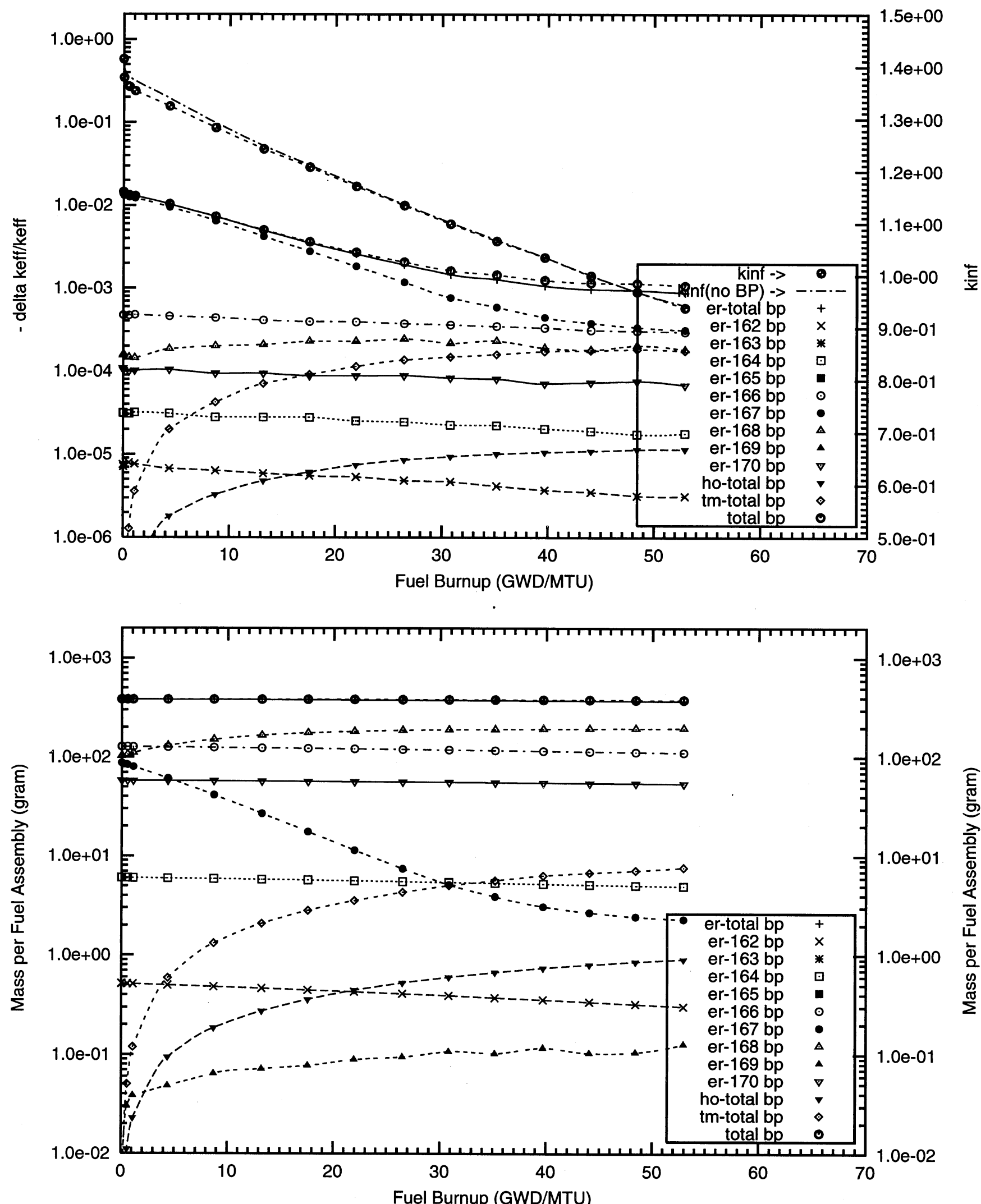
Figure C2.3. Negative Reactivity and Masses BP for Er-nat and transmutation daughters as a function of fuel life for $17 \times 17$ fuel assemblies with 08 poison rods,

$16.0 \mathrm{w} \%$ Er2O3 poison homogeneously mixed in the outer one-third volume of the UO2 pellets Reactor power $3400 \mathrm{MW}$ th, 193 fuel assemblies, initial enrichment $4.5 \mathrm{w} \% \mathrm{U}-235$

(Case : er000_6b_100p_h20_08_bp_fuel_2_o)
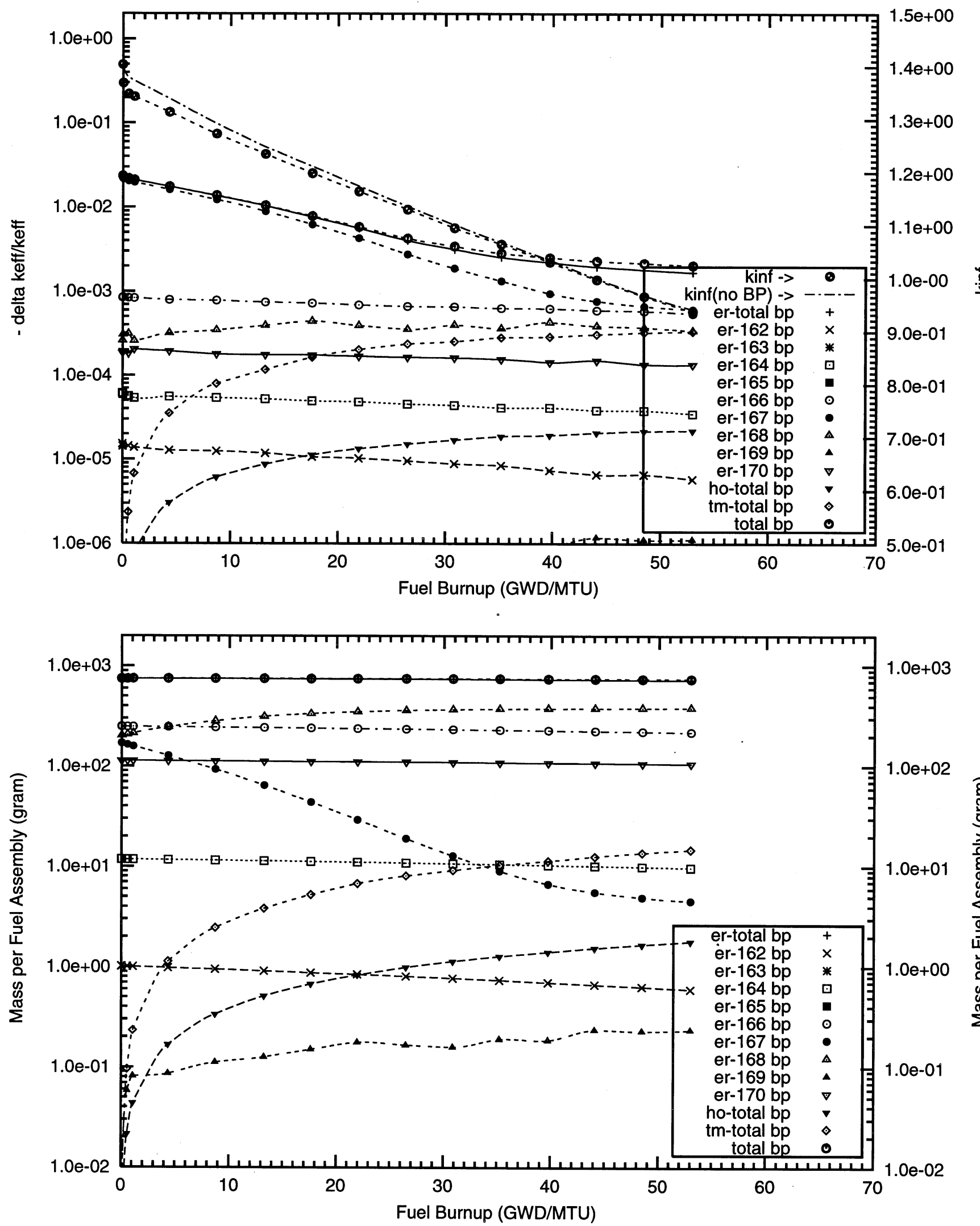
Figure C2.4. Negative Reactivity and Masses BP for Er-nat and transmutation daughters as a function of fuel life for $17 \times 17$ fuel assemblies with 16 poison rods,

$1.0 \mathrm{w} \%$ Er2O3 poison homogeneously mixed in the outer one-third volume of the UO2 pellets

Reactor power $3400 \mathrm{MWth}, 193$ fuel assemblies, initial enrichment 4.5W\%U-235

(Case : er000_1b_100p_h20_16_bp_fuel_2_o)
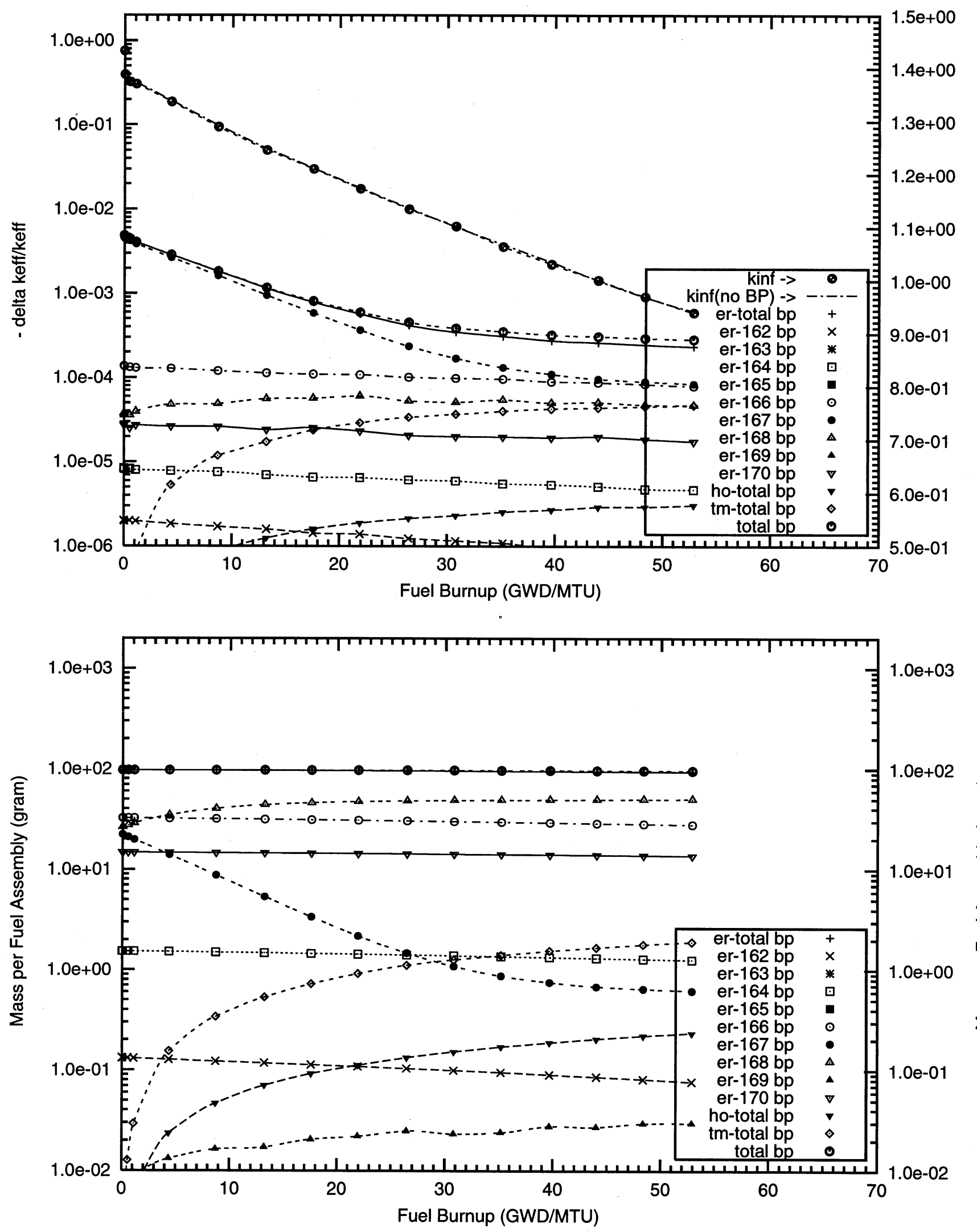
Figure C2.5. Negative Reactivity and Masses BP for Er-nat and transmutation daughters as a function of fuel life for $17 \times 17$ fuel assemblies with 16 poison rods,

$2.0 \mathrm{w} \%$ Er2O3 poison homogeneously mixed in the outer one-third volume of the UO2 pellets

Reactor power $3400 \mathrm{MWth}, 193$ fuel assemblies, initial enrichment $4.5 \mathrm{w} \% \mathrm{U}-235$

(Case : er000_2b_100p_h2o_16_bp_fuel_2_o)
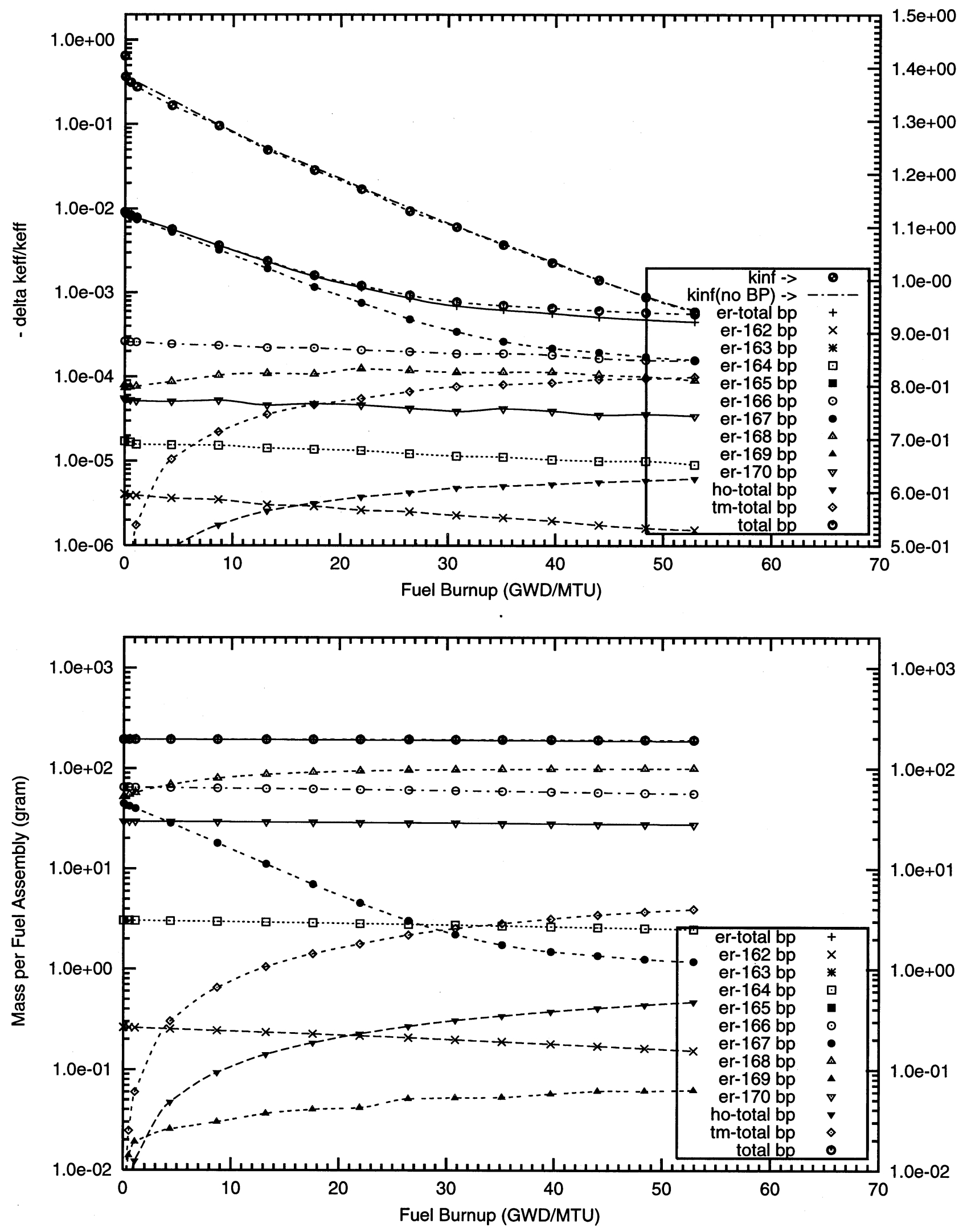
Figure C2.6. Negative Reactivity and Masses BP for Er-nat and transmutation daughters

as a function of fuel life for $17 \times 17$ fuel assemblies with 16 poison rods,

$4.0 \mathrm{w} \%$ Er2O3 poison homogeneously mixed in the outer one-third volume of the UO2 pellets

Reactor power $3400 \mathrm{MWth}, 193$ fuel assemblies, initial enrichment 4.5w\%U-235

(Case : er000_3b_100p_h20_16_bp_fuel_2_0)

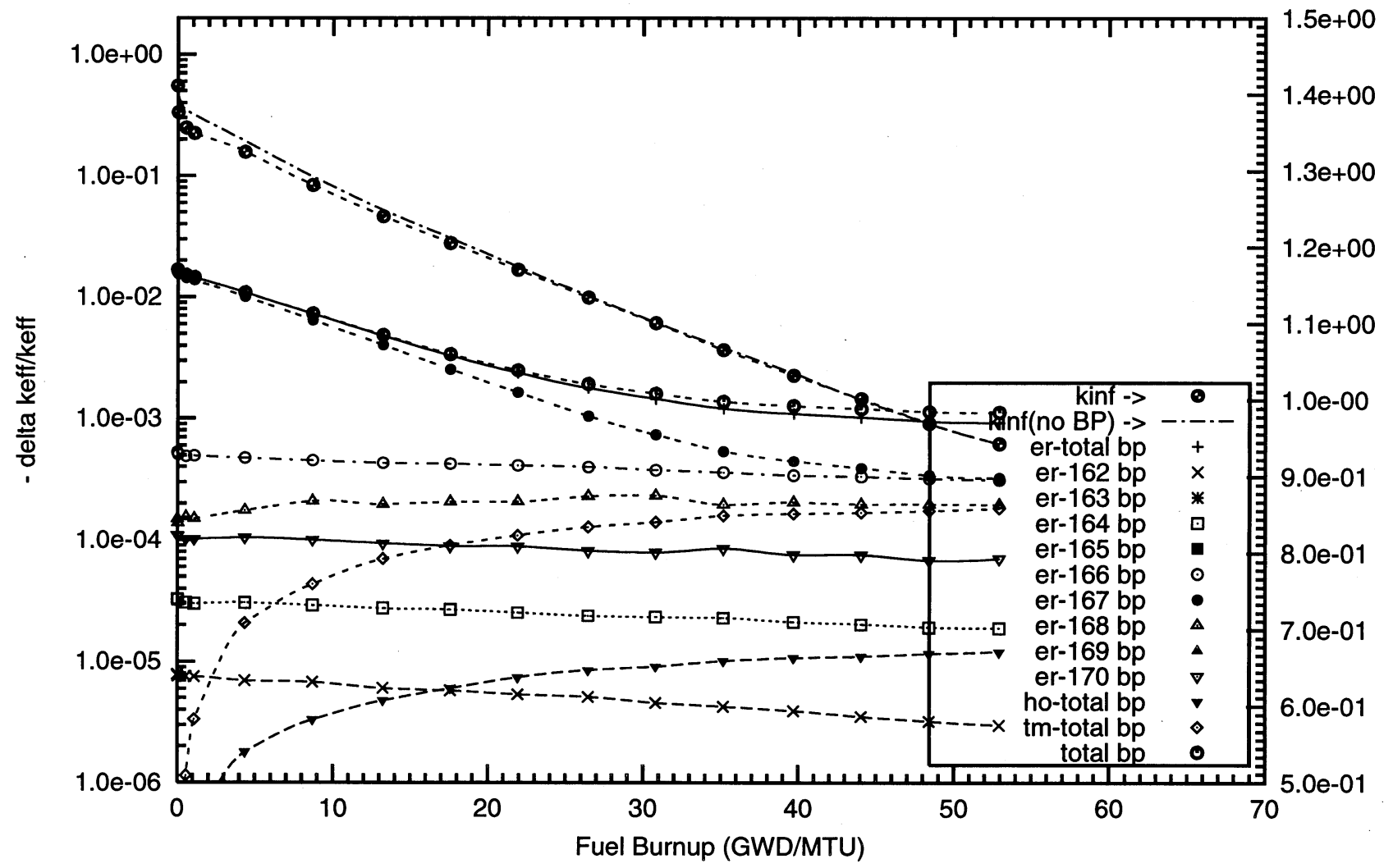

产

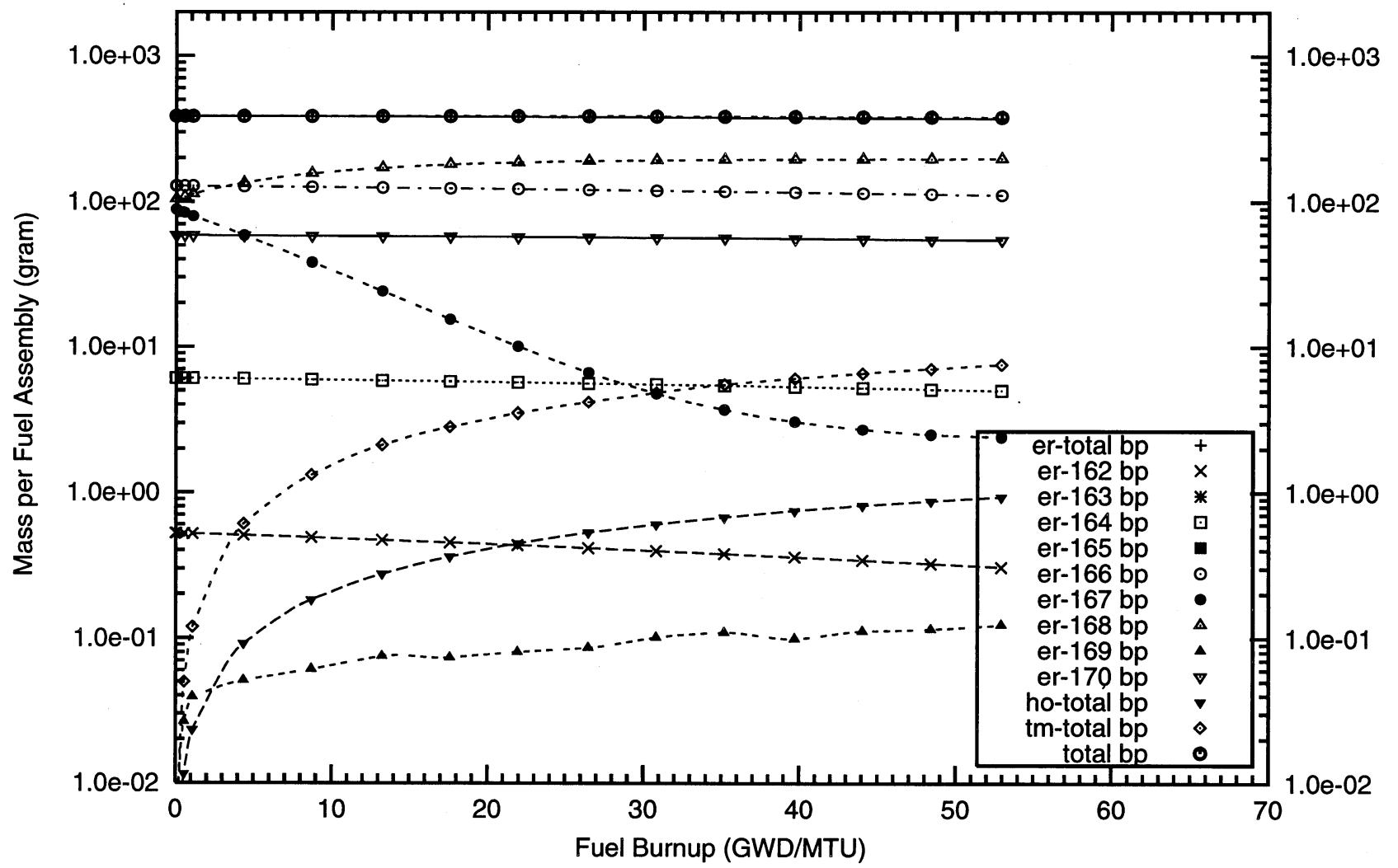


Figure C2.7. Negative Reactivity and Masses BP for Er-nat and transmutation daughters as a function of fuel life for $17 \times 17$ fuel assemblies with 16 poison rods,

$8.0 \mathrm{w} \%$ Er2O3 poison homogeneously mixed in the outer one-third volume of the UO2 pellets

Reactor power $3400 \mathrm{MWth}, 193$ fuel assemblies, initial enrichment 4.5w\%U-235

(Case : er000_4b_100p_h20_16_bp_fuel_2_o)
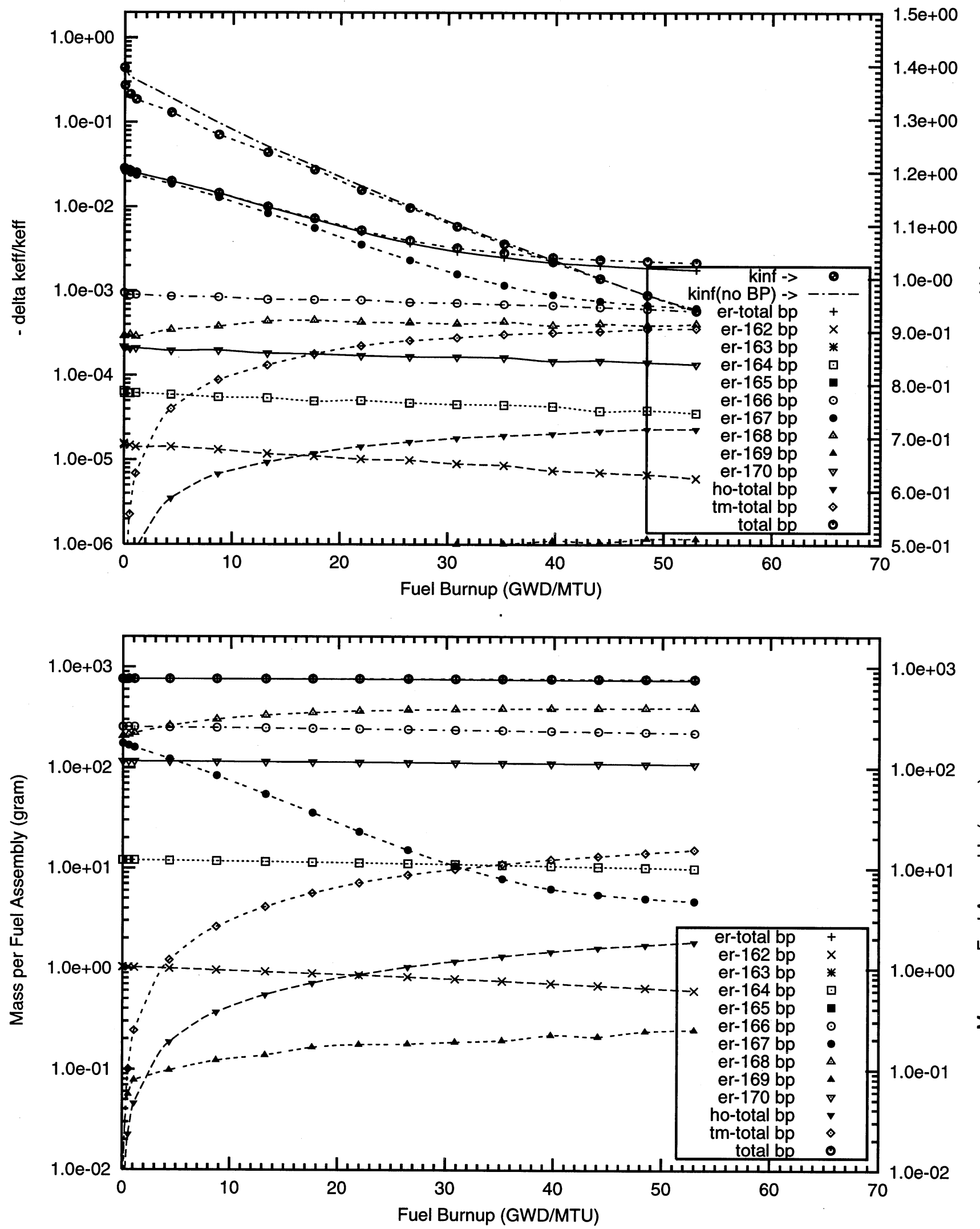
Figure C2.8. Negative Reactivity and Masses BP for Er-nat and transmutation daughters as a function of fuel life for $17 \times 17$ fuel assemblies with 18 poison rods,

$1.0 \mathrm{wt} \%$ Er2O3 poison homogeneously mixed in the outer one-third volume of the UO2 pellets

Reactor power $3400 \mathrm{MW}$ th, 193 fuel assemblies, initial enrichment 4.5wt\%U-235

(Case : er000_1b_16_bp_fuel o)
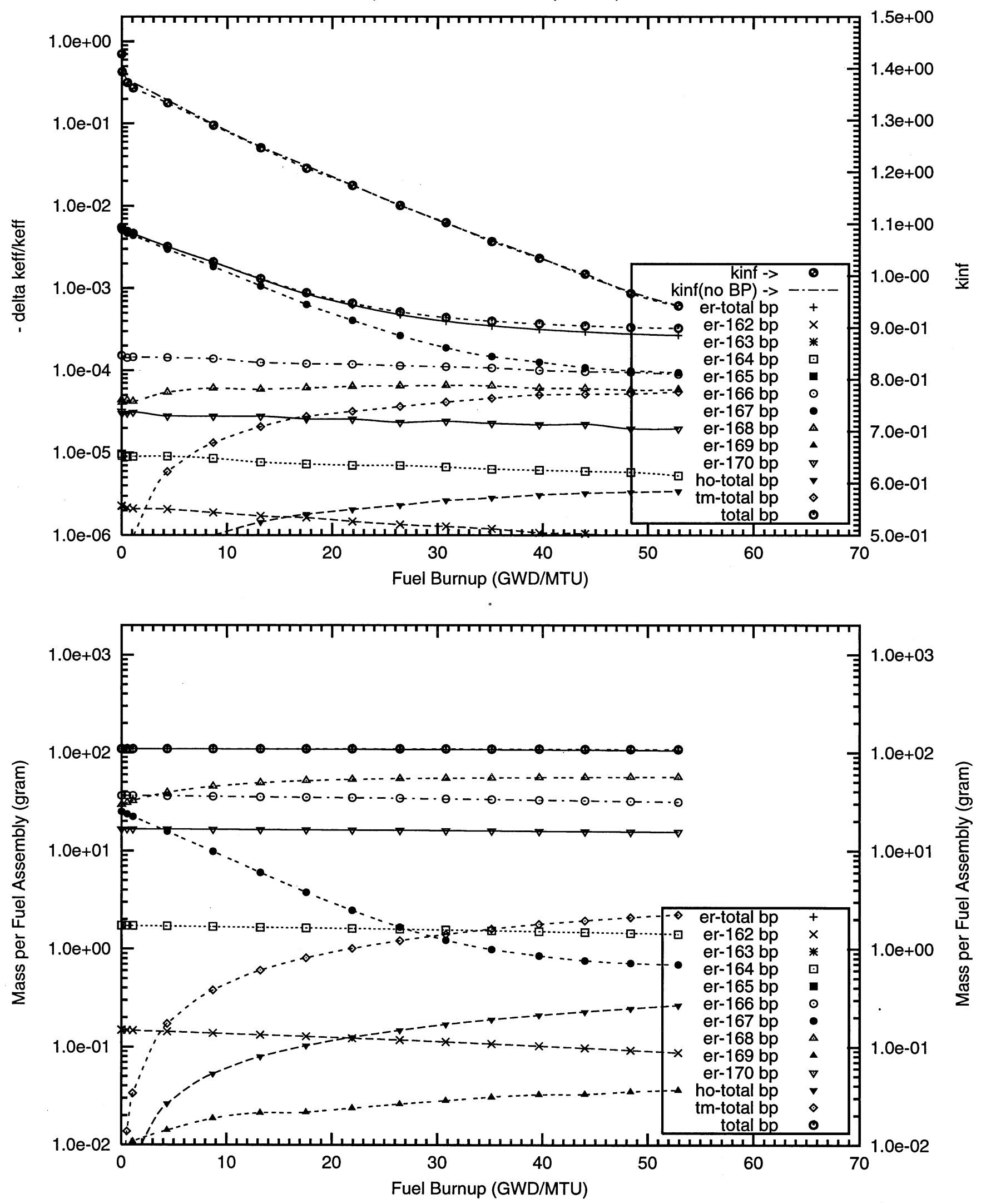
Figure C2.9. Negative Reactivity and Masses BP for Er-nat and transmutation daughters as a function of fuel life for $17 \times 17$ fuel assemblies with 18 poison rods-

4.0wt\% Er2O3 poison homogeneously mixed in the outer one-third volume of the UO2 pellets

Reactor power $3400 \mathrm{MW}$ th, 193 fuel assemblies, initial enrichment 4.5wt\%U-235

(Case : er000_3b_16_bp_fuel o)
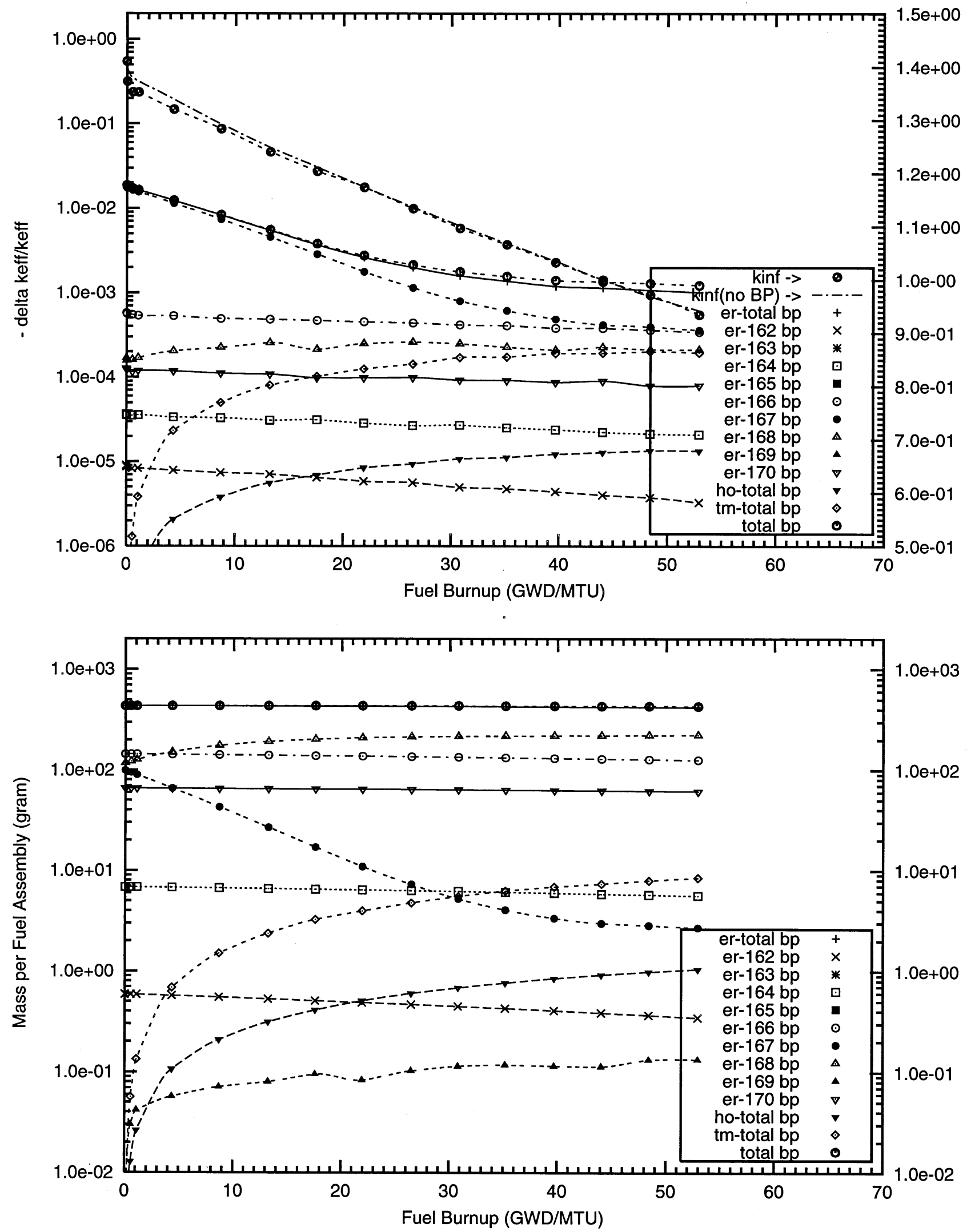
Figure C2.10. Negative Reactivity and Masses BP for Er-nat and transmutation daughters as a function of fuel life for $17 \times 17$ fuel assemblies with 64 poison rods,

$0.5 \mathrm{w} \%$ Er2O3 poison homogeneously mixed in the outer one-third volume of the UO2 pellets

Reactor power $3400 \mathrm{MWth}, 193$ fuel assemblies, initial enrichment 4.5w\%U-235

(Case : er000_0b_100p_h2o_64_bp_fuel_2_o)
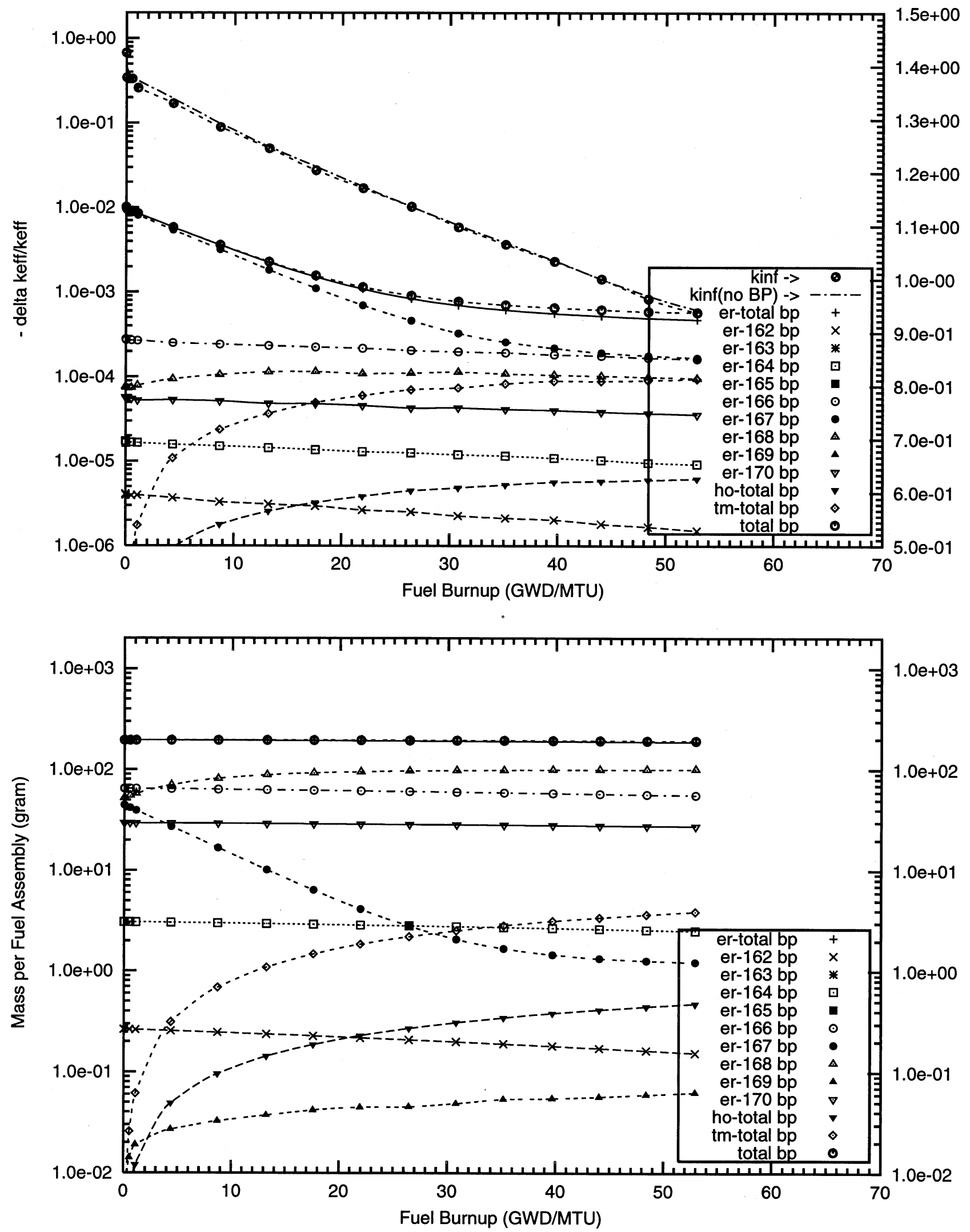
Figure C2.11. Negative Reactivity and Masses BP for Er-nat and transmutation daughters as a function of fuel life for $17 \times 17$ fuel assemblies with 64 poison rods,

$1.0 \mathrm{w} \%$ Er2O3 poison homogeneously mixed in the outer one-third volume of the UO2 pellets Reactor power $3400 \mathrm{MWth}, 193$ fuel assemblies, initial enrichment 4.5w\%U-235

(Case : er000_1b_64_bp_fuel o)
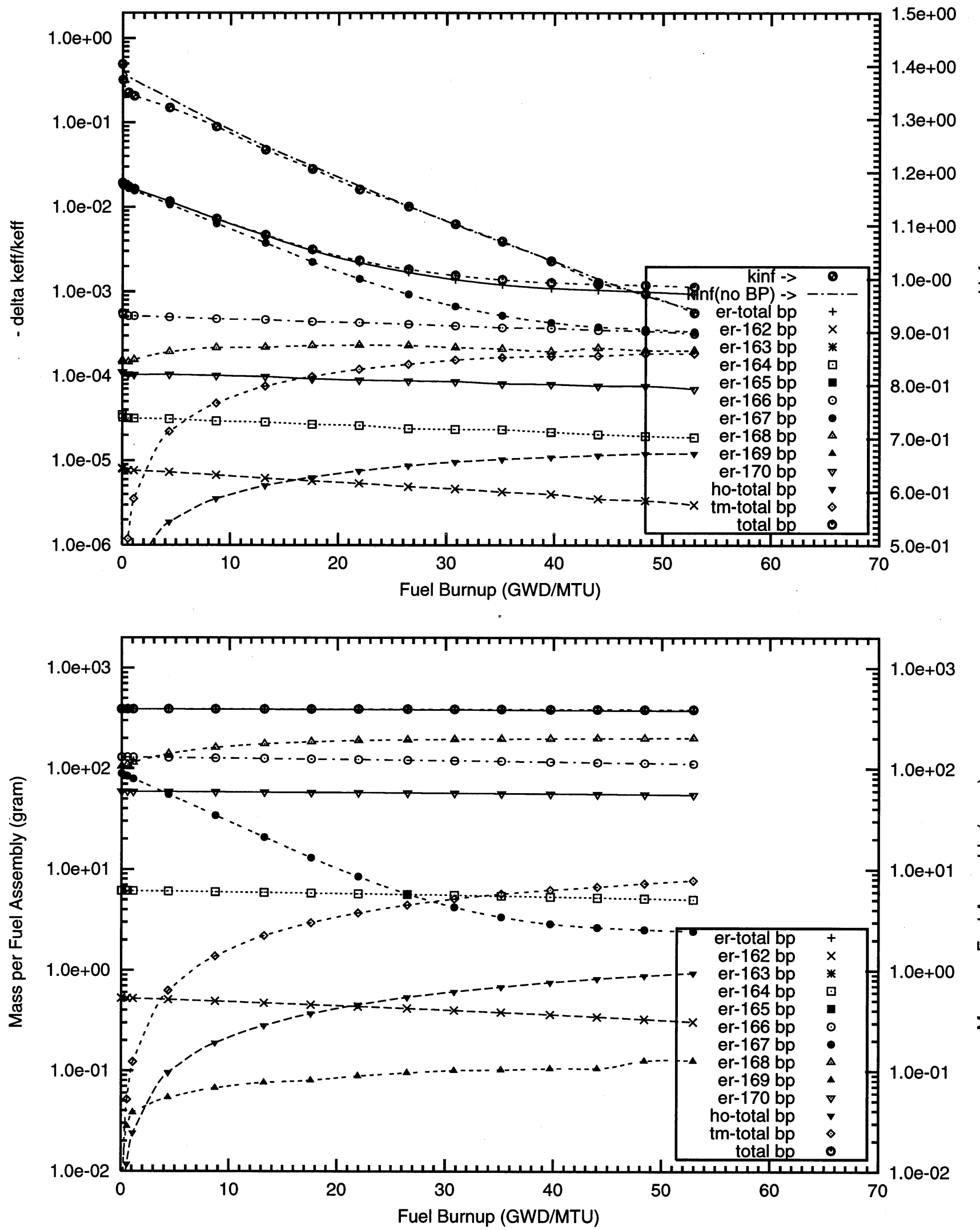
Figure C2.12. Negative Reactivity and Masses BP for Er-nat and transmutation daughters as a function of fuel life for $17 \times 17$ fuel assemblies with 64 poison rods,

$2.0 \mathrm{w} \%$ Er2O3 poison homogeneously mixed in the outer one-third volume of the UO2 pellets Reactor power $3400 \mathrm{MWth}, 193$ fuel assemblies, initial enrichment 4.5w\%U-235

(Case : er000_2b_64_bp_fuel o)
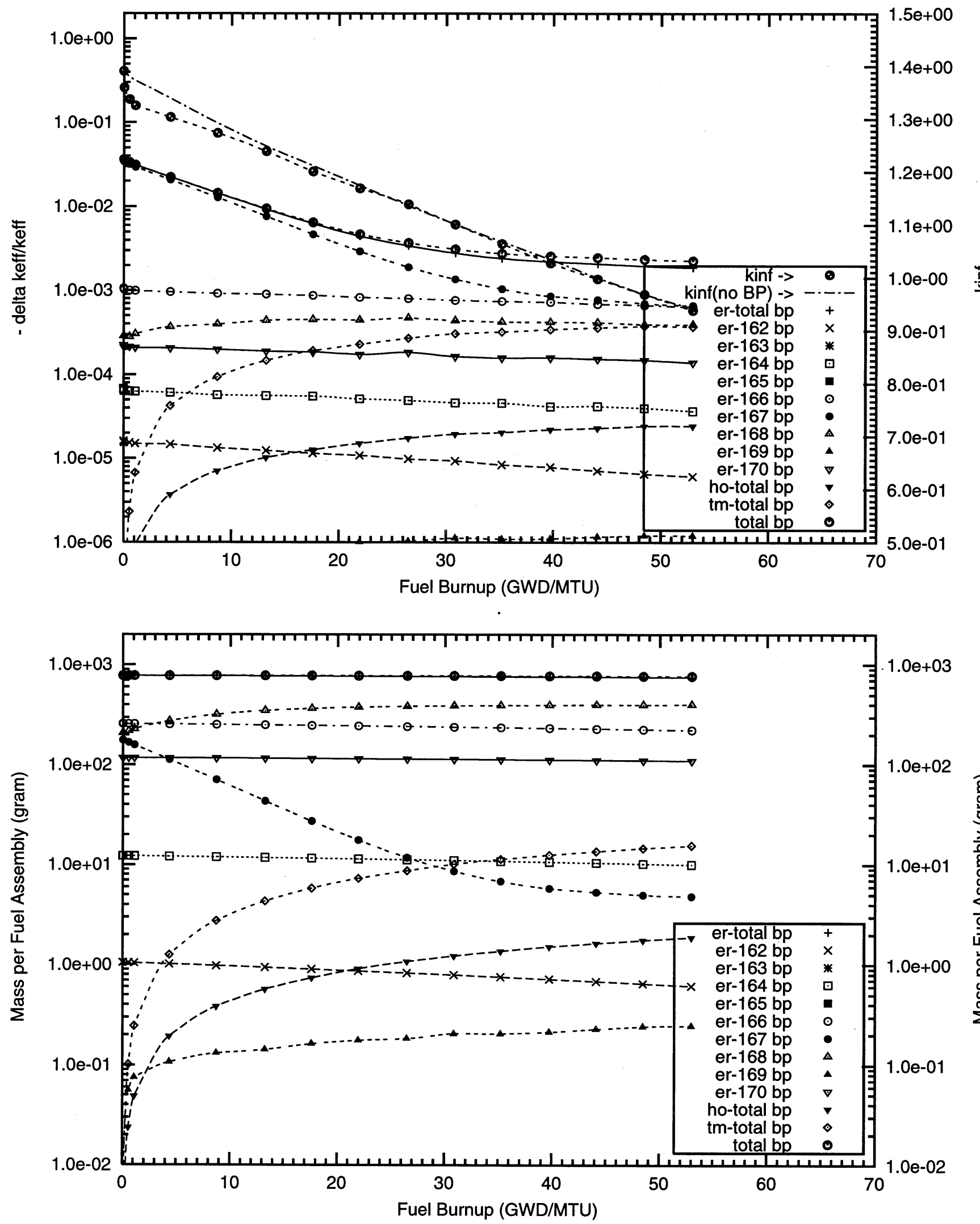
Figure C2.13. Negative Reactivity and Masses BP for Er-nat and transmutation daughters as a function of fuel life for $17 \times 17$ fuel assemblies with 64 poison rods,

$4.0 \mathrm{w} \%$ Er2O3 poison homogeneously mixed in the outer one-third volume of the UO2 pellets Reactor power $3400 \mathrm{MWth}, 193$ fuel assemblies, initial enrichment $4.5 \mathrm{w} \% \mathrm{U}-235$

(Case : er000_3b_64_bp_fuel o)
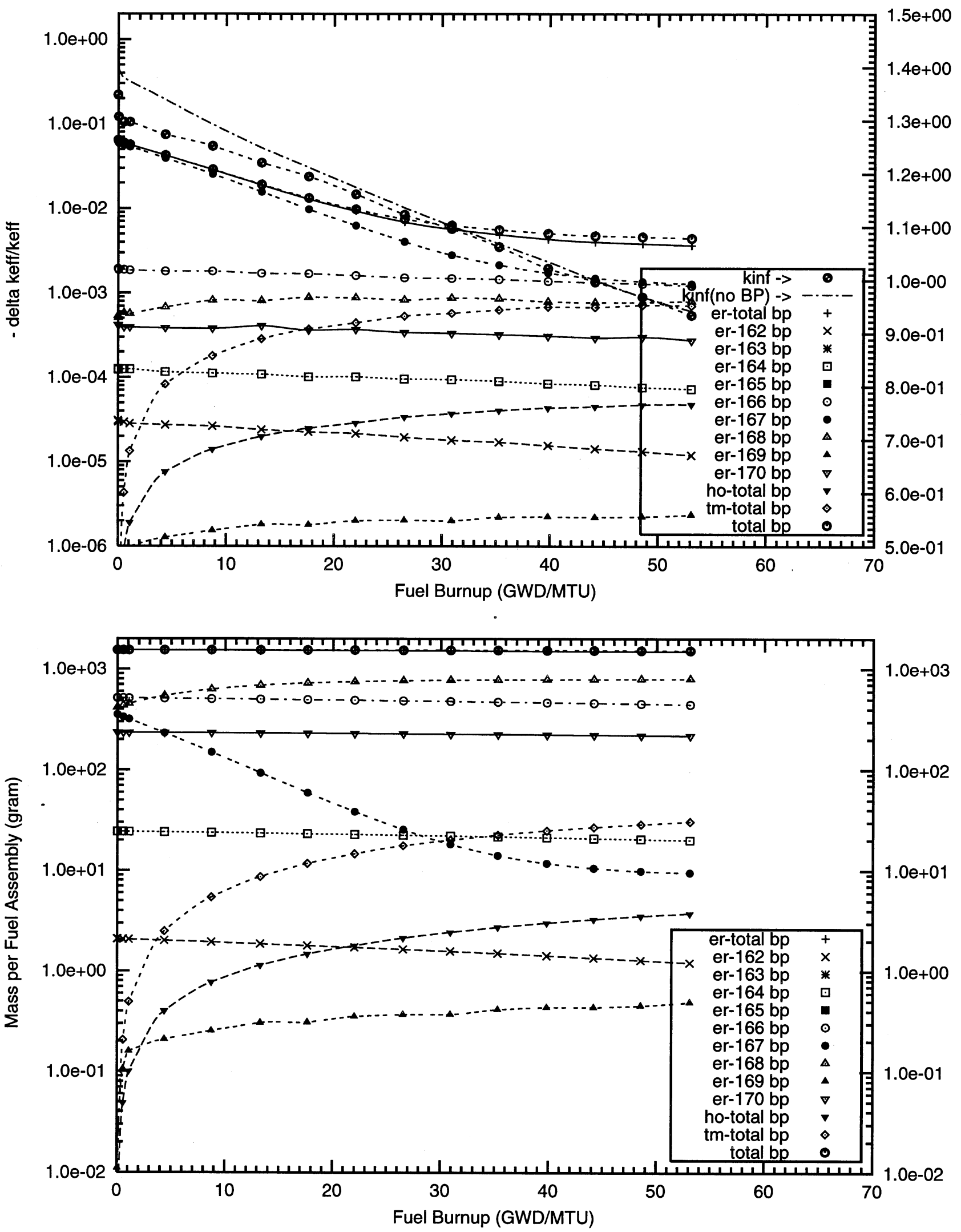
Figure C2.14. Negative Reactivity and Masses BP for Er-nat and transmutation daughters

as a function of fuel life for $17 \times 17$ fuel assemblies with 64 poison rods,

$8.0 \mathrm{w} \%$ Er2O3 poison homogeneously mixed in the outer one-third volume of the UO2 pellets

Reactor power $3400 \mathrm{MW}$ th, 193 fuel assemblies, initial enrichment $4.5 \mathrm{~W} \% \mathrm{U}-235$

(Case : er000_4b_100p_h20_64_bp_fuel_2_o)
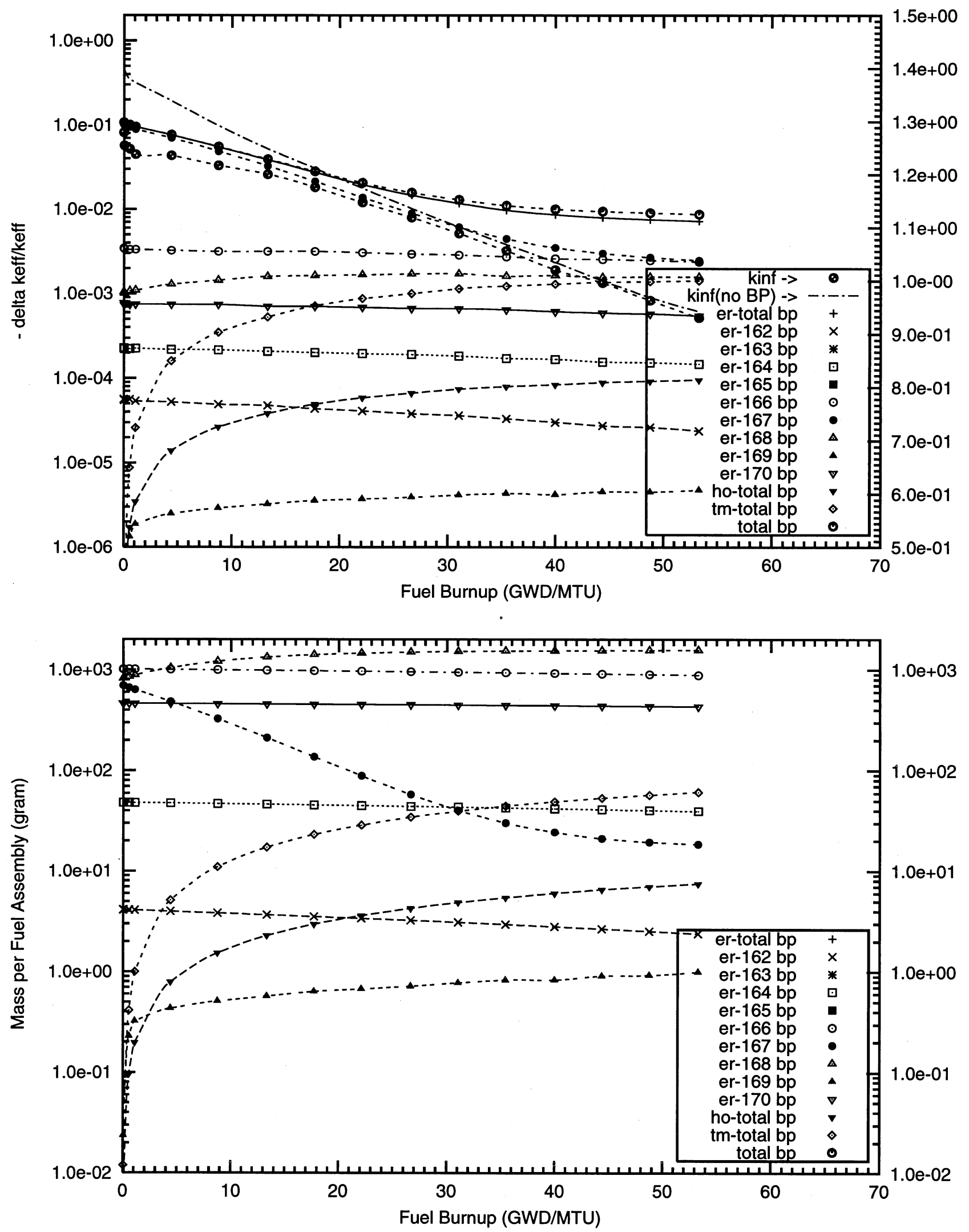
C2 - Erbium Fully Enriched in ${ }^{167} \mathrm{Er}$ 

Figure C2.15. Negative Reactivity and Masses BP for Er-167 and transmutation daughters as a function of fuel life for $17 \times 17$ fuel assemblies with 08 poison rods,

$4.0 \mathrm{w} \%$ Er2O3 poison homogeneously mixed in the outer one-third volume of the UO2 pellets

Reactor power $3400 \mathrm{MWth}, 193$ fuel assemblies, initial enrichment 4.5w\%U-235

(Case : er167_3b_08_bp_fuel o)

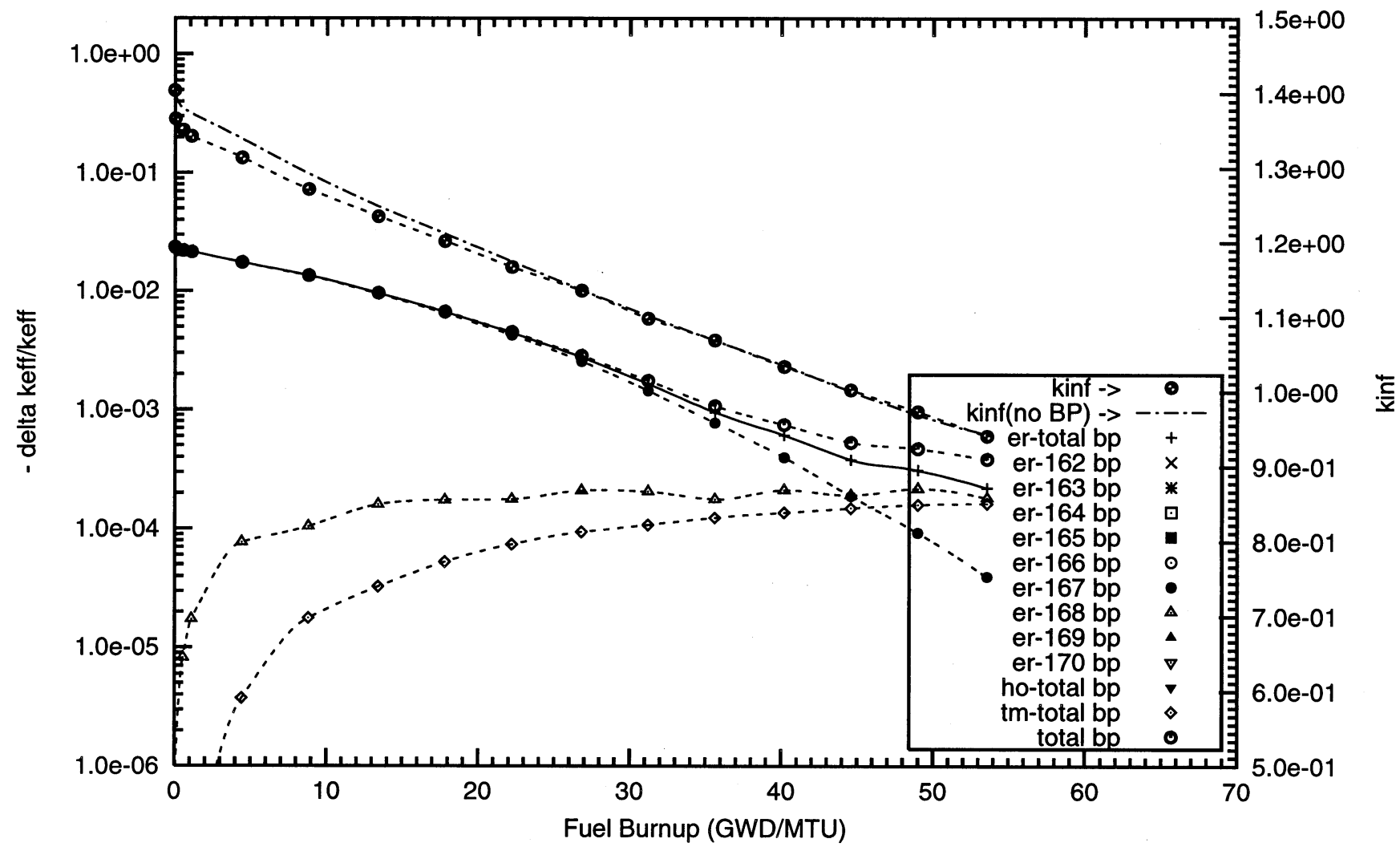


Figure C2.16. Negative Reactivity and Masses BP for Er-167 and transmutation daughters as a function of fuel life for $17 \times 17$ fuel assemblies with 08 poison rods,

8.0w\% Er2O3 poison homogeneously mixed in the outer one-third volume of the UO2 pellets Reactor power $3400 \mathrm{MWth}, 193$ fuel assemblies, initial enrichment $4.5 \mathrm{w} \% \mathrm{U}-235$ (Case : er167_4b_08_bp_fuel o)

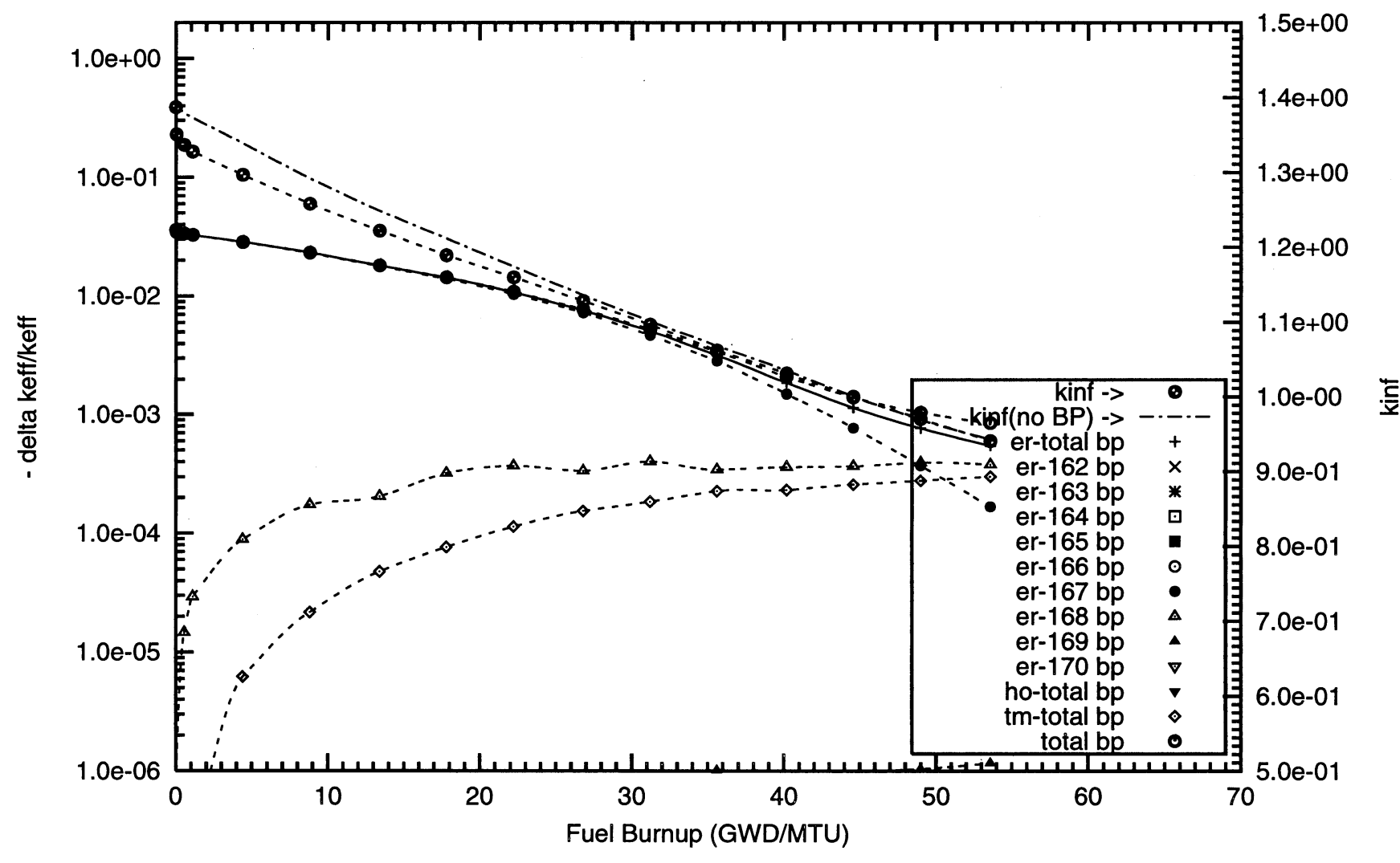


Figure C2.17. Negative Reactivity and Masses BP for Er-167 and transmutation daughters as a function of fuel life for $17 \times 17$ fuel assemblies with 08 poison rods,

$12.0 \mathrm{w} \%$ Er2O3 poison homogeneously mixed in the outer one-third volume of the UO2 pellets Reactor power $3400 \mathrm{MWth}, 193$ fuel assemblies, initial enrichment 4.5w\%U-235

(Case : er167_5b_08_bp_fuel o)

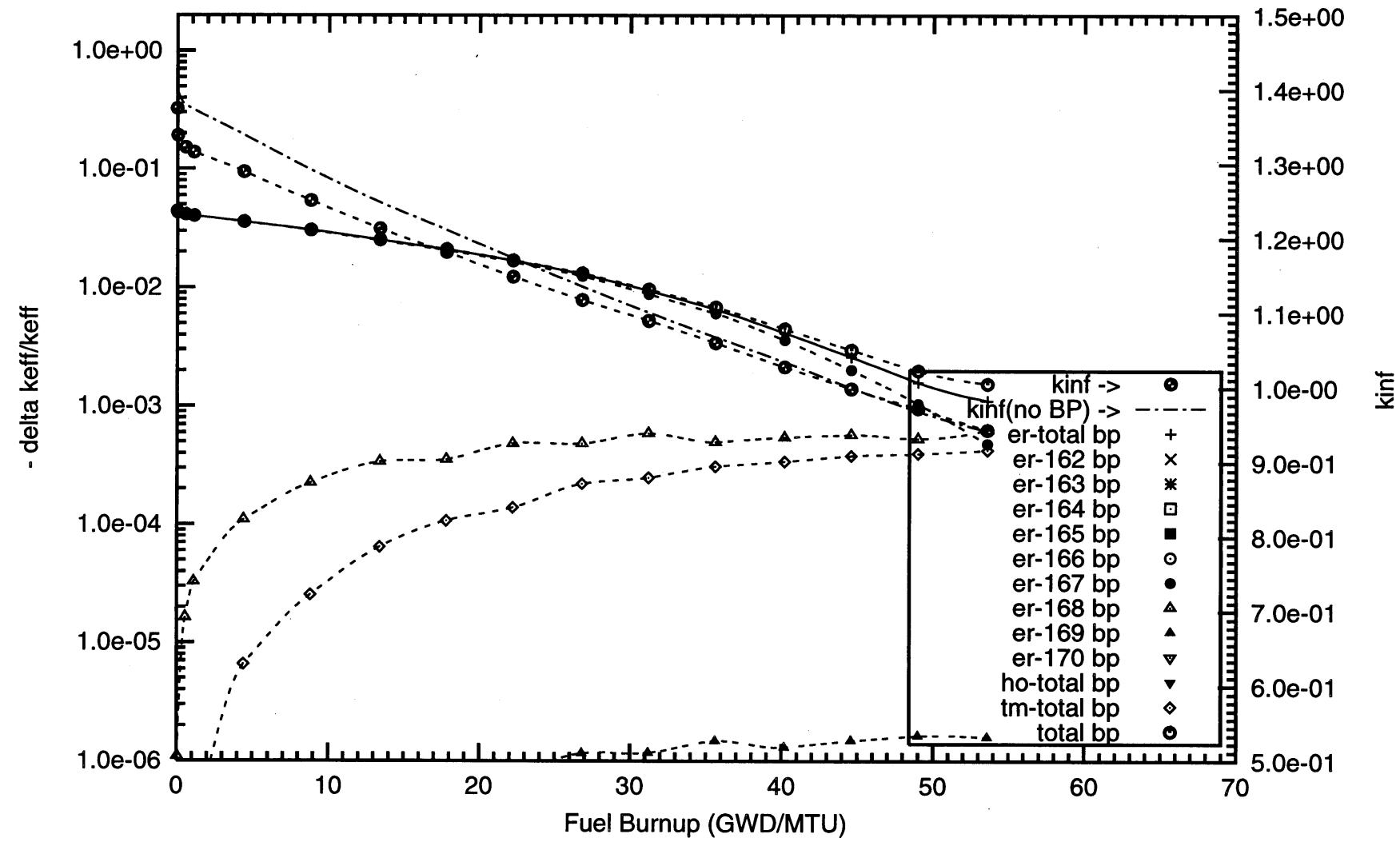


Figure C2.18. Negative Reactivity and Masses BP for Er-167 and transmutation daughters as a function of fuel life for $17 \times 17$ fuel assemblies with 16 poison rods,

$0.2 \mathrm{w} \%$ Er2O3 poison homogeneously mixed in the outer one-third volume of the UO2 pellets Reactor power $3400 \mathrm{MWth}, 193$ fuel assemblies, initial enrichment $4.5 \mathrm{w} \% \mathrm{U}-235$

(Case : er167_8b_100p_h20_16_bp_fuel_2_o)
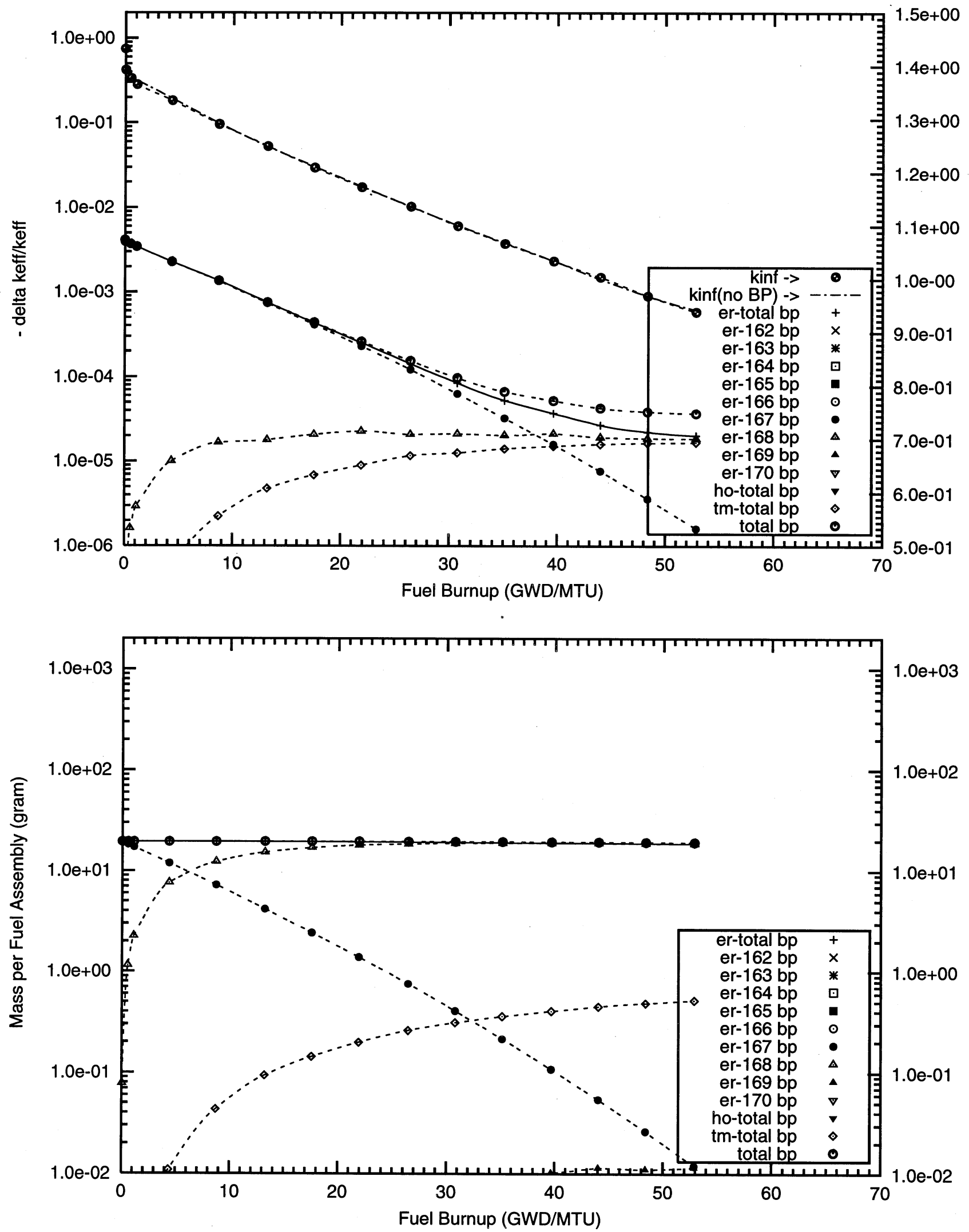
Figure C2.19. Negative Reactivity and Masses BP for Er-167 and transmutation daughters as a function of fuel life for $17 \times 17$ fuel assemblies with 16 poison rods,

$0.5 \mathrm{w} \% \mathrm{Er} 2 \mathrm{O} 3$ poison homogeneously mixed in the outer one-third volume of the UO2 pellets

Reactor power $3400 \mathrm{MW}$ th, 193 fuel assemblies, initial enrichment $4.5 \mathrm{w} \% \mathrm{U}-235$

(Case : er167_0b_100p_h2o_16_bp_fuel_2_o)
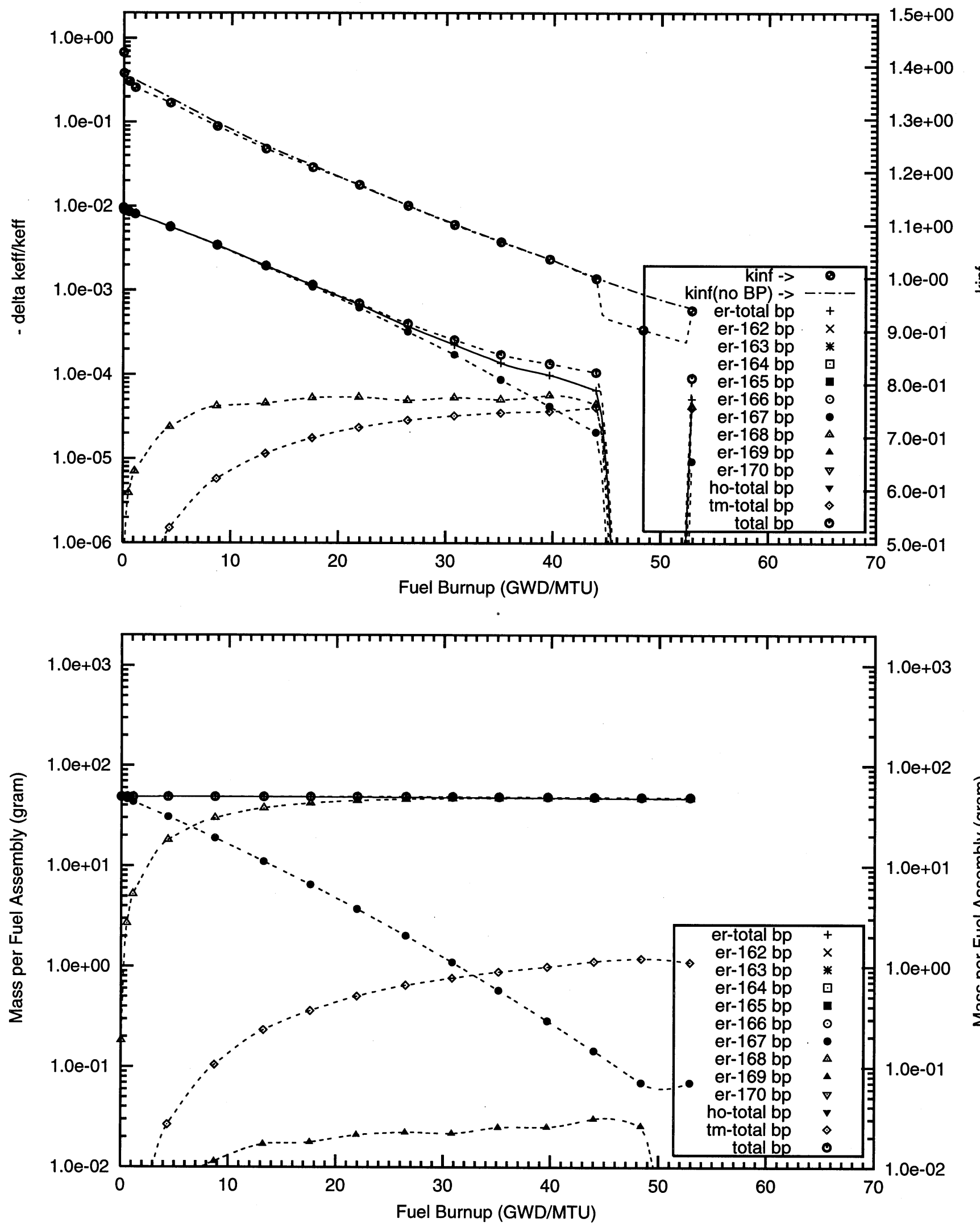
Figure C2.20. Negative Reactivity and Masses BP for Er-167 and transmutation daughters as a function of fuel life for $17 \times 17$ fuel assemblies with 16 poison rods,

$1.0 \mathrm{w} \% \mathrm{Er} 2 \mathrm{O} 3$ poison homogeneously mixed in the outer one-third volume of the UO2 pellets

Reactor power $3400 \mathrm{MWth}, 193$ fuel assemblies, initial enrichment 4.5w\%U-235

(Case : er167_1b_100p_h2o_16_bp_fuel_2_o)
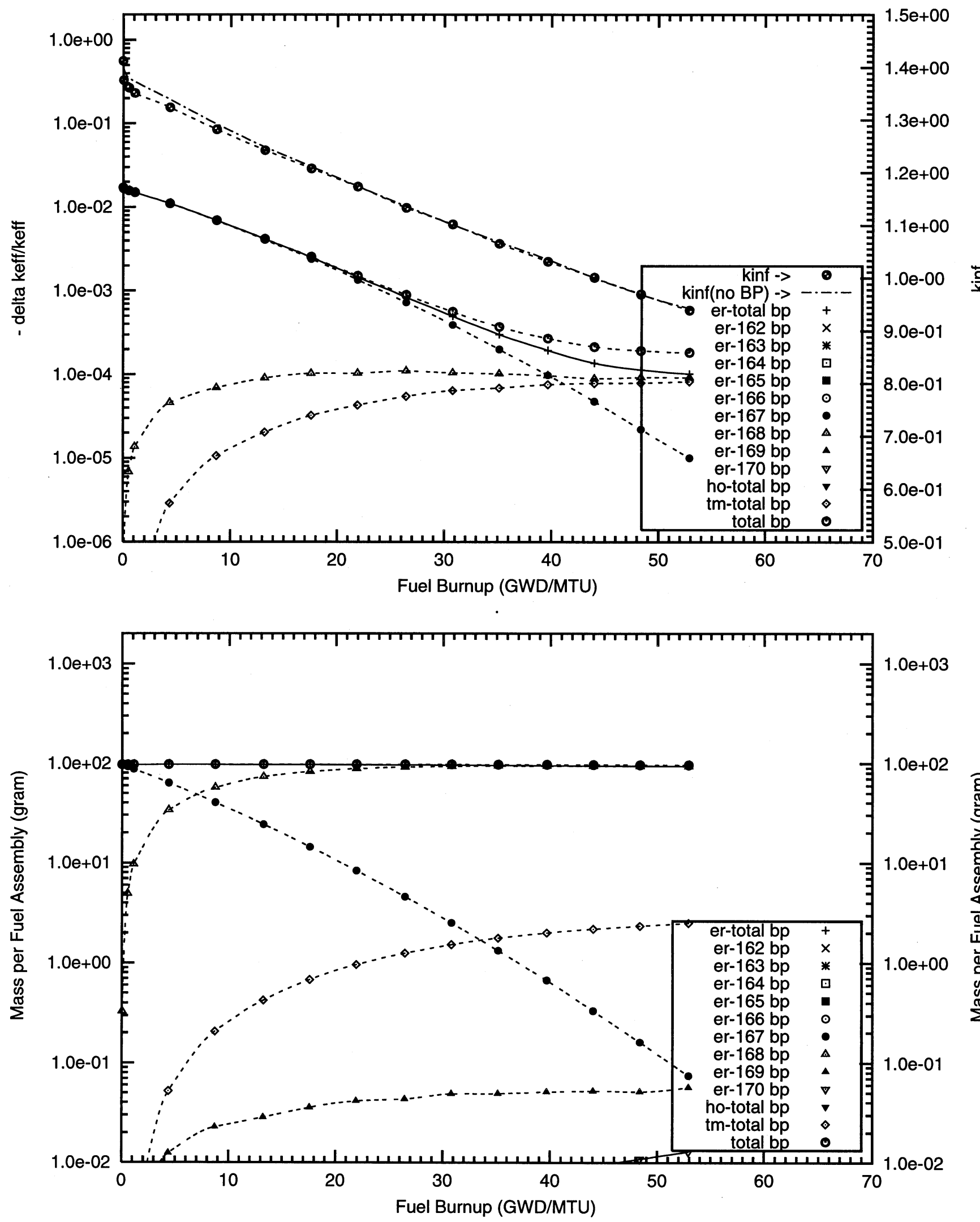
Figure C2.21. Negative Reactivity and Masses BP for Er-167 and transmutation daughters as a function of fuel life for $17 \times 17$ fuel assemblies with 16 poison rods,

$2.0 \mathrm{w} \%$ Er2O3 poison homogeneously mixed in the outer one-third volume of the UO2 pellets

Reactor power $3400 \mathrm{MWth}, 193$ fuel assemblies, initial enrichment 4.5w\%U-235

(Case : er167_2b_100p_h2o_16_bp_fuel_2_o)
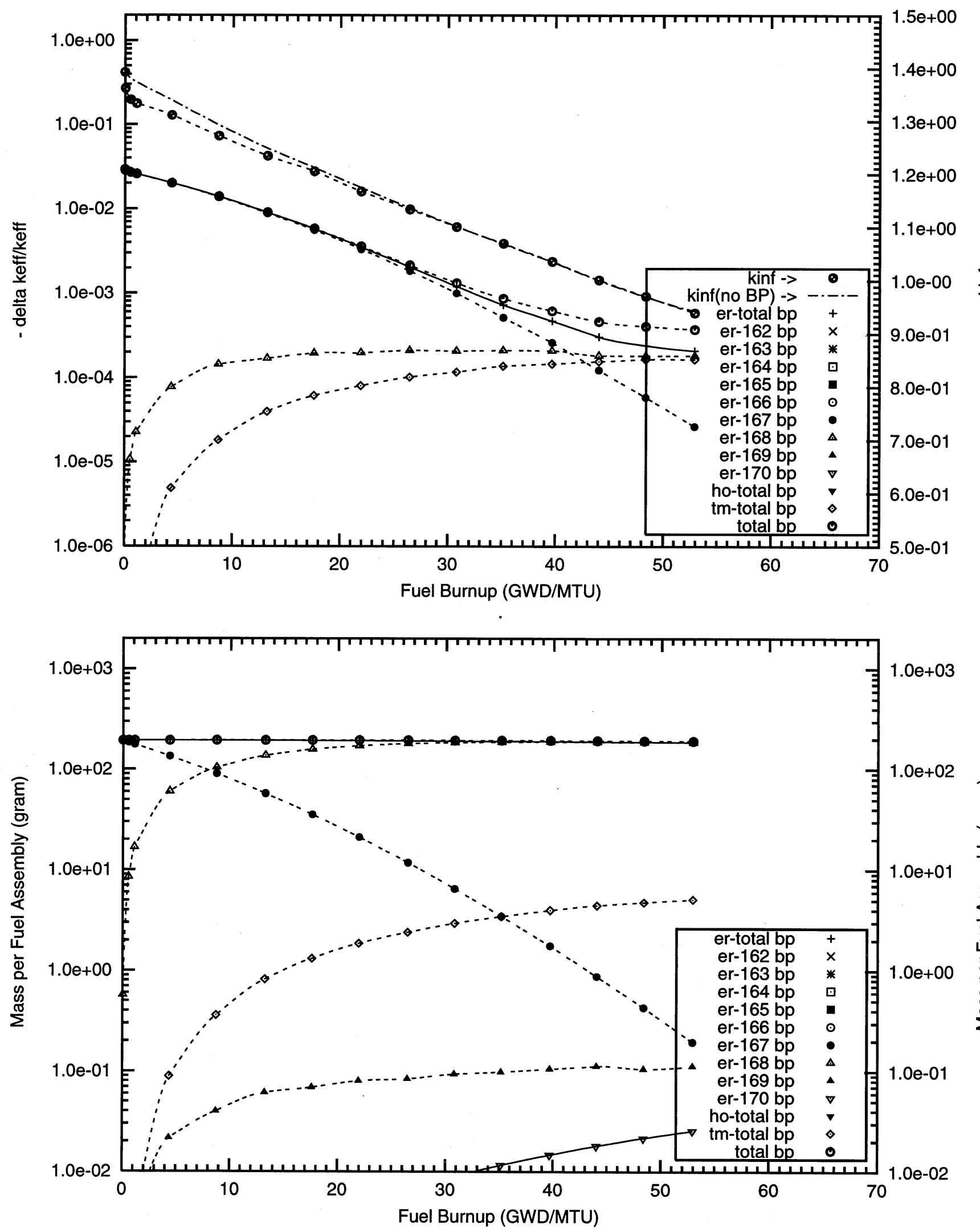
Figure C2.22. Negative Reactivity and Masses BP for Er-167 and transmutation daughters as a function of fuel life for $17 \times 17$ fuel assemblies with 16 poison rods,

$4.0 \mathrm{w} \%$ Er2O3 poison homogeneously mixed in the outer one-third volume of the $\mathrm{UO} 2$ pellets

Reactor power $3400 \mathrm{MWth}, 193$ fuel assemblies, initial enrichment 4.5w\%U-235

(Case : er167_3b_100p_h2o_16_bp_fuel_2_o)
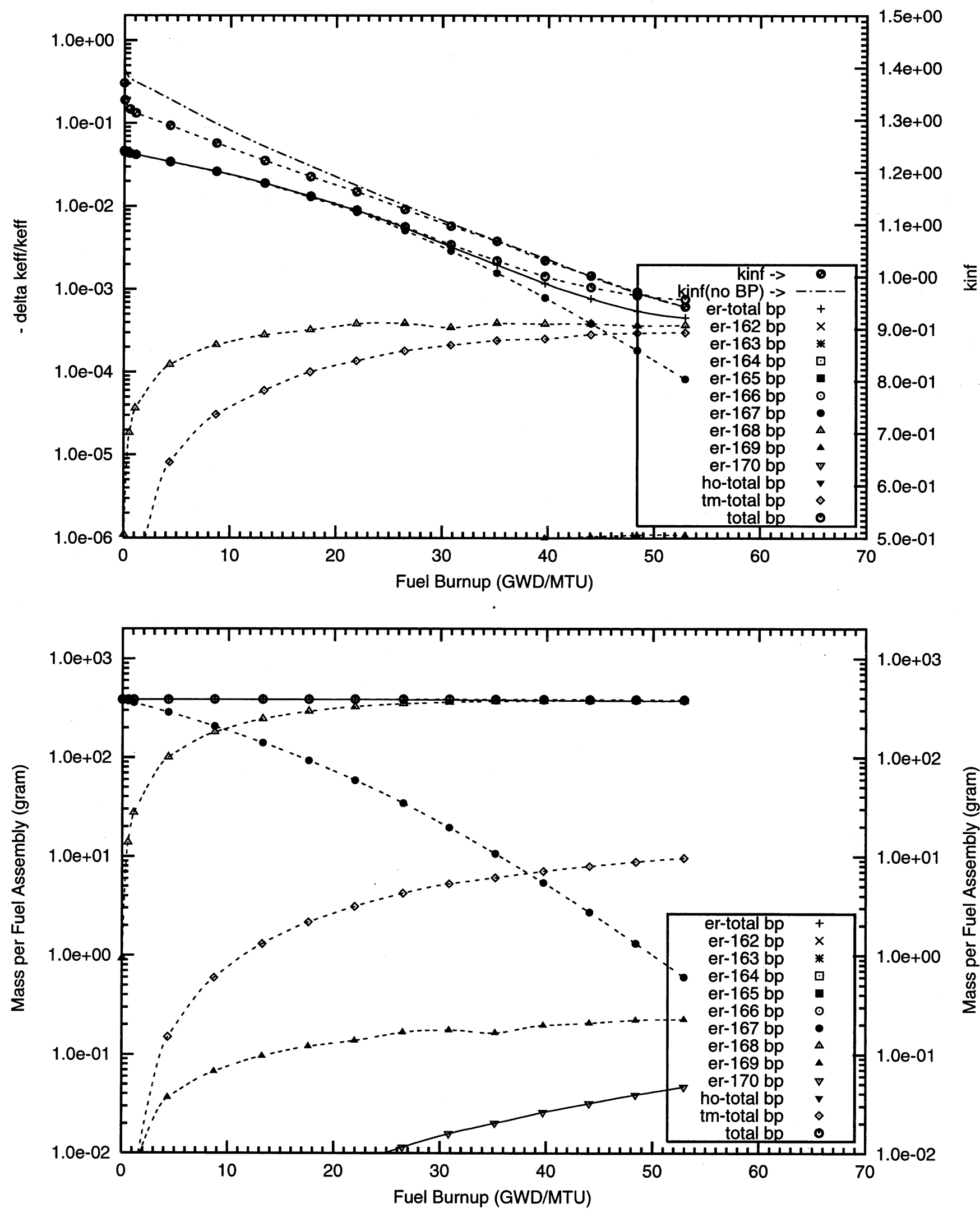
Figure C2.23. Negative Reactivity and Masses BP for Er-167 and transmutation daughters as a function of fuel life for $17 \times 17$ fuel assemblies with 18 poison rods,

4.0w\% Er2O3 poison homogeneously mixed in the outer one-third volume of the $\mathrm{UO} 2$ pellets Reactor power $3400 \mathrm{MWth}, 193$ fuel assemblies, initial enrichment 4.5w\%U-235

(Case : er167_3b_16_bp_fuel o)
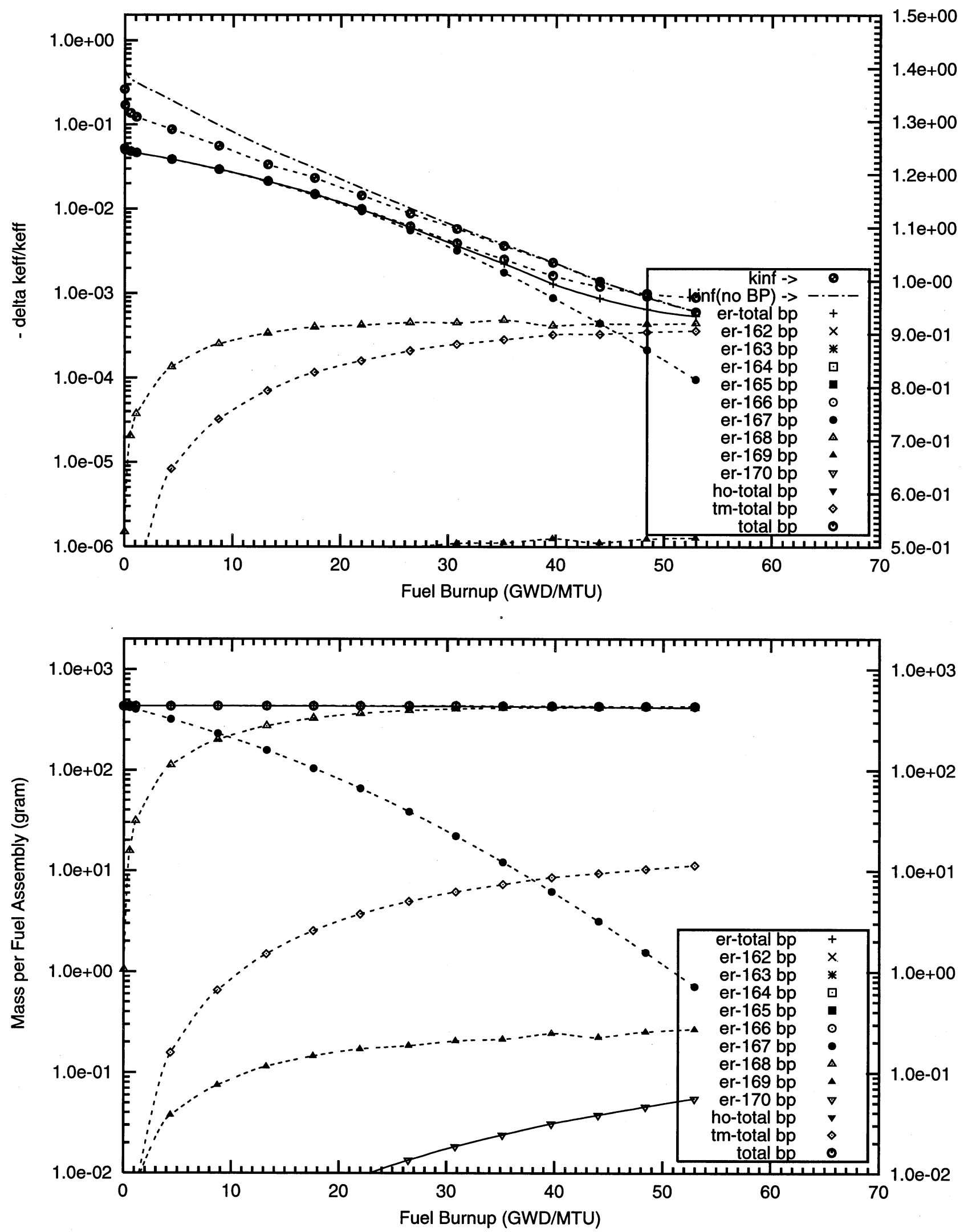
Figure C2.24. Negative Reactivity and Masses BP for Er-167 and transmutation daughters as a function of fuel life for $17 \times 17$ fuel assemblies with 64 poison rods,

$0.5 \mathrm{w} \%$ Er2O3 poison homogeneously mixed in the outer one-third volume of the $\mathrm{UO} 2$ pellets Reactor power $3400 \mathrm{MW}$ th, 193 fuel assemblies, initial enrichment $4.5 \mathrm{w} \% \mathrm{U}-235$

(Case : er167_0b_100p_h2o_64_bp_fuel_2_o)
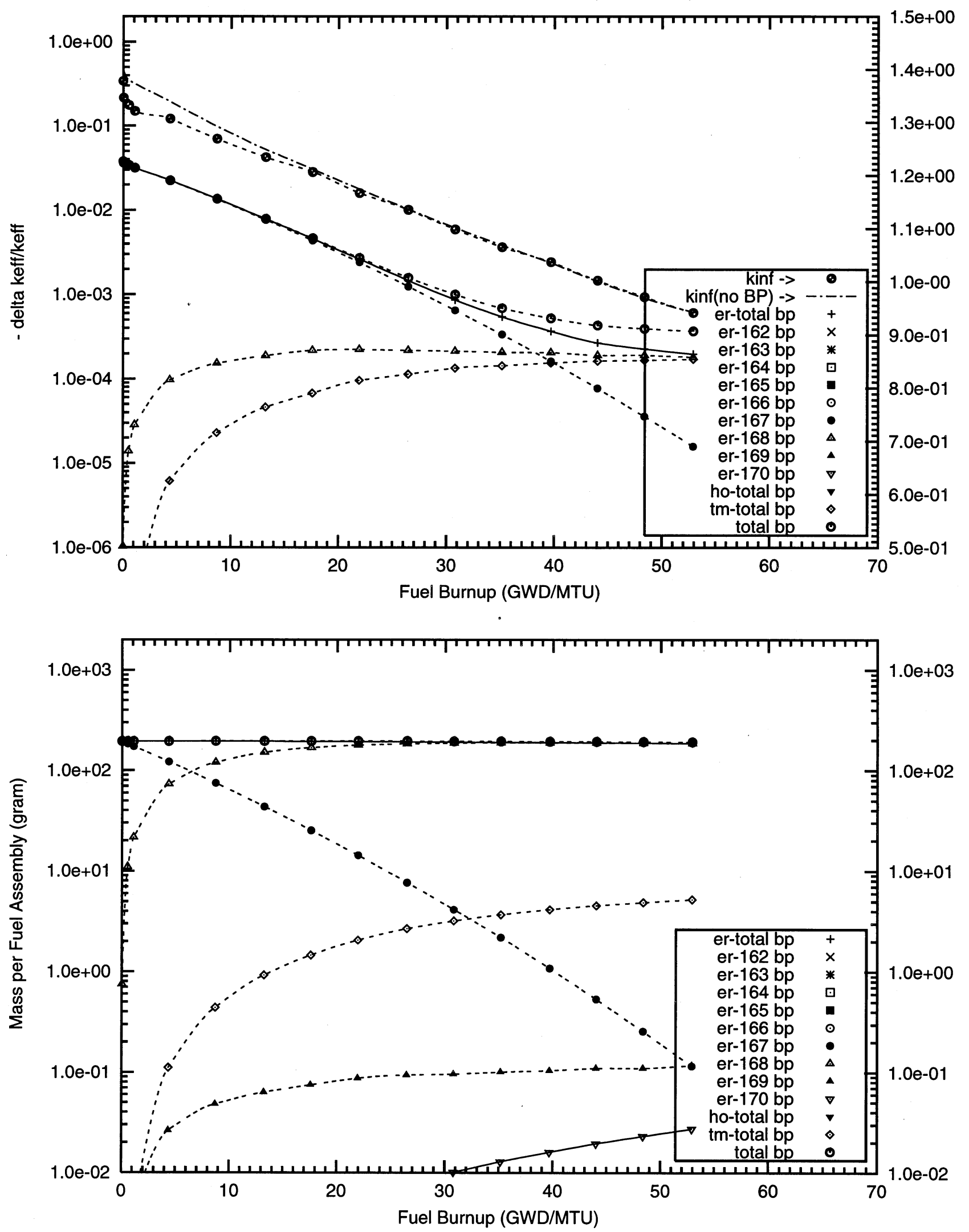
Figure C2.25. Negative Reactivity and Masses BP for Er-167 and transmutation daughters as a function of fuel life for $17 \times 17$ fuel assemblies with 64 poison rods,

$1.0 \mathrm{w} \%$ Er2O3 poison homogeneously mixed in the outer one-third volume of the $\mathrm{UO} 2$ pellets Reactor power $3400 \mathrm{MWth}, 193$ fuel assemblies, initial enrichment 4.5w\%U-235

(Case : er167_1b_64_bp_fuel o)
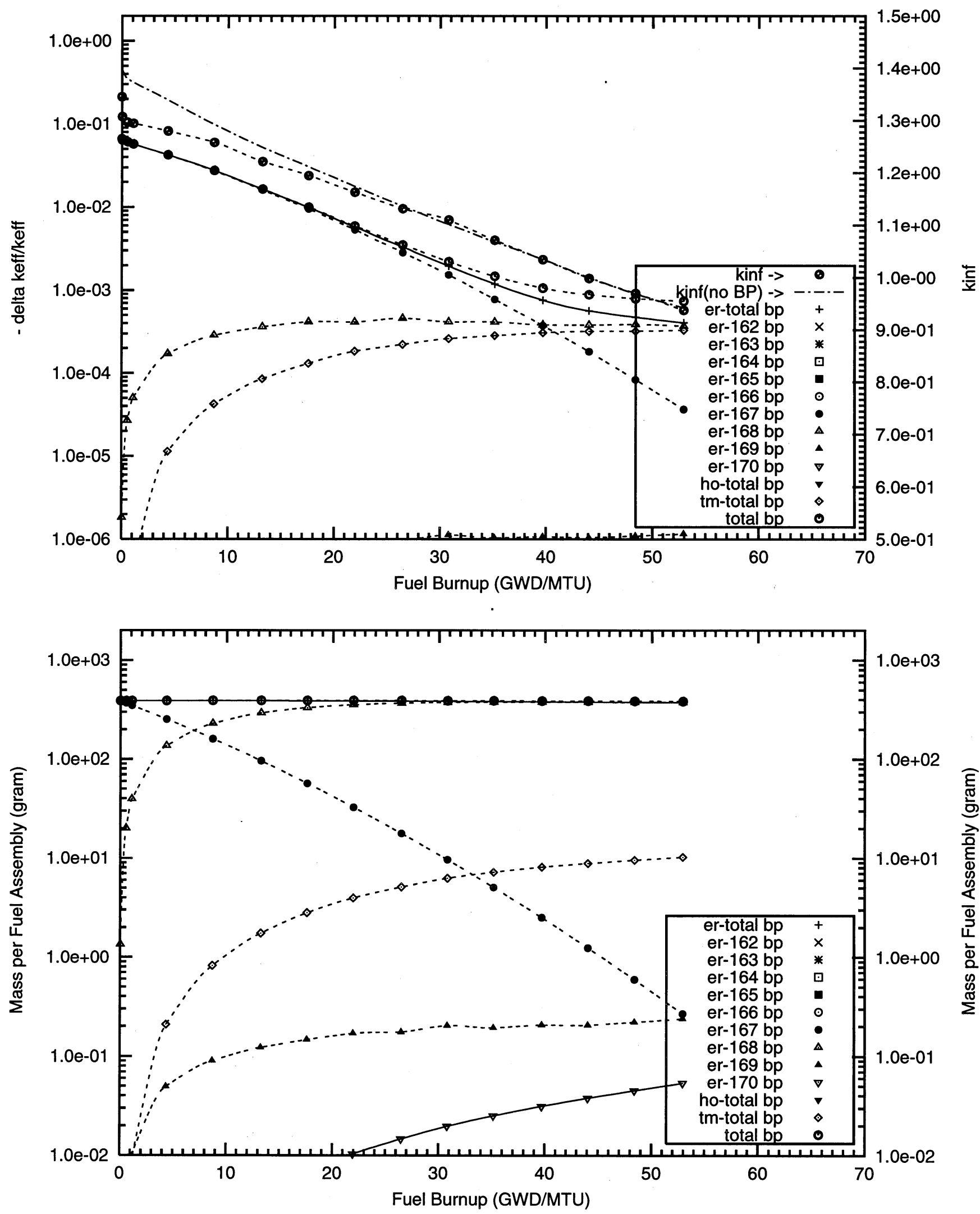
Figure C2.26. Negative Reactivity and Masses BP for Er-167 and transmutation daughters as a function of fuel life for $17 \times 17$ fuel assemblies with 64 poison rods,

$2.0 \mathrm{w} \%$ Er2O3 poison homogeneously mixed in the outer one-third volume of the $\mathrm{UO} 2$ pellets

Reactor power $3400 \mathrm{MWth}, 193$ fuel assemblies, initial enrichment 4.5w\%U-235

(Case : er167_2b_100p_h2o_64_bp_fuel_2_o)
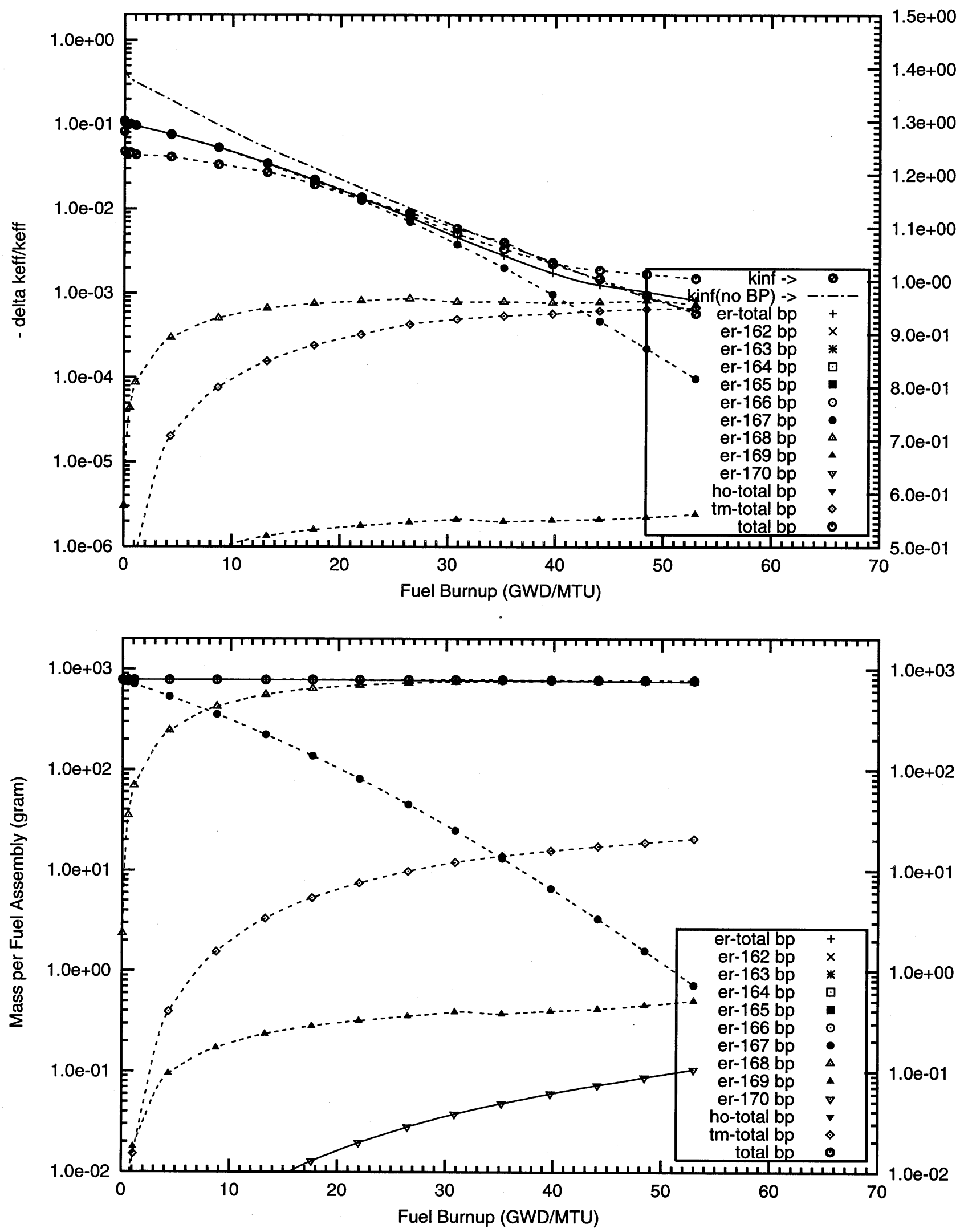
Figure C2.27. Negative Reactivity and Masses BP for Er-167 and transmutation daughters as a function of fuel life for $17 \times 17$ fuel assemblies with 64 poison rods,

$4.0 \mathrm{w} \%$ Er2O3 poison homogeneously mixed in the outer one-third volume of the UO2 pellets Reactor power $3400 \mathrm{MWth}, 193$ fuel assemblies, initial enrichment $4.5 \mathrm{w} \% \mathrm{U}-235$

(Case : er167_3b_64_bp_fuel o)
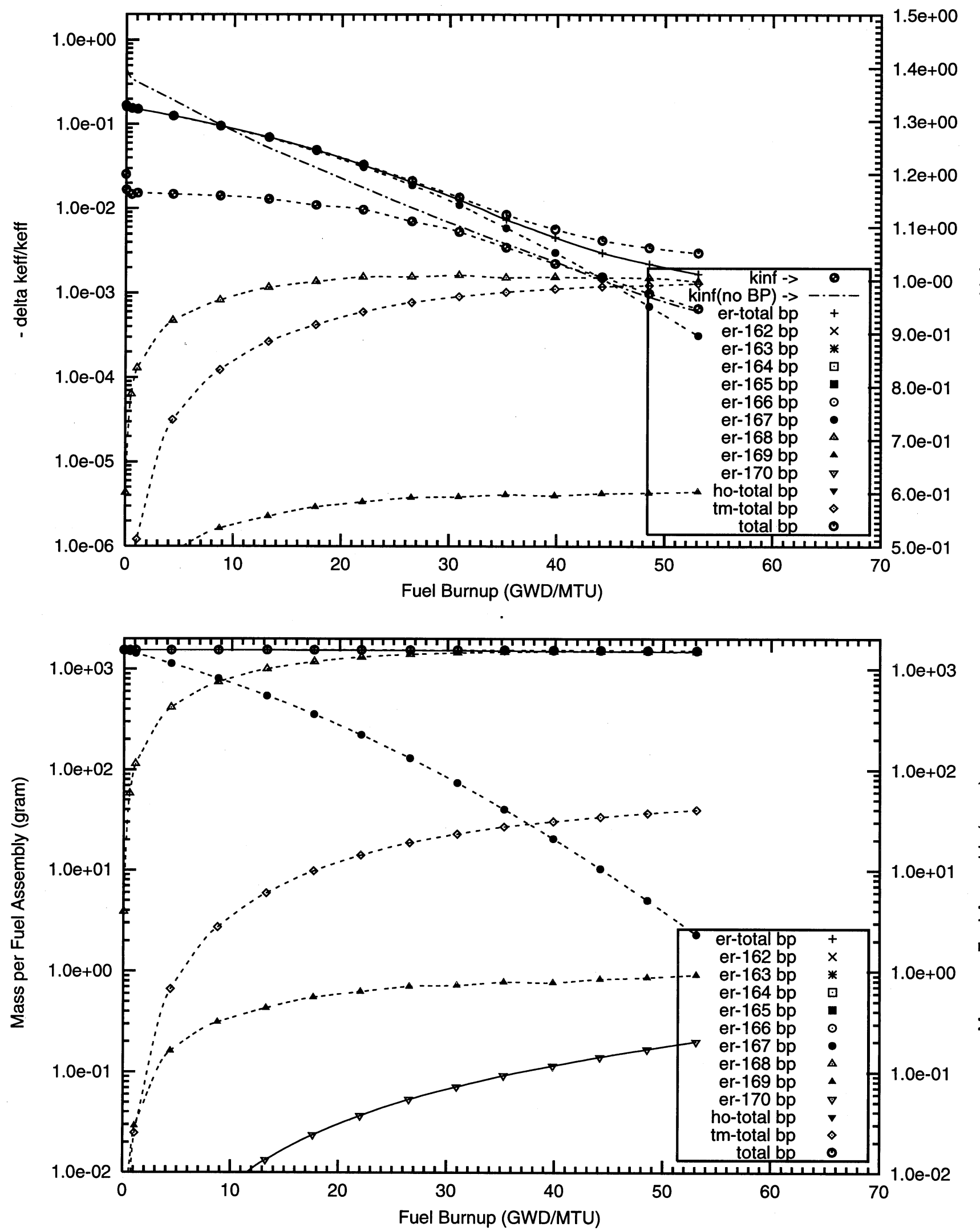


\section{Appendix C3}

Reactivity Worths and Masses of $\mathrm{Er}_{2} \mathrm{O}_{3}$ Coating on the Surface of the Fuel Pellets

\section{Table 15 Cases}



C3 - Erbium with Natural Isotopic Abundances 

Figure C3.1. Negative Reactivity and Masses BP for Er-nat and transmutation daughters as a function of fuel life for $17 \times 17$ fuel assemblies with 16 poison rods,

2.0 mill thick Er2O3 poison coating on the outer radial surface of the UO2 pellets

Reactor power $3400 \mathrm{MWth}, 193$ fuel assemblies, initial enrichment 4.5w\%U-235

(Case : er000_2f_100p_h2o_16_bp_fuel_2_o)
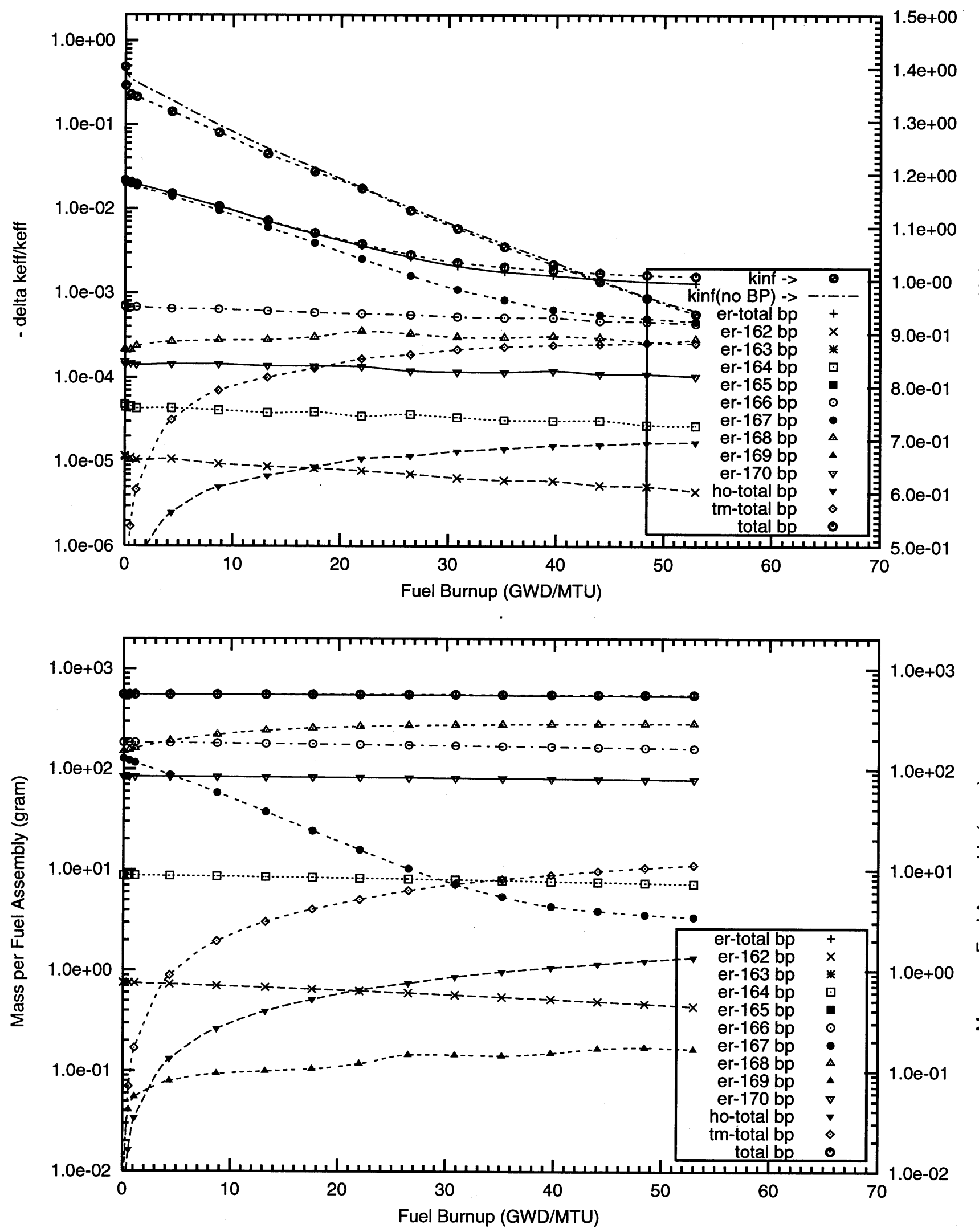
Figure C3.2. Negative Reactivity and Masses BP for Er-nat and transmutation daughters as a function of fuel life for $17 \times 17$ fuel assemblies with 16 poison rods,

4.0 mill thick Er2O3 poison coating on the outer radial surface of the UO2 pellets

Reactor power $3400 \mathrm{MWth}, 193$ fuel assemblies, initial enrichment 4.5w\%U-235

(Case : er000_3f_100p_h2o_16_bp_fuel_2_o)
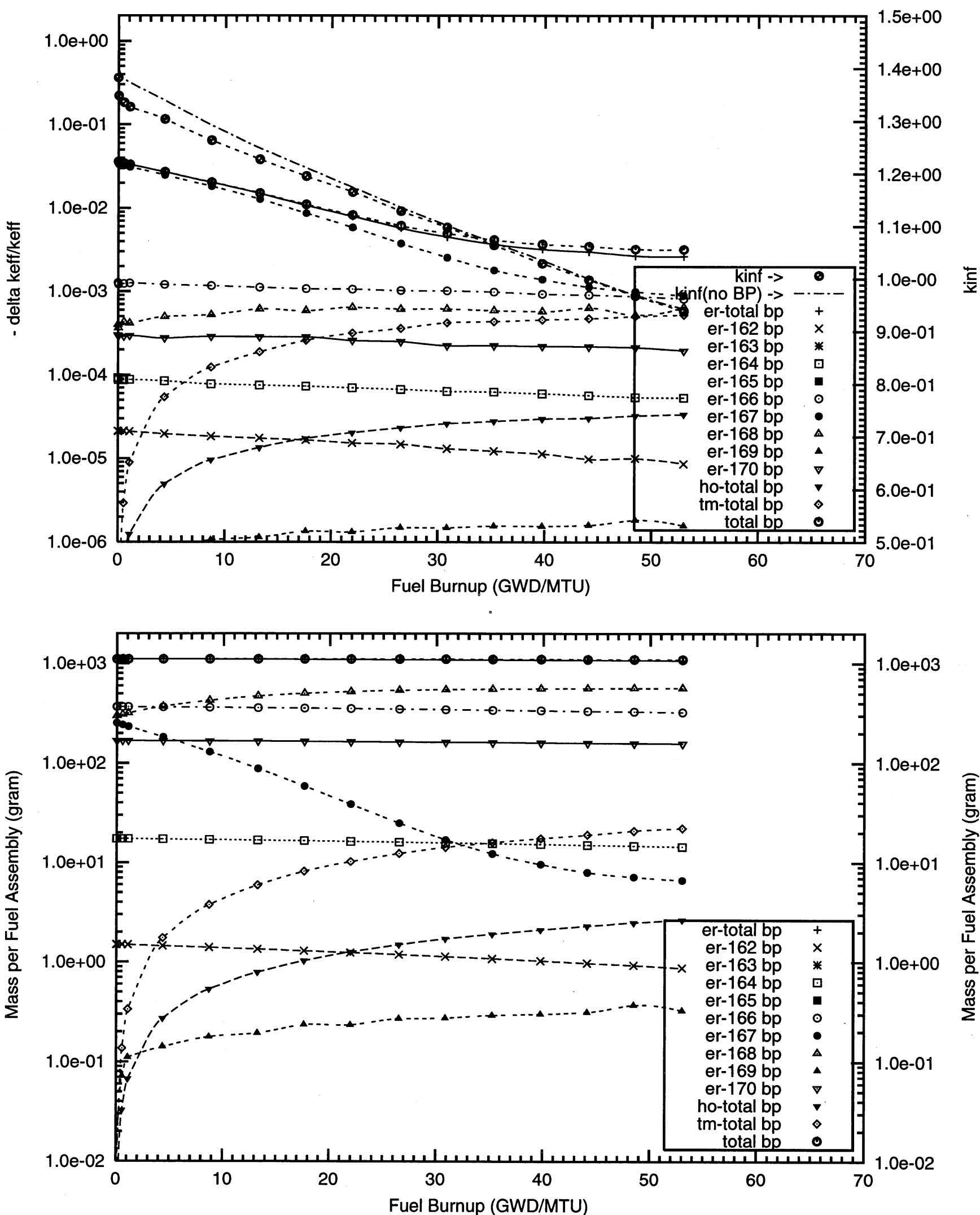
Figure C3.3. Negative Reactivity and Masses BP for Er-nat and transmutation daughters as a function of fuel life for $17 \times 17$ fuel assemblies with 16 poison rods,

8.0 mill thick Er2O3 poison coating on the outer radial surface of the UO2 pellets

Reactor power $3400 \mathrm{MWth}, 193$ fuel assemblies, initial enrichment 4.5w\%U-235

(Case : er000_4f_100p_h2o_16_bp_fuel_2_o)
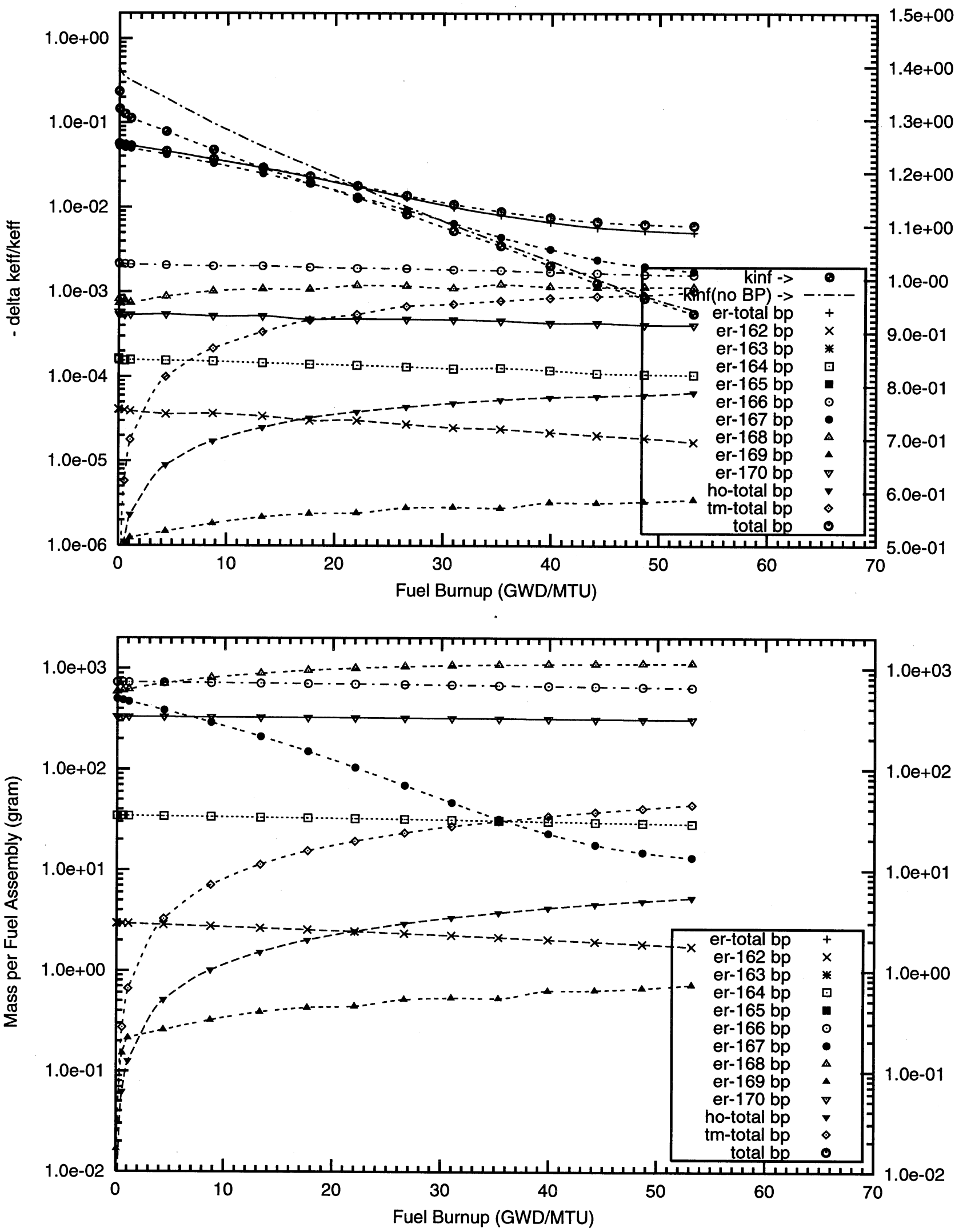
Figure C3.4. Negative Reactivity and Masses BP for Er-nat and transmutation daughters as a function of fuel life for $17 \times 17$ fuel assemblies with 18 poison rods,

2.0 mill thick Er2O3 poison coating on the outer radial surface of the UO2 pellets

Reactor power $3400 \mathrm{MWth}, 193$ fuel assemblies, initial enrichment 4.5w\%U-235

(Case : er000_2f_16_bp_fuel (old) o)
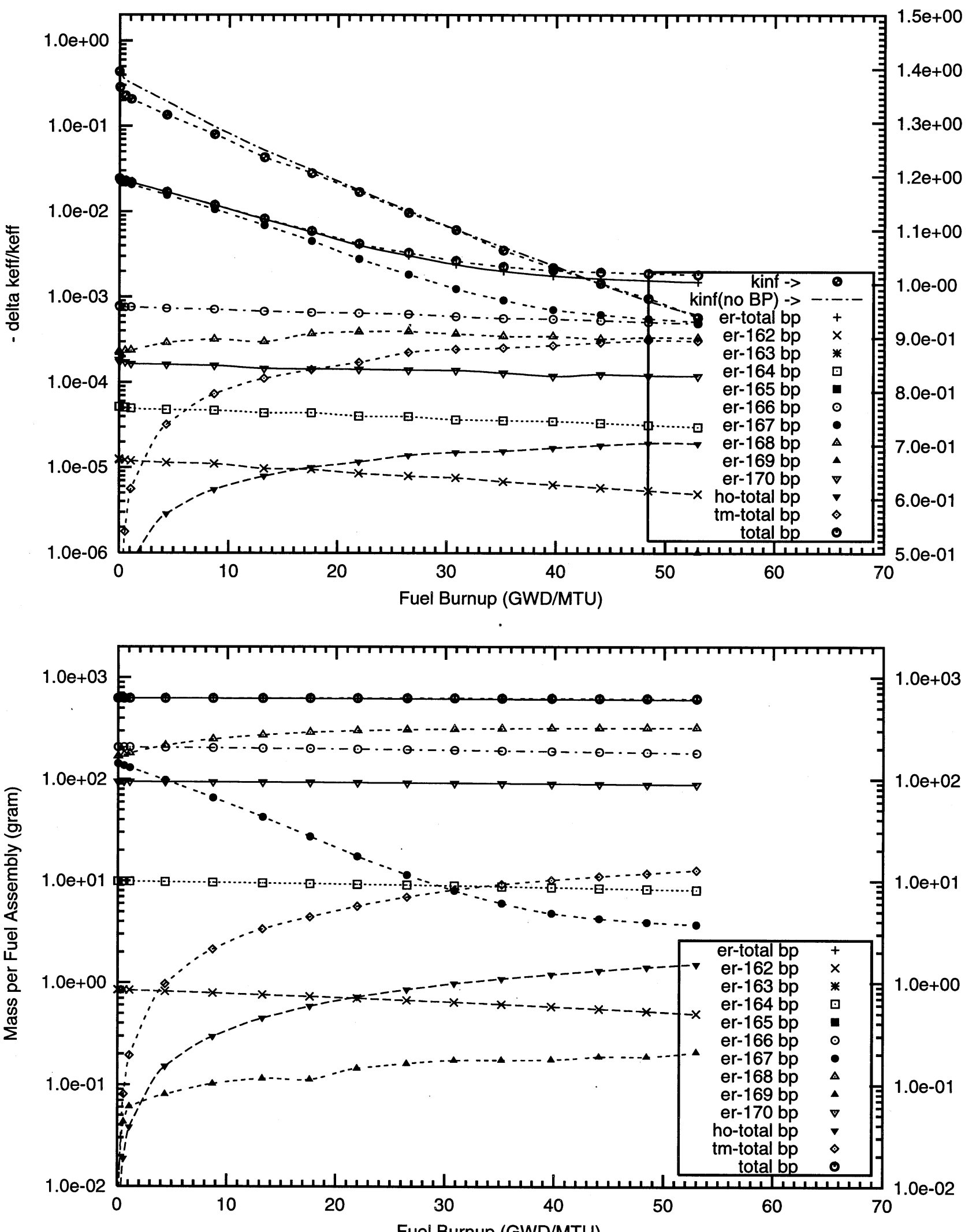
Figure C3.5. Negative Reactivity and Masses BP for Er-nat and transmutation daughters as a function of fuel life for $17 \times 17$ fuel assemblies with 18 poison rods,

4.0 mill thick Er2O3 poison coating on the outer radial surface of the UO2 pellets

Reactor power 3400 MWth, 193 fuel assemblies, initial enrichment 4.5w\%U-235

(Case : er000_3f_16_bp_fuel (old) o)
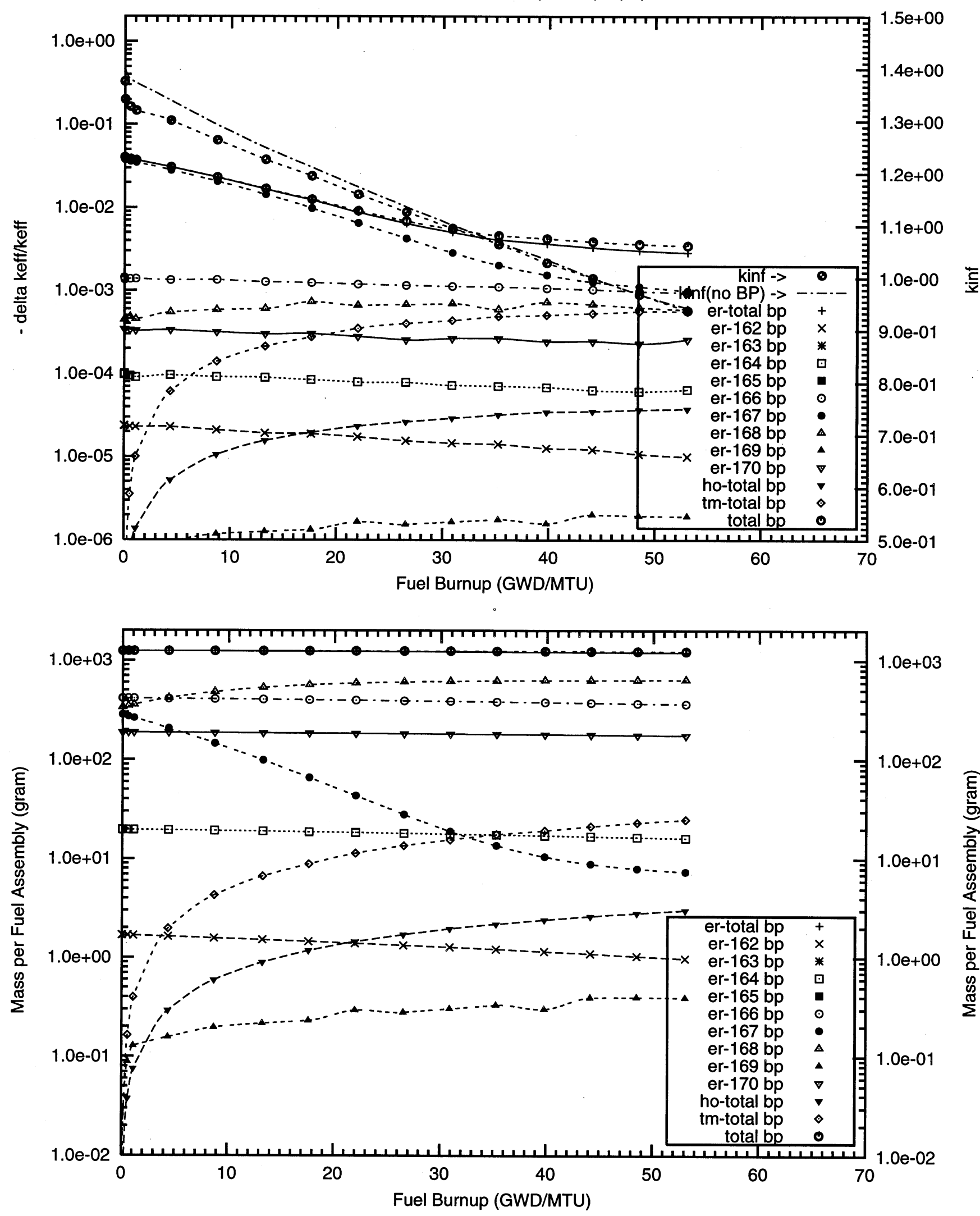
Figure C3.6. Negative Reactivity and Masses BP for Er-nat and transmutation daughters as a function of fuel life for $17 \times 17$ fuel assemblies with 64 poison rods,

1.0 mill thick Er2O3 poison coating on the outer radial surface of the UO2 pellets

Reactor power $3400 \mathrm{MWth}, 193$ fuel assemblies, initial enrichment 4.5w\%U-235

(Case : er000_1f_64_bp_fuel o)
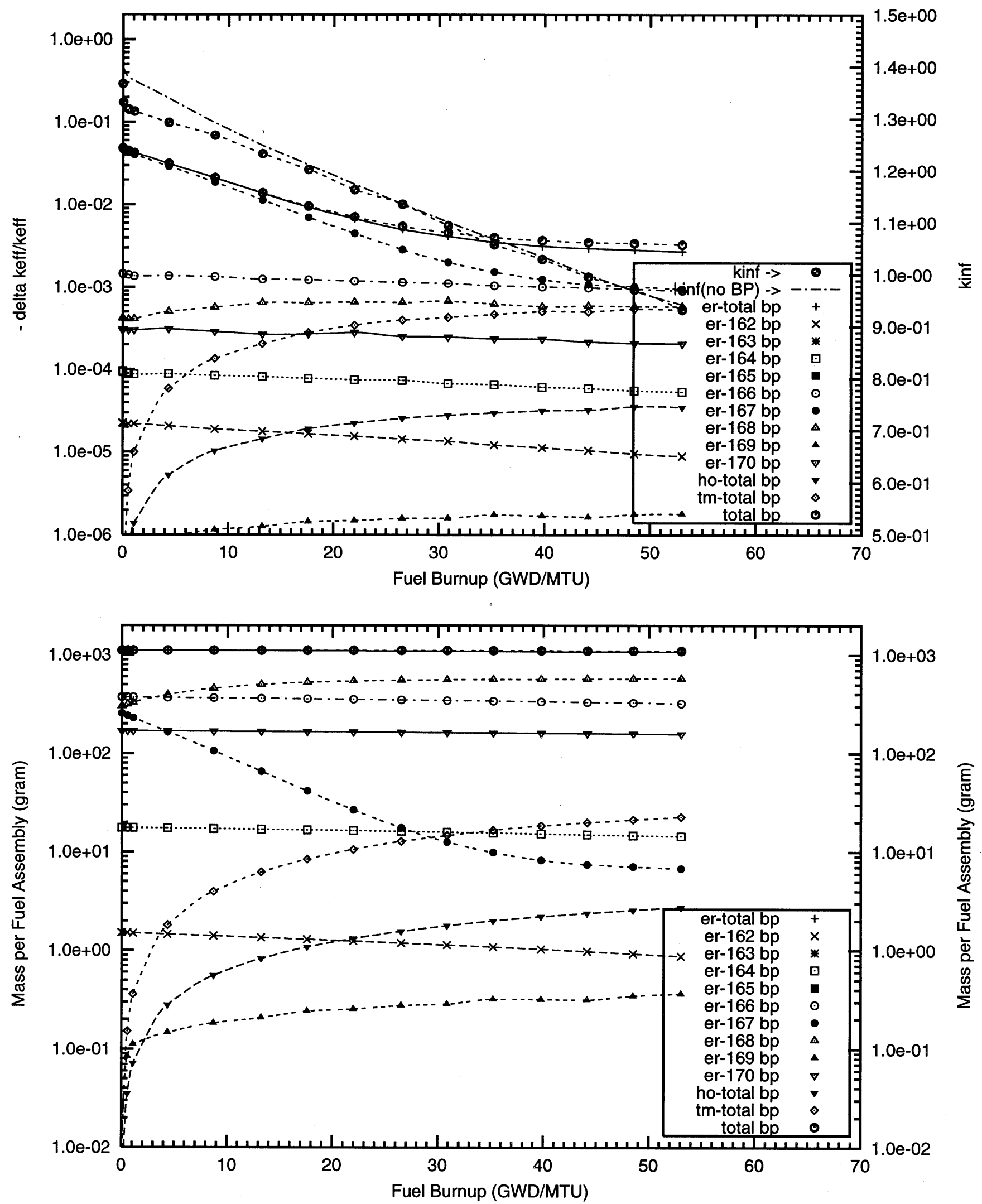
Figure C3.7. Negative Reactivity and Masses BP for Er-nat and transmutation daughters as a function of fuel life for $17 \times 17$ fuel assemblies with 64 poison rods,

2.0 mill thick Er2O3 poison coating on the outer radial surface of the UO2 pellets Reactor power $3400 \mathrm{MWth}, 193$ fuel assemblies, initial enrichment $4.5 \mathrm{w} \% \mathrm{U}-235$

(Case : er000_2f_64_bp_fuel o)
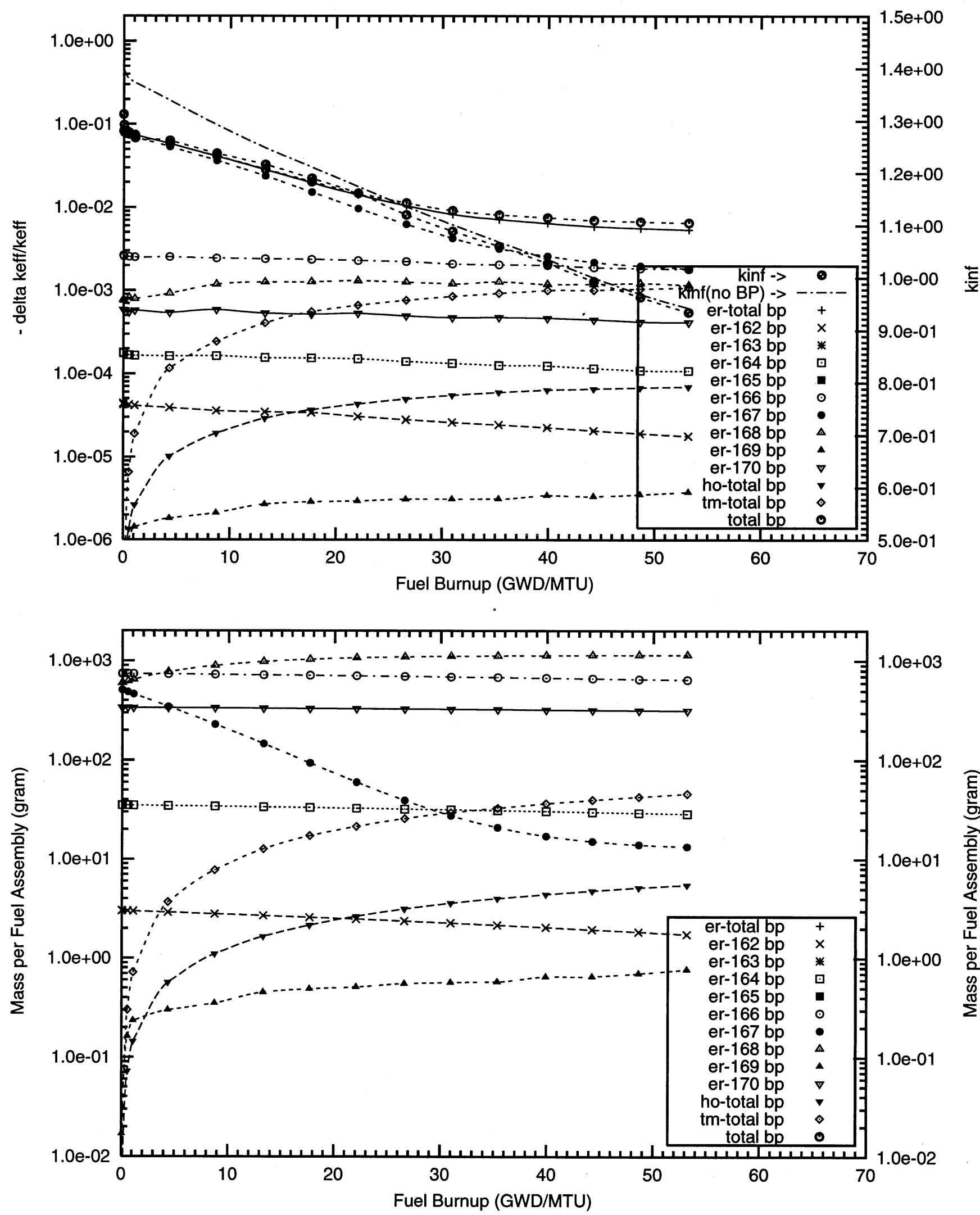
Figure C3.8. Negative Reactivity and Masses BP for Er-nat and transmutation daughters as a function of fuel life for $17 \times 17$ fuel assemblies with 64 poison rods,

4.0 mill thick Er2O3 poison coating on the outer radial surface of the UO2 pellets Reactor power $3400 \mathrm{MWth}, 193$ fuel assemblies, initial enrichment 4.5w\%U-235

(Case : er000_3f_100p_h20_64_bp_fuel_2_o)
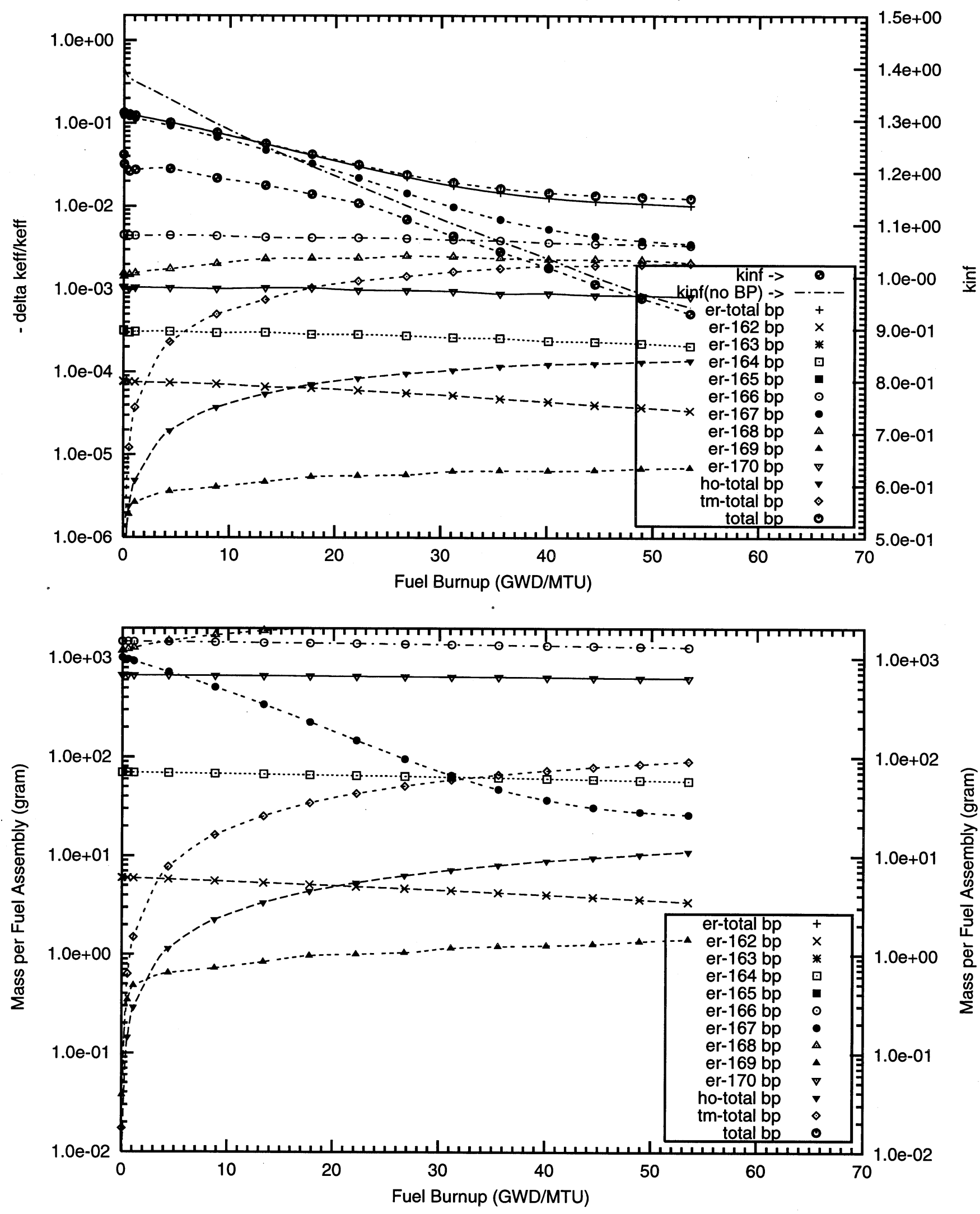
Figure C3.9. Negative Reactivity and Masses BP for Er-nat and transmutation daughters as a function of fuel life for $17 \times 17$ fuel assemblies with 104 poison rods,

1.0 mill thick Er2O3 poison coating on the outer radial surface of the UO2 pellets

Reactor power $3400 \mathrm{MWth}, 193$ fuel assemblies, initial enrichment $4.5 \mathrm{w} \% \mathrm{U}-235$

(Case : er000_1f_104_bp_fuel o)
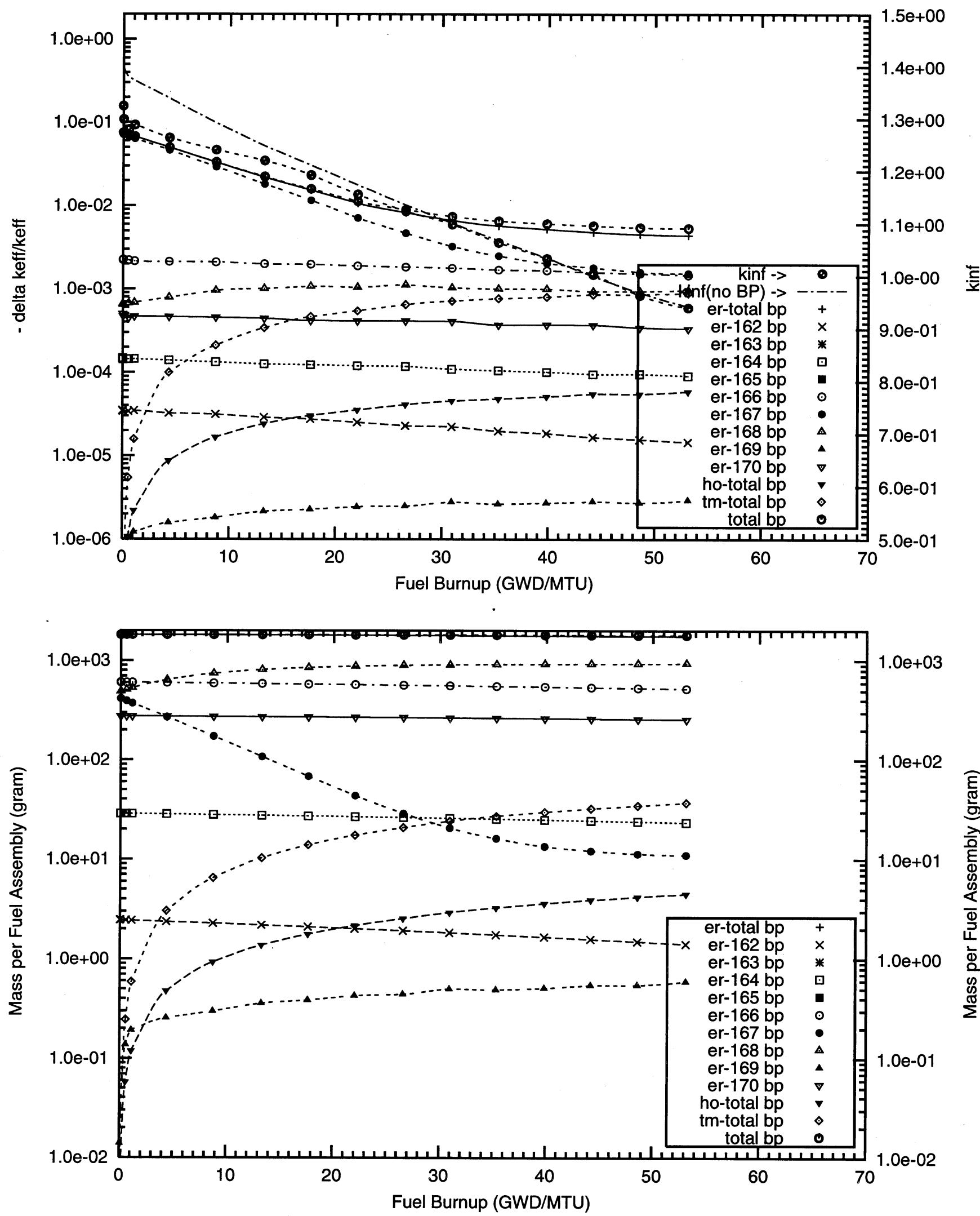

C3 - Erbium Fully Enriched in ${ }^{167} \mathrm{Er}$ 

Figure C3.10. Negative Reactivity and Masses BP for Er-167 and transmutation daughters as a function of fuel life for $17 \times 17$ fuel assemblies with 16 poison rods,

1.0 mill thick Er2O3 poison coating on the outer radial surface of the UO2 pellets

Reactor power $3400 \mathrm{MWth}, 193$ fuel assemblies, initial enrichment $4.5 \mathrm{w} \% \mathrm{U}-235$

(Case : er167_1f_100p_h2o_16_bp_fuel_2_o)

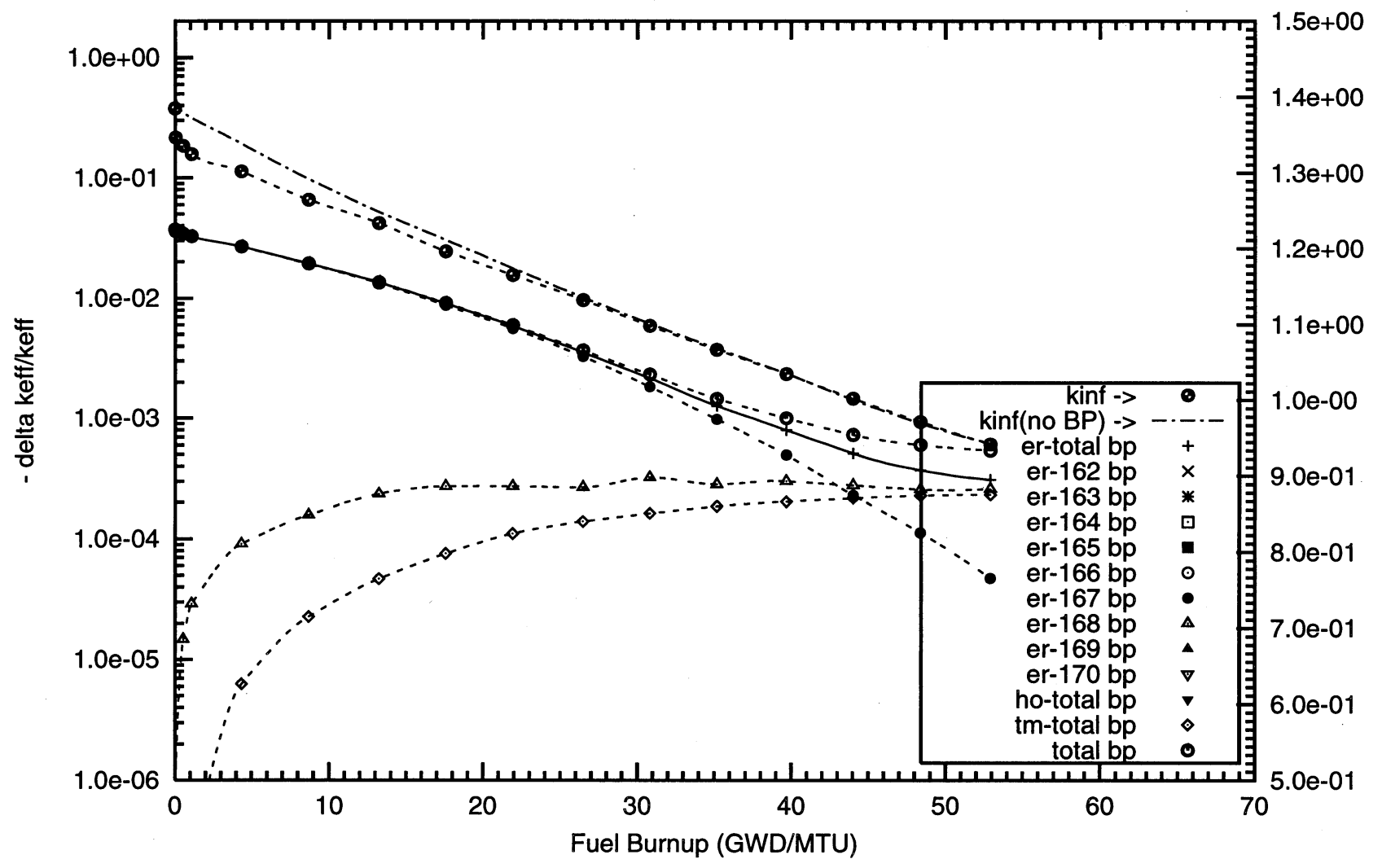

$\overline{\frac{c}{x}}$

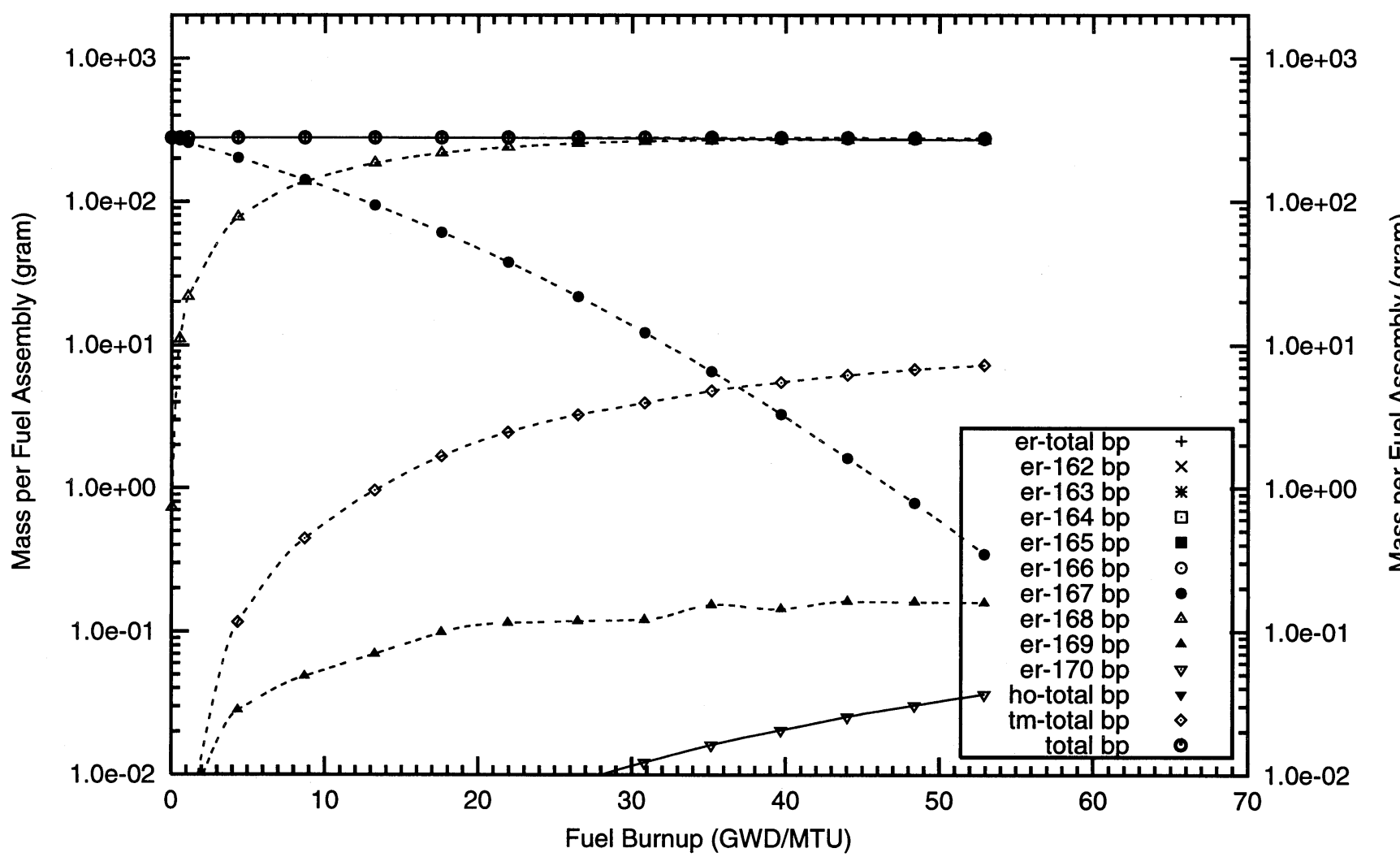


Figure C3.11. Negative Reactivity and Masses BP for Er-167 and transmutation daughters as a function of fuel life for $17 \times 17$ fuel assemblies with 16 poison rods,

2.0 mill thick Er2O3 poison coating on the outer radial surface of the UO2 pellets Reactor power $3400 \mathrm{MWth}, 193$ fuel assemblies, initial enrichment 4.5w\%U-235

(Case : er167_2f_100p_h2o_16_bp_fuel_2_o)
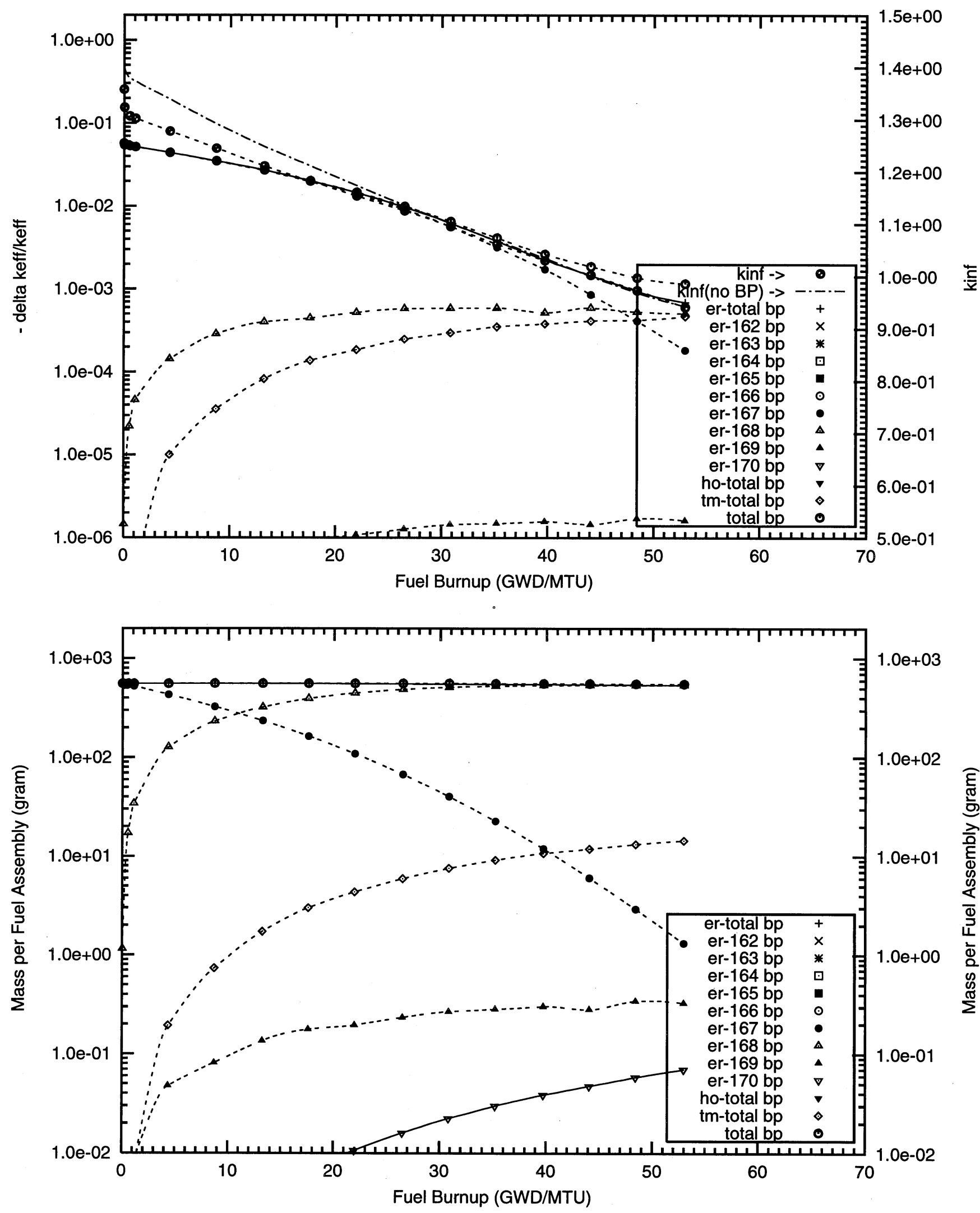
Figure C3.12. Negative Reactivity and Masses BP for Er-167 and transmutation daughters

as a function of fuel life for $17 \times 17$ fuel assemblies with 16 poison rods,

4.0 mill thick Er2O3 poison coating on the outer radial surface of the UO2 pellets

Reactor power $3400 \mathrm{MWth}, 193$ fuel assemblies, initial enrichment $4.5 \mathrm{w} \% \mathrm{U}-235$

(Case : er167_3f_100p_h2o_16_bp_fuel_2_o)

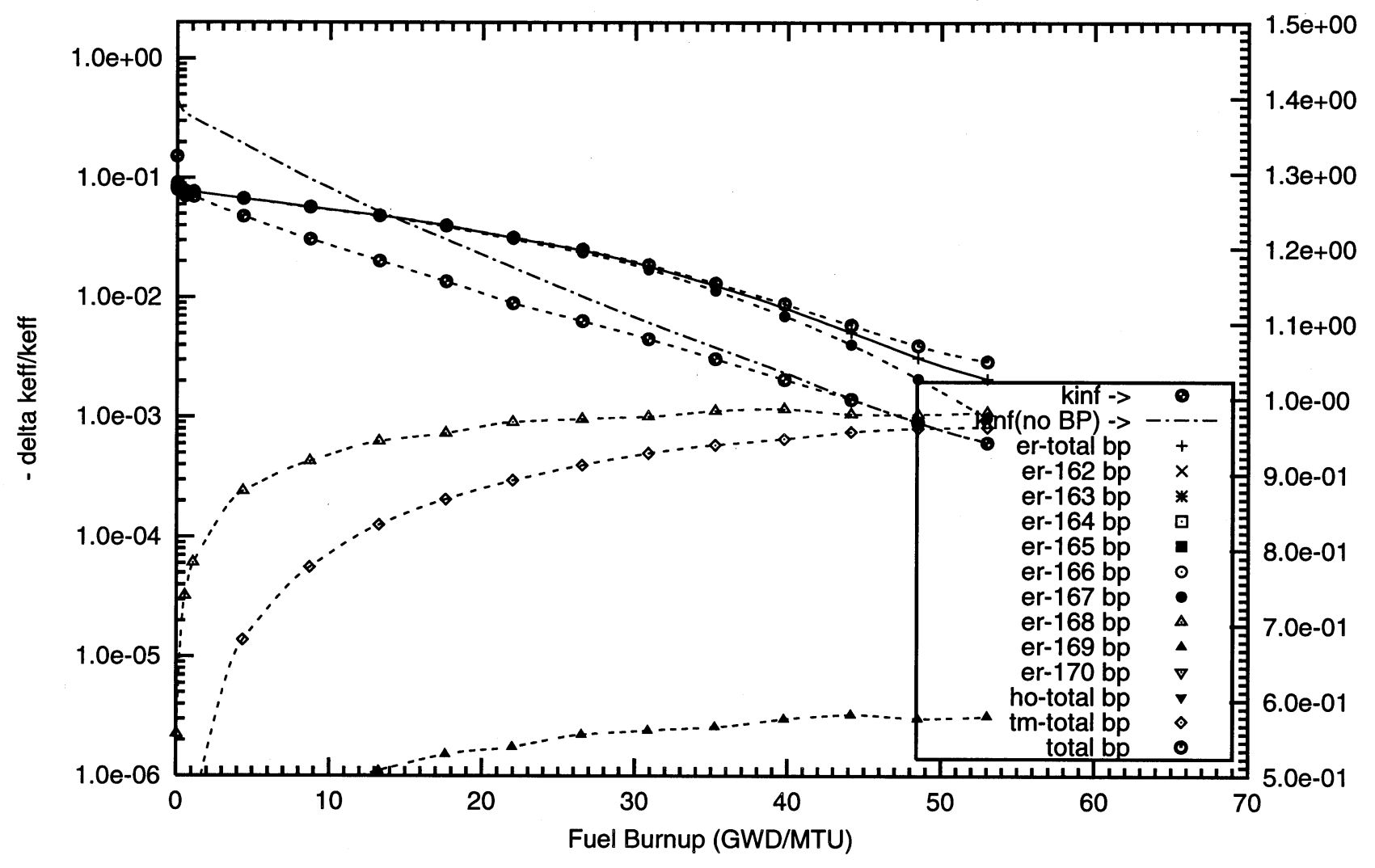

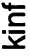

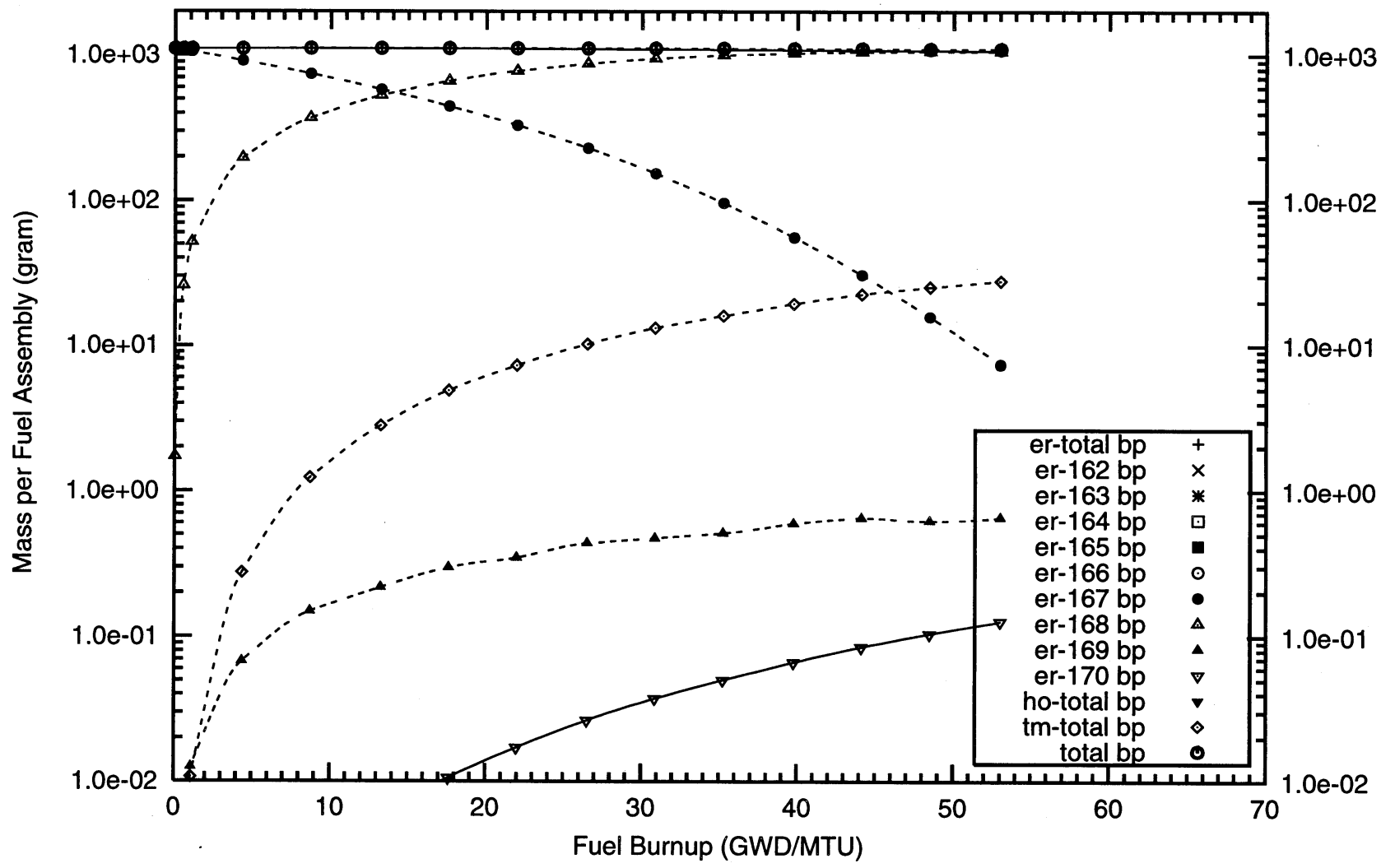


Figure C3.13. Negative Reactivity and Masses BP for Er-167 and transmutation daughters as a function of fuel life for $17 \times 17$ fuel assemblies with 18 poison rods,

1.0 mill thick Er2O3 poison coating on the outer radial surface of the UO2 pellets

Reactor power $3400 \mathrm{MWth}, 193$ fuel assemblies, initial enrichment 4.5w\%U-235

(Case : er167_1f_16_bp_fuel (old) o)
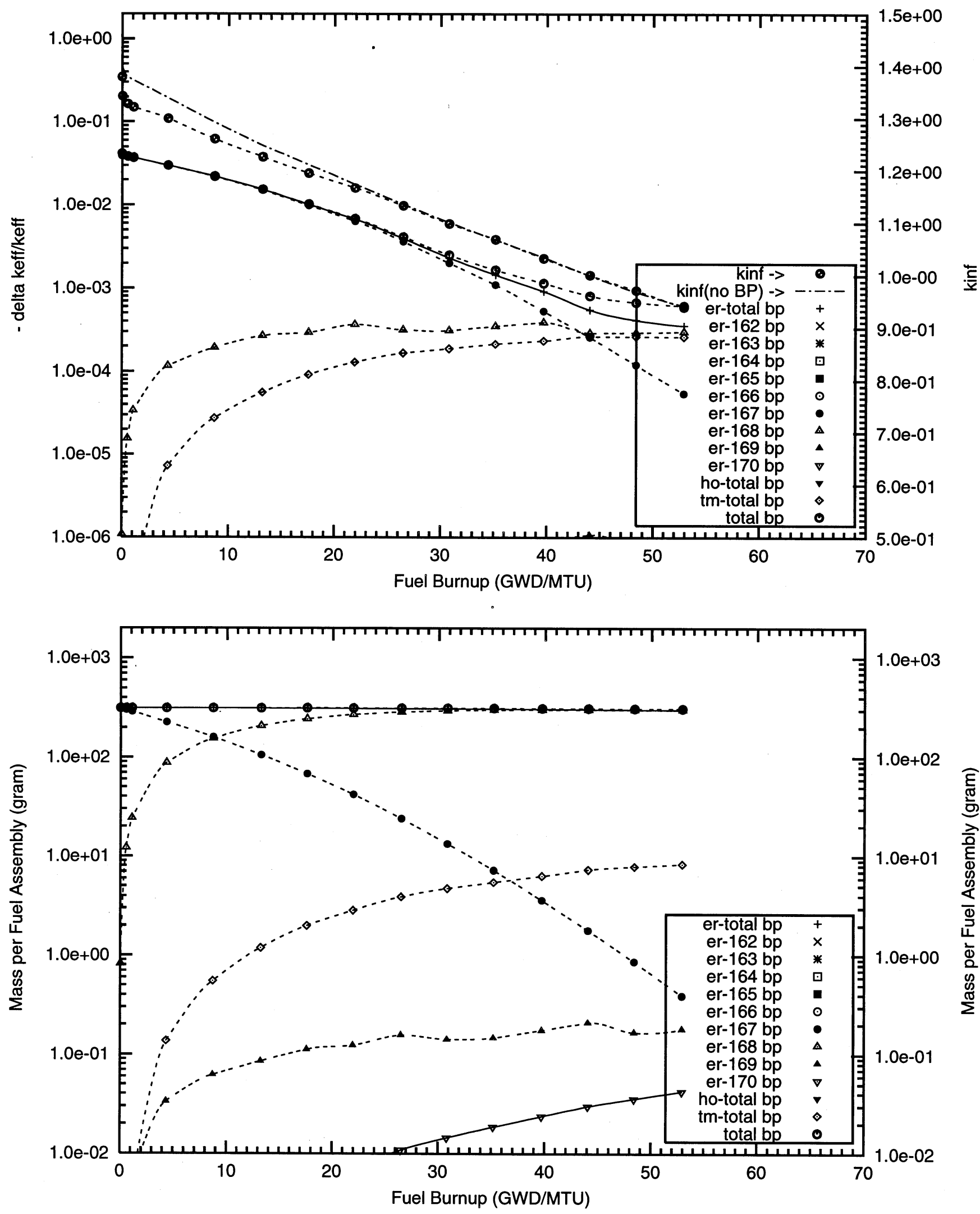
Figure C3.14. Negative Reactivity and Masses BP for Er-167 and transmutation daughters as a function of fuel life for $17 \times 17$ fuel assemblies with 18 poison rods,

2.0 mill thick Er2O3 poison coating on the outer radial surface of the UO2 pellets

Reactor power $3400 \mathrm{MWth}, 193$ fuel assemblies, initial enrichment 4.5w\%U-235

(Case : er167_2f_16_bp_fuel (old) o)
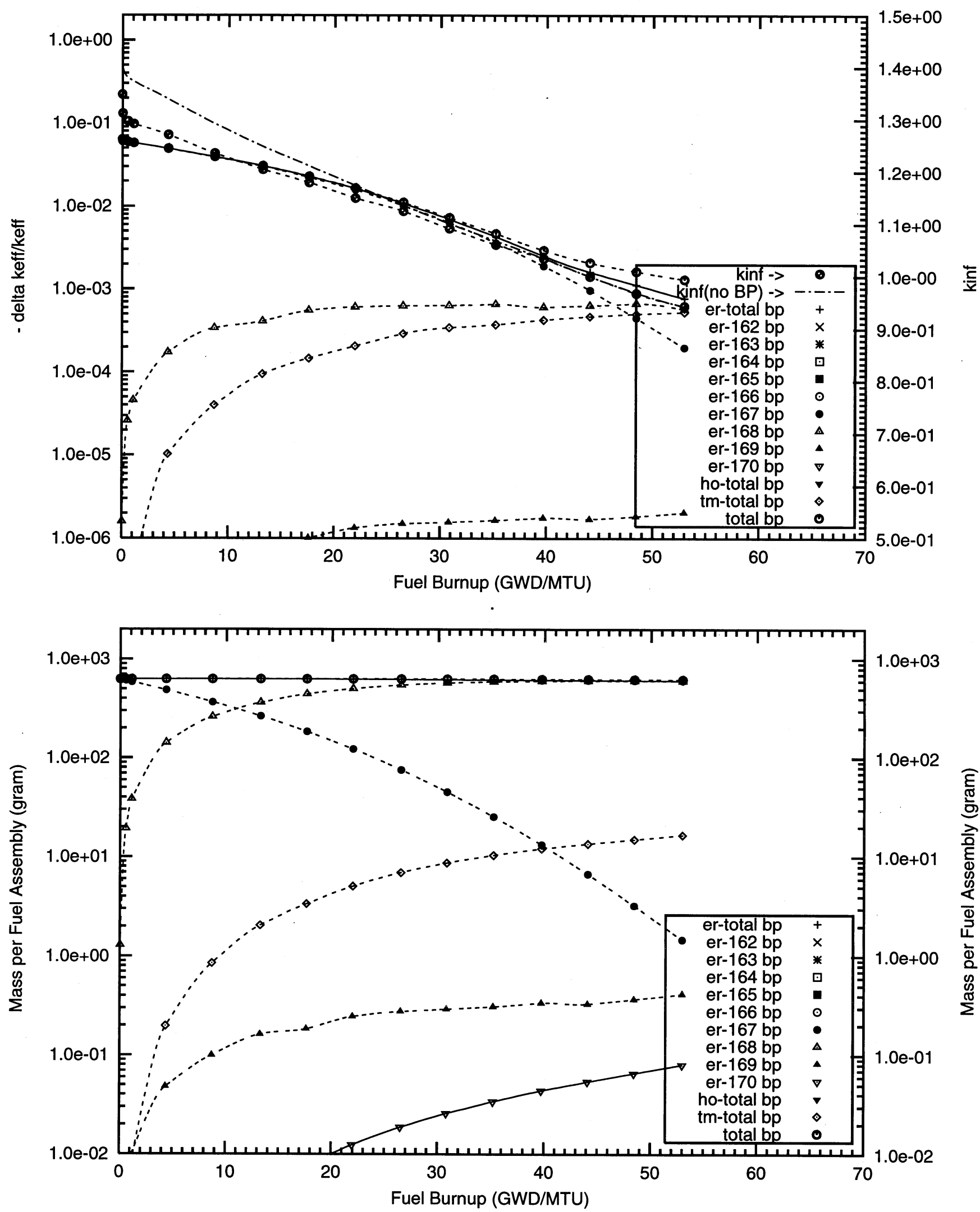
Figure C3.15. Negative Reactivity and Masses BP for Er-167 and transmutation daughters as a function of fuel life for $17 \times 17$ fuel assemblies with 18 poison rods,

4.0 mill thick Er2O3 poison coating on the outer radial surface of the UO2 pellets

Reactor power $3400 \mathrm{MWth}, 193$ fuel assemblies, initial enrichment 4.5w\%U-235

(Case : er167_3f_16_bp_fuel (old) o)

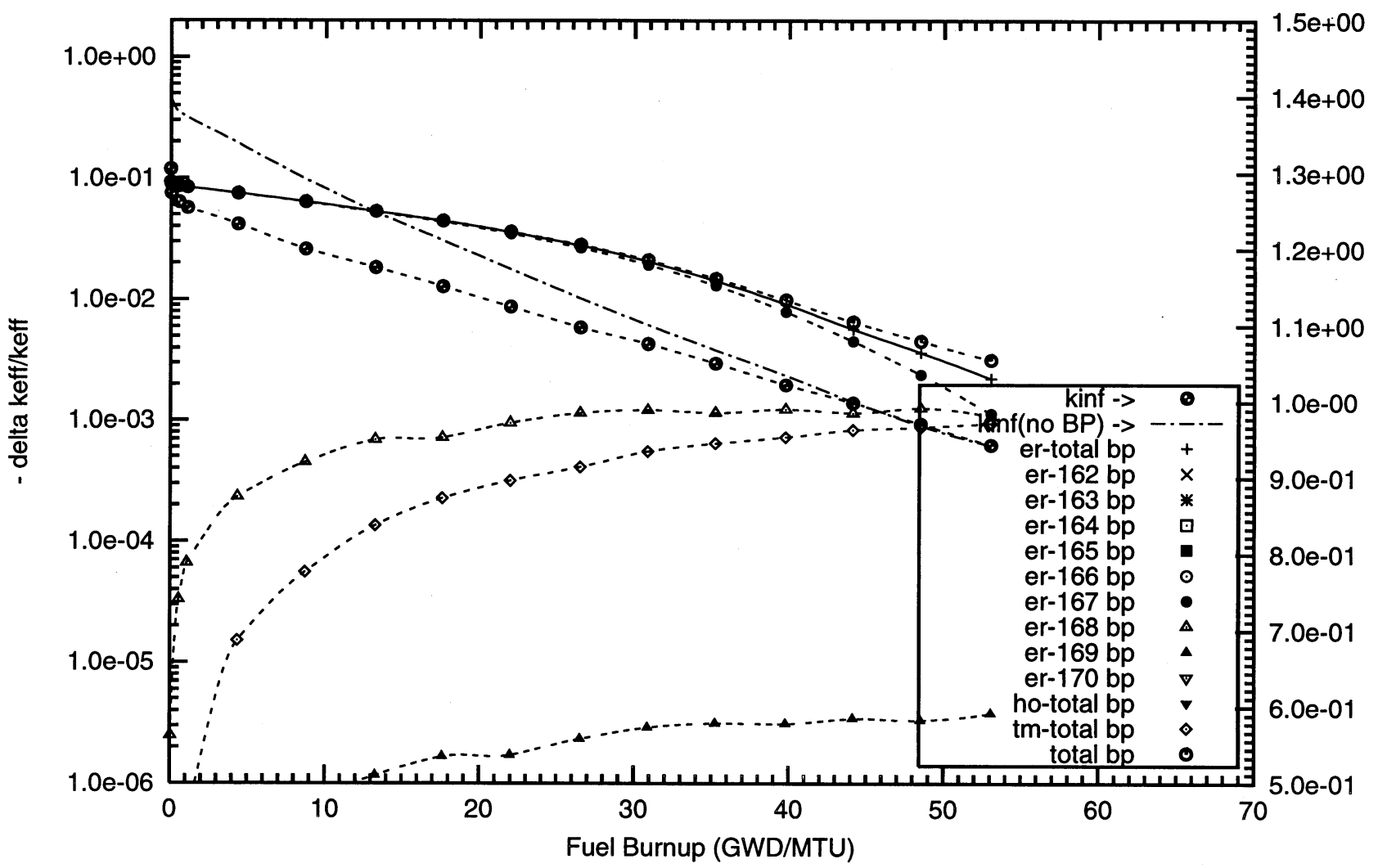

$\frac{\overline{\underline{C}}}{\bar{x}}$

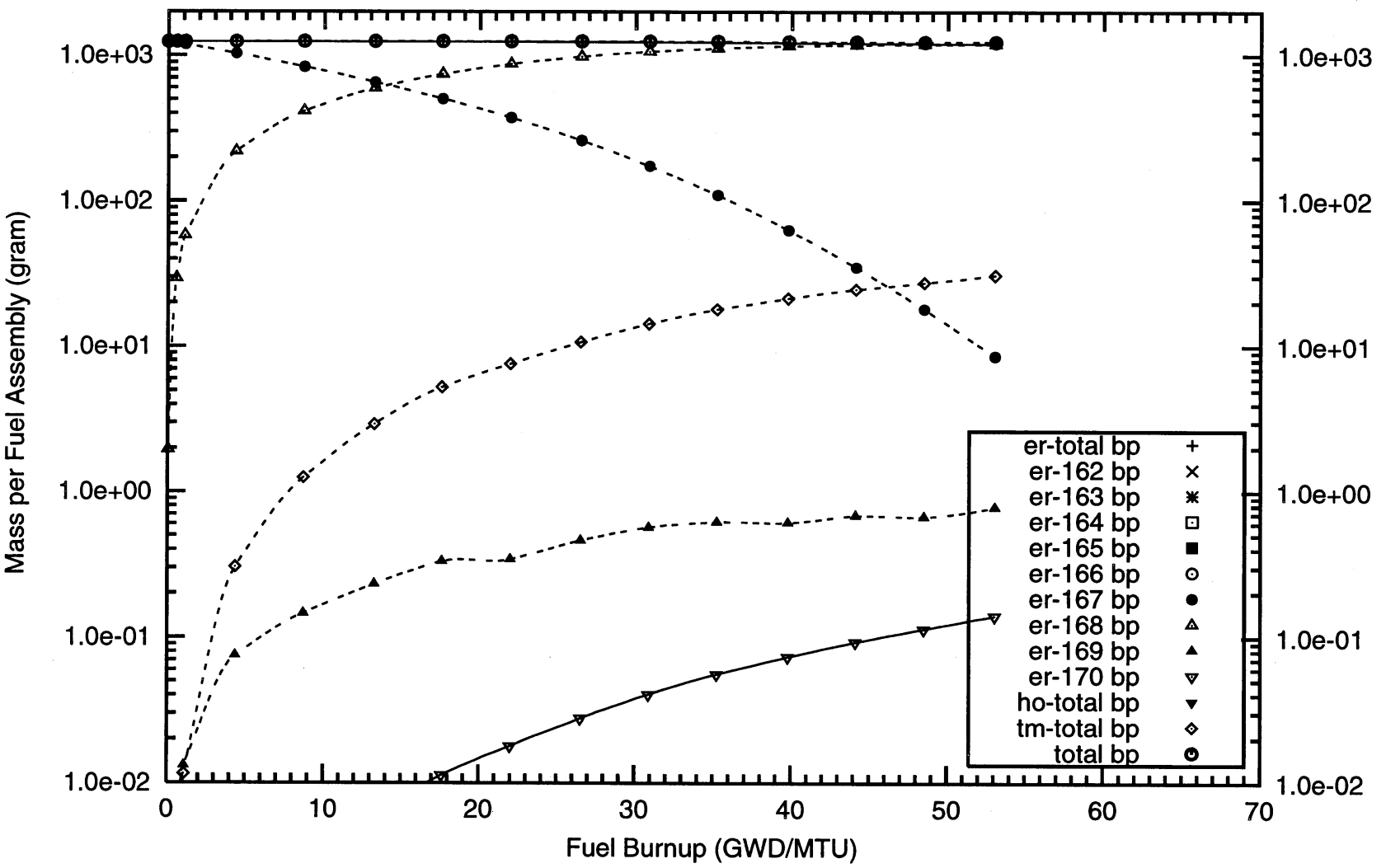


Figure C3.16. Negative Reactivity and Masses BP for Er-167 and transmutation daughters as a function of fuel life for $17 \times 17$ fuel assemblies with 64 poison rods,

0.2 mill thick Er2O3 poison coating on the outer radial surface of the UO2 pellets Reactor power $3400 \mathrm{MWth}, 193$ fuel assemblies, initial enrichment 4.5w\%U-235

(Case : er167_8f_100p_h2o_64_bp_fuel_2_o)
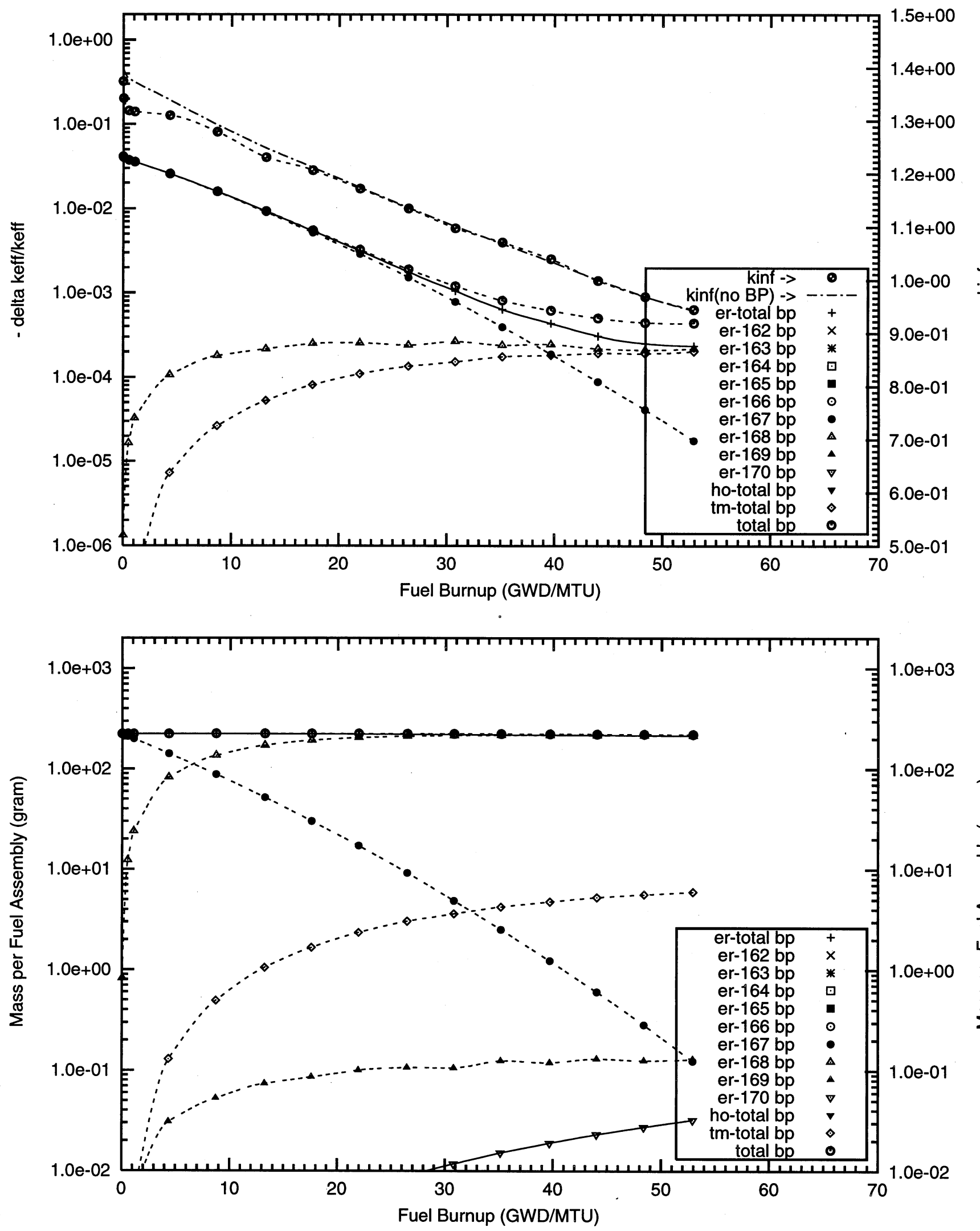
Figure C3.17. Negative Reactivity and Masses BP for Er-167 and transmutation daughters

- as a function of fuel life for $17 \times 17$ fuel assemblies with 64 poison rods,

0.5 mill thick Er2O3 poison coating on the outer radial surface of the UO2 pellets

Reactor power $3400 \mathrm{MWth}, 193$ fuel assemblies, initial enrichment 4.5w\%U-235

(Case : er167_0f_64_bp_fuel o)
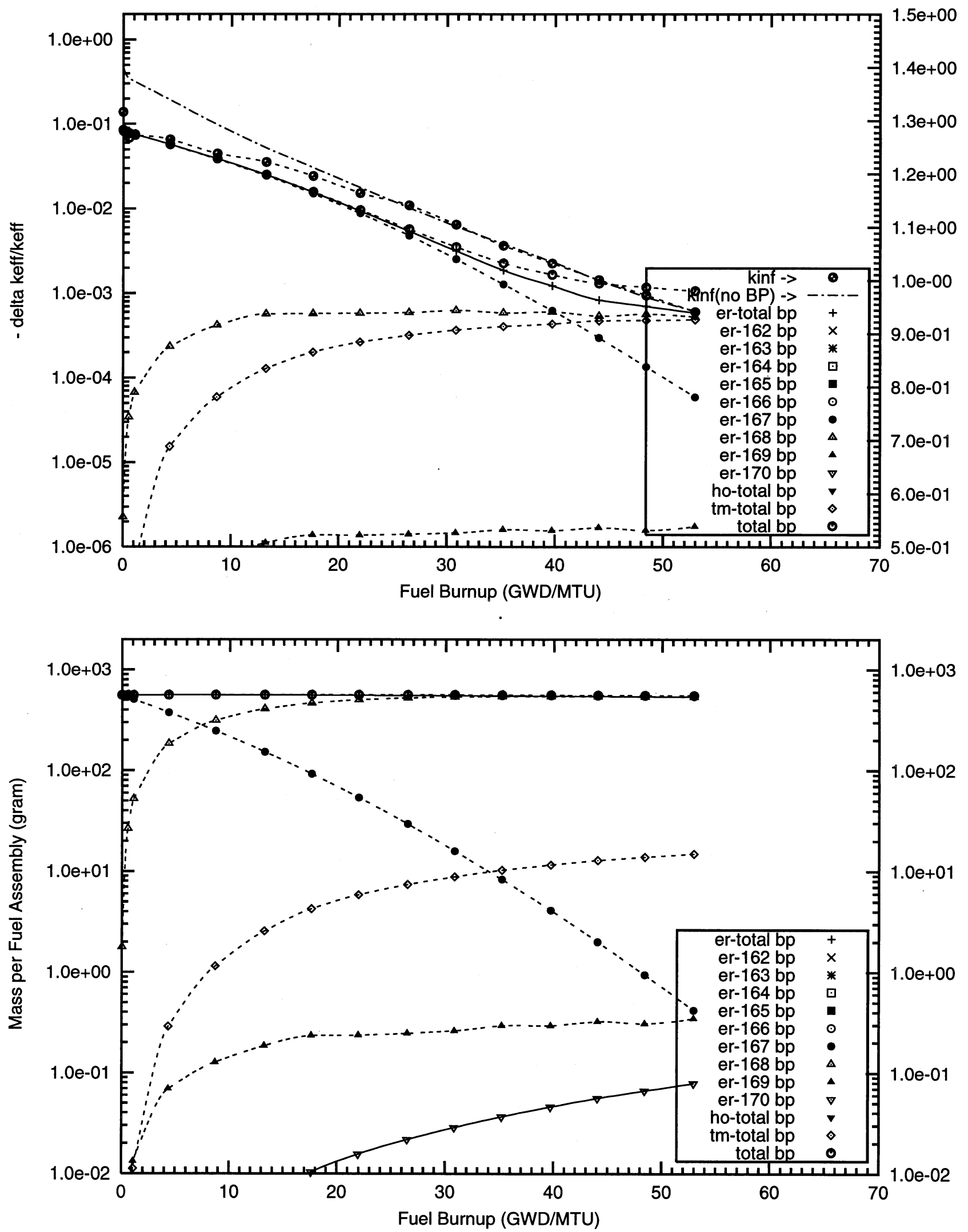
Figure C3.18. Negative Reactivity and Masses BP for Er-167 and transmutation daughters as a function of fuel life for $17 \times 17$ fuel assemblies with 64 poison rods,

1.0 mill thick Er2O3 poison coating on the outer radial surface of the UO2 pellets

Reactor power $3400 \mathrm{MWth}, 193$ fuel assemblies, initial enrichment 4.5w\%U-235

(Case : er167_1f_64_bp_fuel o)
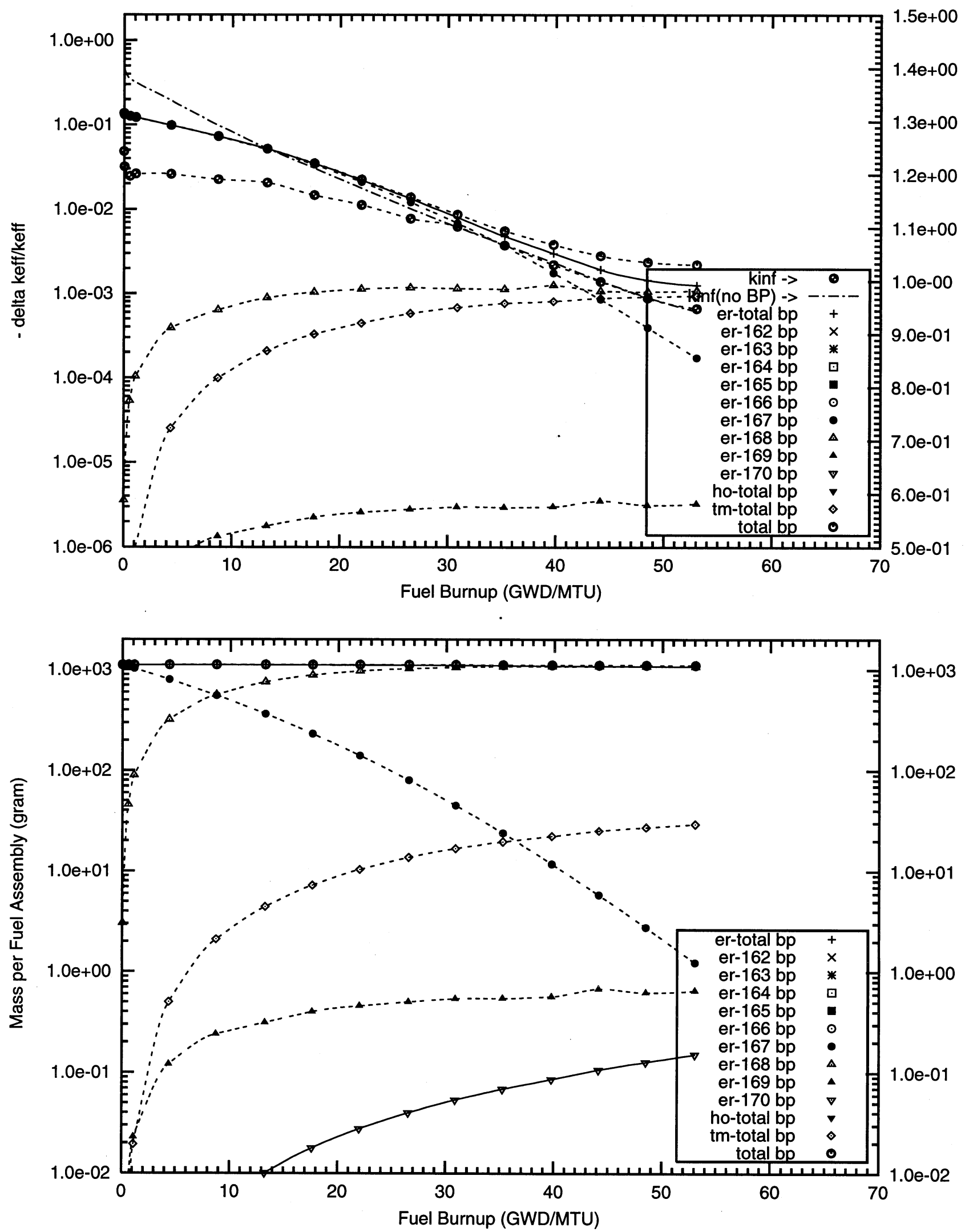
Figure C3.19. Negative Reactivity and Masses BP for Er-167 and transmutation daughters as a function of fuel life for $17 \times 17$ fuel assemblies with 104 poison rods, 0.2 mill thick Er2O3 poison coating on the outer radial surface of the UO2 pellets Reactor power 3400 MWth, 193 fuel assemblies, initial enrichment 4.5w\%U-235 (Case : er167_8f_104_bp_fuel o)
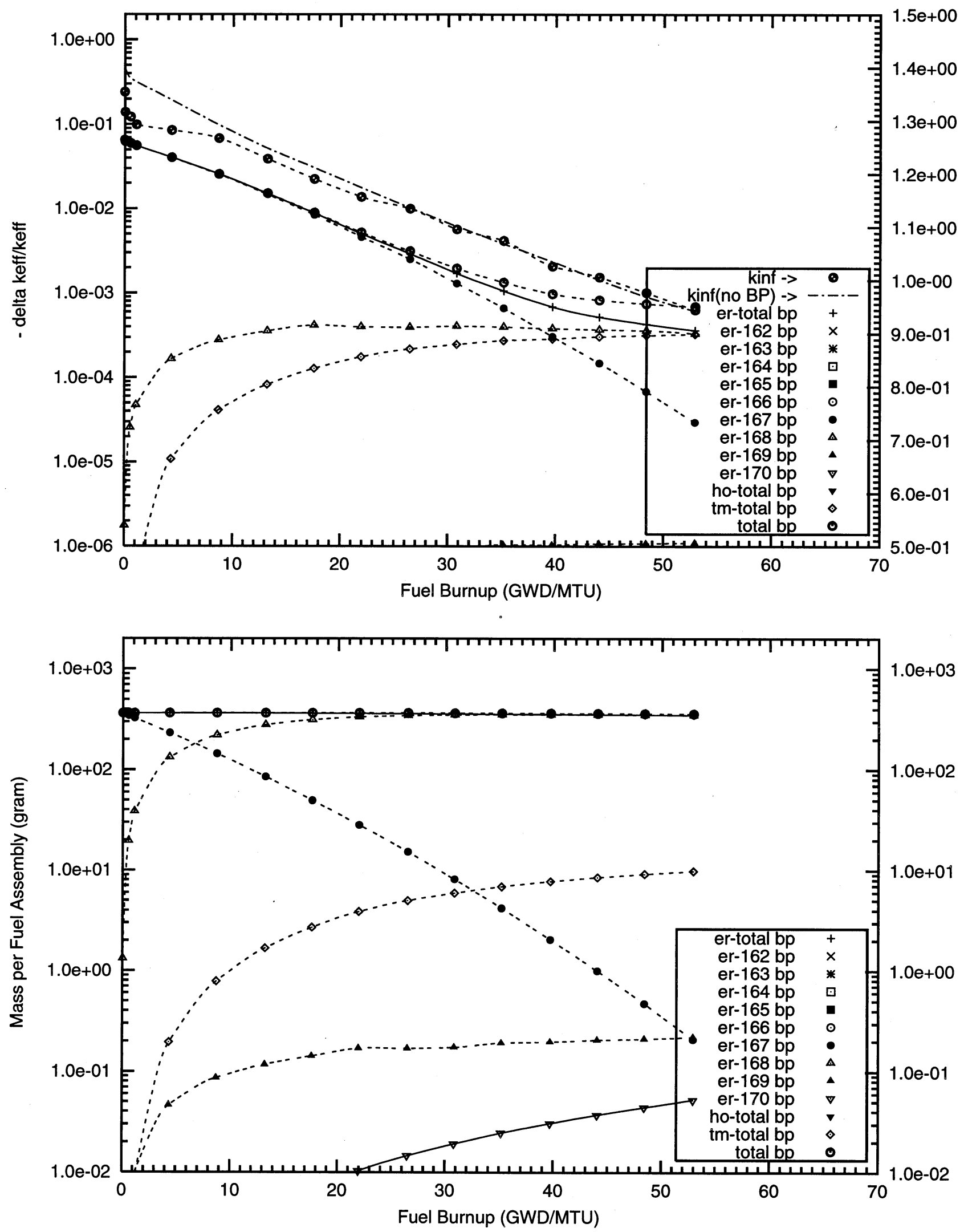
Figure C3.20. Negative Reactivity and Masses BP for Er-167 and transmutation daughters as a function of fuel life for $17 \times 17$ fuel assemblies with 104 poison rods,

0.5 mill thick Er2O3 poison coating on the outer radial surface of the UO2 pellets Reactor power $3400 \mathrm{MWth}, 193$ fuel assemblies, initial enrichment 4.5w\%U-235 (Case : er167_of_104_bp_fuel o)
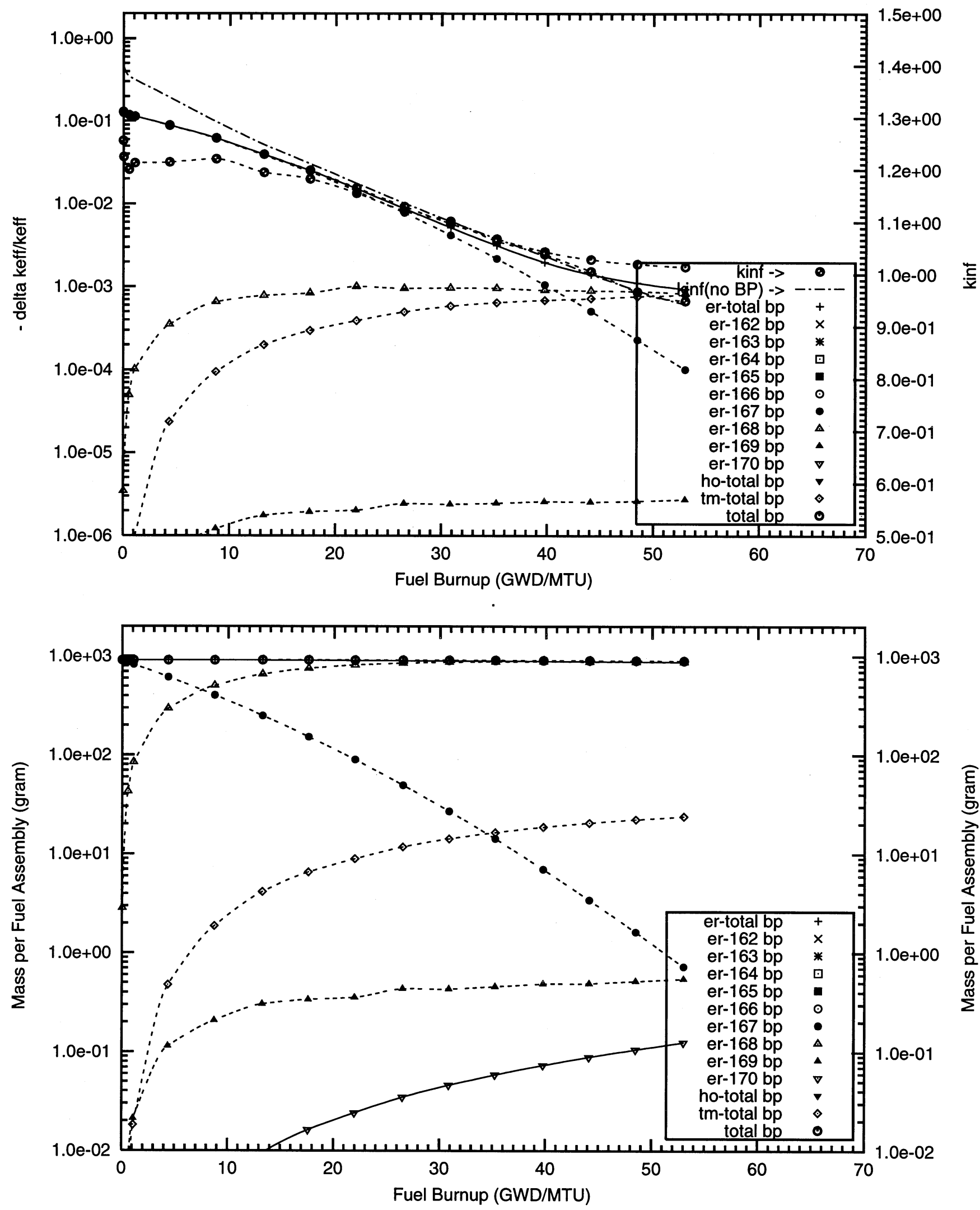


\section{Appendix C4}

Reactivity Worths and Masses of Erbium Alloyed with the Zircaloy Cladding of Fuel Rods

\section{Table 16 Cases}



C4 - Erbium with Natural Isotopic Abundances 

Figure C4.1. Negative Reactivity and Masses BP for Er-nat and transmutation daughters as a function of fuel life for $17 \times 17$ fuel assemblies with 104 poison rods,

$1.0 \mathrm{w} \%$ Er poison homogeneously mixed in the fuel cladding,

Reactor power $3400 \mathrm{MWth}, 193$ fuel assemblies, initial enrichment 4.5w\%U-235

(Case : er000_1c_104_bp_fuel o)
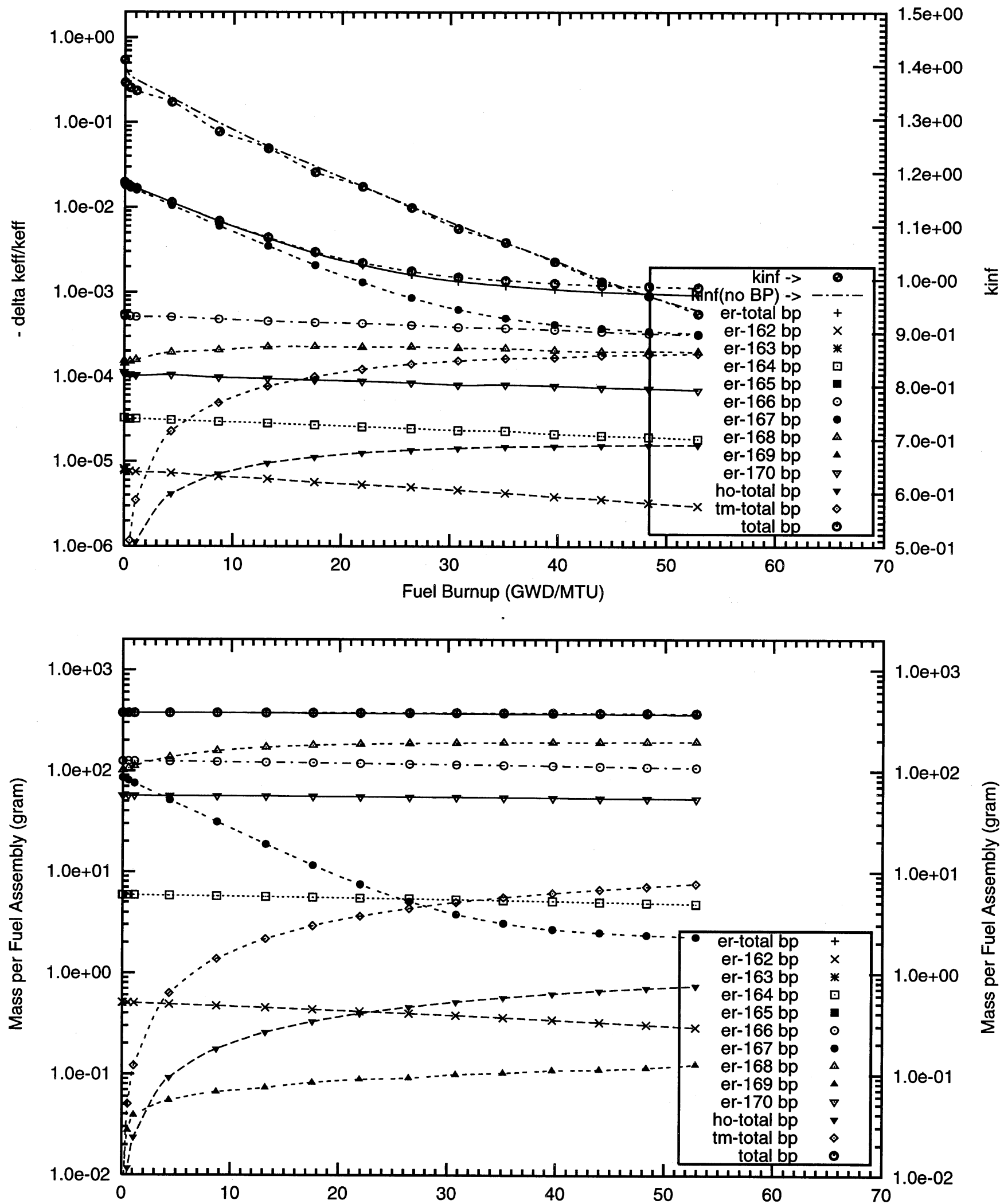

Fuel Burnup (GWD/MTU) 
Figure C4.2. Negative Reactivity and Masses BP for Er-nat and transmutation daughters as a function of fuel life for $17 \times 17$ fuel assemblies with 104 poison rods",

$4.0 \mathrm{w} \%$ Er poison homogeneously mixed in the fuel cladding,

Reactor power 3400 MWth, 193 fuel assemblies, initial enrichment $4.5 w \% U-235$

(Case : er000_3c_100p_h20_104_bp_fuel_2_o)
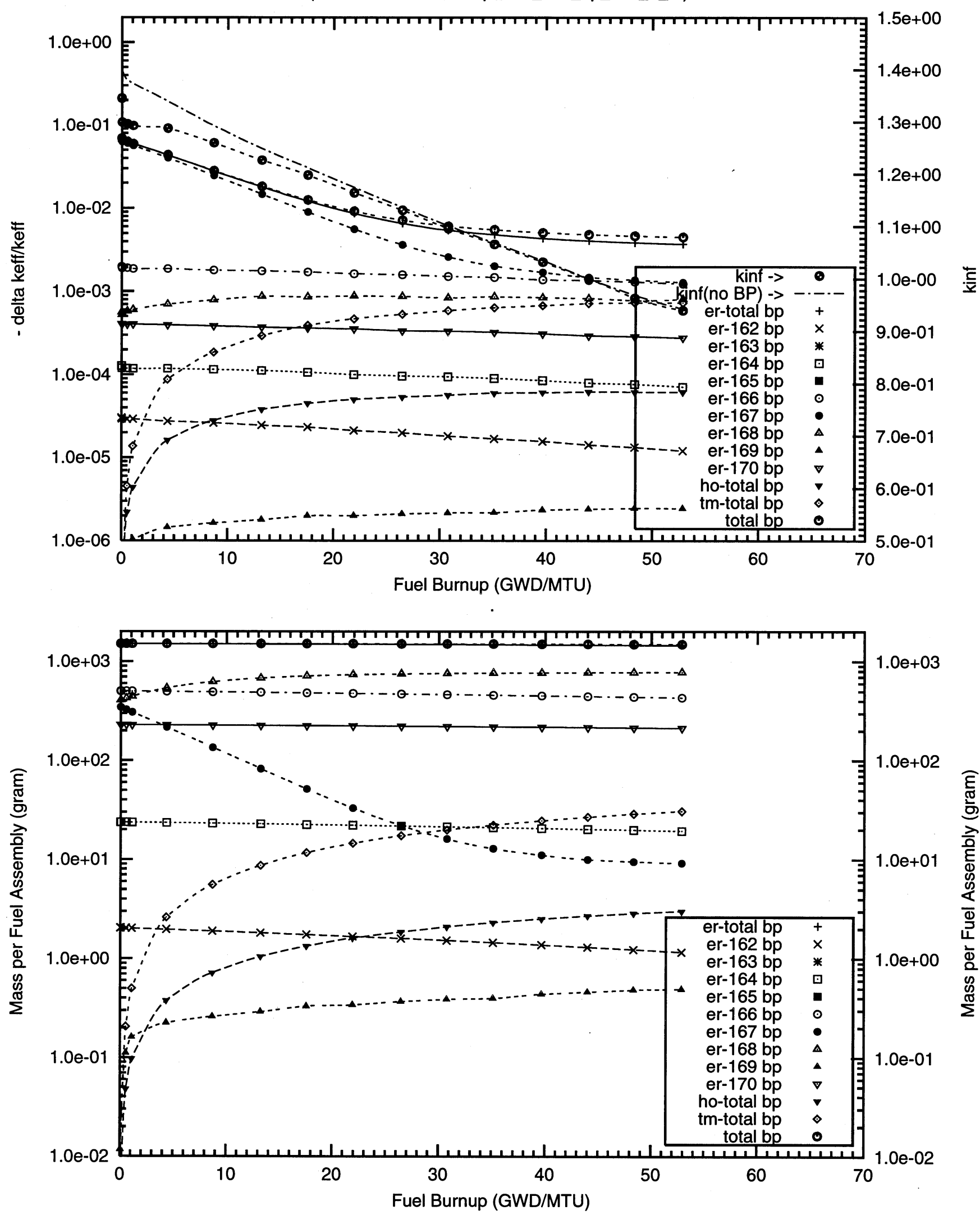
C4 - Erbium Fully Enriched in ${ }^{167} \mathrm{Er}$ 
Figure C4.3. Negative Reactivity and Masses BP for Er-167 and transmutation daughters as a function of fuel life for $17 \times 17$ fuel assemblies with 104 poison rods,

$0.5 \mathrm{w} \%$ Er poison homogeneously mixed in the fuel cladding,

Reactor power $3400 \mathrm{MWth}, 193$ fuel assemblies, initial enrichment $4.5 \mathrm{w} \% \mathrm{U}-235$

(Case : er167_0c_104_bp_fuel o)
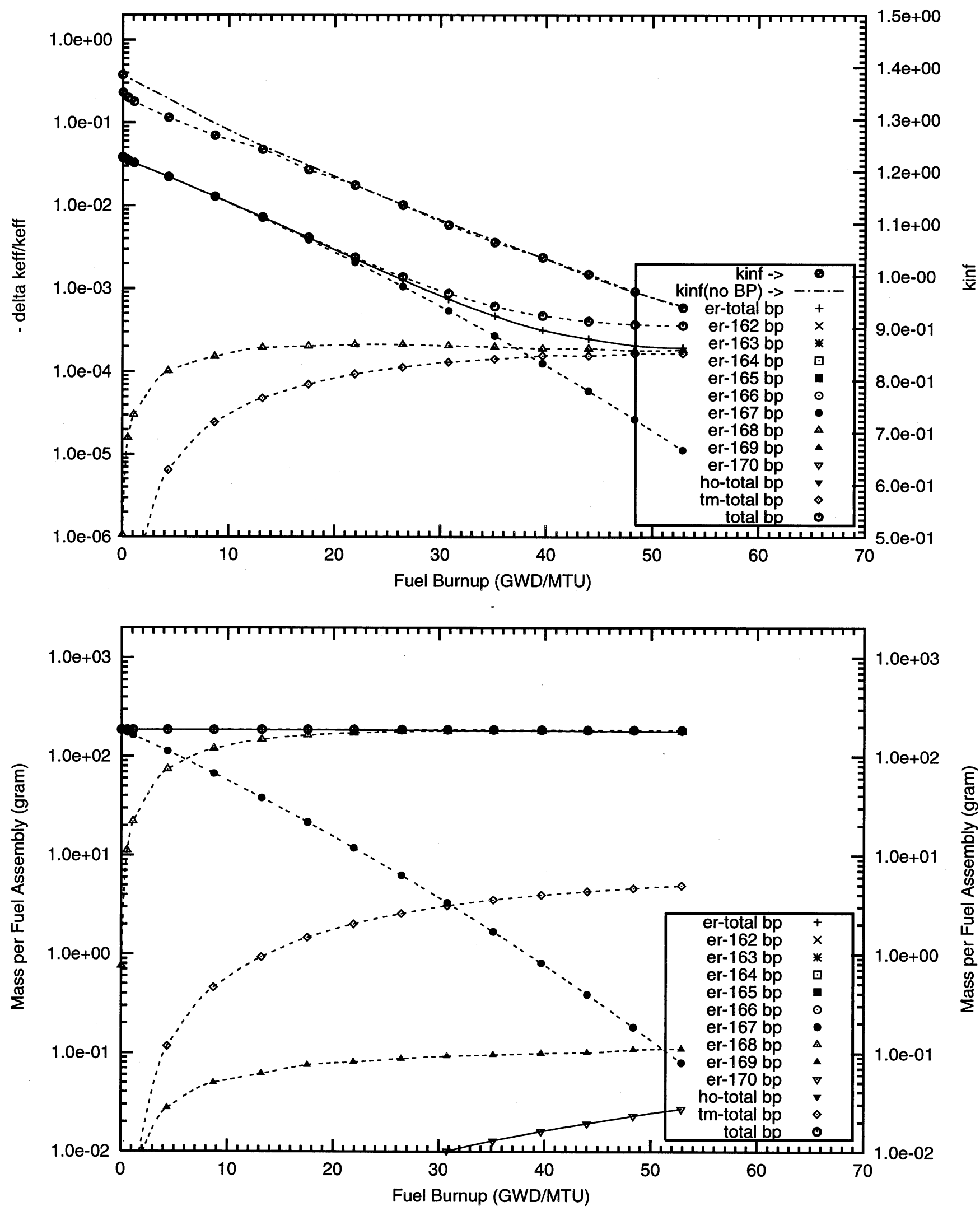
Figure C4.4. Negative Reactivity and Masses BP for Er-167 and transmutation daughters as a function of fuel life for $17 \times 17$ fuel assemblies with 104 poison rods,

$1.0 \mathrm{w} \%$ Er poison homogeneously mixed in the fuel cladding.

Reactor power $3400 \mathrm{MWth}, 193$ fuel assemblies, initial enrichment $4.5 \mathrm{w} \% \mathrm{U}-235$

(Case : er167_1c_100p_h20_104_bp_fuel_2_o)
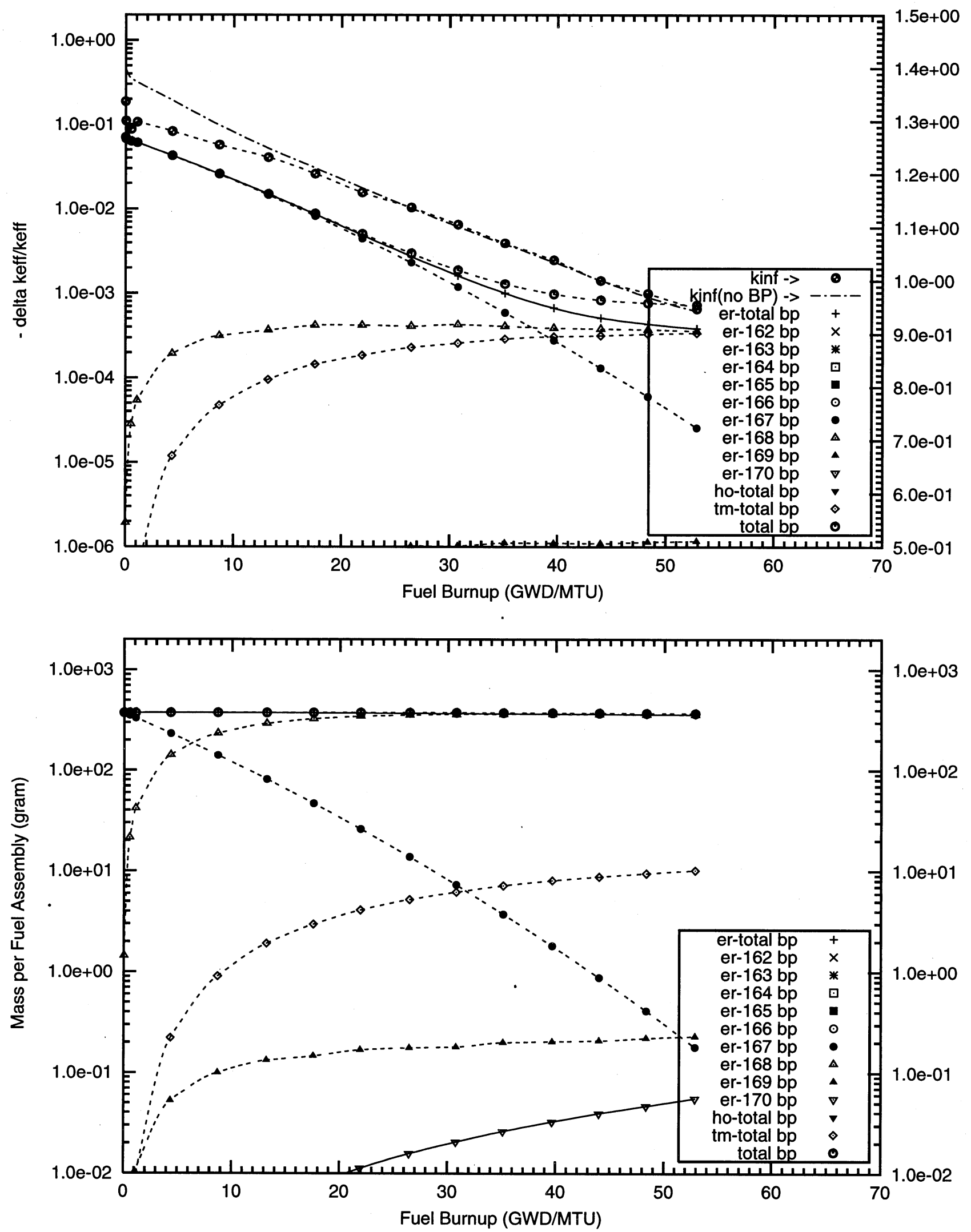
Figure C4.5. Negative Reactivity and Masses BP for Er-167 and transmutation daughters

as a function of fuel life for $17 \times 17$ fuel assemblies with 104 poison rods,

$2.0 \mathrm{w} \% \mathrm{Er}$ poison homogeneously mixed in the fuel cladding,

Reactor power $3400 \mathrm{MWth}, 193$ fuel assemblies, initial enrichment $4.5 \mathrm{w} \% \mathrm{U}-235$

(Case : er167.2c_104_bp_fuel o)
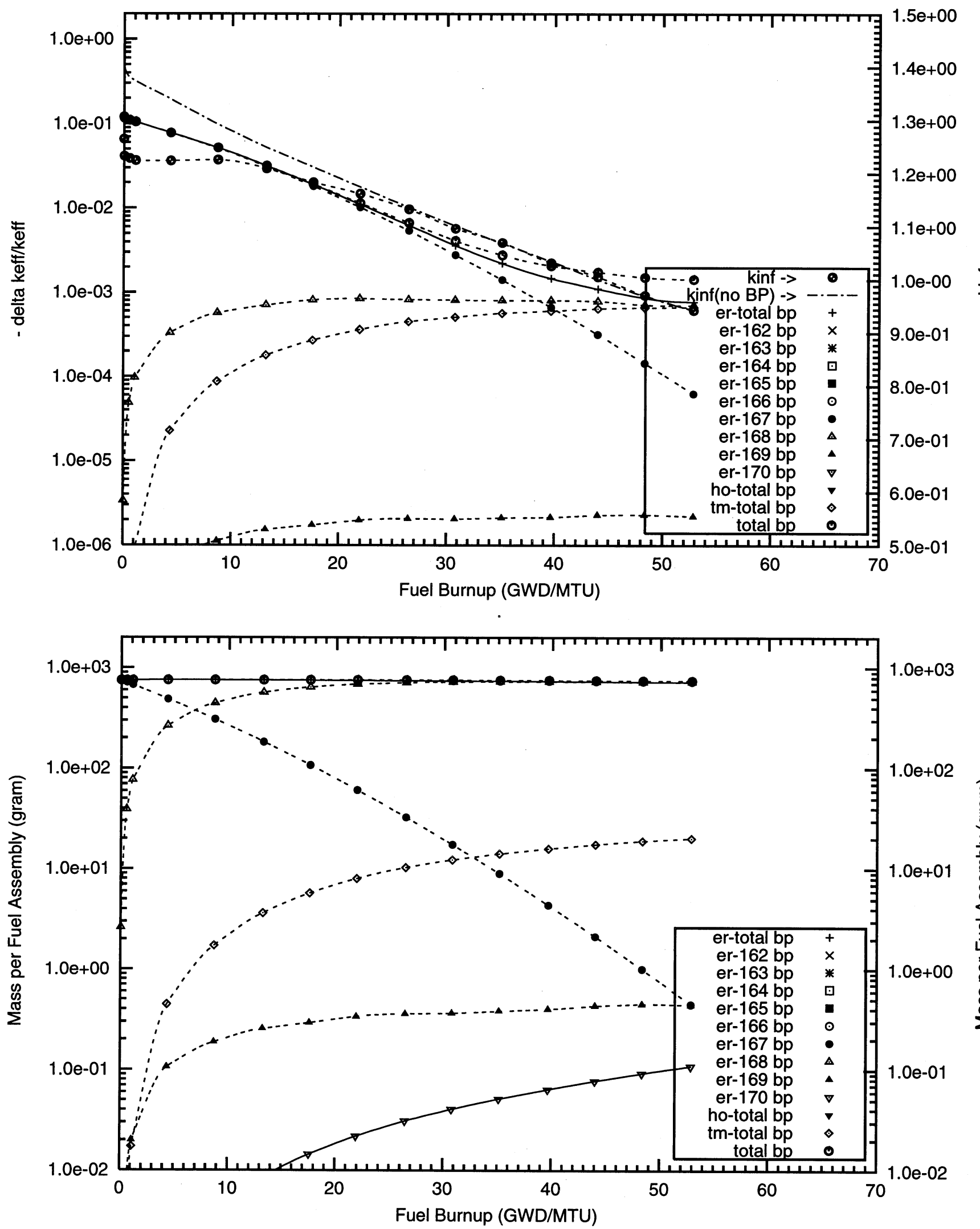
Figure C4.6. Negative Reactivity and Masses BP for Er-167 and transmutation daughters as a function of fuel life for $17 \times 17$ fuel assemblies with 104 poison rods,

$2.0 \mathrm{w} \%$ Er poison homogeneously mixed in the fuel cladding,

Reactor power $3400 \mathrm{MWth}, 193$ fuel assemblies, initial enrichment 6.0w\%U-235

(Case : er167_2c_enru_6p0_104_bp_fuel o)

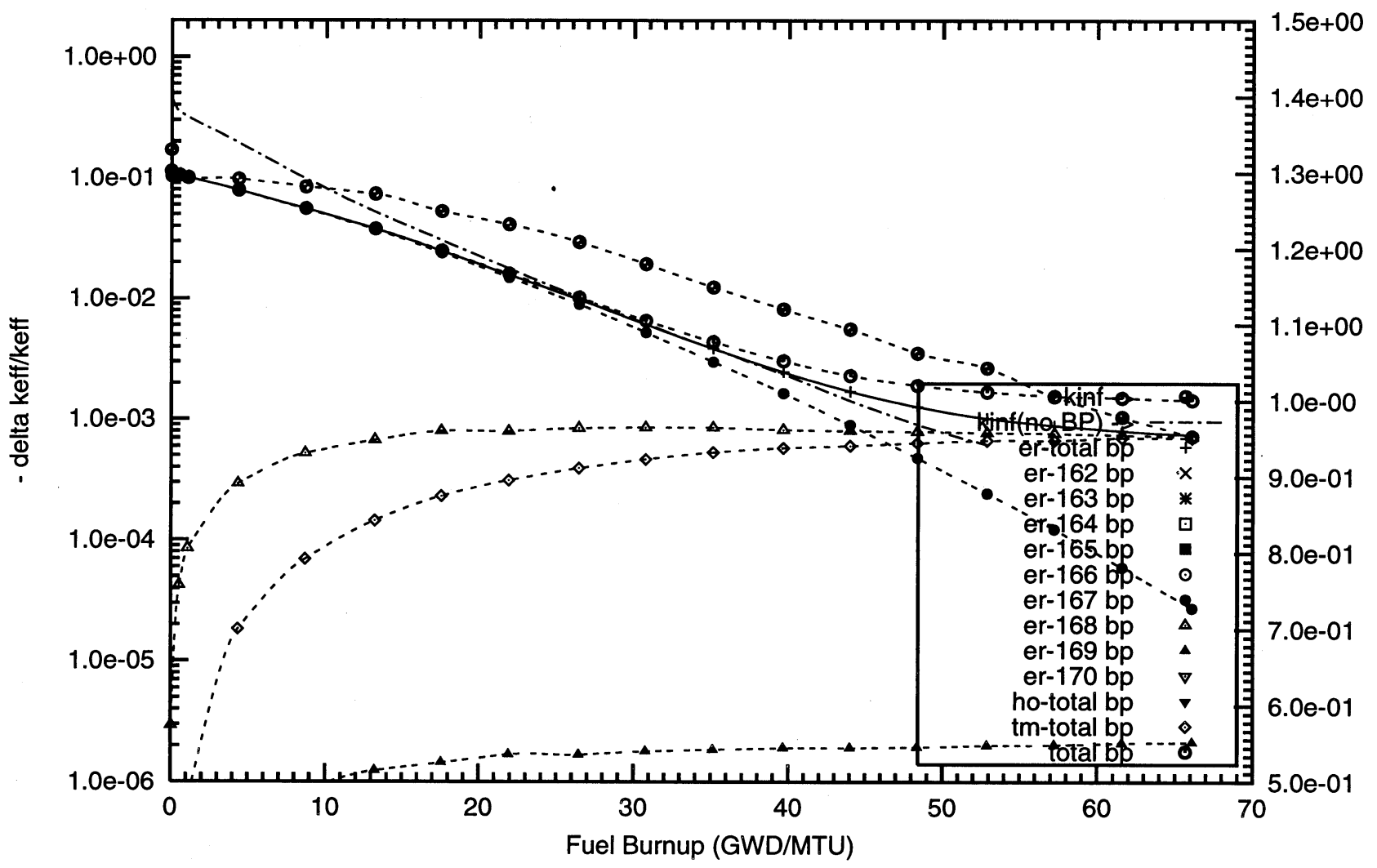

$\frac{\bar{E}}{\bar{x}}$

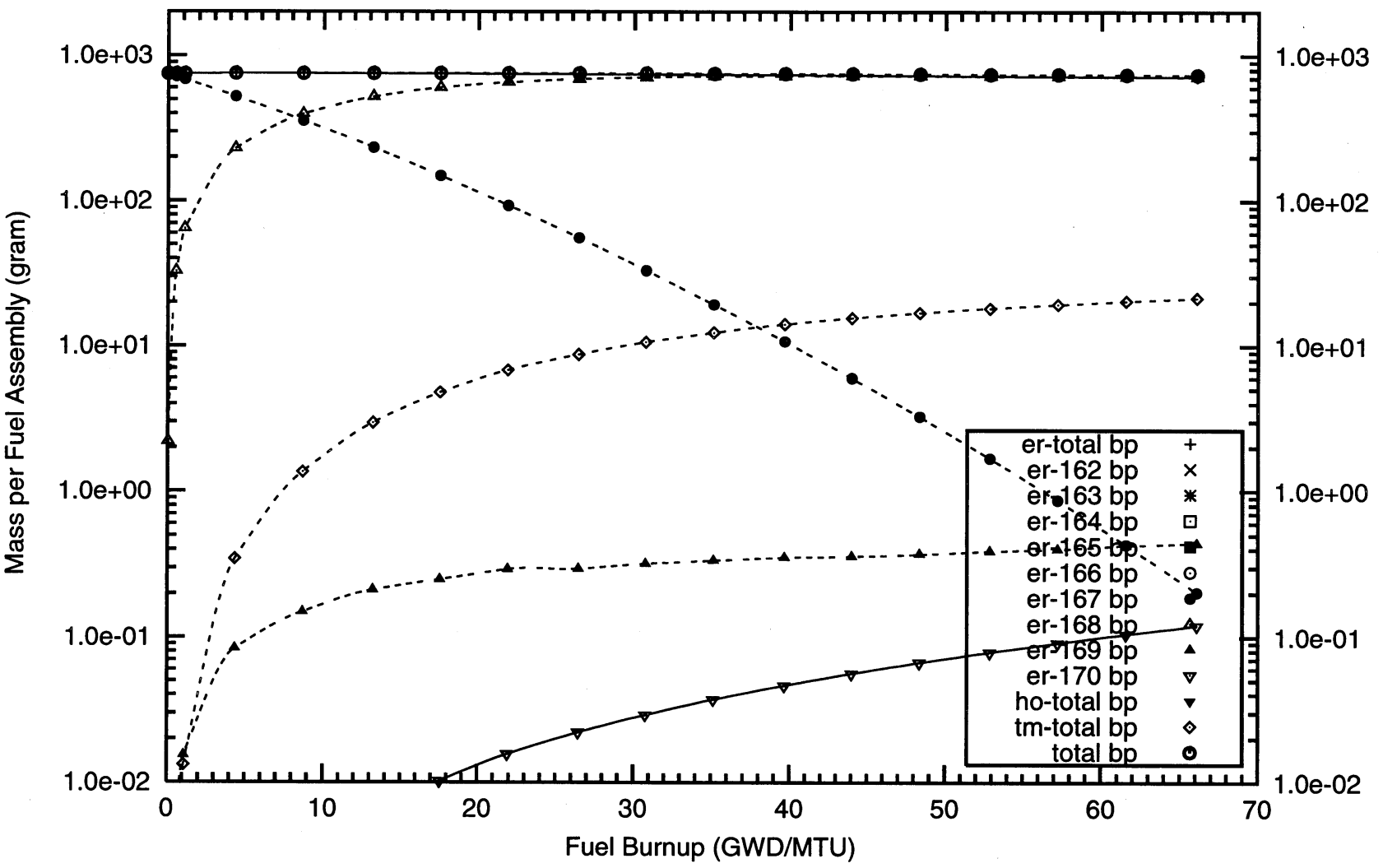




\title{
Appendix D1
}

Reactivity Worths and Masses of $\mathrm{Dy}_{2} \mathrm{O}_{3}$ Burnable Poison Homogeneously Mixed in the Fuel Pellets

\author{
Table 17 Cases
}




\section{D1 - Dysprosium with Natural Isotopic Abundances}


Figure D1.1. Negative Reactivity and Masses BP for Dy-nat and transmutation daughters as a function of fuel life for $17 \times 17$ fuel assemblies with 08 poison rods, $4.0 \mathrm{w} \%$ Dy2O3 poison homogeneously mixed in the UO2 pellets

Reactor power $3400 \mathrm{MWth}, 193$ fuel assemblies, initial enrichment 4.5w\%U-235

(Case : dy000_3_08_bp_fuel o)

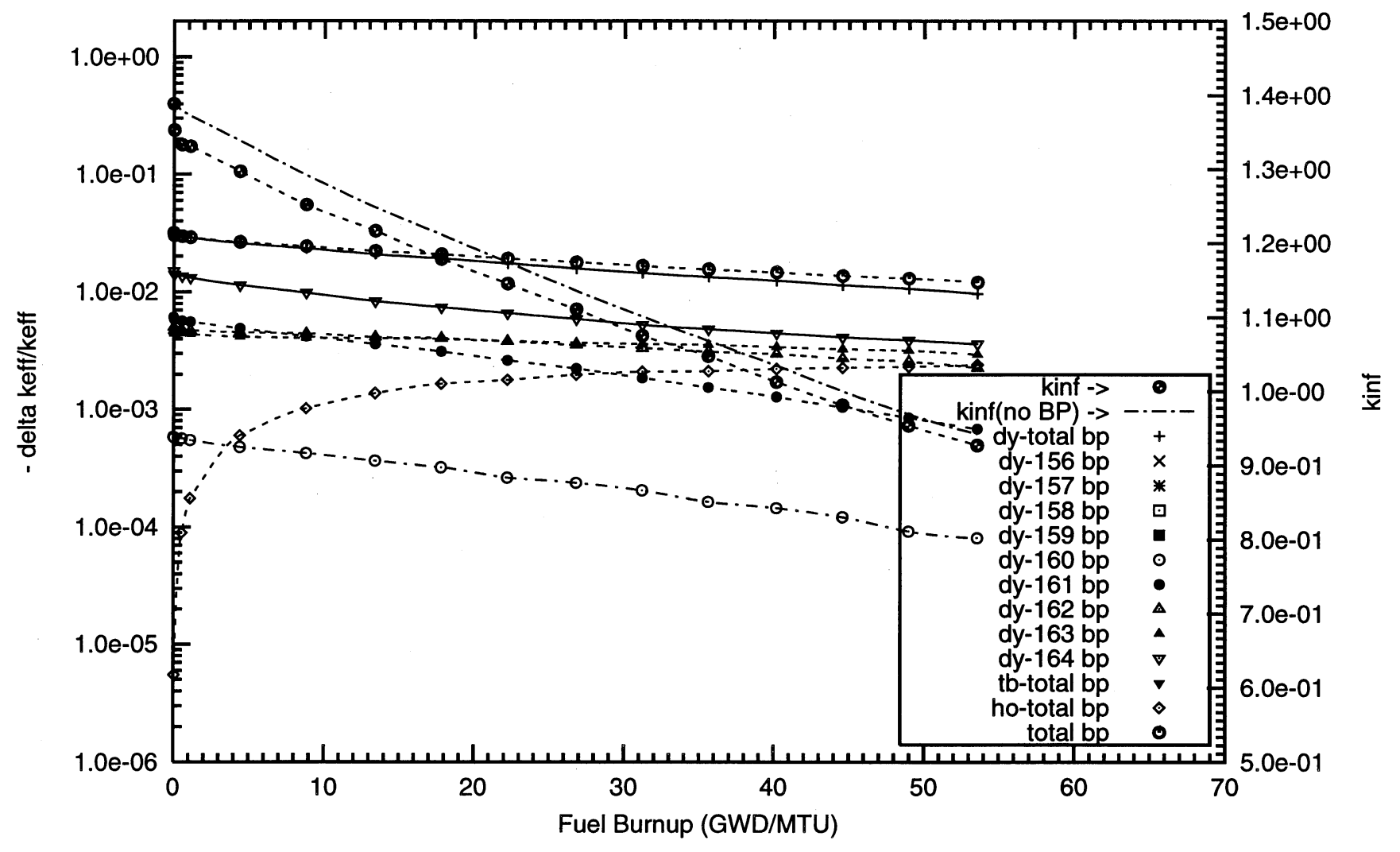


Figure D1.2. Negative Reactivity and Masses BP for Dy-nat and transmutation daughters

as a function of fuel life for $17 \times 17$ fuel assemblies with 16 poison rods, $2.0 \mathrm{w} \%$ Dy2O3 poison homogeneously mixed in the UO2 pellets

Reactor power $3400 \mathrm{MWth}, 193$ fuel assemblies, initial enrichment 4.5w\%U-235

(Case : dy000_2_100p_h2o_16_bp_fuel_2_o)

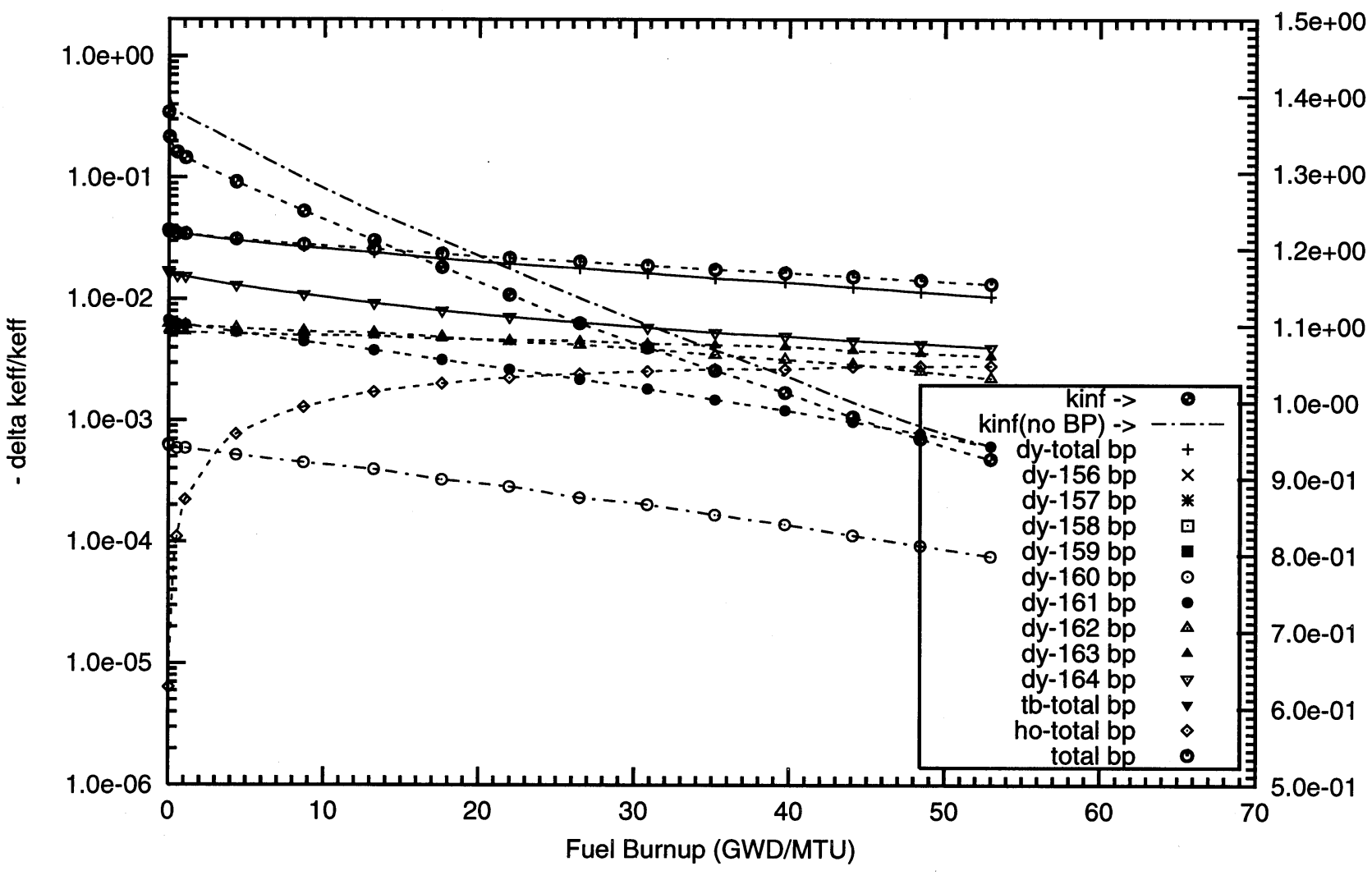

$\overline{\frac{E}{x}}$

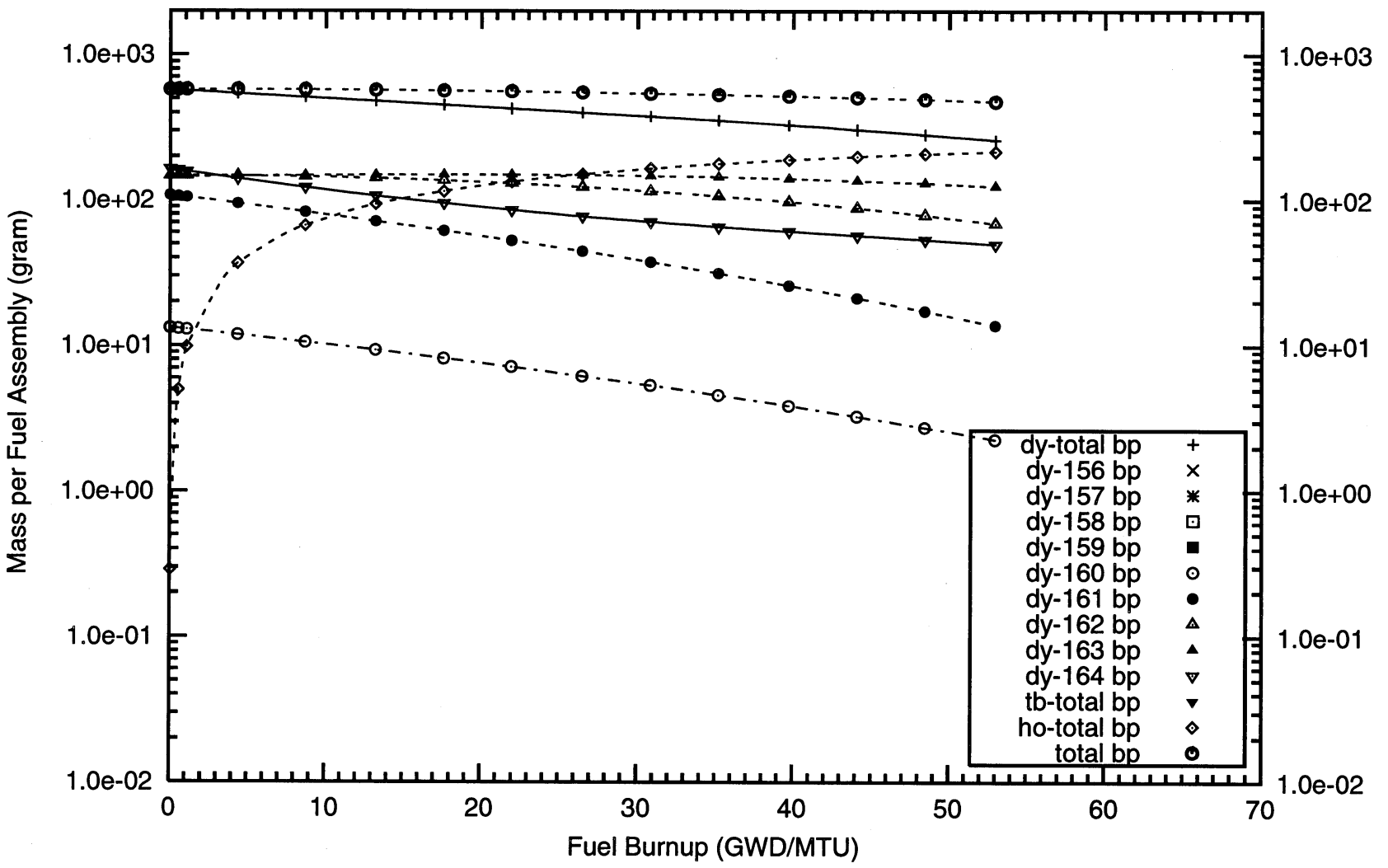


Figure D1.3. Negative Reactivity and Masses BP for Dy-nat and transmutation daughters as a function of fuel life for $17 \times 17$ fuel assemblies with 16 poison rods, $4.0 \mathrm{w} \%$ Dy2O3 poison homogeneously mixed in the UO2 pellets

Reactor power $3400 \mathrm{MWth}, 193$ fuel assemblies, initial enrichment 4.5w\%U-235

(Case : dy000_3_100p_h2o_16_bp_fuel_2_o)
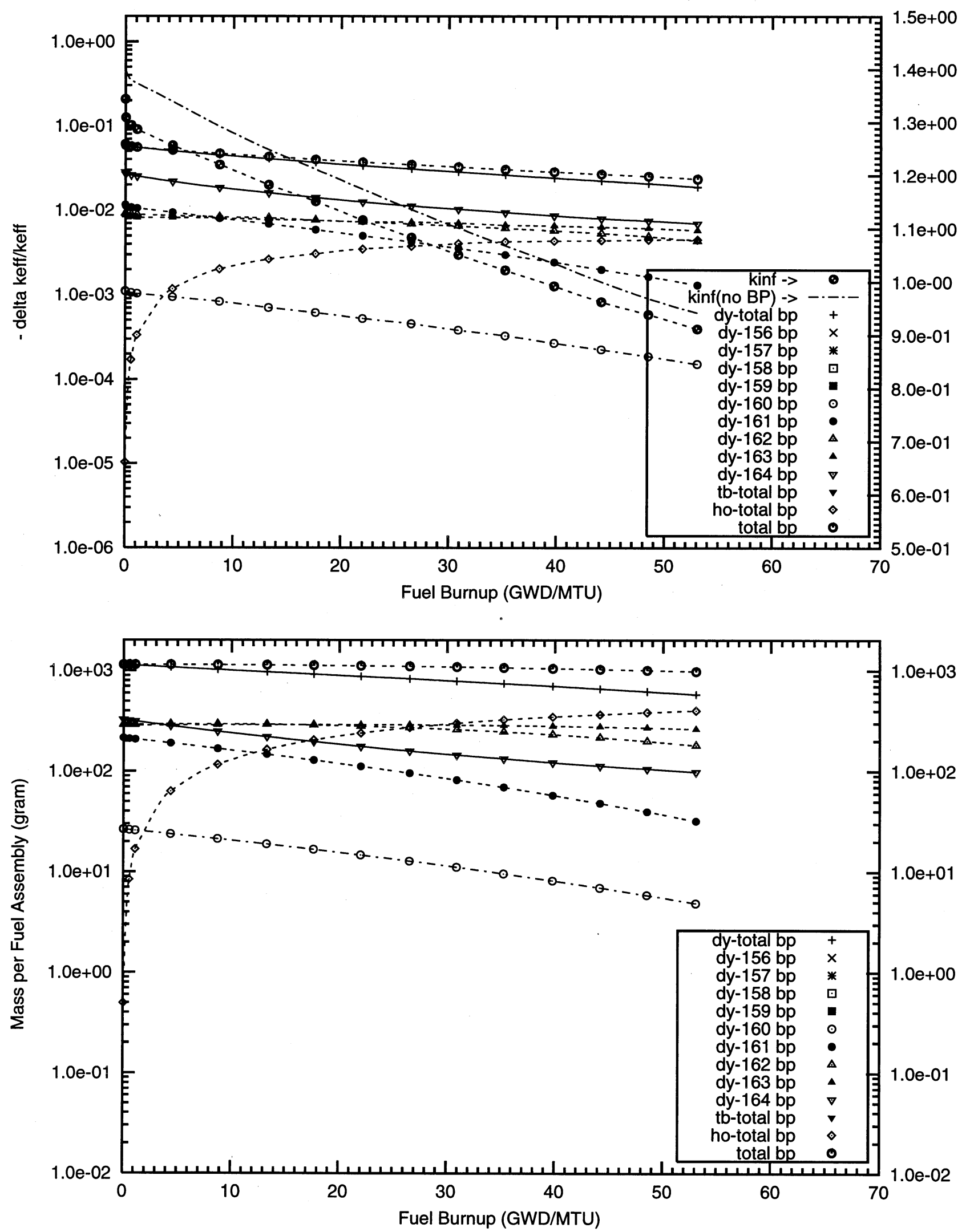
Figure D1.4. Negative Reactivity and Masses BP for Dy-nat and transmutation daughters as a function of fuel life for $17 \times 17$ fuel assemblies with 16 poison rods, 8.0w\% Dy2O3 poison homogeneously mixed in the UO2 pellets

Reactor power $3400 \mathrm{MWth}, 193$ fuel assemblies, initial enrichment 4.5w\%U-235

(Case : dy000_4_100p_h2o_16_bp_fuel_2_o)
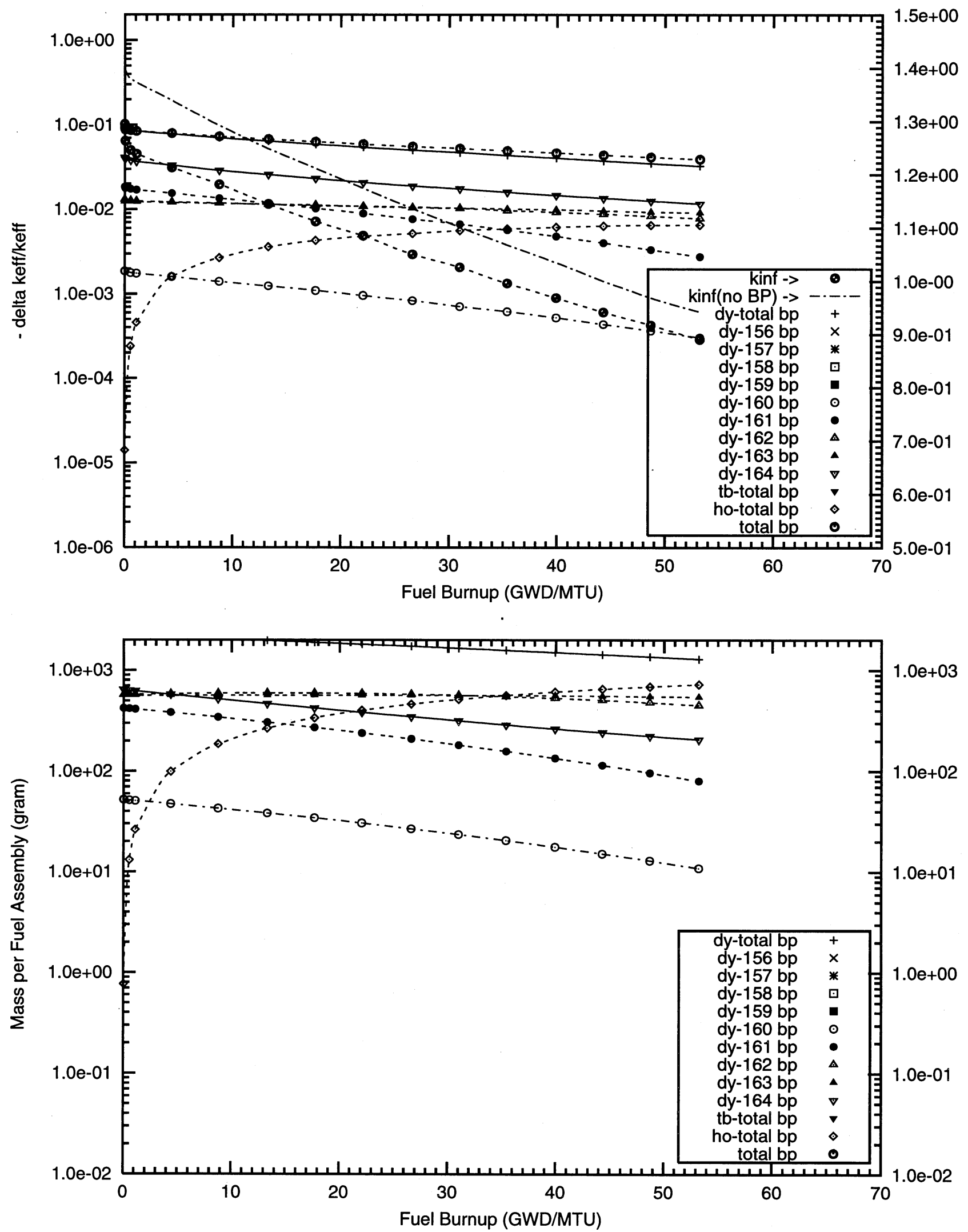
Figure D1.5. Negative Reactivity and Masses BP for Dy-nat and transmutation daughters as a function of fuel life for $17 \times 17$ fuel assemblies with 20 poison rods, $4.0 \mathrm{w} \%$ Dy2O3 poison homogeneously mixed in the UO2 pellets

Reactor power $3400 \mathrm{MWth}, 193$ fuel assemblies, initial enrichment $4.5 \mathrm{w} \% \mathrm{U}-235$ (Case : dy000_3_16_bp_fuel o)

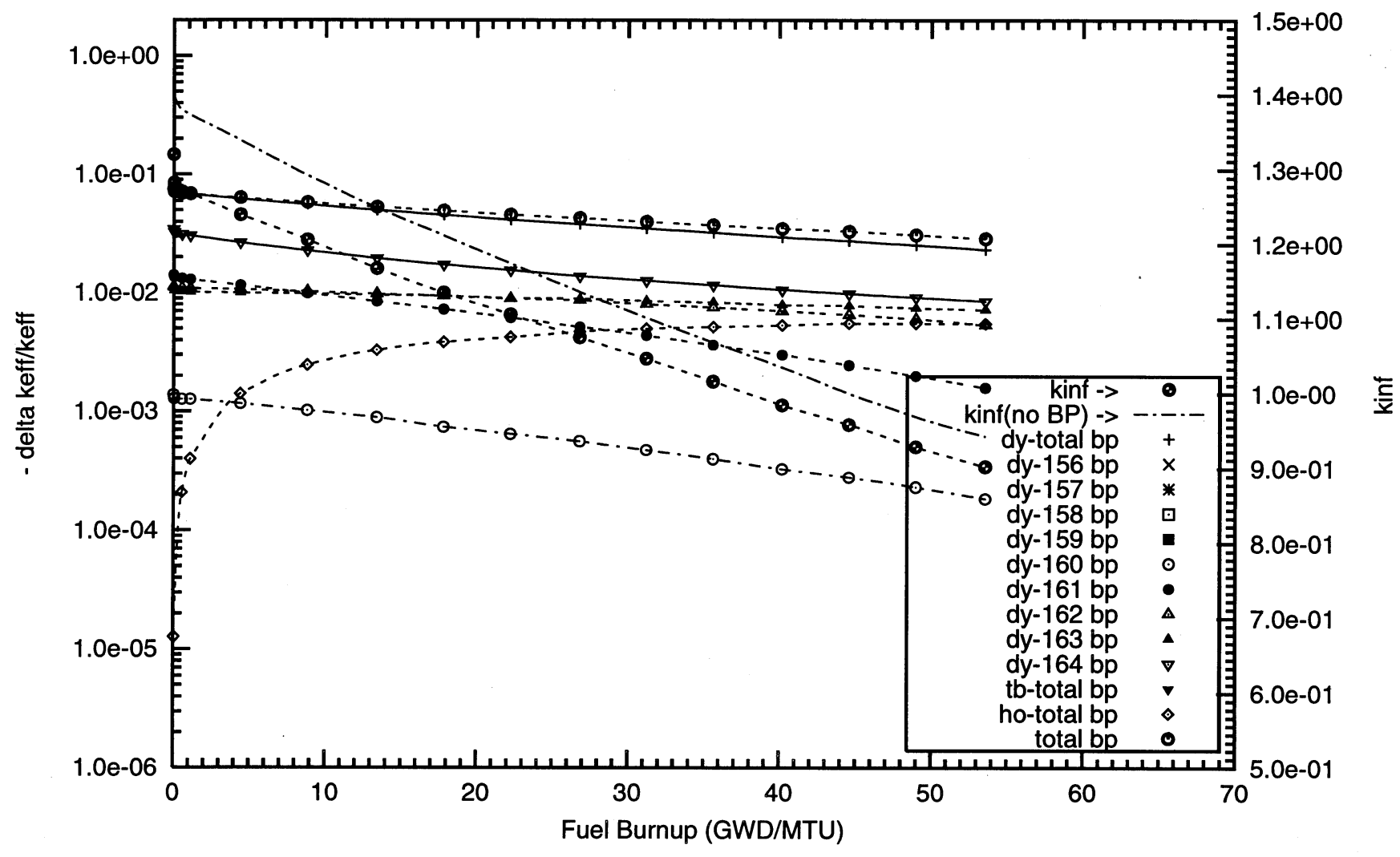


Figure D1.6. Negative Reactivity and Masses BP for Dy-nat and transmutation daughters as a function of fuel life for $17 \times 17$ fuel assemblies with 64 poison rods, $0.5 \mathrm{w} \%$ Dy2O3 poison homogeneously mixed in the UO2 pellets

Reactor power $3400 \mathrm{MWth}, 193$ fuel assemblies, initial enrichment 4.5w\%U-235

(Case : dy000_0_100p_h20_64_bp_fuel_2_o)
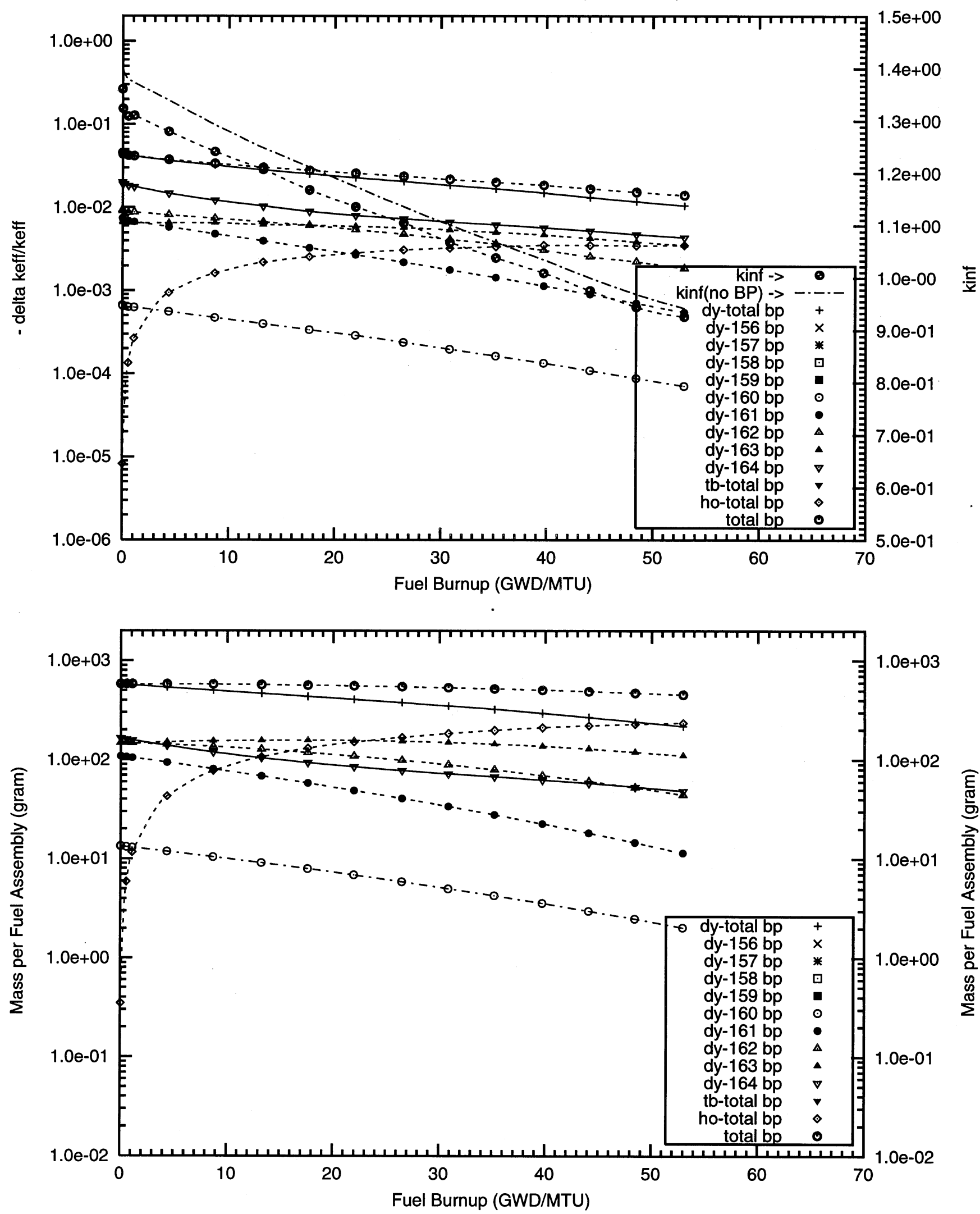

D-10 
Figure D1.7. Negative Reactivity and Masses BP for Dy-nat and transmutation daughters as a function of fuel life for $17 \times 17$ fuel assemblies with 64 poison rods,

$1.0 \mathrm{w} \%$ Dy2O3 poison homogeneously mixed in the UO2 pellets

Reactor power $3400 \mathrm{MWth}, 193$ fuel assemblies, initial enrichment 4.5w\%U-235

(Case : dy000_1_64_bp_fuel o)
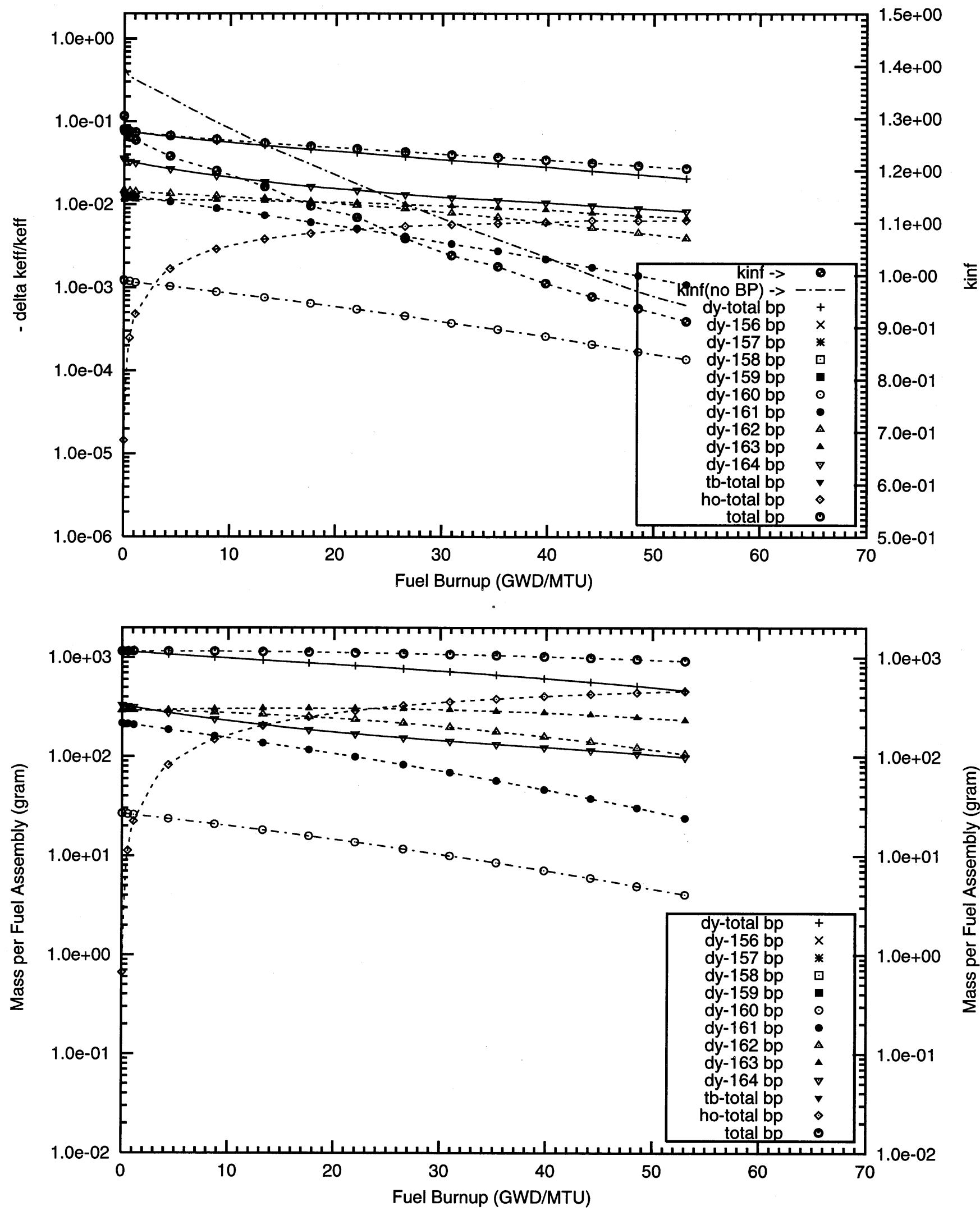
Figure D1.8. Negative Reactivity and Masses BP for Dy-nat and transmutation daughters as a function of fuel life for $17 \times 17$ fuel assemblies with 64 poison rods, $2.0 \mathrm{w} \%$ Dy2O3 poison homogeneously mixed in the UO2 pellets

Reactor power $3400 \mathrm{MWth}, 193$ fuel assemblies, initial enrichment 4.5W\%U-235

(Case : dy000_2_100p_h20_64_bp_fuel_2_o)
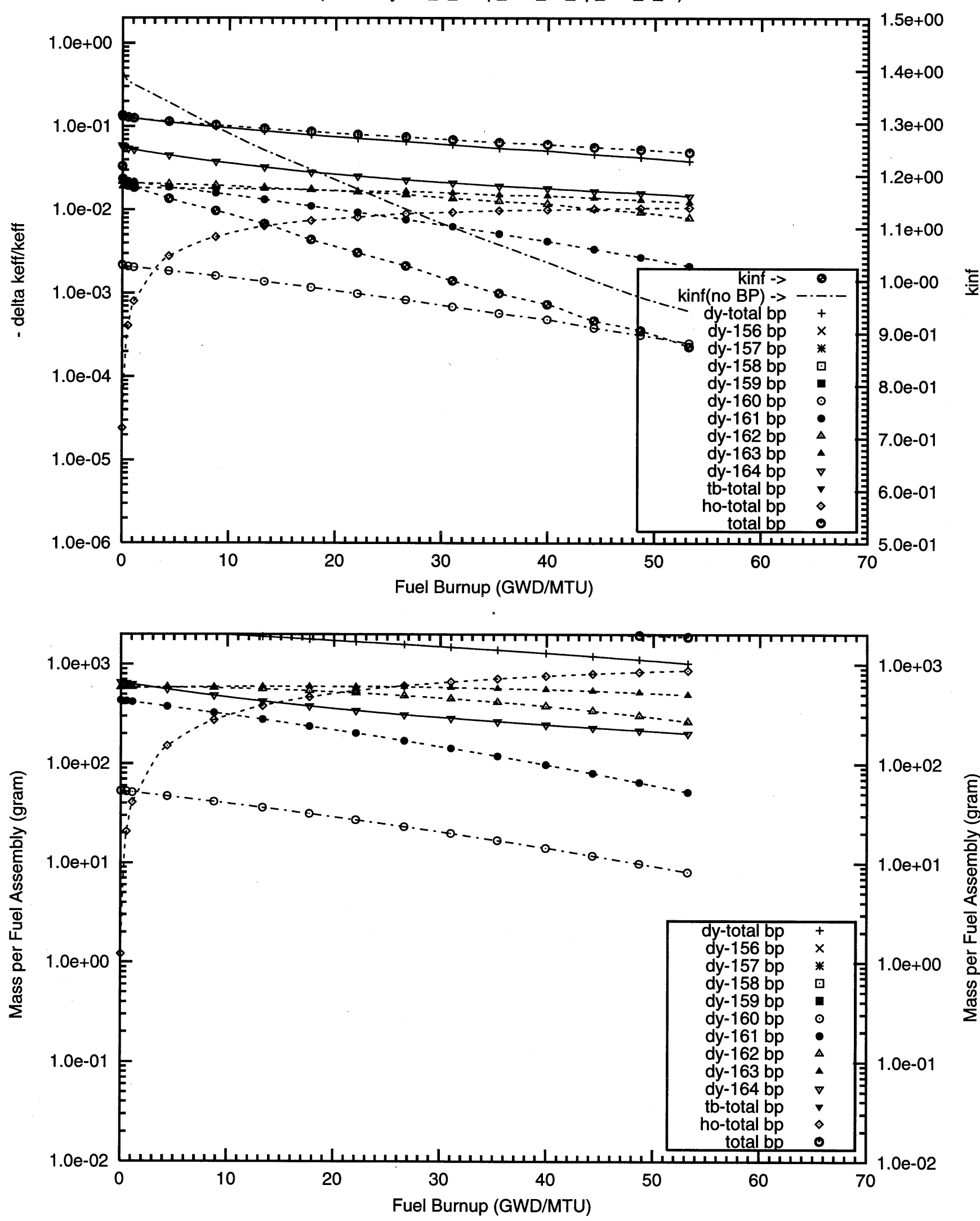


\section{D1 - Dysprosium Fully Enriched in ${ }^{164} D y$}


Figure D1.9. Negative Reactivity and Masses BP for Dy-164 and transmutation daughters as a function of fuel life for $17 \times 17$ fuel assemblies with 08 poison rods,

$4.0 \mathrm{w} \%$ Dy2O3 poison homogeneously mixed in the UO2 pellets

Reactor power $3400 \mathrm{MWth}, 193$ fuel assemblies, initial enrichment 4.5W\%U-235

(Case : dy164_3_08_bp_fuel o)

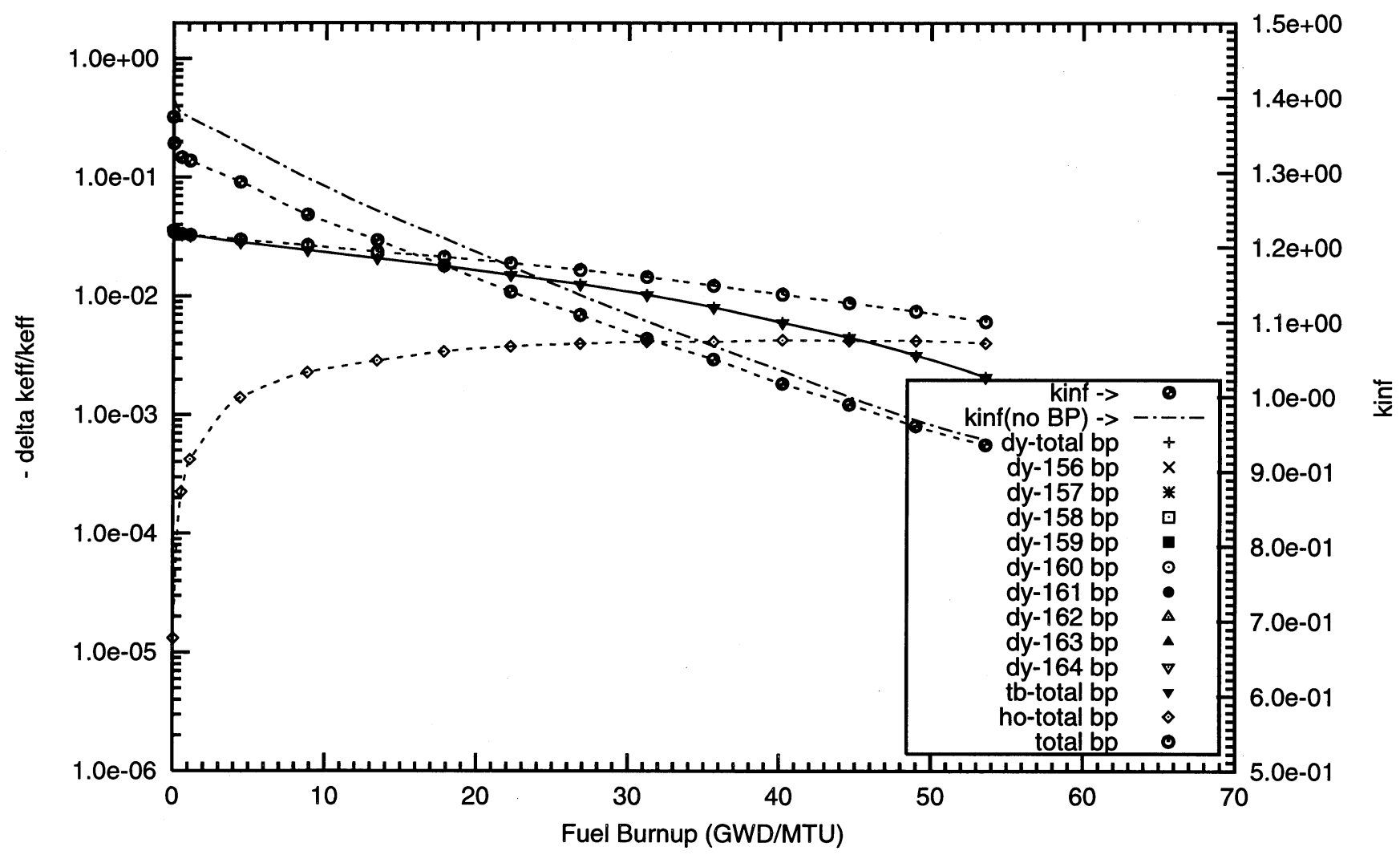


Figure D1.10. Negative Reactivity and Masses BP for Dy-164 and transmutation daughters as a function of fuel life for $17 \times 17$ fuel assemblies with 16 poison rods, $1.0 \mathrm{w} \%$ Dy2O3 poison homogeneously mixed in the UO2 pellets

Reactor power $3400 \mathrm{MWth}, 193$ fuel assemblies, initial enrichment 4.5w\%U-235

(Case : dy164_1_100p_h2o_16_bp_fuel_2_o)
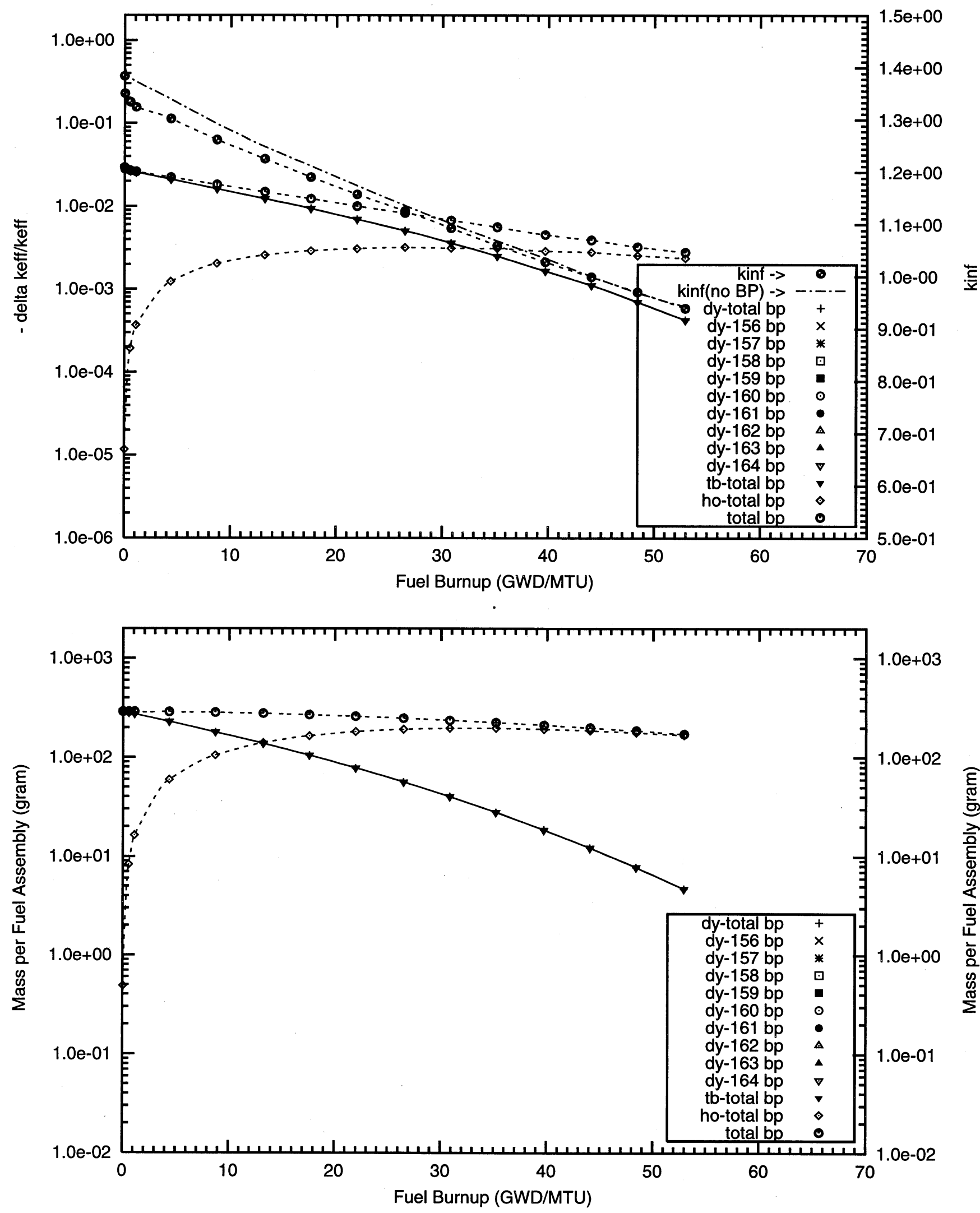
Figure D1.11. Negative Reactivity and Masses BP for Dy-164 and transmutation daughters as a function of fuel life for $17 \times 17$ fuel assemblies with 16 poison rods, $2.0 \mathrm{w} \%$ Dy2O3 poison homogeneously mixed in the UO2 pellets

Reactor power $3400 \mathrm{MWth}, 193$ fuel assemblies, initial enrichment 4.5w\%U-235

(Case : dy164_2_100p_h20_16_bp_fuel_2_o)
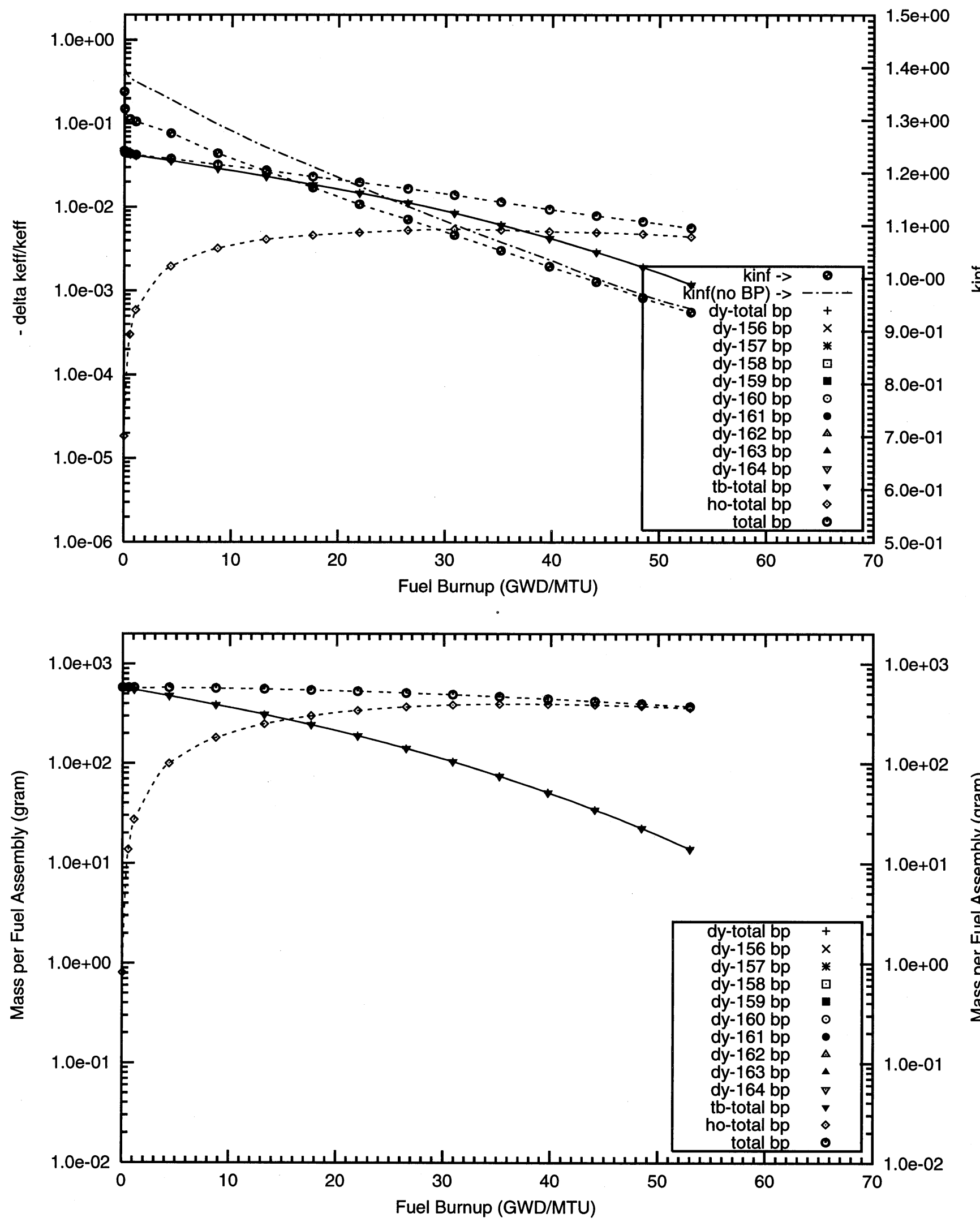
Figure D1.12. Negative Reactivity and Masses BP for Dy-164 and transmutation daughters as a function of fuel life for $17 \times 17$ fuel assemblies with 16 poison rods, $4.0 \mathrm{w} \%$ Dy2O3 poison homogeneously mixed in the UO2 pellets

Reactor power $3400 \mathrm{MWth}, 193$ fuel assemblies, initial enrichment 4.5w\%U-235

(Case : dy164_3_100p_h20_16_bp_fuel_2_o)
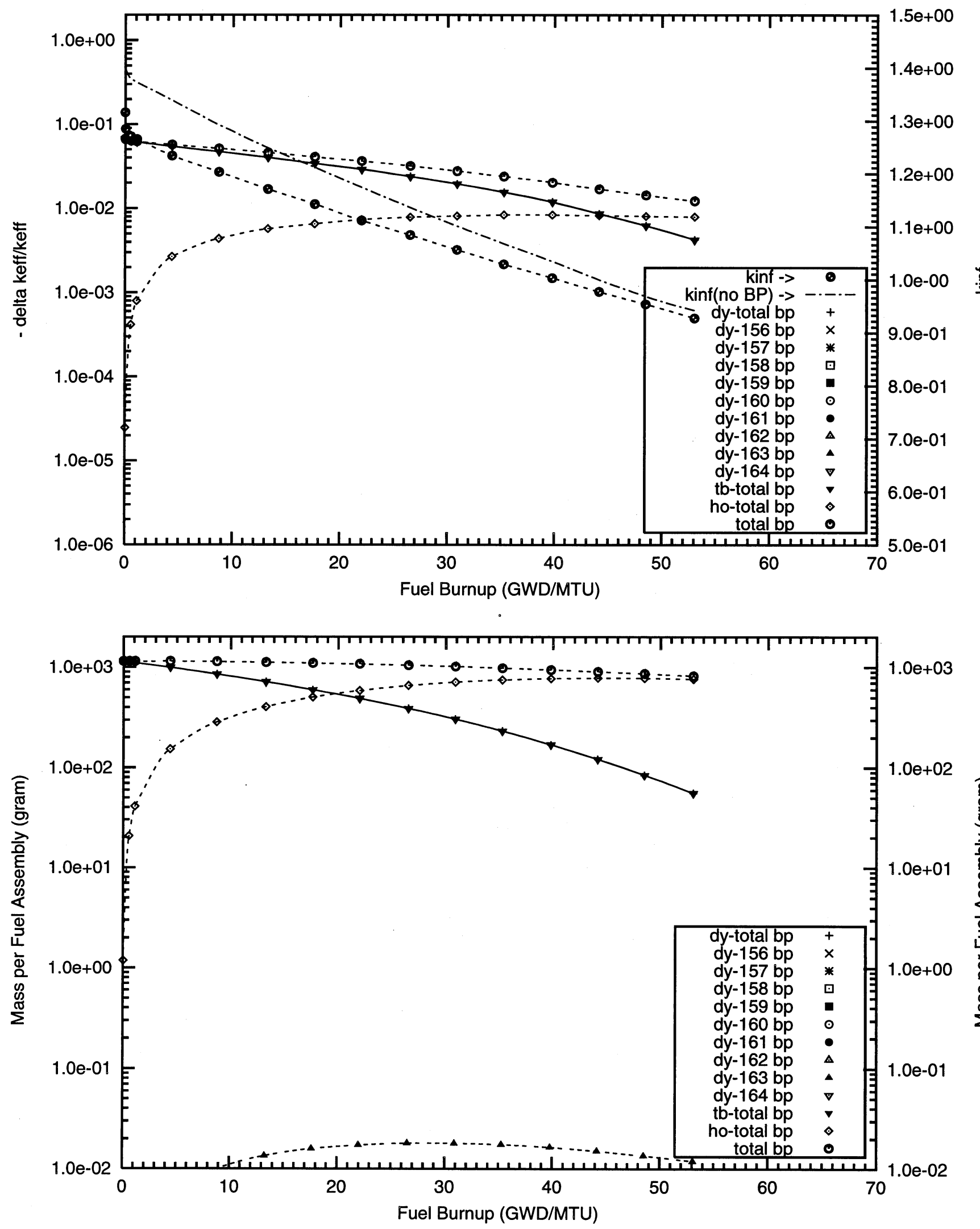
Figure D1.13. Negative Reactivity and Masses BP for Dy-164 and transmutation daughters as a function of fuel life for $17 \times 17$ fuel assemblies with 20 poison rods, $4.0 \mathrm{w} \%$ Dy2O3 poison homogeneously mixed in the UO2 pellets

Reactor power $3400 \mathrm{MWth}, 193$ fuel assemblies, initial enrichment 4.5W\%U-235

(Case : dy164_3_16_bp_fuel o)
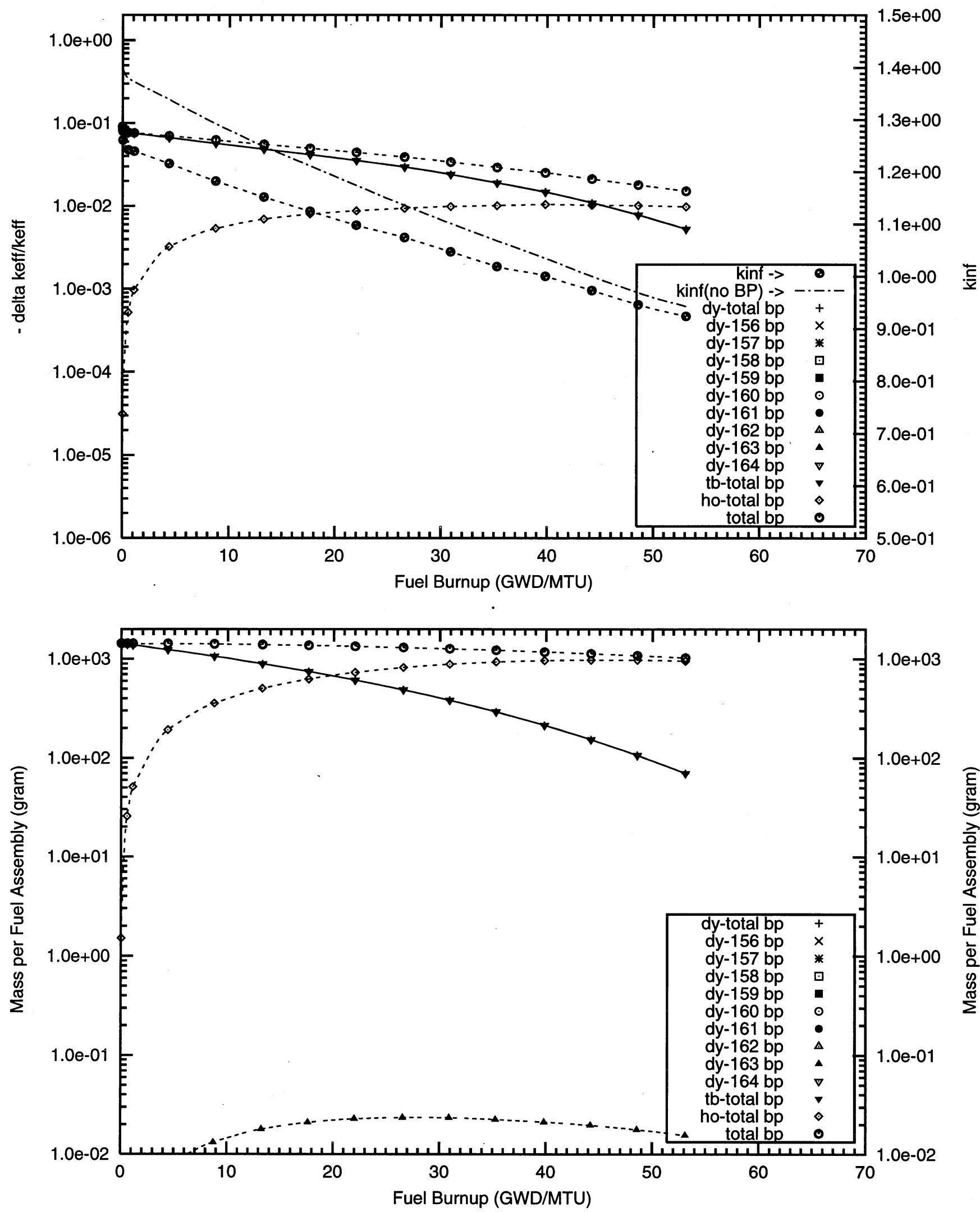
Figure D1.14. Negative Reactivity and Masses BP for Dy-164 and transmutation daughters as a fünction of fuel life for $17 \times 17$ fuel assemblies with 64 poison rods,

$0.5 \mathrm{w} \%$ Dy2O3 poison homogeneously mixed in the UO2 pellets

Reactor power $3400 \mathrm{MWth}, 193$ fuel assemblies, initial enrichment 4.5W\%U-235

(Case : dy164_0_100p_h2o_64_bp_fuel_2_o)
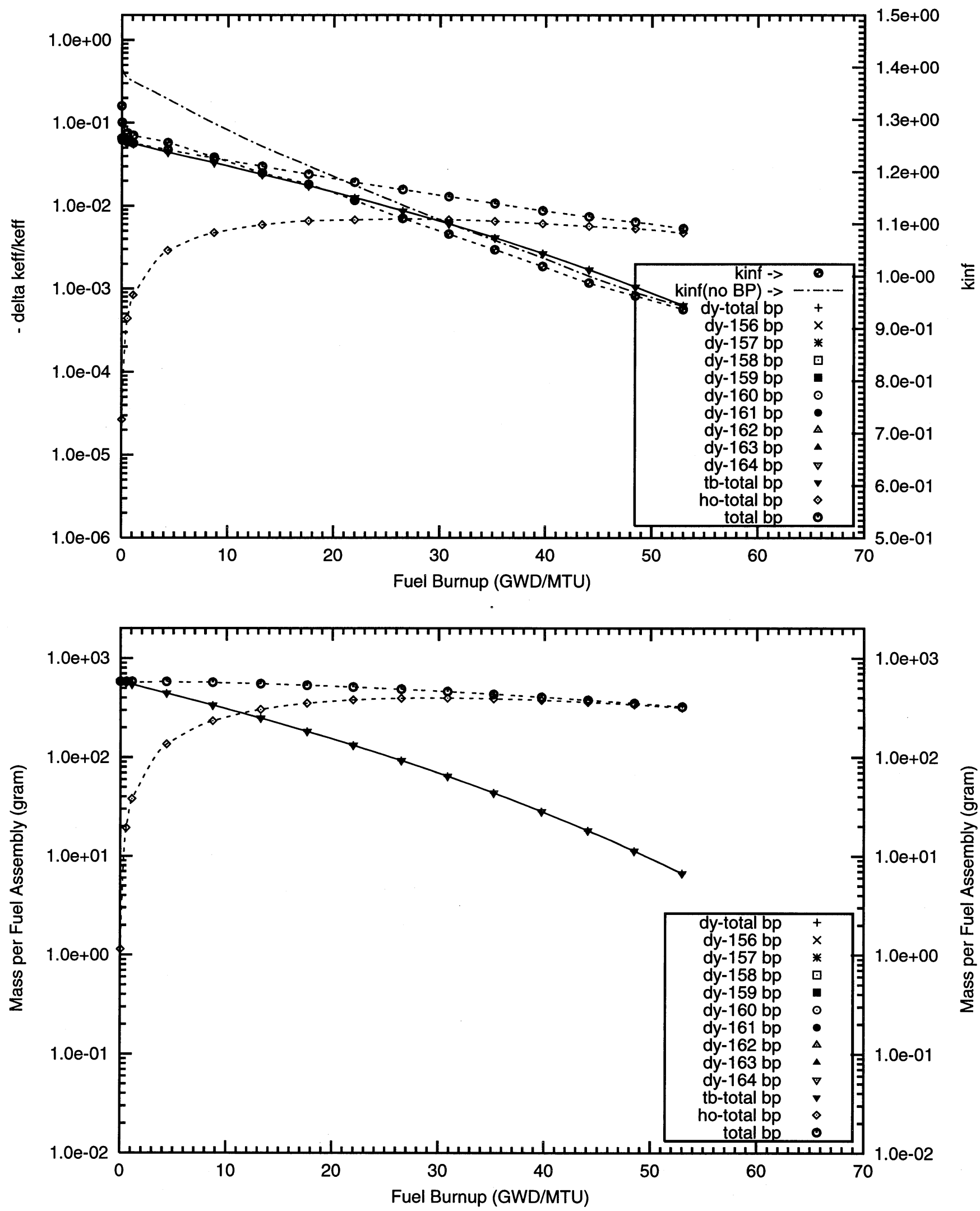
Figure D1.15. Negative Reactivity and Masses BP for Dy-164 and transmutation daughters as a function of fuel life for $17 \times 17$ fuel assemblies with 64 poison rods, $1.0 \mathrm{w} \%$ Dy2O3 poison homogeneously mixed in the UO2 pellets

Reactor power $3400 \mathrm{MWth}, 193$ fuel assemblies, initial enrichment 4.5w\%U-235

(Case : dy164_1_64_bp_fuel o)
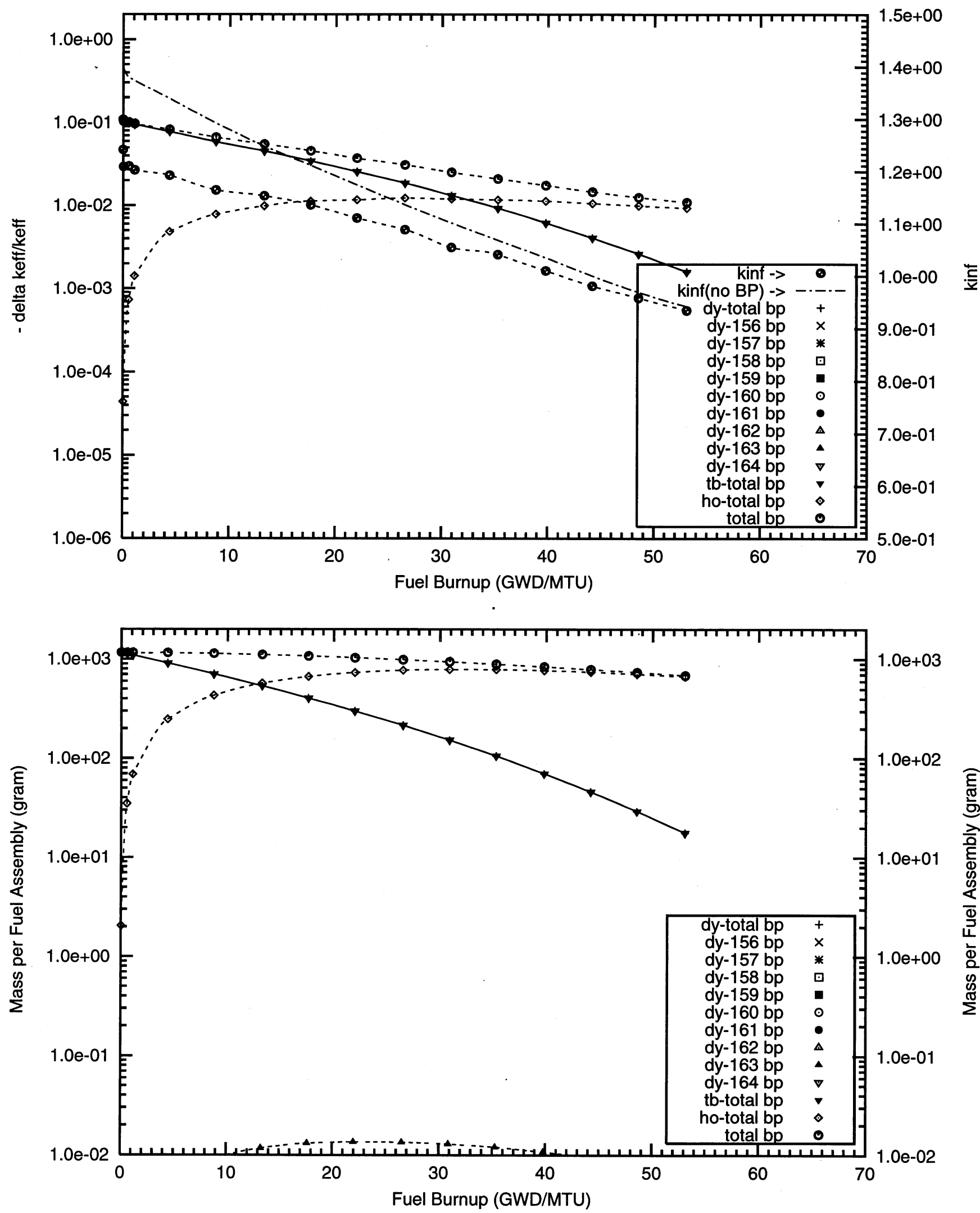


\section{Appendix D2}

Reactivity Worths and Masses of $\mathrm{Dy}_{2} \mathrm{O}_{3}$ Burnable Poison Homogeneously Mixed in the Outer One-Third Volume of the Fuel Pellets

\section{Table 18 Cases}




\section{D2 - Dysprosium with Natural Isotopic Abundances}



Figure D2.1. Negative Reactivity and Masses BP for Dy-nat and transmutation daughters as a function of fuel life for $17 \times 17$ fuel assemblies with 08 poison rods,

$4.0 \mathrm{w} \%$ Dy2O3 poison homogeneously mixed in the outer one-third volume of the UO2 pellets

Reactor power $3400 \mathrm{MWth}, 193$ fuel assemblies, initial enrichment 4.5w\%U-235

(Case : dy000_3b_08_bp_fuel o)

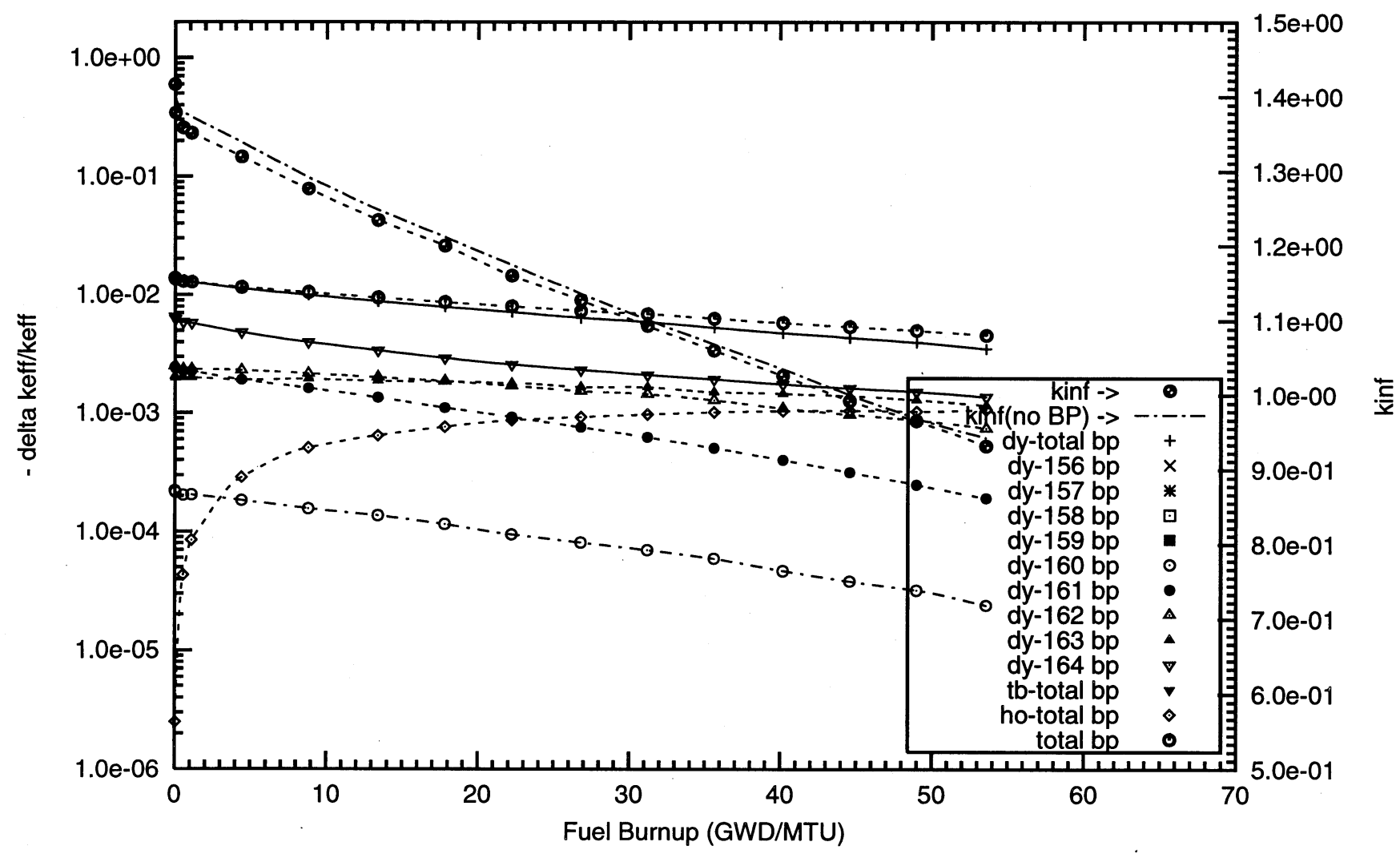


Figure D2.2. Negative Reactivity and Masses BP for Dy-nat and transmutation daughters as a function of fuel life for $17 \times 17$ fuel assemblies with 08 poison rods,

$8.0 \mathrm{w} \%$ Dy2O3 poison homogeneously mixed in the outer one-third volume of the UO2 pellets Reactor power $3400 \mathrm{MW}$ th, 193 fuel assemblies, initial enrichment 4.5w\%U-235 (Case : dy000_4b_08_bp_fuel o)

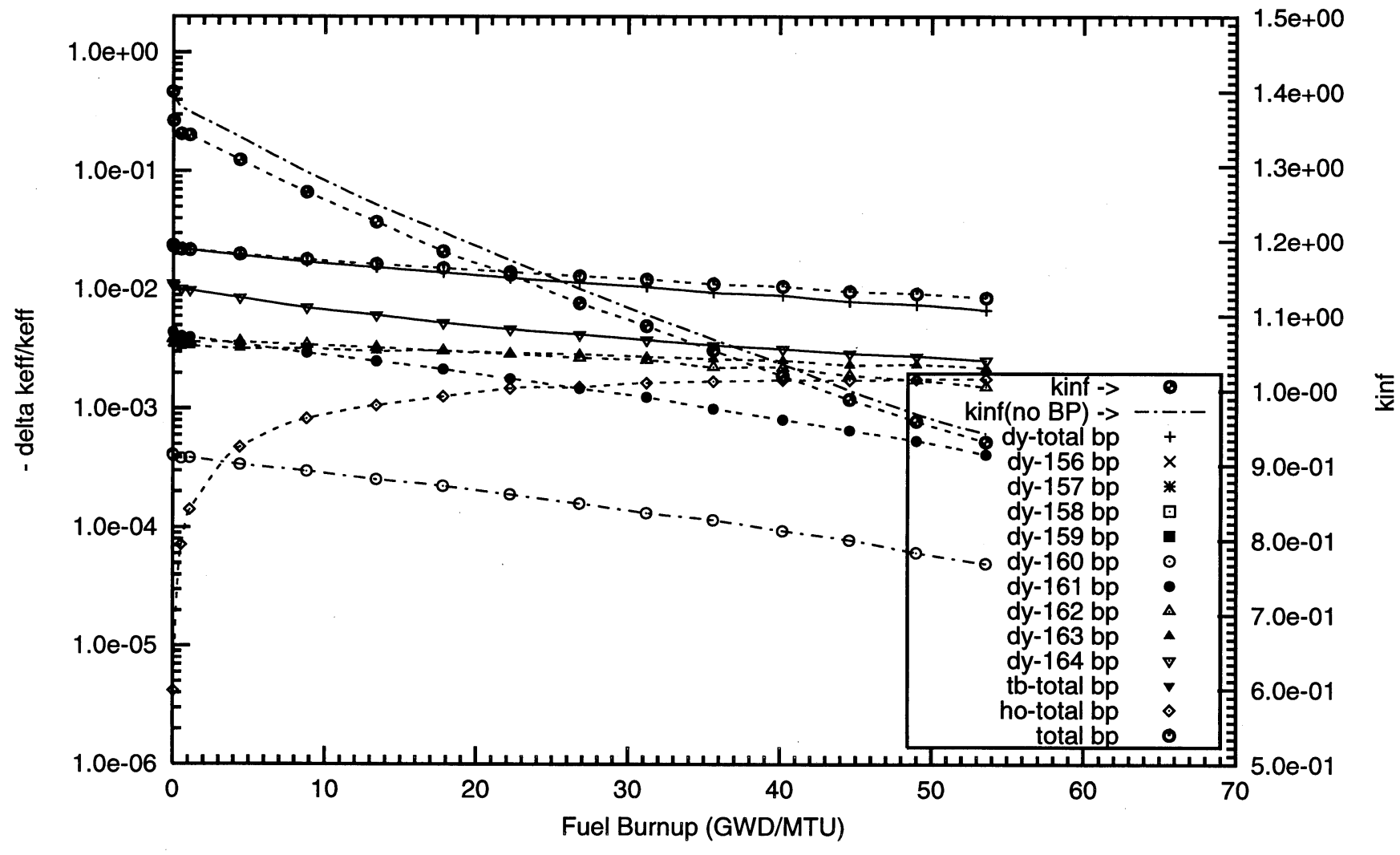


Figure D2.3. Negative Reactivity and Masses BP for Dy-nat and transmutation daughters as a function of fuel life for $17 \times 17$ fuel assemblies with 64 poison rods,

$1.0 \mathrm{w} \%$ Dy2O3 poison homogeneously mixed in the outer one-third volume of the UO2 pellets Reactor power $3400 \mathrm{MWth}, 193$ fuel assemblies, initial enrichment 4.5W\%U-235

(Case : dy000_1b_64_bp_fuel o)
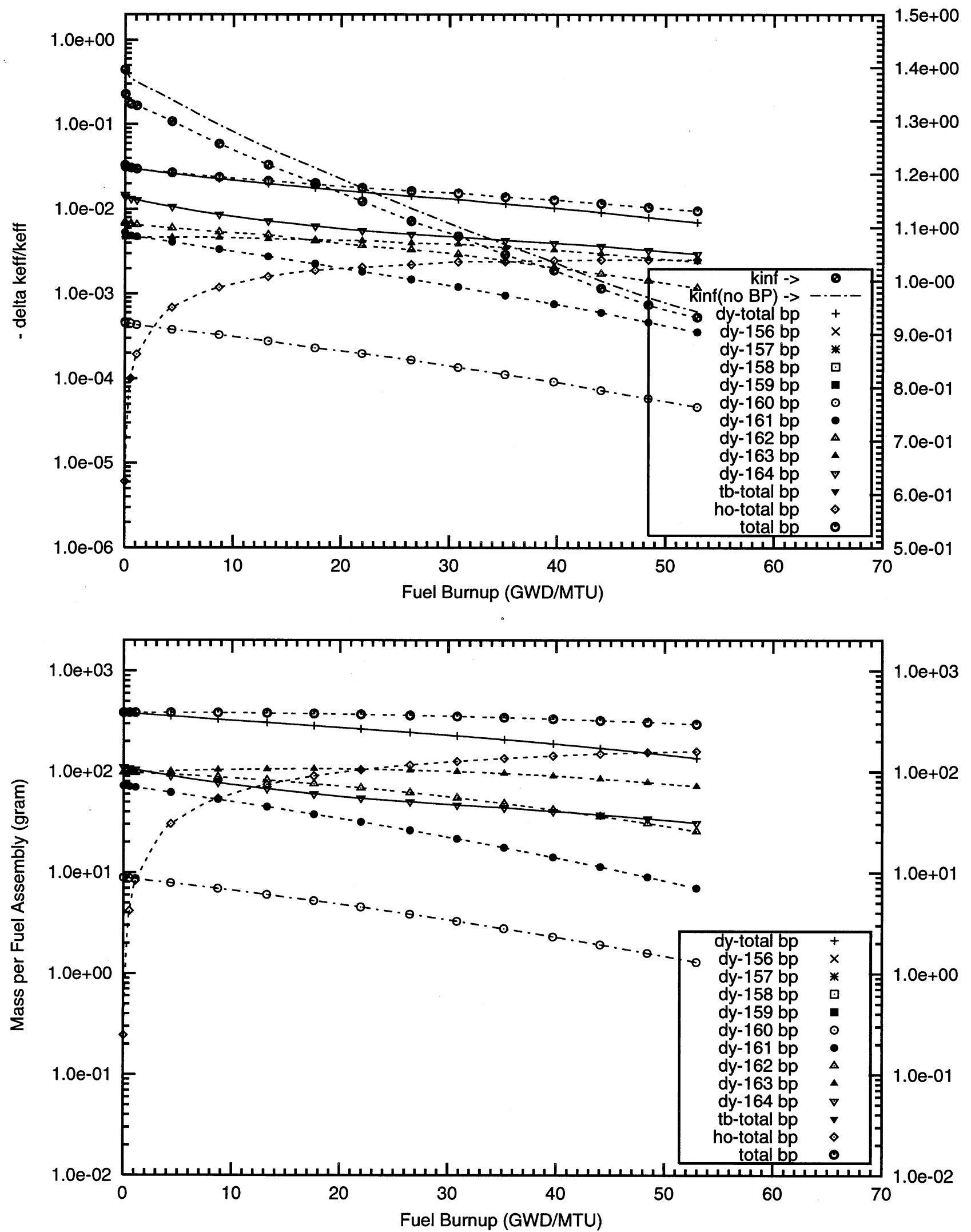



\section{D2 - Dysprosium Fully Enriched in ${ }^{164}$ Dy}



Figure D2.4. Negative Reactivity and Masses BP for Dy-164 and transmutation daughters as a function of fuel life for $17 \times 17$ fuel assemblies with 08 poison rods,

$4.0 \mathrm{w} \%$ Dy2O3 poison homogeneously mixed in the outer one-third volume of the $\mathrm{UO} 2$ pellets Reactor power $3400 \mathrm{MWth}, 193$ fuel assemblies, initial enrichment $4.5 \mathrm{w} \% \mathrm{U}-235$ (Case : dy164_3b_08_bp_fuel o)

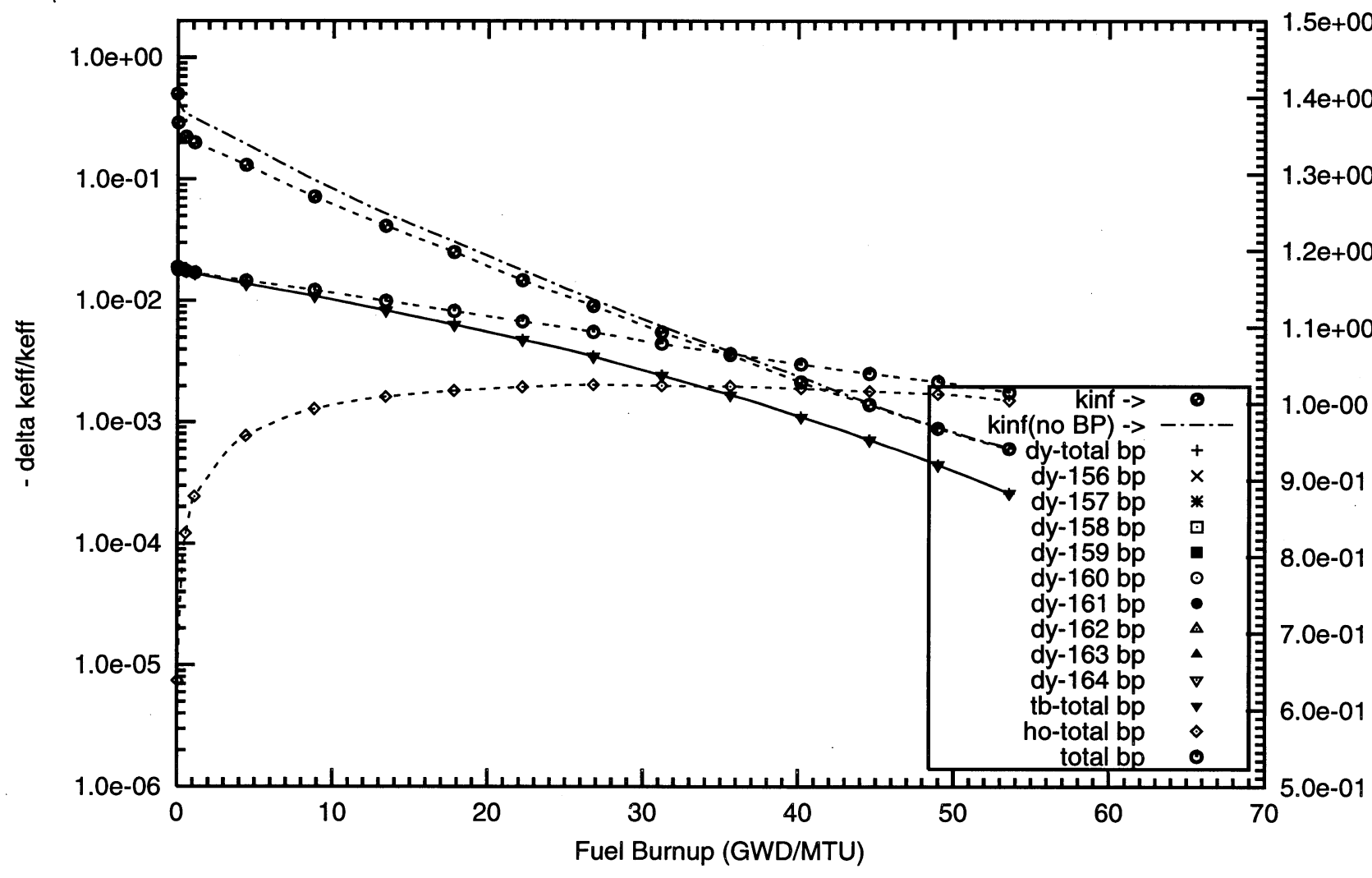


Figure D2.5. Negative Reactivity and Masses BP for Dy-164 and transmutation daughters as a function of fuel life for $17 \times 17$ fuel assemblies with 08 poison rods,

$8.0 \mathrm{w} \%$ Dy2O3 poison homogeneously mixed in the outer one-third volume of the UO2 pellets Reactor power $3400 \mathrm{MWth}, 193$ fuel assemblies, initial enrichment $4.5 \mathrm{w} \% \mathrm{U}-235$ (Case : dy164_4b_08_bp_fuel o)

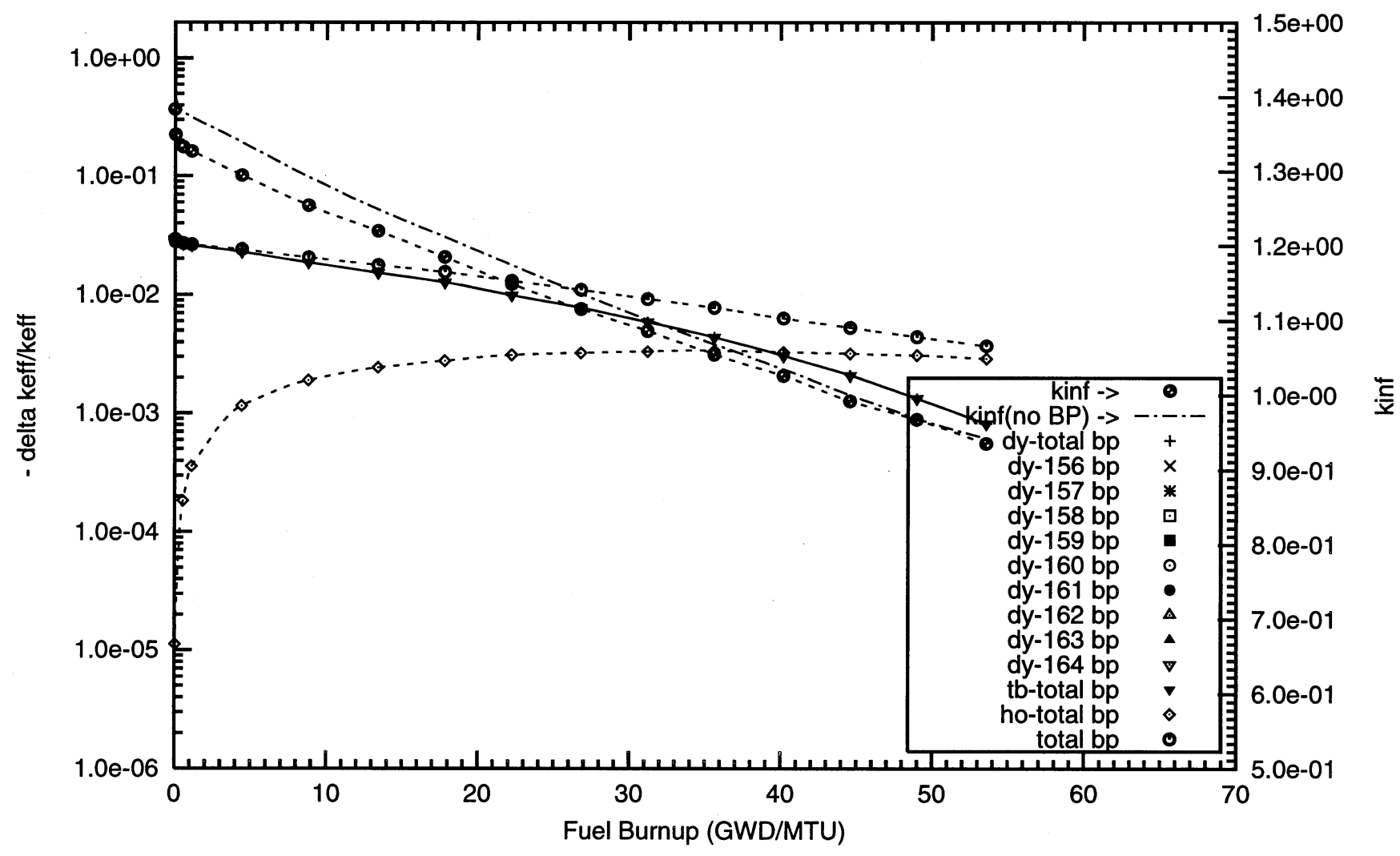


Figure D2.6. Negative Reactivity and Masses BP for Dy-164 and transmutation daughters as a function of fuel life for $17 \times 17$ fuel assemblies with 08 poison rods,

$12.0 \mathrm{w} \%$ Dy2O3 poison homogeneously mixed in the outer one-third volume of the UO2 pellets Reactor power $3400 \mathrm{MW}$ th, 193 fuel assemblies, initial enrichment 4.5w\%U-235

(Case : dy164_5b_08_bp_fuel o)

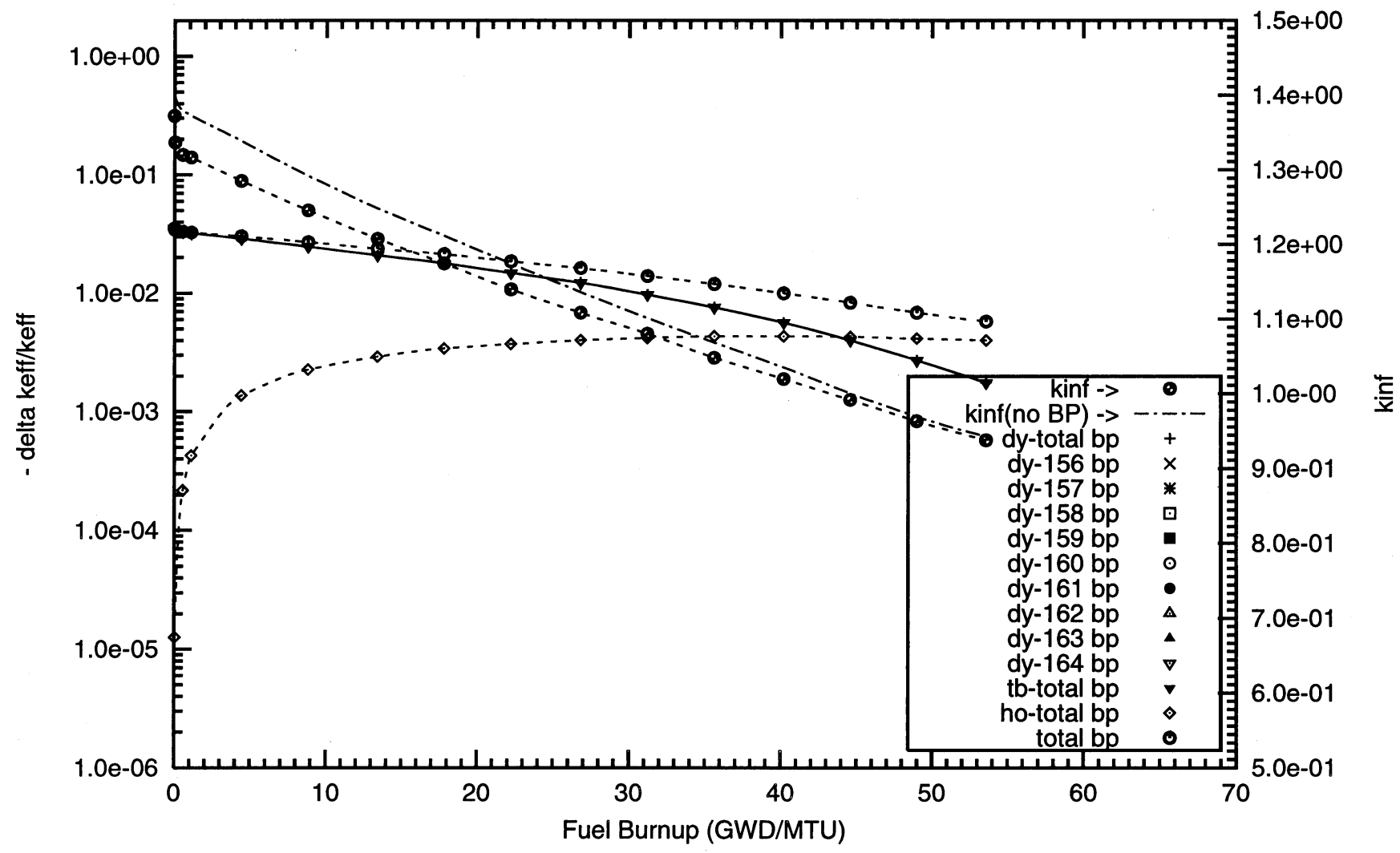


Figure D2.7. Negative Reactivity and Masses BP for Dy-164 and transmutation daughters as a function of fuel life for $17 \times 17$ fuel assemblies with 64 poison rods,

$1.0 \mathrm{w} \%$ Dy2O3 poison homogeneously mixed in the outer one-third volume of the UO2 pellets Reactor power $3400 \mathrm{MWth}, 193$ fuel assemblies, initial enrichment 4.5W\%U-235

(Case : dy164_1b_64_bp_fuel o)
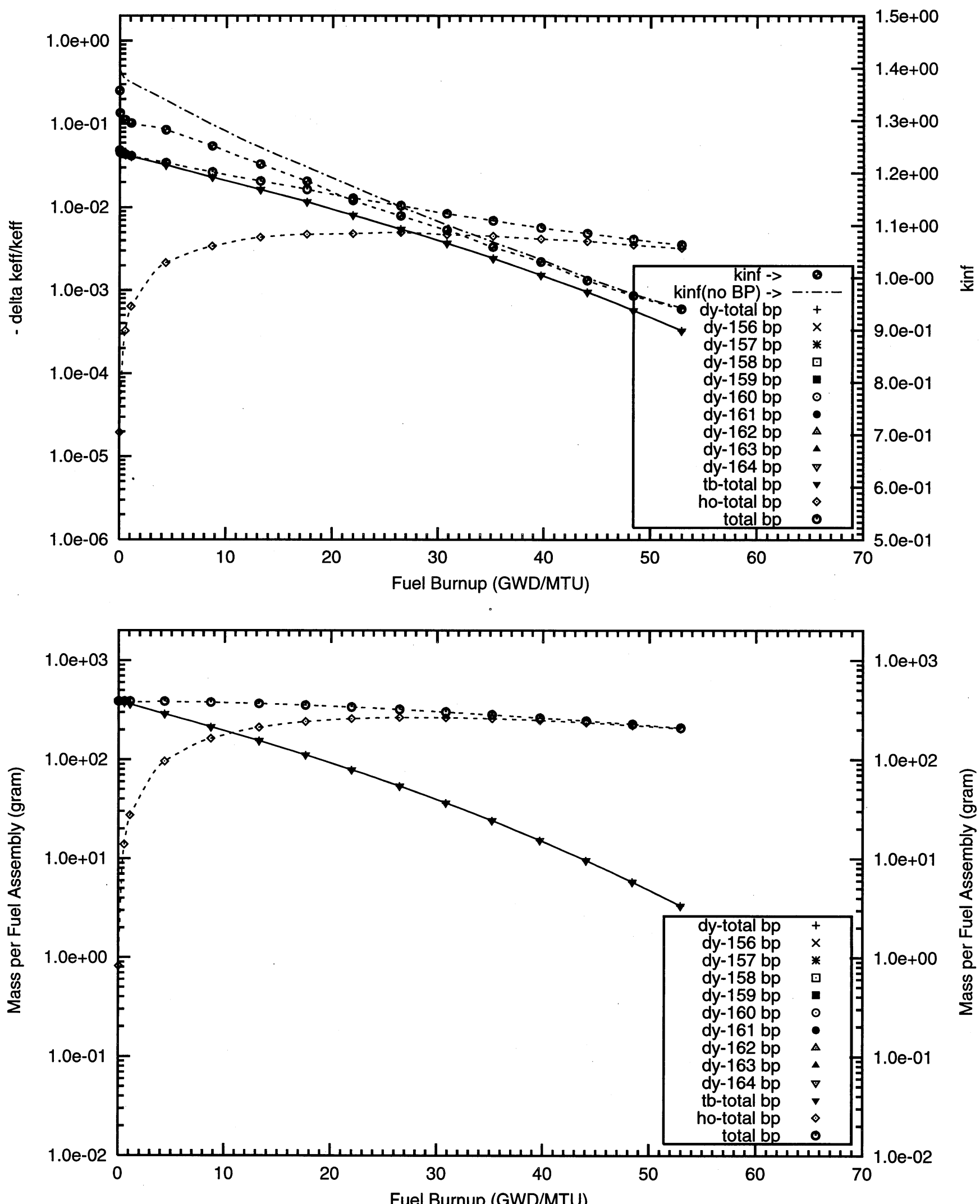

Fuel Burnup (GWD/MTU) 


\title{
Appendix D3
}

Reactivity Worths and Masses of $\mathrm{Dy}_{2} \mathrm{O}_{3}$ Coating on the Surface of the Fuel Pellets

\author{
Table 19 Cases
}





\section{D3 - Dysprosium with Natural Isotopic Abundances}



Figure D3.1. Negative Reactivity and Masses BP for Dy-nat and transmutation daughters as a function of fuel life for $17 \times 17$ fuel assemblies with 18 poison rods, 2.0 mill thick Dy2O3 poison coating on the outer radial surface of the UO2 pellets Reactor power $3400 \mathrm{MWth}, 193$ fuel assemblies, initial enrichment $4.5 \mathrm{w} \% \mathrm{U}-235$

(Case : dy000_2f_16_bp_fuel o)
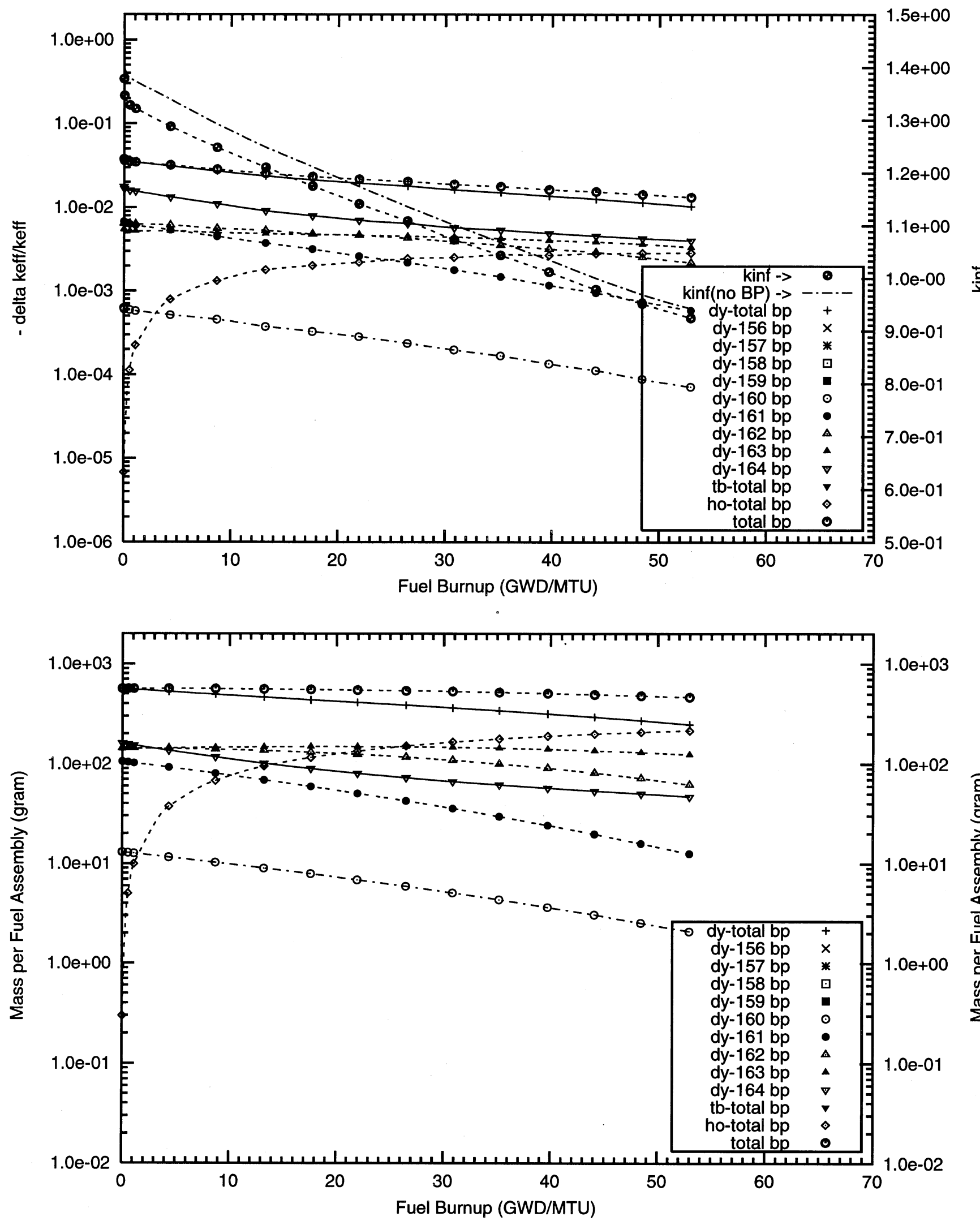
Figure D3.2. Negative Reactivity and Masses BP for Dy-nat and transmutation daughters as a function of fuel life for $17 \times 17$ fuel assemblies with 64 poison rods, 1.0 mill thick Dy2O3 poison coating on the outer radial surface of the UO2 pellets Reactor power $3400 \mathrm{MWth}, 193$ fuel assemblies, initial enrichment 4.5w\%U-235 (Case : dy000_1f_64_bp_fuel o)
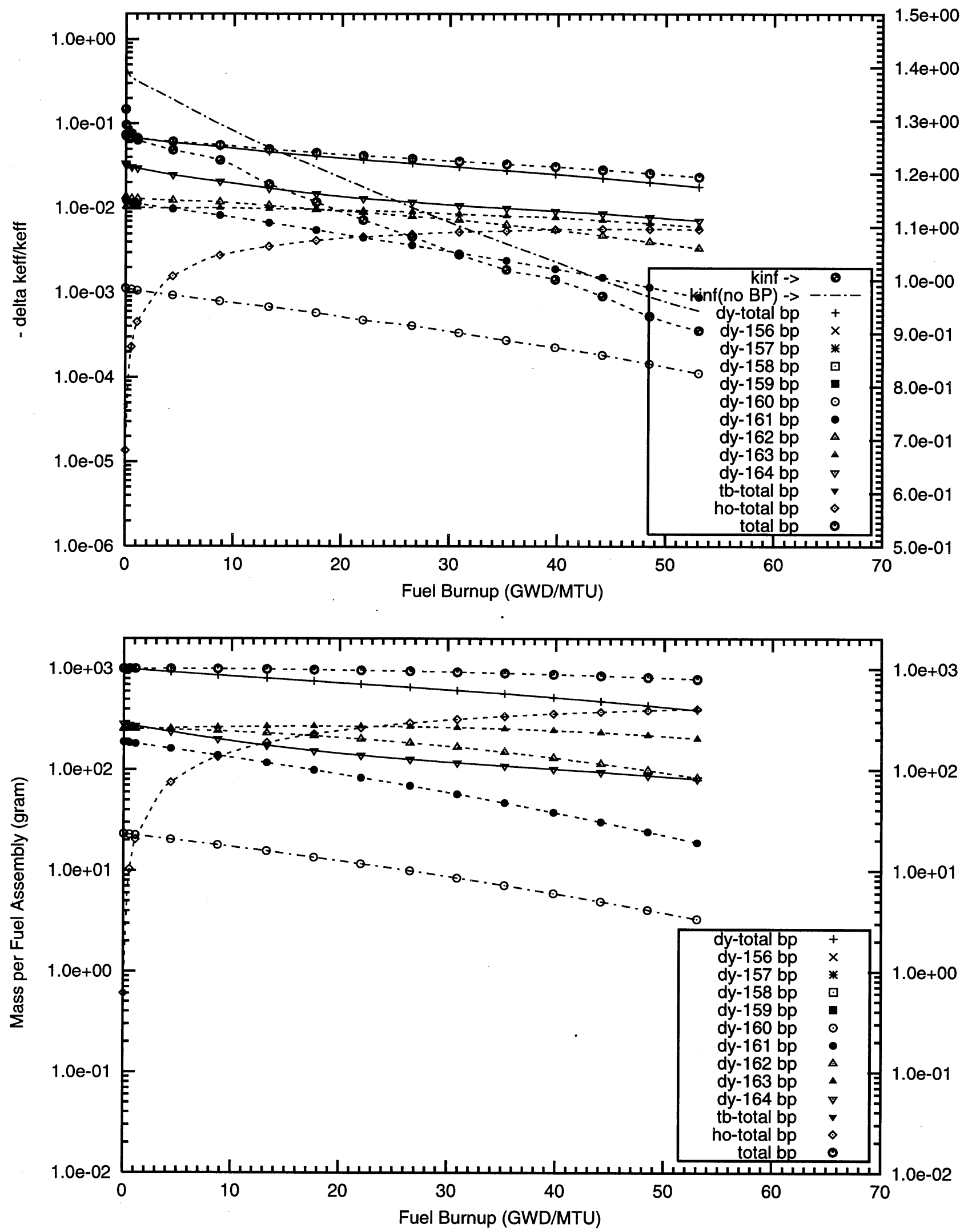


\section{D3 - Dysprosium Fully Enriched in ${ }^{164} D y$}


Figure D3.3. Negative Reactivity and Masses BP for Dy-164 and transmutation daughters as a function of fuel life for $17 \times 17$ fuel assemblies with 08 poison rods,

4.0 mill thick Dy2O3 poison coating on the outer radial surface of the UO2 pellets Reactor power $3400 \mathrm{MWth}, 193$ fuel assemblies, initial enrichment 4.5w\%U-235 (Case : dy164_3f_08_bp_fuel o)

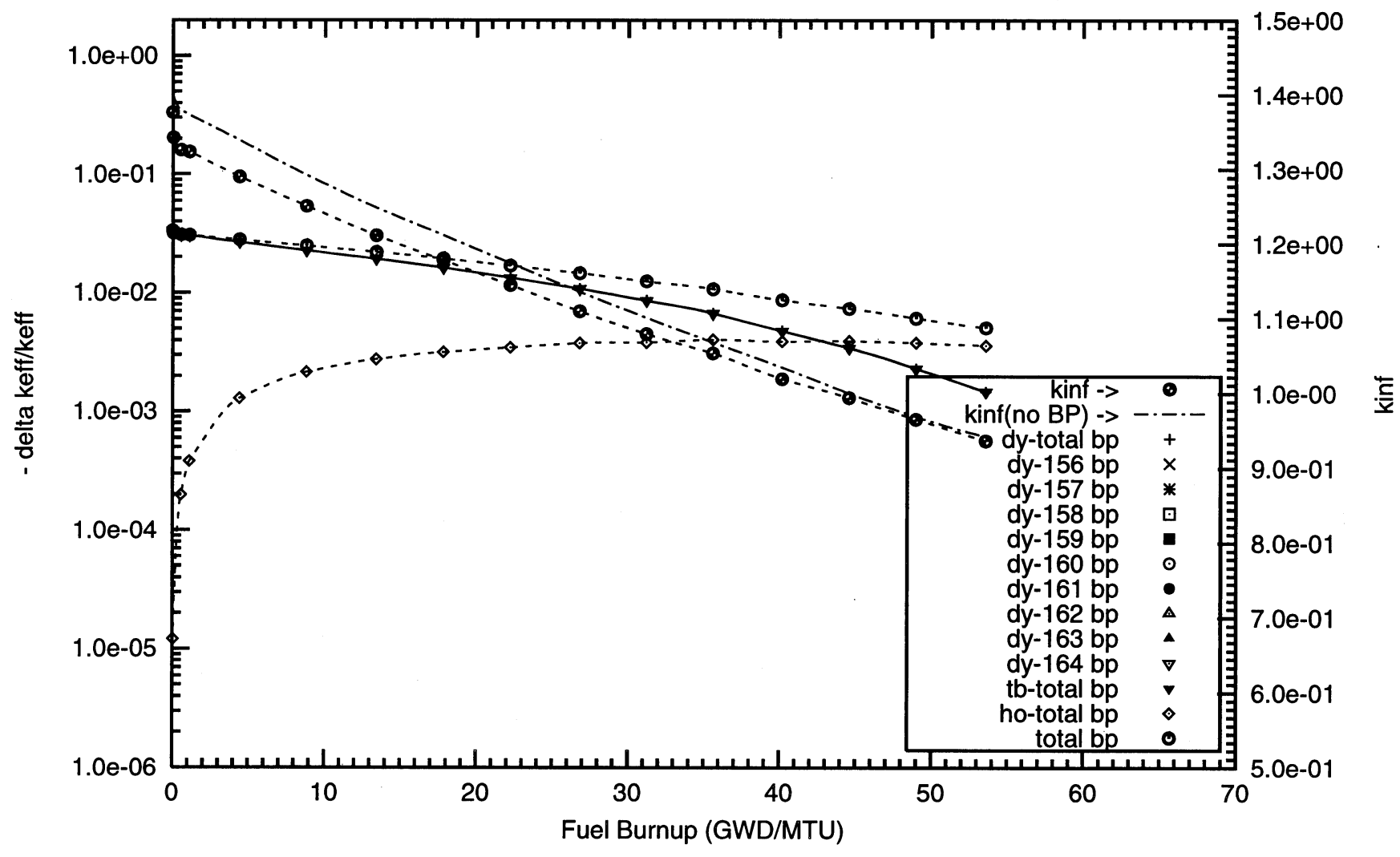


Figure D3.4. Negative Reactivity and Masses BP for Dy-164 and transmutation daughters

as a function of fuel life for $17 \times 17$ fuel assemblies with 08 poison rods,

8.0 mill thick Dy2O3 poison coating on the outer radial surface of the UO2 pellets

Reactor power $3400 \mathrm{MWth}, 193$ fuel assemblies, initial enrichment 4.5W\%U-235

(Case : dy164_4f_08_bp_fuel o)
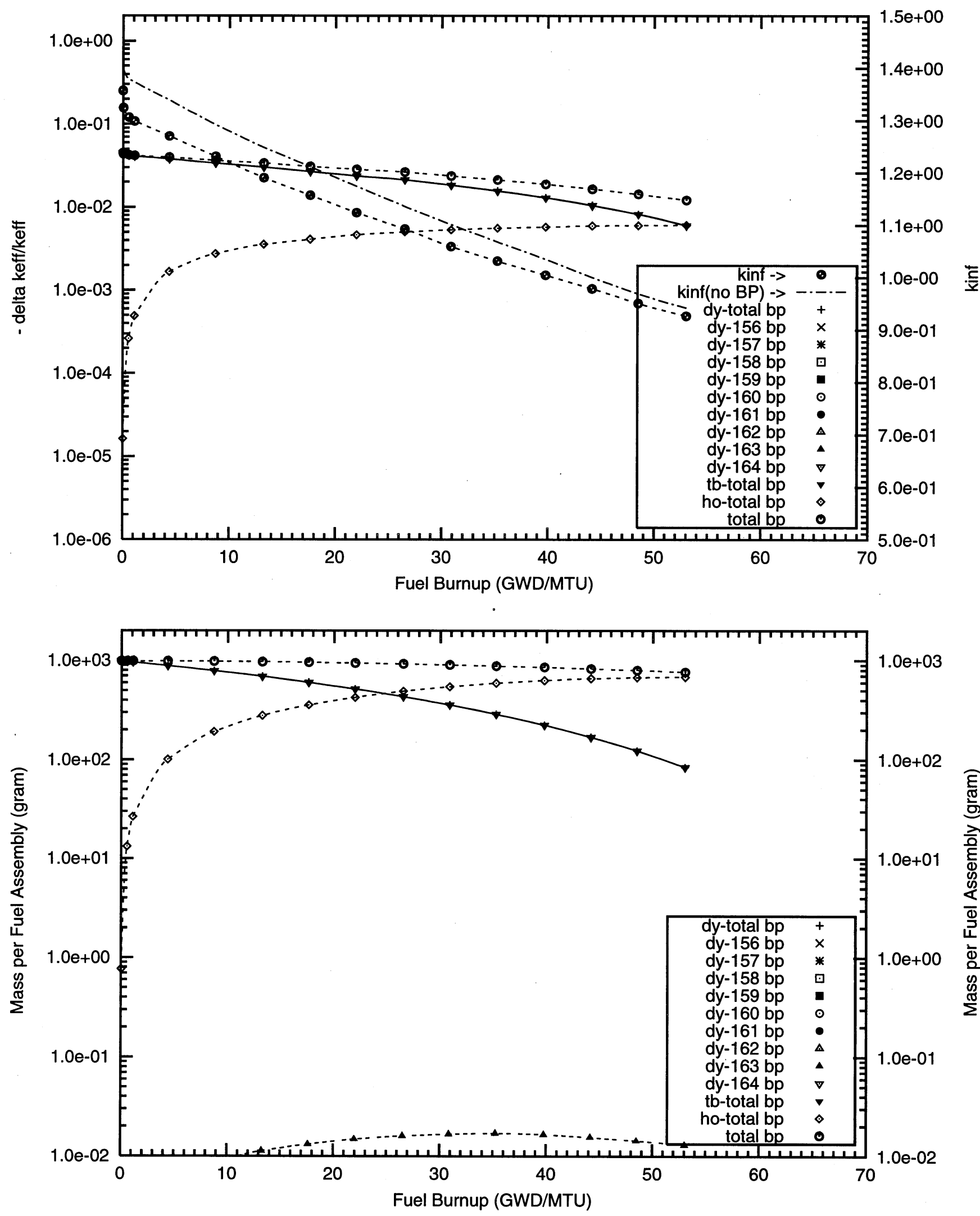
Figure D3.5. Negative Reactivity and Masses BP for Dy-164 and transmutation daughters as a function of fuel life for $17 \times 17$ fuel assemblies with 18 poison rods,

2.0 mill thick Dy2O3 poison coating on the outer radial surface of the UO2 pellets Reactor power $3400 \mathrm{MWth}, 193$ fuel assemblies, initial enrichment 4.5w\%U-235 (Case : dy164_2f_16_bp_fuel o)
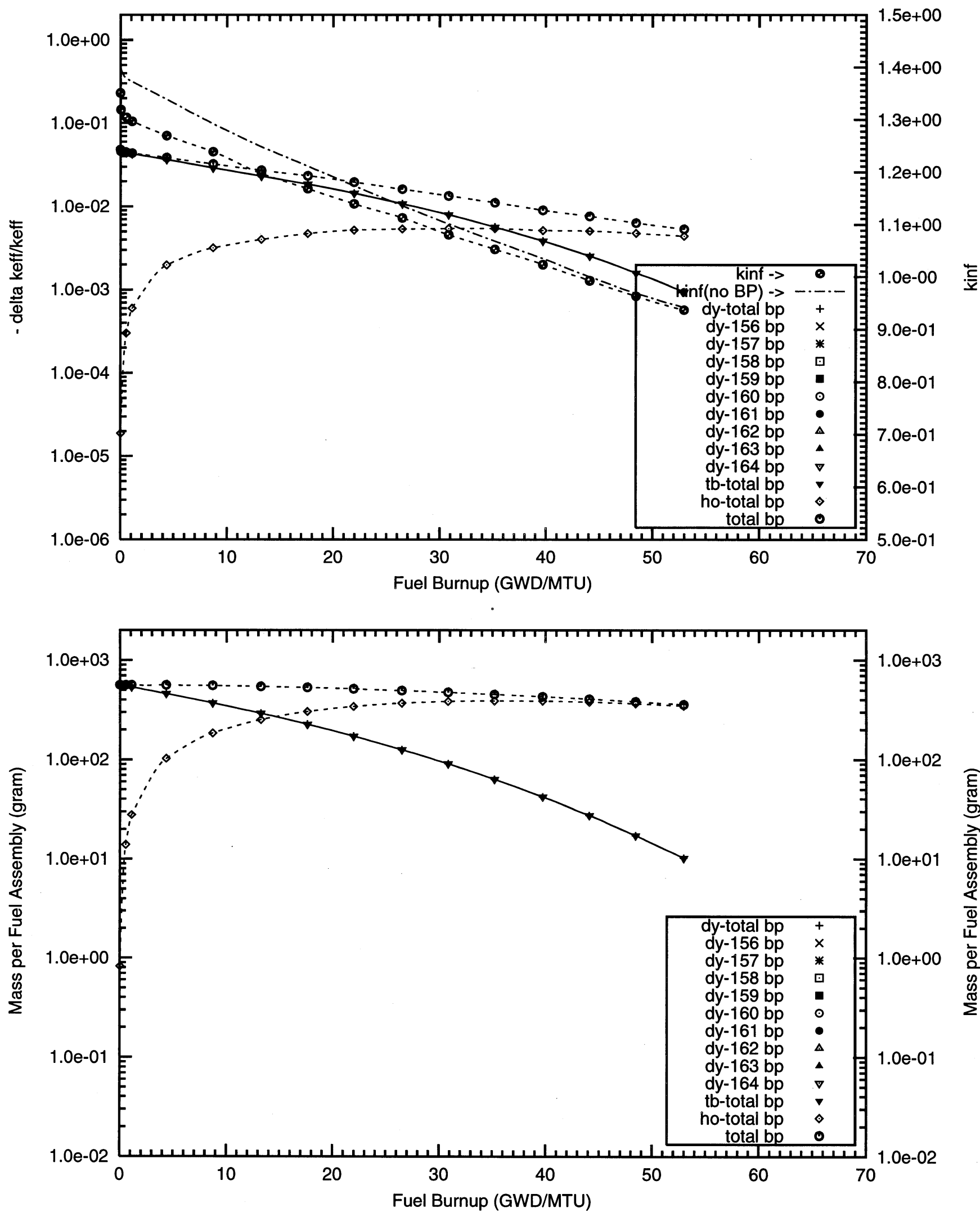
Figure D3.6. Negative Reactivity and Masses BP for Dy-164 and transmutation daughters as a function of fuel life for $17 \times 17$ fuel assemblies with 18 poison rods, 4.0 mill thick Dy2O3 poison coating on the outer radial surface of the UO2 pellets Reactor power $3400 \mathrm{MWth}, 193$ fuel assemblies, initial enrichment $4.5 \mathrm{w} \% \mathrm{U}-235$ (Case : dy164_3f_16_bp_fuel o)

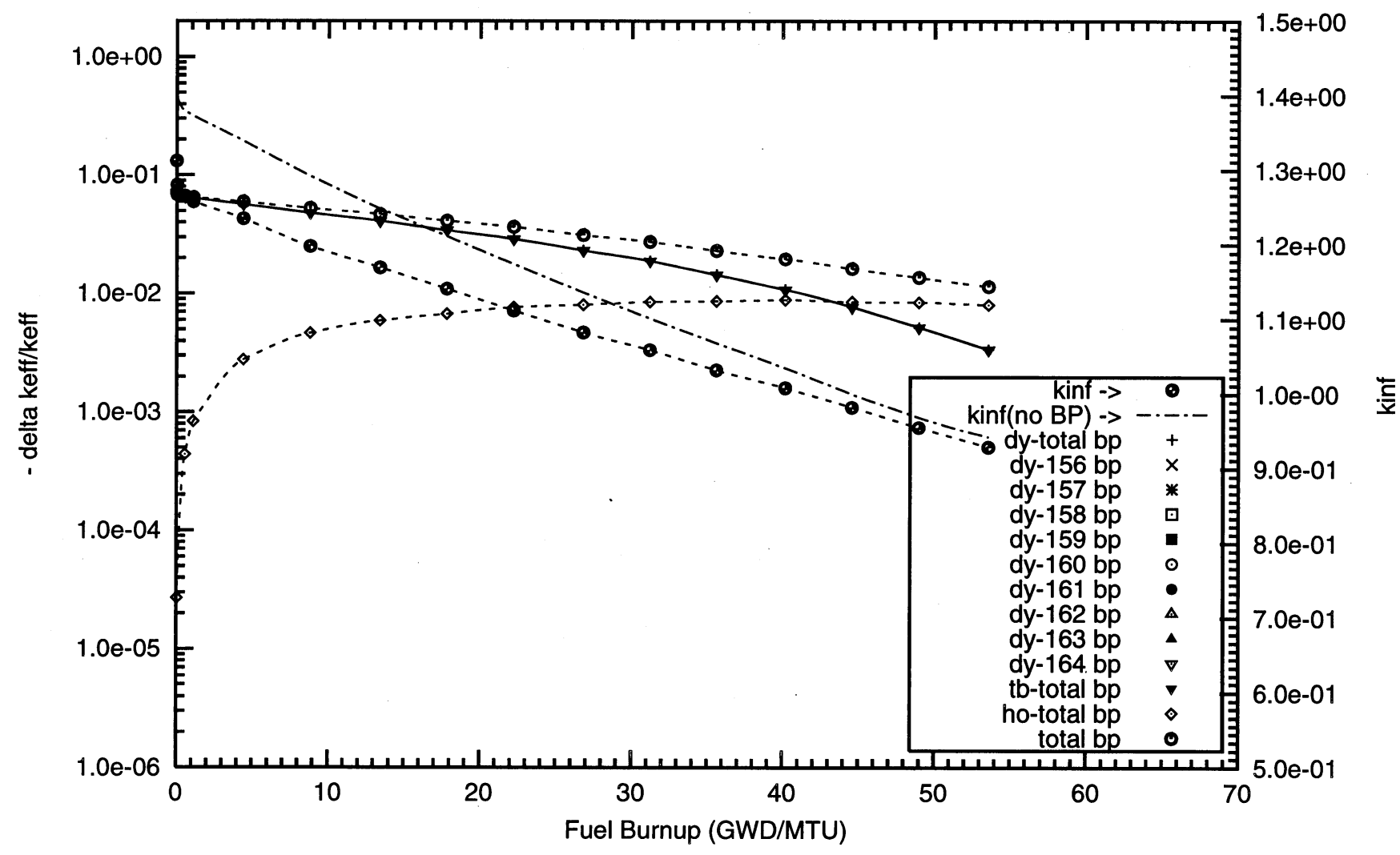


Figure D3.7. Negative Reactivity and Masses BP for Dy-164 and transmutation daughters as a function of fuel life for $17 \times 17$ fuel assemblies with 64 poison rods,

0.5 mill thick Dy2O3 poison coating on the outer radial surface of the UO2 pellets Reactor power $3400 \mathrm{MWth}, 193$ fuel assemblies, initial enrichment 4.5w\%U-235

(Case : dy164_0f_64_bp_fuel o)
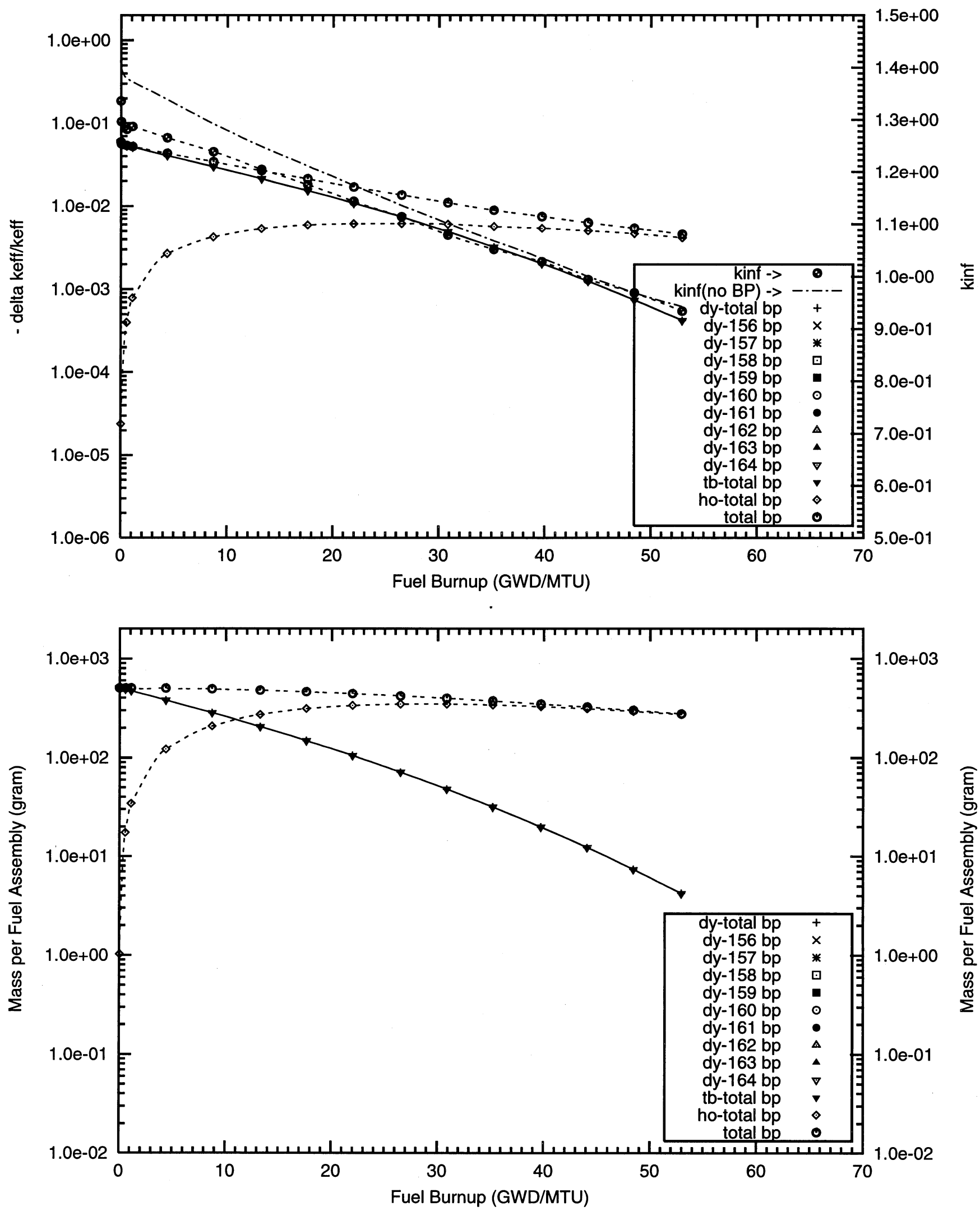
Figure D3.8. Negative Reactivity and Masses BP for Dy-164 and transmutation daughters as a function of fuel life for $17 \times 17$ fuel assemblies with 64 poison rods,

1.0 mill thick Dy2O3 poison coating on the outer radial surface of the UO2 pellets

Reactor power $3400 \mathrm{MWth}, 193$ fuel assemblies, initial enrichment 4.5W\%U-235

(Case : dy164_1f_64_bp_fuel o)
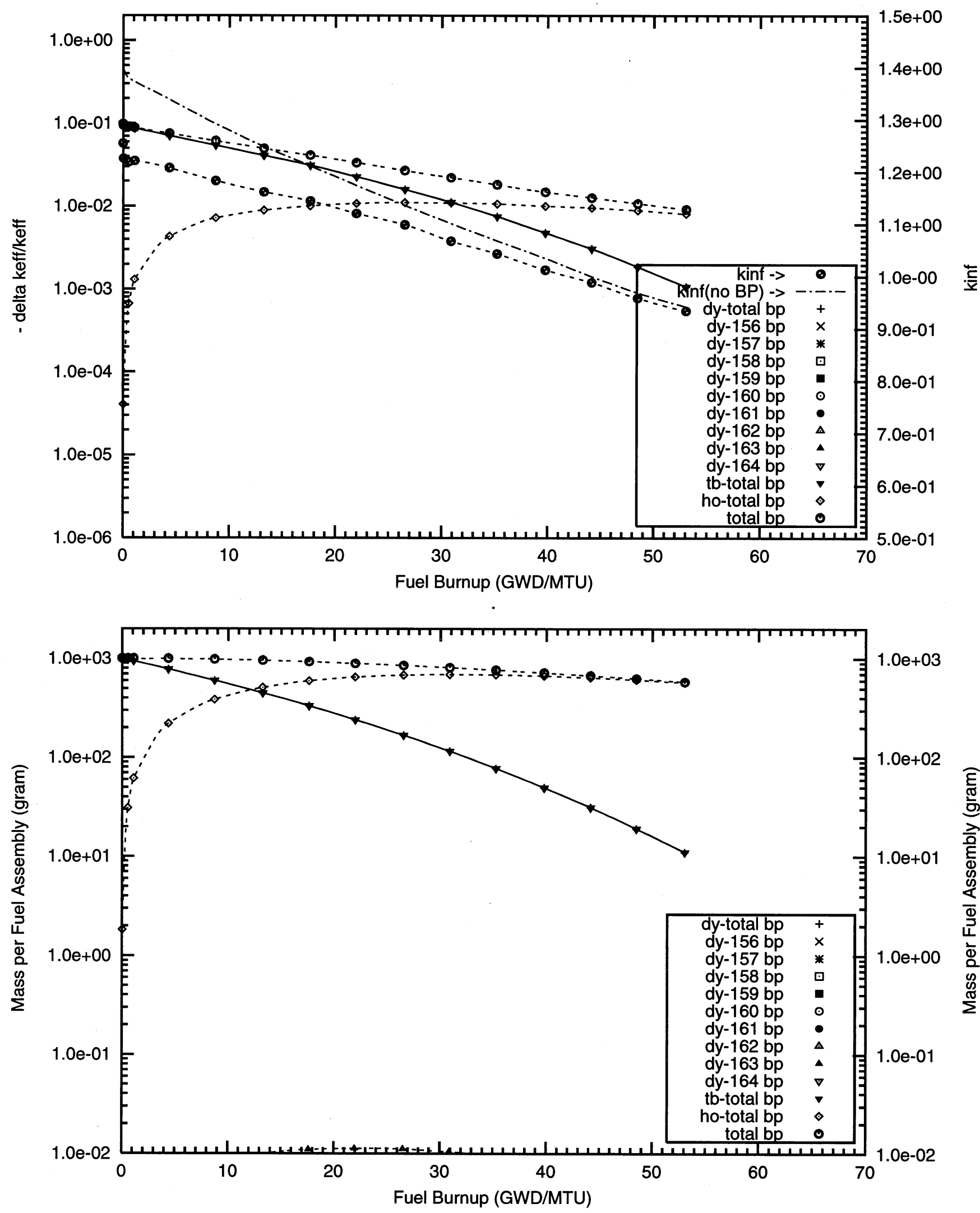
Figure D3.9. Negative Reactivity and Masses BP for Dy-164 and transmutation daughters

as a function of fuel life for $17 \times 17$ fuel assemblies with 64 poison rods,

2.0 mill thick Dy2O3 poison coating on the outer radial surface of the UO2 pellets

Reactor power $3400 \mathrm{MW}$ th, 193 fuel assemblies, initial enrichment $4.5 \mathrm{~W} \% \mathrm{U}-235$

(Case : dy164_2f_64_bp_fuel o)
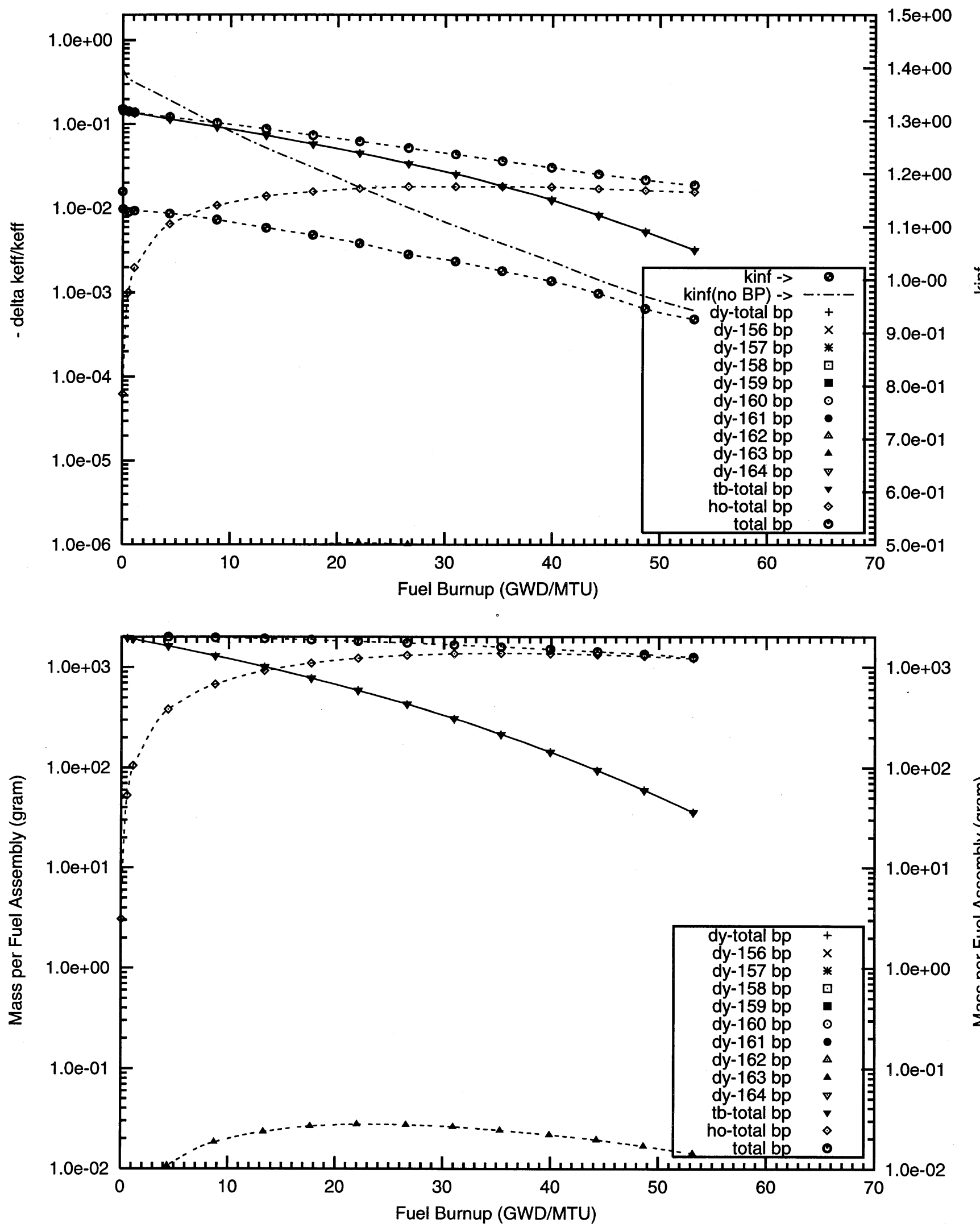
Figure D3.10. Negative Reactivity and Masses BP for Dy-164 and transmutation daughters as a function of fuel life for $17 \times 17$ fuel assemblies with 104 poison rods, 0.5 mill thick Dy2O3 poison coating on the outer radial surface of the UO2 pellets Reactor power $3400 \mathrm{MW}$ th, 193 fuel assemblies, initial enrichment $4.5 \mathrm{~W} \% \mathrm{U}-235$

(Case : dy164_Of_104_bp_fuel o)
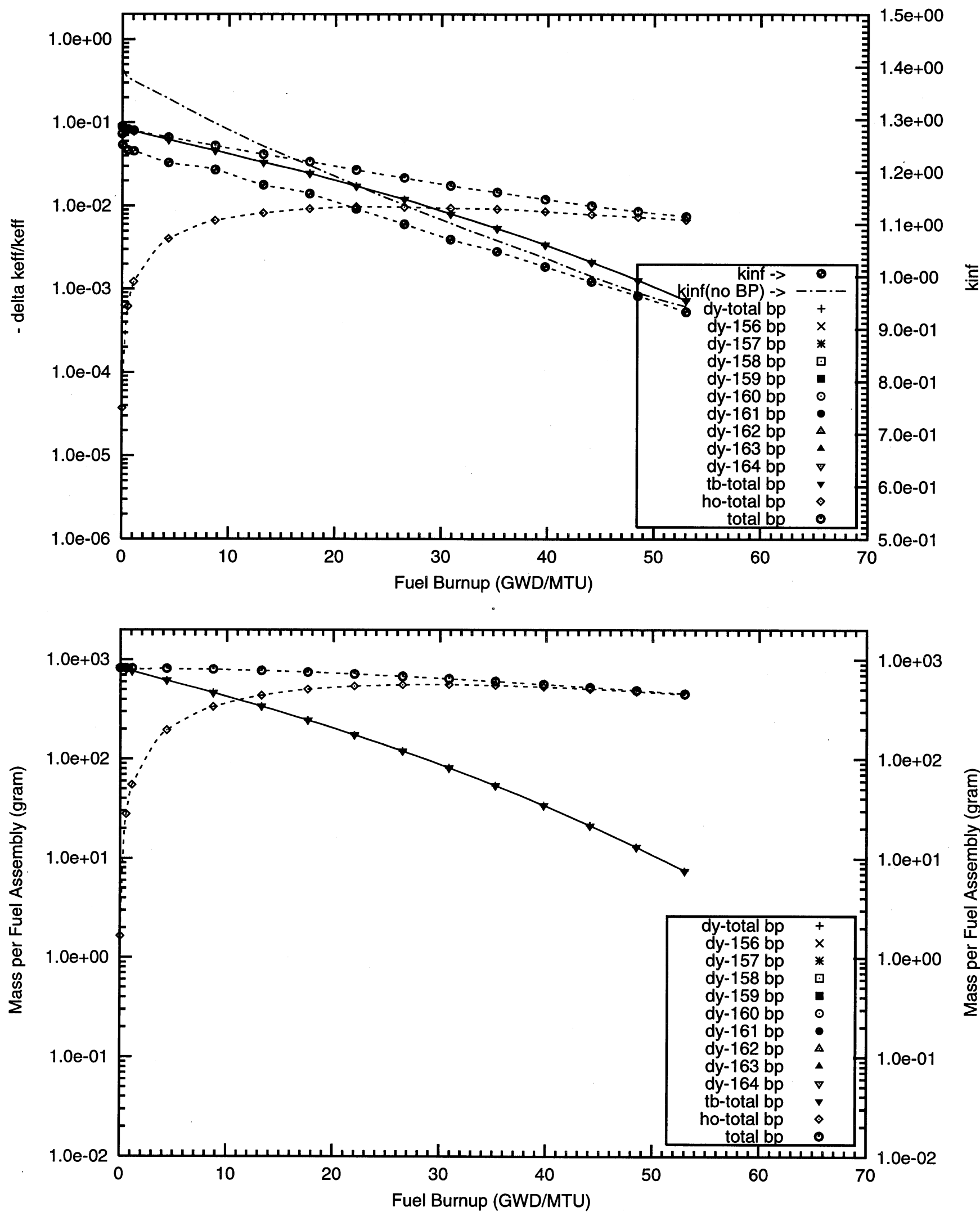


\section{Appendix D4}

Reactivity Worths and Masses of Dysprosium Alloyed with the Zircaloy Cladding of Fuel Rods

\section{Table 20 Cases}




\section{D4 - Dysprosium with Natural Isotopic Abundances}


Figure D4.1. Negative Reactivity and Masses BP for Dy-nat and transmutation daughters as a function of fuel lite for $17 \times 17$ fuel assemblies with 104 poison rods,

$8.0 \mathrm{w} \%$ Dy poison homogeneously mixed in the fuel cladding,

Reactor power $3400 \mathrm{MWth}, 193$ fuel assemblies, initial enrichment $4.5 \mathrm{w} \% \mathrm{U}-235$

(Case : dy000_4c_100p_h2o_104_bp_fuel_2_o)
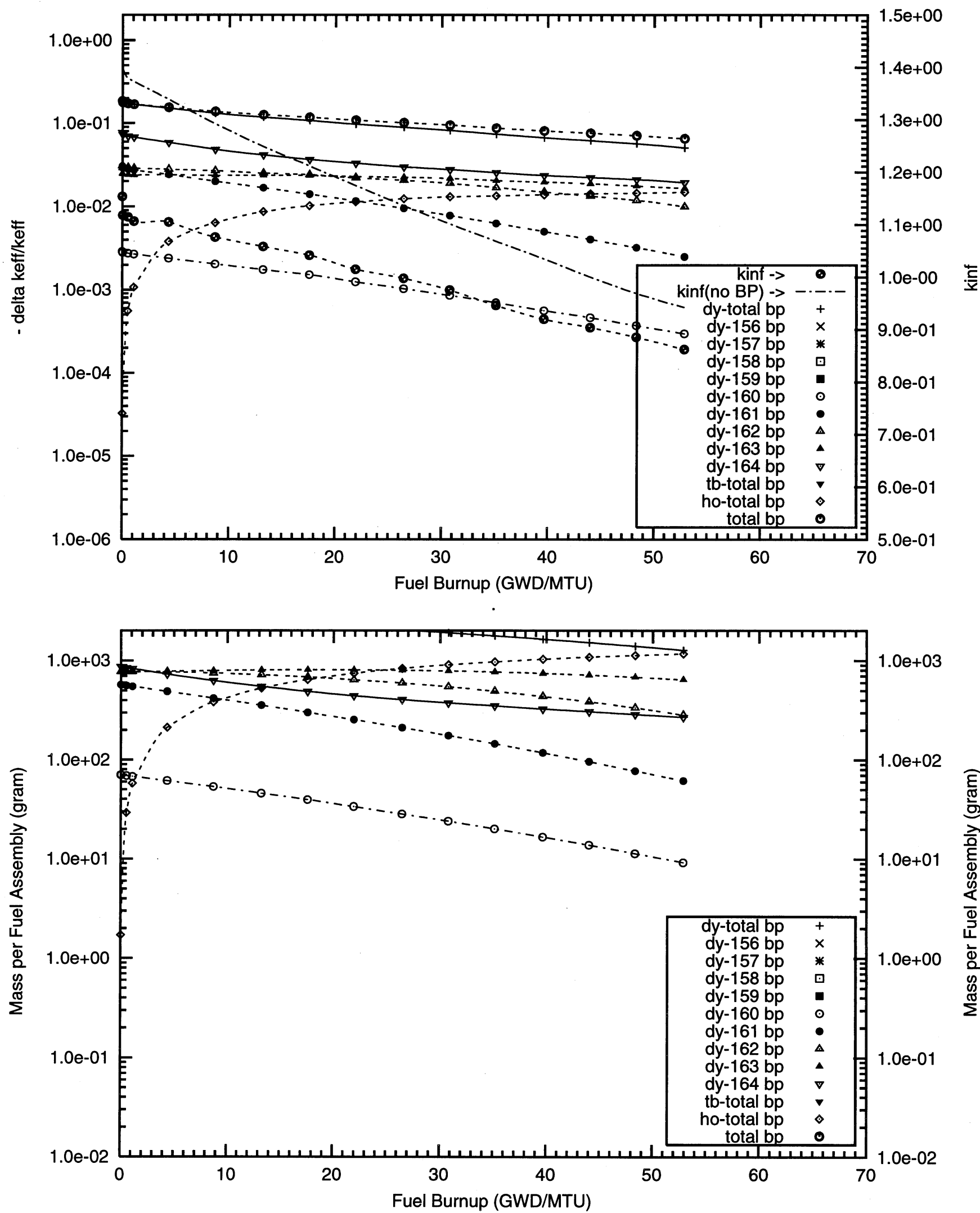



\section{D4 - Dysprosium Fully Enriched in ${ }^{164}$ Dy}


Figure D4.2. Negative Reactivity and Masses BP for Dy-164 and transmutation daughters

as a function of fuel life for $17 \times 17$ fuel assemblies with 64 poison rods,

$2.0 \mathrm{w} \%$ Dy poison homogeneously mixed in the fuel cladding,

Reactor power $3400 \mathrm{MWth}, 193$ fuel assemblies, initial enrichment $4.5 \mathrm{w} \% \mathrm{U}-235$

(Case : dy164_2c_64_bp_fuel o)
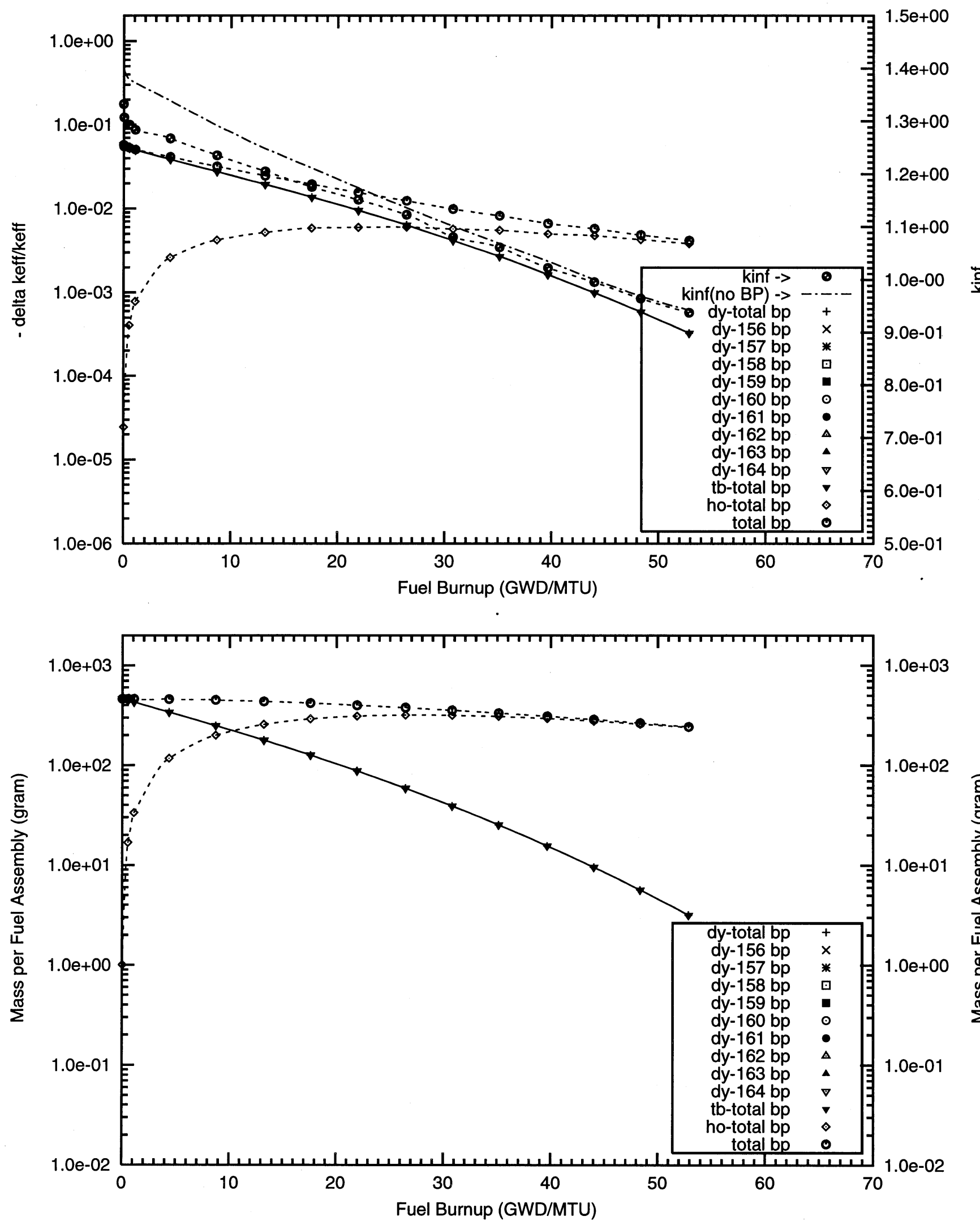
Figure D4.3. Negative Reactivity and Masses BP for Dy-164 and transmutation daughters

as a function of fuel life for $17 \times 17$ fuel assemblies with 104 poison rods,

$2.0 \mathrm{w} \%$ Dy poison homogeneously mixed in the fuel cladding,

Reactor power $3400 \mathrm{MWth}, 193$ fuel assemblies, initial enrichment $4.5 \mathrm{w} \% \mathrm{U}-235$

(Case : dy164_2c_104_bp_fuel o)
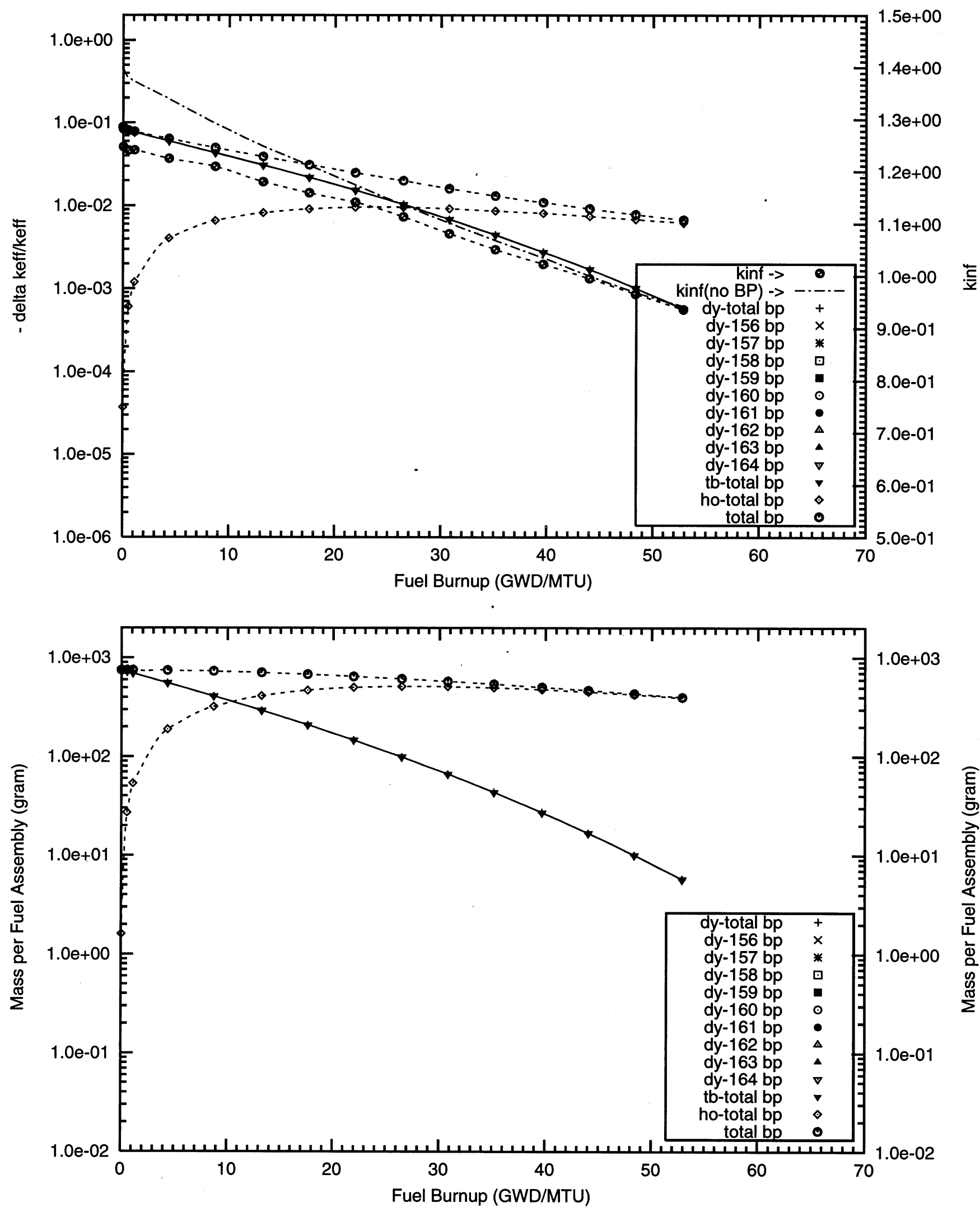

D-62 
Appendix E1

Reactivity Worths and Masses of $\mathrm{Eu}_{2} \mathrm{O}_{3}$ Burnable Poison Homogeneously Mixed in the Fuel Pellets

\section{Table 21 Cases}




\section{E1 - Europium with Natural Isotopic Abundances}



Figure E1.1. Negative Reactivity and Masses BP for Eu-nat and transmutation daughters as a function of fuel life for $17 \times 17$ fuel assemblies with 08 poison rods,

$4.0 \mathrm{w} \%$ Eu2O3 poison homogeneously mixed in the UO2 pellets

Reactor power $3400 \mathrm{MWth}, 193$ fuel assemblies, initial enrichment $4.5 \mathrm{w} \% \mathrm{U}-235$

(Case : eu000_3_08_bp_fuel o)

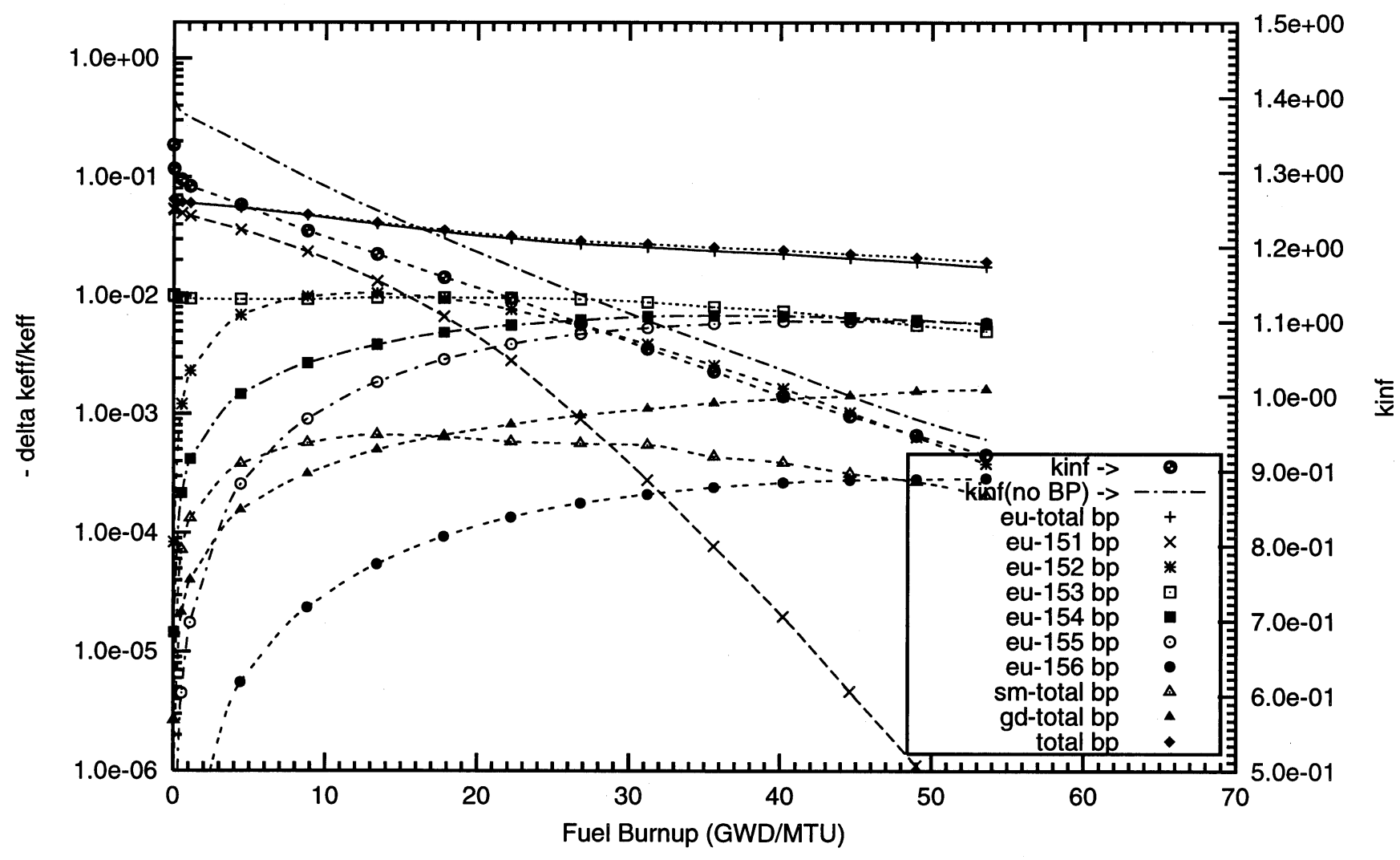





\section{E1 - Europium Fully Enriched in ${ }^{151}$ Eu}


Figure E1.2. Negative Reactivity and Masses BP for Eu-151 and transmutation daughters as a function of fuel life for $17 \times 17$ fuel assemblies with 08 poison rods, $4.0 \mathrm{w} \%$ Eu2O3 poison homogeneously mixed in the UO2 pellets

Reactor power $3400 \mathrm{MWth}, 193$ fuel assemblies, initial enrichment $4.5 \mathrm{w} \% \mathrm{U}-235$ (Case : eu151_3_08_bp_fuel o)

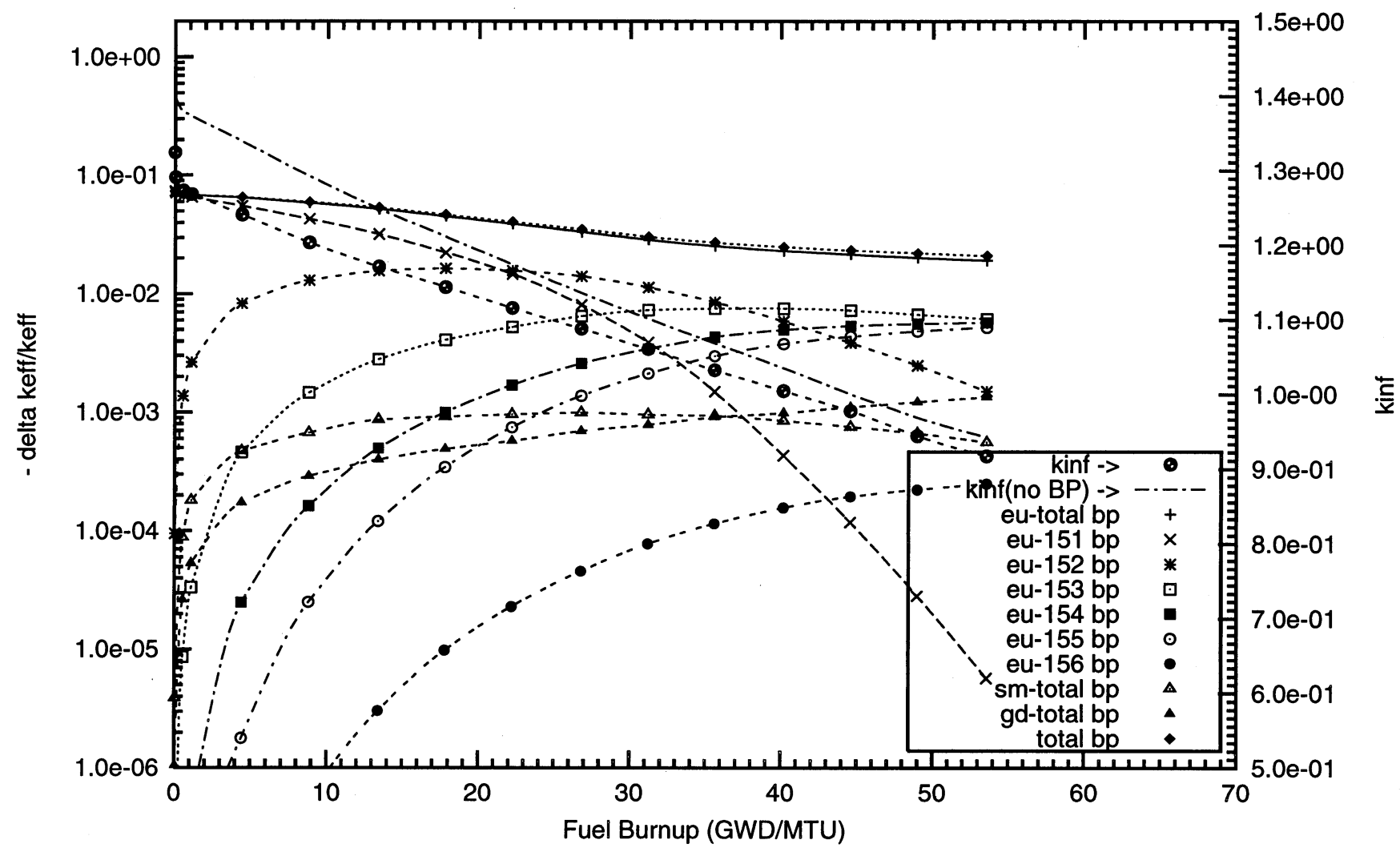





\section{E1 - Europium Fully Enriched in ${ }^{153}$ Eu}



Figure E1.3. Negative Reactivity and Masses BP for Eu-153 and transmutation daughters as a function of fuel life for $17 \times 17$ fuel assemblies with 08 poison rods, $4.0 \mathrm{w} \%$ Eu2O3 poison homogeneously mixed in the UO2 pellets

Reactor power $3400 \mathrm{MWth}, 193$ fuel assemblies, initial enrichment 4.5W\%U-235 (Case : eu153_3_08_bp_fuel o)

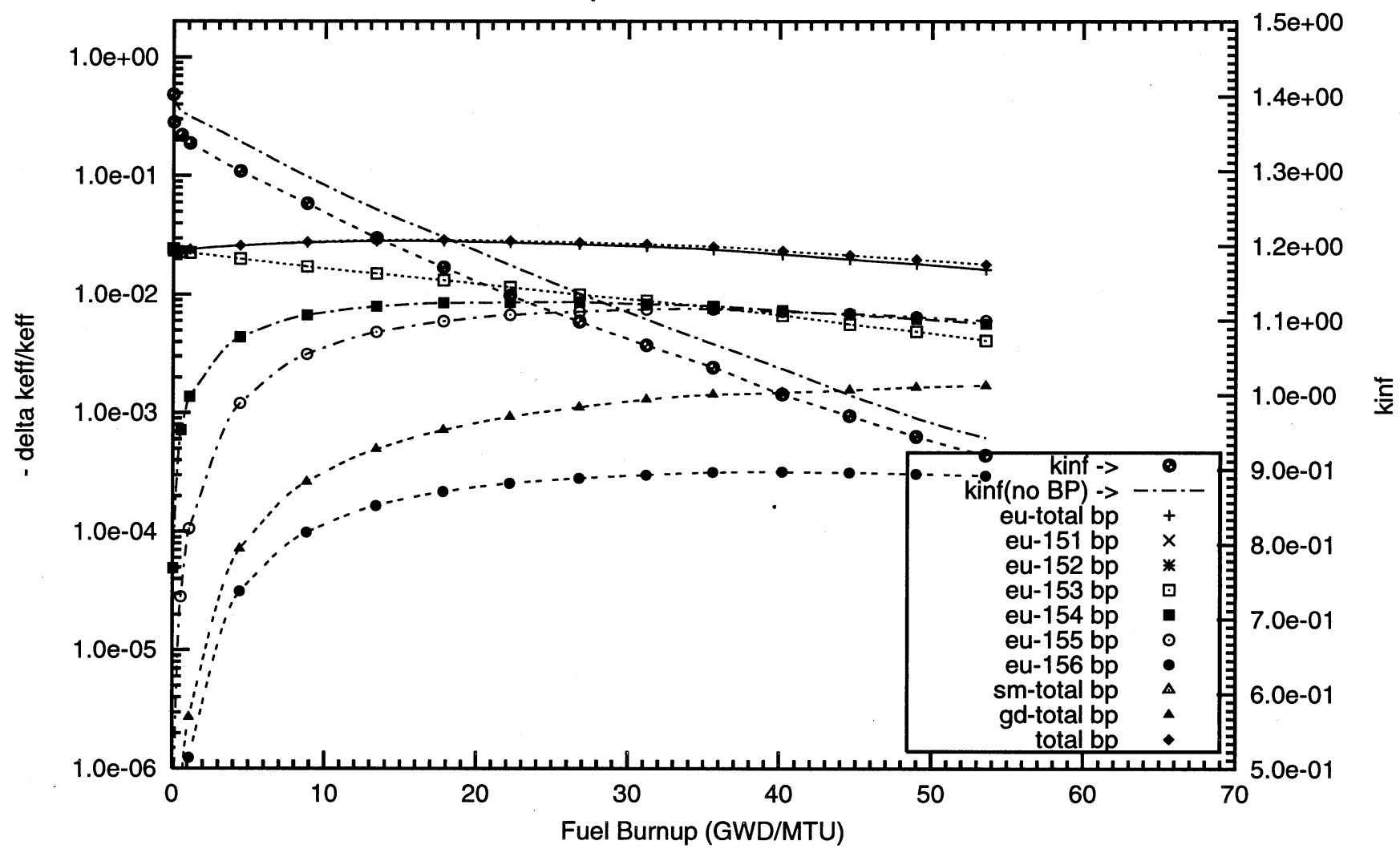




\section{Appendix E2}

Reactivity Worths and Masses of $\mathrm{Eu}_{2} \mathrm{O}_{3}$ Burnable Poison Homogeneously Mixed in the Outer One-Third Volume of the Fuel Pellets

\section{Table 22 Cases}



E2 - Europium with Natural Isotopic Abundances 

Figure E2.1. Negative Reactivity and Masses BP for Eu-nat and transmutation daughters as a function of fuel life for $17 \times 17$ fuel assemblies with 08 poison rods,

$8.0 \mathrm{w} \%$ Eu2O3 poison homogeneously mixed in the outer one-third volume of the UO2 pellets

Reactor power $3400 \mathrm{MWth}, 193$ fuel assemblies, initial enrichment $4.5 \mathrm{w} \% \mathrm{U}-235$

(Case : eu000_4b_08_bp_fuel o)

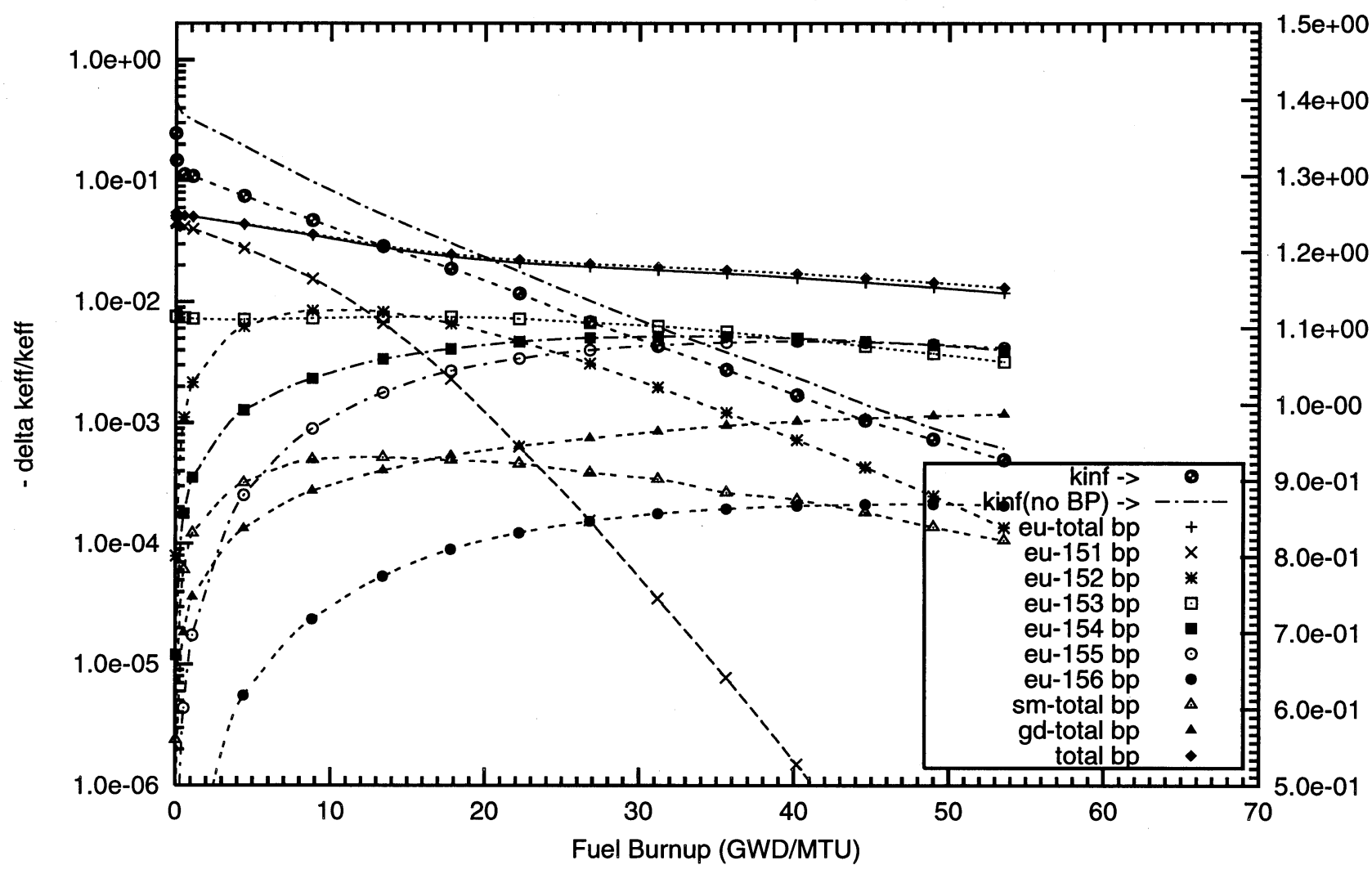


E2 - Europium Fully Enriched in ${ }^{151}$ Eu 

Figure E2.2. Negative Reactivity and Masses BP for Eu-151 and transmutation daughters as a function of fuel life for $17 \times 17$ fuel assemblies with 08 poison rods,

$4.0 \mathrm{w} \%$ Eu2O3 poison homogeneously mixed in the outer one-third volume of the UO2 pellets Reactor power $3400 \mathrm{MW}$ th, 193 fuel assemblies, initial enrichment 4.5w\%U-235

(Case : eu151_3b_08_bp_fuel o)

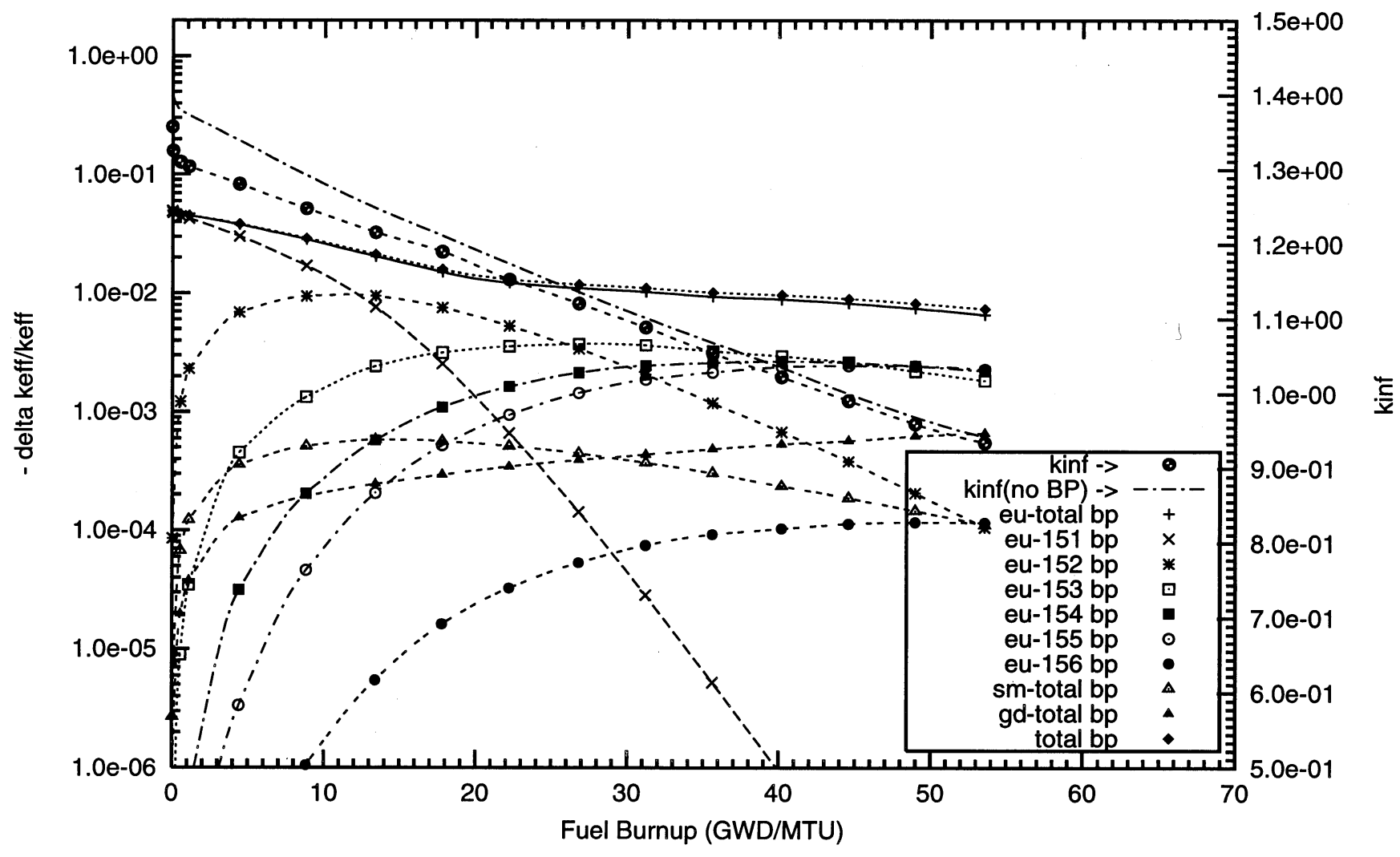





\section{E2 - Europium Fully Enriched in ${ }^{153}$ Eu}



Figure E2.3. Negative Reactivity and Masses BP for Eu-153 and transmutation daughters as a function of fuel life for $17 \times 17$ fuel assemblies with 08 poison rods,

$8.0 \mathrm{w} \%$ Eu2O3 poison homogeneously mixed in the outer one-third volume of the $\mathrm{UO} 2$ pellets Reactor power $3400 \mathrm{MWth}, 193$ fuel assemblies, initial enrichment $4.5 \mathrm{w} \% \mathrm{U}-235$

(Case : eu153_4b_08_bp_fuel o)

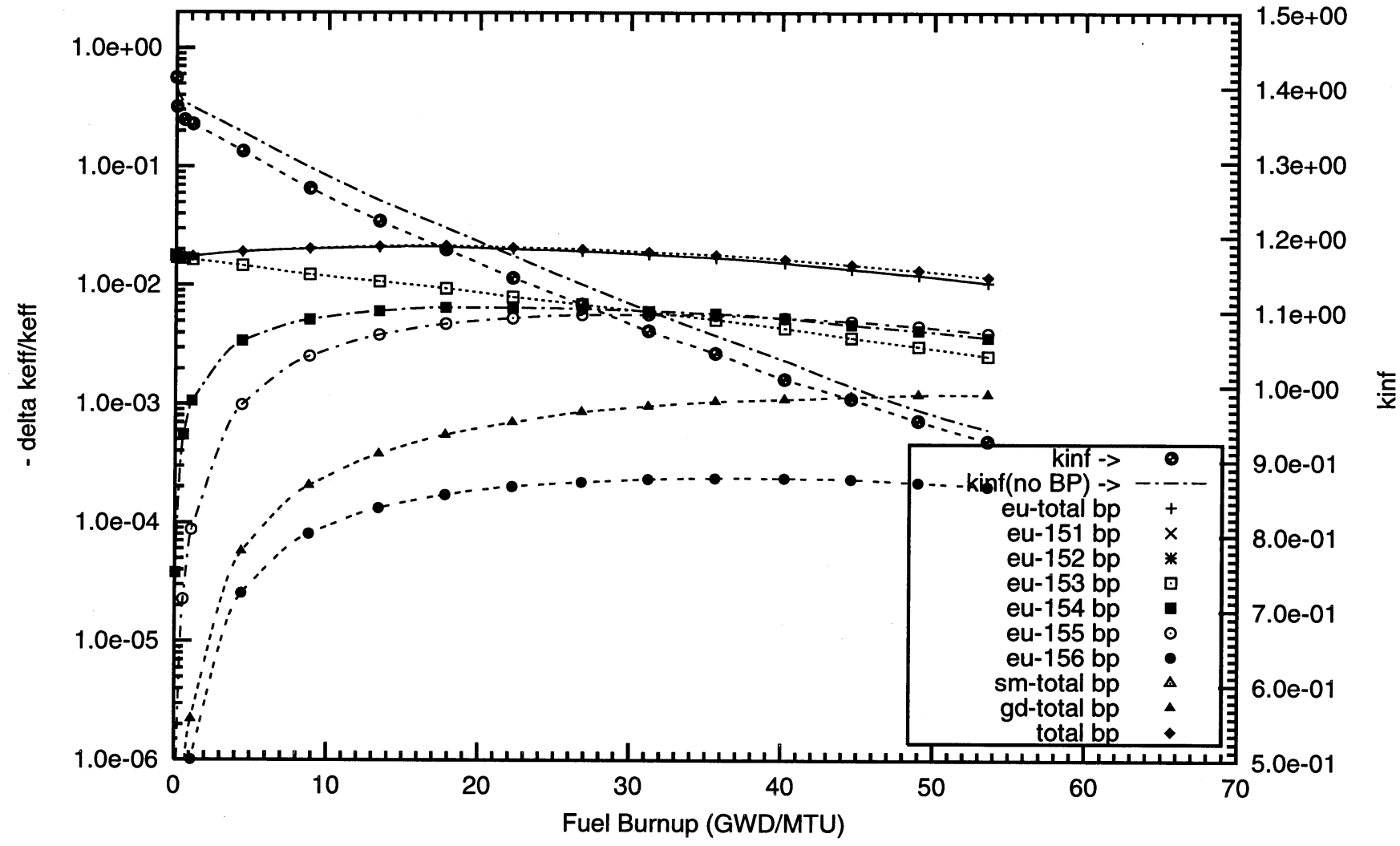


Appendix E3

Reactivity Worths and Masses of $\mathrm{Eu}_{2} \mathrm{O}_{3}$ Coating on the Surface of the Fuel Pellets

\author{
Table 23 Cases
}



E3 - Europium with Natural Isotopic Abundances 

Figure E3.1. Negative Reactivity and Masses BP for Eu-nat and transmutation daughters as a function of fuel life for $17 \times 17$ fuel assemblies with 64 poison rods,

1.0 mill thick Eu2O3 poison coating on the outer radial surface of the UO2 pellets Reactor power $3400 \mathrm{MWth}, 193$ fuel assemblies, initial enrichment $4.5 \mathrm{w} \% \mathrm{U}-235$

(Case : eu000_1f_64_bp_fuel o)
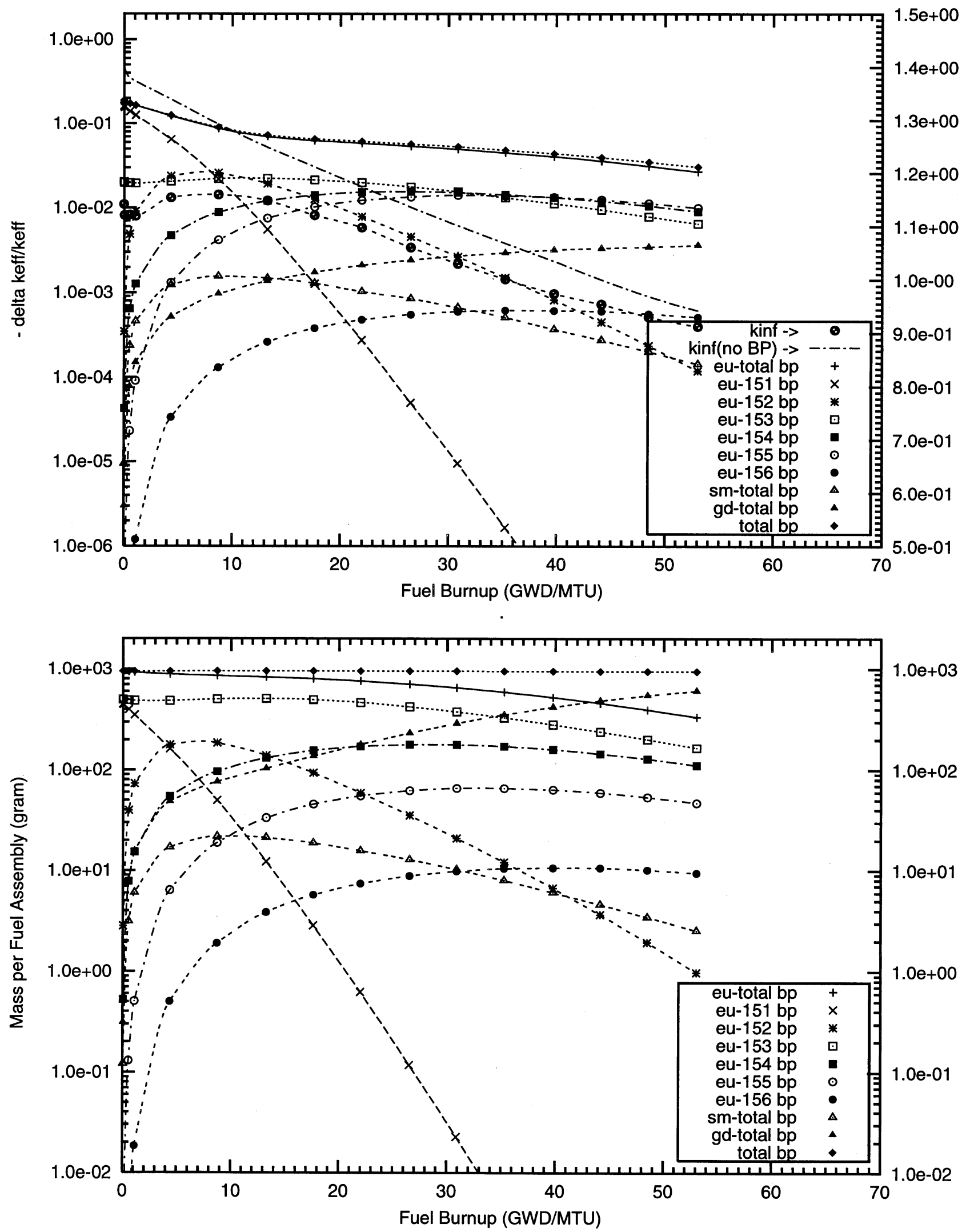



\section{E3 - Europium Fully Enriched in ${ }^{151}$ Eu}


Figure E3.2. Negative Reactivity and Masses BP for Eu-151 and transmutation daughters as a function of fuel life for $17 \times 17$ fuel assemblies with 64 poison rods,

0.2 mill thick Eu2O3 poison coating on the outer radial surface of the UO2 pellets

Reactor power $3400 \mathrm{MWth}, 193$ fuel assemblies, initial enrichment 4.5w\%U-235

(Case : eu151_8f_64_bp_fuel o)
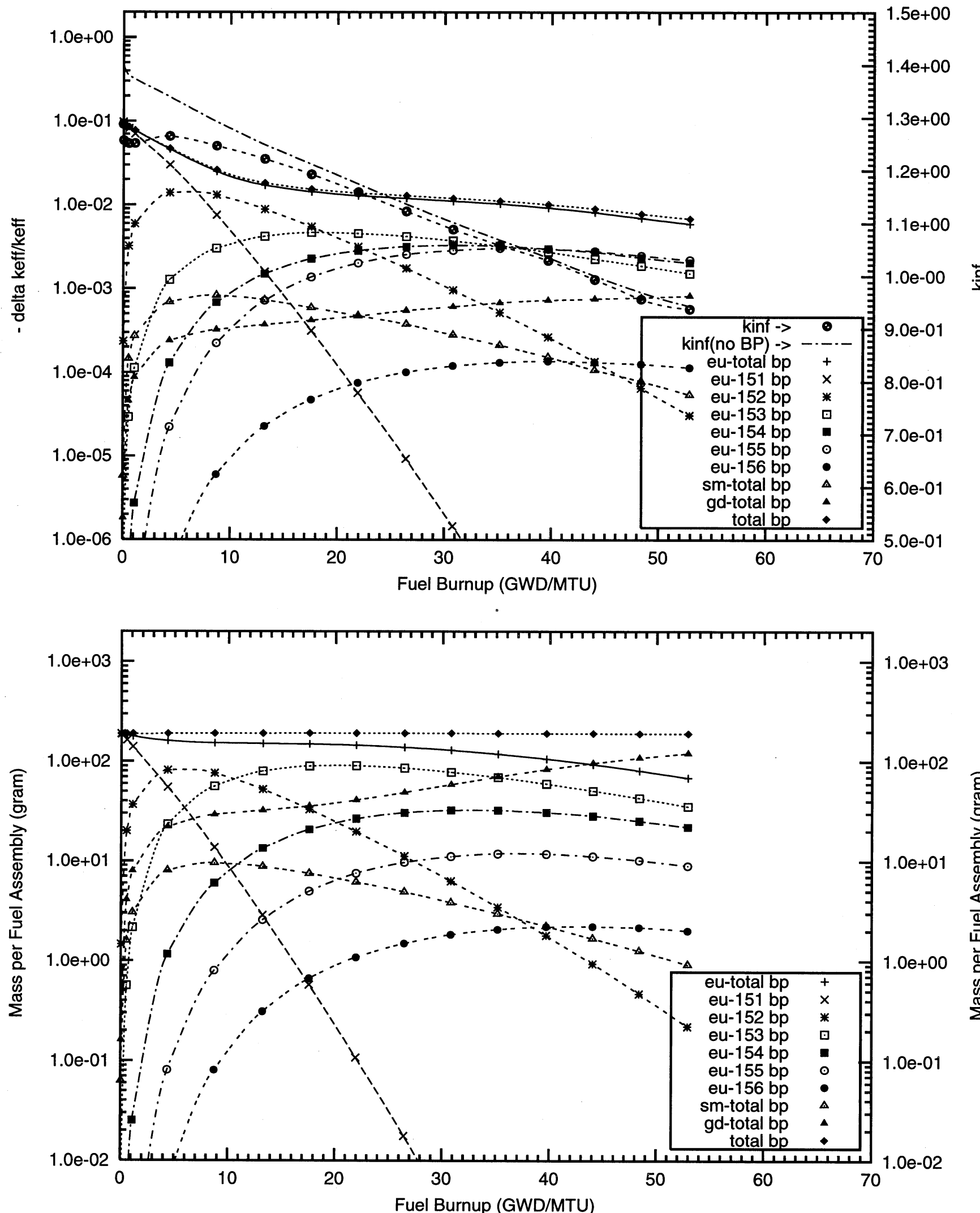

Fuel Burnup (GWD/MTU) 
Figure E3.3. Negative Reactivity and Masses BP for Eu-151 and transmutation daughters as a function of fuel life for $17 \times 17$ fuel assemblies with 64 poison rods,

0.5 mill thick Eu2O3 poison coating on the outer radial surface of the UO2 pellets

Reactor power $3400 \mathrm{MWth}, 193$ fuel assemblies, initial enrichment 4.5w\%U-235

(Case : eu151_0f_64_bp_fuel o)
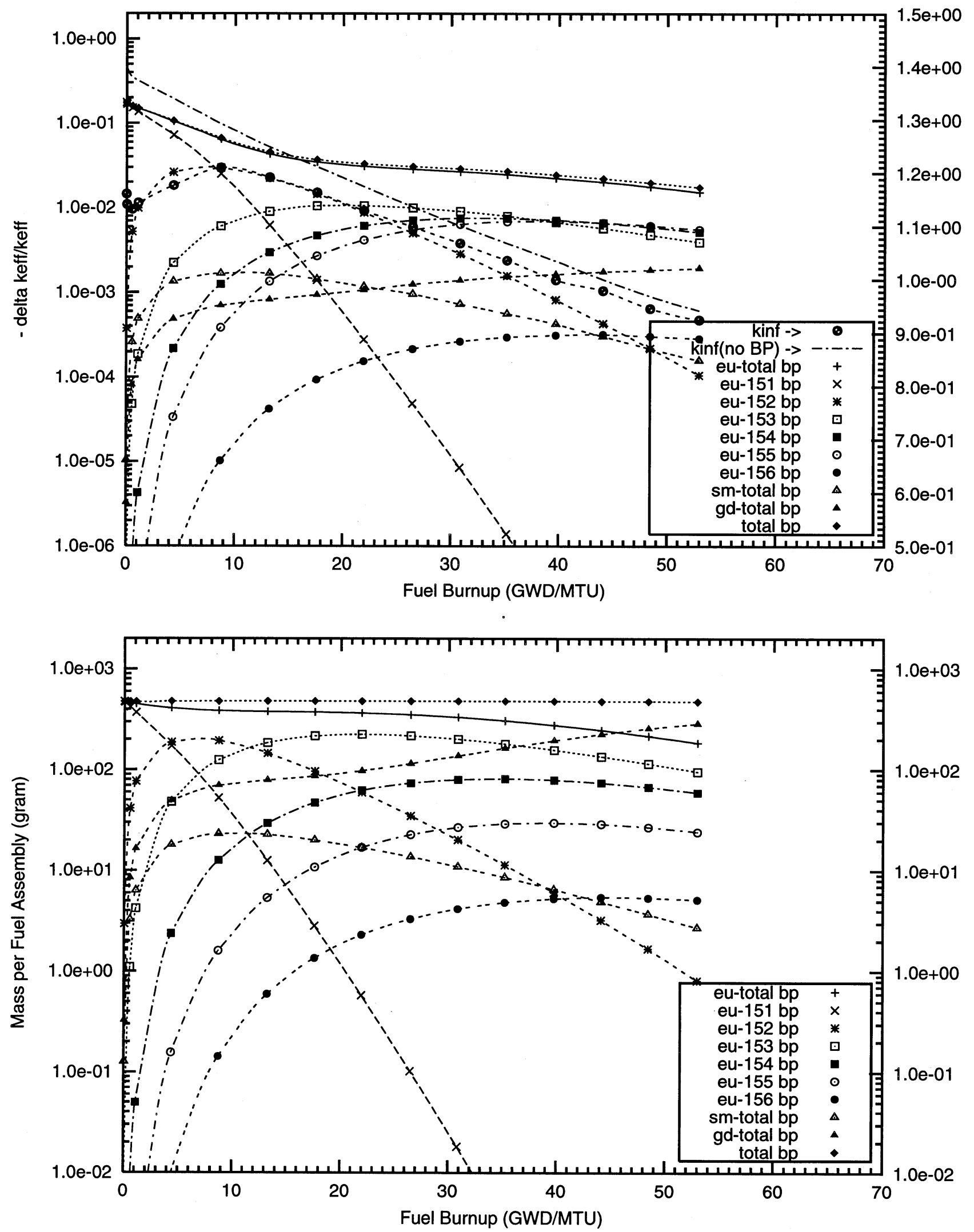
Figure E3.4. Negative Reactivity and Masses BP for Eu-151 and transmutation daughters as a function of fuel life for $17 \times 17$ fuel assemblies with 104 poison rods, 0.2 mill thick Eu2O3 poison coating on the outer radial surface of the UO2 pellets Reactor power $3400 \mathrm{MWth}, 193$ fuel assemblies, initial enrichment 4.5w\%U-235 (Case : eu151_8f_104_bp_fuel o)
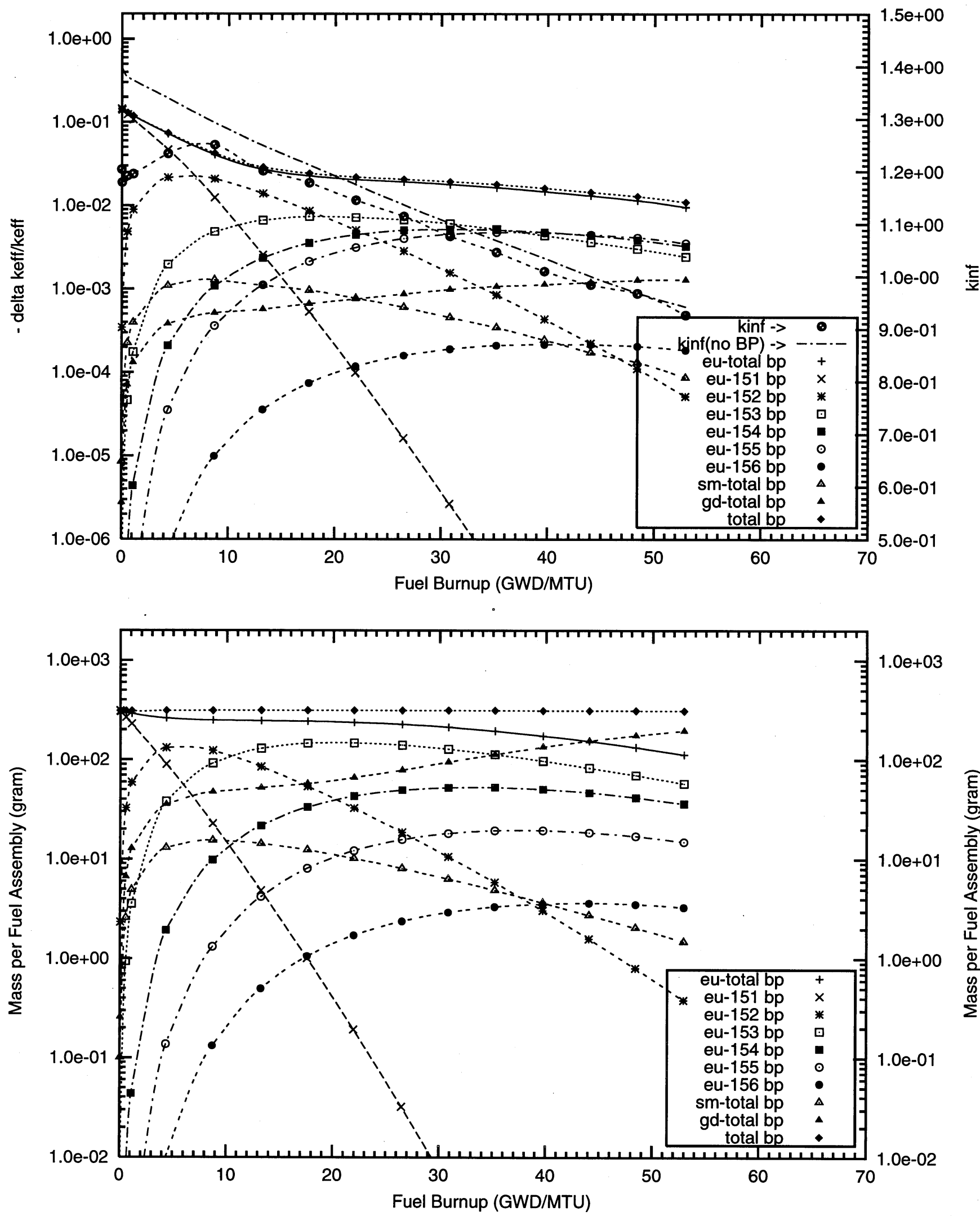



\section{E3 - Europium Fully Enriched in ${ }^{153} \mathbf{E u}$}



Figure E3.5. Negative Reactivity and Masses BP for Eu-153 and transmutation daughters as a function of fuel life for $17 \times 17$ fuel assemblies with 64 poison rods,

0.2 mill thick Eu2O3 poison coating on the outer radial surface of the UO2 pellets

Reactor power $3400 \mathrm{MWth}, 193$ fuel assemblies, initial enrichment 4.5W\%U-235

(Case : eu153_8f_64_bp_fuel o)
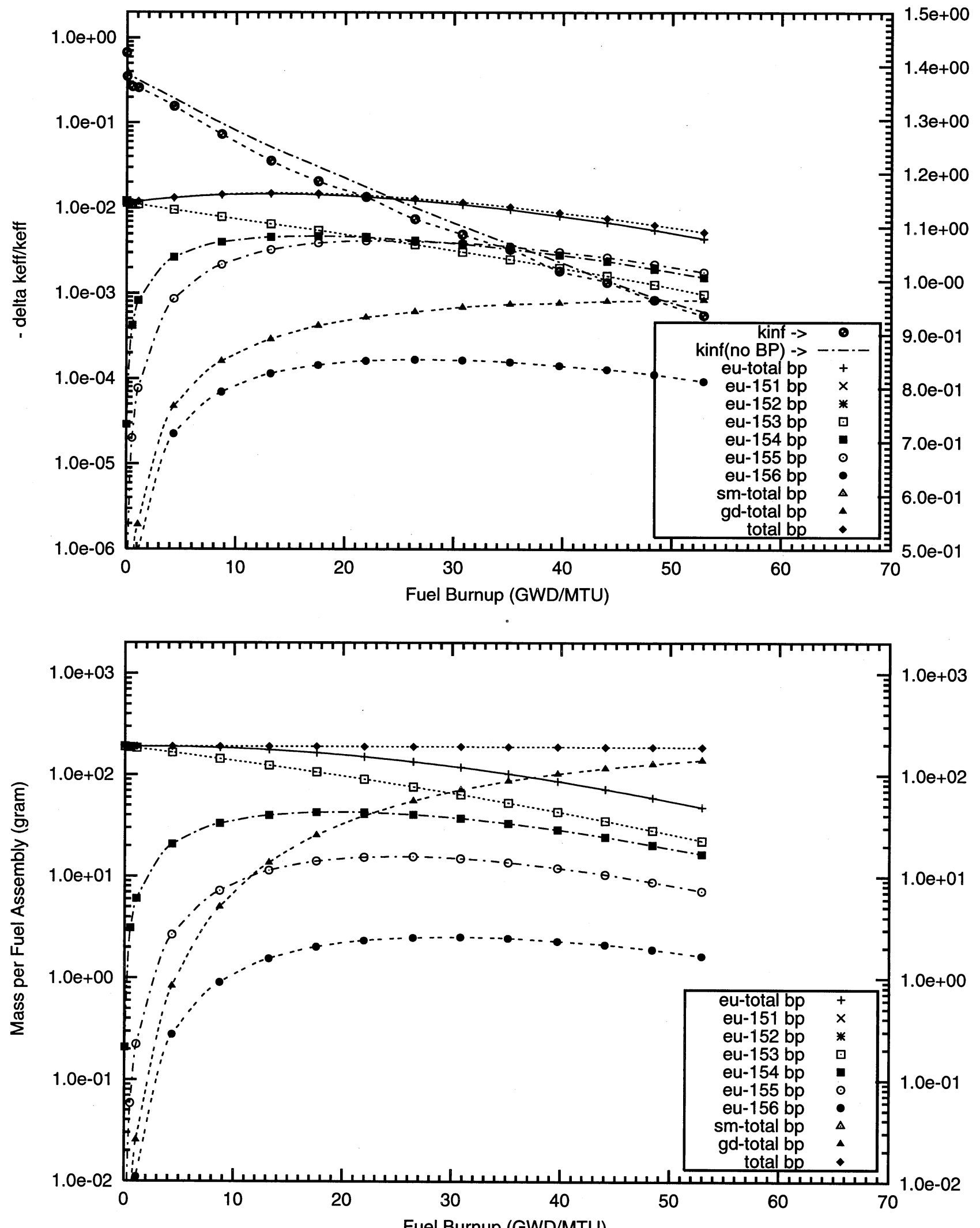


\title{
Appendix E4
}

Reactivity Worths and Masses of Europium Alloyed with the Zircaloy Cladding of Fuel Rods

\author{
Table 24 Cases
}


E4 - Europium with Natural Isotopic Abundances 
Figure E4.1. Negative Reactivity and Masses BP for Eu-nat and transmutation daughters as a function of fuel life for $17 \times 17$ fuel assemblies with 64 poison rods,

$1.0 \mathrm{w} \%$ Eu poison homogeneously mixed in the fuel cladding,

Reactor power $3400 \mathrm{MWth}, 193$ fuel assemblies, initial enrichment $4.5 \mathrm{w} \% \mathrm{U}-235$

(Case : eu000_1c_64_bp_fuel o)
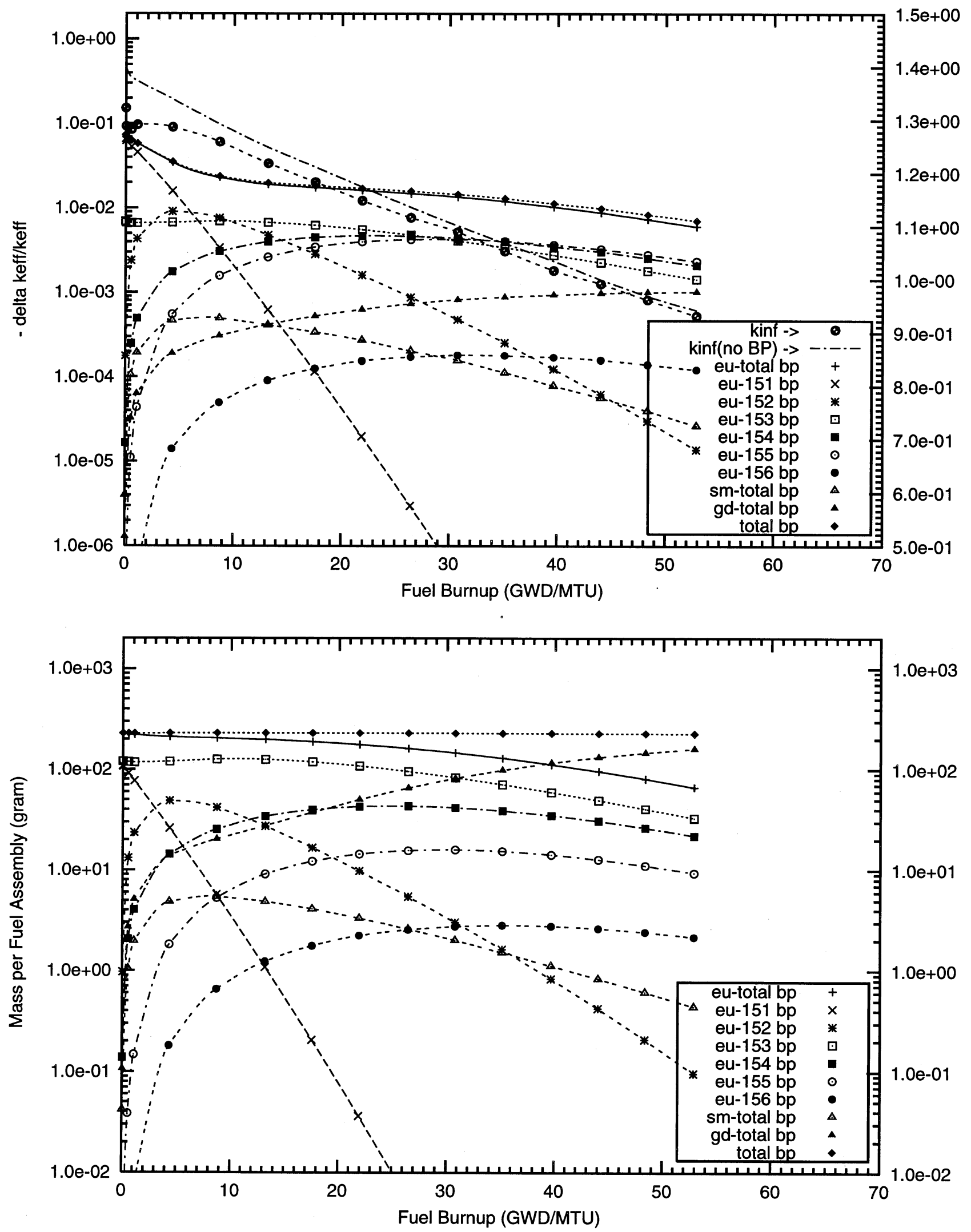
Figure E4.2. Negative Reactivity and Masses BP for Eu-nat and transmutation daughters as a function of fuel life for $17 \times 17$ fuel assemblies with 64 poison rods,

$2.0 \mathrm{w} \%$ Eu poison homogeneously mixed in the fuel cladding,

Reactor power $3400 \mathrm{MWth}, 193$ fuel assemblies, initial enrichment $4.5 \mathrm{w} \% \mathrm{U}-235$

(Case : eu000_2c_100p_h2o_64_bp_fuel_2_o)
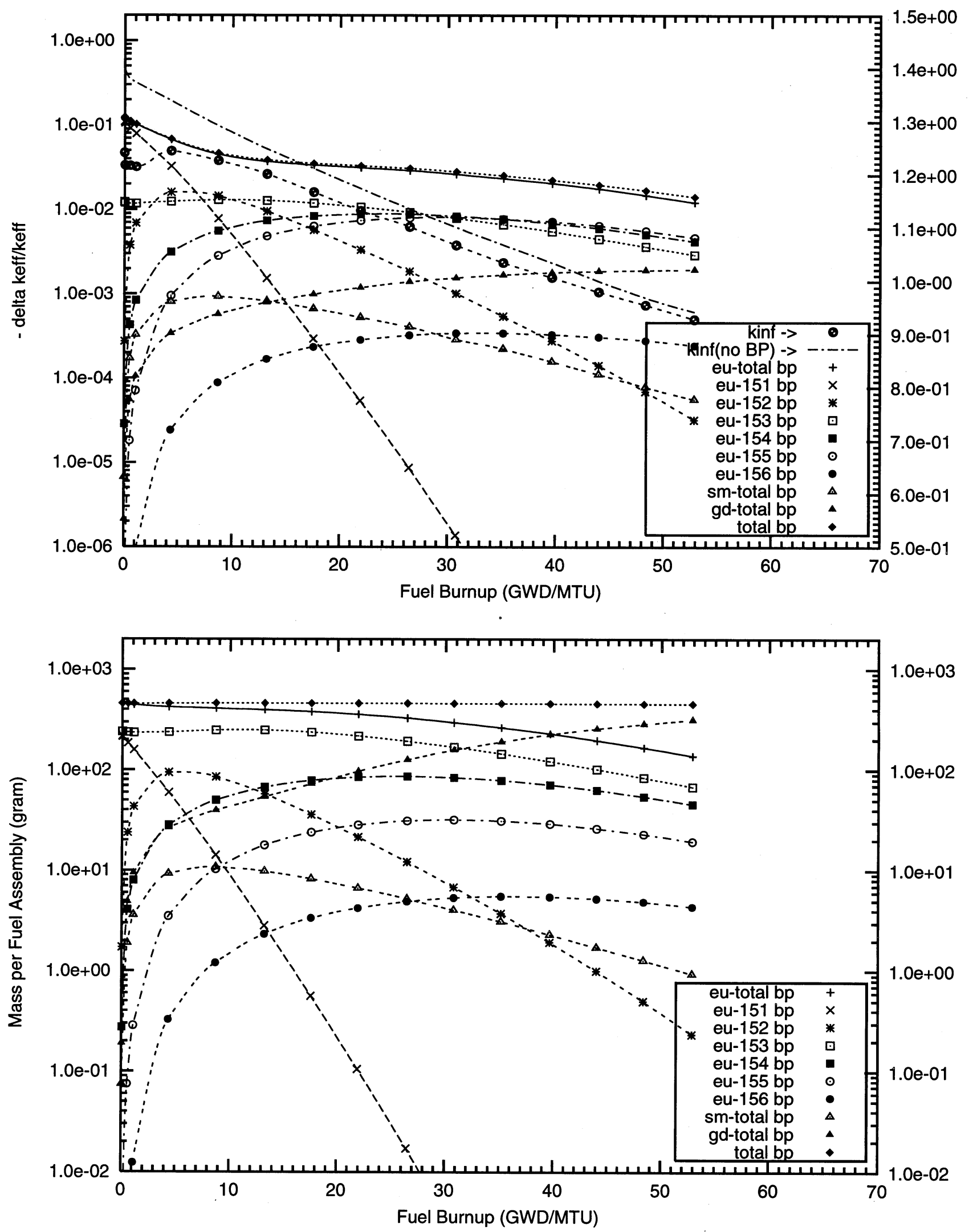
Figure E4.3. Negative Reactivity and Masses BP for Eu-nat and transmutation daughters as a function of fuel life for $17 \times 17$ fuel assemblies with 104 poison rods,

$0.5 \mathrm{w} \%$ Eu poison homogeneously mixed in the fuel cladding,

Reactor power $3400 \mathrm{MWth}, 193$ fuel assemblies, initial enrichment 4.5w\%U-235

(Case : eu000_0c_100p_h2o_104_bp_fuel_2_o)
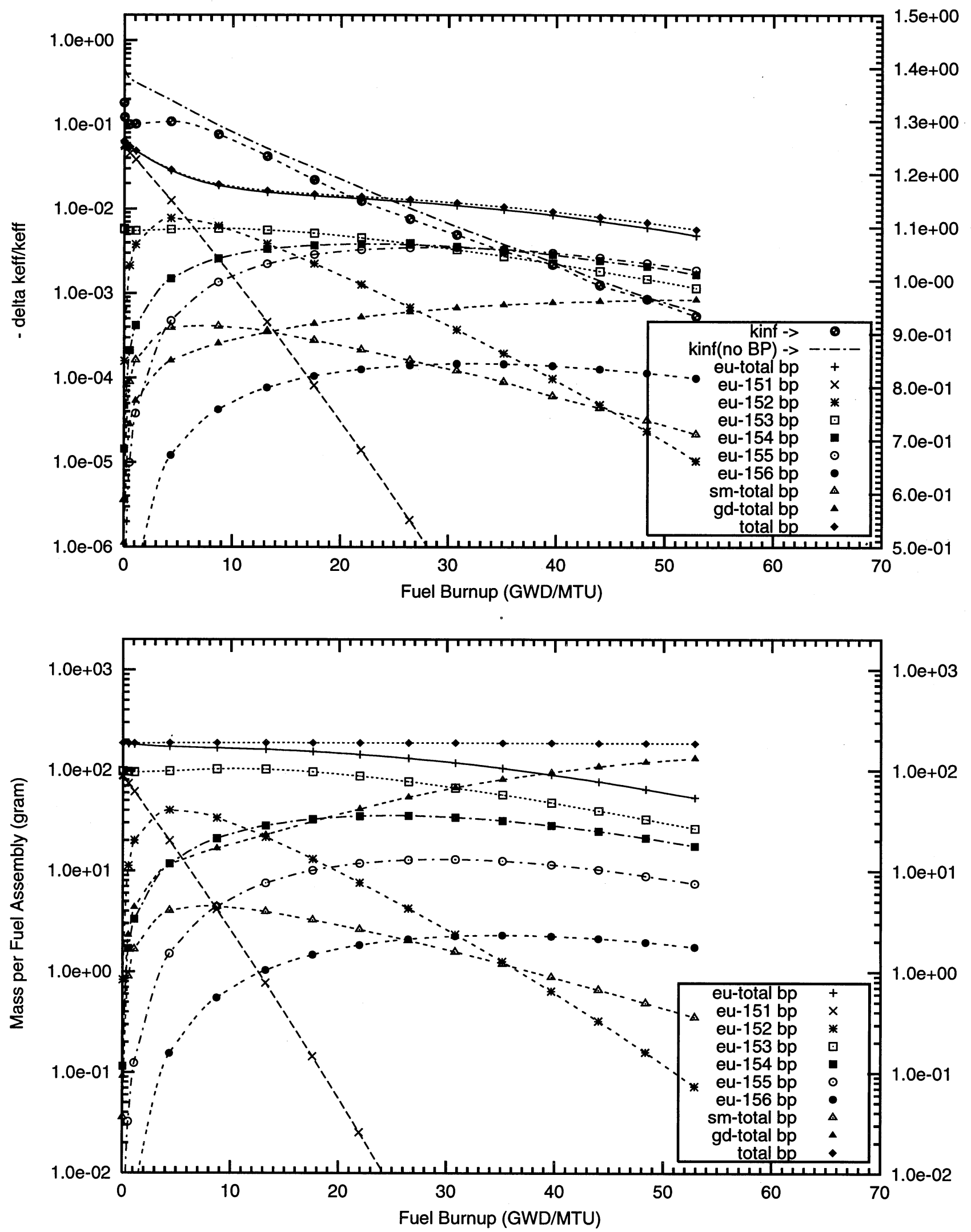
Figure E4.4. Negative Reactivity and Masses BP for Eu-nat and transmutation daughters as a fuñction of fuel life for $17 \times 17$ fuel assemblies with 104 poison rods,

$1.0 \mathrm{w} \%$ Eu poison homogeneously mixed in the fuel cladding,

Reactor power $3400 \mathrm{MWth}, 193$ fuel assemblies, initial enrichment $4.5 \mathrm{w} \% \mathrm{U}-235$

(Case : eu000_1c_100p_h20_104_bp_fuel_2_0)
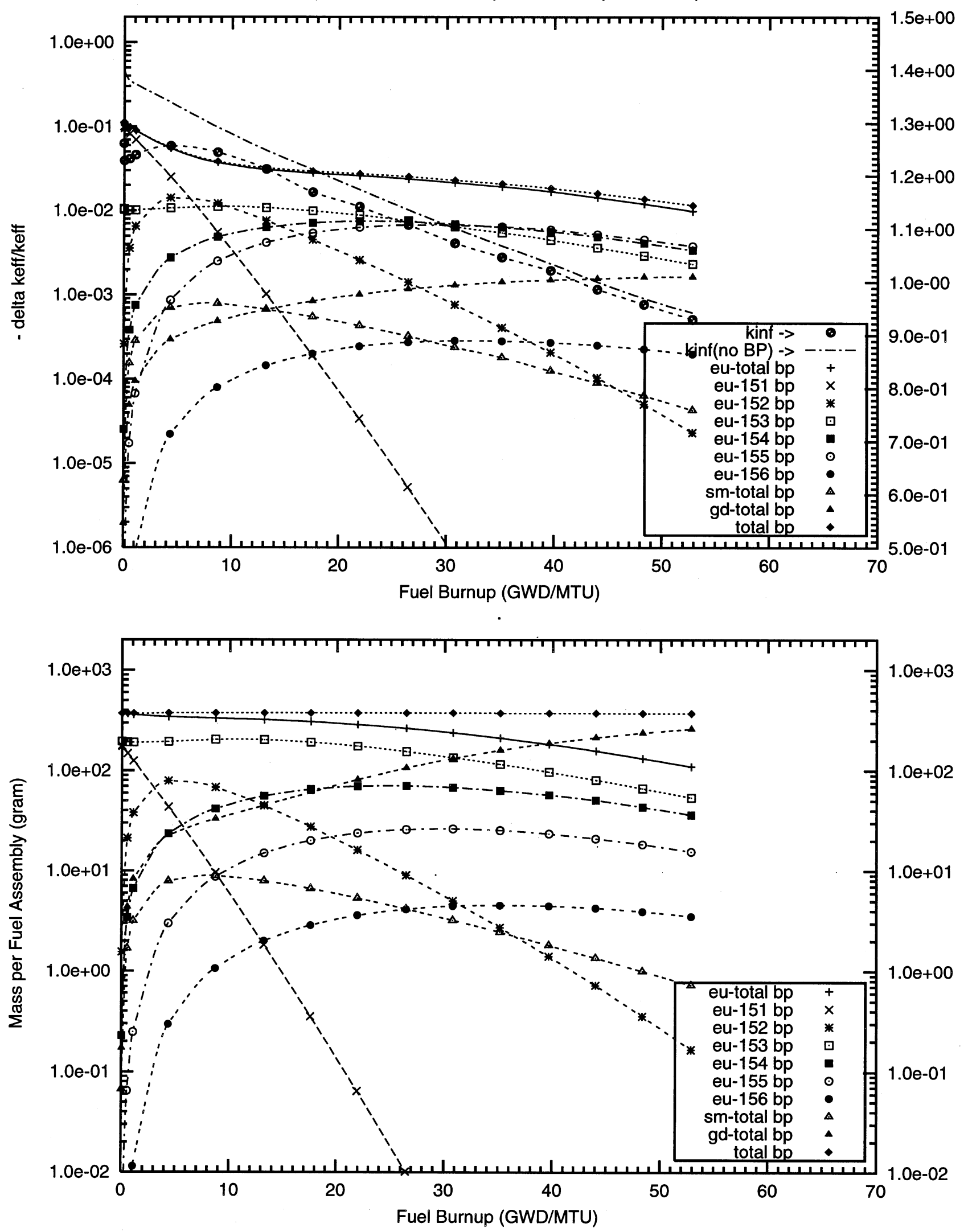


\section{E4 - Europium Fully Enriched in ${ }^{151} \mathbf{E u}$}



Figure E4.5. Negative Reactivity and Masses BP for Eu-151 and transmutation daughters as a fünction of fuel life for $17 \times 17$ fuel assemblies with 64 poison rods,

$0.5 \mathrm{w} \%$ Eu poison homogeneously mixed in the fuel cladding,

Reactor power $3400 \mathrm{MWth}, 193$ fuel assemblies, initial enrichment $4.5 \mathrm{w} \% \mathrm{U}-235$

(Case : eu151_0c_100p_h2o_64_bp_fuel_2_o)
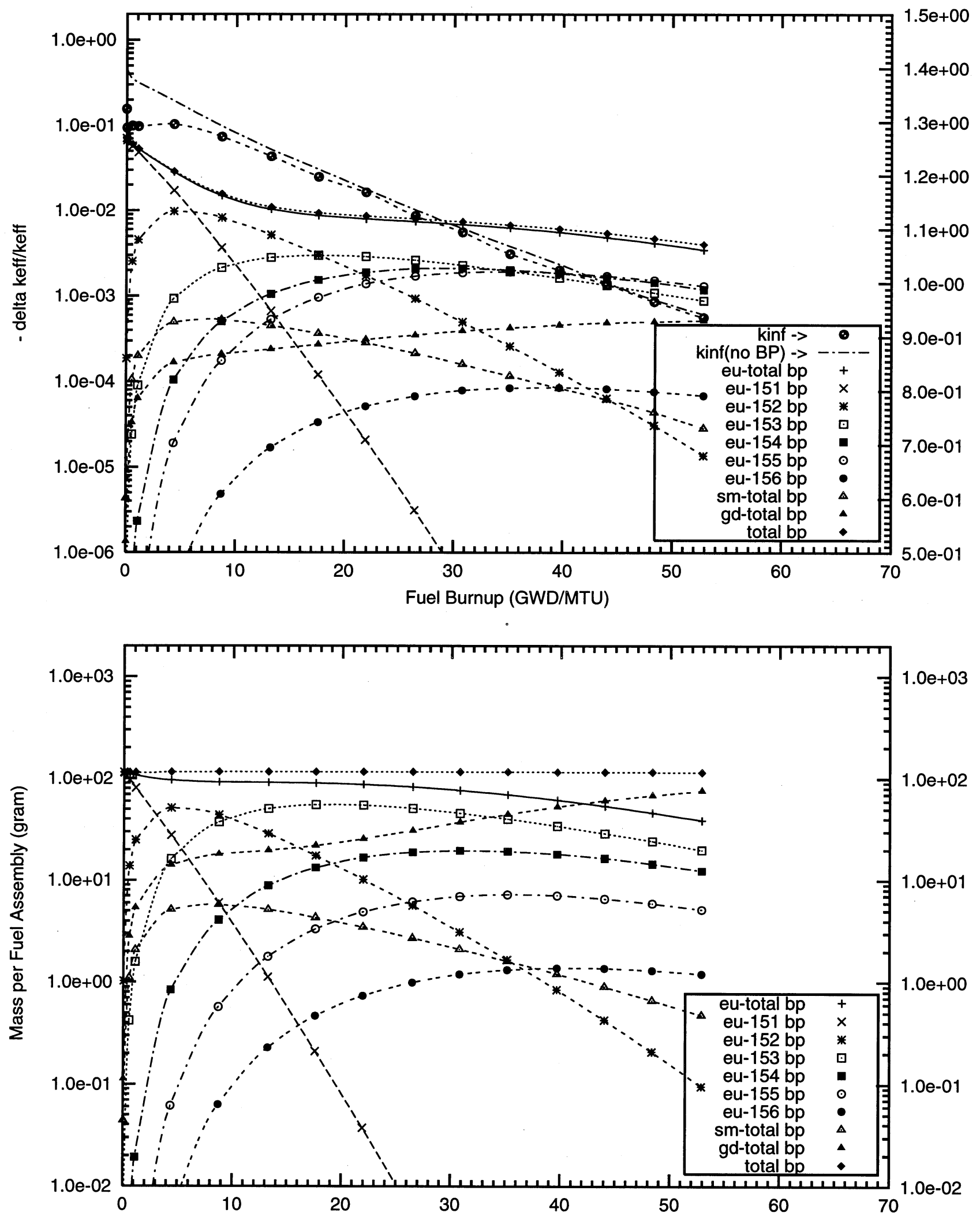

Fuel Burnup (GWD/MTU) 
Figure E4.6. Negative Reactivity and Masses BP for Eu-151 and transmutation daughters as a function of fuel life for $17 \times 17$ fuel assemblies with 64 poison rods,

$1.0 w \%$ Eu poison homogeneously mixed in the fuel cladding,

Reactor power $3400 \mathrm{MWth}, 193$ fuel assemblies, initial enrichment $4.5 \mathrm{w} \% \mathrm{U}-235$

(Case : eu151_1c_100p_h2o_64_bp_fuel_2_o)

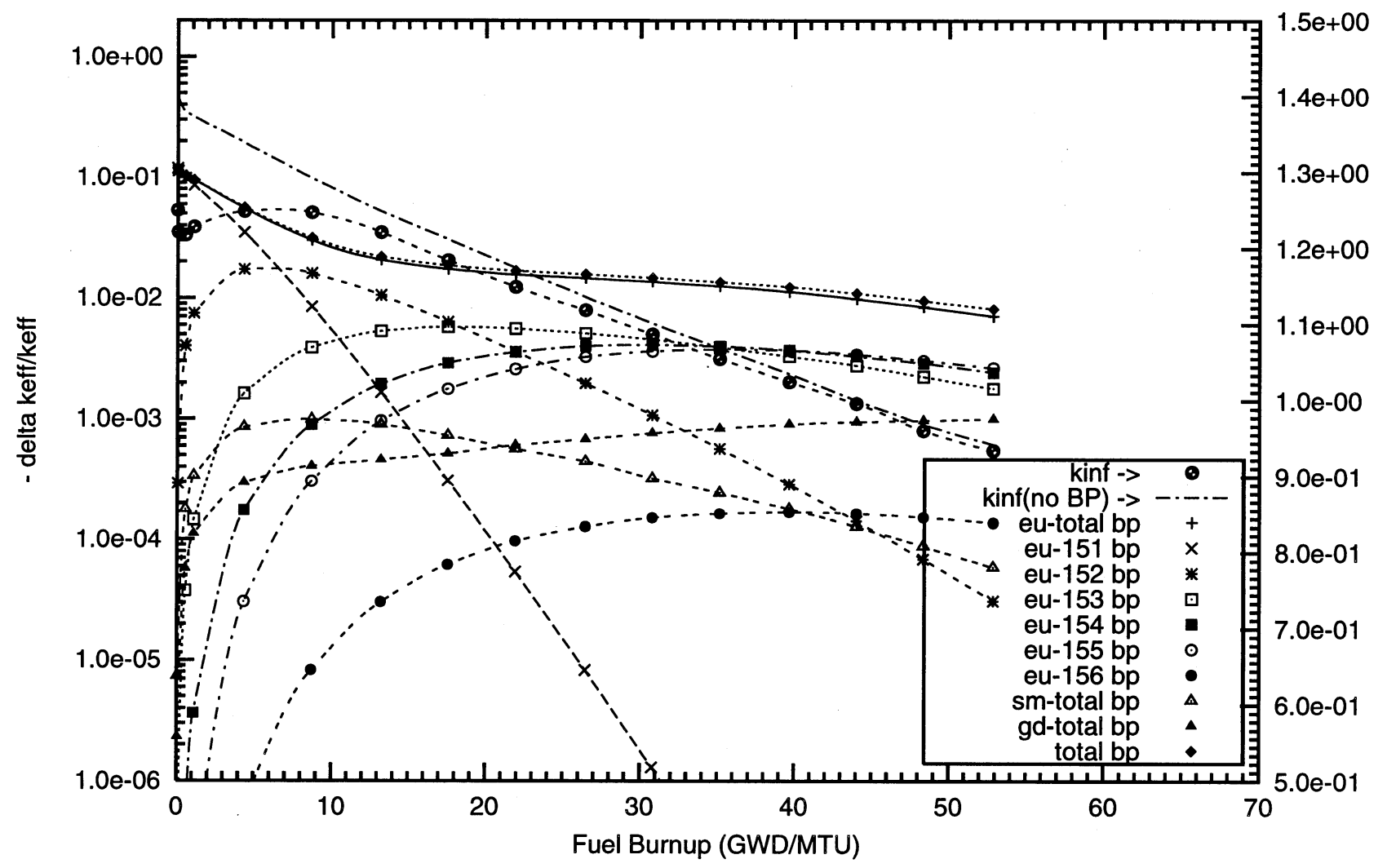

\section{产}

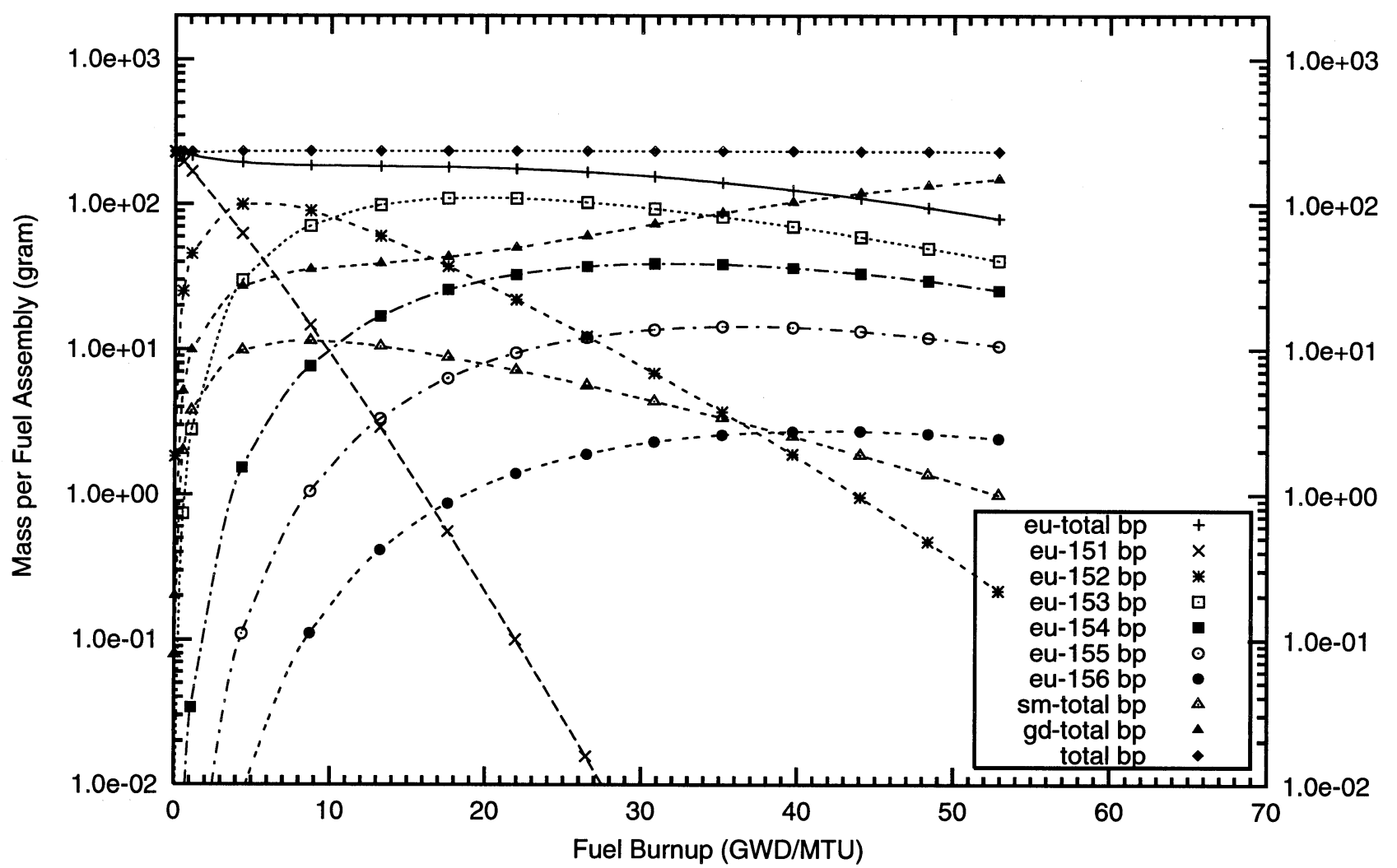


Figure E4.7. Negative Reactivity and Masses BP for Eu-151 and transmutation daughters as a function of fuel life for $17 \times 17$ fuel assemblies with 104 poison rods,

$0.2 w \%$ Eu poison homogeneously mixed in the fuel cladding,

Reactor power $3400 \mathrm{MWth}, 193$ fuel assemblies, initial enrichment $4.5 \mathrm{~W} \% \mathrm{U}-235$

(Case : eu151_8c_104_bp_fuel o)
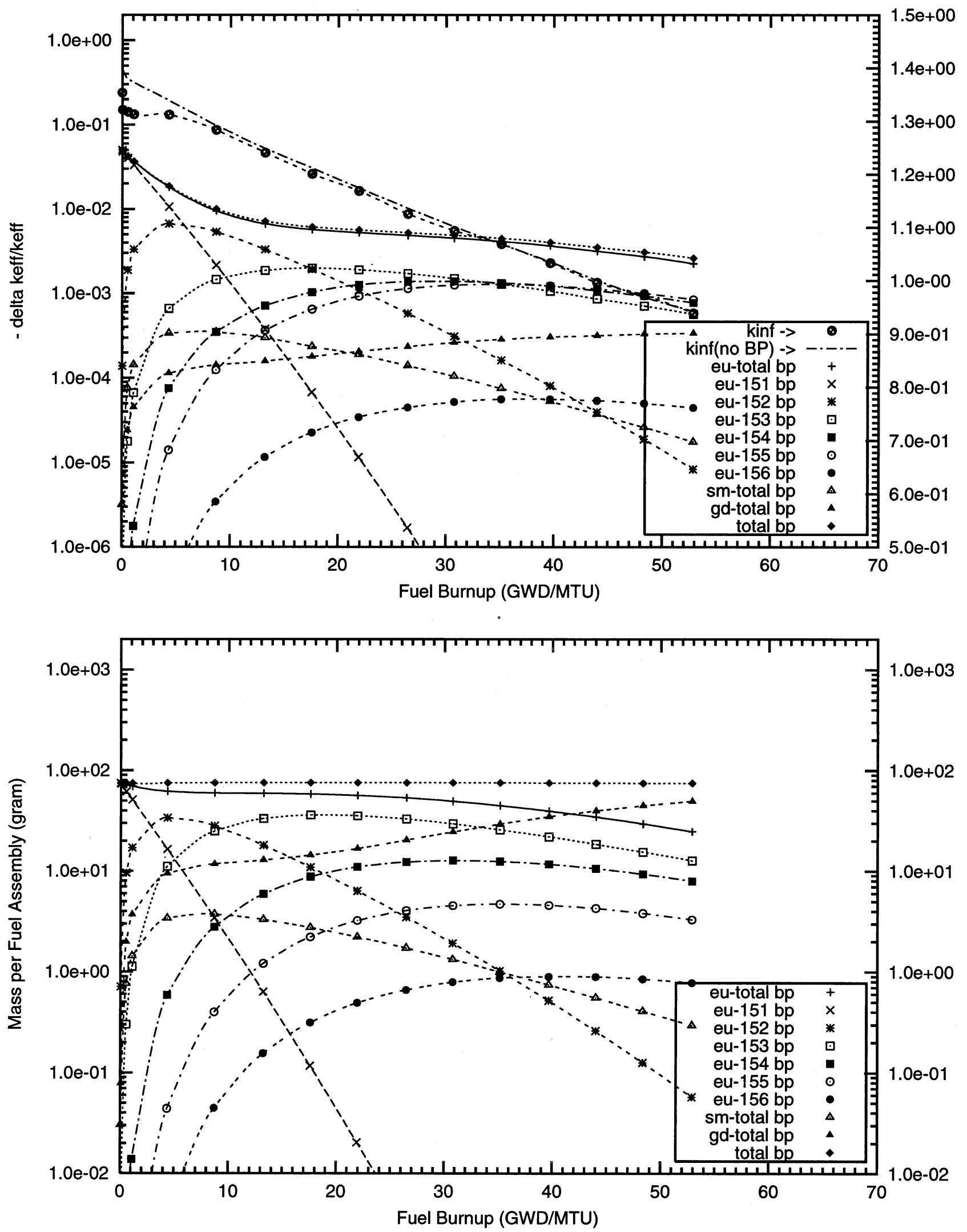
Figure E4.8. Negative Reactivity and Masses BP for Eu-151 and transmutation daughters

as a function of fuel life for $17 \times 17$ fuel assemblies with 104 poison rods,

$0.5 \mathrm{w} \%$ Eu poison homogeneously mixed in the fuel cladding,

Reactor power $3400 \mathrm{MWth}, 193$ fuel assemblies, initial enrichment $4.5 \mathrm{w} \% \mathrm{U}-235$ (Case : eu151_0c_104_bp_fuel o)

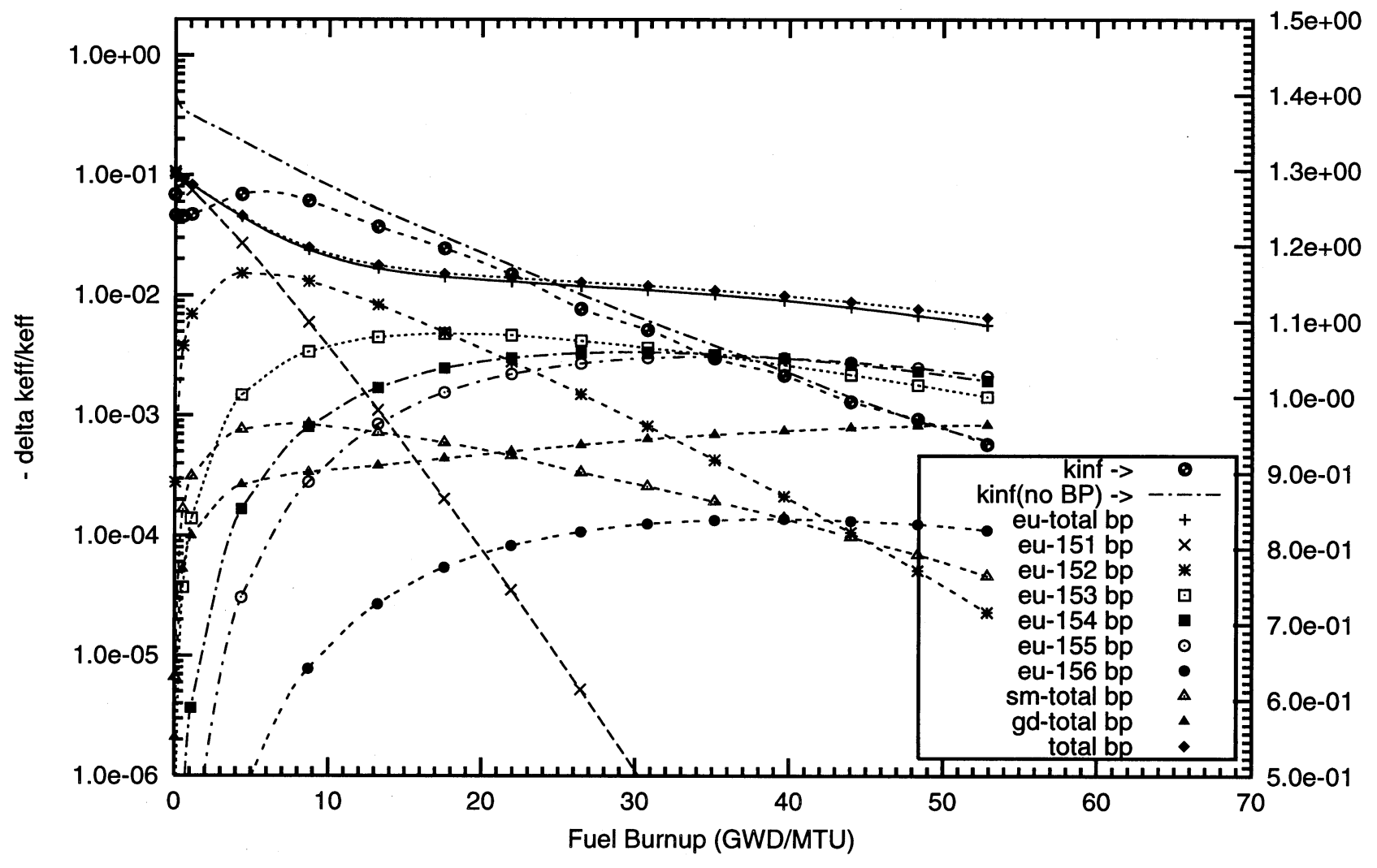

\section{言}

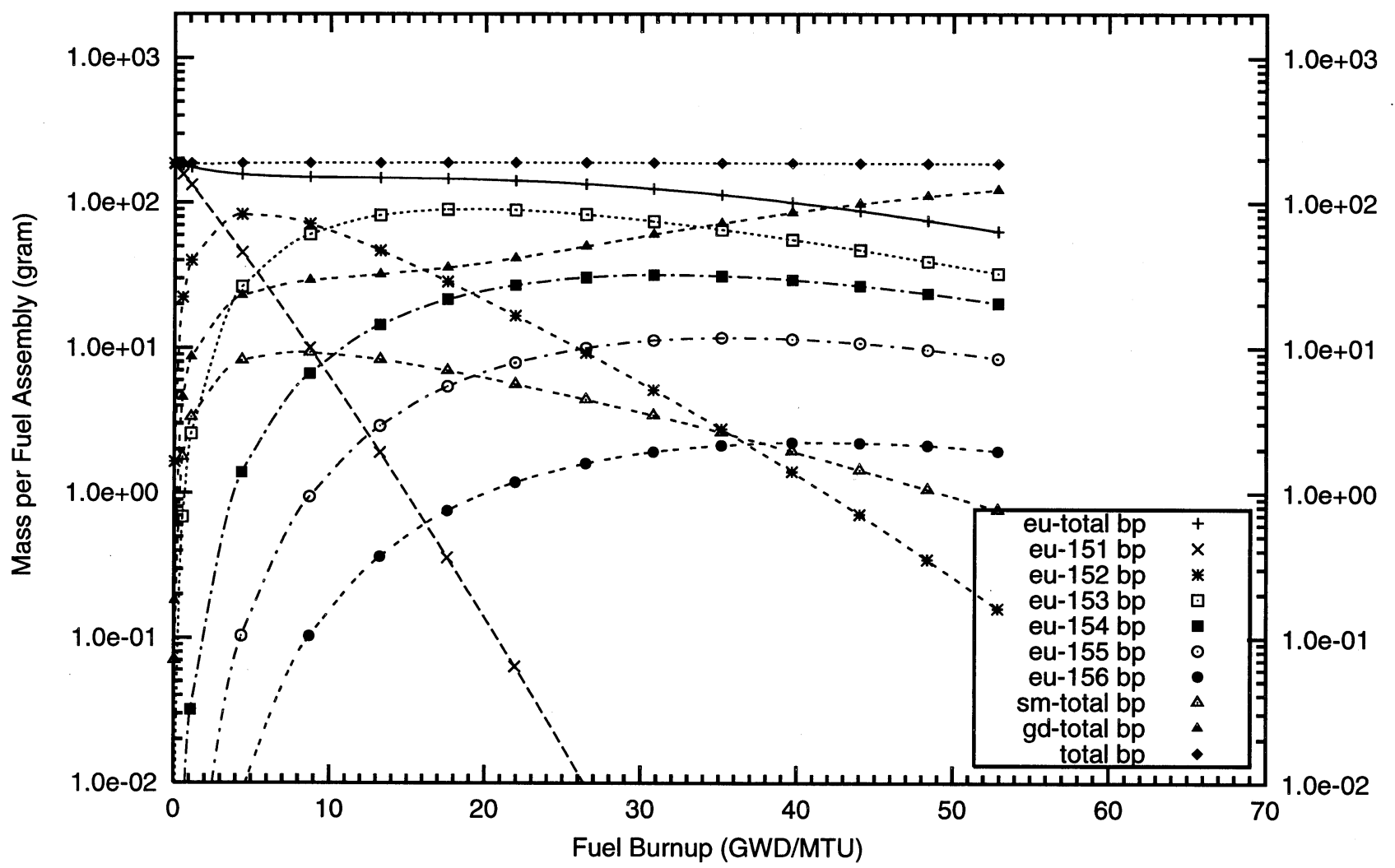


Figure E4.9. Negative Reactivity and Masses BP for Eu-151 and transmutation daughters as a function of fuel life for $17 \times 17$ fuel assemblies with 104 poison rods,

$0.5 \mathrm{w} \%$ Eu poison homogeneously mixed in the fuel cladding,

Reactor power $3400 \mathrm{MWth}, 193$ fuel assemblies, initial fuel enrichment $6.0 \mathrm{w} \% \mathrm{U}-235$

(Case : eu151_0c_enru_6p0_104_bp_fuel o)
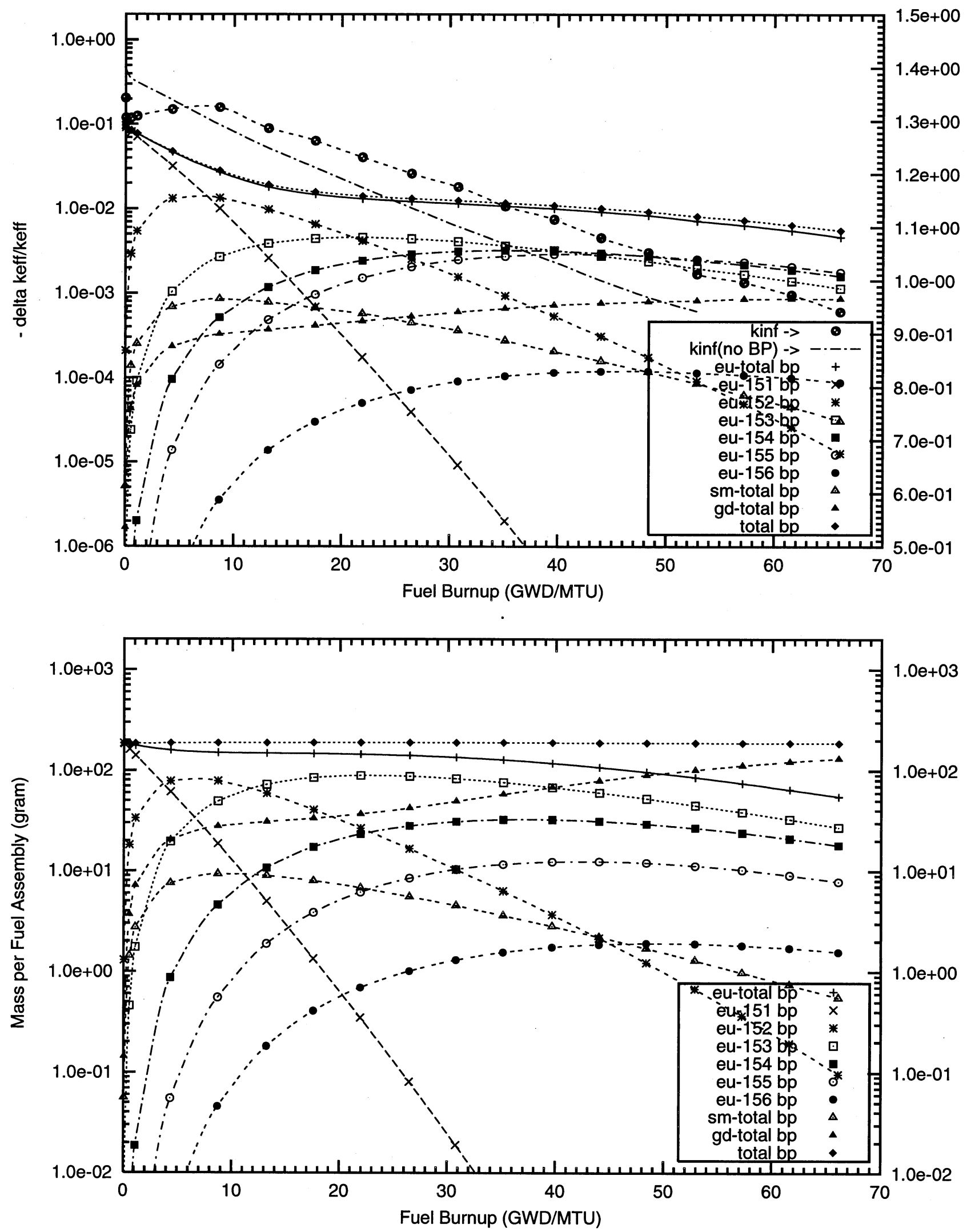
Figure E4.10. Negative Reactivity and Masses BP for Eu-151 and transmutation daughters as a function of fuel life for $17 \times 17$ fuel assemblies with 104 poison rods,

$1.0 \mathrm{w} \%$ Eu poison homogeneously mixed in the fuel cladding,

Reactor power $3400 \mathrm{MWth}, 193$ fuel assemblies initial fuel enrichment $6.0 \mathrm{w} \% \mathrm{U}-235$

(Case : eu151_1c_enru_6p0_104_bp_fuel o)
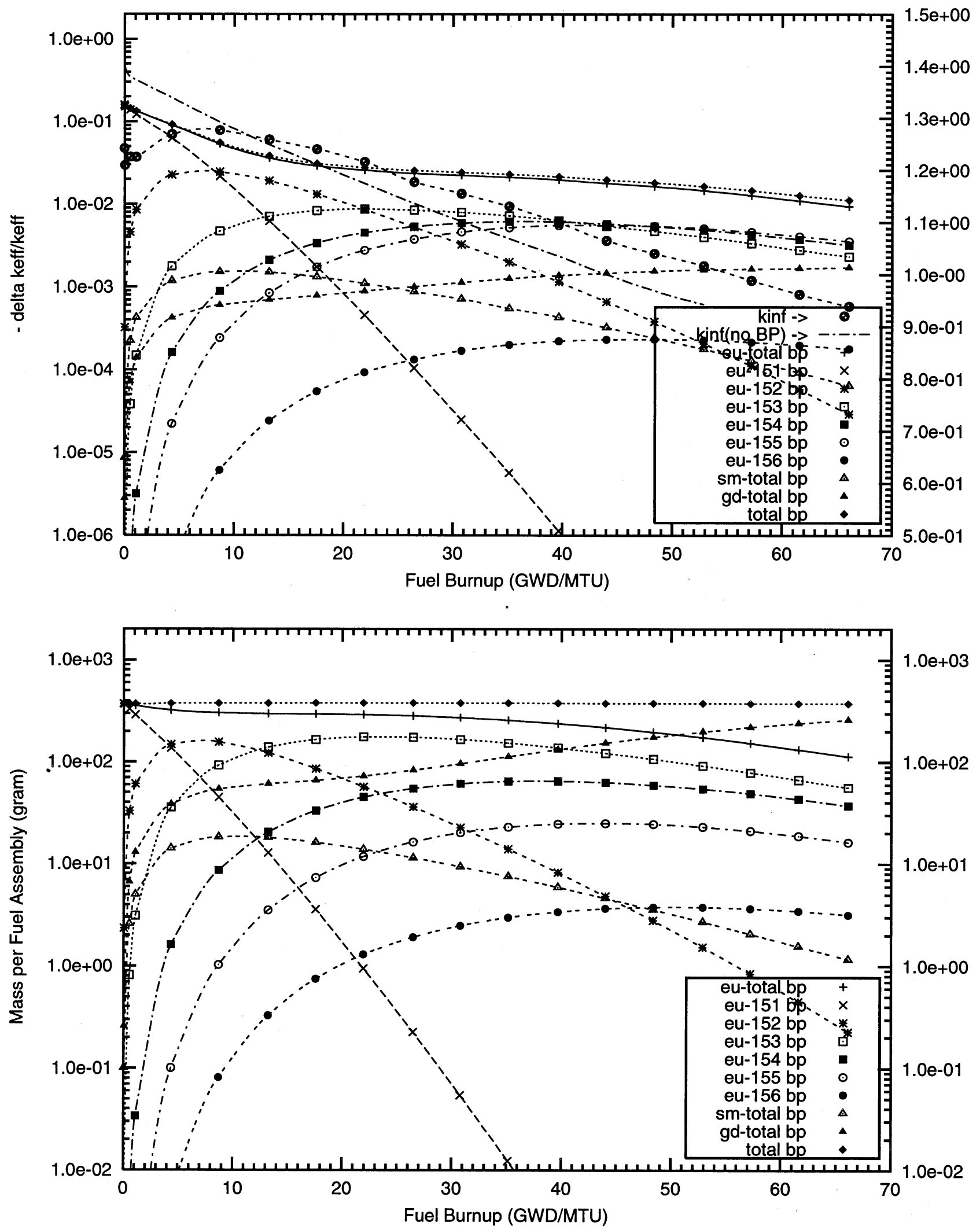


\section{Appendix F1}

Reactivity Worths and Masses of $\mathrm{HfO}_{2}$ Burnable Poison Homogeneously Mixed in the Fuel Pellets

\section{Table 25 Cases}





\section{F1 - Hafnium with Natural Isotopic Abundances}



Figure F1.1. Negative Reactivity and Masses BP for Hf-nat and transmutation daughters as a fünction of fuel life for $17 \times 17$ fuel assemblies with 08 poison rods, $4.0 \mathrm{w} \% \mathrm{HfO} 2$ poison homogeneously mixed in the UO2 pellets

Reactor power $3400 \mathrm{MWth}, 193$ fuel assemblies, initial enrichment $4.5 \mathrm{w} \% \mathrm{U}-235$ (Case : hf000_3_08_bp_fuel o)

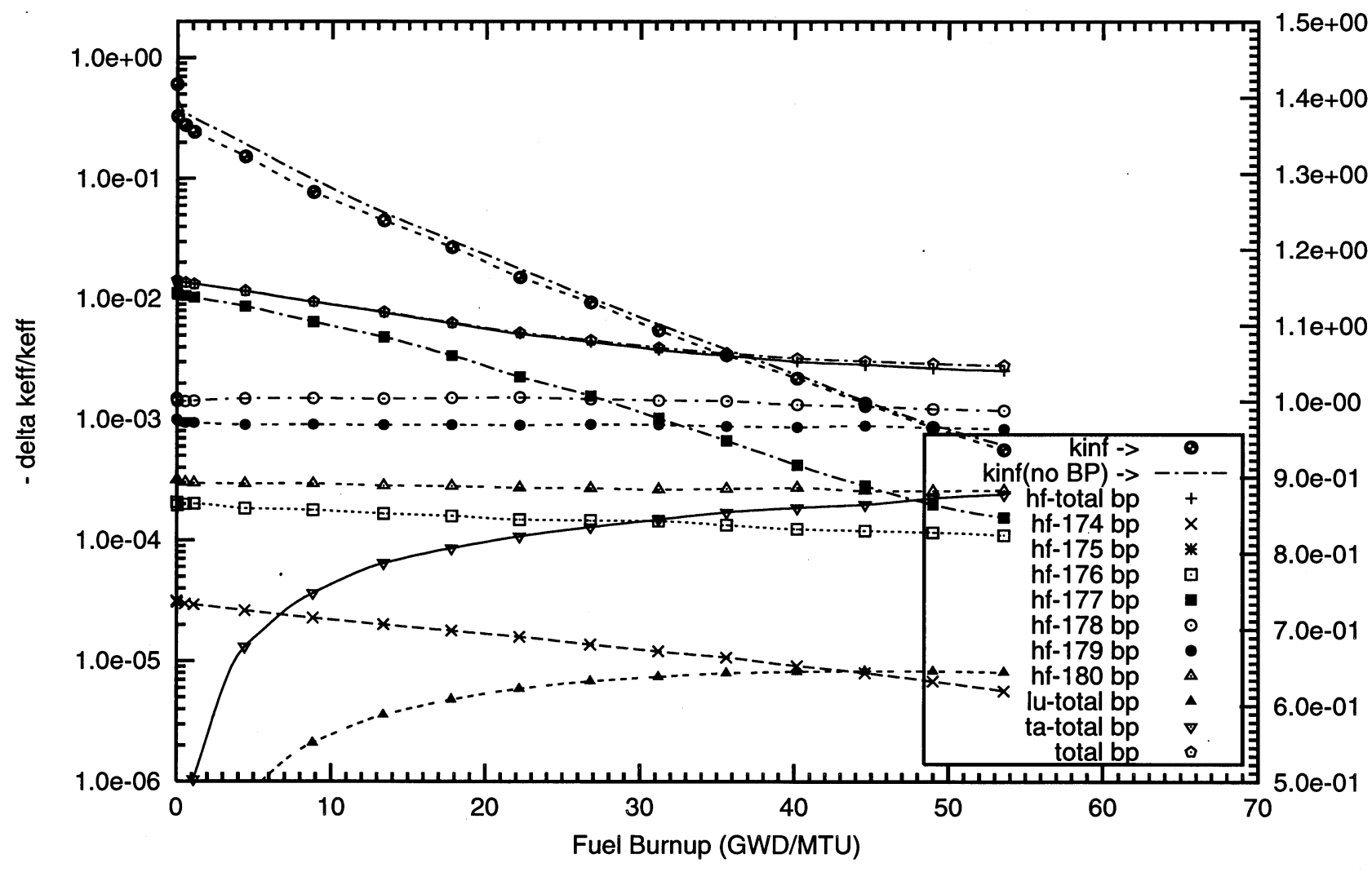




\section{F1 - Hafnium Fully Enriched in ${ }^{174} \mathrm{Hf}$}



Figure F1.2. Negative Reactivity and Masses BP for Hf-174 and transmutation daughters as a function of fuel life for $17 \times 17$ fuel assemblies with 08 poison rods,

$4.0 \mathrm{w} \% \mathrm{HfO} 2$ poison homogeneously mixed in the UO2 pellets

Reactor power $3400 \mathrm{MWth}, 193$ fuel assemblies, initial enrichment 4.5w\%U-235

(Case : hf174_3_08_bp_fuel o)

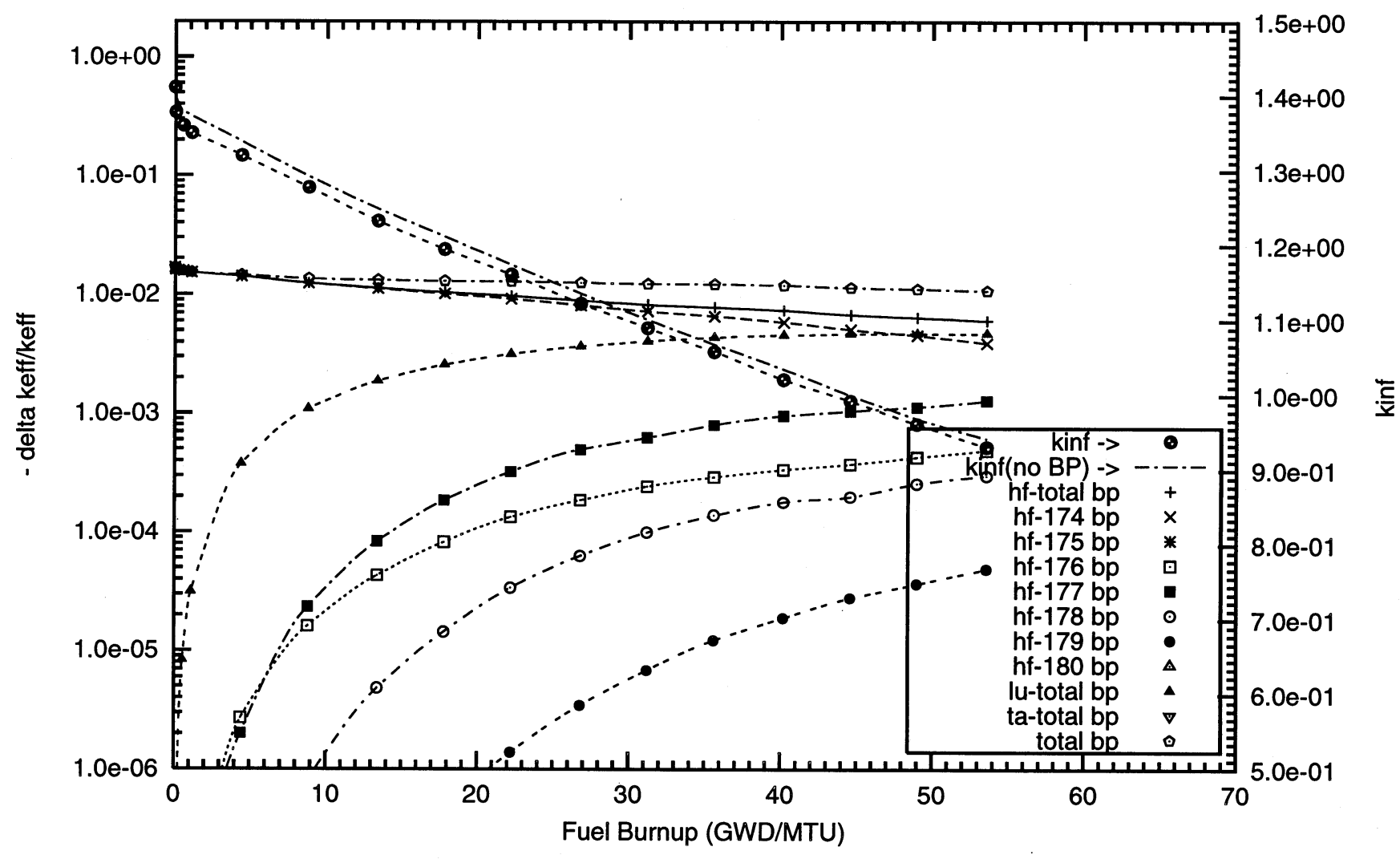




\section{F1 - Hafnium Fully Enriched in ${ }^{177} \mathrm{Hf}$}



Figure F1.3. Negative Reactivity and Masses BP for Hf-177 and transmutation daughters as a function of fuel life for $17 \times 17$ fuel assemblies with 08 poison rods,

$4.0 \mathrm{w} \% \mathrm{HfO} 2$ poison homogeneously mixed in the UO2 pellets

Reactor power $3400 \mathrm{MWth}, 193$ fuel assemblies, initial enrichment $4.5 \mathrm{w} \% \mathrm{U}-235$

(Case : hf177_3_08_bp_fuel o)
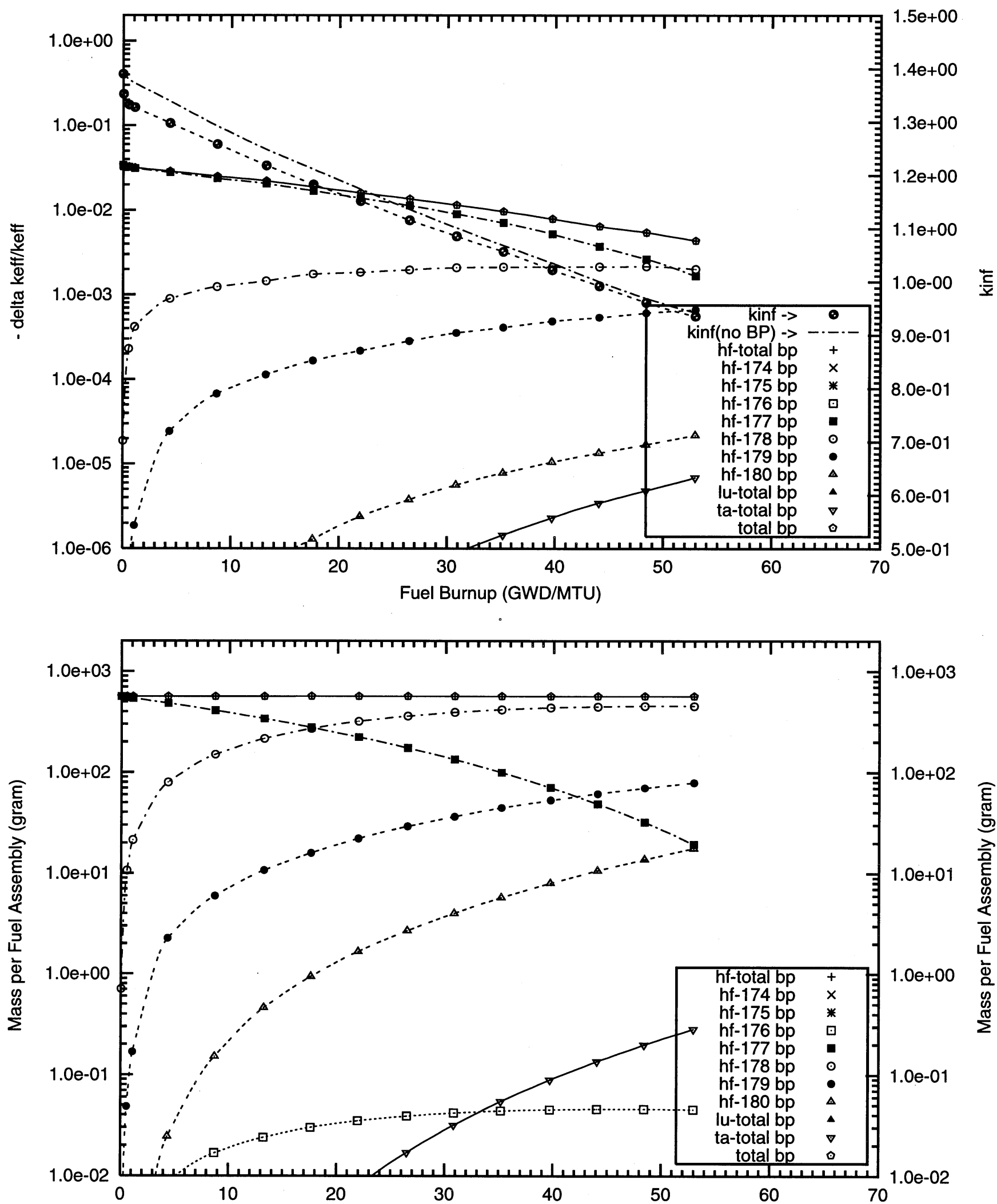

Fuel Burnup (GWD/MTU) 


\title{
Appendix F2
}

\section{Reactivity Worths and Masses of $\mathrm{HfO}_{2}$ Burnable Poison Homogeneously Mixed in the Outer One-Third Volume of the Fuel Pellets}

\author{
Table 26 Cases
}



F2 - Hafnium with Natural Isotopic Abundances 
Figure F2.1. Negative Reactivity and Masses BP for Eu-nat and transmutation daughters as a function of fuel life for $17 \times 17$ fuel assemblies with 08 poison rods,

$8.0 \mathrm{w} \% \mathrm{HfO} 2$ poison homogeneously mixed in the outer one-third volume of the $\mathrm{UO} 2$ pellets Reactor power $3400 \mathrm{MW}$ th, 193 fuel assemblies, initial enrichment 4.5W\%U-235

(Case : hf000_4b_08_bp_fuel o)

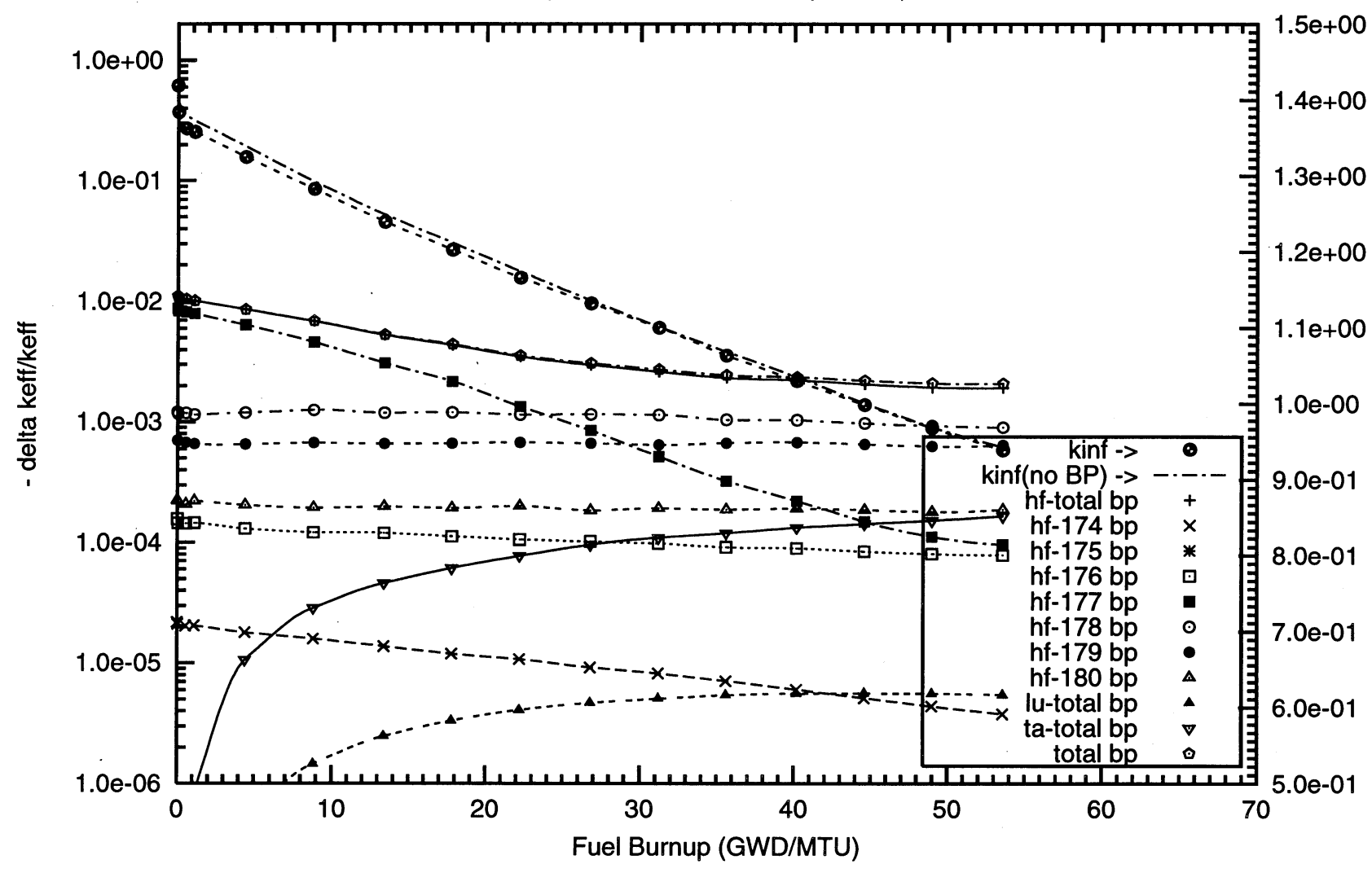



F2 - Hafnium Fully Enriched in ${ }^{177} \mathrm{Hf}$ 

Figure F2.2. Negative Reactivity and Masses BP for Eu-177 and transmutation daughters

as a function of fuel life for $17 \times 17$ fuel assemblies with 08 poison rods,

$8.0 \mathrm{w} \% \mathrm{HfO} 2$ poison homogeneously mixed in the outer one-third volume of the $\mathrm{UO} 2$ pellets

Reactor power $3400 \mathrm{MWth}, 193$ fuel assemblies, initial enrichment 4.5w\%U-235

(Case : hf177_4b_08_bp_fuel o)

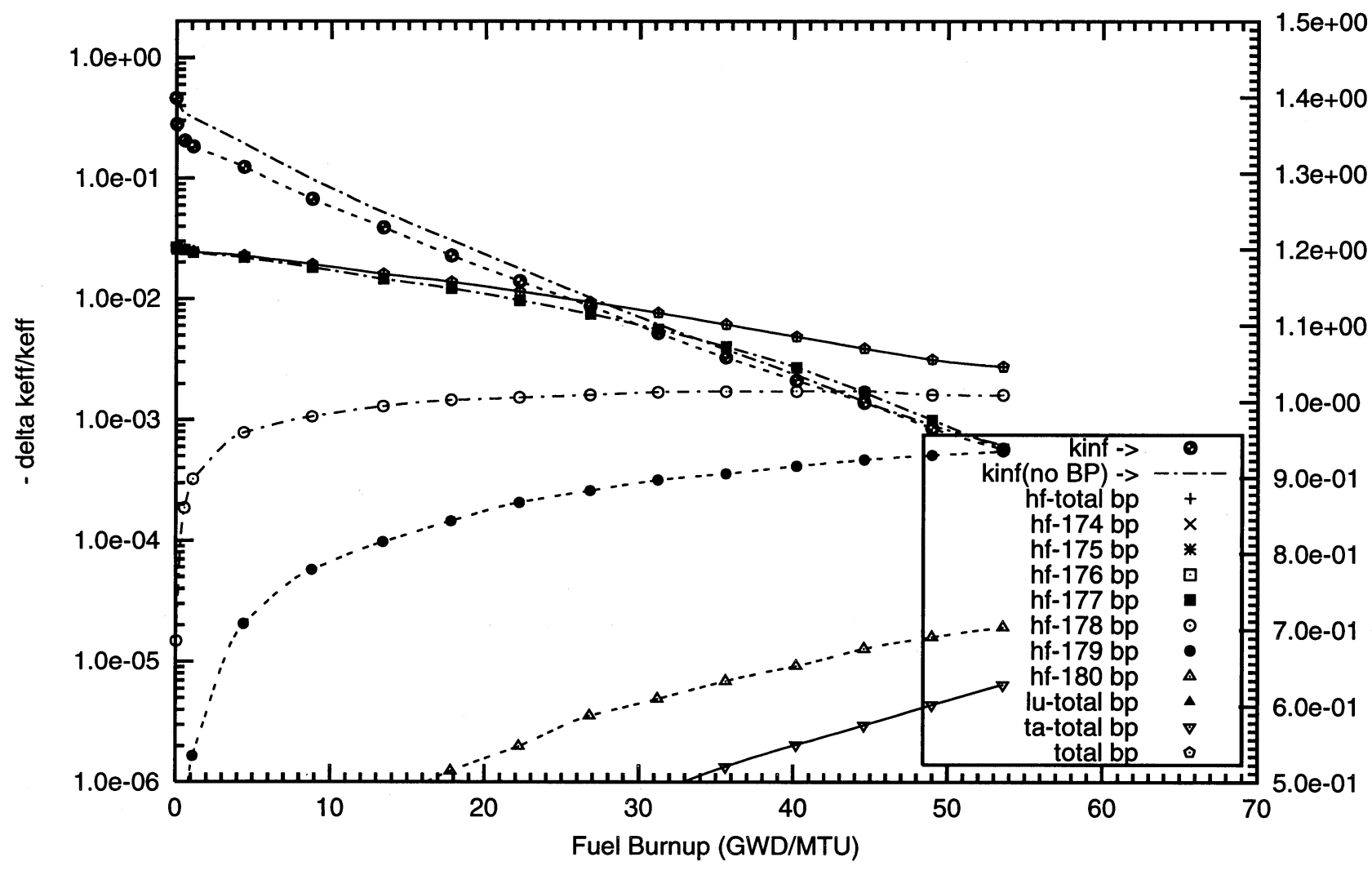


Figure F2.3. Negative Reactivity and Masses BP for Eu-177 and transmutation daughters as a function of fuel life for $17 \times 17$ fuel assemblies with 08 poison rods,

$12.0 \mathrm{w} \% \mathrm{HfO} 2$ poison homogeneously mixed in the outer one-third volume of the $\mathrm{UO} 2$ pellets Reactor power $3400 \mathrm{MWth}, 193$ fuel assemblies, initial enrichment 4.5w\%U-235

(Case : hf177_5b_08_bp_fuel o)

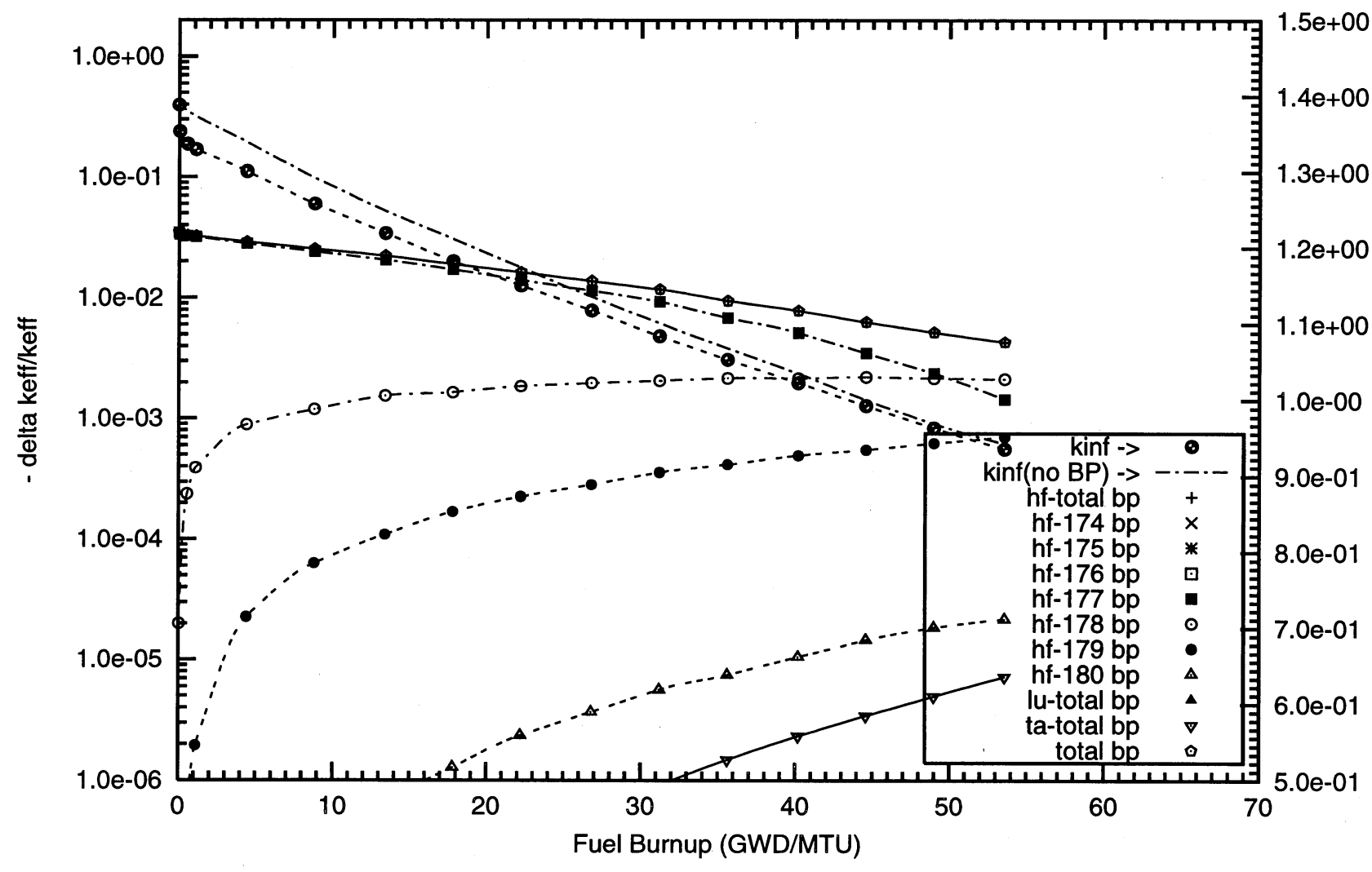




\section{Appendix F3}

\section{Reactivity Worths and Masses of $\mathrm{HfO}_{2}$ Coating on the Surface of the Fuel Pellets}

\section{Table 27 Cases}




\section{F3 - Hafnium with Natural Isotopic Abundances}



Figure F3.1. Negative Reactivity and Masses BP for Hf-nat and transmutation daughters as a function of fuel life for $17 \times 17$ fuel assemblies with 16 poison rods,

4.0 mill thick $\mathrm{HfO} 2$ poison coating on the outer radial surface of the UO2 pellets Reactor power $3400 \mathrm{MWth}, 193$ fuel assemblies, initial enrichment $4.5 \mathrm{w} \% \mathrm{U}-235$

(Case : hf000_3f_100p_h20_16_bp_fuel_2_o)
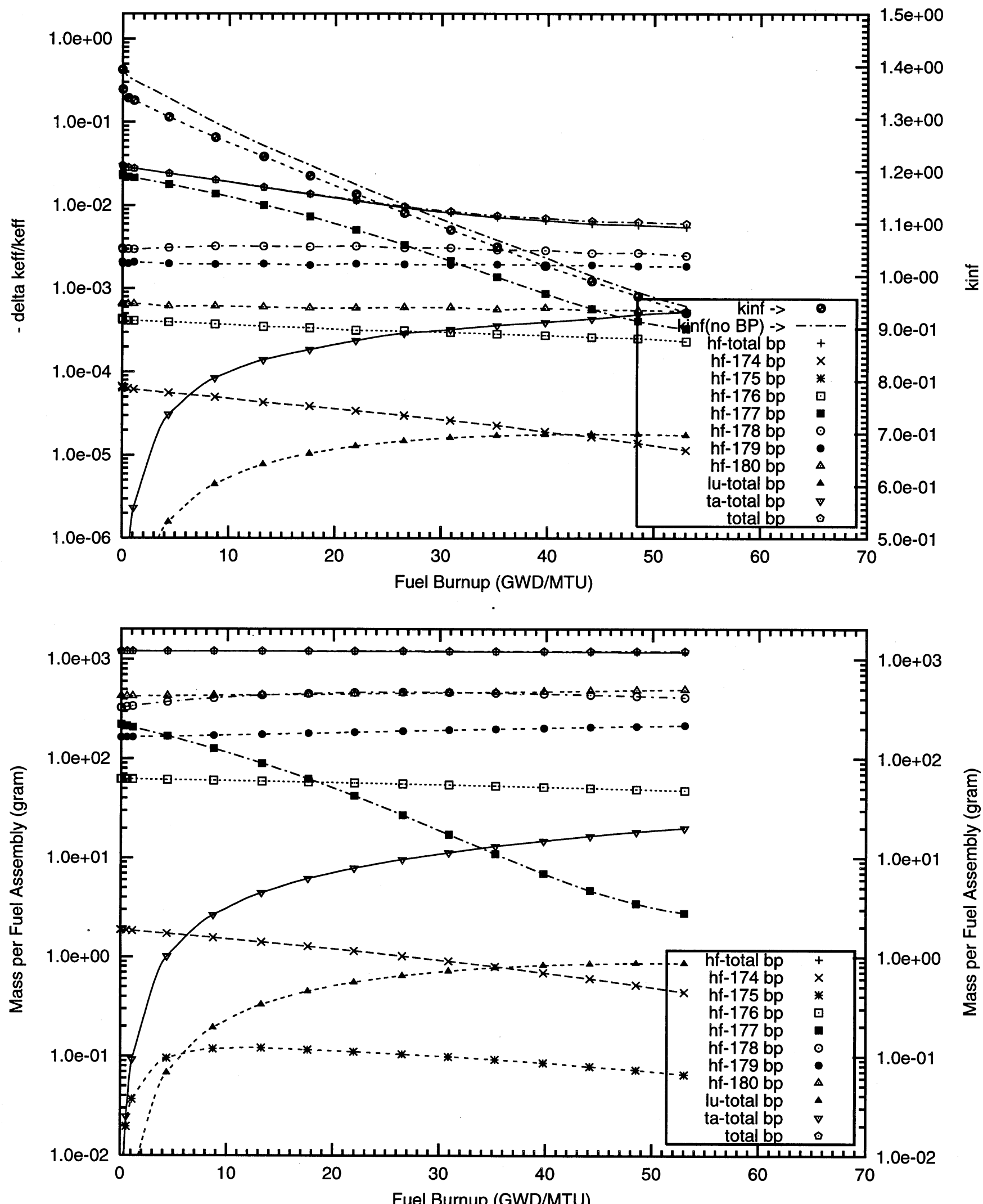

Fuel Burnup (GWD/MTU) 
Figure F3.2. Negative Reactivity and Masses BP for Hf-nat and transmutation daughters as a function of fuel life for $17 \times 17$ fuel assemblies with 16 poison rods,

8.0 mill thick $\mathrm{HfO} 2$ poison coating on the outer radial surface of the UO2 pellets Reactor power $3400 \mathrm{MWth}, 193$ fuel assemblies, initial enrichment 4.5w\%U-235

(Case : hf000_4f_100p_h2o_16_bp_fuel_2_o)
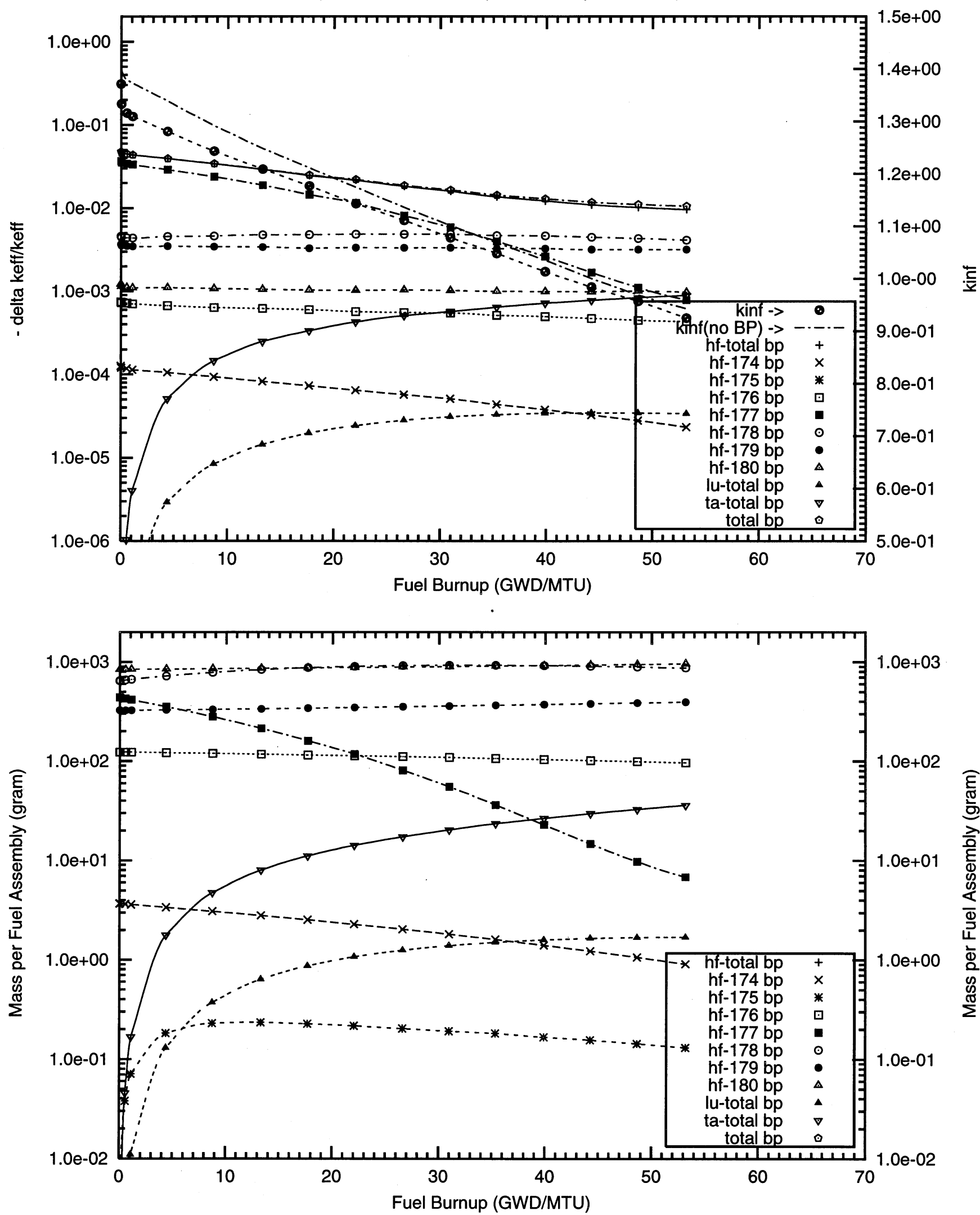
Figure F3.3. Negative Reactivity and Masses BP for Hf-nat and transmutation daughters as a function of fuel life for $17 \times 17$ fuel assemblies with 18 poison rods,

4.0 mill thick $\mathrm{HfO} 2$ poison coating on the outer radial surface of the $\mathrm{UO} 2$ pellets Reactor power $3400 \mathrm{MWth}, 193$ fuel assemblies, initial enrichment 4.5w\%U-235

(Case : hf000_3f_16_bp_fuel (old) 0)
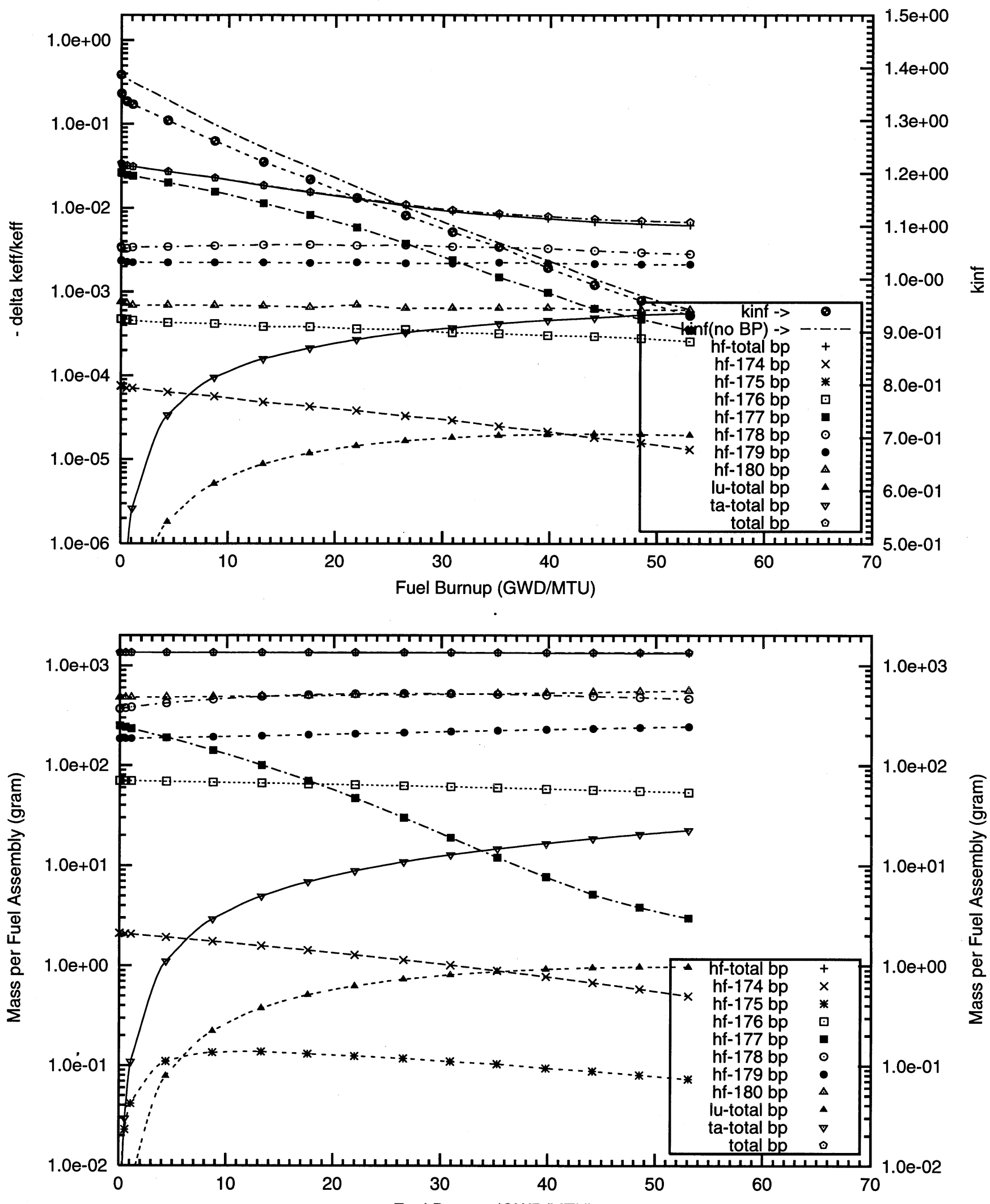

Fuel Burnup (GWD/MTU) 
Figure F3.4. Negative Reactivity and Masses BP for Hf-nat and transmutation daughters as a function of fuel life for $17 \times 17$ fuel assemblies with 64 poison rods, 2.0 mill thick $\mathrm{HfO} 2$ poison coating on the outer radial surface of the UO2 pellets Reactor power $3400 \mathrm{MWth}, 193$ fuel assemblies, initial enrichment 4.5w\%U-235 (Case : hf000_2f_64_bp_fuel o)
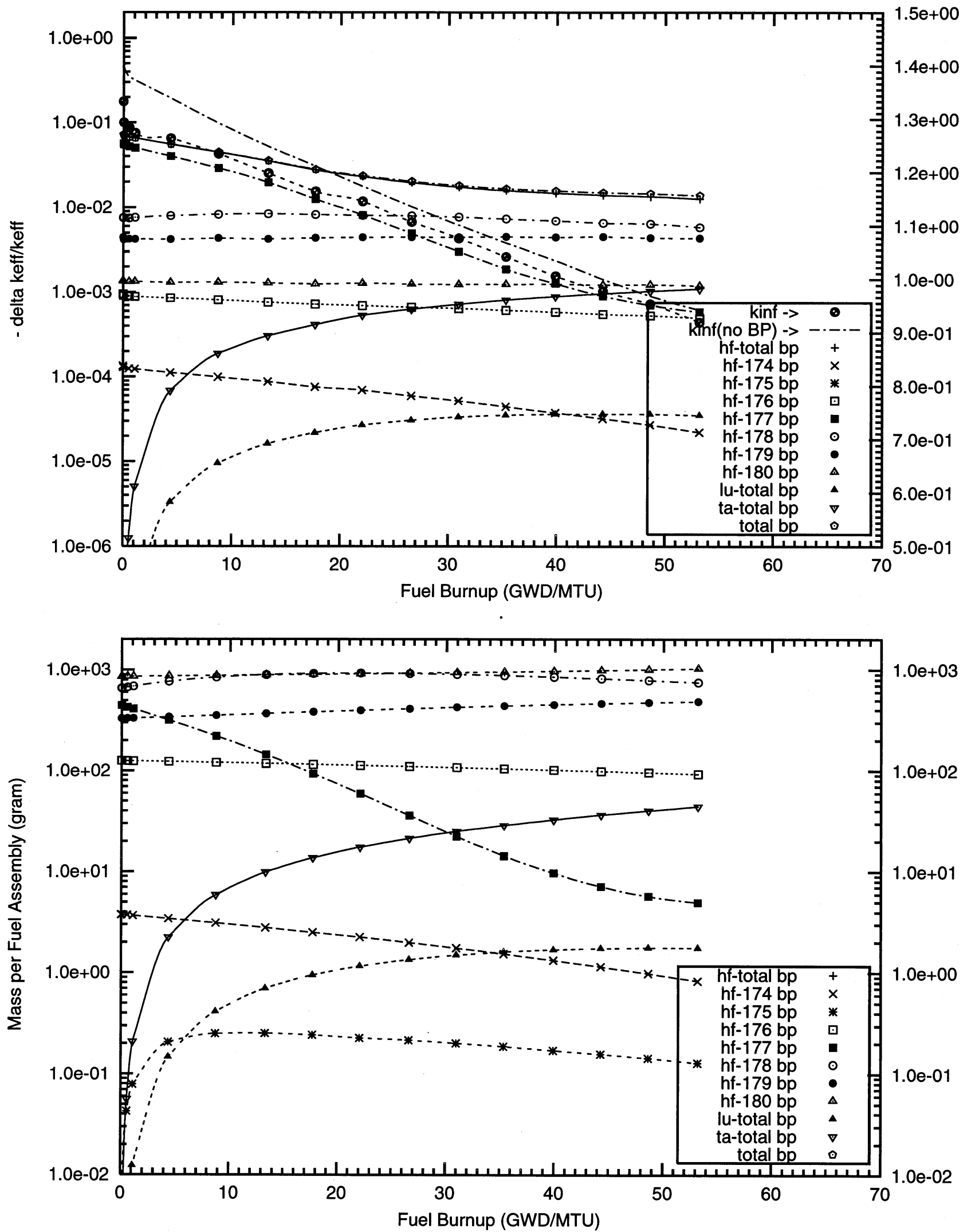
Figure F3.5. Negative Reactivity and Masses BP for Hf-nat and transmutation daughters as a function of fuel life for $17 \times 17$ fuel assemblies with 104 poison rods,

1.0 mill thick $\mathrm{HfO} 2$ poison coating on the outer radial surface of the UO2 pellets Reactor power $3400 \mathrm{MWth}, 193$ fuel assemblies, initial enrichment $4.5 \mathrm{w} \% \mathrm{U}-235$

(Case : hf000_1f_104_bp_fuel o)
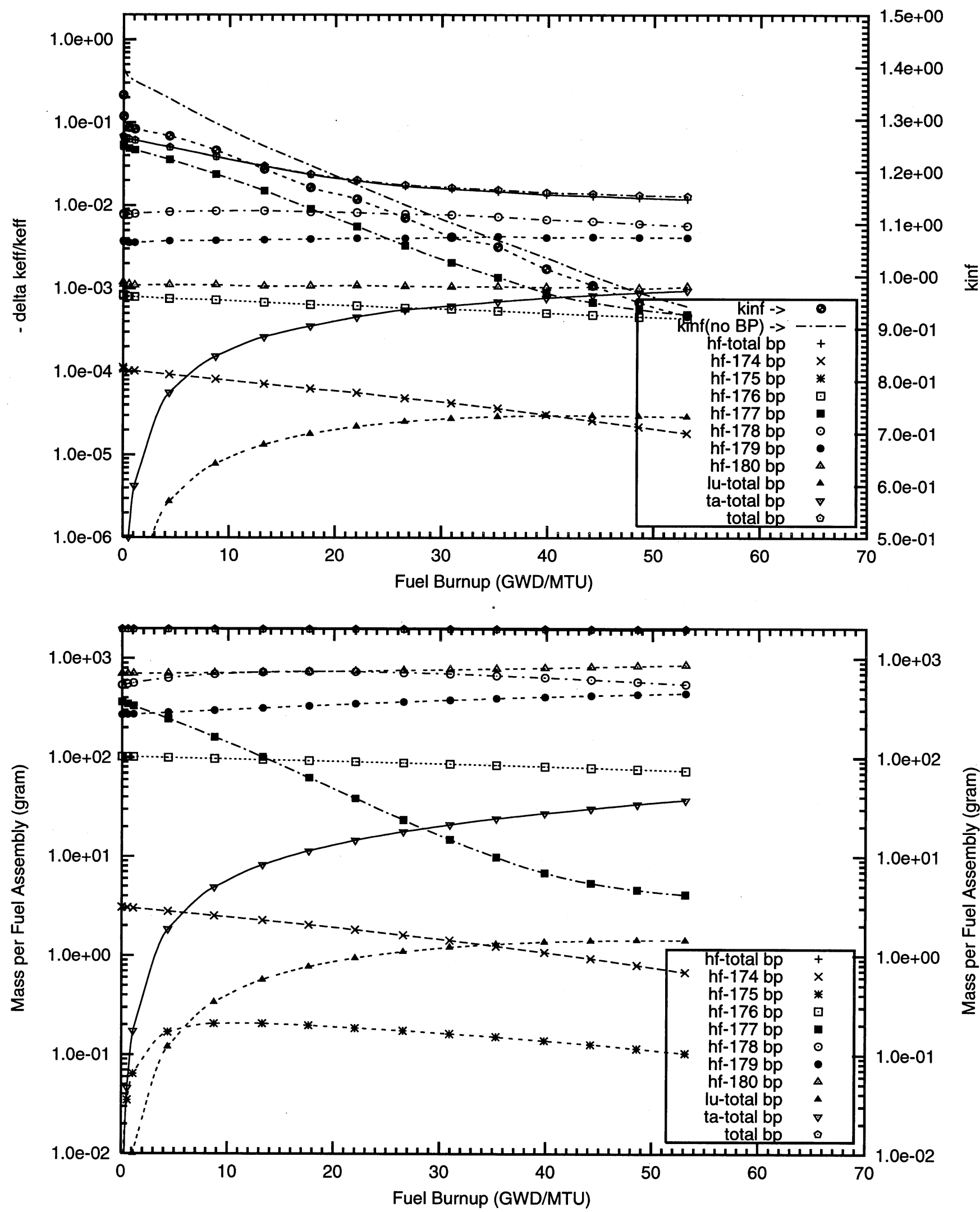
Figure F3.6. Negative Reactivity and Masses BP for Hf-nat and transmutation daughters as a function of fuel life for $17 \times 17$ fuel assemblies with 104 poison rods,

2.0 mill thick $\mathrm{HfO} 2$ poison coating on the outer radial surface of the UO2 pellets Reactor power $3400 \mathrm{MWth}, 193$ fuel assemblies, initial enrichment 4.5w\%U-235

(Case : hf000_2f_100p_h20_104_bp_fuel_2_o)
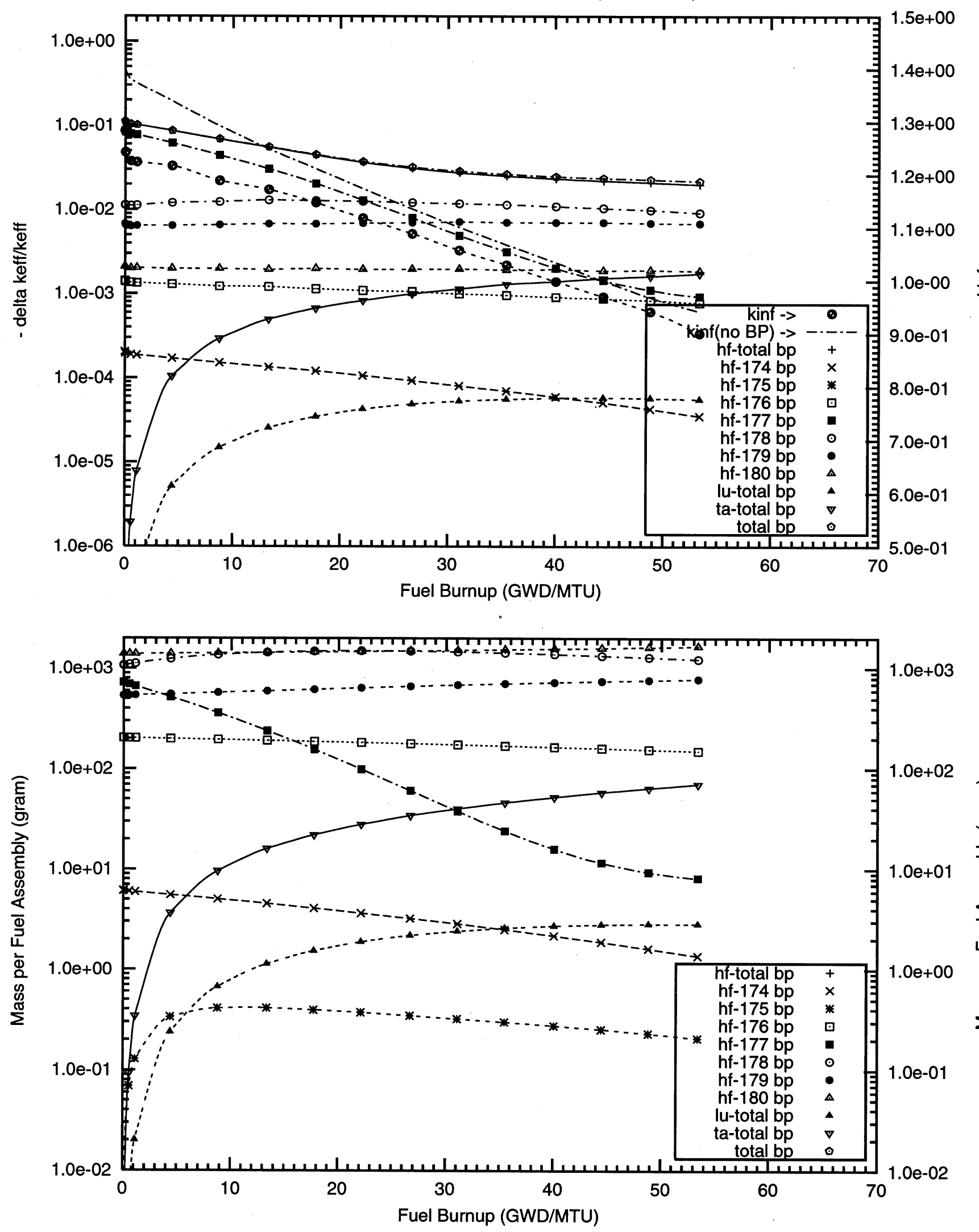
F3 - Hafnium Fully Enriched in ${ }^{177} \mathbf{H f}$ 

Figure F3.7. Negative Reactivity and Masses BP for Hf-177 and transmutation daughters as a function of fuel life for $17 \times 17$ fuel assemblies with 16 poison rods,

2.0 mill thick $\mathrm{HfO} 2$ poison coating on the outer radial surface of the UO2 pellets

Reactor power $3400 \mathrm{MWth}, 193$ fuel assemblies, initial enrichment 4.5w\%U-235

(Case : hf177_2f_100p_h2o_16_bp_fuel_2_o)
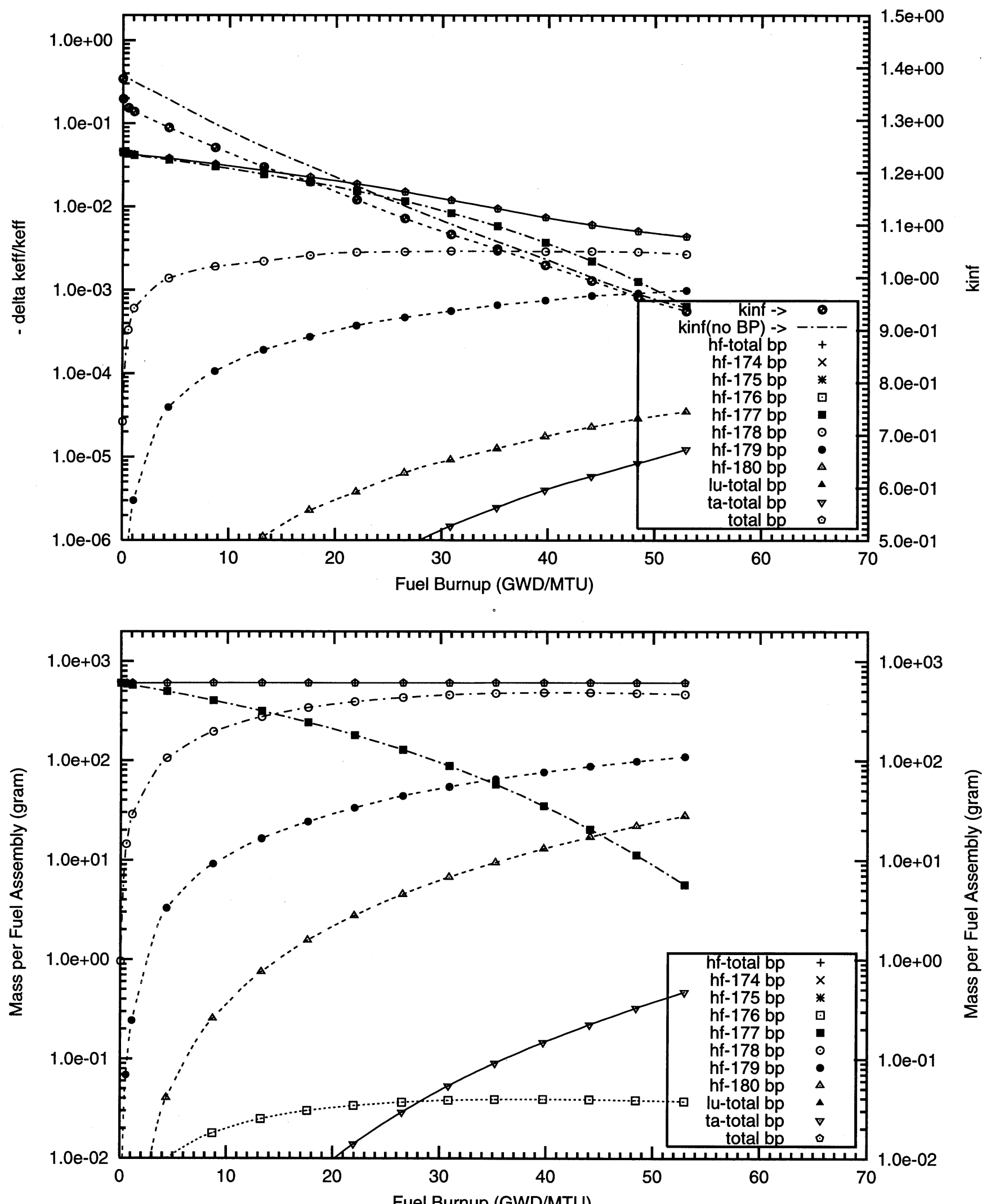

Fuel Burnup (GWD/MTU) 
Figure F3.8. Negative Reactivity and Masses BP for Hf-177 and transmutation daughters as a function of fuel life for $17 \times 17$ fuel assemblies with 16 poison rods,

4.0 mill thick $\mathrm{HfO} 2$ poison coating on the outer radial surface of the UO2 pellets

Reactor power $3400 \mathrm{MWth}, 193$ fuel assemblies, initial enrichment 4.5W\%U-235

(Case : hf177_3f_100p_h2o_16_bp_fuel_2_o)
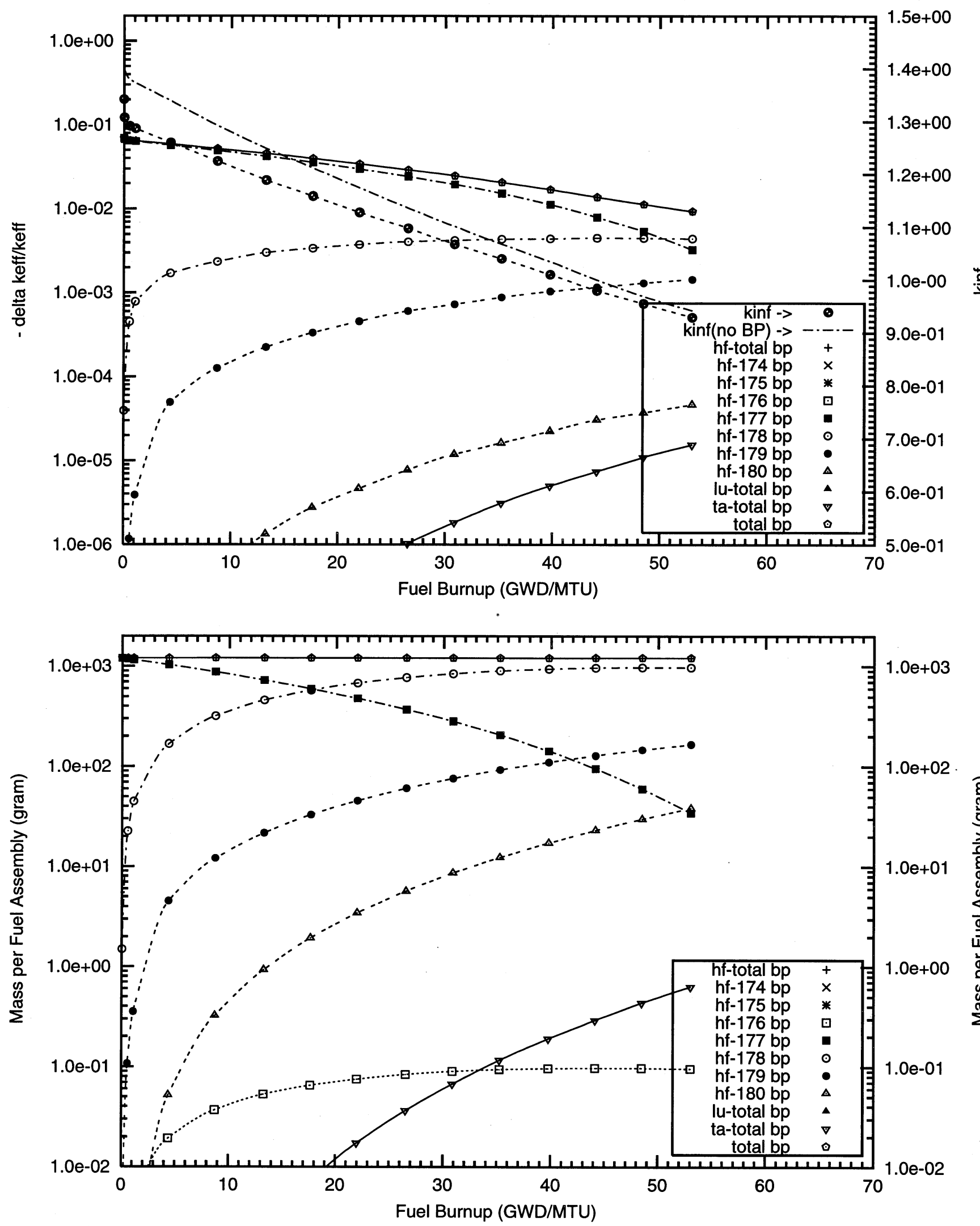
Figure F3.9. Negative Reactivity and Masses BP for Hf-177 and transmutation daughters as a function of fuel life for $17 \times 17$ fuel assemblies with 18 poison rods,

4.0 mill thick $\mathrm{HfO} 2$ poison coating on the outer radial surface of the $\mathrm{UO} 2$ pellets Reactor power 3400 MWth, 193 fuel assemblies, initial enrichment 4.5w\%U-235

(Case : hf177_3f_16_bp_fuel (old) o)
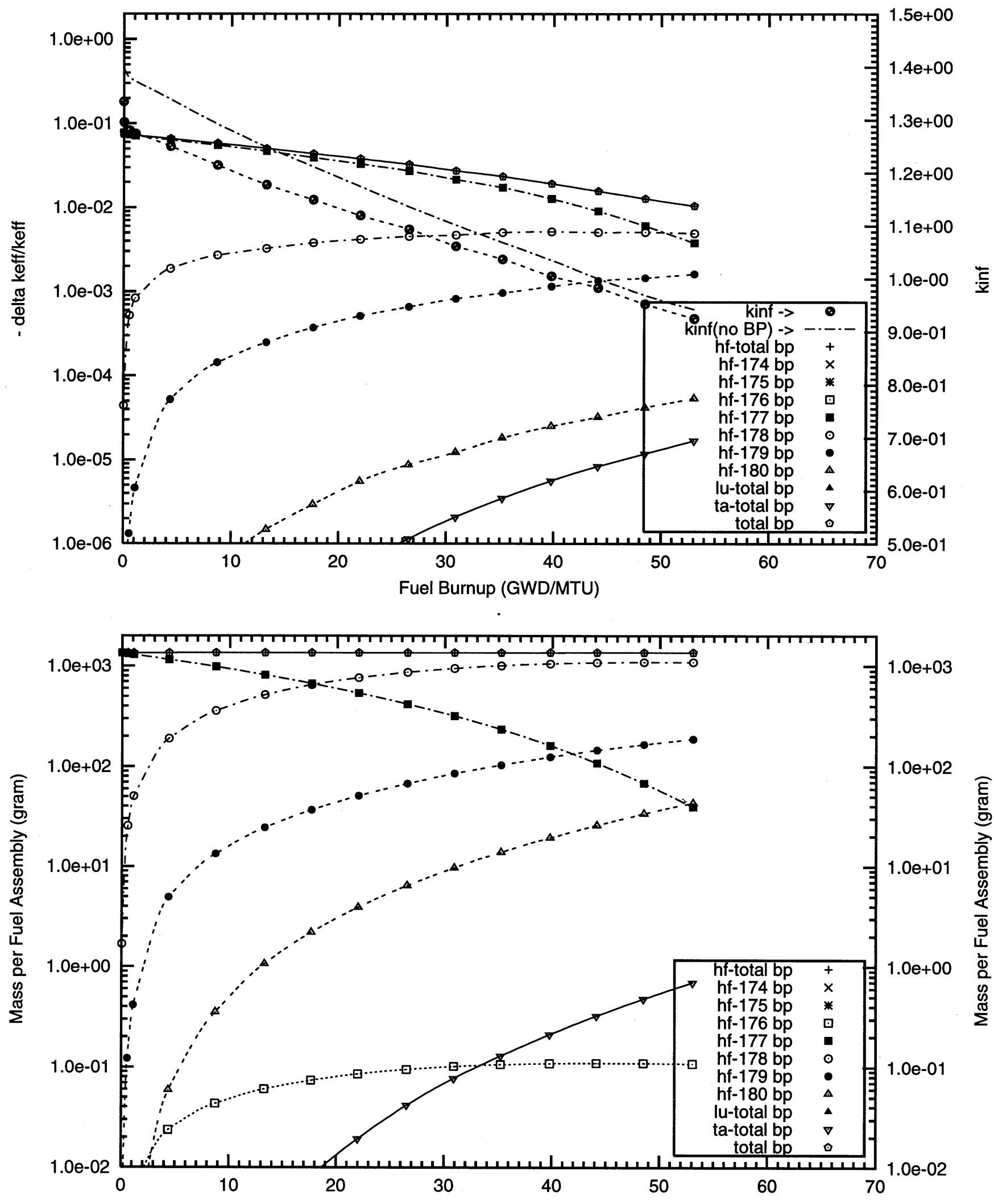

Fuel Burnup (GWD/MTU) 
Figure F3.10. Negative Reactivity and Masses BP for Hf-177 and transmutation daughters as a function of fuel life for $17 \times 17$ fuel assemblies with 64 poison rods,

0.5 mill thick $\mathrm{HfO} 2$ poison coating on the outer radial surface of the $\mathrm{UO} 2$ pellets Reactor power $3400 \mathrm{MWth}, 193$ fuel assemblies, initial enrichment 4.5w\%U-235

(Case : hf177_of_64_bp_fuel o)
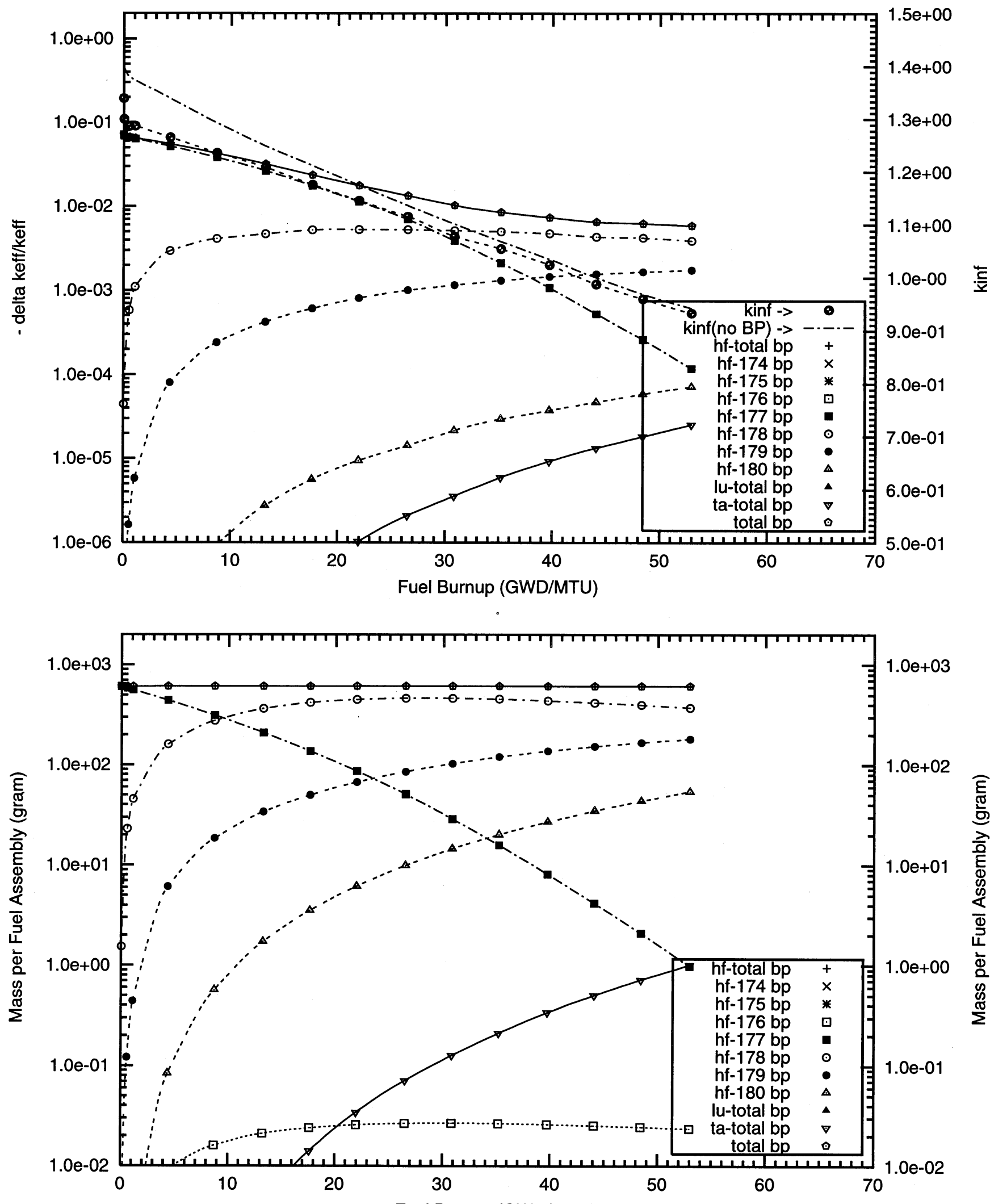

Fuel Burnup (GWD/MTU) 
Figure F3.11. Negative Reactivity and Masses BP for Hf-177 and transmutation daughters as a function of fuel life for $17 \times 17$ fuel assemblies with 64 poison rods,

1.0 mill thick $\mathrm{HfO} 2$ poison coating on the outer radial surface of the $\mathrm{UO} 2$ pellets Reactor power $3400 \mathrm{MWth}, 193$ fuel assemblies, initial enrichment 4.5W\%U-235

(Case : hf177_1f_64_bp_fuel o)
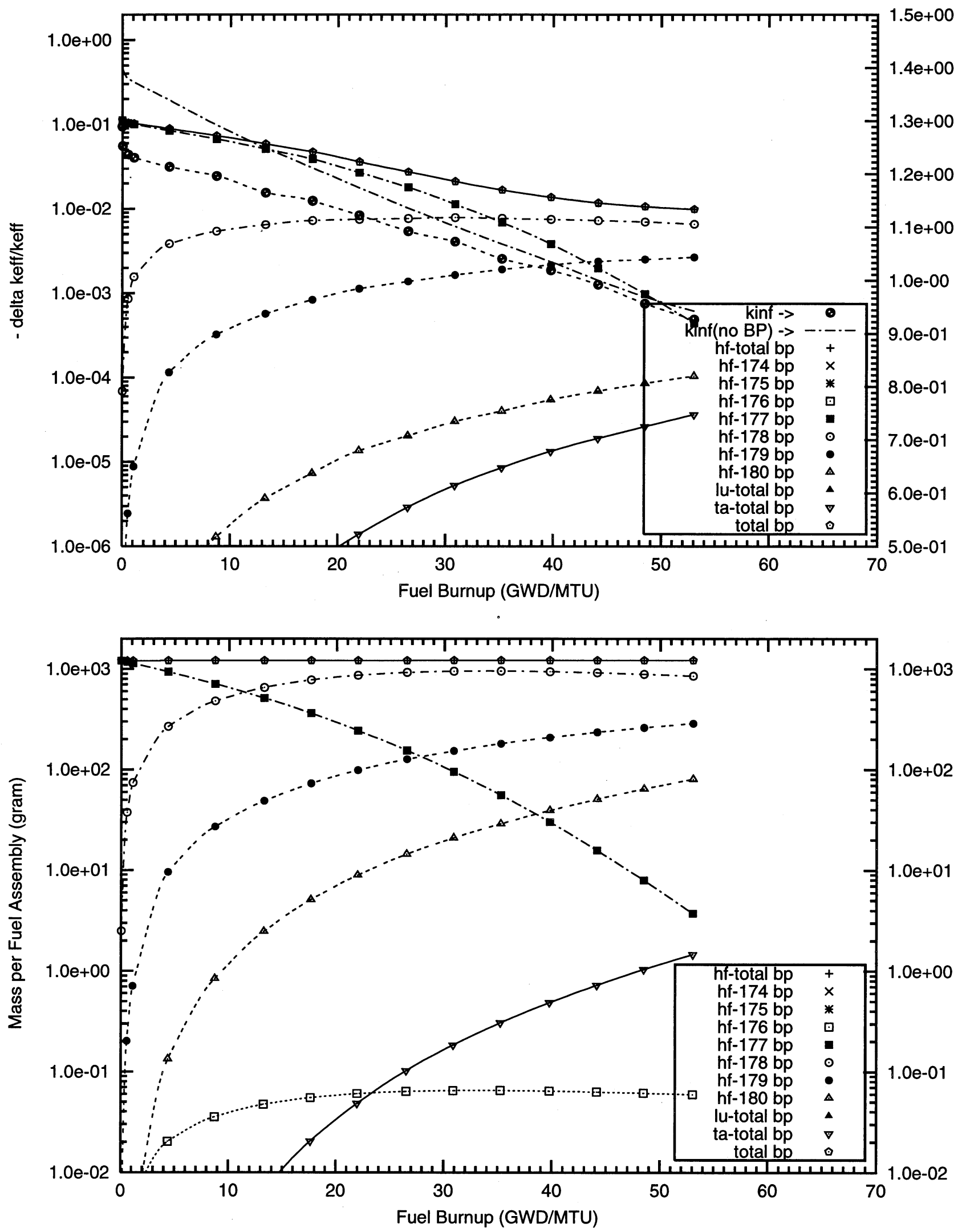
Figure F3.12. Negative Reactivity and Masses BP for Hf-177 and transmutation daughters as a function of fuel life for $17 \times 17$ fuel assemblies with 104 poison rods,

0.2 mill thick $\mathrm{HfO} 2$ poison coating on the outer radial surface of the $\mathrm{UO} 2$ pellets Reactor power $3400 \mathrm{MWth}, 193$ fuel assemblies, initial enrichment 4.5w\%U-235

(Case : hf177_8f_100p_h2o_104_bp_fuel_2_o)
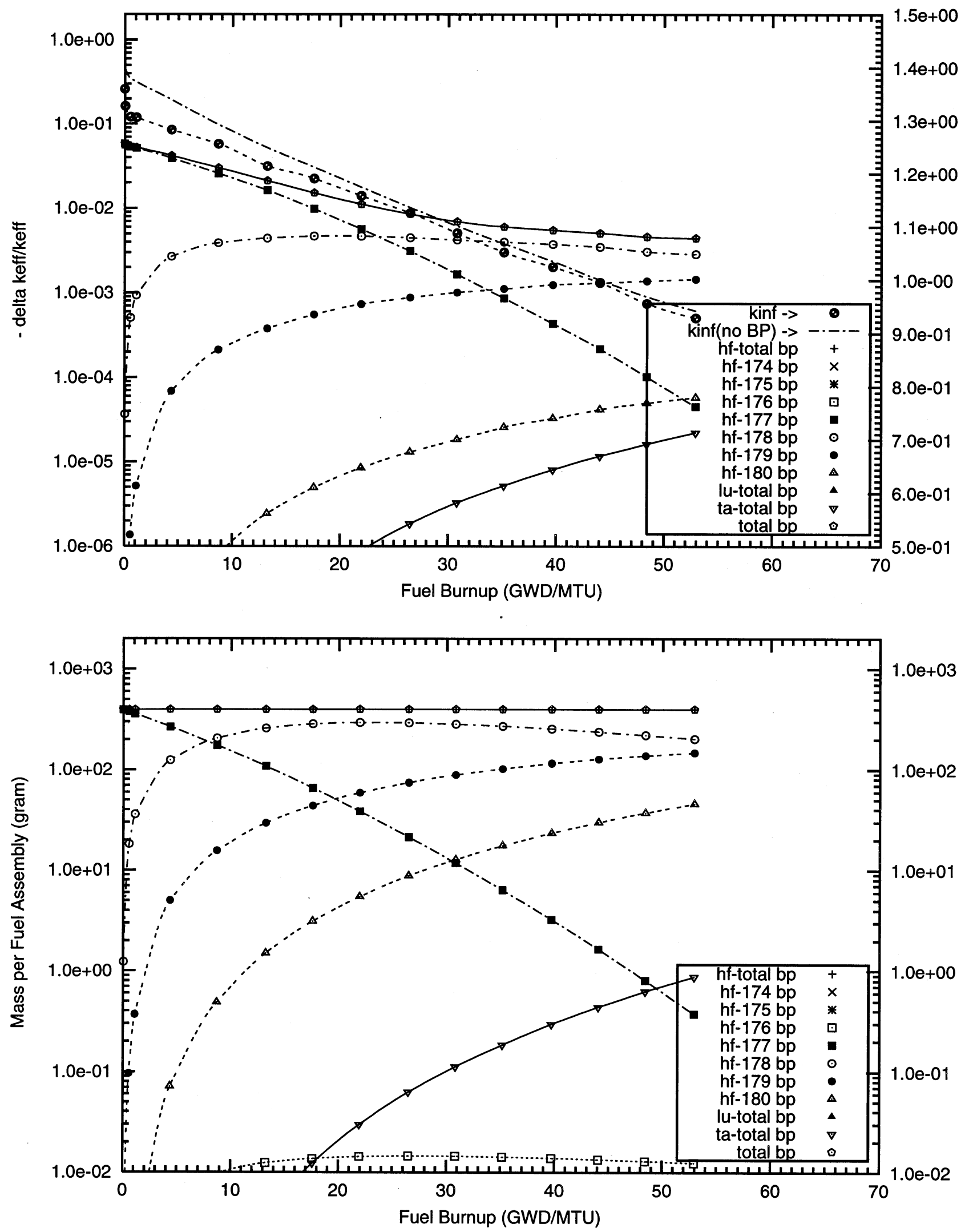
Figure F3.13. Negative Reactivity and Masses BP for Hf-177 and transmutation daughters

as a function of fuel life for $17 \times 17$ fuel assemblies with 104 poison rods,

0.5 mill thick $\mathrm{HfO} 2$ poison coating on the outer radial surface of the $\mathrm{UO} 2$ pellets

Reactor power $3400 \mathrm{MWth}, 193$ fuel assemblies, initial enrichment $4.5 \mathrm{w} \% \mathrm{U}-235$

(Case : hf177_of_104_bp_fuel o)
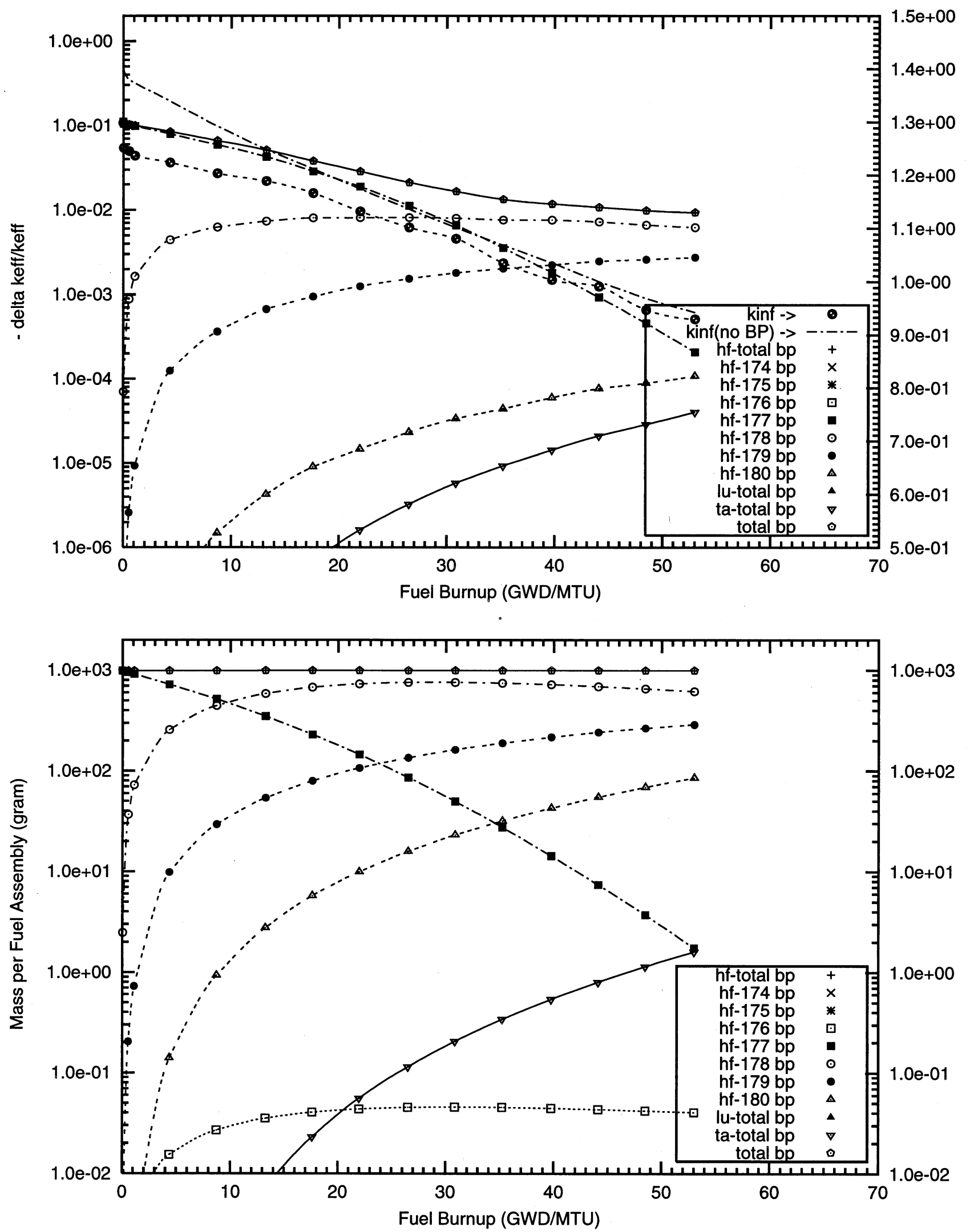
Appendix F4

Reactivity Worths and Masses of Hafnium Alloyed with the Zircaloy Cladding of Fuel Rods

\section{Table 28 Cases}



F4 - Hafnium with Natural Isotopic Abundances 
Figure F4.1. Negative Reactivity and Masses BP for Hf-nat and transmutation daughters as a function of fuel life for $17 \times 17$ fuel assemblies with 64 poison rods,

$1.0 \mathrm{w} \% \mathrm{Hf}$ poison homogeneously mixed in the fuel cladding,

Reactor power 3400 MWth, 193 fuel assemblies, initial enrichment $4.5 \mathrm{w} \% \mathrm{U}-235$

(Case:hf000_1c_100p_h2o_64_bp_fuel_2_o)
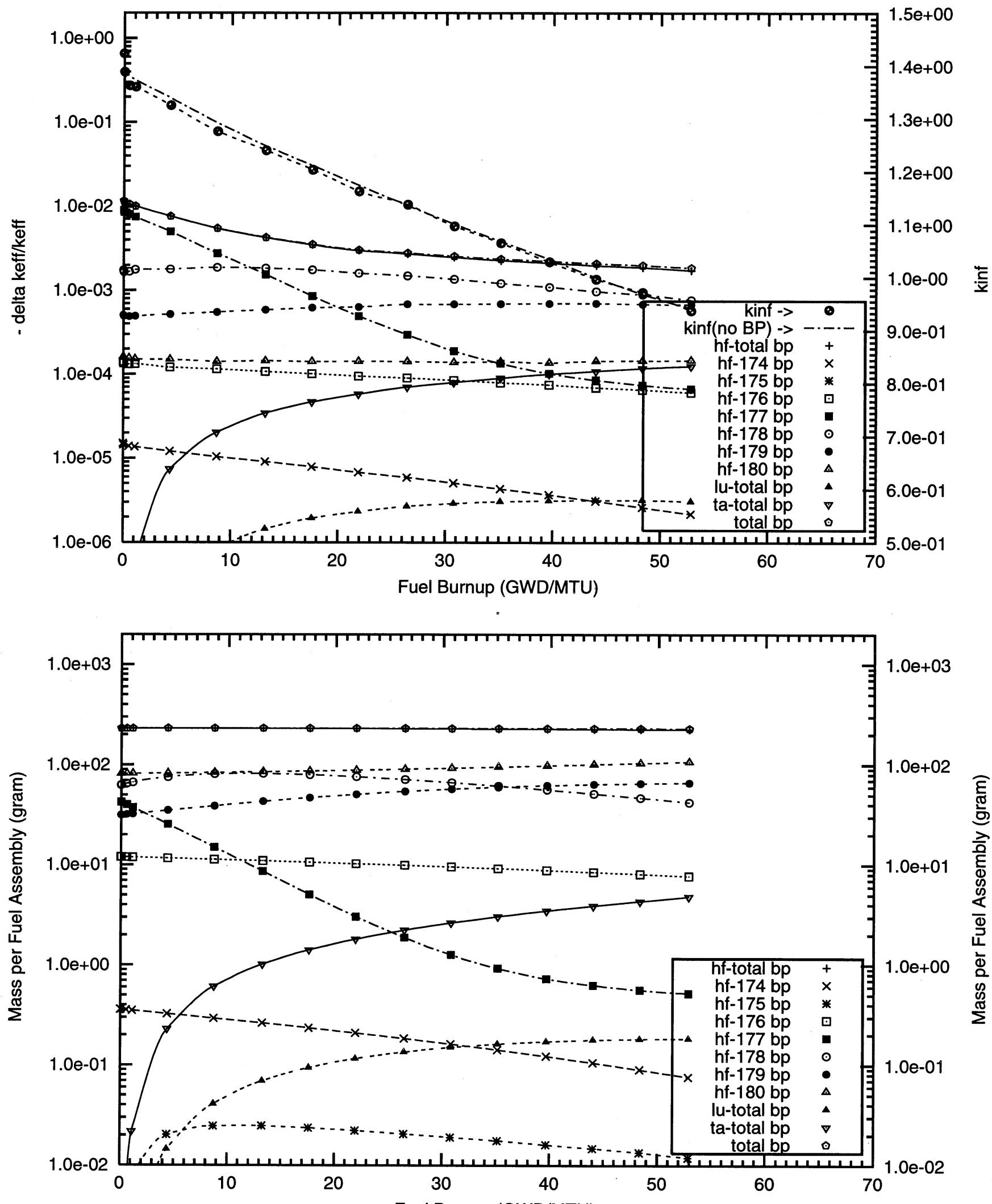

Fuel Burnup (GWD/MTU) 
Figure F4.2. Negative Reactivity and Masses BP for Hf-nat and transmutation daughters as a function of fuel life for $17 \times 17$ fuel assemblies with 104 poison rods, $1.0 \mathrm{w} \% \mathrm{Hf}$ poison homogeneously mixed in the fuel cladding,

Reactor power $3400 \mathrm{MWth}, 193$ fuel assemblies, initial enrichment 4.5w\%U-235 (Case : hf000_1c_100p_h20_104_bp_fuel_2_o)
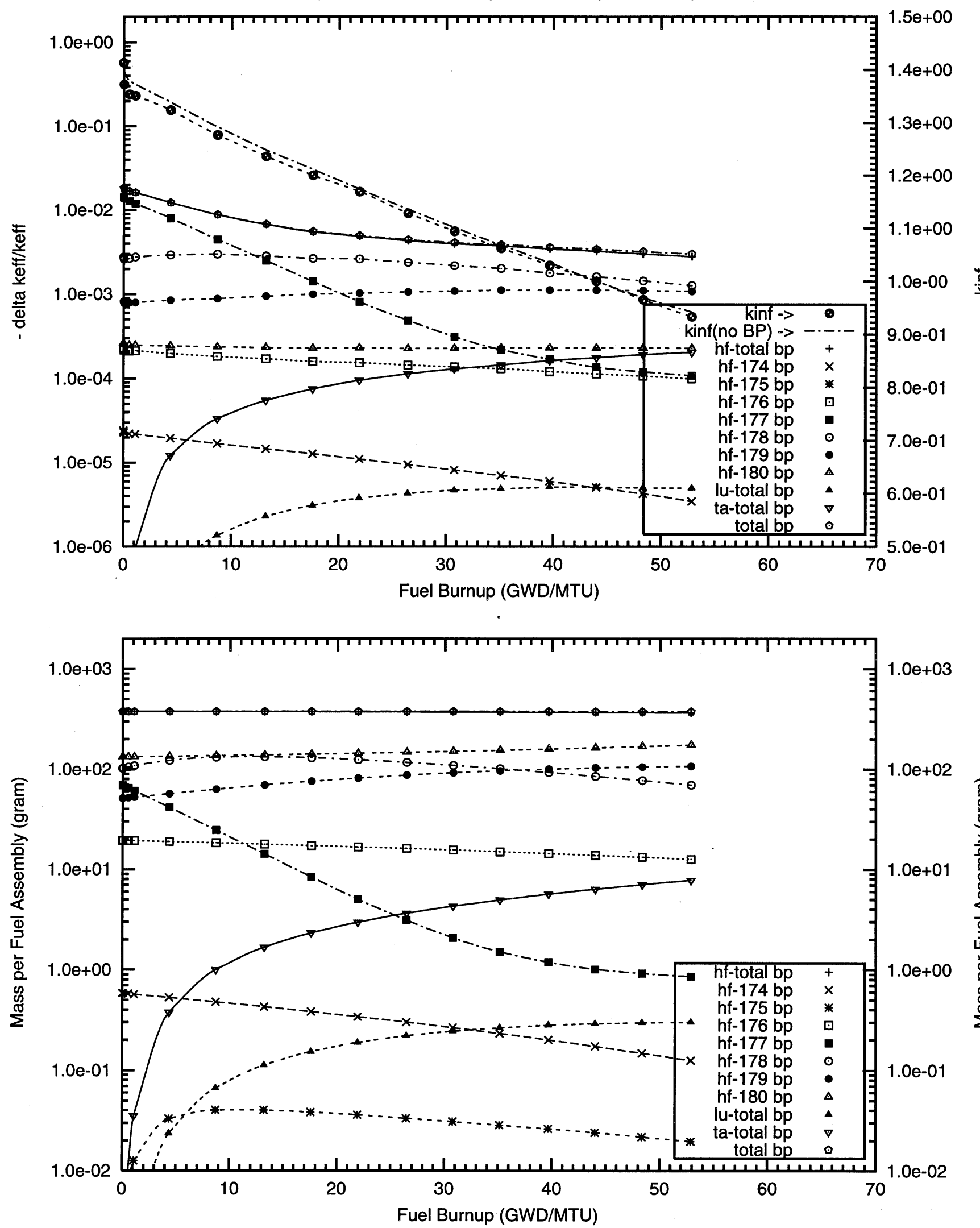
Figure F4.3. Negative Reactivity and Masses BP for Hf-nat and transmutation daughters as a function of fuel life for $17 \times 17$ fuel assemblies with 104 poison rods,

$4.0 \mathrm{w} \% \mathrm{Hf}$ poison homogeneously mixed in the fuel cladding,

Reactor power $3400 \mathrm{MWth}, 193$ fuel assemblies, initial enrichment $4.5 \mathrm{w} \% \mathrm{U}-235$

(Case : hf000_3c_100p_h20_104_bp_fuel_2_o)
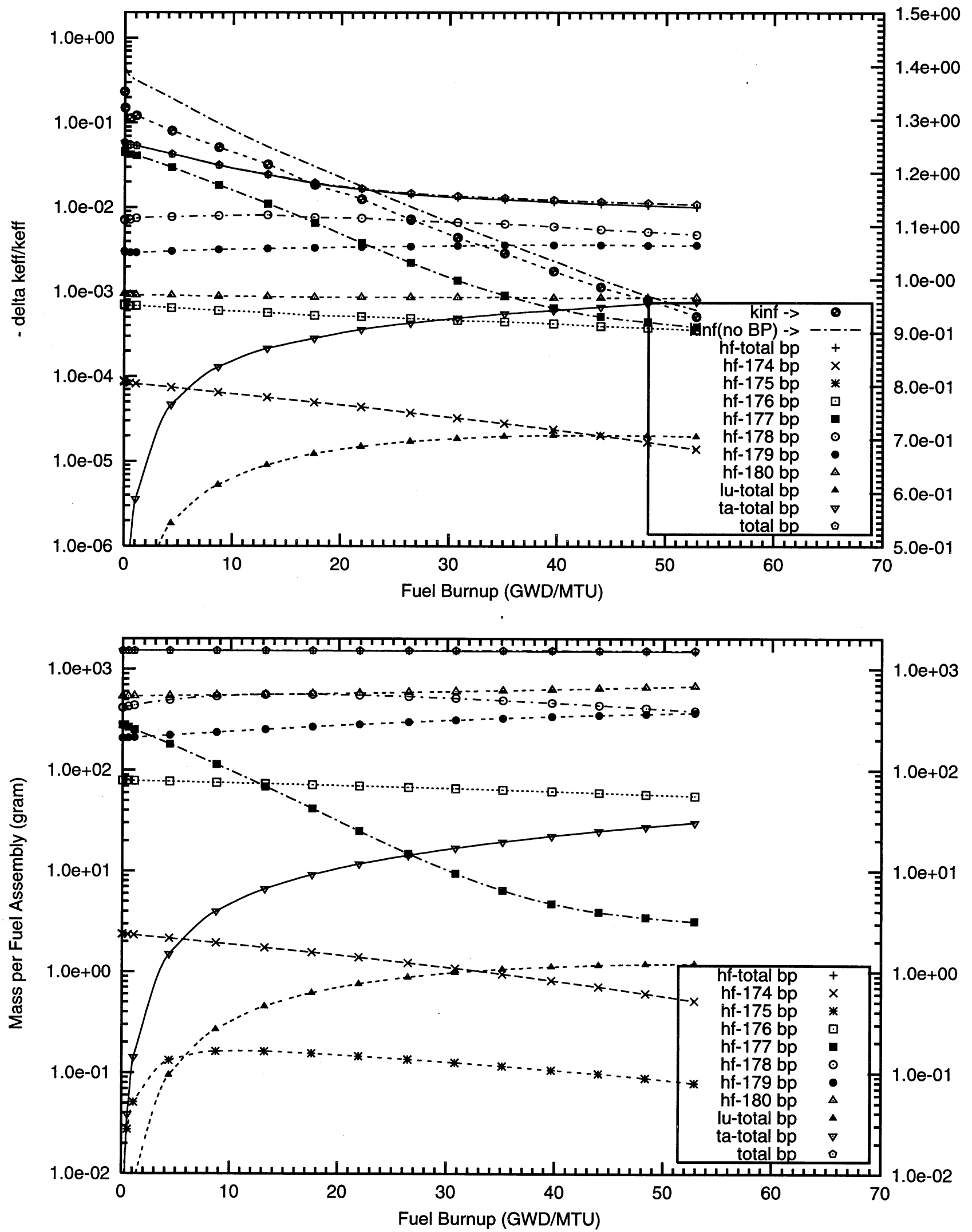

F4 - Hafnium Fully Enriched in ${ }^{177} \mathrm{Hf}$ 

Figure F4.4. Negative Reactivity and Masses BP for Hf-177 and transmutation daughters as a function of fuel life for $17 \times 17$ fuel assemblies with 64 poison rods,

$1.0 \mathrm{w} \% \mathrm{Hf}$ poison homogeneously mixed in the fuel cladding.

Reactor power $3400 \mathrm{MWth}, 193$ fuel assemblies, initial enrichment $4.5 \mathrm{w} \% \mathrm{U}-235$

(Case : hf177_1c_64_bp_fuel o)
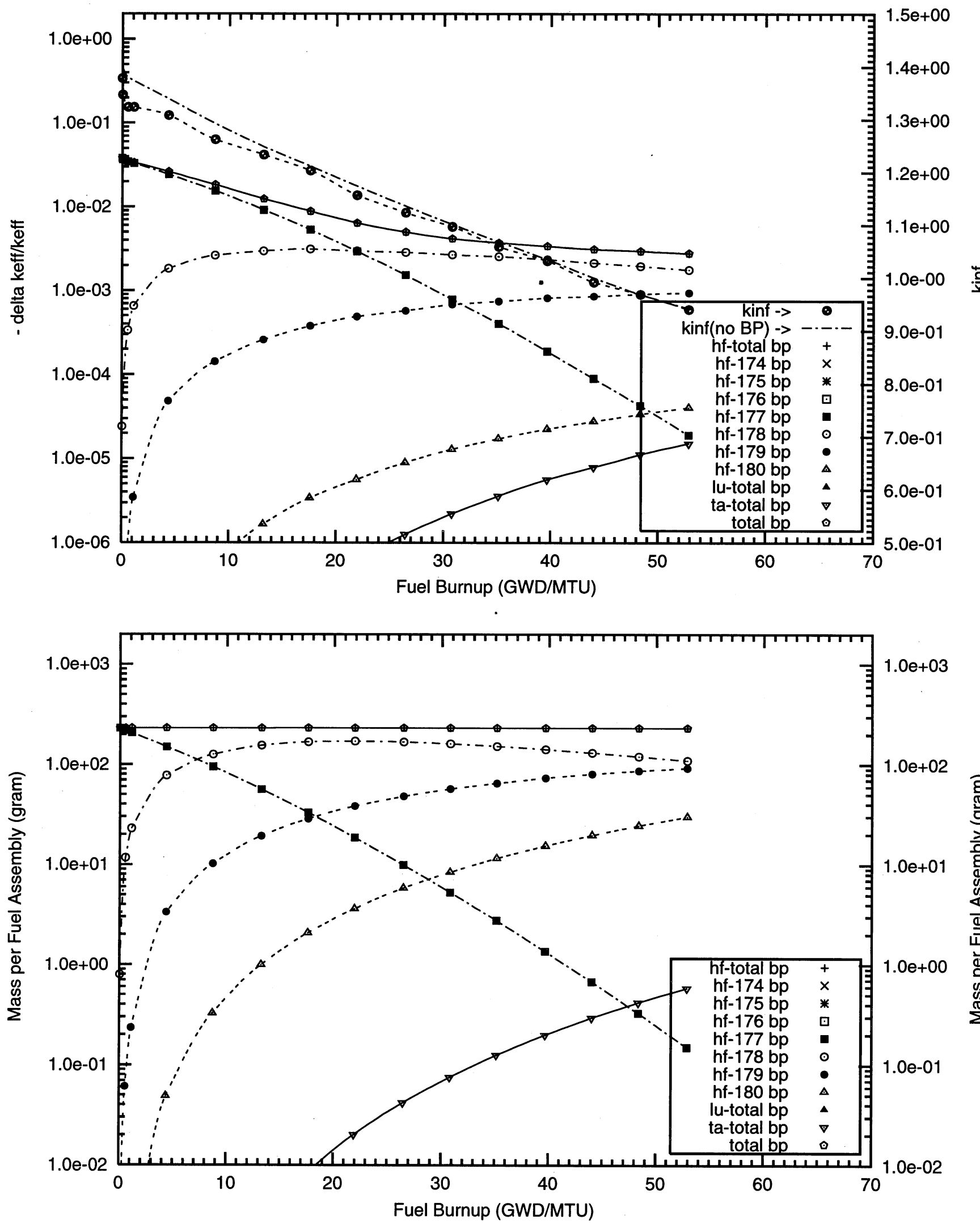
Figure F4.5. Negative Reactivity and Masses BP for Hf-177 and transmutation daughters as a function of fuel life for $17 \times 17$ fuel assemblies with 104 poison rods,

$0.5 \mathrm{w} \% \mathrm{Hf}$ poison homogeneously mixed in the fuel cladding,

Reactor power $3400 \mathrm{MWth}, 193$ fuel assemblies, initial enrichment $4.5 \mathrm{w} \% \mathrm{U}-235$

(Case : hf177_0c_104_bp_fuel o)
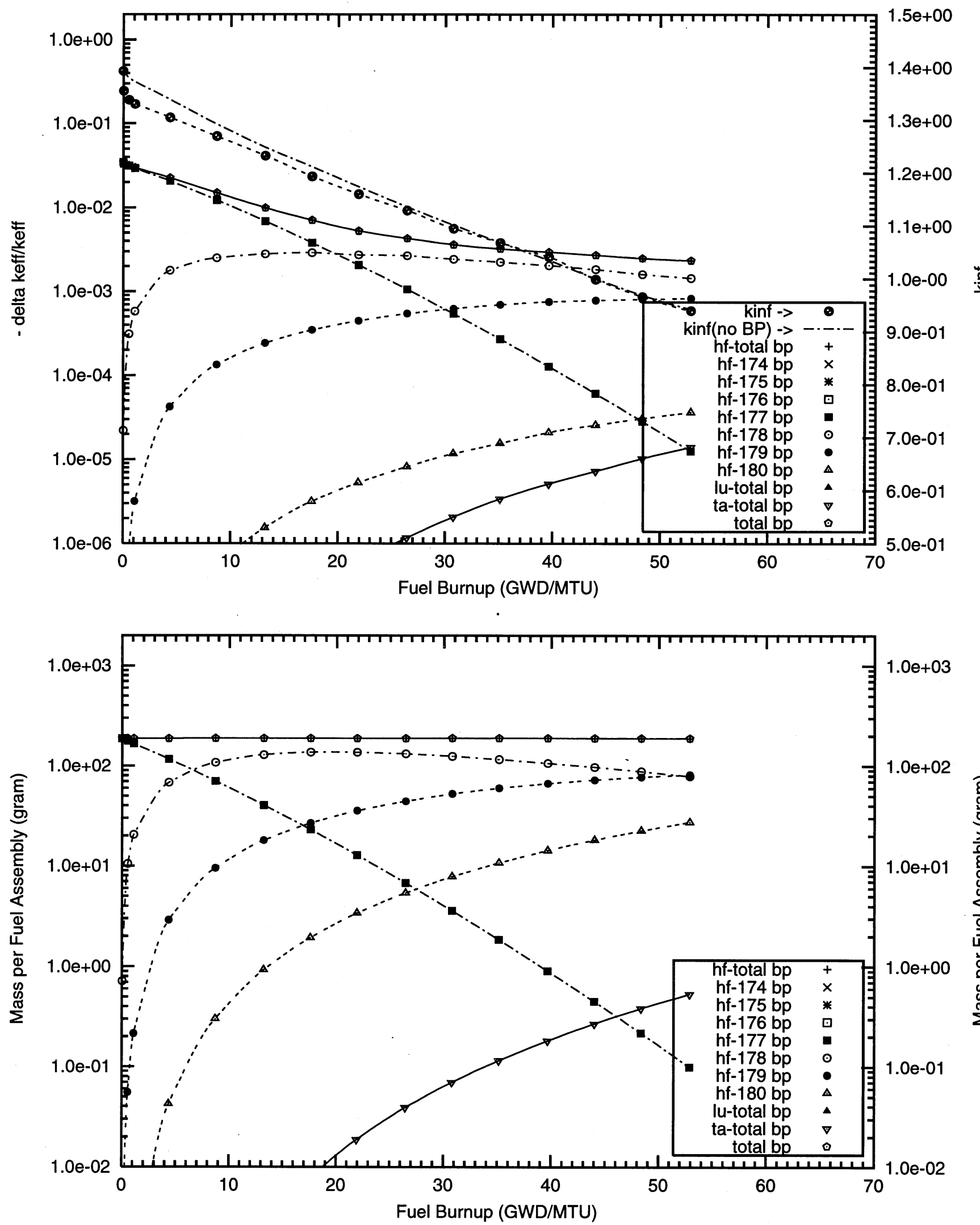
Figure F4.6. Negative Reactivity and Masses BP for Hf-177 and transmutation daughters as a function of fuel life for $17 \times 17$ fuel assemblies with 104 poison rods,

$1.0 \mathrm{w} \% \mathrm{Hf}$ poison homogeneously mixed in the fuel cladding,

Reactor power $3400 \mathrm{MWth}, 193$ fuel assemblies, initial enrichment $4.5 \mathrm{w} \% \mathrm{U}-235$

(Case : hf177_1c_100p_h20_104_bp_fuel_2_o)
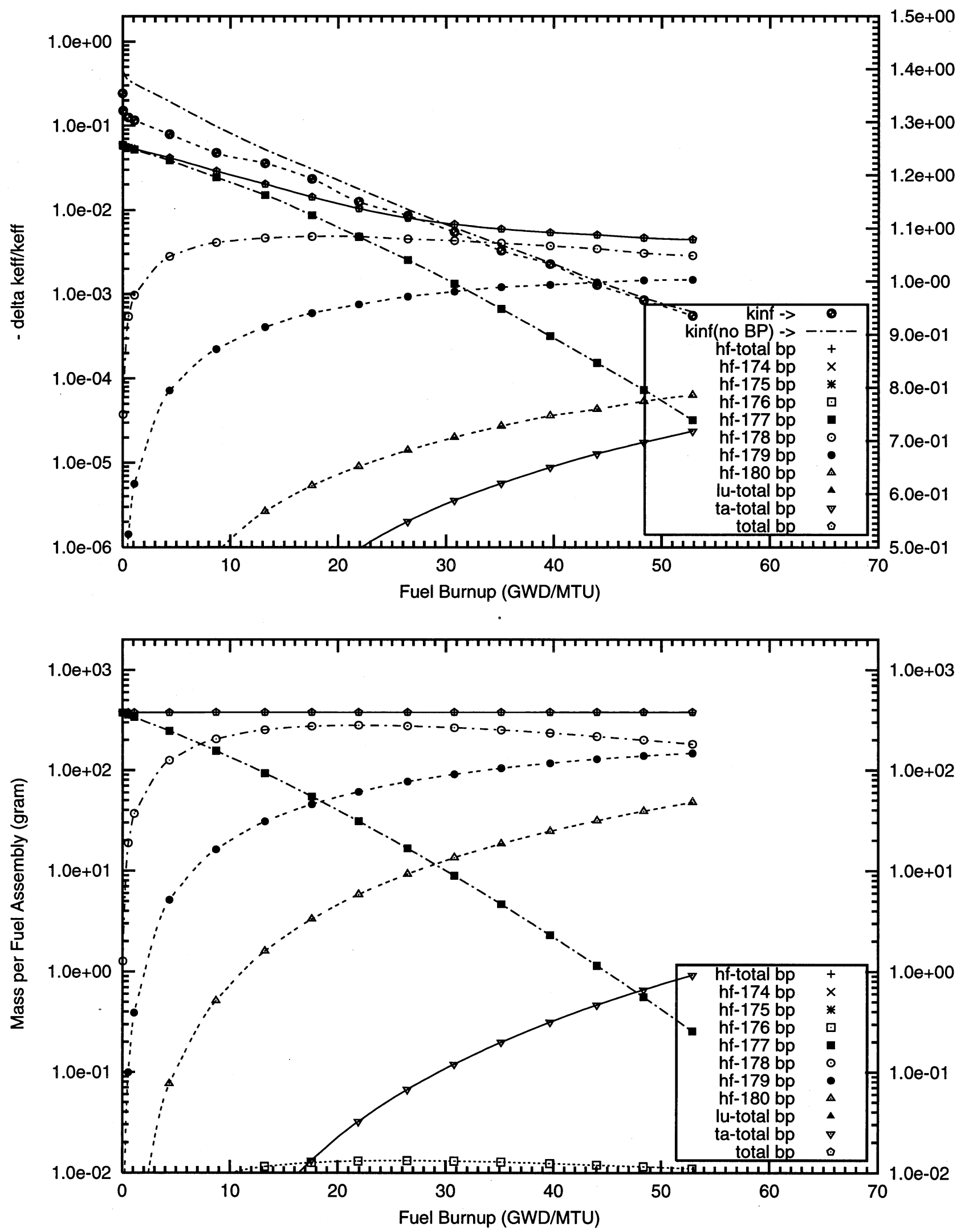
Figure F4.7. Negative Reactivity and Masses BP for $\mathrm{Hf}-177$ and transmutation daughters as a function of fuel life for $17 \times 17$ fuel assemblies with 104 poison rods,

$2.0 \mathrm{w} \% \mathrm{Hf}$ poison homogeneously mixed in the fuel cladding,

Reactor power $3400 \mathrm{MWth}, 193$ fuel assemblies, initial enrichment $4.5 \mathrm{w} \% \mathrm{U}-235$ (Case : hf177_2c_104_bp_fuel o)
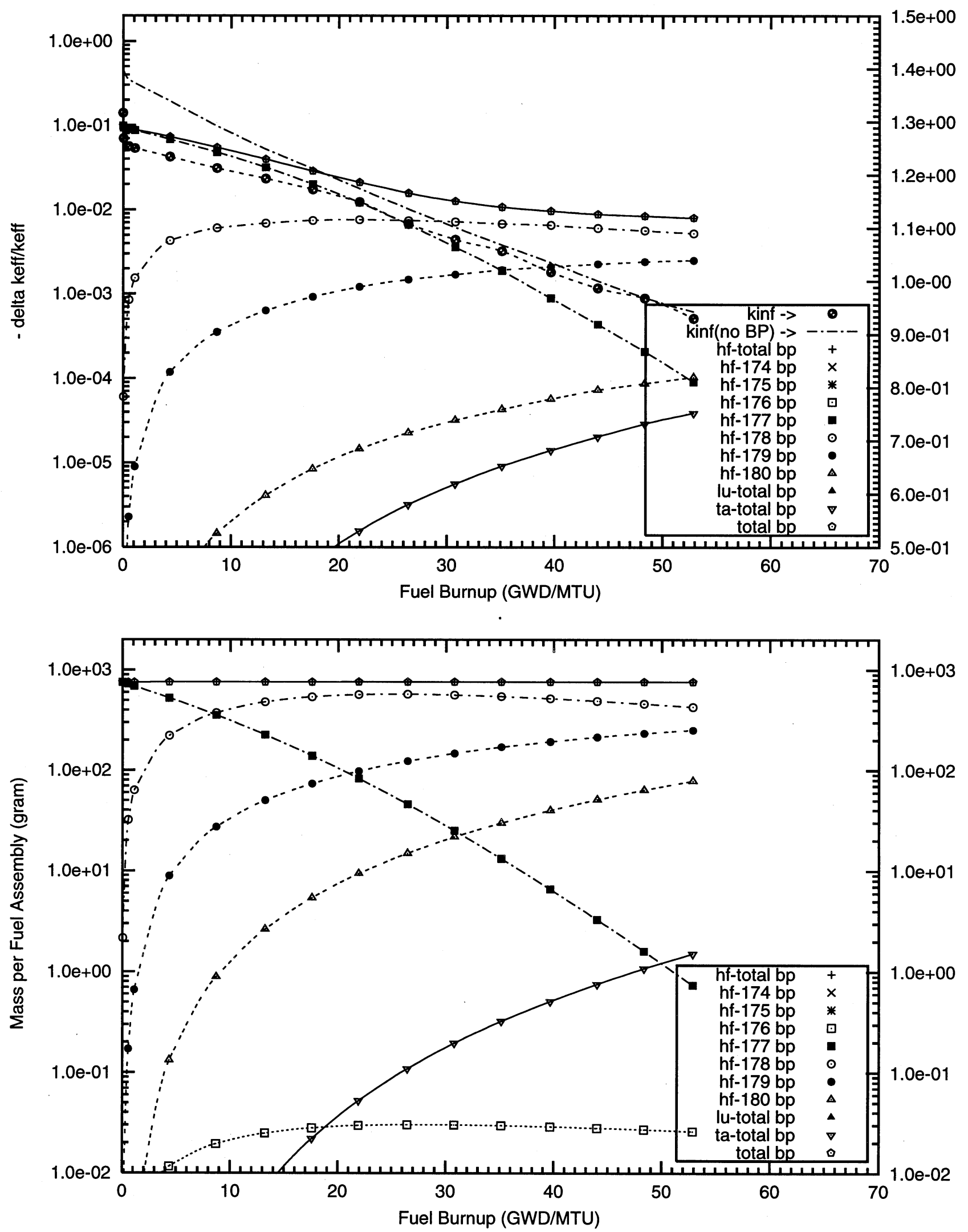


\title{
Appendix G1
}

Reactivity Worths and Masses of $\mathrm{Lu}_{2} \mathrm{O}_{3}$ Coating on the Surface of the Fuel Pellets

\author{
Table 29 Cases
}



G1 - Lutetium with Natural Isotopic Abundances 

Figure G1.1. Negative Reactivity and Masses BP for Lu-nat and transmutation daughters as a function of fuel life for $17 \times 17$ fuel assemblies with 104 poison rods,

1.0 mill thick Lu2O3 poison coating on the outer radial surface of the UO2 pellets

Reactor power $3400 \mathrm{MWth}, 193$ fuel assemblies, initial enrichment 4.5wt\%U-235 (Case : lu000_.1f_104_bp_fuel o)
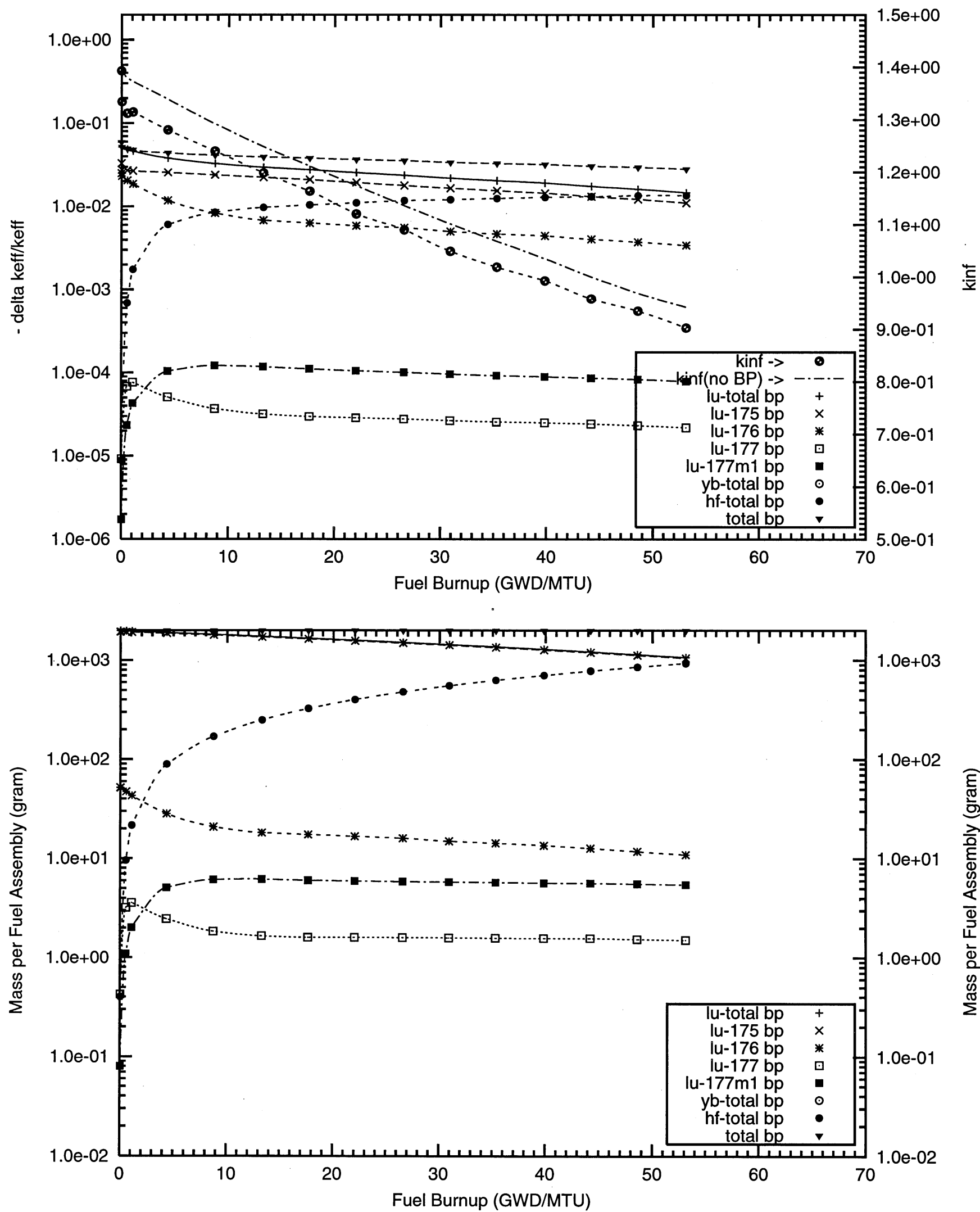
Figure G1.2. Negative Reactivity and Masses BP for Lu-nat and transmutation daughters as a function of fuel life for $17 \times 17$ fuel assemblies with 64 poison rods,

4.0 mill thick Lu2O3 poison coating on the outer radial surface of the UO2 pellets

Reactor power $3400 \mathrm{MWth}, 193$ fuel assemblies, initial enrichment 4.5w\%U-235

(Case : lu000_3f_64_bp_fuel o)
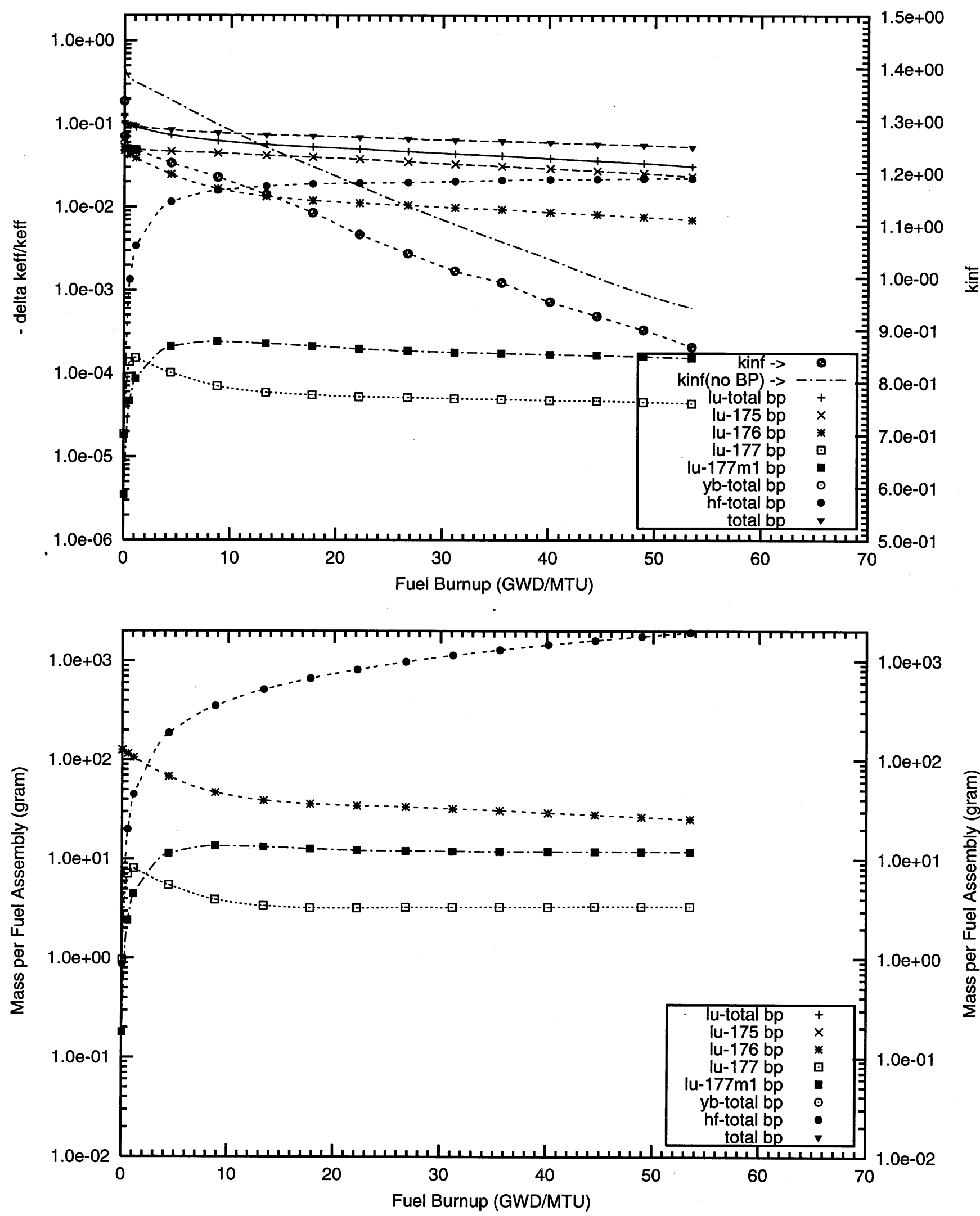
G1 - Lutetium Fully Enriched in ${ }^{176} \mathrm{Lu}$ 

Figure G1.3. Negative Reactivity and Masses BP for Lu-176 and transmutation daughters as a function of fuel life for $17 \times 17$ fuel assemblies with 64 poison rods,

0.5 mill thick Lu2O3 poison coating on the outer radial surface of the UO2 pellets

Reactor power 3400 MWth, 193 fuel assemblies, initial enrichment 4.5w\%U-235

(Case : lu176_0f_64_bp_fuel o)
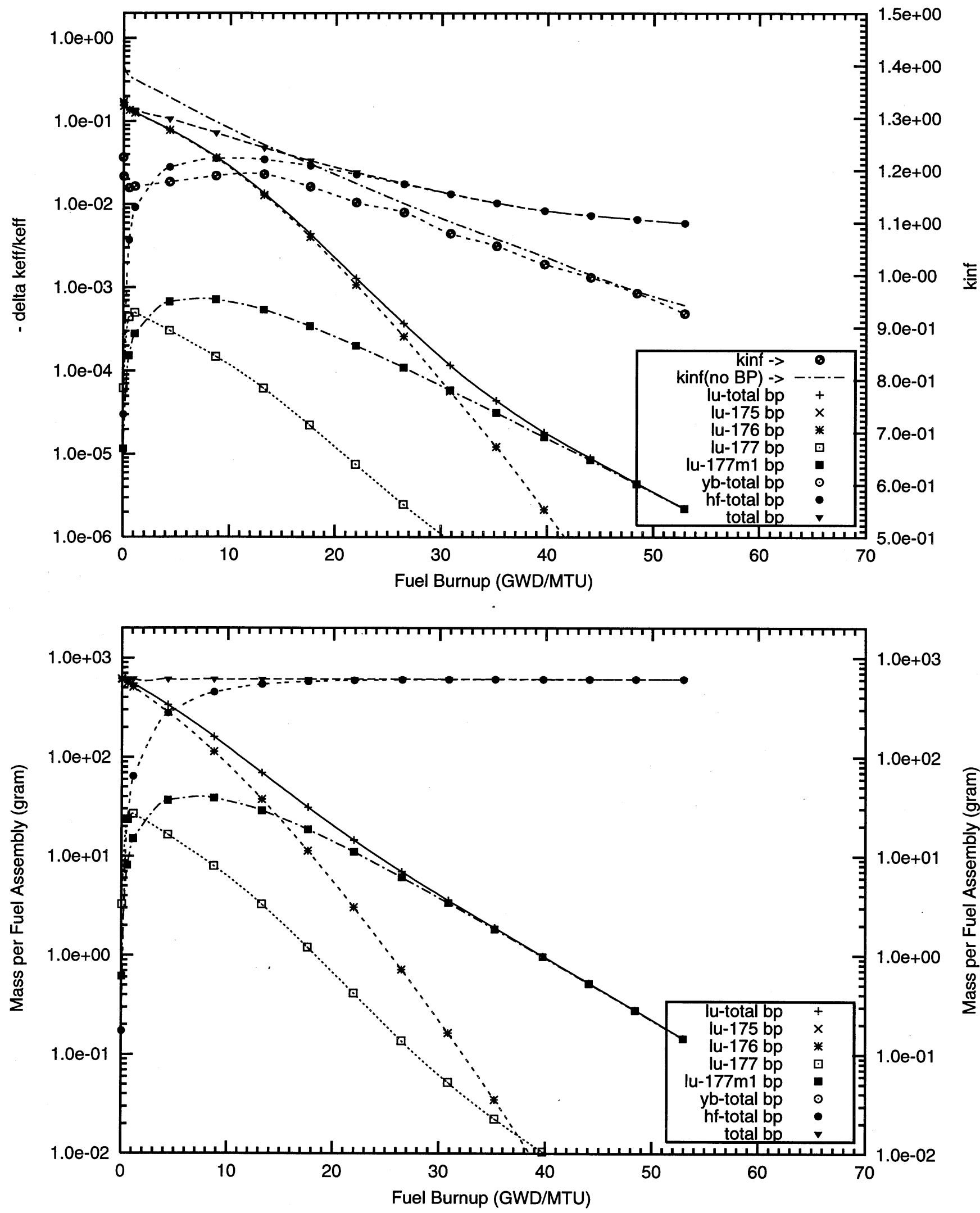
Figure G1.4. Negative Reactivity and Masses BP for Lu-176 and transmutation daughters as a function of fuel life for $17 \times 17$ fuel assemblies with 64 poison rods,

1.0 mill thick Lu2O3 poison coating on the outer radial surface of the UO2 pellets

Reactor power $3400 \mathrm{MWth}, 193$ fuel assemblies, initial enrichment 4.5w\%U-235

(Case : lu176_1f_64_bp_fuel o)
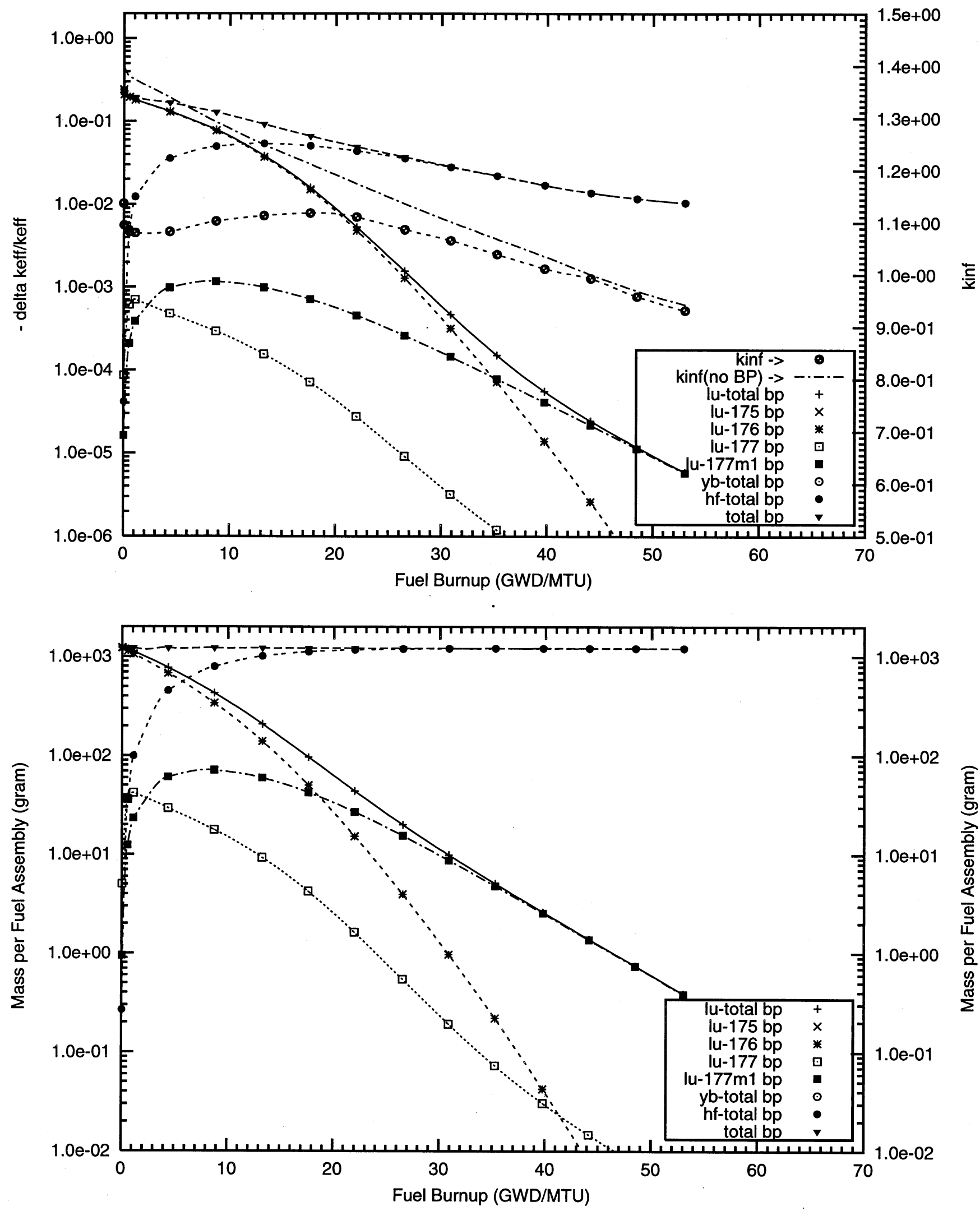
Figure G1.5. Negative Reactivity and Masses BP for Lu-176 and transmutation daughters as a function of fuel life for $17 \times 17$ fuel assemblies with 104 poison rods,

0.2 mill thick Lu2O3 poison coating on the outer radial surface of the UO2 pellets

Reactor power 3400 MWth, 193 fuel assemblies, initial enrichment 4.5w\%U-235

(Case : lu176_8f_104_bp_fuel o)
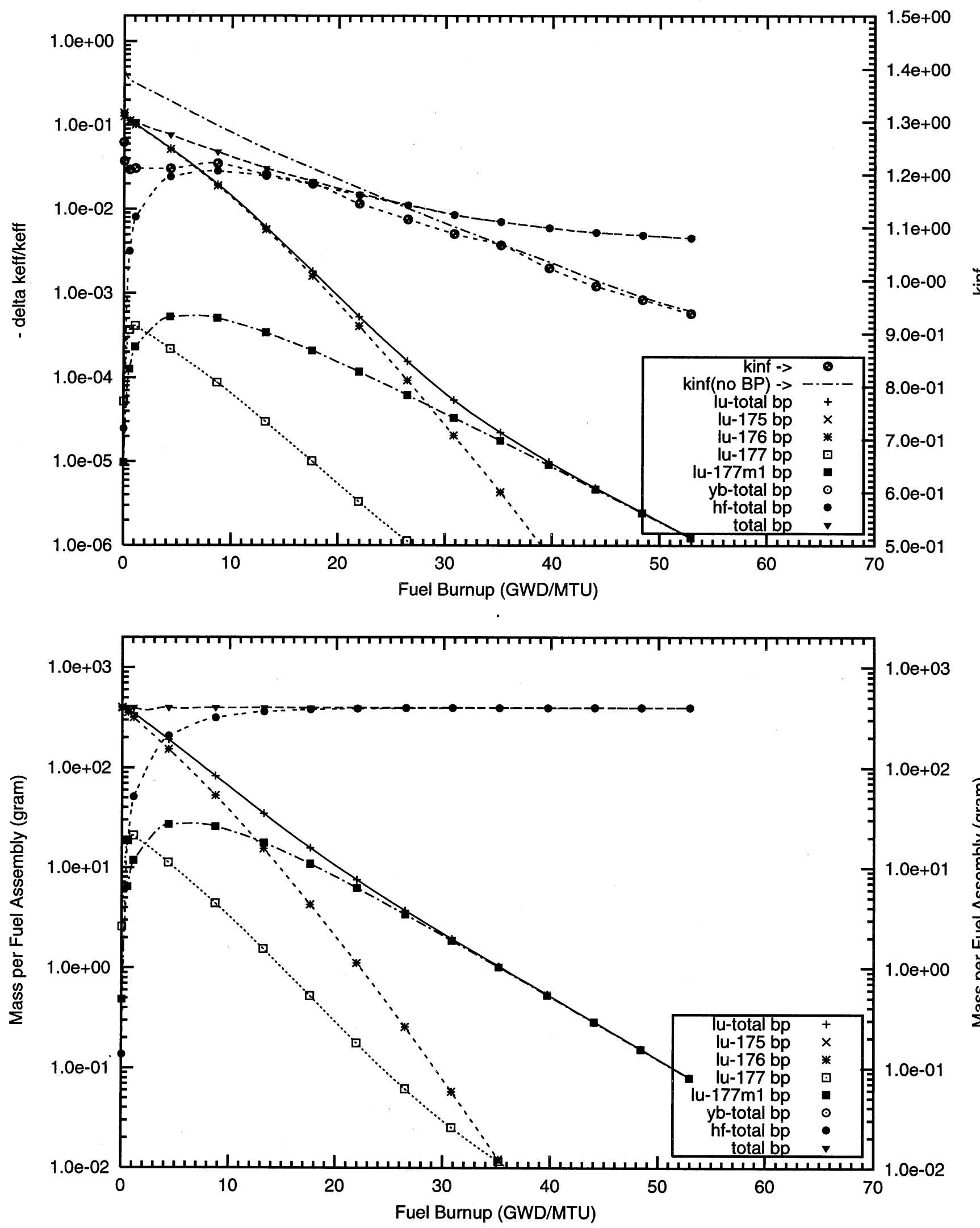
